\title{
The 45th Annual Meeting of the European Society for Blood and Marrow Transplantation: Physicians - Poster Session
}

C Springer Nature Limited 2019

24-27 March 2019 Frankfurt, Germany

Copyright: Modified and published with permission from https://www.ebmt.org/annual-meeting

Sponsorship Statement: Publication of this supplement is sponsored by the European Society for Blood and Marrow Transplantation. All content was reviewed and approved by the EBMT Committee, which held full responsibility for the abstract selections.

Presenting author names are bold type in the contributor lists.

\section{Acute leukaemia}

P001

Low treatment related mortality and relapse leading to excellent outcomes after allogeneic transplantation for acute lymphoblastic leukaemia using a nonmyeloablative conditioning without T CELL depletion

Mary Lynn Savoie ${ }^{1,2}$, Jeff K Davies ${ }^{3,4}$, Jamie Cavenagh ${ }^{3,4}$, Heather Oakervee ${ }^{4}$, Jonathan Sive ${ }^{4}$, Matthew Smith ${ }^{4}$, Deborah Anderson ${ }^{4}$,John Gribben ${ }^{3,4}$, Bela Wrench ${ }^{3,4}$

${ }^{1}$ University of Calgary, Calgary, Canada, ${ }^{2}$ Tom Baker Cancer Centre, Calgary, Canada, ${ }^{3}$ Barts Cancer Institute, Queen Mary University of London, London, United Kingdom, ${ }^{4}$ St Bartholomew's Hospital, Barts Health NHS Trust, Barts Cancer Centre, London, United Kingdom

Background: Allogeneic hematopoietic stem cell transplantation is routinely offered to patients with high-risk or advanced ALL in the hopes of improving outcomes. Use of truly non-myeloablative (NMA) conditioning reduces toxicity in other contexts but outcome data for ALL patients after NMA transplants is lacking. We report the outcomes of 31 patients with ALL transplanted using a NMA conditioning without $\mathrm{T}$ cell depletion.

Methods: First transplant patients between October 2006 and June 2018 were reviewed. These were consecutive patients until 2015 then only those considered unfit for
FMC conditioning as per the UKALL 2014 protocol. All patients were conditioned with fludarabine $25 \mathrm{mg} / \mathrm{m}^{2} /$ day for 5 days and cyclophosphamide $1 \mathrm{~g} / \mathrm{m}^{2} /$ day for 2 days. Short course MTX and ciclosporin were used for GVHD prophylaxis. Standard supportive care was employed. Thirty-one patients with a median age of 43 (23-67) met the criteria for this case review. 30 had B-ALL and 10 were Philadelphia chromosome positive. 24 patients $(77 \%)$ had high risk disease by standard diagnostic criteria. $27(87 \%)$ were in first complete remission (CR1). Matched sibling donors were used in 13 instances with the remaining being fully matched unrelated donors. $58 \%$ of patients had a HCT-CI score of $0,32 \%$ a score of 1 or 2 with 3 patients having a score of 3 or higher. Median CD34 dose was $5.3 \mathrm{x}$ $10^{6} / \mathrm{kg}(0.93-34.12)$ with a median CD3 dose of $2.13 \times 10^{8} \%$ $\mathrm{kg}(0.12-7.37)$

Results: TRM was low at $7 \%$ at 1 year and $11 \%$ at 2 and 3 years respectively. No factors included in a univariate analysis (which included age, diagnosis, disease status, HCT-CI, donor type, CMV risk and cell dose) significantly impacted TRM. The incidence of classical acute (a) GVHD grade 2-4 and $3-4$ was $18 \%$ and $8 \%$ by day 100 and $29 \%$ and $13 \%$ by day 180 if late onset aGVHD is included. 24 out of 30 eligible patients developed chronic GVHD of any stage. Relapse incidence was low (22\% at 3 years in all patients, $17 \%$ in CR1 patients) and was not impacted by any pre-transplant factors including positive MRD post phase 2 induction (present in 6 patients). Notably, in univariate analysis relapse was significantly lower in patients who developed chronic GvHD. 
Event-Free Survival (EFS) and Overall Survival (OS) at 3 years were $70 \%$ and $72 \%$ respectively for the whole cohort and $73 \%$ and $76 \%$ respectively for patients transplanted in CR1. Univariate analysis for pre- and post-transplant factors impacting EFS and OS identified only chronic GvHD which was associated with significantly better EFS and OS.

Conclusions: In conclusion, non-myeloablative T-replete conditioning for ALL transplantation is associated with low TRM and relapse resulting in excellent outcomes (Fig 1). Although this approach is associated with a significant incidence of cGvHD, this was protective against disease relapse consistent with a concomitant and sustained immunological Graft-versus-Leukemia effect.
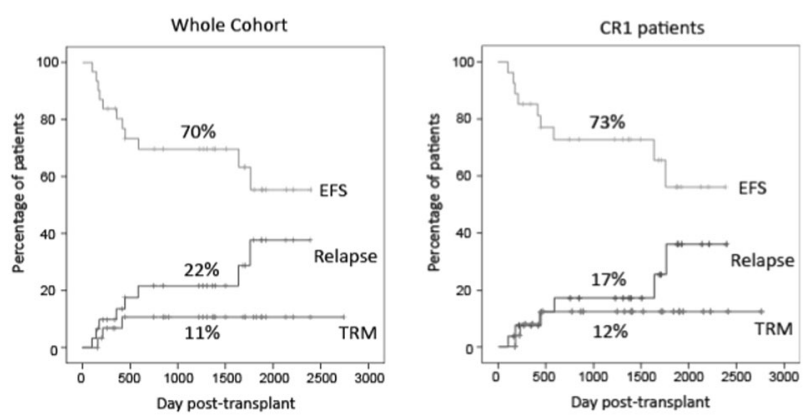

[[P001 Image] 1. Figure 1]

Disclosure: Nothing to declare

\section{P002}

clinical relevance of in vitro generation of dendritic cells of leukemic origin to predict response to immunotherapy in patients with AML and MDS

Markus Freudenreich ${ }^{1}$, Johanna Tischer ${ }^{1}$, Tanja Kroell, Andreas Kremser ${ }^{I}$, Julia Dreyssig ${ }^{1}$, Christine

Grabrucker $^{1}$, Anja Liepert ${ }^{1}$, Hans Jochem Kolb ${ }^{1}$, Christoph Schmid ${ }^{2}$, Helga Schmetzer ${ }^{1,3}$

${ }^{1}$ University Hospital Grosshadern Ludwigs-MaximiliansUniversity, Munich, Germany, ${ }^{2}$ Augsburg University Hospital, Augsburg, Germany, ${ }^{3}$ Helmholtz Center Munich, German Research Center for Environmental Health/Clinical Cooperative Group Haematopoetic Cell Transplantation (CCG-HCT), Munich, Germany

Background: Allogeneic stem cell transplantation (alloSCT) is the treatment of choice for many patients (pts) suffering from acute myeloid leukemia (AML). The graft vs. leukemia effect (GvL), applied by immunocompetent cells of donor origin, is the most important effector mechanism for the eradication of leukemia, The presentation of leukemic or allospecific antigens by malignant blasts is regarded as a crucial trigger for an effective allogeneic immune response. Conversely, insufficient stimulatory capacity by the leukemic blasts is thought to be a relevant escape mechanism from cellular immunotherapy (alloSCT or donor-lymphocyte infusion (DLI)).

The purpose was to test, whether the ability of malignant blasts to differentiate in vitro towards dendritic cells of leukemic origin $\left(\mathrm{DC}_{\text {leu }}\right)$ is associated with response to alloSCT or outcome after immunotherapy (second alloSCT or DLI) for post-transplant relapse in AML.

Methods: Leukemic blasts were isolated from peripheral blood (PB) or bone marrow (BM) samples of AML patients before alloSCT $(n=47)$ or at relapse after alloSCT $(n=22)$. A panel of 6 different assays was used to generate $\mathrm{DC}_{\text {leu }}$ in vitro ( 5 of them containing GM-CSF). Finally, in vitro results were correlated with clinical characteristics and outcome of patients treated with donor lymphocyte infusion and/or alloSCT.

Results: $\mathrm{DC}_{\text {leu }}$ could be generated in vitro from all 69 samples. When correlating proportions of DC-subtypes generated ex vivo with clinical data, significantly higher mean proportions of $\mathrm{DC}_{\text {leu }}$ in the $\mathrm{DC}$-fraction were found in responders vs. non-responders to immunotherapy (76.8\% vs $58.8 \%, \mathrm{p}=0.006$, range: $13 \%-99 \%)$. Vice versa, the chance for response to immunotherapy was significantly higher, if a $\mathrm{DC}_{\text {leu }} / \mathrm{DC}$ ratio of $>=50 \%$ could be reached in vivo $(\mathrm{p}=0.004)$. Those patientswere characterized by a longer time to relapse $(p=0.04)$ and by a higher probability for leukemia-free survival $(\mathrm{p}=0.005)$.

Similarly, generation of higher amounts $(>8 \%, \mathrm{p}=0.04)$ of $\mathrm{DC}_{\text {leu }}$ in the MNC-fraction, and generation of more mature DC ( $>47 \% \mathrm{CD} 83+, \mathrm{p}=0.03$ using the best GM-CSF containing assay) were associated with a longer time to relapse in the respective patients. Moreover, overall survival was improved, if $>70 \% \mathrm{DC}_{\text {leu }} / \mathrm{DC}$ could be generated with the best GM-CSF containing assay $(\mathrm{p}=0.048)$.

Conclusions: In vitro generation of $\mathrm{DC} / \mathrm{DC}_{\mathrm{leu}}$ from leukemic blasts obtained in active stages of AML before alloSCT or at relapse post transplant were associated with clinical outcome. This observation supports a role of antigen presentation by leukemic cells for an allogeneic immune response in AML.

Disclosure: Nothing to declare

\section{P003}

Abstract already published.

P004

Impact of consolidation by autologous HCT as compared to chemotherapy on toxicity of subsequent allogeneic HCT in CR2 in patients with AML

Jakob Passweg ${ }^{1}$, M, Labopin ${ }^{2}$, M. Christopeit ${ }^{3}, J$. Cornelissen $^{4}$, T. Pabst ${ }^{5}$, G. Socié ${ }^{6}$, N. Russell ${ }^{7}$, I. Yakoub- 
Agha $^{8}$, D. Blaise ${ }^{9}$, T. Gedde-Dahl ${ }^{10}$, H. Labussière-

Wallet $^{11}$, R. Malladi ${ }^{12}$, E. Forcade ${ }^{13}$, S. Maury ${ }^{14}$, E.

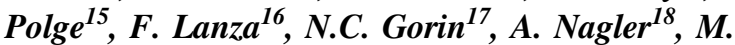
Mohty $^{17}$

${ }^{1}$ Universitätsspital Basel, Basel, Switzerland, ${ }^{2}$ Saint Antoine Hospital, EBMT ALWP Office, Paris, France, ${ }^{3}$ University Hospital Eppendorf, Hamburg, Germany, ${ }^{4}$ Erasmus MC Cancer Institute, University Medical Center Rotterdam, Rotterdam, Netherlands, ${ }^{5}$ University Hospital Bern, Clinic of Oncology, Bern, Switzerland, ${ }^{6}$ Hopital St. Louis, Paris, France, ${ }^{7}$ Nottingham University Hospital NHS Trust, Nottingham, United Kingdom, ${ }^{8} \mathrm{CHRU}$ de Lille, Lille, France, ${ }^{9}$ Institut Paoli Calmettes, Marseille, France, ${ }^{10}$ Oslo University Hospital, Rikshospitalet, Oslo, Norway, ${ }^{11}$ Centre Hospitalier Lyon Sud, Service Hematologie, Lyon, France, ${ }^{12}$ University Hospitals Birmingham, Birmingham, United Kingdom, ${ }^{13} \mathrm{CHU}$ Bordeaux, Pessac, France, ${ }^{14}$ Hôpital Henri Mondor, Creteil, France, ${ }^{15}$ Hôpital Saint Antoine, Paris, France, ${ }^{16}$ Ospedale Civile, Dipartimento di Ematologia, Medicina Trasfusionale e Biotecnologie, Unità Operativa di Ematologia, Ravenna, Italy, ${ }^{17}$ Hospital Saint Antoine, Paris, France, ${ }^{18}$ Chaim Sheba Medical Center, Tel Aviv University, Tel Hashomer, Israel

Background: The role of autologous hematopoetic cell transplantation (HCT) in the treatment of AML is not clear. Trials in the past have shown that autologous HCT consolidation lowers the risk of relapse, however the magnitude of this effect is limited. Autologous HCT is advocated in patients with AML with lower genetic risk in CR1.Many of these patients will eventually relapse and will undergo reinduction followed by allogeneic HCT in CR2.

Methods: The aims of this study is to analyze outcome of allogeneic HCT performed in CR2 comparing patients with prior consolidation by autologous HCT vs. patients with chemotherapy consolidation. Primary outcome is Non relapse mortality (NRM) of allogeneic HCT in CR2 in patients with, or without prior autologous HCT in CR1. Secondary outcomes include leukemia free survival (LFS), relapse rate $(\mathrm{RI})$, graft versus host disease free relapse free survival (GRFS), overall survival (OS), and treatment related toxicities.

Results: 2619 adult patients reigstered with the ALWP of the EBMT with de novo AML were included, receiving a first allogeneic HCT in CR2, in 2000-2017 with $(n=417)$ or without $(n=2202)$ prior autologous HCT. Patient and transplant characteristics are shown in the Table. Patient groups were not entirely comparable, patients with prior autologous HCT were younger, had less often a favorable cytogenetic profile, had more commonly donors other than matched siblings and more often received reduced intensity conditioning (RIC) as compared to MAC conditioning.
Univariate outcomes are shown in the Table with slightly higher NRM risks in patients with prior autologous HCT consolidation. In multivariate analysis NRM risks in patients with prior autologous HCT were 1.34 (1.07-1.67), $\mathrm{p}=0.01$ after adjustment for patient age, cytogenetic risk category, year of transplant, donor type, conditioning intensity, sex matching, time from diagnosis to relapse and time from relapse to allogeneic HCT as compared to patients with chemotherapy consolidation. Similarly, risks of events in LFS and GRFS were higher with prior autologous HCT, 1.17 (1.01-1.35), $\mathrm{p}=0.03$ and 1.18 (1.031.35) $\mathrm{p}=0.02$, respectively, risk of death was also higher 1.13 (0.974-1.32) $\mathrm{p}=0.1$ but this was not statistically significant.

Conclusions: We may conclude that some of the advantages of potentially higher anti-leukemic activity of high dose chemotherapy and autologous HCT when given to patients with AML in CR1(as was shown in a randomized trial by Vellenga $\mathrm{E}$ et al with lower relapse and higher LFS by approximately $10 \%$ but no significant differences in overall survival) may be lost by higher toxicity of allogeneic HCT in CR2 in case of subsequent relapse.

\begin{tabular}{llll}
\hline & $\begin{array}{l}\text { Chemotherapy } \\
\text { consolidation }\end{array}$ & $\begin{array}{l}\text { Autologous HCT } \\
\text { consolidation }\end{array}$ & $\mathrm{P}$ \\
\hline NRM (2y) & $21.3 \%[19.6-23.1]$ & $25.2 \%[21-29.6]$ & 0.008 \\
Relapse (2y) & $28.1 \%[26.1-30]$ & $28.6 \%[24.1-33.2]$ & NS \\
OS (2y) & $58.1 \%[55.9-60.2]$ & $55.2 \%[50.2-60.2]$ & 0.02 \\
LFS (2y) & $50.6 \%[48.4-52.8]$ & $46.2 \%[41.2-51.2]$ & 0.004 \\
GRFS (2y) & $39.7 \%[37.5-41.8]$ & $35.7 \%[30.8-40.5]$ & 0.02 \\
\hline
\end{tabular}

[[P004 Table] 1. Outcome]

Disclosure: none

P005

Abstract already published.

P006

Extramedullary relapse as a poor predictor of survival even in patients with graft-versus-host disease post allogeneic hematopoietic cell transplantation for acute leukemia

Ioanna Sakellari ${ }^{1}$, Eleni Gavriilaki ${ }^{1}$, Ioannis Batsis ${ }^{1}$, Despina Mallouri ${ }^{1}$, Maria Gavriilaki ${ }^{2}$, Chrysa Apostolou ${ }^{1}$,

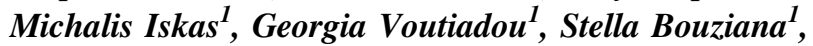
Zoi Bousiou ${ }^{I}$, Varnavas Constantinou ${ }^{I}$, Marianna Masmanidou $^{1}$, Damianos Sotiropoulos ${ }^{1}$, Evangelia Yannaki $^{l}$, Chrysavgi Lalayanni ${ }^{1}$, Maya Pilavaki, Konstantinos Chatziioannou ${ }^{I}$, Sotirios Papayannopoulos $^{1}$, Achilles Anagnostopoulos ${ }^{1}$ 
${ }^{1}$ G. Papanicolaou Hospital, Thessaloniki, Greece, ${ }^{2}$ Aristotle University of Thessaloniki, Postgraduate Course, Medical Research Methodology, Thessaloniki, Greece

Background: Although relapse is a major cause of mortality in patients receiving allogeneic hematopoietic cell transplantation (HCT) for acute leukemia, limited and conflicting data exist on extramedullary relapse (EMR). We aimed to describe the incidence, risk factors, outcomes and prognosis in relapsed HCT recipients.

Methods: We retrospectively reviewed charts of consecutive allogeneic HCT recipients transplanted in our center with the indication of acute leukemia (7/1990-7/ 2018). We recorded: age, gender, disease, previous extramedullary involvement, phase at transplant, type of transplant, donor, conditioning, graft-versus-host-disease (GVHD), infections, treatment-related mortality and relapse mortality. In patients with extramedullary relapses, additional data on clinical manifestations, imaging, cerebrospinal fluid testing, histopathology and management were additionally documented. Incidence of isolated EMR (iEMR) and bone marrow relapse (BMR) was calculated using cumulative incidence (CI) analysis, with each and treatment-related mortality considered a competing risk.

Results: Among 554 alloHCT recipients followed for 1.8 (0.04-27.75) years, $61(11 \%)$ patients presented with EMR. The majority of EMRs involved the central nervous system (CNS, 56\%). Isolated EMR was observed in 38 patients at 9.5 (1.8-67.3) months. 10-year cumulative incidence (CI) of $10.5 \%$ for iEMR was associated only with pre-transplant advanced disease phase $(\mathrm{p}<0.001)$. BMR was observed in 149 patients at 9 (0.3-276 months), with a 10 -year CI of $34.8 \%$. In the multivariate analysis, BMR CI was independently associated with fungal infections $(\mathrm{p}<$ $0.001)$, pre-transplant disease phase $(\mathrm{p}<0.001)$ and lines of treatment $(\mathrm{p}=0.042)$. 10-year TRM of our whole cohort was $33.2 \%$.

The majority of iEMR and BMR (75\% and 81\%, respectively) patients received systemic treatment combined with local radiation for iEMR (26\%) and donor lymphocyte infusions (DLIs, $16 \%$ and $28 \%$ respectively) when feasible. Extensive chronic GVHD was recorded in $47 \%$ of iEMR and $48 \%$ of BMR patients. Outcomes were poor in iEMR, with 10-year overall survival (OS) of $18.3 \%$. Favorable OS in iEMR was associated only with sibling donors $(p=0.049)$ and not with other factors, such as treatment with DLIs or presence of chronic GVHD. Similarly poor outcomes (10year OS of $19.1 \%$ ) were observed in BMR. Favorable OS was independently associated only with the diagnosis of AML $(p=0.050)$ and absence of bacterial infections $(p=0.049)$. In the whole cohort, both iEMR and BMR were independent unfavorable predictors of OS $(p<0.001)$ along with extensive chronic GVHD $(\mathrm{p}=0.012)$.
Conclusions: In a large population with long-term follow-up, incidence of iEMR was relatively high, developed at the late post-transplant period and associated only with disease phase at transplant. Furthermore, iEMR and BMR conferred similarly poor outcomes despite systemic treatment or extensive chronic GVHD. These independent predictors of survival highlight the unmet clinical need of novel approaches either as maintenance or treatment to reduce extramedullary or systemic relapse post alloHCT for acute leukemia.

Disclosure: No competing financial interest.

\section{P007}

Impact of T-cell depletion on outcome in patients undergoing allogeneic hematopoietic stem cell transplantation for acute myeloid leukemia

Sarah Morin ${ }^{\text {, Yan Beauverd }}{ }^{1}$, Mitja Nabergoj ${ }^{1}$, Federica Giannotti $^{1}$, Carole Dantin ${ }^{1}$, Amandine Pradier ${ }^{1}$, Thien-An Tran $^{1}$, Thomas Longval', Maria Anastasiou ${ }^{1}$, Laura Bounaix ${ }^{1}$, Caroline Stephan ${ }^{1}$, Olga Tsopra ${ }^{1}$, Carmen de Ramon Ortiz ${ }^{1}$, Anne-Claire Mamez, Stavroula Masouridi-Levrat ${ }^{1}$, Yves Chalandon ${ }^{I}$

${ }^{1}$ Geneva University Hospitals, Geneva, Switzerland, ${ }^{2}$ APHP, Paris, France

Background: Allogeneic stem cell transplantation (HSCT) remains the only curative option for most patients with acute myeloid leukemia. However, HSCT is associated with significant morbidity and mortality, mainly related to acute or chronic graft-versus-host disease (aGvHD or cGvHD). At the Geneva University Hospital, we use in vitro partially T-cell depleted grafts in selected patients with $<0.1 \%$ Mesurable Residual Disease (MRD) at the time of transplantation. This approach is based on studies that showed reduced incidence of aGvHD and cGVHD with no significant impact on outcome with this procedure. However, this has not been studied specifically in patients with AML.

Methods: We conducted a single-center retrospective analysis to compare outcomes in adult patients who were transplanted with T-cell replete or in vitro partially T-cell depleted grafts (TDEP) for AML in first complete remission between 1998 and 2018, with either HLA-identical or 10/10 matched unrelated donors. In vitro partial T-cell depletion was performed using alemtuzumab.

Results: A total of 145 patients were analyzed. 89 (61\%) and $56(39 \%)$ received partially TDEP and T-cell replete grafts, respectively. Patients in the partially TDEP group were significantly younger (median age 50 vs 58.5 years $(\mathrm{p}<0.05))$. Their EBMT score was significantly lower (EBMT score 1: $11.2 \%$ vs $1.8 \%$; score $2: 46.1 \%$ vs $25 \%$; score 3: $34.8 \%$ vs $60.7 \%$; score $4: 7.9 \%$ vs $7.1 \%$; score 5 : 
$0 \%$ vs $5.4 \%$; $<<0.05)$ and their Disease Risk Index was significantly lower (low: $3.4 \%$ vs $0 \%$; intermediate: $83.1 \%$ vs $69.6 \%$; high: $10.1 \%$ vs $30.4 \%$; $\mathrm{p}<0.05$ ). RIC regimen was used in $31.5 \%$ in the TDEP group in $66.1 \%$ in the Tcell replete group $(\mathrm{p}<0.05)$. Stem cell source was PBSC in $98.9 \%$ in the partially TDEP and $95.6 \%$ in the T cell replete group, respectively. The 3-year overall survival (OS) and progression-free survival (PFS) were $55.9 \%$ and $49.2 \%$ in the patially TDEP group and $42.2 \%$ and $41.6 \%$ in the T-cell replete group ( $\mathrm{p}=0.94$ for OS and 0.53 for PFS). The 3 year cumulative incidence (CI) of grade II-IV aGVHD was $22 \%$ in the TDEP group and $61.7 \%$ n the T-cell replete group $(\mathrm{p}<$ 0.05 ) and for cGVHD it was $11.9 \%$ and $40.1 \%$, respectively $(\mathrm{p}<0.05)$. The CI of non-relapse mortality at 3 years was $27 \%$ in the T-cell replete group and $15.1 \%$ in the TDEP group $(\mathrm{p}=0.124)$ and the $\mathrm{CI}$ of relapse at 3 years was $35.8 \%$ and $31.5 \%$ respectively $(\mathrm{p}=0.53)$. Importantly, partial T-cell depletion had no impact on OS (HR 0.99\%, $\mathrm{p}=0.98$ ) in a multivariate analysis.

Conclusions: Partial T-cell depletion significantly reduced the CI of grade II-IV aGVHD and cGVHD in patients with AML with no significant impact on OS and RI. These results support the use of partial T-cell depletion for HSCT in selected AML patients with negative MRD prior to transplantation.

Clinical Trial Registry: Non applicable

Disclosure: Nothing to declare

\section{P008}

Abstract already published.

\section{P009}

Abstract already published.

\section{P010}

Long-term complete remission in CR1-AML patients after transplantation: Rising survivorship awareness

Maria Teresa Lupo-Stanghellini ${ }^{1}$, Francesca Lorentino ${ }^{\text {, }}$, Matteo Carrabba ${ }^{1}$, Carlo Messina ${ }^{I}$, Daniela Clerici, Simona Piemontese ${ }^{1}$, Francesca Pavesi ${ }^{1}$, Fabio Giglio ${ }^{1}$, Raffaella Greco', Magda Marcatti', Sara Mastaglio ${ }^{1}$, Sarah Marktel, Andrea Assanelli, Luca Vago ${ }^{I}$, Chiara Bonini $^{1,2}$, Consuelo Corti ${ }^{1}$, Jacopo Peccatori, Massimo Bernardi $^{1}$, Fabio Ciceri ${ }^{1,2}$

${ }^{1}$ San Raffaele Scientific Institute, Milano, Italy, ${ }^{2}$ University Vita-Salute San Raffaele, Milano, Italy

Background: After a diagnosis of acute myeloid leukemia (AML) the majority of patients (pts) who achieve complete remission (CR) eventually relapse, with only approximately $30 \%$ of pts maintaining CR for 3 years or longer. Late relapses (after 3 years in CR) occur rarely $(6-10 \%)$ in pts receiving HSCT in CR1 and late effects are followed up by routine surveillance as well as preventative measures.

The purpose of this study was to investigate long-term outcomes in pts with diagnosis of AML undergoing HSCT at our Institution in CR1.

Methods: A standardized follow-up of HSCT-survivors is applied at our center. We analyzed 116 adult pts with AML in CR1 consecutively transplanted between January 2004 and December 2016 at our Institution. A written consent was given for the use of medical records for research. A landmark analysis was adopted for patients in CR at 2-y after HSCT (LTCR - long-term CR).

Results: LTCR was achieved after HSCT in 91/116 patients (male 55, female 36) transplanted in CR1. The median follow-up was 6 years and the median age at transplant 52 years ( $\mathrm{r}$ 20-72). The selected donor was a family haploidentical relative in 29 cases, an HLA identical relative in 21 , a match unrelated donor in 39 and a cordblood in 2 .

In this cohort of LTCR, the 5-year overall survival was 92\% (95\% CI 83-96). Cumulative incidence of relapse evaluated in competing risk with transplant related mortality (TRM) - and TRM - evaluated in competing risk with relapse - were respectively $7 \%$ (95\% CI $1-23$ ) and $2 \%$ for the CR1 cohort. The event-free-survival (EFS) was $91 \%$ (95\% CI 83-95).

The causes of death were relapse $(6 / 10 \mathrm{pts})$, second cancer (3/10 pts) and sepsis (1/10 pts).

The 5-year incidence of dyslipidemia - defined as cholesterol $>/=200 \mathrm{mg} / \mathrm{dl}$, and/or $\mathrm{LDL}>/=115 \mathrm{mg} / \mathrm{dl}$, and/or triglycerides $>/=150 \mathrm{mg} / \mathrm{dl}$ or need for specific treatment - was $24 \%$.

The 5-year incidence of osteopenia / osteoporosis defined as T-score lower than -1 and greater than -2.5 and T-score lower than 2.5 respectively - was $38 \%$.

The 5-year incidence of second cancer was 11\%: 10 nonmelanoma skin cancer, 2 lung carcinoma, 3 cervical intraepithelial neoplasm, 1 thyroid cancer, 1 gastric cancer and 1 colon cancer.

The 2-year incidence of chronic moderate-severe GvHD was $27 \%$ (95\% CI 13-38), with the latest diagnosis performed on day 570 . Of note, $4 / 24$ pts are still on active treatment at last follow-up.

Conclusions: Relapse incidence is low for patient that reached LTCR: patients in CR1 at transplant can obtain excellent OS and EFS once reached the target of LTCR.

A proactive long-term follow-up and strategy of counseling are essential to keep at best quality the survival advantage offered by HSCT in patients with AML in CR1.

Disclosure: Chiara Bonini has research contract with Intellia Therapeutics. The other authors declare that they have no conflicts of interest. 


\section{P011}

Results of hematopoietic stem cells transplantation with TCR $\alpha \beta+/ C D 19+-$ depletion from matched unrelated donors in pediatric acute leukemia patients in complete remission

\section{Ilyushina ${ }^{I}$, Livshits $^{I}$, Larisa Shelikhova ${ }^{I}, Z$} Shekhovtsova $^{I}, D_{\text {Balashov }}{ }^{1}$ I Shipitsina ${ }^{I}, D_{\text {Shasheleva }}{ }^{I}$, $R$ Khismatullina ${ }^{I}, S$ Blagov ${ }^{I}$, A Bogoyavlenskaya ${ }^{I}, S$ Kovrygin $^{1}$, S Kozlovskaya ${ }^{1}, S$ Radygina ${ }^{1}$, Y Skvortsova ${ }^{1}, E$ Kurnikova ${ }^{I}$, Y Muzalevsky ${ }^{1}$, A Kazachenok ${ }^{1}$, D Pershin ${ }^{I}$, M Fadeeva $^{I}$, A Popov ${ }^{I}$, Y Olshanskaya ${ }^{I}$, I Kalinina ${ }^{I}, N$ Miakova $^{l}$, D Litvinov $^{1}, G_{\text {Novichkova }}{ }^{1}$, A Maschan $^{1}, M$ Maschan $^{1}$

1"Dmitry Rogachev National Medical Research Center of Pediatric Hematology, Oncology and Immunology", Moscow, Russian Federation

Background: Relapse, graft-versus-host disease (GvHD) and GvHD-associated mortality are major obstacles to success of transplantation from unrelated (MUD) donors in children with acute leukemia (AL). Negative depletion of $\alpha \beta \mathrm{T}$ cells and CD19+ B lymphocytes, conserves the mature donor-derived natural killer cells and $\gamma \delta \mathrm{T}$ cells in the graft, may improve GvHD control, immune reconstitution and prevent the relapse. We present a retrospect analyses of a cohort of pts with AL in CR transplanted from MUD with depletion.

Methods: A total of 59 children with acute leukemia (34 AML, 25 ALL, 21 female, 38 male, median age 8,5y) underwent allo HSCT from matched unrelated donor between June 2012 and July 2017. All pts were in complete remission $(\mathrm{CR} 1=34, \mathrm{CR} 2=23, \mathrm{CR}>2=2)$.

All pts, except one, received treosulfan-based conditioning. Either melphalan $(n=56)$ or thiophosphamide $(n=2)$ or etoposide $(\mathrm{n}=1)$ were added as a second agent. Fludarabine was used in all pts. Two types of GVHD prophylaxis were used: Type $1(\mathrm{n}=35)$ : hATG $50 \mathrm{mg} / \mathrm{kg}$ and post-HSCT tacro/mtx $(\mathrm{n}=30)$ or without prophylaxis $(\mathrm{n}=5)$; type 2 $(\mathrm{n}=24)$ : thymoglobulin(rATG) $5 \mathrm{mg} / \mathrm{kg}$, rituximab $200 \mathrm{mg} /$ $\mathrm{m}^{2}$ with either bortezomib on days $+2,+5(\mathrm{n}=21)$ or tacro/ $m t x(n=3)$. a $\beta$ T cell depletion with CliniMACS was used in all cases. The median dose of CD34+ cells was $9 \times 10^{6} \%$ $\mathrm{kg}$, a $\mathrm{T}$ cells $-15 \times 10^{3} / \mathrm{kg}$. Median time of follow-up for survivors was 5,3 years (range, 2,3 - 6,5).

Results: Primary engraftment was achieved in $100 \%$ pts., the median time to neutrophil and platelet recovery was 15 and 14 days, respectively. All evaluable pts achieved sustained complete donor chimerism by day +30 .

Early (100 day) mortality was 3,4\% (1pt - bacterial sepsis, $1 \mathrm{pt}$ - ADV fulminant hepatitis), 5-years overall pTRM at 4 years was 13,5\% (95\%CI:7-26). Six late TRM events were due to: viral infection in 2 pts $(\mathrm{CMV}=1, \mathrm{ADV}+\mathrm{CMV}=1)$, bacterial sepsis in 2 pts and 2 pts had bacterial and viral infection, all late deaths were associated with cGvHD and prolonged corticosteroid therapy.

CI of acute GvHD grades II-IV was 36\% (95\% CI: $25-$ 50), acute GvHD grades III-IV 3,7\% (95\% CI:1,5-14,5). CI of cGvHD was $27 \%$ (95\%CI:18-41).

Regimen 2 was more effective in prevention of aGvHD II-IV in comparison with regimen 1: $8 \%$ (95\% CI: 2,2-30) vs $45,7 \%$, respectively, $\mathrm{p}=0,04$. All events with acute GvHD grades III-IV had pts with regimen 1. rATG was also effective in prevention of cGvHD: CI at 4 years after HSCT was $12,5 \%$ vs. $37 \%$, respectively, $p=0,04$. Cumulative incidence of relapse was $25 \%$ (95\%CI: 14-50) without difference between rATG and hATG.

Event-free survival $(\mathrm{EFS})($ event=death or relapse) at 4 years was $61 \%$ (95\%CI: 48-73), overall survival 59\%(95\% CI:47-72), there were no difference between age and diagnosis.

Conclusions: We confirm that the depletion of TCR $\alpha \beta$ $+/ \mathrm{CD} 19+\mathrm{T}$ lymphocytes from the graft ensures high engraftment rate. Transplant-related mortality is caused by infections, mostly associated with cases of chronic GVHD. GvHD prophylaxis including rATG/Rituximab/ Bortezomib improves GvHD control in recipients of TCR $\alpha \beta+/ C D 19+$ depleted grafts in comparison to hATG/ Tacro/MTX apparently without loss of anti-leukemic activity.

Disclosure: No disclosure

\section{P012}

Outcome of relapsed or refractory acute lymphoblastic leukemia patients with extramedullary disease or lymphoblastic lymphoma receiving inotuzumab ozogamicin or standard care in the INO-VATE trial

David I. Marks ${ }^{1}$, Daniel J. DeAngelo ${ }^{2}$, Matthias Stelljes ${ }^{3}$, Michaela Liedtke ${ }^{4}$, Wendy Stock ${ }^{5}$, Nicola Gökbuget ${ }^{6}$, Susan O'Brien $^{7}$, Elias Jabbour ${ }^{8}$, Akil Merchant ${ }^{9}$, Tao Wang $^{10}$, Eric Vandendries ${ }^{11}$, Alexander Neuhof ${ }^{12}$, Anjali Advani $^{13}$, Hagop Kantarjian ${ }^{8}$

${ }^{1}$ University Hospitals Bristol, Bristol, United Kingdom, ${ }^{2}$ Dana-Farber Cancer Institute, Boston, MA, United States, ${ }^{3}$ Universitätsklinikum Münster, Münster, Germany, ${ }^{4}$ Stanford University School of Medicine, Stanford, CA, United States, ${ }^{5}$ University of Chicago, Chicago, IL, United States, ${ }^{6}$ Goethe University Frankfurt, Frankfurt, Germany, ${ }^{7}$ University of California, Chao Family Comprehensive Cancer Center, Irvine, CA, United States, ${ }^{8} \mathrm{MD}$ Anderson Cancer Center, Houston, TX, United States, ${ }^{9}$ University of Southern California, Los Angeles, CA, United States, ${ }^{10}$ Pfizer, Groton, CT, United States, ${ }^{11}$ Pfizer, Cambridge, MA, 
United States, ${ }^{12}$ Pfizer Pharma GmbH, Berlin, Germany, ${ }^{13}$ Cleveland Clinic, Cleveland, $\mathrm{OH}$, United States

Background: Extramedullary relapse is difficult to treat in patients with acute lymphoblastic leukemia (ALL). Inotuzumab ozogamicin (InO) is a humanized anti-CD22 monoclonal antibody conjugated to calicheamicin. In patients with relapsed or refractory B-cell (R/R) ALL, InO showed significant higher response, MRD-negativity rates, and longer progression-free survival (PFS) and overall survival (OS) versus standard of care (SC) chemotherapy. This analysis of the INO-VATE trial investigated the outcomes of R/R ALL patients with extramedullary disease (EMD) or lymphoblastic lymphoma (LBL) at baseline.

Methods: Adult patients with R/R ALL (excluding isolated extramedullary relapse and active central nervous system leukemia) were randomized to $\operatorname{InO}(n=164)$ or SC $(\mathrm{n}=162)$. InO starting dose was $1.8 \mathrm{mg} / \mathrm{m}^{2} /$ cycle $(0.8 \mathrm{mg} /$ $\mathrm{m}^{2}$ on Day $1 ; 0.5 \mathrm{mg} / \mathrm{m}^{2}$ on Days 8 and 15 of a 21-28-day cycle for $\leq 6$ cycles). For patients with complete remission (CR) or CR with incomplete hematologic recover (CRi), InO dose was $1.5 \mathrm{mg} / \mathrm{m}^{2} /$ cycle. SC included fludarabine/ cytarabine $[\mathrm{Ara}-\mathrm{C}] /$ granulocyte colony-stimulating factor [FLAG], Ara-C plus mitoxantrone, or high-dose Ara-C. Outcomes were analyzed by baseline EMD and LBL status.

\begin{tabular}{|c|c|c|c|c|}
\hline Response, n (\%) & $\begin{array}{l}\text { Inotuzumab } \\
\text { ozogamicin }(\mathrm{N}=18)\end{array}$ & $\begin{array}{l}\text { Standard } \\
\text { care }(N=11)\end{array}$ & $\begin{array}{l}\text { Rate } \\
\text { difference }\end{array}$ & $\begin{array}{l}P- \\
\text { value }\end{array}$ \\
\hline $\begin{array}{l}\text { Complete } \\
\text { remission (CR) }\end{array}$ & $4(22.2)$ & 0 & & \\
\hline $\begin{array}{l}\text { CR with incomplete } \\
\text { hematologic } \\
\text { recover (CRi) }\end{array}$ & $8(44.4)$ & $2(18.2)$ & & \\
\hline Partial response (PR) & $1(5.6)$ & $1(9.1)$ & & \\
\hline $\begin{array}{l}\text { Resistant } \\
\text { disease (RD) }\end{array}$ & $4(22.2)$ & $8(72.7)$ & & \\
\hline $\begin{array}{l}\text { Progressive } \\
\text { disease (PD) }\end{array}$ & $1(5.6)$ & 0 & & \\
\hline $\begin{array}{l}\mathrm{CR} / \mathrm{CRi}, \mathrm{n}(\%)(95 \% \\
\text { confidence interval } \\
{[\mathrm{CI}] \text { for rate and }} \\
97.5 \% \mathrm{CI} \text { for rate } \\
\text { difference) }\end{array}$ & 12 (66.7) (41.0-86.7) & $\begin{array}{l}2(18.2) \\
(2.3-51.8)\end{array}$ & $\begin{array}{l}48.5 \\
(12.4- \\
84.5)\end{array}$ & 0.0144 \\
\hline
\end{tabular}

[[P012 Table] 1. Best overall response in patients with extramedullary disease at baseline or lymphoblastic lymphoma]

Results: At baseline, R/R ALL with EMD and LBL were diagnosed in 7 and $11 \mathrm{InO}$ patients and 5 and $6 \mathrm{SC}$ patients. Median (range) age of the $\mathrm{InO}$ and SC patients was 55.5 (2078) and 47.0 (28-64) years, with $8 / 18(44.4 \%)$ and $8 / 11$ (72.7\%) males, respectively. The rate of $\mathrm{CR} / \mathrm{CRi}$ was significantly higher in the InO group (12/18 [66.7\%], 95\% confidence interval [CI]: 41.0-86.7) compared with SC (2/11 [18.2\%], 95\% CI: 2.3-51.8; $P=0.0144)$ (Table). Allogeneic hematopoietic stem cell transplantation was carried out in $6 /$
$18(33.3 \%) \mathrm{InO}$ and $2 / 11$ (18.2\%) SC patients prior to any post-study induction therapy. The PFS hazard ratio [HR] was 0.502 (97.5\% CI: 0.203-1.240; $P=0.0410$ ), with median PFS of 4.4 (95\% CI: 1.9-7.1) months among InO and 1.6 (95\% CI: 0.8-3.7) months in SC patients. The OS HR was 0.661 (97.5\% CI: $0.269-1.621 ; P=0.1478)$, with median OS of 5.9 (95\% CI: 3.4-9.4) months in $\mathrm{InO}$ versus 5.5 (95\% CI: 2.16.7) months in SC patients (Figure). All patients had adverse events (AEs). Serious AEs occurred in 10/18 (55.6\%) InO and 5/11 (45.5\%) SC patients; 4 (22.2\%) InO and 0 SC patients had grade 5 AE. One $(1 / 15,6.7 \%)$ patient in the $\mathrm{InO}$ group died from veno occlusive disease.

Conclusions: Among R/R ALL patients with EMD and LBL, improvement in remission rates, transplant rates, and progression free survival was shown in the $\mathrm{InO}$ group versus the SC group. Although patient numbers were small and limited the ability for a robust comparison, these results support the use of InO in patients in this difficult to treat population with R/R ALL and EMD or LBL.
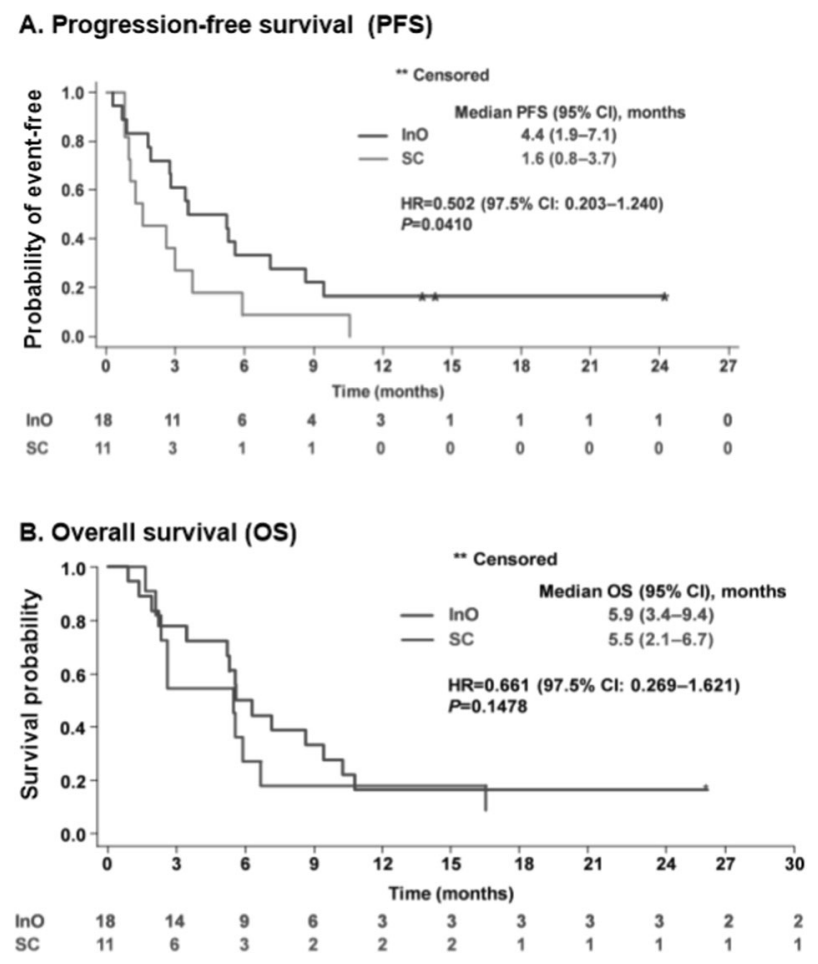

[[P012 Image] 1. PFS and OS in patients with extramedullary disease or lymphoblastic lymphoma]

Clinical Trial Registry: www.clinicaltrials.gov (Identifier: NCT01564784)

Disclosure: DM: Consultancy: Novartis, Pfizer, and Amgen.

DJD: Honoraria: Takeda, Novartis, Shire, Pfizer, Blueprint Medicines, Incyte, Amgen, and BMS. Consultancy: 
Novartis, Pfizer, Amgen, ARIAD, and BMS. Research funding: Blueprint Medicines, Glycomimetics, and ARIAD.

MS: Honoraria: Novartis, Amgen, Jazz Pharmaceuticals, and Pfizer. Consultancy: MSD and Pfizer. Research funding: Pfizer.

ML: Research funding: Amgen/Onyx, BlueBirdBio, Celgene, Genentech/Roche, Gilead, Pfizer, Prothena, and Takeda. Membership on an entity's Board of Directors or advisory committees: Caelum, Gilead, Pfizer, Prothena, and Takeda. Honoraria: Pfizer, Prothena, and Amgen/Onyx. Consultancy: Amgen/Onyx.

WS: Consultancy: Jazz Pharmaceuticals.

NG: Honoraria and research funding: Amgen and Pfizer.

SO: Consultancy: Vaniam Group, Pharmacyclics, Aptose Biosciences, GlaxoSmithKline, Amgen, Astellas, TG Therapeutics, Pfizer, Janssen, Gilead, Celgene, Alexion, Sunesis, and Abbvie. Research funding: Pharmacyclics, Kite Pharma, TG Therapeutics, Pfizer, Gilead, Acerta, Regeneron, and Sunesis.

EJ: Consultancy: Takeda, Pfizer and Bristol-Myers Squibb. Research funding: Novartis, Takeda, Pfizer, Abbvie, and Bristol-Myers Squibb.

AM: Nothing to declare.

TW, EV, and AN: Employment and equity ownership: Pfizer.

AA: Consultancy: Novartis and Glycomimetics. Research funding: Amgen and Pfizer. Honoraria: Pfizer.

HK: Honoraria: Pfizer, Orsenix, Immunogen, BMS, ARIAD, Amgen, Actinium and AbbVie. Research funding: Pfizer, Novartis, BMS, Astex, ARIAD, and Amgen.

\section{P013}

Stroma-mediated resistance to dasatinib of acute lymphoblastic leukemia (ALL) tumor cells can be abrogated by the histone deacetylase inhibitor panobinostat via calcineurin-dependent CXCR4 downregulation

Katia Beider', Olga Landes ${ }^{1}$, Ania Hava GrushchenkoPolaq $^{1}$, Olga Ostrovsky ${ }^{1}$, Valeria Voevoda-Dimenshtein ${ }^{1}$, Ivetta Danylesko $^{1}$, Avichai Shimoni', Amnon Peled ${ }^{2}$, Mohamad Mohty, Arnon Nagler ${ }^{1}$

${ }^{1}$ Sheba Medical Center, Ramat Gan, Israel, ${ }^{2}$ Hadassah Hebrew University Medical Center, Jerusalem, Israel, ${ }^{3}$ Hospital Saint-Antoine, Paris, France

Background: BCR-ABL-targeted tyrosine kinase inhibitors (TKI) revolutionized the outcome of patients inflicted with $\mathrm{Ph}+\mathrm{B}$-ALL. Moreover, addition of TKI may be relevant strategy for Ph-like ALL patients.

Methods: We hypothesized that overcoming the BM microenvironment-mediated protection of ALL cells from
TKI-mediated apoptosis may further enhance the responsiveness to TKI therapy.

Results: In vitro treatment of BCR-ABL-positive ALL cell lines (BV-173, NALM1 and NALM20) with dasatinib resulted in significant dose-dependent cell growth inhibition, with IC50 of $10-15 \mathrm{nM}(\mathrm{p}<0.01)$. Furthermore, dasatinib exhibited significant growth suppression of BCRABL -negative ALL cells (NALM6 and REH), with IC50 of $250 \mathrm{nM}$ and $185 \mathrm{nM}$, respectively. However, when cocultured with bone marrow stromal cells (BMSCs), dasatinib-mediated effect was abrogated in both $\mathrm{Ph}$ - and $\mathrm{Ph}+\mathrm{ALL}$ cells. Furthermore, dasatinib treatment promoted significant upregulation of chemokine receptor CXCR4, on both mRNA and cell surface levels. Elevated CXCR4 expression was accompanied by increased responsiveness of ALL cells to CXCL12 stimulation, resulting in strong and sustained phosphorylation of Erk1/2 and Akt and increased adhesion capacity to BMSCs. Therefore, dasatinib-induced upregulation of CXCR4 promotes stroma-mediated survival advantage of ALL cells upon TKI therapy.

Next, in order to overcome the CXCR4-mediated stromal protection, we choose to combine dasatinib with the histone deacetylase inhibitor panobinostat, for its known ability to deplete CXCR4 in AML cells. Single-agent treatment with panobinostat demonstrated significant inhibition of $\mathrm{Ph}$ - and $\mathrm{Ph}+\mathrm{ALL}$ cell growth at low nanomolar concentrations $(\mathrm{p}<$ 0.01 ). Importantly, combination of panobinostat with dasatinib synergized $(\mathrm{CI}<0.5)$, effectively overcoming the protection provided by BMSCs and inducing the apoptosis of $\mathrm{Ph}-$ and $\mathrm{Ph}+\mathrm{ALL}$ cells, as demonstrated by phosphatidylserine externalization, mitochondrial depolarization and DNA fragmentation. Furthermore, combining panobinostat with dasatinib significantly reduced CXCR4 surface levels in $\mathrm{Ph}-$ and $\mathrm{Ph}+$ ALL cells. Accordingly, CXCL12mediated responses, including Erk1/2 and Akt activation and adhesion to BMSCs were significantly reduced upon combined panobinostat/dasatinib treatment. These data indicate that panobinostat effectively suppresses both basal and dasatinib-induced CXCR4 expression and function in ALL cells overcoming stroma-mediated resistance to dasatinib.

To determine the molecular mechanism, we performed gene and protein expression analysis. Panobinostat, alone or in combination with dasatinib, significantly down-regulated the protein levels of calcineurin, a serine-threonine protein phosphatase previously implicated in T-ALL and B-ALL pathogenesis, as well as of NFATc1, a critical effector of the calcineurin signaling cascade, and NFATc1-regulated target genes. It was previously found that calcineurin signaling positively regulates CXCR4 expression in $\mathrm{T}$ lymphocytes. Additionally, cyclosporin A (CsA) decreased both basal and dasatinib-induced CXCR4 surface levels in 
ALL cells, overcoming the protection of the BMSCs which result in potentiation of the cytotoxic effect of dasatininb and panobinostat. Combining CsA with panobinostat resulted in deeper suppression of NFATc1-regulated target genes. We thus link the effect of panobinostat with calcineurin-dependent downregulation of CXCR4, blocking the ability of the leukemic cells to respond to CXCL12mediated stromal support.

Conclusions: Taken together, our results identify calcineurin signaling pathway as a novel target of panobinostat in ALL cells and indicate that HDAC inhibition with panobinostat may be effective strategy for facilitating the anti-leukemic activity of TKI therapy.

Disclosure: nothing to disclose

\section{P014}

Allogeneic stem cell transplantation (allo-SCT) with sequential conditioning in patients with relapsed/ refractory acute lymphoblastic leukemia: A report from EBMT acute leukemia working party

Abdul Hamid Bazarbachi', Rama Al Hamed ${ }^{1}$, Labopin Myriam $^{1}$, Boris Afanasyev ${ }^{2}$, Rose-Marie Hamladji, Beelen Dietrich $^{4}$, Ganser Arnold ${ }^{5}$, Scheid Christof ${ }^{6}, W u$ Depei $^{7}$, Bunjes Donald ${ }^{8}$, Polina Stepensky ${ }^{9}$, Johanna Tischer $^{10}$, Nicolaus Kröger ${ }^{11}$, Zina Peric ${ }^{12}$, Sebastian Giebel $^{13}$, Arnon Nagler ${ }^{14}$, Mohamad Mohty ${ }^{15}$

${ }^{1}$ EBMT Paris Study Office / CEREST-TC, Saint Antoine Hospital, INSERM UMR 938, Université Pierre et Marie Curie, Paris, France, ${ }^{2}$ First State Pavlov Medical University of St. Petersburg, Raisa Gorbacheva Memorial Research Institute for Paediatric Oncology, Hematology and Transplantation, St Petersburg, Russian Federation, ${ }^{3}$ Centre Pierre et Marie Curie, Service Hématologie Greffe de Moëlle, Alger, Algeria, ${ }^{4}$ University Hospital, Essen, Germany, ${ }^{5}$ Hannover Medical School, Hannover, Germany, ${ }^{6}$ University of Cologne, I. Cologne, Germany, ${ }^{7}$ First Affiliated Hospital of Soochow University, Suzhou, China, ${ }^{8}$ Klinik fuer Innere Medzin III, Universitätsklinikum Ulm, Ulm, Germany, ${ }^{9}$ Hadassah University Hospital, Jerusalem, Israel, ${ }^{10}$ Klinikum Grosshadern, Med. Klinik III, Munich, Germany, ${ }^{11}$ University Hospital Eppendorf, Bone Marrow Transplantation Centre, Hamburg, Germany, ${ }^{12}$ University Hospital Centre, Zagreb, Croatia, ${ }^{13}$ Maria SklodowskaCurie Institute - Oncology Center, Gliwice, Poland, ${ }^{14}$ Chaim Sheba Medical Center, Tel Hashomer, Israel, ${ }^{15}$ Hopital St. Antoine, Paris, France

Background: The treatment of relapsed/refractory acute lymphoblastic leukemia (RR-ALL) remains a clinical challenge with a generally dismal prognosis. Allo-SCT using a sequential conditioning ("FLAMSA"-like regimen) has shown promising results in relapsed/refractory AML, but little is known about the efficacy of this procedure in RR-ALL.

Methods: We identified 115 adult patients (45\% females; median age: $38 \mathrm{y}$; range, 18-66) with ALL in primary refractory phase $(26 \%)$ or in relapse $(74 \%)$, allografted between 2000 and 2017 from a matched sibling (31\%), matched unrelated $(58 \%)$ or haploidentical donor $(11 \%)$ at EBMT participating centers. Almost half $(49 \%)$ of the patients had T-ALL and $23 \%$ had a positive Philadelphia chromosome. Six patients (5\%) underwent a previous autotransplant. Karnofsky score was above 90 in 52\% of patients. Conditioning was myeloablative (MAC) with high dose TBI in $30 \%$ of patients, reduced intensity (RIC) including low dose TBI in $22 \%$, or with chemotherapy alone in $48 \%$. In vivo $\mathrm{T}$ cell depletion was performed in 77 cases $(69 \%)$. Most patients (74\%) and about half of the donors $(47 \%)$ were CMV positive. $14 \%$ of patients were males who received a graft from a female donor. The median follow-up was 37 (range, 13-111) months.

Results: Overall, 14 patients (13\%) failed to engraft, 18 (16\%) died within 100 days after allo-SCT without relapse, and $64(56 \%)$ could achieve complete remission. At day 100 , the cumulative incidences of grade II-IV and grade IIIIV acute GVHD were $30 \%$ and $17 \%$, respectively. The 2 year cumulative incidences of chronic and extensive chronic GVHD were $25 \%$ and $11 \%$, respectively. The 2 -year relapse incidence (RI) and non-relapse mortality (NRM) were $45 \%$ and $41 \%$, respectively. The 2-year leukemia free survival (LFS), overall survival (OS) and GVHD relapsefree survival (GRFS) were 14\%, 17\% and 12\%, respectively. In a multivariable Cox analysis, Karnofosky score below 90 negatively affected RI, LFS, OS and GRFS. Also, conditioning with chemotherapy alone, compared to TBIbased conditioning, negatively affected relapse rates $(\mathrm{HR}=4.13 ; \mathrm{p}=0.0006), \mathrm{LFS}(\mathrm{HR}=2.32 ; \mathrm{p}=0.004)$ and $\mathrm{OS}$ $(\mathrm{HR}=2.29 ; \mathrm{p}=0.006)$.

Conclusions: Allo-SCT using a sequential conditioning regimen is proposed by different teams in RR-ALL, and could be an option, especially when considering a TBIbased regimen. However, the overall 2-year LFS of $14 \%$ suggests that these patients still face extremely dismal outcomes, highlighting that other therapies (e.g. BITE antibodies, inotuzumab, CAR T cells) need to be combined prior and/or after allo-SCT in order to further improve outcome.

Disclosure: no conflict of interest, no funding

\section{P015}

Abstract already published.

\section{P016}

HLA-haploidentical microtransplantation as 
consolidation therapy in older patients with acute myeloid leukemia

Sergey Bondarenko ${ }^{I}$, Olesya Smykova ${ }^{I}$, Elena Darskaya ${ }^{I}$, Olga Pirogova ${ }^{1}$, Tatiana Rudakova ${ }^{1}$, Anna Smirnova ${ }^{2}$, Elena Babenko ${ }^{I}$, Ildar Barkhatov ${ }^{I}$, Tatiana Gindina ${ }^{I}$, Alexander Alyanskiy ${ }^{2}$, Ivan Moiseev ${ }^{1}$, Alexander Kulagin $^{1}$, Boris Afanasyev ${ }^{2}$

${ }^{1}$ Pavlov First Saint Petersburg State Medical University, R. M. Gorbacheva Memorial Institute of Hematology, Oncology and Transplantation, St. Petersburg, Russian Federation, ${ }^{2}$ Pavlov First Saint Petersburg State Medical University, R.M. Gorbacheva Memorial Institute of Hematology, Oncology and Transplantation, St Petersburg, Russian Federation

Background: Clinical outcomes of acute myeloid leukemia (AML) in older patients still remain unsatisfactory. Allogeneic hematopoietic stem cell transplantation significantly improves the prognosis of patients with AML, but it is limited by severe toxicities and high incidence of treatmentrelated mortality in older patients. Microtransplantation (microHCT) is a promising cellular therapy in this group of patients.

Methods: We analyzed data of 65 patients $\geq 55$ y.o. with AML who achieved complete remission (CR). The first group of 44 patients (microHCT group) received 1-3 courses of consolidation therapy, which consisted of cytarabine $0.5-1.0 \mathrm{~g} / \mathrm{m}^{2}$ every $12 \mathrm{~h}$ for 3 days (D-4, -3 , -2), followed by an infusion of G-CSF-mobilized PBSC from haploidentical donor, without any GVHD prophylaxis. The median number of $\mathrm{CD} 3+$ cells infused per course was $1,4$ (range, $1,0-2,5) \times 10^{8} / \mathrm{kg}$. Among this group, 15 patients received nivolumab $100 \mathrm{mg} / \mathrm{d}$ on $\mathrm{D}+5$. Donor chimerism was detected by short tandem repeat PCR and donor microchimerism was detected by quantitative real-time PCR method based on insertion/deletion polymorphism $(\mathrm{D}+3$, $+8,+15$, etc.).

The second group of 21 patients (historical control group) received chemotherapy only as consolidation therapy. Age, sex and cytogenetic risk group were comparable between the 2 groups.

Results: The median follow-up time was 14 months (range, 4-46 months). The 2-year OS and DFS were 56\% and $42 \%$, respectively, in the microHCT group. The probabilities of 2-year DFS was significantly higher in the microHCT group than in the historical control group (42\% vs $14 \%$, respectively, $\mathrm{P}=.02$ ). Donor microchimerism was detected in $38(86 \%)$ patients with the median period of microchimerism persistence 8 days (range, 3-58 days). And 2 patients developed mixed chimerism (12\% and 20\%). One patient who had $12 \%$ of chimerism developed a cytokine release syndrome, this patient failed to respond to treatment and died on $\mathrm{D}+12$. Another patient with $20 \%$ of chimerism developed a skin graft-versus-host-disease (GVHD) grade 3 (histologic confirmation), resolved by therapy with glucocorticosteroids. The regimen was well tolerated with the non-relapse mortality of $2 \%$ and low risk of GVHD. The most common adverse events were fever in the first 48 hours, transient rash and hematological toxicity. Given the small group of patients, it is impossible to assess the effectiveness of nivolumab.

Conclusions: Immunotherapy with HLA-haploidentical microHCT demonstrated higher efficacy compared to the historical control and has acceptable toxicity. Additional studies are necessary to further assess the role of microHCT as consolidation treatment for older patients with AML.

Disclosure: Nothing to declare

\section{P017}

Relapse of acute leukemia after allogeneic haematopoietic stem cell transplantation (HSCT): Combination of pharmacological and cellular approach

Riccardo Boncompagni ${ }^{1}$, Ilaria Cutini ${ }^{1}$, Carlo Nardini ${ }^{1}$, Stefano Guidi ${ }^{I}$, Antonella Gozzini ${ }^{1}$, Chiara Nozzoli ${ }^{1}$, Riccardo Saccardi ${ }^{I}$

${ }^{1}$ Careggi University Hospital, Florence, Italy

Background: Relapse of Acute Leukemia (AL) after Allogeneic Haematopoietic Stem Cell Transplantation (HSCT) is a dramatic event with a dismal prognosis. The availability of new drugs, such as azacitidine (AZA) and blinatumomab $(\mathrm{Bl})$, either alone or in combination with donor T-Lymphocyte infusion (DLI), might improve the outcome. We report the experience of our centre in $\mathrm{AL}$ patients showing an initial relapse after HSCT.

Methods: From January 2012 to January 2017, 103 allogeneic HSCT were performed at our institution. 99 patients (pts) (96\%) affected by AL (myeloid nonpromyelocitic $=71$, lymphoblastic $=27$, undifferentiated $/$ mixed phenotype $=5), 4$ pts $(4 \%)$ were second allogeneic procedures due to a previously relapse ( $3 \mathrm{pts})$, and 1 pts was an engraftment failure. All patients were regularly monitored for both chimerism and Minimal Residual Disease (MRD); patients showing an overt clinical relapse or a loss of full chimerism and/or detection of MRD received a conventional chemo-based reinduction or repeated DLI infusions, possibly interposed to the administrations of either Aza in Myeloid AL or Bl in Lymphoid AL. The median follow-up from HSCT was 22 months. Posttransplant relapse occurred in 33 pts. Ten pts received DLI based therapy: 4 pts alone, 3 pts in combination with Aza, and 3 pts in combination with Bl. The other 23 pts 
received chemotherapy courses, only 2 pts were not treated: 1 pts for the worsening of the general status and the other for invasive fungal infection.

Results: Forty-three pts (42\%) were in complete remission (CR) and negative minimal residual disease (MRD) at the time of HSCT; 16 pts were in active disease (16\%), and 44 (42\%) showed a morphological CR with positive MRD. 41pts (40\%) developed chronic Graft Versus-Host Disease (cGVHD) as followed: 23pts (22\%) mild, 17pts (16\%) moderate, and only 1 sever grade respectfully. Only 1 patient developed cGVHD after DLI. The overall Leukemia Free Survival (LFS) time was 16 months, the absence of cGVHD (Hazard Ratio - HR: 5,968; $\mathrm{p}=0,01$ ) and the pre-HSCT disease status (HR 2,353; p = 0,028) were the most important factors on LFS. All pts treated with chemo-based regimens died due to progression or infective complications. 1 patient of Aza/DLI group is still alive with a extramedullary relapse; 2 pts treated with B1/DLI are in CR. OS was better for the DLI group compared to the chemotherapy group (28 vs 2 months respectfully; $\mathrm{p}<0,001$ ).

Conclusions: DLI after allo-HSCT has exhibited definite anti-leukemic effects in post-transplant patients. $\mathrm{Bl}$ and Aza were reported to increase DLI's Graft vs-Leukemia (GVL) effect. Although cGVHD could be the most important protective factor against the relapse but it remains the main cause of morbidity. Maximising the GVL effect without putting the patient at risk of GVHD still represents an unmet need. Our data show that the combination of either Bl or Aza with DLI infusion is safe and might represent an improvement in disease control in the early phase of relapse.

Disclosure: Nothing to declare

\section{P018}

Increased detection of (Leukemiaspecific) adaptive and innate immune-reactive cells under treatment of AMLdiseased rats and one therapy-refractory AML-patient with blastmodulating, clinically approved response modifiers

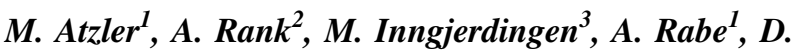 Deen $^{1}$, R. Wang ${ }^{1}$, B. Eiz-Vesper ${ }^{4}$, C. Schmid ${ }^{2}, H$. Schmetzer ${ }^{I}$}

${ }^{1}$ Klinikum Grosshadern, Munich, Germany, ${ }^{2}$ Augsburg University Hospital, Dept Med II, Hematopoetic Stem Cell Transplantation, Augsburg, Germany, ${ }^{3}$ Oslo University Hospital, Rikshospitalet, Oslo, Norway, ${ }^{4}$ Hannover Medical School, Institute for Transfusion-Medicine, Hannover, Germany

Background: Kits (combinations of immune-modulatory compounds:GM-CSF+Picibanil (Kit-I), Prostaglandine
(PG-E2,Kit-K) or +PGE1 (Kit-M),patent 102014014993) convert myeloid blasts into dendritic cells of leukemic origin $\left(\mathrm{DC}_{\text {leu }}\right)$. After stimulation with $\mathrm{DC}_{\text {leu }}$, antileukemic $\mathrm{T}$ cells can be generated ex vivo. The compounds are approved for clinical use and are therefore attractive tools for immunotherapy in myeloid leukemia.

Methods: DC/DCleu-culture from rats'/patients' wholeblood (WB) with kits, mixed lymphocyte culture (MLC) of Tcells with kit-treated blood, functional blast-cytotoxicity and leukemia-specificity assays (CSA/ELISPOT/Degranulation/intracellular cytokine-assays). In addition flowcytometric evaluations of cellular and (leukemia-specific) lymphocyte compositions were performed from rats'/pts' blood in the course of the disease.

\section{Results:}

1) AML-diseased rats: each 3rats were treated with "I", " $\mathrm{K}$ " or " $\mathrm{M}$ " or were untreated (controls). A significant increase of DCleu could be detected in spleen/PB in kit(esp. M) treated compared to untreated animals without induction of blasts' proliferation (Ki67positivity): a significant reduction of blasts was seen with " $M$ " $(p=0.03 /$ 0.0001 in spleen/PB) and "I", but not "K". Successful treatment correlated with an increase of CD62L+Tcells, most likely representing Tmem-cells, $(\mathrm{p}=0.07)$ and a reduction of $\mathrm{CD} 4+$ Treg $(\mathrm{p}=0.037)$.

2) 6 therapy-refractory AML-patients (during the course of decitabine/LD-AraCtreatment): kit-M was shown to ex vivo generate DCleu, activate immunereactive cells and mediate leukemia-specific/antileukemic response. Activated or leukemia-specific lymphocytes were monitored in low proportions in active stages of the disease as well as of two patients during the further course of persisting disease.

One of these patients (72 yo male), was offered an individual systemic salvage-treatment (Kit-M, applied as continuous infusions) for refractory leukemia. after approval from the local ethical commitee,extensive information of the patient about the experimental nature of the treatment and obtaining his written informed consent.

Clinically the treatment was well tolerated and the patient improved clinically. Neutrophils in WBC increased from $10 \%$ to $50 \%$, thrombocytes reached $100 \mathrm{G} / 1$ after 24 days. After 4 weeks of treatment, the patient was discharged in good clinical conditions. 12 days later, progression of $\mathrm{AML}$ was seen with high blast counts in PB and BM. The patient developed severe sepsis and died few days later.

Immune monitoring showed (other than before treatment and in the patients without kit-M-treatment) a continuous increase of proliferating and non-naïve Tcells, NK, CIKand NKT-, TH17 cells, Bmem-cells and DC in PB. The production of IFNY producing T-, CIK and NKT-cells was demonstrated, suggesting an in vivo production/activation of (potentially leukemia-specific) cells. Immune stimulatory effects decreased after discontinuation of therapy. 
Conclusions: Treatment of WB as well as leukemically diseased organisms with blast-modulating kits (especially GM-CSF and PGE1) was well tolerated and induced clinical and immunological improvement (adaptive and innate immune system), whereas low counts of (leukemiaspecific) activated immune-reactive cells were found in non-kit-treated organisms.

Disclosure: Nothing to declare

\section{P019}

Long-term outcomes after allogeneic hematopoietic stem cell transplantation for acute myeloid leukemia with non-myeloablative and myeloablative conditioning: A single-center cohort study of $\mathbf{4 3 8}$ consecutive patients

1Lars Klingen Gjarde, Niels Smedegaard Andersen", Lone Smidstrup Friis ${ }^{1}$, Brian Thomas Kornblit ${ }^{1}$, Søren Lykke Petersen ${ }^{I}$, Ida Schjødt ${ }^{1}$, Henrik Sengeløv ${ }^{I}$

${ }^{I}$ Rigshospitalet, Copenhagen, Denmark

Background: Since 2000, we have at our institution used a non-myeloablative (NMA) conditioning regimen for older (>50 years) or significantly comorbid younger patients undergoing allogeneic hematopoietic stem cell transplantation (allo-HSCT) for acute myeloid leukemia (AML). We aimed to compare the long-term outcomes of NMA conditioned patients with myeloablative (MA) conditioned patients.

Methods: We studied 220 NMA and 218 MA conditioned adult ( $>15$ years) consecutive patients receiving their first alloHSCT for AML from 2000 to 2017 at Rigshospitalet. NMA conditioning consisted mainly of 2 Gy total body irradiation (TBI) and fludarabine $90 \mathrm{mg} / \mathrm{m}^{2}$ (95\% of cases). MA conditioning consisted mainly of cyclophosphamide $120 \mathrm{mg} /$ $\mathrm{kg}$ and either $12 \mathrm{~Gy}$ TBI (86\% of cases) or busulfan $12.8 \mathrm{mg} /$ $\mathrm{kg}$ (5\% of cases), or fludarabine $150 \mathrm{mg} / \mathrm{m}^{2}$ and treosulfan 42 $\mathrm{mg} / \mathrm{m}^{2}$ (6\% of cases). Five percent and 19\% of NMA and MA conditioned patients, respectively, received anti-thymocyte globulin. Patients were followed until death or end-of-followup on October $31^{\text {st }}$, 2018. Cumulative incidences with $95 \%$ confidence intervals (CI) of acute graft-versus-host disease (aGVHD) grade II-IV, chronic graft-versus-host disease (cGVHD), relapse and non-relapse mortality (NRM) were calculated and compared between NMA and MA conditioned patients using Gray's test with death as a competing risk (or relapse when comparing NRM). Overall survival (OS) was estimated by the Kaplan-Meier method.

Results: NMA and MA conditioned patients were comparable when regarding sex $(49 \%$ and $48 \%$ female, respectively) and donor (matched related donor in $34 \%$ and $36 \%$, respectively), but differed, as expected by indication, with regards to age (median of 60 versus 42 years, respectively) and Karnofsky score $(<90$ in $18 \%$ and $11 \%$, respectively). NMA conditioned patients had generally a lower AML stage at transplant $\left(1^{\text {st }}\right.$ complete remission in $68 \%$ versus $49 \%$ of MA conditioned patients) and a lower AML cytogenetic risk (adverse risk in $17 \%$ versus $21 \%$ of MA conditioned patients). Patients were followed for a total of 2090 person-years (median follow-up in surviving patients was 6.2 years). aGVHD grade II-IV occurred less frequently in NMA conditioned patients $(20 \%$ [CI: $15 \%$ $26 \%$ ] versus $38 \%$ [CI: $32 \%-45 \%$ ] in MA conditioned patients, $\mathrm{p}<0.01$ ), while cGVHD occurred in similar rates (50\% [CI: 43\%-56\%] in NMA conditioned patients and $51 \%$ [CI: 44\%-58\%] in MA conditioned patients, $\mathrm{p}=0.77$ ). There was a trend towards a higher relapse rate in NMA conditioned patients (34\% [CI: $28 \%-40 \%$ ] versus $28 \%$ [CI: $22 \%-34 \%]$ in MA conditioned patients, $\mathrm{p}=0.07$ ), and NMA conditioned patients had, however not with statistical significance, lower NRM (20\% [CI: $14 \%-25 \%$ ] versus $25 \%$ [CI: $19 \%-31 \%$ ] in MA conditioned patients, $\mathrm{p}=0.27$ ). OS (Figure) was comparable, with 5-year OS rates of 55\% (CI: 48\%-62\%) in NMA conditioned patients and 54\% (CI: 47\%-61\%) in MA conditioned patients.

Conclusions: Patients with AML undergoing allo-HSCT with NMA conditioning at our institution were older and frailer than MA conditioned patients, but their overall survival after transplantation was comparable. This might be explained by a generally lower AML stage and cytogenetic risk at transplant in NMA conditioned patients.

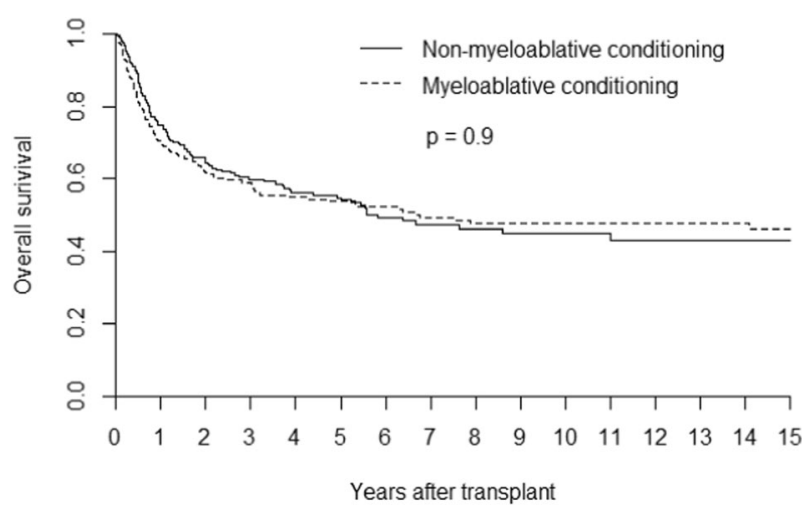

[[P019 Image] 1. Overall survival after allo-HSCT for AML in 220 NMA and 218 MA conditioned patients]

Disclosure: Nothing to declare.

P020

Low dose cytarabine may prevent hematologic relapse prior to hsct in patients with AML in first complete remission with minimal residual disease

Sarah Lindner ${ }^{1}$, Fabian Lang ${ }^{1}$, Heike Pfeifer ${ }^{1}$, Tobias Berg ${ }^{I}$, Salem Ajib ${ }^{I}$, Julia Riemann ${ }^{1}$, Zuzanna 
Jedlickova ${ }^{1}$, Saskia Güller ${ }^{1}$, Rosa Toenges ${ }^{1}$, Juliane Steinmann ${ }^{1}$, Hans Martin ${ }^{1}$, Hubert Serve ${ }^{1}$, Gesine Bug ${ }^{I}$

${ }^{1}$ University Hospital Frankfurt, Frankfurt am Main, Germany

Background: Allogeneic HSCT is urgently indicated in patients with AML in first complete hematologic remission (CHR) after intensive chemotherapy with increasing or recurrent minimal residual disease (MRD). These patients are at high risk of hematologic relapse (HR) during preparation of their transplant and HSCT with active AML was found associated with poor outcome. Azacitidine has recently been shown to substantially delay or even prevent HR in $>50 \%$ of patients (RELAZA2 trial, Platzbecker et al., Lancet Oncology 2018). We here present the outcome of a small cohort of consecutive patients with MRD-positive AML who received low dose cytarabine (LDARAC) as bridging therapy prior to HSCT.

Methods: MRD was assessed by quantitative polymerase chain reaction (qPCR) using mutated NPM1 $(\mathrm{n}=5)$, RUNX1-RUNX1T1 $(\mathrm{n}=2)$, CBFB-MYH11 $(\mathrm{n}=1)$ or KMT2A-PTD $(n=1)$. MRD negativity was defined as ratio of oncogene to control gene (ABL1) $\leq 0,01 \%$ while increased or recurrent MRD required a ratio $>1 \%$ (Shayegi et al., Blood 2013). Primary endpoint of our retrospective analysis was progression to $\mathrm{HR}$ ( $\geq 5 \%$ bone marrow blasts or extramedullary disease); secondary endpoints were achievement of molecular remission prior to HSCT, neutropenia G4 according to CTCAE, thrombocytopenia G4, anemia $\geq \mathrm{G} 3$, admission to hospital, OS and RFS. OS and RFS were calculated from the first dose of LDARAC. LDARAC was self-administered subcutaneously by the patients at home at a flat dose of 20mg BID over 10 days and repeated after 4 weeks if necessary.

Results: Between 12/2015 and 10/2018, nine patients (median age 55, range, 22-68 years) with low $(n=7)$, intermediate $(\mathrm{n}=1)$ or high-risk cytogenetics $(\mathrm{n}=1)$ according to ELN criteria 2017 were treated in continuous CHR for increasing $(n=2)$ or recurrent MRD $(n=7)$ starting at a median of 260 (range, 154-651) days after the last consolidation therapy, i.e., duration of CHR was $>6$ months in all pts. Patients received one $(n=4)$, two $(n=2)$ or three cycles $(n=3)$ of LDARAC prior to HSCT. In three patients, neutropenia G4 occurred and one patient needed platelet transfusion. All patients were managed in the outpatient setting. In eight out of nine patients (89\%), HR was successfully prevented and 3 patients (33\%) even became MRD negative prior to HSCT. One patient (RUNX1RUNX1T1 positive AML) progressed to HR after one cycle of LDARAC and received salvage therapy with high-dose ARAC and mitoxantrone (HAM) prior to HSCT. All patients proceeded to HSCT from a matched related $(n=1)$, unrelated $(n=7)$ or haploidentical donor $(n=1)$ and are still alive (median follow-up of 666 days). Conditioning regimens included fludarabine (FLU)/melphalan (MEL)/TBI $(n=5)$, FLU/MEL $(n=1)$, FLU/TBI $(n=1)$, FLU/busulfan (BU)4 $(n=1)$ and thiotepa/BU3/FLU $(n=1)$. After HSCT, only the LDARAC-refractory patient relapsed, resulting in a probability of RFS of $88 \%$ at 2 years.

Conclusions: Our data suggest that a bridging therapy with up to three cycles of LDARAC prior to HSCT is feasible and was associated with favorable outcomes in patients with NPM1-mutated or core binding factor AML and molecular relapse $>6$ months after achieving a first CHR. The treatment has low costs, can be administered on an outpatient basis and is very well tolerated.

Clinical Trial Registry: Not applicable

Disclosure: Nothing to declare

\section{P021}

Matched sibling vs haploidentical transplant for acute myeloid leukemia: A single centre experience from developing country

Shilpa Prabhu', Sharat Damodar ${ }^{1}$, K.S Nataraj ${ }^{1}$, Sunil Bhat $^{\text {I, V.M Annapandian }}{ }^{2}$, Shobha Badiger ${ }^{1}, K$ VasundharaI, Ruchi Chaudhary $^{I}$

${ }^{1}$ Narayana Health City, Hematology and Stem Cell Transplant, Bangalore, India, ${ }^{2}$ Narayana Hrudayalaya Foundations, Academic Research, Bangalore, India

Background: Allogenic hematopoietic stem cell transplant (HSCT) is the only curative treatment for all the patients with AML. High risk disease qualifies for upfront HSCT irrespective of the presence of matched sibling donor (MSD). In the absence of MSD, haploidentical stem cell transplant is easier option with success rates as high as MSD in a high volume transplant centre. We present our experience from a single centre.

Methods: We analyzed retrospective data of AML patients who have undergone HSCT at our centre between January-2013 and August-2018. For MSD transplant we used Fludarabine + Busulfan or Fludarabine + Melphalan conditioning regimen, in matched unrelated donor transplant (MUD) regime used was Fludarabine + Busulfan + ATG. We followed John Hopkins's protocol for haploidentical HSCT. Cyclosporine + methotrexate was used as GVHD prophylaxis in MSD and unrelated donor group and cyclophosphamide + tacrolimus + mycophenolate was used for haploidentical post-transplant. Day 100 Survival, overall survival (OS), incidence of GVHD and CMV reactivation was computed.

Results: A total of 96 AML patients underwent HSCT during the study period, the basic and clinical characteristics 
of the study patients are presented in Table 1. Conditioning regime did not have significant impact on OS. Survival at day 100 was $78 \%$. The OS function and relapse free survival (RFS) function did not significantly differ between MSD and Haploidentical transplantation $(68.3 \%$ vs $60.0 \%$; $\mathrm{p}=0.225)$ and $(68.3 \%$ vs $75.0 \% ; \mathrm{p}=0.760)$ (Graph 1$)$. Disease status at latest follow up showed that $82 \%$ were in remission and $18 \%$ had relapsed. Overall one year survival and five year survival in the entire cohort was $68 \%$ and $58 \%$ respectively. The average cost of MSD transplant at our centre is INR 10,00,000 ( $€$ 10000-12000), haploidentical transplant is INR 20,00,000 ( $€ 25000-27000$ ) and MUD transplant is INR $32,00,000$ ( $€ 30,000+10000$ for stem cell procurement).

Conclusions: Our study showed comparable outcomes in MSD and Haploidentical transplant with respect to Day100 survival, OS, and rate of GVHD. In a developing country like India where patients are not covered under state health insurance, the additional cost of procurement of stem cells in a MUD transplant would add to the financial burden to the patients. Haploidentical transplant is a feasible option in case of non-availability of MSD, due to ease of donor availability and strong motivation from the family donor to donate the stem cells.

\begin{tabular}{ll}
\hline Total patients $=96$ & $\mathrm{~N}(\%)$ or Mean \pm SD \\
\hline Gender (M / F) & $65(66.7 \%) / 31$ \\
& $(32.3 \%)$ \\
Age (Years) & $28.01 \pm 17.09$ \\
Matched Sibling Donors (MSD), Haplo- & $60(62.5 \%), 30$ \\
identical, Matched Unrelated Donors (MUD) & $(31.3 \%), 6(6.3 \%)$ \\
Mean CD34 cell dose & $6.8 \pm 2.8 \times 106 / \mathrm{mcl}$ \\
Acute GVHD & $50(52.1 \%)$ \\
Chronic GVHD & $9(9.4 \%)$ \\
CMV reactivation & $70(72.9 \%)$ \\
Platelets engraftment day & $14.9 \pm 5.9$ \\
Neutrophil engraftment day & $13.8 \pm 3.3$ \\
\hline
\end{tabular}

[[P021 Table] 1. Basic demographics and clinical details of the study patients]

Disclosure: Nothing to declare

\section{P022}

Favorable-risk acute myeloid leukemia with mutant NPM1: The role of allogeneic hematopoietic stem cell transplantation

Chara Giatra $^{1}$, Ioannis Baltadakis ${ }^{1}$, Ioannis Tsonis ${ }^{1}$, Zois Mellios $^{1}$, Dimitra Oikonomopoulou ${ }^{1}$, Konstantinos Gkirkas $^{2}$, Maria Garofalaki ${ }^{1}$, Eirini Tziotziou ${ }^{1}$, Zoi Arapidou $^{1}$, Tatiana Tzenou ${ }^{1}$, Maria Stamouli', Maria
Bouzani $^{1}$, Sosana Delibasi ${ }^{1}$, Maria Pagoni ${ }^{1}$, Stavros Gigantes $^{1}$, Panagiotis Tsirigotis ${ }^{2}$, Nikolaos Harhalakis ${ }^{1}$, Dimitrios Karakasis ${ }^{I}$

${ }^{1}$ Evangelismos General Hospital, Athens, Greece, ${ }^{2}$ University of Athens, Attikon Hospital, Athens, Greece,

Background: Allogeneic stem cell transplantation (alloHSCT) is not indicated as consolidation of first complete remission (CR1) in favorable-risk acute myeloid leukemia (AML) bearing mutations in nucleophosmin (NPM1) in the absence of FLT3 internal tandem duplication (FLT3-ITD). Nevertheless, a substantial proportion of patients eventually proceed to allo-HSCT beyond CR1 or for chemoresistant minimal residual disease (MRD) while in CR1, which might compromise transplantation outcomes. The study aimed at examining the characteristics and results of allo-HSCT in AML cases with mutated NPMI and wild-type FLT3 (NPMImut/FLT3wt), with special focus on molecular monitoring of MRD following transplantation.

Methods: From 11/2010 until 04/2018, 16 patients (women/men, 9/7) underwent allo-HSCT for NPMlmut/ FLT3wt AML. At transplant, median age of patients was 44.5 years (range, 35-63), and disease phase was CR1 $(n=5), C R 2(n=9)$, or primary refractory $(n=2)$. Among the 13 patients who were transplanted in CR and had available molecular MRD assessments, 10 had detectable mutant NPMI transcripts by real-time quantitative PCR (RQ-RCR). Also, 4 patients fulfilled criteria of molecular relapse (increasing levels of NPM1-mutated transcripts in two successive bone marrow samples), with mutant NPM1 load of 386-4,900 transcripts/10,000 $A B L$ transcripts). The conditioning regimen was myeloablative in the majority of cases $(n=14)$ or reduced-intensity $(n=2)$. The type of donor varied, namely HLA-identical sibling $(n=6)$, matched unrelated $(n=5)$, haploidentical relative $(n=3)$, or double umbilical cord blood $(n=2)$.

Results: Engraftment was achieved in all cases, with a median time to absolute neutrophil count $>500 / \mathrm{uL}$ of 16 days (range, 12-29). Among the 13 patients with posttransplant monitoring of MRD by RQ-PCR, 9 exhibited a stable molecular remission whereas a rising level of NPM1mutated transcripts was observed in 4 cases due to either hematologic $(n=3)$ or molecular $(n=1)$ relapse of disease. The cumulative incidences (CIN) of hematologic relapse and non-relapse mortality (NRM) were $18.75 \%$ and $25 \%$ at 12 months, respectively. No events of relapse or NRM were encountered beyond 6 months from allo-HSCT. Out of 3 patients with hematologic relapse post transplant, 2 died of disease whereas one achieved a stable complete remission after withdrawal of immunosuppression. At a median follow-up time of 40 months (range, 14-89), 10/16 patients continue to be alive in CR. The estimated disease-free 
(DFS) and overall survival (OS) at 3 years is $56.2 \%(95 \%$ CI, 36.5-86.7\%) and $62.5 \%$ (95\% CI, 42.8-91.4\%), respectively.

Conclusions: Although the majority of patients are transplanted in relatively advanced phase of disease, alloHSCT remains a highly curative modality for NPMImut/ FLT3wt AML cases with refractory disease or molecular relapse following conventional therapy. Regular monitoring of MRD by RQ-PCR, as well as identification of additional prognostic molecular markers by next-generation sequencing (NGS) may allow better timing of allo-HSCT with further improvement of outcomes.

Disclosure: Nothing to declare.

\section{P023}

\section{Abstract already published.}

\section{P024}

Pooled clinical safety analysis of CPX-351 versus conventional chemotherapy in patients with newly diagnosed or relapsed/refractory acute myeloid leukemia (AML)

\section{Jorge E Cortes', Robert J Ryan', Arthur C Louie, Michael Chiarella ${ }^{2}$, Geoffrey $L \mathrm{Uy}^{3}$}

${ }^{1}$ University of Texas MD Anderson Cancer Center, Houston, TX, United States, ${ }^{2}$ Jazz Pharmaceuticals, Inc., Palo Alto, CA, United States, ${ }^{3}$ Washington University School of Medicine, St. Louis, MO, United States

Background: CPX-351 (Vyxeos ${ }^{\circledR}$ ) is an advanced liposomal encapsulation of cytarabine/daunorubicin at a synergistic 5:1 molar ratio. CPX-351 is approved by the US FDA and EMA for the treatment of adults with newly diagnosed, therapy-related AML or AML with myelodysplasia-related changes.

Methods: Safety data were pooled from 5 studies of CPX-351 in adults aged 18-75 years with newly diagnosed or relapsed/refractory AML. CPX-351 induction consisted of 100 units $/ \mathrm{m}^{2}$ (cytarabine $100 \mathrm{mg} / \mathrm{m}^{2}+$ daunorubicin 44 $\mathrm{mg} / \mathrm{m}^{2}$ ) on Days 1,3 , and 5 (second induction: Days 1 and 3). CPX-351 consolidation consisted of 65 or 100 units $/ \mathrm{m}^{2}$ (varying by study) on Days 1 and 3. CPX-351 was evaluated against standard-of-care controls.

Results: Baseline characteristics were generally balanced between CPX-351 $(n=375)$ and controls $(n=236)$; the majority of patients were aged $\geq 60$ years $(78 \% ; 87 \%)$ and had secondary AML $(55 \% ; 72 \%)$. Controls included $7+3$ $(n=192)$ and salvage therapy with mitoxantrone/etoposide/ cytarabine $(n=23)$, idarubicin/cytarabine $(n=8)$, other cytarabine-based chemotherapy $(n=12)$, and mitoxantrone/ etoposide $(n=1)$. The treatment-emergent adverse event
(TEAE) profile of CPX-351 100 units $/ \mathrm{m}^{2}$ was comparable to induction controls, but associated with a greater proportion of patients with TEAEs, grade $\geq 3$ TEAEs, and serious TEAEs during consolidation (Table). Therefore, the CPX-351 consolidation dose was reduced to 65 units $/ \mathrm{m}^{2}$ in latter studies; this dose demonstrated an improved TEAE profile similar to consolidation controls. The most frequent system organ class was gastrointestinal disorders for both CPX-351 and controls; a lower incidence was reported for CPX-351 (90\%) versus controls (95\%), with this difference driven by the lower incidence of diarrhea for CPX-351 (46\%) versus controls $(66 \%)$. The most frequently reported grade $\geq 3$ TEAEs were febrile neutropenia (CPX-351: 62\%; controls: $59 \%)$, pneumonia $(16 \% ; 13 \%)$, hypoxia $(10 \%$; $11 \%)$, and bacteremia $(10 \% ; 3 \%)$. Early mortality rates, both overall and by treatment period, appeared lower with CPX-351 versus controls at Day 30 and Day 60 (Table); the majority of early deaths were attributable to TEAEs.

Conclusions: Across the 5 studies comprising the CPX351 clinical development program, CPX-351 demonstrated a safety profile comparable to conventional chemotherapy in adults with newly diagnosed or relapsed/refractory AML.

\begin{tabular}{|c|c|c|c|c|c|c|c|}
\hline \multirow[b]{2}{*}{$\mathrm{n}(\%)$} & \multicolumn{2}{|c|}{ Induction period } & \multicolumn{3}{|c|}{ Consolidation period } & \multicolumn{2}{|c|}{$\begin{array}{l}\text { Overall } \\
\text { treatment period }\end{array}$} \\
\hline & $\begin{array}{l}100 \text { units } / \mathrm{m}^{2} \\
\text { CPX-351 } \\
(\mathrm{n}=375)\end{array}$ & $\begin{array}{l}\text { All } \\
\text { controls } \\
(\mathrm{n}=236)\end{array}$ & $\begin{array}{l}65 \text { units } / \mathrm{m}^{2} \\
\text { CPX- } \\
351(\mathrm{n}=52)\end{array}$ & $\begin{array}{l}100 \text { units } / \mathrm{m}^{2} \\
\text { CPX- } \\
351(\mathrm{n}=63)\end{array}$ & $\begin{array}{l}\text { All } \\
\text { controls }(n=55)\end{array}$ & $\begin{array}{l}\mathrm{CPX}-351 \\
(\mathrm{n}=375)\end{array}$ & $\begin{array}{l}\text { All } \\
\text { controls } \\
(\mathrm{n}=236)\end{array}$ \\
\hline Any TEAE & $375(100)$ & $236(100)$ & $44(85)$ & $61(97)$ & $49(89)$ & $375(100)$ & $236(100)$ \\
\hline $\begin{array}{l}\text { Grade } \\
\geq 3 \text { TEAE }\end{array}$ & $342(91)$ & $211(89)$ & $28(54)$ & $46(73)$ & $30(55)$ & $346(92)$ & $212(90)$ \\
\hline Serious TEAE & $145(39)$ & $76(32)$ & $25(48)$ & $33(52)$ & $24(44)$ & $189(50)$ & $91(39)$ \\
\hline $\begin{array}{l}\text { Treatment- } \\
\text { related TEAE }\end{array}$ & $362(97)$ & $220(93)$ & $36(69)$ & 49 (78) & $39(71)$ & $363(97)$ & $220(93)$ \\
\hline $\begin{array}{l}\text { TEAE leading } \\
\text { to } \\
\text { discontinuation }\end{array}$ & $7(2)$ & $3(1)$ & 0 & 0 & 0 & 7 (2) & $3(1)$ \\
\hline $\begin{array}{l}\text { TEAE leading } \\
\text { to death }\end{array}$ & $28(7)$ & $25(11)$ & $1(2)$ & $5(8)$ & $4(7)$ & $34(9)$ & $29(12)$ \\
\hline $\begin{array}{l}\text { 30-day } \\
\text { mortality }\end{array}$ & $14(4)$ & $15(8)$ & $1(2)$ & $2(3)$ & $2(6)$ & $21(6)$ & $19(10)$ \\
\hline $\begin{array}{l}\text { 60-day } \\
\text { mortality }\end{array}$ & $23(6)$ & 17 (9) & $1(2)$ & $2(3)$ & $2(6)$ & $47(13)$ & $38(20)$ \\
\hline
\end{tabular}

\section{[[P024 Table] 1. Table]}

Clinical Trial Registry: NCT00389428, NCT00788892, NCT00822094, NCT02238925, NCT01696084

Disclosure: JE Cortes has received consulting fees from Jazz, Novartis, Daiichi Sankyo, Pfizer, and Astellas Pharma, and research funding from Jazz, Novartis, Daiichi Sankyo, Pfizer, Astellas Pharma, and Arog. RJ Ryan, M Chiarella, and AC Louie are employees of and hold stock ownership in Jazz; AC Louie additionally has patents/royalties with Jazz. GL Uy has received consulting fees from Curis and GlycoMimetics.

\section{P025}

Comparison of outcomes after HLA-haploidentical related versus unrelated hematopoietic stem cell 
transplantation with post-transplantation cyclophosphamide for acute leukemia

László Gopcsa ${ }^{1}$, Hajnalka Andrikovics ${ }^{2}$, Anikó Barta ${ }^{1}$, Árpád Bátai ${ }^{1}$, Ottó Csacsovszki ${ }^{1}$, Zoltán Csukly ${ }^{1}$, János Dolgos $^{1}$, János Fábián ${ }^{1}$, Zita Farkas ${ }^{1}$, Anikó Fodor ${ }^{1}$, Apor Hardi, Ágnes Király', Gergely Lakatos ${ }^{1}$, Enikö Lehoczki $^{1}$, Lilla Lengyel, Nora Lovas ${ }^{1}$, Zoltán Mátrai ${ }^{1}$, Gábor Mikala ${ }^{1}$, Nora Meggyesi ${ }^{2}$, Melinda Paksi ${ }^{1}$, Mónika Petô $^{1}$, Katalin Rajczy ${ }^{3}$, Marienn Réti ${ }^{1}$, Eszzter Sári ${ }^{1}$, János Sinkó ${ }^{1}$, Andrea Sipos ${ }^{1}$, Anikó Szilvási ${ }^{3}$, Éva Torbágyi ${ }^{1}$, István Vályi-Nagy ${ }^{1}$, Tamás Masszi ${ }^{4}$, Péter Reményi ${ }^{1}$

${ }^{1}$ Central Hospital of Southern-Pest, National Institute of Hematology and Infectious Diseases, Haematology and Stem Cell Transplantation, Budapest, Hungary, ${ }^{2}$ Central Hospital of Southern-Pest, National Institute of Hematology and Infectious Diseases, Molecular Genetic Laboratory, Budapest, Hungary, ${ }^{3}$ National Blood Transfusion Service, Budapest, Hungary, ${ }^{4}$ Semmelweis University 3 rd. Budapest, Hungary

Background: Haploidentical hematopoietic stem cell transplantation (HSCT) with post-transplantation Cyclophosphamide (PGCY) marked improved clinical outcome. Recent studies comparing allogeneic HSCT using unrelated donors versus haplo donors in patients with acute leukemia have suggested equivalent outcomes. The depletion of Tcells with PGCY was subsequently applied for unrelated HSCT setting for patients with unrelated donor.

Methods: We performed a retrospective study on 90 patients with acute leukemia in order to compare the outcome after HLA haploidentical $(n=30)$ and unrelated HSCT $(n=60)$ with PGCY. The main characteristics of patients were similar in both groups. Baseline disease were: 19 AML (63\%) and 11 ALL (37\%) for Haplo group and 33 AML (55\%) and 27 ALL (45\%) for unrelated group. Disease state at time of haplo and unrelated-HSCT were following: 19 and 50 patients in CR1 (63\% and 83\%) and 11 and 10 non CR1 (37\% and 17\%). For AML recipients mainly received thiotepa, busulfan and fludarabine and for ALL recipients received TBI and Etoposide conditioning. All patients who received PBSC graft were treated with rabbit antithymocyte globulin (ATG) on days -2 and -1 .

Results: At the time of analysis, the OS and DFS did not differ between the Haplo and unrelated groups (67\% vs $63 \%$, and $63 \%$ vs $56 \%$ ). Incidence of severe (grade 3-4) acute GVHD was the same in two groups (10\% versus $8 \%$ ). Recipients of Haplo-HSCT transplant were statistical significance less likely to experience disease relapse (3\% vs $28 \%$ ) and chronic GVHD (20\% vs $47,5 \%)$. However, GVHD free relapse free survival (GRFS) rate was slightly higher after Haplo-HSCT (77\% vs 64\%). Addition, cumulative incidence of TRM rate was higher after Haplo-HSCT (30\% vs 15\%).For Haplo and unrelated groups who underwent HSCT in CR1, the OS were $84 \%$ and $67 \%$ versus $22 \%$ and $45 \%$ for those in non CR1. For AML, the OS was same in two groups (Haplo 63\% versus unrelated 67\%). However patients with ALL, the OS was higher in Haplo group compared with unrelated group (72\% versus 59\%). The impact of pretransplant disease state have a more powerfull effect on survival in the Haplo-HSCT setting (for AML CR1 77\% versus non CR1 33\% and for ALL CR1 100\% versus non CR1 0\%). Viral reactivations were significant concern in both groups.

Conclusions: Our retrospective analysis suggests largerly similar OS and DFS with Haplo versus unrelated transplants with PGCY for acute leukemia. Our data indicate that Haplo-HSCT results in a lower incidence relapse and of chronic GVHD and higher GRFS compared with unrelated HSCT. In addtion, the pretransplant disease state have the important effect on the outcomes in both groups. AlloPBSC with ATG can be used safely and effective as graft source in Haplo-HSCT with acceptable post-transplant outcomes and replaced BM in this settings. More statistical data for transplant related characteristics will be provided at the presentation.We emphasize that use the same PGCY GVHD prophylaxis for all types of allogeneic transplant. Based on our results, we recommend Haplo-HSCT with PGCY against unrelated transplant for patients with acute leukemia.

Disclosure: Disclosure of conflict of interest: None.

\section{P026}

Excellent efficacy and tolerability of inotuzumab ozogamicin in B-cell all relapsed after allo-HSCT

Teresa Artola ${ }^{1}$, José Guinea ${ }^{2}$, Lourdes Aguirre ${ }^{1}$, Nagore Argoitial, Nerea Uresandi $^{1}$, Carmen González ${ }^{1}$, María Araiz $^{1}$, M. José Pizarro ${ }^{1}$, Dolores de Juan ${ }^{1}$, Carlos Vallejo $^{I}$

${ }^{1}$ University Hospital Donostia, San Sebastián, Spain, ${ }^{2}$ University Hospital of Araba, Hematology, Vitoria/Gasteiz, Spain,

Background: allo-HSCT is a potentially curative approach for high-risk B-cell ALL. However, relapse after-HSCT continues to be an issue, and the management of this situation is difficult, challenging, and with a very poor survival. Inotuzumab ozogamicin is a humanized CD22 monoclonal antibody linked to the cytotoxic agent calicheamicin. Inotuzumab has shown striking clinical activity even among heavily pretreated relapsed/refractory ALL patients. However, the experience in the post-allo-HSCT setting is very limited. 
Methods: 211 pts underwent first allo-HSCT between January 2015 and October 2018 in our center, including 28 pts with ALL (our series). Median follow-up of the series was 17 months (range: 2-47). Characteristics of the series are shown in table 1. Five pts died in CR from non-relapse mortality (17.9\%); three of those pts died during the first 3 months after HSCT, and the other two at 8 and 15 months post-HSCT. Seven pts $(25 \%)$ relapsed after-HSCT; the disease was $\mathrm{CD} 22$ and $\mathrm{CD} 19$ positive in all the 7 cases. One patient, with a Down syndrome, received palliative care and died due to the relapse. The rest of the patients (6) received treatment for the ALL as reflected in table 2. The only patient with $\mathrm{Ph}+\mathrm{ALL}$ received ponatinib, while the $5 \mathrm{Ph}$ neg patients received treatment with Inotuzumab.

\begin{tabular}{|c|c|c|}
\hline & $\begin{array}{l}\text { Allo-HSCT for } \\
\text { ALL }(\mathrm{n}=28)\end{array}$ & $\begin{array}{l}\text { ALL-relapse after } \\
\text { allo-HSCT }(\mathrm{n}=7)\end{array}$ \\
\hline $\begin{array}{l}\text { Age (years) (median } \\
\text { [range]) }\end{array}$ & $43[7-66]$ & $31[22-53]$ \\
\hline Gender (male/female) & $\begin{array}{l}13(46,4 \%) / 15 \\
(53,6 \%)\end{array}$ & $\begin{array}{l}2 / 13(15,4 \%)--5 / 15 \\
(33,3 \%)\end{array}$ \\
\hline $\begin{array}{l}\text { ALL status at allo-HSCT } \\
\text { (RC1 // RC 2) }\end{array}$ & $\begin{array}{l}17(60,7 \%) / / \\
11(39,3 \%)\end{array}$ & $\begin{array}{l}2 / 17(11,8 \%) / / 5 / 11 \\
(45,5 \%)\end{array}$ \\
\hline Phenotype (B // T) & $\begin{array}{l}25(89,3 \%) / / 3 \\
(10,7 \%)\end{array}$ & $7 / 25(28 \%) / / 0 / 3(0 \%)$ \\
\hline $\begin{array}{l}\mathrm{Ph} / \mathrm{bcr} \text {-abl (negative // } \\
\text { positive) }\end{array}$ & $\begin{array}{l}20(71,4 \%) / / 8 \\
(28,6 \%)\end{array}$ & $\begin{array}{l}6 / 20(30 \%) / / 1 / 8 \\
(12,5 \%)\end{array}$ \\
\hline $\begin{array}{l}\text { Donor (URD // MSD } \\
\text { // Haplo) }\end{array}$ & $16 / / 11 / / 1$ & $\begin{array}{l}4 / 16(25 \%) / / 2 / 11 \\
(18,2 \%) / / 1 / 1(100 \%)\end{array}$ \\
\hline $\mathrm{SC}$ source $(\mathrm{PB} / / \mathrm{BM})$ & $25 / / 3$ & $\begin{array}{l}6 / 25(24 \%) / / 1 / 3 \\
(33,3 \%)\end{array}$ \\
\hline $\begin{array}{l}\text { Conditioning type (TBI-Cy } \\
\text { // TBF // others) }\end{array}$ & $14 / / 11 / / 3$ & $\begin{array}{l}5 / 15(33,3 \%) / / 1 / 11 \\
(9,1 \%) / / 1 / 1(100 \%)\end{array}$ \\
\hline $\begin{array}{l}\text { Conditioning intensity } \\
\text { (Intensive // RIC // NMA) }\end{array}$ & $20 / / 7 / / 1$ & $\begin{array}{l}6 / 20(30 \%) / / 0 / 7(0 \%) \\
/ / 1 / 1(100 \%)\end{array}$ \\
\hline
\end{tabular}

[[P026 Table] 1. TABLE 1: Characteristics of patients and transplants.]

Results: None of the 5 Inotuzumab-treated patients developed VOD/SOS or major toxicities. Of note, majority of the doses were administered in the outpatient setting. As shown in table 3 , all the patients reached a new $\mathrm{CR}$, recovered donor chimerism, and were focused to received anti-CD19 CAR-T cells.

\section{Conclusions:}

1) In our knowledge, this is the first series of ALL pts relapsed after allo-HSCT treated with Inotuzumab;

2) The tolerance of Inotuzumab in the post-transplant setting was very good;

3) The reported treatment approach resulted in an excellent outcome with all the patients reaching a new $\mathrm{CR}$, and recovering the donor chimerism;
4) Anti-CD22 therapy with Inotuzumab was successfully employed as a bridge to CAR-T consolidation strategy with a different leukemic molecular target (CD19);

5) The presented approach seems to be a feasible, welltolerated, and effective strategy for CD22-positive ALL patients who relapse after allo-HSCT.

Disclosure: Nothing to declare.

\section{P027}

Prophylactic donor lymphocyte infusion after haploidentical versus matched-sibling PBSCT in very high-risk acute myeloid leukemia: Similar safety and efficacy for reducing relapse

Xiao-Ning Gao ${ }^{1}$, Ji Lin ${ }^{2}$, Li-Jun Wang ${ }^{1}$, Fei Li ${ }^{1}$, HongHua Li ${ }^{I}$, Shu-Hong Wang ${ }^{1}$, Wen-Rong Huang ${ }^{I}$, Chun-Ji Gao $^{I}$, Li Yu ${ }^{I}$, Dai-Hong Liu ${ }^{I}$

${ }^{1}$ Chinese PLA General Hospital, Beijing, China, ${ }^{2}$ Chinese PLA General Hospital, Institute of Basic Medicine, Beijing, China

Background: Donor lymphocyte infusion (DLI) could be used prophylactically to reduce relapse after allogeneic hematopoietic stem cell transplantation for very high-risk leukemia/lymphoma without effective targeted therapy. To compare the safety and efficacy of prophylactic DLI for prevention of relapse after allogeneic peripheral blood stem cell transplantation from haploidentical donors (HID-SCT) and matched-sibling donors (MSD-SCT) in patients with very high-risk acute myeloid leukemia (AML), we performed a retrospective, observational cohort study enrolled in 21 HID-SCT and 13 MSD-SCT recipients.

Methods: The very high-risk features were defined as:

(i) in the non-remission (NR) state prior to transplantation, including primary induction failure, relapse untreated or refractory to reinduction chemotherapy, or untreated AML evolution from MDS;

(ii) achieving complete remission 1 with $\geq 3$ cycles of induction of chemotherapy;

(iii) carrying TP53, DNMT3a, TET2 or FLT3-ITD gene mutation.

The scheduled time of the prophylactic DLI was +3060 days after transplantation for MSD-SCT recipients and $+60-90$ days for HID-SCT recipients. The G-CSFmobilized peripheral blood stem cells were infused to the recipient at a dose of $2 \times 10^{7} \mathrm{CD}^{+}$cells $/ \mathrm{kg}$. CsA was given at $2 \mathrm{mg} / \mathrm{kg}$ b.i.d from day -3 to day +90 (HID-SCT) or to day +60 (MSD-SCT), and then tapered at 33\% per month to be discontinued on day $+150-180$ (HID-SCT) or on day $+120-150$ (MSD-SCT) unless graft-versus-host disease (GVHD) developed. If the patients received DLI before day +90 (HID-SCT) or day +60 (MSD-SCT), CsA was 
given 8 weeks after DLI in HID group and 4 weeks in MSD group at a though concentration of $150-250 \mathrm{ng} / \mathrm{ml}$ for DLIassociated GVHD prophylaxis, and then tapered and discontinued within 2 weeks unless GVHD developed. If GVHD occurred before the scheduled time of prophylactic DLI, it would be delayed for 8 weeks when GVHD was well controlled.

Results: Prophylactic DLI was administered at a median of 71 (34-240) days for HID-SCT recipients and 53 (35-97) days for MSD-SCT recipients $(p=0.008)$, and both groups displayed similar baseline characteristics except for donor's gender distribution (Table 1). Grade 2-4 acute graft-versushost disease (GVHD) at 100-day post-DLI was higher in HID-SCT group than that in MSD-SCT group (59.5\% vs. $30.8 \%, p=0.05)$. Grade $3-4$ acute GVHD (17.5\% vs. $7.7 \%)$, 1-year chronic GVHD (36.6\% vs. $33.2 \%)$ and severe chronic GVHD (15.3\% vs. $27.3 \%)$ were similar between two groups $(p>0.05)$. One-year non-relapse mortality was higher in HID-SCT group than that in MSD-SCT group with marginal significance $(27.9 \%$ vs. $0.0 \%, p=0.061)$. One-year relapse rate was similar between HID-SCT group and MSD-SCT group (21.6\% vs. 36.5\%, $p>0.05$ ). Estimated 1-year overall survival (OS, $55.1 \%$ vs. $83.9 \%$ ) and relapsefree survival (RFS, $50.1 \%$ vs. $74.0 \%$ ) rates were both similar between HID-SCT group and MSD-SCT group $(p>0.05)$. In multivariate analyses, non-remission status prior to transplant, poor-risk gene mutations and donor's age $\geq 48$ years predicted a higher risk of relapse after DLI. Nonremission status prior to transplant predicted inferior OS and RFS. Patient's age $\geq 40$ years also predicted an inferior OS.

Conclusions: Prophylactic DLI after HID-SCT demonstrated similar tolerance and efficacy for reducing relapse compared to that after MSD-SCT for very high-risk AML.

Disclosure: The authors declare no conflict of interest.

\section{P028}

\section{Prognostic impact of pre-transplant TIM3 levels on} transplant outcome in acute leukemia patients

\section{Zeynep Arzu Yegin ${ }^{1}$, Ferda Can ${ }^{1}$, Lale Aydin Kaynar', Sanem Gokcen ${ }^{I}$, Rezzan Eren Sadoglu', Zubeyde Nur Ozkurt ${ }^{l}$, Ozlem Karacaoglu ${ }^{I}$}

\section{${ }^{1}$ Gazi University Faculty of Medicine, Ankara, Turkey}

Background: $\mathrm{T}$ cell immunoglobulin and mucin domaincontaining protein-3 (TIM3), a negative regulator of T cells, is expressed on a variety of tumors including hematological malignancies like acute myeloid leukemia (AML) and some lymphoma types in which it was shown to be associated with an adverse prognosis. The aim of this study is to identify the prognostic impact of pre-transplant TIM3 levels on early and late transplant related complications as well as post-transplant relapse and survival

Methods: A total of 177 hematopoietic stem cell transplantation (HSCT) recipients with an initial diagnosis of acute leukemia [median age: 36(16-66) years; male/ female: 111/66] were included in the study. AML was the initial diagnosis in 99 patients (55.9\%), acute lymphoblastic leukemia (ALL) in 74 patients $(41.8 \%)$, mixed phenotype acute leukemia in 3 patients (1.7\%) and blastic plasmacytoid dendritic cell neoplasm in 1 patient $(0.6 \%)$. Soluble TIM-3 levels in pre-transplant serum samples were measured with Enzyme Linked Immunosorbent Assay (ELISA).

Results: Median pre-transplant TIM3 level was 955.6 (65.8-3784.4) $\mathrm{pg} / \mathrm{mL}$ in the whole cohort. Pre-transplant TIM3 levels were significantly higher in AML patients when compared to ALL [1063.7(409.5-3784.4) vs 831.4 (65.8-3254.4); $\mathrm{p}=0.01]$. TIM3 levels were significantly lower in patients with abnormal cytogenetics when compared to normal karyotype $(\mathrm{p}=0.017)$. Cytogenetic abnormalities, including mainly a complex karyotype or chromosome 8 abnormalities, were more frequent in patients with low TIM3 levels $(\mathrm{p}=0.053)$. Pre-transplant TIM3 levels were significantly higher in patients who developed post-transplant viral hemorrhagic cystitis $(\mathrm{p}=0.034)$. A positive correlation was demonstrated between TIM3 levels and acute graft versus host disease (GvHD) grade $(\mathrm{p}=0.013 ; \mathrm{r}=0.299)$. At a median follow-up of $14.6(0.2-160.9)$ months, overall survival (OS) was found to be better in low-TIM3 group when compared to highTIM3 group, without statistical significance (\%35.2 vs \% 20.4; $>0.05$ ) (Figure 1). Probability of OS was relatively better in both AML $(42.6 \%$ vs $26.7 \%$; $>0.05)$ and ALL patients $(29.5 \%$ vs $19 \%$; p>0.05) representing low pretransplant TIM3 levels in the subgroup analysis

Conclusions: In this study, elevated levels of pretransplant TIM3 levels in AML patients were compatible with the previous reports which had underlined an increased TIM3 expression on AML stem cells. The possible association of TIM3 expression with cytogenetic features should be confirmed with further studies as there is no adequate data except its relationship with FLT3-ITD mutational status. TIM-3 is also expressed on exhausted $\mathrm{T}$ cells in patients with viral infections, including human immunodeficiency virus, hepatitis B and hepatitis $\mathrm{C}$ virus. It plays an essential role in the regulation of antiviral and antitumor immune responses which may be an explanation for the increased frequency of hemorrhagic cystitis in patients with higher TIM-3 levels. The adverse prognostic impact of TIM3 on GvHD and OS was confirmed without statistical significance which may be related to small sample size. As TIM3 has a wide spectrum of action in the tumor microenvironment including stimulatory and inhibitory 
activities, further clues are required to define the exact role of this molecule in the clinical course of allogeneic HSCT in order to develop targeted therapeutic strategies

Clinical Trial Registry: N/A

Disclosure: Nothing to declare

\section{P029}

Homozygous HLA-C1 is associated with increased risk of relapse after HLA-matched transplantation in recipients with acute lymphoid leukemia: A Japanese national registry study

Nobuyoshi Arima ${ }^{1}$, Junya Kanda ${ }^{2}$, Toshio Yabe ${ }^{3}$, Yasuo Morishima $^{4}$, Junji Tanaka ${ }^{5}$, Shinichi Kako ${ }^{6}$, Hirotoshi Sakaguchi $^{7}$, Motohiro Kato ${ }^{8}$, Kazuteru Ohashi ${ }^{9}$, Yukiyasu Ozawa $^{10}$, Takahiro Fukuda ${ }^{11}$, Shuichi Ota ${ }^{12}$, Heiwa Kanamori $^{13}$, Makoto Onizuka ${ }^{14}$, Tatsuo Ichinohe ${ }^{15}$, Yoshiko Astuta ${ }^{16,17}$, Yoshinobu Kanda ${ }^{6,18}$

${ }^{1}$ Medical Research Institute Kitano Hospital, Osaka, Japan, ${ }^{2}$ Kyoto University, Kyoto, Japan, ${ }^{3}$ Japanese Red Cross Kanto-koshinetsu Block Blood Center, Tokyo, Japan, ${ }^{4}$ Central Japan Cord Blood Bank, Seto, Japan, ${ }^{5}$ Tokyo Women's Medical University, Tokyo, Japan, ${ }^{6}$ Saitama Medical Center, Jichi Medical UniversitySaitama, Japan, ${ }^{7}$ Japanese Red Cross Nagoya First Hospital, Children's Medical Center, Nagoya, Japan, ${ }^{8}$ National Center for Child Health and Development, Children's Cancer Center, Tokyo, Japan, ${ }^{9}$ Tokyo Metropolitan Cancer and Infectious Diseases Center, Komagome Hospital, Tokyo, Japan, ${ }^{10}$ Japanese Red Cross Nagoya First Hospital, Nagoya, Japan, ${ }^{11}$ National Cancer Center Hospital, Tokyo, Japan, ${ }^{12}$ Sapporo Hokuyu Hospital, Sapporo, Japan, ${ }^{13}$ Kanagawa Cancer Center, Yokohama, Japan, ${ }^{14}$ Tokai University School of Medicine, Hematology and Oncology, Isehara, Japan, ${ }^{15}$ Hiroshima University Hospital, Hiroshima, Japan, ${ }^{16}$ Japanese Data Center for Hematopoietic Cell Transplantation, Nagoya, Japan, ${ }^{17}$ Nagoya University Graduate School of Medicine, Nagoya, Japan, ${ }^{18}$ Jichi Medical University, Shimotsuke, Japan

Background: After hematopoietic stem cell transplantation (HSCT), the role of natural killer (NK) cells which express killer-cells immunoglobulin-like receptors (KIRs) and recognize HLA-class 1 ligands is important. KIR2DL1 recognizes not HLA-C ${ }^{\text {Asp80 }}$ (C1), but HLA$\mathrm{C}^{\mathrm{Lys} 80}(\mathrm{C} 2)$ and has polymorphism based on the $245^{\text {th }}$ amino acid of the transmembrane domain. Low frequency of C2 and high frequency of strong KIR2DL1 are characteristics observed in Japanese. By using large transplant database, we reported that homozygous HLA-C1 (C1/C1) recipients displayed lower relapse rates than did $\mathrm{C} 1 / \mathrm{C} 2$ recipients after HLA-matched HSCT for acute myeloid leukemia (AML; HR $=.79, \mathrm{P}=.006$ ) or chronic myeloid leukemia $(\mathrm{CML} ; \mathrm{HR}=.48, \mathrm{P}=.025)$. This effect seemed to be independent of acute graft-versus-host disease (aGVHD) or cytomegalovirus reactivation occurrence (Arima N et al BBMT 2018).

Methods: Relapse rates of Japanese recipients who first underwent HLA-matched HSCT between 1996 and 2016 for the treatment of acute lymphoid leukemia (ALL) were compared between $\mathrm{C} 1 / \mathrm{C} 1$ pairs and $\mathrm{C} 1 / \mathrm{C} 2$ pairs, using data from Japanese Data Center for Hematopoietic Cell Transplantation and adjusting for transplant characteristics. Cord blood transplantation was excluded. Multivariable competing risk regression analyses were performed to evaluate relapses and relapse-free survival (RFS) was estimated using Kaplan-Meier method.

Results: After 61 recipients who did not achieve remission or experienced graft failure and 41 recipients not-expressing $\mathrm{C} 1$ were excluded, resting 2779 recipients aged 0-72 years (median, 31.2 years) were analyzed. The median follow-up period for survivors was 5.0 years. There were 2447 recipients expressing $\mathrm{C} 1 / \mathrm{C} 1$ and 332 recipients expressing $\mathrm{C} 1 / \mathrm{C} 2$, respectively. After HLA-matched HSCT, C1/C1 recipients had higher relapse rates than $\mathrm{C} 1 /$ $\mathrm{C} 2$ recipients $(\mathrm{HR}=1.55, \mathrm{P}=.003)$, resulting in worse RFS among $\mathrm{C} 1 / \mathrm{C} 1$ recipients $(\mathrm{HR}=1.27, \mathrm{P}=.034)$. The frequent relapse in $\mathrm{C} 1 / \mathrm{C} 1$ recipients than in $\mathrm{C} 1 / \mathrm{C} 2$ was noticeable among recipients with aGVHD $(\mathrm{HR}=1.89, \mathrm{P}=$ $.002)$, those without cytomegalovirus reactivation $(\mathrm{HR}=$ $1.84, \mathrm{P}=.002)$, and those with Ph-negative ALL (HR = $1.88, \mathrm{P}=.001$ ).

Conclusions: KIR2DL1-positive NK cells may promote graft-versus-leukemia (GVL) in $\mathrm{C} 1 / \mathrm{C} 1$ recipients with AML or CML but suppress GVL in $\mathrm{C} 1 / \mathrm{C} 1$ recipients with ALL. One interpretation is that transplant-activated NK cells impair antigen-presenting cells or deprive cytotoxic Tlymphocytes of their GVL effects on ALL cells. This hypothesis may be explained by the fact that aGVHD was necessary for the recessive relapse in $\mathrm{C} 1 / \mathrm{C} 1$ recipients with ALL. Furthermore, Ph-positive ALL cells sometimes mimic AML cells in terms of their frequent myeloid antigen expression and might be directly targeted by NK cells. It would be necessary to further clarify in vitro the character of NK cell-affecting in the transplant immunity against residual leukemia cells.

Disclosure: Authors have nothing to declare.

\section{P030}

Hematopoietic stem cell transplantation with sequential conditioning for children with relapsed/refractory acute leukemia

Nao Yoshida , Kazuki Matsumoto ${ }^{1}$, Daiki Yamashita, Yiqing Zhu ${ }^{I}$, Daichi Sajiki ${ }^{l}$, Ryo Maemura ${ }^{I}$, Hirotoshi Sakaguchi $^{l}$, Asahito Hama ${ }^{1}$ 
${ }^{1}$ Children's Medical Center, Japanese Red Cross Nagoya First Hospital, Nagoya, Japan

Background: Patients with acute leukemia who fail to achieve complete remission show a dismal prognosis even with allogeneic hematopoietic stem cell transplantation (HSCT). This study evaluated whether sequential conditioning approach that is cytoreductive chemotherapy applied shortly prior to the main conditioning followed by HSCT can improve prognosis in such high-risk patients.

Methods: We retrospectively analyzed the outcomes of 90 children (median 8 , range $0-18$ years old) with primary refractory $(\mathrm{n}=11)$ or refractory relapsed $(\mathrm{n}=79)$ acute leukemia (AML $n=43$, ALL $n=47$ ) who received HSCT in our department between 1990 and 2016. The stem cell source was related peripheral blood (PB) in 4 patients, related bone marrow in 31 , unrelated bone marrow in 40 , or unrelated cord blood in 15 . The grafts were HLA serologically matched $(n=63)$ or mismatched $(\mathrm{n}=27)$ with the recipient. In total, 29 patients received the sequential conditioning approach. As cytoreductive chemotherapy, fludarabine/cytarabine/idarubicin/G-CSF (FLAG-IDA) was used in 12 patients, mitoxantrone or daunorubicin/cytarabin in 10 , or other regimens in 7 , and 6 of them were combined with gemtuzumab ozogamicin. Without waiting for hematological recovery, the patients promptly underwent HSCT; therefore, the median interval between cytoreductive chemotherapy and main conditioning was 11 days. The main conditioning regimens were total body irradiation-based myeloablative $(\mathrm{n}=22)$, busulfan-based myeloablative $(\mathrm{n}=4)$, or reduced intensity $(\mathrm{n}=3)$.

Results: In 90 children with relapsed/refractory acute leukemia, the 5-year overall survival (OS), leukemia-free survival (LFS), cumulative incidence of relapse (RI), and transplantation-related mortality (TRM) were $24 \%, 21 \%$, $53 \%$, and $26 \%$, respectively. In multivariate analysis, the use of sequential conditioning was identified as the most favorable factor for LFS (hazard ratio [HR] $0.37 ; P=$ 0.001 ), although there were no differences in the outcomes according to the types of cytoreductive chemotherapy or the main conditioning regimen. HLA-matched donor (HR 0.46; $P=0.005)$ and $\mathrm{PB}$ blasts-negative at the beginning of conditioning (HR $0.49 ; P=0.02$ ) were also independently associated with better LFS. With sequential conditioning, leukemia burden prior to the HSCT was significantly reduced; PB blasts became undetectable at the beginning of conditioning in $66 \%$ patients given the approach, while in $38 \%$ patients without the approach $(P=0.02)$. Notably, the outcomes in the patients without PB blasts at the beginning of conditioning who received sequential conditioning were promising; the 5-year OS and LFS reached $73 \%$ and $62 \%$ and the 5-year RI and TRM were $33 \%$ and 5\%, respectively.
Conclusions: Our study reveals that HSCT with sequential conditioning can be an effective and tolerable treatment option for children with relapsed/refractory acute leukemia. The treatment strategies that focus on the reduction of leukemia burden immediately prior to HSCT may contribute to the induction of long-term remissions in patients with high-risk acute leukemia.

Disclosure: This research was funded by Japanese Red Cross, Nagoya 1st. Hospital Research Grant NFRCH180028.

\section{P031}

Use of blinatumomab to achieve remission and consolidation with haploidentical transplant with cyclophosphamide post for the treatment of children with refractory acute lymphoblastic leukemia (ALL)

\section{Alberto Olaya Vargas ${ }^{1}$, Roberto Rivera Luna ${ }^{2}$, Yadira Melchor Vidal ${ }^{3}$, Haydeé Del Pilar Salazar Rosales ${ }^{1}$, Gerardo López Hernández ${ }^{1}$, Rosa María Nideshda Ramírez Uribe ${ }^{I}$}

${ }^{1}$ Instituto Nacional de Pediatria, Mexico City, Mexico, ${ }^{2}$ Instituto Nacional de Pediatria, Hem/Oncology, Mexico City, Mexico, ${ }^{3}$ Medical Center ABC, Mexico City, Mexico

Background: Most of patients with ALL in relapse or refractory to conventional treatment have only $30 \%$ possibilities to achieve long term remission. This report refers to the therapeutic efficacy and adverse events from the blinatumomab to achieve molecular remission in patients with pre-B CD19+ which lead to haploidentical with cyclophosphamide post transplant as a consolidation.

Methods: A pilot study was conducted in children with refractory ALL preB-CD19 +. As a strategy to achieved remission blinatumomab was used at a dose $10 \mu \mathrm{g} / \mathrm{m} 2$ for continous infusion of 48 hours, increasing the dose to $15 \mu \mathrm{g} / \mathrm{m} 2$ during 28 days, patients with a MRD of $<0.002$ Log, after 2 cycles received an haploidentical bone marrow transplant as a consolidation, the conditioning regimen was with total body irradiation scheme at $200 \mathrm{cGy} /$ day/3 days, cyclophosphamide and etoposide. Receiving prophylaxis for GVHD with cyclophosphamide.

Results: A total of 10 patients were included, seven of them achieved complete remission after 2 cycles of blinatumomab, one with partial remission (Table 1), these seven patients, six received an haploidentic transplant achieving graft in 6 of the transplanted patients. One patient had a bone marrow relapse in the first 6 months of the follow-up and 5 patients are free of disease with a follow-up to 20 months (figure 1). As a acute complication the 10 patients presented cytokine release syndrome, during the infusion of blinatumumab 10 patients presented 
tachycardia (Table 3 ) and the 6 patients presented aGVHD after HSCT (5 grade I-II and I grade IV).

Conclusions: Allogeneic bone marrow transplant constitutes a treatment option on those patients that relapse or become refractory to treatment, one of the major problem is basically to identify a HLA-identical donor, the alternative is an haploidentical donor. The most important factor to get these results is the disease status before transplant. The use of blinatumomab has proven to be effective in achieving remission in relapse Acute Linfoblastic Leukemia pre-B CD $19+$ or refractory to treatment.

\begin{tabular}{lll}
\hline CHARACTERISTICS & $\mathrm{N}^{\mathrm{o}}$ & $\%$ \\
\hline Male & 4 & $40 \%$ \\
Female & 6 & $60 \%$ \\
Median age at diagnosis, (range), years & 9.22 & $(7-12)$ \\
Status of disease & & \\
2 o +3 relapse & 6 & $60 \%$ \\
Refractory to primary or salvage therapy & 4 & $40 \%$ \\
Complete remission after Blinatumomab & 7 & $70 \%$ \\
Partial remission after Blinatumomab & 1 & $10 \%$ \\
Active disease & 2 & $20 \%$ \\
\hline
\end{tabular}

[[P031 Table] 1. Table $N^{\circ}$ 1. Demographic characteristics of patients undergoing Blinatumomab $(n=10)]$

Disclosure: A. Olaya-Vargas, R. Rivera-Luna, Y. Melchor-Vidal, H. Salazar-Rosales, G. Lopez-Hernandez, N. Ramirez-Uribe. We wish to confirm that there are no known conflicts of interest associated with this abstact, the only financial support was provided by Mexican Associations that helping children wiht cancer in a few patients.

\section{P032}

Sequential high-dose chemotherapy reinduction followed by myeloablative allogeneic transplant for active acute myeloid leukemias

\section{Lucia Brunello $^{I}$, Roberto Passera ${ }^{1}$, Marco Cerrano ${ }^{1}$, Luisa Giaccone ${ }^{1}$, Ernesta Audisio ${ }^{1}$, Dario Ferrero ${ }^{1}$, Stefano D'Ardia ${ }^{I}$, Semra Aydin', Chiara Maria Dellacasa ${ }^{I}$, Alessandro Busca ${ }^{I}$, Benedetto Bruno ${ }^{I}$ \\ ${ }^{1}$ A.O.U. Città della Salute e della Scienza di Torino, Torino, Italy,}

Background: The sequential use of intensive chemotherapy and reduced-intensity hematopoietic stem cell transplantation (HSCT) represents a possible approach for the treatment of relapsed-refractory acute myeloid leukemias (AML) (Schmid C, JCO 2005; Ringdén O, Br J Haematol. 2017, Mohthy M, Haematologica 2017).
Methods: At our Center, 27 relapsed/refractory AML patients were transplanted during chemo-induced neutropenia after high-dose salvage chemotherapy. Median age at transplant was 52 years (range 21-62). Patients suffered from de novo $(n=18 / 27,67 \%)$ or secondary AML $(n=9 /$ $27,33 \%)$. Genetic risk stratification was reported using stardardized groups proposed by the European Leukemia Net (ELN) in 2010. Favorable, Intermediate I and II and Adverse risk category at diagnosis was observed in 1/27 (4\%), 19/27 (70\%), 7/27 patients (26\%) respectively. All patients had active disease at the time of Sequential Therapy and median marrow blast count was 25\% (range 7-88\%). Patients received a high-dose Cytarabine based (MEC in 17/ $27,63 \%$ ) regimen as salvage therapy. Donors were haploidentical relatives for 15/27 (56\%) patients, identical siblings and matched-unrelated for $6 / 27$ patients $(22 \%)$ and $6 / 27$ (22\%), respectively. A myeloablative conditioning was used to further implement anti-leukemic effects. Conditioning, Thiotepa-Busulfan-Fludarabine in $89 \%$ patients, was started at a median of 8 days (range 3-14) after the last day of chemotherapy. Bone marrow and peripheral blood stem cells were used as graft source in 11/27 (41\%) and 16/27 (59\%) patients. Graft-versus-host disease (GVHD) prophylaxis and supportive care were administered accordingly to each HSCT platform.

Results: All patients engrafted. Median day of neutrophil recovery was day +16 (range 12-23). Median follow-up of survivors was 31 months (range 4-125). Non Relapse Mortality and Relapse Incidences (NRM, RI) were $16 \%$ and $48 \%$ at 1 year and $16 \%$ and $58 \%$ at 3 years, respectively. Overall cumulative incidences of acute and chronic GVHD were $48 \%$ and $43 \%$ at day +100 and +400 . One and 3 year Overall Survival (OS) were $58 \%$ and $34 \%$, while 1 and 3 year Event-Free Survival (EFS) were $35 \%$ and $26 \%$. Significant better OS and EFS were observed in patients with Favorable-Intermediate I-II versus Adverse risk score (1-3 years OS $64 \%$ and $50 \%$ vs $43 \%$ and $0 \% \mathrm{p}=0.022 ; 1-3$ years EFS $43 \%$ and $36 \%$ vs $14 \%$ and $0 \% \mathrm{p}=0.013$ ). Adverse risk had a significant impact on OS (HR 3.24, $\mathrm{p}=0.030$ ) and $\mathrm{EFS}$ (HR 3.33, $\mathrm{p}=0.018$ ) by univariate analysis and on RI (SDHR 3.02, p=0.031) by Fine and Gray Test.

Conclusions: Though small the patient cohort, our findings suggest that sequential therapy with a myeloablative HSCT is feasible in treating relapsed/refractory AML. Transplant-related toxicity was low (16\%) and relapse was the major treatment-failure. However, even with this approach, patients with adverse cytogenetic features have a very dismal prognosis. For these patients, the use of new drugs before HSCT and/or maintenance therapy after transplant is highly encouraged to improve outcomes.

Disclosure: Alessandro Busca: Honoraria from Gilead Sciences, Merck, Pfizer Pharmaceuticals and Jazz 
Pharmaceuticals; speaker for Gilead Sciences, Merck, Pfizer Pharmaceuticals, Astellas Pharma, and Basilea.

Benedetto Bruno Honoraria from: Gilead, Pfizer, Hospira, Honoraria and research funding from Celgene; Research funding from Pierre Fabre, Adienne, Hospira italia, MSD Italia

\section{P033}

The significance of minimal residual disease (MRD) in children AML on outcome after allogeneic stem cell transplantation with myeloablative or reduced intensity conditioning

Zhemal Rakhmanova ${ }^{1}$, Olesya Paina ${ }^{1}$, Polina Kozhoka ${ }^{1}$, Anastasiya Frolova', Kirill Ekushov ${ }^{1}$, Maria Galas ${ }^{1}$, Lyubov Tsvetkova ${ }^{1}$, Aygul Khabirova ${ }^{1}$, Elena Babenko ${ }^{1}$, Tatyana Gindina ${ }^{I}$, Aleksandr Alyanskiy ${ }^{I}$, Ildar Barkhatov $^{I}$, Elena Semenova ${ }^{1}$, Ludmila Zubarovskaya, Boris Afanasyev ${ }^{I}$

${ }^{1}$ First Pavlov Saint Petersburg State Medical University, Raisa Gorbacheva Memorial Research Institute of Pediatric Oncology, Hematology and Transplantation, Saint Petersburg, Russian Federation

Background: In spite of satisfactory results of overall survival (OS) after AlloHSCT in $1^{\text {st }}$ and $2^{\text {nd }}$ CR AML, relapse free survival (RFS) and graft-versus-host-disease free/relapse free survival (GRFS) require further improvement. The detection of MRD is one of the factors which influence on the outcome of AlloHSCT in AML is unclear but identification is important to improve risk-adapted relapse prophylactic treatment after AlloHSCT.

Aim. To evaluate outcomes of AlloHCST in $1^{\text {st }}$ and $2^{\text {nd }}$ CR pediatric AML depending on the level of MRD status before myeloablative (MAC) or reduced intensity conditioning regimens (RIC).

Methods: The data of 72 children with AML in $1^{\text {st }}$ and $2^{\text {nd }} \mathrm{CR}$ underwent AlloHSCT between 2008 and 2018 were analyzed. Median age at the moment of AlloHCST was 8 years old (2-18). MRD negative status had 42 (58\%) patients, 30 (42\%) were MRD positive by flow cytometry. MAC based on busulfan (16 mg/b.w.) received 27 (37\%) patients, on treosulfan - 7 (10\%) patients. RIC based on melphalan received 20 (28\%) patients, based on busulfan (8 $\mathrm{mg} / \mathrm{b}$.w.) - 18 (25\%) patients. Patients received prophylaxis of aGVHD by ATG $20(28 \%)$ or PTCy - $48(66 \%)$ patients plus CsA - $23(32 \%)$ or tacrolimus \pm sirolimus - $43(60 \%)$ patients that depended on source of transplant (related, unrelated or haplo donor).

Results: At the median follow up 3 years in the cohort of MRD positive patients OS is $66 \%$ vs $72 \%$ in MRD negative $(\mathrm{p}>0,05)$. RFS is $56 \%$ vs $83 \%$ accordingly $(p=0,01)$. Graft-versus-host-disease free/relapse free survival (GRFS) in MRD positive patients is $37 \%$ vs $51 \%$ in MRD negative $(p>0,05)$. OS, RFS, GRFS in MRD positive patients after MAC is $57 \%, 42 \%, 30 \%$ vs $75 \%$, $68 \%$, 43\% after RIC accordingly ( $\mathrm{p}>0,05)$. OS, RFS, GRFS in MRD negative patients after MAC is $75 \%, 85 \%$, $55 \%$ vs $65 \%, 82 \%, 47 \%$ after RIC accordingly ( $>00,05)$. OS, RFS in MRD negative patients with/without PTCy is $82 \%, 86 \%$ vs $42 \%, 78 \%$ (p>0,05); GRFS is $62 \%$ vs $28 \%$ accordingly $(\mathrm{p}=0,042)$. OS, RFS, GRFS in MRD positive patients with/without PTCy is $66 \%, 55 \%, 54 \%$ vs $66 \%$, $57 \%, 27 \%(\mathrm{p}>0,05)$.

Conclusions: MRD status does not statistically significant affect on OS that can be related to different approaches to the treatment of relapse after AlloHSCT. MRD positive status statistically significant decreases RFS that underline the necessity of posttransplant therapy improvement. RIC vs MAC in all patients in first and second remission do not show statistically significant impact on OS, RFS, GRFS. PTCy significantly improves GRFS in MRD negative patients.

Disclosure: None of the authors has anything to disclose.

\section{P034}

Allogeneic HSCT in therapy-associated AML and MDS after treatment for lymphoma: A retrospective singlecenter analysis

Daniela Vanessa Wenge ${ }^{1}$, Christian Reicherts ${ }^{1}$, JanHenrik Mikesch ${ }^{1}$, Christoph Groth ${ }^{1}$, Klaus Wethmar ${ }^{1}$, Christoph Schliemann ${ }^{1}$, Rolf Mesters ${ }^{I}$, Torsten Kessler ${ }^{I}$, Cyrus Khandanpour ${ }^{1}$, Norbert Schmitz', Georg Lenz, Matthias Stelljes ${ }^{1}$

${ }^{1}$ University Hospital Muenster, Medicine A, Muenster, Germany

Background: With increasing overall-survival (OS) of lymphoma patients, higher incidences of therapy-related clonal bone marrow diseases, such as acute myeloid leukemia (AML) and myelodysplastic syndrome (MDS) are occuring. Generally, the outcome is considered poor. Allogeneic hematopoietic stem cell transplantation (allo HSCT) often remains the only potentially curative treatment option. Nonetheless, there is only little data available concerning this patient group.

Methods: We retrospectively collected data from 33 patients with therapy-related AML (tAML) and MDS (tMDS) after treatment for Hodgkin's lymphoma (HL; $\mathrm{n}=7$ and $\mathrm{n}=2$ ) or non-Hodgkin's lymphoma (NHL; $\mathrm{n}=10$ and $\mathrm{n}=14$ ), who received an allo HSCT between 2000 and 2018. Median follow-up of surviving patients was 3.1 years (range 2.6 months- 9.4 years). 
Results: Median interval between lymphoma diagnosis and onset of tAML or tMDS was 10 years (range 1.5 years- 38.8 years). On average, patients were transplanted 7 months after diagnosis of tAML/tMDS (range 161 months) at a median age of 56 years (range 37- 69 years). Remission status of tAML-patients at allo HSCT $(\mathrm{n}=17)$ was $1^{\text {st }}$ complete remission $(\mathrm{CR} ; 35 \%), 2^{\text {nd }} \mathrm{CR}$ $(12 \%)$ or relapsed/refractory disease $(53 \%)$. Prior to transplant, patients received dose-reduced conditioning therapy $(n=24)$, melphalan-based sequential conditioning $(n=7)$ or FLAMSA-based conditioning $(n=2)$. Patients were transplanted with peripheral stem cell grafts from 10/10 HLA-matched unrelated donors $(n=21)$, HLAidentical sibling donors $(n=10)$ or haploidentical donors $(n=2)$. Cumulative incidence of relapse for all patients at 3 years was $25.2 \%$ (95\% CI: $12.6 \%$ - 50.5\%). Cumulative incidences of non-relapse mortality (NRM) at 1 year and 3 years were $24.2 \%$ (95\% CI: $13.3 \%-44.3 \%$ ) and $41.9 \%$ (95\% CI: $25.2 \%$ - 69.6\%), respectively. Relapse-free survival and OS at 3 years post-allo HSCT were $42.0 \%$ (95\% CI: $22.6 \%-61.5 \%)$ and $44.4 \%$ (95\% CI: $25 \%$ 63.8\%), respectively.

Patients with a history of HL had a 3-year-OS of $62.2 \%$ compared to $40.7 \%$ and $35.6 \%$ for patients with indolent or aggressive NHL. More than one line of therapy for NHL/HL prior to onset of tAML/tMDS resulted in a trend towards higher NRM rate and lower 3-year-OS (one treatment line: $9.1 \%$ and $70.7 \%, 2$ lines of treatment: $33.3 \%$ and $22.2 \%,>2$ lines of treatment $63.9 \%$ and $32.5 \%$ ). 5 patients died from relapsing tAML/tMDS and 11 patients died in CR after transplantation. The major causes of non-relapse related lethality were early death due to infection/toxicity (death between d0- d20 post-allo HSCT, $n=5$ ), infectious complications without GvHD $(\mathrm{n}=1)$, infections due to GvHD treatment $(\mathrm{n}=2)$ and toxic pneumonitis/severe lung complications $(\mathrm{n}=3$, all patients with history of NHL).

Conclusions: Outcomes in this patient cohort are comparable with those reported for patients transplanted with primary AML or MDS. More than one line of therapy for HL/NHL may be considered as a relevant risk factor for NRM and, consequently, inferior survival following allo HSCT for tAML/tMDS.

Disclosure: Nothing to declare.

\section{P035}

Clinical outcome of sequential conditioning regimen followed by allogeneic stem cell transplantation for refractory acute leukemia: Experience of one center

\author{
Lucia Garcia ${ }^{1}$, Leyre Bento ${ }^{I}$, Bernardo Lopez ${ }^{I}$, Andrés \\ Novo ${ }^{I}$, Antonio Gutiérrez, Maria Antonia Duran', \\ Antonia Sampol
}

${ }^{1}$ Son Espases University Hospital, IdISBa, Palma de Mallorca, Spain

Background: The prognosis of relapsed/refractory acute leukemia $(\mathrm{R} / \mathrm{R} \mathrm{AL})$ is poor and the treatment is challenging. In this setting, allogeneic stem cell transplantation (alloSCT) constitutes the only curative option although the high relapse rate and non-relapse mortality (NRM). The sequential conditioning regimen followed by allo-SCT has been used for persistent disease and aims to improve disease control by intensified chemotherapy, thus conceding more time for the presumed graft-versus-leukemia effect to occur.

Methods: The clinical outcome of $\mathrm{R} / \mathrm{R}$ AL with the sequential conditioning regimen combining a chemotherapy rescue followed by RIC allo-SCT in our center is described. Patients who underwent a sequential allo-SCT from 2005 to 2017 are included. The primary endpoint was progression free survival (PFS) and overall survival (OS) that were estimated by the Kaplan- Meier method. Secondary endpoints were non-relapse mortality (NRM).

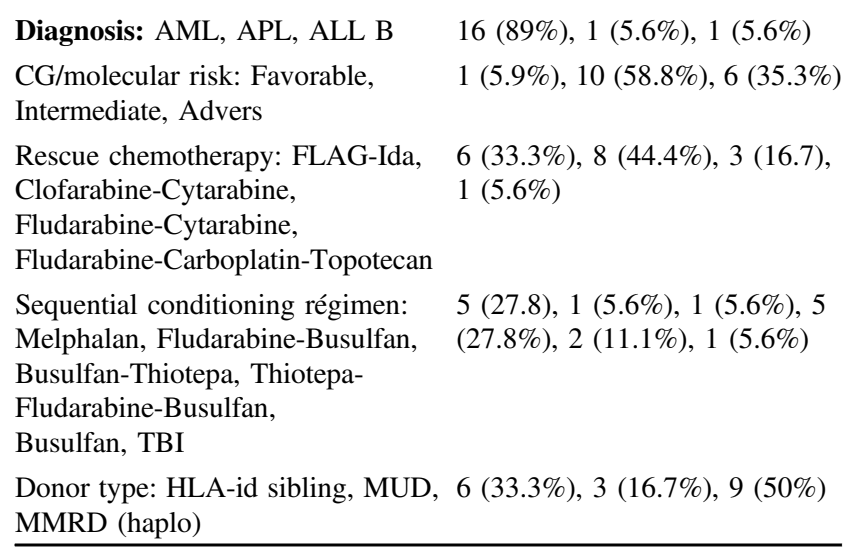

[[P035 Table] 1. Main characteristic of the patients]

Results: We retrospectively analyzed 18 patients with active disease at the time of transplantation. The median age at allo-SCT was 48 years (range 15-65). The main characteristic of the patients are shown in Table 1 . Complete response was achieved in 15 patients $(83 \%)$ at day 100 post SCT. At a median follow-up of 21 months (2.5-53), 50\% relapsed, 5 patients are alive in CR and 13 died. The main causes of death were SCT-related in $33.4 \%$ (27.8\% bacterial and 5.6\% fungal infections) and disease in $38.9 \%$. The median OS was 10.8 months (IC 95\%: 3-18.6) and the median PFS was 5.6 months (IC 95\%: 3.6-7.5). Comparing Clofarabine-Cytarabine with FLAG-Ida as a rescue chemotherapy regimen, the CR rate at day 100 post SCT were similar (87\% and $83 \%$ respectively) but we found interesting results in PFS: 6 months (IC 95\%: 4.9- 7.1) in the group of Clofarabine-Cytarabine and 3.6 months(IC 
95\%: 2.7-4.5) in the group of FLAG-Ida. The median OS was more than double in the group of ClofarabineCytarabine (10.8 vs. 4 months).

Conclusions: The sequential conditioning regimen combining chemotherapy rescue followed by allo-SCT represents a promising approach for patients with R/R AL. We confirm high $\mathrm{CR}$ rate but early relapses taking into account this high risk population. Therefore, there is a need to find new drugs or maintenance strategies in order to prolong the OS and the PFS.

Disclosure: Nothing to declare.

\section{P036}

\section{Abstract already published.}

\section{P037}

Potential role of autologous stem cell transplant in favorable-risk genetics NPM1 positive acute myeloid leukemia - A retrospective single center analysis

Pedro Chorão' ${ }^{1}$, Pedro Medeiros ${ }^{1}$, Eliana Aguiar ${ }^{1}$, Maria Luís Amorim ${ }^{1}$, Maria José Soares ${ }^{1}$, Paula Gomes ${ }^{1}$, Ricardo Pinto ${ }^{1,2}$, Fernando Príncipe ${ }^{1}$, José Eduardo Guimarães $^{1,2}$

${ }^{1}$ Centro Hospitalar de São João, Porto, Portugal, ${ }^{2}$ Faculdade Medicina da Universidade do Porto, Porto, Portugal

Background: Recommendations of the 2017 European Leukemia Net (ELN) for favorable-risk genetics (FRG) acute myeloid leukemia (AML) favor consolidation over transplantation, although reviews suggest advantage of autologous stem cell transplant (ASCT) in event free survival. Our objective was to compare the progression free survival (PFS) and overall survival (OS) of normal karyotype NPM1 mutated without FLT3 ITD or allelic ratio < 0.5 (NPM1+) AML patients treated with consolidation chemotherapy alone (CC), ASCT or allogeneic stem cell transplant (AlloSCT)

Methods: Retrospective review of NPM1+ FRG-AML patients, treated in one institution (2008 to 2017) with the following induction regimens: cytarabine (Ara-C) and VP16 with daunorubicin (ADE) or mitoxantrone (MiCE). Consolidation regimens were Ara-C with daunorubicin (AC-D), idarubicin, VP-16 and Ara-C (mini-ICE) or highdose Ara-C (HiDAC). In ASCT, conditioning regimens were $\mathrm{BuCy}$ or BVAC and in AlloSCT were BuCy or FluBu. PFS and OS were calculated from the start of the last consolidation or stem cell infusion.

Results: A total of 39 patients were evaluated, with a median age of 53 years (y) (23-68y), 69\% female, $95 \%$ with ECOG performance status (PS) $0-1$ and $36 \%$ with ageadjusted Charlson comorbidity index (aaCII) $\geq 2$ at diagnosis. Patients were treated with $\mathrm{CC}$ in $33 \%(\mathrm{n}=13)$, ASCT in $36 \%(n=14)$ and AlloSCT in $21 \%(n=12)$ of cases. There were no differences between groups for age, aaCII, PS, leucocytes at diagnosis or extra-medullary disease. FLT3-ITD was more frequent in AlloSCT group $(64 \%)$ than CC $(23 \%)$ or ASCT $(8 \% ; \mathrm{p}=0.07)$.

At induction, ADE was used in $82 \%$ and MiCE in $18 \%$ of patients, with a complete remission (CR) rate of $95 \%$. There were no differences between groups for induction regimen or $\mathrm{CR}$. In $\mathrm{CC}$ group, consolidation regimens were 1 cycle $(8 \%)$ and 2 cycles AC-D (61\%) and 2 cycles mini-ICE $(31 \%)$. ASCT patients received consolidation with 1-2 cycles AC-D (78\%) and 1 cycle miniICE (22\%), while AlloSCT patients received 1-2 cycles AC-D (84\%), 2 cycles HiDAC (8\%) and no consolidation in $8 \%$.

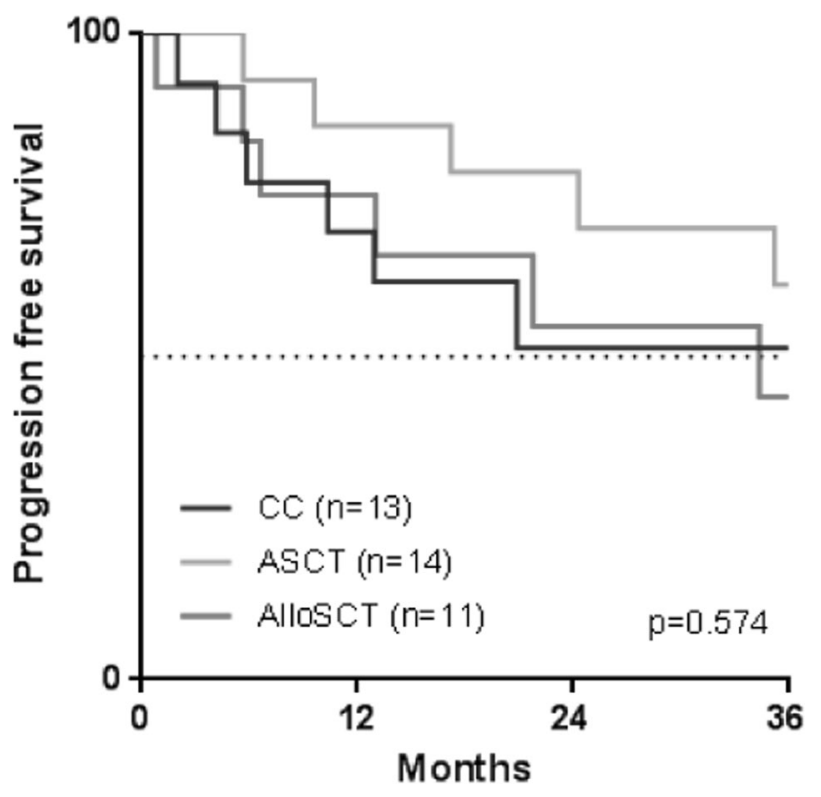

[[P037 Image] 1. Figure 1. PFS at $3 y$ in CC, ASCT and AlloSCT groups.]

Median follow-up was 39 months, PFS at 3y was 53\% and $\mathrm{OS}$ at $3 \mathrm{y}$ was $64 \%$. PFS at $3 \mathrm{y}$ for ASCT group was superior then CC and AlloSCT groups $(61 \%, 51 \%$ and $44 \%$, respectively; Figure 1), although not statistically significant. $\mathrm{OS}$ at $3 \mathrm{y}$ was statistically similar between groups, although inferior in AlloSCT comparing to CC and ASCT (44\%, $77 \%$ and $70 \%$, respectively).

Conclusions: In this historical cohort review, although there was no advantage in OS for ASCT in NPM1+ FRG AML, our data suggests that there might be a PFS improvement in ASCT over CC, which needs to be further addressed in prospective studies.

Disclosure: Nothing to declare 


\section{P038}

Early tapering of immunosuppressive drugs after haploidentical transplantation in patients with high risk leukemia

\section{Seong Kyu Park', Se Hyung Kim ${ }^{1}$, Sung Hee Lim ${ }^{2}$, Chan Kyu Kim², Jong Ho Won ${ }^{3}$, Dae Sik Hong ${ }^{2}$}

${ }^{1}$ Soonchunhyang University Bucheon Hospital, Hematology, Bucheon, Korea, Republic of, ${ }^{2}$ Soonchunhyang University Bucheon Hospital, Bucheon, Korea, Republic of, ${ }^{3}$ Soonchunhyang University Seoul Hospital, Seoul, Korea, Republic of

Background: Disease recurrence is the most important obstacle to achieve long-term survival for patients with advanced acute leukemia after allogeneic hematopoietic stem cell transplantation (HSCT). Several studies showed that early tapering of immunosuppressive agents could achieve graft versus leukemic (GVL) effects at early time after transplantation and the relapse most occurred at the first 6 months after transplantation. In order to reduce the relapse risk and improve the survival, the strategy of early tapering of immunosuppressive agents was retrospectively evaluated in haplo-identical stem cell transplantation patients with high risk leukemia.

Methods: Thirty patients with advanced leukemia received early tapering of immunosuppressive drugs between days 30 and 60 after HSCT according symptoms and signs with no grade II IV acute or significant chronic graft versus host disease (GVHD).

Results: At the time of transplantation, 21 patients reached first or subsequent complete remission $(\mathrm{n}=15$ for $\mathrm{CR} 1, \mathrm{n}=6$ for CR2). The other 9 patients were non-CR or refractory to salvage therapy. Median time to stop immunesuppressants was 66 days after HSCT (32 98 days). Acute GVHD \pm chronic GVHD was developed in 13 patients (43.3\%) at median 27 days (13 113) after stopping immunosuppressive drugs. Only 3 patients had chronic GVHD with limited grade. During median follow-up duration (15.3 months), overall survival was $54.5 \%$ (58\% in $\mathrm{CR}$ vs $50 \%$ in non-CR or refractory, $\mathrm{P}=0.777$ ). Twelve patients $(40 \%)$ died during the follow-up period and relapse was the most frequent cause of death $(n=8,26.7 \%)$. Other causes included secondary cancers $(n=2)$ and acute GVHD $(\mathrm{n}=1)$, and fungal infection $(\mathrm{n}=1)$. In multivariate analysis, risk group based on cytogenetics and CMV reactivation was an independent prognostic factor for better survival; acute and chronic GVHD tended to be associated with better survival.

Conclusions: In patients with high risk acute leukemia, the strategy of early tapering of immunosuppressive agents may facilitate post-transplantation strategies for relapse reduction and can lead to improve survival after allogeneic stem cell transplantation from haplo-identical donor.

Clinical Trial Registry: None

Disclosure: Nothing to declare

\section{P039}

Autologous stem cell transplantation in patients with acute myeloid leukemia. single center experience

Borce Georgievski ${ }^{I}$, Irina Panovska ${ }^{I}$ Stavridis $^{I}$, Aleksandra Pivkova Veljanovska ${ }^{I}$, Zlate Stojanoski, Lidija Cevreska ${ }^{I}$, Dusko Dukovski ${ }^{I}$, Dijana Milovska ${ }^{1}$, Lazar Cadievski ${ }^{1}$

${ }^{1}$ University Clinic for Hematology, Skopje, Skopje, Macedonia, the Former Yugoslav Republic of

Background: Acute myeloid leukemia is a hematological malignant disease that motivates the persistent struggle in the scientific world to provide effective cure that can establish acceptable survival rates in this group of patients. Autologous stem cell transplantation with myeloablative conditioning is still a powerful weapon that can be used against this entity

Methods: We have evaluated retrospectively patients with AML where autologous stem cell transplantation was performed in the period from 2000 till 2018. Our group consisted of 94 patients; male patients 45 (47.8\%), female patients $49(52.2 \%)$. Median age at diagnosis was 44 years (16-68). The average period from time of diagnosis to autologous SCT was 7.05 months.

Results: In the majority of our group, we used myeloablative conditioning regimen with Busulphan-Cyclophosphamide, 60 patients $(63.8 \%)$, in 2 patients $(2.1 \%)$ we have added melphalan to Bu-Cy conditioning, in 22 (23.4\%) patients we used BEAM conditioning and in the rest, 10 patients (10.6\%) we used BAM conditioning regimen. As auto graft we used peripheral blood stem cells (PBSC) in 78 patients (82.9\%), and in 16 patients (17.1\%) we used bone marrow. The main mobilising regimen for PBSC was G-CSF + etoposide and it was performed in 44 patients $(46.8 \%)$, and in the remaining 34 patients $(36.1 \%)$ mobilising of PBSC was performed only with G-CSF. The mean number od apheresis procedures done in our group was 1.55 , and the mean number of collected mononuclear cells was $3.05 \times 10^{8} / \mathrm{kg}$ TT. The mean time to engraftment was 12.8 days (9-23). The transplant related mortality (TRM) was $2.1 \%$. The 5 year Overall Survival of our patients was 46.7 patients. The main reason for death was relapse of the primary disease $(73 \%)$. 20 patients $(21 \%)$ were treated with salvage chemotherapy regimen (FLAG-Ida) because with the standard induction regimen $7+3$ there was absence of adequate therapeutic response, or predominantly 
no Complete remission was achieved. All patients were transplanted in Complete Remission

Conclusions: Autologous stem cell transplantation could be an acceptable therapeutic solution for patients with AML as a consolidation therapy, where neither suitable compatible donor is available nor allogeneic stem cell transplantation could not be performed from various reasons depending on the bone marrow transplant unit

Disclosure: Nothing to declare

\section{P040}

Prophylaxis DLI alone may not prevent relapse of FLT3-ITD positive AML after allogeneic HCT

LiQin Cao ${ }^{1}$, JiMin Shi ${ }^{1}$, YanMin Zhao ${ }^{1}$, Yi Luo ${ }^{1}$, Jian $\mathrm{Yu}^{1}$, Ya-Min Tan ${ }^{1}$, Xiao-Yu Lai', Yuan-Yuan Zhu', GuoQing Wei, Jie Sun ${ }^{1}$, Jing-Song $\mathrm{He}^{1}$, Wei-Yan Zheng ${ }^{1}$, Zhen Cai ${ }^{I}$, He Huang ${ }^{I}$

${ }^{1}$ Zhejiang University, First Affiliated Hospital, Hangzhou, China

Background: Compared with traditional chemotherapy, allogeneic hematopoietic stem cell transplantation (alloHSCT) can improve the prognosis of patients with FLT3ITD positive AML. Relapse after transplantation is still an important factor affecting survival. Prophylaxis donor lymphocyte infusion (DLI) often used to prevent relapse after allo-HSCT. However, its role to patients with FLT3 positive after allo-HSCT had not determined.

Methods: From January of 2014 to December of 2017, 50 patients with FLT3-ITD positive AML who underwent allo-HSCT in our center were taken into this syudy, among them13 patients underwent prophylaxis DLI after alloHSCT.

Results: The estimated three years OS and LFS in all patients was $58.2 \%$ and $69.3 \%$ respectively. The three years cumulative incidence of relapse was $30.3 \%$. 13 of 50 patients underwent prophylaxis DLI, the median time was 105 days (range 63 to 227 days) after allogeneic HSCT. The three years estimated OS was higher in prophylaxis DLI patients than those without prophylaxis DLI, but not reached significant difference (65.9\% VS $35.2 \%, \mathrm{P}=0.86$ ), and the 3 years estimated LFS as well as cumulative incidence of relapse was also similar in two group $(75.2 \%$ VS $64.8 \%, \mathrm{p}=0.66 ; 23.9 \%$ VS $35.1 \%, \mathrm{P}=0.61$ ).

Conclusions: Thus, prophylaxis DLI alone to prevent relapse of patients with FLT3-ITD positive AML after alloHSCT may has limited effect. Additional targeted and coordinated interventions are needed to maintain durable remission after allo-HSCT in this high-risk population.

Clinical Trial Registry: No

Disclosure: Nothing to declare

\section{P041}

Role of allogeneic hematopoietic cell transplantation in acute myeloid leukemia patients with $N P M 1^{W T} A N D$ FLT3-ITD negative group

\section{Sang Kyun Sohn', Ju-Hyung Kim ${ }^{1}$, Jungmin Lee ${ }^{1}$, Ji Yeon $\mathrm{Ham}^{2}$, Jang Soo Suh ${ }^{2}$, Joon Ho Moon ${ }^{1}$}

${ }^{1}$ Kyungpook National University Hospital, Hematology/ Oncology, Daegu, Korea, Republic of, ${ }^{2}$ Kyungpook National University Hospital, Laboratory Medicine, Daegu, Korea, Republic of

Background: One of the most potent prognostic factors affecting outcomes in AML is the presence of cytogenetic and molecular markers which can guide the selection of post-remission therapies. Recently, favorable outcomes of $\mathrm{NPM}^{\mathrm{wt}} /$ FLT3-ITD ${ }^{\text {neg }} /$ non-CEBPA $^{\mathrm{dm}}$ group after allogeneic hematopoietic cell transplantation (allo-HCT) have been reported, that is similar to those of favorable risk by the ELN risk classification. However, the role of allo-HCT compared to consolidation chemotherapy has not yet been elucidated.

Methods: The data of 88 patients who were diagnosed with AML and received intensive induction therapy from 2015 March to 2017 July were included in the current study. To address the time dependence of the allo-HCT, the Simon and Makuch method was used in the graphical representation and the Mantel-Byar test and Andersen and Gill methods for identifying risk factors for long-term survival.

Results: Median age of the patients were 53 years (range 21-69), and 49 patients (56\%) were male. NPM1 mutation was detected in 14 patients (16\%), and FLT3-ITD were none, low, and high ratio in 69 patients $(78 \%), 9(10 \%)$, and $10(12 \%)$, respectively. The ELN risk classification divided the patients into favorable, intermediate, and adverse risk group in 31 patients (35\%), 38 (43\%), and 19 (22\%), respectively. NPN1 and FLT3-ITD both negative group included 29 patients (33\%). Allo-HCT was performed in 48 patients (55\%). Overall, complete response (CR) after induction therapy achieved in 63 patients $(72 \%)$, and 7 patients $(8 \%)$ were primary refractory disease. CR rates did not differ between NPM1 ${ }^{\text {wt }} /$ FLT3-ITD negative group $(\mathrm{n}=17 / 29,58.6 \%)$ and other intermediate risk group $(n=6 / 9,66.7 \% ; p=0.967)$. With median follow-up duration of 12.9 months (range 1.3-39.0 months), one-year OS rate were $100 \%, 83.5 \pm 6.9 \%, 56.1 \pm 12.8 \%$ in favorable, intermediate, and adverse risk group $(\mathrm{p}<0.001)$. Among intermediate risk group, OS rate of NPM1 ${ }^{\mathrm{wt}} / \mathrm{FLT3}$-ITD negative group was similar to other intermediate risk $(\mathrm{p}=0.403)$. Allo-HCT was performed in 11 patients of NPM $1^{\text {wt }} /$ FLT3-ITD negative group. One-year OS rates did not differ between NPM1 ${ }^{\text {wt }} /$ FLT3-ITD negative and other 
intermediate risk (Mantel-Byar test $\mathrm{p}=0.622$ ). In the multivariate analysis, ELN risk group was identified as the only risk factor for OS (HR 2.76, 95\% CI 1.51-5.02, p< 0.001). Allo-HCT was not an independent favorable factor for OS in NPM1wt/FLT3-ITD negative group (HR 0.47, 95\% CI 0.09-2.47, $\mathrm{p}=0.372$ ).

Conclusions: NPM $1^{\mathrm{wt}} / \mathrm{FLT} 3-$ ITD negative group showed similar CR and OS rates compared to other ELN intermediate group. Allo-HCT did not improve the OS rate of this group. Therefore, the implication of allo-HCT to this group needs to be carefully considered considering other high risk factors.

Disclosure: The authors declare no conflict of interest.

\section{P042}

Low-dose decitabine as maintenance after allogeneic hematopoietic stem cell transplantation for high-risk acute myeloid leukemia

Alessandro Bucalossi ${ }^{1}$, Monica Tozzi ${ }^{I}$, Mariapia Lenoci, Francesca Toraldo ${ }^{I}$, Valeria Del Re ${ }^{I}$, Giuseppe Marotta ${ }^{I}$

${ }^{1}$ Azienda Ospedaliera Universitaria Senese, UOC Terapie Cellulari e Officina Trasfusionale, Siena, Italy

Background: Aberrant DNA methylation occurs in Acute Myeloid Leukemia (AML) blasts. Therapy with hypomethylating agents may reverse the abnormal epigenetic silencing to modify the malignant phenotype.

Decitabine incorporates into DNA after phosphorylation and forms irreversible covalent bonds with DNA methyltransferase-1, leading to genome-wide global DNA hypomethylation.

The administration of decitabine at doses of $15-20 \mathrm{mg} /$ sm/die for 5-10 days every months in high-risk AML patients resulted in high frequency of complete remission. However, allogeneic hematopoietic stem cell transplantation (HSCT) remains the only curative therapy for high-risk AML, but disease relapse is the principal cause of treatment failure for these patients, depending on the diagnosis characteristics and status of disease at the time of transplantation. The outcomes of salvage treatments are very poor. Therefore, early maintenance therapy, directed at eliminating minimal residual disease and promoting a graft versus-leukemia (GVL) effect, could be an effective method to improve outcome after allo-HSCT.

Decitabine enhances FOXP3 expression in CD4+CD25+ $\mathrm{T}$ cells ( $\mathrm{T}$ regs). The Tregs are thought to play an important role in modulating graft versus-host-disease (GVHD) without sacrificing the beneficial of GVL effect. These studies provide a rationale for the administration of decitabine after alloHSCT for high-risk AML. Our study, open-label and prospective, aims to expand the current knowledge about the use of low-dose decitabine as maintenance therapy in high-risk AML patients after allo-HSCT.

Methods: Seven patients with high-risk AML (2 with secondary AML, 5 with cytogenetics or molecular adverse factor) in complete remission, were enrolled in the study between day 50 and 80 after allo-HSCT to evaluate the prophylactic effect of decitabine. Other major eligibility criteria included performance status < 2 (ECOG), no grade 3 or 4 acute GVHD, no uncontrolled infections, creatinine $<$ $1.5 \mathrm{x}$ upper limit of normal (ULN), bilirubin $<1.5 \mathrm{x}$ ULN, and hepatic enzymes $<2.5 \times$ ULN.

All patient received a myelo-ablative conditioning regimen with busulfan $(12.8 \mathrm{mg} / \mathrm{kg})$ and fludarabine $(150 \mathrm{mg} /$ $\mathrm{sm})$. The source of graft was peripheral blood in six patients and bone marrow in one patient. Four patients had transplanted using unrelated HLA-compatibile donors and three using HLA-identical sibling. Acute and chronic GVHD prophylaxis it was carried out with Thimoglobulin, Metothrexate and Cyclosporine.

Decitabine was administered as an intravenous infusion over 1 hour at the dose of $10 \mathrm{mg} / \mathrm{sm} /$ day for 5 consecutive days every 6 weeks for up to $8-10$ cycles (to cover the first year post allo-HSCT).

The endpoints of study included toxicity, AML relapse, incidence and severity of GVHD, overall and disease free survival.

Results: All patients completed the treatment without any toxicity and nobody has developed a GVHD. The mean follow-up time was 22 months (16 - 35 months). Five patients are alive and in complete remission, while two patients relapsed (one after 19 months, and 1 after 20 months from allo-HSCT); of the two relapsed patients, one died and the other is alive with active disease.

Conclusions: Our study shows that post-transplant decitabine is safe and may play a role in the maintenance of remission in high-risk AML patients.

Disclosure: Nothing to declare

\section{P043}

Allogenic hematopoietic stem cell transplant consolidation therapy in older acute myeloid leukemia patients - survival and toxicities

Carla Alves ${ }^{1}$, Isabelina Ferreira ${ }^{2}$, Gilda Teixeira ${ }^{2}$, Nuno Miranda $^{I}$, Sofia Jorge ${ }^{I}$, Manuel Abecasis ${ }^{I}$

${ }^{1}$ Instituto Portugues de Oncologia de Lisboa Francisco Gentil, Hematologia, Lisbon, Portugal

Background: Reduced-intensity conditioning (RIC) allowed selected patients (pts), with acute myeloid leukemia (AML) and over 60 years old (y.o.), to become candidates 
for allogenic hematopoietic stem cell transplant (alloHSCT), as a strategy to prolong survival.

Methods: Data from AML pts over 60 years, who underwent RIC allo-HSCT in our institution between September 2011 and September 2017, was retrospectively collected from clinical files to evaluate the overall survival (OS) up to November 2018. We calculated the OS using Kaplan-Meyer curves.

Results: We identified 15 pts, median age 62 y.o. (60-67) and median HTC-I score 2. The median follow-up was 25 months. One patient (pt) had CML blast crisis and was on first major molecular remission. Of the remaining 14 AML pts, 7 were in $1^{\text {st }}$ complete remission (CR), 4 in $2^{\text {nd }}$ $\mathrm{CR}$ and 1 with progressive disease (PD); the other 2 pts could be classified as MDS according to 2016 WHO diagnostic criteria and were in CR1. Donors (D) were: 3 matched unrelated (MUD), 5 mismatched unrelated (MMUD - 9/10), 6 matched siblings and 1 haploidentical. Thirteen pts were infused with peripheral blood HSC and 2 with bone marrow. Conditionings were: FLUBCNUMEL in 5 unrelated donor (UD) pts and 4 siblings, FLUMEL in 1 UD pt and 1 sibling, FLUBU in 2 UD pts and FLUTBI 2Gy in 1 sibling and in the haploidentical. Graft versus host disease (GVHD) prophylaxis was Tacrolimus (TAC) + MMF in 5 UD pts and 1 sibling, TAC + MTX in 2 UD pts and Cyclosporine (CyA) + MMF in 1 UD pt and 5 siblings. All MMUD pts had ATG. PTCy was done in the haploidentical setting with TAC + MMF. The median time to neutrophil and platelet engraftment for the whole cohort was 14 and 11 days, respectively. One pt with secondary engraftment failure required re-infusion of selected CD34+ cells. Ten pts presented with mild acute skin GVHD. Eleven pts had chronic GVHD, 2 classified as severe; 7 required systemic therapy, 5 of those beyond 1 year. The median time on immunosuppressants was 404 days. At 2 years the OS was $63.5 \%$. There were 6 deaths: 3 disease-related (2 relapses at 22 and 58 months and $1 \mathrm{PD}$ at $\mathrm{d}+30$ ), 2 infection complications (2 septic shock) and 1 to secondary neoplasia. Other relevant complications were hypoxemic pneumonia in 5 pts, 1 urinary sepsis, CMV and EBV reactivation respectively in 8 and 4 pts; pulmonary and renal toxicity either in 2 pts. At end of follow-up, 9 pts were in remission, 8 without negative measurable residual disease (MRD), the other MRD negative pt died of septic shock and severe intestinal GVHD.

Conclusions: In this small cohort, chronic GVHD and infectious complications were major causes of morbidity but there were no treatment related deaths before $d+100$. Pts maintaining or achieving MRD negativity after transplant had better survival. Although with only 15 pts, these results suggest that allo-HSCT is feasible as consolidation strategy in selected AML pts over 60 years.

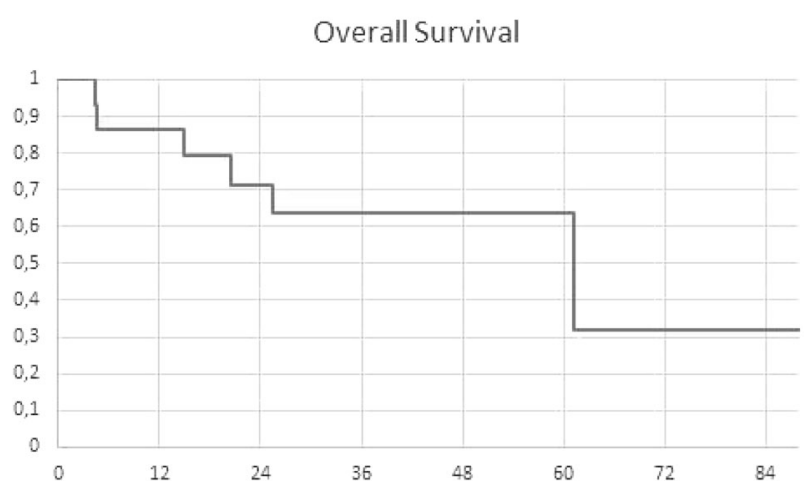

[[P043 Image] 1. Overall Survival]

Disclosure: Nothing to declare.

\section{P044}

Abstract withdrawn.

Aplastic anaemia

\section{P045}

Outcome of hematopoietic stem cell transplantation in fanconi anaemia: A single centre study from Pakistan

Tariq Mehmood Satti, Muhammad Farhan', Syed Kamran Mehmood, Tariq Azam Khattak ${ }^{1}$, Qamar-UnNisa, Chaudhry', Tariq Ghafoor ${ }^{2}$, Mehreen Ali Khan ${ }^{1}$

${ }^{1}$ Armed Forces Bone Marrow Transplant Centre, Rawalpindi, Pakistan, ${ }^{2}$ Armed Forces Bone Marrow Transplant Centre, Paediatrics, Rawalpindi, Pakistan

Background: Hematopoietic stem cell transplantation (HSCT) is the only curative option for Fanconi anaemia (FA); an inherited disorder characterized by congenital anomalies, progressive bone marrow failure (BMF) and a predisposition to develop malignancies.

Methods: We retrospectively analysed the data of 27 consecutive patients that underwent HSCT at this centre from 2001 till June 2018. The data was analysed for variables affecting the outcome in terms of overall survival (OS).

Results: Median age at diagnosis was 10 years (2-20 years). Median age at transplant was 11.3 years (4-25 yrs). All patients at transplant were in aplastic phase. Male to female ratio was 1.2:1. Twenty-four (88.9\%) patients had congenital anomalies along with BMF while 3 were phenotypically normal. Twenty-three $(85.2 \%)$ patients were 10/10 HLA matched with siblings, 2 with parents and 2 with cousins. Eleven (40.7\%) patients had gender mismatch transplant.

Three patients had major and 6 had minor $\mathrm{ABO}$ mismatch. 
Conditioning protocol included, Fludarabine $120 \mathrm{mg} / \mathrm{m}^{2}$, ATG $20 \mathrm{mg} / \mathrm{kg}$ and Cyclophosphamide at a dose of 20-40 $\mathrm{mg} / \mathrm{kg}$. Mean time to neutrophil and platelets engraftment was $12.3 \pm 2.92$ days and $20 \pm 10.3$ days, respectively. Average hospital stay was $18.5 \pm 7$ days. Major transplant complications were neutropenic fever (95\%), hypertension (62\%), mucositis I-II (41\%), azotaemia (27\%) and gut toxicity grade I-II (25\%).

Four patients $(14.8 \%)$ had acute graft versus host disease (GVHD) II-IV of skin and gut. Seven (26\%) patients had chronic GVHD; 4 had limited chronic GVHD of skin, 3 had extensive chronic GVHD of skin, gut, liver and lungs.

Overall survival (OS) after HSCT at 6 months, 1, 5 and 8 years was $67 \%, 63 \%, 59 \%$ and $55 \%$ respectively. OS with $\mathrm{Cy} 30 \mathrm{mg} / \mathrm{kg}$ is $62 \%, \mathrm{Cy} 20 \mathrm{mg} / \mathrm{kg}$ is $33 \%$ and $\mathrm{Cy} 40 \mathrm{mg} / \mathrm{kg}$ is $50 \%$, though the number of patients in Cy20 $(n=6)$ and Cy40 ( $n=2)$ is very small.

Post-transplant mortality is $12(44.4 \%)$. The major causes of mortality include; respiratory infections (04), Primary graft failure (03), grade IV gut GVHD (02), diffused alveolar haemorrhage (01), CMV disease (01) and acute myeloid leukaemia (01) 4.5 years post-transplant.

Overall survival in patients transplanted at younger age $(<$ 11 years) was $81.8 \%$ compared to $37.5 \%$ in older age group $(>11$ years) and was statistically significant ( $\mathrm{p}$ value $=0.03$ )

No statistically significant association was found between OS and ABO mismatch, sex mismatch, time since diagnosis, source of stem cells and GVHD.

Conclusions: Our study demonstrated a survival advantage in Fanconi anaemia when transplanted at a younger age and conditioning with cyclophosphamide $30 \mathrm{mg} / \mathrm{kg}$, fludarabine $120 \mathrm{mg} / \mathrm{m}^{2}$ and ATG $20 \mathrm{mg} / \mathrm{kg}$ an acceptable conditioning protocol.

Disclosure: No conflict of interest

\section{P046}

\section{Abstract already published.}

\section{P047}

Incidence and outcome of severe complications in patients with paroxysmal nocturnal hemoglobinuria: A real life scenario from a single center $\mathbf{3 0}$ years experience

\section{Anna Paola Iori ${ }^{1}$, Luisa Quattrocchi ${ }^{1}$, Stefania De Propris $^{1}$, Walter Barberi', Ursula La Rocca ${ }^{1}$, Matteo Salvini', Maria Lucia De Luca ${ }^{1}$, Giovanni Assanto ${ }^{1}$, Antonella Bruzzese ${ }^{1}$, Sara Pepe ${ }^{1}$, Michela Ansuinelli, Daniel Piamonti ${ }^{1}$, Alessandra Napoli ${ }^{1}$, Marco Brunori', Robin Foà ${ }^{1}$}

${ }^{1}$ Policlinico Umberto I, Sapienza University, Rome, Italy,

Background: Paroxysmal nocturnal hemoglobinuria (PNH) is a rare clonal non-neoplastic hematopoietic stem cell disease whose incidence is 1.5-2.9 cases/million of individuals worldwide. Disease characteristics and natural history have been mostly analyzed by multicenter, retrospective studies, with the limit of heterogeneous approaches. Herein we report the incidence of severe complications and outcome in a real life setting scenario of PNH patients consecutively diagnosed and managed at our PNH referral Center between January 1985 and June 2018.

Methods: Patients received a homogeneous diagnostic and treatment approach according to the period of observation (availability of diagnostic tests and eculizumab). All patients treated with eculizumab received vaccination with conjugated anti-meningococcus ACWYserotypes and, since 2016, conjugated anti-meningococcus B-serotype. In the event of any complication, patients could refer to dedicated Hematology Emergency Rooms (ER) 24 hours daily. The occurrence of renal failure and pulmonary hypertension was specifically evaluated. The renal function was studied according to the Cockcroft-Gault formula and the lung function was prospectively monitored by daytime-on exertion, nocturnal pulsoximetric profiles and complete spirometric tests, with DLCO measurement.

Results: Overall,48 PNH patients, median age 36 years (range 17-84), were analyzed. At diagnosis, 26 patients had classic PNH, 19 aplastic PNH and 3 an intermediate form. The cumulative incidences (CI) of thrombosis, and clonal hematologic neoplasm were $29 \%$, and $6 \%$, respectively. CI of pancytopenia in the 26 patients with classic PNH was $23 \%$. One patient showed a spontaneous disappearance of the PNH clone. Since 2005, eculizumab was administered in 28 patients. After eculizumab treatment $50 \%$ and $32 \%$ of patients reached hemoglobin level $>11 \mathrm{~g} / \mathrm{dL}$ and $>8<11 \mathrm{~g} /$ $\mathrm{dL}$ without transfusion, respectively, while $18 \%$ were nonresponsive. During eculizumab treatment no thrombotic event was observed while two severe infectious episodes (respiratory tract and urinary tract infection) were observed in only one of the 28 patients. Extravascular hemolysis was demonstrated in $50 \%$ of patients. No patient showed a significant reduction of the renal function.Out of 24 patients prospectively monitored for lung function no pathological alteration in any diurnal or nocturnal pulseoximetric test was observed. No patient showed obstructive or restrictive ventilatory deficiency, nor reduced DLCO values. 30-years overall survival (OS) was $90 \%$ (4 patients who died for non$\mathrm{PNH}$ related reasons were censored at the last follow-up).A better OS, even if not statistically significant,was associated to the absence of thrombotic events (90\%vs70\%), and the period of diagnosis (100\% in 2006-2018, $91 \%$ in 1996$2005,75 \%$ in $1985-1995)$.

Conclusions: Our study reports a better OS and lower rate of severe complications in $\mathrm{PNH}$ compared to previous experiences. Although renal failure and lung hypertension have been reported by other groups, we did not observe 
these complications along a prolonged follow-up. We can assume that the availability of a dedicated ER service enabled an early diagnosis and prompt treatment in case of hemoglobinuria crises (reducing the risk or organ damage) or other complications. The use of eculizumab, together with improved supportive approaches, presumably accounts for the trend towards a better survival witnessed over the last decade in the management of PNH patients.

Disclosure: Nothing to declare

\section{P048}

Haploidentical and unrelated allogeneic stem cell transplantation in aplastic anemia:Single center experience

Zafer Gulbas ${ }^{1}$, Elif Birtas Atesoglu, Meral Sengezer ${ }^{1}$, Imran Dora ${ }^{1}$, Cigdem Eren ${ }^{1}$, Suat Celik ${ }^{1}$, Demet Cekdemir ${ }^{I}$

${ }^{1}$ Anadolu Medical Center, Hematology, Kocaeli, Turkey

Background: Aplastic anemia is a syndrome of bone marrow failure characterized by peripheral pancytopenia and marrow hypoplasia. Allogeneic stem cell transplantation from HLAmatched sibling is performed in the firstline setting in young aplastic anemia patients and in elderly patients who are refractory to immunosuppressive treatment. But if the patient does not have a HLA-matched sibling, allogeneic stem cell transplantation is performed from unrelated and haploidentical donors. In this study, we analyzaed and compared the results of aplastic anemia patients who had undergone allogeneic stem cell transplantation either from matched unrelated or haploidentical donors.

Methods: We collected and analyzed data of aplastic anemia patients who had undergone allogeneic stem cell transplantation from matched unrelated or haploidentical donor between 2011 and 2018.

Results: There were 10 patients who had received allogeneic stem cell transplantation from unrelated donors and there were 10 patients who had undergone haploidentical transplantation. But in 4 patients who had undergone haploidentical transplantation, engraftment failure had occurred and they were transplanted from different haploidentical donors fort he second time. So a total of 10 unrelated and 14 haploidentical transplants were performed. The median age of patients who had undergone unrelated transplantation was 29(16-55) and the median age of patients who had undergone unrelated transplantation was 22(19-61). The results of the haploidentical and unrelated transplantations are shown in Table 1. The neutrophil and platelet engraftment times were significantly longer in haploidentical transplantations ( $\mathrm{p}=0,006$ and $\mathrm{p}=0,005$, respectively). However, VOD, GVHD and 100 day mortality rates were not different in the 2 groups. Similarly overall survival (OS) of the patients who had undergone haploidentical or unrelated transplantation were not significantly different $(\mathrm{p}=0,38)$ (Figure 1$)$.

Conclusions: Although the number of patients are low in this study, we can conclude that urelated and haploidentical transplantation in aplastic anemia have comparable toxicity and efficacy.

\begin{tabular}{llll}
\hline & Haploidentical & Unrelated & $\mathrm{p}$ \\
\hline $\mathrm{n}$ & 14 & 10 & \\
Neutrophil engraftment time, day & $17(12-54)$ & $12(10-18)$ & 0,006 \\
Platelet engraftment time, day & $17(14-41)$ & $13(10-26)$ & 0,005 \\
Acute GVHD & $5(35,7 \%)$ & $1(10 \%)$ & 0,34 \\
VOD & 0 & $1(10 \%)$ & 0,41 \\
100 day mortality & $2(14,3 \%)$ & $1(12,5 \%)$ & 0,9 \\
\hline
\end{tabular}

[[P048 Table] 1. Table 1: Results of transplantation groups]

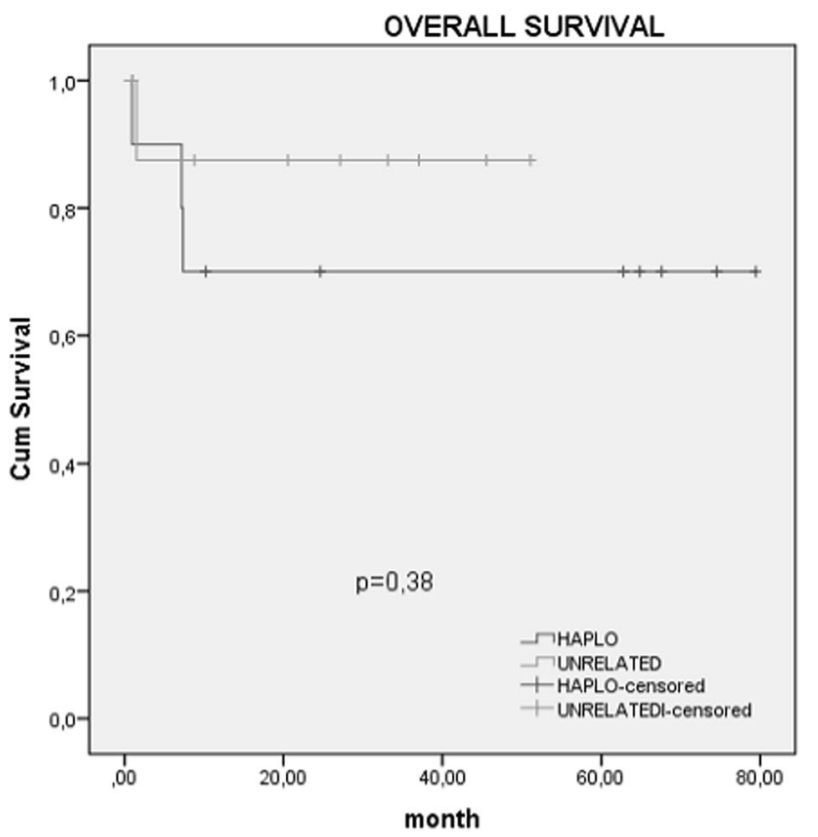

[[P048 Image] 1. Figure 1:Overall Survival]

Disclosure: Nothing to declare

P049

Allogeneic hematopoietic stem cell transplantation in adult aplastic anaemia patients: Promising treatment modality

${ }^{1}$ Eren Arslan Davulcu, ${ }^{1}$ Hale Bülbül, ${ }^{1}$ Yusuf Ulusoy, Nur Akad Soyer ${ }^{I}$, Fatma Keklik Karadağ $\breve{g}^{I}$, Ayșenur Arslan ${ }^{1}$, Fahri Şahin ${ }^{1}$, Mahmut Töbï̈ ${ }^{1}$, Güray Saydam ${ }^{1}$, Murat Tombuloğlu ${ }^{1}$, Filiz Vural ${ }^{1}$ 
${ }^{1}$ Ege University, Izmir, Turkey

Background: Aplastic anaemia (AA) is a rare and serious hematologic disorder. Immunosuppression and allogeneic hematologic stem cell transplantation (ASCT) are two main treatment options. Related and unrelated HLA full-matched ASCT is very promising especially in young patients. Our aim is to retrospectively evaluate clinical characteristics and outcome in AA patients who were undergone ASCT in Ege University Hematology Department Stem Cell Transplantation Unit.

Methods: Patients who were undergone ASCT in our department between January 2012 and September 2018 retrospectively evaluated. Patients demographic data, age at diagnosis, time between diagnosis and ASCT, treatment until ASCT, source of stem cell and number of CD34+ stem cell infused, transplantation regimen, prophylaxis for graft-versus host disease (GVHD), time of neutrophil and thrombocyte engraftment, information about acute and chronic GVHD, overall survival and final status were analyzed. Overall and progression free survival was calculated.

Results: In six years, 13 AA patients were transplanted. 4 patients had severe, 7 of them had very severe, 1 patient had not severe but transfusion depended and unresponsive to immunosupressive treatment AA. One of the patients was Fanconi AA. Paroxysmal nocturnal hemoglobinuria clone detected in two patient's follow-ups, they received eculizumab until ASCT. Male/female ratio was 8/5. Mean age in ASCT was 29.61 years (19-44), mean time from diagnosis to transplantation was 43.61 months (1-192). The source of stem cells was peripheral blood in all donors. 3 of the donors were unrelated. Mean CD34 + stem cell was $8,51 \times 10^{6} / \mathrm{kg}$ (5-14.87). Standard GVHD prophylaxis regimen was methotrexate $15 \mathrm{mg} / \mathrm{m}^{2}$ on day $+1,10 \mathrm{mg} / \mathrm{m}^{2}$ on day $+3,+6,+11$. and cyclosporin $5 \mathrm{mg} / \mathrm{kg}$ starting on day +1 . and titrated according to blood drug level. Patients conditioning regimen and clinical course after ASCT summarized in table 1 . There was no acute GVHD but 5 patients had chronic GVHD. One patient had grade 3 skin GVHD in 6th month of transplantation and eye GVHD in 12 th month. Another patient grade 2 skin GVHD in 4th month and eye GVHD in 5th month. Other two patients had grade 1 skin GVHDs in 15th and 7th months, 1 patient had grade 2 skin GVHD in $5^{\text {th }}$ month. 2-year overall and progression free survival were $82.1 \%$ and $84.6 \%$ respectively.

Conclusions: ASCT is the only curative treatment in AA. In our group mortality rate was $15.4 \%$ and deaths were in early post-transplantation period because of the septic complications. Other patients are alive $(84.6 \%)$ and didn't encounter serious or life-threatening complications. In young patients with HLA matched donors, ASCT is a very effective and safe treatment modality.

Disclosure: No conflict of interest

\section{Autoimmune diseases}

\section{P050}

Management of viral reactivation in multiple sclerosis patients after autologous transplantation with BEAM/ ATG conditioning regimen

Chiara Innocenti', Massimo Di Gioia', Maria Pia Amato ${ }^{1}$, Nunziata Ciccone ${ }^{1}$, Ilaria Cutini, Marta Giannini ${ }^{2}$, Antonella Gozzini', Stefano Guidi', Alice Mariottini', Luca Massacesi ${ }^{1}$, Chiara Nozzoli', Annamaria Repice ${ }^{1}$, Rossolini Gian Maria ${ }^{1}$, Riccardo Saccardi ${ }^{1}$

${ }^{1}$ University Hospital Careggi, Florence, Italy, ${ }^{2}$ Prato Hospital, Prato, Italy,

Background: Autologous stem cells transplantation (aHSCT) is an effective treatment for very aggressive autoimmune diseases such as multiple sclerosis (MS). However, toxicity remains the major concern to a wide application of this approach. Post transplant viral reactivations may induce severe complications and a rigorous monitoring of peripheral blood viral load for a prompt and effective therapy is required. A higher rate of EBV and CMV reactivation has been observed in patients affected by MS and conditioned with BEAM plus ATG compared with a controlled group of lymphoma patients without ATG in the conditioning regimen[1]. We report here the policy of our Center about both monitoring and treatment of such side effect.

Methods: A series of 37 consecutive patients with MS, transplanted between 2014 and 2018 is included in this analysis. All patients were mobilized with Cyclophosphamide 4g/sqm + G-CSF and conditioned with BEAM plus rabbit ATG (Thymoglobulin $@$, $7.5 \mathrm{mg} / \mathrm{Kg}$ ). Monitoring of CMV/EBV DNA on whole blood by quantitative PCR was performed after the engraftment, weekly for at least one month, then at longer intervals. Pre-emptive treatment with valgancyclovir was started in case of CMV viral load ${ }^{3} 1 \times 10^{\wedge} 4$ copies/mL. In case of EBV assay between $1 \times 10^{\wedge} 4$ and $1 \times 10^{\wedge} 5$ copies $/ \mathrm{mL}$ further determinations were performed and Rituximab-based treatment was started if the viral copies exceeded $1 \times 10^{5}$ copies. Patients received treatment in case of symptomatic disease for any value of the PCR of both viruses or EBV titer ${ }^{3} 1 \times 10^{\wedge} 5$ copies $/ \mathrm{mL}$.

Results: Detectable DNA for CMV was observed in 15/ $37(40,5 \%)$ patients at a median time from transplant of 23 days (range 12-36) and 6/37 (16\%) required pre-emptive treatment. All patients promptly responded to treatment within 2 weeks. EBV viral load was detectable in 19/37 
patients $(51,3 \%)$ at a median time of 22 days (range 12-52). One patient out of 19 started the treatment on first determination for high viral load $\left(>1 \times 10^{\wedge} 6 / \mathrm{mL}\right)$; nine presented an EBV viral load over $1 \times 10^{\wedge} 4$ copies $/ \mathrm{ml}$, three of them were treated thereafter for the persistent increase of the viral load $\left(>10^{5} / \mathrm{ml}\right)$. Six patients spontaneously recovered the EBV reactivation. Previous treatments were not predictive of any higher risk of viral reactivation. No impact on engraftment related to the reactivation was observed.

Conclusions: This policy shows that, despite a high rate of CMV and EBV reactivation, no grade III-IV adverse events were observed, suggesting the key role of viral monitoring in these patients and the efficacy of the preemptive treatment. EBV reactivation at low titers should be monitored to identify those cases that could achieve a spontaneous resolution and avoid the induction of further immunosuppression by Rituximab. These data confirm that patients diagnosed with AD undergoing autologous HSCT need a more intense pattern of care than hematological patients.

1. CMV AND EBV REACTIVATION AFTER AUTOLOGOUS HAEMOPOIETIC STEM CELL TRANSPLANTATION WITH BEAM CONDITIONING REGIMEN FOR MULTIPLE SCLEROSIS AND LYMPHOMA: A RETROSPECTIVE SINGLE CENTER CASE-CONTROL STUDY M. Di Gioia1,*, C. Innocenti1, R.Saccardi EBMT2014

Disclosure: Chiara Innocenti: Nothing to declare, Massimo Di Gioia: Nothing to declare, MariaPia Amato: Nothing to declare, Nunziata Ciccone: Nothing to declare, Ilaria Cutini: Nothing to declare, Marta Giannini: Nothing to declare, Antonella Gozzini: Nothing to declare, Stefano Guidi: Nothing to declare, Alice Mariottini: non-financial support from Biogen idec, Sanofi Genzyme, Novartis, Luca Massacesi: non-financial support from Biogen, Novartis, Merck Serono, Genzyme and Teva, Chiara Nozzoli: nothing to declare, Annamaria Repice: personal fees and nonfinancial support from Biogen idec, Sanofi Genzyme and Novartis; non-financial support from Teva; personal fees from Merck

Gian Maria, Rossolini: personal fees, grant and/or financial support from Accelerate Diagnostic, Angelini, Astra-Zeneca, Basilea, Becton-Dickinson, bio-Merieux, Biotest, Cepheid, Elitech, Merck, Novartis, Nordic Pharma, Pfizer, Seegene, Shionogi, Zambon, Roche, Curetis, ThermoFisher, Beckman Coulter, Menarini, Arrow, Symcel. Other with VenatorX (strains sold by the department)

Riccardo Saccardi: personal fees from Sanofi Genzyme

\section{P051}

Autologous haematopoietic stem cell transplantation for autoimmune diseases: Experience of a tertiary referral center in Colombia

Claudia Lucia Sossa Melo ${ }^{1,2}$, Sara Ines Jimenez ${ }^{1,2}$, Angela María Peña ${ }^{1,2}$, Luis Antonio Salazar ${ }^{1,2}$, Manuel Rosales², Jose Patricio López', Manuel Ardila-Báez', Claudia Marcela Chalela ${ }^{I}$, Maria Luna-Gonzalez ${ }^{1}$, Maria Fernanda Ortiz ${ }^{1}$, Diego Saaibi ${ }^{1,2}$, Ivan Mauricio Peña ${ }^{1,2}$

${ }^{1}$ Universidad Autónoma de Bucaramanga, Bucaramanga, Colombia, ${ }^{2}$ Clinica FOSCAL, Bucaramanga, Colombia

Background: Autoimmune diseases are chronic serious conditions that are often refractory to standard therapies. Since 1996, autologous haematopoietic stem cell transplantation (HSCT) has been a very promising alternative that has shown satisfactory long-term results. The aim of this study is to evaluate immune reconstitution and mortality following HSCT in patients with autoimmune disease.

Methods: A retrospective study was conducted on patients with diagnosis of autoimmune diseases that underwent autologous HSCT between July 2012 and January 2018 at a tertiary referral center in Colombia, South America. Descriptive statistics were used to analyze patient's demographic and clinic characteristics.

Results: Seven patients were included, with a mean age of 37 years (range 26-57). Five patients were female (71\%). The indications for HSCT were systemic sclerosis $(n=3)$, multiple sclerosis $(n=2)$, and myasthenia gravis $(n=2)$. The conditioning regimen administered in patients with systemic sclerosis was cyclophosphamide + human anti-T lymphocyte immunoglobulin, BEAM (Carmustine, Etoposide, Cytarabine (Ara-C), and Melphalan) + Human anti-T lymphocyte immunoglobulin in patients with multiple sclerosis, and cyclophosphamide + Human anti-T lymphocyte immunoglobulin in myasthenia gravis. Median time to myeloid engraftment (neutrophils $>0.5 \times 109 / \mathrm{L}$ ) was 12 days post-transplantation, and platelet engraftment, defined as $>20,000$ platelets $/ \mathrm{mm}^{3}$ untransfused, was 11 days postHSCT.

Median time of hospitalization was 21 days (range 1166); longer in-patient management was due to infectious complications. Infectious complications included bacteremia caused by E. Coli and pneumocystis pneumonia that resulted in septic shock and acute respiratory failure, respectively.

Evaluating T-cell immune reconstitution, none of the patients had reached normal CD4+ cell value after one year of HSCT follow-up. Four patients $(57.1 \%)$ reached normal CD8 + cells value at 3 months post-HSCT. Regarding Bcell immune reconstitution, $85.7 \%$ of the patients $(6 / 7)$ had reached both IgG and IgA normal levels at one-month postHSCT, and four patients (57.1\%) had achieved normal IgM 
level at 6 months post-transplantation. In the 10 months to 6 years of post-HSCT follow-up, there has not been evidence of disease relapse in any of the patients. There were no deaths in the present cohort.

Conclusions: Autologous HSCT has proved to be a successful treatment alternative in patients with severe refractory autoimmune disease, achieving sustained remissions for more than 5 years.

Disclosure: Nothing to declare

\section{P052}

Autologous haematopoietic stem cell transplantation in multiple sclerosis - A single centre experience

Adriana Roque ${ }^{1,2}$, Telma Nascimento ${ }^{1}$, Emilia Cortesão $^{1,2}$, Ana Isabel Espadana ${ }^{1}$, Luís Francisco Araújo $^{1,2}$, Raquel Guilherme ${ }^{1,2}$, Maria Carmo Macário ${ }^{1}$, Lívia Sousa $^{1,2}$, Ana Bela Sarmento-Ribeiro ${ }^{1,2}$, Letícia Ribeiro $^{1}$, Catarina Geraldes ${ }^{1,2}$

${ }^{1}$ Centro Hospitalar e Universitário de Coimbra, Coimbra, Portugal, ${ }^{2}$ University of Coimbra, Coimbra, Portugal

Background: Multiple sclerosis(MS) is an inflammatory disease caused by autoimmune reactivity of $\mathrm{T}$ cells against myelin.

There is accumulating evidence of the efficacy of highdose chemotherapy followed by autologous haematopoietic stem cell transplantation(aHSCT) in MS patients who failed response to standard immunotherapy, despite a variability in eligibility criteria, conditioning regimens and outcome.

Methods: We retrospectively reviewed MS patients submitted to aHSCT in our centre(2014-2018). Patient eligibility criteria were active relapsing remitting(RRMS) or secondary-progressive MS (SPMS), with prior failure to treatment with disease-modifying therapies and evidence of disease activity (clinical relapse or new active lesions in magnetic resonance $[\mathrm{MR}])$.

Mobilization of CD34+cells to peripheral blood was performed with granulocyte colony-stimulating factor $(\mathrm{G}-$ CSF $10 \mu \mathrm{g} / \mathrm{kg} / \mathrm{day}$ ) and conditioning regimen according to BEAM protocol.

The severity of MS disability was classified according to the Expanded Disability-Status Scale (EDSS) and aHSCT toxicity was evaluated by CTCAEv5.0.

Results: Seven MS patients had undergone aHSCT (4 female/3 male), with a median age at aHSCT of 39.9 years (32-42).

Median age at MS diagnosis was 31.6 years(27-36) and median time from diagnosis until aHSCT was 6.8 years(3$13)$.

Four patients $(57.1 \%)$ had SPMS and 3(42.9\%) had RRMS, with $4(57.1 \%)$ having MR active lesions. Pre-
aHSCT relapse rate per year was 2(1-4). Median baseline EDSS was 6.5(5.5-7.5).

Median number of previous DMTs was 6(3-9). All patients had been treated with corticosteroids and copaxone, $5(71.4 \%)$ received rituximab, $6(85.7 \%)$ natalizumab, 1 (14.3\%) alemtuzumab, 2(28.6\%) fampridine and 2(28.6\%) fingolimod.

All patients collected enough CD34+ cells in a single apheresis session. Median number of CD34+ cells infused was $7.2 \pm 3.8 \times 10^{\wedge} 6 / \mathrm{kg}$, for a mean DMSO volume of 50.4 $\pm 14.8 \mathrm{~mL}$.

Median inpatient stay during aHSCT was 28 days(21-47). All patients developed febrile neutropenia, one was admitted to intensive care unit due to sepsis. One patient developed an anaphylactic reaction to transfusion and another a self-limited encephalopathy. Two (28.7\%) patients had grade $\geq 2$ oral mucositis, and all had gastrointestinal toxicity (grade 2-3).

Median time until neutrophils $>500 / \mu \mathrm{L}$ was $11(6-12)$ days, to platelets $>20,000 / \mu \mathrm{L}$ was 9 days $(5-12)$, and to engraftment was 13 days(12-14). Patients were transfused with a median of 1(0-2) unit for erythrocytes and 3(1-8) for platelets.

There was no treatment-related mortality and no long term side effects have been observed so far.

For a median post-aHSCT follow-up of 8.7 months, no patient developed new lesions in MR, but 2(28.6\%) experienced symptoms consistent with a clinical relapse, at a median time of 20.5(5-35) months, effectively rescued with corticosteroids. The absence of evidence of disease activity at 6-months was $71.4 \%$.

Although there was no variation concerning EDSS punctuation, $4(57.1 \%)$ patients self-reported significant benefits, especially concerning limb strength and sphincter continence improving.

Conclusions: Our real life results claim a stabilization effect of MS patients with highly active/progressive disease after aHSCT, with no significant toxicity.

The failure in reporting benefits in EDSS punctuation is probably due to a small sample size and short follow-up.

More studies are needed to establish the best patient selection criteria and define the ideal time to include these patients in transplantation programs, as well as to evaluate its long term outcome.

Disclosure: Nothing to declare.

\section{P053}

The direct antiglobulin test in oncohematological patients

Elena Poponina ${ }^{1}$, Elena Butina ${ }^{1}$, Anna Yovdiy ${ }^{1}$, Galina Zaytseva $^{1}$, Natalia Minaeva ${ }^{1}$, Igor Paramonov ${ }^{1}$ 
${ }^{1}$ Kirov Scientific Research Institute of Hematology and Blood Transfusion of the Federal Medical-Biological Agency, Kirov, Russian Federation

Background: The development of oncohematological diseases may be complicated by the autoimmune pathology, including the production of antibodies to own red blood cells. The direct antiglobulin test (DAT) is used to detect antibodies and complement components on the surface of red blood cells. A differentiated DAT allows to semiquantitatively assess the immunoglobulins fixed on red blood cells and determine their subclass.

Methods: The results of DAT were analyzed in 250 oncohematological patients first hospitalized at the Institute clinic in 2018 with the diagnosis: multiple myeloma (MM) - 67, acute myeloid leukemia (AML) - 55, non-Hodgkin lymphoma (NHL) - 44, acute lymphoblastic leukemia (ALL) - 32, Hodgkin disease (HD) - 26, chronic lymphocytic leukemia (CLL) - 19, chronic myelogenous leukemia (CML) - 7 patients. DAT was performed by gel method using anti-IgG/C3d, DC-Screening I and DAT IgG1/IgG3 cards (BioRad, Switzerland). The frequency of positive DAT in patients was compared with the same in 200 healthy residents of the region - donors of blood components.

Results: All blood donors were DAT-negative. Autoantibodies to red blood cells were detected in $15,2 \%$ of oncohematological patients. The most often positive DAT was revealed in patients with MM - 26,9\%. Autoantibodies were detected in 18,2\% patients with NHL, 15,4\% — with HD, 10,9\% - with AML, 10,5\% — in patients with CLL. In patients with ALL and CML the DAT result was negative.

The intensity of the reaction $(+)$ was observed in $57,9 \%$ of patients with positive DAT, $(++)$ - in $34.2 \%,(+++)$ - in $5,3 \%,(++++)-2.6 \%$. In the majority of cases $(92,1 \%)$ $\operatorname{IgG} 2,4$ were fixed on the surface of red blood cells, associated with a low risk of immune hemolysis. Only in 3 patients $(7,9 \%)$, with the DAT reaction $(+++)$ and $(+++$ $+)$, DAT was positive due to IgM and complement components.

Autoantibodies in the plasma of patients can interact not only with their own red blood cells, but also with test and donors red blood cells, being detected in an indirect antiglobulin test (IAT). A positive IAT was in $13,2 \%$ of patients with positive DAT. These patients had difficulties in donors due to non-specific agglutination.

Conclusions: Despite the fact that the development of oncohematological diseases is often accompanied by the production of autoantibodies, not in every case a positive result of DAT should be regarded as a sign of autoimmune hemolysis. To correctly assess the risk of hemolysis, the physicians should analyze other laboratory and clinical data.
Positive DAT may be correlated with the difficulties of selection of the donor for such patient.

Disclosure: Nothing to declare.

Cellular Therapy

\section{P054}

Single cell identification of defined ebv epitope-specific TCR $\alpha \beta$ pairs for adoptive $T$ cell transfer against EBV infection and EBV-associated malignancies

María Fernanda Lammoglia Cobo ${ }^{1}$, Christian Pircher ${ }^{1}$, Kerstin Dietze ${ }^{I}$, Thomas Kammertöns ${ }^{1}$, Regina Gary ${ }^{2}$, Andreas Moosmann ${ }^{3}$, Josef Mautner ${ }^{3}$, Klaus Dornmair, Anna Takvorian ${ }^{I}$, Lars Bullinger ${ }^{1}$, Thomas Blankenstein $^{1,5}$, Armin Gerbitz, Leo Hansmann ${ }^{1}$

${ }^{1}$ Charité - University Medicine Berlin, Berlin, Germany, ${ }^{2}$ University Medicine Erlangen, Erlangen, Germany, ${ }^{3}$ Helmholtz Center Munich, Munich, Germany, ${ }^{4}$ Biomedical Centre and Hospital of the LMU, Institute of Clinical Neuroimmunology, Munich, Germany, ${ }^{5}$ Max Delbrück Centre for Molecular Medicine, Berlin, Germany

Background: Reactivation of Epstein-Barr virus (EBV) represents a potentially life-threatening condition in approximately $30 \%$ of patients after allogeneic stem cell transplantation, with no specific treatment available.

Methods: We have previously developed a manufacturing protocol for the expansion of Cytomegalovirus (CMV) and EBV-specific $\mathrm{T}$ cells by stimulation of G-CSFmobilized stem cell grafts with defined peptide pools (Gary, 2018). This Advanced Therapeutic Medicinal Product is currently investigated in an ongoing phase I/IIa trial (EudraCT Number: 2012-004240-30). However, the expansion of virus-specific $\mathrm{T}$ cells relies on a pre-existing virusspecific memory compartment in the stem cell donor. In virus-seronegative donors, no expansion can be achieved. We therefore aim to identify EBV peptide-specific $\mathrm{T}$ cell receptors (TCRs) that can be translated into off-the-shelf cell products for the treatment and prophylaxis of EBV infection and $\mathrm{EBV}$-associated malignancies.

Leftover cells from five allogeneic stem cell grafts were expanded in vitro in the presence of HLA-B35*01-restricted peptides (HPVGEADYFEY from EBNA1, EPLPQGQLTAY from BZLF1) associated with latent and lytic EBV infection. After expansion, single EBV-specific T cells were FACS sorted using peptide-MHC (pMHC) tetramers, and individual TCR $\alpha$ - and $\beta$-chain pairs were determined with single cell sequencing (Han 2014, Penter 2018). To confirm peptide specificity, dominant TCR pairs were transfected into a murine $58 \alpha^{-} \beta^{-}$reporter T hybridoma cell line with NFAT-driven GFP expression (Siewert, 2012). Functional TCR $\alpha \beta$ candidates were transduced into human peripheral 
blood $\mathrm{T}$ cells and co-cultured with allogeneic and autologous EBV positive lymphoblastoid cell lines (LCL) to test for recognition of EBV-infected cells.

Results: Frequencies of HPV- and EPL-specific T cells, as determined by pMHC tetramer staining, indicated an average 40 -fold increase after in vitro expansion. Three dominant TCR $\alpha \beta$ pairs were re-expressed in $58 \alpha^{-} \beta^{-}$cell lines and recognized their respective target peptides -as presented by NFAT-driven GFP expression and IL-2 secretion- upon co-incubation with HLA-matched peptideloaded antigen presenting cells. After transduction into human $\mathrm{T}$ cells from HLA-matched and unmatched donors, all three TCRs recognized the HLA-B*35:01 pMHC tetramers, and $\mathrm{T}$ cells transduced with two EPL-specific TCRs resulted in IFN- $\gamma$ secretion in the presence of HLAmatched EBV-LCLs.

Among the five HLA-B*35:01 donors, $10 \mathrm{HPV}$ - and EPL-specific TCR clones in total were found in at least 2 patients with different extents of dominance. The repertoires indicate an antigen-directed oligoclonal expansion. Meanwhile, sequence comparison between an in vitro and in vivo expansion after EBV reactivation in one patient revealed the presence of several identical sequences.

Conclusions: We present a system for highly efficient identification of EBV epitope-specific TCRs that can be reexpressed on human $\mathrm{T}$ cells and recognize EBV-infected cells. The expansion of common peptide-specific TCRs across HLA-matched patients and the in vivo expansion upon EBV reactivation suggest high relevance for virus control. The translation of defined sets of TCRs into cellular products for adoptive transfer will help to overcome unmet clinical needs in the treatment and prophylaxis of virus reactivation, primary infection, and EBV-associated malignancies such as post-transplant lymphoproliferative disease (PTLD) and Hodgkin lymphoma. Furthermore, it opens the possibility to treat CMV-reactivation after solid organ transplantation through the identification of CMVspecific TCRs.

Clinical Trial Registry: Not applicable.

Disclosure: Nothing to declare.

\section{P055}

\section{Abstract already published.}

\section{P056}

NKG2D-CAR $T$ cells as an immunotherapy against pediatric hematological malignancies

Lucía Fernández ${ }^{1}$, Adrián Fernández ${ }^{1}$, Adela Escudero ${ }^{2}$, Leila Cardoso ${ }^{2}$, Jaime Valentín ${ }^{3}$, Alejandra Leivas ${ }^{4}$, María Vela ${ }^{3}$, Joaquín Martínez-López ${ }^{4}$, Mar Valés ${ }^{5}$, Antonio Pérez-Martínez,
${ }^{1}$ CNIO, Clinical Research, Madrid, Spain, ${ }^{2}$ INGEMM, Madrid, Spain, ${ }^{3}$ IdiPAZ, Madrid, Spain, ${ }^{4}$ Hospital 12 de Octubre, Hematology, Madrid, Spain, ${ }^{5} \mathrm{CNB}$, Madrid, Spain, ${ }^{6}$ Hospital La Paz, Madrid, Spain

Background: Lymphoid and myeloid acute leukemia are the most frequent cause of cancer related death in children. Interactions between NKG2D receptor, expressed in cytotoxic immune cells, and its ligands (NKG2DL), that are upregulated in many types of tumor cells including leukemic blasts, are important for anti-tumor immune surveillance. Nevertheless, tumor cells may develop immune scape strategies like ligand shedding, which reduces NKG2DL expression and may cause NKG2D receptor downregulation. Engineering T lymphocytes with NKG2D CAR may overcome immune evasion and become an effective therapeutic strategy.

Methods: CD45RA ${ }^{-} \mathrm{T}$ cells were obtained by depletion of non-mobilized apheresis with CD45RA magnetic beads using CliniMACS. NKG2D-CAR T cells were generated by lentiviral (NKG2D-41BB-CD3z) transduction of CD45RA $\mathrm{T}$ cells with $\mathrm{MOI}=2$.

The expression of NKG2D ligands was analyzed in peripheral blood or bone marrow samples from a total of 97 leukemia patients $(\mathrm{AML}=13, \mathrm{~B}-\mathrm{ALL}=52$ and $\mathrm{T}-\mathrm{ALL}=19)$, at different status of the disease (Diagnosis, Remission, Relapse/refractory), and in 10 different leukemia cell lines by qPCR and flow cytometry. Cytotoxicity of NKG2DCAR $T$ cells against leukemia cells was evaluated by performing conventional-4 hours Europium-TDA assays. Soluble NKG2DL (sNKG2DL) concentration was measured in the sera of leukemia patients by ELISA.

To evaluate the effect of sNKG2DL on NKG2D-CAR $\mathrm{T}$ cells, those were cultured in the presence or absence of different concentrations of sNKG2DL for 7 days. One week later, cell proliferation and CAR downregulation were measured by flow cytometry using Cell Trace Violet and NKG2D labeling, respectively. The production of IFN-g and TNF-a was measured in the supernatants by ELISA. The effect on cytotoxicity was evaluated in a 2 hoursdegranulation assay by co-culturing sNKG2DL pretreated NKG2D-CAR T cells against K562 cell line.

Results: NKG2D ligands were expressed in leukemia cell lines and leukemic blasts. NKG2DL expression changed with disease status with a trend to decrease at diagnosis and relapse/refractory compared to remission. NKG2D-CAR $\mathrm{T}$ cells were cytotoxic against $8 / 10$ leukemia cell lines with a percentage of specific lysis over $50 \%$. Myeloid and TALL cell lines were more susceptible to NKG2D-CAR T cells (specific lysis ranging from 50-78\%) compared to BALL cell lines (19-52\%). Physiological concentrations of sNKG2DL caused an increase in NKG2D-CAR expression. However, supra-physiological levels of sNKG2DL 
decreased NKG2D-CAR expression up to 5 times and increased cell proliferation up to 4 times. CD4+ subpopulation was more affected by downregulation, while proliferation had more impact on CD8+ subset. The effects of sNKG2DL were dose-dependent and attenuated by IL-2.

Conclusions: NKG2D-CAR T cells are cytotoxic against leukemia cells, specially AML and T-ALL, and thus could be a novel therapeutic approach for non-B leukemia, or those B-ALL that relapse with undetectable CD19 after CD19-CAR treatment. NKG2D-CAR expression may be downregulated only by supra-physiological levels of sNKG2DL, although antitumor activity is not affected. IL2 softens the negative effects of sNKG2DL inducing NKG2D expression, cell proliferation and cytokines production. The changes observed in NKG2DL surface expression at the different stages of the disease could be related to ligands release and immune escape.

Disclosure: Nothing to declare

\section{P057}

anti-leukaemia and anti-viral T-cell immunotherapy of photodepleted allogeneic $\mathrm{T}$ cells using TH9402 photosensitiser

\section{Denis-Claude Roy ${ }^{1,2}$, Ines Adassi, ${ }^{1,2}$, Céline Leboeuf ${ }^{1,2}$, Vibhuti P. Dave $e^{1,2}$}

${ }^{1}$ Hôpital Maisonneuve-Rosemont Research Center, Montréal, Canada, ${ }^{2}$ University of Montréal, Montréal, Canada

Background: For patients with high-risk leukaemia, allogeneic haematopoietic stem cell transplantation is the only curative treatment. The presence of alloreactive $\mathrm{T}$ cells in the donor graft, however, leads to a high probability of developing graft-versus-host disease (GVHD). T-cell depletion minimises the presence of GVHD-causing alloreactive cells, but often results in an increased incidence of infections and disease relapse. Photodepletion treatment (PDT) can specifically deplete activated alloreactive T cells while conserving resting $\mathrm{T}$ cells. PDT-treated cells have been utilised after T-cell-depleted haploidentical transplant to help reduce infection and relapse. The efficacy and safety of such PDT-treated cells is currently under clinical investigation in a phase III trial (HATCY, NCT02999854; Kiadis Pharma).

Here the reactivity of PDT-treated donor T cells was assessed toward tumour-associated and viral antigenic peptides derived from Wilm's Tumour protein 1 (WT1p), preferentially expressed antigen in melanoma (PRAMEp), and from cytomegalovirus and Epstein-Barr virus (CMV/ EBVp).

Methods: Healthy donor (HLA-A*0201) peripheral blood mononuclear cells (PBMCs) were co-cultured with irradiated PBMCs from another mismatched donor (1:1) in a 4-day mixed lymphocyte reaction. TH9402, a photoactive rhodamine derivative, was added and cells were exposed to visible light to deplete the TH9402-containing activated alloreactive cells. Elimination of alloreactive cells post-PDT was assessed using CD25 and HLA-DR as activation markers. An ex vivo expansion protocol was exploited to evaluate the impact of PDT on reactivity to tumour and viral antigenic peptides. Post-PDT T cells were co-cultured with irradiated autologous monocyte-derived dendritic cells (10:1) pulsed with WT1p, PRAMEp or CMV/EBVp. Antigen-specific T cells were re-stimulated on Days 7 and 14 with WT1p- or PRAMEp-pulsed autologous PBMCs or with CMV/EBVp added directly to the culture. MHCtetramer staining was performed on Days 14 and 21; IFN- $\gamma$ ELISpot was conducted on Day 21.

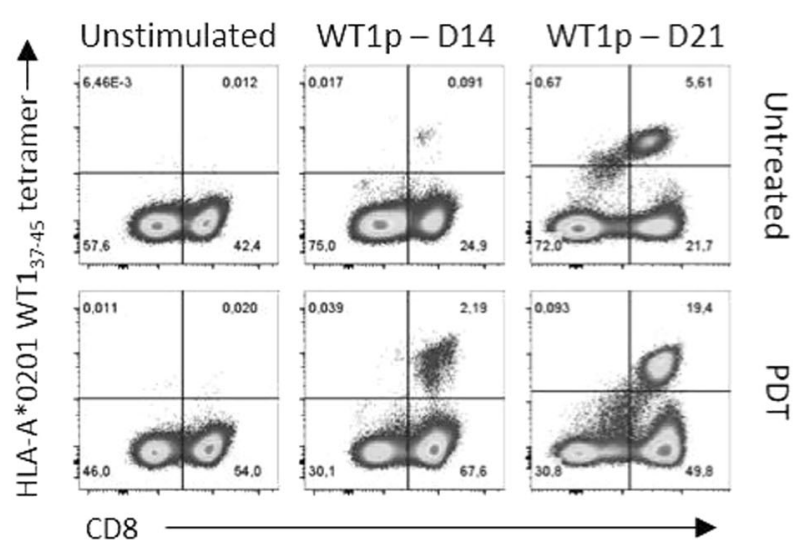

A. Representative example of HLA-A*0201 WT1 $1_{37-45}$ tetramer staining on Days 14 and 21.

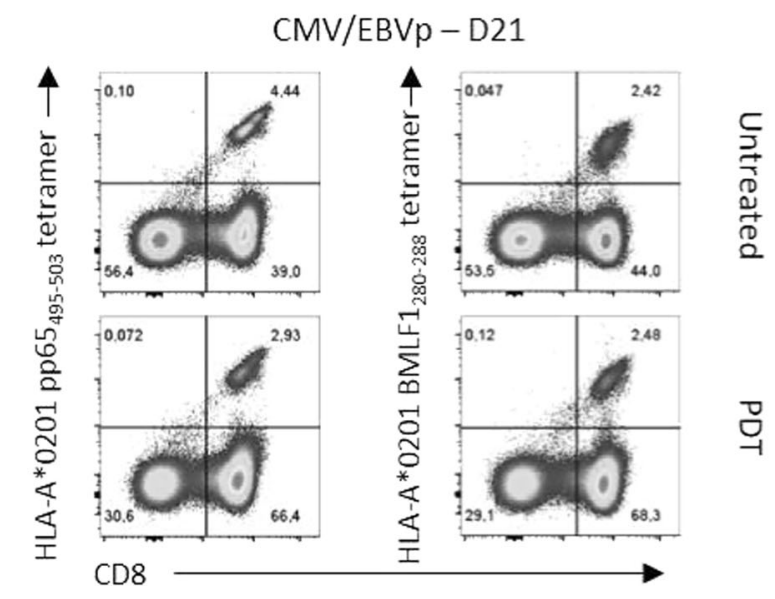

[[P057 Image] 1. Functional WT1-specific and viralspecific $T$ cells can be expanded post-PDT]

Results: PDT resulted in a drastic decrease of CD25 and/ or HLA-DR activation marker-expressing CD4+ and CD8 $+\mathrm{T}$ cells. PDT-treated cells showed a significant increase in 
the frequency and number of $\mathrm{WT}_{37-45}$-specific $\mathrm{T}$ cells on Day 14 of culture, greater than the increase seen in untreated cells, with some cultures continuing expansion on Day 21 (Figure A). PDT-treated WT1 $1_{37-45}$-specific T cells secreted IFN- $\gamma$ upon exposure to $\mathrm{WT} 1_{37-45}$ peptide and were highly cytotoxic against $\mathrm{WT}_{37-45}$-pulsed $\mathrm{T} 2$ cells. These data demonstrate that PDT preserves functional WT1-specific T cells. Detection of T-cell responses to PRAMEp in some donors showed preservation of antileukaemic T cells post-PDT, not restricted to WT1. HLAA*0201 CMV pp65 $495-503$ and HLA-A*0201 EBV $B M L F 1_{280-288}$ tetramers detected significant numbers of specific $\mathrm{T}$ cells in both PDT-treated and untreated cultures on days 14 and 21 of expansion (Figure B). Similar IFN- $\gamma$ secretion was seen in untreated and PDT-treated cells after re-stimulation with pp65 ${ }_{495-503}$ and $B M L F 1_{280-288}$ peptides, demonstrating that PDT did not affect the functionality of the antiviral $\mathrm{T}$ cells.

Conclusions: PDT selectively eliminates alloreactive GVHD-causing $\mathrm{T}$ cells, while preserving functional antileukaemic and antiviral $\mathrm{T}$ cells to prevent both disease relapse and viral infection/reactivation without the need for immunosuppression. These results corroborate the low infection and relapse rates observed in patients treated with PDT-treated cells.

\section{Clinical Trial Registry: N/A}

Disclosure: Funding for this study and medical writing support was provided by Kiadis Pharma. Denis-Claude Roy discloses patents and royalties and is an author on patent at the University of Montreal and Hôpital MaisonneuveRosemont. Denis-Clauide Roy also discloses travel support and consultancy with Kiadis Pharma. No other authors have conflicts of interest to declare.

\section{P058}

Sleeping beauty based engineering of chimeric antigen receptor NK cells for treatment of acute lymphoblastic leukemia

Tobias Bexte ${ }^{1,2}$, Lacramioara Botezatu ${ }^{3}$, Stephan Mueller $^{1,2}$, Hanna Steigerwald ${ }^{1,2}$, Michael Hudecek ${ }^{4}$, Axel Schambach $^{5}$, Ute Modlich ${ }^{2,6}$, Winfried S. Wels ${ }^{7,8}$, Zoltan Ivics $^{3,8}$, Evelyn Ullrich ${ }^{1,2,8}$

${ }^{1}$ Hospital of the Goethe University Frankfurt, Frankfurt am Main, Germany, ${ }^{2}$ Johann Wolfgang Goethe-University, LOEWE Center for Cell and Gene Therapy, Frankfurt am Main, Germany, ${ }^{3}$ Paul Ehrlich Institute, Langen, Germany, ${ }^{4}$ Universitätsklinikum Würzburg, Medizinische Klinik und Poliklinik II, Würzburg, Germany, ${ }^{5}$ Hannover Medical School, Institute of Experimental Hematology, Hannover, Germany, ${ }^{6}$ Paul-Ehrlich-Institute, Research Group Gene Modification in Stem Cells, Langen, Germany, ${ }^{7}$ Georg-
Speyer-Haus, Institute for Tumor Biology and Experimental Therapy, Frankfurt am Main, Germany, ${ }^{8}$ German Cancer Consortium (DKTK), Frankfurt am Main, Germany

Background: Acute lymphoblastic leukemia (ALL) is the most common childhood cancer and relapsed or refractory ALL is still difficult to treat. Engineered T cells equipped with a synthetic chimeric antigen receptor (CAR) targeting CD19 have demonstrated remarkable efficacy to treat ALL. However natural killer (NK) cells are known for their target-independent cytotoxic potential without induction of cytokine release syndrome (CRS) or graft-versus-hostdisease (GvHD), CAR-NK cells can overcome the persisting problem in the therapy with CAR T cells. As the use of viral vector generated CAR NK cells is limited by theire genotoxicity, cost and regulatory demands, we are developing an innovative protocol using non-viral Sleeping Beauty (SB) transposition of third party NK cells as a source to produce 'off the shelf' CAR-engineered cell products.

Methods: NK cells are isolated from peripheral blood mononuclear cells (PBMCs) using CD56 selection kits. They are successfully expanded ex vivo with IL-15 cytokine stimulation under feeder-cell free conditions. After few days of expansion NK cells are electroporated using pmaxGFP. Transfection efficiency and percent of living cells after electroporation is analyzed by flow cytometry. Transposition based nucleofection using an SB100X mRNA and a minicircles (MC) DNA vector is performed at different time points after NK cell isolation. The transient MC-Venus longtime expansion and the viability after SB100X based nucleofection is measured over two weeks.

Furthermore, $\alpha$-retroviral ( $\alpha$-RV) CD19-CAR transduction of NK cells with different viral amounts (MOI) is conducted and the cytotoxicity of the engineered CD19CAR-NK cells against the CD19 positiv cell line SupB15 is addressed.

Results: For an $\alpha-R V$ CD19-CAR transduction of maximal $1 \times 10^{4} \mathrm{NK}$ cells we could show transduction efficiency of $68,96 \%$ for MOI10. The $\alpha$-RV CD19-CAR modified NK cells had a high killing activity against CD19 positiv SupB15 cells (E:T ration 1:1 90,85\%) compared to CD19 negativ K562 cell lines (E:T ration 1:1 16,42\%) and the non-transduced NK cells (E:T ratio 1:1 9,03\%).

In first experiments with pmaxGFP vector based nucleofection, we could show an increasing efficiency of $55,9 \% 48 \mathrm{~h}$ post electroporation with a only slightly decreas of living cells $(21,5 \%)$ comparing to the non-electroporated NK cell viability. Using SB100X mRNA with MC-Venus DNA we electrotransfected $1 \times 10^{6} \mathrm{NK}$ cells after fews days of cultivation and we reached $36,6 \%$ of transfected NK cells $24 \mathrm{~h}$ post electroporation and a transient expression of MC- 
Venus positive NK cells up to $54,6 \%$ efficiency with an increasing rate of live cells over 14 days after electroporation.

Conclusions: The Sleeping Beauty based nucleofection of NK cells is a very promising non-viral method to generate more easy, safer and higher amounts of genetically modified third party NK cells for therapy of ALL and has also a broad range of clinical applications.

Disclosure: Winfried S. Wels is an inventor on a patent describing chimeric antigen receptors with an optimized hinge region. Axel Schambach is an inventor on a patent describing alpharetroviral SIN vectors. Michael Hudecek and Zoltan Ivics are inventors on patents related to Sleeping Beaut gene transfer technology. The remaining authors have nothing to disclose.

\section{P059}

NK and NKT cell doses in allogeneic stem cell grafts affect relapse-free survival and graft-versus-host disease after transplantation

Lia Minculescu ${ }^{1}$, Hanne Marquart ${ }^{1}$, Lars Peter Ryder ${ }^{1}$, Lone Smidstrup Friis ${ }^{I}$, Ida Schjødt ${ }^{l}$, Brian Thomas Kornblit ${ }^{1}$, Niels Smedegaard Andersen ${ }^{1}$, Søren Lykke Petersen $^{1}$, Eva Haastrup ${ }^{I}$, Anne Fischer-Nielsen ${ }^{1}$, Henrik Sengeløv ${ }^{I}$

${ }^{1}$ Copenhagen University Hospital, Rigshospitalet, Copenhagen, Denmark

Background: Mature immune cells from the stem cell graft are essential for the graft-versus-tumor (GVT) effect to eliminate residual malignant cells after hematopoietic stem cell transplantation (HSCT), but donor cells are also involved in complications such as graft-versus-host disease (GVHD).

Methods: We performed a prospective study of the detailed graft composition in 102 recipients of peripheral blood stem cells (PBSC) or bone marrow (BM) in order to identify correlations to clinical outcomes, table 1. Grafts were characterized with concentrations of T cells and NK cells together with a multi-color flow cytometry panel with staining for TCR $\alpha \beta, \mathrm{TCR} \gamma \delta$, V $\delta 1, \mathrm{~V} \delta 2, \mathrm{CD} 3, \mathrm{CD} 4$, CD8, HLA-DR, CD196, CD45RO, CD45RA, CD16, CD56, CD337 and CD314 for detailed immune phenotyping. Cell contents in stem cell grafts were analyzed both as fractions of CD45 positive lymphocytes and as absolute concentrations converted to transplanted cells/ $\mathrm{kg}$. Fractions were evaluated in patients receiving both BM and PBSC $(\mathrm{n}=102)$, while concentrations (cells $/ \mathrm{kg})$ were only analyzed in patients transplanted with PBSC $(\mathrm{n}=88)$.
$\mathrm{N}$

Follow-up, days, median, min-max 662 (386-884)

Age, years, median, min-max 59 (20-74)

Disease, n,percent AML, ALL, 41 (40\%), 14 (14\%), 25 (24\%), 8

MDS, Myelofibrosis,NHL,Chronic (8\%), 5 (5\%), 4 (4\%), 5 (5\%),

leukemia, Other

Graft source, n, percent BM, PBSC 14 (14\%), 88 (86\%)

Donor, n, percent MRD, MUD $23(23 \%), 79(77 \%)$

Donor, HLA-match, n, percent 10/ 91 (89\%), 9 (9\%), 2 (2\%)

10 or $9 / 10$ allele math, $1 \mathrm{Ag}$

mismatch, Haplo-identical

Donor-recipient, sex, n, percent M/ 49 (48\%), 26 (25\%), 17 (17\%),

$\mathrm{M}, \mathrm{M} / \mathrm{F}, \mathrm{F} / \mathrm{F}, \mathrm{F} / \mathrm{M}$

$10(10 \%)$

Conditioning intensity, $\mathrm{n}$, percent 48 (47\%), 54 (53\%)

Myeloablative, Non-myeablative

Conditioning regimen, $\mathrm{n}$, percent $50(49 \%), 26(25 \%), 19(19 \%), 4$ TBI-Flu, Flu-Treo, TBI-Cy, TBI- (4\%), 3 (3\%)

Etopophos, Other

\section{[[P059 Table] 1. Table 1]}
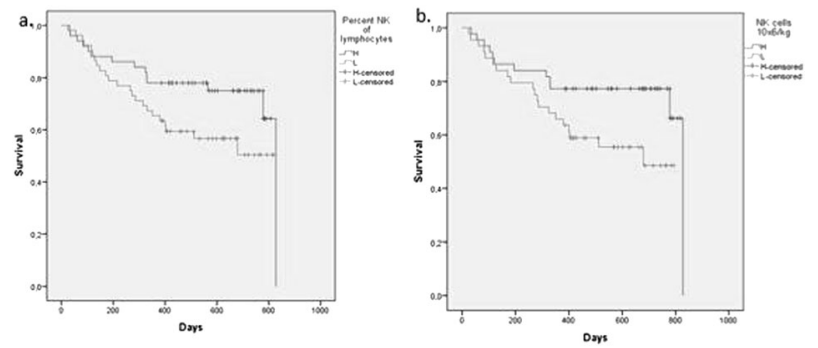

Figure 1. Estimated relapse-free survival in patients transplanted with a) high $(H)$ versus low (L) graft percentages of NK cells out of lymphocytes (median 8.1\%) in all patients $(\mathrm{n}=102)$, and $\mathrm{b})$ high $(\mathrm{H})$ versus low $(L)$ transplanted NK cell concentrations (median $27 \times 10^{6} / \mathrm{kg}$ ) in patients transplanted with PBSC ( $\left.\mathrm{n}=88\right)$.

\section{[[P059 Image] 1. Figure 1]}

Results: We found, that patients transplanted with graft NK cell doses above the median of $27 \times 10^{6} / \mathrm{kg}$ and fractions of NK cells out of lymphocytes above the median of $8.1 \%$ had significantly increased relapse-free-survival compared to patients transplanted with grafts containing NK cell doses below these values, figure 1; results stayed significant in multivariate analyses. Relapse incidence was significantly lower in uni- and multivariate analyses in patients receiving grafts with high NK cell fractions compared with low fractions, $\mathrm{p}=0.01$, with 1 -year relapse rates of $8 \%$ versus $27 \%$ in patients transplanted with high versus low fractions of NK cells, $p=0.01$. Peripheral blood concentrations of NK cells obtained from samples from 17 PBSC donors before G-CSF mobilization were significantly correlated to graft concentrations- and fractions of NK cells.. Analyses of graft contents of NKT cells showed that the incidence of grade IIIV acute GVHD were significantly lower in patients 
transplanted with graft doses below the median of $14.5 \times 10^{6} \%$ $\mathrm{kg} \quad(\mathrm{p}=0.04)$ and $4 \%$ NKT cells out of lymphocytes $(\mathrm{p}=0.03)$ compared to patients transplanted with grafts containing NKT cell doses above these values. Of note, the graft concentrations of CD3-, CD4- or CD8 positive T cells were not associated with overall survival, relapse-free survival or GVHD.

Conclusions: Our observations suggest that a more detailed graft characterization could benefit in risk assessment in HSCT and that donor selection, graft manipulation and additional cell infusions could optimize graft compositions regarding doses of cells beneficial for the transplantation.

Clinical Trial Registry: Not relevant

Disclosure: The authors have nothing to declare

\section{P060}

Extracorporeal photopheresis in the treatment of refractory acute GVHD: Analysis of mononuclear cell infusion

Gillen Oarbeascoa $^{1}$, Maria Luisa Lozano ${ }^{2,3,4}$, Luisa Maria Guerra $^{5}$, Cristina Amunarriz ${ }^{6}$, Nuria Revilla ${ }^{2,3,4}$, Pastora Iniesta $^{2,3,4}$, Cynthia Acosta Fleitas ${ }^{5}$, Jose Luis Arroyo ${ }^{6}$, Eva Martinez Revuelta ${ }^{7}$, Andrea Galego ${ }^{8}$, Dolores Hernandez-Maraver, Mi Kwon ${ }^{1,10}$, Aurora Viejo ${ }^{9}$, Jose Maria Garcia Gala ${ }^{7}$, Concepcion Andon Saavedra ${ }^{8}$, Jose Luis Diez-Martin 1,10,11, Cristina Pascual,10, GEA Grupo Español de Aferesis (GEA) ${ }^{12}$

${ }^{1}$ Hospital General Universitario Gregorio Marañon, Hematology, Madrid, Spain, ${ }^{2}$ Hospital Universitario Morales Meseguer, Murcia, Spain, ${ }^{3}$ Centro Regional de Hemodonacion, Murcia, Spain, ${ }^{4}$ IMIB-Arrixaca, Murcia, Spain, ${ }^{5}$ Hospital Universitario de Gran Canaria Dr Negrin, Las Palmas de Gran Canaria, Spain, ${ }^{6}$ Banco de Sangre y Tejidos de Cantabria - Hospital Universitario Marqués de Valdecilla, Santander, Spain, ${ }^{7}$ Hospital Universitario Central de Asturias, Hematology and Hemotherapy Service, Oviedo, Spain, ${ }^{8}$ Complexo Hospitalario Universitario A Coruña, A Coruña, Spain, ${ }^{9}$ Hospital Universitario La Paz, Madrid, Spain, ${ }^{10}$ Instituto de Investigación Sanitaria Gregorio Marañón, Madrid, Spain, ${ }^{11}$ Universidad Complutense de Madrid, Madrid, Spain, ${ }^{12}$ Grupo Español de Aferesis, Madrid, Spain

Background: Extracorporeal photopheresis (ECP) is an immunomodulatory treatment that has shown efficacy in steroid refractory acute GvHD, but the mechanism of action is only partially understood. There is no clear relationship between the ECP-treated mononuclear cells (MNC) or lymphocyte numbers and response to ECP. The objective of the study was to analyse the relationship between the infused subpopulation cellularity and response.

Methods: 65 patients from 7 different centers with a total of 1008 ECP procedures were retrospectively analized. ECP procedures were performed from January-2011 to June2017. All ECP procedures were performed with the off-line system. The response was defined as Responder (Complete and Partial Response) and Non-responder. Infused cell numbers for lymphocytes, monocytes and mononuclear cells (Lym+Mon, MNC) were calculated. For analytic purposes, the median number of cells infused per procedure until response (or until the median number of procedures until response for non-responding patients) and the cumulative number of cells infused until response (or until the median in non-responders) were calculated for all the subgroups. Same procedures were performed with the number cells infused until day 30 of ECP. Finally, the response and survival impact of infusing a number of cells above or below the median and in different tertiles was assessed until the median number of procedures needed to achieve a response.

\begin{tabular}{|l|c|}
\hline TABLE 1 & $\mathrm{N}=65$ \\
\hline Age (median, range) & $49(14-65)$ \\
\hline Gender & \\
Female & $19(29 \%)$ \\
Male & $46(71 \%)$ \\
\hline Diagnostic & \\
AML & $24(37 \%)$ \\
AU & $8(12 \%)$ \\
MDS & $6(9 \%)$ \\
Others & $27(42 \%)$ \\
\hline Donor & \\
Sibling HLA identical & $22(34 \%)$ \\
Sibling mismatched (except Haplo) & $2(3 \%)$ \\
Matched unrelated donor & $13(20 \%)$ \\
Mismatched unrelated donor & $3(5 \%)$ \\
Haploidentical & $24(37 \%)$ \\
UCB & $1(1 \%)$ \\
\hline Stem cell source & \\
PB & $56(86 \%)$ \\
BM & $8(12 \%)$ \\
UCB & $1(2 \%)$ \\
\hline GvHD prophylaxis & \\
CSA-MTX & $18(27 \%)$ \\
Post-trasplant Cy & $25(39 \%)$ \\
Others & $22(34 \%)$ \\
\hline ECP indication line & \\
2 & $31(48 \%)$ \\
3 & $32(49 \%)$ \\
\hline 3 & $2(3 \%)$ \\
\hline GvHD grade & $19(29 \%)$ \\
Grade 2 & $46(71 \%)$ \\
Grade 3-4 & \\
\hline Involvement & $33(66 \%)$ \\
Skin & $37(57 \%)$ \\
Digestive & $30(46 \%)$ \\
Liver & $34(52 \%)$ \\
\hline Only 1 organ involved & \\
\hline IIP60 Image]1. Table 1: Patient and ECP characteristics] \\
\hline
\end{tabular}

Disclosure: Nothing to declare

[[P060 Image] 1. Table 1: Patient and ECP characteristics] 
Results: The median number of procedures until response was 3. We observed a trend towards a higher median number of monocytes per procedure and cumulative infused monocytes in responding patients (median number infused 19.0 vs $13.5 \times 10^{6} / \mathrm{kg} \mathrm{p}=0.071$, cumulative infused median number 71.5 vs $41 \times 10^{6} / \mathrm{kg} \mathrm{p}=0.067$ ) that was lost in the day 30 of treatment. There was also a trend toward higher median infused MNC until response for responders (54 vs $\left.37 \times 10^{6} / \mathrm{kg} \mathrm{p}=0.087\right)$. We observed no differences in the number of lymphocytes infused, but patients who received a number of lymphocytes per procedure over the first tertile $\left(31 \times 10^{6} / \mathrm{kg}\right)$ presented higher response rates $(75 \%$ vs $45 \%$, $\mathrm{p}=0.0317)$. None of the other analysed parameters showed a significant impact in overall survival.

Conclusions: Patients with acute GvHD who responded to ECP received higher numbers of monocytes and MNC in the early phase of the treatment (a median of the first 3 processes). Also the patients who received higher numbers of lymphocytes in the first procedures achieved a higher response rate. These findings suggest the possibility that higher number of treated and infused cells could influence the response to ECP, but specifically designed prospective studies are need to asses this possibility.

Disclosure: Nothing to declare

\section{P061}

Donor-specific immunomodulation via induction of regulatory $B$ cells by clinical MIC cells infusion

Christian Morath ${ }^{1}$, Anita Schmitt ${ }^{1}$, Christian Kleist ${ }^{1}$, Volker Daniel', Gerhard Opelz, Caner Süsal', Florian $K_{a ̈ l b l e}{ }^{1}$, Claudia Sommerer ${ }^{1}$, Lei Wang ${ }^{1}$, Ming $\mathrm{Ni}^{1}$, Peter Dreger $^{1}$, Angela Hückelhoven-Krauss ${ }^{1}$, Arianeb Mehrabi ${ }^{1}$, Uta Merle ${ }^{1}$, Pego Da Silva Luiza ${ }^{1}$, Carsten Müller-Tidow ${ }^{1}$, Martin Zeier ${ }^{1}$, Matthias Schaier ${ }^{1}$, Michael Schmitt $^{1}$, Peter Terness ${ }^{1}$

${ }^{1}$ University Hospital Heidelberg, Heidelberg, Germany

Background: The field of kidney transplantation has made enormous progress over the last decades towards being a standard treatment for patients with end-stage renal disease. However, administration of immunosuppressive drugs is still one of the major limitations of long-term allograft survival. Therefore, strategies for induction of donorspecific tolerance are highly desirable. To this aim, a clinical phase I study with donor-derived modulated immune cells (MICs) was conducted.

Methods: Donor-derived MICs were manufactured under GMP conditions. Potency of MICs was tested by different in vitro bio-assays. MICs were administered to patients with an escalation from $1.5 \times 10^{6} \mathrm{MICs} / \mathrm{kg}$ on day $-2(\mathrm{~N}=3$, group A), to $1.5 \times 10^{8} \mathrm{MICs} / \mathrm{kg}$ on day $-2(\mathrm{~N}=3$, group $\mathrm{B})$ or on day $-7(\mathrm{~N}=4$, group $\mathrm{C})$ before kidney transplantation accompanied by standard immunosuppressive medication post-transplantation. Frequency of adverse events (AE) was assessed from day 30 until day 360 post-transplant. Dynamic changes of various lymphocyte subsets in patients after MIC therapy were detected by multicolor flow cytometry. Donor-specific immunosuppression was assessed by measuring anti-donor antibodies and mixed lymphocyte reaction (MLR) against donor and thirdparty cells.

Results: In all kidney transplant recipients, we observed a median serum creatinine of $1.4 \mathrm{mg} / \mathrm{dL}$ at day 30 which remained stable until day 360 (median creatinine of $1.4 \mathrm{mg}$ / dL) without significant proteinuria. None of patients experienced rejection episode. 69 AEs were observed while three AEs being severe. Most importantly, none of them was associated with MICs transfusion. Besides two infectious complications, no post-transplant positive cross match results against the donor or titers of de novo donorspecific antibodies were recorded. Notably, immunosuppressive therapy could be reduced without signs of rejection in group $\mathrm{C}$.

After infusion, we observed a dramatic increase of CD19 + B cells up to a median of 300 cells/ $\mu \mathrm{L}$ until day 30 , followed by a reduction to 35 cells/ $\mu \mathrm{L}$ on day 180 in group C. Notably, regulatory B cells significantly increased from a median of $2 \%$ on day 30 to $20 \%$ on day 180 . In parallel, the plasma IL-10/TNF- $\alpha$ ratio increased from a median of 0.05 before cell therapy to 0.11 on day 180 . After MIC cell therapy recipient lymphocytes showed no or only minimal reactivity against irradiated donor PBMCs in vitro, while reactivity against $3^{\text {rd }}$-party-donor PBMCs was not impaired.

Moreover, in vitro MICs product demonstrated their immunomodulatory potency by inducing tolerogenic dendritic cells (tDCs) characterized by low expression of costimulatory (CD80, CD86) and maturation (CD83) as well as high expression of inhibitory marker CD103. Functionally, tDCs could inhibit not only the release of IFN- $\gamma$ but also the proliferation of $\mathrm{CMV}$ specific $\mathrm{CD}^{+} \mathrm{T}$ cells. Moreover, MIC-induced tDCs showed the capacity to inhibit donor-specific allo-reactive $\mathrm{CD}^{+}{ }^{+}$and $\mathrm{CD}^{+} \mathrm{T}$ cell proliferation.

Conclusions: MIC cell therapy modulates the immune system of kidney transplant recipients by increasing the ratio of regulatory $B$ cells and facilitates the reduction of conventional immunosuppressive therapy without allograft injury or rejection episodes. Therefore, MIC cell therapy represents a promising strategy in transplantation medicine. We currently prepare a phase II trial with MIC cell therapy.

Disclosure: Nothing to declare

\section{P062}

genome editing of graft-derived $\mathbf{T}$ cells for post- 
transplant immunotherapy in combination with $\mathrm{TCR} \alpha \beta$ ${ }^{+} / \mathrm{CD}^{+}{ }^{+}$-depleted haploidentical HSCT

\section{Volker Wiebking ${ }^{1}$, Ciaran M. Lee ${ }^{2}$, Nathalie Mostrel ${ }^{1}$,} Rasmus O. Bak ${ }^{3,4}$, Gang Bao ${ }^{2}$, Alice Bertaina ${ }^{1}$, Matthew H. Porteus ${ }^{1}$

${ }^{1}$ Stanford University, Palo Alto, CA, United States, ${ }^{2}$ Rice University, Houston, TX, United States, ${ }^{3}$ Aarhus University, Aarhus C., Denmark, ${ }^{4}$ Aarhus University, Aarhus Institute of Advanced Studies (AIAS), Aarhus C., Denmark

Background: Treatment with $\alpha \beta^{+}$T-cell/CD19 ${ }^{+}$B-cell depleted haploidentical HSCT ( $\alpha \beta$ haplo-HSCT) is now a valid option for children with high-risk leukemia lacking a fully matched related donor. The low risk of transplantrelated mortality and acute or chronic graft-versus-host disease (GvHD) render this approach more and more appealing. However, additional treatments need to be developed to further reduce the frequency of relapse.

Methods: At Lucile Packard Children's Hospital, Stanford, we prospectively collected TCR $\alpha \beta^{+}$T-cells left-over (non-target fraction) during the $\alpha \beta^{+} \mathrm{T}$-cell/CD19 ${ }^{+} \mathrm{B}$-cell depletion process from 6 haploidentical donors. We used genome editing with Cas9 ribonucleoprotein(RNP)/recombinant adeno-associated virus type 6 (rAAV6) to disrupt the T-cell receptor (TCR) and integrate a CD19-specific CAR in-frame into the TRAC locus. We evaluated the functionality of the genome edited CAR T cells in in-vitro and invivo assays with $\mathrm{CD} 19^{+}$target cells. We use bioinformatics and targeted deep sequencing to evaluate the specificity of the genome editing process.

Results: Our data show that after $\alpha \beta$ haplo-HSCT, the nontarget fraction consists of $>90 \% \alpha \beta^{+}$T-cells which can be expanded and genome editing performed achieving high editing frequencies ( $>90 \%$ knockout of the TCR and $>70 \%$ of transgene expression). In addition, the residual TCR $\alpha \beta^{+}$cells can be efficiently depleted by more than 2-log folds with clinically-scalable reagents. After editing, the cells continue to rapidly expand and show antileukemic activity and cytokine production when co-cultured with the $\mathrm{CD} 19^{+}$Nalm6 cell line. Moreover, they are able to eradicate leukemia xenografts in an NSG mouse model, without signs of GvHD.

Conclusions: $\alpha \beta$ haplo-HSCT represents an attractive platform for post-transplant immunotherapy. Our preclinical data demonstrate that the non-target fraction can be efficiently engineered by genome editing to manufacture a new cell product characterized by high antileukemic activity and without the risk for GvHD.

Disclosure: Nothing to declare.

\section{P063}

\section{Abstract already published.}

\section{P064}

Automated manufacturing of clinical grade NKG2DCAR memory $T$ cells using clinimacs prodigy

Adrián Fernández ${ }^{1}$, Lucía Fernández ${ }^{1}$, Isabel Mirones ${ }^{2}$, Adela Escudero $^{3}$, Leila Cardoso ${ }^{3}$, Alejandra Leivas ${ }^{1,4}$, Joaquín Martínez-López ${ }^{1,4}$, Ana Belén Romero ${ }^{5}$, Antonio Marcos $^{5}$, Diego Lanzarot ${ }^{6}$, Raquel De Paz, Antonio Pérez-Martínez ${ }^{5}$

${ }^{1}$ CNIO, Madrid, Spain, ${ }^{2}$ IdiPAZ, Madrid, Spain, ${ }^{3}$ INGEMM, Madrid, Spain, ${ }^{4}$ Hospital 12 de Octubre, Madrid, Spain, ${ }^{5}$ Hospital La Paz, Madrid, Spain, ${ }^{6}$ Miltenyi Biotec GmbH, Madrid, Spain

Background: Immunotherapy using CAR $\mathrm{T}$ cells has shown promising results to fight cancer. However, CAR-T cell production requires specialized infrastructure and operators, which implies high cost and centralized production. Automated production of CAR-T cells in CliniMACS Prodigy device allows clinical-grade manufacturing of CAR $\mathrm{T}$ cells.

Methods: 100 million CD45RA ${ }^{-}$memory $\mathrm{T}$ cells from healthy donors were cultured in TexMACS supplemented with $100 \mathrm{IU} / \mathrm{ml} \mathrm{IL-2.} \mathrm{At} \mathrm{day} 0$ cells were activated with T Cell TransAct for $24 \mathrm{~h}$. At day one, activated CD45RA memory $\mathrm{T}$ cells were transduced with NKG2D-CD8TM41BB-CD3z lentiviral vector at $\mathrm{MOI}=2$. Then, NKG2DCAR $T$ cells were expanded for 10-13 days. NKG2D-CAR $\mathrm{T}$ cell products were next harvested, counted and analyzed for viability, NKG2D-CAR expression and anti-tumor cytotoxicity. Different quality tests including sterility, vector copy number, genetic stability, quantification of viral particles in the supernatant, myc/tert expression and endotoxin detection were performed. Spare cells were cryopreserved either in autologous plasma and 10\% DMSO, M199 35\% Albumin and 9\%DMSO or Hypothermosol. After 12 months, cryopreserved NKG2D-CAR $\mathrm{T}$ cell products were analyzed for viability, NKG2D-CAR expression and cytotoxicity.

Results: NKG2D-CAR memory T cells expanded up to $2076 \pm 697$ million with $77,8 \pm 20 \%$ NKG2D-CAR expression and $76 \pm 10 \%$ viability. Harvested CAR T cells showed $90 \pm 14 \%$ of specific lysis against Jurkat cells and $31 \pm 16 \%$ against 531MII osteosarcoma cell line. No microbiological contamination was observed in final CAR $\mathrm{T}$ cells products. Vector copy number was $\leq 5$ in all validations except for one. CGH and karyotype showed no genetic alterations. Free viral particles were undetectable in the supernatants. No overexpression of myc/tert was found except for one validation. Endotoxins were $\leq 0.25 \mathrm{EU} / \mathrm{ml}$. All cryopreserved NKG2D-CAR T cell products kept NKG2DCAR expression one year after freezing. However, viability 
and cytotoxicity was best preserved using autologous plasma $10 \%$ DMSO.

Conclusions: Automated production of large-scale clinical-grade NKG2D-CAR T cells using CliniMACS Prodigy is feasible and reproducible, allowing a decentralized protocol to generate CAR T cells for clinical use.table.

Disclosure: Nothing to declare

\section{P065}

Safety and outcome of high-dose donor CD45RO+ memory T-cells infusion after allogeneic transplantation

Mercedes Gasior Kabat ${ }^{I}$, David Bueno ${ }^{I}$, Raquel De Paz, Yasmina Mozo ${ }^{1}$, Blanca Rosich ${ }^{1}$, Luisa Sisinni, Antonio Marcos $^{I}$, Ana Belen Romero ${ }^{1}$, Aida Constanzo ${ }^{1}$, Victor Jimenez Yuste ${ }^{1}$, Antonio Perez Martinez ${ }^{I}$

${ }^{1}$ Hospital Universitario La Paz, Madrid, Spain

Background: Immune Reconstitution (IR) is essential to control severe infections after Hematopoietic Stem Cell Transplantation (HSCT). Reconstitution of adaptive immunity may take up to 2 years to recover T-Lymphocytes (LT). Delay in early LT recovery increases the risk of relapse, viral infections and transplant related mortality. Adoptive transfer of selected $\mathrm{T}$ cell subset with low alloreactivity potential is emerging as a strategy to improve IR.

Methods: Depletion of CD45RA+ naive T cells, preserving $\mathrm{CD} 45 \mathrm{RO}+$ memory $\mathrm{T}$ cells could provide functional lymphocytes to protect against infection and leukemia relapse with low risk of graft versus host disease (GvHD). We present our experience with high-dose donor CD45RO + memory $\mathrm{T}$ cell as donor lymphocyte Infusions (DLI) to assess safety and outcome.

A total of 58 DLI of CD45RO+ after HSCT was performed in cases of CMV/EBV reactivation (50\%), mixed chimerism (26\%), persistent lymphopenia $(8,5 \%)$, graft rejection $(3,5 \%)$, relapse $(3 \%)$ or to boost IR $(7 \%)$. DLI product was obtained performing a CD45RA depletion on donor leukapheresis product using the CliniMACS $^{\circledR}$ device.

Results: Twenty-two pediatric patients, median age 11 years (range 3-18), with malignant $(\mathrm{n}=15)$ and nonmalignant diseases (7), received CD45RA $+(n=14)$, TCR alpha/beta $(n=2)$ depleted grafts from haploidentical and CD45RA + depleted grafts from match unrelated $(n=6)$ and match related $(n=2)$ donors. At a median of 97 days (range 15-462) after transplantation, patients received a total of 58 DLI of CD45RO + cells, median 2 (range 1-6), containing a median of $2.87 \times 10^{7} / \mathrm{Kg}$ (range $4.8 \times 10^{4}-2 \times 10^{8} / \mathrm{Kg}$ ), CD3 $+\mathrm{CD} 45 \mathrm{RO}+1.05 \times 10^{7} / \mathrm{Kg}$ (range $4.8 \times 10^{4}-1.09 \times 10^{8} / \mathrm{Kg}$ ) and CD45RA+ cells $5 \times 10^{2} / \mathrm{Kg}$ (range $0-9.8 \times 10^{4} / \mathrm{Kg}$ ). All infusions were well-tolerated and did not develop or worsen GvHD. A total of $11 / 29$ episodes of CMV/EBV viral reactivations decreased viral load, 4/29 cleared viral load and 5/29 showed a clinical improvement. A total of $4 / 5$ patients with persistent lymphopenia there was a slightly increase in total lymphocyte count, but not to normal levels. Prophylactic DLI of CD45RO+ to boost IR increased lymphocyte count in 2 of 3 cases. None of the DLI administered in cases of mixed chimerism, graft failure or relapse were effective in reverting those situations.

Conclusions: Our preliminary data suggest that infusions of high dose CD45RO + memory $\mathbf{T}$ cells are a safe adoptive immunotherapy strategy. Efficacy has been observed in patients with lymphopenia and CMV/EBV reactivation, with no positive results in patients with mixed chimerism, graft failure and relapse. However, to determine the real efficacy of this strategy, prospective studies are required.

Disclosure: Nothing to declare.

\section{P066}

Abstract already published.

P067

Successful treatment of two relapsed/refractory $T(8 ; 21)$ acute myeloid leukemia patients by CD19-directed chimeric antigen receptor $T$ cells

Changju Qu ${ }^{1}$, Zheng $\mathrm{Li}^{1}$, Liqing Kang ${ }^{2}$, Ying Wang ${ }^{3}$, Haiping Dai, Jia Yin ${ }^{1}$, Guanghua Chen ${ }^{1}$, Mingqing $\mathrm{Zhu}^{1}$, Li Yao ${ }^{1}$, Ting $\mathrm{Xu}^{1}$, Xiaopeng Tian ${ }^{1}$, Yang $\mathrm{Xu^{1 }}$, Jia Chen ${ }^{1}$, Xiaming $Z \mathrm{Chu}^{1}$, Lei $\mathrm{Yu}^{1}$, Depei $\mathrm{Wu}^{1}$, Xiaowen Tang ${ }^{1}$

${ }^{1}$ First Affiliated Hospital of Soochow University, Suzhou, China, ${ }^{2}$ East China Normal University, Shanghai UnicarTherapy Bio-medicine Technology Co., Ltd., Shanghai, China, ${ }^{3}$ First People's Hospital of Lianyungang, Lianyungang, China

Background: Increasing clinical trials have confirmed that chimeric antigen receptor $\mathrm{T}$ cells (CAR-T) targeting CD19 antigen (CAR-T-19) is a promising effective approach for the treatment of relapsed/refractory $(\mathrm{R} / \mathrm{R}) \mathrm{B}$-cell lineage malignancies. Considering CD19 is frequently expressed in large part of $\mathrm{t}(8 ; 21)$ acute myeloid leukemia (AML) cells, we suppose that CAR-T-19 may be used as an approach to rescuing $\mathrm{R} / \mathrm{R} \mathrm{t}(8 ; 21)$ AML patients.

Methods: Both patients received lymphodepletion chemotherapy with Decitabine $20 \mathrm{mg} / \mathrm{m}^{2} \times 5 \mathrm{~d}$, Fludarabine $30 \mathrm{mg} / \mathrm{m}^{2} \times 3 \mathrm{~d}$ and Cyclophosphamide $300 \mathrm{mg} / \mathrm{m}^{2} \times 3 \mathrm{~d}$ (DAC + FC). Two days after chemotherapy, autologous/allogeneic CART-19 cells provided by the unicar-therapy biomedicine technology co.(Shanghai, China) at a total dose 
of $5-10 \times 10^{6}$ cells per kilogram $(\mathrm{kg})$ were infused dose escalation within 2 to 3 days. The research protocol was approved by the institutional review boards of the first affiliated hospital of Soochow University and both patients gave written informed consent.

Results: Both cases responded well with transient and reversible toxicities. Case 1 presented with grade 1 cytokine release syndrome (CRS), manifested by intermittent fever and chill from day 4 after CAR-T-19 infusion for half months associated with neutropenia. CAR-T cells expansion were observed in blood without obvious increase of cytokines. After infusion, Case 1 achieved and maintained molecular complete remission (CR) for more than 10 months.

Case 2 presented with grade 3 CRS manifested by continuous high fever, hypotension and grade 1 liver disfunction from day 1 after CAR-T-19 cell infusion for 1 week. Obvious cytokines releasing (peak IL-6 serum concentration $1774.5 \mathrm{pg} / \mathrm{ml}$, peak CRP serum concentration $367 \mathrm{pg} / \mathrm{ml}$ ) were detected which were associated with CART-19 cell expansion in blood and no severe off-tumor effect was observed. After infusion, case 2 achieved hematological CR and cytogenetic CR and got 3 months disease free survival.

Conclusions: Our report implicates that CAR-T-19 is a safe and promising approach to managing $\mathrm{R} / \mathrm{R} \mathrm{t}(8 ; 21) \mathrm{AML}$ with CD19 expression, and may provide a salvage treatment approach for all AML patients with CD19 expression and benefit a certain population with AML besides B-linage malignancies.

\section{Clinical Trial Registry: NA}

Disclosure: Nothing to declare.

This work was supported by research grants from the National Key R\&D Program of China (2016YFC0902800), National natural science Foundation of China $(81873443,81270645,81400155,81500146)$, Priority Academic Program Development of Jiangsu Higher Education Institutions (PAPD), Frontier Clinical Technical Project of the Science and Technology Department of Jiangsu Province (BE2017655), Jiangsu Provincial Medical Talent (ZDRCA2016045),Jiangsu Natural Science Foundation of China (BK20140374) and top-notch young health talents, 5th Suzhou Health professionals program(2018 to CQ)

\section{P068 \\ Challenge of cryopreservation for gmp grade CD19 CAR-T cells}

\author{
Wenjie Gong ${ }^{1}$, Lei Wang ${ }^{1}$, Sanmei Wang ${ }^{1}$, Brigitte \\ Neuber ${ }^{1}$, Leopold Sellner ${ }^{1,2}$, Maria-Luisa Schubert ${ }^{I}$, \\ Angela Hückelhoven-Krauss ${ }^{1}$, Alexander Kunz ${ }^{1}$, Ulrike \\ Gern $^{1}$, Birgit Michels ${ }^{1}$, Mandy Hinkelbein ${ }^{1}$, Stefanie
}

Mechler $^{1}$, Petra Richter ${ }^{1}$, Carsten Müller-Tidow ${ }^{1,2}$, Peter Dreger $^{1,2}$, Michael Schmitt ${ }^{1,2}$, Anita Schmitt ${ }^{1}$

${ }^{1}$ Heidelberg University Hospital, Heidelberg, Germany, ${ }^{2}$ German Cancer Consortium (DKTK), National Center for Tumor Diseases (NCT), Heidelberg, Germany

Background: Chimeric antigen receptor engineered $\mathrm{T}$ (CAR-T) cells have emerged as a powerful cellular therapy to treat malignant disease, which is currently revolutionizing field of cancer immunotherapy. A cryopreservation step postmanufacture is not only a logistical necessity for large scale cell manufacturing processes but also a mandatory request by regulatory authorities. In case relapse after $1^{\text {st }}$ CAR-T cell transplantation, a second application, maybe at a higher dose constitutes a therapeutical option. However, data concerning clinical grade CAR-T cell stability and functionality after months of cryopreservation have not been released by companies so far. To investigate the effect of cryopreservation on CAR-T cells, we performed this study.

Methods: Different batches of CD19 CAR-T cells were manufactured according to GMP requirements at our institution. Final CAR-T products were frozen at concentrations of $1 \times 10^{7}$ cells $/ \mathrm{ml}$ (high batch) and $2 \times 10^{6}$ cells $/ \mathrm{ml}$ (low batch) by a controlled freezing process with the Biofreeze BV40 device and stored in liquid nitrogen tanks below $-150^{\circ} \mathrm{C}$ until release. Quality control tests for sterility, endotoxin and mycoplasma were performed for each batch according to European Pharmacopoeia and United States Pharmacopoeia guidelines. Stability of CD19 CAR-T cells in terms of viability, recovery, transduction efficiency and functional capacity were determined by microscopy, multi-parametric flow cytometry as well as chromium-51 release tests following our SOPs.

Results: All the results of quality controls fully met the requirements of the regulatory authorities. Stability results were highly robust and reproducible over time for all our GMP CAR-T batches. Duration of cryopreservation (up to 90 days) had no negative influence on cell viability, recovery of viable CD19 CAR-T cells and transduction efficiency. However, the cell concentration for cryopreservation has a significant impact on the post-thawing viability (low batches vs. high batches: $96.33 \pm 2.17$ vs. $74.87 \pm$ $8.68, p<0.05$ ) and recovery (low batches vs. high batches: $89.67 \pm 6.76$ vs. $74.90 \pm 9.19, p<0.05$ ) of cryopreserved CD19 CAR-T cells, but not the transduction efficiency. Moreover, we observed four transient side-effects of cryopreservation on the amount of cytokines released by CAR-T cells, the cytokine release on a per-cell basis, the multifunctionality of CAR-T cells and the killing capacity. Of note, functional capacity of cryopreserved CAR-T cells after overnight resting was comparable or even enhanced for INF- $\gamma$ and TNF- $\alpha$ release by $\mathrm{CD}^{+}{ }^{+}$and $\mathrm{CD} 8^{+} \mathrm{CD} 19$ 
CAR-T cells when compared to fresh CAR-T cells. The multi-functionality of CAR-T cells could be preserved. Furthermore, the killing capacity of cryopreserved CD19 CAR-T cells after overnight resting could reach the level of non-cryopreserved/fresh CAR-T cells.

Conclusions: Cryopreservation up to 90 days has no harmful effect on transduction efficiency and functionalities of CAR-T cells. However, the cell number per milliliter freezing medium matters. Dose over 1 x $10^{7}$ cells $/ \mathrm{ml}$ should be avoided. For the conduction of in vitro bio-assays to determine the function of CAR-T cells, an overnight resting process could mimic the situation after clinical application and eliminate the transient side-effects of cryopreservation to fully regain the functional potency of CAR-T cells.

Disclosure: Nothing to declare

\section{P069}

AML-blast conversion to leukemia-derived dendritic cells (DCLEU) in 'DC-culture-media' shifts the (SERUM) chemokine-release to a more 'inflammatory' (in culture) going along with improved antileukemic Tcell-reactivity

Marion Merle ${ }^{1}$, Dorothea Fischbacher ${ }^{1}$, Anja Liepert ${ }^{1}$, Christine Grabrucker', Tanja Kroell', Andreas Kremser', Julia Dreyssig ${ }^{1}$, Markus Freudenreich ${ }^{1}$, F. Schuster ${ }^{2}$, A. Borkhardt $^{2}$, D. Kraemer ${ }^{3}$, C.-H. Koehne ${ }^{2}$, H.J. Kolb ${ }^{4}$, C. Schmid $^{5}$, Helga Maria Schmetzer ${ }^{4}$

${ }^{1}$ University Hospital Munich, Munich, Germany, ${ }^{2}$ University Hospital Düsseldorf, Düsseldorf, Germany, ${ }^{3}$ Municipal Hospital Oldenburg, Oldenburg, Germany, ${ }^{4}$ University Hospital Munich-Großhadern, Munich, Germany, ${ }^{5}$ Municipal Hospital Augsburg, Augsburg, Germany

Background: DC and specific T-cells are important mediators of CTL-responses. We could already show that allogeneic donor- or autologous T-cells obtained from AMLpatients can be stimulated by $\mathrm{DC}_{\text {leu }}$, resulting in a very efficient lysis of naive blasts.

Methods: Chemokine-release (CXCL8, -9, -10, CCL2, -5 , and IL-12) was analysed by cytometric bead array in serum of AML/MDS-pts as well as in supernatants from 5 different DC-generating-methods and correlated with pts' clinical course, DC- and T-cell-interactions as well as specific T-cell-reactions. The lytic activity of DCleu/blast -stimulated T-cells in MLC against naive blasts was quantified in a cytotoxicity assay.

Results: Minimal differences in median chemokine-levels in pts' serum subdivided in subtypes were seen, but higher release of CXCL8, -9, -10 and lower release of CCL2 and -5 tendentially correlated with more favourable subtypes $(<50$ years of age, $<80 \%$ blasts in PB). In persisting disease, a higher serum-release of CCL5 and at relapse a significantly higher CCL2-release were found compared to first diagnosis - pointing to a change of 'disease activity' on a chemokine level. Whereas chemokine-levels in DC-culture supernatants compared to serum were variable, clear correlations with lateron (after stimulating T-cells with DCleu in MLC) improved antileukemic T-cell activity were seen: higher values of all chemokines in DC-culture supernatants always correlated with improved T-cells' antileukemic activity (compared to stimulation with blast-containing $\mathrm{MNC}$ as control) - whereas with respect to the corresponding serum values higher release of CXCL8, -9 , and -10 but lower values of CCL5 and -2 correlated with higher probabilities to improve antileukemic activity of DCleu-stimulated (vs. blaststimulated) T-cells. Predictive significant cut-off-values could be evaluated separating the groups compared. Moreover, correlations with lateron achieved response to immunotherapy and occurrence of GVHD were seen: Higher serum values of CXCL8, -9, -10 and CCL2 and lower values of CCL5 correlated with achieved response to immunotherapy. Predictive cut-off-values could be evaluated separating the groups compared in 'responders' and 'non-responders'. Higher levels of CCL2 and -5 but lower levels of CXCL8, -9, -10 correlated with occurrence of GVHD.

Conclusions: We conclude, that in AML-pts' serum higher values of CXCL8, -9, -10 and lower values of CCL5 and in part of CCL2 correlate with more favorable subtypes and improved antitumor'-reactive function. Since in DCculture supernatants higher values of all chemokines correlated with improved antileukemic T-cell reactivity we conclude a change of functionality of CCL5 and -2 from an 'inflammatory' or 'tumor-promoting' to an 'antitumor'reactive function. This knowledge can contribute to develop immune-modifying strategies that promote antileukemic adaptive immune-responses.

Disclosure: Nothing to declare

\section{P070}

Leukemia control after allogeneic hematopoietic cell transplantation through ex vivo expanded invariant natural killer $\mathbf{T}$ cells from donor lymphocyte infusions (DLI-INKTS)

Simona Jahnke ${ }^{I}$, Hannes Schmid ${ }^{1}$, Kathy-Ann Secker ${ }^{1}$, Silke Duerr-Stoerzer ${ }^{1}$, Hildegard Keppeler ${ }^{1}$, Rebecca Baur $^{2}$, Michael Schumm ${ }^{3}$, Rupert Handgretinger ${ }^{3}$, Wolfgang Bethge ${ }^{I}$, Lothar Kanz ${ }^{I}$, Corina Schneidawind ${ }^{1}$, Dominik Schneidawind ${ }^{1}$

${ }^{1}$ University Hospital Tuebingen, Tuebingen, Germany, ${ }^{2}$ University of Erlangen-Nuremberg, Erlangen, Germany, ${ }^{3}$ Children's University Hospital, Tuebingen, Germany 
Background: Allogeneic hematopoietic cell transplantation (allo-HCT) is a curative treatment option for patients suffering from hematologic malignancies. Infusion of donor lymphocytes (DLIs) can induce sustained remission in case of minimal residual disease or relapse through potent graftversus-leukemia (GVL) effects, although graft-versus-host disease (GVHD) represents a common dose-limiting toxicity. As invariant natural killer T (iNKT) cells are known to prevent GVHD while promoting beneficial anti-tumor effects, we investigated the role of iNKT cells for successful DLIs.

Methods: We analyzed DLI samples by flow cytometry. iNKT cells were identified by staining with PBS57-loaded CD1d tetramers. Culture-expanded and purified DLI-iNKTs were then tested against tumor cell lines and primary leukemia cells in an ex vivo tumor control model. Tumor cell viability after coincubation with DLI-iNKTs was measured by flow cytometry using 7-AAD.

Results: iNKT cells represent $0.05 \%$ (range 0.001 $0.55 \%$ ) of donor lymphocytes and can be expanded 300fold following a two-week protocol with a preferential expansion of CD4+ iNKT cells. Tumor cell lines such as Jurkat were efficiently lysed after coincubation with DLIiNKTs. CD107a as a marker of degranulation was significantly upregulated on DLI-iNKTs after stimulation by Jurkat. In addition, increased concentrations of TNF$\alpha$, IFN- $\gamma$, sFasL and Perforin were measured after coincubation of DLI-iNKTs with Jurkat. We observed that tumor cell lysis correlated with the expression of the MHC-I-like molecule CD1d. Consequently, adding a CD1d antibody to the coculture abrogated the DLI-iNKTmediated kill of tumor cells. DLI-iNKTs also efficiently lysed primary leukemia cells such as AML blasts: expression of CD1d on these AML blasts significantly correlated with DLI-iNKT-mediated tumor cell lysis $\left(\mathrm{r}^{2}=0.7, \mathrm{p}=0.03\right)$.

Conclusions: Ex vivo expansion of DLI-iNKTs and subsequent DLI enrichment is an immunotherapeutic approach that could improve leukemia control and thus, prevent relapse after allo-HCT without exacerbating GVHD.

Disclosure: Nothing to declare.

\section{P071}

Generation of antigen-specific cytotoxic $T$ lymphocytes targeting WT1 using activated B cells

\section{Sun Ok Yun ${ }^{1}$, Kyung Won Baek ${ }^{1}$, Hee Young Shin', Hyoung Jin Kang ${ }^{1}$}

${ }^{1}$ Seoul National University, Seoul, Korea, Republic of
Background: The Wilms tumor antigen 1 (WT1) is highly expressed in many malignancies including leukemia and targeting WT1 as a Tumor Associated Antigen (TAA) in cancer immunotherapy is attractive. In this study, we generated WT1-specific cytotoxic T lymphocytes to confirm if activated $\mathrm{B}$ cells can act as a cancer antigen presenting cell and induce CTLs.

Methods: For the induction of CTLs against WT1, activated B cells were used as an antigen presenting cells. B cells were isolated from PBMCs of normal healthy donors and activated with $\alpha$-galactosylceramide $(\alpha$-GalCer) and nucleofected with WT1-coding plasmid DNA. Activated B cells were the cultured with PBMCs for 17days in vitro and harvested for assay.

Results: Cells expanded about 3 times after 17 days of culture. We examined characteristic of WT1-specific CTLs by their surface markers. WT1-specific CTLs had more than 90\% CD3 + marker, and ratio of CD8 to CD4 was 1.7-5.8. We also examined NKT cell markers to see if NKT cells were activated by IL-15, a cytokine used in the induction of CTLs, and the portion of NKT cells was about $2 \%$. The CTLs showed a decrease in naïve cell $(C D 62 L+C D 45 R A$ $+)$ and an increase in effector memory (CD62L+CD45RA) and central memory (CD62L-CD45RA-) compared with non-stimulated PBMCs. Subsequently, the IFN- $\gamma$ ELISPOT (Enzyme-linked immunospot) assay was performed to confirm the response of the induced WT1-specific CTLs to the WT1 antigen. When WT1-specific CTLs encounters a target that does not have a WT1 antigen, it did not produce IFN- $\gamma$, but when it encounters a target cells loaded WT1 antigen, it responded to secrete IFN- $\gamma$. Killing assays were also performed to determine the immunogenicity of induced CTLs. The induced WT1 CTLs was found to be killing more than $90 \%$ when the E:T ratio was 10:1 when the autologous PBMC met the target with WT1 pepmix. In addition, we found that WT1 CTLs has killing activity when it encounters leukemia cell lines that express WT1 and matched HLA-A*0201.

Conclusions: In this study, we can induce antigenspecific CTLs that specifically react to WT1 using activated $\mathrm{B}$ cells as antigen-presenting cells. These observations confirmed that B cells activated by $\alpha-G a l C e r$ can act as a TAA presenting cell to induce TAA specific CTLs as viral antigen, such as pp65 and IE1, and consequently WT1specific CTLs could be induced. Moreover, CTLs induced activated B cells had ability to recognize and kill the target cells expressing WT1 correctly. Our results demonstrate that these in vitro expanded WT1-specific CTLs using activated B cells can be a promising candidate for adoptive immunotherapy against cancer.

Disclosure: Nothing to declare 


\section{P072}

Validation of the clinimacs prodigy $T$ cell transduction process to generate $\mathrm{CD19-chimeric}$ antigen receptor

$T$ cells for the use in clinical trials

Judith Böhringer ${ }^{1}$, Michael Schumm ${ }^{1}$, Christiane Braun ${ }^{1}$, Marina Schmidt ${ }^{\text {, Patrick Schlegel }}{ }^{I}$, Christian Seitz, Murat Aktas $^{2}$, Georg Rauser ${ }^{2}$, Sandra Karitzky ${ }^{2}$, Peter Lang $^{1}$, Rupert Handgretinger ${ }^{I}$

${ }^{1}$ University Children's Hospital Tübingen, Tübingen, Germany, ${ }^{2}$ Miltenyi Biotec GmbH, Bergisch Gladbach, Germany

Background: $\mathrm{T}$ cells with chimeric antigen receptors (CARs) on their surface facilitate to target specific surface expressed antigens. Research and clinical trials with CD19CAR T cells show impressive remission induction rates and increased survival in heavily pretreated patients. Therefore, CAR $T$ cells are introduced as new potent cellular therapeutics in the clinical routine. In order to establish the manufacture of CD19-CAR T cells, validation runs with the fully automated CliniMACS Prodigy $T$ cell transduction process have been performed using the Miltenyi anti-CD19CAR lentiviral vector.

Methods: Unmobilized leukaphereses from 3 donors $(2 \mathrm{x}$ healthy, $1 \mathrm{x}$ ALL) were used for the CliniMACS Prodigy T cell transduction process. Leukocytes undergo a $\mathrm{CD} 4^{+}$/ $\mathrm{CD}^{+} \mathrm{T}$ cell enrichment via magnetic beads, followed by stimulation with MACS $\otimes_{\text {GMP }} \mathrm{T}$ cell TransAct ${ }^{\mathrm{TM}}$, transduction with an anti-CD19-CAR lentiviral vector, expansion with IL7 and IL15, and final formulation to the cellular product. During and after the manufacture, FACS analyses were performed as well as cytotoxicity assays after CD19-CAR T cell production.

Results: Total volumes of leukaphereses were between 60 and $280 \mathrm{ml}$ with $2.6-4.0 \times 10^{9}$ total mononuclear cells. After enrichment $100 \times 10^{6} \mathrm{CD}^{+} / \mathrm{CD}^{+} \mathrm{T}$ cells were transduced with anti- CD19-CAR lentiviral vector and were further expanded. Cells were harvested on day 12. The final cell counts of the cellular products were $6.1,7.2$ and $5.0 \mathrm{x}$ $10^{9}$ mononuclear cells from two healthy volunteers and the ALL-patient, respectively. The transduction efficiency of the CD19-CAR $\mathrm{T}$ cells was $48.6 \%, 43.4 \%$ and $32.0 \%$ among viable $\mathrm{CD} 3^{+}$cells. The final count of CAR $\mathrm{T}$ cells was therefore 2.9, 3.0 and $1.5 \times 10^{9}$ cells. The final products exerted excellent cytolytic activity against $\mathrm{CD} 19^{+} \mathrm{BCP}-$ ALL cell line NALM-6. Importantly, CD19-CAR T cells generated from the ALL patient demonstrated complete eradication of autologous blasts at 0.3 to $1 \mathrm{E}: \mathrm{T}$ ratio after 24 hours incubation.

Conclusions: The CliniMACS Prodigy T cell transduction process has been shown to run a fully-automated manufacturing process over 12 days without any deviations in a clean room environment on a single device. The user interaction was reduced to activities at only 3 days to set up the system and provide fresh medium and reagents. The transduction process yielded a high number of $\mathrm{T}$ cells with a high frequency of CD19-CAR T transduced cells. The results were comparable for both unmobilized leukaphereses from healthy donors and showed expected slightly lower results in the patient. Finally, these results demonstrate that the CliniMACS Prodigy $\mathrm{T}$ cell transduction process is well suited to provide the clinical MB-CART19.1 $\mathrm{r} / \mathrm{r} \mathrm{CD} 19+\mathrm{BCM}$ study with appropriate investigational medical products.

Disclosure: Murat Aktas: Employment Miltenyi Biotec GmbH

Georg Rauser: Employment Miltenyi Biotec GmbH

Sandra Karitzky: Employment Miltenyi Biotec GmbH

\section{P073}

Donor-lymphocyte infusion (DLI) after hematopoietic stem cell transplantation with post-transplant cyclophosphamide (PTCY) as GVHD prevention treatment

Susanna Gallo ${ }^{I}$, Daniela Caravelli, Paolo Becco ${ }^{I}$, Alessandra Polo ${ }^{2}$, Milena Salierno ${ }^{2}$, Karol Giancaspero², Delia Rota Scalabrini ${ }^{1}$, Dario Sangiolo ${ }^{3}$, Elena Vassallo ${ }^{4}$, Pio Manlio Mirko Frascione ${ }^{1}$, Stefano Poletto ${ }^{1}$, Luca Paruzzo $^{1}$, Luca Crotto ${ }^{5}$, Massimo Aglietta ${ }^{1}$, Franca Fagioli $^{4}$, Fabrizio Carnevale Schianca ${ }^{1}$

${ }^{1}$ Medical Oncology, Turin Metropolitan Transplant Center, Candiolo Cancer Institute-FPO, IRCCS, Candiolo, Italy, ${ }^{2}$ Collection and Processing Laboratory, Candiolo Cancer Institute-FPO, IRCCS, Candiolo, Italy, ${ }^{3}$ Medical Oncology, Candiolo Cancer Institute-FPO, IRCCS, Candiolo, Italy, ${ }^{4}$ Pediatric Onco-Hematology, Turin Metropolitan Transplant Center, A.O.U. Citta' della Salute e della Scienza di Torino, Ospedale Infantile Regina Margherita, Torino, Italy, ${ }^{5}$ Clinical Research Office, Candiolo Cancer Institute-FPO, IRCCS, Candiolo, Italy

Background: Allogeneic hematopoietic stem cell transplantation (alloHCT) is an effective strategy in the long term control of several hematologic diseases, however, patients could experience complications, as graft versus host disease (GVHD) and disease relapse. Recently, the introduction of post-transplant Cyclophosphamide (ptCy) allowed to significantly reduce GVHD, but disease relapse remains an important issue. Donor-lymphocyte infusion (DLI) is an established adoptive cell therapy for disease relapse after alloHSCT, but, in order to be efficient and safe, patients have to be off immunosuppression treatments and 
GVDH-free. Here we report our data about efficacy and safety of DLI infusion as treatment for disease relapse in patients who received peripheral blood stem cell transplantation (alloPBSCT) from HLA-matched unrelated/related plus ptCy as GVDH prophylaxis in our clinical trial (NCT 02300571).

Methods: We collected data from 13 patients, treated with $\mathrm{ptCy}(50 \mathrm{mg} / \mathrm{kg} / \mathrm{die}$, days $+3+4)$, mofetil mycophenolate (MMF) and tacrolimus (T) as GVHD prophylaxis after allo-PBSCT, who received DLI infusions. They were treated between January 2013 and October 2018. We report data about overall response rate (ORR), disease control rate (DCR), and DLI-related mortality and morbidity. Diagnosis were as follow: 5 had multiple myeloma, 3 had acute myeloid leukemia, 3 had acute lymphoblastic leukemia and 2 had lymphomas. All patients but one, who had chimerism loss, received DLI because of disease relapse.

Results: Median time between transplant and DLI was 9 (range 3-87) months. Median number of DLI infusions was 2 (range 1-13). 10 patients (77\%) received Cyclophosphamide $300 \mathrm{mg} / \mathrm{m} 2$ preparative regimen the day before the cryopreserved DLI infusions, while in the other 3 cases DLI were associated with Lenalidomide, Ponatinib and 5Azacitidine.

The overall response rate (ORR) was 50\%, while disease control rate (DCR) was achieved in $75 \%$. The patient who received DLI because of loss of chimerism converted it in full donor after 2 infusions. After DLI treatment the incidence of acute GVHD grade I-III was $54 \%$, while was $46 \%$ for grade II-III and patients were started on short course of systemic immunosuppression treatments . None of these patients died because of DLI adverse events. Estimated 1-year overall survival was $77 \%$ with a limited follow-up length (6 months).

Conclusions: The infusion of non-manipulated lymphocytes from allogeneic donors is a valuable and safe strategy of treatment for patients relapsing after alloPBSCT with ptCy. ptCy showed high efficacy in GVHD prevention, allowing early discontinuation of immunosuppression drugs. Because of this, we can reach the goal to transform transplant in a platform where we could add early DLI infusions as a new strategy for disease control.

Clinical Trial Registry: NCT 02300571

Disclosure: Nothing to declare

\section{P074}

Extracorporeal photopheresis in the treatment of refractory chronic GVHD: Analysis of mononuclear cell infusion

Gillen Oarbeascoa ${ }^{1}$, Maria Luisa Lozano ${ }^{2,3,4}$, Luisa Maria Guerra $^{5}$, Cristina Amunarriz ${ }^{6}$, Nuria Revilla ${ }^{2,3,4}$, Pastora Iniesta $^{2,3,4}$, Cynthia Acosta Fleitas ${ }^{5}$, Jose Luis Arroyo ${ }^{6}$,
Eva Martinez Revuelta ${ }^{7}$, Andrea Galego ${ }^{8}$, Dolores Hernandez-Maraver ${ }^{9}$, Mi Kwon $^{1,10}$, Aurora Viejo ${ }^{9}$, Jose Maria Garcia Gala ${ }^{7}$, Concepcion Andon Saavedra ${ }^{8}$, Jose Luis Diez-Martin ${ }^{1,10,11}$, Cristina Pascual, ${ }^{1,10}$, GEA Grupo Español de Aferesis (GEA) ${ }^{12}$

${ }^{1}$ Hospital General Universitario Gregorio Marañon, Hematology, Madrid, Spain, ${ }^{2}$ Hospital Universitario Morales Meseguer, Murcia, Spain, ${ }^{3}$ Centro Regional de Hemodonacion, Murcia, Spain, ${ }^{4}$ IMIB-Arrixaca, Murcia, Spain, ${ }^{5} \mathrm{Hos}-$ pital Universitario de Gran Canaria Dr Negrin, Las Palmas de Gran Canaria, Spain, ${ }^{6}$ Banco de Sangre y Tejidos de Cantabria - Hospital Universitario Marqués de Valdecilla, Santander, Spain, ${ }^{7}$ Hospital Universitario Central de Asturias, Hematology and Hemotherapy Service, Oviedo, Spain, ${ }^{8}$ Complexo Hospitalario Universitario A Coruña, A Coruña, Spain, ${ }^{9}$ Hospital Universitario La Paz, Madrid, Spain, ${ }^{10}$ Instituto de Investigación Sanitaria Gregorio Marañón, Madrid, Spain, ${ }^{11}$ Universidad Complutense de Madrid, Madrid, Spain, ${ }^{12}$ Grupo Español de Aferesis, Madrid, Spain

Background: Extracorporeal photopheresis (ECP) is an immunomodulatory treatment that has shown efficacy in steroid refractory chronic GvHD, but the mechanism of action is only partially understood. In some studies, a correlation has been suggested between treated Mononuclear cells (MNC) or lymphocytes and response to ECP. The objective of the study was to analyze the relationship between the infused cellularity and response in chronic GvHD.

Methods: 48 patients from 7 different centers with a total of 930 ECP procedures were retrospectively analyzed. ECP procedures were performed from January-2011 to June2017. All ECP procedures were performed with the off-line system. The response was defined as Responder (Complete and Partial Response) and Non-responder. Infused cell numbers for lymphocytes, monocytes and mononuclear cells (Lym+Mon, MNC) were calculated. For analytic purposes, the median number of cells infused per procedure until response (or until the median number of procedures until response for non-responding patients) and the cumulative number of cells infused until response (or until the median in non-responders) were calculated for all the subgroups. Same procedures were performed with the number cells infused until day 30 of ECP. Finally, the response and survival impact of infusing a number of cells over or below the median and in different tertiles (T1, T2 and T3) was assessed until the median number of procedures needed to achieve a response.

Results: The median number of procedures until response was 3 . We observed no differences in the median number of lymphocytes, monocytes or MNCs infused until response or 
until day 30 between responding and non-responding patients. There were no differences in response if patients received lymphocytes or monocytes above or below the median number. Nevertheless, patients that received a total absolute number of MNCs above the median ( $64 \times 108$ cells) showed a trend towards a higher response rate $(75 \%$ vs $61 \%, \mathrm{p}=0.09$ ). The patients that received a cumulative number of lymphocytes in the 3 first ECP procedures above the median showed improved overall survival (OS) (2y OS $85 \%$ vs $55 \%, \mathrm{p}=0.024)$. Patients that received a number of monocytes above the median showed a trend towards better survival $(\mathrm{p}=0.09)$, that was significant when the number of monocytes infused surpassed the first tertile (2y OS $38 \%$ for $\mathrm{T} 1,79 \%$ for $\mathrm{T} 2,92 \%$ for $\mathrm{T} 3, \mathrm{p}=0.003$ ). Finally, the patients that received a cumulative number of MNCs above the first tertile also showed improved survival (2y OS $47 \%$ for T1, $74 \%$ for $\mathrm{T} 2,94 \%$ for $\mathrm{T} 3, \mathrm{p}=0.015$ ).

Conclusions: There were no differences in the infused cellularity between responding and non-responding patients with chronic GvHD. At the same time, we found that except for a trend toward better response with higher MNCs infused, there was no relationship between lymphocytes and monocytes with the response rate as other previous studies have suggested. However, even if there is no relationship with the response rate, the patients receiving the highest numbers of lymphocytes, monocytes and MNCs in the cohort showed an improved survival, suggesting that larger quantities of cells could exhibit a protective effect. Nevertheless, prospective studies that address this relationship are needed.

Disclosure: Nothing to declare

\section{P075}

Comparative analysis of the cytotoxic potential of cytokine-induced killer and natural killer cells for neuroblastoma therapy

\section{Annekathrin Heinze ${ }^{1}$, Beatrice Grebe ${ }^{1}$, Eva Mudry ${ }^{1}$, Jochen Früh ${ }^{1}$, Bushra Rais ${ }^{1}$, Claudia Cappel ${ }^{1}$, Sabine Hünecke $^{I}$, Eva Rettinger ${ }^{I}$, Thomas Klingebiel ${ }^{I}$, Peter Bader $^{1}$, Evelyn Ullrich ${ }^{1,2}$}

${ }^{1}$ University Hospital Frankfurt, Frankfurt, Germany, ${ }^{2}$ German Cancer Consortium (DKTK) partner site:, Frankfurt, Germany

Background: Neuroblastoma (NB) is the most common solid extracranial tumor in childhood. Despite therapeutic progress, prognosis for high-risk NB is poor and innovative therapies are of medical need. Therefore, we investigated the cytotoxic potential of interleukin (IL)-activated natural killer (NK) cells compared to activated cytokine-induced killer (CIK) cells against different human NB cell lines in vitro.
Methods: NK cells were isolated from peripheral blood mononuclear cells (PBMCs) using CD56 enrichment or CD3/CD19 depletion kits. They were successfully expanded ex vivo with different cytokine combinations such as IL-2, IL-15, IL-18 and/or IL-21 under feeder-cell free conditions. In contrast, CIK cells were generated from PBMCs by ex vivo stimulation with interferon- $\gamma$, IL-2, OKT-3 and IL-15. A comparative analysis of expansion rate, purity, phenotype and cytotoxic activity against different NB cell lines following different culturing protocols was performed.

Results: CD56 enriched NK cells showed a median expansion rate of 4.3 -fold after 10 to 12 days in culture with a final frequency up to $99.0 \%$ NK cells and a median frequency of $0.5 \% \mathrm{CD}^{+} \mathrm{CD}^{-} 6^{-} \mathrm{T}$ cells. In contrast, the starting cell product after CD3/CD19 depletion consisted of a median frequency of $43.5 \% \mathrm{NK}$ cells that expanded significantly faster with 7.5 -fold and also reached up to $98.6 \%$ purity without any relevant $\mathrm{T}$ cell contamination. CIK cells expanded with a median rate of 30.8 -fold and contained $3.3 \% \mathrm{NK}, 84.2 \% \mathrm{~T}$ and $6.2 \%$ NK-like T cells. Interestingly, NK cells, particularly after CD3/CD19, showed a significantly higher median cytotoxic capacity against NB cells depletion (46.6\% for CD56 enrichment, $53.7 \%$ for $\mathrm{CD} 3 / \mathrm{CD} 19$ depletion) compared to CIK cells that induced $7.2 \%$ killing of $\mathrm{NB}$ cells with E:T ratio $5: 1$ in a 3 hours' co-incubation assay. Interestingly, prolonging the ex vivo stimulation after $\mathrm{CD} 3 / \mathrm{CD} 19$ depletion to 15 days enhanced the median expansion rate to 12.3 -fold with a slightly reduced cytotoxic potential $(40.9 \%$ for 11 days' ex vivo expansion, $31.1 \%$ for 15 days' ex vivo expansion, comparison of the same donors). The addition of an IL21boost prior harvesting increased the expansion rate to median 12.6-fold (compared to 11.7-fold for the same donors) with an improved cytotoxicity of 51.5\% (compared to $45.8 \%$ ). Fortunately, all NK cell products showed a high viability and no relevant $\mathrm{T}$ or $\mathrm{B}$ cell contamination (median $<0.2 \%$ ). Interestingly, further optimization of the culturing procedure with use of another cell culture medium led to an improved median 24.4-fold (compared to 9.6-fold) NK cell expansion rate in 15 days, also resulting in comparable cytotoxicity of $52.5 \%$.

Conclusions: NK and CIK cell products may offer an innovative immune therapeutic option for patients with high-risk NB after allogenic stem cell transplantation. Our study revealed that NK cells have a significantly higher cytotoxic potential to combat NB. Interestingly, the use of IL-15 expanded and IL-21 activated NK cells developed from a CD3/19 depleted apheresis product is highly promising as additional immunotherapy in combination with haploidentical stem cell transplantation of children with NB.

Disclosure: Nothing to declare. 


\section{P076}

Quantitative determination of donor allo-reactive T-cells in haploidentical donor-recipient pairs by enzymelinked immunospot (ELISPOT) and mixed lymphocyte culture (MLC) assays

\section{Yana Bayzyanova ${ }^{I}$, Maria Efimenko ${ }^{1}$, Natalia Khripkova $^{I}$, Sergei Kovrygin ${ }^{1}$, Ruslan Nikolaev ${ }^{I}$, Rimma Khismatullina $^{1}$, Elena Osipova ${ }^{1}$, Michael Maschan ${ }^{1}$}

${ }^{1}$ Dmitry Rogachev National Research Center of Pediatric Hematology, Oncology and Immunology, Moscow, Russian Federation

Background: T-cell alloreactivity is responsible not only for graft versus host disease and morbidity, associated with hematopoietic stem cell transplantation (HSCT) but also for graft-versus-leukemia (GVL) activity. In this regard, monitoring and quantitation of alloreactive T-cells (allo-T) may potentially provide valuable information for individualized clinical management of transplant recipients. The aim of this study was the optimization of allo-T detection and comparison of the ELISPOT and MLC assays.

Methods: Allo-T were determined in 20 haploidentical donor-recipient pairs before HSCT. Donor mononuclear cells (MNC) served as effector cells (EC). Patient CD3depleted MNC were used as stimulatory cells (SC).The ratio EC:SC were 5:1 and 10:1. The frequency of allo- $T$ in donor peripheral blood was tested in ELISPOT assay and MLC. ELISPOT provides the detection and quantitation of activated T-cells on the basis of cytokine secreted by each cell. The co-incubation time was $24 \mathrm{~h}$ for IFN-gamma and $48 \mathrm{~h}$ for IL-2 detection. In MCL assay donor MNC were labeled with CFSE and allo-T, proliferating in response to stimulation with alloantigens, were determined by flow cytometry on day 5 .

Results: The median number of IFN-gamma producing allo-T per 300000 donor MNC was 143,5 (2-1469; EC:SC ratio - 5:1) and 75,0 (1-1440; EC:SC ratio - 10:1). The median frequency of allo-T was $0,056 \%(0,00076-0,538$; EC:SC ratio - 5:1) and 0,019\% (0,00019 - 0,632; EC:SC ratio - 10:1) among lymphocytes. IL-2-producing allo-T were less frequent in donor MNC in comparison with IFNgamma-producing allo-T. The median number per 300000 MNC was 7,5 (0,5-356; EC:SC ratio - 5:1) and 6,0 (0-169; EC:SC ratio - 10:1). The median frequency of IL-2- allo-T was $0,0028 \%(0,0002-0,130$; EC:SC ratio - 5:1) and 0,0022\% (0 - 0,0619; EC:SC ratio - 10:1) among lymphocytes. The EC:SC ratio 10:1 is enough for stimulation of IL-2 producing by MNC in ELISPOT assay, but for optimal stimulation of IFN-gamma producing cells EC:SC ratio 5:1 is preferable. This suggests that allo-T are predominantly IFN-gamma producing cells. Alloreactive proliferating T-clones were detected in MLC in 10 of 14 donor-recipient pairs on 5 day of cocultivation. Median percentage of proliferating T-clones were 9,5\% (2,1 - 32,5; EC:SC ratio - 5:1) and 7,6\% (3,0 - 24,1; EC:SC ratio - 10:1) among lymphocytes. However, MLC assay only permit a qualitative analysis that confirmed the presence of alloreactive T-clones, giving no information on their frequency within the culture. Results of ELISPOT and MCL assay directly correlated.

Conclusions: Allo-T were detected in $100,0 \%$ of assayed haploidentical donor-recipient pairs by ELISPOT and only in $71,4 \%$ by MLC. This difference in detection is due to the fact that ELISPOT allows to detect single cytokine secreting cell whereas MLC can reveal proliferating allo-T clones. The analysis of allo- $\mathrm{T}$ in haploidentical donor-recipient pairs may provide rationale to manipulate the allo-immune response and to exploit the powerful ability of allo-T to control hematologic malignancies.

Disclosure: Nothing to declare

\section{P077}

Allogeneic mesenchymal stromal cell as rescue therapy in an infant with life-threatening respiratory syndrome due to a filamin a mutation

\section{Stefania Croce ${ }^{1}$, Elisa Lenta ${ }^{2}$, Melissa Mantelli ${ }^{2}$, Maria Antonietta Avanzini', Aurelio Lillo Ferraro ${ }^{3}$, Laura Catenacci $^{2}$, Gloria Acquafredda ${ }^{2}$, Valeria Calcaterra ${ }^{1}$, Gloria Pelizzo ${ }^{3}$}

${ }^{1}$ University of Pavia, San Matteo Hospital, Pavia, Italy, ${ }^{2}$ Policlinico S. Matteo Pavia, Pavia, Italy, ${ }^{3}$ Arnas Civico Di Cristina Benfratelli, Palermo, Italy

Background: Cell-based therapy has gained attention in the respiratory system diseases and encouraging results are reported following mesenchymal stromal cells (MSCs) administration. Due to their capacity to produce and secrete a variety of paracrine factors and bioactive macromolecules, MSCs became a key player in lung tissue injuries and function, reducing fibrosis, promoting the normal development of alveoli and pulmonary vessels. For the first time we used the MSC infusions as rescue therapy in a pediatric patient with FLNA gene mutation and life-threatening respiratory syndrome.

Methods: A child with a new pathogenic variant of the FLNA gene c.7391_7403del; (p.Val2464AlafsTer5) at the age of 18 months, due to the serious and irreversible chronic respiratory failure and dismal prognosis, was treated with 4 intravenous infusions of allogeneic bone marrow (BM)MSCs at the dose of $1 \times 10^{6} \mathrm{MSCs} / \mathrm{kg}$ body weight. BMMSCs were produced at "Cell Factory", Fondazione IRCCS Policlinico S. Matteo, Pavia,isolated and expanded ex vivo 
from healthy donor BM, following a previously reported protocol. Premedication with antistaminic drug, $30 \mathrm{~min}$ before every infusion to avoid any potential reaction was performed. The evolution of the respiratory condition was detected. Peripheral blood were collected before each MSC treatment for Treg and Th17 monitoring. Treg, defined as CD4+ CD127neg CD25+ cells expressing the forkhead box P3 (FoxP3) transcription factor, and Th17, defined as CD4+ cells expressing intracellular IL-17, evaluation was performed by flow cytometry (FACSCanto; BD Biosciences, San Diego, CA) as previously reported, following standard procedures.

Results: No acute adverse events related to MSCs infusion was recorded. During follow-up, patient maintained a good general condition and showed a regular growth. No systemic or respiratory infections occurred. After the second infusion, the child experienced a progressive improvement of his clinical respiratory condition, with a good adaptation to mechanical ventilation, in the absence of episodes of respiratory exacerbations. The baby maintains adequate volumes of exchange with substantial reduction of the inspiratory support. A reduction of trigger sensitivity was also obtained. Thorax CT scan showed a recovery of the basal parenchyma bilaterally and the improvement of the anatomical-functional alignment and aerial penetration. After the first MSC administration, an enrichment of Treg and Th17 percentage in peripheral blood, was observed. While, after the second MSC infusion a significant increase in Treg/Th17 ratio was noted.

Conclusions: This report suggest that MSC serial infusions are a promising therapy in aiding the respiratory failure, even in a pediatric patient with FLNA mutation. Intravenous administrations of allogeneic MSCs are feasible and safe without toxicity. Our results suggest that to mitigate lung injury, MSCs may act as regulators of Treg and Th17 balance. Further investigations are upcoming to establish the useful of this therapeutic proposal in interstitial lung diseases in children.

Disclosure: Nothig to declare

\section{P078}

feasibility of IL-15 stimulated donor NK cells manufacturing for early infusion in patients with high risk acute myeloid leukemia undergoing haploidentical transplantation

Gonzalo Pérez Balsera $^{1,2}$, Nieves Dorado Herrero ${ }^{1,2}$, Ana Pérez Corral ${ }^{1,2}$, Mi Kwon ${ }^{1,2}$, Mariana Bastos Oreiro, Cristina Pascual Izquierdo $^{1,2}$, Laura Solán Blanco ${ }^{1,2}$, Rebeca Bailen Almorox ${ }^{1,2}$, Carmen Falero Ruíz, ${ }^{1,2}$, María Eugenia Fernandez Santos ${ }^{1,2}$, Antonio Pérez Martínez ${ }^{3}$, Cristina Muñoz Martínez ${ }^{2}$, Pascual Balsalobre ${ }^{1,2}$, José Luis Díez Martin ${ }^{1,2}$, Javier Anguita Velasco ${ }^{1,2}$
${ }^{1}$ Instituto de Investigación Sanitaria Gregorio Marañón, Madrid, Spain, ${ }^{2}$ Hospital General Universitario Gregorio Marañón, Madrid, Spain, ${ }^{3}$ Hospital Universitario La Paz, Madrid, Spain

Background: NK cells provide a potent antitumor effect in the setting of manipulated haploidentical hematopoietic stem cell transplant (Haplo-HSCT). We propose a novel strategy to enhance the antitumor effect of allogeneic transplant through the infusion of NK cells stimulated with IL-15 exvivo in adult high-risk acute myeloid leukemia (AML) patients undergoing unmanipulated Haplo-HSCT. The objective of this study was to provide efficiency and productivity data obtained in the manufactured cellular products infused.

Methods: Selection criteria included patients with highrisk AML undergoing unmanipulated Haplo-HSCT. Lymphoapheresis of the haploidentical donor was performed using Spectra Optia (Terumo ${ }^{\circledast}$ BCT) on days +6 and +13 after transplant. From the obtained product a double immunomagnetic cellular selection with Clinimacs system (Miltenyi Biotec $^{\oplus}$ ) was performed in two steps: CD3+ depletion followed by positive $\mathrm{CD} 56+$ selection. The obtained an enriched cellular product of CD3-CD56+ NK cells was incubated with IL-15 $(10 \mathrm{ng} / \mathrm{mL})$ between 12 and 18 hours at $37^{\circ} \mathrm{C}$ and $5 \% \mathrm{CO} 2$ in GMP conditions. Quality and microbiological controls were performed at the end of each manufacturing step. DxH cellular counters (Beckman Coulter $^{\circledR}$ ) and multiparametric flow cytometry were used for lymphocyte subpopulations and viability analysis (Navios cytometer; Beckman Coulter ${ }^{\circledR}$, conjugated Monoclonal antibodies; Miltenyi Biotec ${ }^{\circledR}$ ). The final product was infused intravenously to the patient on days +8 and +15 if manufacturing conditions were met (range of $0.5-100 \times 10^{6}$ $\mathrm{NK} / \mathrm{Kg}$, purity $\geq 80 \%$, viability $\geq 70 \%$ and $<1 \times 10^{4} \mathrm{CD} 3+$ cells/Kg). If not, it was discarded. NK cell activation in the product was measured by the expression of $\mathrm{CD} 25$ and CD69.

Results: Between November 2017 and April 2018, 3 patients were included in this ongoing trial. Two products were manufactured for 2 of the patients, and only one for the first patient, due to transplant complications between first and second infusion. One product did not meet minimum viability criteria and was discarded. In the infused final products mean and SEM of NK cell purity, recovery and viability were $83.7 \% \pm 4.4,30.9 \% \pm 4$ and $76.3 \% \pm 17.4$, respectively. Log $\mathrm{CD} 3+$ depletion ranged between -5.48 and -6.03. Median infused doses of NK cells and CD3+ cells per $\mathrm{kg}$ were $3.78 \times 10^{6}\left(2.8 \times 10^{6}-4.57 \times 10^{6}\right)$ and $114(87-$ 532). Complete manufacturing data of all 5 procedures are shown in Table 1. Mean expression of activation markers was: CD69 (basal 15.6\% 2.6, after IL-15 69\% \pm 12.7 ), 
CD25 (basal 0.1\% \pm 0.04 , IL-15 33\% \pm 7.75 ). No patient had immediate adverse effects with product infusion.

Conclusions: We demostrate the feasibility of the manufacturing process of IL-15 estimulated NK cells from haploidentical donors and early post-transplant infusion in adult AML patients in the setting of unmanipulated HaploHSCT. Achieved doses of NK cells for adult patients are promising, with safe content of contaminant $\mathrm{T}$ lymphocytes less than $1 \times 10^{4} / \mathrm{Kg}$. More procedures need to be analyzed in order to confirm this observations.

Clinical Trial Registry: NCT03669172

https://clinicaltrials.gov/ct2/show/study/NCT03669172

Disclosure: This study is funded by Instituto de Salud Carlos III (PI 15/00879). None of the authors have something to declare.

\section{P079}

Improving clinical manufacturing of cytokine-induced killer (CIK) cells

Melanie Bremm ${ }^{1}$, Eva Rettinger ${ }^{1}$, Claudia Cappel, Verena Katzki ${ }^{1}$, Stephanie Erben ${ }^{1}$, Sibille Betz ${ }^{I}$, Andrea Quaiser $^{2}$, Halvard Bonig ${ }^{3}$, Michael Schmidt ${ }^{3}$, Lisa-Marie Pfeffermann $^{1}$, Thomas Klingebiel ${ }^{1}$, Peter Bader ${ }^{1}$, Sabine Huenecke ${ }^{I}$

${ }^{1}$ University Hospital Frankfurt/Clinic for Children and Adolescents, Frankfurt am Main, Germany, ${ }^{2}$ University Hospital Leipzig, Institute for Clinical Immunology, Leipzig, Germany, ${ }^{3}$ Institute for Transfusion Medicine and Immunohematology, Frankfurt am Main, Germany

Background: Cytokine-induced killer (CIK) cells are a promising immunotherapeutic approach to combat relapse following allogeneic hematopoietic stem cell transplantation (HSCT) for acute leukemia or myelodysplastic syndrome. To show safety and efficacy, a multicenter clinical study with 20 pediatric and 20 adult patients including up to eight CIK cell applications with escalating doses is ongoing.

Methods: We favor single large scale CIK cell generation with the aim to apply fresh CIK cells and cryopreserve ready-for-use doses according to the study protocol in contrast to recurrent manufacturing. Therefore cryopreserved CIK cells were tested against freshly generated CIK cells to approve equivalence. Furthermore, an alternative medium supplement for CIK cell culturing was investigated to avoid supply bottlenecks in AB-Serum.

Results: Fresh Frozen Plasma (FFP), platelet lysate (PL) and $\mathrm{AB}$-serum in $\mathrm{CIK}$ cell culture showed median expansion rates of 10 -fold, whereas cultivation without medium additive resulted in significantly lower proliferation $(\mathrm{p}<0.01)$. CIK cell composition including T cells, NK like $\mathrm{T}$ cells and a minor part of NK cells was not significantly influenced by changing the medium additive. Moreover, neither cytotoxicity against THP-1 cells nor CD25 expression on NK like T cells were significantly influenced by the different medium additives. For CIK cell generation either ficollized peripheral blood (PB) or unstimulated leukapheresis (LP) products were utilized. With regard to repeated manufacturing within the clinical study, also cryopreserved LP and PBSC as starting material came into the focus of interest. Comparing CIK cell expansion rates, no significant differences for the entire CIK cells and the subgroup of $\mathrm{T}$ cells were detected between the four starting materials. Cryopreservation of CIK cells had no significant effect on CIK cell composition, cytotoxicity and CD25 expression on NK like T cells. A small, albeit not significant effect of cryopreservation on viability was detected, which was $86.1 \%$ before and $79.4 \%$ after freezing and thawing.

Conclusions: The challenge was an efficient time-, personal- and cost saving production of CIK cells within the clinical study. Introducing FFP enabled CIK cell manufacturing for an increased patient cohort by avoiding supply bottlenecks in AB-serum. Furthermore, cryopreservation allows the storage of ready-for-use CIK cell doses fulfilling the demands of the clinical study.

Clinical Trial Registry: EudraCT Number 2013005446-11

Disclosure: Nothing to declare.

\section{P080}

Automated generation of CD45RA depleted donor lymphocyte infusion (DLI) with the clinimacs prodigy ${ }^{\circledR}$ CD45RA system

Joanna Justyna Stenzel ${ }^{I}$, Stephanie Soltenborn ${ }^{I}$, Carina Thießen ${ }^{1}$, Felix Hebbeker ${ }^{1}$, Kirsten Langeveld ${ }^{\prime}$, René Meißner', Michaela Malchow ${ }^{1}$, Eleni Papanikolaou ${ }^{1}$, Andreas Bosio ${ }^{1}$, Mario Assenmacher ${ }^{1}$, Julia Dzionek ${ }^{I}$

${ }^{1}$ Miltenyi Biotec GmbH, Bergisch Gladbach, Germany

Background: Donor-derived $\mathrm{CD}^{2} 5 \mathrm{RO}^{+}$memory $\mathrm{T}$ cells have been described to provide defense against pathogens threatening immunocompromised patients after allogeneic hematopoietic stem cell transplantation (HSCT) while bearing a reduced GvHD risk; thus, in the recent years, DLIs depleted of naïve CD45RA ${ }^{+} \mathrm{T}$ cells by CliniMACS ${ }^{\circledR}$ technology have been infused posttransplant to prevent infections in haploidentical $\mathrm{HSCT}^{1,2}$

Methods: The current CliniMACS CD45RA System was developed for graft engineering. Up to 20x10e9 magnetically labeled CD45RA + cells from leukapheresis products can be depleted from up to 50x10e9 white blood cells (WBC). We developed a new CliniMACS Prodigy ${ }^{\circledR}$ process in order to ease the procedure for routine-use, to reduce the 
specifications according to reported cell numbers for DLI applications, and to enable the use of peripheral blood products with high amounts of red blood cells (RBC). The new system was tested by performance runs. An new fluorescent flow analysis protocol was developed.

Results: The resulting CliniMACS Prodigy PB-45RA System is an automated procedure with integrated labeling and washing steps. The new application software PB-45RA Depletion enables to deplete up to $1.8 \times 10 \mathrm{e} 9 \mathrm{CD}_{4} \mathrm{RA}^{+}$cells from up to $5 \times 10 \mathrm{e} 9$ total $\mathrm{WBC}$ from peripheral blood products. A major difference of this process is the RBC removal option based on an integrated camera for cell pellet detection. The final cell product is provided in physiologic saline. Verification runs with peripheral blood products $(n=6$ in total, $\mathrm{n}=3$ with whole blood, $\mathrm{n}=3$ with leukapheresis products) resulted in a mean depletion of $5.0 \log$ (range 3.9 5.7) for CD45RA ${ }^{+} T$ cells in the CD45RA depleted product. Viability of the target products was always above $96 \%$, and mean WBC recovery was $66 \%$. The mean process time was $3 \mathrm{~h} 23 \mathrm{~min}$ (range $3 \mathrm{~h}$ to $3 \mathrm{~h} 50 \mathrm{~min}$ ) without including the manual steps, i.e. tubing set installation and downstream analysis of blood products by flow cytometry. This data were in line with preceding evaluation runs $(\mathrm{n}=6)$, and results obtained in cooperation with an external beta test site. ${ }^{3}$ The performance results were furthermore in line with results obtained on CliniMACS Plus instrument runs. For quality control of CD45RA depleted products we developed a flow cytometric analysis strategy for fast, accurate, and convenient analysis of even rare counts of remaining unwanted cells. It allows to determine naïve $\mathrm{T}$ cells at two different levels of subset staining. The minimum requirement for the flow cytometric analysis includes 4 colors to define viable $\mathrm{CD} 3+\mathrm{CD} 45 \mathrm{RA}+$ cells. For further evaluation of the naïve $\mathrm{T}$ cell subsets 3 additional colors are used to define viable CD3+CD45ROCD95-CD62L+CD197+ cells.

Conclusions: The automated CliniMACS Prodigy PB45RA System process is capable to deplete CD45RA + cells efficiently from peripheral blood products within 4 hours. The new process is a fast, convenient, and regulatory compliant method for the preparation of ready-to-use CD45RA-depleted cell products for clinical applications. The submission to an European notified body for CE certification is an important next step.

1 Maschan, M et al. (2017) Bone Marrow Transplant. 53:264.

2 Triplett, BM et al. (2018) Transpl Infect Dis. 20: e12823.

3 Mueller, N et al. (2018) Cytotherapy 4:532

Disclosure: All authors are employees of Miltenyi Biotec GmbH, Germany

\section{P081}

Extracorporeal photopheresis treatment for steroid resistant graft versus host disease in pediatrics: Single center experience

Başak Adaklı Aksoy', Yunus Emre Savcl' ${ }^{2}$, Azize Mergen ${ }^{2}$, Selime Aydoğdu', Gürcan Dikme ${ }^{2}$, Ceyhun Bozkurt ${ }^{1}$, Tunç Fuşgın ${ }^{3}$

${ }^{1}$ Istinye University, Medical Park Hospitals, Bahçelievler, Istanbul, Turkey, ${ }^{2}$ Medical Park Hospitals, Bahçelievler, Istanbul, Turkey, ${ }^{3}$ Altinbas University, Medical Park Hospitals, Bahçelievler, Istanbul, Turkey

Background: Steroid resistant graft vs host disease (GVHD) is defined as unresolved or worsened symptomatology of GVHD at day 14 despite effective immunosuppression with steroid therapy. Second line immunosuppressive agents, extracorporeal photopheresis (ECP) and/or mesenchymal stem cell therapies are being tried as salvage therapy.

Methods: We performed a retrospective single center trial between February 2016 - September 2018 with 25 out of 173 stem cell transplanted patients diagnosed with steroid resistant GVHD and treated with ECP.

Results: 25 patients diagnosed with steroid resistant GVHD, age between 2-17 years, 15 male and 10 female with diagnosis of AML, ALL, thalassemia major, aplastic anemia, NHL, MDS, HLH and CGD before transplant. Transplant characteristics were given on.

\begin{tabular}{ll} 
Donor (MFD:84, MUD: 77, & MFD:8(\%32) MUD:15(\%60) \\
Haploidentical: 12) & Haploidentical: $2(\% 8)$ \\
HLA match & 10/10: 10 (\%40) 9/10: 13 (\%52) \\
& Haploidentical: 2 (\%8) \\
Stem cell source & BM: 3 (\%12) PSC: 20 (\%80) BM + \\
& PSC: $2(\% 8)$ \\
CD34 + cells(x10e6/ kg) & Median: 5,8 (3,9-19) \\
Neutrophil engraftment (days) & Median: $13(10-29)$ \\
Platelet engraftment (days) & Median: $17(10-38)$ \\
Chimerism (Full blood) & Full donor: 21(\%84) Mixed: $4(\% 16)$ \\
\hline
\end{tabular}

\section{[[P081 Table] 1. Transplant Characteristics]}

Myeloablative conditioning regimen was preferred for 24 patients out of 25. GVHD prophylaxis regimens are CSA +MTX: 20(\%80), CSA+MMF: 2(\%8), CSA only: 1(\%4). ATG was given 20 patients. Despite been given GVHD prophylaxis $47(\% 27,1)$ patients out of 173 transplanted patients had GVHD features. Of 47 patients, 25 had experienced steroid resistant GVHD after transplantation, including $17(\% 68)$ grade 3 and $8(\% 32)$ grade 4. ECP treatment was started mean 11 days after diagnosis of steroid resistant GVHD and $2(\% 8)$ patients had complete response while $12(\% 48)$ patients had partial and $11(\% 44)$ 
patients had no response to ECP treatment on day 28 . Sixteen out of 25 patients had also received mesenchymal stem cell therapy as salvage therapy. Only one patient had experienced hypocalcemic tetany, a complication of ECP procedure. Thirteen patients had died and 12 were directly related with steroid resistant GVHD. Other conditions like relapse of primary disease or PRES syndrome also played role in death.

Conclusions: Extracorporeal photopheresis is a reliable and effective second line treatment modality in steroid resistant GVHD. Starting ECP sessions as soon as GVHD symptoms occur increases its effectivity. Mesenchymal stem cell administration with ECP for 16 (\%64) patients limits our study to reach o conclusion for efficacy of ECP itself. Need for hemodialysis catheters, the prolonged sessions while adequate flow is not possible and catheter related infections are the lmitations for feasibility of ECP.

Disclosure: nothing to declare

\section{P082}

Donor lymphocyte infusion administrations after allogeneic stem cell transplantations in pediatrics: $A$ single center experience

Selime Aydoğdu', Azize Mergen ${ }^{1}$, Başak Aksoy ${ }^{2}$, Hazal Ş. Akbay $^{3}$, Funda Çipe ${ }^{1}$, Gürcan Dikme ${ }^{1}$, Tunç Fuşgın ${ }^{3}$, Ceyhun Bozkurt ${ }^{2}$

${ }^{1}$ Medical Park Hospitals, Bahçelievler, Istanbul, Turkey, ${ }^{2}$ Istinye University, Medical Park Hospitals,Bahçelievler, Istanbul, Turkey, ${ }^{3}$ Altinbas University, Medical Park Hospitals, Bahçelievler, Istanbul, Turkey

Background: Loss of chimerism is one of the major problems after allogeneic stem cell transplantation(SCT). Donor- lymphocyte infusions(DLI) are used as a treatment after taper or stopping immunosuppression. In this study, DLI experience in 20 patients with loss of chimerism after SCT due to various benign and malign hematological diseases was presented.

Methods: Between July 2015- August 2018, twenty patients, detected chimerism loss and received DLI after SCT were evaluated retrospectively. Patients received myeloablative or reduced intensity conditioning, ATG, cyclosporine A and methotrexate for GVHD prophylaxis. Chimerism analyses were performed with short tandem repeat(STR) method from peripheral blood. Results below 95\% were considered as mixed chimeric and below $\% 5$ were nonchimeric. When patients considered as mixed chimeric, immunosuppression therapy was ceased immediately and treated with DLI. Donor lymphocyte infusions were performed at two-week intervals with chimerism follow-up. Student T, Mann Whitney U, Ki Kare tests and Kaplan-Meier analysis were used.

Results: Between 1-16 ages (median 4), 7 female, 13 male patients were evaluated. The initial diagnoses were thalassemia major(10), aplastic anemia(3),ALL(4), AML (3). DLI initiation time was $119.65+-92.71$ days after SCT, total number of DLI administrations were $3.5+-2.19$. Dose of DLI was $1 \times 10^{5}-63.6 \times 10^{6} / \mathrm{kg}$ (mean $20.5 \times 10^{6} / \mathrm{kg}$ ). Nine patients' chimerism out of 20 , fell below $95 \%$ at first month after transplant; 4 patients were nonchimeric, 2 of them were complet chimeric and 3 were mixed chimeric. Eleven patients' chimerism were below 95\% between 1-6 months after SCT, 5 patients were nonchimeric and 6 were mixed chimeric. Early mixed chimerism was found relevant with graft rejections $(p=0.04)$. Patients were followed up for 91645 days. Eight patients' chimerism increased after DLI infusion and continued to decrease in 12 patients. After DLI, acute GVHD has been seen in both group.The group with decreased chimerism after DLI, dose was mean $12 \times 10^{6}$ $\pm 23 \times 10^{6} / \mathrm{kg}$ while the group with increased chimerism had DLI dose mean $22 \times 10^{6} \pm 19 \times 10^{6} / \mathrm{kg}$. Although the difference was not statistically significant, numerical value revealed significantly different. Eventually 10 patients out of 20 were mixed chimeric, 6 patients were complete chimeric and 4 were none. In thalassemic patients, 7 patients with thalassemia-trait donor were mixed chimeric, In 3 patients whose donors were normal, 2 of them were complete chimeric and one of them was nonchimeric. The difference was significant $(\mathrm{p}=0.02)$. The $\mathrm{CD} 34$ infusion doses revealed mean $6.61 \pm 5.06 \times 10^{6} / \mathrm{kg}$ in mixed chimeric patients, $7.16 \pm 3.31 \times 10^{6} / \mathrm{kg}$ in complete chimeric patients and $6.625 \pm 1.37 \times 10^{6} / \mathrm{kg}$ in the patients with loss of chimerism. CD34 amount was seen high as numerical value in complete chimerics but no statistical significance was found. Overall survival was $85 \%$, disease-free survival was $25 \%$.

Conclusions: We evaluated the efficacy of DL for patients with mixed chimerism in our patient group. We concluded that chimerism loss in patients with early decreased chimerism is similar to those in literature in spite if DLI practices. Dose and application frequency were greater in patients with increased chimerism. The small number and the heterogeneity of the patients limited our study. In this regard, studies with larger series and homogeneous groups are acquired.

Disclosure: Nothing to declare

\section{P083}

Phase I clinical trial of repeated administrations of bone-marrow derived mesenchymal stem cells in steroid-refractory chronic graft-versus-host disease patients 
Nayoun Kim ${ }^{1}$, Young-Woo Jeon ${ }^{2}$, Jae-Deog Jang ${ }^{1}$, KeonIl Im ${ }^{1}$, Nak-Gyun Chung ${ }^{2}$, Young-Sun Nam ${ }^{1}$, Yunejin Song ${ }^{1}$, Jun-Seok Lee ${ }^{1}$, Seok-Goo Cho ${ }^{1,2}$

${ }^{1}$ The Catholic University of Korea, Seoul, Korea, Republic of, ${ }^{2}$ Seoul St. Mary's Hospital, The Catholic University of Korea, Catholic Blood and Marrow Transplantation Center, Seoul, Korea, Republic of

Background: Chronic graft-versus-host disease (cGHVD) is the most common long-term complication of allogenic hematopoietic stem cell transplantation which is associated with poor quality of life and increased risk of morbidity and mortality. Currently, there is no standardized treatment available for patients who do not respond to steroids. As an alternative to immunosuppressive drugs, mesenchymal stem cells (MSCs) have been used to treat and prevent steroidrefractory acute GVHD patients. These studies and reports have also provided a basis for using MSCs in steroid refractory cGVHD patients.

Methods: To evaluate the safety and efficacy of repeatedinfusions of MSCs, we enrolled ten severe steroid-refractory cGVHDs patients. Steroid refractory was defined as either no response to steroids lasting at least 4 weeks or progression of disease during treatment or tapering lasting at least 2 weeks. Patients were intravenously administered with MSCs produced from third-party bone marrow donors at a 2-week interval for a total of four doses. Each dose contained $1 \times 10^{6}$ cells per kg body weight and all four doses consisted of MSCs from the same donor and same passage.

Results: We enrolled ten patients ( 3 female/ 7 male, with a median age of 41.5(range 17-68). Median of cGVHD affected organs was 3 (range 2-4) including the skin $(n=4)$, eyes $(n=8)$, oral cavity $(n=9)$, lung $(n=1)$, liver $(n=2)$ and joints $(n=6)$. All ten patients received their planned four doses of MSCs, administering a total of 40 infusions. Median time from initial cGVHD diagnosis to first MSC treatment was 709 days (range 222-4413). MSC infusions were well tolerated with no immediate or delayed toxicities. After 8 weeks of the first MSC infusion, all ten patients showed partial response showing alleviation in clinical symptoms and increased quality of life. Organ responses were seen in skin $(n=2)$, eyes $(n=5)$, oral cavity $(n=8)$, liver $(\mathrm{n}=1)$, and joint $(\mathrm{n}=5)$. However, one patient died of progressive GVHD and one patient relapsed from primary disease.

Conclusions: Repeated infusions of MSCs was feasible and safe and may be an effective salvage therapy in patients with steroid-refractory cGVHD. Further large-scale clinical studies with long-term follow up is needed in the future to determine the role of MSCs in cGVHD.
Clinical Trial Registry: This study was registered and approved by Clinical Research Information Service, Republic of Korea, in the WHO Registry Network (KCT0001894).

Disclosure: Authors have nothing to declare

\section{P084}

Therapeutic use of umbilical cord blood stored in the polish stem cell bank for standard and experimental therapy

\section{Izabela Zdolińska-Malinowska ${ }^{I}$, Maciej Rojek ${ }^{I}$,Dariusz Boruczkowski ${ }^{1}$}

\section{${ }^{I}$ The Polish Stem Cell Bank, Warsaw, Poland}

Background: The majority of pregnant Polish women (84\%) have heard of cord blood banking. However, most doctors do not have sufficient knowledge about the possibility of using cord blood in order to respond to their potential concerns. Only $16.5 \%$ of healthcare professionals were aware that cord blood could be used to treat haematological diseases. In order to make doctors aware of this issue and provide patients with the information they expect, we would like to present data on the use of cord blood stored in our blood bank for haematological and nonhaematological therapies.

Methods: The table presented below has been created using data from the general database of the Polish Stem Cell Bank, Warsaw, Poland. No data regarding umbilical cord blood data have been excluded. All patients were planned to be assessed on day 1 , day 28 , on discharge, 100 days after transplantation and 1, 2, 3, 4, and 5 years after transplantation, but in some cases, patients were lost from follow-up due to a persistent lack of reports from transplantation centres.

Results: In 32 cases, the therapeutic use of CB was transplantation (replacement of patients' own tissue); in 13 cases it was administration (infusion without destruction of patients' own tissue). Thirty-three were administered as standard therapy and 13 as experimental therapy.

Conclusions: The survey study cited above, indicated low awareness of cord blood use among healthcare professionals. On the other hand, one study indicated that the expectations placed in cord blood banking may be unreasonable. As a private cord blood bank, we support recommendations which underline the importance of patient education. In Poland, cord blood has been approved as standard therapy in approx. 80 diseases; most of them are rare, but Polish law allows the use of cord blood for the siblings, parents and grandparents of a donor. Additionally, 168 active and planned clinical trials throughout the world evaluate the therapeutic efficacy of cord blood in such areas as haematology, neurology, cardiology, diabetology, congenital paediatric disorders, ophthalmology, 
dermatology, gastroenterology, HIV infection, and the quality of life during aging. Therefore, further indications may be expected in the future.

\begin{tabular}{|c|c|c|c|c|c|c|c|c|c|}
\hline Indication & $\begin{array}{l}\text { Number of } \\
\text { allogeneic } \\
\text { transplantat- } \\
\text { ions/ } \\
\text { administrat- } \\
\text { ions for } \\
\text { related } \\
\text { recipients }\end{array}$ & $\begin{array}{l}\text { Number of } \\
\text { allogeneic } \\
\text { transplant- } \\
\text { ations/ } \\
\text { administr- } \\
\text { ations for } \\
\text { unrelated } \\
\text { recipients } \\
\text { (public } \\
\text { bank) }\end{array}$ & $\begin{array}{l}\text { Number } \\
\text { of } \\
\text { autologo- } \\
\text { us } \\
\text { transplan- } \\
\text { tations/ } / \\
\text { administ- } \\
\text { rations }\end{array}$ & $\begin{array}{l}\text { Only } \\
\text { cord } \\
\text { blood }\end{array}$ & $\begin{array}{l}\text { Cord } \\
\text { blood }+ \\
\text { bone } \\
\text { marrow/ } \\
\text { placental } \\
\text { blood }\end{array}$ & $\begin{array}{l}\text { Complete } \\
\text { remission }\end{array}$ & $\begin{array}{l}\text { Partial } \\
\text { remission/ } \\
\text { improve- } \\
\text { ment }\end{array}$ & $\begin{array}{l}\text { Lack of } \\
\text { improv- } \\
\text { ement/ } \\
\text { Relapse } \\
\text { without- } \\
\text { death }\end{array}$ & Death \\
\hline Leukaemias & 10 & 1 & 0 & 8 & 3 & 7 & 1 & 1 & 2 \\
\hline Anaemias & 4 & 0 & 1 & 3 & 1 & 5 & 0 & 0 & 0 \\
\hline $\begin{array}{l}\text { Cerebral } \\
\text { palsy / } \\
\text { ischemia }\end{array}$ & 0 & 0 & 5 & 5 & 0 & 0 & 5 & 0 & 0 \\
\hline Autism & 1 & 0 & 7 & 8 & 0 & 0 & 8 & 0 & 0 \\
\hline Thalassemia & 7 & 0 & 0 & 0 & 7 & 7 & 0 & 0 & 0 \\
\hline $\begin{array}{l}\text { More than } \\
\text { one } \\
\text { indication }\end{array}$ & 2 & 0 & 0 & 0 & 2 & 2 & 0 & 1 & 0 \\
\hline $\begin{array}{l}\text { Other } \\
\text { diseases }\end{array}$ & 7 & 0 & 0 & 4 & 3 & 5 & 0 & 1 & 1 \\
\hline Total & 31 & 1 & 13 & 28 & 16 & 26 & 14 & 3 & 3 \\
\hline
\end{tabular}

[[P084 Table] 1. Therapeutic use of umbilical cord blood in particular diseases]

Disclosure: All authors are employees of the Polish Stem Cell Bank

\section{P085}

Cytokine release syndrome treatment and management flowchart

Jaydine Carr' ${ }^{1}$, Sonia Berzosa ${ }^{I}$

${ }^{1}$ Bristol Oncology and Haematology Centre, Ward D703, Bristol, United Kingdom

Background: Cytokine release syndrome (CRS) has been identified as a clinically significant, on target, off tumour side effect of the chimeric antigen receptor (CAR) T-Cell therapies. It is clinically increased in interkeukin 6 and elevations in other cytokines, lactate dehydrogenase (LDH), C-reactive protein (CRP), and ferritin. These side effects can include fever, fatigue, headache, encephalopathy, hypertension, tachycardia, coagulopathy, nausea, capillary leak and multi organ dysfunction. CRS symptoms can appear as early as one day after infusion and can resolve quickly or last for weeks. It's severity to be related to the disease burden prior to CAR T-Cell therapy.

Methods: The Bristol Oncology and Haematology centre will be providing CAR T-Cell therapy to patients in early 2019. On collating from the leading consultant on the ward and fellow nursing team members it was apparent that an effective way at managing patients post CAR T-Cell therapy side effects is to provide an educational and informative poster depicting a flow chart that will aid the practitioner to recognise and effectively treat/manage a patient with CRS symptoms.
Results: None as of yet as this is a prospective tool ready for our first patient in early 2019

Conclusions: Through continuing reading and study days prior to the ward receiving its first CAR T-Cell patient it is increasingly important that the entire multi disciplinary team recognise CRS and understand the importance of early detection, careful monitoring and early intervention.

Clinical Trial Registry: N/A

Disclosure: Nothing to declare

Chronic leukaemia and other myeloproliferative disorders

P086

Reduced intensity allogeneic stem cell transplantation for younger patients with myelofibrosis

Daniele Mannina $^{1,2}$, Christine Wolschke ${ }^{1}$, Tatjana Zabelina ${ }^{I}$, Gaby Zeck ${ }^{1}$, Ute-Marie von Pein ${ }^{1}$, Francis Ayuk ${ }^{1}$, Nicolaus Kröger ${ }^{1}$

${ }^{1}$ Hamburg University Hospital, Hamburg, Germany, ${ }^{2}$ Ospedale San Raffaele, Milano, Italy

Background: Allogeneic stem cell transplantation (alloSCT) is the only curative procedure for primary and secondary myelofibrosis (PMF, SMF). Elderly people are mainly affected, limiting the feasibility of intensive myeloablative chemotherapy regimens. The introduction of reduced-intensity conditioning (RIC) made alloSCT feasible and effective for old patients. Nevertheless, the incidence of PMF and SMF is not negligible in young patients, theoretically able to tolerate also high-intensity therapy. Very few data are available about the efficacy of RICalloSCT in the particular setting of young-aged MF patients.

Methods: This study includes 56 Myelofibrosis young patients (age $<55 \mathrm{y}$ ) who received alloSCT between 2002 and 2016 at the University Hospital Hamburg/Germany. Four patients were previously splenectomized. Patients mostly fall into intermediate risk groups according to DIPSS. Four patients belonged to the high-risk triple-negative category (JAK2/CALR/MPL-). ASXL1-mut was tested in 50 patients (pos: 17). In 96\% graft source was PBSC, 2 patients received BMSC. Only $30 \%$ of patients had a 10/10 HLA-matched sibling, the others were transplanted from fully-matched (36\%) or partially-matched $(34 \%)$ unrelated donor. All transplants were conditioned according the EBMT protocol with Busulfan (10 mg/Kg PO or $8 \mathrm{mg} / \mathrm{Kg} \mathrm{IV})$, Fludarabine $(150 \mathrm{mg} / \mathrm{m} 2)$, ATLG (Grafalon ${ }^{\circledR}$ Neovii, Germany) administered in 3 days at a dose of $20 \mathrm{mg} / \mathrm{Kg}$ die for MUD, $10 \mathrm{mg} / \mathrm{Kg}$ die for MRD transplants, followed by CylosporinA, and Mycophenolate in the first 28 days.

Results: Engraftment rate was $98 \%$, median neutrophil engraftment time 15 days. Platelet engraftment was reached 
by 51 patients (91\%, median 19 days). Four patients (7\%) developed poor graft function, successfully treated with CD34+ selected PBSC-boost. After a median follow up of 8.6 years, estimated 5y-PFS and OS were $68 \%$ and $82 \%$ respectively. DIPSS-risk and donor HLA-matching resulted the only significant impacting factors on OS. Neither cytogenetic nor molecular abnormalities were significantly related to OS. Twenty-five patients (44\%) experienced aGVHD grade $>1$. C-GVHD was observed in 34 patients $(65 \%)$, mostly (82\%) beginning in the first 300 days after transplantation. Cumulative incidence of TRM was $7 \%$ at 1 year, with a plateau after the first year $(5 \mathrm{y}$ TRM $=12 \%)$. TRM was observed only in patients with maximal grade (3) of marrow fibrosis. Furthermore, TRM never occurred in previously splenectomized patients $(\mathrm{p}=0.00)$, but no significant impact from splenectomy on OS was observed $(\mathrm{p}=0.32)$. After transplant, 11 patients $(20 \%)$ relapsed: 1 died without any treatment because of infection, 9 received DLI (3 durable CR), 7 patients (6 after DLI) underwent a second alloSCT, with long-term survival in 5 cases.

Conclusions: RIC followed by allogeneic SCT is a curative treatment approach for younger patients with myelofibrosis with a low NRM. The most important outcome-determining factor is donor HLA-matching. Interestingly, marrow high grade fibrosis showed to significantly impact TRM. Biological markers such as ASXL1 mutation and cytogenetics, largely known as highly predictive for poor prognosis in the disease natural course, did not show any impact on survival, suggesting that patients harboring these abnormalities could get the greatest benefit from alloSCT. Further data collection, and a prospective randomized trial are needed to confirm our conclusion.

Disclosure: Nothing to declare

\section{P087}

\section{Abstract already published.}

\section{P088}

Splenectomy as a risk factor for relapse after allogeneic hematopoietic stem cell transplantation in patients with myelofibrosis - retrospective cohort study

\section{Rashit Bogdanov ${ }^{1}$, Markus Ditschkowski ${ }^{1}$, Nina-Kristin Steckel ${ }^{1}$, Vesna Klisanin ${ }^{1}$, Michael Koldehoff ${ }^{1}$, Lambros Kordelas $^{1}$, Tobias Liebregts ${ }^{1}$, Nikolaos Tsachakis-Mueck ${ }^{1}$, Rudolf Trenschel ${ }^{1}$, Dietrich W. Beelen ${ }^{1}$}

${ }^{1}$ University Hospital Essen, West-German Cancer Center, Essen, Germany

Background: Splenectomy is a common procedure in patients (pts) with myelofibrosis (MF) performed to achieve improvement in blood cell counts and reduce B-symptoms.
However, it has also been shown that splenectomy may adversely predispose to leukemic transformation in this setting.

Aim: To evaluate in a single-center retrospective analysis the long-term impact of pre- or post-transplant splenectomy on transplant outcome regarding overall survival and relapse risk.

Methods: This retrospective analysis comprises the data of 163 pts (93 male and 70 female) with primary $(n=108)$ or secondary $(\mathrm{n}=55) \mathrm{MF}$ after allo-HSCT from HLA-matched sibling $(n=48)$ or unrelated $(n=115)$ donors in our center between 1994 and 2018. The median age was 56 years (range, 22 to 75 years). A myeloablative conditioning regimen was performed in 142 pts, while 21 pts where treated with a reduced intensity conditioning. Peripheral blood stem cells $(n=154)$ or bone marrow $(n=9)$ with a median of $7.0 \times 10^{6} \mathrm{CD} 34^{+}$cells $/ \mathrm{kg}$ bodyweight (BW) (range, 1.0 to 30 ) were transplanted. Splenectomy was performed in 41 of 163 pts: 21 pts were splenectomized prior to and 20 pts after allo-HSCT. Relapse was diagnosed in $22(14 \%)$ of 163 pts. The median duration to relapse after transplantion was 13 months (range, 3-99 months).

Results: The median duration of follow-up of this cohort was 28 months (range, 2-120 months), the 10-year overall survival (OS) was 46\%. 74 pts died, including 7 pts who relapsed and $67 \mathrm{pts}$ who died of treatment related causes. The observed probability of relapse was significantly higher in splenectomized pts than in non-splenectomized pts: $37 \%$ versus $10 \%$ (relative risk (RR) 3.7, 95\% CI, 1.5-9.4, $\mathrm{p}=0.007$ ). At 10 years, the OS was $50 \%$ in nonsplenectomised and 39\% in splenectomised pts $(\mathrm{p}=0.35)$ (Fig.1). The relapse rate in splenectomised pts was independent of pre- (5 of 21 pts, $24 \%$ ) or post-transplant ( 6 of 20 pts, $30 \%$ ) treatment (RR 1.3, 95\% CI, 0.34-5.5, $\mathrm{p}=0.73$ ).

Conclusions: On the basis of our cohort, we could assert that pre- and post-allo-HSCT splenectomy was equally and significantly associated with an increased relapse ratio in patients with MF, which also tends to negatively affect overall survival.

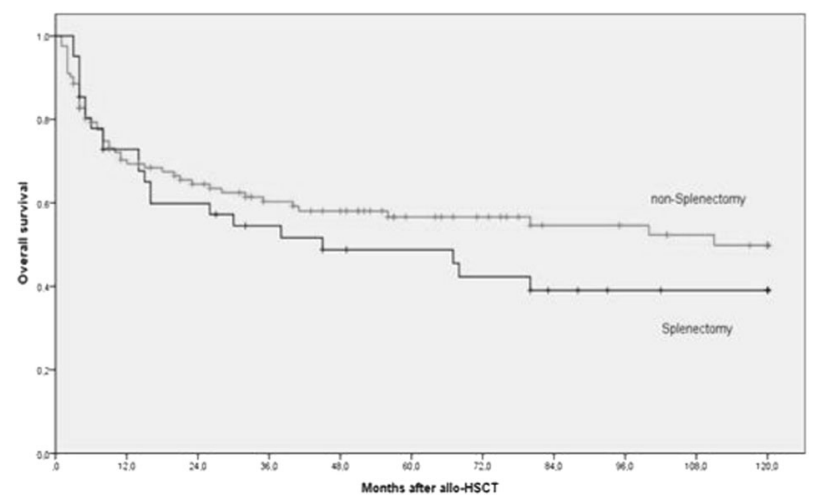

[[P088 Image] 1. Figure 1: The overall survival after allo-HSCT in patients with myelofibrosis.] 
Clinical Trial Registry: no

Disclosure: Nothing to declare

\section{P089}

Three consecutive cases illustrating that Rituximab can induce deep responses in ibrutinib-resistant B-cell prolymphocytic leukemia for bridging to allogeneic stem cell transplantation

Nora Liebers $^{1,2}$, Sascha Dietrich ${ }^{1,2,3}$, Michael Hundemer ${ }^{1}$, Susanne-Annegret Hain ${ }^{4}$, Martin Schiller ${ }^{1}$, Eckhart Weidmann $^{5}$, Tobias Roider ${ }^{1}$, Monika Brüggemann ${ }^{6}$, Carsten Müller-Tidow ${ }^{I}$, Peter Dreger ${ }^{I}$

${ }^{1}$ University of Heidelberg, Heidelberg, Germany, ${ }^{2}$ National Center for Tumor Diseases and German Cancer Research Centre, Heidelberg, Germany, ${ }^{3}$ European Molecular Biology Laboratory (EMBL), Heidelberg, Germany, ${ }^{4}$ St. Marien-Krankenhaus, Medizinische Klinik III, Siegen, Germany, ${ }^{5}$ Krankenhaus Nordwest, Klinik für Onkologie und Hämatologie, Frankfurt, Germany, ${ }^{6}$ Universitätsklinikum Schleswig-Holstein, Klinik für Innere Medizin II, Kiel, Germany

Background: B-cell prolymphocytic leukemia (B-PLL) is a very rare lymphoproliferative disorder. Although allogeneic stem cell transplantation (alloSCT) could be a curative option, patients often do not qualify for this consolidation treatment due to an aggressive course of disease. In this case study, we report on three patients who failed ibrutinib therapy but achieved complete remission and even MRD negativity after treatment with the chimeric CD20-antibody Rituximab, enabling them to undergo alloSCT.

Methods: Clinical data and follow-up data were collected by chart review.

Results: All three patients (pt\#1: male, 42 years; pt\#2: female, 66 years; pt\#3: female, 64 years) were referred with B-PLL harboring highly complex aberrant karyotypes, including $17 \mathrm{p}$ abnormalities in $\mathrm{pt} \# 1$ and $\mathrm{pt} \# 2$. A TP53 mutation could be detected in pt\#2 and pt\#3. All three patients had symptomatic disease with rapidly increasing hyperleukocytosis and massive splenomegaly. Two of them were treatment-naive and one relapsed after chemoimmunotherapy. All patients were put on ibrutinib $420 \mathrm{mg}$. Despite initial response to treatment, two patients developed progressive disease after $4(\mathrm{pt \# 1)}$ and 9 months (pt\#2) on ibrutinib, whereas $\mathrm{pt} \# 3$ remained in partial remission with persisting leukocytosis, precluding consolidating alloSCT as originally intended. In pt\#1, ibrutinib was replaced by venetoclax, but without response. In order to control rapid lymphoproliferation, rituximab was added to venetoclax. Grade 4 infusion reaction / tumor lysis syndrome (TLS) (fever, tachycardia and hypotonia requiring intravenous vasopressors) followed each rituximab administration despite fractionating rituximab to small doses. However, continuation of rituximab $(100 \mathrm{mg} / \mathrm{d}$ over $10 \mathrm{~d})$ led to complete and durable clearance of hyperleukocytosis (from 250/nl to MRD negativity) despite venetoclax cessation. A similar pattern was observed in pt\#2, who received rituximab while showing rapidly increasing leukocytosis upon ibrutinib. Again, complete clearance of B-PLL cells in the peripheral blood (from 148/nl to MRD negativity) occurred after initial grade 4 TLS despite only modest CD20 expression on tumor cells in this patient. Also, pt\#3 achieved profound B-PLL cell depletion (from 38/nl to a MRD rate of $1.1 \%$ ) upon addition of rituximab to ibrutinib (without TLS in this case). Subsequently, all three patients were able to undergo alloSCT after conditioning with fludarabine and total body irradiation with $8 \mathrm{~Gy}$. Pt\#1 received stem cells from a HLA-ident sibling donor, whereas Pt\#2 and Pt\#3 had unrelated donors (HLA-ident and HLA-matched respectively). With follow-up times of 17 and 11 months post-transplant, pt\#1 and pt\#2 are currently in ongoing MRD-negative remission. Pt\#1 developed an acute graft-versus-host disease (GvHD) of the liver (grade 3), nevertheless the clinical course was well controlled by immunosuppression. In pt\#2 a chronic GvHD of the skin occurred. Pt\#3, who achieved MRD negativity after alloSCT, developed acute and chronic steroidrefractory GvHD of the skin and gastrointestinal tract. Nine months post-transplant, GvHD deteriorated and after further complications the patient died of pneumonia 11 months post-transplant.

Conclusions: Supplementary treatment with rituximab can induce deep remissions in patients with ibrutinibresistant, genetically poor-risk B-PLL, thereby enabling them to undergo successful consolidation with alloSCT. A high risk of life-threatening infusion reactions / TLS associated with the addition of rituximab has to be taken into account.

Disclosure: Nora Liebers: consultant for Takeda

Peter Dreger: consultant for AbbVie und Janssen

All other authors: no conflicts of interest

\section{P090}

Safety and efficacy of the BCR inhibitors after allogeneic stem cell transplantation for CLL

Lucrecia Yáñez ${ }^{1}$, Christelle Ferra ${ }^{2}$, Miguel Alcoceba ${ }^{3}$, Marcos Gonzalez ${ }^{3}$, Rafael Andreu, Ildefonso Espigado 5 , Javier Loscertales ${ }^{6}$, Jose Antonio Garcia Marco ${ }^{7}$, Maria José Terol ${ }^{8}$, Carlos Solano ${ }^{8}$, Francesc Bosch ${ }^{9}$

${ }^{1}$ Hospital Universitario Marqués de Valdecilla, Santander, Spain, ${ }^{2}$ Trias i Pujol, Institut Català d'Oncologia (ICO), Badalona, Spain, ${ }^{3}$ Hospital Universitario de Salamanca, 
Salamanca, Spain, ${ }^{4}$ Hospital Universitari i Politècnic La Fe, Valencia, Spain, ${ }^{5}$ Hospital Universitario Virgen del Rocio, Sevilla, Spain, ${ }^{6}$ Hospital de La Princesa, Madrid, Spain, ${ }^{7}$ Hospital Puerta de Hierro, Madrid, Spain, ${ }^{8}$ Hospital Clinic de Valencia, Valencia, Spain, ${ }^{9}$ Hospital Vall d'Hebron, Barcelona, Spain

Background: There is little experience on the use of the newer targeted therapies in CLL patients relapsed after allogeneic stem cell transplantation (allo-SCT). Against this background, we evaluate the safety and efficacy of the BCR inhibitors (BCRi), ibrutinib and idelalisib, administered after allo-SCT for the purpose of treating the CLL relapse.

Methods: Data from 11 CLL pts who relapsed after SCT, and were subsequently treated with ibrutinib $(n=6)$, idelalisib $(n=3)$ or both $(n=2)$, were retrospectively collected in collaboration with the Spanish group of CLL (GELLC) and the Spanish group of Stem Cell Transplantation (GETH).

Results: Transplant characteristics are summarized in table 1.

Eight patients received the BCRi as the first salvage treatment after SCT relapse, whereas 3 patients had received $\geq 2$ prior lines of treatment. At the time of the onset of the BCRi, 7 patients had Rai 4 stage and 6 patients had a lymph node size $\geq 5 \mathrm{~cm}$. Del17p was present in 4 patients and del11q and complex karyotype in 2 patients, respectively. TP53 gene mutation was detected in 3 patients (all with del17p13). Median time from SCT to BCRi therapy was 53.9 months, being shorter in patients treated with ibrutinib $(\mathrm{n}=8$, median 51 months) than in those treated with idealisib ( $\mathrm{n}=3$, median 111 months).

Median time on ibrutinib and on idelalisib was 7.3 months (4.1-18.8) and 6 months (3.0-23.4), respectively. The best overall response rate (ORR) obtained with ibrutinib was $75 \%$ (1 CR, $4 \mathrm{PR}, 1 \mathrm{PR}+\mathrm{L}$ ) whereas it was of $40 \%$ for patients receiving idelalisib $(1 \mathrm{CR}, 1 \mathrm{PR}+\mathrm{L})$.

Among the 8 patients treated with ibrutinib, 7 (87.5\%) presented an adverse event (AE), being diarrhea $(n=3)$, asthenia $(n=2)$ and infections $(n=5)$ the most frequent. Hypertension was seen in 1 patient and none patient developed atrial fibrillation. Five patients stopped ibrutinib treatment, due to toxicity $(n=4)$ or progression $(n=1)$. After ibrutinib discontinuation, 4 patients were newly treated with idelalisib $(n=2)$ or venetoclax $(n=2)$. All patients treated with idelalisib developed at least one $\mathrm{AE}$, being diarrhea $(n=3)$, pneumonitis $(n=2)$ and neutropenia $(n=2)$ the most common. Four patients discontinued idelalisib because progression $(n=3)$ or toxicity $(n=1)$. Venetoclax was given after idelalisib in 3 patients.

Although acute and/or chronic GVHD before BCRi was documented in $7(63.6 \%)$ and $5(45.5 \%)$ patients, respectively, only one patient (treated with idelalisib) reactivated a mild chronic GVHD. None patient received infusion lymphocyte from donor after BCRi and one patient underwent a second SCT.

With a median follow-up of 14.3 months (5.9-33.9) after the onset of the BCRi treatment, 4 patients had died, all of them due to CLL progression (3 Richter's transformation), whereas 5 patients remained in response ( $3 \mathrm{CR}, 2 \mathrm{PR}$ ). The overall survival probability of the whole series at 12 months was $77.8 \% \pm 13.9 \%$.

Conclusions: In our study, ibrutinib and idelalisib, administered in CLL patients relapsed after SCT did not increase the risk of GVHD reactivation but they show high incidence of adverse events. Nevertheless, BCRi offers a possibility of disease control in these patients with poor prognosis. Further studies are needed to confirm these data.

\begin{tabular}{lr}
\hline Variable & $\mathrm{N}(\%)$ \\
\hline Gender (male) & $7(63.6)$ \\
Age at transplant, years, median (range) & $52(45-60)$ \\
$\geq 2$ prior lines of treatment & $7(63.6)$ \\
BCRi prio SCT & $2(18.2)$ \\
Stem cell source, peripheral blood & $9(81.8)$ \\
Matched related donor & $6(54.5)$ \\
Matched unrelated donor & $3(27.7)$ \\
Reduced intensity conditioning & $10(90.9)$ \\
\hline
\end{tabular}

[[P090 Table] 1. Patients and transplant characteristics, $N=11$.

Disclosure: Dr. Yañez reports travel grants and conferences with Janssen, Roche, Gilead, Merck and Pfizer.

Dr. Ferra reports travel grants and conferences with Gilead, Jansen and Abbvie.

Dr. González reports travel grants and conferences with Gilead, Jansen, Roche and Abbvie. The rest of authors declare no conflicts of interest.

\section{P091}

Long-term survival of CML patients who received autologous HCT before and after the tyrosine kinase inhibitors ERA. Study on behalf of the EBMT CMWP
Mauricette Michallet ${ }^{1}$, Mohamad Sobh ${ }^{1}$, Sheree
Hazelaar $^{2}$, Jane Apperley ${ }^{3}$, Noel Milpied ${ }^{4}$, Hélène
Labussière-Wallet ${ }^{5}$, Didier Blaise ${ }^{6}$, Roberto Foa ${ }^{7}$, Jorge
Sierra $^{8}$, Eric Deconinck ${ }^{9}$, Emanuele Angelucci ${ }^{10}$, Kristina
Carlson $^{11}$, Rocio Parody Porras ${ }^{12}$, Arnold Ganser ${ }^{13}$,
Jaime Sanz ${ }^{14}$, Mohamad Mohty ${ }^{15}$, Rizzoli Vittorio ${ }^{16}$,
Richard E Clark ${ }^{17}$, Amit Patel ${ }^{17}$, Martin Mistrik ${ }^{18}$, Grant McQuaker $^{19}$, Patrice Chevallier ${ }^{20}$, Tsila Zuckerman ${ }^{21}$, Achilles Anagnostopoulos $^{22}$, Franck E Nicolini ${ }^{1}$, Yves Chalandon $^{23}$, Ibrahim Yakoub-Agha ${ }^{24}$ 
${ }^{1}$ Centre Leon Berard, Hematology, Lyon, France, ${ }^{2}$ EBMT data office Leiden, Leiden, Netherlands, ${ }^{3}$ Imperial College London, London, United Kingdom, ${ }^{4} \mathrm{CHU}$ Bordeaux, Pessac, France, ${ }^{5}$ Centre Hospitalier Lyon Sud, Pierre Benite, France, ${ }^{6}$ Institut Paoli-Calmettes, Marseille, France, ${ }^{7}$ University 'La Sapienza', Roma, Italy, ${ }^{8}$ Hospital Santa Creu i Sant Pau, Barcelona, Spain, ${ }^{9}$ Hopital Jean Minjoz, Besancon, France, ${ }^{10}$ Ospedale San Martino, Genova, Italy, ${ }^{11}$ University Hospital Uppsala, Uppsala, Sweden, ${ }^{12}$ ICO Hospital Duran i Reynals, Barcelona, Spain, ${ }^{13}$ Hannover Medical School, Hannover, Germany, ${ }^{14}$ Hospital Universitari i politècnic La Fe, Valencia, Spain, ${ }^{15}$ Hospital Saint Antoine, Paris, France, ${ }^{16}$ Centro Trapianti Unico Di CSE Adulti e Pediatrico A. O Brotzu, Parma, Italy, ${ }^{17}$ Royal Liverpool University Hospital, Liverpool, United Kingdom, ${ }^{18}$ University Hospital Bratislava, Bratislava, Slovakia, ${ }^{19}$ Beatson, West of Scotland Cancer Centre, Glasgow, United Kingdom, ${ }^{20} \mathrm{CHU}$ Nantes, Nantes, France, ${ }^{21}$ Rambam Medical Center, Haifa, Israel, ${ }^{22}$ George Papanicolaou General Hospital, Thessaloniki, Greece, ${ }^{23}$ Hôpitaux Universitaires de Genève and Faculty of Medicine, University of Geneva, Geneva, Switzerland, ${ }^{24} \mathrm{CHRU}$ de Lille, Lille, France

Background: Prior to the introduction of tyrosine kinase inhibitors (TKI), median survival of chronic phase chronic myeloid leukemia (CP-CML) patients was approximately 60 months and the standard treatment with interferon-alpha resulted in complete cytogenetic responses in about $30 \%$ of the patients. Autologous stem cell transplantation (autoSCT) was first attempted for patients in transformation in order to restore a second $\mathrm{CP}$ and was introduced secondarily in $\mathrm{CP}$ to try to prolong the response. The main rational for autografting in $\mathrm{CP}$ resides on the reduction of the tumor burden and the number of leukemic cells at risk of developing blastic transformation. Nevertheless, auto-SCT alone was not able to maintain a long-term remission. Nowadays, TKIs represent the state-of-the-art therapy for CML and the concept of Auto-SCT has only little interest while long-term follow-up and outcome in this setting are currently unknown.

The aim of our study is to evaluate at a first time the longterm outcome of CML patients who received auto-SCT in chronic phase, and to evaluate at a second time in a subgroup analysis, the outcome of those who received TKI after having been auto-transplanted, mainly for disease progression/loss of response and/or to enhance disease response.

Methods: We found a total of 969 patients who received auto-SCT for CP-CML in Europe between years 1989 and 2004, $578(60 \%)$ were males, median age at auto-SCT was 47 years (range: 19-67), the median time between diagnosis and auto-SCT was 19 months, stem cells source was peripheral blood in $84 \%$ of patients, most frequent conditioning regimen was busulfan $4 \mathrm{mg} / \mathrm{Kg} / \mathrm{day} 4$ days + 1 day of melphalan $140 \mathrm{mg} / \mathrm{m}^{2}$ one day prior to the cells reinfusion. Information about receiving TKI post auto-SCT was available only for 103 patients, first TKI was imatinib for $89(86 \%)$ patients, dasatinib for $8(8 \%)$, nilotinib for 6 $(5 \%)$ and ponatinib for one $(1 \%)$ patient.

Results: After a median follow-up of 9.5 years (range: 127) from time of auto-SCT for the whole population, the probability of overall survival (OS) at 10 years was $50 \%$ (95\% CI: 46-53); there was $540(56 \%)$ patients who relapsed after a median time of 16 months after auto-SCT. There was a total of 530 patients transplanted before the TKI era and survived until the availability of TKIs. When we performed a landmark analysis evaluating the outcome of patients who received auto-SCT, survived until the TKI era and received TKI $(\mathrm{N}=103)$, the 10 years OS probability of these patients from TKI treatment was 70\% (95\% CI: 5878). Additional data requests will be sent to centers querying about prognosis, molecular responses, treatment and disease details.

Conclusions: We demonstrate here with these preliminary results that the introduction of TKI has improved survival of CML patients. In addition, patients who received auto-SCT, survived until the TKI era and also received TKI, had encouraging rates of long-term survival. An extensive analysis will be performed when additional data will be available and the study will be updated with more results.

Disclosure: Nothing to declare

\section{P092}

A 35 year single center transplant experience in chronic myeloid leukemia

Antonella Bruzzese ${ }^{1}$, Walter Barberi ${ }^{1}$, Isabella Sperduti', Roberto Latagliata ${ }^{1}$, Massimo Breccia ${ }^{1}$, Luisa Quattrocchi ${ }^{I}$, Ursula La Rocca ${ }^{I}$, Michela Ansuinelli, Sara Pepe ${ }^{I}$, Robin Foà ${ }^{1}$, Anna Paola Iori ${ }^{I}$

${ }^{1}$ Sapienza University, Roma, Italy, ${ }^{2}$ Istituto Nazionale dei Tumori, Roma, Italy

Background: Allogeneic hematopoietic stem cell transplantation (HSCT) has been considered for decades the only curative approach for patients with chronic myeloid leukemia (CML). In the tyrosine kinase inhibitors (TKIs) era, HSCT for CML has been reserved only to patients not achieving a cytogenetic remission or showing progressive disease after multiple TKI treatment lines. However, a progressive improvement in the long-term survival has been obtained in the overall HSCT population. The present study aimed at evaluating whether in CML patients transplanted at 
our Center over a long time period - from 1983 to 2018 - the outcome improved over time.

Methods: 136 consecutive patients who underwent a transplant between 1983 and 1999 were compared to 43 patients who received the transplant between 2000 and 2018. Overall survival (OS), leukemia-free survival (LFS) and graft-leukemia-free survival (GLFS) were estimated using the Kaplan-Meier method and the log-rank test was used to compare risk factors categories.

Results: Of the 179 patients [median age 35 years (range 7-66)], $148(82.7 \%)$ were in $1^{\text {st }}$ or $2^{\text {nd }}$ chronic phase, $25(13.9 \%)$ in accelerated phase and six (3.4\%) in blast crisis. Matched related donors and alternative donors (matched unrelated donors, cord blood or mismatched related donors) were used in 156 and 23 cases, respectively. As stem cell source, bone marrow was used in 142 patients, peripheral blood in 33 and umbilical cord blood in 4 . TBIbased conditioning regimens were used in 89 patients, while in the other 90 cases irradiation-free conditioning regimens were used.

Both in univariate and multivariate analysis, irradiationfree conditioning regimens (HR 1.8; 95\%CI 1.1-3.0, $\mathrm{p}=.0014$ ) and transplants performed in $1^{\text {st }}$ chronic phase (accelerate phase HR 2.1; 95\%CI 1.2-3.8, $\mathrm{p}=.008-2^{\text {nd }}$ chronic phase HR 4.9; 95\%CI 2.3-10.3, $\mathrm{p}=.0001$ - blast crisis HR 2.5; 95\%CI 1.0-6.4, p<.05) were associated with a better OS. Patients transplanted before 2000 had a worse OS (HR 6.5; 95\%CI 2.7-15.5, p < .0001) and DFS (HR 2.2; $95 \%$ CI 1.0-4.8, $\mathrm{p}=.045)$. A trend for a worse GLFS was observed in univariate analysis (HR 1.6; 95\%CI 1.0-2.7, $\mathrm{p}=0.05)$, in the first period of observation.

Conclusions: Our single center experience confirms that higher OS, DFS and GLFS are observed in CML patients allografted in more recent years. Improvement of conditioning regimens, use of TBI-free conditioning regimens and supportive therapy, have presumably contributed to these results, together with the more recent strategy of close monitoring of minimal residual disease, and prompt use of TKI or donor lymphocyte infusion in case of relapse. HSCT is nowadays a safer therapeutic procedure in CML patients that should be considered timely in TKI-resistant patients to avoid progression into a more advanced disease phase.

Disclosure: The authors declare no conflict of interest.

\section{P093}

Reduced-intensity transplantation (RIT) in patients with high-risk or advanced chronic lymphocytic leukemia in last 5 years: Improvement of transplant outcomes single centre experience

\author{
Michal Karas ${ }^{I}$, Katerina Steinerova ${ }^{I}$, Marcela \\ Hrabetova $^{1}$, Daniel Lysak ${ }^{1}$, Jiri Sramek ${ }^{1}$, Alexandra \\ Jungova ${ }^{I}$, Pavel Jindra ${ }^{1}$
}

${ }^{1}$ University Hospital Pilsen, Pilsen, Czech Republic

Background: Allogeneic hematopoietic stem cell transplantation is the only documented curative treatment of chronic lymphocytic leukemia (CLL) however with reported higher transplant-related morbidity and mortality. New targeted drugs dramatically improved the results of highrisk CLL treatment, leading to a decrease in the number of CLL transplanted patients (pts). In recent years outcome of RIT has also improved, but actual published results in CLL patients are limited. To evaluate the current results of RIT for CLL we retrospectively analysed the results of pts with CLL transplanted in our centre during last 5 years and we also tried to compare these results with our historical results and published EBMT results in period 2000-2010 (van Gelder, 2017).

Methods: From 6/2013 to 6/2018 20 consecutive pts with median of age 61 years (range: 36-68 years) with high-risk or advanced CLL (17p-/p53 mutation, chemoresistant CLL including 3 pts resistant to BCRi, relapse $\leq 24$ moths, $\geq 3$ treatment lines) underwent RIT (25\% HLA identical related, $60 \%$ HLA matched unrelated, 25\% HLA mismatched unrelated). HCT-CI $\geq 3$ was in $20 \%$ of pts. Source of stem cells was peripheral blood in $80 \%$ and bone marrow in $20 \%$ of pts. The median of infused CD $34+$ cells was $5,4 \times 10^{\wedge} 6 /$ $\mathrm{kg}$. The conditioning regimen consisted of fludarabine and melphalan (+ATG in unrelated donor). GVHD prophylaxis were cyclosporine and methotrexate.

Results: All pts engrafted. None of 3 pts in CR before RIT progressed at day +30 after RIT and among 17 pts beyond CR before RIT all of them achieved at least PR at day +30 after RIT. 13 pts $(65 \%)$ developed acute GVHD (2 pts grade III-IV) and among 19 evaluable pts 10 (53\%) of them developed chronic GVHD (6 mild, 2 moderate, 2 severe). With median follow-up 50 months (range 6-63 months) 15 pts $(75 \%)$ are alive in CR. 3 pts $(15 \%)$ relapsed or progressed 5, 19 and 21 months after RIT and 2 of them died. Last relapsed patient achieved next CR after ibrutinib. 3 pts $(15 \%)$ died due to NRM. NRM till day +100 after RIT was $5 \%$. The estimated probabilities of 2-years CGRFS, PFS and OS are 55\%, $73 \%$ and $83 \%$.

Conclusions: In spite of relatively small number of evaluated pts and retrospective type of analysis our data show that RIT in pts with high-risk or advanced CLL has achieved promising results (2- and 5- years PFS and OS $73 \%$ and $55 \%$ resp. 83 and $57 \%$ ) in recent years and these results are better than outcomes of our historical patient cohort from period 2010-2012 (2- and 5-years PFS $41 \%$ and $26 \%$ resp. OS $56 \%$ and $35 \%, \mathrm{p}=0.009)$ or EBMT published data of pts transplanted for CLL in period 2000-2010 (2and 5-years PFS $46 \%$ and $35 \%$ resp. OS $62 \%$ and $45 \%$ ). Current results of transplantation should be taken into account in our future decision-making process on 
indications for transplantation in pts with high-risk CLL, of course also in the context of new or updated results of targeted CLL treatment and its complications.

Disclosure: No conflict of interest and funding

\section{P094}

\section{Abstract already published.}

\section{P095}

Childhood chronic myeloid leukemia in Singapore: Is there a role for hematopoietic stem cell transplantation in the TKI era?

Ah Moy Tan ${ }^{1}$, Mya Soe Nwe ${ }^{1}$, Vijayakumari ${ }^{1}$, Met Yoke Chan $^{1}$, Vinod Gunasekaran', Prasad Iyer ${ }^{1}$, Poh Lin Tan ${ }^{2}$

${ }^{1}$ KK Women's and Children's Hospital, Singapore, Singapore, ${ }^{2}$ National University Hospital, Singapore, Singapore

Background: Chronic Myeloid Leukemia (CML) in children is rare. It constitutes $<1 \%$ of all paediatric leukemia's. The availability of Tyrosine Kinase Inhibitors (TKI) from the early 2000's has revolutionized the care as well as quality of life for children with CML. TKI became available for children in Singapore from 2004. The role of upfront HSCT for CML in the TKI-era is controversial.

Methods: Retrospective data and treatment outcomes were collected from the Singapore Childhood Cancer Registry (SCCR). Most children with cancer in Singapore receive therapy at one of the two public paediatric cancer centers (KKH or NUH). A total of thirty two cases were diagnosed with CML and received treatment in either of these centers over a twenty year period (1997-2016).

Results: The age at diagnosis of the thirty two children ranged from 4 to 17 years (median 12.5years). Six patients in the pre-TKI era were treated with an upfront HSCT. The remainder twenty six patients were initially started on a TKI. Of these 12/26 (46\%) had a HSCT at a median period of 22.5 months from diagnosis (range 5-43 months). The reason for HSCT in ten out of the twelve children was due to high risk features i.e. accelerated/blastic phase/ no CCR/no CMR. The remaining two patients had a HSCT due to parent and patient preference for attempt at upfront cure rather than the use of life-long and expensive TKI therapy. Non-compliance to TKI therapy was a major finding in our teenage cohort.

Eleven of the eighteen transplants used a matched sibling donor. Three patients had cord blood as their stem cell source. One patient had a single antigen mismatched related donor and three patients had a mismatched unrelated donor for their HSCT.

All patients except one had myeloablative conditioning with Busulfan and Cyclophosphamide. ATG was added according to physician preference. One patient had Cy/TBI conditioning because of pre-transplant lymphoid blast crisis. Anti GvHD medications included cyclosporine/ methotrexate or tacrolimus and methylprednisolone in the cord transplant patients.

Six of the eighteen (33\%) patients who had a HSCT died. Four died due to treatment related mortality ( 2 infections, 1 Acute GvHD and 1 pulmonary fibrosis). One patient died due to an early relapse and one had a late relapse related mortality. For the pre-TKI era, HSCT related 5 and 10 year OS was $83 \%$ and $67 \%$ respectively. Post-TKI era 5 and 10 year OS was $75 \%$. For the entire cohort, the 10 year OS was $70 \%$.

Conclusions: The post-TKI era transplant outcomes from our two centers is comparable to leading centers in the world. Outcomes for patients with mismatched unrelated donors was poor in our cohort. We recommend a haploidentical related donor transplant or an unrelated cord blood stem cell source for patients when a matched sibling or unrelated donor is not available.

Clinical Trial Registry: NA

Disclosure: We have nothing to disclose.

\section{P096}

Fludarabine, busulfan, and thiotepa may be a promising conditioning regimen for myelofibrosis patients undergoing allogeneic stem cell transplantation

Roni Shouval', Yakov Vegal, Joshua A Fein ${ }^{1}$, Ivetta Danyleskol, Noga Shem Tov ${ }^{1}$, Ronit Yerushalmi ${ }^{l}$, Arnon Nagler $^{\text {, }}$, Avichai Shimoni ${ }^{1}$

${ }^{1}$ Chaim Sheba Medical Center, Tel Aviv University, Hematology and Bone Marrow Transplantation, Ramat Gan, Israel

Background: Allogeneic stem-cell transplantation (SCT) is a curative therapy for patients with myelofibrosis. However, recurrent disease and non-relapse mortality (NRM) are frequent causes of treatment failure. The optimal conditioning regimen for SCT in this disease has not been defined.

Methods: We retrospectively analyzed transplantation outcomes of all adult patients given SCT for myelofibrosis between 2003 and 2018 at a single large academic medical center. Patients $(n=59)$ were treated with several conditioning regimens that were grouped according to conditioning intensity. Myeloablative conditioning (MAC) included busulfan $12.8 \mathrm{mg} / \mathrm{kg}$ and cyclophosphamide $120 \mathrm{mg} / \mathrm{kg}$ (BuCy, $\mathrm{n}=10$ ), fludarabine and busulfan $12.8 \mathrm{mg} / \mathrm{kg}$ (Flu/ $\mathrm{Bu} 4, \mathrm{n}=9$ ) and fludarabine and treosulfan $30-36 \mathrm{~g} / \mathrm{m}^{2}$ (Flu/ Treo, $\mathrm{n}=6$ ). Reduced-intensity conditioning included fludarabine and busulfan $6.4-8.0 \mathrm{mg} / \mathrm{kg}(\mathrm{Flu} / \mathrm{Bu} 2, \mathrm{n}=22)$. More recently we adopted the TBF regimen including 
fludarabine, busulfan $6.4-9.6 \mathrm{mg} / \mathrm{kg}$ and thiotepa $5-10 \mathrm{mg} /$ $\mathrm{m} 2(\mathrm{n}=12)$. All patients were also given anti-thymocyte globulin during conditioning, irrespective of donor source.

Results: The median age was 59 years (interquartile range [IQR] 52-63). The majority of patients had documented splenomegaly $(81 \%)$ and were not previously exposed to ruxolitinib $(71 \%)$. Donor type was an HLA-matched sibling (41\%), $10 / 10(51 \%)$ or $9 / 10(8 \%)$ matched unrelated donor. The DIPPS + score distribution was intermediate-1 (16\%), intermediate-2 $(45 \%)$, or high $(\mathrm{n}=39 \%)$. The median followup was 3.2 years (IQR 1.7-5.5). At 3 years the probability for overall survival (OS), NRM, and relapse were $56 \%$ [95\%CI: 42-70], 32.9\% [19.8-46.1] and 21.0\% [10.4-31.6]), respectively. In a univariate analysis, conditioning type was strongly associated with OS; at 1-year, the probability of OS was highest with TBF (92\% [77-100]) compared with Bu/Cy (80\% [59-100]), FB4 (68\% [51-91\%]), Flu/Treo (33\% [11-100]), and FB2 (68\% [51-91]), $\mathrm{P}=0.001$. TBF benefit was maintained in a multivariable Cox model when compared to MAC (hazard ratio [HR] 0.10 [0.01-0.93], $\mathrm{P}=0.043$ ). Relapse rates at 1-year were lowest with TBF $(8 \%$ [1-54], $\mathrm{P}=0.33)$ with a trend to a lower risk in the multivariable analysis (HR 0.14 [0.01-1.41], $\mathrm{P}=0.096$ ). In a univariate and multivariable analysis, NRM risk was not increased with $\mathrm{TBF}(\mathrm{P}=0.234$ and $\mathrm{P}=0.642$, respectively). Other variables associated with inferior 1-year OS included hypoalbuminemia at SCT $(\mathrm{p}=0.05)$, a high DIPSS + score $(\mathrm{p}=0.01)$, and an HLA mismatched unrelated donors $(\mathrm{p}=0.001)$. Age, the degree of splenomegaly and prior ruxolitinib were not significant prognostic factors.

Conclusions: Outcomes following Allogeneic SCT are strongly dependent on the conditioning regimen. TBF may be an ideal regimen for myelofibrosis patients, providing promising rates of survival and disease control, without excessive NRM. This regimen should be further explored in prospective comparative studies.

Disclosure: Nothing to declare

\section{P097}

Outcome of patients with chronic myeloid leukemia transplanted with HLA-identical sibling donor and partial T-cell depletion. a single center experience with a very long follow-up

\section{Stavroula Masouridi Levrat ${ }^{I}$, Sarah Morin ${ }^{1}$, Federica Giannotti $^{1}$, Carole Dantin ${ }^{1}$, Amandine Pradier ${ }^{1}$, Thien-An Tran $^{I}$, Thomas Longval, Maria Anastasiou ${ }^{I}$, Laura

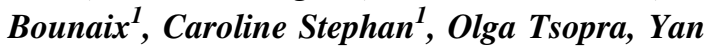 Beauverd $^{1}$, Mitja Nabergoj ${ }^{1}$, Carmen de Ramon Ortiz ${ }^{I}$, Anne-Claire Mamez ${ }^{I}$, Yves Chalandon ${ }^{I}$}

${ }^{1}$ Geneva University Hospitals, Geneva, Switzerland
Background: Since the success of tyrosine kinase inhibitors (TKIs), transplant-related mortality is considered too high to justify allogeneic hematopoietic stem cell transplantation (alloHSCT) as first-line treatment for chronic myeloid leukemia (CML) patients in chronic phase (CP). AlloHSCT is currently considered for patients failing to at least 2 TKIs or with disease in advanced phase. Nevertheless, the optimal timing for transplant referral is still not well defined.

Methods: We performed a retrospective analysis on 23 consecutive patients with $\mathrm{CML}$ in $\mathrm{CP}$ receiving first transplants from an HLA-identical sibling donor with partially T-cell depleted grafts from 1998 to 2016 at our center. Partial T-cell depletion (pTD) consisted of in vitro alemtuzumab incubation of a part of the graft for infusion at day 0 while the rest, containing $100 \times 10^{6} \mathrm{CD} 3+$ cells $/ \mathrm{kg}$ was given as a T-cell add-back at day 1 . Donor lymphocyte infusions (DLIs) were provided, in the absence of GvHD, in case of disease relapse or mixed chimerism. Molecular monitoring was performed by 3-month BCR-ABL1 RTqPCR testing in peripheral blood during at least a 5-year period after HSCT. Thereafter, 3-month testing schedule was maintained where possible, or followed by a 6-month one. Kaplan-Meier method was employed to determine the probability of overall survival (OS) and leukemia free survival (LFS) since alloHSCT.

Results: Median age at HSCT was 36 years (range, 1858). All patients were in first CP but one who was in second CP. Twelve patients were TKI-naïve at HSCT (1998-2001 period), 4 patients had presented suboptimal response or/ and intolerance to imatinib (2002-2004 period), while the last seven patients had presented suboptimal response or/ and intolerance to imatinib, dasatinib and nilotinib (20052016 period). The time interval from diagnosis to transplant was $<12$ months in 11/23 (48\%) patients. $15(65 \%)$ patients had an EBMT risk score of 0-2, while $8(35 \%)$ patients of 34. The conditioning regimen was myeloablative for all but one patients. The stem cell source was PBSC for 22 patients and bone marrow for one. All patients engrafted. 14 patients presented molecular relapse and one patient hematological relapse with a median interval from transplant to relapse of 9 months (range, 5-70). 17 patients received DLIs (15 for relapse and 2 for mixed chimerism), while 3 patients in relapse also received TKI. Without prior administration of DLI, 3(13\%) patients presented grade II aGvHD and 2 patients moderate cGvHD. After DLI, aGvHD occurred in 3 and $\mathrm{cGvHD}$ in 4 patients. One patient died of disease progression 3 years after HSCT and one of myocardial infarction 19 years after HSCT. With a median follow-up of 14.4 years (range 2.3-20.6), 15-year OS and LFS were $95 \%$. At the time of the analysis $21 / 23$ patients were alive and in major molecular response.

Conclusions: These results of excellent long-term survival and no transplant-related mortality suggest that 
pTCD improves the outcome of CP-CML patients transplanted from an identical sibling donor and they can be useful for deciding risk-adapted strategies. We believe that pTCD could allow earlier transplant referral of patients failing TKIs and having an identical sibling donor.

Disclosure: Nothing to declare

\section{P098}

Single tertiary centre experience in allogeneic haematopoietic stem cell transplantation (allo-HSCT) for primary and secondary myelofibrosis (MF)

Anna Tsoulkani, Sarah Louise Wharin', Mamta Garg', Katherine Hodgson ${ }^{1}$, Ann E. Hunter ${ }^{1}$, Alexander Murray Martin $^{I}$

${ }^{1}$ University Hospitals of Leicester, Leicester, United Kingdom, ${ }^{2}$ University Hospitals of Leicester, Haematology, Leicester, United Kingdom

Background: Myelofibrosis (MF) is a category of myeloproliferative neoplasms (MPN) according to the WHO 2016 classification which is divided into primary MF (PMF) and secondary MF incorporating post-essential thrombocythaemia (post-ET) and post-polycythaemia vera (post-PV) MF. Secondary MF occurs in up to $10 \%$ and $20 \%$ of patients diagnosed with ET and PV respectively. Overall survival in overt PMF across all risk groups is variable at 3-7 years. The only curative option for fit patients is allo-HSCT. Novel therapy is emerging but current recommendation is that eligible patients with life expectancy less than 5 years should be considered for allografting.

Methods: We retrospectively looked at the clinical features and outcomes of all allo-HSCT for MF performed in our centre since 2010.

Results: 12 patients (10 male, 2 female) aged between 29-68 years old (median age 60) with intermediate-2 or high-risk MF as per the International Prognostic Scoring System (IPSS) or Dynamic IPPS (DIPPS) were transplanted in our centre since 2010. 6 of them (50\%) were diagnosed with PMF and the remaining 50\% with secondary MF; 4 post-ET and 2 post-PV MF. 2/12 of our patient group received a sibling allograft and 10/12 a matched unrelated donor allograft (70\% received a 10/10 Human Leukocyte Antigen (HLA)-matched transplant, 20\% a 9/10 HLAmatched and $10 \%$ a 8/10 HLA-matched graft). All patients received a reduced intensity conditioning (RIC); 3/12 patients with Fludarabine/ Melphalan/ Campath (FMC), 8/ 12 Fludarabine/ Busulphan/ ATG (FBATG) and 1 Fludarabine/ Ara-C/ Campath (FLAG/ Campath); all received peripheral blood as source of HSC. Engraftment occurred between day 13-48, with a median of $\mathrm{D}+17$. One late graft rejection occurred. All patients were alive at $\mathrm{D}+100.9$ patients are currently alive; Overall survival (OS) is $75 \%$. Transplant related mortality (TRM) was $16.6 \%$ at 1 year, $25 \%$ at 3 years. 1 patient died of graft versus host disease (GvHD) and 2 patients of septicaemia leading to multiorgan failure. Acute GvHD grade II skin occurred in 5 patients, grade III and above in 2 patients. 5 patients have limited chronic GvHD. 2/12 patients received Donor Lymphocyte Infusion (DLI) for mixed chimerism (one of which had 2nd graft failure). Out of these 2 patients 1 developed acute grade 4 GvHD and died.

Response rate: $6 / 9$ alive patients i.e $66.6 \%$ exhibit no fibrosis in trephine biopsies, 1/9 alive patients had residual fibrosis but $100 \%$ donor chimerism, 1/9 alive patients had residual fibrosis with mixed donor chimerism, other patient non-assessable.

Conclusions: Allo-HSCT remains the only potentially curable option for myelofibrosis. In our centre which serves 1.1 million population, with 12 new cases per year, 12 patients were transplanted since 2010. Our data suggest that close collaboration between MPN-treating haematologists and transplant physicians is required so that all suitable patients have a transplant assessment early in their disease course. Novel molecular prognostic systems are likely to identify those best placed to benefit in future but this series currently supports allo-HSCT survival and cure.

Clinical Trial Registry: N/A

Disclosure: Nothing to declare

\section{P099}

Hematopoietic cell transplantation in myelofibrosis patients: A single centre experience

\section{Annalisa Natale ${ }^{I}$, Stella Santarone ${ }^{I}$, Gabriele Papalinetti $^{1}$, Paola Olioso ${ }^{1}$, Mauro Di Ianni', Paolo Di Bartolomeo $^{1}$}

${ }^{1}$ Ospedale Civile Dipartimento di Ematologia, Medicina Trasfusionale e Biotecnologie, Pescara, Italy

Background: Allogeneic hematopoietic cell transplantation (HCT) is the only potentially curative therapy available for myelofibrosis (MF) patients. The aim of this study is to evaluate the outcome of MF patients after HCT performed in our Centre.

Methods: We retrospectively analyzed 21 consecutive MF patients transplanted in our Center between November 1986 and January 2018.

Results: Median age at time of HCT was 53 years (range, 33-67). 15 patients were male. 13 patients had primary MF, 6 secondary MF (6 post Essential Thrombocytemia, 2 post Polycythemia Vera). Median time from diagnosis to HCT was 413 days (range, 105-4624). Dynamic international prognostic scoring system (DIPSS) score at the time of HCT was intermediate- 2 or high risk in 20 patients $(95 \%)$, 
intermedate-1 in 1 patient. Molecular evaluation was available in 14 out of 21: JAK2 V617F mutation was detectable in 8 patients, MPL-W515K in 1 patient, CARL in 1 patient. 4 patients were "triple negative" for driver mutations. Cytogenetics information was available for 6 out of 21; among which 3 patients had complex karyotype, 1 trisomy 8 and 1 trisomy 9.3 patients underwent splenectomy before HCT. Ruxolitinib was administered in 4 patients before HCT. $10(48 \%)$ patients received stem cells from an HLA identical sibling, 9 (42\%) from a matched unrelated donor and 2 (10\%) from an haploidentical sibling. Graft source was bone marrow in 7 patients (34\%) and peripheral blood in $14(66 \%)$. Conditioning was myeloablative in 16 patients $(76 \%)$, reduced intensity in 5 (24\%). All patients engrafted. Acute graft versus host disease was absent in 12 patients (57\%), grade I-II in 7 (34\%), grade III-IV in $2(9 \%)$. In 18 evaluable patients chronic graft versus host disease was limited in $3(17 \%)$, extensive in $4(22 \%)$ and absent in $11(61 \%)$. Transplantrelated mortality at 180 days was $24 \%$. Main causes of death were: acute GvHD in 2 patients, chronic GvHD in 1, pancreatitis in 1, pulmonary aspergillosis in 1. Relapse occurred in 7 patients and was the main cause of death in 4 of them. Notably, 2 patients experienced late relapse after 6.6 and 17.6 years after HCT. Both of them are living while receiving Ruxolitinib therapy. After a median follow up of 581 days (range, 38-11660), 10 out of 21 patients are alive. 7 of them $(33 \%)$ are disease-free and 3 are living. The Kaplan-Meyer overall survival and disease-free survival at 10 years was $40 \%$ and $35 \%$, respectively.

Conclusions: Our experience confirms that HCT is a valid option to achieve cure in one third of MF patients. Two patients experienced very late ( $>5$ years) recurrence of MF. The rarity of this condition limits the amount of data and cases available for evaluation and study. Life-long follow-up of all MF transplanted patients is warranted to better understand this rare event.

Disclosure: Nothing to declare

\section{P100}

\section{Allogeneic stem cell transplantation results of patients with myelofibrosis:Single center experience}

\section{Elif Birtas Atesoglu ${ }^{1}$, Meral Sengezer ${ }^{1}$, Imran Dora ${ }^{1}$, Cigdem Eren ${ }^{1}$, Suat Celik', Demet Cekdemir' ${ }^{1}$, Zafer Gulbas ${ }^{1}$}

${ }^{1}$ Anadolu Medical Center, Hematology, Kocaeli, Turkey

Background: With existing treatment strategies high risk myelofibrosis patients have short survival rates. In fact allogeneic stem cell transplantation is the only treatment modality which has a curative potential. Therefore, we decided to analyze the results of patients who had undergone allogeneic stem cell transplantation with diagnosis of myelofibrosis in our center.

Methods: We collected and analyzed the data of myelofibrosis patients who had undergone allogeneic stem cell transplantation with a conditioning regimen consisting of Fludarabine-Busulfan-ATG between March 2015 and May 2018.

\begin{tabular}{ll}
$\mathrm{n}$ & 13 \\
\hline Age, median (range) & $59(42-$ \\
& $65)$ \\
F/M & $6 / 7$ \\
Donor type of Allogeneic Stem Cell Transplantation HLA- & 751 \\
matched sibling Matched Unrelated Haploidentical & \\
Neutrophil engraftment time, day;median(range) & $21(14-$ \\
& $26)$ \\
Platelet engraftment time, day;median(range) & $21(14-$ \\
& $37)$ \\
Bacterial infection & 7 \\
& $(53,8 \%)$ \\
100 day mortality & 2 \\
1 year OS & $(15,4 \%)$ \\
\hline
\end{tabular}

[[P100 Table] 1. Table1: Characteristics of the patients]

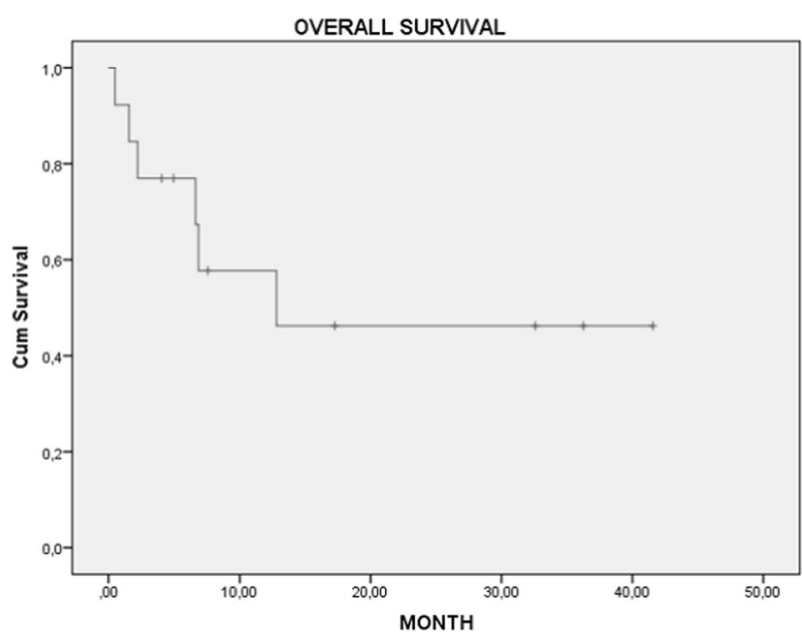

[[P100 Image] 1. Figure 1:Overall Survival]

Results: There were 13 patients. The characteristics of the patients are summarized in Table 1 . While, 7 out of 13 patients were transplanted from HLA-matched siblings, in 5 of the patients the donors were matched unrelated and 1 patient were transplanted from a haploidentical donor. 100 day mortality rate was $15,4 \%$ and 1 year Overall Survival was $57 \%$ (Table 1 and Figure 1). 
Conclusions: Allogeneic stem cell transplantation with a conditioning regimen of Fludarabine-Busulfan-ATG has an acceptable toxicity profile and survival rate in myelofibrosis patients and should be considered in high risk myelofibrosis patients.

Disclosure: Nothing to declare

\section{P101}

Allogeneic haematopoietic stem cell transplantation in patient with primary myelofibrosis and splanchnic vein thrombosis

Maria Barabanshikova ${ }^{1}$, Elena Morozova', Julia Vlasova', Ivan Moiseev ${ }^{1}$, Olga Pirogova ${ }^{1}$, Vadim Baikov ${ }^{1}$, Alexander Shvetsov ${ }^{1}$, Alexander Smirnov', Elena Darskaya $^{1}$, Boris Afanasyev ${ }^{1}$

${ }^{1}$ Pavlov First Saint Petersburg State Medical University, Saint Petersburg, Russian Federation

Background: Primary myelofibrosis (PMF) is BCR-ABLnegative myeloproliferative neoplasm with progressive clinical course and usually poor prognosis. Allogeneic stem cell transplantation (alloHSCT) is currently the only treatment option with curative potential in patients with PMF. Thrombotic episodes are frequent events in MF, especially thrombosis of splanchnic veins. Usually it leads to portal hypertension, esophageal variceal bleeding (EVB) and may complicate alloHSCT. To our knowledge there are no data about the feasibility of alloHSCT in myelofibrosis complicated by splanchnic vein thrombosis. Here we report the outcome of alloHSCT in PMF patient with subacute BuddChiari syndrome and portal vein thrombosis.

Methods: A 37-years old female was diagnosed with $J A K 2 V 617 F$-positive PMF, 46XX, IPSS low risk, DIPSSplus intermediate -2 risk, subacute Budd-Chiari syndrome and portal vein thrombosis four years before alloHSCT. To reduce the splenomegaly and constitutional symptoms we performed pre-transplant ruxolitinib therapy $30 \mathrm{mg}$ daily. After three months of therapy the patient achieved clinical improvement (ELN criteria). Contrast-enhanced computer tomography and magnetic resonance imaging showed enlarged intrahepatic collateral vessels and signs of portal vein thrombosis with cavernous transformation and multiple dilated collateral veins. Gastroscopy documented enlarged esophageal veins. Allogeneic stem cell transplantation was performed from 10/10 -HLA matched unrelated donor with peripheral stem cells $\left(6.1\right.$ x $10^{9}$ CD34+ cells $\left./ \mathrm{kg}\right)$. Conditioning regimen consisted of fludarabine $(180 \mathrm{mg} /$ $\mathrm{m}^{2}$ ), busulfan (10 mg/kg p.o.). Post-transplant cyclophosphamide was administered at $100 \mathrm{mg} / \mathrm{kg}$ at day $+3,+4$, and ruxolitinib $15 \mathrm{mg}$ was used from $\mathrm{D}+5$ till $\mathrm{D}+100$ as graft versus host disease prophylaxis.
Results: Starting $\mathrm{D}+1$ the patient experienced eight episodes of EBV some of them with severe blood loss. To treat the bleeding episodes Blackmore tube was placed six times with temporary effect. To place Blackmore tube the patient was two times intubated and required mechanical ventilation. At D+18 leukocyte and neutrophil engraftment, full donor chimerism and molecular remission were achieved. Platelet engraftment was documented only at D +42 and poor graft function was present due to cytomegalovirus reactivation $(\mathrm{D}+41)$ and parvovirus $\mathrm{B} 19$ reactivation $(\mathrm{D}+62)$. EVB was stopped at $\mathrm{D}+95$ only after two esophageal veins ligations, and two procedures of gastric veins sclerotherapy. Soon $(D+111)$ the patient achieved complete platelet recovery (more than $50 \times 10^{9} / 1$ ) and became red blood cells transfusion independent.

At day +180 complete remission was confirmed by splenomegaly resolution, regression of bone marrow fibrosis, full donor chimerism, JAK2V617F-negative molecular status. CBC showed $\mathrm{Hb} 130 \mathrm{~g} / \mathrm{l}$, platelets $73 \times 10^{9} / 1$, leucocytes $3,8 \times 10^{9} / 1$. Ultrasound examination after transplant documented portal vein thrombosis recanalization. At day +180 she developed mild $(\mathrm{NIH})$ chronic graft versus host disease with eyes and mouth involvement, which was managed with topical steroids. At D+958 after transplant the patient is alive in complete remission and has no recurrent bleedings.

Conclusions: Splanchnic vein thrombosis can significantly complicate the course of alloHSCT in PMF. Easy access to surgical, intensive care unit and endoscopic teams is required to make alloHSCT more feasible in this group of patients.

Disclosure: authors declare no conflict of interest

Conditioning regimen

\section{P102}

Treosulfan-based conditioning regimens for allogeneic haematopoietic stem cell transplantation in paediatric non-malignant diseases

\section{Samar Elbahy ${ }^{1,2}$, Denise Bonney ${ }^{1}$, Helen Campbell,} Tasneem Khaled ${ }^{1}$, David Deambrosis ${ }^{1}$, Robert Wynn ${ }^{1}$

${ }^{1}$ Royal Manchester Children's Hospital, Manchester, United Kingdom, ${ }^{2}$ Benha University, Benha, Egypt

Background: Haematopoietic stem cell transplantation (HSCT) represents the definitive treatment for many nonmalignant diseases. Treosulfan is considered a safe and effective conditioning drug compared to other conventional myeloablative conditioning(MAC) regimens, especially in patients with comorbidities.

Methods: We present the results of 94 transplants in 92 Patients(62\% males and 38\% females) transplanted for 
various non-malignant conditions at a median age of four years(Range, 1 month-18 years).

All patients received Treosulfan-based MAC regimens, Treosulfan(Total dose, $36-42 \mathrm{gms} / \mathrm{m} 2$ ) was given in combination with different conditioning drugs. The most commonly used regimen was Treosulfan, Fludarabine $(150 \mathrm{mgs} / \mathrm{m} 2)$ and Thiotepa $(10 \mathrm{mgs} / \mathrm{kg})$ referred to as FTT that was used in $59 \%(\mathrm{n}=55)$. Serotherapy was given in $93 \%$ of patients $(\mathrm{n}=87)$, as either Alemtuzumab or Antithymocyte globulin in $82 \%(\mathrm{n}=77)$ and $11 \%(\mathrm{n}=10)$, respectively. post-transplant Graft-versus-Host disease (GVHD) prophylaxis was given in all patients, based mostly on ciclosporin.

46 patients $(49 \%)$ received the transplant from identicalrelated donors, 46 patients(49\%) received the transplant from matched-unrelated donors, and two patients $(2 \%)$ had haploidentical transplants. $90 \%$ of the patients $(n=85)$ were fully HLA-matched. All stem cell sources were used as bone marrow in $59 \%(\mathrm{n}=55)$, peripheral blood stem cells in $33 \%(\mathrm{n}=31)$, and umbilical cord blood in $8 \%(\mathrm{n}=8)$.

This Treosulfan-based conditioning was given as the $1^{\text {st }}$ transplant in $92 \%(\mathrm{n}=85)$, and as the $2^{\text {nd }}$ transplant after the failure of a first procedure in $8 \%(\mathrm{n}=9)$. Two patients received Treosulfan-based conditioned transplant twice.

Results: Neutrophil engraftment and platelet engraftment occurred at a median of 13 days and 18 days respectively. Chimerism was full donor in $55 \%(\mathrm{n}=52)$, high donor in $18 \%(\mathrm{n}=17)$, and mixed donor in $9 \%(\mathrm{n}=8)$.

GVHD developed in $43 \%$ of patients $(n=40)$, with acute GVHD grade I/II and grade III/IV developed in $29 \%(\mathrm{n}=27)$ and $2 \%(n=2)$, respectively. Chronic GVHD grade I/II and grade III/IV developed in $13 \%(\mathrm{n}=12)$ and $2 \%(\mathrm{n}=2)$, respectively.

All chronic GVHD were mild, limited, non-extensive, and resolved completely. None of our patients had persistent GVHD necessitating long-term systemic immunosuppression.

Mild VOD occurred in $13 \%(\mathrm{n}=12)$, and severe VOD occurred in $2 \%(\mathrm{n}=2)$. One of them died but was believed to be related to the underlying disease (Wolman Syndrome).

Viral reactivation occurred in $55 \%$ of patients $(n=52)$, with CMV, EBV, and Adenovirus reactivation was found in $34 \%, 21 \%$, and $14 \%$, respectively. Five patients had invasive adenoviraemia that contributed to death in two of them.

Primary graft failure happened in two patients(2\%) due to adenoviraemia. Seven patients(7\%) had secondary graft failure with autologous reconstitution. Graft failure was significantly lower $(\mathrm{P} 0.045)$ in the FTT group than other conditioning groups.

At a median follow-up of 35 months (Range, two-174 months), eleven patients(11.9\%) died, with Overall survival of $88.1 \%$, and event-free survival of $80.9 \%$. Five patients died due to complications related to their original disease, while six patients died due to transplant-related causes (transplant-related mortality $6.5 \%$ ).

Immune reconstitution in alive patients was achieved at a median of eight months. This time was significantly longer (P0.034) in FTT group.

Conclusions: This study demonstrates that Treosulfan is a safe and effective conditioning drug that can achieve engraftment, with low rates of graft failure, transplantrelated mortality and morbidity, even if it is used twice in the same patient.

Disclosure: Nothing to declare

\section{P103}

Abstract already published.

P104

Abstract already published.

P105

Abstract already published.

P106

Busulfan, etoposide, cytarabine and melphalan (BUEAM) as a conditioning regimen for autologous stem cell transplantation in patients with non-hodgkin lymphoma (NHL)

Jae-Cheol Jo ${ }^{1}$, Jin-Seok Kim ${ }^{2}$, Je-Hwan Lee ${ }^{3}$, Jung-Hee $\mathrm{Lee}^{3}$, Seong Nam Im ${ }^{4}$, Won-Sik Lee ${ }^{5}$, Sang-Min Lee ${ }^{5}$, Sung-Soo Yoon ${ }^{6}$, In-Ho Kim ${ }^{6}$, Yun Suk Choi ${ }^{1}$, Seong $\mathrm{Hwa} \mathrm{Bae}{ }^{7}$

${ }^{1}$ Ulsan University Hospital, University of Ulsan College of Medicine, Hematology and Oncology, Ulsan, Korea, Republic of, ${ }^{2}$ Severance Hospital, Yeonsei University College of Medicine2, Seoul, Korea, Republic of, ${ }^{3}$ Asan Medical Center, University of Ulsan College of Medicine, Seoul, Korea, Republic of, ${ }^{4}$ Haeundae Paik Hospital, Inje University College of Medicine, Busan, Korea, Republic of, ${ }^{5}$ Busan Paik Hospital, Inje University College of Medicine, Busan, Korea, Republic of, ${ }^{6}$ Seoul National University Hospital, Seoul National University College of Medicine, Seoul, Korea, Republic of, ${ }^{7}$ Daegu Catholic University Medical Center, Daegu Catholic University College of Medicine, Daegu, Korea, Republic of

Background: High-dose chemotherapy (HDC) followed by autologous stem cell transplantation (ASCT) is the treatment of choice for the patients with relapsed or high risk NHL. Although the high-dose conditioning regimens commonly used in patients with non-Hodgkin lymphoma (NHL) are BEAM (BCNU, etoposide, cytarabine, and melphalan), 
BEAC (BCNU, etoposide, cytarabine, and cyclophosphamide), survival of patients with NHL received above high-dose chemotherapy followed by ASCT was still unsatisfactory.

Methods: We prospectively evaluated the efficacy and toxicity of busulfan, etoposide, cytarabine and melphalan (BuEAM) including iv busulfan instead of BCNU of standard BEAM as a conditioning for ASCT in patients with NHL. The high-dose chemotherapy consisted of $\mathrm{Bu}$ (3.2 $\mathrm{mg} / \mathrm{kg}$ i.v. q.d. from day -6 to day -5$)$, E $\left(200 \mathrm{mg} / \mathrm{m}^{2}\right.$ i.v. b.i.d. on day -4 and day -3$)$ A $\left(1 \mathrm{~g} / \mathrm{m}^{2}\right.$ i.v. q.d. on day -4 and day -3$)$ and $M\left(140 \mathrm{mg} / \mathrm{m}^{2}\right.$ i.v. q.d. on day -2) at 7 centers in Korea.

Results: Two hundred five patients were enrolled onto the study. Main subgroup was diffuse large $B$ cell lymphoma $(\mathrm{n}=104,50.7 \%)$, T cell lymphomas $(\mathrm{n}=59$, $29.8 \%)$, and NK/T cell lymphoma $(n=22,10.7 \%)$. Upfront ASCT was performed in 160 patients $(78.0 \%)$, and salvage ASCT in 45 patients $(22.0 \%)$. The disease status of the patients before HDT/ASCT consisted of 133 patients $(64.8 \%)$ with complete response and 72 patients $(35.2 \%)$ with partial response. Treatment related toxicities included nausea in 149 patients (72.7\%), diarrhea in 127 patients $(62.0 \%)$, anorexia in 107 patients $(52.2 \%)$ and stomatitis in 97 patients $(47.3 \%)$, which were grade I or II in the majority of cases. The common grade III toxicities were stomatitis $(6.9 \%)$, diarrhea $(5.9 \%)$, and anorexia $(5.4 \%)$. There were no VOD, and transplant-related mortality occurred in 4 patients $(1.95 \%)$, due to infection. One hundred fifty three patients $(74.6 \%)$ achieved a complete response and 13 patients $(6.3 \%)$ after ASCT, while 28 patients $(13.7 \%)$ showed progressive disease. At a median follow-up duration of 38.6 months, the estimated 3-year overall survival and progression free survival for all patients was $74.5 \%$ and $56.6 \%$, respectively.

Conclusions: The conditioning regimen of BuEAM for ASCT was well tolerated and seemed to be effective in patients with relapsed or high risk NHL.

Disclosure: None of declare

\section{P107}

Post-transplant cyclophosphamide combined with ATG as GVHD prophylaxis for allogeneic HCT in high risk AML and MDS

\section{Wael Alanazi ${ }^{1}$, Jeffrey H Lipton ${ }^{1}$, Dennis D Kim ${ }^{1}$, Auro Viswabandya $^{1}$, Santhosh Thyagu ${ }^{1}$, Rajat Kumar ${ }^{1}$, Wilson Lam $^{I}$, Arjun D. Law ${ }^{1}$, Fotios V Michelis ${ }^{1}$}

${ }^{1}$ Hans Messner Allogeneic Transplant Program, Princess Margaret Cancer Centre, University Health Network, University of Toronto, Toronto, Canada
Background: Allogeneic hematopoietic cell transplantation (HCT) is potentially curative for high risk acute myeloid leukemia (AML) and myelodysplastic syndrome (MDS), however both GvHD and disease relapse remain major challenges. We recently introduced a combination of posttransplant cyclophosphamide (PTCy) and ATG $(4.5 \mathrm{mg} / \mathrm{kg})$ as graft-versus-host disease (GvHD) prophylaxis. The purpose of our study was to compare outcomes between PTCy/ ATG and other GvHD prophylaxis regimens for high risk AML and MDS.

Methods: We retrospectively investigated outcomes of 159 patients that underwent allogeneic HCT between January 2014 and July 2017 for high risk AML $(n=120$, $75 \%)$ and $\operatorname{MDS}(\mathrm{n}=39,25 \%)$. GvHD prophylaxis regimens were compared for overall survival (OS), cumulative incidence of relapse (CIR) and non-relapse mortality (NRM) in univariate and multivariable analysis. High risk AML was defined as secondary AML, therapy related AML, high risk cytogenetics (ELN criteria) in CR1, good/ intermediate cytogenetic risk AML in CR2 and primary induction failure; high risk MDS was defined as high/very high risk WPSS score.

Results: Median age of patients was 56 years (range 2273 years). Donors were matched related in $52(33 \%)$ patients, matched unrelated in $89(56 \%)$ patients and haploidentical in $18(11 \%)$ patients. Graft source was peripheral blood stem cells in 158 patients (99\%). Myeloablative conditioning was used in 54 patients (34\%), reduced intensity regimens in $105(66 \%)$ patients. PTCy combined with ATG was used in 69 (43\%) patients, other GvHD prophylaxis regimens were used in $90(57 \%)$ patients. Both donor and recipient were CMV negative in $18(11 \%)$ patients.

Median follow-up of survivors was 29 months (range 1456 months). Univariate analysis demonstrated OS of the entire cohort at 2 years was $49 \%$ (95\% CI 41-57\%), CIR at 2 years was $22 \%$ (95\%CI 16-29\%) and NRM at 2 years was $32 \%$ (95\%CI 25-39\%). Concerning GvHD prophylaxis regimen, 2-year OS for PTCy/ATG versus others was $46 \%$ (95\%CI 33-58\%) versus 51\% (95\%CI 40-61\%) $(\mathrm{p}=0.87$, Figure), 2-year CIR for PTCy/ATG versus other was $31 \%$ (95\%CI $20-43 \%)$ versus $16 \%(95 \%$ CI $9-24 \%)(\mathrm{p}=0.02)$ and 2-year NRM for PTCy versus other was $28 \%$ (95\%CI 18$39 \%$ ) versus $34 \%(95 \%$ CI $25-44 \%)(p=0.35)$. Grade II-IV acute GvHD was seen in $23 \%$ of PTCy/ATG patients versus $59 \%$ using other regimens $(\mathrm{p}<0.0001)$. Chronic GvHD was observed in $20 \%$ of PTCy/ATG patients versus $42 \%$ using other regimens $(\mathrm{p}=0.004)$.

Multivariable analysis for OS confirmed that the GvHD prophylaxis regimen has no influence $(p=0.19)$, while the predominant predictor of survival was age at HCT (HR 1.03, 95\%CI 1.01-1.05, $\mathrm{p}=0.01)$. For CIR, the PTCy/ATG combination had no influence compared to other GvHD 
prophylaxis regimens $(\mathrm{p}=0.6)$, while RIC conditioning was the predominant predictor of relapse (HR 3.05 for RIC, 95\% CI $1.26-7.37, \mathrm{p}=0.01$ ). For NRM, the ATG with PTCy combination demonstrated no significant difference $(\mathrm{p}=0.12)$, while age at HCT was the predominant predictor ( $\mathrm{HR}=1.04,95 \% \mathrm{CI} 1.01-1.07, \mathrm{p}=0.02$ ).

Conclusions: The PTCy/ATG combination for GvHD prophylaxis has demonstrated on multivariable analysis similar OS, CIR and NRM with other previously used regimens at our center. A decrease in ATG dose may potentially decrease the relapse rate while retaining the advantage of decreased GvHD.

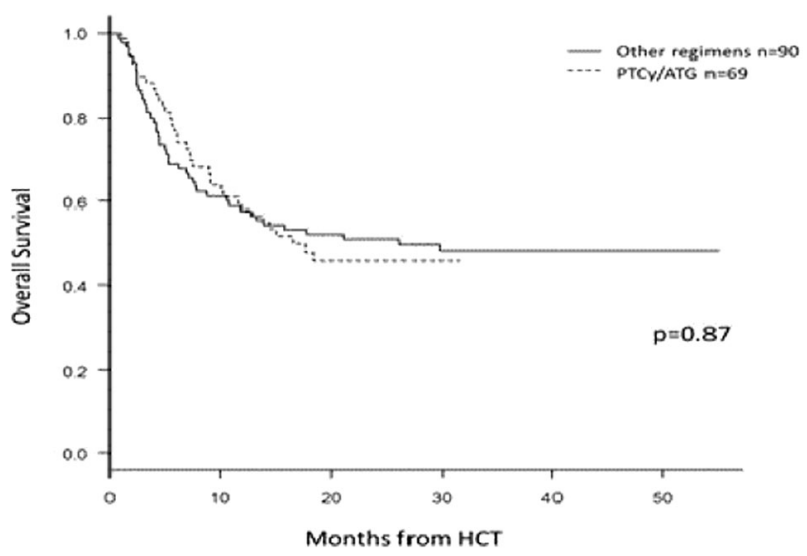

[[P107 Image] 1. Overall survival]

Disclosure: No disclosures

\section{P108}

Frequency and impact of early mixed chimerism after myloablative conditioning regimen with fludarabine and busulfan (FB4) and ATG for allogeneic transplantation in myeloid malignancies

Anna Grassi ${ }^{\text {, Chiara Pavoni }}{ }^{1}$, Caterina Micò ${ }^{1}$, Alessandra Algarotti ${ }^{1}$, Maria Chiara Finazzi ${ }^{1}$, Marta Bellini $^{1}$, Federico Lussana ${ }^{1}$, Federica Delaini, Elena Oldani $^{1}$, Anna Salvi ${ }^{1}$, Orietta Spinelli, Alessandro Rambaldi $^{1}$

\section{${ }^{1}$ ASST Papa Giovanni XXIII, Italy}

Background: The combination of fludarabine with myeloablative doses of busulfan (FB4) represents a standard of care conditioning regimen before allogeneic transplantation in patients with myeloid malignancies (Giralt, S.: The Lancet Oncology 2015). FB4 has potent antileukemic activity and is associated with low transplantrelated mortality and acute GvHD. However, early after transplantation (days 30-90), a proportion of patients may not convert to a full donor haemopoietic chimerism, particularly if anti-T lymphocyte globulin (ATG) is used as GvHD prophylaxis (Rambaldi A, et al.: The Lancet Oncology 2015)

Methods: We retrospective analyzed 104 patients who underwent an allogeneic stem cell transplantation after FB4 conditioning regimen at our hospital, from November 2007 to August 2018. The median age was 51 years (range 22-67) and diagnoses were AML 76\%, MDS 18\% CML 5\% MFI 1\%). The disease status at transplantation was: CR1 in 59\%, CR2 in 5\% and active disease in $36 \%$ of patients. The stem cell source was represented by PBSC in more than $95 \%$ of cases and anti-T lymphocyte globulin (ATG) was part of the conditioning regimen in more than $95 \%$ of cases at a dose of $5 \mathrm{mg} / \mathrm{Kg}$. The donor was a HLA identical sibling (26\%), a matched unrelated $(65 \%)$ or mismatched (one allele or one antigen mismatched) unrelated, 9\%. Hematopoietic chimerism was molecularly evaluated by Variable Number of Tandem Repeats (VNTR) on bone marrow (BM) mononuclear cells or peripheral blood (PB) T lymphocytes, purified by immunomagnetic positive selection (Miltenyi, Biotec). The analysis was performed at day 30, 60, 90, 180 and 360 after transplantation

Results: After 30, 60 and 90 days from transplantation, the proportion of patients with a full BM chimerism was $94 \%, 87 \%$ and $83 \%$, respectively. At the same time points, the PB T cell chimerism was $47 \%, 65 \%$ and $69 \%$. Before day 100, 10 patients required the infusion of DLI to treat a pending or overt hematologic relapse and 12 patients to convert the lymphoid chimerism from mixed (median 44\%, range 0-76), to complete (successfully in 7 cases). After day 100, 13 additional patients required DLI to treat disease relapse or progression and 8 patients to improve the chimeric status or the immune reconstitution. At 5 years, the Overall Survival is $64 \%$, with a relapse and non-relapse mortality of $21 \%$ and $11 \%$, respectively (Figure 1). By uni and multivariable analysis, AML diagnosis and a mixed BM chimerism before day 100 were associated with Relapse and Overall Survival while age $>50$ was the only factor significantly associated with NRM. A mixed PB T-lymphoid chimerism before day 100 does not adversely impact on non-relapse mortality, cumulative incidence of relapse, leukemia-free and overall survival.

Conclusions: After FB4 and ATG, a progressive increase of PB lymphoid donor chimerism develops gradually after transplantation, in most of cases without the need of DLI. Early mixed lymphoid chimerism does not compromise the main long-term clinical outcomes and may at least partially explain the low non-relapse mortality. An incomplete BM chimerism within the first 3 months strongly correlates with early disease progression or relapse. 


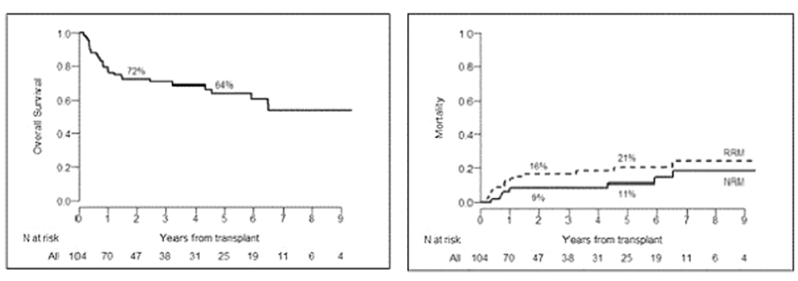

[[P108 Image] 1. Fig.1]

Disclosure: Nothing to declare

\section{P109}

Busulfan/sulfolane metabolic ratio on day three of conditioning may predict the event-free survival in children receiving busulfan based conditioning prior to allogeneic hematopoietic stem-cell transplantation

Chakradhara Rao S Uppugunduri ${ }^{1,2}$, Aziz Rezgui ${ }^{3}$, Patricia Huezo-Diaz Curtis ${ }^{1,2}$, Tiago Nava ${ }^{1,2}$, Simona Mlakar $^{1,2}$, Yves Theoret ${ }^{3}$, Henrique Bittencourt ${ }^{3}$, Maja Krajinovic $^{3}$, Marc Ansari, ${ }^{1,2}$

${ }^{1}$ University Hospitals of Geneva, Geneva, Switzerland, ${ }^{2}$ CANSEARCH Research Laboratory, Faculty of Medicine, University of Geneva, Geneva, Switzerland, ${ }^{3}$ CharlesBruneau Cancer Center, CHU Sainte-Justine Research Center, Montreal, Canada,

Background: Busulfan $(\mathrm{Bu})$ is widely used as a component of myeloablative conditioning regimen before hematopoietic stem cell transplantation (HSCT) in children. Bu has a narrow cumulative exposure window. The relation of $\mathrm{Bu}$ exposure with toxicity is well established, but the link between the exposure and EFS is not clear due to conflicting reports especially in pediatric patients. Obtaining the ratio of $\mathrm{Bu}$ to its metabolite i.e. metabolic ratio (MR) may serve as an indicator of $\mathrm{Bu}$ GSH conjugating capacity of an individual, thus cumulative exposure of $\mathrm{Bu}$ for a particular day that could be used along with AUC as a marker to predict EFS. The present investigation is aimed at evaluating the utility of $\mathrm{Bu}$ MR to predict EFS in children undergoing allogeneic HSCT.

Methods: Two different cohorts with children receiving $\mathrm{Bu}$ in four times daily (QID, $\mathrm{n}=44$ ) and once daily doses $(\mathrm{QD}, \mathrm{n}=13$ ) at St. Justine's Hospital, Montreal were studied. $\mathrm{Bu}$ and $\mathrm{Su}$ levels were measured on day 3 of the conditioning regimen at the end of infusion (dose 9 in QID or dose 3 in QD dosing). EFS was defined from the time of transplant until death, relapse, or rejection, whichever occurred first. A receiver-operator characteristic curve (ROC) for Bu MRs measured was plotted to show the trade-off in sensitivity vs. 1- specificity rates for EFS, as the cut-off of the test was shifted from low to high. Cutoff values were defined based on the Youden's J statistic (i.e. sensitivity + specificty-1).

Results: Twenty-two males and 22 females aged from 0.1 to 19.9 years $($ mean \pm SD: $7.2 \pm 5.7)$ from Bu QID cohort had the mean MR of 5.9 (SD: 3.2). A cut off value of 4.9 in MR was chosen in ROC analysis in this cohort, with better sensitivity $(71 \%)$ and specificity $(70 \%)$ for EFS prediction $(\mathrm{p}=0.01, \mathrm{AUC}=0.7(95 \% \mathrm{CI}=0.6-0.8)$. In QD cohort nine females, and four males aged between 0.4 and 15.8 years $(6.7 \pm 5.1)$ had the mean MR of 29.3 (SD: 16.6). In ROC analysis, a cut off value of 25.06 was chosen with better sensitivity $(100 \%)$ and specificity $(100 \%)$ for EFS prediction $(\mathrm{p}=0.003$; $\mathrm{AUC}=1.0)$.

Conclusions: The $\mathrm{Bu}$ MR on day 3 above 4.973 and 25.06 were associated with worse EFS in children undergoing HSCT and received $\mathrm{Bu}$ in QID and QD dosing schedules, respectively.

Clinical Trial Registry: NCT01257854

Disclosure: Nothing to declare

\section{P110}

Population pharmacokinetics of treosulfan in pediatric patients undergoing hematopoietic stem cell transplantation

Eileen van der Stoep ${ }^{I}$, Juliette Zwaveling ${ }^{I}$, Alice Bertaina $^{2}$, Franco Locatelli ${ }^{2}$, Henk-Jan Guchelaar ${ }^{1}$, Arjan Lankester ${ }^{3}$, Dirk Jan Moes ${ }^{1}$

${ }^{1}$ Leiden University Medical Center, Clinical Pharmacy and Toxicology, Leiden, Netherlands, ${ }^{2}$ Bambino Gesù Childrens Hospital IRCCS, Rome, Italy, ${ }^{3}$ Leiden University Medical Center, Leiden, Netherlands

Background: Treosulfan is an alkylating agent increasingly used prior to hematopoietic stem cell transplantation (HSCT). The main objective of this study was to develop a population pharmacokinetic model of treosulfan in pediatric HSCT recipients and to explore the effect of different covariates on treosulfan pharmacokinetics (PK). Also, a limited sampling model (LSM) was developed.

Methods: In this multicentre study, 91 patients, receiving a dose of 10,12 or $14 \mathrm{~g} / \mathrm{m}^{2}$ treosulfan a day, administered during 3 consecutive days, were enrolled. A population pharmacokinetic model was developed using nonlinear mixed effect modelling (NONMEM version 7.3.0, using PsN toolkit 4.7.0 and Piraña version 2.9.7 as modelling environment). Demographic factors, as well as laboratory parameters, were included as covariates.

Results: Treosulfan PK was best described by a twocompartment model. A bodyweight-based allometric model improved the model more than a model incorporating body 
surface area (BSA). Clearance (CL) and intercompartmental clearance parameters were $6.07 \mathrm{~L} / \mathrm{h} / 15.6 \mathrm{~kg}$ (95\%CI 5.46$6.68)$ and $2.15 \mathrm{~L} / \mathrm{h}$ (95\%CI 1.39-2.91). Typical volumes of distribution of the central and peripheral compartments were $8.00 \mathrm{~L} / 15.6 \mathrm{~kg}(95 \% \mathrm{CI} 6.88-9.12)$ and $2.05 \mathrm{~L}$ (95\%CI 1.52-2.58). A model-based dosing table based on bodyweight is created to achieve a target exposure of 1540 $\mathrm{mg} * \mathrm{hr} / \mathrm{L}$ (Table 1), which was the median exposure of our population. Estimated glomerular filtration rate (eGFR) was shown to be the only parameter that significantly reduced interpatient variability in CL from $36.5 \%$ to $34.8 \%$. A limited sampling model with 3 samples (taken at 1.5, 4 and 7 hours after start of infusion) accurately estimated pharmacokinetic parameters of treosulfan.

Conclusions: To the best of our knowledge, this is the largest cohort of pediatric patients treated with treosulfan used for a population pharmacokinetic study. We developed a two-compartment model with weight and eGFR as covariates influencing treosulfan PK. Recently we showed a relationship between treosulfan exposure and early toxicity. Patients with an exposure $>1650 \mathrm{mg} * \mathrm{hr} / \mathrm{L}$ have an increased risk of developing grade 2 or higher mucositis and skin toxicity. Another study in 87 pediatric patients with thalassemia major reported an association between treosulfan clearance $\left(<7.97 \mathrm{~L} / \mathrm{h} / \mathrm{m}^{2}\right)$ and poor overall survival. Our model, together with the limited sampling strategy, can be used to adjust the dose, prior to or during treosulfan administration. Ongoing studies conducted in different disease settings will determine if treosulfan exposure can influence patient outcome. Subsequently, the optimal target exposure can then be established.

\begin{tabular}{ll}
\hline Weight $(\mathrm{kg})$ & Treosulfan dose $(\mathrm{mg})$ per day \\
\hline 10 & 6500 \\
20 & 11000 \\
30 & 15000 \\
40 & 19000 \\
50 & 22500 \\
60 & 25500 \\
70 & 29000 \\
\hline
\end{tabular}

[[P110 Table] 1. Table 1: Recommended treosulfan dose for different weight categories]

Disclosure: This study was supported by a grant (no. 213) from the Dutch Foundation Kinderen Kankervrij (KiKa). An unrestricted research grant by Medac GmbH was provided to AL. All other authors declare no conflict of interest.

\section{P111}

Phase II study for safety and efficacy of BEB (bendamustine, etoposide, busulfan) conditioning regimen for ASCT in non-hodgkin lymphoma

\section{Ho-Jin Shin ${ }^{1}$}

${ }^{1}$ Pusan National University Hospital, Busan, Korea, Republic of

Background: Autologous stem cell transplant (ASCT) is an effective treatment method for non-Hodgkin lymphoma (NHL). Until recently, carmustine, etoposide, cytarabine and melphalan (BEAM) was the most commonly used conditioning regimen. Despite acceptable efficacy with BEAM, carmustine is associated with major pulmonary toxicity. For this reason, the aim of this study was to investigate the safety and efficacy of BEB conditioning regimen for ASCT in NHL.

Methods: We conducted a prospective, multicenter, phase II study for BEB conditioning regimen for ASCT in NHL patients. A total of 33 patients were enrolled from 3 centers. They underwent ASCT with BEB conditioning regimen (Busulfan $3.2 \mathrm{mg} / \mathrm{kg}$ for 3days, Etoposide $400 \mathrm{mg} /$ $\mathrm{m}^{2}$ for 2 days, Bendamustine $200 \mathrm{mg} / \mathrm{m}^{2}$ for 2days) between 2016 and 2018.
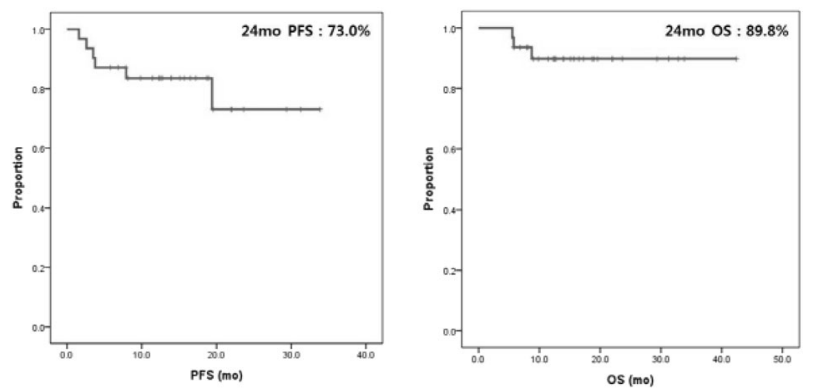

[[P111 Image] 1. Two year progression-free survival and overall survival.]

Results: The median age was 52 years (range 21-66) and 16 patients $(48.5 \%)$ were men. The most common type was diffuse large $B$ cell lymphoma $(n=23,69.7 \%)$ and more than half of patients $(n=19,57.6 \%)$ were classified as IPI score 3 or 4 . Eight patients (27.3\%) had a history of relapse and 19 patients $(57.6 \%)$ received more than 2 lines of chemotherapy before ASCT. Most patients $(n=27,81.8 \%)$ were complete remission (CR) state at ASCT. A median number of $5.85 \times 10^{6} / \mathrm{kg}$ CD34 cells were infused (range 2.018.6). All patients engrafted after a median time of 11 days (range 10-14). Twelve patients (36.4\%) experienced neutropenic fever and 16 patients $(48.5 \%)$ had grade 3 toxicities during ASCT. However, no one had a documented infection, veno-occlusive disease, or treatment-related 
death. Three months CR rate was $81.8 \%$. During a median follow-up period of 10.2 month, 7 patients (21.2\%) exhibited relapse or progression, while 1 patient $(3.0 \%)$ died of the disease. The estimated 2-year PFS and OS rate were $73.0 \%$ and $89.8 \%$, respectively (Figure 1 ).

Conclusions: The BEB conditioning regimens for ASCT is a feasible with tolerable toxicity in patients with NHL.

Disclosure: Nothing to declare

\section{P112}

\section{Long-term report of total marrow or total lymphoid} IMRT in advanced leukemia, myeloma and lymphoma

\section{Stefano Vagge ${ }^{I}$, Alida Dominietto ${ }^{I}$, Fabio Guolo ${ }^{I}$, Roberto Lemoli, Andrea Bacigalupo ${ }^{2}$, Emanuele Angelucci ${ }^{1}$, Renzo Corvò ${ }^{I}$}

${ }^{1}$ IRCCS Ospedale Policlinico San Martino, Genova, Italy, ${ }^{2}$ Università Cattolica del Sacro Cuore; Fondazione Policlinico Universitario 'A.Gemelli'-IRCCS, Haematology, Roma, Italy

Background: During the last three decades, total body irradiation (TBI) continues to play an important role in the conditioning regimens for patients undergoing stem-cell transplant (SCT) for a wide variety of advanced hematological malignancies. However, TBI showed boundaries in dose limits for toxicity in allogenic and moreover in autologous stem cell transplantation. Currently, the choice of conditioning regimen is based on the use of the least-toxic regimen to achieve the optimal therapeutic result. This report aims to assess the feasibility of a conditioning strategy based on high dose chemotherapy and whole-body radiotherapy focused on selective extensive tumor burden irradiation, both in allogeneic and autologous stem cell transplantation.

Methods: Since December 2009, sixty-two patients (pts) have been irradiated by helical tomotherapy (HT) to extensive target before allogeneic or autologous transplantation. Selected total marrow irradiation (TMI) schedules were planned to treat patients with high risk acute leukemia (ALL or AML) or multiple myeloma $(\mathrm{MM})$ as a part of conditioning regimen. Total lymphoid irradiation (TLI) was planned for patients with refractory or relapsed (R/R) Hodgkin (HD) or Non-Hodgkin lymphomas (NHL).

Results: TMI and TLI allowed delivering therapeutic dose over extensive selected targets with wide reduction of toxicity to all the organs at risk (OARs). The higher radiation doses rate to the OARs is reduced from $30 \%$ to $70 \%$. Allogenic conditioning regimen was TLI (4Gy x $3 \mathrm{fx}$ ) than fludarabine + endoxan for patients with HD (4 $p t s)$. TMI $(4 \mathrm{~Gy} \times 3 \mathrm{fx})+$ fludarabine + melphalan for patients with MM (4 pts). TMI (4Gy x 2 fx) + thiotepa + fludarabine + busulfan for advanced LAM patients (4 $p t s)$. TMI as the boost (2-3Gy) after conventional TBI was (12 Gy in 6 bi-fractionated doses) by cyclophosphamide (18 pts). Autologous preparation to SCT consisted of TLI (4Gyx $3 \mathrm{fx}$ ) followed by high-dose bendamustine and melphalan for patients older than 40 years and conventional FEAM (Fotemustine, Etoposide, Cytarabine, and Melphalan) for younger patients, in HD e NHL (20 pts). While TMI (4Gy x $3 \mathrm{fx}$ ) plus melphalan was delivered for autologous SCT in MM and LAM (12 pts). No unexpected acute toxicity was found. In the allogenic setting, all the patients' engraftment was achieved in all patients. No acute graft versus host disease increasing was detected. Within the autologous setting, only $33 \%$ developed grade $3 / 4$ mucositis. None experienced grade $3 / 4$ extra-hematological toxicity. Outcomes of the specific disease will be reported.

Conclusions: The current report describes the clinical feasibility of using HT to deliver TMI or TLI in the setting of autologous transplantation or during allogenic stem cell conditioning regimen, to allow all patients (old, fragile or with high tumor burden) to achieve an ablative regimen before SCT. To our knowledge, this single institution experience describes data from one of the largest cohort of patients treated in Europe since the development of this irradiation techniques.

Disclosure: Nothing to declare

\section{P113}

Intravenous busulphan plus melphalan versus melphalan alone as conditioning regimen for newly diagnosed patients with multiple myeloma: Long-term follow-up of a matched case-controlled study

Margarita Blanes $^{1}$, Jose Ignacio Lorenzo ${ }^{2}$, Paz Ribas ${ }^{3}$, Ana Jiménez, José David González $z^{5}$, María José Cejalvo $^{3}$, Carlos Solano $^{6}$, Adrian Alegre ${ }^{7}$, Javier de la Rubia ${ }^{3}$

${ }^{1}$ University Hospital General de Elda, Hematology, Elda, Spain, ${ }^{2}$ University Hospital La Fe, Hematology, Valencia, Spain, ${ }^{3}$ University Hospital Doctor Peset, Hematology, Valencia, Spain, ${ }^{4}$ University Hospital 12 de Octubre, Hematology, Madrid, Spain, ${ }^{5}$ University Hospital Insular de Canarias, Hematology, Las Palmas de Gran Canaria, Spain, ${ }^{6}$ University Hospital Clínico, Hematology, Valencia, Spain, ${ }^{7}$ University Hospital La Princesa, Hematology, Madrid, Spain

Background: Autologous stem cell transplantation (ASCT) is considered the standard consolidation therapy for younger patients with newly diagnosed multiple 
myeloma (MM). We have previously reported the results of a matched case-control study comparing the outcome of a series of patients with newly diagnosed MM undergoing ASCT after intravenous (iv) busulfan (BU) and melphalan (BUMEL) or melphalan $200 \mathrm{mg} / \mathrm{m}^{2}$ (MEL200) as preparative regimen (Blanes et al, BBMT 2013). Here we update the progression free survival (PFS) and overall survival (OS) data from this casecontrol study.

Methods: Between June 2005 and September 2009, 51 patients with newly diagnosed MM underwent ASCT after BUMEL in five Spanish centers. These patients were compared with a control group of 102 pair mates included in the study of Grupo Español de Mieloma GEM2000. Induction therapy in both groups of patients was based in polychemotherapy without the use of new drugs. Case matching was performed according to age, clinical stage at diagnosis, and response to induction therapy. Conditioning regimen consisted of iv BU at a dose of $3.2 \mathrm{mg} /$ $\mathrm{kg}$ once a day on days -5 to -3 followed by MEL at a dose of $-140 \mathrm{mg} / \mathrm{m}^{2}$ on day -2 in the BUMEL group versus MEL200 in the control group. Maintenance therapy after transplant consisted of interferon and steroids in the majority of patients.

Results: The cut-off date for this update was June 30, 2018. After a median follow-up of 56 and 63 months in the BUMEL and MEL200 groups respectively, 35 patients had relapsed in the BUMEL group and 82 patients in the control group. Median PFS was $33(95 \%$ CI, 25.4-48.3) months in the BUMEL and 24 (95\% CI, 20.1-32.7) months in the MEL200 group $(P=0.04)$ (Figure 1). In this update, 12 patients in the BUMEL group are in maintained response and 7 of them are in continuous CR (two with negative status for minimal residual disease) between 9 and 12 years after transplantation. Ten-year OS was not significantly different between both groups, being 41 (95\% CI 30-58) months in the BUMEL and 29 (95\% CI 18-47) months in the control group.Transplant-related mortality was similar in both groups of patients (4\% in the BUMEL and $2 \%$ in the MEL200 group). Regarding toxicity, BUMEL was associated with a higher incidence of mucositis and liver toxicity than the melphalan-only approach but no patient in our series developed sinusoidal occlusive syndrome and the hepatic toxicity observed was only grade I/II. Finally, no long-term side effects have been reported among BUMEL recipients.

Conclusions: This long-term follow-up analysis confirms that a therapeutic strategy including BUMEL as conditioning regimen beforeASCT in patients with newly diagnosed $\mathrm{MM}$ is highly active and safe in these patients.

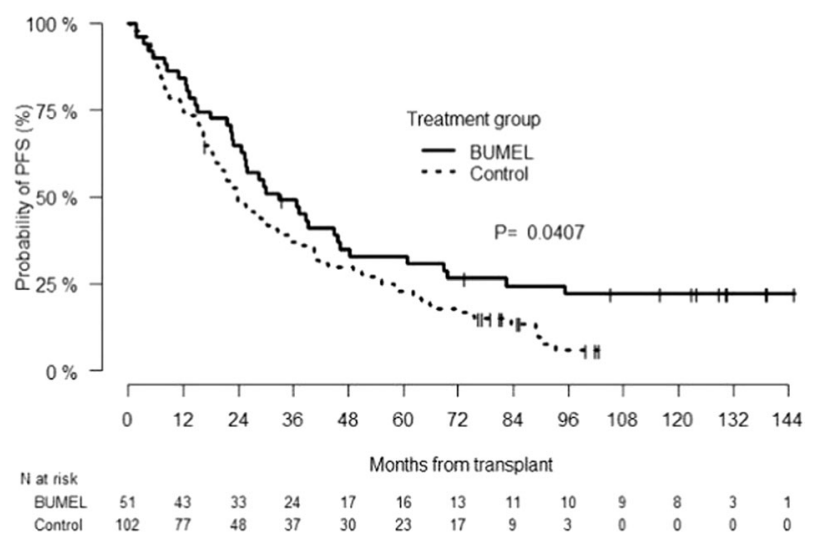

[[P113 Image] 1. Figure 1. Progression free survival in the BUMEL (__ and control group (......).]

Clinical Trial Registry: The GEM2000 protocol and BUMEL clinical trial were registered at ClinicalTrials.gov as NCT00560053 and NCT00804947

Disclosure: None of the authors has anything to disclose.

\section{P114}

A phase I trial evaluating carfilzomib, bendamustine, and melphalan (CBM) conditioning prior to autologous hematopoietic stem cell transplantation for multiple myeloma- final results

\section{Muneer Abidi ${ }^{1,2}$, Kelli Cole ${ }^{3}$, Marlee Muilenburg ${ }^{1}$, Jessica Parker $^{1}$, Stephanie Williams ${ }^{1,2}$, Samer Al-Homsi ${ }^{3}$}

${ }^{1}$ Spectrum Health Cancer Center, Grand Rapids, MI, United States, ${ }^{2}$ Michigan State University College of Human Medicine, Internal Medicine, Grand Rapids, MI, United States, ${ }^{3}$ NYU Langone Health, Internal Medicine, Hematology, New York, NY, United States,

Background: Autologous hematopoietic cell transplantation (AHCT) \& high-dose melphalan (M) based conditioning remains the standard of care for transplant eligible patients with multiple myeloma (MM). Efforts to combine radiation \& other chemotherapeutic drugs with $M$ have resulted in increased toxicity. We hypothesized that integrating a Proteasome inhibitor, another alkylating agent and $\mathrm{M}$ in the conditioning is safe \& may improve complete remission $(\mathrm{CR})$ \& overall survival (OS) rates.

Methods: We enrolled MM patients (pts), eligible to receive AHCT in this phase I dose escalation study from 05/ 2014 to 10/2017. Carfilzomib (C) was added to bendamustine (B) \& M. All patients received a fixed dose $\left(20 \mathrm{mg} / \mathrm{m}^{2}\right)$ of C on days (d) $-29,-28,-22,-21,-15, \&-14$. C was done in dose escalation, per table I, on $\mathrm{d}-2,-1,+5$ and +6 . Dose limiting toxicity (DLT) was defined as: lack of neutrophil \& platelet 
engraftment by $d+22$ and +35 respectively, \& any G 4 GI toxicity or any $\geq \mathrm{G} 3$ non-hematologic toxicity as probably related to the study protocol. Due to DLT in cohort 2, the study was amended after first 6 pts \& the M was reduced to $140 \mathrm{mg} / \mathrm{m}^{2} \&$ the $\mathrm{d}+6$ dose of $\mathrm{C}$ was omitted.

Results: Eighteen pts were enrolled, 11 males \& 7 females. Median age was 58 years (39-68). Performance status was $\geq$ $70 \%$ (KPS) in all pts. Per the International Staging System (ISS), 3 pts had stage I disease, 6 had stage II, \& 7 had stage III. Staging was indeterminate in 2 pts. Five pts had undergone a prior AHCT. Disease status at enrollment was stable disease $(\mathrm{SD})(\mathrm{n}=3)$, partial response $(\mathrm{PR})(\mathrm{n}=9)$, very good partial response (VGPR) $(n=5), \&$ one pt had progressive disease (PD). Median $\mathrm{CD} 34^{+}$cell dose infused was $3.24 \times 10^{6} / \mathrm{kg}\left(2.23-8.42 \times 10^{6}\right)$. Median follow-up was 25.1 months (1.4-44.4). Median time to neutrophil engraftment was $12 \mathrm{~d}$ (11-15). and platelet engraftment was $16 \mathrm{~d}(12-$ 20). One pt died (cohort 2 ) on $d+44$ before achieving platelet engraftment. Ten pts received maintenance therapy. Posttransplant disease status was assessed per protocol by SPEP, SPIF, serum free light chains \& ratio. Seventeen pts were evaluable for response assessment at d $+100, d+180 \& d+$ 365. At d+100: 4 pts had CR, 8 had VGPR, 1 pt had PR \& 4 pts had SD. At d+365: 4 pts had CR, 5 had VGPR, 3 pts had PR and 5 pts had PD. OS probability at last follow up was $60.3 \%$ (95\% CI $27.6-82 \%$ ) and progression free survival $66.3 \%$ (95\% CI 29.8-87\%).

Conclusions: The combination of CBM (cohort 3B) for AHCT appears feasible, with manageable toxicities. Longer follow-up \& a phase II study are warranted to determine response rates and long-term outcomes.

\begin{tabular}{|c|c|c|c|c|}
\hline \multicolumn{3}{|c|}{ CohortPts $(\mathrm{n}) \mathrm{C}(\mathrm{mg} / \mathrm{m} 2)$ days } & \multirow{2}{*}{$\begin{array}{l}\text { B (mg/m2) days } \\
(120) \text { d }-2 \text { \& } \\
(100) \text { d }-1\end{array}$} & \multirow{2}{*}{$\begin{array}{l}\begin{array}{l}\text { M (mg/ } \\
\mathrm{m} 2) \text { days }\end{array} \\
\begin{array}{l}(100) \mathrm{d}-2 \&- \\
1\end{array}\end{array}$} \\
\hline 1 & 3 & $\begin{array}{l}(15) \mathrm{d}-2,-1,+5, \\
\&+6\end{array}$ & & \\
\hline 2 & 3 & $\begin{array}{l}(20) \mathrm{d}-2,-1,+5 \\
\& \mathrm{~d}+6\end{array}$ & $\begin{array}{l}(120) d-2 \& \\
(100) d-1\end{array}$ & $\begin{array}{l}(100) d-2 \&- \\
1\end{array}$ \\
\hline $2 \mathrm{~B}$ & 3 & (20) d $-2,-1, \&+5$ & $\begin{array}{l}\text { (120) d }-2 \text { \& } \\
\text { (100) d -1 }\end{array}$ & $(140) \mathrm{d}-1$ \\
\hline 3B & 9 & (27) d $-2,-1, \&+5$ & $\begin{array}{l}(120) d-2 \& \\
(100) d-1\end{array}$ & $(140) \mathrm{d}-1$ \\
\hline
\end{tabular}

\section{[[P114 Table] 1. Table I]}

Disclosure: Muneer Abidi: Research funding/honorarium from following pharmaceutical companies: Amgen, Celgene, Onyx, Millennium, Sobi. Other authors have Nothing to declare.

\section{P115}

The effect of TGFB1 polymorphism after allogeneic hematopoietic stem cell transplantation
Hajnalka Andrikovics ${ }^{1}$, Petra Kovy ${ }^{1}$, Nora Meggyesi ${ }^{1}$, Livia Varga ${ }^{I}$, Katalin Balassa ${ }^{I}$, Andras Bors ${ }^{l}$, Laszlo Gopcsa ${ }^{I}$, Melinda Paksi ${ }^{1}$, Aniko Barta ${ }^{1}$, Eszter Vad ${ }^{1}$, Arpad Batai ${ }^{1}$, Attila Tordai ${ }^{2}$, Tamas Masszi ${ }^{2}$, Janos Sinko ${ }^{1}$, Peter Remenyi ${ }^{1}$

${ }^{1}$ Central Hospital of Southern Pest (DPC), Budapest, Hungary, ${ }^{2}$ Semmelweis University, Budapest, Hungary

Background: Allogeneic hematopoietic stem cell transplantation (allo-HSCT) is a potentially curative therapeutic option for malignant hematopoietic diseases. Cytokines including transforming growth factor $\beta 1$ (TGFB1) play a pivotal role in immune reconstruction, and the development of graft versus host disease (GvHD) or infections.

Methods: The role of TGFBI gene $-1347 \mathrm{C}>\mathrm{T}$ variant was investigated in a cohort of 409 adult recipient-donor pairs [178 female and 231 male, median age: 43 years (range 19-73)]. The majority of the patients suffered from acute myeloid leukaemia $(155 / 409,38 \%)$ and 254 patients from other malignant hematopoietic disorders. During transplantation $210(52 \%)$ patients received graft from their siblings, 199 (48\%) from matched unrelated donors (MUD). Myeloablative conditioning (MAC) was applied in 261 (64\%) and reduced intensity conditioning (RIC) in 148 (36\%) cases. For GvHD prevention tacrolimus plus sirolimus (TAC\&SIR) were most commonly applied in $238(58 \%)$, cyclosporine (CSA) in $95(23 \%)$ and TAC as single agent in 76 (19\%) cases. TGFB1 variant was analysed from genomic DNA with LightCycler hybridisation probe method.

Results: We did not find any association of recipient TGFB $1-1347 \mathrm{C}>\mathrm{T}$ with HSCT outcome parameters (acute and chronic GvHD, cytomegalovirus infection [CMV], transplant associated thrombotic microangiopathy [TATMA]) and survival (transplant related mortality, [TRM]; overall survival, $[\mathrm{OS}])$ neither in the whole HSCT cohort nor in any subgroups. In the whole cohort donor $-1347 \mathrm{C}>\mathrm{T}$ genotype did not impair survival $(\mathrm{p}=0.4)$. Diagnosis, donor type, conditioning and GvHD prophylaxis were tested for interaction with donor TGFB1 genotype. Although donor TGFB $1-1347 \mathrm{C}>$ genotype influenced OS in the subgroups of patients with acute leukaemia, or treated with MAC conditioning or with TAC-based GvHD prophylaxis in multivariate analyses, test for interaction indicated the differential effect of TGFB1 genotypes in MAC versus RIC subgroups $(\mathrm{p}=0.035)$. In the MAC cohort, patients transplanted with -1347TT genotype donor had unfavourable outcome (60-month OS CC: $62.1 \% \pm 4.8 \%$, TC: $46.8 \%$ $\pm 4.8 \%$ and TT: $35.6 \% \pm 9.3 \%, \mathrm{p}=0.032$; which was independent from age, donor type and GvHD prophylaxis, $\mathrm{p}=0.003$, HR: $2.35,95 \%$ CI: $1.35-4.10)$. In case of myeloablative conditioning, relapse rate (CC: $18 \%$ vs. CT 
$28 \%$ vs. TT $18 \%$ ) was not different in subgroups stratified by donor TGFB1 genotype. Frequency of acute GvHD grade III-IV [CC: $11 \%$; CT: 17\%; TT: $24 \%, \mathrm{p}=0.057]$, and transplant-related mortality was higher in TT-carriers (CC:26\%; CT:28\%; TT:46\%, p=0.02 CC\&CT vs TT). TA-TMA, CMV infection/reactivation and cGvHD were also not different according to donor genotypes. Fungal infections occurred more frequently as causes of death in carriers (CC: $9.7 \%$ vs. CT: $38.1 \%$ vs TT: $33.3 \%, \mathrm{p}=0.022$ ).

Conclusions: Our results suggest that donor TGFB1 $-1347 \mathrm{C}>\mathrm{T}$ may exert an adverse influence on the outcome of myeloablative conditioning. Our finding might be explained by the combination therapy of calcineurin and mTOR inhibition in GvHD prophylaxis in myeloablative conditioning.

Disclosure: Nothing to declare.

\section{P116}

Treosulfan-based reduced intensity conditioning in HLA-haploidentical transplantation using PTCY as GVHD prophylaxis in high-risk MDS /AML of the elderly

\section{Alessia Fraccaroli, Dusan Prevalsek ${ }^{1}$, Sarah Haebe ${ }^{1}$, Veit Bücklein $^{1}$, Christoph Schulz ${ }^{1}$, Heidrun Drolle ${ }^{1}$, Susanne Fritsch ${ }^{1}$, Georg Ledderose ${ }^{1}$, Johanna Tischer ${ }^{1}$ \\ ${ }^{1}$ University Hospital of Munich, LMU, München, Germany}

Background: Standard conditioning regimens prior to allogeneic hematopoietic stem cell transplantation (allo-HSCT) are often associated with a considerable risk of severe adverse events, especially in elderly patients suffering from high-risk (HR) MDS/AML. Previous clinical studies have demonstrated feasibility of treosulfan-based reduced-intensity conditioning (RIC) by stable engraftment, low non-relapse mortality (NRM), and favorable survival in elderly patients undergoing HLA-matched related or unrelated allo-HSCT (Beelen et al, ASH 2017 \#0521). However, data for treosulfan-based conditioning in the T-cell-replete HLAhaploidentical (haplo-HSCT) setting in high-risk AML/MDS patients are rare. Here we report on the outcome of eleven patients treated with a treosulfan-based conditioning undergoing haplo-HSCT using exclusively post-transplantation cyclophosphamide (PTCY) as GvHD prophylaxis.

Methods: Eleven patients with high-risk (HR) AML $(n=9) / \operatorname{MDS}(n=2)$ who underwent haplo-HSCT using treosulfan for reduced intensity conditioning (RIC) and PTCY as GvHD prophylaxis were retrospectively analyzed with respect to outcome and toxicity. All patients were $>55$ years old and transplanted between January 2016 and February 2018 at our institution. The majority of the patients (9/11) suffered from active disease at time of treatment initiation, only two patients presented in CR. All but one received sequential conditioning with cytoreductive chemotherapy using FLAMSA applied shortly prior to treosulfan-based RIC (10g/m² over 3 days). A bone marrow graft was used in 9/11 patients. Post-grafting immunosuppression consisted of cyclophosphamide, tacrolimus and MMF. National Cancer Institute Common Terminology Criteria for Adverse Events version 3.0 were used for nonhematologic toxicity assessment starting from sequential therapy initiation or conditioning until day +30 .

Results: Median age of the entire cohort was 63 years (range: 58-71). The HCT-CI was $\geq 2$ in eight pts (median HCT-CI=2, range: 0-5). No graft rejection occurred. Neutrophil and platelet engraftment were achieved in $100 \%$ and $91 \%$ of the patients at a median of 20 (16-23) and 26.5 (13-30) days, respectively. Acute GvHD grade IIIV occurred in $18 \%$ of the patients, exclusively involving the skin. No one developed severe ( ${ }^{\circ}$ III-IV) acute GvHD. No patient died prior to haplo-HSCT. Severe nonhematologic regimen-related toxicities ( ${ }^{\circ}$ III-IV) occurred in $2 / 11$ patients, predominately affecting the gastrointestinal tract. No patient suffered from $\geq$ two $^{\text {III-IV }}{ }^{\circ}$ toxicities. All patients developed fever during treatment course, four with positive blood cultures. CMV reactivated in $6 / 7$ patients at risk. No EBV reactivation or PTLD occurred. Six patients had clinical and radiological signs of pneumonia (probable invasive aspergillosis) without detection of aspergillus/antigen in the bronchoalveolar lavage. CI of NRM at day +180 was $0 \%$. Four patients relapsed within the first year after haplo-HSCT, with two of them dying due to relapse. At last follow-up (Dec 2018) 9/11 patients were alive. With a median follow-up of 5 months (2-31) estimated 1-year OS and DFS were $80 \%$ and $59 \%$, respectively.

Conclusions: Treosulfan-based unmanipulated HLAhaploidentical allo-grafting using PTCY as GvHD prophylaxis in HR MDS and AML patients aged over 55 years is safe and well tolerated resulting in stable engraftment and a favorable toxicity profile. Our preliminary data further show promising outcome with low NRM, no severe acute GvHD and favorable survival offering an attractive alternative in RIC for haplo-HSCT of the elderly.

Disclosure: nothing to declare

\section{P117}

Comparison of outcomes of total body irradiation (TBI) vs non-TBI conditioning regimens in acute lymphoblastic leukemia for allogeneic transplantation

Huseyin Saffet Bekoz, Omur Gokmen Sevindik ${ }^{1}$, Tulay Ozcelik $^{2}$, Mutlu Arat ${ }^{2}$, Ipek Yonal Hindilerden ${ }^{3}$, Sevgi Kalayoglu Besisik ${ }^{3}$, Meliha Nalcaci, ${ }^{3}$ Ant Uzay, Suleyman Sami Karti ${ }^{4}$, Demet Kivanc ${ }^{1}$, Deniz Sargin ${ }^{1}$ 
${ }^{1}$ Medipol University, Istanbul, Turkey, ${ }^{2}$ Istanbul Bilim University Medical Faculty, Hematology and Adult BMT, Istanbul, Turkey, ${ }^{3}$ Istanbul University Medical Faculty, Istanbul, Turkey, ${ }^{4}$ Acibadem University, Hematology and Adult BMT, Istanbul, Turkey

Background: In adult patients diagnosed acute lymphoblastic leukemia (ALL) long-term results are poor with intensive chemotherapy.

Allogeneic stem stem cell transplantation is the potential treatment that provides cure for these patients. Myeloablative preparation regimens include total body irradiation (TBI) + cyclophosphamide $(\mathrm{Cy})$ and busulfan + cyclophosphamide.In adult ALL patients WBI/Cy widely used, but the toxicity rate is higher. The aim of this study is to compare the result and effect of the TBI/Cy and busulfan/Cy regimens in Allogenic bone marrow transplantation in ALL patients.

Methods: Between 1993 -2018 there were 137 ALL patients who underwent transplantation using myeloablative preparation regimen with or without addition TBI in the adult bone marrow transplantation units of Medipol Medical Faculty, Istanbul University Istanbul Medical Faculty, Sisli Florence Nightingale Hospital, Atakent Acıbadem Hospital adult bone marrow units . We analyzed overall survival (OS), progression free survival (PFS), veno occlusive disease, acute and chronic graft versus disease development rates in these patients.

Results: Demographic characteristics of patients summarized in table -1

There was no significant difference between groups in donor age, gender, stem cell source.

It was observed that the relapse rate was not statistically significant in both group.There was no statistically significant difference between the patients who underwent myeloablative regimen and myeloablative regimen with TBI in relaps, death, OS, PFS.

(figure-1)

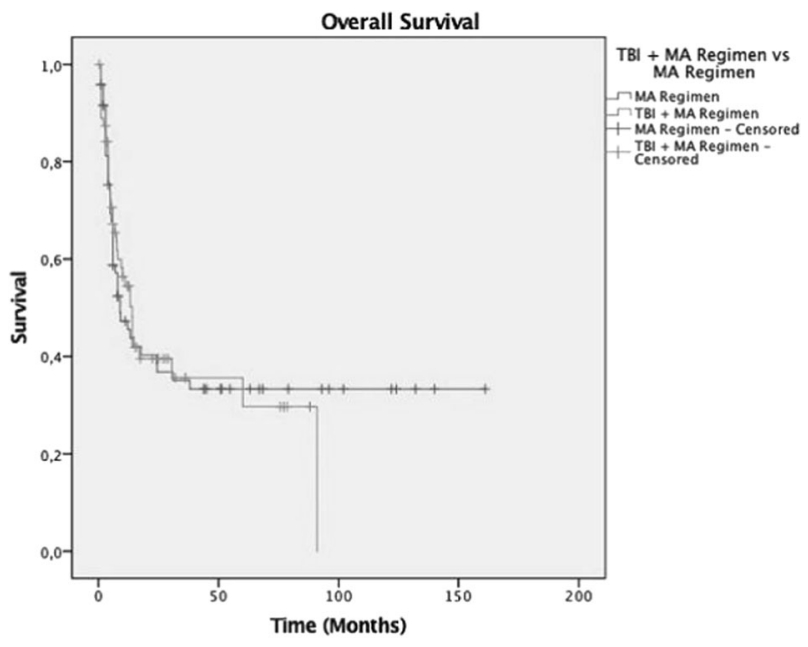

\section{[[P117 Image] 1. Figure 1]}

In terms of transplant complications there was also no respectable difference in development of VOD and acute and chronic graft versus disease but VOD was more common in the group that did not use TBI (P: 0.068) (table-1)

\begin{tabular}{lllll}
\hline & $\begin{array}{l}\text { total } \\
\text { (n:137) }\end{array}$ & $\begin{array}{l}\text { MA } \\
\text { regimen }\end{array}$ & $\begin{array}{l}\text { TBI+MA } \\
\text { regimen }\end{array}$ & P value \\
\hline Age(year) & $\begin{array}{l}34(17- \\
64)\end{array}$ & $\begin{array}{l}34(17- \\
62)\end{array}$ & $34(19-64)$ & 0.907 \\
Gender (M/F) & $90 / 47$ & $49 / 23$ & $41 / 24$ & 0.591 \\
transplant timing (TR1/TR2/ & $75 / 50 /$ & $39 / 28 / 5$ & $36 / 22 / 7$ & 0.286 \\
primary refractory & 12 & & & \\
donor (MSD/MUD) & $115 / 21$ & $58 / 13$ & $57 / 8$ & 0.588 \\
donor gender(M/F) & $72 / 65$ & $39 / 33$ & $33 / 32$ & 0.734 \\
donor age(year) & $33(8-$ & $35(8-66) 33(8-57)$ & 0.535 \\
& $66)$ & & & \\
Median follow up(month) & $8(1-$ & $8(1-161)$ & $10(1-91)$ & 0.412 \\
& $140)$ & & & \\
relaps(No/Yes) & $92 / 45$ & $46 / 26$ & $46 / 19$ & 0.467 \\
Death(No/Yes) & $57 / 80$ & $29 / 43$ & $28 / 37$ & 0.862 \\
\hline
\end{tabular}

\section{[[P117 Table] 1. Table -1]}

Conclusions: Although there are contradictory data in the literature, in our multicentre study, it was revealed that the addition of TBI in the myeloablative preparation regimen compared with myeloablative preparation regimen alone did not have a positive or negative effect on overall survival.We think that if we can prepare a good VOD prophylaxis approches, we can give up TBI in future.

Disclosure: Nothing to declare

\section{P118}

Fludarabine-based reduced intensity conditioning for fanconi anemia - A single center experience

Ehud Even-Or', Hodaya Cohen ${ }^{1}$, Adeeb NaserEddin ${ }^{1}$, Bella Shadur', Batia Avni', Sigal Grisariu ${ }^{1}$, Irina Zaidman $^{I}$, Polina Stepensky ${ }^{1}$

${ }^{1}$ Hadassah Hebrew University Medical Center, Bone Marrow Transplantation, Jerusalem, Israel,

Background: Fanconi anemia (FA) is a rare genetic disease characterized by chromosomal fragility, somatic abnormalities, bone marrow failure and a tendency to develop malignancies. Hematopoietic stem cell transplantation (HSCT) is currently the only curative treatment in FA patients who develop bone marrow failure or AML/MDS. HSCT for patients with FA is challenging as these patients are exquisitely sensitive to genotoxic chemotherapy agents 
and ionizing radiation, and to the inflammatory side effects of graft versus host disease. Since the mid-1990's Fludarabine based reduced intensity conditioning regimens are used at our center for HSCT of patients with FA.

Methods: In this retrospective study we collected data from all HSCT done at Hadassah Medical Center for patients with FA using fludarabine based conditioning regimens. The collected data included patient's clinical data and transplant data. The study was approved by the institutional Helsinki committee.

Results: Since June 1996 until August 2018, 35 patients with FA underwent 39 HSCT at Hadassah Medical Center with fludarabine based conditioning regimens. The median age of the patients at transplant was 9.1 years (range 3.2 30.8). All patients had bone marrow failure with severe aplastic anemia, 16 of which were transfusion dependent at the time of transplant and eight of the patients developed MDS. The conditioning regimen consisted fludarabine 180 $\mathrm{mg} / \mathrm{m}^{2}$ cyclophosphamide (20 to $40 \mathrm{mg} / \mathrm{kg}$ ) and antithymocyte globulin. Patients with unrelated donors received additional low dose busulfan $(n=7)$ or additional TBI $(\mathrm{n}=3)$. For GVHD prophylaxis, cyclosporine A was given either alone $(n=21)$, with MMF $(n=13)$ or with methotrexate $(n=1)$. The graft source was bone marrow $(B M)$ in most cases $(n=31)$, PBSC in seven cases, matched sibling cord $+\mathrm{BM}$ in two cases and one matched related cord. Twentyfive of the donors were family donors and ten were unrelated. Twenty-nine of the donors were 10/10 HLA matched, six were 9/10 mismatched and one haploidentical. Four patients had engraftment failure and required a second transplant, two of them were re-transplanted with cyclophosphamide and TBI, one with fludarabine, busulfan and campath, and one with no conditioning. Thirty of the 35 patients are alive (86\%). Four patients died of transplant complications and one died of metastatic squamous cell carcinoma. Eight survivors are mixed chimeras (81\%-94\% donor) and are all doing well, none of them developed any GVHD. Nine patients developed acute GVHD, four of them with grade 3-4. Seven of these patients later developed chronic GVHD, two of them have extensive disease.

Conclusions: Our results show a high survival rate of $86 \%$, with a low rate of engraftment failure and reasonable rates of GVHD. Only one of our patients died of late effects of HSCT for FA. Mixed chimerism does not seem to present a problem. We conclude that reduced intensity fludarabine based conditioning regimens are a good treatment option for patients with Fanconi anemia undergoing HSCT.

Disclosure: Nothing to declare

\section{P119}

Total marrow irradiation + bendamustine as reducedtoxicity myeloablative conditioning prior to allohsct for younger patients with multiple myeloma
Malgorzata Sobczyk-Kruszelnicka ${ }^{1}$, Tomasz Czerw ${ }^{1}$, Wlodzimierz Mendrek ${ }^{l}$, Jacek Najda ${ }^{I}$, Maria SadusWojciechowska ${ }^{I}$, Malgorzata Ociepa-Wasilkowska ${ }^{I}$, Andrzej Frankiewicz ${ }^{I}$, Katarzyna Michalak, Anastazja Szlauer-Stefanska ${ }^{1}$, Malgorzata Krawczyk-Kulis ${ }^{1}$, Lukasz Dolla $^{l}$, Grzegorz Wozniak ${ }^{1}$, Lukasz Kleszyk ${ }^{1}$, Bozena Jochymek $^{1}$, Jerzy Holowiecki ${ }^{l}$, Krzysztof Slosarek ${ }^{I}$, Leszek Miszczyk ${ }^{1}$, Sebastian Giebel ${ }^{1}$

${ }^{1}$ Maria Sklodowska-Curie Institute - Cancer Center, Gliwice Branch, Gliwice, Poland

Background: The prognosis of patients with multiple myeloma (MM) has improved markedly over the last two decades. Despite that, alloHSCT remains the only treatment option with curative potential. However, its use is limited due to high incidence of non-relapse mortality (NRM) after myeloablative conditioning while insufficient efficacy of reduced-intensity regimens. We developed a new protocol characterized by reduced toxicity while preserved myeloablative potential, based on the use of total marrow irradiation (TMI) in combination with bendamustine. The aim of this study was to evaluate its safety and efficacy in a singlecenter experience.

Methods: Between years 2013-2018, MM patients below 55 years old were offered tandem auto-alloHSCT as part of first-line therapy. The decision was based on individual patient preferences after detailed description of potential risks. AutoHSCT was preceded by melphalan $200 \mathrm{mg} / \mathrm{m}^{2}$ iv. The conditioning prior to alloHSCT consisted of TMI performed using helical tomotherapy at the dose of $4 \mathrm{~Gy} / \mathrm{d}$ on days $-3,-2,-1$ (total $12 \mathrm{~Gy}$ ) and bendamustine 140-220 $\mathrm{mg} / \mathrm{m} 2 / \mathrm{d}$ iv. on days $-5,-4$ (total $280-440 \mathrm{mg} / \mathrm{m} 2$ ). The immunosuppressive therapy consisted of cyclosporine + methotrexate +/- ATG. Peripheral blood was used as a source of stem cells.

Results: The analysis included 18 patients (women - 9, men - 9). The median follow-up was 28 (4-68) months. The median age at alloHSCT was 44 (26 - 53) years. The disease stage before alloHSCT was as follows: CR-6, VGPR-4, PR6. Patients were treated with HSCT from either HLAmatched siblings $(n=7)$ or unrelated donors $(n=11)$. The interval between autoHSCT and alloHSCT was 5 (4-23) months.

All patients engrafted after alloHSCT with median time of neutrophil and platelet recovery of 14 and 12 days, respectively. One patient $(6 \%)$ experienced grade 2 acute GvHD, while there were no cases of grade 3-4 acute GvHD. The incidence of mild, moderate and severe chronic GvHD was $17 \%, 0 \%$ and $6 \%$, respectively. The rate of grade 3 non-hematological toxicities was $11 \%$. One patient died of late bacterial infection. The incidence of TRM was 5\%. Grade 4 adverse events were not reported. Disease status 
3 months after alloHSCT was: CR-10, VGPR-5, PR-3. The probability of OS and PFS after 30 months was $94 \%(+/-$ $6 \%$ ) and $77 \%(+/-12 \%)$, respectively. The incidence of progression and TRM was $17 \%$ and $6 \%$, respectively.

Conclusions: AlloHSCT using TMI $12 \mathrm{~Gy}+$ bendamustine conditioning protocol is characterized by good tolerance and low risk of GvHD. It may be used for younger patients with $\mathrm{MM}$ as part of tandem autoalloHSCT strategy. Encouraging results reported in this study should be confirmed in prospective clinical trials.

Disclosure: Nothing to declare

\section{P120}

Comparison between two reduced intensity conditioning regimens in patients with a myeloid malignancy: $A$ single center experience comparing FB2 with flumel

Mitja Nabergoj ${ }^{1}$, Anne-Claire Mamez, Sarah Morin ${ }^{1}$, Carmen de Ramon Ortiz ${ }^{l}$, Carole Dantin ${ }^{I}$, Amandine Pradier $^{1}$, Thien-An Tran ${ }^{1}$, Thomas Longval, Maria Anastasiou $^{I}$, Laura Bounaix ${ }^{1}$, Caroline Stephan ${ }^{I}$, Yan Beauverd $^{I}$, Federica Giannotti ${ }^{1}$, Stavroula MasouridiLevrat $^{\text {, }}$, Yves Chalandon ${ }^{1}$

${ }^{1}$ Geneva University Hospitals, Geneva, Switzerland

Background: Hematopoietic Stem cell transplantation (HSCT) remains the only curative option for high-risk myeloid neoplasms. The optimal reduced-intensity conditioning (RIC) is still debated.

Methods: A single-center retrospective analysis was conducted at our institution to compare two different RIC regimens in adult patients transplanted for myeloid malignancy from 2001 to 2018. A total of 137 patients were analysed, 74 of them treated with Busulfan-based (Fludarabine $150 \mathrm{mg} / \mathrm{m}^{2}$, Busulfan $6.4 \mathrm{mg} / \mathrm{kg}, \mathrm{FB} 2$ ) and 63 with Melphalan-based conditioning regimen (Fludarabine $150 \mathrm{mg} / \mathrm{m}^{2}$, Melphalan $140 \mathrm{mg} / \mathrm{m}^{2}$, FluMel). Antithymocyte globulin (ATG) was administered in all patients while no one received TBI. Partial in vitro T-cell depletion was performed using alemtuzumab for low risk patients.

Results: The two groups were well balanced with a median age of 61 and 62 years in the FB2 and FluMel group, respectively, and a median follow up of 46 months. The most frequent indication for transplant in both groups was AML (59.5 and $69.8 \%$ for FB2 group and FluMel group, respectively) and the stem cell source was peripheral blood in 94.6 and $96.8 \%$ of patients. More patients in the first group had near to significant worst Karnosfky status (< 90) at transplant compared to second (35.1 vs $19 \%$, $\mathrm{p}=0.057)$ and more patients received a T-partial depleted graft (54.1 vs 33.3\%, $\mathrm{p}=.028)$. The neutrophil engraftment was significantly shorter after FluMel (15 vs 18 days, $\mathrm{p}<$
$.01)$. The 3-year overall survival (OS) and disease-free survival (DFS) were of 43.0 and $36.5 \%$, respectively, after FB2 and 54.9 and $52.0 \%$ after FluMel, respectively, and were not significantly different ( $\mathrm{p}=.41$ for OS and .15 for DFS), with a Karnofsky $>=90$ being the only factor significantly associated in univariate analysis with better OS and DFS ( $\mathrm{p}=.02$ for both). The cumulative incidence (CI) of grade 2 to 4 acute graft-versus-host disease (aGVHD) was $16.2 \%$ after FB2 and $38.3 \%$ after FluMel $(p<.001)$ and was associated in multivariate analysis with both $\mathrm{T}$ depletion and RIC type ( $\mathrm{p}<.001$ and .005 , respectively). The CI of chronic GVHD at 3 years was $13.9 \%$ in FB2 and $22.1 \%$ in FluMel group $(\mathrm{p}=.24)$. The CI of Non-relapse mortality at 3 years was $18.7 \%$ after FB2 and $29.6 \%$ after FluMel $(\mathrm{p}=.11)$. The CI of relapse at 3 years was $44.8 \%$ for the first and $18.4 \%$ for the second group $(\mathrm{p}<.001)$ and was associated with conditioning regimen in multivariate analysis $(\mathrm{p}=.02)$. No difference in 3-years GVHD-free/ relapse-free survival (GRFS) was observed between the two group (25.5\% for FB2 and 37.6\% for FluMel, $\mathrm{p}=.48$ ).

Conclusions: When comparing two RIC regimens for myeloid neoplasms, we observed a higher incidence of aGVHD after FluMel whereas no statistical difference was noted for the cGVHD occurrence. While the toxicity appears to be higher after FluMel, this result is counterbalanced by a higher proportion of relapse after FB2, accounting for no difference in OS, DFS and GRFS between the two groups. These findings could be partially explained by a larger proportion of patients receiving a partial T-depletion after FB2 RIC, but a larger trial is needed to clarify this issue.

Disclosure: Nothing to declare.

\section{P121}

Once-daily vs 4-times daily intravenous busilvex in conditioning regimen before allogeneic stem cell transplantation for patients with myeloid malignancies: Safety and efficacy

Nour Ben Abdeljelil ${ }^{\text {, Ons Hrizi }}{ }^{\text {, Maroua Ben Hmida }}{ }^{\text {, }}$ Amel Lakhal, Rym El Fatmi ${ }^{1}$, Lamia Torjemane ${ }^{1}$, Dorra Belloumi $^{1}$, Saloua Ladeb ${ }^{1}$, Tarek Ben Othman ${ }^{1}$

${ }^{1}$ Centre National de Greffe de Moelle Osseuse, Tunis, Tunisia

Background: Busilvex $(\mathrm{Bu})$ is part of standard conditioning regimen before allogeneic stem cell transplantation (ASCT) for patients with myeloid malignancies and usually administered as an intravenous (iv) infusion 4-times daily. This study aimed to compare the saftey and efficacy of this schedule to a once-daily iv $\mathrm{Bu}$. 
Methods: We conducted a retrospective study in adult patients ( $\geq 18$ years) with myeloid malignancies who received ASCT from HLA-identical sibling donors between January 2011 and June 2018 following iv Bu-based preparative regimens. Graft-versus host disease (GVHD) prophylaxis consited of cyclosporine and short course of methotrexate. Intravenous $\mathrm{Bu}$ was administered 4-times daily $(0.8 \mathrm{mg} / \mathrm{kg}$ every 6 hours $\mathrm{x} 12$ to 16 doses $)$ or oncedaily in a 3 -hour infusion ( $3.2 \mathrm{mg} / \mathrm{kg} \times 3$ to 4 days) since June 2015

Results: Ninty-nine patients were enrolled (54 men and 45 women). Median age was 35 years (range, $18-50$ y). The median time from diagnosis to ASCT was 5 months (range, 51days -7 years). Diagnosis were acute myeloid leukemia $(\mathrm{n}=79,80 \%)$, chronic myeloid leukemia $(\mathrm{n}=8,8 \%)$, myelodysplasic syndrome $(\mathrm{n}=6,6 \%)$, primitive myelofibrosis $(\mathrm{n}=4,4 \%)$ and chronic myelomonocytic leukemia $(\mathrm{n}=2,2 \%)$. Thirty-seven $(37.3 \%)$ patients had EBMT-score $\geq 2$. Sixty-five (65.6\%) patients were transplanted in CR1, 5 (5\%) beyond CR1 and 24 (24.2\%) had active disease. Conditioning regimens consisted of Bu/cyclophosphamide in 86 patients $(86.8 \%)$, Bu/fludarabine in 13 patients (13.1\%). Four-times daily $\mathrm{Bu}$ was given to 58 patients (58.5\%, groupe1) and once-daily Bu to 41 patients (41.5\%, groupe 2). Stem cell source were BM in 46 patients (46.5\%) and PBSC in 53 patients (53.5\%). Globally, patients characteristics were well balanced between the two groups. The rates of severe complications were similar between the two groups with no statistically significant differences except oral mucositis (table1).

\begin{tabular}{lccr}
\hline & $\begin{array}{l}\text { 4-times daily } \\
\mathrm{Bu}(\mathrm{n}=58)\end{array}$ & $\begin{array}{l}\text { Once-daily } \\
\mathrm{Bu}(\mathrm{n}=41)\end{array}$ & $\mathrm{p}$ \\
\hline Severe infections & $22(37.9 \%)$ & $11(26.8 \%)$ & 0.25 \\
Hepatic cytolysis & $33(56.9 \%)$ & $26(63.4 \%)$ & 0.51 \\
$\begin{array}{l}\text { Hepatic cholestasis } \\
\text { Sinusoidal occlusion }\end{array}$ & $19(32.8 \%)$ & $20(48.8 \%)$ & 0.11 \\
syndrome & $3(5.2 \%)$ & 0 & 0.27 \\
$\begin{array}{l}\text { Oral mucositis grade III-IV } \\
\text { Median time to }\end{array}$ & $16(65.5 \%)$ & $35(85.4 \%)$ & 0.03 \\
engraftemnt (days) & $19(33.3 \%)$ & $14(35 \%)$ & 0.87 \\
$\begin{array}{l}\text { Acute GVHD grade II- } \\
\text { IV (n=97) }\end{array}$ & $15(26.3 \%)$ & $13(32.5)$ & 0.51 \\
$\begin{array}{l}\text { CMV reactivation/ } \\
\text { infection (n=97) }\end{array}$ & $14($ range, $11-25)$ & 0.18 \\
Chronic GVHD (n=91) & $29(54.7 \%)$ & $16(42.1 \%)$ & 0.24 \\
\hline
\end{tabular}

\section{[[P121 Table] 1. Table1. Complications post ASCT]}

Non-relapse mortality (NRM) was comparable in the two groups $(21 \%$ and $17 \%$ in groups 1 and 2 , respectively, $\mathrm{p}=0.65$ ). The relapse rate was $24 \%$ and $32 \%$, respectively $(\mathrm{p}=0.4)$. After a median follow-up of 2 years (range, 5 days
- 7years), the OS was not significantly different between groups 1 and $2: 64 \%$ vs $56 \%(p=0.09)$. However, the RFS was significantly better in the groupe $1: 62 \%$ vs $56 \%$ $(\mathrm{p}=0.03)$.

Conclusions: Once-daily iv Bu regimen seems to be an efficient and safe alternative to the 4-times daily protocol. However, results should be interpreted with caution because the historical comparison and lack of Bu pharmacokinetics studies.

Disclosure: No conflict of interest

\section{P122}

Long-term follow-up of children with JMML underwent allogenic HSCT with busulfan or treosulfan-based conditionin

Veronika Konstantinova ${ }^{1}$, Elena Machneva ${ }^{I}$, Yulia Skvortsova $^{2}$, Kirill Kirgizov ${ }^{2}$, Alexandra Burya ${ }^{1}$, Ekaterina Prinstanskova ${ }^{1}$, Natalia Sidorova ${ }^{1}$, Olga Filina $^{1}$, Elena Skorobogatova ${ }^{1}$

${ }^{1}$ The Russian Children's Research Hospital, Moscow, Russian Federation, ${ }^{2}$ Dmitry Rogachev National Medical Research Center of Pediatric Hematology, Oncology and Immunology, Moscow, Russian Federation

Background: Standard therapy of the most patients with juvenile myelomonocytic leukemia (JMML) is allogeneic hematopoietic stem cell transplantation (aHSCT). The choice of optimal conditioning regimen for patients with JMML is crucial as well as long-term observation. We aimed to estimate the long-term follow-up and survival rates of patients with JMML after aHSCT with the help of Busulfan or Treosulfan-based conditioning regimens.

Methods: Thirty eight patients with JMML underwent aHSCT in 2002-2018. We compared equal groups of patients received Busulfan $(\mathrm{n}=19)$ and Treosulfan-based $(n=19)$ conditioning regimen. $M: F=28: 11$. Median of age at HSCT was 2.5 (0.6-5). Donor type: HLA-related 10/10 29\% ( $\mathrm{n}=11)$, HLA-related 9/10 - 2.6\% $(\mathrm{n}=1)$, HLAunrelated 10/10 - 39.4\% ( $\mathrm{n}=15)$, HLA-unrelated 9/10 $15.8 \%(n=6)$, and haploidentical - 13.2\% $(n=5)$. Stem cell source: BM - 55.3\% $(n=21)$, PBSC - 26.3\% $(n=10)$, UCB $10,5 \%(\mathrm{n}=4)$, and $\mathrm{UCB}+\mathrm{BM}-7.9 \%(\mathrm{n}=3)$. Disease status on HSCT: CR $-73.7 \%(n=28)$, Refractory $-26.3 \%(n=10)$.

Results: Median follow-up 15.5 months (1-129 months). The estimated 10-year overall survival (OS) probability in patients received Busulfan-based conditioning was 48,8 $\pm 13,4 \%$ in comparison with $68,0 \pm 10,8 \%$ in patients with Treosulfan-based regimen $(p=0,458)$. Event-free survival (EFS) was $42,1 \pm 11,3 \%$ in group with Busulfan-based regimen and $57,0 \pm 11,5 \%$ in patients with Treosulfanbased conditioning $(p=0,224)$. 


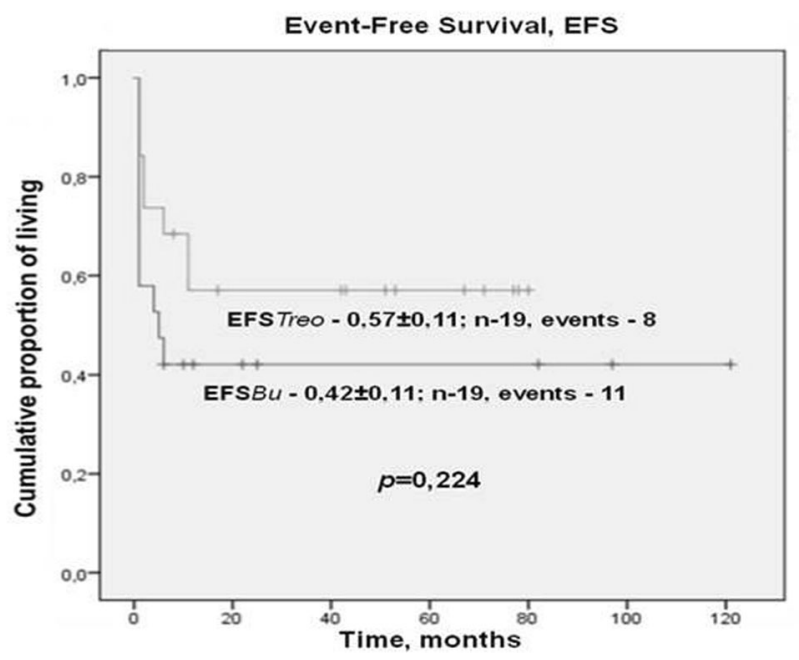

[[P122 Image] 1. EFS of patients with JMML after aHSCT]

Relapse-free survival (RFS) in patient's group with Busulfan-based regimen was $72,7 \pm 12,0 \%$, with Treosulfan-based conditioning - 81,5 $\pm 9,8 \% \quad(p=0,505)$. No statistical difference shown.

Conclusions: Long-term follow-up of patients with JMML underwent aHSCT showed satisfactory OS, EFS and RFS. There we no statistical difference shown between Busulfan and Treosulfan-based conditioning regimens.

Disclosure: Nothing to declare

\section{P123}

High dose sequential conditioning regimen followed by allogeneic hematopoietic transplantation for high-risk acute myeloid leukemia, myelodysplastic syndrome, myeloproliferative neoplasia patients: A multicenter study in Singapore

Elson Neo ${ }^{1,2}$, Michelle Poon ${ }^{1}$, Yeh Ching Linn ${ }^{3}$, William Hwang $^{3}$, Aloysius $\mathrm{Ho}^{3}$, Lip Kun Tan', Davanaliz R. Del Rosario ${ }^{1}$, Liang Piu Koh ${ }^{1,2}$

${ }^{1}$ National University Cancer Institute, Singapore, Singapore, ${ }^{2}$ National University of Singapore, Yong Loo Lin School of Medicine, Singapore, Singapore, ${ }^{3}$ Singapore General Hospital, Singapore, Singapore

Background: Post-transplant relapse remains the leading cause of treatment failure in high risk (HR) acute myeloid leukemia (AML), myelodysplastic syndrome (MDS), myeloproliferative neoplasia (MPNs) receiving allogeneic hematopoietic cell transplantation (allo-HCT), especially for patients with relapsed or refractory AML. Recently, a sequential transplant approach, as developed by the Munich group, comprising of intensive cytoreductive chemotherapy
FLAMSA (Fludarabine/Amsacrine/cytarabine) to decrease leukemia cell burden shortly prior to conditioning regimen, has been successfully used for high-risk (HR) AML/MDS with promising results.

Methods: We studied 48 patients (median age 53 years, range 26 - 68) with HR AML $(n=38)$, as defined by refractory, relapsed disease, secondary leukemia, or high/ very risk disease risk index risk, and $\operatorname{HR}$ MDS $(n=10)$ according to IPSS-R, undergoing allo-HCT using the sequential transplant approach in 2 institutions between January 2009 and October 2018. The sequential transplant approach combined a cytoreductive chemotherapy, which consisted of either FLAMSA $(n=17)$, FLAG +/- Ida (fludarabine/cytarabine/Granulocyte colony stimulating factor /Idarubicin) $(\mathrm{n}=23)$, or Clo-AraC (Clofarabine/Cytarabine) $(n=8)$, followed by reduced (RIC) $(\mathrm{N}=43)$ or myeloablative (MAC) $(\mathrm{N}=5)$ conditioning regimen. All patients received peripheral blood stem cell from matched related donors $(\mathrm{N}=27)$ matched unrelated donors $(\mathrm{N}=14)$, or mismatched unrelated donors $(\mathrm{N}=7)$. Post-grafting immunosuppression consisted of calcineurin inhibitor and mycophenolate mofetil in all patients. Thymoglobulin was added for GVHD prophylaxis for unrelated donor transplant.

Results: The median time to neutrophil $>1000 / \mu \mathrm{L}$ was 10 days (range, 9-25). With a median follow-up of 28.2 months (range, 1.4 to 103.1 months), the KaplanMeier estimate of leukemia-free (LFS) and overall survival (OS) at 5 years were $45 \%$ (95\% CI, 8-30), 46\% (95\% CI, 830), respectively. Patients receiving FLAG or Clo-AraC based sequential regimen showed a trend towards more favourable overall survival (OS) as compared to patients given FLAMSA ( 5 year OS: $53 \%$ vs $31 \%$; $\mathrm{p}=0.236$ ). At 2 years, the cumulative incidences of relapse and non-relapse mortality (NRM) were $46 \%$ (95\% CI, 31-60\%) and $15 \%$ (95\% CI, 7- $21 \%$ ), respectively. In multivariate analysis, the type of sequential conditioning regimen did not show any significant impact on LFS, OS, NRM or relapse.

Conclusions: Sequential transplant conditioning with FLAMSA, FLAG or Clo-AraC followed by allo-HCT is an effective strategy in overcoming the dismal prognosis of HR AML and MDS, and enabling long-term disease free survival. More studies on effective strategies such as posttransplant maintenance therapy of prophylactic donor lymphocyte infusion, are needed to further eliminate the risk of relapse, without increasing risk of treatment related toxicity.

Disclosure: Nothing to declare

\section{P124}

Optimization of the blood sampling procedure for busulfan therapeutic drug monitoring (TDM) 
Vera Domingos $^{1}$, Paulo Paixão ${ }^{2}$, Nuno Miranda, Margarida Pereira $^{2}$, Vera Pires ${ }^{1}$, Ana Sofia Jorge ${ }^{1}$, Elsa Oliveira $^{I}$, António Melo Gouveia ${ }^{1}$, Manuel Abecasis ${ }^{I}$

${ }^{1}$ Instituto Português de Oncologia de Lisboa, Lisbon, Portugal, ${ }^{2}$ Faculdade Farmácia da Universidade de Lisboa, Lisbon, Portugal

Background: We implemented the busulfan TDM program in 2014 and in January 2017 we switched from the multiple daily dosing (MDD) to the once daily dosing (ODD) regimen .

To optimize our sampling scheme (15 minutes, 1, 2, 3, 4, 6, 8 and 10 hours after the end of a 3-hour infusion), we reduced the number of blood samples collected, reducing nursing and laboratory staff time and increasing patient convenience.

This study aims to show the performance of a simplified sampling protocol which includes the first 5 samples from the original protocol.

Methods: Individual PK parameters were retrospectively estimated using 5 samples (simplified protocol) and were compared with those obtained after 8 samples (original protocol).

Individual PK parameter values for a one compartment model were estimated using a maximum likelihood estimation modelling algorithm (ADAPT 5.0) and the statistical analysis of the results was performed (Statgraphics Centurion XV).

Results: 43 patients [18 females, 25 males, 18 children and 25 adults, mean age 30.1 (10 months-62 years)] were included. 38 patients had malignant disease and 5 nonmalignant disease.

BU Clearance (CL) and AUCs values obtained for both protocols are summarized in table 1 .

\begin{tabular}{lll}
\hline & $\begin{array}{l}\text { Original Protocol - } \\
\text { Mean (SD) }\end{array}$ & $\begin{array}{l}\text { Simplified Protocol - } \\
\text { Mean (SD) }\end{array}$ \\
\hline CL D1 (L/h) & $8.07(3.10)$ & $8.12(3.14)$ \\
CL D2 (L/h) & $8.17(2.98)$ & $8.23(3.03)$ \\
CL D3 (L/h) & $6.51(2.54)$ & $6.53(2.56)$ \\
$\begin{array}{l}\text { AUC D1 } \\
(\mathrm{mg} * \mathrm{~h} / \mathrm{L})\end{array}$ & $21.2(5.3)$ & $21.1(5.1)$ \\
$\begin{array}{l}\text { AUC D2 } \\
(\mathrm{mg} * \mathrm{~h} / \mathrm{L})\end{array}$ & $19.7(3.8)$ & $19.6(3.8)$ \\
$\begin{array}{l}\text { AUC D3 } \\
(\mathrm{mg} * \mathrm{~h} / \mathrm{L})\end{array}$ & $23.6(6.5)$ & $23.5(6.5)$ \\
\hline
\end{tabular}

\section{[[P124 Table] 1. Busulfan CL and AUC values]}

Based on the approved dosage recommendations, mean (SD) initial dose was 171.1(69.3) mg. After TDM, mean (SD) calculated dose at day 1 for the remaining days (to achieve the defined target cumulative AUC) was 158,5 $(72,6) \mathrm{mg}$ obtained from the original protocol. According to the simplified protocol the result would be $159,8(73,7) \mathrm{mg}$. The median and the mean variation of the calculated dose were $0 \%$ and $1 \%(0-8 \%)$ between protocols.

A strong relationship between the CL of the day 1- 3 obtained from the original protocol and the simplified protocol is observed $\left(\mathrm{R}^{2}=0.9985\right)$.

This high correlation is also observed for patients with busulfan $t_{1 / 2}>3 h\left(R^{2}=0.9936\right)$, a population were the reduction of sampling could be more problematic.

ANOVA test for the $\log \mathrm{CL}$ with the factors: patient, day of busulfan and type of sampling protocol was performed. Sampling protocol was determined as non-statistically significant $(\mathrm{p}=0.7248)$.

Conclusions: Results suggest that both protocols are equivalent concerning to the busulfan $\mathrm{CL}$ estimation and calculated AUC. Variation between protocols regarding the calculated dose at day 1 for the remaining days to achieve the defined target cumulative AUC is considered acceptable.

We verified a strong relationship between busulfan CL obtained from both protocols and sampling protocol doesn't influence CL statistically.

A reduced sampling collection of 5 determinations until 4 $h$ after the end of the infusion is shown to be sufficient for the TDM of busulfan, so this was implemented in our centre in line with published data.

Disclosure: Nothing to declare

\section{P125}

Impact of anti-thymocyte globulin doses in unrelated hematopoietic stem cell transplantation for patients with myeloid neoplasm

Sung Hwa Bae ${ }^{1}$, Jun Yeb Nam ${ }^{1}$, Min Kyoung Kim ${ }^{2}$, Myung Soo Hyun ${ }^{2}$, Joon Ho Moon ${ }^{3}$, Yoo Jin Lee ${ }^{3}$, Sang Kyun Sohn ${ }^{3}$

${ }^{1}$ Daegu Catholic University Hospital, Daegu, Korea, Republic of, ${ }^{2}$ Yeungnam University Hospital, Daegu, Korea, Republic of, ${ }^{3}$ Kyungpook National University Hospital, Daegu, Korea, Republic of

Background: Anti-thymocyte globulin (ATG) is widely used for the prophylaxis of graft-versus-host disease (GVHD) in hematopoietic stem cell transplantation (HSCT). However, there is still controversy regarding the optimal dose of ATG. Therefore, we analyzed the impact of ATG doses in unrelated HSCT for patients with myeloid neoplasm.

Methods: This was a retrospective multi-center study that assessed the impact of ATG doses on clinical outcomes in 
patients with acute myeloid leukemia (AML) or myelodysplastic syndrome (MDS) undergoing an unrelated HSCT. The patients who received peripheral blood stem cells (PBSC) transplantation after conditioning regimens containing i.v. busulfan (BU), fludarabine and rabbit ATG between 2010 and 2017 were included in this study.

Results: A total of 96 patents, median age 45 years, with AML $(n=74)$ or MDS $(n=22)$ were included in our analyses. 66 patients $(69 \%)$ received a myeloablative regimen (i.v. BU>6.4 mg/kg). High-ATG (ATG $9 \mathrm{mg} / \mathrm{kg}$ ), intermediate-ATG (ATG $4.5-5 \mathrm{mg} / \mathrm{kg}$ ) and low-ATG (ATG $3 \mathrm{mg} / \mathrm{kg}$ ) were given in 11, 49 and 36 patients, respectively. After a median follow-up of 23 months, the cumulative incidence of extensive chronic GVHD was $9.1 \%$ in the high-ATG group, $13.8 \%$ in the intermediate-ATG group and $29.7 \%$ in the low-ATG group $(\mathrm{p}=0.31)$. The rate of 2 year relapse-free survival was significantly higher in the intermediate-ATG group than other groups $30 \%$ in the high-ATG group vs. $73.2 \%$ in the intermediate-ATG group vs. $53.8 \%$ in the low-ATG group, $\mathrm{p}=0.048$ ). The rate of 2year overall survival was similar $(45.5 \%, 68.5 \%$ and $44.8 \%$, respectively; $\mathrm{p}=0.09$ ). The rate of chronic GVHD-free and relapse-free survival at 2 years was significantly higher in the intermediate-ATG group (18.2\% in the high-ATG group vs. $53.8 \%$ in the intermediate-ATG group vs. $18.8 \%$ in the low-ATG group, $\mathrm{p}=0.0086$ ).

Conclusions: Our study shows that the incidence of extensive chronic GVHD was similar regardless of the doses of ATG after transplantation of PBSC from unrelated donor for patients with AML or MDS. However, the rate of relapsefree survival and the rate of a composite end point chronic GVHD-free and relapse-free survival were significantly higher in the intermediate dose $(4.5-5 \mathrm{mg} / \mathrm{kg})$ of ATG group.

Disclosure: Nothing to declare

\section{P126}

Autologous stem cell transplantation with reduced-dose beam/beac is feasible for patients $\geq 70$ years old and yields similar results as full-dose conditioning in patients aged 65-69

\section{Israel Henig ${ }^{1}$, Dana Yehudai-Ofir ${ }^{1}$, Ronit Leiba ${ }^{1}$, Noa Lavi $^{1}$, Shimrit Ringelstein-Harlev ${ }^{I}$, Tsila Zuckerman ${ }^{1,2}$}

${ }^{1}$ Rambam Health Care Campus, Haifa, Israel, ${ }^{2}$ Technion, Rappaport Faculty of Medicine, Haifa, Israel

Background: Autologous hematopoietic stem cell transplantation (ASCT) with BEAM/ BEAC conditioning is widely used in patients with lymphoma. There are no clear guidelines for dose adjustment in frail patients aged 65 and older. We aimed to study the feasibility of reduced-dose conditioning in this patient population.
Methods: We retrospectively retrieved data from the electronic medical records for consecutive patients aged 65 and older, who underwent an ASCT for lymphoma over the last 10 years at our institution.

Results: Forty four patients $\geq 65$ years old underwent ASCT between 1 Aug 2008 and 31 Aug 2018. Twenty eight of them received a reduced-dose conditioning (median $25 \%$, range 20\%-33\% dose reduction). The dose was reduced for $92 \%$ of patients $\geq 70$ years old and for $53 \%$ of patients aged 65-69. The outcomes of the following three groups of patients were compared: A) age $\geq 65$; without dose reduction, B) age 65-69; with dose reduction and C) age $\geq 70$; with dose reduction (table 1). Only one patient aged 70 received full-dose conditioning. There was no significant difference between the groups in the number of previous chemotherapy cycles (median 2, range 1-3). However, significantly more patients at the age of 65-69 were in complete remission $(\mathrm{CR})$ pre-transplant in both full and reduced-dose conditioning groups (A and $\mathrm{B}$ ). No significant intergroup differences were observed in the occurrence of complications (mucositis and infections), day 30 transplantrelated mortality (TRM) or engraftment day. Similarly, no significant differences were found either in the 1-year progression-free survival (PFS), which was 50\%, 64\% and $50 \%$, or 1-year non-relapse mortality (NRM), which was $17 \%, 7 \%$ and $10 \%$, respectively for groups A, B and C. The 1-year overall survival (OS) tended to be higher in group B (85\%), compared to groups A $(66 \%)$ and $\mathrm{C}(70 \%)$.

Conclusions: BEAM/BEAC conditioning dose reduction was not found to adversely affect 1-year PFS and OS rates. Despite the fact that $2 / 3$ of the patients in the age group $\geq 70$ underwent ASCT in partial remission and had dose reduction, theier achieved TRM, PFS and OS rates were similar to those of patients aged 65-69. BEAM/BEAC conditioning at a 75\%dose may be a suitable option for patients in their seventh decade requiring ASCT. This strategy should be further evaluated in prospective clinical trials.

\begin{tabular}{|c|c|c|c|c|}
\hline Group & $\begin{array}{l}\text { A) age } \geq 65,100 \% \\
\text { dose }(n=16)\end{array}$ & $\begin{array}{l}\text { B) age } 65-69,75 \% \\
\text { dose }(n=17)\end{array}$ & $\begin{array}{l}\text { C) age } \geq 70,75 \% \\
\text { dose }(\mathrm{n}=11)\end{array}$ & P-value \\
\hline BEAM/BEAC & $69 \% / 31 \%$ & $82 \% / 18 \%$ & $81 \% / 19 \%$ & ns* \\
\hline CR pre-ASCT & $80 \%$ & $65 \%$ & $27 \%$ & $\begin{array}{l}\mathrm{P}<0.001^{*} \\
(\mathrm{~A} \text { vs. C) }\end{array}$ \\
\hline Day 30 TRM & $6 \%$ & $6 \%$ & $9 \%$ & ns* \\
\hline $\begin{array}{l}\text { Mucositis/infection } \\
\text { complications }\end{array}$ & $69 \% / 31 \%$ & $53 \% / 35 \%$ & $54 \% / 45 \%$ & $\mathrm{~ns} *$ \\
\hline $\begin{array}{l}\text { CD34 }(* 106 / \\
\mathrm{kg}) \text { dose }\end{array}$ & $\begin{array}{l}6.6 \text { (range 2.9- } \\
25.5 \text { ) }\end{array}$ & 5.75 (range $2.5-30$ ) & $\begin{array}{l}5.9 \text { (range 5.1- } \\
11.8 \text { ) }\end{array}$ & $\mathrm{ns} \ddagger$ \\
\hline Engraftment day & 10 (range 9-12) & 11 (range 8-16) & 11 (range 9-21) & nsł \\
\hline 1-year PFS & $50 \%$ & $64 \%$ & $50 \%$ & $\mathrm{~ns} *$ \\
\hline 1-year OS & $66 \%$ & $85 \%$ & $70 \%$ & ns* \\
\hline 1-year NRM & $17 \%$ & $7 \%$ & $10 \%$ & $\mathrm{~ns}^{*}$ \\
\hline
\end{tabular}

[[P126 Table] 1. Patient information; NRM - Nonrelapse mortality; * Chi-square test, $\neq$ One way ANOVA]

Clinical Trial Registry: N/A 
Disclosure: Nothing to declare

\section{P127}

Reduction in autologous transplant related mortality by modifying conditioning regimen protocol

\section{Natasha Ali, Kashif Shahzad ${ }^{1}$, Bakhtawar Altaf ${ }^{1}$, Ahmed Raheem $^{1}$, Mohammad Usman Shaikh ${ }^{1}$, Salman Adil ${ }^{1}$}

${ }^{1}$ Aga Khan University, Karachi, Pakistan

Background: The transplant related mortality in autologous transplants for lymphoma and multiple myeloma, reported worldwide ranges from 0-5\%. From 2004-2015, the TRM at our center for these two diseases was approximately $20 \%$. We introduced changes in mobilization schedule, conditioning regimens and drug dosages to determine whether these changes affected the transplant related mortality and overall survival.

Methods: From April 2004- December 2015, we used BEAM (BCNU: $300 \mathrm{mg} / \mathrm{m}^{2}$ on day -6 ; Etoposide $200 \mathrm{mg} /$ $\mathrm{m}^{2}$ on days -5 to -2 , Cytarabine $200 \mathrm{mg} / \mathrm{m}^{2}$ on days -5 to -2 and Melphalan $140 \mathrm{mg} / \mathrm{m}^{2}$ on day -1 as conditioning chemotherapy for patients admitted in transplant unit for autologous transplants in Hodgkin's and Non-Hodgkin's Lymphoma. In patients with multiple myeloma high dose Melphalan $\left(200 \mathrm{mg} / \mathrm{m}^{2}\right)$ was used. The mobilization protocol consisted of Cyclophosphamide $1.5 \mathrm{gm} / \mathrm{m}^{2}$ followed by GCSF $5 \mu \mathrm{gm} / \mathrm{kg}$ twice daily till stem cell collection was completed. From January 2016, we changed the BEAM protocol to BendaEAM with dose modifications that included: bendamustine $150 \mathrm{mg} / \mathrm{m}^{2}$ on days -5 and -4 , cytarabine $150 \mathrm{mg} / \mathrm{m}^{2}$ on days -5 to -2 , etoposide $150 \mathrm{mg} / \mathrm{m}^{2}$ on days -5 to -2 and melphalan $100 \mathrm{mg} / \mathrm{m}^{2}$ on day -1 . For multiple myeloma Melphalan was reduced to $150 \mathrm{mg} / \mathrm{m}^{2}$. We used only GCSF for mobilization of stem cells, which was continued till stem cell harvest was complete. Response to treatment was evaluated by comparing TRM and overall survival for two time periods: 2004-2015 and from 2016 till date.

Results: From April 2004 till December 2015, n=78 autologous transplants were performed. The male:female ratio was 2.4:1. Fifty seven patients underwent transplant for lymphomas, $n=16$ for multiple myeloma and $n=5$ for other diagnosis. Median age was 23 \pm 14.6 (2-64 years). The mean MNC was $4.7 \times 10^{8} \pm 1.7 / \mathrm{kg}$. Engraftment was achieved in $80 \%$ of patients. The transplant related mortality was $19.5 \%$ and overall survival was $72 \%$ (follow up: 104 months). Since January 2016 till March 2018 we have performed $n=18$ autologous transplants of which $n=17$ were males. Fifteen transplants were performed for lymphomas (NHL:8, HD:7) and $n=3$ for multiple myeloma. Median age was $24 \pm 15$ (20 - 64 years). The mean mononuclear cell count was $5.8 \times 10^{8} / \mathrm{kg}$ and the mean CD34 count was $3.7 \times 10^{6} / \mathrm{kg}$. Engraftment was achieved in all patients. The transplant related mortality was $0 \%$ and the overall survival was $83 \%$ (follow up 22 months).

Conclusions: We were able to reduce the autologous transplant related mortality to $0 \%$ by decreasing dosages of conditioning chemotherapy and changing the mobilization protocol. Long follow-up is needed to determine late mortality and late relapse in comparison to standard chemotherapy dosages

Disclosure: Nothing to declare

\section{P128}

Risk and benefit of thiotepa based conditioning followed by autologous stem cell transplantation in high risk lymphomas

\section{Zoltan Csukly ${ }^{1}$, Laszlo Gopcsa ${ }^{1}$, Aniko Barta ${ }^{2}$, Melinda} Paksi $^{2}$, Eszter Sari ${ }^{2}$, Mariann Reti ${ }^{2}$, Peter Remenyi ${ }^{2}$

${ }^{1}$ South-Pest Hospital Centre, Haematology, Budapest, Hungary, ${ }^{2}$ South-Pest Hospital Centre, Budapest, Hungary

Background: The purpose of this study was to assess transplantation related mortality, overall survival (OS) and disease free survival (DFS) of patients with high risk Hodgkin's (HD), primary refractory diffuse large B cell lymphoma (DLBCL) and primary central nervous system lymphoma (PCNSL) who received thiotepa based high-dose chemotherapy and autologous stem-cell transplantation (ASCT).

Methods: Data were reviewed of $15 \mathrm{HD}, 11 \mathrm{DLBCL}$ and 11 PCNSL patients who received ASCT between 2013 and 2018. Patients caracteristics was 16 men and 21 women, median age at diagnosis 36 years (16-62). Stage (AnnArbor) at diagnosis of HD/DLBCL/PCNSL: stage IE $n=0 /$ $0 / 10(0 / 0 / 91 \%)$, stage II $n=7 / 2 / 0(47 / 18 / 0 \%)$, stage III $n=2 /$ $0 / 1(13 / 0 / 9 \%)$, stage IV $n=6 / 9 / 0(40 / 82 / 0 \%)$. Median time from diagnosis to ASCT HD/DLBCL/PCNSL: 25/11/ 6 month (4-72). Induction treatment in HD patients was ABVD, in most DLBCL patients R-CHOP and in PCNSL patients high dose methotrexate and cytosin arabinoside. Tumor status at ASCT HD/DLBCL/PCNSL: complete metabolic remission (CMR) $n=4 / 1 / 11(27 / 9 / 91 \%)$ and from PCNSL patient's $n=8(73 \%)$ were in first complete remission (CR1). Type of stem cell graft was periferial blood stem cell in all case. Conditioning: thiotepa $(250 \mathrm{mg} /$ $\mathrm{m} 2$ on days -9 to -7 , busulphan $3,2 \mathrm{mg} / \mathrm{kg}$ on days -6 to -4 and cyclophosphamide $60 \mathrm{mg} / \mathrm{kg}$ on days -3 to -2 plus rituximab $500 \mathrm{mg} / \mathrm{m} 2$ on day -10 in DLBCL and PCNSL. Median follow up from ASCT 711 days (31-1554). Tumor stage at ASCT was defined with computer tomography with positron emission tomography (PET-CT). 
Results: Median time of engraftment was 10 days (9-14). Thiotepa caused toxicoderma appeared at 9 (24\%) patients. Cytomegalovirus (CMV) reactivation was seen in $3(8 \%)$ cases with low DNA content $(284,454,5500$ copies $/ \mathrm{ml})$ and responded completely to oral valgancyclovir therapy. Transplantation related mortality HD/DLBCL/PCNSL $\mathrm{n}=$ $2 / 3 / 1(9 / 27 / 13 \%)$, in 4 cases bacterial sepsis and one systemic mycoses and one pulmonary fibrosis. Incidence of long-lasting grade III-IV thrombocytopenia and anaemia: $\mathrm{n}=15(40,5 \%)$ and $\mathrm{n}=3(8 \%)$, median time of duration from transplantation 71 days (31-720) and 35 days (31-85) respectively. Two-year OS of HD/DLBCL/PCNSL: 87/55/ $82 \%$. Two-year DFS of HD/DLBCL/PCNSL: $73 / 27 / 82 \%$.

Conclusions: Conditioning caused side effects (toxicoderma, CMV reactivation, long-lasting cytopenias) are frekvent but a managable problem. Life threatening comlications such as bacterial and fungal infections are more frekvent than after $\mathrm{B}(\mathrm{T}) \mathrm{EAM}$ conditioning. TRM was much highest in DLBCL patients (27\%) wich is associated with high number of active tumor status at transplantation (91\%). In contrast to DLBCL patients with poor DFS, patients with PCNSL and HD can gain benefit with this conditioning. For PCNSL patients is important to transplant in CR1 and it must be carried out as soon as possible after induction treatment.

Disclosure: Nothing to declare

\section{P129}

Busulfan and thiotepa conditioning regimen for autologous stem cell transplantation in patients with multiple myeloma

Sung-Hoon Jung ${ }^{1}$, Seo-Yeon Ahn ${ }^{1}$, Deok-Hwan Yang ${ }^{1}$, Hyeoung-Joon Kim ${ }^{1}$, Jin Seok Kim ${ }^{2}$, Kihyun Kim ${ }^{3}$, Hyeon-Seok Eom ${ }^{4}$, Ho-Young Yhim ${ }^{5}$, Yoo Jin Lee ${ }^{6}$, Shin Young Hyun ${ }^{7}$, Je-Jung Lee ${ }^{I}$

${ }^{1}$ Chonnam National University Hwasun Hospital, Hwasungun, Korea, Republic of, ${ }^{2}$ Severance Hospital, Yonsei University College of Medicine, Seoul, Korea, Republic of, ${ }^{3}$ Samsung Medical Center, Seoul, Korea, Republic of, ${ }^{4}$ National Cancer Center, Goyang-si, Korea, Republic of, ${ }^{5}$ Chonbuk National University Medical School, Jeonju, Korea, Republic of, ${ }^{6}$ Kyungpook National University Hospital, Daegu, Korea, Republic of, ${ }^{7}$ Wonju Severance Christian Hospital, Wonju, Korea, Republic of

Background: This study evaluated the efficacy and toxicity of intravenous busulfan and thiotepa as a conditioning regimen for autologous stem cell transplantation (ASCT) in patients with multiple myeloma (MM).

Methods: We retrospectively analyzed the data of 68 patients with MM who received the intravenous busulfan and thiotepa conditioning for ASCT between November 2016 and April 2018 in Korea.

Results: The median time to transplant was 5.4 months, and 66 patients $(97.1 \%)$ underwent ASCT within 12 months of the diagnosis. The overall response rate after ASCT was $95.6 \%$, including $55.9 \%$ with complete response, $22.1 \%$ with very good partial response, and $17.6 \%$ with partial response. The most common severe non-hematologic toxicity (grade 34) was infection $(44.1 \%)$. Three patients $(4.4 \%)$ developed venous-occlusive disease. One patient $(1.5 \%)$ died due to severe pneumonia after ASCT. After a median follow-up of 13.0 months, the median progression-free survival (PFS) and overall survival (OS) were not reached.

Conclusions: In conclusion, a conditioning regimen of intravenous busulfan and thiotepa was effective and tolerable.

Clinical Trial Registry: Not applicable

Disclosure: The authors have declared no conflicts of interest.

\section{P130}

Myeloablative haploidentical bone marrow transplantation with post-transplant cyclophosphamide in paediatric patients with haematological malignancies

Santanu Sen ${ }^{I}$, Sameer Tulpule

${ }^{1}$ Kokilaben Dhirubhani Ambani Hospital, Mumbai, India

Background: Haploidentical transplants have been shown to be safe and effective in treating haematological malignancies in the paediatric population. We have previously reported on our experience of using reduced intensity conditioning with post Transplant Cyclophosphamide in Haploidentical patients. We herein report our experience of using a TBI based Myeloablative conditioning to treat our first 8 patients with haematological malignancies.

Methods: 8 patients were enrolled in the study, 5 with relapsed Acute Lymphoblastic Leukemia (ALL) and 3 with relapsed/resistant Acute Myeloid Leukemia (AML). All AML patients had genetic markers of high risk disease and all ALL patients had very early relapses (either on therapy or within 6 months of stopping therapy). All patients were conditioned with an identical protocol using TBI-based myeloablative preparative regimen (Fludarabine $30 \mathrm{mg} / \mathrm{m}^{2} / \mathrm{d} \times 4 \mathrm{~d}$ and TBI $150 \mathrm{cGy}$ bid on $\mathrm{d}-4$ to -1 [total dose $1200 \mathrm{cGy}$ ]) followed by an infusion of unmanipulated peripheral blood stem cells from a Haploidentical family donor. Postgraft immunosuppression consisted of Cyclophosphamide $50 \mathrm{mg} / \mathrm{kg} / \mathrm{day}$ on days 3 and 4, Mycophenolate Mofetil through day 35, and Tacrolimus through day 180.

Results: Median time of neutrophil and platelet engraftment was 11 and 19 days, respectively. All patients 
achieved sustained complete donor chimerism by day +28 . Acute GVHD, grades II-IV and III-IV, was seen in $75 \%$ and $25 \%$, respectively. Disease progression occurred in 2 patients: $8 \& 10$ months after transplant and there was one death due to severe fungal infection. Estimated twoyear survival and relapse were $75 \%$ and $24 \%$, respectively. 2 patients had severe BK viremia and CMV reactivation occurred in 4 patients. All patients were successfully managed with appropriate supportive and antiviral therapy.

Conclusions: We report good outcome with a myeloablative conditioning in haploidentical transplants with excellent engraftment and hopefully a longer life expectancy. With small number of patients, it is difficult to state whether using a myeloablative conditioning would lead to better long term outcomes in this cohort of patients with very haematological malignancies, but we certainly showed that it is possible to achieve excellent early results.

Disclosure: Nothing to declare

\section{P131}

Fludarabine in combination with melphalan and ATG can be the best conditioning for hematopoietic stem cell transplant of children with hemophagocytic lymphohistiocytosis

\section{Gholamreza Bahoush Mehdiabadi ${ }^{I}$}

\section{${ }^{1}$ Iran University of Medical Sciences (IUMS), Pediatrics,} Tehran, Iran, Islamic Republic of

Background: Allogeneic hematopoietic cell transplantation (HCT) with myeloablative conditioning for disorders associated with excessive inflammation such as hemophagocytic lymphohistiocytosis (HLH) is associated with early mortality. The superior overall survival rate of HCT using reduced intensity conditioning (RIC) regimen for HLH patients compared to myeloablative conditioning were reported by various group since 2006 .

Methods: In this prospective study, we analyzed the outcome of two pediatric patients with HLH who had received HSCT, using reduced-intensity conditioning (RIC) regimen. They received the same RIC regimen based on the use of Fludarabine (30 mg/m $/$ day for 5 days) in combination with melphalan $\left(70 \mathrm{mg} / \mathrm{m}^{2} /\right.$ day for 2 days) and horse antithymocyte globulin (ATG $10 \mathrm{mg} / \mathrm{kg} / \mathrm{d}$ for 4 days). Cyclosporine and Methotrexate were used as graft-vs.-host disease (GvHD) prophylaxis.

Results: A 2 months boy with primary HLH (FHL2) was transplanted from his mother and a 4 years girl with secondary HLH was transplanted from her brother. Both of donors were HLA match with their recipients. They were received $6 \times 10^{6} / \mathrm{kg}$ and $10 \times 10^{6} / \mathrm{kg} \mathrm{CD} 34^{+}$cells from the harvested peripheral blood stem cells, respectively. They achieved full neutrophil and platelet recovery. The time to neutrophil recovery was 13 and 11 days, respectively. Full chimerism was achieved for both of them. In addition, they was developed grade 3 and 2 of acute GvHD, respectively. GvHD was completely controlled with prednisolone. They are alive and in complete remission without any significant complications after 36 and 14 months, respectively.

Conclusions: It appears that Fludarabine in combination with Melphalan and ATG may be the best conditioning regimen for hematopoietic stem cell transplant of children with HLH. Due to a few number cases of this study, a study with sufficient sample size is required.

Disclosure: "Nothing to declare"

Early complications / late effects \& quality of life / fertility

\section{P132}

Associations among depression, antidepressant use, survival, and quality of life in hematopoietic cell transplant recipients

Anna Baratal, Brian D. González $z^{2}$,Jun-Min Zhou ${ }^{3}$, Jongphil Kim ${ }^{3}$, Hailey Bulls ${ }^{2}$, Areej El-Jawarhi ${ }^{4}$, Heather Jim $^{2}$

${ }^{1}$ Hospital de la Santa Creu i Sant Pau and Josep Carreras Leukemia Research Institute, Barcelona, Spain, ${ }^{2}$ Moffitt Cancer Center, Tampa, FL, United States, ${ }^{3}$ Moffitt Cancer Center, Tampa, FL, United States, ${ }^{4}$ Massachusetts General Hospital, Boston, MA, United States

Background: Hematopoietic cell transplant (HCT) recipients often report depression and impaired quality of life (QOL) before transplant. Mixed evidence suggests depression may be a risk factor for greater mortality and worse QoL. Inconsistent findings may be due to the fact that previous studies have not evaluated antidepressant use. The aim of the study was to compare pre-transplant patientreported physical functioning and post-transplant overall survival (OS) between four groups of HCT recipients: 1) non-depressed/taking antidepressant (treated depression), 2) depressed/taking antidepressant (undertreated depression), 3) depressed/not taking an antidepressant (untreated depression), and 4) not depressed/not taking an antidepressant (control). It was hypothesized that physical functioning and OS would be worse among patients with untreated and undertreated depression relative to those with treated depression and controls.

Methods: This retrospective case-control study included patients completing depression (PHQ-8) and quality of life (SF-12) questionnaires at pre-transplant. Analyses were conducted separately for allogeneic and autologous recipients. 
Results: Participants $(\mathrm{N}=1,797)$ were $58 \%$ men, mean age 57 years (19-79), 39\% allogeneic recipients. Regarding depression and antidepressant use, 146 (21\%) allogeneic patients were characterized as having treated depression, 47 (7\%) as untreated depression, $49(7 \%)$ as undertreated depression, and $461(65 \%)$ as controls. Hierarchical linear regression models indicated that after adjusting for significant univariate factors (performance status, disease status, and regimen intensity), allogeneic patients with treated depression $(B=-2.58,95 \% \quad C I=-4.63,-0.54)$ reported better physical functioning than patients with undertreated depression $(\mathrm{B}=-$ $6.06,95 \% \mathrm{CI}=-9.43,-2.70)$ and untreated depression $(\mathrm{B}=-$ $7.48,95 \% \mathrm{CI}=-10.70,-4.25)$ but worse physical functioning than controls ( $\mathrm{p}$ values <0.05). Cox regression models indicated depression/antidepressant usage was not associated with OS among allogeneic patients ( $\mathrm{p}$ values $\geq 0.10$ ).Among autologous patients, $195(17.82 \%)$ were characterized as having treated depression, $83(7.59 \%)$ as untreated depression, $77(7.04 \%)$ as undertreated depression, and $739(67.55 \%)$ as controls. Hierarchical linear regression models indicated that after controlling for significant univariate factors (gender, performance status, diagnosis, and disease status), autologous patients with treated depression $(B=-2.97,95 \% \quad C I=-4.71$, -1.23) reported better physical functioning than patients with undertreated depression $(\mathrm{B}=-8.63,95 \% \mathrm{CI}=-11.23,-6.03)$ and untreated depression $(B=-8.62,95 \% \mathrm{CI}=11.15,-6.08)$, but worse physical functioning than controls ( $\mathrm{p}$ values $<0.05$ ). Cox regression models showed depression/antidepressant usage was associated with OS ( $\mathrm{p}$ values $\leq 0.05$ ), with patients with treated depression demonstrating significantly worse OS than other groups ( $p=0.05)$, but this association was no longer significant in multivariate analyses controlling for diagnosis and disease status $(\mathrm{p}=0.09)$.

Conclusions: Patients with untreated or undertreated depression pre-transplant may benefit from depression screening and treatment to improve physical functioning.

Disclosure: HSLJ: Consultant for RedHill Biopharma and Janssen Scientific Affairs

\section{P133}

Eltrombopag (EPAG) induces a high percentage of responses in patients WITH post allo-HSCT poor graft function (PGF) and no active GVHD

\section{Lourdes Aguirre', Aitziber Lizardi, Pilar Bachiller', Brigida Esteban ${ }^{1}$, Carmen González ${ }^{1}$, Nagore Argoitia ${ }^{1}$, María Araiz ${ }^{1}$, Aranzazu Aguirre ${ }^{1}$, Anunciación Urquía ${ }^{1}$, Carlos Vallejo ${ }^{1}$}

${ }^{1}$ University Hospital Donostia, San Sebastián, Spain

Background: Persistent cytopenia is a life-threating complication after HSCT. Several causes can lead to this situation (viruses, GVHD, drugs, etc). A specific entity is the one called "poor graft function (PGF)", which is diagnosed in pts with $\geq 2$ cytopenias after day +30 , in the presence of donor chimerism and the absence of GVHD or relapse. PGF is more frequent after alternative allo-HSCTs, such a haplo-identical, mismatched, or UCB. Several therapeutic approaches for PGF, with poor results, have been tested. Recently, EPAG has been shown to improve platelet counts in the post-allo-HSCT setting. In this study, we analysed the efficacy of EPAG in pts with post-transplant persistent cytopenias.

Methods: The population analyzed includes all 175 pts who underwent allo-HSCT from June 2015 through May 2018 in our Unit. Median age was 52 years (12-69). 102 were male $(58.3 \%)$ and 73 female $(41.7 \%)$. Baseline diseases were: 69 AML, 39 LPD, 20 ALL, 18 MDS, 15 MPD, 9 MM, and 5 BMF. Donor was unrelated in $101(54.3 \%)$ and was family in $74(42.3 \%)$ (including 25 haplo-identical). Conditioning was RIC in $95(54.3 \%)$ and intensive in $80(45.7 \%)$. SC source was PB in $164(93.7 \%)$ and BM in $11(6.3 \%)$. Median followup was 24 months (6-41). EPAG was initiated at some point during the first 6-month post-HSCT period in 12 pts $(6.9 \%$ of the series) due to thrombocytopenia $(<20000 / \mathrm{mcL})$ plus, at least, one other cytopenia. Patients characteristics shown in table 1. EPAG was started at $50 \mathrm{mg} /$ day and escalated each 2 weeks to 75,125 and $150 \mathrm{mg} /$ day if platelet count was < 20000/mcL. Global response was considered when, after EPAG, the patient needed no transfusions and reached the three of the following: platelets $>50000 / \mathrm{mcL}, \mathrm{Hgb}>10 \mathrm{~g} / \mathrm{dL}$, and ANC $>1000 / \mathrm{mcL}$. EPAG was tapered off in responders and discontinued if no response was reached after 16 weeks.

Results: At EPAG initiation, all the 12 pts had thrombocytopenia $(<20000 / \mathrm{mcL}), 10$ had anemia $(\mathrm{Hgb}<$ $10 \mathrm{~g} / \mathrm{dL}$ ), and 5 had neutropenia (ANC $<1000 / \mathrm{mcL}$ ). Counts pre and post and response to EPAG are shown in table 2. Among the 8 responders, all but one (who relapsed from thrombocytopenia and died from bleeding) were alive at analysis close (87.5\%). Among the 4 non-responders, three pts had GVHD-associated cytopenias, and finally died from infectious complications; the other patient relapsed from her AML, reached a new CR after treatment, and is alive and well 27 months afterwards. EPAG was tapered off and discontinued in 6/8 pts who responded; $2 / 8$ responders are still on EPAG. EPAG was discontinued in the $4 / 4$ pts who did not respond. Rest of treatment details shown in table 2 .

Conclusions:

1) EPAG worked striking well in subjects with PGF, an otherwise a life-threatening situation for patients.

2) EPAG induced impressive responses in platelets, but strong bilinear and trilinear responses were also seen.

3) EPAG did not improve GVHD-associated cytopenias. 
4) To confirm these innovative and transcendent results, we have just initiated a multicenter prospective study on the role of EPAG for treatment of post-HSCT PGF.

\begin{tabular}{ll}
\hline & $\begin{array}{l}\text { EPAG after allo- } \\
\text { HSCT (n=12) }\end{array}$ \\
\hline $\begin{array}{l}\text { Age (years) (median [range]) } \\
\text { Gender (male/female) }\end{array}$ & 59 [24-69] \\
$\begin{array}{l}\text { Baseline disease (AML // NHL // ALL // } \\
\text { MDS // MF // AA) }\end{array}$ & $3(58.3 \%) / / 5(41.7 \%)$ \\
Donor (URD* // Haplo** // MSD) & $\mathbf{6} 1 / / 1 / / 1$ \\
Conditioning intensity (RIC // Intensive) & $9(75 \%) / / 3(25 \%)$ \\
$\begin{array}{l}\text { SC source (PB // BM) } \\
\text { Pre-HSCT transfusion load (PBRC) } \\
\text { (median [range]) }\end{array}$ & $11(91.7 \%) / / 1(8.3 \%)$ \\
$\begin{array}{l}\text { Pre-HSCT ferritin level (ng/mL) (median } \\
\text { [range]) }\end{array}$ & $1232[22-122]$ \\
\hline
\end{tabular}

* Five out of the six URDT were mismatched

** The donor was a woman in the six cases: three sisters and three daughters

[[P133 Table] 1. Characteristics of patients and transplants

\begin{tabular}{ll}
\hline & $\begin{array}{l}\text { EPAG after allo- } \\
\text { HSCT (n=12) }\end{array}$ \\
\hline $\begin{array}{l}\text { EPAG initiation (post-HSCT) } \\
\text { (median [range]) }\end{array}$ & Day $+103[17-178]$ \\
$\begin{array}{l}\text { EPAG treatment duration (median } \\
\text { [range]) }\end{array}$ & 19 weeks [2-84] \\
$\begin{array}{l}\text { EPAG maximum dose (median } \\
\text { [range]) }\end{array}$ & 150 mg/day [125-150] \\
$\begin{array}{l}\text { Platelets pre-EPAG // post-EPAG* } \\
\text { (n=8) (median [range]) }\end{array}$ & $13000[1000-18000] / /$ \\
$\begin{array}{l}\text { Hgb pre-EPAG // post-EPAG* } \\
\text { (n=3) (median [range]) }\end{array}$ & $104000[39000-171000]$ \\
$\begin{array}{l}\text { ANC pre-EPAG // post-EPAG* } \\
\text { (n=3) (median [range]) }\end{array}$ & $16.1]$ \\
Global response to EPAG & 470 [70-660] // 3020 [1830- \\
$\quad$ - Poor graft function (no & $5200]$ \\
GVHD) (N=9) & \\
- GVHD-associated cytopenia (N=3) & \\
\hline
\end{tabular}

*In responders

[[P133 Table] 2. EPAG treatment and response]

Disclosure: Nothing to declare

\section{P134}

Pooled safety analysis of defibrotide treatment in patients with HEPATIC veno-occlusive disease/ sinusoidal obstruction syndrome (VOD/SOS) following hematopoietic stem cell transplantation (HSCT)

Paul Richardson', Angela Smith ${ }^{2}$, Nancy Kernan ${ }^{3}$, Leslie Lehmann $^{4}$, Robert Ryan ${ }^{5}$, William Tappe ${ }^{5}$, Stephan Grupp $^{6}$

${ }^{1}$ Jerome Lipper Multiple Myeloma Center, Dana-Farber Cancer Institute, Harvard Medical School, Boston, MA, United States, ${ }^{2}$ University of Minnesota, Minneapolis, MN, United States, ${ }^{3}$ Memorial Sloan Kettering Cancer Center, New York, NY, United States, ${ }^{4}$ Center for Stem Cell Transplantation, Dana-Farber Cancer Institute, Harvard Medical School, Boston, MA, United States, ${ }^{5}$ Jazz Pharmaceuticals, Palo Alto, CA, United States, ${ }^{6}$ The Children's Hospital of Philadelphia, Philadelphia, PA, United States

Background: Hepatic VOD/SOS with multi-organ dysfunction (MOD; typically, renal or pulmonary) may be associated with $>80 \%$ mortality. Defibrotide is approved for treating severe hepatic VOD/SOS post-HSCT in patients aged $>1$ month in the EU, and for hepatic VOD/SOS with renal or pulmonary dysfunction post-HSCT in the US. This analysis provides an overview of the safety results from 3 studies of patients with VOD/SOS, with or without MOD, who received defibrotide $25 \mathrm{mg} / \mathrm{kg} /$ day.

Methods: Safety data were pooled from patients with VOD/SOS post-HSCT treated with defibrotide in a phase 3 trial $(n=102)$ and a phase 2 , randomized dose-finding trial $(\mathrm{n}=74$ receiving $25 \mathrm{mg} / \mathrm{kg} /$ day). Safety data for historical controls (HC) from the phase 3 study $(n=32)$ also are provided. Reported separately, due to differences in patient population and data monitoring protocol, are AEs from the expanded-access program (T-IND) in patients with VOD/ SOS with and without MOD ( $\mathrm{n}=1000$ post-HSCT). VOD/ SOS was diagnosed by Baltimore criteria/biopsy for the phase $2 / 3$ studies; diagnosis by Baltimore or modified Seattle criteria was permitted in the T-IND.

Results: Median patient age at HSCT for the phase 2/ 3 studies was 24.0 years, 18.0 years for the $\mathrm{HC}$, and 14.0 years for the T-IND.

In the phase $2 / 3$ studies defibrotide-treated group $(\mathrm{n}=176), 169(96.0 \%)$ experienced AEs; most common (>10\%) were hypotension (36.9\%), diarrhea $(24.4 \%)$, and multi-organ failure $(21.6 \%)$. Treatment-related AEs were at least possibly related to defibrotide (Table). Any hemorrhage (an AE of special interest) occurred in 101 patients (57.4\%); most commonly epistaxis (13.6\%), gastrointestinal and pulmonary alveolar hemorrhage and hematuria $(8.5 \%$ each), and conjunctival hemorrhage $(6.3 \%)$.

All $32 \mathrm{HC}$ experienced an AE; most common (>25\%) were hypotension $(50.0 \%)$, tachycardia $(43.8 \%)$, diarrhea $(37.5 \%)$, nausea $(31.3 \%)$, and pyrexia, agitation, and 
petechiae (28.1\% each). Any hemorrhage occurred in 24 patients $(75.0 \%)$ : most common $(>10 \%)$ were petechiae (28.1\%); hematuria, epistaxis, and pulmonary alveolar hemorrhage (15.6\% each); and lip hemorrhage (12.5\%).

In the T-IND $(\mathrm{n}=1000), 385 / 512$ patients with MOD (75.2\%) and 324/488 patients without MOD (66.4\%) had an $\mathrm{AE}$; other than VOD/SOS and MOD, most commonly (>10\% in either subgroup) hypotension $(15.2 \%$ and $8.4 \%$, respectively). TRAEs occurred in 210 patients (21.0\%) (Table). Any treatment-emergent hemorrhage occurred in 166 patients with MOD (32.4\%) and 124 patients without MOD (25.4\%); most commonly ( $>5 \%$ in either subgroup) pulmonary hemorrhage ( $8.2 \%$ and $4.7 \%$, respectively) and gastrointestinal hemorrhage (5.5\% and $4.3 \%$, respectively).

Conclusions: The incidence and type of AEs were as expected in these critically ill patients. Of the pooled patients, 96\% had AEs; $57.4 \%$ had a hemorrhage. All HCs had an AE, with $75.0 \%$ having a hemorrhage. In the T-IND, patients with MOD had higher rates of AEs.

Support: Jazz Pharmaceuticals

\begin{tabular}{lclc}
\hline Event, n(\%) & $\begin{array}{l}\text { Phase 2/3 studies } \\
(\mathrm{n}=176)\end{array}$ & $\begin{array}{l}\text { T-IND } \\
\text { MOD } \\
(\mathrm{n}=512)\end{array}$ & $\begin{array}{l}\text { T-IND } \\
\text { No MOD } \\
(\mathrm{n}=488)\end{array}$ \\
\hline Total TEAEs & $169(96.0)$ & $385(75.2)$ & $324(66.4)$ \\
Total TRAEs & $58(33.0)$ & $118(23.0)$ & $92(18.9)$ \\
$\begin{array}{l}\text { Most Common by } \\
\text { PT: Pulmonary }\end{array}$ & $10(5.7) \mathrm{a}$ & $25(4.9)$ & $21(4.3)$ \\
hemorrhage & $10(5.7)$ & $16(3.1)$ & $4(0.8)$ \\
$\begin{array}{l}\text { Hypotension } \\
\text { GI hemorrhage }\end{array}$ & $7(4.0)$ & $16(3.1)$ & $14(2.9)$ \\
Epistaxis & $8(4.5)$ & $11(2.1)$ & $12(2.5)$ \\
\hline
\end{tabular}

[[P134 Table] 1. Table. Safety Summary]

Clinical Trial Registry: ClinicalTrials.gov: NCT00003966, NCT00358501, and NCT00628498

Disclosure: Paul G. Richardson has served on advisory committees and as a consultant, and has received research funding from Jazz Pharmaceuticals.

Angela R. Smith and Leslie Lehmann have nothing to disclose.

Nancy A. Kernan received grants from Gentium during the conduct of the study, and her research was supported by The National Cancer Institute of the National Institutes of Health under award number P30 CA008748; the content is solely the responsibility of the author and does not necessarily represent the official views of the National Institutes of Health. She has a research grant from Jazz Pharmaceuticals.

Robert Ryan and William Tappe are employees of Jazz Pharmaceuticals and hold stock and/or stock options in Jazz Pharmaceuticals plc.
Stephan A. Grupp has served on a steering committee and as a consultant to Jazz Pharmaceuticals.

P135

Defibrotide for treatment of adults with hepatic VOD/ SOS with or without multiorgan failure after hematopoietic cell transplantation: Results of a systematic review/meta-analysis

\section{Mohamed Kharfan-Dabaja ${ }^{1}$, Tea Reljic ${ }^{2}$, Hemant Murthy $^{3}$, Athanasios Tsalatsanis ${ }^{2}$, Mohamad Mohty ${ }^{4}$, Ambuj Kumar ${ }^{2}$}

${ }^{1}$ Mayo Clinic, Blood and Marrow Transplantation Program, Jacksonville, FL, United States, ${ }^{2}$ University of South Florida, Morsani College of Medicine, Program of Comparative Effectiveness Research, Tampa, FL, United States, ${ }^{3}$ University of Florida Health Cancer Center, Gainesville, FL, United States, ${ }^{4}$ University Pierre \& Marie Currie and Inserm UMRs 938, Paris, France

Background: Although hematopoietic cell transplantation (HCT), autologous or allogeneic, is potentially curable in various hematologic malignancies, the procedure is associated with serious and potentially life-threatening complications, among them veno-occlusive disease/sinusoidal obstructive syndrome (VOD/SOS) of the liver. Several studies, prospective or retrospective, have reported outcomes of defibrotide, when used as prophylaxis or treatment, in a mixed population of adult and pediatric patients. In this systematic review/meta-analysis, we analyze outcomes of defibrotide when specifically used for treatment of adult patients with hepatic VOD/SOS with or without multiorgan failure.

Methods: A comprehensive search of 3 large databases (Medline/Pubmed, Cochrane and EMBASE) on November 2, 2018 identified 642 publications. Analysis was restricted only to adult patients (defined as median age older than 16 years) who received defibrotide for treatment of VOD/SOS and were reported in prospective or retrospective (which included $\geq 5$ patients) studies published in full manuscript form. There were no limitations based on language. Data were extracted in relation to benefits [complete remission (CR) rate and overall survival (OS)] and harms (hemorrhage, any site or organ-specific). A total of 15 studies (prospective $=6$; retrospective $=9$ ) with 1437 patients met inclusion criteria.

Results: The median year of publication of prospective studies was 2013 (2002-2018) and for retrospective ones 2016 (2000-2018). The prescribed starting dose of defibrotide varied among studies ranging from $6.25 \mathrm{mg} / \mathrm{kg} /$ day to $80 \mathrm{mg} / \mathrm{kg} /$ day, mostly for a 21-day course. The pooled CR rate was $39 \%(95 \% \mathrm{CI}=28-49 \%)$ for prospective and $54 \%$ 
$(95 \% \mathrm{CI}=39-69 \%)$ for retrospective studies. The pooled day $+100 \mathrm{OS}$ rates were $43 \%(95 \% \mathrm{CI}=37-48 \%)$ and $65 \%(95 \%$ $\mathrm{CI}=53-75 \%$ ) for prospective and retrospective studies, respectively. The pooled rates of hemorrhage (any site) were $15 \%(95 \% \mathrm{CI}=2-35 \%)$ for prospective and $21 \%(95 \%$ $\mathrm{CI}=4-43 \%$ ) for retrospective studies. When analyzing organ-specific hemorrhage, 3 prospective studies $(n=1091$ patients) reported pooled rates of pulmonary alveolar (PA) hemorrhage of $2 \%(95 \% \mathrm{CI}=1-3 \%)$ and of $5 \%(95 \% \mathrm{CI}=3-$ $7 \%$ ) for gastrointestinal (GI) hemorrhage. Only one retrospective study ( $\mathrm{n}=14$ patients) reported an incidence of PA hemorrhage of $7 \%(95 \% \mathrm{CI}=0-34 \%)$ and a different study ( $\mathrm{n}=14$ patients) reported an incidence of GI hemorrhage of $14 \%(95 \% \mathrm{CI}=2-43 \%)$. None of the 15 studies reported cerebral hemorrhage as a complication of defibrotide therapy.

Conclusions: This systematic review/meta-analysis confirms the efficacy of defibrotide for treatment of VOD/SOS with or without multiorgan failure, yielding CR rates of 39$54 \%$ and day +100 OS rates of $43-65 \%$. The purportedly higher pooled CR and OS rates observed with retrospective (vs. prospective) studies are likely due to assignment-bias inherent to observational studies. Moreover, although the pooled hemorrhage (any site) rates of $15-21 \%$ is considered proportionally significant, the pooled rates of PA and GI hemorrhage were $\leq 5 \%$, in prospective studies.

Clinical Trial Registry: Not applicable

Disclosure: M.A.K-D: Consultancy for Pharmacyclics

M.M: received lectures honoraria and research support from Jazz Pharma

\section{P136}

Efficacy and safety of defibrotide in the treatment of hepatic veno-occlusive disease/sinusoidal obstruction syndrome following hematopoietic stem cell transplantation: Interim results from the defifrance study

Mohamad Mohty', Myriam Labopin ${ }^{2}$, Delphine Lebon ${ }^{3}$, Ana Berceanu ${ }^{4}$, Charlotte Jubert ${ }^{5}$, Ibrahim YakoubAgha $^{6}$, Stéphane Girault ${ }^{7}$, Marie Detrait ${ }^{8}$, Cécile Pochon', Fanny Rialland ${ }^{10}$, Virginie Gandemer ${ }^{11}$, Régis Peffault de Latour $^{12}$, David Michonneau' ${ }^{12}$, Floriane Delaval ${ }^{13}$, Gerard Michel $^{14}$, Anne Sirvent ${ }^{15}$, Laurence Clement ${ }^{16}$, Anne-Lise Menard ${ }^{17}$, Anne Huynh ${ }^{18}$, Virginie Bouvatier $^{19}$, Raj Hanvesakul ${ }^{20}$, Zakaria Medeghri ${ }^{19}$, Jean-Hugues Dalle ${ }^{21}$

${ }^{1}$ Hôpital Saint-Antoine, Paris, France, ${ }^{2}$ Hôpital SaintAntoine, Paris, France, ${ }^{3} \mathrm{Chu}$ Amiens Picardie Hôpital, Amiens, France, ${ }^{4}$ Hôpital Jean Minjoz, Besançon, France, ${ }^{5}$ Hôpital des Enfants, Bordeaux, France, ${ }^{6}$ Hôpital Claude Huriez, Lille, France, ${ }^{7}$ Hôpital Dupuytren - CHU Limoges,
Limoges, France, ${ }^{8} \mathrm{CHU}$ Nancy, Nancy, France, ${ }^{9}$ Hôpital D'Enfants, Nancy, France, ${ }^{10}$ Hôtel-Dieu, Nantes, France, ${ }^{11}$ CHU Hôpital Sud, Rennes, France, ${ }^{12}$ Hôpital Saint-Louis, Paris, France, ${ }^{13} \mathrm{Jazz}$ Pharmaceuticals, Paris, France, ${ }^{14}$ Hôpital de la Timone, Marseille, France, ${ }^{15}$ Hôpital Arnaud de Villeneuve, Montpellier, France, ${ }^{16}$ Hôpital Haut-Lévèque, Pessac, France, ${ }^{17}$ Centre Henri Becquerel, Rouen, France, ${ }^{18}$ IUCT Oncopole, Toulouse, France, ${ }^{19}$ Jazz Pharmaceuticals, Lyon, France, ${ }^{20}$ Jazz Pharmaceuticals, Oxford, United Kingdom, ${ }^{21}$ Hôpital Robert Debré, Paris, France

Background: Hepatic veno-occlusive disease/sinusoidal obstruction syndrome (VOD/SOS) is a potentially lifethreatening complication of conditioning for hematopoietic stem cell transplant (HSCT) but may occur after nontransplant chemotherapy alone. VOD/SOS with multi-organ dysfunction (MOD) may be associated with $>80 \%$ mortality with supportive care alone. Diagnosis of VOD/SOS was traditionally based on Baltimore or modified Seattle criteria; however, the EBMT recently published separate diagnostic criteria for adults and children. Defibrotide is approved for treating severe hepatic VOD/SOS post-HSCT in patients aged $>1$ month in the EU, and for hepatic VOD/SOS with renal or pulmonary dysfunction post-HSCT in the USA. The goal of the DEFIFRANCE study, requested by the French health authorities, is to collect real-world data on safety and efficacy in a broader patient population in France, including all indications. This is the first interim analysis of the largest current evaluation of defibrotide for the treatment of VOD/SOS in Europe.

Methods: DEFIFRANCE is an observational, multicenter, post-marketing study that includes any patient treated with defibrotide from HSCT centers in France. This interim analysis is based on all patients treated with defibrotide, including those with severe and very severe post-HSCT VOD/SOS. VOD/SOS was diagnosed using traditional criteria. Day+100 survival, complete remission (CR; total serum bilirubin $<2 \mathrm{mg} / \mathrm{dL}$ and resolution of MOD), and safety profile are reported.

Results: A total of 324 patients treated with defibrotide were included retrospectively and prospectively between July 2014 and October 2018 from 36 French sites. VOD/SOS was diagnosed in a subset of 140 patients ( 99 adults, 41 pediatric patients). Of these, 109 patients (74 adults; 35 pediatric patients) had severe or very severe VOD/SOS, divided in 50 severe VOD/SOS (31 adults; 19 pediatric patients) and 59 very severe VOD/SOS (43 adults; 16 pediatric patients).

Overall in the 140 VOD/SOS patients, Kaplan-Meier estimated Day+100 survival was $58.2 \%$ (95\% CI, 49.2\%$66.1 \%$ ). Kaplan-Meier estimated Day+100 survival was $55.9 \%$ (95\% CI, $45.8 \%-64.9 \%$ ) in the combined severe/very severe VOD/SOS group (severe: $77.2 \%$ [95\% CI, 62.6\%$86.7 \%]$; very severe: $37.2 \%$ [24.6\%-49.8\%]). CR rates 
were: overall, 52.8\%; combined severe and very severe VOD/SOS: $52.0 \%$; severe, $73.9 \%$; very severe: $33.3 \%$. The Table shows the safety profile.

Conclusions: This interim analysis of the DEFIFRANCE registry demonstrated estimated Day +100 survival of $58.2 \%$ overall in the patients with VOD/SOS and 55.9\% in patients with confirmed severe or very severe VOD/SOS. The higher estimated survival for severe $(77.2 \%)$ versus very severe $(37.2 \%) \mathrm{VOD} / \mathrm{SOS}$ highlights the importance and impact of earlier intervention. Survival, CR rate, and safety findings, consistent with prior clinical trials, provide supportive evidence for the clinical utility of defibrotide for treatment VOD/SOS patients.

Support: Jazz Pharmaceuticals

\begin{tabular}{lll}
\hline $\begin{array}{l}\text { Events of Interest } \\
(>2 \%) \mathrm{n}(\%)\end{array}$ & $\begin{array}{l}\text { Overall Patients } \\
\text { with VOD/SOS } \\
(\mathrm{n}=140)\end{array}$ & $\begin{array}{l}\text { Patients with Severe or } \\
\text { Very Severe VOD/SOS } \\
(\mathrm{n}=109)\end{array}$ \\
\hline Total & $75(53.6)$ & $57(52.3)$ \\
Infection & $34(24.3)$ & $26(23.9)$ \\
Hemorrhage & $28(20.0)$ & $20(18.3)$ \\
Thromboembolic event & $6(4.3)$ & $2(1.8)$ \\
Low blood pressure & $4(2.9)$ & $4(3.7)$ \\
\hline
\end{tabular}

\section{[[P136 Table] 1. Table]}

\section{Disclosure:}

Mohamad Mohty: has received honoraria and research funding from Jazz Pharmaceuticals, Delphine Lebon: nothing to disclose, Ann Berceanu: none, Charlotte Jubert: has received funding from Jazz Pharmaceuticals, Ibrahim Yakoub-Agha: has received honoraria from Jazz Pharmaceuticals, Stéphane Girault: none, Marie Detrait: has received research funding from Jazz Pharmaceuticals, Cécile Pochon: none, Fanny Rialland: none, Virginie Gandemer: none, Jean-Hugues Dalle: has received honoraria from Jazz Pharmaceuticals, Régis Peffault de Latour: has received research grant / honoraria / board from Pfizer, Novartis, Alexion; research grant Amgen; and honoraria from Jazz Pharmaceuticals, David Michonneau: has received honoraria from Jazz Pharmaceuticals, Myriam Labopin: has received honoraria from Jazz Pharmaceuticals, Floriane Delaval: employee of Jazz Pharmaceuticals and holds stock and/or stock options in Jazz Pharmaceuticals plc, Gerard Michel: none, Anne Sirvent: none, Laurence Clement: none

Anne-Lise Menard: none, Anne Huynh: has received honoraria from Jazz Pharmaceuticals, Virginie Bouvatier: employee of Jazz Pharmaceuticals and holds stock and/or stock options in Jazz Pharmaceuticals plc, Raj Hanvesakul: employee of Jazz Pharmaceuticals and holds stock and/ or stock options in Jazz Pharmaceuticals plc, Zakaria
Medeghri: employee of Jazz Pharmaceuticals and holds stock and/or stock options in Jazz Pharmaceuticals plc

\section{P137}

Incidence and predictors of severe cardiotoxicity in patients with severe aplastic anemia after haploidentical hematopoietic stem cell transplantation

Zheng-Li Xu ${ }^{I}$, Lan-Ping $X u^{I}$, Yuan-Yuan Zhang ${ }^{1}$, Yi-Fei Cheng ${ }^{1}$, Xiao-Dong Mo ${ }^{1}$, Feng-Rong Wang ${ }^{1}$, Yu-Hong Chen ${ }^{I}$, Wei Han ${ }^{I}$, Chen-Hua Yan ${ }^{I}$, Yu-Qian Sun ${ }^{I}$, TingTing Han ${ }^{I}, Y u$ Wang $^{I}$, Xiao-Hui Zhang ${ }^{I}$, Xiao-Jun Huang ${ }^{I}$

${ }^{1}$ Peking University Institute of Hematology, Peking University People's Hospital, Beijing, China

Background: Severe cardiotoxicity after hematopoietic stem cell transplantation (HSCT) is a rare but fatal complication. The aim of this study was to evaluate the frequency of severe cardiac complications and to assess the ability of various factors to predict these complications in patients with aplastic anemia after haploidentical transplantation., this is the first study evaluating the values of both clinical and imaging factors in the prediction of severe cardiotoxicity among SAA patients after haploidentical transplantation.

Methods: A retrospective study was conducted in 216 consecutive aplastic anemia patients who received haploidentical transplantation from 2006 to 2017. All patients received a unified regimen including busulfan, cyclophosphamide (CTX) and antithymocyte globulin at our single center.

Results: A total of 12 (5.6\%) patients developed grade III or IV cardiac toxicity. Patients with cardiotoxicity had significantly poorer overall survival (OS) than those without cardiotoxicity $(12.5 \%$ vs. $89.6 \%, \mathrm{P}<0.001)$. Our multivariable model identified four independent adverse predictors of severe cardiotoxicity, including pre-transplant ECOG score $(\geq 2)$, abnormal ST-T wave on 12-lead electrocardiogram (ECG), hyperlipemia and recalculated CTX dose $(\geq 1.8 \mathrm{~g} / \mathrm{m} 2 / \mathrm{d})$. A predictive risk model was refined as low risk (0-1 factor), intermediate risk (2 factors) and high risk (3-4 factors). The respective incidences of severe cardiotoxicity were $50.0 \%, 6.0 \%$, and $1.3 \%$ in the high-, intermediate- and low-risk groups $(\mathrm{P}<0.001)$. The corresponding OS rates were $49.0 \%, 80.4 \%$, and $90.3 \%$ in the three groups $(\mathrm{P}<0.001)$ at the last follow-up.

Conclusions: Patients with high risk scores had the poorest outcomes and should be monitored closely. A reduced intensity conditioning might be recommended for these patients.

Disclosure: There are no conflicts of interest to declare. 


\section{P138}

Impact of acute renal failure in transplant-related mortality and development of chronic renal disease in allogeneic stem-cell transplantation

Marta Garrote ${ }^{1}$, Jesus Villarreal ${ }^{1}$, Roger Borràs ${ }^{2}$, Ana Moreno-Castaño ${ }^{1}$, Montse Rovira ${ }^{1}$, María Suárez-Lledó ${ }^{1}$, Luis Gerardo Rodríguez-Lobato ${ }^{1}$, Carmen Martínez ${ }^{1}$, Laura Rosiñol', Noemi Llobet ${ }^{1}$, Carla Ramos ${ }^{1}$, Álvaro Urbano-Ispizua ${ }^{1}$, Francesc Fernández-Avilés ${ }^{1}$, Luis Fernando Quintana ${ }^{1}$, Gonzalo Gutiérrez-García ${ }^{1}$

${ }^{1}$ Hospital Clínic de Barcelona, Haematology, Barcelona, Spain, ${ }^{2}$ Institut de Recerca Biomèdica August Pi i Sunyer (IDIBAPS), Barcelona, Spain

Background: Allogeneic stem-cell transplantation (alloSCT) is associated with significant transplant-related mortality (TRM). Acute renal failure (ARF) is a frequent complication and usually presents early after the procedure, compromising its feasibility. The aim of this study is to analyse the incidence of ARF, its risk factors and its potential impact on TRM after allo-SCT.

Methods: 422 patients were included (244 males [58\%]; median age 43 years, range 16-67) treated with Allo-SCT consecutively between January 2001 and April 2012 in a single institution. Patient characteristics are detailed in table 1. Median follow-up was 1.8 years (range, 1.0-2.7). Renal function was evaluated using creatinine and data was collected pre-transplant (baseline) and at the point when ARF was developed after allo-SCT. ARF was evaluated using AKIN criteria, being AKIN-1 an increase 1.5- to 1.9-fold from baseline, AKIN-2 an increase 2.0- to 2.0-fold and AKIN-3 an increase $\geq 3$-fold. Chronic renal disease was evaluated one year after the date of ARF using KDIGO criteria.

Results: Cumulative incidence of ARF at 1 year was $63 \%$ (AKIN-1, 25\%; AKIN-2, 27\%; AKIN-3, 15\%). In the multivariate analysis, ARF (AKIN-1/2) was associated with: non-use of antithymocyte globulin in conditioning chemotherapy, $\mathrm{p}=0.02$ (HR 2.3, 0.2 to 0.9 ) and development of severe aGvHD, $\mathrm{p}=0.04(\mathrm{HR}=1.5,1$ to 2.3$)$. In patients with ARF AKIN-3, the most important variables in the multivariate analysis were: use of methotrexate (MTX) plus cyclosporine vs mycophenolate mofetil plus cyclosporine as GvHD prophylaxis, $\mathrm{p}=0.009$ ( $\mathrm{HR}=1.9,1.2$ to 3.1); myeloablative conditioning vs reduced intensity, $\mathrm{p}=0.03(\mathrm{HR}=1.7,1$ to 2.8$)$ and use of total irradiation therapy in conditioning, $\mathrm{p}=0.02(\mathrm{HR}=1.7,1.1$ to 2.8$)$. TRM at 1 year increased significantly according to AKIN: AKIN-1, 25\%; AKIN 2, 35\%; AKIN 3, 51\%; $\mathrm{p}=0,003$; $\mathrm{HR}=11.2$. Overall survival at 3 years according to AKIN was: AKIN 1, 52\%, AKIN 2, $45 \%$ and AKIN 3, 29\%; $\mathrm{p}=0,004$ (figure 1). The incidence of chronic renal disease at 1 year after allo-SCT according to ARF was: no ARF (8\%), AKIN-1 (11\%), AKIN-2 (15\%) and AKIN-3 (16\%); $\mathrm{p}=0.006$.

Conclusions: ARF is a frequent complication during the first year after allo-SCT and is associated with several factors. ARF AKIN-3 was associated with more intensive strategies received during conditioning, meanwhile AKIN$1 / 2$ were related to development of GvHD. There is an association of ARF (AKIN-1, 2 or 3) with development of chronic renal disease.

\begin{tabular}{|c|c|}
\hline Diagnosis & $\begin{array}{l}\text { Acute leukemia, } 189 \text { (44); MDS, } 41 \text { (10); } \\
\text { lymphoma, } 102 \text { (25); other, } 90 \text { (21) }\end{array}$ \\
\hline Donor type & Sibling, 232 (55); unrelated, 190 (45) \\
\hline HLA $8 / 8$ identity & $357(85)$ \\
\hline Stem-cell source & $\begin{array}{l}\text { Peripheral blood, } 333 \text { ( } 80) \text {; bone marrow, } 63 \text { (15); } \\
\text { umbilical cord, } 22 \text { (5) }\end{array}$ \\
\hline Conditioning & $\begin{array}{l}\text { Myeloablative, } 208 \text { (49); reduced intensity, } \\
214 \text { (51) }\end{array}$ \\
\hline $\begin{array}{l}\text { Total body } \\
\text { irradiation }\end{array}$ & $188(45)$ \\
\hline GvHD prophylaxis & $\begin{array}{l}\text { Cyclosporine + MTX, } 191 \text { (46); cyclosporine }+ \\
\text { mycophenolate, } 183 \text { (44); other, } 41 \text { (10) }\end{array}$ \\
\hline $\begin{array}{l}\text { Risk for CMV } \\
\text { reactivation }\end{array}$ & $\begin{array}{l}\text { Low, } 158 \text { (41); intermediate, } 127 \text { (33); high, } \\
102 \text { (26) }\end{array}$ \\
\hline
\end{tabular}

[[P138 Table] 1. Table 1. Patients characteristics, N(\%)]

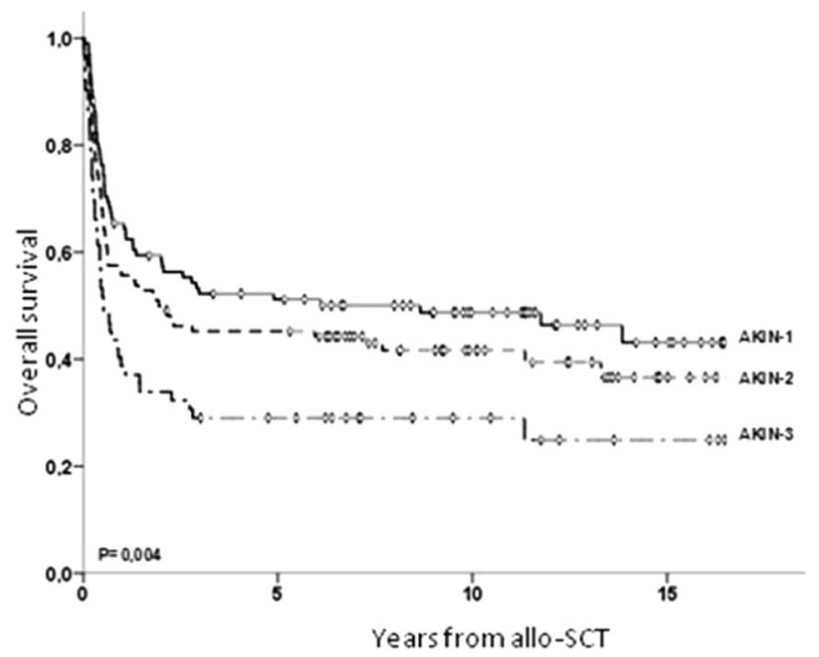

[[P138 Image] 1. Figure 1. Overall survival according to acute renal failure AKIN criteria]

\section{Disclosure:}

Gonzalo Gutiérrez-García: honoraria from Gilead. Grant from Jazz Pharmaceutical.

The other authors do not have any disclosure to declare. 


\section{P139}

Allogeneic stem cell transplantation at-home program in the post-transplant cyclophosphamide GVHD prophylaxis era

Gonzalo Gutiérrez-García ${ }^{1,2,3}$, Montserrat Rovira ${ }^{1,2,3}$, Pilar Ayora ${ }^{1}$, Ariadna Domenech ${ }^{1}$, Cristina Gallego ${ }^{1}$, María Teresa Solano ${ }^{1}$, Ana B Moreno-Castaño ${ }^{1,2,3}$, María Suárez-Lledó ${ }^{1,2,3}$, Nuria Borràs ${ }^{1}$, Luis Gerardo Rodríguez-Lobato ${ }^{1,2,3}$, Julio César Solano-Vega ${ }^{1}$, Laura Rosiñol $^{1,2,3}$, Carmen Martínez ${ }^{1,2,3}$, Pedro Marín ${ }^{1,2,3}$, María Adelina Hernando ${ }^{1}$, Álvaro Urbano-Ispizua ${ }^{1,2,3}$, Francesc Fernández-Avilés ${ }^{1,2,3}$

${ }^{1}$ Hospital Clínic de Barcelona, Hematology, Barcelona, Spain, ${ }^{2}$ University Barcelona, Barcelona, Spain, ${ }^{3}$ Institut de Recerca Biomèdica August Pi i Sunyer (IDIBAPS), Barcelona, Spain

Background: The introduction of cellular therapies such as CAR-T and modalities of GvHD-prophylaxis with posttransplant/cyclophosphamide (PtCy) that increase the number of admission days have boosted the pressure of available beds in the BM-units. In this sense, our centre started an at-home allogeneic stem cell transplantation (alloSCT) program to follow aplasia from the $\mathrm{D}+1$ until independent ambulatory patient. To evaluate the feasibility and safety of alloSCT, we compared two groups: alloHSCT/athome (AH-Group) vs. alloHSCT/in-patient (IP-Group).

Methods: We included 78 patients receiving alloSCT (January 2014-November 2018) in a single centre: 39 patients, AH-Group and 39, IP-Group. All patients received conditioning at the hospital. GvHD-prophylaxis consisted in tacrolimus (TK) plus mycophenolate (MPM) or methotrexate, or PtCy $(\mathrm{D}+3, \mathrm{D}+4)$ plus $\mathrm{TK}(\mathrm{D}+5)$. All patients received prophylaxis with levofloxacin, fluconazole and acyclovir. Besides that, AH-Group patients received prophylaxis with ceftriaxone $1 \mathrm{~g} / 24 \mathrm{~h}$ IV or ertapenem $1 \mathrm{~g} /$ 24h IV, and Aspergillus-prophylaxis with inhaled liposomal amphotericin-B or posaconazole during neutropenia. Patients of AH-Group since $\mathrm{D}+1$ or $\mathrm{D}+6$ (in PtCyprophylaxis) received a nurse visit at-home once daily. The visits by the physician were performed at the hospital and only during complication events. First-line therapy of neutropenic fever was meropenem $1 \mathrm{~g} / 8 \mathrm{~h}$ in both groups, using a portable infusion pump in AH-Group. In this group, the absence of focal infection or signs of severe sepsis allowed returning home after the initiation of antibiotics. The platelets support was performed at-home and the red blood support at hospital.

Results: The median (range) age (years) of the series was 54 (19-70). The median follow-up of the series has been not achieved. The source of the SCT was peripheral blood in all cases. We didn't find statistical differences between two groups (AH vs IP) in terms of age, diagnosis, type of donor, intensity of conditioning, GvHD-prophylaxis, toxicity (mucositis, acute renal injury, neutropenia and thrombocytopenia), aGvHD, Aspergilosis and TRM. Interestingly, a significant reduction of neutropenic fever was observed resulting the lower use of meropenem in the AH-group than IP-group. The admission median days were similar in the both groups and it represented 21-23 days the reduction in the total economic cost of the AH-group. The whole analysis of the results are detailed in table:

\begin{tabular}{|c|c|c|c|}
\hline $\mathrm{N}=78$ & $\begin{array}{l}\text { In-Patient Group, } \\
(\mathrm{N}=39), \mathrm{N}(\%)\end{array}$ & $\begin{array}{l}\text { At-Home Group, } \\
\mathrm{N}=39, \mathrm{~N}(\%)\end{array}$ & $\mathrm{P}$ \\
\hline $\begin{array}{l}\text { Donor: Sibling, URD, } \\
\text { Haploidentical }\end{array}$ & $\begin{array}{l}19(49 \%), 19 \\
(49 \%), 1(2 \%)\end{array}$ & $\begin{array}{l}14(36 \%), 24 \\
(62 \%), 1(2 \%)\end{array}$ & 0.5 \\
\hline Conditioning: MAC & $14(36 \%)$ & $17(44 \%)$ & 0.5 \\
\hline $\begin{array}{l}\text { GvHD prophylaxis: TK/MMF; } \\
\text { TK/MTX; PtCy/TK }\end{array}$ & $\begin{array}{l}23(59 \%), 4(10 \%), \\
21(31 \%)\end{array}$ & $\begin{array}{l}26(68 \%), 2(5 \%), \\
11(28 \%)\end{array}$ & 0.6 \\
\hline $\begin{array}{l}\text { Neutropenia }<0.5 \times 10 \mathrm{e} 9 / \mathrm{L} \\
\text { (Median-range,days) }\end{array}$ & $17(11-37)$ & $17(8-23)$ & 0.8 \\
\hline $\begin{array}{l}\text { Neutropenic fever and } \\
\text { Aspergillosis }\end{array}$ & $35(90 \%), 1(3 \%)$ & $11(39 \%), 1(3 \%)$ & $\begin{array}{l}<0.0001 \\
\text { and } 0.6\end{array}$ \\
\hline $\begin{array}{l}\text { Mucositis (grade 3-4) and } \\
\text { Acute renal injury }\end{array}$ & $10(26 \%), 24(62 \%)$ & $5(13 \%), 27(69 \%)$ & $\begin{array}{l}0.3 \\
\text { and } 0.5\end{array}$ \\
\hline $\begin{array}{l}\text { Re-admission rate, only for At- } \\
\text { Home group }\end{array}$ & - & $3(8 \%)$ & - \\
\hline aGVHD & $27(69 \%)$ & $30(77 \%)$ & 0.4 \\
\hline 1-year-TRM and 2-year- OS & $26 \%$ and $69 \%$ & $21 \%$ and $66 \%$ & $\begin{array}{l}0.5 \\
\text { and } 0.8\end{array}$ \\
\hline
\end{tabular}

\section{[[P139 Table] 1. Results]}

Conclusions: In our experience, at home alloSCT, including PtCy-GvHD prophylaxis, is a feasible and safe procedure reflected in similar TRM and Aspergillosis incidence. At-home allo-SCT is associated with a significant lower risk of neutropenic fever than in-patient group, as well as a very low readmission rate.

Disclosure:

Gonzalo Gutiérrez-García: honoraria from Gilead. Grant from Jazz Pharmaceutical and Janssen.

Laura Rosiñol: honoraria from Takeda, Janssen, Amgen and Celgene.

The others author do not have any disclosures to declare.

\section{P140}

Stable renal function in children and adolescents with sickle cell disease after non-myeloablative conditioning hematopoietic stem cell transplant

Sasia Julie Volden Pedersen ${ }^{1}$, Dania Monagel', Cherry Mammen $^{3}$, Victor A. Lewis ${ }^{4}$, Greg M. T. Guilcher ${ }^{4}$, Aisha Bruce $^{5}$

${ }^{1}$ University of Alberta Hospital/Stollery Children's Hospital, Edmonton, Canada, ${ }^{2}$ Alberta Children's Hospital, 
Calgary, Canada, ${ }^{3}$ British Columbia Children's Hospital, Vancouver, Canada, ${ }^{4}$ Alberta Children's Hospital, Calgary, Canada, ${ }^{5}$ University of Alberta Hospital/Stollery Children's Hospital, Edmonton, Canada

Background: Renal complications in sickle cell disease (SCD) include episodes of acute kidney injury (AKI), progressive chronic kidney disease (CKD) and hyperfiltration, defined by abnormally high glomerular filtration rates (GFRs). Hematopoietic stem cell transplant (HSCT) from an HLA identical sibling donor is a well-established curative treatment for SCD, but traditional myeloablative conditioning (MAC) regimens pose risks of kidney injury due to intensive use of chemotherapeutic agents, infectious risks, and use of calcineurin inhibitors (CNIs). AKI and subsequent fluid overload (FO) are common in pediatric HSCT with reported AKI incidence of 21\%-50\% (KyungNam Koh et. Al., 2017). We report renal outcomes in pediatric patients with SCD who received HSCT following a non-myeloablative conditioning (NMA) regimen without CNI exposure.

Methods: Retrospective chart review describing renal outcomes in pediatric patients (18 years of age or younger) with SCD (HbSS) who underwent NMA HSCT in Alberta, Canada from July 2013 to February 2018. The NMA regimen is illustrated in Figure 1. Reported renal outcomes: 1) measured GFR (DTPA) pre-HSCT, 2) AKI (KDIGO definition) post-HSCT by reviewing all serum creatinine levels from pre-HSCT to one month post-HSCT, 3) \%FO calculated: (max post HSCT weight - baseline weight)/ baseline weight $\mathrm{x} 100$ for the two first weeks post-HSCT, and 4) estimated GFR (eGFR) using the pediatric Schwartz formula at last follow-up post-HSCT, CKD defined as eGFR $<60 \mathrm{~mL} / \mathrm{min} / 1.73 \mathrm{~m}^{2}$, mildly reduced GFR: $60-$ $90 \mathrm{~mL} / \mathrm{min} / 1.73 \mathrm{~m}^{2}$, and hyperfiltration: GFR $\geq 150 \mathrm{~mL} /$ $\min / 1.73 \mathrm{~m}^{2}$.

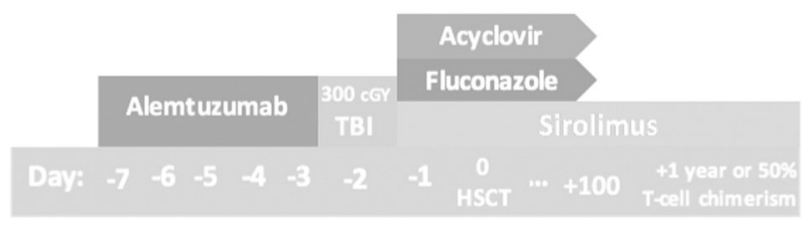

\section{[[P140 Image] 1. Figure 1: NMA regimen used]}

Results: Eighteen patients (33\% male, 3-18 years old at transplant) were included. Most common pre-morbid events: vaso-occlusive crisis $(n=17)$, acute chest syndrome $(\mathrm{n}=8)$, splenic sequestration $(\mathrm{n}=6)$, and cholelithiasis $(n=4)$. Median follow-up time: 27 months (range: 7 62 months). All patients engrafted successfully with no acute or chronic GVHD.
Baseline measured GFRs were all $>60 \mathrm{~mL} / \mathrm{min} / 1.73 \mathrm{~m}^{2}$ (range: 79-227) with mildly reduced GFR and hyperfiltration seen in one (5.6\%) and $12(66.7 \%)$ patients respectively.

At baseline (pre-HSCT), the only AKI event was one transplant related AKI secondary to delayed hemolytic reaction after exchange transfusion in preparation for transplant. Post-HSCT, there were no AKI events. Additionally, no substantial \%FO post-HSCT was observed. Average $\% \mathrm{FO}$ week one post-HSCT: $+0.01 \%$ (Range: $-4.2 \%-+1.0 \%)$ and week two post-HSCT: $+0.04 \%$ (Range: $-4.24 \%-+1.5 \%$ ).

Post-HSCT eGFR remained $>90 \mathrm{~mL} / \mathrm{min} / 1.73 \mathrm{~m}^{2}$ at last follow-up in all patients. Hyperfiltration was present in 5 $(27.8 \%)$ of the patients.

Conclusions: This is the first study describing stable kidney function in children with SCD after the present NMA HSCT regimen with alemtuzumab/300 cGy total body irradiation (TBI) with prolonged post-HSCT Sirolimus. No episodes of AKI or significant fluid overload were observed during the first month post-HSCT, and no patient developed CKD during follow-up. Further prospective studies are needed to confirm our findings and to determine if stable renal function persists during longer-term followup.

Disclosure: Nothing to declare.

\section{P141}

Lung microbiota in patients with idiopathic pneumonia syndrome (IPS) after HCT

Sachiko Seo ${ }^{1,2}$, Andrew Connell ${ }^{3}$, John Everett ${ }^{3}$, Joshua Hill $^{1,4}$, Terry Stevens-Ayers ${ }^{1}$, Keith Jerome ${ }^{1,4}$, Frederic Bushman $^{3}$, Collman Ronald ${ }^{3}$, Michael Boeckh ${ }^{1,4}$

${ }^{1}$ Fred Hutchinson Cancer Research Center, Seattle, WA, United States, ${ }^{2}$ Dokkyo Medical University, Tochigi, Japan, ${ }^{3}$ University of Pennsylvania Perelman School of Medicine, Pennsylvania, PA, United States, ${ }^{4}$ University of Washington, Seattle, WA, United States

Background: Idiopathic pneumonia syndrome (IPS) is a non-infectious pulmonary complication after hematopoietic cell transplantation (HCT) and the etiology remains unknown. Recent studies have reported that various diseases are associated with changes of microbiota. The aim of this study was to evaluate the lung microbiota in HCT recipients with IPS and identify microorganisms potentially associated with IPS.

Methods: Frozen bronchoalveolar lavage (BAL) samples from HCT recipients with IPS $(\mathrm{N}=18)$ and research BAL samples from asymptomatic HCT recipients as controls $(\mathrm{N}=12)$ were retrospectively analyzed. All samples were 
negative for common viruses by quantitative PCR. Sequencing libraries were made with 1 ng of input DNA per sample (Nextera XT, Illumina). Samples were pooled and sequenced by HiSeq 2000 to obtain 100-bp paired end data. Sequence data analysis and read classification were performed with Sunbeam and the quality control and read classification were performed using Komplexity and Kraken, which classifies bacterial, archeal, and viral genomes. We used sequence data of bronchoscope prewashes from a separate cohort as controls for environmental sources $(\mathrm{N}=24)$. Bray-Curtiss dissimilarity among samples was calculated using the Vegan $\mathrm{R}$ packages. PERMANOVA and a two-sided Wilcoxon rank sum test were used to compare between the study groups.

Results: BAL samples started at a median of $22 \times 10^{6}$ raw read pairs per sample and reduced to $21 \times 10^{3}$ reads assignable to microbial taxa following quality control. The bacterial phyla Proteobacteria and Firmicutes were most abundant followed by Bacteroidetes and Actinobacteria in both BAL and bronchoscope prewash samples. Separation of BAL and prewash microbiota using Bray-Curtiss dissimilarity plots showed that BAL samples were distinguished by sequences assigned to Staphylococcus, Acidovorax, and Bradyrhizobium species, while prewash samples were distinguished mostly by Pseudomonas and Elizabethkingia species, consistent with environmental sources (Figure). Within BAL samples, Staphylococcus species were the main drivers of separation between IPS cases and the controls ( $p=0.002$, PERMANOVA, Figure). Consistent with this, a linear discriminant analysis to identify taxa best distinguishing cases and controls identified Staphylococcus, especially S. epidermidis, in IPS cases with Lactobacillus and Streptococcus species in controls. We then compared relative abundances of $\mathrm{S}$. epidermidis between all study groups. IPS case samples were significantly enriched in S. epidermidis compared to control ( $\mathrm{p}<0.001$, two-sided Wilcoxon rank sum test) and prewash samples $(\mathrm{p}<0.001)$. Viruses were classified by category as human pathogens, non-human pathogens, and bacteriophages. Torque teno viruses (TTV) was the most commonly detected virus among viruses that replicate on human cells, and there was a trend towards higher abundance in IPS case samples than controls.

Conclusions: Lung microbial sequences in HCT recipients predominantly consisted of Proteobacteria and Firmicutes, and had considerable overlap with environmental background. Patients with IPS had significantly more Staphylococcus sequences detected than asymptomatic HCT patients. These results suggest that patients with acute lung injury post-HCT show distinct patterns of lung microbiota, although heterogeneity of sample collection and processing cannot be excluded and no singular organism was uniquely associated with IPS. A prospective study is required to confirm these findings and define the clinical significance of differences in abundance patterns.

Disclosure: Nothing to declare

\section{P142}

Abstract withdrawn.

\section{P143}

Romiplostim for the treatment of thrombocytopenia after allogeneic stem cell transplantation

Maria Liga $^{1}$, Nikos Spyridis ${ }^{1}$, Dimitris Aggelinas ${ }^{1}$, Chara Korovila $^{I}$, Evangellia Triantafyllou ${ }^{l}$, Panagiotis Bountouris $^{1}$, Markos Marangos ${ }^{1}$, Alexandros Spyridonidis $^{1}$

${ }^{1}$ University Hospital of Patras, Patras, Greece

Background: Thrombocytopenia is a common complication after allogeneic stem cell transplantation (allo-HCT). with variable possible causes, such as drug side effects, infections, poor graft function, Graft vs Host disease (GvHD) and immune mediated. The purpose of this study was to evaluate the efficacy of romiplostim, a thrombopoietin receptor agonist, in patients with prolonged thrombocytopenia with no obvious cause after allogeneic transplantation.

Methods: Retrospective analysis of allo-HCT patients who received romiplostim at a single BMT Unit between November 2015 and November 2018. Romiplostim was given because of prolonged ( $>3$ weeks) thrombocytopenia $(<60,000 \mu \mathrm{L})$ that couldn't be explained by obvious causes such as administration of drugs (antibiotics/antivirals), infection or GvHD. All patients were in complete remission and had complete chimerism. Response to romiplostim treatment was considered transfusion independence or PLT $>80.000 / \mu \mathrm{L}$.

Results: In total, 19 patients (median 45 years, 19-67) received romiplostim. Patients (10 male, 9 females) had AML (10 pts), ALL (8), MDS (2) or Hodgkin (1), received a myeloblative (busiphex-based: 16, TBI-based:1) or RIC (2) conditioning and were transplanted from a sibling (5), VUD (11) or haploidentical (3) donor with PBSC (16) or BM (3). All patients revealed primary neutrophil (median 14 days, range $10-19$ ) and $>20.000 / \mu \mathrm{L}$ platelet (13 days, 7 31) engraftment. Romiplostim was started at median day +104 (range 58-419) with a median dose $5 \mu \mathrm{g} / \mathrm{kg}$ (1-5). The median platelet count before commencement of treatment with romiplostim was $24.000 / \mu \mathrm{L}$ (range 12.000-57.000) and 10 them (59\%) were transfusion-dependent. In total 14/17 (82\%) patients responded to romiplostim treatment. Eight out of the $10(80 \%)$ transfusion dependent patients responded to the administration of romiplostim. Six out of 
the 7 patients $(86 \%)$ who were transfusion independent at romiplostin initiation (PLT median 28.000/ $\mathrm{LL}$, range 19.000-56.000) responded. The median duration of treatment was 74 days (15-253) and the median follow up from the commencement of romiplostim was 177 days (15-1080). Three out of $17(18 \%)$ patients experienced relapse of thrombocytopenia after discontinuation of romiplostim and re-initiation of romiplostim was commenced in all of them, of which 2 responded and 1 didn't. The administration of romiplostim was done on an external basis and was well tolerated by the patients. Two patients experienced GvHD during romiplostim treatment (both patients transplanted from 7/8 unrelated donor, 25 and 42 days after initiation treatment with romiplostim). $3 / 19$ patients interrupted romiplostim due to disease relapse. $11 / 19$ patients receiving romiplostim are alive in complete remission and 8 died (3 due to relapse, and 5 due to TRM).

Conclusions: We present high response rates to romiplostim in patients with prolonged thrombocytopenia after allogeneic transplantation. In this retrospective study there were no side effects from the administration of romiplostim. However, the administration of romiplostim after allo-HCT should be controlled in prospective trials.

Disclosure: Nothing to declare

\section{P144}

Thrombopoietin receptor agonists for management of poor graft function in adult patients after allogeneic HSCT

Tatiana Rudakova', Alexander Kulagin', Olesya Klimova ${ }^{1}$, Irina Golubovskaya ${ }^{1}$, Tatiana Bykova ${ }^{1}$, Elena Darskaya', Sergey Bondarenko ${ }^{I}$, Ivan Moiseev ${ }^{I}$, Ildar Barkhatov $^{I}$, Elena Babenko ${ }^{\prime}$, Alexander Alyanskiy ${ }^{I}$, Boris Afanasyev ${ }^{1}$

${ }^{1}$ Pavlov First Saint Petersburg State Medical University/ Raisa Gorbacheva Memorial Institute of Children's Oncology, Hematology and Transplantation, St. Petersburg, Russian Federation

Background: Poor graft function (PGF) is a lifethreatening complication of allo-HSCT. Currently there is no standard therapy for treatment PGF. Thrombopoietin receptor (TPO) agonists showing desirable results in severe aplastic anemia may be a possible strategy to resolve severe PGF (sPGF) after allo-HSCT. The aim of our study was to assess an efficacy of TPO agonists for PGF after allo-HSCT in adult patients.

Methods: We report a single-center analysis of 29 adult patients (median age 22 years, range 18-57, M/F 13/16), receiving TPO agonists for isolated severe thrombocytopenia $(n=7)$ and sPGF $(n=22)$ after allo-HSCT. Primary diagnoses were AML (10), ALL (6), MDS (7), PMF (4), MDS/MPN (4), SAA (4), CML (1), NHL (1). Severe PGF was defined as cytopenia in $\geq 2$ lineages (platelet $<20 \times$ $10^{9} / \mathrm{L}$, ANC $<0.5 \times 10^{9} / \mathrm{L}$, hemoglobin $<70 \mathrm{~g} / \mathrm{L}$ any time after sustained engraftment), full or stable mixed donor chimerism $>90 \%$ and no signs of relapse. Median dose of romiplostim was 5 (range, $3-5$ ) $\mathrm{mcg} / \mathrm{kg}$ weekly, eltrombopag - 50 (range, 50-150) $\mathrm{mg} /$ day. Overall response (OR) included $\mathrm{CR}$ (platelet $\geq 100 \times 10^{9} / \mathrm{L}, \mathrm{ANC} \geq 1.5 \times 10^{9} / \mathrm{L}$, and hemoglobin $\geq 100 \mathrm{~g} / \mathrm{l}$ ) and $\mathrm{PR}$ (platelet $>20 \times 10^{9} / \mathrm{L}$, ANC $\geq 0,5 \times 10^{9} / \mathrm{L}$, hemoglobin $\left.>70 \mathrm{~g} / \mathrm{l}\right)$.

Results: Median time from PGF diagnosis to treatment with TPO agonists was 14 days (0-119), median treatment duration was 3 weeks (1-43). TPO agonists were well tolerated with no cases of grade III-IV toxicity. TPO agonists were combined with rituximab $(n=4)$, rituximab and DLI $(n=3)$ and HSC boost $(n=1)$ in $8(28 \%)$ patients. A total of $14(48 \%)$ patients met criteria of response (CR: $\mathrm{n}=4,14 \%$; PR: $\mathrm{n}=10,34 \%$ ). Combination therapy showed no difference in OR compared to TPO agonists alone. OR was not depended on the TPO agonist used nor the time to therapy initiation. Median increase in ANC in responders was $3.4 \times 10^{9} / \mathrm{L}(0.8-6.0)$, in platelet count $-48 \times 10^{9} / \mathrm{L}(21-$ 205). A total of 15 patients died due to relapse $(n=2)$, GVHD III-IV grade $(n=3)$ and infection $(n=10)$. Two-year OS from the start of TPO agonist therapy was $44 \%(95 \%$ CI, 25-62) with a significant difference between responders and non-responders: $71 \%(95 \%$ CI, 33-90) vs. $18 \%(95 \%$ CI, 3-40) $(\mathrm{p}=0,002)$.

Conclusions: This study showed promising results of TPO agonists for management of sPGF. Further studies are warranted to specify optimal timing and dosing regimen, predictors of response.

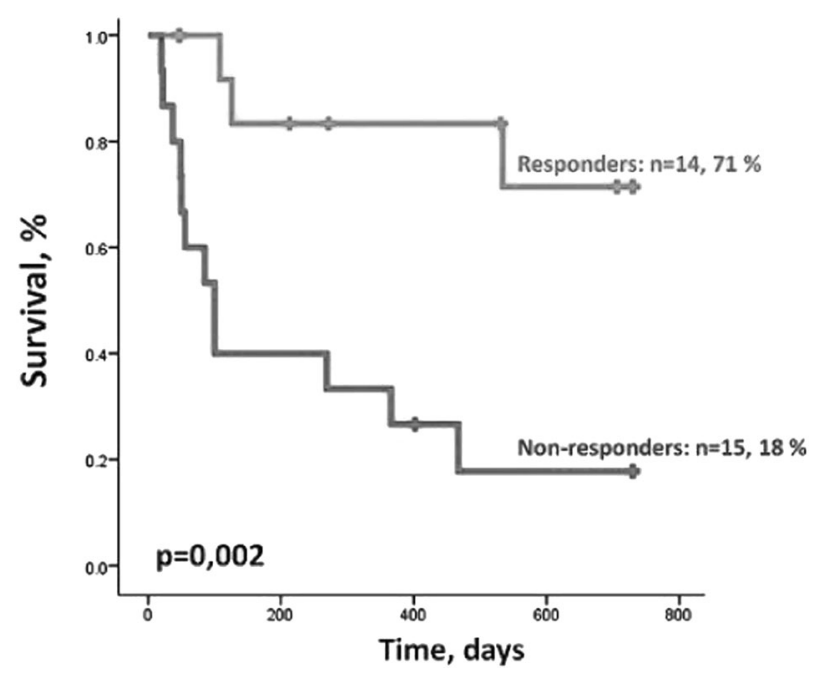

[[P144 Image] 1. Two-year OS in responders and nonresponders to TPO agonist therapy] 
Disclosure: There are conflicts of interest to disclose

\section{P145}

Cytomegalovirus reactivation kinetics and peak titers as novel predictors of survival and relapse after allogeneic cell transplantation for hematologic malignancies

Saskia Leserer ${ }^{I}$, Evren Bayraktar ${ }^{I}$, Nikolaos TsachakisMück ${ }^{1}$, Michael Koldehoff ${ }^{1}$, Lara Kasperidus ${ }^{1}$, Esteban Arrieta-Bolanos $^{I}$, Mirko Trilling ${ }^{I}$, Katharina Fleischhauer $^{I}$, Dietrich W. Beelen ${ }^{1}$, Amin T. Turki ${ }^{1}$

${ }^{1}$ University Hospital Essen, Essen, Germany,

Background: After allogeneic hematopoietic cell transplantation (HCT), human Cytomegalovirus (CMV) reactivation associates with non-relapse mortality (NRM) but also with reduced relapse in patients with leukemia, as shown by numerous studies that evaluated CMV reactivation as a qualitative yes/no parameter in the first months posttransplant. We hypothesized that longitudinal quantitative assessment of CMV reactivation kinetics and virus loads might improve patient-specific clinical outcome associations.

Methods: This retrospective study included 705 patients with HCT for hematologic malignancies treated between 01/2012 and 12/2017 at University Hospital Essen, Germany. CMV titers were monitored weekly by quantitative PCR (qPCR); CMV reactivation was defined by a cutoff of $>500$ genome copies per ml. Patients were included for analysis, if at least 5 measurements were available during the first 200 days after HCT. In total, 11,508 samples were analyzed. Subgroup analyses were performed according to the time of CMV reactivation (before/after $+30 \mathrm{~d}$ ) or the CMV viremia titer $(>100,000,20,000-100,000$ and 500 - 20,000 copies/ml).

Results: CMV reactivation was detected in 350 (median age 58 years; range 17-76 years) out of 705 patients. Baseline characteristics (age, gender, underlying disease, transplant) of patients without CMV reactivation were comparable. CMV reactivation kinetics followed a Gaussian normal distribution with a median first reactivation at $+33 \mathrm{~d}$ and peak titers at $+47 \mathrm{~d}$. All except 1 patient reactivated before $100 \mathrm{~d}, 40 \%$ before $+30 \mathrm{~d}$. Overall survival (OS) of the CMV reactivation group as a whole did not significantly differ from the non-reactivation group (34 vs. 38 months).

However, in subgroup analyses OS was significantly reduced in patients with very early $(<+30 \mathrm{~d})$ compared to later reactivation ( 17 vs. 59 months, $\mathrm{p}=0.040$ ). Moreover and importantly, OS was significantly reduced in patients with CMV reactivation at high titers of $>100,000$ copies $/ \mathrm{ml}$ compared to those with lower titers ((10 vs. 45 months $) \mathrm{p}<$
0.0001).Cox regression analyses confirmed significantly reduced OS for patients with CMV reactivation $>100,000$ copies $/ \mathrm{ml}$ and $<$ day +30 as compared to the other cohorts (HR 2.03, 95\%CI 1.45-2.86, $\mathrm{p}<0.0001$ and HR 1.36, 95\% CI 1.01-1.83, $\mathrm{p}=0.041$ ) respectively). The NRM was consistently higher (HR 2.59; 95\%CI, 1.69-3.97, p< 0.0001 ) for patients with CMV copies $>100,000 / \mathrm{ml}$. The risk of hematologic relapse was exclusively reduced in patients with a peak CMV viremia between 20,000 and 100,000 copies/ml (HR 0.55, 95\% CI 0.32-0.95; $\mathrm{p}=0.033$ ) as compared to patients without CMV reactivation. For other levels of CMV reactivation this effect was not observed.

Conclusions: Our data showed that CMV reactivations before $+30 \mathrm{~d}$ or with high titers of $>100,000$ copies $/ \mathrm{ml}$ associated with significantly reduced OS, while CMV reactivations at intermediate titers between 20,000 and 100,000 copies $/ \mathrm{ml}$ had a positive impact on relapse incidence. These findings underline the complexity of CMV reactivations after HCT outcome, and support longitudinal evaluation of CMV titers and individualized quantitative kinetics models for risk assessment after HCT to distinguish the advantageous from the detrimental aspects of CMV reactivation.

Disclosure: ATT has received lecture fees from Jazz Pharmaceuticals and travel subsidies from Neovii Biotech outside the submitted work. The other authors declare no competing financial interests within the submitted work.

\section{P146}

Association of serum ferritin levels before start of conditioning with mortality after alloSCT - a prospective, non-interventional study of the EBMT transplant complication working party

Olaf Penack ${ }^{1}$, Christophe Peczynski², Steffie van der Werf $^{3}$, Mohammad Mohty ${ }^{4}$, Ibrahim Yakoub-Agha ${ }^{5}$, Sylvia Montoto $^{6}$, Jürgen Finke ${ }^{7}$, Arnold Ganser ${ }^{8}$, Helene Schoemans $^{9}$, Jane Apperley ${ }^{10}$, Ritta Niittyvuopio ${ }^{11}$, Wilfried Schroyens $^{12}$, A Unal ${ }^{13}$, Armin Gerbitz ${ }^{14}$, Michael Schaap $^{15}$, J Sierra ${ }^{16}$, Agostino Cortelezzi ${ }^{17}$, Gerald Wulf ${ }^{18}$, Pascal Turlure $^{19}$, Montserat Rovira ${ }^{20}$, Z Ozkurt ${ }^{21}, M$ Pascual Cascon ${ }^{22}$, Johannes Clausen ${ }^{23}$, Hildegard Greinix $^{24}$, Rafael Duarte ${ }^{25}$, Grzegorz Basak ${ }^{26}$

${ }^{1}$ Charite Campus Virchow Klinikum, Hematology, Berlin, Germany, ${ }^{2}$ EBMT Paris Study Office/CEREST-TC, Hôpital Saint Antoine, Paris, France, ${ }^{3}$ EBMT Leiden Data Office, Leiden, Netherlands, ${ }^{4}$ Hospital Saint Antoine, Paris, France, ${ }^{5} \mathrm{CHRU}$ Lille, Lille, France, ${ }^{6} \mathrm{St}$. Bartholomew's and the Royal London NHS Trust, London, United Kingdom, ${ }^{7}$ University Freiburg, Freiburg, Germany, ${ }^{8}$ University Hannover, Hannover, Germany, ${ }^{9}$ University Hospital 
Gasthuis, Leuven, Belgium, ${ }^{10}$ Imperial College London, London, United Kingdom, ${ }^{11} \mathrm{HUCH}$ Comprehensive Cancer Center, Helsinki, Finland, ${ }^{12}$ Antwerp University Hospital, Antwerp, Belgium, ${ }^{13}$ Erciyes Medical School, Kayseri, Turkey, ${ }^{14}$ Charité Univerisätsmedizin Berlin, Berlin, Germany, ${ }^{15}$ Radboud University, Nijmegen, Netherlands, ${ }^{16}$ Hospital Santa Creu i Sant Pau, Barcelona, Spain, ${ }^{17}$ Fondazione IRCCS - Ca'Granda, Milan, Italy, ${ }^{18}$ Universitätsklinikum Göttingen, Göttingen, Germany, ${ }^{19} \mathrm{CHRU}$ Limoge, Limoge, France, ${ }^{20}$ Hospital Clinic, Barcelona, Spain, ${ }^{21}$ Gazi University Faculty of Medicine, Ankara, Turkey, ${ }^{22}$ Hospital Regional de Málaga, Malaga, Spain, ${ }^{23}$ Ordensklinikum, Linz, Austria, ${ }^{24}$ University Hospital Graz, Graz, Australia, ${ }^{25}$ Hospital Universitario Puerta de Hierro, Madrid, Spain, ${ }^{26}$ Central Clinical Hospital, Warsaw, Poland

Background: Elevated serum ferritin levels occur due to iron overload or during inflammation and macrophage activation. A correlation of high serum ferritin levels with increased mortality after alloSCT has been suggested by several retrospective analyses as well as by two smaller prospective studies.

Methods: This international multicentric study aimed to study the association of ferritin serum levels before start of conditioning with alloSCT outcome. Patients with acute leukemia, lymphoma or MDS receiving a matched sibling alloSCT for the first time were considered for inclusion, regardless of conditioning. Data were prospectively collected between $8 / 2014$ and 2/2018. A comparison of outcomes between patients with high and low ferritin level was performed using univariate analysis and multivariate analysis using cause-specific Cox model. Variables included in the multivariate analyses were age, sex mismatch, diagnosis, disease status, Karnofsky score, number of CD34 cells given, intensity of conditioning, type of GVHD prophylaxis, ATG use, time from diagnosis to transplant, year of transplant and CMV status.

Results: Twenty centers from 10 European countries reported data on 385 alloSCT recipients. Patient characteristics are given in Table 1. The ferritin cut off point was determined at $1500 \mu \mathrm{g} / \mathrm{l}$ (median of measured ferritin levels). Overall survival of alloSCT recipients with ferritin levels above cut off measured before start of conditioning was significantly shorter (Figure $1 \mathrm{~A}$, univariate $\mathrm{HR}=2.3$ $\mathrm{CI}=1.4-3.6 \mathrm{p}=0.00041$; multivariate $\mathrm{HR}=2.5, \mathrm{CI}=1.5$ $4.1, \mathrm{p}=0.0005)$. Progression-free survival was also shorter (Figure 1B, univariate $\mathrm{HR}=2.1 \mathrm{CI}=1.4-3.2 \mathrm{p}=0.00014$; multivariate $\mathrm{HR}=2.4, \mathrm{CI}=1.6-3.8, \mathrm{p}<0.0001)$. Excess mortality in the high ferritin group was due to both higher relapse incidence (univariate $\mathrm{HR}=1.7 \mathrm{CI}=1-2.8 \mathrm{p}=0.03$; multivariate $\mathrm{HR}=2.2, \mathrm{CI}=1.2-3.8, \mathrm{p}=0.007)$ and increased non-relapse mortality (univariate $\mathrm{HR}=3.1 \mathrm{CI}=1.5-6.3$ $\mathrm{p}=0.002 ;$ multivariate $\mathrm{HR}=3.1, \mathrm{CI}=1.5-6.4, \mathrm{p}=0.002$ ). Non-relapse mortality was driven by significantly higher infection-related mortality in the high ferritin group (univariate $\mathrm{HR}=3.9 \mathrm{CI}=1.6-9.7 \mathrm{p}=0.003$; multivariate $\mathrm{HR}=3.9, \mathrm{CI}=1.6-9.7 \mathrm{p}=0.003)$. Acute and chronic GVHD incidence or severity were not associated to serum ferritin levels.

Conclusions: Ferritin levels before start of conditioning can serve as routine laboratory biomarker to predict mortality after alloSCT.

\begin{tabular}{|c|c|c|c|}
\hline & $\begin{array}{l}\text { Ferritin } \\
<=1500 \mu \mathrm{g} / \mathrm{l} \\
(\mathrm{n}=153)\end{array}$ & $\begin{array}{l}\text { Ferritin } \\
>1500 \mu \mathrm{g} / 1 \\
(\mathrm{n}=145)\end{array}$ & $\begin{array}{l}\mathrm{p}- \\
\text { value }\end{array}$ \\
\hline $\begin{array}{l}\text { Year of transplant median(range) } \\
\text { [IQR] }\end{array}$ & $\begin{array}{l}\text { 2016(2014- } \\
2018)[2015- \\
2017]\end{array}$ & $\begin{array}{l}2015(2014- \\
2018)[2015- \\
2016]\end{array}$ & 0.6 \\
\hline $\begin{array}{l}\text { Patient age (years) median(range) } \\
\text { [IQR] }\end{array}$ & $\begin{array}{l}52(17.1-71.3) \\
{[38.1-60.2]}\end{array}$ & $\begin{array}{l}53.2(19-70.9) \\
{[42.9-62.3]}\end{array}$ & 0.3 \\
\hline $\begin{array}{l}\text { Time from diagnosis to transplant } \\
\text { (months) median (range)[IQR] }\end{array}$ & $5(1-71)[3-8]$ & $4(1-61)[3-6]$ & 0.05 \\
\hline $\begin{array}{l}\text { Number of CD34+ cells infused (e } \\
+06) \text { median (range) [IQR] }\end{array}$ & $\begin{array}{l}5.9(0.9-10.7) \\
{[4.5-7.2]}\end{array}$ & $\begin{array}{l}5.5(0.6-10.2) \\
{[4.2-6.7]}\end{array}$ & 0.1 \\
\hline $\begin{array}{l}\text { Sex mismatch Female to male Other } \\
\text { combination }\end{array}$ & $\begin{array}{l}27(18 \%) 123 \\
(82 \%)\end{array}$ & $\begin{array}{l}43(30 \%) 98 \\
(70 \%)\end{array}$ & 0.012 \\
\hline $\begin{array}{l}\text { Diagnosis Acute leukaemia } \\
\text { Lymphoma MDS }\end{array}$ & $\begin{array}{l}93(61 \%) 19 \\
(12 \%) 41(27 \%)\end{array}$ & $\begin{array}{l}107(74 \%) 12 \\
(8 \%) 26(18 \%)\end{array}$ & 0.058 \\
\hline Disease status CR Not in CR & $\begin{array}{l}94(64 \%) 54 \\
(36 \%)\end{array}$ & $\begin{array}{l}98(70 \%) 42 \\
(30 \%)\end{array}$ & 0.2 \\
\hline ATG No Yes & $\begin{array}{l}77(50 \%) 76 \\
(50 \%)\end{array}$ & $\begin{array}{l}79(54 \%) 66 \\
(46 \%)\end{array}$ & 0.5 \\
\hline $\begin{array}{l}\text { Conditioning intensity MAC/ } \\
\text { Chemo MAC/TBI RIC }\end{array}$ & $\begin{array}{l}30(20 \%) 26 \\
(17 \%) 95(63 \%)\end{array}$ & $\begin{array}{l}32(22 \%) 25 \\
(17 \%) 87(61 \%)\end{array}$ & 0.9 \\
\hline
\end{tabular}

[[P146 Table] 1. Table 1]

Disclosure: The authors declare no confict of interest related to this study

\section{P147}

Prediction of reduced lung function and acute GVHD by surfactant protein $D$ in allogeneic stem cell transplantation

Anne Mols Krarup ${ }^{1}$, Hilde Hylland Uhlving ${ }^{I}$, Rudi Steffensen ${ }^{2}$, Katrine Kielsen ${ }^{1}$, Carsten Heilmann ${ }^{1}$, Marianne Ifversen ${ }^{1}$, Kim Gjerum Nielsen ${ }^{1}$, Henrik Sengeløv ${ }^{1}$, Frederik Buchvald ${ }^{1}$, Grith Lykke Sorensen ${ }^{3}$, Klaus Gottlob Müller ${ }^{I}$

${ }^{1}$ Rigshospitalet, University of Copenhagen, Copenhagen, Denmark, ${ }^{2}$ Aalborg University Hospital, Aalborg, Denmark, ${ }^{3}$ Institute of Molecular Medicine, University of Southern Denmark, Odense, Denmark

Background: Obstructive impairment of lung function is a complication following allogeneic hematopoietic stem cell 
transplantation (HSCT) and may progress to bronchiolitis obliterans that has a high mortality rate.

Surfactant protein D (SP-D) is an innate defense molecule involved in immune regulation at the epithelial surfaces, particularly in the lungs, and elevated levels have been associated with exacerbation of chronic obstructive pulmonary disease (COPD). The aim of this study was to investigate, whether SP-D plasma levels and variants in the gene encoding SP-D may predict the development of reduced lung function after allogenic HSCT.

Methods: We performed a population-based, singlecenter study of children (aged 6-18 years) treated with allogeneic HSCT. The study consisted of 1) a prospective study of serial plasma SP-D levels and rs721917 genotypes in 55 patients during the first 6 months after HSCT, and 2) a retrospective study of rs721917 genotypes within the SP-D gene in 247 patients transplanted between 1990-2017. Pulmonary function tests were performed regularly as part of the clinical monitoring.

Results: At the day of graft infusion (day 0) SP-D levels were reduced compared to levels before start of treatment with conditioning chemotherapy, defined as baseline (615 $\mathrm{ng} / \mathrm{ml}$ (quartiles 441-1132) at day $0 \mathrm{vs} 771 \mathrm{ng} / \mathrm{ml}$ (5421348) at baseline, $p<0.01$ ). From day +7 SP-D levels increased and remained increased during the whole study period $(771 \mathrm{ng} / \mathrm{ml}(542-1348)$ at baseline vs $1287 \mathrm{ng} / \mathrm{ml}$ (713-2549) at 6 months, $\mathrm{p}<0.01)$. Acute GvHD (aGvHD) occurred in 25 patients, of those 17 patients with grade 2-4. High SP-D levels at day +14 were associated with the development of aGvHD (1402 $\mathrm{ng} / \mathrm{ml}$ (1244-2023) vs 839 $\mathrm{ng} / \mathrm{ml}(523-1630), \mathrm{p}<0.01$ ) (fig. 1).

The C/C genotype was associated with generally low SPD levels and low FEV1/FVC at all time intervals compared to the other genotypes, significantly 24-36 months postHSCT $(p=0.02)$. There was no overall correlation between SP-D levels and lung function, but stratifying for genotype, high baseline SP-D levels were predictive for reduced FEV1/FVC at 8-24 months in CC and TT homozygous individuals.

Conclusions: Patients with a genotype causing low capacity for SP-D production are at increased risk of developing pulmonary impairment after HSCT. In addition, our data lend support to other studies indicating that SPD production may increase during inflammatory pulmonary disease, acting as a reactive, protective mechanism. Further research is warranted to define the role of SP-D levels and genotypes as a prognostic tool for lung function and aGvHD.

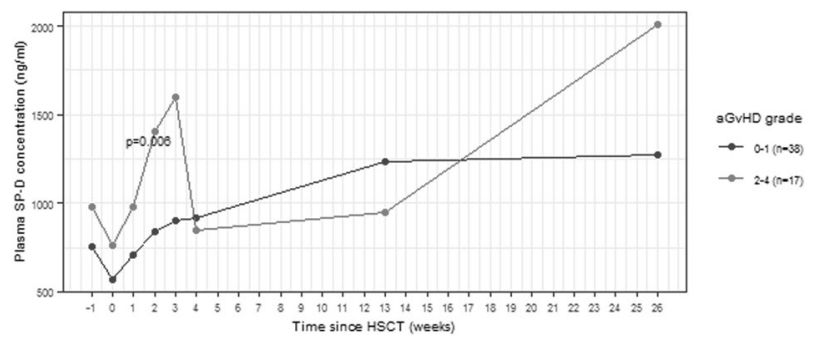

[[P147 Image] 1. Figure 1: SP-D levels in patients with and without $a G v H D$ by time from the transplant.]

Disclosure: Nothing to declare.

\section{P148}

Positive effects of whole body vibration on patient's physical capacity and quality of life during allogeneic hematopoietic cell transplantation - A randomized controlled study

Antonia Pahl, Anja Wehrle ${ }^{1}$, Sarah Kneis ${ }^{1}$, Albert
Gollhofer $^{1}$, Jürgen Finke ${ }^{1}$, Hartmut Bertz

${ }^{1}$ University of Freiburg, Germany, Freiburg, Germany

Background: Allogeneic hematopoietic cell transplantation (alloHCT) means a long period of restricted mobility and a range of therapy related side effects on muscle function. In this context patients demonstrated a huge decline of physical capacity and muscle mass in particular, accompanied with a decrease of quality of life (QoL). Resistance training could maintain muscle mass but is limited by patients' blood values (platelet-count) and well-being. Whole body vibration (WBV) was shown to maintain muscle mass during bed rest and has less impact on blood pressure than conventional resistance exercises. Furthermore it was also shown to be feasible in patients during high dose chemotherapy. Therefore the aim of our study was to examine the effects of WBV during alloHCT on patients physical and functional performance as well as QoL.

Methods: 43 patients receiving alloHCT were randomly allocated to either a WBV exercise group (IG) or an active control group (CG) doing stretching and mobilization. Both groups exercised during the whole time of hospitalization for 5 times per week and underwent pre-, post- and followup-assessment. Physical capacity was determined by maximum oxygen consumption $\left(\mathrm{VO}_{2 \text { peak }}\right)$ and maximum power $\left(\mathrm{P}_{\max }\right)$ during cardiorespiratory exercise test and by maximum strength of the knee extensors and flexors $\left(\mathrm{EX}_{\max }, \mathrm{FLEX}_{\max }\right)$ during isokinetic strength test. Functional performance was assessed by jumping height during counter movement jump (CMJ) and time of chair rising test (CRT) as well as power output during both tests. QoL was assessed by questionnaires of the EORTC. 
Results: During alloHSCT $\mathrm{VO}_{2 \text { peak }}$ and $\mathrm{P}_{\max }$ decreased in both groups but till follow-up an increase is seen in the IG $(P=0.035 ; P=0.011)$. At day $+180 /$ follow-up a $\mathrm{VO}_{2 \text { peak }}$ group difference is seen $(P=0.034)$. $\operatorname{EX}_{\max }(P=0.003)$ and FLEX $_{\max }(P=0.044)$ were only reduced in the CG during hospitalization. Jumping height and power output decreased in the CG during hospitalization $(P=0.005, P=0.039)$ and a difference between groups were seen in changes of jumping height from pre- to follow-up-assessment $(P=0.033)$ : increase in the IG and decrease in the CG. The IG showed a decrease in time from baseline to follow-up $(P=0.022)$ in the CRT and an increase of power output $(P=0.009)$. QoL decreased only in the CG during hospitalization $(P=0.015)$ while during follow-up QoL increased in both groups (IG: $P=0.013$; $\mathrm{CG}: P=0.037)$. In the $\mathrm{CG}$ physical functioning decreased during intervention $(P=0.001)$ whereas an increase was seen in the IG from pre- to follow-upassessment $(P=0.035)$. Body image was significant worse in the $\mathrm{CG}$ compared to the IG at hospital discharge $(P=0.007)$ as well as at follow-up measurement $(P=0.030)$ where it got worse over time $(P=0.036)$.

Conclusions: WBV was shown to maintain maximum strength, jumping performance and QoL during alloHCT. Although cardiorespiratory fitness could not be maintained by WBV during hospitalization, it seems in the follow up period till day +180 that recovery of the cardiorespiratory system is enhanced by WBV carried out during alloHST. Nevertheless reasons for this changes in recovery have to be analyzed in further studies as well as treatment effects of WBV compared to conventional resistance training.

Disclosure: Supported by a grant of the faculty of medicine and Comprehensive Cancer Center Freiburg

\section{P149}

Respiratory virus infection within 1 year after of AlloSCT is the significant risk factor of obstructive ventilatory disturbance

Kosei Kageyama', Michiho Ebihara', Mitsuhiro Yuasa', Daisuke Kaji ${ }^{1}$, Aya Nishida ${ }^{1}$, Shinsuke Takagi ${ }^{1}$, Hisashi Yamamoto $^{1}$, Go Yamamoto ${ }^{1}$, Yuki Asano-Mori', Naoyuki Uchida $^{1}$, Atsushi Wake ${ }^{1}$, Akiko Yoneyama', Shigeyoshi Makino ${ }^{1}$, Shuichi Taniguchi ${ }^{1}$

${ }^{1}$ Toranomon Hospital, Hematology, Tokyo, Japan,

Background: Obstructive ventilatory disturbance (OVD) is one of the major life-threading complication at the chronic phase of allogeneic stem cell transplantation (Allo-SCT). Bronchiolitis obliterans has been the most established etiology as a part of chronic graft-versus-host disease and major cause of late non-relapse mortality of Allo-SCT. But other etiologies impact on respiratory function after AlloSCT and risk factor of OVD have not been well understood.

Methods: To address these issues, we retrospectively reviewed the medical record of 747 consecutive patients who first Allo-SCT at Toranomon Hospital between 2009 and 2017. To detect OVD, forced expiratory volume in 1 second (FEV1.0) showed less than $80 \%$ of predicted in spirometry test was defined as positive. In the recipients who showed FEV1.0 less than $80 \%$ in pre-transplant test, more than $20 \%$ reduction of FEV1.0 was regarded as positive. Nasopharyngeal swab of those who had upper respiratory tract symptoms were tested for the presence of respiratory viral antigens (ADV, PIV, and RSV). Patients with ECOG performance status of 4, had active infection at transplant were excluded from this analysis. The cases of early death or relapse before 30 days post-transplant, and the cases of graft failure were also excluded.

Results: The median age was 55 years (range, 16-74). Underlying diseases were AML in 403, MDS/MPD in 73, CML in 26, ALL in 82, ATL in 19, HL in 12, NHL in 104, and others in 28 . Five hundred twenty-nine $(71 \%)$ were not in remission at the time of transplant. Five hundred eightythree patients $(78 \%)$ were conditioned with myeloablative regimens, whereas 164 patients received reduced-intensity regimens. Donor sources consisted of related peripheral blood /bone marrow (BM) $(n=85)$, unrelated BM (153) and cord blood (509). One hundred seventeen developed respiratory virus infection on median of 45(0-363) days post-transplant. ADV in 3, PIV in 61, RSV in 38, ADV/PIV in $4, \mathrm{ADV} / \mathrm{RSV}$ in $1, \mathrm{PIV} / \mathrm{RSV}$ in 10 . With median observation period of survivors of 1438 (30-3390) days, 490 recipients $(66 \%)$ underwent spirometry at least once. Forty-six developed OVD on median of 198 (60-804) days post-transplant. Cumulative incidence of OVD was $6.4 \%$ in total population. In 490 recipients those who could spirometry, overall survival at 5 years was $73.2 \%$ in patients who developed OVD and was comparable with those who did not develop it $(64.3 \%, p=0.486)$. In univariate analysis, disease status (CR/AA or nonCR), recipient age (age $<55$ or $\geq 55$ ), prior autologous stem cell transplantation (yes or no), intensity of conditioning regimen (MAC or RIC), TBI dose ( $<8$ Gy or $\geq 8 \mathrm{~Gy}$ ), busulfan dose $(<9.6 \mathrm{mg} / \mathrm{kg}$ or $\geq 9.6 \mathrm{mg} / \mathrm{kg})$, donor source (cord blood or non-cord) had no impact on the incidence of OVD. Patients who developed respiratory virus infection showed significantly higher incidence of OVD compared to those who did not developed it $(12.4 \%$ vs $5.3 \%$, p< 0.01$)$. In multivariate analysis, respiratory virus infection was the only significant risk factor for the development of OVD $(\mathrm{HR}=2.43,95 \%$ CI 1.30-4.57, $\mathrm{p}<0.01)$.

Conclusions: Respiratory virus infection within 1 year after Allo-SCT is the significant risk factor of OVD.

Disclosure: Nothing to declare. 


\section{P150}

Abstract already published.

\section{P151}

increased risk of metabolic syndrome in male survivors after allo-HSCT in childhood despite normal BMI at long-term follow-up

Ena Muhic ${ }^{1}$, Sidsel Mathiesen ${ }^{1}$, Marianne Ifversen ${ }^{I}$, Kaspar Sørensen ${ }^{1}$, Anders Juul ${ }^{1}$, Klaus Müiller ${ }^{1}$

${ }^{1}$ Rigshospitalet, University of Copenhagen, Copenhagen, Denmark

Background: Metabolic Syndrome (MetS) is related to increased risk of cardiovascular disease and type-2 diabetes (DM-2) and usually seen in overweight individuals in the general population. We investigated MetS and clinical risk factors two decades after HSCT.

Methods: All male survivors treated with myeloablative allo-HSCT during childhood (< 17 years) between 19802010 in Denmark were invited to a follow-up study. MetS was defined as the presence of at least three NCEP ATP III criteria: fasting plasma triglyceride $(\mathrm{TG}) \geq 1.7 \mathrm{mmol} / \mathrm{L}$, high density lipoprotein (HDL) $<1.03 \mathrm{mmol} / \mathrm{L}$ or medical treatment of hyperlipidemia; fasting plasma glucose (FPG) $\geq 5.6 \mathrm{mmol} / \mathrm{L}$; abdominal circumference $(\mathrm{AC})>102 \mathrm{~cm}$; BP $\geq 130 \mathrm{mmHg}$ (systolic) / $\geq 85 \mathrm{mmHg}$ (diastolic) or medical treatment for hypertension.

Patients with overt DM-2 were included into the MetS group. Furthermore, patients were examined for chronic graft-versus-host disease (cGvHD) by the NIH-criteria at the time of follow-up and high sensitivity C-reactive protein (hsCRP) was measured. The prevalence of MetS was compared to a Nordic reference group (Hildrum et al. 2009).

Results: We included 49 out of 97 eligible males (participation rate 51\%) aged 18-44 years, median 29 years. Median (range) follow-up was 21 (8-32) years. Of these 49 males, $74 \%$ had a malignant diagnosis and $65 \%$ were treated with TBI-based conditioning. Donors were matched siblings $(n=21)$, matched relatives $(n=3)$ or matched unrelated donors $(n=25)$.

MetS was more prevalent $(33 \%)$ in the young adult survivors compared to the prevalence reported for 20-39year-olds in the Nordic reference (16\%). Instead the prevalence was comparable to that reported for the 50-69year-olds $(32 \%)$. Of the components of Mets, elevated TG (51\%), hypertension (47\%), and decreased HDL (40\%) were frequent, while FPG was elevated in $16 \%$. Importantly, only $4 \%$ of those with MetS had increased AC and mean BMI $\left(23.8 \mathrm{~kg} / \mathrm{m}^{2}\right)$ of the HSCT survivors was within normal range in contrast to features of MetS observed in the background population.

Having MetS was significantly associated with TBI (RR $=7.9,95 \% \mathrm{CI}(1.1-55.3), \mathrm{p}=0.004)$ as was the following single components of MetS (mean in TBI group vs. mean in non-TBI group): elevated TG $(2.34 \mathrm{mmol} / \mathrm{L}$ vs. $0.93 \mathrm{mmol} /$ $\mathrm{L}, \mathrm{p}=0.006)$, lower HDL (1.04 mmol/L vs. $1.38 \mathrm{mmol} / \mathrm{L}$, $\mathrm{p}=0.001)$ and higher diastolic $\mathrm{BP}(80 \mathrm{mmHg}$ vs. $72 \mathrm{mmHg}$, $\mathrm{p}=0.03$ ).

MetS was only demonstrated in one patient who received non-TBI based conditioning.

Sixteen of 49 patients had cGvHD of which nine were moderate to severe cases, but cGvHD was not associated with MetS. However, low-grade inflammation measured by hsCRP was related to increased AC ( $\mathrm{rho}=0.41, \mathrm{p}=0.004)$ and $\mathrm{TG}$ ( $\mathrm{rho}=0.34, \mathrm{p}=0.028)$.

Conclusions: Our results indicate that male long-term survivors of allo-HSCT during childhood have a high risk of MetS at an earlier age than the general population. The presence of MetS despite normal BMI in several patients suggests unconventional etiologies like the effect of TBI and low-grade inflammation.

Disclosure: Nothing to declare.

\section{P152}

current practice in vitamin $D$ management across adult and paediatric allogeneic haematopoietic stem cell transplant centres: A survey by transplant complications working party of EBMT

Jose Ros-Soto ${ }^{1}$, John A. Snowden ${ }^{2}$, Nina Salooja ${ }^{3}$, Maria Gilleece $^{4}$, Anne Parker ${ }^{5}$, Diana Greenfield ${ }^{2}$, Chloe Anthias $^{6}$, Arun Alfred ${ }^{7}$, Alenca Harrington ${ }^{8}$, Christophe Peczynski $^{8}$, Karl Peggs ${ }^{9}$, Alejandro Madrigal, Grzegorz W. Basak ${ }^{10}$, Hélène Schoemans ${ }^{11}$

${ }^{1}$ Anthony Nolan, London, United Kingdom, ${ }^{2}$ Sheffield Teaching Hospitals NHS Trust, Sheffield, United Kingdom, ${ }^{3}$ Imperial College London, London, United Kingdom, ${ }^{4} \mathrm{St}$ James's Hospital, Leeds, United Kingdom, ${ }^{5}$ Queen Elizabeth University Hospital, Glasgow, United Kingdom, ${ }^{6}$ Royal Marsden NHS Trust, London, United Kingdom, ${ }^{7}$ The Rotherham NHS Foundation Trust, Rotherham, United Kingdom, ${ }^{8}$ EBMT Data Office, Paris, France, ${ }^{9}$ University College London Hospital, London, United Kingdom, ${ }^{10}$ Medical University of Warsaw, Warsaw, Poland, ${ }^{11}$ University Hospital Leuven and KU Leuven, Leuven, Belgium

Background: Vitamin D (VD) has immune-regulatory properties and can affect the course of haematopoietic stem cell transplantation (HSCT). 
Methods: To describe the current clinical practice of diagnosis and treatment of VD deficiency in allogeneic HSCT patients, directors of 326 EBMT affiliated allogeneic transplant centres from 42 countries were invited to participate in an online survey comprising 34 questions.

Results: This survey was completed by transplant directors (46\%), transplant consultants (41\%), nonconsultant grade physicians (8\%), HSCT clinical nurses specialists (CNS) $(3 \%)$ and other (2\%) from 114 centres in 24 countries. $58 \%$ of the centres are adult-only, $21 \%$ paediatric-only and $21 \%$ treat adult and paediatric patients (mixed centres). $46 \%$ are located higher than 50 degrees latitude (northern countries) and 54\% lower than this latitude (southern countries). At the time of the survey $84 \%$ were members of the European Union (EU).

Measurement of serum VD is routinely performed in $47 \%$ of the centres prior and in $70 \%$ after allogeneic HSCT. The main clinical indications are known osteopaenia/osteoporosis $(86 \%)$, previous fracture $(71 \%)$, treatment with steroids (68\%), premature menopause $(46 \%)$ and established menopause (32\%). Monitoring occurs every 3 months (39\%), every 6 months (24\%), once a year (18\%) or at other time-points (19\%). In this regard, seasonality is not taken into account in the majority of the centres (94\%). Local and national/international guidelines (NICE) are only followed by $19 \%$ and $18 \%$ of the centres, respectively. The most common cut-off value of serum VD for commencing on replacement is $50 \mathrm{nmol} / \mathrm{L}$ ( $32 \%$ ). Northern countries tend to use values of $\geq 75 \mathrm{nmol} / \mathrm{L}$ whereas southern countries $\leq 50$ $\mathrm{nmol} / \mathrm{L}$. $15 \%$ do not use cut-off values.

Following HSCT, $83 \%$ of centres prescribe VD supplements to maintain calcium metabolism and bone health (92\%), enhance immune reconstitution post-HSCT (24\%), GvHD prevention (17\%), enhance immune-suppression to treat GvHD (10\%), treat depression/fatigue (3\%) and reduce relapse risk $2 \%$. A "loading" dose is administrated in $30 \%$ (54\% adult, $25 \%$ mixed and $22 \%$ paediatric), with a mean duration of 4 weeks (1-52). The median daily loading dose is 2,000 IU (286-20,000). The median "maintenance" daily dose is $800 \mathrm{IU}(67-10,000)$. There are not remarkable differences between adult and paediatric centres or northern and southern countries.

VD replacement is prescribed by transplant physicians (75\%), family physicians (10\%), endocrinologists (3\%), CNS $(3 \%)$, others $(4 \%)$ and in $5 \%$ of the centres, patients are advised to buy it over-the-counter. VD is prescribed combined with calcium carbonate in 52\% and alone in $48 \%$ of the centres. It is eventually discontinued by $69 \%$ of the centres when therapeutical levels of VD are reached (69\%), DEXA scan returns to normal $(12 \%)$ and symptomatic improvement (9\%).

Conclusions: This survey has demonstrated discrepancies in monitoring and replacement of VD across EBMT allogeneic HSCT Programmes. Although awareness has arisen over the last decade, there is still lack of evidence about the optimal levels of VD required for immunemodulation post-HSCT. This survey emphasises the need for specific guidelines to harmonise the current management of VD deficiency in adult and paediatric HSCT setting.

Disclosure: Nothing to declare

\section{P153}

Poor graft function after unmanipulated haploidentical SCT with post-transplant high dose cyclophosphamide

Ignacio Gómez-Centurión ${ }^{1}$, Pascual Balsalobre ${ }^{1,2}$, Ana Perez Corral ${ }^{1,2}$, Nieves Dorado ${ }^{1,2}$, Laura Solán $^{1,2}$, Rebeca Bailén $^{1,2}$, Silvia Monsalvo ${ }^{1,2}$, Carolina Martinez. Laperche $^{1,2}$, Ismael Buño ${ }^{1,2,3}$, Javier Anguita ${ }^{1,2}$, José Luis Diez-Martin $^{1,2}$, Mi Kwon ${ }^{1,2}$

${ }^{1}$ Hospital General Universitario Gregorio Marañón, Madrid, Spain, ${ }^{2}$ Instituto de Investigación Sanitaria Gregorio Marañón, Madrid, Spain, ${ }^{3}$ Unidad de Genómica, Hospital General Universitario Gregorio Marañón, Madrid, Spain

Background: The use of unmanipulated Haploidentical SCT (Haplo-SCT) with post-transplant Cyclophosphamide (PT-Cy) as GVHD prophylaxis has widely extended. Primary and secondary graft failure are relatively uncommon complications. However, poor graft function (PGF) after Haplo-SCT with PT-Cy has not been described thoroughly. The objective of this study is to describe characteristics, treatments and outcomes of patients with PGF after HaploSCT with PT-Cy.

Methods: We retrospectively analyzed 132 Haplo-SCT with PT-Cy consecutively performed between 2011 and 2017 in our centre. PGF was defined as either occurring after initial engraftment: persistent neutropenia (ANC < $500 / \mathrm{uL}$ ) with the need of at least 3 doses of G-CSF and/or thrombocytopenia (platelets $<20.000 / \mathrm{uL}$ ) with platelet transfusion dependence, with complete donor chimerism and without concurrent severe GVHD or disease relapse.

Results: Nineteen patients were excluded from the analysis due to early mortality (death before day +30 ), primary graft failure (absence of neutrophil engraftment by day +28 , with mixed chimerism) or secondary graft failure (development of severe cytopenias and mixed chimerism after initial achievement of neutrophil engraftment). Thirty one patients $(27,5 \%)$ were diagnosed with PGF. Main characteristics of these patients are summarized in Table 1 . Twenty six patients (84\%) presented with neutropenia and were treated with G-CSF, while 5 patients $(16 \%)$ only developed severe thrombocytopenia without neutropenia, and were treated only with platelet transfusion. Twenty four patients $(77,5 \%)$ had at least $1 \mathrm{CMV}$ reactivation, 15 
patients $(48 \%)$ had 2 or more CMV reactivations and 21 patients $(67 \%)$ received valganciclovir for CMV reactivation treatment. Although most patients achieved adequate peripheral blood counts (PBC) with initial salvage therapy, 6 patients $(19 \%)$ had persistent cytopenias in spite of G$\mathrm{CSF}$, platelet transfusion, CMV reactivation resolution and myelotoxic drugs withdrawal. Four of them were treated with a boost of CD34+ selected peripheral blood donor cells at a median of 170 days after HSCT (range 150-190). Median CD34+ cells infused was $3,42 \times 10^{6} / \mathrm{kg}$. These 4 patients achieved adequate $\mathrm{PBC}$ after salvage therapy and two developed GVHD. The other 2 patients were treated with increasing doses of thrombopoietin (TPO) receptor agonist (TRA) eltrombopag. One patient started treatment 160 days after HSCT with $25 \mathrm{mg}$ daily and increased dose to $125 \mathrm{mg}$ daily, with complete recovery of PBC 6 months after initiating TRA. The second patient started treatment 110 days after HSCT with $25 \mathrm{mg}$ daily and increased dose to $100 \mathrm{mg}$ daily, with complete recovery of PBC 2 months after initiating TRA. Twenty one patients $(67 \%)$ with PGF diagnosis had long term survival.

\begin{tabular}{ll} 
Number of patients & 31 \\
Median age, y (range) & $46(18-64)$ \\
Female/male, $\mathrm{n}$ & $12 / 19$ \\
Diagnosis & \\
AML/MDS & 13 \\
ALL & 5 \\
NHL & 5 \\
HL & 5 \\
Other & 3 \\
CMV serostatus & \\
D+/R+ & 16 \\
D+/R- & 4 \\
D-/R+ & 6 \\
D-/R- & 5 \\
Conditioning, MA/RIC & $13 / 18$ \\
Median CD34+ cells/kg infused & $5.46 \times 10 \mathrm{e} 6$ (2.24-11.4) \\
CMV reactivation & \\
1 episode & 24 \\
2 or more episodes & 15 \\
Treatment & \\
G-CSF & 26 \\
Only platelet transfusion & 5 \\
Boost CD34+ & 4 \\
TPO receptor agonist & \\
\hline
\end{tabular}

[[P153 Table] 1. Characteristics, treatments and outcomes of PGF patients]

Conclusions: Poor graft function is a frequent complication after Haplo-SCT with Cy-post. CMV reactivation and myelotoxic drugs could be the most relevant factors associated with development of this entity. Although most patients recover PBC without specific therapies beyond G$\mathrm{CSF}$ and platelets transfusion, there is a small group of patients with persistent cytopenias. Boost of CD34+ selected cells is effective in reverting this condition, with GVHD as main complication of this procedure. Use of TRA seems to be an interesting option for these patients, although more experience is needed to draw definitive conclusions.

Disclosure: Nothing to declare.

\section{P154}

Late complications and quality of life assessment (FACT-BMT, HADS, NCCN distress thermometer) for survivors after allogeneic hematopoietic stem cell transplantation; tablet-PC based surveys

Yunsuk Choi ${ }^{I}$, Jaekyoung Cheon ${ }^{I}$, SuJin Koh ${ }^{I}$, Jin-Ho Baek $^{1}$, Young Ju Min', Ji Hyun Lee ${ }^{2}$,Jae-Cheol Jo'

${ }^{1}$ Ulsan University Hospital, University of Ulsan College of Medicine, Ulsan, Korea, Republic of, ${ }^{2}$ Dong-A University College of Medicine, Busan, Korea, Republic of

Background: Allogeneic hematopoietic stem-cell transplantation (allo-HCT) is an only curative modality for hematologic malignancies. Allo-HCT, however, is physically and psychosocially demanding and can induce long term complications. Therefore, we aimed to investigate the unmet needs for physical and phycological late complications and clinical factors affecting the quality of life for alloHCT survivors.

Methods: The survivorship surveys for allo-HCT recipients have been conducted to build survivorship care plan on regular follow up visit at the survivorship outpatient clinic of Ulsan University Hospital, Ulsan, Korea, using Tablet-PC based patients report forms. We retrospectively analyzed the survivorship survey data in adult allo-HCT survivors over two year post-transplant between 2015 and 2018 at Ulsan University Hospital, Ulsan, Korea. A total of 69 patients completed the Tablet-PC surveys for quality of life (FACT-BMT, Korean language version), anxiety (HADS-A), depression (HADS-D), distress (NCCN distress thermometer [DT]), fatigue (Brief Fatigue Inventory), physical and psychological symptoms, function (physical, social, sexual, cognitive, work); life styles (exercise, tobacco use, alcohol, nutrition); and health screening.

Results: The median age was 46 years (range, 20-70). The most common symptom was fatigue $(80.6 \%)$. Oral pain (34.3\%), ocular and oral dryness (37.3\% and $22.4 \%)$, insomnia $(34.3 \%)$, memory disturbance (29.9\%), joint stiffness or pain $(20.9 \%)$, cough or sputum $(26.9 \%)$, skin pruritis (19.4\%), dyspepsia (19.4\%), headache $(13.4 \%)$ 
were also frequently observed. The high risk patients for anxiety (HADS-A score $\geq 8$ ) and depression (HADS-D score $\geq 8$ ) was found in $14.9 \%$ and $13.6 \%$, respectively. $10.4 \%$ of patients was in high distress status (NCCN DT score $\geq 4)$. We found that younger age $(<60$ years $)$ was significantly associated with poor quality of life score (FACT-BMT $) \quad(\mathrm{P}=0.001)$ and high risk of fatigue $(\mathrm{P}=0.008)$, anxiety (HADS-A) $(\mathrm{P}=0.001)$, and depression (HADS-D) $(\mathrm{P}=0.025)$. Female sex was significantly related to lower physical well-being score and higher distress score $(\mathrm{P}=0.046$ and $\mathrm{P}=0.05$, respectively). Acute lymphoblast leukemia (ALL) survivors after allo-HCT showed significantly worse quality of life score (FACT-BMT) $(\mathrm{P}=0.006)$ and higher depression score (HADS-D) $(\mathrm{P}=0.028)$ compared to those with other disease. Chronic graft versus host disease (GVHD) and continuous immunosuppressant usage also have significant adverse impact on lower FACT-BMT score $(\mathrm{P}=0.024$ and $\mathrm{P}=0.033$, respectively) and higher HADS-D score $(\mathrm{P}=0.015$ and $\mathrm{P}=0.019$, respectively). But there was no significant difference in FACT BMT, HADSA, HADS-D, NCCN DT according to donor type, conditioning intensity, anti-thymocyte globulin use, acute GVHD. Smoking and alcohol drinking was continued in $7.5 \%$ and $17.9 \%$ of allo-HCT survivors. $20.9 \%$ of survivors did not exercise regularly. Regular health screening tests have been done only in 40 patients $(59.7 \%)$.

Conclusions: Allo-HCT survivors over 2 years following allo-HCT still have many physical and psychological symptoms. Younger patients ( $<60$ years), female, ALL, chronic GVHD, and sustained use of immunosuppressant were significant risk factors for poor quality of life and anxiety. We need to build more active survivorship care plan after allo-HCT especially for those patients.

Disclosure: All authors have nothing to declare.

\section{P155}

Evaluation of the new EBMT criteria for the diagnosis of VOD/SOS in 693 consecutive transplant patients using an electronic patient record analysis system

\section{Asha Aggarwal, Nicola Gray ${ }^{1}$, Oliver Lomas ${ }^{1}$, Katalin Balassa $^{1}$, Nadjoua Maouche ${ }^{1}$, Robert Danby ${ }^{1,2}$, Andy Peniket $^{1}$, Grant Vallance ${ }^{I}$}

${ }^{1}$ Oxford University Hospitals, Oxford, United Kingdom, ${ }^{2}$ Anthony Nolan Research Institute, London, United Kingdom

Background: Veno-Occlusive disease (VOD), or sinusoidal obstruction syndrome (SOS), is a recognised complication of haematopoietic stem cell transplantation. Hepatic vasculature endothelial cells are damaged by conditioning chemotherapy, leading to venous occlusion and centrilobar necrosis. The EBMT criteria for diagnosis of VOD are bilirubin $>=34$ with two of painful hepatomegaly, $>5 \%$ weight gain and ascites. VOD is often under-diagnosed, and as a result, treatment may be delayed.

Integrated electronic patient record (EPR) systems are now widely used, and provide an opportunity to retrospectively audit practice to identify patients in whom VOD may have been un-diagnosed or in whom treatment was delayed. In addition these systems have potential for alerting clinicians to the potential diagnosis of VOD.

Methods: We have developed software to analyse the data downloaded from EPR to identify patients in whom VOD was a possible diagnosis according to the new EBMT criteria.

In order to identify patients who may have had VOD we first screened for patients with a bilirubin of $>=34 \mathrm{mmol} / \mathrm{l}$ (which is an absolute requirement for the clinical diagnosis of VOD) within the first 50 days of transplantation. EPR data was then used to assess whether patients had $>5 \%$ weight gain. Radiology reports were reviewed for patients who had bilirubin $>=34 \mathrm{mmol} / \mathrm{L}$ to ascertain if they revealed ascites or painful hepatomegaly.

Results: 652 patients underwent 693 transplant procedures (January 1st 2013 to July 31st 2018). 162 of all transplant patients (23.4\%) were found to have a bilirubin of $>=34 \mathrm{mmol} / \mathrm{l} .39$ of 403 (9.6\%) autograft patients and 123 of $249(49.4 \%)$ allograft patients had an elevated bilirubin at this level. These 162 patients were assessed for evidence of $5 \%$ weight gain. This was the case in 30 patients overall$1 \%$ of autograft patients, $10.5 \%$ of allograft patients. Seven patients ( 2 autograft and 5 allograft) had radiological evidence of ascites. Two patients had a recording of painful hepatomegaly (both post allograft).

Overall our analysis identified 5 patients $(0.7 \%$ overall) fulfilling the EBMT diagnostic criteria for classic early VOD all of whom received defibrotide. All patients had received allogeneic transplants. We failed to identify any cases of late onset VOD or any undiagnosed patients over this period.

Conclusions: This analysis enabled us to efficiently perform a complete audit of our practice to identify patients with VOD. We would recommend using electronic patient records to retrospectively audit practice in this way. The tool that we have created for this analysis will be made freely available for public use and the details will be presented at the EBMT meeting.

We now plan to extend the function of our EPR system to provide alerts to clinicians when VOD is a possible diagnosis and may lead to more rapid treatment of these patients. Our data suggests that elevation of bilirubin and weight gain of $>5 \%$ will be the most frequently occurring criteria on which to base these alerts. 
Disclosure: G.Vallance has performed consultancy work for Jazz pharmaceuticals.

\section{P156}

Endothelial activation and stress index in predicting outcome of allogeneic stem cell transplantation- A retrospective cohort analysis

\section{Zinaida Peric ${ }^{1}$, Tomislav Taborsak ${ }^{1}$, Nadira Durakovic ${ }^{1}$, Lana Desnica ${ }^{1}$, Alen Ostojic ${ }^{1}$, Ranka Serventi-Seiwerth ${ }^{1}$, Radovan Vrhovac ${ }^{I}$}

${ }^{1}$ University Hospital Centre Zagreb, Zagreb, Croatia

Background: Endothelial dysfunction is a common pathophysiology of major complications after allo-SCT, such as graft-versus-host disease, veno-occlusive disease, thrombotic microangiopathy and sepsis. Endothelial Activation and Stress Index (EASIX) is a simple score comprised of standard laboratory parameters (creatinine, LDH and thrombocytes) developed as a potential tool to predict allo-SCT mortality by Luft and colleagues. A recent validation of EASIX included three retrospective cohorts and showed that EASIX taken before start of conditioning can be used as an independent predictor of survival after allo-SCT.

Methods: The aim of our study was to retrospectively evaluate pre-transplant EASIX in our cohort of consecutive patients who underwent allo-SCT in the University Hospital Centre Zagreb between 2012 and 2017. With the use of a cut-off used in the validation cohorts, we compared two groups of patients for overall survival (OS) and transplantrelated mortality (TRM). Group comparisons were done using the log-rank test or Gray test for competing risks outcomes. A multivariate analysis evaluated the association of OS with relevant variables by using a Cox's proportionalhazard regression model.

Results: Our study group included 313 patients and comprised 180 males (57\%) and 133 females (43\%, with a median age of 48 years (range, 18 to 67 years) at the time of transplantation. The most frequent malignancies in our population were acute leukemia (196 patients, 63\%) and myelodysplastic/myeloproliferative neoplasm (451 patients; $16 \%)$. The donor was an identical sibling for 106 patients (34\%), matched unrelated donor for 176 patients $(56 \%)$ and haploidentical for 31 patients (10\%). 104 patients (33\%) received a myeloablative conditioning regimen while 209 patients $(67 \%)$ received a reduced-intensity conditioning regimen. With a median follow-up of 16 months (range, 1260 ) for the whole study group, the OS at 24 months was $60 \%$, (95\%CI 54-68) in the group of patients with low EASIX score and $43 \%(95 \%$ CI 33-56) in the group of patients with high EASIX score $(\mathrm{p}=0.004)$. This difference was mainly attributed to higher TRM in the group with high
EASIX score $(32 \%, 95 \%$ CI $22-45$ at 12 months) compared to the group with low EASIX score $(18 \%, 95 \%$ CI $13-23$ at 12 months $)(\mathrm{p}=0.009)$. In the multivariate analysis which included EASIX, patients' age, intensity of conditioning, diagnosis (lymphoid vs myeloid), status of the disease at transplant and type of the donor, worse OS was independently associated only with older age of patients (HR 1.66; 95\% CI, 1.07-2.59, $\mathrm{p}=0.02)$ and high EASIX score (HR $1.51 ; 95 \%$ CI, 1.01-2.24, $\mathrm{p}=0.04)$.

Conclusions: Our retrospective data support previous data and suggest that EASIX could potentially serve as a valid tool for prediction of allo-SCT outcomes. As a simple biomarker panel, EASIX could easily be implemented in clinical decision making in the field of allo-SCT. These retrospective data need validation in a prospective study which is currently being conducted.

Clinical Trial Registry: NA

Disclosure: NO

\section{P157}

Management of veno-occlusive disease: Virtual simulation improves clinical performance of hematologists/oncologists and advanced practice providers

\section{Lauren Willis ${ }^{1}$, Gwen Littman ${ }^{1}$}

${ }^{1}$ Medscape Oncology, LLC, New York, NY, United States

Background: Veno-occlusive disease (VOD) is a potentially devastating complication that can occur after hematopoietic stem cell transplant (HSCT) and in severe cases can lead to multi-organ failure. (Mohty 2016) Defibrotide has been proven to be effective to prevent and treat VOD, and it is critical that clinicians are aware of how to diagnose and treat this serious complication of HSCT. This study was conducted to determine if an online, simulation-based continuing medical education (CME) intervention could improve performance of hematologists/oncologists (hem/ onc) and advanced practice providers (nurse practitioners and physician assistants, APPs) in the diagnosis and treatment of patients with VOD.

(Reference: Mohty M, et al. Revised diagnosis and severity criteria for sinusoidal obstruction syndrome/venoocclusive disease in adult patients: a new classification from the European Society for Blood and Marrow Transplantation. Bone Marrow Transplant. 2016 Jul;51(7):906-912).

Methods: A CME certified virtual patient simulation (VPS) was made available via a website dedicated to continuous professional development. The VPS consisted of 2 cases presented in a platform that allows clinicians to assess the patients and make diagnostic and therapeutic decisions supported by an extensive database of diagnostic 
and treatment possibilities, matching the scope and depth of actual practice. Clinical decisions were analyzed using a sophisticated decision engine, and tailored clinical guidance (CG) employing up-to-date evidence-based and faculty recommendations was provided after each decision. One case was about VOD and the other case was about acute myeloid leukemia (AML). Decisions were collected postCG and compared with each user's baseline (pre-CG) decisions using a 2-tailed paired t-test to determine p-values $(P<.05$ indicates significance). Data were collected between 9/21/2017 and 11/7/2018.

Results: At the time of assessment, 115 hem/oncs and 409 APPs had fulfilled the participation criteria for completing the VOD case simulation.

\begin{tabular}{|c|c|c|}
\hline & Hem/Oncs & APPs \\
\hline Diagnose VOD & $\begin{array}{l}24 \% \text { RI }(\mathrm{p}<0.001) \\
\text { post-CG } 88 \%\end{array}$ & $\begin{array}{l}53 \% \text { RI }(\mathrm{p}<0.001) \\
\text { post-CG } 49 \%\end{array}$ \\
\hline Baltimore Criteria & $\begin{array}{l}1 \% \text { RI }(\mathrm{p}<0.103) \\
\text { post-CG } 77 \%\end{array}$ & $\begin{array}{l}11 \% \text { RI }(\mathrm{p}<0.103) \\
\text { post-CG } 50 \%\end{array}$ \\
\hline Seattle Criteria & $\begin{array}{l}5 \% \text { RI }(\mathrm{p}<0.140) \\
\text { post-CG } 69 \%\end{array}$ & $\begin{array}{l}11 \% \text { RI }(\mathrm{p}<0.140) \\
\text { post-CG } 41 \%\end{array}$ \\
\hline \multicolumn{3}{|l|}{ Treat VOD } \\
\hline Order defibrotide & $\begin{array}{l}103 \% \text { RI }(\mathrm{p}<0.001) \\
\text { post-CG } 63 \%\end{array}$ & $\begin{array}{l}209 \% \text { RI }(\mathrm{p}<0.001) \text {; } \\
\text { post-CG } 34 \%\end{array}$ \\
\hline $\begin{array}{l}\text { Order } \\
\text { intravenous fluids }\end{array}$ & $\begin{array}{l}116 \% \text { RI }(\mathrm{p}<0.001) \\
\text { post-CG } 54 \%\end{array}$ & $\begin{array}{l}83 \% \text { RI }(\mathrm{p}<0.001) \\
\text { post-CG } 44 \%\end{array}$ \\
\hline Continue ursodiol & $\begin{array}{l}11 \% \text { RI }(\mathrm{p}=0.015) \\
\text { post-CG } 91 \%\end{array}$ & $\begin{array}{l}8 \% \text { RI }(\mathrm{p}<0.001) \\
\text { post-CG } 90 \%\end{array}$ \\
\hline \multicolumn{3}{|c|}{$\mathrm{RI}=$ relative improvement pre-CG to post-CG } \\
\hline
\end{tabular}

[[P157 Table] 1. Table 1. Post-CG Diagnosis and Treatment Decisions]
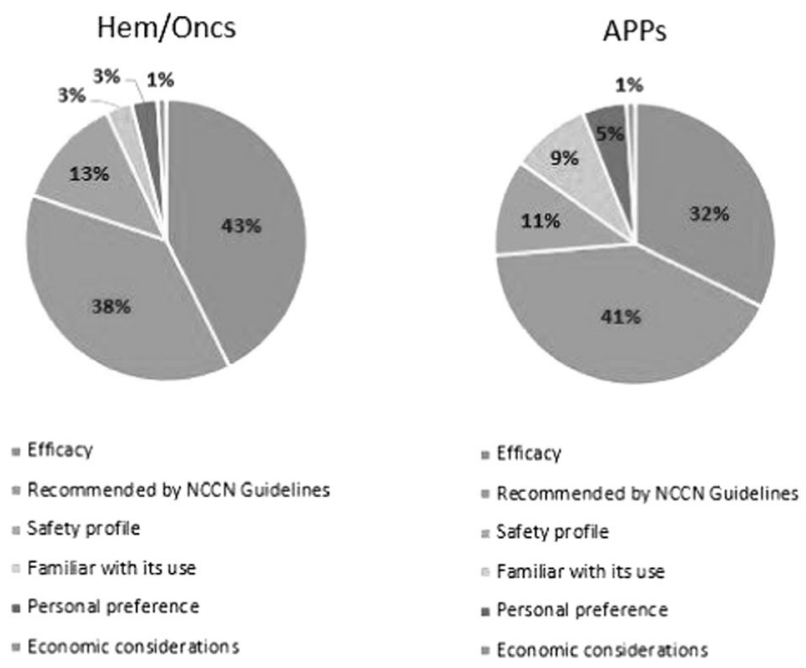

[[P157 Image] 1. Figure 1. Rationales for Ordering Defibrotide (can select up to 2 choices)]
Conclusions: This study demonstrates that VPS that immersed and engaged clinicians in an authentic and practical learning experience improved evidence-based clinical decisions related to the management of VOD. This VPS increased the percentage of clinicians who utilized standardized criteria to diagnose VOD and who ordered defibrotide and IV fluids for VOD management. However, further education is needed to increase the competence and performance of clinicians, particularly APPs, in these areas in order to positively impact patients.

Disclosure: Nothing to declare.

\section{P158}

A nationwide retrospective study of hematopoietic stem cell transplantation in solid organ transplant recipients: On behalf of JSHCT, transplant complications working group

Akihito Shinohara ${ }^{1}$, Kumi Oshima ${ }^{2}$, Shigeo Fuji, Katsutsugu Umeda ${ }^{4}$, Atsuta Yoshiko ${ }^{5}$, Takahiro Fukuda, Junji Tanaka ${ }^{1}$, Masao Ogata ${ }^{7}$

${ }^{1}$ Tokyo Women's Medical University, Tokyo, Japan, ${ }^{2}$ Tokiwa Hospital, Fukushima, Japan, ${ }^{3}$ Osaka International Cancer Institute, Osaka, Japan, ${ }^{4}$ Kyoto University Hospital, Kyoto, Japan, ${ }^{5}$ Japanese Data Center for Hematopoietic Cell Transplantation, Aichi, Japan, ${ }^{6}$ National Cancer Center Hospital, Tokyo, Japan, ${ }^{7}$ Oita University Hospital, Oita, Japan

Background: The outcome of hematopoietic stem cell transplantation (HSCT) in solid organ transplant remain unclear. To address this issue, we conducted a retrospective survey of the 404 Japan Society for Hematopoietic Stem Cell Transplantation centers.

Methods: To address this issue, we conducted a nationwide retrospective survey of the Japan Society for Hematopoietic Stem Cell Transplantation (JSHCT) centers. A first questionnaire was emailed to JSHCT centers requesting information on cases of HSCT in SOT recipient. Patients' data about SOT were collected by sending a second questionnaire to the centers with the patient. Based on these reports, patients' data about HSCT was identified in the Japan transplant outcomes registry database by the Transplant Registry Unified Management Program (TRUMP), confirmed in 2017.

Results: Of the 404 JSHCT centers, 238 responded to the survey $(58.9 \%) .14$ of the responding centers reported a total of 19 patients who had undergone SOT from living donor, and subsequent HSCT. They consist of three autologous HSCT (auto-HSCT) and 13 allogeneic HSCT (allo-HSCT). In auto-HSCT, all patients had received liver transplant for hapatoblastoma. They achieved neutrophil 
engraftment at 30 days after HSCT, and two of three patients were alive at one year after HSCT. In allo-HSCT $(n=16)$, seven patients had received liver transplants, and nine patients had received kidney transplants. Five patients received HSCT from unrelated donor, and 11 patients received HSCT from related donor; two donors were identical in SOT. Their stem cell sources were seven peripheral blood stem cell, six bone marrow, and three cord blood. All but one patients achieved neutrophil engraftment at 30 days after HSCT. Five-year overall survival (5yOS) was $37.5 \%$. While $5 y \mathrm{OS}$ in patients with bone marrow failure $(n=4)$ was $100 \%$, that in patients with malignant disease $(n=12)$ was $16.7 \%$; all but one patients with malignant disease received allo-HSCT in non-remission. Seven of nine kidney-transplant recipients experienced dialysis, and three patients experienced renal rejection after HSCT. On the contrary, no liver-transplant recipient experienced hepatic rejection.

Conclusions: In SOT recipients, the outcome of alloHSCT for malignant disease was poor, partly due to disease status before allo-HSCT. Severe renal complications were common in kidney-transplant recipients, suggesting renal care with caution during and after allo-HSCT.

Disclosure: This work was supported in part by the Practical Research Project for Allergic Diseases and Immunology (Research Technology of Medical Transplantation) from Japan Agency for Medical Research and Development, AMED.

\section{P159}

High incidence but low mortality of EBV related PTLD after T-cell replete allo-peripheral blood HCT with aggressive monitoring and without pre-emptive rituximab

Maria Queralt Salas ${ }^{1}$, Shruti Prem ${ }^{1}$, Wilson Lam ${ }^{1}$, Zeyad Al-Shaibani ${ }^{1}$, David Loach ${ }^{1}$, Santhosh Thyagu ${ }^{1}$, Fotios V Michelis ${ }^{1}$, Dennis (Dong Hwan) Kim ${ }^{1}$, Jeffrey Howard Lipton $^{1}$, Rajat Kumar ${ }^{1}$, Auro Viswabandya ${ }^{1}$, Arjun Datt Law $^{1}$

${ }^{1}$ Princess Margaret Cancer Centre, Hans Messner Allogeneic BMT Program, Toronto, Canada

Background: The aim of the study is to report the incidence and outcome of post-transplant lymphoproliferative disorder (PTLD) in the setting of allogeneic peripheral blood hematopoietic stem cell transplantation (allo-HSCT) combining post-transplant cyclophosphamide (PTCy) and anti-thymocyte globulin (ATG) as graft versus host disease (GVHD) prophylaxis.

Methods: Between October 2015 and May 2018, 195 adult patients diagnosed with hematological malignancies underwent a first T-cell replete allo-HSCT in our center. All patients received a reduced intensity conditioning regimen with fludarabine, busulfan, and 200cGy of total body irradiation, combined with rabbit-ATG, PTCy and cyclosporine (CsA).

EBV titres were monitored weekly by quantitative PCR in plasma samples. The cut-off value for test positivity was $>600$ copies of EBV DNA/ml of plasma. Last follow up was November 2018. Median follow up for patients known to be alive was 19 months (range 5-35).

Results: Patient information is summarized in Table 1. EBV reactivation was documented in 117 (60\%) patients. Median time to EBV reactivation and the diagnosis of presumed/proven (P/P)-PTLD were 75 (16-326) days and 97 (54-306) days [3 (0-10) months], respectively. Median time between first EBV reactivation to P/P-PTLD was 21 (0-175) days.

Seventeen (14\%) of the 117 patients developed P/PPTLD. Median age was 55 years (22-73). Two (12\%) received MRD, 9 (53\%) 10/10 MUD, 1 (6\%) 9/10 MUD, and 5 (29\%) haploidentical donor grafts. Twelve (71\%) were on therapeutic cyclosporine at diagnosis.

Pre-Emptive therapy was not given to any case and only Probable or Proven PTLD were given rituximab. Treatment was based on reduction of the immunosuppression in 3 patients and with the addition of weekly Rituximab $375 \mathrm{mg} /$ $\mathrm{m}^{2}$ in 15 cases. Fifteen $(88 \%)$ achieved complete clinical responses with PCR negativity. Two (12\%) patients died secondary to PTLD.

Conclusions: ATG based conditioning is associated with increased viral reactivations. Frequent EBV monitoring and pre-emptive treatment may lead to rapid disease control. Further research is required to optimize monitoring and management strategies in allo-HSCT recipients.

Disclosure: Nothing to declare

\section{P160}

Acoustically enriched extracellular vesicles as potential markers for allogeneic hematopoietic stem cell transplantation complications

\section{Hooi-Ching Lim ${ }^{1}$, Robert Palmason ${ }^{2}$, Stig Lenhoff ${ }^{2}$, Thomas Laurell, Stefan Scheding ${ }^{1,2}$}

${ }^{1}$ University of Lund, Lund, Sweden, ${ }^{2}$ Skåne University Hospital Lund, Lund, Sweden

Background: Extracellular vesicles (EVs) contain a number of condition-specific proteins, DNA and RNA types and might therefore be used for the early detection of posttransplant complications. However, traditional EV isolation (ultracentrifugation) is time consuming and requires large sample volumes thus making it difficult to perform 
longitudinal studies on larger patient cohorts. We therefore investigated whether recently-developed acoustic trapping could be applied to isolate EVs from patient plasma for biomarker development.

Methods: Plasma samples were collected from 10 consecutive patients before and up to 3 months after allogeneic hematopoietic stem cell transplantation. Patients (age: 22-58 years) with high-risk or refractory/relapsed diseases were transplanted with mobilized PBSC from related $(n=2)$ and unrelated donors $(n=8)$ after standard conditioning. GvHD prophylaxis was cyclosporine and methotrexate. Plasma samples were frozen and thawed for EV enrichment using a novel acoustofluidic-based technology (acoustic trapping). Acoustic trapping uses ultrasound as a local $\lambda / 2$ acoustic standing wave produced by a piezoelectric transducer over a capillary. First, $12 \mu \mathrm{m}$ polystyrene beads are captured which serve as seeding particles. After washing, target particles (EVs) are then captured ("trapped") in the acoustic field. A semi-automatic trapping device (AcouTrap) was used to isolate EVs from diluted plasma (1:2 in PBS). The number of EVs and size distribution were analyzed by nanoparticle tracking analysis. miRNA analysis was performed by qPCR.EVs were enriched in duplicate from 50 $\mu \mathrm{l}$ and $300 \mu \mathrm{l}$ of diluted plasma for nanoparticle tracking analysis and qPCR analysis, respectively.

Results: EVs were successfully isolated from all plasma samples. A total of 89 plasma samples were processed. Numbers of trapped EVs ranged from $3.7 \times 10^{8}-5.5 \times 10^{9}$ before conditioning to $4.4 \times 10^{8}-1.5 \times 10^{10}$ per $50 \mu$ diluted plasma after transplantation. The maximum change in EV numbers in individual patients compared to pretransplantation values ranged from 2-fold to 7-fold. Most patients showed slight increases in EV size after transplantation. Eight of the patients showed signs of infection and received i.v. antibiotics. Increased levels of EVs (> 2-fold) were recorded in three patients during these episodes. Furthermore, increased EV numbers were observed in a patient who required i.v. antiviral therapy for CMV reactivation. Acute grade I GvHD was observed in five patients of which two had increased EV numbers ( $>2$-fold). One patient developed grade IV GvHD which was accompanied by a 4-fold increase in EV numbers. Interestingly, progressively increasing EV numbers preceded the detection of early relapse in a pre-B ALL patient by three weeks. RNA isolation from trapped EVs yielded sufficient material for miRNA profiling. Here, first miRNA profiling data demonstrated that miRNAs were detected in EV samples (miR-103a, -23a, -30c, -142 and -451a), and that acoustically enriched EVs were not affected by hemolysis in contrast to the corresponding whole plasma samples (dCq of miR-23a and miR-451a).
Conclusions: Acoustic trapping allows for efficient and rapid enrichment of EVs from small

volume plasma samples. Trapped EV samples contain sufficient amounts of miRNA for downstream analysis and are thus promising candidates for biomarker development in transplantation.

Disclosure: Laurell and Scheding are founders and board members of Acousort AB, a Lund-based biotech SME that develops particle and cell sorting methods based on ultrasound.

\section{P161}

The incidence, risk factors and outcomes of primary poor graft function after allogeneic hematopoietic stem cell transplantation

Fei Gao ${ }^{1,2}$, Jimin Shi, ${ }^{1,2}$, Yi Luo ${ }^{1,2}$, Yamin Tan ${ }^{1,2}$, Xiaoyu Lai $^{1,2}$, Jian Yu ${ }^{1,2}$, He Huang ${ }^{1,2}$, Yanmin Zhao ${ }^{1,2}$

${ }^{1}$ Bone Marrow Tranplantation Center, the First Affiliated Hospital, School of Medicine, Zhejiang University, Hangzhou, China, ${ }^{2}$ Institute of Hematology, Zhejiang University, Hangzhou, China

Background: Allogeneic hematopoietic stem cell transplantation (allo-HSCT) is a curative therapy for both hematologic malignancy and many other blood disease. While, primary poor graft function (PGF) is still a severe complication following HSCT which lead to poor prognosis. Up to now, the incidence and risk factors of PGF have not been totally revealed.

Methods: From January 2013 to December 2017, a total of 647 patients who received allo-HSCT in our center were analyzed retrospectively. There were 9 males $(47.4 \%)$ and 10 females $(52.6 \%)$ with a median age of 36.21 years (2149 years). PGF was defined as persistent neutropenia $\left(\leq 0.5 \times 10^{9} / \mathrm{L}\right)$, thrombocytopenia (platelets $\left.\leq 20 \times 10^{9} / \mathrm{L}\right)$, and $/$ or hemoglobin $\leq 70 \mathrm{~g} / \mathrm{L}$ after engraftment with hypocellular bone marrow and full donor chimerism, without concurrent graft-versus-host disease or disease relapse. Incidence was calculated from all patients. Of the 647 total patients, nineteen (2.94\%) developed primary PGF. A 1:4 ratio of nested case control study using the good graft function (GGF) subjects transplanted in the same year with the same sex and age of \pm 5 years was carried out.

Results: Data was analyzed by univariate and multivariate logistic regression, and univariate analysis identified disease species, the time from diagnosis to transplantation, disease states, myelofibrosis, splenomegaly, serum ferritin (SF) level, CMV infection, mononuclear and CD34+ cells in graft as potential risk factors $(\mathrm{P}<0.1)$ for PGF. multivariate analysis identified 3 elements as the independent risk factors $(\mathrm{P}<0.05)$, including $\mathrm{CD} 34+$ cells $<5 \times 10^{5}$ / 
$\mathrm{kg} \quad(\mathrm{P}=0.013, \quad \mathrm{OR}=4.316, \quad 95 \% \quad$ confidence interval (CI):1.362-13.682), serum ferritin (SF) levelå $2000 \mathrm{ng} / \mathrm{ml}$ $(\mathrm{P}=0.017, \mathrm{OR}=4.173,95 \% \mathrm{CI}: 1.285-13.551)$ and splenomegaly $(\mathrm{P}=0.044, \mathrm{OR}=3.679,95 \% \mathrm{CI}: 1.036-13.062)$. Furthermore, cox regression model suggests PGF (P $<0.001, \mathrm{OR}=9.311,95 \% \mathrm{CI}: 3.784-22.913)$, together with $\mathrm{SF}$ level $(\mathrm{P}=0.032, \mathrm{OR}=2.595,95 \% \mathrm{CI}: 1.084-6.210)$ strongly influence the one-year overall survival for all patients. Five patients who suffered from primary PGF received second donor stem cell infusion (DSI), but only one of them responded, indicating DSI did not improve the hematologic recovery. After a median follow-up of 6.08 months (1-48 months), three out of 19 PGF patients $(15.8 \%)$ both have achieved hematologic recovery after supportive therapy and survived till now. Compared to cases with GGF, patients with primary PGF experienced poor overall survival (one-year OS: 26.3 vs. $88.2 \%, \mathrm{P}<$ 0.001). The reason for death is as follow: 10 patients died of multiple organ failure owing to severe infection, other 4 patients perished because of disease relapse, intracranial or gastrointestinal hemorrhage.

Conclusions: In conclusion, primary PGF is a rare but life-threatening complication after allo-HSCT. Low CD34+ cells in graft, high SF level and splenomegaly are major risk factors for PGF, and effective therapies need to be explored.

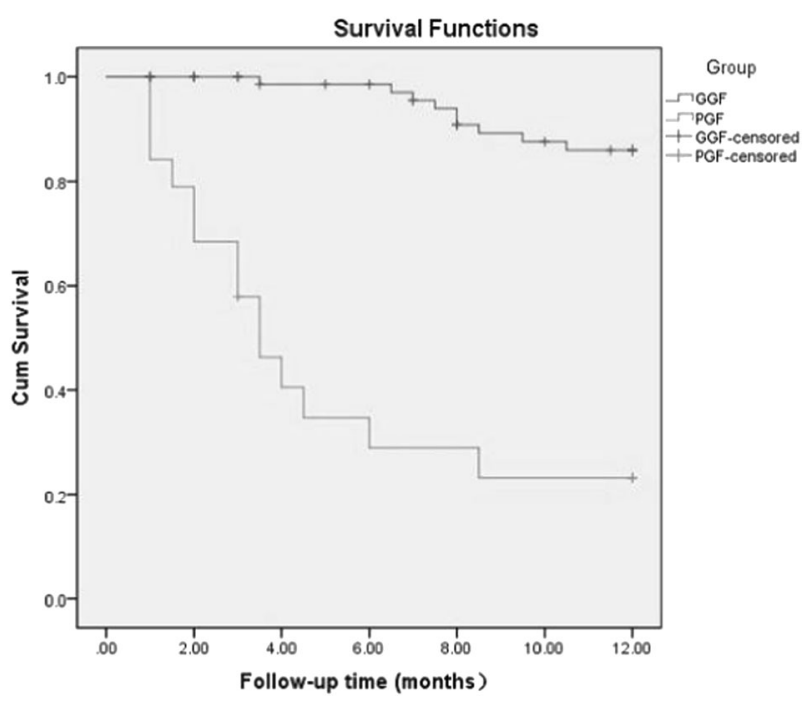

[[P161 Image] 1. One-year OS PGF VS GGF]

Disclosure: Nothing to declare

\section{P162}

Utilization of opioid analgesics in a large cohort of patients affected by severe chronic graft-versus-host disease
Ana Zelic Kerep ${ }^{1,2}$, Filip Pirsl ${ }^{1}$, Seth Steinberg ${ }^{3}$, Sandra Mitchell, Lauren Curtis ${ }^{1}$, Ann Berger ${ }^{5}$, Laura ParsonsWandell $^{1}$, Megan Kenyon ${ }^{1}$, Judy Baruffaldi, Jeannette Nashed $^{1}$, Drazen Pulanic ${ }^{1,6,7}$, Kristin Baird ${ }^{8}$, Ronald Gress $^{1}$, Steven Pavletic ${ }^{1}$

${ }^{1}$ Experimental Transplantation and Immunology Branch, Center for Cancer Research (CCR), National Cancer Institute (NCI), National Institutes of Health (NIH), Bethesda, MD, United States, ${ }^{2}$ University Hospital Center Zagreb, Zagreb, Croatia, ${ }^{3}$ Biostatistics and Data Management Section, National Cancer Institute, Center for Cancer Research, National Institutes of Health, Bethesda, MD, United States, ${ }^{4}$ Outcomes Research Branch, NCI, NIH, Rockville, MD, United States, ${ }^{5}$ Clinical Center, NIH, Bethesda, MD, United States, ${ }^{6}$ University of Zagreb School of Medicine, Zagreb, Croatia, ${ }^{7}$ Faculty of Medicine Osijek, J.J. Strossmayer University of Osijek, Osijek, Croatia, ${ }^{8}$ Pediatric Oncology Branch, CCR, NCI, NIH, Bethesda, MD, United States

Background: Transplant survivors affected by cGVHD usually take one or more immunosuppressants, as well as prophylactic antimicrobials; use of multiple medication classes concurrently poses a risk for drug-drug interactions or amplified side-effects. The use of medications other than cGVHD-direct immunosuppressive therapies has not been well-characterized. This study aims to evaluate patterns of opioid analgesic use in a cohort of patients severely affected by cGVHD.

Methods: Patients ( $n=335)$ with cGVHD were consecutively enrolled in a cross-sectional natural history study (NCT00092235) from 10/2004-12/2016 at the NIH. Participants underwent a comprehensive evaluation including a detailed history and physical examination (including current medications), multidisciplinary evaluations, and laboratory and diagnostic testing. For this analysis, respondents were classified as receiving or not receiving an opioid analgesic. Following the initial screening by univariate methods $(n=335)$, multivariable logistic regression analysis (MLR) was used to identify a set of factors which could jointly impact opioid use. For MLR data were divided into a training $(n=167$ patients) and a validation set $(n=168)$.

Results: Study participants' median age was 48.5 years (19-75), 44\% were female, $74 \%$ had severe cGVHD per NIH scoring criteria, and $77 \%$ were currently receiving high or moderate levels of systemic immunosuppression. Approximately one third (33\%) were taking opioid analgesics $(\mathrm{OA})$. Based on the univariate screening results ( $\mathrm{p}<=0.05$ ), a set of 24 parameters was evaluated by univariate logistic regression in the 167-patient training set, and the following parameters retained their significance and were included in the MLR model: NIH average score per 
organ, total LSS, patient impression of severity, NIH cGVHD severity, presence of skin erythema, Karnofsky performance score (KPS, ) clinician's therapeutic intent, NIH joint score, and with the presence of several cGVHD symptoms including rashes, mouth sores, avoidance of food, vomiting, weight loss, joint and muscle aches, joint limitation, energy loss, need for naps, fevers, anxiety. Multivariable logistic regression identified KPS $<77 \%$ as predictive of OA use, OR $0.92,95 \%$ CI $0.89-0.95$. In the training set $56.4 \%$ of pts using opioids were correctly identified, $78.2 \%$ of those not taking opioids were identified, an overall fraction of correctly identified pts was $70.9 \%$ (95\% CI $63.3-77.7 \%$ ), while in the testing set, $45.5 \%$ of those using opioids were correctly identified, and $67.9 \%$ of those not taking opioids were correctly identified, with overall $60.5 \%$ (95\% CI: 52.6-68.0\%) classification accuracy.

Conclusions: This study showed the burden of OA in this cGVHD cohort. Lower KPS was significantly associated with OA use, as well as self-reported symptoms and a more severe cGVHD disease, which could be of interest in the development of non-pharmaceutical interventions in this patient population. Additional, prospective studies are needed to explore the indications for and effectiveness of $\mathrm{OA}$ in this population of survivors.

Disclosure: No conflict of interest to declare.

\section{P163}

Patients and caregivers inform the design of a mobile app platform for hematopoietic cell transplantation survivorship care

\section{Jaime M. Preussler ${ }^{1,2}$, Ellen M. Denzen ${ }^{1,2}$, Navneet $S$. Majhail $^{3}$, K. Scott Baker ${ }^{4}$, Meggan McCann ${ }^{1}$, Linda J. Burns $^{1,2}$, Jean C. Yit, Karen L. Syrjala}

\section{${ }^{1}$ National Marrow Donor Program/Be The Match, Minnea- polis, MN, United States, ${ }^{2}$ Center for International Blood and Marrow Transplantation, Minneapolis, MN, United States, ${ }^{3}$ Cleveland Clinic, Cleveland, OH, United States, ${ }^{4}$ Fred Hutchinson Cancer Research Center, Seattle, WA, United States}

Background: Technology is improving the ability of patients to participate in their own health care and to access survivorship tools as they need them. INSPIRE (INteractive Survivorship Program with Information and REsources) is an online health program that includes a mobile app, website, and individualized survivorship care plans for adult autologous and allogeneic hematopoietic cell transplant (HCT) survivors. The INSPIRE program will be tested for efficacy in a randomized control trial (RCT) at 13 transplant centers. The project integrates two previously effective
RCTs that tested an internet-based program and patientcentered survivorship care plans for HCT survivors. Patient and caregiver input is essential to inform the design and features for the mobile app platform so that it is usable and engaging for those it targets.

Methods: Using a qualitative research design, we conducted telephone focus groups of adult patients and caregivers in the United States. Adult (age $>18$ years at the time of study entry) HCT recipients had to be at least oneyear post-HCT to participate. Participants had to be able to communicate in English, and could have received a HCT for any diagnosis, and from any donor source or stem cell type. Those who had multiple transplants were included. Participants were asked to review printed and online visual presentations of the mobile app before the focus groups so they were prepared to discuss their responses to the materials during the call. Focus groups were conducted to saturation, when no new qualitative content was offered.

Results: Three focus groups were conducted with 22 total participants (20 patients, two caregivers/patient advocates). All patients received an allogeneic HCT; average time since HCT was 8 years (range: 2-22 years).The majority of participants were female (77.3\%). Participants had differing perspectives on the usefulness of the app to track follow-up appointments, lab values, and other health care plans. There was high interest in having the app tailored to meet specific needs of patients, including tracking information over time (e.g. test results, medications), and having health information available specific to their needs. To minimize duplication of information and data entry, participants recommended syncing the app with their calendars and online patient portals they already use. Reasons provided for not using the app included perception that the materials repeated information already received, side effects such as graft-versus-host disease that restricted vision or motor skills, and lack of comfort with apps for some older participants.

Conclusions: Many health technology and mobile apps are being created to improve patients' health and survivorship care. In this study, HCT survivors and caregivers identified a variety of features that they would want in an app or website, in particular, features tailored to individual needs. Health technologies provide an opportunity to improve survivorship care, but patients and caregivers should be engaged in the process of developing these tools to assure the technology fits their needs and will be used. Given the effort required to maintain these technologies, they require testing for health benefits in rigorous clinical trials.

Clinical Trial Registry: ClinicalTrials.gov: NCT03125070

Disclosure: Nothing to disclose 


\section{P164}

Determinants of the physical performance less than 1 year following allogenic hematopoietic stem cell transplantation: A retrospective study about 59 patients

\section{Virgile Pinelli, Anne Huynh ${ }^{2}$, Philippe Marque ${ }^{3}$, Marc Labrunée ${ }^{3}$}

${ }^{1}$ IUCT Oncopole, Supportive Care, Toulouse, France, ${ }^{2}$ IUCT Oncopole, Hematology, Toulouse, France, ${ }^{3} \mathrm{CHU}$ Toulouse, Physical Medicine and Rehabilitation, Toulouse, France

Background: Thanks to allogeneic stem cell transplantation (allo-HSCT) patients suffering from hematologic malignancies have seen an increase in there life duration expectancy, but they are many side effets including decreasing in physical performance and in quality of life. The intensity of physical performance decrease is variable between patients, and today we did not know why. The aim of our study was first to characterize the physical performance of subjects less than 1 year following allo-HSCT by the use of a cardiopulmonary exercise testing (CPET), and then to determine the predictive factors of exercise performance.

Methods: We did a retrospective analysis from 59 patients who had an allo-HSCT at hematology department of Toulouse-Oncopole and CPET from 01/2015 to 09/2017. The CPET was performed using a cycle ergometer with $\mathrm{O} 2$ and $\mathrm{CO} 2$ analyzer breath by breath, (Masterscreen CPX CareFusion, San Diego, USA), a continuous 12-lead electrocardiogram, and a blood pressure monitoring. The protocol included a 2-min rest period, a 2-min warm-up of $20 \mathrm{~W}$ pedaling followed by a $10 \mathrm{~W} / \mathrm{min}$ incremental phase, up to exhaustion, then a 2-min active recovery of $20 \mathrm{~W}$ pedaling, then a 2-min passive recovery. Three exercise markers were analysed: the peak of oxygen uptake (peak $\mathrm{VO}_{2}$ ), the $\mathrm{VE} / \mathrm{VCO}_{2}$ slope and the first ventilatory threshold $\left(\mathrm{VT}_{1}\right)$. Data relative to conditioning regimen, short-term complications, impairment at CPET day, and physical activity since allo-HSCT were gathered.

Results: After allo-HSCT, nearly 3 over 4 patients reported fatigue, a half reported dyspnea, and 1 over 4 or more reported pain, muscular, neurological or psychological impairment. More than $60 \%$ of patients suffer from moderate or severe physical intolerance, particularly when myeloablative conditioning regimen was used. Only $37 \%$ of patients followed rehabilitation sessions supervised by a physiotherapist, and non- supervised physical activity has been performed by $87 \%$ of patients. Despite normal lung function tests and echocardiography findings in most patients, $80 \%$ had exercise intolerance (EI), $85 \%$ exercise deconditioning, and $55 \%$ had abnormal ventilatory efficiency. Patients with moderate and severe impaired exercise capacity were significantly younger at diagnosis and at allo-HSCT, such as patients with severe deconditioning

Conclusions: Based on a retrospective study, we reported for the first time complete results from CPET and detailed clinical evaluation concerning deficiency and disability following first year after allo-HSCT. These results confirm that exercise impairment is very frequent with more than a half of patients suffering from alterations of one or more of the three performance markers, despite being active.

Disclosure: Nothing to declare

\section{P165}

Demyelinating disorders: A paradigm of immunity disorders after hematopoietic stem cell transplantation

\section{Anna Amelia Colombo ${ }^{1}$, Oscar Borsani ${ }^{2}$, Daniela Caldera $^{1}$, Paolo Bernasconi ${ }^{2}$}

${ }^{1}$ University of Pavia, San Matteo Hospital, Hematology Oncology, Pavia, Italy, ${ }^{2}$ University of Pavia, San Matteo Hospital, Molecular Medicine, Pavia, Italy

Background: Neurologic complications are a major problem in patients who undergone Hematopoietic Stem Cell Transplantation (HSCT). Given the higher survival of transplanted patients, the burden of neurological complications is increasing in the last years. A significant reduction in overall survival was demonstrated in patients who developed neurological complication after HSCT, irrespectively of the hematopoietic stem cell (HSC) source. Neurologic disorders in transplanting setting comprise a wide variety of ethiologies including demyelinating disease, which are caused by immune and non-immune mechanisms. Here, we analyzed the clinical presentation and the underlie ethiologies of patients developing HSCT-related demyelinating disorders in order to give diagnostic and prognostic clues useful to manage these severe but treatable complications in the transplant setting.

Methods: A total of 45 patients of our department which developed neurological complications after HSCT were consecutively collected and $33(73 \%)$ of them, namely those having a diagnosis of a demyelinating disorder, were grouped and described according to the ethiologies of their neurological disorder.

Results: In 14/33 (42\%) patients, an immune-mediated process was found, while 10/33 (30\%) were diagnosed as having an infective etiology and 2/33 (6\%) were supposed to have a demyelinating disorder caused by toxic exposition. A definitive etiologic diagnosis was not formulated in the remaining $7 / 33(21 \%)$ patients. When patients who developed an immune-mediated demyelinating disorder 
(10/33) were compared to those in which a clear immune pathogenic mechanism was not detected (23/33), a higher incidence of acute graft-versus-host disease (aGVHD) was detected in the former than in the latter (20\% vs $8 \%$ ). Moreover, comparison of these two groups revealed that those with no evidence of immune-mediated process have a slight higher prevalence of T-cell depleted HSCT thanthose with an immune-mediated demyelinating disorder $(60 \%$ vs $50 \%$ ). Finally, a lymphoproliferative disorder pre-existing the HSCT was detected in 5/14 $(36 \%)$ patients with immune-mediated demyelinating disorder but only in 4/19 (21\%) of those without evidence of immune-mediated processes.

Conclusions: Demyelinating disorders may be responsible of near $40 \%$ of neurologic complications in the posttransplant setting and, among them, an immune-mediated process is likely to be involved in more than $40 \%$ of cases. Our results suggest that the immune mechanism that underliesthe aGVHD may also be involved in developing demyelinating disease in transplanted patients. It also may be possible that the lymphoproliferative disorder preexisting the HSCT is a risk factor able to increase the risk to develop an immune-mediated demyelinating disorder in the post-transplanting setting. Using a T-cell depleted HSCT can increase the risk of immune-mediated disorders in at least a small fraction of transplanted patients.

Despite our results should be validated on a larger cohort of patients, we can speculate on the possible connections between the wide range of complex and still poorly defined immunity disorders which can influence the prognosis and course of transplanted patients.

Disclosure: Nothing to declare

\section{P166}

\section{Abstract already published.}

\section{P167}

\section{Risk factors and implications of oral mucositis in} recipient of allogeneic hematopoietic stem cell transplantation: A prospective single center study

\section{Roni Shouval, Liza Kouniavsky ${ }^{1}$, Joshua A. Fein ${ }^{1}$, Ivetta Danylesko ${ }^{1}$, Noga Shem Tov ${ }^{1}$, Ronit Yerushalmi ${ }^{1}$, Avichai Shimoni', Arnon Nagler ${ }^{1}$}

\section{${ }^{1}$ Chaim Sheba Medical Center, Tel Aviv University, Ramat Gan, Israel}

Background: Injury to the mucosal barrier and subsequent development of oral mucositis (OM) is among the most common toxicities of allogeneic stem cell transplantation (SCT). Despite the high prevalence of OM and its debilitating nature, prospective studies evaluating determinants of
$\mathrm{OM}$ are scarce. We therefore prospectively evaluated the occurrence of OM following SCT. Risk factors for OM and its implications short and long-term outcomes were assessed.

Methods: OM was prospectively evaluated on a weekly basis in patients undergoing allogeneic HSCT. The grade of OM was determined based on the National Cancer Institute Common Toxicity Criteria for Adverse Events (CTCAE) scale (v. 4.0). Severe OM was defined as grade II to IV. Conditioning regimens were evaluated individually and according to intensity; myeloablative (MAC), reduced intensity (RIC) or reduced toxicity (RTC). The latter category included only patients receiving Fludarabine and Treosulfan at dose of 30-42 g/m2 (Flu/Treo). Risk factors for the development of severe OM were initially identified by a univariate analysis and then analyzed in a multivariate logistic regression model. Association of OM with peritransplant infectious complications, IV morphine consumption, hospitalization length, neutrophil engraftment, acute and chronic graft-versus-host disease (GVHD), non-relapse mortality (NRM) and overall survival were assessed in a univariate analysis. Competing events were considered in analyzing engraftment, GVHD, and NRM.

Results: 115 patients who underwent an allogeneic SCT between 2016 and 2017 were included. Median follow-up was 316 days. Leading indications for transplantation were acute myeloid leukemia (49\%), lymphoma (16\%), and myelodysplastic syndrome $(15 \%)$. The majority of patients received an allograft from a matched sibling or unrelated donor (88\%) and methotrexate GVHD prophylaxis (74\%). The median time to $\mathrm{OM}$ onset was 7 (interquartile range [IQR] 5-9) days. Prevalence of grade II-IV OM was $60 \%$. The median duration OM was 10 [6-13] days, and IV morphine was administrated for a median of 5 [6-12] days for patients with grades III-IV OM (45\%). In a univariate analysis a younger age $(\mathrm{p}=0.023)$, lower BMI $(\mathrm{p}=0.01)$, recent smoking history $(\mathrm{p}=0.08)$, recent antibiotics exposure $(\mathrm{p}=0.018)$, MAC $(\mathrm{p}<0.001)$, and use of methotrexate $(\mathrm{p}=0.009)$ were associated with an increased risk for grade II-IV OM. In a multivariable model the risk for grade II-IV OM was lower with RTC (i.e., Flu/Treo) vs. MAC (odds ratio [OR] 27.31; $\mathrm{p}<0.001)$ and $\mathrm{RTC}$ vs $\mathrm{RIC}(\mathrm{OR} 7.25$; $\mathrm{p}=0.001)$, mycophenolate mofetil vs. methotrexate (OR $3.43 ; \mathrm{p}=0.027)$ and recent smoking (OR 4.7; $\mathrm{p}=0.075)$. Compared to lower grades, grade II-IV OM was associated with a longer hospitalization duration (median 29 days vs. 27 days; $p=0.006$ ), delayed neutrophil engraftment (median 14 vs. 12 days; $\mathrm{p}=-0.004)$, and more gastrointestinal related infections ( $10 \%$ vs. $0 \%$; $\mathrm{p}=0.045$ ). Grade II-IV OM was not associated with increased risk of bloodstream infections, acute or chronic GVHD, non-relapse mortality, and increased mortality. 
Conclusions: Oral mucositis is prevalent among allogeneic-SCT recipients. Importantly, fludarabine-treosulfan, which is considered a myeloablative is associated with a markedly reduced risk for OM. Consequences of $\mathrm{OM}$ include prolongation of hospitalization, delay in neutrophil engraftment, and a tendency for gastrointestinal infections, but does not increase the risk for GVHD and mortality.

Disclosure: Nothing to declare

\section{P168}

Hepatic focal nodular hyperplasia after hematopoietic stem cell transplantation: A single centre retrospective analysis

\section{Alessandro Cattoni', Anna Fornari', Adriana Balduzzi ${ }^{1}$}

${ }^{1}$ San Gerardo Hospital, University of Milano-Bicocca, Monza, Italy

Background: The advent of recent diagnostic techniques for the assessment of iron overload (T2*- MRI) and their systematic use as screening tools in the setting of secondary hemochromatosis have led to an increased awareness that focal nodular hyperplasia $(\mathrm{FNH})$ represents a possible incidental finding after hematopoietic stem cell transplantation (HSCT).

Methods: Clinical and radiological features of patients undergoing HSCT in a single pediatric institution have been retrospectively reviewed for $\mathrm{FNH}$. In order to provide an estimate of the prevalence of FNH after HSCT, we analysed all the T2*-MRI scans performed during the last 5 years in our Centre and recorded the number of patients with FNH (group A). In addition, data about patients incidentally diagnosed with FNH at abdominal imaging performed for different clinical indications have been collected (group B).

Results: Eight out of 118 (7\%) transplanted patients who underwent at least one T2*-MRI scan from September 2013 to September 2018 were incidentally diagnosed with FNH. Group B included 3 subjects with FNH incidentally found at ultrasound or non-T2* MRI scans performed before 2013. Overall, 11 transplanted patients (5 males, 45\%), transplanted for AL (9 cases) or bone marrow failure (2 cases) at a median age of 13.1 \pm 3.6 years, were diagnosed with FNH between 0.6 and 12.8 years after HSCT, namely $3.2 \pm 2.6$ years in Group A and 7.0 \pm 4.1 years in Group B.

A variable degree of iron overload was demonstrated in all patient (LIC: 230-340 $\pm 50 \mathrm{microg} / \mathrm{g}$; baseline serum ferritin: $685-3189 \mathrm{ng} / \mathrm{mL}$ ). The potential risk factors for FNH are reported in table 1.

In 8/11 patients, the radiological finding was pathognomonic; in $1 / 11$ the diagnosis of $\mathrm{FNH}$ was confirmed histologically, while 2/11 subjects were labelled as "FNHlike", although a potential diagnosis of hepatic adenoma could not be ruled out. In 3/11 patients, FNH presented with an isolated lesion, while $8 / 11$ had 2 to more than 10 hepatic nodules at diagnosis. The size of nodules at diagnosis ranged from 3 to $41 \mathrm{~mm}$. In unenhanced MRI scans, lesions were predominantly hyperintense on both $\mathrm{T} 1$ - and $\mathrm{T} 2$ weighted sequences. In dynamic studies with contrast medium, all lesions strongly enhanced during the arterial phase, with a variable degree of wash-out in the late venous scans. Hepatic function tests were normal in all the enrolled patients at diagnosis of FNH. Among the 9/11 patients for whom at least a follow-up scan was available, 1 presented a complete regression, 3 a reduction and 3 an increase in the size and/or number of lesions, while in 2 patients the nodules remained substantially unchanged after a mean radiological follow-up of $4.5 \pm 3.3$ years. No malignant transformations were observed.

Conclusions: FNH represents a relatively frequent incidental finding after HSCT. Although a malignant transformation is rare, given the demonstrated variable evolution of the hepatic nodules, a radiological follow-up is highly recommended.

Disclosure: Nothing to disclose.

\section{P169}

Incidence and risk factors for hepatic sinusoidal obstruction syndrome after allogeneic transplantation: Retrospective multicenter study of Turkish hematology research and education group (THREG), updated data

Nur Soyer', Mehmet Gunduz ${ }^{2}$, Emre Tekgunduz, ${ }^{3}$, Burak Deveci $^{4}$, Hakan Ozdogu ${ }^{5}$, Handan Haydaroglu Sahin ${ }^{6}$, Esra Ermis Turak ${ }^{7}$, Mufide Okay ${ }^{8}$, Irfan Kuku', Ipek Yonal Hindilerden $^{10}$, Pervin Topcuoglu ${ }^{2}$, Feviz Altuntas ${ }^{3}$, Ihsan Karadogan ${ }^{4}$, Mustafa Pehlivan ${ }^{6}$, Ali Unal', Hakan Goker $^{8}$, Mehmet Ali Erkurt ${ }^{9}$, Sevgi Kalayoglu Besisık ${ }^{10}$, Filiz Vural $^{1}$

${ }^{1}$ Ege University Medical Faculty, Izmir, Turkey, ${ }^{2}$ Ankara University, Ankara, Turkey, ${ }^{3}$ Ankara Oncology Hospital, Ankara, Turkey, ${ }^{4}$ Medstar Antalya Hospital, Antalya, Turkey, ${ }^{5}$ Baskent University Medical Faculty, Adana, Turkey, ${ }^{6}$ Gaziantep University, Gaziantep, Turkey, ${ }^{7}$ Erciyes University, Medical Faculty, Kayseri, Turkey, ${ }^{8}$ Hacettepe University, Ankara, Turkey, ${ }^{9}$ Inonu University, Malatya, Turkey, ${ }^{10}$ Istanbul University, Istanbul, Turkey

Background: Hepatic sinusoidal obstruction syndrome (HSOS) is a potentially life-threatening complication of allogeneic hematopoietic stem cell transplantation (alloHSCT). The mean incidence of HSOS was found to be $13.7 \%(0 \%-40 \%)$ in the literature. We examined the incidence and risk factors for HSOS after allo-SCT. 
Methods: Ten centers from Turkey were enrolled in the study. We retrospectively evaluated the medical records of patients who were treated with allo-SCT between January 2012 and December 2015. A Baltimore criterion was used for assessment of HSOS. Four hundred twenty six (97.2\%) of 438 patients who were treated with prophylaxis with defibrotide alone or one or more of the N-Acetylcysteine, diuretics and heparin used defibrotide (10-25 mg/kg/day).

Results: The study included 1153 patients $(687$ males/ 466 females) with median age of 38 (15-71) years. The demographic and clinical characteristics of patients were summarized in Table 1. The incidence of HSOS was $7.5 \%$ (86). Prophylaxis for HSOS was used in $42(48.1 \%)$ of patients, who developed HSOS. Defibrotide as prophylaxis was received by 34 of $42(81 \%)$ of patients. HSOS developed in a median of 13 (0-34) days after stem cell infusion. Seventy-eight $(90.7 \%)$ of patients who developed HSOS had infection at the time of diagnosis. Forty-eight of them had ascites, 65 had hepatomegaly and, 76 had weight gain.

\begin{tabular}{llll}
\hline Characteristics & $\begin{array}{l}\text { Patients without } \\
\text { HSOS (n=1067) }\end{array}$ & $\begin{array}{l}\text { Patients with } \\
\text { HSOS (n=86) }\end{array}$ & Statistics \\
\hline $\begin{array}{l}\text { Age, median (range) } \\
\text { Male (n; \%) }\end{array}$ & $\begin{array}{l}68.68 \pm 13.77 \\
\text { MAC regimen as }\end{array}$ & $\begin{array}{l}39.06 \pm 12.33 \\
\text { conditioning (n; \%) }\end{array}$ & $\begin{array}{l}56(65.1) \\
\mathrm{P}=\mathrm{NS}\end{array}$ \\
$\begin{array}{l}\text { HSOS prophylaxis } \\
\text { yes/no (n; \%) }\end{array}$ & $396 / 671(37.1)$ & $42 / 44(48.8)$ & $\mathrm{P}=\mathrm{NS}$ \\
$\begin{array}{l}\text { MTX/CsA as GVHD } \\
\text { prophylaxis (n; \%) }\end{array}$ & $905(84.8)$ & $81(94.1)$ & $\mathrm{P}=0.031$ \\
$\begin{array}{l}\text { Use of PB as stem cell } \\
\text { source (n; \%) }\end{array}$ & $1022(95.8)$ & $78(90.7)$ & $\mathrm{P}=\mathrm{NS}$ \\
$\begin{array}{l}\text { Use of matched- } \\
\text { related donor (n; \%) }\end{array}$ & $854(80)$ & $64(74.4)$ & $\mathrm{P}=0.042$ \\
$\begin{array}{l}\text { Successful } \\
\text { engraftment (n; \%) }\end{array}$ & $949(88.9)$ & $58(67.4)$ & $\mathrm{P}=0.000$ \\
\hline
\end{tabular}

[[P169 Table] 1. Patients' characteristics (*Peripheral blood: PB, Bone marrow: BM, MAC: Myeloablative conditioning, NS: not significant, RIC: Reduced-intensity condi)]

Seventy-three (84.9\%) of patients with HSOS were treated with defibrotide after diagnosis. The median time of starting defibrotide in these patients was 13.5 (2-29) days. Thirty-seven $(43 \%)$ of patients with HSOS recovered completely and forty-nine (57\%) of them died as a result of multi organ failure. The incidence of HSOS-related mortality in allo-HSCT cohort was found to be $4.2 \%$. In univariate analysis, statistically significant associations were not found between HSOS incidence and age/sex of recipient, type of conditioning regimen, stem cell source and type of GVHD prophylaxis. On the other hand donor type, engraftment status and prophylaxis for HSOS were significantly associated with HSOS development. HSOS prophylaxis was significantly decreased HSOS-associated mortality $(\mathrm{p}=0.001)$.

Conclusions: HSOS still remains a serious lifethreatening complication of allo-SCT. Although the incidence is low, HSOS is associated with increased 100-day non-relapse mortality. HSOS prophylaxis especially with defibrotide, seems to reduce HSOS associated mortality in high risk patients.

Disclosure: Nothing to declare

\section{P170}

Prophylaxis with defibrotide in adults at very high risk of veno-occlusive disease: Results in 11 patients

Fabio Giglio ${ }^{I}$, Elisabetta Xue ${ }^{I}$, Lorenzo Lazzari, Raffaella Greco ${ }^{I}$, Teresa Daniela Clerici, ${ }^{I}$, Sarah Marktel $^{I}$, Maria Teresa Lupo-Stanghellini ${ }^{I}$, Andrea Angelo Assanelli, Magda Marcatti ${ }^{1}$, Consuelo Corti, Massimo Bernardi ${ }^{1}$, Simona Piemontese ${ }^{1}$, Jacopo Peccatori $^{1}$, Fabio Ciceri $^{1,2}$

${ }^{1}$ IRCCS San Raffaele Scientific Institute, Milano, Italy, ${ }^{2}$ University Vita-Salute San Raffaele, Milano, Italy

Background: Hepatic Sinusoidal Obstruction Syndrome/ Veno-Occlusive Disease (SOS/VOD) is a life threatening complication that can occur after Hematopoietic Stem Cell Transplantation (HSCT). Severe SOS/VOD rapidly evolves in Multiple Organ Dysfunction Syndrome (MODS), associated with a mortality rate exceding $80 \%$. Precocity of defibrotide (DF) treatment is the leading factor for efficacy. Prophylactic use of DF is recommended in children, but its value has not been validated in the adult population, although factors for individual risk assessment for VOD are debated. We here present a real-world experience of DF prophylaxis in adult patients at very high risk of SOS/VOD receiving allogeneic HSCT.

Methods: From 2016 to 2018 we treated with prophylactic Defibrotide and Ursodeoxycholic Acid (UDCA) 11 patients, median age 30 years (range 21-59). Nine patients received allogeneic HSCT for acute lymphoblastic leukemia (8 B-ALL and 1 T-ALL), one patient for Severe Aplastic Anemia, one patient for Primary Myelofibrosis. They were all at high risk for SOS/VOD because of previous hepatotoxicity (3 patients), previous HSCT (6 patients), double alkylating agent (5 patients) or previous treatment with Inotuzomab Ozogamicin (IO; 7 patients). Of the 7 patients treated with IO, 6 received 2 cycles of IO, and 1 received 1 cycle, with the last IO dose administered a median 41.5 days before HSCT (range 34-61 d). Defibrotide 
was administered in 4 daily doses for a total dose of $25 \mathrm{mg} /$ $\mathrm{kg}$ per day and UDCA at the dose of $300 \mathrm{mg}$ twice per day, starting from day -6 prior transplant. All patients received Treosulfan-Fludarabine based conditioning. In 5 patients Thiotepa was added to the conditioning and in 2 patients a low dose 4Gy TBI. GvHD prophylaxis included posttransplant cyclophosphamide, rapamycin and mycophenolate in all patients, except one patient with Aplastic Anemia receiving $\mathrm{ATG}$, rapamycin and mycophenolate. Donor source was PBSC in all cases. Seven patients received family haploidentical (MMRD) transplant, 1 patient a MRD transplant and 3 patients a MUD transplant.

Results: The median duration of defibrotide therapy was 34 days (range 32-64 days). Documented non-severe gastrointestinal bleeding occurred in 2 patients requiring defibrotide temporarily discontinuation, no other significant bleedings were experienced. Four patients developed grade II-IV acute GVHD and no transplant-associated thrombotic microangiopathy were diagnosed. Overall, SOS/VOD occurred in 3/11 cases within 21 days after HSCT (days 9, 10 and 13) and no late-onset SOS/VOD were diagnosed. SOS/VOD was very severe, causing MODS and death in all 3 cases. All 3 patients were characterized by a common pattern of very high risk factors for SOS/VOD by prior HSCT and salvage treatment for B-ALL with 2 cycles of IO close to HSCT. Furthermore, they all received a fully myeloablative conditioning regimen with Treosulfan and Thiotepa and a MMRD transplant.

Conclusions: Defibrotide prophylaxis was safe and well tolerated with no severe related complications.

SOS/VOD occurred despite continuous DF prophylaxis in 3/7 patients treated with Inotuzomab Ozogamicin close before undergoing $2^{\text {nd }}$ transplant. To reduce the incidence of severe VOD, pre allo-HSCT treatment with Inotuzomab Ozogamicin should prompt avoidance of other cumulative risk factors for VOD, such as use of double alkylating agents.

Disclosure: Nothing to declare

\section{P171 \\ Pulmonary injury in children receiving high-dose busulfan}

\section{Ron Rabinowicz ${ }^{1}$, Patrick Stafler ${ }^{1,2}$, Anat Yahel ${ }^{1}$, Daniel Kurnik $^{3}$, Jerry Stein ${ }^{1,2}$}

${ }^{1}$ Schneider Children's Medical Center, Petah Tikva, Israel, ${ }^{2}$ Tel Aviv University, Sackler Faculty of Medicine, Tel Aviv, Israel, ${ }^{3}$ Rambam Medical Center, Haifa, Israel

Background: Busulfan is the backbone of many preparative regimens administered to children undergoing allogeneic and autologous hematopoietic stem cell transplantation (HSCT). Among its many long-term adverse effects, Busulfan can cause various degrees of pulmonary injury. Although well described in adults, there are few large series exploring pulmonary toxicity of Busulfan in children.

We describe long-term pulmonary follow-up in a large group of children treated at a single center who had received high-dose Busulfan and examine the relationship of systemic drug exposure and lung function over time.

Methods: All surviving children who had received highdose Busulfan between 1993-2013 in the context of HSCT at the Schneider Children's Medical Center, were referred for serial pulmonary function monitoring (including spirometry, plethysmography and diffusing capacity for carbon monoxide [DLCO]. Pre-transplant testing was available for children who were old enough to perform the procedure. Spirometry results were adjusted according to the revised Global Lung Initiative formulas for age, gender, and height. Pulmonary injury was defined as a $\mathrm{Z}$ score below -1.96 for spirometry, or $<80 \%$ of predicted for the other parameters. Busulfan levels were monitored following the second drug dose. All patients received Busulfan in four daily doses. Area Under the Curve (AUC) calculations were performed by Bayesian calculations.

Results: Between 1993-2013, 263 patients aged 0-18 years were diagnosed with malignant or non-malignant diseases and treated with high-dose Busulfan. Of 130 shortterm survivors, 75 had at least one post-transplant pulmonary function evaluation. The mean age at treatment with Busulfan was 7.9 years (range, 0.4-27 years). Of these 75 children, 22 children had undergone autologous transplantation and 53 children had an allogeneic transplant. 7 of these patients eventually relapsed and 3 died. 26 children had one or more pulmonary risk factors before HSCT - chest or upper abdomen radiation (13), chest wall tumors or lung metastasis (8), chest surgery (5), prior administration of pulmonary-toxic drug (3) or asthma (2). During follow-up (up to 14 years, median 5.5 years), FEV1 and FVC spirometry tests both decreased significantly $(\mathrm{p}=0.002$ and 0.001 , respectively), while the decrease in DLCO was not statistically significant. $35 \%$ of patients had abnormal pulmonary function tests and seven children had symptomatic disease which in two may have been manifestations of GVHD. Interestingly, no correlation was found between Busulfan AUC, Busulfan peak levels, the number of Busulfan doses administered, the type of transplantation (autologous vs. allogeneic) or primary disease to pulmonary injury. Even after censoring of children with pre-transplant pulmonary risk factors we noted a decrease of FEV1 and FVC.

Conclusions: As in adults, pulmonary injury is observed in children treated with high-dose Busulfan prior to HSCT. No correlation was observed between Busulfan AUC and 
pulmonary injury. Follow-up of children who receive this drug should include regular pulmonary monitoring, referral to a pulmonologist when subclinical pulmonary compromise is found, and counseling regarding measures that might prevent or ameliorate pulmonary damage. Continued follow-up of this cohort of patients should inform our pretransplant patient information sessions, and the future use of Busulfan in children.

Disclosure: Nothing to declare

\section{P172}

Predictors of survival in patients with transplantassociated thrombotic microangiopathy

Mikhail M. Kanunnikov, Zhemal Z. Rakhmanova, Olesya V. Paina, Elena I. Darskaya, Tatiana A. Bykova, Asmik

G. Gevorgian, Elena V. Morozova, Ludmila S.

Zubarovskaya, Sergey N. Bondarenko, Ivan S. Moiseev, Boris V. Afanasyev

${ }^{1}$ First Pavlov Saint Petersburg State Medical University, Raisa Gorbacheva Memorial Research Institute of Pediatric Oncology, Hematology and Transplantation, Saint Petersburg, Russian Federation

Background: Transplant-associated thrombotic microangiopathy (TA-TMA) is a specific complication of allogeneic hematopoietic stem cell transplantation (HSCT).

Post-HSCT TMA has been attributed to the vascular endothelial damage caused by high-dose chemotherapy, calcineurin inhibitors (CNIs), graft-versus-host disease (GVHD), infections. There is a little evidence published regarding the efficacy and factors influencing the outcome of withdrawal of CNIs.

Methods: The analysis comprised a total of 54 patients, with diagnosed hematologic malignancy (AML (16), ALL (12), MDS (4), Hodgkin lymphoma (5), CML (3) and Neuroblastoma (1) received allo-SCT, from a matched related, unrelated or haploidentical donor between 2007 and 2018. Patients were diagnosed with TA-TMA based on Cho criteria. The median age of patients was 23 (37 adults, 17 children). GVHD prophylaxis was performed with tacrolimus (Tac) in 42 , cyclosporine $\mathrm{A}(\mathrm{CsA})$ in 11 , combination tacrolimus+sirolimus (Sir) in 12. 24 patients received ATG and 26 PTCy. Withdrawal of CNIs was accompanied by administration of systemic steroids (21 patients) or substitution with Sir after reaching levels of CsA $<100$ $\mathrm{ng} / \mathrm{ml}$ or Tac $<3 \mathrm{ng} / \mathrm{ml}$ in 13 . The target concentration of Sir was 3-9 ng/ml. In pediatric patients who received combination Tac + Sir, the Tac was discontinued in one step while Sir continued. Median time to development TMA was 31,5 days after allo-SCT (range 1-408). Median follow-up of surviving patients was 395 days. The primary outcome was overall survival (OS) up to 2 years after development of TA-TMA.

Results: The following significant predictors of 2-year OS were identified: Tac replacement with $\operatorname{Sir}(\mathrm{p}<0,001)$, PTCy in prophylaxis $(\mathrm{p}<0,001)$, acute GVHD (aGVHD) grade 2-4 $(p=0,029)$, previous sepsis $(p=0,003)$, level of $\mathrm{LDH}$ in debut $(\mathrm{p}=0,001)$, combination $\mathrm{Sir}+\mathrm{Tac}$ in prophylaxis $(\mathrm{p}=0,05)$, major $\mathrm{AB} 0$-mismatch $(\mathrm{p}=0,022)$, severity of CNS symptoms $(\mathrm{p}<0,001)$. There was no significant difference in OS according to patients' age, sex, "salvage" disease status at transplantation, previous VOD, viral (HHV 1,2,6, CMV, EBV) reactivations, count of $\mathrm{CD} 34+$ cells transfused, $\mathrm{LDH}$ level, shizocytes and creatinine in the debut of TA-TMA. In the multivariate analysis replacement of CNIs with Sir (HR $0.27,95 \%$ CI $0.08-0.96, p=0.018$ ) and baseline LDH level (HR 1.01, 95\%HR 1.00-1.01, $\mathrm{p}=0.029$ ) were associated with survival differences. The cut off for $\mathrm{LDH}$ was $4 \mathrm{xUNL}$. aGVHD grade 2-4 (HR 1.96, 95\%CI 0.92-4.17, $\mathrm{p}=0,081$ ) and use of PTCy (HR 0.535, 95\%CI 0.16-1.82, $\mathrm{p}=0.317$ ) were not significant in the multivariate analysis (figure 1). TA-TMA cases after PTCy were significantly less frequently associated with clinically significant aGVHD (19\% vs $68 \%$, $\mathrm{p}<0,001)$. The survival was higher after PTCy (74\% vs $15 \%)$, but not significant due to sample size and other TA-TMA factors. Leading causes of death were: GVHD progression (11\%), bacterial infection (11\%), TMA (17\%) and other (19\%).

Conclusions: Replacing Tac by Sir is an effective therapeutic strategy in a group of patients with debut of TA-TMA at least after PTCy, where it is less likely to be associated with aGVHD. There is a significant overlap of populations with PTCy prophylaxis and substitution with Sir, thus the study is not powered to provide guidance for patients on conventional prophylaxis with TA-TMA.

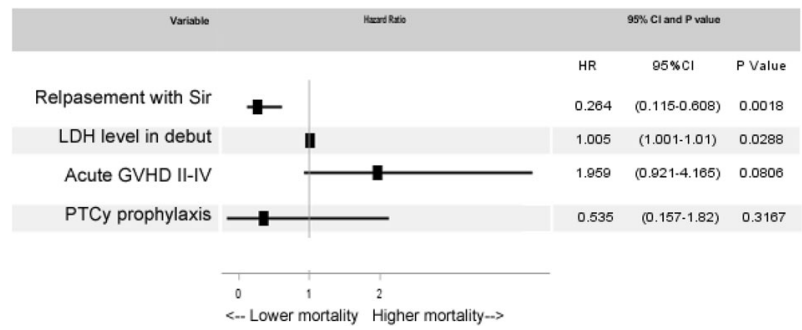

[[P172 Image] 1. Figure 1. Factors influencing overall survival in TA-TMA (multivariate analysis)]

Disclosure: None of the authors has anything to disclose.

\section{P173}

Donor-recipient AB0 mismatch effect on the allogeneic hematopoietic stem cell transplantation outcome: A single-center retrospective study 
Giorgia Saporiti $^{1}$, Andrea Busani, ${ }^{1}$, Giorgia Levati ${ }^{1}$, Elena Tagliaferri $^{I}$, Federica Grifoni ${ }^{I}$, Cristina Manera ${ }^{I}$, Luca Baldini ${ }^{\text {, Francesco Onida }}{ }^{I}$

${ }^{1}$ Fondazione IRCCS Ca' Granda Ospedale Maggiore Policlinico di Milano - University of Milan, Milano, Italy

Background: Because transmission of major histocompatibility complex and blood group system genes is independent from each other, approximately $40-50 \%$ of all allogeneic hematopoietic stem cell transplantations (alloHSCT) are realized crosswise the AB0-blood group boundary. However, due to the widespread expression of $\mathrm{AB} 0$ antigens on a variety of human tissues other than erythrocytes, $\mathrm{AB} 0$ incompatibility may have an impact on the outcome of allogeneic HSCT that goes beyond the wellknown immune-hematological complications such as immediate hemolysis due to the presence of isoagglutinins and delayed hemolysis due to passenger B lymphocytes. Here we aimed to assess the donor-recipient $\mathrm{AB} 0$ mismatch effect on the allo-HSCT outcome, comprising non-relapse mortality (NRM), overall and relapse-free survival, posttransplant PRC transfusion requirement, as well as relapse rate, incidence of graft- failure and acute GvHD.

Methods: Clinical and laboratory data from 180 consecutive patients undergoing allogeneic HSCT between 01/2008 and 06/2018 at the Fondazione IRCCS Ca' Granda Maggiore Policlinico Hospital in Milan, Italy, were retrospectively collected. Kaplan Meier estimates were used for the analysis of survival outcomes while NRM, relapse and acute GvHD cumulative incidences were investigated by competing risk analysis.

Results: The patient series included 105 AB0-match, 31 major $\mathrm{AB} 0$-mismatch, 34 minor $\mathrm{AB} 0$-mismatched and 10 bidirectionally $\mathrm{AB} 0$-mismatch transplants. Indication for allo-HSCT were mainly AML/MDS (77 pts), ALL (34 pts) and T-NHL/CTCL (32 pts). Mean overall survival for groups of patients undergoing $\mathrm{AB} 0$-identical, major $\mathrm{AB} 0$ mismatch and minor AB0 mismatch HSCT were 66 months (95\% CI [55;77]), 47 months (95\% CI [28;65) and 46 months $(95 \%$ CI $[31 ; 61])$, respectively. NRM in the three groups were significantly different, with point estimates of $12 \%, 29 \%$ and $26 \%$ at 5 years, respectively, whereas no significant differences were observed for relapse rate and graft failure incidence. Although not statistically different, incidence of acute grade III-IV GvHD was twice as high in patients transplanted from minor $\mathrm{AB} 0$ mismatched donors than in the $\mathrm{AB} 0$ identical group (16\% vs $8 \%$ ). Following transplantation, PRC transfusion requirement was significantly higher in the major $\mathrm{AB} 0$ mismatch then in the AB0-match transplanted patients (median $14 \mathrm{vs}$ $7, p=0.01$ ), with a marginal positive correlation between the anti-donor $\mathrm{A} / \mathrm{B}$ IgG titers measured prior HSCT and the total number of PRC transfused during the first year following transplantation.

We observed only one case of PRCA occurring in a 50year-old $0+$ woman who was transplanted from a 32-yearold male A + HLA-identical sibling using peripheral blood as the stem cell source following a myeloablative conditioning for AML in first complete remission. Anti-A $\mathrm{IgG}$ isoagglutinin titers prior to transplantation were 1:256. During the first year post transplantation, the patient required a total of 46 PRC transfusions, with gradual resolution occurring only after introduction of danazole treatment.

Conclusions: In our patient cohort, both major and minor AB0 mismatch associated to a significantly higher NRM. Major AB0 mismatch associated to a higher PRC transfusion requirement. A more frequently occurring severe acute GvHD was also suggested in minor AB0-mismatch transplants. Altogether, our results suggest that allo-HSCT outcome may be significantly affected by AB0 blood group mismatch.

Disclosure: Nothing to declare

\section{P174}

Intestinal transplant-associated thrombotic microangiopathy (TA-TMA): histopathological review. An infradiagnosed complication

Javier Carrillo Checa ${ }^{I}$, Oscar Javier Blanco Nuñez ${ }^{I}$, Luis Miguel Chinchilla Tabora ${ }^{1}$, Alejandro Avendaño Pita ${ }^{1}$, Estefanía Pérez López ${ }^{1}$, Mónica Cabrero Calvo ${ }^{1}$, Ana África Martín López ${ }^{1}$, Fermín Sánchez-Guijo Martín ${ }^{1}$, Lourdes Vázquez López ${ }^{1}$, Almudena Navarro Bailon ${ }^{1}$, Antonio Velasco Guardado ${ }^{1}$, Félix López Cadenas ${ }^{1}$, María Diez Campelo ${ }^{1}$, Oriana López Godino ${ }^{2}$, Miguel Alcoceba Sánchez ${ }^{1}$, Dolores Caballero Barrigon ${ }^{1}$, Lucía López Corral ${ }^{1}$

${ }^{1}$ Complejo Asistencial Universitario de Salamanca-IBSALGerencia Regional de Salud de Castilla y Leon, Salamanca, Spain, ${ }^{2}$ Hospital Morales Meseguer, Murcia, Spain

Background: AT-TMA is a severe endothelial injury complication and it may involve the intestinal vasculature. Intestinal TMA could be fatal and missdiagnosed. Clinical and pathological criteria to differentiate from intestinal GVHD are needed. The aim of this study was to analyze the incidence and histological characteristics of intestinal TMA in patients diagnosed of systemic TMA.

Methods: We analyzed the incidence of TMA in 555 patients who underwent allo-HSCT in our institution between january 2010-august 2018. TMA diagnosis was based on Ho criteria. We do a pathological review in 103 biopsies from 25 out of 52 patients in whom an endoscopy 
have been performed 30 days before and 60 days after the diagnosis of TMA for suspicious of GVHD. Review was performed by a pathologist expert in GVHD, who examined the biopsies in search of hystopathological features of GVHD, TMA or viral infection. Diagnosis of gastrointestinal GVHD was stablished according to Mcdonald and Sales criteria, while intestinal TMA diagnosis was stablished by Warren et al criteria.

Results: 52 out of 555 patients $(9,4 \%)$ were diagnosed of TMA. Transplant characteristics and TMA data of patients with systemic TMA are shown in image. 47 out of 52 patients with TMA (90\%) had been diagnosed with prior/ simultaneous acute GVHD, 20 of them grade III-IV, and $80 \%$ with gastrointestinal GVHD.

Intestinal TMA have been reported only in 7 out of 25 patients (28\%) at diagnosis, whereas when review based on Warren criteria was performed, in 19 patients $(76 \%)$ the pathologist found at least 1 of the criteria of endothelial damage and $48 \%$ of the patients 3 or more Warren criteria were founded. The most frequent features were endothelial cell swelling $(\mathrm{n}=16,64 \%)$ and perivascular mucosal hemorrhage $(n=15,60 \%)$. Review hystological features of biopsies are shown in table 3 of the image.

Regarding GVHD, it was found in 21 patients (84\%) at diagnosis and in $23(92 \%)$ at pathological review.

With a median follow-up of 10 months (1-73) 32 patients of the 52 with systemic TMA (62\%) are dead. 9 of the deaths $(41 \%)$ were related to TMA (3 TMA, 3 TMA + GVHD, and 3 TMA+infection). Patients with 3 or more Warren criteria in pathological review had poor outcome compared with patients less than 3 criteria (30\% alive VS $51 \%$ at 12 months, $\mathrm{p}=0.9$ ).

Conclusions: Intestinal TMA is a life-threatening underdiagnosed entity. Only 7 patients of 25 patients were diagnosed of intestinal TMA.

We found that most of our patients had endothelial damage in the gastrointestinal biopsy pathological reviews. GVHD histological criteria were present in most of the patients, mainly histological grade I-II. Prognosis of these patients is poor and pathologist effords in diagnosed the entity is guarranted.

Disclosure: Nothing to disclosure

\section{P175}

Strategies to reduce neutropenic fever and hospital readmission in multiple myeloma patients managed at home after autologous stem cell transplantation

Luis Gerardo Rodríguez-Lobato ${ }^{1}$, Alexandra MartínezRoca ${ }^{I}$, Sandra Castaño-Díez, Alicia PalominoMosquera $^{I}$, Gonzalo Gutiérrez-García ${ }^{I}$, María SuárezLledó $^{l}$, Montserrat Rovira ${ }^{I}$, Carmen Martínez ${ }^{I}$, Laura Rosiñol ${ }^{1}$, Alexandra Pedraza-Navarrete ${ }^{I}$, Ana Moreno-
Castaño $^{I}$, Cristina Gallego ${ }^{I}$, Adelina Hernando ${ }^{I}$, Susana Segura ${ }^{I}$, Álvaro Urbano-Ispizua ${ }^{1}$, Francesc FernándezAvilés ${ }^{1}$

${ }^{1}$ Hospital Clínic, Institut d'Investigacions Biomèdiques August Pi i Sunyer (IDIBAPS), Josep Carreras Leukemia Research Institute (IJC), Barcelona, Spain

Background: Neutropenic fever (NF) is the most frequent cause of readmission in the outpatient autologous stem cell transplantation (ASCT) programs. In our at home model for multiple myeloma patients, we added primary prophylaxis with ceftriaxone, decreasing the incidence of fever during aplasia phase from $85 \%$ to $57.6 \%$. The aim of this study was to analyze the addition of two strategies to reduce the non-infectious NF: withdrawal of G-CSF and the addition of primary prophylaxis for engraftment syndrome with corticosteroids after ASCT.

Methods: Between January 2002 and August 2018111 myeloma patients were managed at-home since day +1 of ASCT. All were conditioned with Mel200. All patients received prophylaxis with quinolone, fluconazole, aerolized pentamidine, low-dose acyclovir (HVS + ), and ceftriaxone (since day +4 ). The patients were classified into 3 groups: group A ( $\mathrm{n}=33$; G-CSF since day +7 without corticosteroid), group $\mathrm{B}(\mathrm{n}=32$; no G-CSF and no corticosteroid), group C ( $\mathrm{n}=46$; no G-CSF with prednisone $0.5 \mathrm{mg} / \mathrm{kg} /$ day since day +7 until granulocyte recovery). First-line therapy at home of NF was piperacillin-tazobactam $4.5 \mathrm{~g} / 6 \mathrm{~h}$ i.v. using a portable intermittent infusion pump. Fever was an indication of immediate medical consultation and those patients presenting signs of focal infection or severe sepsis were admitted. Other indications for readmission were: willingness of the patient or caregiver, uncontrolled nausea, vomiting or diarrhoea, and mucositis requiring total parenteral nutrition or i.v. morphics.

Results: The main characteristics of the patients and outcomes are shown in table 1 . There were no differences between groups regarding age, gender, immunological subtype, response before ASCT, HCT-CI, and CD34 cell dose infused. There were more patients with advanced disease (ISS III) in group C compared to group A (19.5\% vs. $6.1 \% ; \mathrm{P}=0.003)$. The duration of neutropenia was longer in those groups that did not receive G-CSF (A: 8 days, B: 11 days, $\mathrm{C}: 10$ days; $\mathrm{P}<0.01$ ). Comparing group $\mathrm{A}$ with group $\mathrm{C}$, we observed that the incidence of $\mathrm{NF}$ and the readmissions rates were lower in group C (NF: $57.6 \%$ vs. $23.9 \%$; $\mathrm{P}=0.002$; relative risk reduction: 0.41 , and number needed to treat 2.97 ; readmissions: $12.1 \%$ vs. $2.2 \%$; $\mathrm{P}=0.07$, respectively). The 10-day cumulative incidence of $\mathrm{NF}$ were $54.5 \%$ in group $\mathrm{A}, 40.6 \%$ in group $\mathrm{B}$, and $23.9 \%$ in group $\mathrm{C} ; \mathrm{P}=0.009$. The non-administration of $\mathrm{G}-$ CSF with the addition of prophylactic corticosteroid did not 
modify the incidence and grade of mucositis, the first day and duration of fever, nor the number of bacterial infections documented. In the multivariate analysis, this combination (no G-CSF with corticosteroid) maintained its protective effect for the development of NF and hospital readmission (OR 0.07; $\mathrm{P}=0.001$ and $\mathrm{OR} 0.07 ; \mathrm{P}=0.05$, respectively).

Conclusions: The non-use of G-CSF and the addition of prophylactic corticosteroid in MM patients managed at home after ASCT minimize the incidence of non-infectious fever and optimize hospital resources by reducing hospital readmissions.

Disclosure: Nothing to declare.

\section{P176}

\section{Abstract already published.}

\section{P177}

Safety of vaccines in a cohort of allogeneic stem cell transplantation recipients

Clara Targhetta ${ }^{1}$, Maria Pina Simula ${ }^{1}$, Cristina Depau ${ }^{1}$, Adriana Vacca ${ }^{1}$, Eugenia Piras ${ }^{1}$, Tania Durzu ${ }^{1}$, Rodrigo Rojas $^{2}$, Valentina Serreli ${ }^{1}$, Sara Cardani ${ }^{1}$, Giorgio La Nasa ${ }^{1}$

${ }^{1}$ Ospedale A. Businco, Azienda Ospedaliera G. Brotzu, U. O. Ematologia e CTMO, Cagliari, Italy, ${ }^{2}$ Università degli Studi di Sassari, UOC Ematologia, Sassari, Italy

Background: Antibody titers to vaccine-preventable diseases decline during the 1-10 years after allogeneic Hematopoietic Stem Cell Transplantation (HSCT) if the recipient is not revaccinated. It is therefore considered best practice to try to offer HSCT recipients the same level of protection against all vaccine preventable diseases as the general population. Few data in the literature are available concerning vaccine-related problems in HSCT recipients. We performed a farmacovigilance evaluation in a cohort of allotransplanted patients followed in our clinic during a 1 year period.

Methods: From October 2017 to November 2018 we administered a list of recommended vaccines to 49 HSCT recipients attending our routine out patient clinic who fulfilled the following criteria: CD4 T cells $>200 / \mu l, C D 19$ B cells $>20 / \mu l$, anti-CD20 antibody infusion $>6$ months, IVIG therapy $>2$ months, no active and severe Graft-versus-HostDisease (GvHD), no chemotherapy or biological therapeutic agents on going. Vaccines suggested were Influenza, Pneumococcal conjugate (PCV13), Polio (inactivated polio vaccine), Diphteria, Tetanus, Acellular pertussis, Hepatitis B, Hepatitis A, Haemophilus influenzae type B, Meningococcal quadrivalent (MCV4), Human papillomavirus, Meningococcal B, Measles-Mumps-Rubella (MMR), Varicella. Live vaccines (MMR and Varicella) were not recommended before 2 years after HSCT and in patients with chronic GvHD. All the patients were asked to take the list to the local health facilities in order to have the vaccines injected and a vaccination table arranged with the doses already received and those to receive. We checked the vaccination tables at each visit and monitored potential side effects and GvHD status at 3, 6, and 12 months after the first vaccine injection.

Results: Twenty-nine out of 49 patients were evaluable (table 1), 16 without GvHD and 13 with chronic GvHD (5 mild, 4 moderate, 4 severe). Median time after HSCT was 34 months (16-240). Median number of vaccines received was 8 (1-14). As regards patients without chronic GvHD, 2 out of 16 experienced fever after vaccine injections; 1 out of 16 developed transient mild reduction of platelet count; 1 patient reported headache and otalgia after vaccine injection, while another one transient joint pain; 1 out of 16 patients presented signs of mouth chronic GvHD (score 1 $\mathrm{NIH}$ ) and transaminase increase (grade 1 according to World Health Organization Toxicity Scale) 3 months after the first vaccine dose, so that cyclosporine dose had to be augmented. As regards patients with chronic GvHD, 4 out of 13 experienced fever after vaccine injections; 2 patients with mild chronic GvHD of the mouth presented hepatic flare two and three months after the first vaccine dose, respectively. In both cases a new increase of cyclosporin and methylprednisolone doses determined progressive normalization of liver enzymes.

Conclusions: These data show that vaccines were globally well tolerated in HSCT recipients, even when they suffered from chronic GvHD. However, close monitoring is warranted in order to better evaluate possible vaccine side effects in this setting of patients.

Disease: AML 11 ALL 5 NHL 3 HL 3 MDS 2 SAA 2 PMF 1 CML 1 SCD 1

Conditioning Regimen: MAC 19 Non MAC 10

Donor: Sibling 18 MUD 7 Haplo 4

[[P177 Table] 1. Table 1]

Disclosure: Nothing to disclose

\section{P178}

Allogeneic HSCT in the treatment for patients $60+$ with AML: Is good QOL attainable, and how can we select for this?

Rachel Wright ${ }^{1}$, Maximilian Oremek ${ }^{2}$, David Davies ${ }^{3}$, Emma Kempshall, Keith Wilson ${ }^{3,4}$, Wendy Ingram $^{3}$

${ }^{1}$ Taunton \& Somerset NHS Trust, Taunton, United Kingdom, ${ }^{2}$ Rheinische Friedrich-Wilhelms-Universität, Bonn, 
Germany, ${ }^{3}$ University Hospital of Wales, Cardiff, United Kingdom, ${ }^{4}$ Cardiff University School of Medicine, Cardiff, United Kingdom

Background: Allogeneic HSCT improves survival for AML patients over the age of 60 years of age when compared to chemotherapy alone. The haematopoietic stem cell transplantation comorbidity index (HCT-CI) and EBMT score predict for non-relapse mortality and overall survival, yet little is known about whether QoL is preserved in this patient group and whether HCT-CI and other performance scores pre-BMT correlate with QoL post allo-HSCT.

Methods: We conducted a retrospective analysis of patients 60 years and older who underwent RIC allo-HSCT at the University Hospital of Wales, Cardiff between September 2011 and December $2017(\mathrm{n}=41)$. HCT-CI, Karnofsky Performance Score (KPS) and EBMT scores were calculated prior to transplant and QoL measured using the FACT BMT (version 4) questionnaire, which was completed at 3,6 and 12 months post transplant. Patients were grouped at the 3-, 6- and 12-month time points for each of the different performance indices, allowing group comparison against compound sub scores using the MannWhitney U test.

Results: 41 patients were included in this study, with median age 65 years (range 60-74). Patient characteristics, including conditioning, donor type, pre-transplant HCT-CI and KPS scores are summarised in table 1 . The 2 year and 5 year overall survival (OS) for the patient cohort was $65.4 \%$ and $48.7 \%$ respectively. HCT-CI of $\geq 3$ vs 0 was significantly associated with poorer BMT-related QoL domains at 3 months $(\mathrm{p}=0.035)$ and general $\mathrm{QoL}$ domains at 6 months $(\mathrm{p}=0.025)$ post-transplant. While EBMT score showed no correlation with QoL parameters, patients with KPS of 100 vs $\leq 90$ showed significant differences in both general $(\mathrm{p}=0.01)$ and BMT-related QoL $(\mathrm{p}=0.04)$ at 3 months and in all QoL domains at 6 months (symptomrelated QoL $\mathrm{p}=0.05$, general QoL $\mathrm{p}=0.01$, BMT-related QoL $\mathrm{p}=0.01$ ). Importantly neither the HCT-CI nor the KPS pre-transplant predicted for QoL at 12 months post transplant.

Conclusions: Patient selection is key to ensuring maximum benefit from allo-HSCT both in terms of overall survival but also with regards to QoL and survivorship. We note that while patients with HCT-CI 33 or KPS $\leq 90$ had significantly poorer QoL at 6 months post allo-HSCT, QoL was recovered by 12 months post transplant, with this significant difference no longer seen. Our data shows that in selected AML patients over the age of 60 years with good performance status and low comorbidity index, a favourable outcome can be achieved with good QoL maintained throughout the post transplant period.

\begin{tabular}{|c|c|c|c|c|c|}
\hline Characteristics & Total $(\%)$ & Characteristics & Total $(\%)$ & Characteristics & Total $(\%)$ \\
\hline $\begin{array}{l}\text { Median age, } \\
\text { yr (range) }\end{array}$ & $\begin{array}{l}65(60- \\
74)\end{array}$ & Donor & & HCT-CI & \\
\hline Gender, male & 24 (58.5) & Sibling & $9(22.0)$ & 0 & $17(41.5)$ \\
\hline Conditioning & & $\begin{array}{l}\text { Matched } \\
\text { unrelated donor } \\
(10 / 10)\end{array}$ & $21(51.2)$ & $1-2$ & $9(21.9)$ \\
\hline FMC & $32(78.0)$ & $\begin{array}{l}\text { Mismatched } \\
\text { unrelated donor } \\
(\leq 9 / 10)\end{array}$ & $11(26.8)$ & $\geq 3$ & 15 (36.6) \\
\hline FB-ATG & $5(12.2)$ & Disease Risk & & KPS & \\
\hline Other & $4(9.8)$ & High Risk & $25(61.0)$ & 100 & $22(53.7)$ \\
\hline Diagnosis & & Standard Risk & 15 (36.6) & 90 & $14(34.1)$ \\
\hline AML & $28(68.3)$ & Good Risk & $1(2.4)$ & $\leq 80$ & $5(12.2)$ \\
\hline sAML & 13 (31.7) & & & & \\
\hline
\end{tabular}

\section{[[P178 Table] 1. Table 1: Patient Characteristics]}

Disclosure: Nothing to declare.

P179

High prevalence of vertebral fractures and bone disease within the first 24 months post-alloSCT

María Suárez-Lledó $^{1}$, Ana Monegal, Teresa Solano ${ }^{1}$, Gonzalo Gutiérrez-Garcia ${ }^{l}$, Alexandra PedrazaNavarrete ${ }^{I}$, Alejandra Patricia Martínez-Roca ${ }^{1}$, Ana Moreno-Castaño ${ }^{1}$, Gerardo Rodriguez-Lobato ${ }^{1}$, Laura Rosiñol ${ }^{1}$, Francesc Fernández-Avilés ${ }^{1}$, Alvaro UrbanoIspizua $^{1}$, Montserrat Rovira ${ }^{I}$, Carmen Martinez ${ }^{I}$

${ }^{1}$ Hospital Clínic de Barcelona, Hematology, Barcelona, Spain

Background: Advances in alloSCT technology, supportive care, and use of reduced intensity conditioning regimens for older patients have led to significant improvements in longterm survival after transplant. The survivors have an elevated probability of late morbidity and mortality, including abnormalities in phosphocalcic metabolism and bone disease. Rapid and progressive bone loss occurs within the first 6-12 months after transplant, and this is followed by a slow process of recovery, with bone loss persisting for 48 to 120 months. Bone fractures can worsen the quality of life of alloSCT survivors, but the real burden of the disease is unknown. The objective of the study is to ascertain the prevalence of bone pathology and vertebral fractures early after transplant in our center.

Methods: This is a retrospective and observational study. Forty-nine patients $(25$ male/24 female, median age $54 \mathrm{y}$, range 19-69) that underwent alloSCT were included in the study in the period of 6 to 24 months after transplant (May 2016-December 2017). Pre- and post-transplant risk factors associated with bone disease were recorded: age $\geq 65$ years, female sex, menopause, hormone replacement therapy, previous treatment with steroids, previous fractures, weight 
$<40 \mathrm{~kg}, \mathrm{BMI}<20-25$, low physical activity, low calcium intake, smoking, alcohol intake, and history of femoral fractures in parents. In all patients laboratory data (including serum calcium, 25-hydroxyvitamin D, and PTH), lumbar and femoral BMD (DXA), and spinal x-ray were also evaluated. A vertebral fracture was defined as a reduction of $\geq 20 \%$ in the anterior, middle or posterior high of the vertebral body.

Results: We identified vertebral fractures in 12 (24\%) patients. Five patients had fractures prior to transplantation, and 7 patients presented "de novo" vertebral fractures following transplantation; therefore, the prevalence of "de novo" postransplant fractures was 7/49 (14\%). Most (85\%) of these fractures were asymptomatic at the time of diagnosis. Most patients (64\%) with vertebral fractures had $\geq 3$ pre-SCT risk factors (median risk factors pre-SCT 3, range 2-6), the most frequent being low calcium intake, steroid exposure, presence of previous fractures, and menopause. Those patients with fractures and less than 3 risk factors pre-TPH, added new risk factors after transplant, mainly steroid treatment. Forty-four patients $(90 \%)$ had vitamin D insufficiency $(<30 \mathrm{ng} / \mathrm{ml}), 15$ (32\%) had osteopenia and 9 (18\%) had osteoporosis. Vitamin D insufficiency and bone disease were more frequent in women than in men $(98 \%$ vs. $84 \%$ for vitamin D, $37 \%$ vs. $28 \%$ for osteopenia, $29 \%$ vs. $8 \%$ for osteoporosis, and $25 \%$ vs. $20 \%$ for vertebral fractures, respectively).

Conclusions: The prevalence of post-transplant bone disease and vertebral fractures in our series is high. Most fractures appearing "de novo" after alloSCT were asymptomatic and were diagnosed by $\mathrm{x}$-ray. Patients who presented vertebral fractures frequently had more than 3 risk factors identified pre-SCT. Patients undergoing alloSCT should have their bone health assessed early in their treatment and, if indicated, should start preventative therapy to avoid bone loss and fractures. Other measures such as physical exercise, vitamin $\mathrm{D}$ and calcium supplementation, and DXA and spinal x-ray at baseline and following transplantation are also highly recommended.

Disclosure:

María Suárez-Lledó received a grant from DKMS-Spain Foundation.

Other Authors have Nothing to declare

\section{P180}

The use of G-CSF in selected patients after autologous stem cell transplantation is associated with low incidence of engraftment syndrome

Lucrecia Yañez ${ }^{1,2}$, Miriam Sanchez Escamilla ${ }^{2}$, Cristina Amunárriz ${ }^{3}$, Guillermo Martín Sánchez ${ }^{1,2}$, Mercedes Colorado $^{1,2}$, Sara Garcia Avila ${ }^{1}$, Mónica López Duarte ${ }^{1,2}$, José Luis Arroyo ${ }^{3}$, Enrique Ocio ${ }^{1,2}$, Arancha Bermudez ${ }^{1,2}$
${ }^{1}$ Hospital Universitario Marqués de Valdecilla, Santander, Spain, ${ }^{2}$ Instituto de investigación Marqués de Valdecilla IDIVAL -, Santander, Spain, ${ }^{3}$ Banco de Sangre y Tejidos de Cantabria, Santander, Spain

Background: The use of G-CSF after autologous stem cell transplantation (ASCT) accelerates neutrophil recovery, however it has been related to an increased risk of engraftment syndrome (ES) development in some studies. For this reason, we do not routinely prescribe G-CSF after ASCT and we only use it in patients with significant complications (enterocolitis, severe sepsis, atrial fibrillation) after stem cell infusion.

The main objective of this study is to evaluate the incidence of ES in patients who receive ASCT for monoclonal gammopathies (MG), non-Hodgkin lymphoma (NHL) and Hodgkin lymphoma (HL) and receive G-CSF only if needed. As secondary objectives we evaluate differences in the engraftment day as well as the length of inpatient stay.

Methods: We retrospectively analyzed patients with MG or lymphoma, who underwent ASCT conditioned with high dose melphalan $\left(140-200 \mathrm{mg} / \mathrm{m}^{2}\right)$ or BEAM, respectively, between 2015 and 2017 in our center. Specific clinical features for ES according to Spitzer and Maiolino criteria were evaluated between 3 days before and 7 days after the engraftment. Statistical analysis was performed with SPSS v. 15.0 .

Results: Thirty-one patients with MG and 34 patients with lymphoma were analyzed. Median age at transplant was 56.8 years (48.7-65.4) and 41 patients $(63.1 \%)$ were male. Median prior lines of treatment in patients with GM or lymphoma were 1 (1-3) and 2 (1-5), respectively. Table 1 shows patients'characteristics.

Mobilization with G-CSF \pm Plerixafor was performed in 27 patients $(36 \%)$ and chemotherapy + G-CSF \pm Plerixafor in 38 patients $(64 \%)$. Median CD34 x $10^{6} / \mathrm{Kg}$ cells infused was $3.6(2.7-5.3)$.

Eleven patients (16.9\%) received G-CSF, 5 due to infection ( 2 enterocolitis, 1 listeriosis, 1 acute hepatitis, 1 septic shock) and 6 because of atrial fibrillation or fibrilloflutter. Median time from SCT to first day of G-CSF was 5 days (5-7) and median time on G-CSF treatment was 5 days (4-7). Patients who received G-CSF showed a short time to neutrophil engraftment $\left(\geq 0.5 \times 10^{6} / \mathrm{L}\right), 10$ days vs. 13 days, $\mathrm{p}<0.001$ but longer duration of hospitalization, 18 days vs. 15 days, $p=0.050$. Non-relapse mortality at day $+30,+100$ and +180 was $0 \%$.

ES was diagnosed in $4(6.2 \%)$ patients, 1 amyloidosis, 2 multiple myeloma and 1 plasmablastic lymphoma. There was not statistical difference in the incidence of ES between patients who received G-CSF $(9.1 \%)$ and patients who did not $(5.6 \%), p=0.533$. Analyzed by disease, ES appeared in 
1 of 6 patients who received G-CSF in the lymphoma group (16.6\%) but none of the 7 patients with MG that received GCSF developed it. We did not find statistical differences between patients who developed ES and those who did not in age (49 years vs. 56 years, $p=0.314$ ), length of hospitalization (19 days vs. 15 days, $\mathrm{p}=0.185)$ and the number of CD34 x $10^{6} / \mathrm{Kg}$ cells infused (3.65 vs. 4.62, $\mathrm{p}=0.408$ ).

Conclusions: The use of G-CSF in selected patients is associated with low incidence of ES. Our study confirms that the use of G-CSF accelerates neutrophil recovery but it is unclear if it can increase the incidence of ES, especially in patients with lymphoma.

\begin{tabular}{|c|c|}
\hline Varlable & $N(\%)$ \\
\hline Gender, Male & $41(63.1)$ \\
\hline Median age, years (IQL range) & $60.3(53.5-67.5)$ \\
\hline \multicolumn{2}{|l|}{ Disease } \\
\hline - Multiple Myeloma & $30(46.1)$ \\
\hline - Amyloidosis & $1(1.6)$ \\
\hline$=$ NHL & $29(44.6)$ \\
\hline - Hodgkin Disease & $5(7.7)$ \\
\hline Prior lines of treatment, median (range) & $\begin{array}{l}\text { Monoclonal gammopathy, } 1 \text { (1-3) } \\
\text { Lymphoma } 2 \text { (1-5) }\end{array}$ \\
\hline \multicolumn{2}{|l|}{ Status disease at transplant } \\
\hline \multicolumn{2}{|l|}{ Monoclonal gammopathy, } \\
\hline sCR, CR, VGPR, PR & $4(12.9), 2(6.5), 14(45.2), 11(35.5)$ \\
\hline Lymphoma, & \\
\hline $1^{\text {st }} C_{R}, 2^{\text {nd }} C R, P R$ & $17(50), 8(23.5), 9(26.5)$ \\
\hline
\end{tabular}

[[P180 Image] 1. Table 1. Patient's characteristics]

Disclosure: Nothing to declare

\section{P181}

Recipient T-memory cell subsets count before ATG as the main prognostic factor of primary graft failure after allo-HSCT

Mikhail Drokov ${ }^{I}$, Natalia Popova ${ }^{1}$, Yulia Davydova ${ }^{1}$, Nikolay Kapranov ${ }^{1}$, Ekaterina Mikhaltsova ${ }^{1}$, Olga Koroleva ${ }^{1}$, Vera Vasilyeva ${ }^{1}$, Darya Dubnyak ${ }^{1}$, Zoya Konova ${ }^{1}$, Ekterina Usikova ${ }^{1}$, Anna Dmitrova ${ }^{1}$, Maria Nareyko ${ }^{1}$, Valentina Dvirnyk ${ }^{1}$, Irina Galtseva ${ }^{1}$, Larisa Kuzmina', Elena Parovichnikova ${ }^{I}$, Valery Savchenko ${ }^{1}$

${ }^{1}$ National Research Centre for Hematology, Moscow, Russian Federation,

Background: Graft failure is one of the TOP-3 problems of allo-HSCT (after GVHD and relapse). The problem of graft failure becomes more significant due to increasing number of allo-HSCT with RIC conditioning regimen from haploidentical and HLA-mismatched unrelated donors. Role of $\mathrm{T}$ cells in graft failure is well known. Here we report an impact of T-memory cell subsets count before
Antithymocyte globulin (ATG) administration on primary graft failure after allo-HSCT.

Methods: Sixteen patients with acute leukemia transplanted in National Research Center for Hematology were included on this prospective study. All patients received horse ATG at dose $10 \mathrm{mg} / \mathrm{kg} /$ day from day -4 to -1 before allo-HSCT as GVHD prophylaxis and were balanced by other factors that could affect engraftment. Detailed patients characteristics are listed in Table 1. Peripheral blood samples were collected on day -4 before allo-HSCT (before ATG injection) in EDTA-tubes. Flow cytometry analysis was performed on BD FACS Canto II (Becton Dickinson, USA) to define T-memory subsets: T-naive and T-stem cell memory (Tnv+scm) - CD45R0-CCR7+CD28+; T-central memory (Tcm) - CD45R0+CCR7+CD28+; T-transitional memory (Ttm) - CD45R0+CCR7-CD28+; T-effector memory (Tem) - CD45R0+CCR7-CD28-; T-terminal effector (Tte) - CD45R0-CCR7-CD28-, among CD4+ and CD8+ T-cells . Sysmex XE-2100 was used to calculate absolute count of different T-cell subsets. Mann-Whitney U test was used for nonparametric data analysis between two groups. Fisher's exact test was used for $2 \times 2$ tables. P-value less than 0.05 was considered statistically significant.

Results: An influence of T-memory cell subsets count before ATG administration on primary graft failure is shown in Figure 1. According to our data high absolute number of CD4+Ttm and CD4+Tte is associated with primary graft failure.

Conclusions: Based on these findings high absolute number of CD4+Ttm and CD4+Tte could be one of the prognostic factors of primary graft failure after allo-HSCT. Optimizing ATG dose due to recipient absolute T-memory cell subsets count before ATG administering may prevent graft failure and improve posttransplant results.

\begin{tabular}{|c|c|c|c|}
\hline & Engrafted $(n=13)$ & $\begin{array}{l}\text { Primary graft } \\
\text { failure }(n=3)\end{array}$ & $\begin{array}{l}\mathrm{p}- \\
\text { value }\end{array}$ \\
\hline $\begin{array}{l}\text { Age (median } \\
\text { with range) }\end{array}$ & $34(23-61)$ & $49(25-58)$ & 0,331 \\
\hline Male / Female & $8 / 5$ & $2 / 1$ & 0,99 \\
\hline ALL / AML & $8 / 5$ & $2 / 1$ & 0,99 \\
\hline $\mathrm{CR} 1 / \mathrm{CR} 2+$ & $7 / 6$ & $3 /-$ & 0,25 \\
\hline MAC / RIC & $1 / 12$ & $-/ 3$ & 0,99 \\
\hline $\begin{array}{l}\text { MMUD / MUD / } \\
\text { Haplo / MRD }\end{array}$ & $3 / 2 / 3 / 5$ & $2 /-/-/ 1$ & 0,44 \\
\hline $\mathrm{BM} / \mathrm{PBSC}$ & $3 / 10$ & $1 / 2$ & 0,99 \\
\hline ATG / ATG +PT-CY & $7 / 6$ & $1 / 2$ & 0,99 \\
\hline
\end{tabular}

[[P181 Table] 1. Table 1. Characteristics of patients]

Disclosure: No relevant conflicts 


\section{P182}

Budesonide - a not so topical glucocorticosteroid? - The relevance of drug interactions

Catherine Prodger ${ }^{1}$, Katalin Balassa ${ }^{1,2}$, Nicola Gray ${ }^{1}$, Oliver Lomas', Asha Aggarwal', Nadjoua Maouche ${ }^{1}$, Vanderson Rocha ${ }^{1,2}$, Robert Danby ${ }^{1,3}$, Andy Peniket ${ }^{1}$

${ }^{1}$ Oxford University Hospitals NHS Foundation Trust, Oxford, United Kingdom, ${ }^{2}$ NHS Blood and Transplant, Oxford, United Kingdom, ${ }^{3}$ Anthony Nolan Research Institute, London, United Kingdom

Background: Upper gastrointestinal graft-versus-host disease (GI GVHD) has been an increasingly recognised entity following allogeneic stem cell transplantation (SCT). Budesonide, widely used in inflammatory bowel conditions, has also been found beneficial in GI GVHD. The major benefit of Budesonide is attributable to its poor absorption and extensive first-pass metabolism via cytochrome $\mathrm{P} 450$ (CYP) 3A4, which translates to less systemic steroid-related effects. However, transplant patients are often exposed to multiple drugs, among which some agents act as CYP3A4 inhibitors and therefore can increase Budesonide bioavailability and might lead to systemic toxicity. Azole antifungal drugs are probably the most common concomitantly used CYP3A4 inhibitors in transplant recipients.

Methods: We reviewed allogeneic SCT records for patients treated with oral Budesonide for GI GVHD at our transplant centre between 2015 and 2018 retrospectively. The aim of the work was to assess the development of adrenal suppression with or without clinical features of iatrogenic Cushing's syndrome. The standard dose of Budesonide was $3 \mathrm{mg}$ three times a day. Patients receiving Prednisolone or other glucocorticosteroids and those with no available serum cortisol level measurements were excluded.

Results: Our analyses identified four allogeneic SCT patients in whom adrenal suppression was diagnosed with undetectable serum cortisol levels during oral Budesonide treatment. Of these patients two developed iatrogenic Cushing's syndrome and both patients were treated with CYP3A4 inhibitors concomitantly:

1. Clarithromycin and Fluconazole;

2. Clarithomycin and Voriconazole.

The development was rapid (within 3 and 4 weeks). Symptoms included morphological features such as moon face, high blood pressure, weight gain, peripheral oedema and proximal myopathy. Symptoms resolved gradually following cessation of azole antifungal agents and on gradual weaning of Budesonide.

Conclusions: Although single agent Budesonide treatment given for GI GVHD is rarely associated with systemic side effects, patients on azole antifungal drugs and macrolide antibiotics are at higher risk of systemic toxicity due to drug interactions. Patients who are allergic to Penicillin and receive macrolide-based prophylaxis can be especially vulnerable. To our knowledge the number of cases reported in literature about systemic effects of oral Budesonide in transplant recipients is less than 10. Our observation supports previous reports on the potential of oral Budesonide to induce systemic effects. We therefore advise careful monitoring of patients treated with Budesonide in combination with CYP3A4 inhibitors, including antimicrobial agents routinely used in SCT.

Disclosure: None

\section{P183}

Implemented strategies to overcome barriers in the establishment of a consolidated hematopoietic stem cell transplantation program in a developing country

\section{Monica Rivera Franco ${ }^{1}$, Eucario Leon Rodriguez,}

${ }^{1}$ Instituto Nacional de Ciencias Medicas y Nutricion Salvador Zubiran, Hematology and Oncology, Mexico City, Mexico

Background: The National Institute of Medical Sciences and Nutrition "Salvador Zubiran" is a National Health Institute located in Mexico City. Although Mexico is considered an upper-middle income country, more than $50 \%$ of the population lives in poverty without health care coverage and patients within this social stratum are referred to our Institution. The first hematopoietic stem cell transplantation (HSCT) in Mexico was performed at our Institution in 1980. From this year until 1997, HSCT were sporadically performed $(n=33)$, showing a poor overall survival (OS) and high non-relapse mortality (NRM). These outcomes resulted from an unstructured HSCT Program, limitedresources, patient low socioeconomic status, and paucity of population-adapted procedures. In 1998, according to these results, a decision to establish a HSCT Program was made. Therefore, in order to set up a successful HSCT Program, implementation of financial and medical strategies were necessary. The objectives of this study were to describe the barriers and implemented strategies for the establishment of a HSCT Program in Mexico along with the outcomes of patients undergoing this procedure throughout the reorganization of the Program.

Methods: This study is a health services research. Barriers were detected based on the results of the HSCT Program from 1980-1997 (not shown). Table 1 shows the financial, medical, and research strategies that were implemented for each barrier. 
Results: From November 1998 to November 2018, 363 HSCT have been performed in 322 patients at our Institution. Most HSCT were autologous $(\mathrm{n}=213,59 \%)$. Forty one patients underwent 2 HSCT. From the 322 patients, most were males $(n=196,61 \%)$ and the median age was 33.5 years (range, 15-65). The most frequent underlying diseases for auto-HSCT were lymphomas $(\mathrm{n}=68,36 \%)$, non-seminomatous germ cell tumors $(\mathrm{n}=42$, $22 \%)$, and multiple myeloma $(\mathrm{n}=42,22 \%)$. Acute leukemias $(\mathrm{n}=41,34 \%)$, aplastic anemia $(\mathrm{n}=25,21 \%)$, and myelodysplastic syndromes $(n=20,17 \%)$ were the most frequent diagnosis for patients undergoing allo-HSCT; and acute leukemia was the most frequent diagnosis for patients undergoing haploidentical HSCT $(n=10,83 \%)$. Acute and chronic GVHD were present in 25\% (Grades I-II 89\%) and $35 \%$ (limited 76\%), respectively. For allo-HSCT, 30, 100day, and 1-year NRM was $2.5 \%, 8 \%$, and $12 \%$, respectively; 30 and 100-day NRM in auto-HSCT was 1.5\%; 10year OS was $63 \%$ and $56 \%$ for auto and allo-HSCT, respectively.

Conclusions: Future perspectives of the HSCT Program include the acquisition of funds for unrelated donors; to improve outcomes of patients undergoing haploidentical HSCT, and to increase the number of in-patient rooms. We conclude that despite paucity of resources and other limitations, the implementation of financial, medical, and research strategies have shown that barriers can be effectively overcome in a developing country in order to establish a consolidated and nationally renowned HSCT Program, providing good outcomes for patients.

Disclosure: None of the authors have any conflict of interest to disclose.

\section{P184}

The effect of protective buffering on daily stress and relationship quality in dyads following hematopoietic stem cell transplantation: Results from daily process methodology

\section{Malgorzata Sobczyk-Kruszelnicka ${ }^{1}$, Aleksandra Kroemeke ${ }^{2}$, Zuzanna Kwissa-Gajewska ${ }^{2}$, Sebastian Giebel ${ }^{1}$}

${ }^{1}$ Maria Sklodowska-Curie Institute - Cancer Center, Gliwice Branch, Gliwice, Poland, ${ }^{2}$ SWPS University of Social Sciences and Humanities, Warszawa, Poland

Background: Cancer-related support communication (e.g., protective buffering) may impact the risk for psychological and relationship distress in patients following hematopoietic stem cell transplantation (HSCT) and their caregivers. Previous studies have revealed that protective buffering (i.e., hiding one's concerns and denying one's worries) has mixed effects: is beneficial (for "protected" person), costly (especially for the person using it), or unrelated to dyadic wellbeing. There has been, however, little evidence linking dyadic protective buffering with distress using daily process methodology. We assessed (1) the relationship between daily protective buffering, and same- and next-day stress and relationship quality in patient-caregiver dyads following HSCT and (2) whether similarity or complementarity in protective buffering between dyads is adaptive.

Methods: Two hundred patients (after first autologous or allogeneic HSCT) and their caregivers (spouse or another relative) independently completed measures of daily protective buffering, daily relationship quality, and daily stress for 28 consecutive evenings after patients' hospital discharge. Actor-Partner-Interdependence Model (i.e., both partners' and caregivers' reports regarding support communication and distress were studied) was used to test study hypotheses.

Results: For both patients and caregivers, multilevel structural equation modeling showed a significant positive relationship between daily protective buffering and sameday relationship quality. Association of protective buffering with same-day stress level was negative. In next-day analyses, patient-reported protective buffering was related to patient's higher relationship quality, whereas caregiverreported protective buffering increased patient's daily stress. Complementarity in protective buffering was related to higher immediate same-day relationship quality for both patients and caregivers, while benefits from similarity have delayed effects, although only in patients.

Conclusions: Contrary to previous studies, protective buffering rather has a beneficial effect in dyads following HSCT. Protection of the partner and relationship against revealing negative emotions and powerlessness was not related to costs in both parties. The findings suggest that the effect of daily protective buffering in dyads following HSCT depends on support timing (same- or next-day effect) and differs for both parties. Patients seem to benefit the most from the similarity in protective buffering, while caregivers from complementarity. The "fit" between patient and caregiver in support communication ought to be taken into consideration in the practical approach.

Disclosure: Nothing to declare.

\section{P185}

Virus reactivation and low dose of $\mathrm{CD34}+$ cell were associatied with secondary poor graft function within the first 100 days after allogeneic stem cell transplantation

Yuqian Sun ${ }^{1}$, Xiao-Jun Huang ${ }^{1}$ 
${ }^{1}$ Peking University People's Hospital, Peking University Institute of Hematology, Beijing Key Laboratory of Hematopoietic Stem Cell Transplantation, Collaborative Innovation Center of Hematology, Beijing, China

Background: Secondary poof graft function (sPGF) was defined as the secondary cytopenia after initial engraftment of HSCT. It was shown to be associated with poor prognosis, however there are very few reports on the incidence, risk factors and outcomes of SPGF.

Methods: Patients who received transplantation from Peking University People's Hospitial during January, 2015 to December, 2015 were retrospectively reviewed if they fulfilled the following conditions:

(1) diagnosed with acute leukemia or myelodysplastic syndrome;

(2) received allo-SCT from either matched sibling donor (MSD) or haploidentical related donor (HID). PGF was defined as persistent neutropenia $\left(\leq 0.5 \times 10^{9} / \mathrm{L}\right)$, thrombocytopenia (platelets $\leq 20 \times 10^{9} / \mathrm{L}$ ), and/or hemoglobin $\leq 70 \mathrm{~g} / \mathrm{L}$ for at least 3 consecutive days, transfusion-dependence, associated with hypoplastic-aplastic bone marrow (BM), and complete donor chimerism without concurrent graftversus-host disease (GVHD) or disease relapse. Primary PGF was defined as the failure to achieve initial engraftment by days 28 after transplantation, while secondary PGF was defined as the fulfillment of the criteria after initial engraftment HSCT.

Results: During January, 2015 to December, 2015, 564 patients who received transplantation from Peking University People's Hospitial were retrospectively reviewed. Among the 490 patients who achieved initial engraftment, 28 patients developed sPGF. The cumulative incidence of sPGF on day 100 was $5.7 \%$. The median time of secondary PGF was 54.5 (34-91) days after transplantation. Low ( $<$ median) CD34+ cell dose $(\mathrm{p}=0.019$, HR 3.07(95\%CI, 1.207-7.813)), EBV reactivation ( $\mathrm{p}=0.009$, HR 3.648(95\%CI, 1.382-9.629)) and CMV reactivation $(\mathrm{p}=0.003$, HR 7.827 (95\%CI, 2.002-30.602)) were identified as independent risk factors with sPGF. There is no significant difference of PGF incidence in MSD group and HID patients $(\mathrm{p}=0.44)$. The overall survival of patients with SPGF at 1 year after transplantation was significantly poor than patients with GGF $(50.5 \%$ versus $87.2 \%, \mathrm{p}<0.001)$.

Conclusions: In conclusion, sPGF develop in 5.7\% patients after allo-SCT, especially in patients with CMV, EBV reactivation or infused with low dose of $\mathrm{CD} 34+$ cell. The prognosis of sPGF is still poor due to lack of standard treatment.

Disclosure: There is no conflict of interet

\section{P186}

Thiotepa with treosulfan and busulfan based conditioning are significantly more gonadotoxic than treosulfan

\section{Nuthana Prathivadi Bhayankaram ${ }^{I}$, Denise Bonney ${ }^{I}$, Helen Campbell, Cheryl Fitzgerald ${ }^{2}$, Robert Wynn ${ }^{1}$}

${ }^{1}$ Royal Manchester Children's Hospital, Manchester, United Kingdom, ${ }^{2}$ St Mary's Hospital, Manchester, United Kingdom

Background: Haematopoietic stem cell transplant (HCT) can be used to treat malignant and non-malignant conditions. Prior to HCT, patients have conditioning with chemotherapy to ablate their bone marrow to make space for transplanted cells.

Previous studies suggest that busulfan results in long-term gonadal toxicity. No previous studies have compared gonadal toxicity outcomes after treatment with busulfan with treosulfan, a newer agent with similar marrow toxicity to busulfan but with reduced non-marrow toxcitiy.

Our aim was to determine whether there are differences in pubertal and fertility outcomes in paediatric patients treated with treosulfan compared with busulfan.

Methods: Inclusion criteria were patients who had received either busulfan or treosulfan or treosulfan with thiotepa, only one HCT and were aged 14 years and above in August 2018. Eligible patients were reviewed in clinic as part of their routine follow-up, thus research ethical approval was not required.

Follice Stimulating Hormone, Luteinising Hormone, oestradiol, and pubertal history were noted. Ovarian reserve was estimated in female patients by measuring serum AntiMullerian Hormone (AMH). Male patients had serum testosterone measured and were also offered semen analysis.

Results: Thirty-five patients met the inclusion criteria, of which twenty-five wanted to be reviewed $(71 \%)$; seventeen females and eight males. Mean age at HCT was 13 years, mean age at review was 19 years and mean years since HCT was 5 years.

Female patients treated with busulfan or treosulfan with thiotepa $(n=14)$ had minimal AMH and none of these patients were having regular periods. Females treated with treosulfan $(n=3)$ had normal AMH and regular periods without needing hormone replacement.

Only four male patients opted for a semen analysis and all had significantly reduced sperm counts.

Conclusions: Our results suggest that females treated with treosulfan have minimal (if any) reduction in ovarian reserve compared to other conditioning regimens which casue significant compromise. Although this was a small 
study, and thus not suitable for statistical analysis, the clinical findings are marked. Future studies should further investigate optimal doses of treosulfan that could be used to achieve bone marrow engraftment and limit long-term effects on fertility.

Disclosure: Nothing to declare

\section{P187}

Hematopoietic stem cell transplantation patients admitted in the intensive care unit: Organ support and mortality

Walter Javier Zambrano Márquez ${ }^{I}$, Paula Lopez ${ }^{I}$, Jose Carlos Iglesias ${ }^{\prime}$, Paola Carolina Tamayo Arroyo ${ }^{1}$, Laura Escalada $^{1}$, Christel Castañon ${ }^{1}$, Laura Francisca Avila Idrovo $^{1}$, Antonio Sole Magdalena ${ }^{1}$, Lucia Morais Bras ${ }^{1}$, Tamara Arias Fernandez ${ }^{2}$, Ariana Fonseca ${ }^{I}$, Soledad Gonzalez Muñiz ${ }^{1}$, Joud Zanabili Al Sibai ${ }^{1}$, Ana Julia Gonzalez Huerta ${ }^{1}$, Pilar Palomo Moraleda ${ }^{1}$, Ana Pilar Gonzalez $^{1}$

${ }^{1}$ Hospital Universitario Central de Asturias, Hematology, Oviedo, Spain, ${ }^{2}$ Centro Comunitario de Sangre y Tejidos de Asturias, Oviedo, Spain, ${ }^{3}$ Hospital Universitario Central de Asturias,

Background: Autologous and allogenic hematopoietic stem cell transplantation (HSCT) are potentially curative treatments for hematological malignancies. Patients with related complications may need admission to the intensive care unit (ICU) for specific therapy and organ support. Mortality risk factors, supportive care and principal causes of admission in ICU are described in our cohort of patients (pts).

Methods: We retrospectively studied 326 pts, 185 male, with a median age of 56,63 years (range: 18-73) who underwent allo-HSCT in our center between July 2014 and October 2018. Two hundred and twenty-seven $(69,6 \%)$ pts received autologous HSCT (auto-HCT) and $99(30,4 \%)$ allogenic HSCT (allo-HSCT); 50 from unrelated donor, 38 from identical sibling, and the remainder, mismatched related donor 11.

Twenty-three $(7,1 \%)$ out of 326 pts were admitted in the ICU in the transplant procedure admission.

Results: Fifteen $(65,2 \%)$ out of 23 pts were male with a median age of 55 years (range: 28-69). Patients' baseline diseases were: multiple myeloma $(34,8 \%)$, non-Hodgkin's Lymphoma (26,1\%), Hodgkin's Lymphoma $(8,7 \%)$, acute lymphoblastic leukemia $(8,7 \%)$, myelodisplasic syndrome $(8,7 \%)$, solid tumor $(8,7)$ and acute myeloblastic leukemia $(4,3 \%)$. Fifteen $(65,2 \%)$ pts received auto-HSCT, $5(21,7 \%)$ allo-HSCT from unrelated donor, $2(8,7 \%)$ allo-HSCT from identical sibling, and the remainder haploidentical HSCT
(1) $(4,3 \%)$. So, $6,6 \%$ of auto-HSCT pts and $8 \%$ of alloHSCT were admitted in the ICU. The median stay in the ICU was 5 days (range: 1-30) and reasons for admission were: respiratory insufficiency $(60,8 \%)$, septic shock $(30,4 \%)$, renal insufficiency $(4,3 \%)$ and multi-organic failure $(4,3 \%)$. Twenty-one $(91.3 \%)$ pts required respiratory support with: nasal cannula or oxygen mask (C/M) (19\%), non-invasive mechanical ventilation (NIMV) $(66,7 \%)$ and invasive mechanical ventilation (IMV) (14,3\%). Fourteen $(60 \%)$ pts needed inotropic agents for shock treatment. Finally, $4(4,5 \%)$ pts required substitutive renal therapy with hemodialysis or haemofiltration (HD/HF). Eleven $(47,8 \%)$ out of 23 pts died, $7(63,6 \%)$ were male with a median age of 55 years (range: 24-64). Ten of them $(90,9 \%)$ needed IMV and were treated with inotropic agents. All patients who required HD/HF $(n=4)$ died. IMV and treatment with inotropic agents were associated with ICU mortality (OR 6,$5 ; \mathrm{p}=0,03$, OR 7; $\mathrm{p}=0,008$; respectively).

Conclusions: In our series of pts, 7,1\% needed admission in the ICU, presenting a mortality rate of $48 \%$ approximately. There were no differences in the prevalence of ICU admission regarding HSCT donor. Main reason for admission was respiratory failure with IMV requirement in $14,3 \%$ of pts. IMV and treatment with inotropic agents were associated with ICU mortality. An early identification of pts at risk of ICU admission could have a beneficial impact on survival improvement

Disclosure: Nothing to declare

\section{P188}

Is there any association between thrombotic risk factors and veno-oclusive disease in childhood allogeneic hematopoietic stem cell transplantation?

\section{Meric Kaymak Cihan', Fatma Belgin Atac ${ }^{2}$, Ozlem Arman Bilir ${ }^{I}$, Tekin Aksu ${ }^{I}$, Serife Mehtap Kanbur ${ }^{I}$, Namık Ozbek ${ }^{1}$}

${ }^{1}$ University of Health Sciences, Ankara Pediatrics and Pediatric Hematology and Oncology Hospital, Ankara, Turkey, ${ }^{2}$ Baskent University Hospital, Medical Biology, Ankara, Turkey

Background: Veno-oclusive disease (VOD) is a major complication of hematopoietic stem cell transplantation (HSCT). In some studies levels of fibrinolytic factors especially plasminogen activator inhibitor-1 (PAI-1) level were found associated with VOD. However, little is known about the relationship between thrombophilia risk factors and VOD. In this study we aimed to investigate association of major thrombophilic gene mutations on VOD in pediatric HSCT patients. 
Methods: We reviewed retrospectively 35 patients with VOD who underwent HSCT between 2010-2018 in Ankara Pediatrics and Pediatric Hematology-Oncology Training and Education Hospital, Bone Marrow Transplantation Unit, Turkey. Fifty-one patients who did not develop VOD and transplanted during the study period were accepted as control group. We evaluated plasma homocysteine and lipoprotein a level, protein $\mathrm{S}$ and $\mathrm{C}$ activity and antigen levels and factor $\mathrm{V}$ G1691A mutation, prothrombin G20210A mutation, methylenetetrahydrofolatereductase (MTHFR) C677T and A1298C mutations, plasminogen activator inhibitor-1 -675 4G/5G polymorphism before HSCT. We also evaluated the patients' hospital files and noted the demographic values and complications of HSCT. Statistical investigations were done with SPSS statistics 17.0 for windows and $\mathrm{p}<0.05$ has been accepted as significant.

Results: There was no difference between control and VOD groups as regard to age, sex, diagnosis, donor type, conditioning regimen, HSC source, and HLA typing. There was no difference between the groups according to homocysteine, lipoprotein a, protein $\mathrm{S}$ and $\mathrm{C}$ activity and antigen levels. We did not find any relation between the genetic variations of thrombophilia and VOD (Table 1). In VOD group there were 6 patients $(17.1 \%)$ with acute graft versus host disease (aGVHD) and in control group there were $7(15.9 \%)$ patients with aGVHD $(\mathrm{p}=0.046)$. Febrile episodes were more frequent in VOD group compared to the controls (respectively; $n=30,85.7 \%$ vs. $n=23,54.8 \%$, $\mathrm{p}=0.006$ ). 8-year overall survival was $\% 77.1$ in VOD group and $100 \%$ in control group $(\mathrm{p}=0.001)$. Disease free survival was also different between VOD and control groups (respectively; $74.3 \%$ vs. $97.3 \%, \mathrm{p}=0.001$ ).

Conclusions: In literature there are recent studies showing higher PAI-1 levels in patients with VOD. However, in our study we did not find any relationship between congenital thrombophilia factors and VOD. New studies with larger sample groups is necessary to better evaluate the association of congenital thrombophilia factors and VOD.

Disclosure: Nothing to declare

\section{P189 \\ Different strategies of chemotherapy-induced nausea and vomiting (CINV) prevention in hematological patients receiving an autologous hematopoietic stem cell transplantation: A single center experience}

Ilaria Cutini, Riccardo Boncompagni, Chiara Nozzoli, Antonella Gozzini ${ }^{1}$, Stefano Guidi ${ }^{I}$, Chiara Innocenti, Massimo Di Gioia ${ }^{1}$, Lorenzo Tofani ${ }^{1}$, Riccardo Saccardi ${ }^{1}$

${ }^{1}$ AOU Careggi, Florence, Italy
Background: Despite the improvements of pharmacological control, CINV still represents a major problem in patient undergoing hematopoietic stem cell transplantation (HSCT). We present here a comparison of two pharmacological strategies for preventing CINV in Multiple Myeloma (MM), Hodgkin (HL), and Non-Hodgkin Lymphoma (NHL) patients who received an autologous HSCT in our Institution.

Methods: From January 2015 to July 2018, we retrospectively analyzed 250 consecutive patients, median age 58 years (22-71yo), diagnosed with MM, HL, and NHL, who underwent an autologous HSCT following a Melphalan $200 \mathrm{mg} / \mathrm{sqm}$ and BEAM/FEAM condition regimens, respectively. The first 122 patients received CINV prophylaxis with palonosetron i.v and dexamethasone $8 \mathrm{mg}$ die (Regimen A), whilst the following 128 were administered with fosaprepitant iv, ondansetron iv and dexamethasone 8 $\mathrm{mg}$ die (Regimen B) Both CINV prophylaxis was administered the day of melphalan infusion (day -1 form transplant). Emesis breakthroughs were treated with alizapride and metoclopramide. Nausea and vomiting were assessed through the CTCAE 4.0 score system. Categorical variables were compared with Pearson Chi-square test.

Results: The overall incidence of nausea was 78\%, (55\% grade $1,41 \%$ grade 2 , and $4 \%$ grade 3 , respectively). In Regimen A was shown to be $80 \%$, (56\% grade1, $41 \%$ grade 2 , and $3 \%$ grade 3, respectively) while in Regimen B was $77 \%$ (54\% grade $1,41 \%$ grade 2 , and $5 \%$ grade 3 , respectively). Pearson Chi-square test did not show any differences between the 2 groups $(p=0.679)$. The overall observed vomit was $32 \%$ (83\% grade $1,16 \%$ grade 2 , and $1 \%$ grade 3 ). In Regimen A it was $(47 \%$ (84\% grade $14 \%$ grade 2 , and $2 \%$ grade 3 ), and $17 \%$ in Regimen B (77\% grade 1 and $23 \%$ grade 2). Conditioning regimens didn't' have any significant impact on either nausea or vomit.

Patientsyounger then median (58 yrs), were reported to have higher incidence of both nausea, $(\mathrm{p}=0.028)$ not related to CINV treatment, and vomit ( $40 \%$ vs $24 \%, \mathrm{p}=0.012$ ). In multivariate analysis the overall incidence of nausea is related to age (younger patients have higher probability to develop nausea (OR 2,282; $\mathrm{p}=0,024)$ whilst the higher incidence of vomit is related to: Regimen A (OR 3.958; $\mathrm{p}<$ $0,001)$, previously reported nausea (OR 4,506; $\mathrm{p}<0,001)$, and no smoking habits (OR 2,761; $\mathrm{p}=0,02)$.

Conclusions: Both regimens are equally effective for nausea control however Regimen B evidenced a better vomiting control. This finding is particularly relevant when the Center policies include an early discharge program, therefore improving both patient's Quality of Life and procedure cost-effectiveness.

Clinical Trial Registry: None

Disclosure: Nothing to declare 


\section{P190}

Prospective single center analysis of a professionallyfacilitated support group for patients after allogeneic hematopoietic cell transplantation

\section{Karsten Geeck ${ }^{1}$, Daniela Heidenreich ${ }^{1}$, Sebastian Kreil ${ }^{1}$, Wolf-Karsten Hofmann ${ }^{1}$, Stefan A. Klein ${ }^{1}$}

${ }^{1}$ Universitätsmedizin Mannheim, III. Med. Klinik, Mannheim, Germany

Background: Patients who underwent an allogeneic hematopoietic cell transplantation (HCT) are challenged by medical, psychological and social complications. Support groups might help HCT-survivors to cope with these challenges. However, the existing literature about post-HCT support groups is scarce. Moreover, data on professionallyfacilitated support groups do not exist. The aim of this project was (1) to establish a professionally-facilitated support group and (2) to assess the discussed topics.

Methods: From 11/2013 until 6/2017 all patients who received an allogeneic HCT at the adult stem cell transplantation program of the University clinic Mannheim were invited to participate in a professionally-facilitated support group. Additionally, spouses and life partners were invited. A theologian who is also a physician served as facilitator. He had no further function within the transplant team. The format of the group was unstructured without any rules regarding regular attendance. The facilitator did not provide topics or a curriculum. During the first year the group met every 14 days followed by a monthly schedule. From the fifth until the 39th meeting the attendance and the discussed topics were minuted by the facilitator. The content of the minutes was analysed by a combination of an inductive and a deductive approach. All participants provided their informed consent for the study.

Results: Altogether 23 patients (female: $n=10$; male: $\mathrm{n}=13$ ) and 10 spouses/life partners (female: $\mathrm{n}=9$; male: $n=1)$ participated. 13 patients $(57 \%)$ and 6 spouses $(60 \%)$ attended more than one meeting. Among those who participated in $\geq 2$ meetings the median time of participation was 16 months. The median count of participations was eight. $30 \%$ of the participants attended the meetings longer than one year, $9 \%$ longer than three years. There was no sex difference with respect to the frequency and the duration of participation. However, the frequency of participation decreased significantly the longer a participant was attending the meetings. During 35 group meetings the facilitator recorded 5138 thematically different contributions to the discussions divided in 37 distinct topics. These topics were grouped into 5 main categories [(a) medical topics, (b) private life and environment, (c) human relationships, (d) physical and mental condition and (e) the support group itself] and eight further categories [(1) compliance, (2) economic issues, (3) religion, (4) sexuality, (5) death and dying, (6) support and coping, (7) objectives and needs and (8) not otherwise specified issues] which could not be grouped in one of the main categories. The most frequent issues were medical topics $(34 \%)$, human relationships (16\%), physical and mental condition (15\%), private life and environment (14\%), financial issues (5\%), the support group itself (4\%), support and coping (4\%) and objectives and needs (4\%). Noteworthy, death and dying $(0.5 \%)$ were rare topics and sexuality was never mentioned.

Conclusions: To our knowledge, this is the first prospective and systematic analysis of a professionallyfacilitated support group for HCT-survivors. These data might help to establish support groups and to identify psychosocial needs of patients and targets for specific support.

Disclosure: Nothing to declare

\section{P191}

Endothelial dysfunction in multiple myeloma patients who underwent autologous hematopoietic stem cell transplant and developed engraftment syndrome

Ana Moreno-Castaño', Marta Palomo, Maribel DíazRicart $^{2,1}$, Ginés Escolar ${ }^{1}$, Sergi Torramadé-Moix', Julia Martínez-Sanchez ${ }^{2}$, Enric Carreras ${ }^{2}$, Montserrat Rovira ${ }^{1}$, María Suárez-Lledó ${ }^{1}$, Francesc Fernández-Avilés ${ }^{1}$, Gonzalo Gutiérrez-García ${ }^{1}$

${ }^{1}$ Hospital Clinic, IDIBAPS, Barcelona, Spain, ${ }^{2}$ Hospital Clínic de Barcelona, Josep Carreras Leukemia Research Institute (IJC), Barcelona, Spain

Background: Endothelial damage is associated with inflammatory complications that appear early after HSCT, such as sinusoidal obstruction syndrome or acute GVHD. Engraftment syndrome (ES) is an inflammatory condition diagnosed by Maiolino clinical score. Potentially, ES can exhibit high morbidity and mortality, especially after autologous-HSCT in multiple myeloma (MM) patients since the introduction of new drugs such proteasome inhibitors and immunomodulatory drugs (IMiDs).

The objective of the present study was to evaluate if ES is associated with endothelial dysfunction in patients with MM who underwent auto-HSCT.

Methods: We included six patients with MM who received induction treatment including new drugs and consolidated their response with an autologous-HSCT. We analysed comparatively the effect of incubating endothelial cells in vitro with serum samples from patients with ES vs. no ES. Serum samples were collected before (PRE), and after 5, 7, and 10 days from the transplant. An additional 
sample was collected at the ES onset and at the discharge day (no ES group). Endothelial cells (HMEC) in culture were exposed to media containing $20 \%$ of serum from each patient for $24 \mathrm{~h}$. Cell growth was controlled morphologically. Expression of the adhesion receptor ICAM-1 on the cell surface was analysed by immunofluorescence, and activation of the inflammation related p-38 MAPK signalling pathway was evaluated by SDS-PAGE and western blot.

Results: Exposure of HMEC monolayers to sera from patients who developed ES (onset day, $n=4$ ) resulted in an increased ICAM-1 expression on the cell surface, higher that the observed with sera from patients who did not develop ES (discharge day, $n=4)(26.4 \%$ of labelled area vs. $6.4 \%$, respectively). In addition, in experiments with sera from patients not developing ES, ICAM-1 expression on cells exposed to sera from day +10 was reduced with respect to the observed with sera from day +5 , probably due to the corticosteroid used as a prophylaxis in our centre. This reduction was not observed in ES patients. Regarding phosphorylation of p-38, it was significantly higher in cells exposed to sera from ES patients than in response to sera from patients who did not develop ES.

Conclusions: The increase in the expression of the adhesion receptor ICAM-1 on the surface and the intracellular activation of p38MAPK in endothelial cells exposed to sera from patients developing ES indicates the existence of endothelial activation in association with ES. Interestingly, the prophylaxis of ES with corticosteroid seems to be less effective in patients who developed ES than in patients who did not develop this complication. These results need to be validated in a higher number of patients and modifications in additional markers of endothelial dysfunction should be investigated.

Disclosure: Gonzalo Gutiérrez-García: Honoraria from Gilead. Grant from Jazz Pharmaceutical

The other authors do not have any disclosure to comment.

\section{P192}

association between uric acid levels before and after allogeneic haematopoetic stem cell transplant and transplant outcomes: A single centre experience

Anna Torrent ${ }^{1}$, Maria Jose Jimenez-Lorenzo ${ }^{1}$, Christelle Ferrá $^{1}$, Mireia Morgades ${ }^{1}$, Susana Vives ${ }^{I}$, Miriam Moreno $^{I}$, Montserrat Batlle ${ }^{I}$, Blanca Xicoy ${ }^{I}$, Juan Manuel Sancho ${ }^{I}$, Laura Abril ${ }^{1}$, Gladys Ibarra ${ }^{1}$, Albert Oriol $^{I}$, Marta Peña ${ }^{1}$, Marta Sitges ${ }^{1}$, Josep Maria Ribera ${ }^{1}$

${ }^{1}$ Institut Català d'Oncologia-Hospital Germans Trias i Pujol, Josep Carreras Leukemia Research Institute, Badalona, Spain
Background: Uric acid (UA) is a known endogenous danger signal which activates the NOD-like receptor protein (NLRP) 3 inflasome.UA is released from injured cells during conditioning in allogeneic stem cell transplantation (HSCT). A pre-clinical study has demonstrated that NLRP3 inflasome-mediated IL-1 production regulates graft-versushost disease (GVHD). The UA role in inflammation and GVHD is unclear. There are discordant reports in the literature about a potential protective role of UA on GVHD after a HSCT.

Methods: We performed a retrospective study to assess the association between serum UA levels pre- and postHSCT and transplant outcomes (overall survival [OS], cumulative incidence [CI] of grade II-IV acute GVHD [aGVHD], relapse and non-relapse mortality [NRM]) in 142 patients undergoing a $8 / 8$ matched related or unrelated donor allogeneic peripheral blood HSCT from January 2008 to December 2017. UA levels were collected before conditioning and on days $0,+7$ and +14 after HSCT.

\begin{tabular}{|c|c|c|}
\hline \multicolumn{2}{|l|}{ Characteristics } & \multirow{2}{*}{$\begin{array}{l}\mathrm{N}=142(\%) \\
68(48) / 18(12) 17(12) / \\
4(3) / 4(2) 12(8) / 5(4) / 7 \\
(5) 4(3) 3(2)\end{array}$} \\
\hline $\begin{array}{l}\text { Hematologic } \\
\text { disease }\end{array}$ & $\begin{array}{l}\text { AML/ALL MDS/CML/MF } \\
\text { NHL/HL/Chronic } \\
\text { lymphoproliferative disease } \\
\text { Myeloma Other }\end{array}$ & \\
\hline Conditioning & Myeloablative & $70(49)$ \\
\hline HSCT & MRD/MUD & $97(68) / 45(32)$ \\
\hline $\begin{array}{l}\text { Donor/ } \\
\text { Recipient sex } \\
\text { combination }\end{array}$ & $\begin{array}{l}\text { High risk (female Donor/male } \\
\text { Receptor) }\end{array}$ & $27(19)$ \\
\hline $\begin{array}{l}\text { GVHD } \\
\text { prophylaxis }\end{array}$ & $\begin{array}{l}\mathrm{CsA}+\mathrm{MTX} / \mathrm{CsA}+\mathrm{MMF} / \\
\mathrm{CsA}+\text { alemtuzumab } / \mathrm{CsA}+ \\
\text { prednisone / Other }\end{array}$ & $\begin{array}{l}120(85) / 8(6) / 6(4) / 6 \\
(4) / 2(1)\end{array}$ \\
\hline $\begin{array}{l}\text { Stage of } \\
\text { disease }\end{array}$ & Advanced & $44(31)$ \\
\hline
\end{tabular}

[[P192 Table] 1. Table 1]

Results: The characteristics of the 142 patients are shown in Table 1. Median age was 52 years (range 15-69), and 80 patients $(56 \%)$ were male. Twenty-seven patients $(19 \%)$ received low doses ATG as part of GVHD prophylaxis. Allopurinol was from the day before start of conditioning therapy until day 0 . The median levels of UA were $4,8 \mathrm{mg} /$ $\mathrm{dL}$ before conditioning, $2,85 \mathrm{mg} / \mathrm{dL}$ at day $0,3,1 \mathrm{mg} / \mathrm{dL}$ at day +7 and $3,2 \mathrm{mg} / \mathrm{dL}$ at day +14 . There was no impact between the UA levels and OS at any time of the HSCT. UA levels at day +7 were associated with a higher CI relapse at 5 years (34\% [95\% CI, 20-49\%] for UA level > $3,1 \mathrm{mg} / \mathrm{dL}$, and $17 \%$ [95\% CI, $8 \%-30 \%$ ] for UA level $\leq 3,1$ $\mathrm{mg} / \mathrm{dL}[\mathrm{p}=0,046])$. There was a trend for a higher CI of grade II-IV aGVHD for the subgroup of patients not treated with ATG with UA $<4,8 \mathrm{mg} / \mathrm{dL}$ ( $48 \%$ vs $30 \%$; $\mathrm{p}=0,083$ ) 
on day -8 and a higher NRM with $\mathrm{UA}<2,85$ on day 0 (50\% vs $30 \%$; $=0,080$ ).

Conclusions: In our study the UA levels showed no impact on OS, and only a tendency for CI of grades II-IV aGVHD grades II-IV and NRM for the subgroup of patients not treated with ATG. Surprisingly, high levels of UA at day +7 of HSCT were associated with a significant higher incidence of relapse.

Disclosure: DKMS Foundation, PI14/01971 (Instituto Carlos III) and SGR288 (GRC), Generalitat de Catalunya.

\section{P193}

early and late onset VOD prevalence and outcomes in a single centre retrospective study over 5 years: The King's BMT experience

Shreyans Gandhi, Simon Tetlow ${ }^{1}$, Varun Mehra ${ }^{1}$, Hugues deLavallade ${ }^{I}$, Austin Kulasekararaj ${ }^{l}$, Pramila Krishnamurthy ${ }^{1}$, Judith Marsh ${ }^{1}$, Sarah Ware ${ }^{1}$, Michelle Kenyon $^{I}$, Ghulam Mufti ${ }^{1}$, Victoria Potter ${ }^{I}$, A Pagliuca ${ }^{I}$

${ }^{1}$ King's College Hospital NHS Foundation Trust, London, United Kingdom,

Background: Veno-occlusive disease (VOD) is an early, uncommon but serious complication of stem cell transplantation (SCT) that is associated with high morbidity and mortality. Defibrotide is the only licensed treatment for VOD, and time to start of treatment (TsT) affects outcomes. Minor differences exist between the Seattle, Baltimore and classical EBMT (2016) criteria, which may trigger different start points for treatment. Late onset VOD ( $>21$ days) is less recognised and we hypothesize, may have worse outcomes with longer time to diagnosis, and more limited treatment options across different healthcare systems.

Methods: Electronic patient records from Sept.2013 Oct.2018 at King's BMT centre and pharmacy databases were reviewed, timepoint to clinical and bio-chemical manifestation of VOD, diagnosis, TsT, survival and longterm outcomes were analysed.

Results: 30 of the 532 patients(5.6\%) who underwent an allogeneic SCT, developed VOD, including 2 paediatric cases. None of the Autologous SCT patients developed VOD. The paediatric and Autologous SCT patients were not analysed any further.

28 adult patients (Male $=22 ; 78.5 \%$ ) developed VOD at a median age of 56 years(range 26-72), of whom 21 developed $<21$ days and 7 patients had late-onset VOD as per EBMT criteria(range 22-93 days). 24 cases classed as severe and 4 as moderate VOD.

10 patients received Defibrotide at diagnosis, 7 patients within 3 days, 5 patients between 4-7 days, and 6 patients received treatment after 7 days.
Overall mortality for this cohort was $50 \%(14 / 28) .12 / 21$ $(57.1 \%)$ of patients with early onset VOD and $2 / 7(28.5 \%)$ patients with late-onset VOD died. Of the 14 deaths, 10 died of liver failure and a further 2 patients had VOD as a likely contributing factor in their deaths. 1 patient died with subarachnoid haemorrhage and 1 with relapsed disease.

Patients that received Defibrotide after 7 days, 5/6 patients $(83.3 \%)$ died, as compared to $3 / 5(60 \%)$ for treatments between 4-7 days, 6/17(35.2\%) for treatments within 3 days. The lone surviving patient who received treatment after 7 days has severe chronic liver disease and it's complications.

Of the 21 patients who fit Seattle criteria for early-onset VOD, only 6 fit the Baltimore or EBMT criteria for classical VOD. 4 of these 6 patients met the Baltimore criteria later than the Seattle criteria were met(range $=2-9$ days).

Conclusions: VOD carries high morbidity and mortality, and beyond the known risk factors and with the caveat of limited numbers in this study, we strongly suspect this is further increased when time to definitive treatment with Defibrotide is delayed, particularly beyond 7 days.

Nearly a quarter of cases with VOD are late-onset as per classical EBMT criteria. However contrary to our hypothesis, their overall outcomes and mortality do not appear worse, with time to treatment again emerging as a strong predictive factor. Conditioning treatment related factors, which play a stronger role in endothelial dysfunction in the hepato-portal circulation, may not be as much at play, perhaps for late-onset disease.

Uniformity in the use of diagnostic criteria, and high degree of vigilance, even beyond 21 days, leading to early treatments may improve outcomes in VOD.

Disclosure: Nothing to declare

\section{P194}

Outcome of eculizumab treatment in stem cell transplantation - associated thrombotic microangiopathy in children: A single centre experience

David Bueno ${ }^{1}$, Yasmina Mozo ${ }^{1}$, Luisa Sisinni, Blanca Rosich $^{1}$, Pilar Nozal ${ }^{1}$, Alejandro Zarauza ${ }^{1}$, Antonio PérezMartínez $^{1}$

${ }^{1}$ La Paz University Hospital, Madrid, Spain

Background: HSCT-associated thrombotic microangiopathy (TA-TMA) affects $10-30 \%$ of patients receiving an allogenic SCT, with a high mortality up to $80-90 \%$ in severe cases. Endothelial injury mediated by complement activation has been atribuited a major role in the pathogenesis, and blockade of $\mathrm{C} 5$ with Eculizumab offers promising results. 
Methods: We present our experience with 6 pediatric cases of TA-TMA treated with Eculizumab. The diagnosis of TA-TMA was stablished attending to Jodele et al criteria. Clinical data were collected retrospectively from medical records.

Results: All cases were diagnosed between August 2016 and April 2018, with a median age of 11 years $(2.5-17)$ at time of diagnosis. Primary disease was acute leukemia in 2 cases (1 ALL and 1 AML), severe aplastic anemia in 3, and primary immunodeficiency in 1 . They received their first SCT in all cases, 3 from MUD and 3 from MMRD (CD45RA + depleted haploidentical grafts), with MAC regimen in 2 cases, and RIC in 4 cases. 3 of them received calcineurin inhibitors (cyclosporine) as GVHD prophylaxis. All patients developed aGVHD (grade 2 or higher in 3 cases). and 5 patients presented viral reactivation. Hypertension was present in 4 cases at TMA diagnosis, requiring 2 or more antihypertensive drugs in 3 of them. All patients had renal injury consisting of less-than-normal glomerular filtration rate (median of 41 (20-59)) and proteinuria, with urine protein-to-creatinine ratio higher $\tan 2 \mathrm{mg} / \mathrm{mg}$ in 2 cases (data not available in 2 patients). Serum haptoglobin was decreased in just 2 cases at diagnosis, and schistocytes were detected in 3 patients. Cutaneos signs were present in all cases, digestive symptoms in 2, neurological affection in 2 , and notoriously all of them developed polyserositis. C3 and $\mathrm{C} 4$ were normal in all cases, with sC5b9 higher than $244 \mathrm{ng} / \mathrm{mL}$ in 2 patients and lower in 1 (data not available in 3 cases). All patients received defibrotide as treatment, and 4 cases received also rituximab, associated to therapeutical plasma exchange in 3. All of them received Eculizumab, as first line in 2 cases (median of 40 days between diagnosis and Eculizumab start). Treatment was correctly monitorized with CH50 levels in 3 cases (not available quick enough in other 3). Median number of doses needed in induction therapy was 8 , and median interval between doses was 7 days. 2 patients required reduced interval and higher doses to maintain $\mathrm{CH} 50$ supressed. 2 patients did not respond, and died because of TMA. 4 patients had hematological response, with chronic renal injury in 3 of them and resolution of acute renal failure in 1 case. Nevertheless 1 patient responding to Eculizumab died because of TMA related complications, and 1 because of an invasive fungal infection. 2 patients are alive, with a median follow up of 6 months from treatment start.

Conclusions: Our experience supports promising results of Eculizumab based treatment for TA-TMA, highlighting the importance of an early treatment and a careful therapy monitoring by $\mathrm{CH} 50$ supression. Prospective studies are needed to achieve a better knowledge of this pathology and its treatment.

Disclosure: Nothing to declare

\section{P195}

AB0-mismatched allogeneic hematopoietic stem cell transplantation and associated complications

Yayra M Pichardo-Cepin ${ }^{1}$, Brenda Lizeth Acosta Maldonado ${ }^{\text {, Liliana M Rivera-Fong }}{ }^{1}$, Andrea I MilanSalvatierra ${ }^{1}$, Karen D. Pérez-Gómez ${ }^{I}$, Perla K AcostaMaldonado ${ }^{I}$, Luis M Valero-Saldaña ${ }^{l}$

${ }^{1}$ National Cancer Institute, México City, Mexico

Background: Approximately 40-50\% of Allogeneic Hematopoietic Stem Cell Transplant (Allo-HSCT) are made with some sort of $\mathrm{ABO}$ blood group system incompatibility.

An HSCT ABO donor-recipient incompatibility implies risks of complications during the process of infusion as acute hemolytic anemia ( $\mathrm{AH})$, delayed graft and other later complications due to the presence of isohemaglutinins (Pure Red Cell Aplasia or passenger lymphocyte syndrome). Also, ABO incompatibility could impact on graft versus host disease (GVHD) incidence, and could be associated with Not Relapse Mortality (NRM) and overall survival (OS).

There are not concluded evidence about the $\mathrm{ABO}$ incompatibility impact, so the aim of this study was to identify complications and response associated with $\mathrm{ABO}$ incompatibility in patients undergoing Allogeneic Hematopoietic Stem Cell Transplantation.

Methods: A retrospective study was performed on patients who receive an allo-HSCT between January 2014 and August 2018. Two groups were performed according to the presences or not of ABO incompatibility. Demographic and clinical information was collected from physical and electronic medical records, and information was analyzed in SPSS v 21

Results: Sixty-eight patients were enrolled in the study, $54 \%$ male, the median age was 34 years (19-61) with the following diagnoses: acute lymphoblastic leukemia $44 \%$, acute myeloblastic leukemia $26.5 \%$, granulocytic chronic leukemia $17.6 \%$, myelodysplastic syndrome $4.4 \%$, dendritic cell neoplasm $4.4 \%$, aplastic anemia $2.9 \%$. Ninety-one percent of the patients received a transplant from an identical HLA donor and $8.8 \%$ received a haploidentical transplant. Fifty-two patients $(76 \%)$ were ABOcompatibility (G1) and 16 patients (24\%) had ABOincompatibility (G2). None patient with ABOincompatibility received a haploidentical transplant.

The contrast between groups didn't show differences in fever, infections, bacterial isolation, presence and degree of acute or chronic GVHD and relapse of the disease. Graft failure was $6 \%(\mathrm{G} 1)$ vs $20 \%(\mathrm{G} 2)(\mathrm{p}=0.27)$, intermediate risk CMV serostatus $79 \%(\mathrm{G} 1)$ vs $87(\mathrm{G} 2)(\mathrm{p}=0.35)$. The most relevant characteristics and complications are described in 
table 1. Contrast analysis between G1 vs G2 showed that within the whole group there were 29 deaths (40\% vs $50 \%$ respectively) $(\mathrm{p}=0.69)$, the overall survival 1 -year was $74 \%$ vs $66 \%(\mathrm{p}=0.58)$ with a median of 29 vs 34 months respectively; mortality associated with relapse was $68 \%$ vs $87 \%$ respectively, and mortality related with transplantation was $35 \%$ vs $13 \%$ respectively.

\begin{tabular}{|c|c|c|c|}
\hline Characteristics & $\begin{array}{l}\mathrm{ABO} \\
\text { compatibility } \\
\mathrm{n}=52 \mathrm{n}(\%)\end{array}$ & $\begin{array}{l}\mathrm{ABO} \\
\text { incompatibility. } \\
\mathrm{n}=16 \mathrm{n}(\%)\end{array}$ & $\mathrm{p}$ \\
\hline \multicolumn{2}{|l|}{ Mismatch ABO Major/minor0 } & $8(50) / 8(50$ & - \\
\hline R Male/D Female & $11(21)$ & $8(50)$ & 0.13 \\
\hline Neutrophil recovery (days) & $14 \pm 5$ & $12 \pm 2$ & $0.041 *$ \\
\hline \multicolumn{4}{|c|}{ Transfusion requirement of erythrocyte concentrates } \\
\hline Before HSCT $>10$ & $18(35.3)$ & $2(14.3)$ & 0.23 \\
\hline After $\mathrm{HSCT}>10$ & $5(15)$ & $3(27)$ & 0.62 \\
\hline $\begin{array}{l}\text { Acute complications } \\
\text { (hemolytic anemia/ } \\
\text { delayed graft) }\end{array}$ & $0 / 1(2)$ & $1(6) / 1(6)$ & 0.12 \\
\hline $\begin{array}{l}\text { Acute complications (Pure } \\
\text { Red Cell Aplasia) }\end{array}$ & 0 & $1(6)$ & 0.23 \\
\hline $\begin{array}{l}\text { Chimerism day }+100 \text { : } \\
\text { incomplete }(<94 \%)\end{array}$ & $3(6)$ & $3(21)$ & 0.22 \\
\hline
\end{tabular}

[[P195 Table] 1. Table1. Relevant characteristics and complications.]

Conclusions: ABO incompatibility did not show association with complications related with the infusion, but there was a higher tendency of graft failure in the ABO incompatibility group. It has no statistical significance, but it is important to expand its study.

Disclosure: None declared

\section{P196}

Prevalence of metabolic syndrome following autologous stem cell transplantation for non-hodgkin lymphoma

\section{Lerryn Edghill, Naa Amua Quaye ${ }^{1}$, Sheridan Thompson', Wendy Ingram ${ }^{2}$, Emma Kempshall', Keith Wilson $^{1,2}$}

${ }^{1}$ Cardiff University School of Medicine, Cardiff, United Kingdom, ${ }^{2}$ University Hospital of Wales, Cardiff, United Kingdom

Background: Metabolic syndrome (MetS) is a wellrecognised late effect of transplantation which has been largely overlooked in the autologous transplant setting. This service evaluation aimed to explore the prevalence of MetS in patients with Non-Hodgkin Lymphoma (NHL) treated with autologous stem cell transplantation (ASCT) within the
South Wales Blood and Bone Marrow Transplant (SWBMT) Programme.

Methods: Retrospective data for 142 NHL patients who underwent ASCT between 1999 and 2017 was analysed. Patients were identified using the SWBMT database and data on MetS was collected using paper and electronic hospital records. Forty-eight patients were excluded due to loss of follow-up, inaccessible/incomplete records, or death. Cause of death was not determined. The NCEP-ATPIII definition of MetS was used. This requires $\geq 3$ of 5 criteria to be met. A BMI of $\geq 30 \mathrm{~kg} / \mathrm{m}^{2}$ and $\mathrm{HbA} 1 \mathrm{c}$ of $\geq 42 \mathrm{mmol} / \mathrm{L}$ were used to replace central obesity and impaired fasting glucose, respectively. Other criteria include triglycerides (TGs) $\geq 1.7 \mathrm{mmol} / \mathrm{L}$ or treatment, high density lipoprotein cholesterol (HDL-C) $<1.0 \mathrm{mmol} / \mathrm{L}$ (male), $<1.3 \mathrm{mmol} / \mathrm{L}$ (female) or treatment and blood pressure $>130 \mathrm{mmHg}$ systolic or $>85 \mathrm{mmHg}$ diastolic, or treatment.

Results: The prevalence of MetS in the cohort was 33\% $(n=31)$. Eighty-two percent of patients $(n=77)$ met one or more criterion for MetS. Twenty-seven percent $(n=25)$ fulfilled only one criterion, $23 \% \quad(n=22)$ fulfilled two criteria, $20 \% \quad(n=19)$ three criteria, 12\% $(n=11)$ four criteria, and $1 \%(\mathrm{n}=1)$ five criteria.

The greatest prevalence of MetS was in the $60+$ age group, accounting for 17 out of $31(55 \%)$ patients with MetS. Overall prevalence decreased with declining age (Table 1). The number of patients aged $<20$ years was too small to make any judgement on risk.

Raised triglycerides was the criterion most frequently met (61/94 patients), followed by hypertension (48), raised BMI (26), low HDL-C (23) and an increased HbA1c (15).

\begin{tabular}{lllll}
\hline $\begin{array}{l}\text { Age } \\
\begin{array}{l}\text { Category } \\
\text { (Years) }\end{array}\end{array}$ & $\begin{array}{l}\text { Total } \\
\text { of Patientswith MetS }\end{array}$ & $\begin{array}{l}\text { Number of } \\
\text { Patients }\end{array}$ & $\begin{array}{l}\text { Prevalence of } \\
\text { MetS per Age } \\
\text { Category }(\%)\end{array}$ & $\begin{array}{l}\text { Prevalence of } \\
\text { MetS per Cohort } \\
(\mathrm{n}=31)(\%)\end{array}$ \\
\hline $0-19$ & 2 & 1 & 50 & 3 \\
$20-39$ & 15 & 2 & 13 & 6 \\
$40-59$ & 49 & 11 & 22 & 35 \\
$\geq 60$ & 28 & 17 & 61 & 55 \\
\hline
\end{tabular}

[[P196 Table] 1. Table 1. Prevalence of Metabolic Syndrome (MetS) by Age]

Conclusions: The prevalence of MetS in our cohort $(33 \%)$ was higher than the estimated worldwide prevalence of $25 \%$, with the majority in the $60+$ age category. This is in keeping with other post-transplant studies, which show an increase in prevalence of MetS after transplantation. Moreover, the overall prevalence of MetS was greater in the older population, which could be associated with the cumulative effect of ageing on the decline of normal metabolic homeostatic mechanisms. 
This study supports the need for late effects screening in survivors of autologous transplantation for NHL. Early recognition of aberrant parameters will allow for timely and targeted lifestyle and/or pharmacological treatment to help prevent diabetes and cardiovascular disease.

Clinical Trial Registry: None

Disclosure: Nothing to declare

\section{P197}

Incidence and prognostic significance of acute renal failure in myeloablative and non-myeloablative allogeneic stem cell transplant. single center experience

Marta Sitges ${ }^{1}$, Christelle Ferrà ${ }^{1}$, Jordi Soler ${ }^{2}$, Mireia Morgades', María José Jimenez ${ }^{1}$, Inés Hernández ${ }^{1}$, Anna Torrent $^{I}$, Marta Peña ${ }^{1}$, Georgina Gener ${ }^{1}$, Mireia Franch ${ }^{1}$, Mireia Santos ${ }^{1}$, Eloi Cañamero ${ }^{1}$, Martina Comes ${ }^{1}$, Andrea Espasa ${ }^{1}$, Montserrat Batlle ${ }^{1}$, Miriam Moreno ${ }^{1}$, Susana Vives ${ }^{1}$, Juan Manuel Sancho ${ }^{1}$, Blanca Xicoy ${ }^{\prime}$, Albert Oriol', Laura Abril' ${ }^{1}$, Gladys Ibarra ${ }^{1}$, Jose María Ribera $^{1}$

${ }^{1}$ Institut Català d'Oncologia-Hospital Germans Trias i Pujol, Josep Carreras Research Institute, Universitat Autònoma de Barcelona, Badalona, Spain, ${ }^{2}$ Hospital Germans Trias i Pujol, Badalona, Spain

Background: Acute renal failure (ARF) is a frequent complication in the early post-allogeneic hematopoietic stem cell transplant (alloHSCT) period with either myeloablative (MA) or non-myeloablative (NMA) conditioning regimens. The aim of this study was to compare the incidence of ARF in both types of HSCT and to evaluate its impact on overall survival (OS) and non-relapse mortality (NRM).

Methods: All alloSCT performed in one center between 2010 and 2018 were included in this study. AlloHSCT from cord blood and from haploidentical donors were excluded. The renal function and the incidence of the main complications after alloSCT from day 0 to day +90 were evaluated. ARF was defined according to KDIGO (Kidney Disease Improving Global Outcomes) classification; the relative increase of serum creatinine levels was considered a marker of kidney damage.

Results: Seventy-seven patients received a MA alloHSCT and 72 a NMA alloHSCT. Recipients of NMA alloHSCT had a higher median age (61 years [range: 18-69] vs. 41 years [15-55], $\mathrm{p}<0.001)$, higher frequency of arterial hypertension $(29 \%$ vs. $6 \%, \mathrm{p}<0.001)$ and showed most frequently active disease at alloSCT (42\% vs. $18 \%$, $\mathrm{p}=0.002)$. In both groups the most frequent graft-versushost disease (GVHD) prophylaxis regimen was cyclosporine $\mathrm{A}$ and methotrexate. The median follow-up time was 2.3 years for the NMA group and 3.6 years for the MA group. Patients from the MA group had higher incidence of grade 3-4 mucositis $(60 \%$ vs. $22 \%$, p < 0.001$)$ and acute GVHD of any grade $(70 \%$ vs. $53 \%, \mathrm{p}=0.029)$ than patients from the NMA alloHSCT. The incidence of ARF was similar in both groups (72\% in NMA and $71 \%$ in MA). In the NMA group arterial hypertension (HR 2.05, $\mathrm{p}=0.018$ ), obesity (HR 4.16, $\mathrm{p}<0.001)$ and prior pneumonia $(\mathrm{HR} 3.19,<0.001)$ were predisposing factors for ARF by multivariate analysis, whereas any factor was identified in the MA group. ARF had no impact on 2-year OS in both groups (28\% vs. $35 \%$ $\mathrm{p}=0.406$ for the NMA group and $45 \%$ vs. $40 \% \mathrm{p}=0.623$ for the MA group). However, worse OS were observed in patients with grade 2-3 ARF in the NMA group (18\% vs. $43 \%, \mathrm{p}=0.048)$ and in patients with grade $3 \mathrm{ARF}$ in the MA group ( $25 \%$ vs. $59 \%, \mathrm{p}=0.011)$. In turn, ARF had no influence on NRM in the MA group but was associated with a trend for higher NRM in the NMA group (59\% vs. $35 \%$, $\mathrm{p}=0.084$ ).

Conclusions: ARF is a frequent complication in patients receiving alloHSCT irrespective of the intensity of the conditioning regimen. Moderate and severe ARF had negative impact on OS.

Disclosure: Supported by grants from: Asociación Española Contra el Cáncer, AECC (GC16173697BIGA), Instituto Carlos III (PI14/01971 FI), 2017-SGR288 (GRC), CERCA Program from Generalitat de Catalunya, and "La Caixa" Foundation.

\section{P198}

Treatment and risk factors of hepatic veno-occlusive disease after pediatric hematopoietic stem cell transplantation: A single-center experience

\section{Barbaros Sahin Karagün', Ilgen Sasmaz, , Ali Bülent Antmen ${ }^{1}$}

${ }^{1}$ Acibadem University, Pediatric Hematology and Oncology, Istanbul, Turkey, ${ }^{2}$ Cukurova University, Pediatric Hematology and Oncology, Adana, Turkey

Background: Defibrotide emerged as a promising treatment option for hepatic veno-occlusive disease, a significant cause of mortality in recipients of HSCT. As VOD diagnosis is quite difficult even with the recently introduced EBMT 2017 criteria, studies which report treatment outcomes and response to prophylaxis are required. Our aim was to evaluate the efficacy of defibrotide prophylaxis in HSCT recipients at our center.

Methods: A total of 236 transplants in 210 patients from January 2013 to July 2018 were included in this study. All patients had factors that increased the risk of VOD and all received $25 \mathrm{mg} / \mathrm{kg} /$ day prophylaxis. Patients' coagulation, 
renal and liver function test were monitored daily and all clinical findings and complaints were recorded. Diagnoses were made via the EBMT 2017 VOD criteria and patients who developed VOD received treatment with increased DF dose $(40 \mathrm{mg} / \mathrm{kg} / \mathrm{day})$ and supportive interventions. After complete remission of VOD findings, patients were returned to the prophylaxis dose. Close follow-up of patients was performed until 100 days.

Results: In total, 17 patients developed VOD (7.2\%), none of the cases were severe (13 mild, 4 moderate). Median age was 8.5 (1-22) years and the most common clinical findings were weight increase, hepatomegaly, right upper quadrant pain and ascites development. In those with VOD, treatment with $40 \mathrm{mg} / \mathrm{kg} /$ day $\mathrm{DF}$ was initiated and average duration of treatment with this dosage was 7.4 (511) days. No adverse events were reported in any of the patients.

Conclusions: Our findings are consistent with previous studies on this topic, and we believe that the use of DF as a prophylactic agent for VOD is beneficial for pediatric patients with risk factors.

Disclosure: The authors report no conflicts of interest in this work.

\section{P199}

\section{Donor-recipient major ABO-incompatibility might impact on the allo-HSCT outcome}

Carmen González ${ }^{1}$, Montserrat Lozano ${ }^{1}$, Nerea Rodríguez, $^{1}$, Lourdes Aguirre ${ }^{I}$, Jose Javier Ferreiro ${ }^{I}$, Clara Lombardi, Laida Ondarra ${ }^{1}$, María José Pizarro ${ }^{1}$, María Araiz, Carlos Vallejo ${ }^{1}$

${ }^{1}$ University Hospital Donostia, Donostia, Spain,

Background: Several factors might influence outcome of allo-HSCT. Analysis of the impact of donor-receptor blood group-incompatibility have been performed in different series not always showing the same results. As a consequence, its clinical impact remains controversial. Minormismatch is characterized by the ability of donor B lymphocytes to produce anti-recipient antibodies. In majormismatch cases, antibodies against donor antigens are present in the recipient.

Methods: 343 pts underwent allo-HSCT between May 2011 and August 2018 in our center. Median age was 52 years (range: $7-69) .193$ pts were male (56.3\%) and 150 female $(43.7 \%)$. Baseline diseases were: 138 AML, 76 LPD, 44 MDS, 41 ALL, 21 MPD, 16 MM, and 7 BMF. Donor was unrelated in 191, and related in 152 cases (including 36 haplo-identical). Donor-recipient ABO compatibility was as follows: $69(20.1 \%)$ majormismatched (including 11 bidirectional), and 274
(79.9\%) nonmajor-mismatched (including 68 minormismatched and 206 matched). Donor-recipient $\mathrm{Rh}$ compatibility was as follows: $50(15.6 \%)$ major-mismatched, and 293 (84.4\%) nonmajor-mismatched (including 34 minor-mismatched and 259 matched). The impact of donor-recipient $\mathrm{ABO}$ and $\mathrm{Rh}$ compatibility on transfusion needs (PRBC and platelet concentrates) and survival by day +100 was analyzed.

Results: For the global series the median number transfusions by day +100 was: $4(0-81)$ PRBC and 4 (092) platelets concentrates. Day +100 overall mortality was 9.3\%. Rh-incompatible and nonmajor $\mathrm{ABO}$ incompatible cases showed no different results. However, major ABOmismatched cases needed more PRBC transfusions (median: 6; range: 0-49) and more platelet transfusions (median: 7 ; range: $0-60)$, and had higher day +100 mortality (18.8\%) $(\mathrm{p}<0.05)$ (see table).

Conclusions: Our analysis showed: 1) Donor-recipient Rh-incompatibility, as well as minor ABOincompatibility had no impact on PRBC and platelet concentrates transfusion needs nor on 100-day mortality;

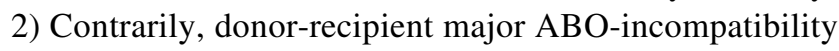
had a significant adverse impact on PRBC and platelet concentrates transfusion needs and 100-day mortality. 3) Donor-recipient Rh-incompatibility and minor ABOincompatibility.might be considered of marginal importance at the time to choose a potential donor. 4) Donorrecipient major ABO-incompatibility should probably be a factor to be considered, along with other features, to choose the best donor

\begin{tabular}{|c|c|c|c|c|}
\hline & $\mathrm{N}$ & $\begin{array}{l}\text { PRBC } \\
\text { (media } \\
\text { [range]) }\end{array}$ & $\begin{array}{l}\text { Platelets (median } \\
\text { [range]) }\end{array}$ & $\begin{array}{l}\text { 100-day } \\
\text { mortality }\end{array}$ \\
\hline Global & 343 & $4[0-81]$ & $4[0-92]$ & $9,30 \%$ \\
\hline \multicolumn{5}{|c|}{ Donor-recipient major } \\
\hline \multicolumn{5}{|c|}{ Rh-incompatibility } \\
\hline$-\mathrm{No}$ & $\begin{array}{l}293 \\
(85,4 \%)\end{array}$ & $4[0-81]$ & $4[0-92]$ & $9,60 \%$ \\
\hline -Yes & $\begin{array}{l}50 \\
(14,6 \%)\end{array}$ & $4[0-81]$ & $4[0-92]$ & $8 \%$ \\
\hline \multicolumn{5}{|c|}{ Donor-recipient major } \\
\hline \multicolumn{5}{|c|}{ ABO-incompatibility } \\
\hline$-\mathrm{No}$ & $\begin{array}{l}274 \\
(79,9 \%)\end{array}$ & $3[0-81]$ & $4[0-92]$ & $6,90 \%$ \\
\hline -Yes & $\begin{array}{l}69 \\
(20,1 \%)\end{array}$ & $6[0-49]$ & $7[0-60]$ & $18,80 \%$ \\
\hline
\end{tabular}

[[P199 Table] 1. Day +100 transfusion needs and overall mortality.]

Disclosure: "Nothing to declare" 


\section{P200}

Incidence for arterial hypertension in adults following haematopoietic stem cell transplantation

Claudia Lucia Sossa Melo ${ }^{1,2}$, Claudia Marcela Chalela ${ }^{1}$, Sara Ines Jimenez ${ }^{1,2}$, Luis Antonio Salazar ${ }^{1,2}$, Maria Luna-Gonzalez ${ }^{1}$, Manuel Rosales ${ }^{2}$, Manuel Ardila-Báez, ${ }^{1}$, Sergio Serrano ${ }^{1}$, Katherine Espinosa ${ }^{2}$, Julián Baez, David Leonardo Reyes ${ }^{2}$, Angela María Peña ${ }^{1,2}$

${ }^{1}$ Universidad Autónoma de Bucaramanga, Bucaramangaga, Colombia, ${ }^{2}$ Clinica FOSCAL, Bucaramanga, Colombia

Background: Survivors of haematopoietic stem cell transplantation (HSCT) are at significant risk of developing treatment-related complications, including cardiovascular risk factors such as arterial hypertension, that could eventually lead to cardiovascular disease. The aim of this study is to evaluate the incidence and risk factors of hypertension following HSCT in a Colombian population.

Methods: A retrospective cohort study was conducted to assess the incidence and risk factors of hypertension in 220 consecutive adult HSCT recipients who underwent transplantation between 2009 and 2017 at a tertiary referral center in Colombia, South America. Blood pressure data, from two different measures, were collected at 7 time points: day of mobilization for autologous HSCT and day 0 before infusion for allogeneic transplantation, day 7, and months 1, 3, 6 and 12 post-transplantation. Hypertension was defined as having a systolic blood pressure $>=140 \mathrm{mmHg}$ and/or a diastolic blood pressure $>=90$ $\mathrm{mmHg}$. Patients with history of arterial hypertension were excluded.

Results: One hundred and seventy-five patients were included, with a mean age of 44 years (range 15-67). Ninety-one patients (52\%) were male. One hundred and sixteen patients $(66.3 \%)$ underwent autologous HSCT and 59 (33.7\%) allogeneic HSCT. The most common indication for HSCT was acute leukemia (26.3\%), followed by nonHodgkin lymphoma (23.4\%) and multiple myeloma (22.9\%). Twelve patients $(6.9 \%)$ had medical history of type 2 diabetes mellitus (DM), 11 (6.3\%) dyslipidemia, 24 (13.7\%) alcohol consumption, and $25(14.3 \%)$ tobacco smoking. Only two of the patients with history of tobacco smoking were active smokers at time of transplantation.

Twenty-four patients $(13.7 \%)$ had developed hypertension by the end of the first year post-HSCT follow-up. Two patients $(8.3 \%)$ had systolic and diastolic, $12(50 \%)$ had only systolic, and $10(41.7 \%)$ had only diastolic hypertension. Only one patient was hypertensive at more than two time points. The incidences of hypertension at each time point were $2.3 \%$ on day 7 post-HSCT, $6.9 \%$ at first month, $9.8 \%$ at three months, $12.1 \%$ at 6 months, and $13.8 \%$ at one-year post-transplantation. Allogeneic HSCT $(\mathrm{P}<0.01)$, therapy with calcineurin inhibitors $(\mathrm{P}<0.01)$, pre-HSCT fasting glucose levels $(\mathrm{P}<0.05)$, acute GvHD $(\mathrm{P}<0.05)$, chronic GvHD $(\mathrm{P}<0.05)$, and media of diastolic blood pressure $(\mathrm{P}<0.05)$ were significantly associated with the development of arterial hypertension. However, age, history of type $2 \mathrm{DM}$, history of tobacco consumption, volume of infusion, prophylactic treatment for GvHD with mycophenolate, chronic GvHD, serum creatinine level on day of HSCT, and being overweight or obese at time of transplantation were not significantly associated with the development of hypertension.

Conclusions: Arterial hypertension is a fairly common complication in HSCT recipients. Similar to findings reported in previous studies, association between allogeneic stem cell transplantation, therapy with calcineurin inhibitors, and acute and chronic GvHD, and post-HSCT hypertension was found in the present cohort. Further studies are needed to assess the link between HSCT and developing long-term cardiovascular complications.

Disclosure: Nothing to declare

\section{P201}

Tramadol-based pain management of oral and esophageal mucositis in pediatric HSCT recipients

Ekaterina Goncharova ${ }^{1,2}$, Portniagin Ivan ${ }^{3}$, Ilya Kazantsev ${ }^{3}$, Asmik Gevorgian ${ }^{3}$, Maxim Bogomolny ${ }^{3}$, Maxim Kucher ${ }^{3}$, Lyudmila Zubarovskaya ${ }^{3}$, Boris Afanasyev $^{3}$

${ }^{1}$ Pavlov First Saint Petersburg State Medical University/ Raisa Gorbacheva Memorial Institute of Children's Oncology, St. Petersburg, Russian Federation, ${ }^{2}$ Pavlov First Saint Petersburg State Medical University, Valdman Institute of Pharmacology, St. Petersburg, Russian Federation, ${ }^{3}$ Pavlov First Saint Petersburg State Medical University/Raisa Gorbacheva Memorial Institute of Children's Oncology, St. Petersburg, Russian Federation

Background: Mucositis is one of the most common early HSCT complications seen in about $70 \%$ transplant recipients with $20 \%$ of patients developing Gr III-IV mucositis. Mucositis is characterized by painful gastrointestinal mucosa lesions impairing the solid and liquid foods intake and increased risk of infections, bleeding, and intestinal paresis. Thus, it greatly decreases the quality of life of a transplant recipient. According to WHO recommendations, the moderate pain control in pediatric patient is based on the use of low-dose morphine. However, there are some factors such as genetic polymorphisms causing variable morphine pharmacokinetics in children, side effects, and social factors (caregivers' general unwillingness to use narcotic 
analgesics), which cause the need for alternative pain relief options in pediatric practice. Tramadol, which has both opioid and non-opioid mechanisms of action, may be a feasible option in mild to moderate pain. It may be delivered via patient-controlled analgesia (PCA), although there is no consensus on its optimal parameters in pediatric practice.

Methods: A total of 69 pediatric patients with a median age of 8 (range 2 to 18 ) years receiving an autologous or allogeneic HSCT in our clinic as part of the treatment regimen for solid tumor $(n=40)$, leukemia $(n=24)$, acquired aplastic anemia $(n=3)$ or inherited condition $(n=2)$ were included. Conditioning regimens were myeloablative (MAC) in 54 and reduced-intensity (RIC) in 15 patients. All patients had oral and/or esophageal mucositis accompanied by moderate pain. The pain severity was assessed using the scales corresponding to patient's age and varied from 3 to 6 points. The pain control was based on intravenous tramadol administration using patientcontrolled analgesia (PCA) approach. The following PCA parameters were used: loading dose of $0.5 \mathrm{mg} / \mathrm{kg}$ (not exceeding $25 \mathrm{mg}$ ), basal infusion rate of $0.25 \mathrm{mg} / \mathrm{kg}$ (not exceeding $12.5 \mathrm{mg}$ ), a bolus of $0.25 \mathrm{mg} / \mathrm{kg}$ (not exceeding $12.5 \mathrm{mg}$ ), lockout interval of $25 \mathrm{~min}$. The maximal daily dose was $8 \mathrm{mg} / \mathrm{kg} / \mathrm{day}$. The pain control was considered adequate if a patient was satisfied or the basic and breakthrough pain score values were not higher than 3 and, accordingly. In case of inadequate pain control NSAIDs were added. Non-responders were switched to morphine. All patients were divided into 2 groups based on conditioning regimen intensity.

Results: As a whole, $46 \%$ of patients did not require pain control measures escalation. The tramadol pain control rate was slightly higher for RIC $(n=9,60 \%)$ compared to MAC $(\mathrm{n}=23,47 \%)$ recipients. In most cases the inadequate pain control was due to progressive mucosal lesions. The PCA regimen used was characterized by very few complications. Drowsiness was observed in $4(7 \%)$ of patients, in all cases the patients also had anemia. There was only 1 (7\%) patients with severe nausea requiring switching to morphine.

Conclusions: Tramadol is an effective pain control option in transplant recipients with mild to moderate pain due to oral and esophageal mucositis without progressive mucosal lesions. The PCA allows achieving a very low complication rate. Therefore, this option may be considered for both MAC and RIC recipients.

Disclosure: No

\section{P202}

Immune reconstitution of lymphocyte subsets after allogenic stem cell transplant (SCT) and vaccination
Gala Vega ${ }^{I}$, Agustín Penedo ${ }^{I}$, Sara Martin ${ }^{I}$, Ines Martinez-Alfonzo ${ }^{I}$, Jose Luis López-Lorenzo ${ }^{1}$, Cristina Serrano $^{1}$

${ }^{1}$ Hospital Universitario Fundación Jiménez Díaz, Hematology, Madrid, Spain

Background: Infectious diseases are a major cause of morbidity and mortality after allogenic stem cell transplant (SCT).

Vaccines constitute an effective strategy to prevent infections but the optimal timing to start vaccinating is not well stablished.

In order to individualize the early vaccination schedule, we studied the lymphocyte subsets involved in generating enough response to produce protective serological levels.

Methods: We studied retrospectively 20 patients that had undergone allogenic SCT at our hospital.

Patient distribution - Age range: 21-68 years-old; diagnosis: acute leukaemia/myelodysplastic syndrome/ chronic myeloid leukemia (16 patients), lymphoma (4 patients).

Analytic parameters: TCD4+, TCD8,$+ \mathrm{NK}$, total B and functional B lymphocyte subsets (naïve $\operatorname{IgD}+\mathrm{CD} 27-$, memory $\operatorname{IgD}+\mathrm{CD} 27+$ and IgD-CD27+, and effectors $\mathrm{CD} 27++\mathrm{CD} 38++)$. Immunoglobulin levels (IgG, IgA, $\operatorname{IgM}$ ) and specific IgG for pneumococcus, tetanus, HBV, chickenpox, measles, rubella and mumps.

Clinical parameters were collected from medical records.

Results: We distributed patients in two groups, based on the timing of lymphocyte analyses:

- Less than 12 months since SCT (5 patients)

No patient showed complete immune reconstitution, although 2 had enough $\mathrm{T}$ and functional B lymphocytes to generate response to vaccination. In these patients, vaccination for pneumococcus was completed and they generated sufficient protection antibody levels, despite being under immunosuppressive treatment.

- More than 12 months since SCT (15 patients)

Before the beginning of vaccination, we collected specific antibodies of 7 patients.

We compared the serological status before and after SCT and observed that protection against tetanus was the most frequently preserved (6 patients) and HBV the least frequent (2 patients).

Other than one patient treated with alemtuzumab, all patients in this group had minimum absolute count of TCD4 $+(>200$ cells/microl), TCD8+ (>200 cells/microl), NK ( $>100$ cells/microl) and B cells ( $>100$ cells/microl).

We also observed presence of B effector and B memory cells, with predominance of IgD-CD27+ memory cells. Immunoglobulin levels were within the normal range. 
In this group, we registered vaccination in 13 patients. All of them were vaccinated against flu, and 11 against pneumococcus and HBV. The rest of vaccines administered were heterogeneous in type and timing.

6 patients were under immunosuppressive treatment at the time of vaccination and were able to generate enough specific antibodies for pneumococcus.

\section{Conclusions:}

Immune reconstitution was not completed 12 months after SCT, although minimal immunological reconstitution was observed

TCD4+ and no-switching memory B lymphocytes were the last ones to reach minimum normal values according to patient age.

Some patients maintain serological protection after allogenic SCT.

Immunoglobulin levels were normal, suggesting no need for immunoglobulin administration to prevent infections. Flu, pneumococcus and HBV vaccines were the most frequently administered.

Pneumococcus vaccination generated a much larger serological response than HBV. This seroconversion occurred in patients under immunosuppressive treatment. The analysis of lymphocyte T, NK, B total and B functional subsets could be useful when programming an early vaccination schedule after SCT.

Completion of the vaccination schedule was heterogeneous despite giving specific indications. Therefore a more rigorous supervision of the process may be required.

Disclosure: No conflict of interests

\section{P203}

Metabolic syndrome is not uncommon complication post allogeneic stem cell transplantation: A single center experience

\section{Panayotis Kaloyannidis ${ }^{1}$, Eshrak Al Shaibani', Deia Awami $^{1}$, Solaf Kanfar ${ }^{1}$, Khalid Bakhit ${ }^{1}$, Ashraf Suhebeh ${ }^{1}$, Belal Blowi', Ahmed Bahrani, Rabab Attas', Hani Al Hashmi', Khalid Al Anezi ${ }^{1}$}

${ }^{1}$ King Fahad Specialist Hospital, Dammam, Saudi Arabia

Background: The significant advances that have been achieved in the allogeneic transplantation (alloHCT) field, have resulted in better post-transplant outcome and therefore complications other than the Graft vs. Host disease (GvHD) or disease recurrence become increasingly important. The post transplant metabolic syndrome (PT-MS), which caused by several factors (i.e. immunosuppressive agents, chemo-radiotherapy, anti-viral, and biologic therapies) is a well known post transplant complication in pediatric allografted long-term survivors however, only few studies have evaluated the prevalence of the PT-MS in adults. In this retrospective study, we sought to evaluate the incidence, the risk factors and the impact of the PT-MS on the alloSCT outcome.

Methods: Since 2011, 42 patients (25 males and 17 females) with adequate clinical and laboratory data and a minimum follow-up of 6 months were included in the study. Their median age was 35.5 (17-62) years and after a myeloablative $(n=28)$ or a reduced intensity $(n=14)$ regimen they received either mobilized peripheral blood stem cells $(n=34)$ or marrow graft $(n=8)$, originated from full-matched siblings $(n=35)$ or haploidentical donors $(n=7)$. Calcineurin inhibitors plus either short-term Methotrexate or Mycophenolate Mofetil were given as GvHD prophylaxis. The diagnosis of PT-MS was based on the NCEP-ATPIII criteria; for patients with unknown data for abdominal circumference the body mass index (BMI) $\geq 25 \mathrm{~kg} / \mathrm{m}^{2}$ was consider as a criterion for PT-MS diagnosis. The independent T-test, logistic regression analysis and logrank tests were used for the statistical analysis.

Results: Twenty (47.6\%) patients (12 males, 8 females) assessed to have PT-MS within the first 6 months following the allograft. Seventeen diagnosed after the $1^{\text {st }}$ trimester post alloSCT and additional 3 patients after $2^{\text {nd }}$ trimester. Sixteen out of 20 patients had elevated glucose and BMI $>25 \mathrm{~kg} / \mathrm{m}^{2}, 13 / 20$ elevated triglycerides levels, 12/20 low HDL levels and 10/20 hypertension. Four (20\%) had already known history of MS before alloSCT (for 10 patients no data were available for MS diagnosis before alloSCT). Interestingly, for $7 / 20(35 \%)$ patients who had diagnosed with PT-MS either in the $1^{\text {st }}$ or in the $2^{\text {nd }}$ trimester the syndrome was reversible and did not fulfill the criteria for PT-MS beyond 6 months post alloSCT. Patients' gender, age, BMI, the type of conditioning regimen and GvHD co-existence evaluated as potential predisposing factors for PT-MS diagnosis. In univariate and multivariate analysis only the: $\mathrm{BMI}>25 \mathrm{~kg} / \mathrm{m}^{2}$ and age $>35$ years were detected as significant risk factors $(\mathrm{p}<0.03)$. The PT-MS did not affected negatively the survival or the NRM incidence post alloSCT

Conclusions: In our study, in agreement with other publications, we demonstrated that the PT-MS is not an uncommon complication post in the early post transplant period however, for a significant number of patients the syndrome was a reversible. For patients with high risk features $\left(\mathrm{BMI}>25 \mathrm{~kg} / \mathrm{m}^{2}\right.$, age $>35$ years, known history of diabetes-mellitus, dyslipidemia, hypertension) apart of close monitoring, specific diet and encouragement for adequate exercise might help to reduce the incidence and the severity of PT-MS. Nevertheless, prospective and well design trials 
are warranted to determine the accurate incidence, severity and the impact of PT-MS on the alloSCT outcome.

Disclosure: No conflict ofinterest

\section{P204}

Experience of a single center in the humanization of the hospitalization process: Technology and team training impact on the QOL of the patient and family

\section{Maria Claudia Moreira ${ }^{I}$, Marcia Rejane ${ }^{I}$, Marcia Garnica $^{I}$, Andrea Ribeiro ${ }^{I}$, Paulo Cesar Dias ${ }^{I}$, Ilza Fellows $^{l}$}

${ }^{1} \mathrm{CHN}$, Niteroi, Brazil

Background: Hematopoietic stem cell transplantation (HSCT) is one of the most aggressive therapeutic modalities of internal medicine, making it a highly stressful experience for the patient and his family. The duration of hospitalization can be prolonged by several intercurrences, frequently generating anxiety in the patient and their caregiver, which may lead to confinement and reactive depression. Interventions in the hospital environment, in addition to the continuous training of the multidisciplinary team, can have a positive impact in this process with improvement in the process of discharge and quality of life of the patient and his / her family.

Methods: The objective of this research was to evaluate the impact of a reformulation in the unit, completed in May 2018, which modified the facilities with availability of hermetic balconies in each room, with a view of an internal garden. There was also the addition of a screen in the corridor of the floor with images - technology known as videoowall, interconnected to motion sensors (kinects) that allow interaction between patients and families, besides facilitating physiotherapy and physical exercise. There was re-training of the multidisciplinary team with emphasis on the practice of humanization. The methodology consisted in the application of questionnaires of satisfaction to patients and their families during the period of hospitalization in a bone marrow transplant unit in the third quarter of 2018 . The items evaluated ranged from the quality of the information provided by the medical team and nursing, to the cordiality and agility with which the patient and his patient were treated by the global team. The results were compared with a similar period of the same unit in the previous year and with the indices collected simultaneously in another unit of the same hospital (cardio-intensive).

Results: Overall and segmental satisfaction scores in the various items surveyed were higher when compared to the previous period of the same unit and were also higher in those obtained in a high complexity unit of the same hospital, composed of patients submitted to mental and psychological stress similar to onco- hematologicos.A reports of "free speech" were also obtained anonymously, in order to guarantee the authenticity and free expression of the subjects analyzed.

Conclusions: The results obtained allowed the validation of the technical and professional team initiatives, bringing indicators that will allow better monitoring and support of these patients and their relatives in this difficult time of treatment. They served as an initial tool in the continuous process of humanization and stimulated the multidisciplinary team to continuously improve this process.

Disclosure: No disclosures

\section{P205}

Bortezomib: Successful treatment of refractory pure red cell aplasia after $\mathrm{ABO}$-incompatible stem cell transplantation

\section{Stefan Lukic ${ }^{1}$, Helge Menzel ${ }^{1}$, Janka Benk ${ }^{1}$, Xenia} Sitskaya ${ }^{I}$, Petra Drewniock ${ }^{I}$, Nadezda Basara ${ }^{I}$

${ }^{1}$ St Franziskus Hospital, Akademical Hospital of UKSH, Internal Medicine, Flensburg, Germany

Background: Pure red cell anemia (PRCA) is a rare complication of ABO-incopatible hematopoetic stem cell transplantation characterized by anemia, reticulocytopenia and absence of erythroid precursors in patient's bone marrow. Most patients with PRCA resolve spontaneously within months, however a small number of patients requires continued red blood cell (RBC) transfusions. The treatment of this complication is difficult and not standardized. Different approaches has been used such as Rituximab, donor lymphocytes, plasma exchange with different outcome. Recently, a remarkable response to treatment with bortezomib has been described in a case of PRCA.

Methods: We reviewed 146 patients who received an allogeneic hematopoetic stem cell transplant (HCT) between Januar 2012 and August 2018 at our institution. Sixty eight patients received a major ABO-mismached HCT. PRCA was defined as a completely absence of erythroid precursors on day +30 bone marrow puncture, with absence of donor red cells and the recipient requiring RBC transfusion.

Results: Only one patient developed PRCA (1.5\%). A 18 years old male received a myeloablative HLA-matched ABOmismatched sibling donor transplant (brother, 12 years) for acute myeloid leukemia (AML), with $\mathrm{t}(8 ; 21)$ CR1,MRD positive (RUNX1-RUNX1T1). The donor was blood type A $\mathrm{Rh}$ positive and the patient $0 \mathrm{Rh}$ positive. The patient had no complication after transplant. The day +30 bone marrow puncture has shown only few erythroid precursors and day +100 puncture and biopsy no erythroid precursors, he had transfusion dependent anemia requiring a RBC transfusion 
every two weeks and retukulocytopenia. Parvo virus and cytomegalovirus were negative. Due to very high ferritin level $(>4.000 \mathrm{u} / \mathrm{l})$ and increased luiver enzymes without signs of GvHD, the treatment with deferasirox has been started. The patient has achived CR1, MRD negative, and has evidence of complete chimerism. High titers of anti-A and anti-B issohemagglutinin was present.We started the treatment with Rituximab $375 \mathrm{mg} / \mathrm{m} 2$ weekly, 4 weeks, however without response. The pathogenesis of the PRCA is thought to be due to the recipients plasma cells, bortezomib, a proteasome inhibitor inducing apoptosis of plasma cells has been given s. c. $1,3 \mathrm{mg} / \mathrm{m} 2$ two times weekly, for two weeks. The patient responded to the treatment two weeks later with increase in $\mathrm{Hb}$, which was $12,9 \mathrm{~g} / \mathrm{dl}$ and increase in retikulocyte number. The patient has continued to be well at the last control.

Conclusions: PRCA aplasia is a rare but serious complication after ABO-incompatible HCT. Bortezomib is an effective treatment for this complication if mediated by residual host isohemeagglutinins after HCT and should be recommended as standard of care.

Clinical Trial Registry: no clinical trial

Disclosure: no disclosure

\section{P206}

\section{Analysis of survival and mortality in patients} undergoing hematopoietic stem cell transplant in solca guayaquil-ecuador

\section{Julie Abifandi-Valverde ${ }^{1}$, María Santos-Bonilla ${ }^{1}$, Bella Maldonado-Guerrero ${ }^{2}$, Mayhua Lam-Rodriguez ${ }^{2}$, Fuad Huamán-Garaicoa ${ }^{3}$}

${ }^{1}$ Universidad Católica de Santiago de Guayaquil, Guayaquil, Ecuador, ${ }^{2}$ Instituto Oncológico Juan Tanca Marengo, UTMO, Guayaquil, Ecuador, ${ }^{3}$ Instituto Oncológico Juan Tanca Marengo, Anatomía Patológica, Guayaquil, Ecuador

Background: According to the World Health Organization (WHO), 50,000 TPH are carried out worldwide and this figure increases per year; however, this therapeutic approach is limited for Latin American countries. In Ecuador, there have been conducted since 2006 to nowadays about 200 hematopoietic stem cell transplants (HSCT), the $90 \%$ of them performed by SOLCA Institution. Due to the importance of this therapeutic technique and the lack of information about its development in our country; we consider relevant conduct this study in order to provide information needed for future research.

Methods: This work is retrospective, observational, cross-sectional and analytical. It included all patients who received HSCT at Stem Cell Transplantation Unit (UTMO, by its Spanish acronym) at SOLCA-Guayaquil, between the years 2009 -2016.We use the Kaplan-Meier method to analyze the survival rate between the autologous and allogeneic transplant. The information collected for this study was obtained from the database of the SOLCAy Institute and the review of the files of the patients included.

Results: At least, 150 patients have been undergoing to HSCT between 2009-2016 years. According to the type of HSCT, $42.1 \%$ received an autologous transplant and $57.9 \%$ received an allogeneic transplant, from which $79.3 \%$ were from a related donor. The main source of transplant was peripheral blood in $86.67 \%$, followed by $12 \%$ obtained from umbilical cord blood and $1.33 \%$ by bone marrow aspiration. The most frequently reported pathologies were acute lymphoblastic leukemia (ALL) (34\%), multiple myeloma (MM) (22\%) and acute myeloid leukemia (AML) $(13.33 \%)$. The overall survival was $68 \%$ (IC: $95 \%$ ). The $82.53 \%$ of patients that were undergoing to autologous transplant have survive, meanwhile the patients that were undergoing allogeneic transplant only the $57.47 \%$ have survived $(\mathrm{p}<0.05)$. The highest death rate occurred during the first year after HSCT, and decreased considerably after that period. The main cause of mortality related to transplant (MRT) was the graft-versus-host disease (GVHD) (8\%); however, the main cause of mortality in the study population $(n=150)$ was relapse in $12.66 \%$ of the patients, presented more frequently in ALL.

Conclusions: The results showed that $68 \%$ of patients undergoing to HSCT have survived. A high rate of deceased patients in this study, have died in the first year before the transplant $(26.6 \% \%)$, due to relapse. The main cause of deceased in the study is not related to HSCT, and was the relapse in $12 \%$ of patients, in compare the GVHD was the main cause of MRT ( $8 \%)$. We consider that HSCT is a technique that is still under development in Ecuador, but despite the short time it has been taking and the institutional and medical limitations present in the health field, has presented excellent results comparable to studies conducted in developed countries.

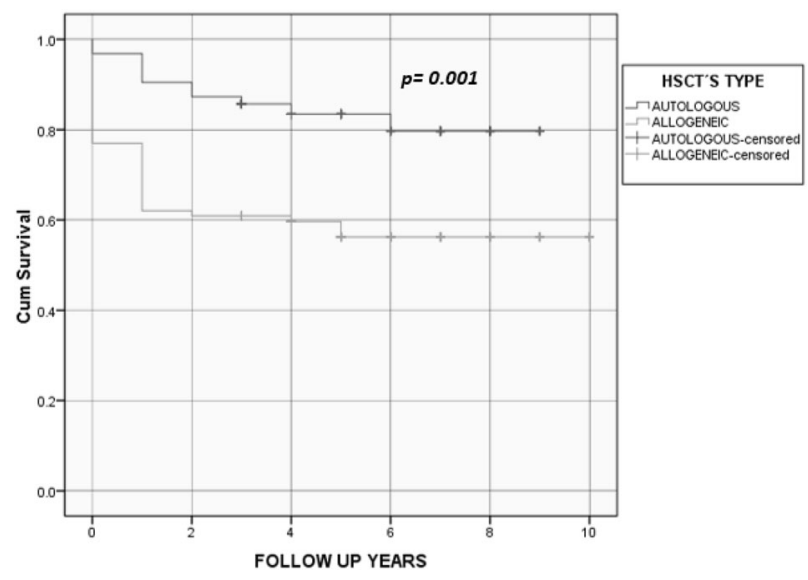

[[P206 Image] 1. Survival According HSCT's type] 


\begin{tabular}{lccccc}
\hline $\begin{array}{l}\text { Age } \\
\text { Group }\end{array}$ & Living Males & $\begin{array}{l}\text { Living } \\
\text { Females }\end{array}$ & Deceased Males & $\begin{array}{l}\text { Deceased } \\
\text { Females }\end{array}$ & Results \\
\hline Children & $16(10.67 \%)$ & $14(9.33 \%)$ & $12(8 \%)$ & $2(1.33 \%)$ & $44(29.33 \%)$ \\
Teenagers & $9(6 \%)$ & $6(4 \%)$ & $3(2 \%)$ & $3(2 \%)$ & $21(14 \%)$ \\
Adults & $33(22 \%)$ & $23(15.33 \%)$ & $16(10.67 \%)$ & $12(8 \%)$ & $84(56 \%)$ \\
Elderly & $1(0.67 \%)$ & $0(0 \%)$ & $0(0 \%)$ & $0(0 \%)$ & $1(0.67 \%)$ \\
\hline
\end{tabular}

[[P206 Table] 1. Survival and Mortality According to Age Group and Gender]

Disclosure: Nothing to declare

\section{P207}

Metastatic progression of pigmented epithelioid melanocytoma after stem cell transplantation in patient with fanconi anemia

Svetlana Radygina ${ }^{I}$, Dmitry Balashov ${ }^{1}$, Irina Kletskaya ${ }^{2}$, Konstantin Mitrakov ${ }^{I}$, Svetlana Kozlovskaya ${ }^{I}$, Nickolay Ershov $^{I}$, Ilia Zakharov ${ }^{I}$, Irina Shipitsina ${ }^{I}$, Michael Maschan $^{1}$, Alexey Maschan ${ }^{3}$

${ }^{1}$ Dmitry Rogachev National Medical Research Center of Pediatric Hematology, Oncology and Immunology, Moscow, Russian Federation, ${ }^{2}$ Russian Children's Clinical Hospital, Moscow, Russian Federation

Background: Pigmented epithelioid melanocytoma (PEM, early known as 'Animal type' melanoma) is a rare tumor with unpredictable clinical behavior and metastatic potential. PEM generally has favorable prognosis. Involvement of regional lymph nodes is not rare. Extranodal and distant nodal metastases are extremely rare. We report about patient with Fanconi anemia (FA) and PEM with developed distant metastases in the early term after allogeneic hematopoietic stem cell transplantation (HSCT).

Methods: 10-years old boy with FA was hospitalized for HSCT. The blue-black painless nodulus $15 \times 15 \mathrm{~mm}$ was noted on the left cheek. This lesion was observed from early childhood and during life only slightly increased in size.

There were no distant and regional metastases on computerized tomography (CT) and scintigraphy with ${ }^{99 \mathrm{~m}} \mathrm{Tc}$. The nodulus and regional lymph nodes were radically removed before HSCT. The resection margin was within the normal tissue. Microscopically the derma and subcutaneous fat were infiltrated with epithelioid and spindle cells with total expression of S100, MelanA, MHB45, CyclinD1. Ki-67 expression level was 15-20\%. Histological structure was specific for PEM.

HSCT with TCR $\alpha \beta+/ C D 19+$ graft depletion from match unrelated donor was performed. The conditioning regimen included total lymphoid irradiation 2Gy, fludarabin $150 \mathrm{mg} /$ $\mathrm{m}^{2}$, cyclophosphamide $40 \mathrm{mg} / \mathrm{kg}$, rabbit ATG $5 \mathrm{mg} / \mathrm{kg}$ and rituximab $100 \mathrm{mg} / \mathrm{m}^{2}$.

Results: At +45 day after HSCT was detected the tumor on the left cheek and parotid region with a histological structure identical to the primary lesion. On CT in S6 segment of the left lung was detected focus $20 \times 20 \mathrm{~mm}$ with a cavity. Invasive aspergillosis was suspected and empirical antifungal treatment was started. But in 15 days the lung lesion increased in size to $52 \times 30 \times 32 \mathrm{~mm}$ and penetrated in the bronchus. After bronchoscopy with biopsy, PEM metastasis was histologically confirmed. Moreover, the tumor on the face continued to grow.

Therapy with cobimetinib and vemurafenib was not effective and patient died from progression of PEM on +98 day after HSCT.

Conclusions: PEM was early described as indolent tumor with rare distant metastasis and favorable prognosis. We suspect that PEM may acquire an aggressive course in the absence of immunological control, especially in high immunocompromised patients after HSCT.

Disclosure: Nothing to declare

\section{P208}

\section{Abstract withdrawn}

\section{P209}

\section{B-cells reconstitution after autologous stem cell} transplantation

\section{Lidia Gartcheva ${ }^{I}$, Antoaneta Mihova ${ }^{I}$, Penka Ganeva, Margarita Guenova ${ }^{I}$, Branimir Spassov ${ }^{I}$}

${ }^{1}$ Specialized Hospital for Active Treating of Haematological Diseases, Sofia, Bulgaria, Laboratory of haematopathology and immunology, Sofia, Bulgaria

Background: The main objective of the study is to assess the dynamics of quantitative and qualitative changes in the parameters of the B cell population and the production of immunoglobulins in patients after autologous transplantation of hematopoietic stem cells in the course of recovery of the immune system.

Methods: 56 patients with hematological neoplasms undergoing autologous transplantation were included in the study: 30 women and 26 men, with an average age of 31 years. Patients were diagnosed with lymphoma $(n=30)$, multiple myeloma $(n=7)$, leukemia $(n=7)$ and solid tumors $(n=12)$. At the time of transplantation, 16 patients were in complete clinical remission or at least with very good partial response, 30 patients were in partial remission and 10 patients - with progression. All patients were evaluated in nine time points through 356 examinations by 
clinical-laboratory, flow cytometric and immunochemical methods.

Results: The percentage of CD19 (+) B cells reached the minimum values one month after transplantation then began to increase in the second month reaching a plateau around the mean values in the period 6-12 months after transplantation. The absolute number remained low during the entire period of observation. The amounts of $\operatorname{IgG}$ and IgM serum immunoglobulins gradually increased within the reference range throughout the entire period, while the $\operatorname{IgA}$ level varied around the lower reference range.

Conclusions: Implementation of an adequate humoral immune response is hampered by the reduction of circulating B cells, suppressed proliferative potential and functional deficits. Restoration of B-cell function occurs over a period of 6 months to 2 years after autologous transplantation.

Clinical Trial Registry: no clinical trials

Disclosure: Nothing to declare

\section{P210}

Incidence and general outcome of allogeneic stem cells transplantation in infants

Justyna Miśkiewicz - Bujna ${ }^{1}$, Zofia Szmit ${ }^{1}$, Jowita Fraczkiewicz ${ }^{1}$, Matgorzata Salomonowicz ${ }^{1}$, Izabella Miśkiewicz ${ }^{1}$, Monika Mielcarek ${ }^{1}$, Krzysztof Katwak ${ }^{1}$, Marek Ussowicz ${ }^{I}$, Ewa Gorczyńska ${ }^{I}$

${ }^{1}$ Wroclaw Medical University, Wrocław, Poland

Background: Allogeneic hematopoietic stem cells transplantation (allo-HSCT) is a life-saving and well established therapy for wide range of diseases. However, it is still uncommon treatment for infants less than 12 months of age. The data about indications and outcome of allo-HSCT in the youngest group of patients is sparse.

The primary objective of this study was to assess the incidence, indications, post- HSCT complications and general outcome of allo-HSCT among infants not older than 12 months. Latter sequelae of HSCT such as physical and cognitive development were secondary aim of this study.

Methods: We retrospectively analyzed data of 63 patients who underwent allo-HSCT before 1 year of age in Department of Pediatric Hematology, Oncology and Bone Marrow Transplantation in Wrocław during years 19992017. Clinical and epidemiological features as well as indications for transplantation, early and late complications and general outcome were assessed.

Results: Infants who underwent HSCT in our Department comprise $8.2 \%$ of all patients undergoing HSCT in analyzed period of time. Thirty-one $(49.2 \%)$ patients received stem cells from matched unrelated donor (MUD), 26 (41.3\%) from mismatched (haploidentical) related donor (MMRD) and $6(9.5 \%)$ from a sibling donor (MSD). Non-malignant disorders were indication for transplant in $49(77.8 \%)$ patients and malignant diseases in $14(22.2 \%)$. Acute graft versus host disease (aGvHD) occurred in 33 (52\%) infants, chronic graft versus host disease (cGvHD) in 15 (24\%). Majority of graft rejections were seen in infants transplanted from MMRD 7(63.6\%), whereas the rest 4 (36.4\%) was associated with MUD. Median follow-up in study cohort was 860 days, 1148 days for alive patients (range 72 days$19.5 \mathrm{yrs}$ ) and 72 days for those deceased (range 3 days-341 days). Overall survival (OS) in study cohort was 0.682 and transplant related mortality (TRM) was 0.317 . In children with malignancy 5 (35.2\%) patients died comparing to 15 $(30.6 \%)$ patients in non-malignant group respectively. Main cause of death in analyzed group of infants was infection $(60 \%)$.

Conclusions:

1. Allo-HSCT is rarely performed in children less than 12 months of age.

2. Majority of those patients receive stem cells due to non-malignant disorder.

3. Among youngest HSCT recipients, haploidentical transplant are more common than in general pediatric transplant population.

4. Graft rejection is a significant problem in infants transplanted from MMRD.

Disclosure: nothing to declare

\section{P211}

Unusual non-infectious lung complication after allogeneic haematopoietic stem cell transplantation

Claudia Lucia Sossa Melo ${ }^{1,2}$, Manuel Rosales ${ }^{2}$, Francisco Fernando Naranjo Junoy ${ }^{1,2}$, Sara Inés Jiménez ${ }^{1,2}$, Luis Antonio Salazar ${ }^{1,2}$, Angela María Peña ${ }^{1,2}$, María Angélica Chacón Manosalva ${ }^{3}$, Maria Luna-González ${ }^{1}$, Claudia Marcela Chalela ${ }^{1}$, Manuel Ardila-Báez ${ }^{1}$

${ }^{1}$ Universidad Autónoma de Bucaramanga, Bucaramanga, Colombia, ${ }^{2}$ Clinica FOSCAL, Bucaramanga, Colombia, ${ }^{3}$ Universidad Industrial de Santander, Bucaramanga, Colombia

Background: Pulmonary Alveolar Proteinosis (PAP) is a very low incidence disease characterized by an accumulation of pulmonary surfactant at the alveoli, which can produce an alteration in gas exchange. Three types of this disease have been described: autoimmune, secondary and congenital. Secondary PAP can occur posterior to a haematopoietic stem cell transplantation (HSCT) as a noninfectious complication, or associated with Pneumocystis 
jirovecii infections, viral infections or Nocardia. We describe the case of a patient with Acute Lymphoblastic Leukemia (ALL) diagnosis with PAP associated to a HSCT and pulmonary pneumocystis.

Methods: A 55-year-old Colombian female patient diagnosed with B-precursor ALL of high-risk in January 2017, positive Philadelphia chromosome, positive BCR / ABL in February 2017, infiltration to the Central Nervous System (CNS), $2.53 \%$ of lymphoblasts, and karyotype without legible metaphases. Refractory to induction according to the PETHEMA protocol (Vincristine, Daunorubicin, Prednisone, L-asparaginase) with presence of $13.9 \%$ blasts at the end of the induction. Re-induction was performed with the FLAG-IDA protocol (Idarubicin, Fludarabine, Cytarabine) achieving complete remission, obtaining Minimum Residual Disease $(M R D)<0.01$. Dasatinib was initiated by BCR / ABL expression and CNS involvement at the time of diagnosis.

An allogeneic HSCT was performed, from a male brother donor, with low intensity conditioning TT BUFLU and prophylaxis of Graft-versus-Host Disease (GVHD) with Tacrolimus and Sirolimus. Patient showed early posttransplant complications, given the reactivation of cytomegalovirus and hemorrhagic cystitis grade I due to adenovirus. Late complications such us GVHD at the cutaneous level and subsequent hepatic and gastrointestinal involvement were seen too, for which immunosuppressive therapy was administered with high doses of systemic corticosteroid.

Results: Patient was hospitalized on day +270 posttransplantation due to febrile neutropenia and respiratory symptoms, with normal chest $\mathrm{CT}$, and $\mathrm{CT}$ of paranasal sinuses with acute pansinusitis, for which she received Meropenem 1 gr intravenously every 8 hours plus Vancomycin 1 gr intravenously every 12 hours during 14 days with symptom resolution.

She remained hospitalized for cytopenias with normal bone marrow and $100 \%$ chimerism. On day +291 posttransplant she presented fever and leukocytosis, with acute respiratory failure with chest CT that showed bilateral alveolar occupation, "crazy-paving" pattern and frosted glass (see image), so diagnostic fibro-bronchoscopy was performed, reporting postoperatively for Pneumocystis jirovecii.

She received 21 days of Trimethoprim-sulfamethoxazole, with a torpid evolution requiring mechanical ventilation and tracheostomy, persisting with hypoxemia. The report of cultures for fungi, mycobacteria, and respiratory panel of FilmArray were negative. A pathology report was obtained with $30 \%$ neutrophils, as well as PAS staining with acellular pink material and elevated serum LDH, with a diagnosis of secondary PAP.
The patient continued with poor general condition, refractory hypoxemia, high ventilatory parameters and hemodynamic instability, due to which she was not able to be a candidate for treatment with total pulmonary lavage; leading to multi-organ failure and later death.

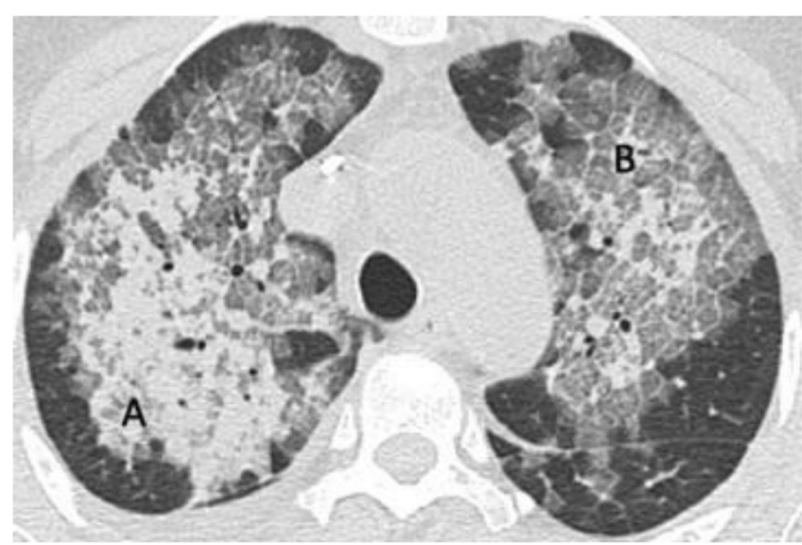

[[P211 Image] 1. High resolution chest CT. Sample opacification in frosted glass (A) and pattern "Crazypaving" (B)]

Conclusions: The importance of considering the diagnosis of PAP as a noninfectious pulmonary complication in patients with allogenic HSCT despite its low incidence is recognized.

Disclosure: Nothing to declare

\section{P212}

Blood samples for monitoring of trough levels can be drawn from the PICC line in patients with intravenous tacrolimus

\section{José Pizarro ${ }^{1}$, Lourdes Aguirre ${ }^{1}$, Carmen Tejado ${ }^{1}$, Maider Rojo ${ }^{1}$, Itxaso Muguruza ${ }^{1}$, Nerea Caminos ${ }^{1}$, Esther Pérez $^{1}$, Naiara Manzanares ${ }^{1}$, Maider Valencia ${ }^{1}$, Carlos Vallejo $^{1}$}

${ }^{1}$ University Hospital Donostia, Hematology, San Sebastián, Spain

Background: Monitoring of calcineurin inhibitors [cyclosporin A (CsA) and tacrolimus (Tac)] blood levels are critical to assure effectiveness and to avoid toxicity in patients undergoing allogeneic HSCT. However, it is not clear if samples have to be drawn from a peripheral site (through venipuncture) or can be obtained from the central line. Blood draws from central line are preferred by the patients, but there are doubts related to accuracy of the value. We aimed to check if there were differences in the 
blood levels of Tac in samples drawn through a PICC line compared to those obtained by venipuncture.

Methods: Once the project was approved by the Clinical Trials and Ethics Committee, 154 pairs of blood samples were drawn (154 from PICC line and 154 from venepuncture) from 33 voluntary allo-HSCT recipients who were receiving continuous infusion tacrolimus from February through August 2018. The pts had inserted a double-lumen polyurethane PICC. Tacrolimus was always administered through the red line, and the blood draw always performed through the purple line. All of the patients signed the informed consent. 22 were male and 11 women. Median age was 55 years (30-68). 24 of the venepunctures were carried out in the arm where the PICC was set, and the other 130 from the contralateral arm. A limited group of nurses performed the extractions of the samples.

Results: As shown in the table, tacrolimus trough levels determined in blood from venepuncture were similar to those in blood drawn through the PICC (median: 10.7 vs $11.1 \mathrm{ng} / \mathrm{mL}$ ). When comparing one by one in the individual patients, the differences were not significant, and changed the dosing prescription in no cases.

Conclusions: In our experience, there are not significant differences in tacrolimus levels draw from the PICC line, compared with a peripheral site. So, in our opinion, if the line for tacrolimus infusion is properly identified and the one used for the sample draw is the alternative one, venepunctures to obtain sample from peripheral sites are not justified for tacrolimus levels measurements.

\begin{tabular}{|c|c|c|c|}
\hline & $\begin{array}{l}\text { Venipuncture } \\
(\mathrm{N}=154)\end{array}$ & $\begin{array}{l}\text { PICC (purple } \\
\text { line) }(\mathrm{N}=154)\end{array}$ & $\mathrm{P}$ \\
\hline $\begin{array}{l}\text { Tacrolimus trough blood level } \\
\text { [median (range)] }\end{array}$ & $\begin{array}{l}10.7 \mathrm{ng} / \mathrm{mL} \\
(1.8-23.6)\end{array}$ & $\begin{array}{l}11.1 \mathrm{ng} / \mathrm{mL} \\
(1.7-24.1)\end{array}$ & Ns \\
\hline $\begin{array}{l}\text { Differences PICC - } \\
\text { venipuncture [median (range)] }\end{array}$ & $\begin{array}{l}-0.3 \mathrm{ng} / \mathrm{mL} \\
(-3.3-+3.2)\end{array}$ & & \\
\hline
\end{tabular}

[[P212 Table] 1. Tacrolimus levels: venipuncture versus PICC line]

Disclosure: Nothing to declare

\section{P213}

Retrospective analysis of intensive care unit admission and outcome of haematopoietic stem cell transplant patients at ST. George's hospital

\section{Ke Xü, Matthias Klammer ${ }^{I}$, Mickey Koh ${ }^{1}$}

${ }^{1}$ St. George's Hospital, Haematology, London, United Kingdom
Background: Patients undergoing a HSCT may require ICU admission due to transplant-related toxicities. The aim of this study was to analyse a single centre experience with HSCT patients requiring ICU admission and the factors affecting outcome.

Methods: We included all adult patients (age $>=18$ ) who had an allogeneic or autologous HSCT during 2017 (D0 between 1-1-2017 to 31-12-2017) at St. George's Hospital. Data was retrospectively collected from patients' notes. ICU outcome and 100-day survival were analysed. For those patients who were admitted to ICU more than once, outcome was analysed from their last ICU admission.

Results: 20 allograft patients were included. 14 were male, with a median age 47 years (range 23-68 years). 6 were female, with median age 61 years (range 51-67 years). Diagnosis N (\%) includes ALL 3 (15\%), AML 7 (35\%), aCML 1 (5\%), CMML 3 (15\%), HL 1 (5\%), MDS 2 (10\%), MDS/MPN 1 (5\%), FL 1 (5\%), SCD 1 (5\%). Sixteen (80\%) patients received their first transplant, $4(20 \%)$ received second transplant. Eight (40\%) patients had sibling donor, 12 patients $(60 \%)$ had unrelated donor. Sixteen $(80 \%)$ patients had 10/10 matched donor, 2 (10\%) patients had 9/ 10 matched donor, 2 (10\%) patients had 8/10 matched donor. Nineteen (95\%) received Reduced Intensity Conditioning (RIC), one (5\%) received Myeloablative (MA) conditioning. Majority of RIC allo-HSCT patients were conditioned with Fludarabine, Mephalan, Campath (FMC). A small number were conditioned with busulfan, fludarabine and ATG. The MA allo-HSCT patient was conditioned with TBI, cyclophosphamide. GvHD prophylaxis was ciclosporin alone starting on day -1 with a target level of 150-250 ug/L for all RIC and ciclosporin and methotrexate for the MA patients.

Two (10\%) allograft patients were admitted to ICU on three occasions. Both patients were male, 58 and 68 years old. One had MMUD allograft for MDS/MPN. The other had $2^{\text {nd }}$ MUD allograft for relapsed AML. The reasons for ICU admission include sepsis, cardiac arrest and respiratory failure. The median duration of ICU admission was 5 days (range 2-9). There were 2 deaths within 100 days of transplant. One patient died on day +11 during his second ICU admission with multi organ failure (MOF). One patient died after ICU discharge on day +23 with relapsed disease, bronchopneumonia with disseminated fungal infection. ICU mortality rate was $50 \%$, and 100 -day mortality rate was $10 \%$.

Nineteen autologous patients were included (median age 61 (range 36-71 years)), 16 (84\%) were myeloma patients who were conditioned with melphalan, $3(16 \%)$ were lymphoma patients who were conditioned with BEAM. The ICU admission was 0\%. The 100-day mortality rate was $0 \%$. 
Conclusions: Our centre's ICU admission rate, ICU mortality rate, cause of ICU admission in allo-HSCT patients and autologous patients is comparable to literature reports. Autologous transplant is safe with no deaths and ICU admissions despite an older age. The mortality rate for allo-HSCT patients requiring ICU admission remain high. All patients were appropriately referred to ICU and there was no one who was denied ICU admission. This analysis is being extended to preceding years.

Disclosure: Nothing to declare

\section{P214}

Liposomal doxorubicin for the treatment of iatrogenic kaposi sarcoma following hematopoietic stem cell transplantation

Benedetta Rambaldi ${ }^{1}$, Michele Malagola ${ }^{I}$, Federica Cattina $^{1}$, Valeria Cancelli, Lisa Gandolfi, Mirko Farina $^{1}$, Alessandro Turra ${ }^{1}$, Nicola Polverelli, Enrico Morello $^{1}$, Domenico Russo ${ }^{1}$

${ }^{1}$ Università degli Studi di Brescia, Brescia, Italy

Background: Iatrogenic Kaposi's Sarcoma (iKS) represent a rare complication after hematopoietic stem cell transplantation (HSCT), related to HHV-8 infection in HIVnegative immunocompromised patients (pts).

Methods: We describe a case of iKS occurred after an allogeneic HSCT and we provide a review of the literature using Pub Med.

Results: A 70-year-old man, HIV-negative, received full HLA-matched related HSCT after a reduced intensity conditioning regimen for relapsed AML. GvHD prophylaxis was based on ATG (Fresenius $30 \mathrm{mg} / \mathrm{Kg}$ ), cyclosporine (Cya) and methotrexate. No severe complication occurred in the first 100 days after transplant. Shortly after Cya withdrawal, he developed grade I acute GvHD. GvHD resolved after restarting Cya. At fifth month after transplant, the patient developed several red and purple angiomatous plaque and nodules involving the skin of both lower limbs, right arm and the nose (figure 1). Skin biopsy revealed multiple localizations of $\mathrm{iKS}$ and positive HHV-8 viremia was detected in the peripheral blood. A visceral involvement was excluded. Patient was treated with Cya tapering and nine courses of liposomal doxorubicin $20 \mathrm{mg} / \mathrm{m} 2$ every 15 days, obtaining a negativity of HHV-8 viremia and partial response of the skin lesions. At last follow up, at 15 months after transplant, the patient was in complete remission (CR) for AML, Cya-free without signs of GvHD recurrence and with his single stable residual iKS lesion on his left limb, currently waiting for local radiotherapy.

We found additional $18 \mathrm{iKS}$ published cases after HSCT. Most of post-HSCT iKS were secondary to an allogeneic-
HSCT (16 out of $19,84.2 \%)$ and occurred in adult $(78,9 \%)$ and male $(68,4 \%)$ pts. Median age at the time of iKS diagnosis was 47.5 years (range $7-70)$. Thirteen pts $(68.4 \%)$ had Mediterranean origin. The most frequent underlying disease was AML (47.4\%). GvHD prophylaxis was primary based on calcineurin inhibitor. Half of the pts developed GvHD and were treated with steroid and other immune suppressive drugs. Median time between the HSCT and the occurrence of iKS was 8.5 months (range 3-56). Cutaneous iKS was the prevalent form of manifestation, however visceral involvement was reported in 7 pts $(36.8 \%)$. In four cases $(21.1 \%)$ an $\mathrm{HHV}-8$ associated BM failure was report. Immune suppression drugs tapering (36.8\%) and chemotherapy (26.3\%) were the most frequent actions taken after the diagnosis of iKS. In most cases, liposomal doxorubicin was used as chemotherapy. CR rate was high, $63.2 \%$, whereas progression disease occurred in 5 out 19 pts (26.3\%), all of which had visceral involvement. In 3 pts (15.8\%), iKS was the cause of death.

Conclusions: Withdrawn of immune suppression drugs and anthracycline based chemotherapy can represent a feasible treatment option for pts with iKS after HSCT.

Clinical Trial Registry: Not applicable

Disclosure: Nothing to declare

\section{P215}

Acquired haemophilia a after haploidentical bone marrow transplantation: A case report

\section{Oscar Borsani ${ }^{1}$, Anna Amelia Colombo ${ }^{1}$, Roberta Sciarra $^{1}$, Daniela Caldera ${ }^{1}$, Paolo Bernasconi ${ }^{1}$}

${ }^{1}$ University of Pavia, San Matteo Hospital, Pavia, Italy

Background: Acquired Haemophilia A (AHA) is an autoimmune disease caused by the spontaneous production of neutralizing immunoglobulin $\mathrm{G}$ (IgG) autoantibodies (inhibitors) targeting endogenous FVIII. Treatment of these inhibitors presents additional challenges in a hematopoietic stem cell transplantation (HSCT) recipient, because preservation of the graft that restores a normal hematopoiesis is critical. Here we describe the management of a case of AHA in an acute myeloid leukemia patient following HSCT.

Methods: The clinical, laboratory and molecular aspects of a 56-year-old Italian male who developed AHA after allogenic bone marrow transplantation were collected and presented in order to show how we diagnose and manage this severe but rare complication within the special setting of HSCT.

Results: A 56-years-old man with a FLT-3 ITD, NPM1, RUNX1-RUNX1T1 and CBFB-MYH11 negative, not differentiated, chromosomally normal acute myeloid 
leukemia (AML) in third complete remission (CR) was submitted to a hematopoietic stem cell transplantation (HSCT) from his haploidentical son. The conditioning regimen consisted of Oncothiotepa, Busulfan and Fludarabine and was followed by the infusion of a T-cell depleted bone marrow graft. GVHD prophylaxis consisted of Cyclosporine A (CsA) and mycophenolate mophetyl (MMF). Neutrophil engraftment occurred on day +24 . Recipient's autoimmunity was negative. At 27 months post-transplantation the patient received an antipneumococcal vaccination. Fifteen days post-vaccination the patient was admitted to our in-patient ward due to general malaise, diffuse muscle and joint pains, cutaneous bleedings, oedemas, hyperchromic urines and constipation. Physical examination revealed diffuse ecchymosis, swelling of deep muscles with a progressive functional disability due to hematomas and hemorrhagic suffusions of the tongue frenulum. A progressive reduction of hemoglobin values occurred on the subsequent days. A bone marrow aspiration revealed the persistence of CR, confirmed by immune-phenotypic studies, and a complete donor chimera. The coagulation panel revealed the following values: INR 0.84 (normal values 0.90-1.20), activated partial thromboplastin time (aPTT) $50.10 \mathrm{sec}-$ onds (normal values 20-32) with PTT ratio 1.75 (normal values 0.70-1.12), Factor VIII coagulant (FVIII:C) $0.46 \%$ (normal values $70-120 \%$ ), Von Willebrand antigen $235.74 \%$ (normal values 50-160), von Willebrand coagulant $185.90 \%$ (50-200), fibrinogen $568 \mathrm{mg} / \mathrm{dl}(170-410)$ and anti-factor VIII inhibitors $6.46 \mathrm{BU} / \mathrm{ml}$ (high titers $>5$ $\mathrm{BU} / \mathrm{ml}$ ). Thus, a diagnosis of acquired autoimmune Haemophilia A was made and treatment with FEIBA combined with prednisone was started.

Patient's clinical conditions dramatically improved as he referred an improvement of movements and the resolution of joint and muscle pains despite the persistence of deep hematomas just after one day of treatment that had determined an increase of FVIII:C value to $1.32 \%$ and an improvement of aPTT to 47.06 seconds. On the following medical checks physical examination showed the progressive disappearance of deep muscle hematomas, and normal values of FVIII:C.

Conclusions: AHA is a rare but severe complication following HSCT and it could appear years afterengraftment. A prompt diagnosis and an early treatment with FEIBA and corticosteroid are necessary to avoid life-threatening sequelae. The inclusion of the coagulation panel in the laboratory exams performed during the follow-up is advisable in order to early detect this life-threatening complication.

Disclosure: Nothing to declare
P216

Successful treatment with defibrotide of sinusoidal obstruction syndrome after allogeneic transplantation in a patient with myelofibrosis, extreme splenomegaly and splanchnic thrombosis

\section{Vera Radici ${ }^{I}$, Alessandra Sperotto ${ }^{I}$, Chiara Cigana, Michela Cerno ${ }^{I}$, Fabrizia Colasante ${ }^{1}$, Roberta De Marchi $^{I}$, Renato Fanin ${ }^{I}$, Francesca Patriarca ${ }^{I}$}

${ }^{1}$ Azienda Sanitaria Universitaria Integrata di Udine, Udine, Italy

Background: Splanchnic thrombosis is an uncommon complication of myelofibrosis and a controindication to proceed to hematopoietic stem cell transplantation (HSCT) due to the risk of additional vascular and endothelial complications. We present a patient with myelofibrosis (MF) that proceeded to HSCT from an unrelated donor, despite splanchnic thrombosis unresolved after heparin treatment and unable to proceed to surgical treatment due to severe thrombocytemia.

Methods: A 67-year woman with MF secondary to essential thrombocythemia, with intermediate-2 score according Dynamic International Prognostic Staging System (DIPSS) and with extreme splenomegaly (maximum diameter $30 \mathrm{~cm}$ ), refractory to ruxolitinib, showed an extensive thrombosis of the portal and splenic veins, unresolved after 4-week heparin therapy, at the time of availability of an HLA (8/8) and ABO matched unrelated donor.

She received a conditioning regimen including fludarabine and thiotepa and a GvHD prophylaxis with ATG thymoglobuline, cyclosporine and methotrexate, followed by the reinfusion of $5.8 \times 10^{6} / \mathrm{kg} \mathrm{CD} 34+$ PBSC. At the time of transplant we were aware of an high risk of developing SOS, on the basis of the older age of the recipient, the unrelated donor, the advanced stage of myelofibrosis and the ferritin serum level of $2.223 \mathrm{ng} / \mathrm{mg}$.

Results: On day +9 after HSCT SOS complicated the aplasia phase, characterized by jaundice, ascites, weight gain, progressive increase in creatinine and bilirubin serum levels. An ultrasound of abdomen confirmed an unchanged thrombosis extension and the development of ascites.

On day +10 the patient was categorized as very severe SOS stage, according to EBMT severity criteria, because of doubling of bilirubin serum level in 48 hours and a $20 \%$ increase in comparison with her baseline weight. Therefore, defibrotide was promptly started in association with diuretic therapy. The treatment was continued for 3 weeks and allowed gradual restoration of the water balance and normalization of bilirubin serum level. 
At the last follow-up, 6 months after HSCT, the patient shows the persistence of a non-transfusion dependent anemia, platelets $80.000 \times 10^{\wedge} 3 / \mathrm{uL}$, palpable spleen $4 \mathrm{~cm}$ below the rib, $>95 \%$ allogeneic chimerism in the granulocytic compartment and $90 \%$ in the T lymphocyte compartment. Splanchnic thrombosis is partially recanalized and replaced by collateral circles with cavernous aspects. The patient is on treatment with fondaparinux and has shown neither significant infectious episodes or acute or chronic GvHD.

Conclusions: We conclude that defibrotide treatment allowed to perform a successfull allogeneic transplant in a patient with MF associated with an overt picture of splanchnic thrombosis.

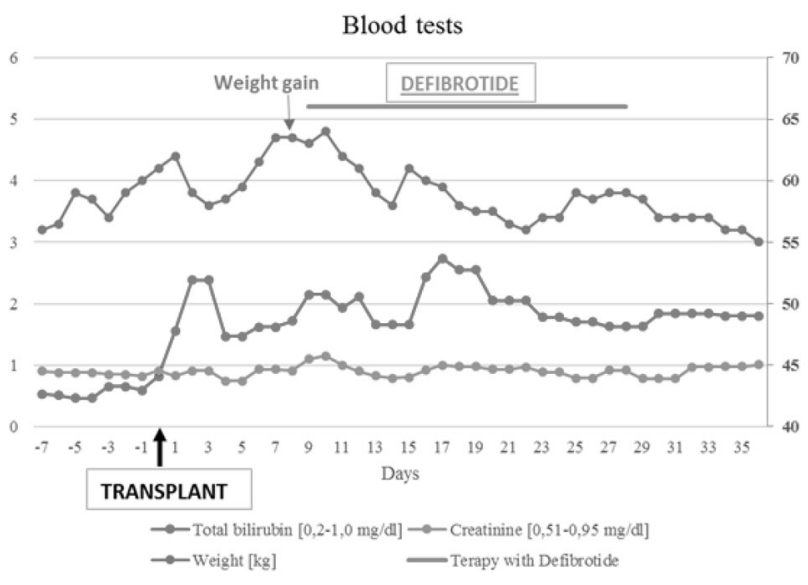

[[P216 Image] 1. Blood tests]

Disclosure: Nothing to declare

\section{P217}

Squamous cell carcinoma: A rare secondary malignancy in pediatric allogeneic hematopoietic stem cell transplant receptors

Pilar Palomo ${ }^{1}$, José Antonio Villegas ${ }^{1}$, Ana De Lucio', Almudena Navarro ${ }^{1}$, Víctor Álvarez ${ }^{1}$, Álvaro De Dios ${ }^{1}$, Joud Zanabili, Ana Julia González', Ana Pilar González", Ana Vivanco ${ }^{I}$, Soledad González.

\section{${ }^{1}$ Hospital Universitario Central de Asturias, Oviedo, Spain}

Background: Hematopoietic stem cell transplantation (HSCT) is associated with an increased incidence of secondary malignancies including skin cancer. Squamous cell carcinoma (SCC) is the most common type in patients who are receiving immunosuppressive therapy and chronic graft-versus-host disease (cGVHD) appears to be an important risk factor for its development. Recent studies describe voriconazole exposure as an independent factor that may contribute to this increased risk as well. In our best knowledge, no cases of SCC have been reported in pediatric allogeneic HSCT to date.

Methods: We present a case report of a 9 year-old boy who developed a SCC with high-risk features six years after undergoing hematopopoietic stem cell transplant.

Results: A 9 year-old boy with acute lymphoblastic leukemia (ALL) underwent a matched unrelated bone marrow transplant 7 years ago. He developed grade IV aGVHD followed by extensive cGVHD with generalized scleroderma. He required intensive and continued immunosuppressive therapy and was on prolonged antifungal prophylaxis with voriconazole. In March 2017, he developed SCC involving left temporal region that was completely excised. Two months later, more lesions in scalp and nose were noted and intralesion treatment with methotrexate was started. However, an unfavorable evolution was noted and he was put on systemic treatment including Cisplatin and Cetuximab receiving the whole scheme from January to March 2018 and continuing only with Cetuximab, ten doses in total, until May, for unaceptable and severe tubulopathy that required admission at the hospital in several ocassions. He achieved a very good partial response but progression was noted shortly in follow up. At this point, non curative therapeutic options were found and he was put on intralesion methotrexate and photodynamic theraphy in a weekly basis with palliative intention. Unfortunately, tumor growth was fast and patient passed away in August 2018, fifteen months after squamous cell carcinoma diagnosis, due to tumoral progression.

\section{Conclusions:}

1) SCC is a rare, non-previously described, secondary malignancy in children undergoing HSCT.

2) High-risk features SCC constitutes an aggresive disease with a median overall survival below 1 year.

3) cGVHD appears to be an important risk factor for its development.

4) Voriconazole induced-photosensitivity might have played a role.

5) Cisplatin based regimens +/-Cetuximab are a therapeutic option in disseminated and/or high risk cases. As outcomes are unsatisfactory in these cases, alternative therapeutic options need to be explored.

Disclosure: Nothing to declare

\section{P218}

Successful pregnancy after allogeneic stem cell transplantation in acute myeloid leukemia

Tobias Rachow ${ }^{1}$, Friederike Hunstig ${ }^{1}$, Ekkehard Schleußner ${ }^{2}$, Andreas Hochhaus ${ }^{1}$, Inken Hilgendorf ${ }^{I}$ 
${ }^{1}$ Universitätsklinikum Jena, Klinik für Innere Medizin II, Abteilung Hämatologie und Internistische Onkologie, Jena, Germany, ${ }^{2}$ Universitätsklinikum Jena, Klinik für Geburtsmedizin, Jena, Germany

Background: Pregnancy is a rare event after allogeneic stem cell transplantation (SCT) for acute leukemia. Here we report, to the best of our knowledge, for the first time on a successful pregnancy after treosulfan-based conditioning.

Methods: A 29-year old woman was diagnosed with acute myeloid leukemia (AML) secondary to chronic myelomonocytic leukemia in July 2015. Ovarian preservation was performed by leuprolide acetate depot injection prior to cytostatic chemotherapy. Of note, no cryopreservation of oocytes or ovarian tissue was conducted. She received two cycles of chemotherapy consisting of idarubicine $\left(12 \mathrm{mg} / \mathrm{m}^{2}\right.$ on day 1-3) and cytarabine ( $1000 \mathrm{mg} / \mathrm{m}^{2}$ b.i.d. on days $1,3,5$ and 7 ). Due to secondary origin of AML SCT was performed in first complete remission of AML after conditioning with treosulfan $(14 \mathrm{~g} /$ $\mathrm{m}^{2}$ days $\left.3-5\right)$ and fludarabine $\left(30 \mathrm{mg} / \mathrm{m}^{2}\right.$ days $\left.1-5\right)$. She received $4.94 \times 10^{6} \mathrm{CD} 34$-positive cells per kilogram body weight from a HLA-matched unrelated donor.

Results: Follow-up bone marrow aspirates showed continuous complete remission of AML. Seven months after SCT she became pregnant, but decided for induced abortion. In January 2018, 25 months after HSCT she became pregnant again and desired the child. Medical examinations were performed monthly on an outpatient basis in stringent cooperation with the maternity clinic. The course of pregnancy was unremarkable, although she was hospitalized due to premature labor in the $34^{\text {th }}$ week of pregnancy. However, gynecological examination showed no clinical significant findings, so that section was planned and she could be discharged again. In the $38^{\text {th }}$ week of pregnancy she gave birth to a healthy girl $(50 \mathrm{~cm}, 2810 \mathrm{~g})$ by cesarean section. Peripartum she developed hypoethesia of the left body half. Neurological examination showed no abnormalities and she recovered immediately. There were no other postpartum complications. Breastfeeding was established but additional food was necessary for a sufficient nutrition of the child.

Conclusions: This case of successful pregnancy following SCT demonstrates that fertility can recover after treosulfan-based conditioning. However, detailed studies of ovarian function and fertility are necessary to gain more insight into the risk of premature ovarian failure.

Disclosure: Nothing to declare.

Experimental stem cell transplantation

\section{P219}

CD19-cart therapy before allo-HSCT in children and adolescents patients who diagnosed R/R B-all with E2APBX1
Zhang Jianping ${ }^{I}$, Li Nannan ${ }^{I}$, Liu Deyan ${ }^{I}$, Lu Yue ${ }^{1}$, Zhou Jiarui ${ }^{1}$, Xiong Min ${ }^{1}$, Sun Ruijuan ${ }^{1}$, Wei Zhijie ${ }^{1}$, Cao Xingyu $^{I}$, Zhao Yanli ${ }^{I}$, Fan Lijun ${ }^{I}$, Gao Xinxia ${ }^{I}$, Yin Dongxu $^{1}$, He Junbao ${ }^{1}$, Chen Huihong ${ }^{1}$, Su Wei, Pang Xuebing ${ }^{I}$, Lu Daopei ${ }^{I}$

${ }^{1}$ Beijing Ludaopei Hospital, Beijing, China

Background: B-ALL with E2A-PBX1 in children and adolescents is described with favourable prognosis. But there are more than $10 \%$ patients with E2A-PBX1 diagnosed as relapsed or refractory. The results of Allo-HSCT in children and adolescents with this group leukemia in our center was analyzed in order to understand the therapeutic effect of CD19-CART on the patients.

Methods: Retrospective analysis, from June 1st, 2012 to July 31,2018, all children and adolescents diagnosed relapse or refractory B-ALL with E2A-PBX1 who received AlloHSCT, total 30 cases. All patients was divided into two groups depending on whether or not accepted CD19-CART before Allo-HSCT. According to FCM-MRD and E2APBX1 level before Allo-HSCT, OS LFS and cumulative recurrence rate were analyzed. $\mathrm{R}$ 3.2.0 was used as Statistical analysis software.

Results: CART vs no-CART: 1 yr OS $69.3 \%$ vs $33.3 \%$, $3 \mathrm{yr}$ OS $60.6 \%$ vs $33.3 \%, \mathrm{P}=0.1$; $1 \mathrm{yr}$ LFS $=1 \mathrm{yr}$ OS, $3 \mathrm{yr}$ $\mathrm{LFS}=3 \mathrm{yr}$ OS; FCM-MRD $=0$ vs FCM-MRD $>0$ : $1 \mathrm{yr}$ OS $57.9 \%$ vs $63.6 \%$; $3 \mathrm{yr}$ OS $59.5 \%$ vs $45.5 \%, \mathrm{P}=0.8 ; 1 \mathrm{yr}$ $\mathrm{LFS}=1 \mathrm{yr}$ OS, 3 yr LFS $=3 \mathrm{yr}$ OS; E2A-PBX1 $=0$ vs E2APBX1>0: $1 \mathrm{yr}$ OS $83.33 \%$ vs $33.33 \% 3 \mathrm{yr}$ OS $83.33 \%$ vs $33.33 \%, \mathrm{P}=0.03$; $1 \mathrm{yr} \mathrm{LFS}=1 \mathrm{yr}$ OS, 3yr LFS=3yr OS; The main cause of morbidity was relapse of leukemia.

Conclusions:

1. For R/R B-ALL with E2A-PBX1 in children and adolescents, FCM-MRD pre-transplant hasn't obvious effect on the outcome of Allo-HSCT, while the level of E2APBX1 has obvious effect. The out come of E2A-PBX1 negative group was obviously better than positive group.

2. CD19-CART can obviously improve the OS and LFS, it is mainly because of CD19-CART can makes more patients fusion to zero.

3. For R/R B-ALL with E2A-PBX1 in children and adolescents, if chemotherapy can't make the fusion to zero. It is suggested to accept CD19-CART therapy to make the fusion zero. It can improve the outcome of OS and LFS.

Disclosure: No interest conflict

P220

Clonal contribution of bone marrow subpopulations during reconstitution

Frauke Gotzhein ${ }^{1}$, Tim Aranyossy ${ }^{2}$, Lars Thielecke ${ }^{3}$, Ingmar Glauche $^{3}$, Kerstin Cornils ${ }^{1}$ 
${ }^{1}$ University Medical Center Hamburg Eppendorf, Hamburg, Germany, ${ }^{2}$ University Medical Center Hamburg Eppendorf, Hamburg, Germany, ${ }^{3}$ Technische Universität Dresden, Institute for Medical Informatics and Biometry, Faculty of Medicine Carl Gustav Carus, Dresden, Germany

Background: Currently, Hematopoietic stem cell transplantation (HSCT) represents the only curative treatment for numerous hematopoietic malignancies like leukemias, immune deficiencies or metabolic diseases. CD34 serves a quality marker for stem cell grafts, which is not solely expressed on stem cells but also on a variety of progenitors. The role and the impact of these subpopulations remains unknown. We made use of our genetic barcode system to analyze the influence and contribution during reconstitution on a clonal level.

Methods: Fluorescence activated cell sorting (FACS) was used to sort hematopoietic stem and progenitor populations, namely HSCs, MPPs, CMPs and CLPs, which were lentivirally transduced with our previously established BC32 barcoding system. After mixing the marked cells with bone marrow support, lethally irradiated recipient animals were and transplanted and monitored over 16 weeks. We focused on bone marrow, blood, spleen and thymus, on chosen endpoints $(1 \mathrm{w}, 3 \mathrm{w}, 8 \mathrm{w}, 16 \mathrm{w})$ and samples were used to analyze the contribution of the subpopulations during the reconstitution process based on fluorescent protein (FP) expression. To investigate the clonal contribution in different organs, we performed next generation sequencing (NGS) and frequencies of unique barcodes in a sample were analyzed by bioinformatical approaches.

Results: A maximum of $15 \%$ of cells expressed the encoded FPs, which were mostly derived from the HSCs and MPPs. CMP-derived cells were only detected 1 week after transplantation in the myeloid compartment. Cells derived from the CLPs were not detected at any time point. We analyzed the barcode content of the differently marked cells after next-generation-sequencing. In accordance with the FACS data, the majority of the clones during the 16 weeks of observation are derived from HSCs and MPPs. CMP-derived clones were only contributing during the first weeks and CLP-derived clones are barely detectable. We did not observe any major differences with regard to age of donor or recipient, despite the total number of clones is higher in the group, which received the "aged" graft, independently from the transduced cell population.

Conclusions: Here we show the suitability of our highly complex multi-color barcode system to study the clonal contribution of HSCs and three progenitor populations after HSCT. Our results will contribute to a better understanding how these different populations interact to support the establishment of a new hematopoietic system. Emphasized by the variability in data of graft and recipient age, this comprehensive analysis gives rise to an impression to the necessity of personalized graft composition, by which treatment success could be influenced.

Disclosure: Nothing to declare

\section{P221}

Survival and fate of adipose derived mesenchymal stem cells in a rat brain injury model

\section{Aggeliki Giannakopoulou ${ }^{1,2}$, Spyros Petrakis ${ }^{3,4}$}

${ }^{1}$ George Papanicolaou Hospital, Haematology, Thessaloniki, Greece, ${ }^{2}$ Aristotle University of Thessaloniki, Laboratory of Anatomy, Histology and Embryology, Faculty of Veterinary Medicine, Thessaloniki, Greece, ${ }^{3}$ Biohellenika Biotechnology Company, Thessaloniki, Greece, ${ }^{4}$ Institute of Applied Biosciences (INAB), Centre for Research and Technology Hellas (CERTH), Thessaloniki, Greece

Background: Mesenchymal stem cells have been identified as promising candidates in the treatment of Central nervous System (CNS) injury through neurotrophic support and immunomodulation. Adipose tissue is an attractive source of mesenchymal stromal/stem cells (ASCs) for regenerative therapeutic applications because they can be harvested from autologous donors with minimally invasive methods, can be rapidly expanded ex vivo, show low immunogenicity if allogeneic, and can be used in autologous or heterologous settings. The present study examines the fate and effects of intracerebroventricularly (ICV) transplanted ASCs in a traumatic brain injury (TBI) model.

Methods: ASCs were isolated from inguinal fat pad of adult Wistar rats under sterile conditions and cultured according to standard procedures. ASCs at passage $2\left(2 \times 10^{5}\right.$ cells) were seeded and transfected with Sleeping Beauty transposase and pT2 Venus-neo ${ }^{R}$ plasmids. Selection with G418 antibiotic resulted in the generation of a homogeneous ASC population which expressed fluorescent Venus protein for several passages, Phenotypic characterization showed that these cells were $99.6 \%$ double positive for CD44 and CD90 stem cell markers, verifying their mesenchymal origin. TBI was induced by stereotactic surgery under deep anaesthesia and subsequently ICV transplantation of Venus + ASCs was performed on adult Wistar rats. Normal ASCs-transplanted and TBI-saline transplanted rats were used as controls. The proliferation, migration, survival and fate of transplanted ASCs and their effect on injury restoration were examined six weeks post transplantation (PT).

Results: Six weeks PT ASCs expressed the fluorescence Venus protein and therefore were identified in brain parenchyma. Their presence into brain was also confirmed by Masson trichrome staining, which revealed their 
collagen depositions. ASCs were found in lesser numbers compared to those transplanted and exhibited no proliferative activity. ASCs were found scattered distributed in brain as individual cells, and there were no aggregates of ASCs or mass formation into lateral ventricles. Extensive migration of ASCs was mainly performed through white matter tracks in the corpus callosum and fimbria of hippocampus. Six weeks PT ASCs retained the characteristics of mesenchymal cells and did not differentiate into cells of neural lineage. ASCs exhibited limited long-term survival, which is restricted in perivascular areas probably contributing to vascular formation. Homing of ASCs into peri-injured area was detected in half of the animals and achieved through the corpus callosum, as revealed by the collagen depositions, in this white matter track. Transplanted ASCs reduced the area of TBI cavity and did not enhance the astroglial scarring in peri-injured area. In TBI +ASCs transplanted animals, the cortical injury site, showed a significantly smaller volume and lower $\%$ tissue loss compared to that of TBI+vehicle animals (1.90 $\pm 0.38 \mathrm{~mm}^{3}$ and $12.25 \pm 2.83 \%$ respectively, versus 1.12 $\pm 0.34 \mathrm{~mm}^{3}$ and $6.57 \pm 1.67 \%, \quad \mathrm{P}=0.015$ and $\mathrm{P}=0.019$ respectively).

Conclusions: Considering the effects of ASCs on inflammation and regeneration, we suggest that their transplantation after brain injury may promote host brain repair mechanisms. ASCs transplantation may be beneficial in TBI, however some of its effects need careful and indepth evaluation.

Disclosure: Nothing to declare

\section{P222}

Cytoprotective autophagy in endothelial cells may promote hematopoiesisin poor graft function patients after allo-transplant

\section{Xie-Na Cao ${ }^{I}$, Yuan Kong ${ }^{I}$, Zhong-Shi Lyu ${ }^{1,2}, Q i W^{1} n^{I}$, Min-Min Shi, , Qian-Yu Sun ${ }^{1}$, Yu-Hong Chen ${ }^{1}, Y u$ Wang $^{1}$, Lan-Ping X ${ }^{1}$, Xiao-Hui Zhang ${ }^{1}$, Xiao-Jun Huang ${ }^{1,2}$}

${ }^{1}$ Peking University People's Hospital, Peking University Institute of Hematology, Beijing Key Laboratory of Hematopoietic Stem Cell Transplantation, Collaborative Innovation Center of Hematology, Peking University, Beijing, China, ${ }^{2}$ Peking-Tsinghua Center for Life Sciences, Academy for Advanced Interdisciplinary Studies, Peking University, Beijing, China

Background: Poor graft function (PGF) remains a serious complication after allogeneic hematopoietic stem cell transplantation (allo-HSCT). Our previous work reported that abnormal bone marrow (BM) endothelial cells (ECs) were involved in the pathogenesis of PGF patients after allo-HSCT (BBMT2013; BMT 2016; Blood2016), but the explicit mechanism requires further clarification. Autophagy is a self-degradative process responsible for the elimination of cytosolic components including proteins and damaged organelles. Recent findings demonstrated that stimulation of autophagy could reduce oxidative status and angiogenic potential in ECsafter high-glucose exposure, from diabetic patients.However, little is known regarding the autophagy of BM ECs in PGF patients. Therefore, the current study was performed to evaluate whether autophagy in BM ECs play a role in the pathogenesis of PGF. Moreover, to investigate the effects of autophagic regulation on ECs and thereby regulating hematopoietic stem cell (HSCs).

Methods: In the prospective case-control study, the autophagy levels were compared in BM ECs from PGF patients, and their matched good graft function (GGF) patients. The expression levels of autophagy-related markers (LC3, Beclin1, and P62), and intracellular autophagosomes were detected by immunohistochemical staining, flow cytometry, western blot and transmission electron microscopy. Subsequently, rapamycin (the autophagy activators) or hydroxychloroquine (HCQ, the autophagy inhibitor) were administrated tothe 7-day cultivated BM ECs and Human Umbilical Vein Endothelial Cells (HUVECs), respectively.The autophagic vacuoleswere detected by Monodansylcadaverine (MDC) staining assay. The BM ECsand HUVECs were evaluated by cell counting, DiI-AcLDL and FITC-lectin-UEA-1 double staining, migration, cell proliferation, and levels of reactive oxygen species (ROS). To explore whether autophagy would affect the ability of BM ECs to support HSCs in vitro, BM CD34+ cells from healthy donors were co-cultured with cultivated BM ECs and HUVECs. Colony-forming unit (CFU) and the apoptosis of co-cultured HSCs were analyzed.

Results: The defective autophagy in BM ECs, characterized by decreased intracellular autophagosomes and autophagic vacuoles, decreased expression of LC3-II and Beclin1, and high level of P62, were observed in PGF patients compared with GGF patients. Moreover, the coculture of BM CD34+ cells with BM ECs showed significant deficient CFU plating efficiency, and increased apoptosis of CD34+ cells in PGF patients. In vitro upregulation of autophagy by rapamycin quantitatively and functionally improved BM ECsand HUVECs, which manifested as more DiI-Ac-LDL and FITC-lectin-UEA-1 double stained cells, increased capacities of migration, lower levels of ROS and apoptosis via regulating Beclin1 pathway, whereas inhibition of autophagy by HCQ aggravated the HUVECs and BM ECs from PGF patients. Furthermore, in vitro upregulation of autophagy by rapamycin significant improved CFU plating efficiency, 
and decreased apoptosis in BM HSCs co-cultured with HUVECs and BM ECs from PGF patients.

Conclusions: These findings suggest that defective autophagy in BM ECs may be involved in the pathogenesis of PGF. The effect of rapamycin in PGFpatients is potentially mediated by improving the dysfunctional BM ECsto support HSCs. Therefore, it would be of value to investigate whether upregulating of cytoprotective autophagy of BM ECs may ameliorate PGF, thereby providing a novel clinical intervention for PGF in the future.

Clinical Trial Registry: None

Disclosure: Nothing to declare

\section{P223}

Role of heparanase gene single nucleotide polymorphisms in allogeneic stem cell transplantation

Elisabetta Metafuni, Gessica Minnella ${ }^{1}$, Monica Rossi, ${ }^{1}$, Sabrina Giammarco ${ }^{1}$, Silvia Bellesi ${ }^{I}$, Maria Giovanna De Ritis $^{I}$, Sara Marietti ${ }^{I}$, Simona Sica ${ }^{I}$, Andrea Bacigalupo ${ }^{I}$, Patrizia Chiusolo ${ }^{I}$

${ }^{1}$ Fondazione Policlinico Universitario A.Gemelli, Rome, Italy

Background: Heparanase (HPSE) in an endo$\beta$-glucuronidase that specifically cleaves the saccaride chains of heparan sulphate proteoglycans (HS), leading to a loss of integrity of the extracellular matrix and to release of HS-bound cytokines, chemokines, angiogenic and growth factors. HPSE gene is polymorphic and includes approximately 300 SNPs. The combination of two SNPs, rs4693608 and rs4364254, are involved in the regulation of HPSE expression with an inverse correlation between mRNA expression and protein levels: GG-CC, GG-CT, GG-TT, GA-CC (Low group) expressed high HPSE concentration; GA-CT and GA-TT (Median group) expressed intermediate HPSE levels; AA-TT and AA-CT expressed low HPSE concentration (High group). We studied HPSE SNPs in the allogeneic stem cell transplantation (HSCT) setting to evaluate a possible association with post-HSCT outcomes.

Methods: We enrolled 228 patients submitted to HSCT in our department since 2005 to 2016. For each couple recipient-donor, rs4693608 SNP was genotyped using restriction fragment lenght polymorphism assay, whereas for rs4364254 SNP an allele-specific polimerase chain reaction was applied. HPSE genotype distribution was compared in different groups according to post-HSCT outcome: graft-versus-host disease (GvHD), transplantrelated mortality (TRM), overall survival (OS), infectious complication and disease-free survival (DFS). Statistical analysis was performed using NCSS 10.
Results: Distribution of rs4693608 SNP was as follows: GG $16.7 \%$, GA $49.8 \%$ and AA $33.5 \%$ among recipients and $17.6 \%, 53.3 \%$ and $29.1 \%$ among donors, respectively. Hardy-Weinberg equilibrium (HWe) was respected. Distribution of rs4364254 SNP was as follows: CC $15.1 \%$, CT $37.8 \%$ and TT $37.8 \%$ among recipients and $15.3 \%, 36 \%$ and $48.7 \%$ among donors, respectively. rs4364254 SNP distribution did not respect the HWe.

An association was found between recipient rs4364254 SNP and the cumulative incidence of aGvHD among patients submitted to a reduced intensity conditioning (RIC): $37.3 \%$ for TT genotype and $69 \%$ for CT or CC genotype $(\mathrm{p}=0.03)$. on the other hand, an association was identified between donor rs4693608/rs4364254 SNPs combination and the cumulative incidence of aGvHD: $81.5 \%$ for Low group donor, $48 \%$ for Median group donor and $40.8 \%$ for High group donor $(\mathrm{p}=0.04)$.

Conversely, AA genotype for donor rs4693608 resulted independent risk factor for cGvHD de novo development $(p=0.049$, OD 2.1) together to donor-recipient sex mismatch (female donor to male recipient vs. others: $p=0.005$, OD 3.56). Considering CMV reactivation rate after HSCT, an association was observed according to recipient rs4364254 SNP: $82 \%$ for CC genotype, $63.5 \%$ for CT genotype and $57.1 \%$ for TT genotype $(\mathrm{p}=0.049)$. Multivariate analysis confirmed recipient rs4364254 SNP as independent risk factor for CMV reactivation after HSCT $(\mathrm{p}=0.04$, OD 2.62) together with recipient CMV serostatus at transplant (positive vs. negative: $\mathrm{p}<0.01$, OD 8.49).

Conclusions: HPSE role was widely studied in the setting of inflammation, autoimmune diseases, hematological disease and tumor. However, it still remains debated the inducing or protective activity of HPSE in the setting of GvHD. Obviously, our results need to be confirmed in a validation cohort.

Clinical Trial Registry: NA

Disclosure: Nothing to declare

\section{P224}

Novel protocol for autologous HSCT in patients with high risk of complications: Ambulatory chemomobilization and transplantation of fresh hematopoietic stem cells with backup storage

Emilian Snarski', Anna Waszczuk Gajda ${ }^{1}$, Michat Górka $^{1}$, Martyna Maciejewska ${ }^{I}$, Elżbieta Urbanowska ${ }^{1}$, Karol Lis ${ }^{I}$, Magdalena Feliksbrot Bratosiewicz ${ }^{I}$, Kamila Skwierawska ${ }^{1}$, Hubert Heleniak, Mateusz Ziarkiewicz, ${ }^{1}$, Król Matgorzata ${ }^{1}$, Maria Króll, Wiestaw Wiktor Jędrzejczak ${ }^{I}$

${ }^{1}$ Medical University of Warsaw, Warsaw, Poland 
Background: Autologous hematopoietic stem cell transplantation (AHSCT) is standard of treatment in many patients with high risk of complications: dialysed patients, patients with heart and kidney amyloidosis or patients with systemic sclerosis. We introduced recently a novel protocol for AHSCT: combination of ambulatory mobilization with very low doses of Ara-C and G-CSF connected with direct AHSCT with fresh cells. This protocol allowed us to reduce the transplant risk in various patient groups traditionally connected with high risk of complications. In this work we summarize the experience in such high risk patients.

Methods: The prospectively collected database of patients after AHSCT was searched for patients who underwent AHSCT after chemomobilization with Ara-C and transplantation with fresh cells and who fulfilled at least one study inclusion criteria: a) dependence on dialysis b) amyloidosis c) systemic sclerosis d) disqualification from transplantation at other centre due to the high risk of complications. There were together 19 patients selected for this analysis - 9 with amyloidosis (6 with $\geq 2$ organs involved), 9 dialysed, 2 with systemic sclerosis, 2 unfit at other centre. The database included prospectively recorded serious adverse events during the mobilization and transplantation.

Results: There were 20 transplantations performed in this group of patients. Mortality was $0 \%$ at 100 days. All patients underwent successful ambulatory mobilization. All patients received Mephalan conditioning with single infusion with median dose of $200 \mathrm{mg} / \mathrm{m} 2$ (MIN 140, MAX 200). Mean engraftment was 10.5 days for white blood cells and 12.7 days for PLT over $20 \mathrm{G} / \mathrm{L}$. The rate of complications was low with 7 cases of neutropenic fewer, 1 single bacterial culture with Staphylococcus Epidermidis without clinical signs of infection, median mucositis grade of 0.4 and without patients on parenteral nutrition. The median time of hospitalization was 19 days (MIN 14, MAX 29).

Conclusions: We present here novel protocol of transplantation combining chemomobilization and AHSCT with fresh cells with excellent safety profile among most severely ill patients allowing for safe and efficient transplants. With this protocol we were able to overcome multiple risk factors and perform full intensity transplantation in very fragile patients.

Disclosure: Nothing to declare

\section{P225}

Single umbilical cord blood transplantation provides durable disease remission of advanced hematological malignancies in elderly patients

\section{Takashi Mitsuki ${ }^{I}$, Yuki Taya ${ }^{I}$, Kazuya Ishiwata ${ }^{I}$, Daisuke Kaji $^{1}$, Aya Nishida ${ }^{1}$, Shinsuke Takagi ${ }^{1}$, Hisashi}

Yamamoto $^{I}$, Go Yamamoto ${ }^{I}$, Naoyuki Uchida ${ }^{I}$, Shuichi Taniguchi $^{1}$, Atsushi Wake ${ }^{I}$

${ }^{1}$ Toranomon Hospital, Kajigaya, Kawasaki, Japan,

Background: Although allogeneic hematopoietic stem cell transplantation (allo HSCT) is potentially curative therapy in a variety of hematological malignancies, little has been reported of the outcome for elderly patients who are not in remission at transplantation. But it has been pointed out that recipient age alone can not be regarded as contraindication for allo HSCT in the literature recently, supported by suitable donor, conditioning regimens and appropriate management of complications. We conducted a retrospective study of elderly patients who had advanced hematologic malignancies to elucidate the outcome of single umbilical cord blood transplantation (sUCBT) in Toranomon Hospital Kajigaya, Japan.

Methods: We retrospectively investigated the outcomes of 19 patients aged over 65 who underwent their first UCBT from June 2013 to December 2017 in our medical center.

Results: Diseases included acute myelogenous leukemia $(\mathrm{n}=12)$, myelodysplastic syndrome $(\mathrm{n}=3)$, adult $\mathrm{T}$-cell leukemia/lymphoma $(\mathrm{n}=2)$, myelofibrosis $(\mathrm{n}=1)$ and chronic lymphocytic leukemia $(\mathrm{n}=1)$. The median age at transplantation was 69 years (range, 65-75) and follow-up for survivor post transplantation was 642 day (range, 391767). All patients were not in complete remission (CR) at the time of transplantation. Reduced intensity conditioning (RIC) regimens were used in 10 patients. All patients received tacrolimus and mycophenolate mofetil as graftversus-host disease (GVHD) prophylaxis. All cases except 4 early death achieved neutrophil recovery at median 18 days (range, 13-28). At 1 year, overall survival (OS) rate and disease free survival (DFS) were $31,6 \%$ (95\% confidence interval (CI), 12.9-52.2). We performed univariate analysis to identify the factor that influenced OS at 1 year, but no statistical significance was demonstrated at the age of transplantation (Aged 65 to 69 vs. $\geqq 70,33.3 \%$ (95\% $\mathrm{CI}, 10.3-58.8$ ) vs. $42.9 \%$ (95\% CI, 9.8-73.4), $\mathrm{p}=0.68$ ). The cumulative incidence of non-relapse mortality (NRM) at 100 days was $47.4 \%$ (95\% CI, 23.6-67.9\%) and relapse at 1 year was $9.1 \%$ (95\% CI, $0.0-24.6 \%$ ). Only two patients developed acute GVHD(II-IV) and one developed severe GVHD at 49 days after transplantation. The main causes of death was infection $(n=10)$, including sepsis $(n=8)$ and viral encephalitis $(n=2)$, followed by idiopathic pneumonia syndrome $(n=2)$ and thrombotic microangiopathy $(n=1)$ during the early phase of transplantation. In contrast, no patients died of recurrence.

Conclusions: Although our report consisted relapsed/ refractory disease of elderly patients at the time of sUCBT, durable remission and lower incidence of GVHD could be 
noteworthy compared with previous reports. Further strategies to reduce the rate of NRM and longer duration of follow up would be warranted.

Disclosure: Nothing to declare

Gene Therapy

\section{P226}

Autologous CD34 + cells enriched with normal mitochondria for non-inherited mitochondrial deletion syndromes

Elad Jacoby ${ }^{1,2}$, Omer Bar Yoseph ${ }^{1,2}$, Moria Blumkin ${ }^{1}$, Noah Gruber ${ }^{1,2}$, Nira Varda-Bloom ${ }^{1}$, Einat Lahav ${ }^{2,1}$, Julia Pansheen ${ }^{1}$, Sharon Barak', Etzyona Eisenstein ${ }^{1}$, Jaana Ahonniska-Assa ${ }^{1}$, Gal Dubnov-Raz ${ }^{1,2}$, Bella Bielorai $^{1,2}$, Hana Golan ${ }^{1,2}$, Michal Besser ${ }^{2,1}$, Jacob Schachter ${ }^{2,1}$, Noa Sher ${ }^{3}$, Natalie Yivgi Ohana ${ }^{1}$, Yair Anikster $^{2,1}$, Amos Toren ${ }^{1,2}$

${ }^{1}$ Sheba Medical Center, Ramat Gan, Israel, ${ }^{2}$ Sackler Faculty of Medicine, Tel Aviv University, Tel Aviv, Israel, ${ }^{3}$ Minovia Therapeutics, Tirat Hacarmel, Israel

Background: Pearson syndrome and Kearns-Sayre syndrome are metabolic disorders caused by a de-novo deletion in the mitochondrial DNA (mtDNA). Allogeneic stem cell transplantation has shown to improve metabolic function in distal organs in several metabolic disorders, but bears significant morbidity and mortality, especially for patients with mitochondrial disorders. Novel gene therapies may correct diseases rising from genomic DNA mutations, but targeting the mitochondrial DNA is complex. Mitochondria are able to transfer into cells and between cells, as seen in preclinical models of mitochondrial and other metabolic disorders. Here, we introduce a novel concept of mitochondrial augmentation therapy (MAT) of autologous CD34+ cells in 4 children with mitochondrial deletion syndromes.

Methods: Patients were treated under a compassionateuse program, approved by the Sheba Medical Center IRB and the Israeli Ministry of Health. Briefly, mobilization was performed using GCSF alone $(n=1)$ or in addition to plerixafor $(n=3)$. CD34+ cells were isolated via Miltenyi CliniMACS system and co-cultured with maternal mitochondria, drawn from peripheral blood and confirmed nondeleted, for 24 hours, and re-infused to the patient without any conditioning. Patients were followed for clinical and metabolic parameters.

Results: All four patients presented with different deletions in mitochondrial DNA, and different baseline characteristics, and were treated at the age of $6.5,7,11$ and 14 years. Despite normal CBC, significant bone marrow hypocellularity was seen in 3 evaluated patients $(20 \%, 30 \%$ and $50 \%$ cellularity at age 7,11 and 14 ), which correlated with low colony forming unit capacity of patients and low yield of CD34+ mobilization in the leukapheresis product. Patients received on average $2 \times 10^{6}$ enriched cells $/ \mathrm{kg}$ (range, 1.1 - 2.8), and the median enrichment of CD34+ cells was $135 \%$ (range, $103-162 \%$ ). No infusion reactions occurred, and the only severe adverse events of this cellular therapy were leukapheresis-related anemia, hypokalemia, hypocalcemia and alkalosis, all resolved promptly with proper supplementation. Follow-up duration is variable, ranging 522 months. We were able to show improvement in mitochondrial heteroplasmy (proportion of deleted mtDNA of total mitochondrial DNA) and in normal mtDNA content, starting 1-5 months from cell therapy, which correlated with improved ATP production in peripheral blood derived mononuclear cells. Clinically, patients showed improvement in aerobic function and endurance (measured by the half-Bruce protocol, sit-to-stand test and 6-minute walk test), muscle strength (hand-held dynamometry), and in quality of life, measured by the international pediatric metabolic disability scale. No metabolic crises occurred following cell infusion.

Conclusions: Patients with deletion in mtDNA have metabolic dysfunction, including poor bone marrow cellularity and function. Hematopoietic stem cells in patients with mtDNA deletions can be enriched with normal mitochondria, via MAT, as first shown in our patients. This novel process is safe and results in increase in the normal mtDNA in peripheral blood of patients, and in improved metabolic and clinical function.

Clinical Trial Registry: Clinicaltrials.gov NCT03384420

Disclosure: Moria Blumkin, Noa Sher and Natalie Yivgi Ohana - Minovia Therapeutics, employment

\section{P227}

High cytotoxic efficiency of alpharetrovirally engineered CD19-specific chimeric antigen receptor natural killer cells for treatment of acute lymphoblastic leukemia

Stephan Müller ${ }^{1}$, Tobias Bexte ${ }^{1}$, Annekathrin Heinze ${ }^{1}$, Franziska Schenk ${ }^{2}$, Axel Schambach ${ }^{3}$, Winfried $S$. Wels $^{4,5}$, Ute Modlich ${ }^{2}$, Evelyn Ullrich ${ }^{1,5}$

${ }^{1}$ Experimental Immunology, University Hospital Frankfurt, Goethe University, Frankfurt am Main, Germany, ${ }^{2}$ Research Group for Gene Modification in Stem Cells, Paul-Ehrlich Institut, Langen, Germany, ${ }^{3}$ Institute of Experimental Hematology, Hannover, Germany, ${ }^{4}$ Georg Speyer Haus, Institute for Tumor Biology and Experimental Therapy, Frankfurt am Main, Germany, ${ }^{5}$ German Cancer Consortium (DKTK), Frankfurt am Main, Germany 
Background: Autologous chimeric antigen receptormodified (CAR) T cells with specificity for CD19 showed potent antitumor efficacy in clinical trials regarding relapsed and refractory acute lymphoblastic leukemia (ALL). Natural killer (NK) cells are cytotoxic lymphocytes that are capable to kill their targets in a non-specific manner and additionally do not cause GvHD. Therefore, using CD19-CAR-NK cells exhibits several advantages, such as safety in clinical use, possible allogenic settings and the potential to also attack heterologous leukemia cells which lost CD19. Previous approaches used CD19-CAR-NK cells pre-stimulated by feeder cells, bearing potential risks. Thus, we focused on the optimization of generating CD19-CAR-NK cells by viral transduction under feeder-cell free conditions.

Methods: Human NK cells were isolated from healthy donor peripheral blood mononuclear cells via CD56 negative selection. After a feeder-cell free expansion phase with interleukin 15, transductions were performed with an EGFP or a CD19-CAR encoding vector at different multiplicities of infection (MOI). To optimize gene modification different transduction enhancers (Retronectin and Vectofusin-1) and viral vector systems (lentiviral and alpharetroviral) were compared. Finally, generated CD19CAR-NK cells were tested in their ability to kill CD19positive and CD19-negative cell lines.

Results: NK cells transduced with a lentiviral EGFP encoding vector or a lentiviral CD19-CAR vector using Retronectin and Vectofusin-1 showed similar transduction efficiencies for both transduction enhancers (EGFP: Retronectin MOI 10: 12.9\%; Vectofusin-1 MOI 10: $12.8 \%$; CD19-CAR: Retronectin MOI 5: 10.7\%, MOI 10: 9.2\%; Vectofusin-1 MOI 5: 11.3\%, MOI 10: 14.4\%). The generated CD19-CAR-NK cells showed increased cytotoxic capacity against CD19-positive cells compared to nontransduced (NT) NK cells $(72.7 \%$ vs. $23.6 \%$, effector to target (E:T) ratio 1:1). Both NK cell populations were equally efficient in killing CD19-negative cells (30.5\% vs. $25.7 \%$ ).

Alpharetroviral transduction of NK cells with an EGFP encoding vector showed higher transduction rates with Vectofusin-1 than with Retronectin (Retronectin MOI 1: 5.3\%, MOI 5: 9.7\%; Vectofusin-1 MOI 1: 55.3\%, MOI 5: $51.6 \%$ ). Further using Vectofusin-1, similar transduction efficiencies could be achieved with an alpharetroviral CD19-CAR encoding vector (MOI 1: 11.1\%, MOI 5: $49.2 \%$, MOI 10: $68.9 \%$ ), outperforming the efficiencies of lentivirally generated CD19-CAR-NK cells in the same experiments (MOI 1: 1.5\%, MOI 5: 8.4\%, MOI 10: 14.9\%). Additionally, alpharetroviral CD19-CAR-NK cells showed a higher cell killing activity against CD19-positive cells than lentiviral CD19-CAR-NK cells or NT-NK cells $(90.5 \%$ vs. $62.5 \%$ vs. $9 \%$, E:T ratio 1:1). Interestingly, similar killing activities were achieved with an E:T ratio of $0.5: 1$
( $88.9 \%$ vs. $58.3 \%$ vs. $10.3 \%$ ) and alpharetroviral CD19CAR-NK cells remained a stable cytotoxicity level at lower cell concentrations down to an E:T ratio of $0.1: 1$. All three NK cell populations were equally efficient in killing CD19negative cells ( $16.4 \%$ vs. $23.6 \%$ vs. $12.3 \%$, E:T ratio $1: 1)$.

Conclusions: CD19-CAR-NK cells can be successfully generated under feeder-cell free conditions using different transduction enhancers and viral vector systems. These data suggest the usage of Vectofusin-1 in combination with alpharetroviral vectors to genetically modify NK cells to achieve sufficient amounts of transduced cells. These CD19-CAR-NK cells mediate high cytotoxicity and therefore may offer a new therapeutic option in the treatment of ALL.

Disclosure: Axel Schambach is an inventor on a patent describing alpharetroviral SIN vectors. Winfried S. Wels is an inventor on a patent describing chimeric antigen receptors with an optimized hinge region. The remaining authors have nothing to disclose.

Graft-versus-host disease - clinical

\section{P228}

Treatment of acute refractory gastrointestinal graftversus-host-disease by fecal microbiota transplantation

Walter Spindelböck ${ }^{1}$, Bianca Huber-Krassnitzer ${ }^{1}$, Barbara Uhl, Gregor Gorkiewicz ${ }^{I}$, Hildegard Greinix ${ }^{I}$, Christoph Högenauer ${ }^{I}$, Peter Neumeister ${ }^{I}$

${ }^{1}$ Medical University of Graz, Graz, Austria

Background: Steroid-refractory acute gastrointestinal (GI) graft-versus-host disease (aGvHD) is a severe complication of allogeneic hematopoietic stem cell transplantation (alloHSCT) associated with a high mortality rate. Loss of intestinal bacterial diversity is thought to be associated with severity of GI-aGvHD and an impaired intestinal microbiota with reduced diversity is an independent predictor of mortality.

Methods: The fecal microbiota transplantation (FMT) procedures were performed according to a protocol approved by the local ethical committee (29-027ex 16/17) after obtaining informed consent. Donors were healthy adult subjects screened for potential infections by serologic and microbiologic tests according to local standards. Donor stool was diluted with saline and homogenized to a volume of $\sim 250 \mathrm{ml}$ fecal solution for instillation into the terminal ileum and caecum via colonoscope. Microbiota sequencing analysis of $16 \mathrm{~s}$ rDNA was performed before FMTs and afterwards at predefined timepoints.

Results: We report the outcome of nine patients refractory to 3-6 lines of immunosuppressive therapies with lower GI-stage III $(n=1)$ or IV $(n=8)$ aGvHD following 
repetitive FMTs from a single donor. All patients had received an allo-HSCT for MDS $(n=3)$, AML $(n=4)$, PMF $(\mathrm{n}=1)$ and $\mathrm{MM}(\mathrm{n}=1)$ following a reduced intensity $(\mathrm{n}=5)$ or MAC $(n=4)$ conditioning regimen using PBSC as stem cell source. After an onset of lower GI aGvHD between 11465 days after allo-HSCT, nine patients refractory to several lines of immunosuppressive therapies received 1-6 FMTs (6 patients were treated with more than 2 FMTs, in 3 patients FMT was only administered once or twice) mostly in weekly intervals. Five patients achieved a clinical complete response with resolved diarrhea and no gastrointestinal complaints, and four of these could be discharged without GvHD symptoms. Two patients (PR, NC) were discontinued after 2 or 3 FMTs in PR or NC due to concomitant infections (Metapneumoviral pneumonia, CMV gastroenteritis), the 2 other non-responders succumbed to GvHDrelated infectious complications. The establishment of donors' microbiota with the emergence of new taxa, an increase in bacterial richness/diversity, and the disappearance of the "enterococcus signature" were associated with disease control and response to FMT. Except the possible transmission of Adenovirus by FMT in one patient, no other immediate procedure-related infections or other side effects were observed.

Conclusions: Restoration of dysbiosis by FMT might represent a promising novel therapeutic approach for a subset of patients with refractory lower GI-aGvHD. Vigorous donor screening for infectious disease is mandatory.

Clinical Trial Registry: Local ethical committee $(\mathrm{N}=$ 29-027ex 16/17) https://www.medunigraz.at/ ethikkommission/Graz/

Disclosure: Nothing to declare

\section{P229}

GVHD prophylaxis with KRP203, a sphingosine-1phosphate receptor modulator, is safe and may have the potential to improve survival in patients undergoing allogeneic stem cell transplantation

Dominik Heim ${ }^{1}$, Jakob Passweg ${ }^{1}$, Michael Medinger ${ }^{1}$, Urs Schanz $^{2}$, Jürgen Finke ${ }^{3}$, Julia Stomper ${ }^{3}$, Ernst Holler ${ }^{4}$, Julia Schmid ${ }^{4}$, Inken Hilgendorf ${ }^{5}$, Nils Winkelmann ${ }^{5}$, Udo Holtick, Christoph Scheid ${ }^{6}$, Gérard Socié ${ }^{7}$, Marie Robin $^{7}$, Noel-Jean Milpied ${ }^{8}$, Edouard Forcade ${ }^{8}$, Ibrahim Yakoub-Agha ${ }^{9}$, Leonardo Magro ${ }^{9}$, Philip Jarvis ${ }^{10}$, Julie Jones $^{10}$, David Sanchez ${ }^{10}$, Navon Mahajan ${ }^{10}$, Malika Souquieres $^{10}$, Christoph Bucher ${ }^{10}$, Peter Gergely ${ }^{10}$

${ }^{1}$ University Hospital Basel, Basel, Switzerland, ${ }^{2}$ University Hospital Zürich, Zurich, Switzerland, ${ }^{3}$ University of Freiburg, Freiburg, Germany, ${ }^{4}$ University Hospital Regensburg, Regensburg, Germany, ${ }^{5}$ Universitätsklinikum Jena, Jena,
Germany, ${ }^{6}$ University of Cologne, Cologne, Germany, ${ }^{7}$ Hôpital Saint Louis, Paris, France, ${ }^{8} \mathrm{CHU}$ Bordeaux, Hôpital Haut-Leveque, Pessac, France, ${ }^{9}$ Lille University Hospital, INSERM U 995, Lille, France, ${ }^{10}$ Novartis Institute of Biomedical Research, Basel, Switzerland

Background: Migration of allo-activated donor effector Tcells from lymphoid tissues to target organs is an important step in acute graft versus host disease (GvHD). The sphingosine-1-phosphate-1 (S1P1) receptor plays a crucial role in lymphocyte trafficking. Data from animal models suggest that pharmacological modulation of the S1P1 receptor reduces GvHD and improves mortality. We investigated this mode of action by using the secondgeneration S1P1 modulator KRP203 for the prophylaxis of GvHD in a pilot clinical trial in patients undergoing allogeneic HSCT.

Methods: A multi-centric, Phase 1b, prospective, open label, two-part study was conducted to evaluate the safety, tolerability and pharmacokinetics of KRP203 in patients undergoing allogeneic HSCT for hematological malignancies. Primary endpoint was safety. Initial efficacy was explored based on the incidence of GvHD, mortality and relapse. Part 1 was a single arm open label study to investigate the safety of $3 \mathrm{mg} / \mathrm{day} \mathrm{KRP} 203$ added to standard of care GvHD prophylaxis (CsA/MTX) in 10 patients. Part 2 was a randomized two-arm open label study to compare the safety, efficacy and PK of $3 \mathrm{mg} /$ day of KRP203 in combination with tacrolimus/MTX to $1 \mathrm{mg} /$ day of KRP203 in combination with CsA/MTX in 13 patients. In both parts, treatment with KRP203 was initiated 10 days before HSCT and continued for an additional 100 days. Patients were followed up for up to 2 years.

Results: 23 patients were included in the study. 16 of 23 patients completed the 110-day treatment with KRP203 at the assigned doses. Median duration of follow-up was 264 days (range 153 to 271 days). KRP203 was safe and well tolerated. 11 serious adverse events (SAEs) suspected to be related to KRP203 were observed. Macular edema $(\mathrm{n}=3)$ and peripheral edema $(\mathrm{n}=1)$ as S1P related adverse events occurred and resolved without sequelae. Of note, the incidence of macular edema in HSCT recipients is unknown. Neutrophil engraftment was confirmed in all patients with a median of 16 days (range 12 to 45 days). 5 of 23 patients presented with Grade III or IV acute GvHD (on Days 50, 56, 68, 99 and 102). No GvHD or infection related death occurred during the first 100 days. 100-day survival was $96 \%$, with no death occurring during KRP203 treatment. 1 death occurred on Study Day 90 due to lymphoma relapse. A second death occurred on Study Day 121 due to liver GvHD. Four patients died in the follow-up period due to gastrointestinal GVHD (Day 265), aspiration pneumonia (Day 327) and relapse (Day 533 and Day 877). 
The Kaplan-Meier estimate of overall survival at 1 year was 0.75 . When comparing the data from the two dose groups (1 and $3 \mathrm{mg} \mathrm{KRP203),} \mathrm{no} \mathrm{major} \mathrm{differences} \mathrm{in} \mathrm{safety,}$ engraftment, GvHD rate or mortality were observed.

Conclusions: This clinical trial was the first to test S1P modulation in this population. Our data suggest that KRP203 had no negative impact on engraftment and overall, was safe, and well tolerated. Based on exploratory data, when comparing to matched historical mortality data, KRP203 may have favorable effects on overall survival (Figure 1).

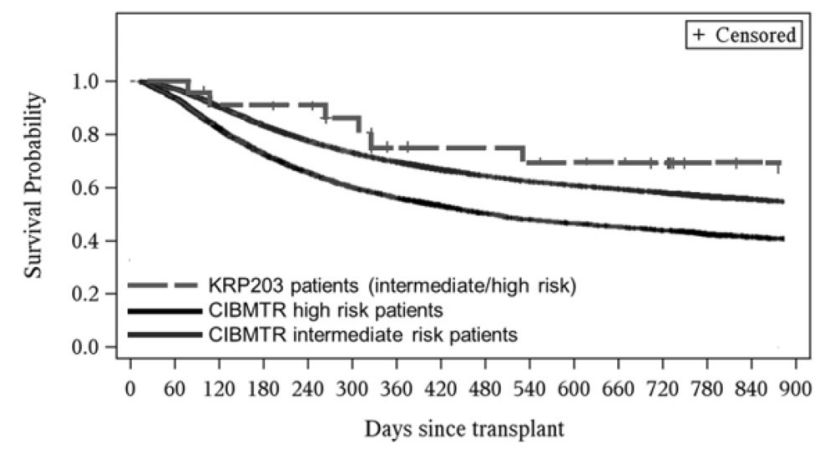

[[P229 Image] 1. Overall survival on KRP203 vs CIBMTR database]

\section{Clinical Trial Registry: NCT01830010}

Disclosure: Dominik Heim: no conflict of interest, Jakob Passweg: no conflict of interest, Michael Medinger: no conflict of interest, Urs Schanz: no conflict of interest, Jürgen Finke: Novartis: Consultancy, Honoraria, travel grants and Research Funding, Julia Stomper: no conflict of interest

Ernst Holler: no conflict of interest, Julia Schmidt: no conflict of interest, Inken Hilgendorf: Novartis: Travel support and Research Funding, Nils Winkelmann: no conflict of interest, Udo Holtick: no conflict of interest, Christoph Scheid: Novartis: Honoraria and Research Funding, Noel-Jean Milpied: no conflict of interest, Edouard Forcade: no conflict of interest, Ibrahim YakoubAgha: no conflict of interest, Leonardo Magro: no conflict of interest, Philip Jarvis: Novartis employee, Julie Jones: Novartis employee, David Sanchez Ramos: Novartis employee, Navin Mahajan: Novartis employee

Malika Souquieres: Novartis employee, Peter Gergely: Novartis employee, Christoph Bucher: past Novartis employee

\section{P230}

Association of uric acid levels before start of conditioning with mortality after alloSCT - a prospective, non-interventional study of the EBMT transplant complication working party
Olaf Penack ${ }^{1}$, Christophe Peczynski ${ }^{2}$, Steffie van der Werf ${ }^{3}$, Mohammad Mohty ${ }^{4}$, Ibrahim Yakoub-Agha ${ }^{5}$, Sylvia Montoto ${ }^{6}$, Jürgen Finke ${ }^{7}$, Arnold Ganser ${ }^{8}$, Helene Schoemans $^{9}$, Jane Apperley ${ }^{10}$, Ritta Niittyvuopio ${ }^{11}$, Wilfried Schroyens $^{12}$, A Unal ${ }^{13}$, Armin Gerbitz ${ }^{1}$, Michael Schaap $^{14}$, J Sierra ${ }^{15}$, Agostino Cortelezzi ${ }^{16}$, Gerald Wulf ${ }^{17}$, Pascal Turlure $^{18}$, Montserat Rovira ${ }^{19}, Z^{2}$ Ozkurt $^{20}, M$ Pascual Cascon ${ }^{21}$, Johannes Clausen ${ }^{22}$, Hildegard Greinix $^{23}$, Rafael Duarte ${ }^{24}$, Grzegorz Basak ${ }^{25}$

${ }^{1}$ Charité Univerisätsmedizin Berlin, Berlin, Germany, ${ }^{2}$ EBMT Paris Study Office/CEREST-TC, Hôpital Saint Antoine, Paris, France, ${ }^{3}$ EBMT Leiden Data Office, Leiden, Netherlands, ${ }^{4}$ Hospital Saint Antoine, Paris, France, ${ }^{5} \mathrm{CHRU}$ Lille, Lille, France, ${ }^{6}$ St. Bartholomew's and the Royal London NHS Trust, London, United Kingdom, ${ }^{7}$ University Freiburg, Freiburg, Germany, ${ }^{8}$ University Hannover, Hannover, Germany, ${ }^{9}$ University Hospital Gasthuis, Leuven, Belgium, ${ }^{10}$ Imperial College London, London, United Kingdom, ${ }^{11}$ HUCH Comprehensive Cancer Center, Helsinki, Finland, ${ }^{12}$ Antwerp University Hospital, Antwerp, Belgium, ${ }^{13}$ Erciyes Medical School, Kayseri, Turkey, ${ }^{14}$ Radboud University, Nijmegen, Netherlands, ${ }^{15}$ Hospital Santa Creu i Sant Pau, Barcelona, Spain, ${ }^{16}$ Fondazione IRCCS - Ca'Granda, Milan, Italy, ${ }^{17}$ Universitätsklinikum Göttingen, Göttingen, Germany, ${ }^{18}$ CHRU Limoge, Limoge, France, ${ }^{19}$ Hospital Clinic, Barcelona, Spain, ${ }^{20}$ Gazi University Faculty of Medicine, Ankara, Turkey, ${ }^{21}$ Hospital Regional de Málaga, Malaga, Spain, ${ }^{22}$ Ordensklinikum, Linz, Austria, ${ }^{23}$ University Hospital Graz, Graz, Australia, ${ }^{24}$ Hospital Universitario Puerta de Hierro, Madrid, Spain, ${ }^{25}$ Central Clinical Hospital, Warsaw, Poland

Background: Uric acid is a danger signal contributing to inflammation. Relevance to alloSCT has been demonstrated in preclinical models: the depletion of uric acid led to improved survival and reduced GVHD (J Exp Med. 2013 Sep 23;210(10):1899-910). Results of a clinical pilot trial suggested that peri-transplant uric acid depletion reduce acute GVHD incidence (BBMT 2014 May;20(5):730-4).

Methods: This international multicentric study aimed to study the association of uric acid serum levels before start of conditioning with alloSCT outcome. Patients with acute leukemia, lymphoma or MDS receiving a matched sibling alloSCT for the first time were considered for inclusion, regardless of conditionning. Data were prospectively collected between $8 / 2014$ and $2 / 2018$. A comparison of outcomes between patients with high and low uric acid level was performed using univariate analysis and multivariate analysis using cause-specific Cox model. Variables included in the multivariate analyses were age, sex mismatch, diagnosis, disease status, Karnofsky score, number of CD34 cells given, intensity of conditioning, 
type of GVHD prophylaxis, ATG use, time from diagnosis to transplant, year of transplant and CMV status.

Results: Twenty centers from 10 European countries reported data on 385 alloSCT recipients. Patient characteristics are given in Table 1. The uric acid cut off point was determined at $4.3 \mathrm{mg} / \mathrm{dl}$ (median of measured uric acid levels). Overall survival (OS) and progression free survival (PFS) of alloSCT recipients with uric acid levels above cut off measured before start of conditioning were significantly shorter (Figure 1A, OS univariate $\mathrm{HR}=2.4$ $\mathrm{CI}=1.6-3.7 \mathrm{p}<0.001$; multivariate $\mathrm{HR}=2.8, \mathrm{CI}=1.7-4.7$, $\mathrm{p}<0.0001$ ) (Figure 1B, PFS univariate $\mathrm{HR}=2 \mathrm{CI}=1.1-3.7$ $\mathrm{p}=0.02$; multivariate $\mathrm{HR}=2.7, \mathrm{CI}=1.4-5, \mathrm{p}=0.003$ ). Nonrelapse mortality was significantly increased in alloSCT recipients with high uric acid levels prior to start of conditioning (univariate $\mathrm{HR}=2 \mathrm{CI}=1.1-3.7 \mathrm{p}=0.018$; multivariate $\mathrm{HR}=2.65, \mathrm{CI}=1.41-5.01, \mathrm{p}=0.003)$. In addition, there was a non-significant trend towards higher acute GVHD incidence (GVHD Grades II-IV univariate $\mathrm{HR}=1.2 \mathrm{CI}=0.8-1.9 \mathrm{p}=0.4$; multivariate $\mathrm{HR}=1.5 \mathrm{CI}=1$ $2.4, \mathrm{p}=0.08$ ) in alloSCT recipients with uric acid levels above cut off before transplantation. Finally, the incidence of relapse after alloSCT was moderately increased in the cohort with higher uric acid levels (univariate $\mathrm{HR}=1.6$ $\mathrm{CI}=1-2.5 \mathrm{p}=0.09$; multivariate $\mathrm{HR}=1.59, \mathrm{CI}=1.02-2.49$, $\mathrm{p}=0.04$ ).

Conclusions: High uric acid levels before start of conditioning correlate with high mortality after alloSCT. Our results can serve as rationale for clinical trials on depletion of uric acid during alloSCT.

\begin{tabular}{|c|c|c|c|}
\hline & $\begin{array}{l}\text { Uric acid } \\
<=4.3 \mathrm{mg} / \mathrm{dl} \\
(\mathrm{n}=186)\end{array}$ & $\begin{array}{l}\text { Uric acid } \\
>4.3 \mathrm{mg} / \mathrm{dl} \\
(\mathrm{n}=180)\end{array}$ & $\begin{array}{l}\mathrm{p}- \\
\text { value }\end{array}$ \\
\hline $\begin{array}{l}\text { Year of transplant median(range) } \\
{[\mathrm{IQR}]}\end{array}$ & $\begin{array}{l}\text { 2015(2014-2018) } \\
{[2015-2017]}\end{array}$ & $\begin{array}{l}\text { 2015(2014-2018) } \\
{[2014-2016]}\end{array}$ & 0.1 \\
\hline $\begin{array}{l}\text { Patient age (years) median } \\
\text { (range)[IQR] }\end{array}$ & $\begin{array}{l}51.8(17.1-71) \\
{[40.7-60.7]}\end{array}$ & $\begin{array}{l}54.6(19.7-71.3) \\
{[44.4-62.2]}\end{array}$ & 0.1 \\
\hline $\begin{array}{l}\text { Time from diagnosis to } \\
\text { transplant (months) median } \\
\text { (range)[IQR] }\end{array}$ & $4(1-61)[3-6]$ & $5(1-71)[3.5-7.5]$ & 0.09 \\
\hline $\begin{array}{l}\text { Number of CD34+ cells infused } \\
(\mathrm{e}+06) \text { median (range) }[\mathrm{IQR}]\end{array}$ & $\begin{array}{l}5.8(1.2-11)[4.5- \\
7.2]\end{array}$ & $\begin{array}{l}5.7(0.6-10.7)[4.5- \\
7.1]\end{array}$ & 0.5 \\
\hline $\begin{array}{l}\text { Sex mismatch Female to male } \\
\text { Other combination }\end{array}$ & $\begin{array}{l}30(17 \%) 148 \\
(83 \%)\end{array}$ & $\begin{array}{l}52(29 \%) 125 \\
(71 \%)\end{array}$ & 0.005 \\
\hline $\begin{array}{l}\text { Diagnosis Acute leukaemia } \\
\text { Lymphoma MDS }\end{array}$ & $\begin{array}{l}130(70 \%) 17(9 \%) \\
39(21 \%)\end{array}$ & $\begin{array}{l}118(66 \%) 19 \\
(10 \%) 43(24 \%)\end{array}$ & 0.7 \\
\hline Disease status CR Not in CR & $\begin{array}{l}120(66 \%) 61 \\
(34 \%)\end{array}$ & $\begin{array}{l}125(72 \%) 48 \\
(28 \%)\end{array}$ & 0.2 \\
\hline ATG No Yes & $\begin{array}{l}100(54 \%) 86 \\
(46 \%)\end{array}$ & $\begin{array}{l}106(59 \%) 74 \\
(41 \%)\end{array}$ & 0.3 \\
\hline $\begin{array}{l}\text { Conditioning intensity MAC } \\
\text { MAC/TBI RIC RIC/TBI }\end{array}$ & $\begin{array}{l}46(25 \%) 36(20 \%) \\
89(48 \%) 13(7 \%)\end{array}$ & $\begin{array}{l}37(21 \%) 26(15 \%) \\
98(55 \%) 17(9 \%)\end{array}$ & 0.3 \\
\hline
\end{tabular}

[[P230 Table] 1. Table 1]

Disclosure: The authors declare no relevant conflict of interest

\section{P231}

Donors' CTLA-4 gene polymorphism is associated with increased relapse rate after hematopoietic stem cell transplantation

\section{Nora Meggyesi ${ }^{1}$, Petra Kovy ${ }^{1}$, Livia Varga ${ }^{1}$, Katalin Balassa $^{1}$, Arpad Batai ${ }^{1}$, Eva Torbagyi ${ }^{1}$, Laszlo Gopcsa ${ }^{1}$, Melinda Paksi', Aniko Barta ${ }^{1}$, Tamas Masszi², Hajnalka Andrikovics ${ }^{1}$, Peter Remenyi ${ }^{1}$}

${ }^{1}$ Central Hospital of Southern Pest, National Institute of Hematology and Infectious Diseases, Budapest, Hungary, ${ }^{2}$ Semmelweis University, Budapest, Hungary

Background: Single nucleotide polymorphisms (SNPs) in cytotoxic T-lymphocyte antigen-4 (CTLA-4) gene have been reported to influence the function of CTLA-4, which plays a well established role in negative regulation of $\mathrm{T}$ cell activation, thus in the development of graft versus host disease (GvHD) after hematopoietic stem cell transplantation (HSCT).

Methods: A cohort of 397 donors and 397 adult patients, who underwent first allo-HSCT between January 2007 and December 2013 at our single center, were investigated for CTLA-4 +49A $>$ G (rs231775) SNP and allo-HSCT outcome. Myeloablative conditioning (MAC) was applied in 259, and reduced intensity conditioning (RIC) in 138 patients. Donor type was HLA-matched sibling in 202 and matched unrelated donors (MUD) in 195 patients. For identification of CTLA-4 from genomic DNA LightCycler melting curve analysis (LightCycler 480II, Roche Diagnostics) was performed.

Results: We found significant correlation between donors' CTLA- $4+49 \mathrm{~A}>\mathrm{G}$ polymorphism and HSCT outcome. Genotype AA was present in 170 donors, AG in 183 donors and 44 donors was homozygous for $\mathrm{G}$ allele. Recipients who received graft from $\mathrm{G}$ allele carrier donors showed significantly increased cumulative incidence of relapse (at 24 months AA: $20.7 \%$, AG: $23.6 \%$ and GG: $34.3 \% ; \mathrm{p}=0.04)$. On contrary, the frequency of the acute GvHD grades III-IV and cytomegalovirus (CMV) reactivation/disease decreased according to the presence of the $\mathrm{G}$ allele in the donor CTLA-4 genotype [aGvHD: AA: $20 \%$, AG: $12 \%$, GG: $5 \%$; $=0.014$; CMV: AA: $24 \%$, AG: $16 \%$, GG: $9 \% ; p=0.039]$. Cumulative incidence of aGvHD was also markedly decreased among patients with $G$ allele carrier donors (at 100 days AA: $19.9 \%$, AG: $10.4 \%$, GG: $6.4 \% ; p=0.01$ ). Donor genotype similarly influenced HSCT outcome in MUD donor and MAC conditioning subgroups. Overall survival (OS) was not different in patient subgroups according to donor genotypes [OS at 24 months: AA: 55.5 $\pm 3.8 \%$, AG: $54.4 \pm 3.7 \%$, GG: $49.4 \pm 7.6 \%$; $=0.68$ ]. We did 
not find any correlation between recipients' CTLA-4 +49A $>$ G polymorphism and HSCT outcome.

Conclusions: Several CTLA-4 SNPs have previously been described to be associated with relapse rate, incidence of aGvHD and OS, but results are often contradictory in the publications. In our study, CTLA-4 $+49 \mathrm{~A}>\mathrm{G}$ polymorphism of HSCT donors influenced risk of relapse, aGvHD, $\mathrm{CMV}$ and cause of death, but not overall survival. The genotyping of CTLA- $4+49 \mathrm{~A}>\mathrm{G}$ polymorphism in donors may help in the risk assessment process and the choice of personalised therapy.

Disclosure: Nothing to declare.

\section{P232}

Ruxolitinib plus etanercept for patients with steroidrefractory acute graft versus host disease after allogeneic stem cell transplantation

Yanmin Zhao ${ }^{1}$, Jinmin Shi, Yi Luo ${ }^{1}$, Yamin Tan', Jianping Lan', Lieguang Chen ${ }^{3}$, He Huang ${ }^{1}$

${ }^{1}$ Zhejiang University; The First Affiliated Hospital, Hangzhou, China, ${ }^{2}$ Zhejiang Provincial People's Hospital, Hangzhou, China, ${ }^{3}$ Yinzhou People's Hospital, Ningbo, China

Background: Although steroids remain first-line therapy for the treatment of acute graft versus host disease (aGVHD), response rates in patients with grade III-IV disease are poor, with no apparent improvement in survival over the past 15 years. We performed a prospective, multicenter trial to assess the efficacy and safety of the combination of ruxolitinib and etanercept as a novel approach to treat grades III-IV SR-aGVHD .

Methods: Forty malignant hematologic disease patients with grades III-IV SR-aGVHD after allo-SCT from three centers in East China were enrolled from January 2017 to June 2018. Ruxolitinib was initiated at a dose of 5-10 mg BID for 2 months, and then tapered gradually for another one month. Etanercept was administrated at $25 \mathrm{mg}$ BIW for 2-8 weeks.

Results: The median age of patients was 25 (range 15-59) years. At day 30 after the combination treatment, the overall response rate (ORR) was $90 \%$ including $30 \mathrm{CRs}(75 \%)$ and 6 PRs $(15 \%)$. The median time to the optimal response was 13 (range 3-34) days. The incidences of CR per organ were $95.7 \%$, $80.8 \%$, and $80 \%$ for skin, liver, and gut, respectively. The aGVHD relapse rate was analyzed for the patients who had achieved CR or PR and survived beyond 60 days. Relapses in aGVHD occurred in $9.38 \%$ (3/ 32) of responsive patients.

The patients who received ruxolitinib within 14 days after aGVHD onset have a significant higher CR rate that those with delayed ruxolitinib therapy $(96.2 \%$ vs. $42.9 \%$, $\mathrm{p}=0.001)$. And the patients without gut infections have a significant higher CR rate than infected cohort $(92.6 \%$ vs. $46.2 \%, \mathrm{p}=0.002)$. By logistic regression analysis, the time from aGVHD to ruxolitinib $(\mathrm{RR}=4.17, \mathrm{p}=0.011)$ and gut infection ( $R R=3.31, p=0.031)$ were independent predictors for incomplete response.

Thirteen patients $(13 / 40,32.5 \%)$ suffered from at least 1 infectious episode after the start of the combination therapy, and pulmonary infectious diseases was a frequent complication $(9 / 40,22.5 \%)$. III-IV Cytopenia and CMVreactivation were observed in $30 \%$ and $47.5 \%$ of patients.

The 1-year overall survival (OS) after initiation of the combination therapy were $76.8 \%$. The 1-year NRM and relapse incidence was $17.9 \%$ and $19.9 \%$, respectively. Patients with complete response on day 30 had significantly higher OS probability than non-CR patients (1-year OS: $86.1 \%$ vs $48.0 \%, \mathrm{p}=0.01)$.

Compared with the historical cohort of basiliximab and etanercept for SR-aGVHD in our center $(n=31)$, no significant difference was found on the baseline. Although the ORR in patients treated with ruxolitinib and etanercept is identical with the historical cohort, ruxolitinib group achieved rapider remissions in liver aGVHD and gut aGVHD than the historical cohort (gut aGVHD: 11 days vs. 17 days, $\mathrm{p}=0.026$; liver aGVHD: 21 days vs. 28 days, $\mathrm{p}=0.039$ ), Thus, with regard to hospital stay after aGVHD onset, the ruxolitinib cohort stayed shorter (median: 18 days vs. 29 days, $p=0.005$ ) than basiliximab cohort.

Conclusions: Combined treatment with ruxolitinib and etanercept resulted in a rapid CR to visceral aGVHD and meanwhile reserve graft anti-leukemia (GVL) effect as the relapse rate of primary disease is relatively lower. The various infection complications associated with ruxolitinib merit more attention.

Disclosure: Nothing to declare

\section{P233}

Post-transplant cyclophosphamide for graft-versus-host disease prophylaxis in patients with inherited disorders undergoing allogeneic stem cell transplantation (alloHSCT)

Tatiana Bykova ${ }^{1}$, Anna Osipova ${ }^{1}$, Varvara Ovechkina ${ }^{1}$, Alexander Galimov', Alexander Alyanskyi ${ }^{1}$, Ivan Moiseev ${ }^{1}$, Elena Semenova ${ }^{1}$, Ludmila Zubarovskaya ${ }^{I}$, Boris Afanasyev ${ }^{1}$

${ }^{1}$ The First Pavlov State Medical University of St. Petersburg R.M. Gorbacheva Memorial Research Institute, SaintPetersburg, Russian Federation 
Background: Graft-versus-host disease (GVHD) remains one of the main life-threatening complications after alloHSCT, especially in patients with non-malignant diseases. The standard GVHD prophylaxis strategy is mostly based on the use of calcineurin inhibitors alone or in combination with other immunosuppressive (IS) Post-transplant cyclophosphamide (PTCy) is effective GVHD prophylaxis optiont for adult patients (pts), but has limited data in children.

Methods: The study aim was to evaluate PTCy as GVHD prophylaxis in pediatric pts with inherited disorders undergoing allo-HSCT. 96 pts, the most of them are pediatric age (median age -3 y.o., range 7 month - 30 y.o.) with different types inherited disorders ( $\beta$-thalassemia - 10 , bone marrow failure syndromes - 26, storage diseases - 46, primary immunodeficiencydisorders - 14) were inrolled in retrospective study. Donor type was: matched/mismatched unrelated (MUD/MMUD) - 69, matched related donor (MRD)- 15, haploidentical (haplo) - 12. Conditioning regimen was: myeloablative (MAC) - 43, reduce-intensity (RIC) - 53. Graft sourse was: bone marrow (BM) - 68, peripheral blood stem cells (PBSC) - 26, combintions BM $+\mathrm{PBSC} / \mathrm{BM}+$ cord blood -2 . PTCy $50 \mathrm{mg} / \mathrm{kg}$ days $+3,+4$ based GVHD prophylaxis recived 33 pts., standart GVHD prophylaxis based on calcineurin inhibitors - 63 pts.

Results: Cumulative incidence (CI) of aGVHD was $48 \%$. Grade 2-4, 3-4 aGVHD were $40 \%$ and $22 \%$ respectively. PTCy based GVHD prophylaxis reduced CI of aGVHD ( $35 \%$ vs $56 \%, \mathrm{p}=0,025)$. Another reduce CI of aGVHD factors were MAC ( $31 \%$ vs $59 \%$ in RIC pts group, $\mathrm{p}=0,044)$, MRD ( $13 \%$ vs $44 \%$ in haplo group vs $56 \%$ in MUD/MMUD group, $\mathrm{p}=0,024), \mathrm{BM}$ as a transplant source ( $44 \%$ vs $62 \%$ in PBSC group, $\mathrm{p}=0,05$ ). In a multivariate analysis MAC (HR 2,6 95\%CI 1,7-6, $\mathrm{p}=0,02$ ), time from diagnosis to allo-HSCT less then 22 month (HR 2,6 95\% CI $1,1-6,2, p=0,03$ ) were predictive for reducing CI aGVHD. For aGVHD 2-4 st. significant factor increase CI was female donor both in univariate $(51 \%$ vs $34 \%, \mathrm{p}=0,04)$ and multivariate analysis (HR 0,7 95\% CI 0,2-0,8, p=0,02).5 years overall survival (OS) was $60 \%$. Improving OS factors were: transplant age younger then 5 y.o. ( $80 \%$ vs $35 \%$, $\mathrm{p}=0,000$ ), time from diagnosis to allo-HSCT less then 22 month (72\% vs $38 \%, \mathrm{p}=0,000)$, engraftment $(72 \%$ vs $22 \%, \mathrm{p}=0,000)$. In a multivariate analysis only transplant age younger then 5 y.o. (HR 3,3 95\%CI 1,4-8, p=0,006) and engraftment (HR $0,395 \% \mathrm{CI} 0,1-0,7, \mathrm{p}=0,005)$ were predictive for OS.

Conclusions: PTCy-based GVHD prophylaxis can be effective options for reduce risk of acute GVHD. Using unrelated donors, bone marrow as transplant source and MAC can reduce CI of GVHD. Performing allo-HSCR earlier from diagnos and in earlier age can improve OS patients with inherited disorders
Disclosure: Nothing to declare

\section{P234}

Abstract withdrawn

\section{P235}

Fecal calprotectin as a useful tool in the differential diagnosis of new onset diarrhea after allogeneic stem cell transplantation

\section{Irene García-Cadenas ${ }^{I}$, Rodrigo Martino ${ }^{1}$, Albert Esquirol $^{l}$, Anna Bosch ${ }^{1}$, Laura Martínez-Martínez ${ }^{1}$, JM Portos $^{I}$, Silvana Saavedra ${ }^{I}$, Rahi Awoll, Salut Brunet ${ }^{I}$, Cándido Juárez ${ }^{I}$, Jordi Sierra ${ }^{l}$}

${ }^{1}$ Hospital de la Santa Creu i Sant Pau, Sant Pau and Jose Carreras Leukemia Research Institutes, Autonomous University of Barcelona, Barcelona, Spain,

Background: Diarrhea is a frequent complication after alloSCT. At onset it is often difficult to differentiate GI GvHD from other causes of enterocolitis. Recently, non-invasive tests, such as fecal calprotectin (FC), have been validated as markers of gut inflammation in patients with Inflammatory Bowel Disease, but only a few studies have been published regarding its use as a diagnostic marker in GI GvHD.

Methods: Our aim in this study was to explore the levels of FC in allo-SCT recipients with new-onset diarrhea. So far we have included 43 allo-SCT recipients who developed acute diarrhea $\geq$ stage 2-4 at a median of 75 days (range:12328) post allo-SCT. Stool samples were analyzed as soon as possible after the onset of diarrhea. FC levels were determined in addition to an extensive microbiological panel for infectious enterocolitis (including norovirus PCR and C. difficile associated diarrhea). Endoscopies for histologic analysis were performed according to the treating physicians' discretion $(\mathrm{n}=15)$.

Results: Patients characteristics are summarized in Table 1. Median follow-up for survivors was 524 days (range:151-1834). Twenty-eight patients (65\%) were diagnosed of GI-GvHD. The additional causes of diarrhea were: drug-related enterotoxicity $(n=6)$, viral enteritis $(n=2)$, food intolerance $(\mathrm{n}=2)$, C.jejuni-enteritis $(\mathrm{n}=1)$, and non-specific causes $(n=4)$.

The concentration of FC was higher in patients with GI GvHD vs. other causes of diarrhea $(544 \mu \mathrm{g} / \mathrm{g}+/-71$ vs. $58 \mu \mathrm{g} / \mathrm{g}+/-33, \mathrm{p}=0.03$ ). Patients who did not develop severe enterocolitis had normal to slightly raised calprotectin at the onset of diarrhea $[<100-150$ in 16 out of 19 $(89 \%)$ cases], including $100 \%(6 / 6)$ of patients with enterotoxic drug-related diarrhea.

Among the 28 patients with GI-GvHD, 13 (30.2\%) were later found to be steroid-resistant. As shown in Figure 1, we 
found a significant association between high FC $(\geq 400 \mu \mathrm{g} / \mathrm{g})$ and severe-refractory GvHD ( $\mathrm{HR} 5.7, \mathrm{p}=0.01)$. Of note, high values of $\mathrm{FC}$ were also found in 3 patients with severe infectious enteritis (norovirus, adenovirus and C.jejuni infections), with baseline FC>800 $\mu \mathrm{g} / \mathrm{g}$, respectively.

Overall survival was 78\% (IC95\%:65-91) at 12 months. Hypoalbuminemia and thrombocytopenia were the only variables linked to 1-yr OS in univariate analysis, regardless of the cause of enterocolitis.

Conclusions: In the absence of standarized (and expensive) biomarker panels for analyzing and predicting GVHD onset and outcomes, the FC test may be an useful tool in the allo-SCT setting. Our initial results show that FC is helpful in predicting mild causes of diarrhea and to identify patients with a high probability of developing severe (and potentially steroid-refractory) GI GvHD, although high levels are also found in severe infectious enteritis.

\begin{tabular}{lc} 
Median age, years (range) & $55(17-72)$ \\
Gender male, n (\%) & $24(56 \%)$ \\
Underlying disease, n (\%)- AL and MDS & $34(79 \%)$ \\
Advanced disease status at HSCT, n (\%) & $11(26 \%)$ \\
$\begin{array}{l}\text { Donor type, n(\%) MUD/MMUD or } \\
\text { haploidentical }\end{array}$ & $17 / 11(39.5 \% / 25.6 \%)$ \\
$\begin{array}{l}\text { Conditioning regimen, n (\%) Reduced- } \\
\text { Intensity strategies }\end{array}$ & $25(58 \%)$ \\
$\begin{array}{l}\text { Graft source, n (\%) Peripheral stem cells } \\
\text { GVHD prophylaxis, n (\%)High-dose } \\
\text { cyclophosphamide-based/Sirolimus- }\end{array}$ & $40(93 \%)$ \\
Tacrolimus & $14 / 14(32.6 \% / 32.6 \%)$ \\
\hline
\end{tabular}

[[P235 Table] 1. Patient characteristics $(N=43)]$

Disclosure: Nothing to declare

\section{P236}

Long term follow-up of a pilot study using placentaderived decidua stromal cells for severe acute graftversus-host disease

Olle Ringden ${ }^{1}$, Mats Remberger ${ }^{1}$, Britt Gustafsson ${ }^{1}$, Jacek Winiarski $^{1}$, Bita Khoein ${ }^{1}$, Lena Klingspor ${ }^{1}$, Magnus Westgren $^{1}$, Jonas Mattsson ${ }^{1}$, Behnam Sadeghi ${ }^{1}$

${ }^{1}$ Karolinska Institutet, TCR, CLINTEC, Stockholm, Sweden

Background: There is an urgent need for effective therapy for severe acute GVHD. Results of GVHD therapies beyond 6 months are rarely reported. We here report a median follow-up of 4 years.
We introduced mesenchymal stromal cells as therapy for severe acute GVHD, with a dramatic response in some, but not all patients. The placenta protects the fetus from the mothers haploidentical immune system during pregnancy. We found that maternal stromal cells from the fetal membrane, so called decidua stromal cells (DSCs) were more immunosuppressive than other sources of stromal cells.

Methods: We treated 21 patients, median 49 years of age (range 1.6-72) for severe acute GVHD. All had biopsy proven gastro-intestinal GVHD. All were steroid refractory, 11 after $>7$ days or with progression and 10 after $>3$ days. We used an improved protocol where DSCs were thawed and infused in a buffer with $5 \%$ albumin. DSCs were given at a median dose of $1.2(0.9-2.9) \times 10^{6}$ cells $/ \mathrm{kg}$ and 2(1-6) doses, given one week apart. Viability of frozen and thawed DSCs was 95\% (89-100) and cell passage was 4 (2-4).

Results: Complete resolution of GVHD was seen in 11 patients and 10 had a partial response. The cumulative incidence of chronic GVHD was 52\%. Six had mild, 4 moderate and one severe $\mathrm{NIH}$ overall GVHD severity scoring. Nine patients died, 3 from relapse, 1 acute GVHD and septicemia, 1 zygomycetes infection, 1 liver insufficiency, 1 cerebral hemorrhage, 1multiorgan failure and 1 chronic GVHD with obstructive bronchiolitis. Four years transplant related mortalliy was $28.6 \%$ and overall survival was $57 \%$. Survival was not significantly worse $(p=0.33)$ than $66 \%$ for all 293 patients undergoing allogeneic hematopoietic cell transplantation during the same period 2012-2015.

Conclusions: To conclude, DSCs seems to be a promising therapy for severe acute GVHD. Randomized trials are under way.

Disclosure: Nothing to declare

P237

Anti-apoptotic protein $B C L-2$ is upregulated in graftversus-host disease

Johanna Strobl', Ram Vinay Pandey ${ }^{1}$, Fiona Tinnefeld ${ }^{1}$, Lisa Kleißl', Merima Herac ${ }^{2}$, Philipp Wohlfarth', Judith Stift $^{2}$, Margit Mitterbauer ${ }^{2}$, Peter Kalhs ${ }^{2}$, Werner Rabitsch $^{2}$, Georg Hopfinger ${ }^{2}$, Georg Stary ${ }^{1,3,4}$

${ }^{1}$ Medical University of Vienna, Vienna, Austria, ${ }^{2}$ Medical University of Vienna, Clinical Institute for Pathology, Vienna, Austria, ${ }^{3}$ Ludwig Boltzmann Institute for Rare and Undiagnosed Diseases, Vienna, Austria, ${ }^{4}$ Center for Molecular Medicine (CeMM), Vienna, Austria

Background: Graft-versus-host disease (GVHD) remains a major cause of mortality after allogeneic hematopoietic 
stem cell transplantation (allo-HSCT) with $30-80 \%$ developing either acute or chronic GVHD. Recently, Bcl-2 inhibitor Venetoclax was approved for treatment of chronic lymphocytic leukemia. Induction of apoptosis and depletion of lymphocyte subpopulations e.g. follicular B-cells or CD4 + and CD8+ T-cells led to further exploration in autoimmune disease.

Methods: To establish expression levels of genes in the Bcl-2 pathway, low-input RNA sequencing was performed on $\mathrm{T}$ cells isolated from non-inflamed skin and peripheral blood of HSCT recipients at 5 different time points before until 1 year after transplantation. Furthermore, we analyzed blood, lung, gut and skin samples of 105 patients post alloHSCT with and without previously untreated acute or chronic GVHD by RT-PCR, flow cytometry and tissue immunofluorescence.

A
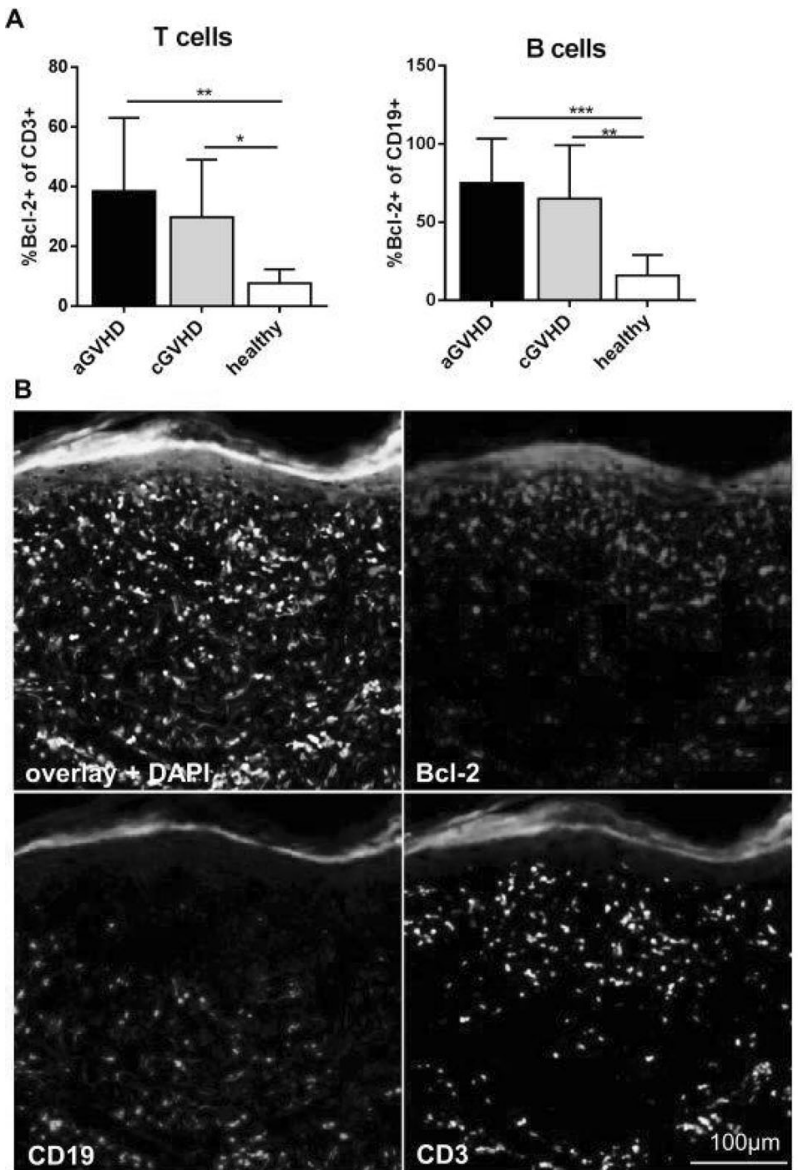

[[P237 Image] 1. Bcl-2 is up-regulated in $T$ and $B$ lymphocytes of acute and chronic GVHD lesions.]

Results: RNA-sequencing revealed that $\mathrm{T}$ cells upregulated $\mathrm{Bcl}-2$ upon conditioning treatment (day 0) and cells of patients who later developed GVHD failed to downregulate $\mathrm{Bcl}-2$ after transplantation (day +14 , day +100$)$.
Bcl-2 protein levels were elevated in overall leukocytes and pathogenic cell subsets including monocytes, CD8+ T lymphocytes and NKT cells showed significantly higher expression of Bcl-2 in peripheral blood of GVHD patients as compared to healthy controls. These results could be recapitulated in tissue samples, where disease-promoting lymphocytes (T, B, NK, NKT) were numerically expanded and expressed Bcl-2 in acute and chronic GVHD skin lesions. Notably, non-pathogenic cell types such as keratinocytes did not exhibit increased Bcl-2 expression compared to control samples from HSCT recipients and healthy donors. While Bcl-2 RNA expression did not depend on type of conditioning (MAC vs. RIC) or GVHD grade, it correlated to disease severity and was significantly elevated in biopsies of patients with steroidrefractory GVHD.

Conclusions: We could show exclusive upregulation of Bcl-2 in GVHD-mediating cell types in peripheral blood and tissue samples affected by GVHD, correlating to GVHD severity and response to first-line therapy. Thus, Bcl-2 inhibition may present a novel and urgently needed targeted therapy in treatment of steroid-refractory acute and chronic GVHD.

Disclosure: Supported by a DOCmed fellowship od the Austrain Academy of Sciences

\section{P238}

Post-transplant cyclophosphamide combined with ATG for GVHD prophylaxis improves survival and lowers non-relapse mortality in older patients undergoing allogeneic hematopoietic cell transplantation

Ivan Pasic ${ }^{1}$, Jeffrey Lipton, Dennis Kim ${ }^{1}$, Auro Viswabandya ${ }^{1}$, Santhosh Thyagu ${ }^{1}$, Rajat Kumar ${ }^{1}$, Wilson Lam $^{1}$, Arjun Law ${ }^{1}$, Fotios Michelis ${ }^{1}$

\section{${ }^{1}$ Princess Margaret Cancer Centre, Toronto, Canada}

Background: Graft-versus-host disease (GVHD) represents a major contributor to morbidity and mortality in recipients of allogeneic hematopoietic cell transplants (HCT). Several therapeutic strategies exist for GVHD prophylaxis and include post-transplant cyclophosphamide (PTCy) and antithymocyte globulin (ATG). While several groups have described the use of PTCy in younger patients, there is a paucity of data about the efficacy of PTCy in older individuals, particularly when combined with ATG. We investigated the combined effect of PTCy with ATG on transplant outcomes in older patients at Princess Margaret Cancer Centre, Toronto, Canada.

Methods: This retrospective study included all patients age $\geq 60$ who underwent allogeneic HCT for any indication at our centre between December 2013 and July 2017. 
Overall survival (OS) was calculated using Kaplan-Meier analysis and multivariable Cox proportional hazards regression. Cumulative incidence of relapse (CIR) and non-relapse mortality (NRM) were calculated using competing risk regression (Fine and Gray method). Incidences of acute (aGVHD) and chronic (cGVHD) were compared using the Fisher's exact test.

Results: Of 133 patients, 84 (63\%) were male. Median age was 65 (range 60-74) and median follow-up among survivors was 28 months (range 6-60). Acute myeloid leukaemia (AML) was the most common indication for HCT (57 patients, $43 \%$ ), followed by myelodysplastic syndrome (37 patients, 28\%) and myelofibrosis (17 patients, 13\%). Eightyfour $(63 \%)$ patients had a matched unrelated donor, 37 (28\%) had a matched related donor and $12(9 \%)$ had a haploidentical donor. One hundred twenty-five (94\%) patients received reduced intensity conditioning. Sixty-two (47\%) patients received PTCy combined with ATG $(4.5 \mathrm{mg} / \mathrm{kg})$ while 71 (53\%) received other forms of GVHD prophylaxis. OS at 2 years was $46 \%$ (95\% confidence interval (CI) 37-54) in the entire cohort. Patients who received PTCy with ATG had a superior 2-year OS compared with other GVHD prophylaxis regimens (Figure 1A): $57 \%$ (95\% CI 44-69) vs. 37\% (95\% CI 26-49), respectively ( $\mathrm{HR}=0.6,95 \% \mathrm{CI} 0.4-0.9, \mathrm{P}=0.02)$. The 2-year NRM for the entire cohort was 37\% (95\% CI, 29-46). Patients who received PTCy with ATG had a lower 2-year NRM compared to those who did not (Figure 1B): $23 \%$ (95\% CI $13-34)$ vs. $49 \%$ (95\% CI 37-60), respectively ( $\mathrm{HR}=0.4$, 95\% CI 0.2-0.7, $\mathrm{P}=0.002$ ). The 2-year $\mathrm{CIR}$ in the whole group was 24\% (95\% CI 17-32). Use of PTCy with ATG was associated with a modest increase in CIR at two years (Figure 1C): $35 \%$ (95\% CI $22-49)$ vs. $16 \%$ (95\% 8-25), respectively $(\mathrm{HR}=2.1,95 \%$ CI 1.0-4.0, $\mathrm{P}=0.04)$. There was a trend toward lower incidence of grade II-IV aGVHD among patients who received PTCy with ATG compared to those who did not: $15 \%$ vs. $30 \%(\mathrm{P}=0.06)$. The incidence of grade II-IV cGVHD was lower in individuals who received PTCy with ATG compared to those who did not: $26 \%$ vs. $45 \%$ $(\mathrm{P}=0.03)$.

Conclusions: In older HCT recipients, use of PTCy combined with ATG is associated with improved OS, lower NRM, decreased risk of both aGVHD and cGVHD and a modest increase in relapse risk. Therefore the PTCy with ATG combination represents an effective strategy for GVHD prophylaxis in older allogeneic HCT recipients.

Disclosure: The authors have no conflict of interest to declare.

\section{P239}

Outcome of severe graft versus host disease in pediatric patients with nonmalignant diseases after allogeneic bone marrow transplantation. A single center experience
Irina Zaidman ${ }^{1}$, Sigal Grisariu ${ }^{1}$, Batia Avni, Ehud Even$\mathrm{Or}^{1}$, Bella Shadur ${ }^{I}$, Adeeb Nasereddin ${ }^{1}$, Polina Stepensky ${ }^{1}$

${ }^{1}$ Hadassah Hebrew University Medical Center, Jerusalem, Israel

Background: Hematopoietic Stem Cell Transplantation (HSCT) remained the only curative option for many nonmalignant diseases in pediatric patients. Survival after HSCT has improved the last few years due to significant advancement in human leukocyte antigens (HLA) typing techniques, less toxic conditioning regimens and better supportive care and resulted to $90 \%$ survival and cure in some non malignant diseases. Graft-versus-host disease (GVHD) remains a major complication of HSCT and leading cause of morbidity and mortality. Prognosis of patients with high grade GVHD is dismal and survival rate varies between $25 \%$ to $55 \%$ in pediatric patients.

Methods: The retrospective study included patients with non malignant diseases who underwent allogeneic HSCT at Hadassah Medical Center from 2008 to 2018. The collected data included patient's clinical data and transplant characteristics. The study was approved by the institutional Helsinki committee.

Results: 182 children with nonmalignant diseases underwent 194 Allogeneic Bone Marrow Transplantations in Hadassah University Hospital during ten years period. Fifty seven patients (31\%) developed AGVHD grade 1-4, twenty five of them (13.7\%) grade 3-4. Median age was 6.34 (range 0.37-17.7), most patients were males (17 males, 8 females). 9 patients underwent BMT from fully matched family members, 6 children were transplanted from matched unrelated donors and 10 from mismatched donors.

Twenty one of 25 patients with severe GVHD (83\%) survived. Four patients $(17 \%)$ died from severe GVHD and complications of immunosuppressive treatment. 3 of 4 deceased patients were transplanted from mismatched donor, in 3 of 4 cases the age of donor was advanced, 2 of 4 patients developed severe GVHD and died after second HSCT. All 4 patients were refractory to different treatment modalities. Three of 4 patients died in 2012 and one in 2015, it was no death from severe GVHD in 12 patients that were transplanted and developed high grade GVHD after 2015.

Conclusions: The results of this study show a high survival rate of $83 \%$ in pediatric patients with non malignant diseases and severe GVHD. Significant risk factors for mortality in our group included mismatched donor, advanced age of donor and second transplant. Trend to better survival was observed after 2015. Additional multicentral studies analyzed the outcomes of aGVHD in pediatric patients with nonmalignant diseases are urgently required. 
Disclosure: Nothing to declare

P240

High levels of FVIII and von willebrand factor in chronic graft-versus-host disease patients

Drazen Pulanic ${ }^{1}$, Antonela Samardzic ${ }^{I}$, Lana Desnica ${ }^{1}$, Renata Zadro ${ }^{1}$, Milan Milosevic ${ }^{1}$, Ranka Serventi Seiwerth ${ }^{I}$, Nadira Durakovic ${ }^{I}$, Zinaida Peric ${ }^{I}$, Desiree Coen Herak ${ }^{I}$, Marija Milos ${ }^{1}$, Lucija Kraljevic ${ }^{1}$, Marinka Mravak Stipetic ${ }^{1}$, Ervina Bilic ${ }^{1}$, Romana Ceovic ${ }^{1}$, Tajana Klepac Pulanic ${ }^{1}$, Igor Petricek ${ }^{1}$, Nina Saban ${ }^{1}$, Ana Zelic Kerep ${ }^{I}$, Dina Ljubas Kelecic ${ }^{I}$, Tamara Vukic ${ }^{I}$, Sanja Mazic $^{1}$, Ines Bojanic ${ }^{1}$, Ernest Bilic ${ }^{1}$, Drago Batinic ${ }^{1}$, Radovan Vrhovac ${ }^{1}$, Steven Z. Pavletic ${ }^{2}$

${ }^{1}$ University Hospital Center Zagreb, Zagreb, Croatia, ${ }^{2}$ National Cancer Institute, National Institutes of Health, Bethesda, MD, United States

Background: Chronic Graft-versus-Host Disease (cGvHD) is a serious late complication after allogeneic hematopoietic stem cell transplantation (alloHSCT) with heterogeneous presentation and still poorly understood pathophysiology including inflammation and endothelial dysfunction. Factor VIII (FVIII) and von Willebrand Factor (VWF) are coagulation factors but also known indicators of endothelial dysfunction and inflammation in different settings, and therefore could serve as interesting candidate biomarkers of cGvHD.

Methods: Since 2013 patients after alloHSCT were assessed by the Multidisciplinary cGvHD team at the University Hospital Center Zagreb, Croatia, using established NIH cGvHD-related measurements. An extensive history, physical and laboratory evaluations were performed, including FVIII, VWF:Ag and VWF:Ac analysis. Descriptive statistic and non-parametric analyses were performed. Variables that showed significant univariate correlations were used in multivariate logistic regression (MLR) to identify the most predictive for FVIII, VWF:Ag and VWF:Ac in cGvHD patients.

Results: 70 cGvHD patients and 41 controls (subjects after alloHSCT without cGvHD) were analysed. Median age of cGvHD patients was 42 (9-65) years, 50\% females, $91.5 \%$ underwent alloHSCT for hematologic malignancies, $55.7 \%$ had myeloablative conditioning and 52.9\% matched related donor. Median time from HSCT to study was 450.5 days and from cGvHD diagnosis to study 82 days. There were no demographic neither transplant related significant differences between cGvHD patients and controls beside stem cell source (peripheral blood $71.4 \%$ vs $51.2 \%, \mathrm{p}=0.041)$ and history of acute GvHD $(70.0 \%$ vs $22.0 \%, \mathrm{p}<0.001)$. Majority of patients had moderate
$(52.9 \%)$ or severe $(42.6 \%)$ NIH global cGvHD score, $57.2 \%$ active cGvHD by clinician's impression. Median number of organs involved by cGvHD was 3 (1-6), and the most frequently involved organs were mouth, skin and eyes (52.0\% each). cGvHD patients compared to controls had higher FVIII levels (median 206 (52-453)\% vs 182 (51406)\%, $\mathrm{p}=0.044$, reference range $50-149 \%$ ) and higher VWF:Ag (median 261.6 (76.6-601)\% vs 203.2 (51.9$600) \%, p=0.030$, reference range $50-160 \%$ ), while VWF: Ac showed a trend toward higher levels among patients (median 253.4 (54-601)\% vs $178(48.6-601) \%, \mathrm{p}=0.084$, reference range 50-150\%). Patients had higher GGT $(\mathrm{p}=0.002)$, lower anticardiolipin $\operatorname{IgG}(\mathrm{p}=0.001)$ and $\operatorname{IgM}$ $(\mathrm{p}=0.003)$, and lower albumin $(\mathrm{p}=0.018)$ than controls, without differences between other laboratory parameters. Univariate analysis showed that among cGvHD patients higher FVIII was associated with worse Karnofsky score (KS) $(\mathrm{p}=0.031)$ and performance score (PS) $(\mathrm{p}=0.030)$, higher leukocytes $(\mathrm{p}=0.031)$, cholesterol $(\mathrm{p}=0.003)$, triglycerides, AST, ALT, GGT, LDH, and lower albumin. Higher VWF:Ag and VWF:Ac in cGvHD patients were associated with worse KS and PS $(p<0.001)$, with more active $c G v H D$ $(\mathrm{p}<0.001)$, worse NIH cGvHD liver $(\mathrm{p}=0.042 ; \mathrm{p}=0.039)$ and NIH cGvHD mouth $(\mathrm{p}=0.012 ; \mathrm{p}=0.009)$, higher total NIH score $(p=0.044 ; p=0.005)$, higher number organs involved ( $p=0.013 ; p=0.003)$, higher ESR, monocytes, Ddimers, AST, ALT, GGT, LDH, triglycerides, $\beta$-2-microglobulin, ferritin, total proteins, $\operatorname{IgA}$ and lower albumin. MLR analysis showed leukocytes $(\mathrm{p}=0.018)$ and cholesterol $(\mathrm{p}=0.010)$ as the strongest predictor of FVIII $(\mathrm{r} 2=49.8 \% ; \mathrm{p}<0.001)$, while strongest predictor of VWF: Ac was number of organs involved by cGvHD ( $\mathrm{r} 2=71.7 \%$; $\mathrm{p}=0.031$ ).

Conclusions: Results of this study detected high FVIII and VWF levels in cGvHD patients with possible reflections to cGvHD manifestations, what needs to be further confirmed in larger longitudinal studies.

Disclosure: This work was supported, in part, by the Unity Through Knowledge Fund project entitled "Clinical and biological factors determining severity and activity of chronic Graft-versus-Host disease after allogeneic hematopoietic stem cell transplantation", and also, in part, by the Croatian Science Foundation project entitled "New biomarkers for chronic Graft-versus-Host disease".

Antonela Samardzic - Work financed by the Croatian Science Fondations` "Young researchers` career development project - training of doctoral students"

\section{P241}

Abstract withdrawn.

P242

Abstract already published. 


\section{P243}

Abstract already published.

\section{P244}

Intestinal thrombotic microangiopathy: A distinct entity in the spectrum of graft-versus-host disease

Eleni Gavriilaki ${ }^{1}$, Ioanna Sakellari ${ }^{1}$, Ioanna Karafoulidou $^{I}$, Nikoleta Pasteli ${ }^{I}$, Ioannis Batsis ${ }^{1}$, Despina Mallouri $^{1}$, Andriana Lazaridou ${ }^{1}$, Michalis Iskas ${ }^{1}$, Anna Vardi $^{1}$, Apostolia Papalexandri, ${ }^{1}$, Styliani

Papaemmanouil $^{1}$, Anastasios Ilias ${ }^{I}$, Achilles Anagnostopoulos ${ }^{1}$

${ }^{1}$ G. Papanicolaou Hospital, Thessaloniki, Greece

Background: Thrombotic microangiopathy (TMA) is a severe complication of allogeneic hematopoietic cell transplantation (HCT) with multisystem involvement. A few recent reports have recognized evidence of TMA in the intestinal vasculature (intestinal TMA/iTMA) of patients with graft-versus-host disease (GVHD) with or without TMA. We aimed to identify patients with iTMA and describe histological, clinical and prognostic features.

Methods: We prospectively evaluated available endoscopic samples (stomach and/or colon) from consecutive adult HCT recipients for previously described histopathologic signs of iTMA (January 2017-September 2018). Systemic TMA was diagnosed according to the International Working Group Criteria. We compared findings among 3 clinical groups: GVHD/systemic TMA, GVHD/no systemic TMA and no GVHD/no TMA.

Results: We studied 20 patients, 5 classified as GVHD/ systemic TMA, 11 GVHD/no systemic TMA and 4 no GVHD/no TMA. Baseline transplant characteristics (age, donor, HLA matching, conditioning) did not differ significantly among groups.

Histological features of iTMA, including loss of glands, total denudation of mucosa, apoptosis and detachment of endothelial cells, intraluminal fibrin, intraluminal microthrombi and mucosal hemorrhage were found in 6 patients. Previously described features of intraluminal schistocytes were not observed in our patients. Interestingly, loss of glands, total denudation of mucosa, apoptosis and detachment of endothelial cells were also found in patients with GVHD and no iTMA, suggesting that these features are not pathognomonic of iTMA.

Among 6 iTMA patients, two patients were classified in the clinical group of acute GVHD/systemic TMA, while the other 4 patients had clinical and histopathological features of iTMA and severe grade III-IV steroid-refractory acute GVHD ( 3 patients) or extensive chronic GVHD (1 patient) but no evidence of systemic TMA. In the majority of patients (5/6), iTMA occurred during the early posttransplant period at $1.7(0.9-4)$ months. Clinical features (gastrointestinal bleeding, diarrhea, pain, nausea) presented no differences between patients with or without iTMA. Prognosis was poor for patients with iTMA who suffered from a significantly higher mortality rate of $83 \%$ compared to the rest patient population $(\mathrm{p}=0.014)$.

With a median follow-up of 11.1 (2.1-67.5) months, 1year overall survival probability (OS) was 22.2 for iTMA, $55 \%$ for GVHD and $60 \%$ for systemic TMA. Unfavorable predictive factors for OS were iTMA ( $\mathrm{p}=0.048)$, HLA mismatched donors $(\mathrm{p}=0.008)$ and gastro-intestinal bleeding $(\mathrm{p}=0.021)$.

Conclusions: Intestinal TMA has emerged as a novel distinct entity in patients with GVHD and/or systemic TMA. Distinct histological features may be useful in differential diagnosis of these severe HCT complications. Mortality rates higher than those of systemic TMA highlight the need of proper recognition of iTMA that needs to be further studied in terms of diagnostic and therapeutic potential.

Disclosure: E.G. was supported by the European Hematology Association Clinical Research Grant. The remaining authors declare no competing financial interest.

\section{P245}

The beneficial effects of thrombomodulin gene polymorphisms after hematopoietic stem cell transplantation

Livia Varga ${ }^{1}$, Petra Kövy ${ }^{2}$, Nora Meggyesi ${ }^{2}$, Andras Bors ${ }^{2}$, Katalin Balassa ${ }^{2}$, Eva Torbagyi ${ }^{2}$, Melinda Paksi ${ }^{2}$, Laszlo Gopcsa $^{2}$, Attila Tordai ${ }^{1}$, Tamas Masszi ${ }^{1}$, Peter Remenyi ${ }^{2}$, Hajnalka Andrikovics ${ }^{2}$

${ }^{1}$ Semmelweis University, Budapest, Hungary, ${ }^{2}$ Central Hospital of Southern Pest (DPC), Budapest, Hungary

Background: The endothelial membrane protein, thrombomodulin is an important regulator of coagulation and innate immunity. Functional single-nucleotide polymorphisms (SNPs) within the thrombomodulin gene (THBD) might contribute to endothelial dysfunction, thereby abnormal reactivity during graft versus host disease (GvHD) after allogenic hematopoietic stem cell transplantation (HSCT).

Methods: We analyzed the impact of THBD SNPs (rs1962, rs1042579 and rs1042580) after HSCT $[\mathrm{n}=400$; 207 sibling/ 193 HLA-matched donor; 256 myeloablative (MAC)/ 144 reduced intensity conditioning (RIC)]. DNA samples for SNP analyses were collected before allo HSCT at the center Department of Hematology and Stem Cell Transplantation, Central Hospital of Southern Pest in 
Hungary between January 2007 and December 2013. Clinical data were analyzed retrospectively. SNPs were genotyped from genomic DNA by melting curve analysis (LightCycler 480 II, Roche Diagnostics).

\section{Figure 1}

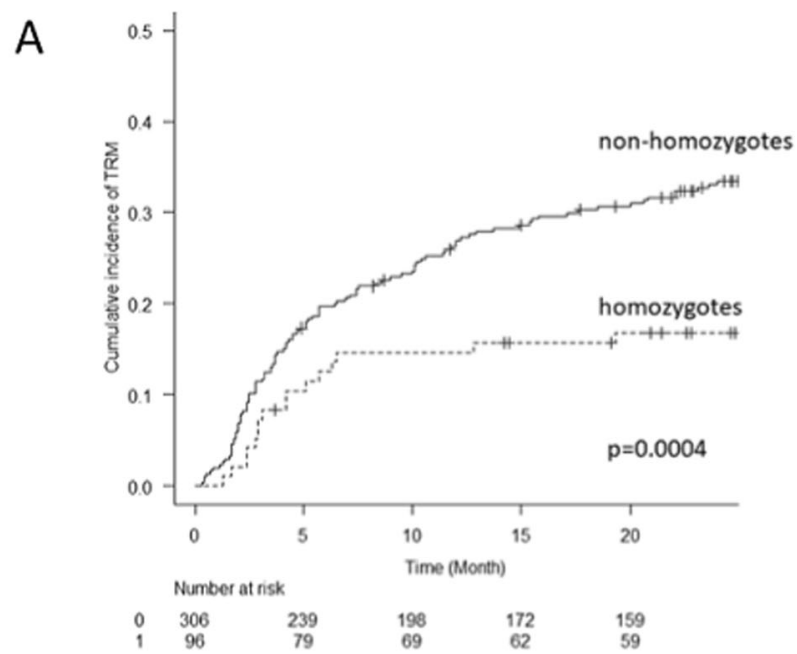

B

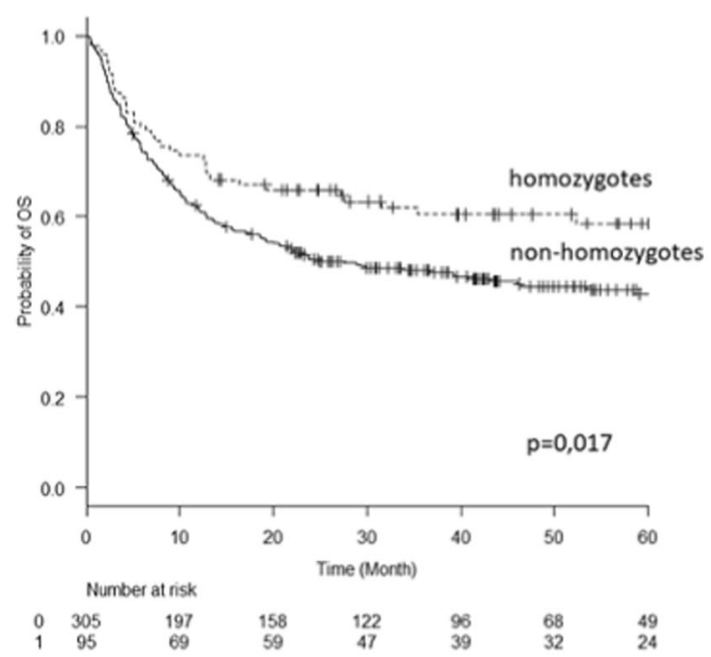

[[P245 Image] 1. Figure 1 A) Transplant related mortality (TRM) and B) overall survival (OS) stratified according to THBD homozygosity.]

Results: Homozygosity for either THBD SNP influenced acute GvHD grades III-IV [8.4\% (8/95) in homozygotes vs. $16.7 \%(51 / 305)$ in non-homozygotes, $p=0.048]$ and cumulative incidence of transplant related mortality (TRM) [at 24 months $15.9 \%(9.4-24.1 \%)$ vs. $33.6 \%$ (28.3-38.9\%), $\mathrm{p}=0.0004$, Figure 1A]. Transplantassociated thrombotic microangiopathy occurred at similar frequency in both genotype groups [14.7\% (14/95) in homozygotes vs. $19.3 \%$ (59/305) in non-homozygotes, $\mathrm{p}=0.36]$. Relapse rate was not different [26.3\% (25/95) vs. $26.2 \%(80 / 305), p=1.0]$. In homozygous patients, favorable overall survival was observed (OS at 60 months: $58.5 \pm 5.4 \%$ vs. $42.8 \pm 3.1 \%, p=0.017$, Figure $1 B$ ). The prognostic effect of $T H B D$ homozygosity was independent from other favorable factors like younger age at HSCT or sibling donor ( $\mathrm{p}=0.025$, HR: $0.66,95 \% \mathrm{CI}$ : 0.46-0.95). The impact of the combination of homozygous variants was pronounced in MAC, but not in RIC (test for interaction: $\mathrm{p}=0.054$, MAC: $\mathrm{p}=0.009$, HR: 0.49 , 95\%CI: 0.29-0.84; RIC: $p=0.89$, HR: $0.97,95 \% C I: 0.58-1.61)$.

Conclusions: Our results suggest that recipient homozygosity for $T H B D$ rs1962, rs1042579 or rs1042580 play protective role after HSCT. THDB homozygosity may propitiously influence the development of acute GvHD, transplant related mortality and the overall survival in case of myeloablative conditioning.

Disclosure: No relevant conflicts of interest.

\section{P246}

Hedgehog blockade in the treatment of steroidrefractory sclerodermatous chronic graft-versus-host disease

Vedran Radojcic ${ }^{1}$, Catherine Lee ${ }^{1}$, Maria Pletneva ${ }^{2}$, Kolleen Hicks ${ }^{1}$, Stefanie Sarantopoulos ${ }^{3}$, Daniel Couriel ${ }^{1}$

${ }^{1}$ University of Utah Huntsman Cancer Institute, Salt Lake City, UT, United States, ${ }^{2}$ University of Utah, Salte Lake City, UT, United States, ${ }^{3}$ Duke University Medical Center, Duke Cancer Institute, Durham, NC, United States

Background: Chronic graft-versus-host disease (cGVHD) remains the major cause of late morbidity and mortality after allogeneic blood and marrow transplantation. Treatment options for cGVHD, particularly its sclerotic forms remain limited. Active Hedgehog $(\mathrm{Hh})$ signaling was shown as a therapeutic target in both mouse and human cGVHD, with limited efficacy and significant toxicities described in a published clinical trial (DeFilipp, 2017).

Methods: Adult patients with steroid refractory sclerodermatous cGVHD, defined as requiring $>0.5 \mathrm{mg} / \mathrm{kg} /$ day of prednisone dose equivalent (PDE), or need for second- or third-line therapy beyond corticosteroids and calcineurin inhibitors or sirolimus were eligible for this open label study of vismodegib, a first generation $\mathrm{Hh}$ pathway inhibitor. Primary endpoint was failure free survival, defined as absence of non-relapse mortality, no recurrent malignancy, steroid dose at 6 months $=<0.2 \mathrm{mg} / \mathrm{kg} /$ day of PDE, and no addition of new systemic treatment. Vismodegib was administered orally for 6-12 months, with dose reductions 
at development of toxicities. Peripheral blood mononuclear cells were isolated from samples collected at treatment initiation and every three months thereafter. The immune profile of circulating $B$ cells was analyzed by flow cytometry and $\mathrm{T}$ helper polarization by qRT-PCR of sortpurified CD4+ T cells.

Results: At the time of interim analysis, 6 patients were evaluated. 3 patients completed 6 months of treatment and five patients completed 3 months of treatment. Therapy was discontinued in 3 patients prior to 6 months due to treatment-related $(n=2)$ and unrelated $(n=1)$ side effects. Most patients experienced grade 2 toxicities (muscle cramps and dysgeusia), with only a single grade 3 toxicity (weight loss). 5 patients who completed 3 months of therapy demonstrated partial response, and overall, the primary endpoint was reached in $50 \%(3 / 6)$ of patients. In 2 patients who discontinued vismodegib, cGVHD worsened acutely after discontinuation. Correlative analysis of immune cellular subsets in peripheral blood in 4 paired samples (pre-treatment and month 3 of therapy) documented modulation of B cell subsets pathogenic in cGVHD (pregerminal center and plasmablast-like B cells) and diminished $\mathrm{T}$ helper 2 polarization in CD4 $\mathrm{T}$ cells.

Conclusions: Overall, use of vismodegib was associated with potential clinical efficacy in sclerodermatous cGVHD with possible mechanistic evidence arising in correlative studies. While side effects were common, further studies of $\mathrm{Hh}$ inhibition in cGVHD are warranted. Future studies should employ adjusted dosing regimens, along with supportive care interventions to offset side effects, and testing of novel Hh inhibitors with enhanced safety profiles.

Clinical Trial Registry: NCT02337517

www.clinicaltrials.gov

Disclosure: Nothing to disclose

\section{P247}

Tracking determinants of the GVH/GVL effect by a comprehensive, pre- and post-SCT, whole-exome sequencing of donor/recipient pairs

\section{Mathieu Leclerc $^{1,2,3}$, Abdel Aissat ${ }^{3,1,2}$, Rabah Redjoul ${ }^{1}$, Dominique Bories ${ }^{1}$, Florence Beckerich ${ }^{1}$, Christine Robin $^{1,3}$, Dan Avi Landau ${ }^{4,5}$, José Laurent Cohen ${ }^{1,2,3}$, Ivan Sloma ${ }^{1}$, Sébastien Maury ${ }^{1,2,3}$}

${ }^{1}$ Hôpital Henri Mondor, Créteil, France, ${ }^{2}$ Inserm U955, Créteil, France, ${ }^{3}$ Université Paris Est, Créteil, France, ${ }^{4}$ Weill Cornell Medical College, Cornell University, New York City, NY, United States, ${ }^{5}$ New York Genome Center, New York City, NY, United States,

Background: Graft-versus-host disease (GVHD) results from recognition of host antigens by donor $\mathrm{T}$ cells following allogeneic hematopoietic stem cell transplantation (SCT). We tested the hypothesis that somatic neomutations occurring after SCT from donor and/or recipient DNA may trigger GVHD.

Methods: We longitudinally analyzed both constitutive and somatic mutations by whole exome sequencing (WES) in 2 patients who received SCT from a sex-matched HLAidentical sibling for NPM1 mutated acute myeloid leukemia (Pt\#1) and JAK2 V617F mutated primary myelofibrosis $(\mathrm{Pt} \# 2)$. Both patients were initially refractory to alloreactivity, i.e. not displaying any signs of GVHD, even after several donor lymphocyte infusions. Acute gut GVHD finally occurred after a further DLI preceded by a lymphodepleting chemotherapy. In Pt\#2, GVHD correlated with a graft-versus-tumor effect. WES was performed on DNA from recipient saliva and donor PBMCs (germline samples) and from sequential post-SCT PBMCs samples on a HiSeq2500 Illumina with $2 \times 100$ bp paired-end reads at a mean depth of coverage of 190-210X. Germline and somatic mutations were determined using in-house bioinformatic pipelines (named Ewok from the Curie Institute and Smaug from the Henri Mondor hospital), using briefly GATK as variant caller for germline samples, and a combination of 3 variant callers for matched normaltumor pairs. We adjusted parameters to detect somatic mutations at a minimal variant allelic frequency (VAF) of $5 \%$ compared to recipient and donor germline for all variations (minimal coverage $=8 \mathrm{X}$ for germline and $5 \mathrm{X}$ for tumor sample).

Results: WES allowed detecting somatic driver mutations explaining AML and PMF for both patients in the initial timepoint and all these driver mutations disappeared at the following timepoints. As expected, the somatic variant rate was 10x higher in Pt\#1 with AML than in Pt\#2 with PMF at each timepoint, except for the final GVHD timepoint. Indeed, at this final point, the somatic variant rate dramatically decreased by $80 \%$ as compared to previous timepoints. By subtracting variants detected pre- and postSCT from those identified at the ultimate time-point of GVHD occurrence, we created 2 sets of 5 and 7 variants respectively for each patient (keeping only variants with at least 4 reads of mutated DNA). These variants can be classified in 2 categories: (i) those with only with a slight increase at time of GVHD, i.e. $\leq 2$-fold compared to highest previous VAF (LRRC43, OR8U1, OR10G9, ALPP, FRG1, $F R G 2 B$ and LILRB3 genes), and (ii) those with a significant increase at that time, i.e. $>2$-fold compared to highest previous VAF (PHF2, SMPD1, ERCC8 and KRTAP9-1 genes). None of the variants or genes involved was common between the 2 patients. Ontology classification of mutated genes showed the implication of some of them in cell death, regulation of MAP kinase activity, mRNA splicing and immune system process, making them good candidates for 
further studies. Identification of variants appearing preGVH and turning off at time of GVHD is ongoing to unveil putative neoantigens that could trigger the alloreactive response.

Conclusions: Using a comprehensive, pre- and post-SCT, WES of donor/recipient pairs, we identified several neomutations from donor and/or recipient DNA correlating with GVH/GVT effect development.

Disclosure: Nothing to declare

\section{P248}

Risk factors for chronic graft-versus-host disease after haploidentical hematopoietic stem cell transplantation in acute myeloid leukemia

Wen-Jing Yu ${ }^{1}$, Xiao-Dong Mo ${ }^{1}$, Xiao-Hui Zhang ${ }^{1}$, LanPing Xu', Yu Wang ${ }^{1}$, Chen-Hua Yan ${ }^{1}$, Huan Chen ${ }^{1}$, YuHong Chen ${ }^{I}$, Kai-Yan Liu', Xiao-Jun Huang ${ }^{I}$

${ }^{1}$ Peking University People's Hospital, Beijing, China

Background: Chronic graft-versus-host disease (cGVHD) is a major complication following haploidentical hematopoietic stem cell transplantation (haplo-HSCT), which may lead to severe morbidity and mortality. However, the risk factors for cGVHD in haplo-HSCT were still unknown. Thus, we aimed to identify the risk factors for chronic graftversus-host disease after haplo-HSCT in acute myeloid leukemia (AML).

Methods: This study included all consecutive patients who underwent unmanipulated haplo-HSCT for AML (complete remission [CR]1 or CR2) at the Institute of Hematology, Peking University, Beijing, China between January 2006 and December 2011. Patients who received donor lymphocyte infusion (DLI) after transplantation were excluded. The final study cohort comprised of 276 patients. Diagnosis of cGVHD was in accordance with the NIH consensus criteria.

Results: A total of 169 patients experienced cGVHD, and mild, moderate, and severe cGVHD were observed in 66, 67 , and 36 patients, respectively. The 2-year cumulative incidence of total cGVHD was $60.5 \%$ (95\% CI, 54.7$66.3 \%$ ), and the 2-year cumulative incidence of moderate to severe and severe cGVHD was $36.6 \%$ (95\% CI, 30.9$42.3 \%$ ) and $12.7 \%$ (95\% CI, 8.8-16.6\%), respectively. The patients who had 3 loci mismatched had a higher 2-year cumulative incidence of total cGVHD (66.2\% vs. $54.5 \%$, $P=0.025)$ and moderate to severe cGVHD (42.3\% vs. $30.6 \%, P=0.028$ ) compared to those of the patients who had 1-2 loci mismatched. The patients who had maternal donors had a higher 2-year cumulative incidence of moderate to severe cGVHD (50.8\% vs. $32.7 \%, P=0.018$ ) compared to that of the patients who had other donors. The patients who had grade III to IV acute graft-versus-host (aGVHD) had a higher 2-year cumulative incidence of total cGVHD $(91.7 \%$ vs. $50.0 \%, P<0.001)$ and moderate to severe cGVHD $(70.8 \%$ vs. $26.3 \%, P<0.001)$ compared to those of the patients without aGVHD. In multivariate analysis, grade III to IV aGVHD was the only independent risk factor for total $\mathrm{cGVHD}(\mathrm{HR}=2.6,95 \% \mathrm{CI}, 1.6-4.2 ; P<$ $0.001)$ and moderate to severe cGVHD (HR=3.8, 95\%CI, 2.1-6.7; $P<0.001)$. In the model excluding aGVHD, maternal donor was the risk factor for moderate to severe cGVHD $(\mathrm{HR}=1.5,95 \% \mathrm{CI}, 1.1-2.3 ; P=0.030)$.

Conclusions: We observe that severe aGVHD was the most important risk factors for cGVHD after haplo-HSCT, and further interventions should be considered in these patients to prevent severe cGVHD.

Disclosure: None of the authors have any potential financial conflict of interest related to this manuscript.

\section{P249}

\section{Crosstalk between NK cells and ECP therapy}

Ming $\mathrm{Ni}^{1,2}$, Lei Wang ${ }^{1}$, Mingya Yang ${ }^{1}$, Brigitte Neuber ${ }^{1}$, Leopold Sellner ${ }^{1}$, Angela Hückelhoven-Krauss ${ }^{1}$, MariaLuisa Schubert ${ }^{1}$, Thomas Luft, Ute Hegenbart ${ }^{1}$, Stefan Schoenland ${ }^{1}$, Patrick Wuchter ${ }^{3}$, Bao An Chen ${ }^{4}$, Volker Eckstein $^{1}$, William Krïger ${ }^{5}$, Ronit Yerushalmi ${ }^{6}, K_{\text {Katia }}$ Beider $^{6}$, Arnon Nagler ${ }^{6}$, Carsten Müller-Tidow ${ }^{1}$, Peter Dreger $^{1}$, Michael Schmitt ${ }^{1}$, Anita Schmitt ${ }^{1}$

${ }^{1}$ University Clinic Heidelberg, Internal Medicine V, Heidelberg, Germany, ${ }^{2}$ Guizhou Medical University, Gui Yang, China, ${ }^{3}$ Heidelberg University, German Red Cross Blood Service Baden-Württemberg-Hessen, Institute of Transfusion Medicine and Immunology, Mannheim, Germany, ${ }^{4}$ Zhongda Hospital, Southeast University, Nan Jing, China, ${ }^{5}$ University Clinic Greifswald, Greifswald, Germany, ${ }^{6}$ Chaim Sheba Medical Center Tel Hashomer, Tel Hashomer, Israel

Background: Extracorporeal photopheresis (ECP) has been successfully used for the treatment of graft-versus-host disease (GvHD). ECP therapy might restore the balance between effector and regulatory cells which is severely impaired in GvHD. NK cells are the first lymphocyte subset to be reconstituted after allogeneic hematopoietic stem cell transplantation (allo-HSCT). As an important innate immune cell population, NK cells can temporally bridge the transient period of T-cell deficiency post allo-HSCT, by protection from opportunistic infections and prevention of leukemic relapse by graft-versus-leukemia (GvL) effect. NK cells not only preserve homeostasis through targeted killing of allo-reactive $\mathrm{T}$ cells and thereby control GvHD but also enhance inflammation by secretion of $\mathrm{TNF}-\alpha$ and 
IFN- $\gamma$ and thereby promote GvHD. Therefore, we investigated here the role of NK cells in GvHD patients under ECP therapy.

Methods: Thirty four patients with steroid-refractory/ resistant $\mathrm{aGvHD} \geq \mathrm{II}^{\circ}$ and moderate to severe cGvHD received ECP therapy which performed according to the guidelines. Glucksberg and NIH criteria were used for clinical staging of aGvHD and cGvHD under ECP therapy, respectively. The comprehensive phenotypical analysis of NK cells was evaluated by multicolor flow cytometry. NK activity in terms of killing function, cytokine release capacity and proliferation function was monitored by chromium-51 release assay, intracellular cytokine staining and CFSE staining, respectively.

Results: Five different NK cell subsets were defined based on CD56 and CD16 expression. CD56 ${ }^{\text {bri }}$ NK cells displayed an immature and activation profile with high expression of CD62L and NKG2D. aGvHD patients had a higher frequency of CD56 $6^{\text {bri }} \mathrm{NK}$ cells when compared with HDs and cGvHD patients, who were characterized by significant increase of the $\mathrm{CD} 56^{\mathrm{dim}} \mathrm{CD} 16^{+}$and CD56 CD16 + NK cell subsets with high expression of differentiation markers $\mathrm{CD} 11 \mathrm{~b}$ and CD57. Of note, CD56 ${ }^{\text {bri }} \mathrm{CD} 16^{-} \mathrm{NK}$ cells could serve as a novel predictive biomarker for the response of aGvHD patients to ECP treatment.

In responding aGvHD patients, an increase of CD56 ${ }^{\text {bri }}$ NK cells was observed already during the early ECP treatment phase, suggesting immune reconstitution. After priming of the progenitors, ECP could differentiate immature CD56 $6^{\text {bri }}$ NK cells into mature CD56 $6^{\mathrm{dim}} \mathrm{NK}$ cells with reduction of CD62L on CD56 $6^{\text {bri }}$ NK cells. Moreover, $\mathrm{CD}^{\text {dim }}$ NK cells could further be matured through upregulation of CD57 expression by ECP. Notably, ECP therapy could shift the NK cells from a cytotoxic to a regulatory phenotype within the $\mathrm{CD} 56^{\text {bri }} \mathrm{NK}$ cells. In spite the immunomodulatory effect of ECP on NK cells, NK activity could be kept intact under ECP therapy. The killing activity of NK cells was stable as confirmed by a ${ }^{51} \mathrm{Cr}$ release assay. ECP therapy had no negative effect on the quantity and quality of cytokine release by NK cells upon K562 stimulation. Especially, the polyfunctionality of NK cells was not altered significantly by the ECP therapy.

Conclusions: NK cells play an important role in GvHD and could serve as a predictive cell population for the clinical response to ECP therapy. In the current study, ECP influenced the differentiation, maturation and education of NK cells ameliorating GvHD without comprising the antiviral immune defense and GvL effect.

Disclosure: The authors declare no competing financial interests, except the following: Therakos Mallinckrodt gave a financial support to AS and MS for the documentation of the clinical course and for the analysis of immune cells of the patients, PW has Honoraria and membership on Advisory Boards for Sanofi-Aventis.

P250

Abstract withdrawn.

\section{P251}

Cyclosporine levels $>200 \mu \mathrm{G} / \mathrm{L}$ on day 10 post-transplant was associated with significantly reduced acute graftversus-host disease following allogeneic hematopoietic stem cell transplantation

Monica Bianchi ${ }^{I}$, Dominik Heim ${ }^{I}$, Claudia Lengerke ${ }^{I}$, Martina Kleber ${ }^{1}$, Dimitrios Tsakiris ${ }^{1}$, Jakob Passweg ${ }^{1}$, Alexandar Tzankov ${ }^{2}$, Michael Medinger ${ }^{I}$

${ }^{1}$ University Hospital Basel, Basel, Switzerland, ${ }^{2}$ University Hospital Basel, Institute of Medical Genetics and Pathology, Basel, Switzerland

Background: Acute Graft-versus-host disease (aGvHD) remains a major complication of allogeneic hematopoietic stem cell transplantation (allo-HSCT). Affected patients, especially with steroid-refractory aGvHD, have a very poor prognosis. Prophylaxis with cyclosporine A (CsA) is the backbone of GvHD prevention in most conditioning regimens.

Methods: In a retrospective analysis of patients treated with allo-HSCT, we correlated CsA levels at the day of transplantation (day 0 ) and day +10 with the incidence of acute and chronic GvHD. We postulate that higher target CsA levels $>200 \mu \mathrm{g} / \mathrm{L}$ will result in a lower incidence rate especially of aGvHD after allo-HSCT.

Results: We assessed 660 patients with either AML $\mathrm{n}=248$, lymphoma/myeloma $\mathrm{n}=127$, MDS/MPN $\mathrm{n}=124$, ALL $n=79$, CLL $n=36$, CML $n=23$, or bone marrow failure $n=22$. In patients with clinically relevant aGvHD grade $\geq 2$, mean CsA levels was lower on day 0 and day +10 $(142 \pm 88 \mu \mathrm{g} / \mathrm{L}$; and $183 \pm 64 \mu \mathrm{g} / \mathrm{L}$; respectively) compared to patients without aGvHD $(156 \pm 81 \mu \mathrm{g} / \mathrm{L}$; and $207 \pm 67 \mu \mathrm{g} / \mathrm{L}$; respectively; day $0: p=0.003$; day $+10: p=7.57 \times 10^{-9}$ ). In patients with CsA level $<200 \mu \mathrm{g} / \mathrm{L}$, the incidence of aGvHD was significantly more frequent compared to patients with CsA levels $>200 \mu \mathrm{g} / \mathrm{L}[(234 / 356 ; 66 \%)$ versus $91 / 248(37 \%) ; p=1.34 \times 10^{-12}$ ]. In patients with cGvHD, there was no significant difference between CsA levels < $200 \mu \mathrm{g} / \mathrm{L}(128 / 330)$ compared to CsA levels > $200 \mu \mathrm{g} / \mathrm{L}$ (96/ 233; $p=0.312$ ). The optimal CsA cut-off level for the prevention (i.e. roughly $50 \%$ incidence reduction) of aGvHD was $>201 \mu \mathrm{g} / \mathrm{L}$ at day 0 and $>195 \mu \mathrm{g} / \mathrm{L}$ at day +10 (Figure 1) 


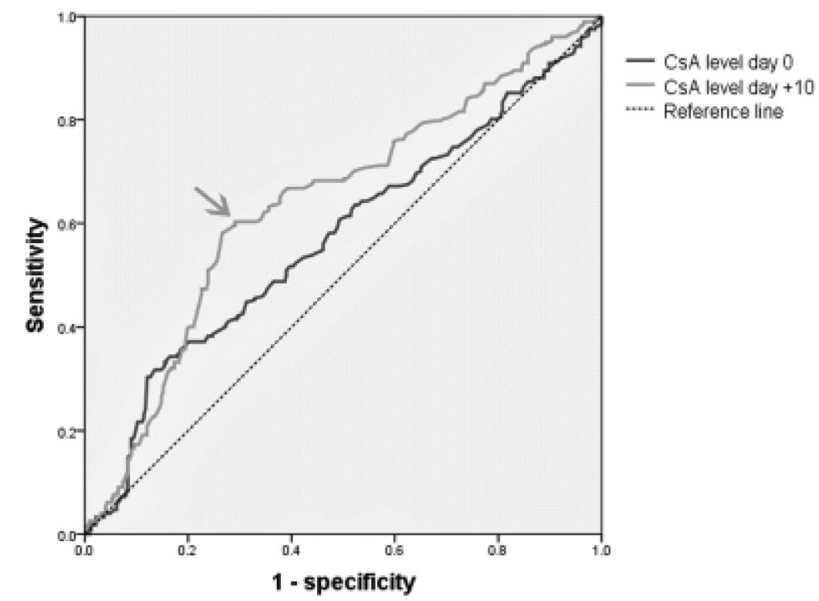

[[P251 Image] 1. Figure 1: Receiver operating characteristics (ROC)-curve of cyclosporine A levels on day 0 and +10$]$

In a competing risk analysis, time to aGvHD grade $\geq 2$ (using death of other causes as competing risk) was associated with CsA levels $>200 \mu \mathrm{g} / \mathrm{L}$ on day 0 and on day 10, unrelated donors, myeloablative conditioning (MAC), and for the diagnosis lymphoma/myeloma.

Conclusions: Our data support close monitoring with active adjustments of CsA dosing to maintain therapeutic CsA levels above $195 \mu \mathrm{g} / \mathrm{L}$ in the first 10 days of alloHCST to reduce aGvHD.

Disclosure: Noting to declare.

\section{P252}

Liposomal cyclosporine a for inhalation (L-CSA-I) to treat bronchiolitis obliterans syndrome: Novel formulation with therapeutic potential for patients with BOS following allo-HSCT

\section{Noreen Roth Henig ${ }^{1}$, Emilie Hofstetter ${ }^{2}$, Dominik Kappeler $^{3}$, Gerhard Boerner ${ }^{3}$}

${ }^{1}$ Breath Therapeutics Inc., Menlo Park, CA, United States, ${ }^{2}$ HealthStrat Consulting, Munich, Germany, ${ }^{3}$ Breath Therapeutics $\mathrm{GmbH}$, Clinical Science, Munich, Germany

Background: Bronchiolitis obliterans syndrome (BOS) is a rapidly progressive lung disease caused by T-cell mediated inflammation that leads to blockage of bronchioles, leading to respiratory failure and death shortly after diagnosis. Approximately $4 \%$ to $10 \%$ of patients who undergo allogeneic hematopoietic stem cell transplant (allo-HSCT) will develop BOS, with $72-100 \%$ developing BOS as a respiratory form chronic graft-vs-host disease (cGVHD) in addition to other signs of cGVHD. Mean time to BOS diagnosis ranges from 273 to 547 days post-transplant. The histopathology of BOS after allo-HSCT and lung transplantation is identical. Early studies of L-CsA-i for the prevention of BOS in lung transplant recipients demonstrated therapeutic benefit. L-CsA-i is a novel, liposomal formulation of cyclosporine administered via a PARI investigational eFlow $^{\hat{a}}$ nebulizer which delivers a potent immunosuppressant to the site of disease. Pharmacokinetics and tolerability of L-CsA-i is presented.

Methods: Retrospective review of two clinical studies of L-CsA-i (isotonic, $4 \mathrm{mg} / \mathrm{mL}$ ) for BOS associated with lung transplantation. Both studies had a control arm and results reported here are for patients who received L-CsA-i. Subjects received $5 \mathrm{mg}$ (single lung transplant) and $10 \mathrm{mg}$ (double lung transplant) bid via inhalation. Blood samples for pharmacokinetic analysis of cyclosporine A concentrations were collected before inhalation, immediately after inhalation, and thereafter in intervals of 15, 30, $60 \mathrm{~min}$ and 2, 4, 8 and 12 hours. Local and general tolerability of LCsA-i was investigated.

Results: Between the two studies, 85 subjects received either 5 or $10 \mathrm{mg}$ bid of L-CsA-i. Pharmacokinetic models predict a constant drug level in the lung. Maximum serum cyclosporine A concentration after inhalation was $57.42 \pm$ $34.26 \mathrm{ng} / \mathrm{ml}$. Trough levels for up to 2-years of daily administration was $4-5 \mathrm{ng} / \mathrm{ml}$ with no evidence of accumulation following repeated exposure. Tolerability data was assessed from 1068 patient-month exposure to L-CsA-i. Reported symptoms were: pharyngeal soreness $1 \%$; cough $22 \%$; dyspnoea $7 \%$; and wheezing $1 \%$. No subject discontinued due to intolerability. Inhalation time is on average 9-13 min.

Conclusions: L-CsA-i provides high and constant concentrations to the airways of the lungs and the site of BOS. L-CsA-i is well tolerated in lung transplant patients. Use of L-CsA-i instead of augmentation of systemic CsA reduces the total drug exposure. A multicentre Phase 2 safety and exploratory efficacy trial for the treatment of BOS in alloHSCT recipients is underway.

\section{Disclosure:}

Noreen Henig, Breath Therapeutics

Emilie Hofstetter, Nothing to declare

Dominik Kappeler, Breath Therapeutics

Gerhard Boerner, Breath Therapeutics

\section{P253}

Screening for predictive biomarkers in related and unrelated transplantations with posttransplantation cyclophosphamide

Ivan Moiseev ${ }^{1}$, Elena Babenko ${ }^{I}$, Olga Epifanovskaya ${ }^{\text {, }}$ Vladislav Sergeev ${ }^{I}$, Anna Dotcenko ${ }^{I}$, Elena Surkova ${ }^{I}$, Daria Kuznetcova ${ }^{1}$, Sergey Lapin ${ }^{1}$, Olga Pirogova ${ }^{1}$, 
Alexandr Alyanskii ${ }^{1}$, Elena Darskaya ${ }^{1}$, Elena Morozova ${ }^{1}$, Sergey Bondarenko ${ }^{1}$, Boris Afanasyev ${ }^{I}$

${ }^{1}$ Pavlov First Saint Petersburg State Medical University, Saint Petersburg, Russian Federation

Background: There are a number of biomarkers that predict non-relapse mortality (NRM), graft-versus-host disease (GVHD) and relapse incidence (RI) after conventional GVHD prophylaxis based on calcineurin inhibitors with or without antithymocyte globulin. Currently there is limited data whether the conventional predictive biomarkers work with posttransplantation cyclophosphamide (PTCy) prophylaxis.

Methods: Prospective single-center study in 2015-2016 enrolled 79 adult patients with acute leukemia in CR (34\% with ALL, 66\% with AML). 26 received matched related bone marrow (BM) graft with single-agent PTCy and 53 received unrelated peripheral blood stem cell graft (PBSC) with PTCy, tacrolimus and MMF. The grafts were studied by flow cytometry (FACS Aria II, antibodies by Miltenyi biotec). The following populations were analyzed: CD3, CD4, CD8, CD16CD56, NKT, iNKT, Treg, double-positive T-cells, double-negative T-cells, TCRalpha/beta, TCR v11 memory cells. The crypreserved plasma from were analysed by ELISA (commercial kits by eBioscience and Critical Diagnostics) for VEGF A soluble TNF receptor (sTNFr), IL-8, IL-6, soluble IL-2 receptor, ST2, IL-17 and sTNFr. The above mentioned biomarkers were tested in logistic regression with ROC analysis, assays with AUC $>0.600$ were selected for analysis in Fyne-Gray regression with competing risks. Cut off levels were determined for significant parameters.

Results: Median follow-up was 19 months (range 12-30). In the whole group overall survival (OS) was $77 \%$, eventfree survival (EFS) 73\%, grade II-IV acute GVHD 13\%, moderate and severe (m\&s) chronic GVHD 20\%, NRM 6\%, mortality in patients with GVHD 0\%, RI 20\%. There was no difference between $\mathrm{BM} /$ related and $\mathrm{PBSC} /$ unrelated grafts in the incidence of GVHD, NRM and RI ( $>>0.11$ ). The only significant predictor of acute GVHD were low levels of IL-8 level on day +7 ( $p=0.0490,0 \%$ vs $15 \%$ with the cut off $40 \mathrm{pg} / \mathrm{ml}$ ). M\&s chronic GVHD was predicted only by the high percentage of iNKT cells in the graft $(p=0.0003,31 \%$ vs $12 \%$ with the cut off $0.03 \%)$. There was a correlation between IL-8 levels and number of NK cells in the graft $(p=0.02)$. NRM was related to infectious complications, nonetheless high levels of VEGF A on day $0(p=0.0458,16 \%$ vs $0 \%$ with the cut off $100 \mathrm{ng} / \mathrm{ml})$, ST2 on day $+30(p=0.0041,11 \%$ vs $0 \% \%$ with the cut off 40 $\mathrm{ng} / \mathrm{ml}$ ) and low percentage of CD16+CD56- cells in the graft $(p=0.0215,22 \%$ vs $2 \%$ with the cut off $1.3 \%$ ). The identified biomarkers of NRM had no association with the pre-transplant CRP and ferritin levels $(\mathrm{p}>0.4)$. The only significant parameter for RI was the level of CD34 cells in the graft $(p=0.0434)$. None of the identified biomarkers significantly predicted overall survival $(p>0.09)$.

Conclusions: In the related and unrelated grafts with PTCy the study of biomarkers has low clinical utility due to very low GVHD-related mortality. However ST2 and VEGF A can predict infection-related mortality. Also the study verified previous observations that high level of IL-8 is associated with reduced GVHD incidence after PTCy ${ }^{1}$ and identified the importance of NK and iNKT cells in the induction of tolerance with PTCy.

References: Pirogova OV et al.Cytokine.2017 Nov;99:148-153.
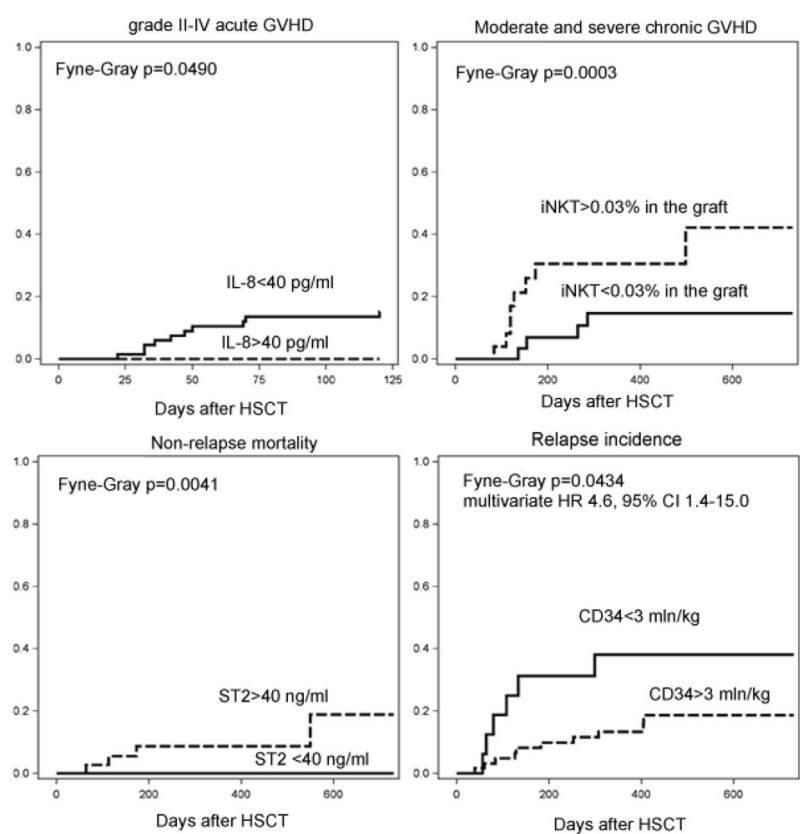

[[P253 Image] 1. Biomarkers with predictive value for GVHD, NRM and relapse]

Disclosure: Nothing to declare

\section{P254}

Abstract withdrawn.

\section{P255}

Abstract already published.

\section{P256}

Post-transplant cyclophosphamide plus short course of tacrolimus/mycophenolate mofetil association guarantee long-term control of GVHD after allogeneic peripheral blood hematopoietic stem cell transplantation from HLA-matched donors 
Paolo Becco $^{I}$, Daniela Caravelli, Susanna Gallo ${ }^{1}$, Alessandra Polo $^{2}$, Milena Salierno ${ }^{2}$, Karol Giancaspero ${ }^{2}$, Delia Rota Scalabrini ${ }^{1}$, Dario Sangiolo ${ }^{3}$, Elena Vassallo ${ }^{4}$, Massimo Berger ${ }^{4}$, Alessandro Cignetti, Valentina Gaidano $^{5}$, Pio Manlio Mirko Frascione ${ }^{1}$, Stefano Poletto ${ }^{I}$, Luca Paruzzo ${ }^{1}$, Luca Crotto ${ }^{6}$, Giuseppe Saglio ${ }^{5}$, Massimo Aglietta $^{1}$, Franca Fagioli ${ }^{4}$, Fabrizio Carnevale Schianca ${ }^{1}$

${ }^{1}$ Medical Oncology, Turin Metropolitan Transplant Center, Candiolo Cancer Institute-FPO, IRCCS, Candiolo, Italy, ${ }^{2}$ Collection and Processing Laboratory, Candiolo Cancer Institute-FPO, IRCCS, Candiolo, Italy, ${ }^{3}$ Medical Oncology, Candiolo Cancer Institute-FPO, IRCCS, Candiolo, Italy, ${ }^{4}$ Turin Metropolitan Transplant Center, A.O.U. Citta' della Salute e della Scienza di Torino, Ospedale Infantile Regina Margherita, Torino, Italy, ${ }^{5}$ University Mauriziano Umberto I Hospital, Torino, Italy, ${ }^{6}$ Clinical Research Office, Candiolo Cancer Institute-FPO, IRCCS, Candiolo, Italy

Background: Hematopoietic cell transplantation (HCT) is the only curative approach for many hematological malignancies but life-threatening toxicities, such as graft-versushost disease (GVHD) and infections, still limit its fullpotential impact on the disease. Strategies for keeping alloHSCT more effective and safe are needed in order to reduce morbidity while improving its immunological effect to control disease relapse. Post-transplant Cyclophosphamide (ptCy) has been demonstrated to improve acute GVHD (aGVHD) and chronic GVHD (cGVHD) control in allogeneic bone-marrow HCT from identical and haploidentical donor. The use of ptCy, after peripheral blood stem cell transplantation (alloPBSCT) from HLA-matched unrelated/related donors, has been investigated by our group in a clinical trial (NCT 02300571) and preliminary results were published last year. Here we report updated efficacy and safety data about the expanded cohort of patients treated with ptCy followed by tacrolimus and mycophenolate mofetil (T/MMF).

Methods: We analysed data about 71 consecutive patients with high-risk hematologic malignancies received alloPBSCT from HLA-matched unrelated/related donors between March 2011 and August 2018. GVHD prophylaxis was ptCy $50 \mathrm{mg} / \mathrm{kg}$ (days $+3+4$ ), Tacrolimus from day +5 and MMF from day +5 to day +28 . Primary objectives were cumulative incidence of aGVHD and cGVHD. Secondary objectives were event-free survival (EFS), cGVHD-EFS, overall survival (OS) and non-relapse mortality (NRM).

Results: Patients median age at transplant was 50 (range 19-74) years. 34 (48\%) patients were transplanted in first complete response (CR), 16 (23\%) patients in second/third $\mathrm{CR}$, the others in disease control. A median dose of 7.0 (range 2-15) x $10^{\wedge} 6 \mathrm{CD} 34 / \mathrm{kg}$ was infused. Primary graft failure was observed in one patient. All patients were off MMF on day +28 , the median day of Tacrolimus discontinuation was 112 (range 39-467). Eight out of 71 (11\%) patients developed aGVHD, $6(8 \%)$ of them were grade II-III; median day of onset was day 68 (range 22-98). No grade IV was observed. No cases of late-onset aGVHD were reported. Cumulative incidence of cGVHD was $8 \%$ (6/ 71), median day of onset was 162 (range 140-268). Systemic treatments were required, but all patients were able to discontinue immunosuppression (IS). With a median follow-up of 22 (range 4-94) months, EFS was 58\%, cGVHD-EFS was $52 \%$ and OS was $72 \%$. Non-relapse mortality (NRM) was 3\% (2/71): 2 patients died because of multidrug resistant bacteria septicemia. Nowadays 41 patients are alive with no evidence of disease, being continuously off IS and completely reintegrated in their normal daily life activities.

Conclusions: The updated reported results confirm, in a larger cohort of patients with a longer follow-up, that ptCy after PBSC-HCT is highly active in aGVHD and cGVHD prevention with extremely limited NRM. This strategy, not only allowed earlier discontinuation of immunosuppression, but also reduced the overall time of exposure to IS for most of the patients. All these features might contribute, in the future, to transform HCT into a safe immunologic platform that may be combined with advanced form of cellular therapies (CAR-Tcells), aiming to increase safely the graftversus-tumor effect.

Clinical Trial Registry: NCT02300571

Disclosure: Nothing to declare

\section{P257}

CD80/86 blockade with abatacept is safe in pediatric patients undergoing unrelated donor hematopoietic cell transplantation for bone marrow failure and likely augments graft-versus-host disease prophylaxis

\section{Elizabeth Stenger ${ }^{1}$, Kelsey Rogowski, Kuang-Yueh Chiang $^{2}$, Ann Haight ${ }^{1}$, Muna Qayed ${ }^{I}$, Yvonne Suessmuth ${ }^{1}$, Leslie Kean ${ }^{3}$, Horan John ${ }^{1}$}

${ }^{1}$ Emory University, Aflac Cancer \& Blood Disorders Center, Atlanta, GA, United States, ${ }^{2}$ University of Toronto, The Hospital for Sick Children, Toronto, Canada, ${ }^{3}$ Boston Children's Hospital, Dana Farber Cancer Institute, Boston, MA, United States

Background: Donor $\mathrm{T}$ cell activation after hematopoietic cell transplantation [HCT] occurs partly through CD28: CD80/86 signaling, with inhibition using abatacept (CTLA4-Ig) showing promise in abrogating graft-versushost disease [GVHD] following unrelated donor HCT for malignant disease. We hypothesized that abatacept GVHD 
prophylaxis could be safely extended into the nonmalignant disease [NMD] setting.

Methods: Pediatric patients (0-21 years) with NMD undergoing unrelated HCT were eligible for this single center, phase I trial. Following reduced intensity conditioning, abatacept $(10 \mathrm{mg} / \mathrm{kg}$ IV on days $-1,+5,+14,+28)$ was added to standard GVHD prophylaxis (cyclosporine, mycophenolate mofetil $[\mathrm{MMF}])$. Patients were followed for 2 years for standard HCT outcomes.

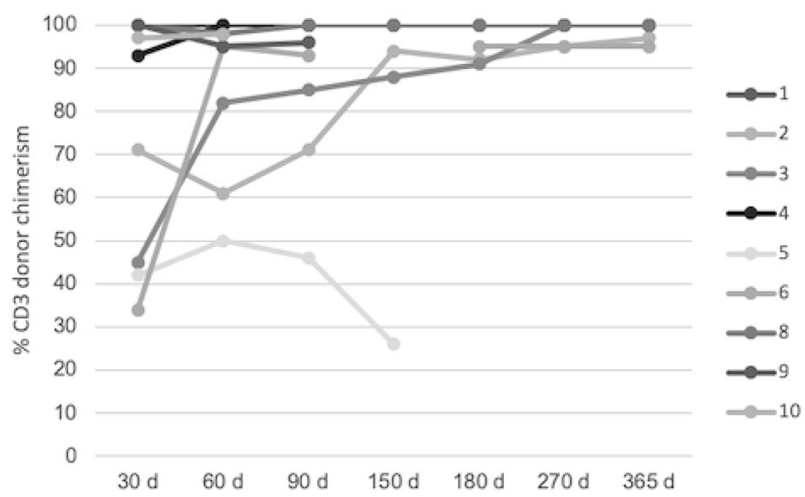

[[P257 Image] 1. Figure 1: Donor CD3 Chimerism is Mixed but Increases Over Time in Majority]

\begin{tabular}{llllllllll}
\hline Pt ID & $\begin{array}{l}\text { Age } \\
\text { (yr) }\end{array}$ & Indication & Conditioning & Donor & $\begin{array}{l}\text { Time to } \\
\text { ANC> } \\
=500(\mathrm{~d})\end{array}$ & $\begin{array}{l}\text { Time to } \\
\text { plts>l } \\
=20(\mathrm{~d})\end{array}$ & $\begin{array}{l}\text { Max } \\
\text { aGVHD }\end{array}$ & $\begin{array}{l}\text { Max } \\
\text { cGVHD }\end{array}$ & $\begin{array}{l}\text { Last } \\
\text { f/u (yr) }\end{array}$ \\
\hline 1 & 7 & DBA & rATGFluCyTBI & $8 / 8$ & 19 & 34 & Gr.II & Mod. & 1.5 \\
2 & 9 & FA & rATGFluCy & $7 / 8$ & 15 & 15 & - & - & 4.0 \\
3 & 11 & SAA & rATGFluCyTBI & $7 / 8$ & 20 & 16 & - & - & 3.9 \\
4 & 6 & SAA & rATGFluCyTBI & $7 / 8$ & 22 & 11 & - & - & 3.8 \\
5 & 2 & CDA & rATGFluCyTBI & $8 / 8$ & 20 & 25 & - & - & 3.6 \\
6 & 6 & FA & rATGFluCy & $8 / 8$ & 22 & 20 & - & - & 3.4 \\
8 & 5 & FA & rATGFluCy & $7 / 8$ & 15 & 17 & Gr.II & Mod. & 0.2 \\
9 & 9 & SCN & AlemFluTTMel & $8 / 8$ & 20 & 16 & Gr.II & N/A & 0.4 \\
10 & 0.4 & CAMT & AlemFluTTMel & $8 / 8$ & 22 & 15 & - & N/A & 0.2 \\
\hline
\end{tabular}

[[P257 Table] 1. Table 1: Baseline Patient Characteristics and Transplant Outcomes]

Results: Since June 2014, 10 patients have been enrolled and transplanted (Table 1, excluding \#7). Donor source was bone marrow in all. With median follow-up of 3.5 years, 8 of 10 patients survive without disease. Initial engraftment was successful in 9, at a median of 20 and 16 days, for neutrophils and platelets respectively. One patient (5) had secondary graft rejection in the setting of viral reactivation (CMV/EBV), with successful engraftment following a $2^{\text {nd }}$ unrelated HCT. In 8 engrafted patients, myeloid (CD33) chimerism was $100 \%$ at all timepoints; T-lymphoid (CD3) chimerism was mixed but reached $>/=95 \%$ (Figure 1 ). One patient (7) with SAA had primary graft rejection in the setting of inadequate TNC dose $\left(0.5 \times 10^{8} / \mathrm{kg}\right)$ and died from marrow aplasia/infection despite $2^{\text {nd }}$ HCT. A second death from Wilms' tumor occurred 17 months post successful HCT, in a patient (1) with DBA and constitutional chromosome abnormality. Except patient 7, all patients received 4 doses of abatacept, which was well tolerated, with all severe adverse events expected for a HCT population. CMV and EBV reactivation occurred in 3 patients each, with resolution using standard anti-viral therapy. One patient (6) was diagnosed with EBV-driven post-transplant lymphoproliferative disease, which responded to rituximab and immune suppression withdrawal. No patients developed severe acute (grade III-IV) or chronic GVHD (Table 1), and no patients required systemic immune suppression at $>1$ year.

Conclusions: These preliminary data suggest that abatacept can be safely added to cyclosporine and MMF GVHD prophylaxis in pediatric patients with bone marrow failure undergoing unrelated donor HCT, with encouraging rates of GVHD despite half of patients having a mismatched (7/8) donor. Given the higher risk of graft rejection in this non-malignant cohort, rejection (in addition to GVHD) will be a primary focus in our subsequent multi-center, phase 2 trial.

Clinical Trial Registry: ClinicalTrials.gov Identifier: NCT01917708

Disclosure: Kean, Leslie: Regeneron Pharmaceuticals, Inc. (research funding), Magenta Therapeutics (consultancy), Bristol Myers Squibb (research funding, other), Kymab (consultancy), Bioverativ (consultancy), Equilium (consultancy), EMDSerono (consultancy)

All other authors: Nothing to declare

\section{P258}

Abstract already published.

\section{P259}

Evaluation of acute graft versus host disease of the esophagus

\section{Andreas Kreft ${ }^{1}$, Helmut Neumann ${ }^{1}$, Danjell Sartorius von} Bach $^{1}$, Eva Maria Wagner-Drouet

\section{${ }^{1}$ University Medical Center Mainz, Mainz, Germany}

Background: Acute intestinal graft-versus-host disease (GvHD) is a serious threat after allogeneic hematopoietic stem cell transplantation (alloHSCT). Although the clinical and histological characteristics of acute GvHD are wellknown for most parts of the gastrointestinal tract, acute esophageal GvHD is not well recognized so fare and not listed in the latest NIH consensus. However since chronic esophageal GvHD in an established diagnostic category, characterized by an esophageal web, acute esophageal may also occur after alloHSCT, possibly preceding the chronic 
GvHD and may to have be separated from from toxicity to infectious complications in the early phase after alloHSCT.

Methods: From our files we identified 65 patients which had upper gastrointestinal tract endoscopy after alloHSCT in with biopsies were taken from the esophagus, stomach and duodenum simultaneously. Of these patients 14 were excluded because of infection, reflux disease or drug toxity and the remaining 51 patients were included in our study. We evaluated the routine stained esophageal biopsies, applied a grading scheme and compared the histological findings with those within the stomach and duodenum, the endoscopic findings and the clinical course.

Results: In 32 of 51 biopsy samples of the esophagus, we identified histological features of acute GvHD, ranging from vacuolar degeneration (grade 1) and single-cell apoptosis (grade 2) to the formation of clefts (grade 3) and mucosa denudation in advanced cases (grade 4), resembling epithelial lesions in acute GvHD of the skin. These findings correlated with GvHD involving the stomach and duodenum and the clinical manifestations of GvHD in other organs. Endoscopically patients with GvHD revealed signs of inflammation, ranging from erythema to ulceration in the more advanced cases, sometimes reminiscent of reflux or infection. Clinically these patients had abdominal discomfort ranging from inappetence to nausea, accompanied by emesis or diarrhea and weight loss.

Conclusions: We have shown that acute esophageal GvHD occurs after alloHSCT and is correlated with acute GvHD in stomach and duodenum. It could be diagnosed and graded histologically. The endoscopic findings are signs of inflammation. Our results may help to establish the histological diagnosis of acute GvHD using endoscopic biopsies from the esophagus and to explain the alterations observed in the esophageal mucosa in patients after alloHSCT.

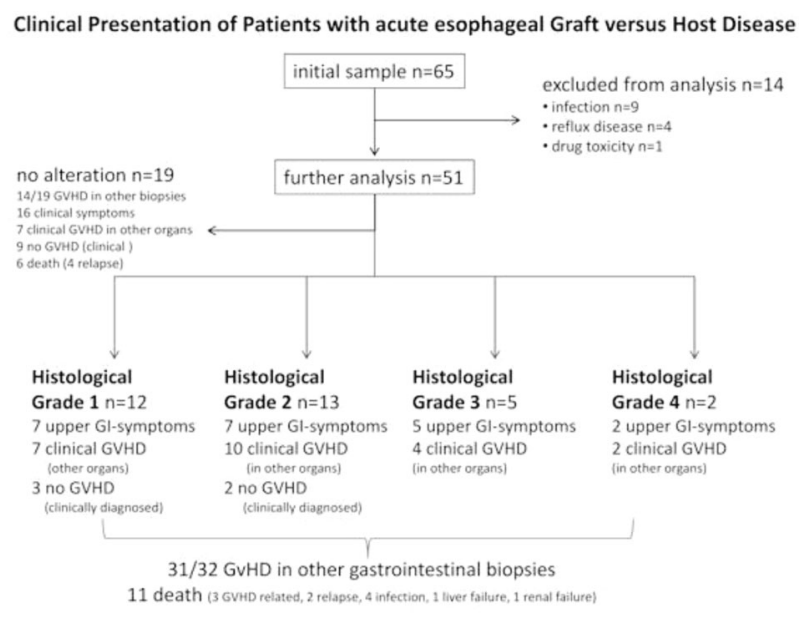

[[P259 Image] 1. Clinical Presentation of Patients with acute esophageal Graft versus Host Disease]

\section{Disclosure: none}

\section{P260}

Histological versus clinical grading of acute intestinal graft versus host disease regarding prediction and prognosis

\section{Andreas Kreft ${ }^{1}$, Helmut Neumann ${ }^{1}$, Mario Schindeldecker ${ }^{1}$, Eva Maria Wagner-Drouet ${ }^{I}$}

${ }^{1}$ University Medical Center Mainz, Pathology, Mainz, Germany

Background: Intestinal acute graft versus host disease (aGvHD) is a major thread after allogenic hematological stem cell transplantation (alloHSCT), with a high mortality in patients which were refractory to steroid treatment in particular. Recent papers point to a correlation of histological grading of intestinal GvHD and prognosis in patient after alloHSCT. However a comparison with clinical scores has not been performed so far.

Methods: In this analysis, retrospective data from 89 patients who underwent endoscopy due to clinical signs of aGVHD (day +20 to +200 after alloHSCT) were evaluated. Of each patient least 3 biopsies from different sites of the colon which were taken simultaneously. Of each biopsy series the maximum histological grad of aGvHD according to the Lerner scheme was obtained and compared with the Glucksberg stage of the lower gastro intestinal tract (GSLGI) and the overall Glucksberg grade (OGG). These three grades were compared for non-relaps related mortality using the Log-Rank test and for sensitivity to steroid treatment applying the Receiver Operating Characteristic for the patients who received steroid treatment. For these patients the non-relaps related mortality for responder and non-responder were calculated using also Log-Rank test.

Results: The histological grade strongly correlated with the survival $(\mathrm{p}=0.009)$. A statistical significant correlation was also found for the GSLGI ( $\mathrm{p}=0.02)$, whereas the OGG revealed no significant correlation $(\mathrm{p}=0.09)$. Non-relaps related mortality was mainly related to infection or sepsis (in 32/56 patients who died). - Eighty-one of the patients received steroid therapy. The sensitivity to the steroid therapy correlated with each of the three scores $(p<0.0001)$ but was the strongest for GSLGI (Area under the curve (AUC) 0.829), compared to OGG (AUC 0.795) and the histological score (AUC 0.691). The survival of the patients, which were sensitive to steroid treatment was significantly better than those of steroid refractory patients $(\mathrm{p}=0.005)$.

Conclusions: We found that histological and clinical grading in patients after alloHSCT with intestinal GvHD was correlated with survival and respond to steroid 
treatment. Histological scoring may predict survival more precisely than OGG and GSLGI but did not add substantial information to the prediction of treatment response.

Non Relaps Mortality in acute intestinal GvHD: Histological Grade versus Clinical Grades

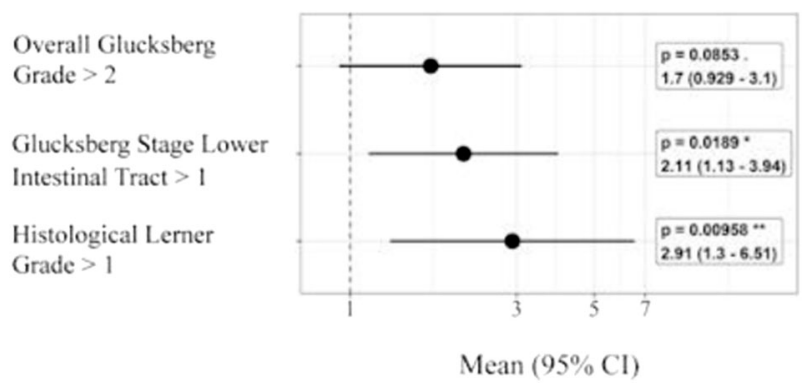

[[P260 Image] 1. Non Relaps Mortality in acute intestinal GvHD: Histological Grade versus Clinical Grades]

Disclosure: none

\section{P261}

Assessment of $B$ cell subsets in patients receiving hematopoietic allogeneic stem cell transplantation

\section{Silvia Bellesi ${ }^{1}$, Elisabetta Metafuni ${ }^{1}$, Federica}

Marchionni ${ }^{1}$, Francesco Corrente ${ }^{I}$, Pier Luigi Puggioni, Sabrina Giammarco ${ }^{I}$, Simona Sica ${ }^{I}$, Valerio De Stefano ${ }^{I}$, Andrea Bacigalupo ${ }^{1}$, Patrizia Chiusolo ${ }^{1}$

${ }^{1}$ Fondazione Policlinico Universitario Agostino Gemelli, Rome, Italy

Background: In human both CD $19+\mathrm{CD} 24^{\text {hi }} \mathrm{CD} 38^{\text {hi }} \mathrm{CD} 1 \mathrm{~d}^{\text {hi }}$ transitional $\mathrm{B}$ cells and $\mathrm{CD} 19+\mathrm{CD} 24{ }^{\mathrm{hi}} \mathrm{CD} 38^{\text {neg }} \mathrm{CD} 27^{\text {pos }}$ memory $\mathrm{B}$ cells have been identified as regulatory subsets. Emerging evidences suggest that regulatory B cells (Bregs) play essential roles in inflammation, autoimmune diseases and tumors. Few data exist about the role of Bregs in the contest of hematopoietic allogeneic stem cell transplantation (HSCT). Some authors have observed that Bregs from patients with chronic graft-versus-host disease (cGVHD) were less frequent and less likely to produce IL-10 than Bregs as compared to healthy donors or patients without cGVHD. These findings suggest that Bregs may be involved in cGVHD pathogenesis. The purpose of our study was to evaluate a possible role of B cell subsets on GVHD occurrence.

Methods: Lymphocyte subset enumeration was performed by AQUIOS CL Flow Cytometer (Beckman
Coulter), a quantitative automated analyzer that performs two diagnostic panels: Tetra-1 CD45-FITC/CD4-RD1/ CD8-ECD/CD3-PC5 and Tetra-2 CD45-FITC/CD56 +CD16-RD1/CD19-ECD/CD3-PC5. B cell subsets (Memory, Mature and Transitional B cells) on peripheral blood samples were analyzed by AQUIOS Designer Software, a tool for the creation of user-defined applications. Panel-1 CD19-FITC/CD10-PE/CD38-ECD/CD24-PC5/CD27-PC7 and Panel-2 CD19-FITC/CD5-PE/CD38-ECD/CD24-PC5/ CD20-PC7 were specifically designed by Beckman Coulter for our center. The flow cytometric analysis was performed as follows: in donors and patients at basal level; on graft products and in patients at days $+30,+60,+90,+120$ after HSCT. Statistical significance was assessed with Prism software (GraphPad) by Mann Withney test. P $<0.05$ was considered statistically significant.

Results: Actually we enrolled 84 patients submitted to HSCT in our center from November 2017. A preliminary statistical analysis was performed on 55 patients. Stem cells source was peripheral blood (PB) in 27 cases and bone marrow $(B M)$ in the others 28 . The conditioning regimen was myeloablative in 26 patients and RIC in 29 patients. aGVHD was diagnosed in 22 patients (40\%). No associations were found between $B$ cell subsets in donors and patients at baseline and the occurrence of aGVHD. However we found a higher median percentage of transitional B cells in graft products in patients without aGVHD (9.6\%, 4.5-26.7) compared to patients with aGvHD $(6.9 \%, 1.5-21.1)(\mathrm{p}=0.02$, Fig 1A). In addition, patients without aGvHD showed a lower median percentage of memory B cells (24.2\%, range 11.6-49) in graft product as compared to patients with aGvHD (34.2\%, range 10.368.15) ( $\mathrm{p}=0.03$, Fig.1B). Finally in the subgroup of patients receiving $\mathrm{PB}$ as stem cell source we observed a higher percentage of CD3+ lymphocytes in graft product in patients with aGVHD (75\%; range 68-80) compared to patients without aGVHD (71\%; range 62-78) $(\mathrm{p}=0.01)$.

In the monitoring of $\mathrm{B}$ cells reconstitution we observed that $\mathrm{CD} 19+$ events did not appear before day +90 after HSCT and these were B transitional immature events predominantly.

Conclusions: Our data suggest a possible protective link between transitional B cells and aGvHD development. These results data need to be confirmed in a larger cohort of patients. Moreover, it will be interesting to evaluate the relationship between transitional B cells at day +90 and the occurrence of cGVHD.

Clinical Trial Registry: NA

Disclosure: Nothing to declare

\section{P262}

Clinical characteristics, inpatient care, and mortality of patients with acute graft-versus-host disease (AGVHD) 
with suboptimal response to corticosteroids: results from a retrospective multicenter study

\section{Jingbo Yu ${ }^{I}$, Shernan G. Holtan ${ }^{2}$, Becky Hanna ${ }^{3}$, Dilan Paranagama $^{1}$, Jackson Tang ${ }^{3}$, Ahmad Naim ${ }^{1}$, John Galvin $^{4}$}

${ }^{1}$ Incyte Corporation, Wilmington, DE, United States, ${ }^{2}$ University of Minnesota, Minneapolis, MN, United States, ${ }^{3}$ Asclepius Analytics, New York, NY, United States, ${ }^{4}$ University of Illinois, Chicago, IL, United States

Background: aGVHD is a major complication of allogeneic hematopoietic stem cell transplant (HSCT) and a risk factor for post-HSCT mortality. The objective of this analysis is to describe patients with aGVHD who had a suboptimal response to corticosteroids.

Methods: Patients who developed IBMTR Severity Index II-IV aGVHD after first HSCT between 1/1/14 to 6/ $30 / 16$ were included in an ongoing chart review at centers in the United States. Patients who had ever participated in a GVHD prophylaxis trial or used JAK inhibitors were excluded from the study. Suboptimal response to corticosteroids was defined as use of additional systemic antiGVHD therapy, inability to taper high-dose steroids ( $\geq 1 \mathrm{mg}$ / $\mathrm{kg}$ ) by $\geq 25 \%$, or tapered corticosteroids by $\geq 25 \%$ but not to $<10 \mathrm{mg} /$ day.

Results: The analysis included 64 patients with suboptimal response to corticosteroids. Mean age was 55 years; $66 \%$ were male. Median time from transplant to aGVHD diagnosis was 33 days. At the time of maximum aGVHD grade, $33 \%$ of patients were grade II and $66 \%$ were grade III-IV; $58 \%$ had lower GI involvement, and 59\% had $\geq 2$ organs involved. From time of diagnosis to maximum aGVHD grade, $52 \%$ of patients had new organ involvement or an increase in aGVHD grade. Median time from diagnosis to maximum grade was 5.5 days, and was 2.5 days for patients with lower GI involvement. Systemic corticosteroids were initiated on the day of diagnosis for $73 \%$ of patients. Average starting daily dose was $93 \mathrm{mg}$ (1.1 $\mathrm{mg} / \mathrm{kg}$ ) for prednisone and $136 \mathrm{mg}(1.6 \mathrm{mg} / \mathrm{kg})$ for methylprednisolone. Steroid dose was increased for $44 \%$ of patients during follow-up; $80 \%$ were unable to taper below $10 \mathrm{mg} /$ day. Among patients who received additional systemic anti-GVHD therapy $(\mathrm{n}=32), 41 \%$ increased their corticosteroid dose before initiation of additional antiGVHD therapy. Median time from initiation of corticosteroids to additional therapy was 16.5 days. Frequently used therapies were mycophenyalate mofetil (25\%), ATG (16\%), extracorporeal photophoresis (13\%), tocilizumab (13\%), etanercept $(9 \%)$, and sirolimus $(9 \%)$. aGVHD recurred in $31 \%$ of patients and was managed by increasing corticosteroid dose in $70 \%$ of patients. $44 \%$ had any infection within first 100 days post-HSCT. Forty patients (63\%) required hospital readmission(s); $40 \%$ had $\geq 2$ readmissions within 100 days post-HSCT, with a mean inpatient lengthof-stay of 28 days. Relapse of underlying malignancy was reported for $13(20 \%)$ patients. Two-thirds $(66 \%, \mathrm{n}=40)$ patients died at a median of 87 (interquartile range (IQR): 44-180) days from aGVHD diagnosis; a higher proportion (76\%) of patients with maximum grade III-IV aGVHD died at a median of 63 (IQR: 41.5-186.5) days; majority (81\%) of patients with lower GI aGVHD died at a median of 68.5 (IQR: 44-137) days.

Conclusions: A majority of patients with aGVHD who had suboptimal response to systemic corticosteroids had severe and rapidly progressing disease and resulted in a high mortality rate $(66 \%)$; progression was more rapid and mortality increased for patients with lower GI involvement. Most patients required readmission to the hospital with extended length-of-stay. An urgent need exists for effective and tolerable therapies that quickly resolve life-threatening aGVHD in early stages of disease.

\section{Disclosure:}

Jingbo Yu, Dilan Paranagama, and Ahmad Naim are employees of Incyte Corporation.

Shernan G. Holtan has served as a consult for Incyte and BMS. Asclepius Analytics (Jackson Tang and Becky Hanna) received funding for this project from Incyte Corporation.

John Galvin has received consulting fees from Incyte.

\section{P263}

\section{Abstract already published.}

\section{P264}

Impact of extracorporeal photopheresis (ECP) in patients with bronchiolitis obliterans (BO) after allogeneic stem cell transplantation (ALLOHCT): Single center experience

\section{Beatriz Aguado ${ }^{1}$, Patricia Lopez-Pereira ${ }^{1}$, Elena Sola ${ }^{1}$, Isabel Vicuña ${ }^{1}$, Adrián Martinez-Vergara ${ }^{1}$, María Churruca $^{l}$, Rosa Girón ${ }^{1}$, Ángela Figuera ${ }^{1}$, Adrián Alegre $^{I}$}

${ }^{1}$ Hospital Universitario de la Princesa, Madrid, Spain

Background: $\mathrm{BO}$ is the pulmonary manifestation of chronic graft-versus-host disease (cGVHD), with high morbidity and mortality. There is no standard treatment and the management depends on the experience of each center. One therapeutic option is ECP, although few studies have analyzed its efficacy in this setting. 


\begin{tabular}{|c|c|c|}
\hline TOTAL $(\mathrm{n}=46)$ & ECP $32.6 \%(n=15)$ & $\begin{array}{l}\text { NO ECP } \\
67.4 \%(n=31)\end{array}$ \\
\hline $\begin{array}{l}\text { AGE AT HCT } \\
\text { (years) }\end{array}$ & $46(29-64)$ & $49(22-68)$ \\
\hline $\begin{array}{l}\text { GENDER • Male } \\
\text { - Female }\end{array}$ & $\begin{array}{l}53.3 \%(\mathrm{n}=8) / \\
46.7 \%(\mathrm{n}=7)\end{array}$ & $\begin{array}{l}58.1 \%(\mathrm{n}=18) / \\
41.9 \%(\mathrm{n}=13)\end{array}$ \\
\hline $\begin{array}{l}\text { UNDERLYING } \\
\text { DISEASE・MDS } \bullet \\
\text { AML・ALL・NHL } \\
\cdot \text { Others }\end{array}$ & $\begin{array}{l}33.3 \%(\mathrm{n}=5) / 46.7 \% \\
(\mathrm{n}=7) / 13.3 \%(\mathrm{n}=2) \\
/ 6.7 \%(\mathrm{n}=1) / 0 \%\end{array}$ & $\begin{array}{l}25.8 \%(\mathrm{n}=8) / 29 \% \\
(\mathrm{n}=9) / 12.9 \%(\mathrm{n}=4) / \\
3.2 \%(\mathrm{n}=1) / \\
29 \%(\mathrm{n}=9)\end{array}$ \\
\hline $\begin{array}{l}\text { CONDITIONING } \\
\text { REGIMEN・MAC } \\
\cdot \text { RIC }\end{array}$ & $\begin{array}{l}60 \%(\mathrm{n}=9) / \\
40 \%(\mathrm{n}=6)\end{array}$ & $\begin{array}{l}48.4 \%(\mathrm{n}=15) / \\
51.6 \%(\mathrm{n}=16)\end{array}$ \\
\hline $\begin{array}{l}\text { STEM CELL } \\
\text { SOURCE } ・ \text { Peripheral } \\
\text { blood } ・ \text { Bone Marrow }\end{array}$ & $100 \%(\mathrm{n}=15) / 0 \%$ & $\begin{array}{l}90.3 \%(\mathrm{n}=28) / \\
9.7 \%(\mathrm{n}=3)\end{array}$ \\
\hline $\begin{array}{l}\text { DONOR } \bullet \text { Matched- } \\
\text { related } \bullet \text { Matched- } \\
\text { unrelated } \bullet \text { Mismatch- } \\
\text { unrelated } \bullet \\
\text { Haploidentical }\end{array}$ & $\begin{array}{l}60 \%(\mathrm{n}=9) / 33.3 \% \\
(\mathrm{n}=5) / 6.7 \% \\
(\mathrm{n}=1) / 0 \%\end{array}$ & $\begin{array}{l}51.6 \%(\mathrm{n}=16) / 32.3 \% \\
(\mathrm{n}=10) / 12.9 \%(\mathrm{n}=4) \\
/ 3.2 \%(\mathrm{n}=1)\end{array}$ \\
\hline $\begin{array}{l}\text { ABO } \\
\text { COMPATIBILITY } \bullet \\
\text { Compatible • Major/ } \\
\text { Bidirectional } \bullet \text { Minor }\end{array}$ & $\begin{array}{l}73.3 \%(n=11) / 20 \% \\
(n=3) / 6.7 \%(n=1) /\end{array}$ & $\begin{array}{l}51.6 \%(n=16) / 16.1 \% \\
(n=5) / 32.2 \%(n=10)\end{array}$ \\
\hline $\begin{array}{l}\text { CMV STATUS } \bullet \mathrm{D} \\
+/ \mathrm{R}+\cdot \mathrm{D}+/ \mathrm{R}-\cdot \mathrm{D}-/ \mathrm{R} \\
+\cdot \mathrm{D}-/ \mathrm{R}-\end{array}$ & $\begin{array}{l}46.7 \%(n=7) / 0 \% \\
40 \%(n=6) / \\
13.3 \%(n=2)\end{array}$ & $\begin{array}{l}64.5 \%(\mathrm{n}=20) / 19.3 \% \\
(\mathrm{n}=6) / 16.1 \% \\
(\mathrm{n}=5) / 0 \%\end{array}$ \\
\hline $\begin{array}{l}\text { GVHD } \\
\text { PROPHYLAXIS } \bullet \\
\mathrm{CSA}+\mathrm{MTX} \cdot \mathrm{CSA}+ \\
\mathrm{MMF} \cdot \mathrm{CSA}+ \\
\mathrm{MTX}+\mathrm{ATG}\end{array}$ & $\begin{array}{l}93.3 \%(n=14) / 6.6 \% \\
(n=1) / 0 \%\end{array}$ & $\begin{array}{l}77.4 \%(\mathrm{n}=24) / 6.4 \% \\
(\mathrm{n}=2) / 16.1 \%(\mathrm{n}=5)\end{array}$ \\
\hline
\end{tabular}

[[P264 Table] 1. Table 1. Demographic and Transplant characteristics]

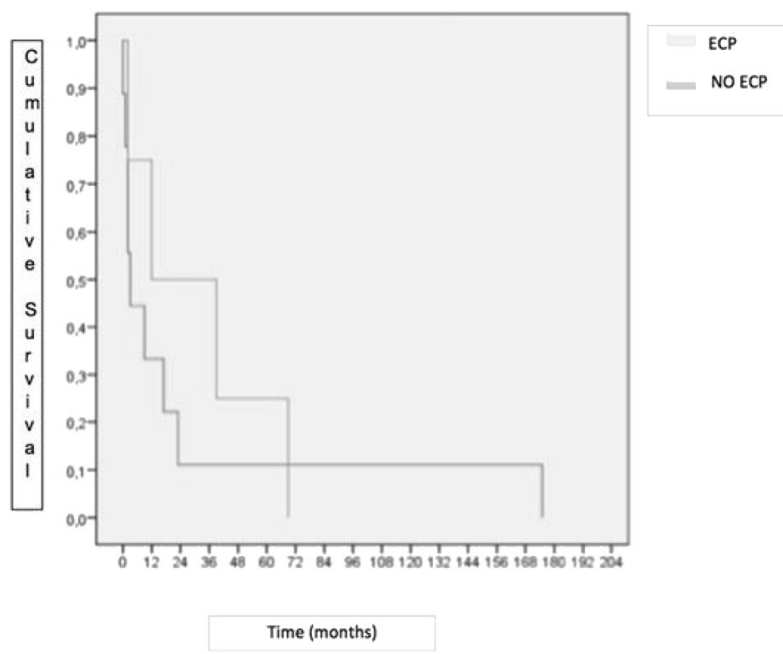

[[P264 Image] 1. Figure 1. Survival curves]
Methods: We retrospectively analyzed 289 alloHCT from January 2009 to December 2017, of which 46 (15.9\%) developed BO (NIH 2014 criteria) (Table 1). Patients who received $\mathrm{ECP}$ as a second line treatment for $\mathrm{BO}$ are analyzed independently. ECP procedures were performed with Therakos Cellex device.

Results: Median time to onset of BO from alloHCT was 6.9 months (range 0.6-31.2). Previous acute GVHD in $52.2 \%(\mathrm{n}=24)$ [grades III-IV $29.2 \%(\mathrm{n}=7)$ ]. In $23.9 \%(\mathrm{n}$ $=11$ ) $\mathrm{cGVHD}$ had exclusive lung involvement, while the other 35 patients $(76 \%)$ had other organs affected. At diagnosis of BO, $69.6 \%(n=32)$ were under immunosuppressive treatment.

$32.6 \%(\mathrm{n}=15)$ of patients with BO received ECP as second-line treatment. Median duration of treatment was 22 months (1.5-36.8) and time to response 9.9 months (1.930.2). Median of sessions was 40 (4-108). Evaluation of response was based on the evolution of FEV1 measurement: 26.6\% $(\mathrm{n}=4)$ complete response; $40 \%(\mathrm{n}=6)$ partial response and $20 \%(\mathrm{n}=3)$ stable disease. One patient did not get any response and another was not evaluable. $66.6 \%$ of patients $(\mathrm{n}=10)$ could reduce immunosuppression, and in one case it was completely discontinued. There is a trend for early separation between survival curves in favor of ECP (Figure 1). One patient had sepsis secondary to central venous catheter infection as complication related to ECP.

Conclusions: ECP has emerged as a promising treatment for BO after alloHCT. In our experience, ECP was effective to stabilize or improve the disease in many patients and allowed to taper esteroids with minimal associated complications. However, prospective studies and longer follow-up are needed to support these findings.

Disclosure: Nothing to declare

\section{P265}

Hypofibrinogenemia and an infection-masking effect of tocilizumab in patients treated due to graft vs. host disease or cytokine release syndrome

\section{Alicja Sadowska-Klasa ${ }^{1}$, Agnieszka Piekarska ${ }^{1}$, Patrycja Mensah-Glanowska ${ }^{2}$, Maria Bieniaszewska ${ }^{I}$, Jan Maciej Zaucha $^{l}$}

${ }^{1}$ Medical University of Gdansk, Gdansk, Poland, ${ }^{2}$ Collegium Medicum Jagiellonian University, Krakow, Poland

Background: The key role of IL-6 signaling in acute graft vs. host disease (aGVHD) and cytokine release syndrome (CRS) has evoked growing use of tocilizumab, an anti-IL6 receptor (IL6-R) antibody, in these settings. Apart from regulation of $\mathrm{T}$ - and $\mathrm{B}$-cell differentiation, immune cells migration to inflammatory sites and T-cell recruitment, IL-6 complex with IL6-R through gp130 upregulates production 
of fibrinogen $(\mathrm{Fg})$ and other acute phase proteins, including $\mathrm{C}$-reactive protein (CRP).

Methods: We retrospectively analyzed data of 7 patients treated with tocilizumab $(8 \mathrm{mg} / \mathrm{kg})$ due to steroid-refractory (SR) aGvHD and 4 patients because of CRS. Median age was 37 and 47 years, respectively. Seven patients were transplanted from unrelated donors (MUD/MMUD) and 4 from sibling donors. Eight patients received myeloablative and 3 reduced intensity conditioning regimen. Analyzed data included concentrations of Fg, CRP, an incidence of infections at tocilizumab administration and in weeks following the infusion.

Results: Stage II aGvHD was diagnosed in 1 patient, stage III in 4, and stage IV in 2 patients. Involvement of the gastrointestinal tract (GI) was observed in $85 \%$ of cases. The median Fg concentration before tocilizumab administration was $2.56 \mathrm{~g} / \mathrm{l}$ (range, 1.4-7) and CRP $21 \mathrm{mg} / \mathrm{dl}$ (range, $1-260)$ and $60 \%$ of patients had an active infection. After infusion of the antibody, we observed a decline of $\mathrm{Fg}$ and CRP levels. The median level of Fg was $1.02 \mathrm{~g} / \mathrm{l}$ (range, 0.46-1.7) 7 - 14 days after the tocilizumab infusion with no severe bleeding complications. A median CRP value was $1.82 \mathrm{mg} / \mathrm{dl}$ (range, 0.1-36) despite confirmed infectious complications. Three weeks after infusion of tocilizumab Fg raised to the normal range in $85 \%$ of patients (Fig 1). Five patients with SR aGVHD achieved a complete response, and 2 had a partial response after tocilizumab therapy.

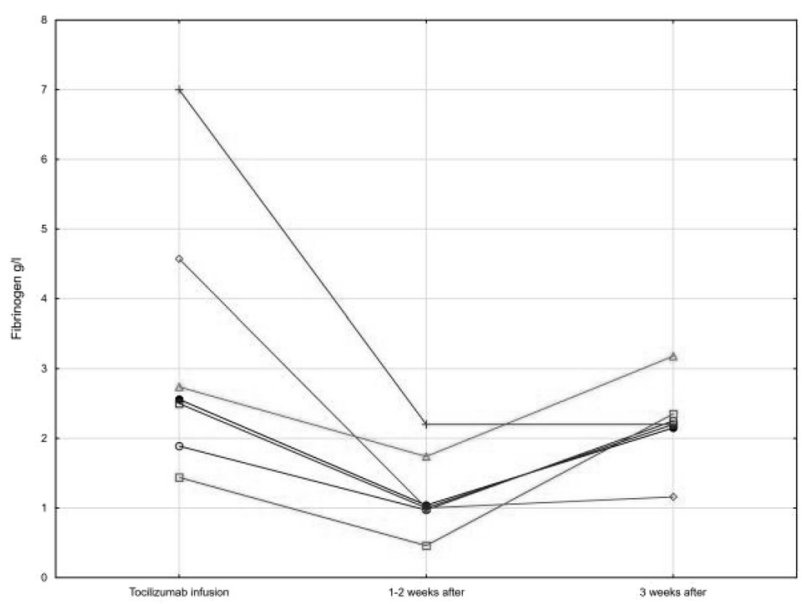

[[P265 Image] 1. Fibrinogen levels in GvHD patients following tocilizumab infusion.]

A group treated with tocilizumab due to CRS had higher initial levels of Fg $5.1 \mathrm{~g} / \mathrm{l}$ (range, 4.1-6.6) and CRP $143 \mathrm{mg} /$ dl (range, 80-227) before administration of the drug. Reduced Fg and CRP levels from a baseline value were also observed in this group. However, concentrations were higher than in GVHD patients: Fg $2.02 \mathrm{~g} / \mathrm{l}$ (range 1.2-3.4) and CRP $3.2 \mathrm{mg} / \mathrm{dl}$ (range 2.7-11). In all patients, a differential diagnosis of disseminated intravascular coagulation was excluded.

Conclusions: 1. Fibrinogen declines after tocilizumab therapy due to its cytokine-regulated production in the liver. Coagulation monitoring should be performed during the first 3 weeks after administration of the antibody to avoid serious bleeding complications.

2. CRP concentrations remain low despite the presence of active infections following infusion of tocilizumab. CRP fails as a marker of infection during 3 weeks following the therapy.

3. Tocilizumab is an effective therapy in patients with aGvHD, especially with the GI involvement.

Disclosure: Nothing to declare

\section{P266}

Vanishing bile ducts after allogenic HSCT: Is it really GVHD?

Antonio Grasso', Lorenzo D'Antiga', Aurelio Sonzogni', Massimo Gregori ${ }^{3}$, Alessandra Maestro ${ }^{3}$, Roberto Simeone $^{3}$, Natalia Maximova ${ }^{3}$

${ }^{1}$ University of Trieste, Trieste, Italy, ${ }^{2}$ Hospital Papa Giovanni XXIII, Bergamo, Italy, ${ }^{3}$ Institute for Maternal and Child Health IRCCS Burlo Garofolo, Trieste, Italy, ${ }^{6}$ Institute for Maternal and Child Health, IRCCS 'Burlo Garofolo, Trieste, Italy

Background: Evaluation of liver GVHD was historically based by elevation of bilirubin levels and by reduction and degeneration of small bile ducts on histological samples of post-transplant liver biopsy. However, there is a lack of studies that compared histological finding of ductopenia between post-autologous HSCT and post-allogenic HSCT. Studying severity of ductopenia following allogenic HSCT, we aimed to demonstrate lack of correlation between ductopenia and clinical signs of liver GVHD.

Methods: We retrospectively collected a series of 72 allogeneic HSCT performed from 2005 to 2017 in the Institute Burlo Garofolo. All patients undergo percutaneous liver biopsy in most cases at three months, one year and three or more years after HSCT. Indications for biopsy were alteration noted at 2 weekly follow-up assessments of at least one clinical or laboratory marker of liver impairment or cholestasis. Ductopenia was defined by number of portal tracts with no interlobular bile duct divided by the total number (severe if the ratio was less than 0.2). Clinical GVHD was defined by NIH consensus criteria

Results: Our population involved $64 \%$ males and $36 \%$ females with oncological (70\%) and non-oncological underlying disease $(30 \%)$. Clinical signs of liver GVHD 
were present in $18 \%$ of the patients $(n=13), 8 \%$ with contextual intestinal involvement, $8 \%$ with cutaneous and intestinal involvement.

71 patients underwent biopsy at a mean time of 110 +/-32 days after HSCT, 45 patients underwent a biopsy at 12 months after HSCT and 35 patients after three or more years from HSCT. Results of biopsies are showed in table 1. No difference in incidence of ductopenia were found between liver GVHD group and no GVHD.

\begin{tabular}{|c|c|c|c|}
\hline $\begin{array}{l}\text { All } \\
\text { patients }(n=72)\end{array}$ & $\begin{array}{l}\text { Biopsy at } 3-6 \\
\text { months }(n=71)\end{array}$ & $\begin{array}{l}\text { Biopsy at } \\
12 \text { months }(n=45)\end{array}$ & $\begin{array}{l}\text { Biopsy at } 3 \text { or } \\
\text { more } \\
\text { years }(n=35)\end{array}$ \\
\hline $\begin{array}{l}\text { Any degree of } \\
\text { Ductopenia }\end{array}$ & $90 \%$ & $94 \%$ & $95 \%$ \\
\hline $\begin{array}{l}\text { Severe } \\
\text { Ductopenia }\end{array}$ & $57 \%$ & $66 \%$ & $58 \%$ \\
\hline $\begin{array}{l}\text { Liver } \\
\text { GVHD }(n=13)\end{array}$ & $\begin{array}{l}\text { Biopsy at } 3-6 \\
\text { months }(n=13)\end{array}$ & $\begin{array}{l}\text { Biopsy at } \\
12 \text { months }(n=7)\end{array}$ & $\begin{array}{l}\text { Biopsy at } 3 \text { or } \\
\text { more } \\
\text { years }(n=5)\end{array}$ \\
\hline $\begin{array}{l}\text { Any degree of } \\
\text { Ductopenia }\end{array}$ & $84 \%$ & $100 \%$ & $100 \%$ \\
\hline $\begin{array}{l}\text { Severe } \\
\text { Ductopenia }\end{array}$ & $54 \%$ & $57 \%$ & $60 \%$ \\
\hline $\begin{array}{l}\text { No Liver } \\
\text { GVHD }(\mathrm{n}=59)\end{array}$ & $\begin{array}{l}\text { Biopsy at } 3-6 \\
\text { months }(\mathrm{n}=58)\end{array}$ & $\begin{array}{l}\text { Biopsy at } \\
12 \text { months }(n=38)\end{array}$ & $\begin{array}{l}\text { Biopsy at } 3 \text { or } \\
\text { more } \\
\text { years }(n=30)\end{array}$ \\
\hline $\begin{array}{l}\text { Any degree of } \\
\text { Ductopenia }\end{array}$ & $88 \%$ & $92 \%$ & $93 \%$ \\
\hline $\begin{array}{l}\text { Severe } \\
\text { Ductopenia }\end{array}$ & $55 \%$ & $65 \%$ & $70 \%$ \\
\hline
\end{tabular}

[[P266 Table] 1. Table 1: Incidence of ductopenia 3-6 months, 12 months and 3 or more years after HSCT in total population, GVHD group and no-GVHD group]

The group that not received chemotherapy prior the HSCT had an overall incidence of ductopenia of $77 \%$ (severe ductopenia of $42 \%$ ) statistically significative in comparison with the oncological underlying disease group (94\% of ductopenia and $64 \%$ of severe ductopenia).

Furthermore, a little sub-group of 16 patients extrapolated from our population received liver biopsy before HSCT for diagnostic assessments: of the 10 with an oncological underlying disease $70 \%$ already showed ductopenia, while no signs of ductopenia were found in the others with a nononcological disease.

Conclusions: There is no correlation between incidence of GVHD and histologically finding of ductopenia on liver biopsy. Ductopenia may be caused in the first place by chemotherapy treatment received before HSCT and myeloablative conditioning for HSCT and it's not related with GVHD. This hypothesis is strengthened by the subgroup analysis of pre-HSCT biopsy.
Disclosure: Nothing to declare

P267

Ruxolitinib plus extracorporeal photopheresis for steroid refractory acute graft versus host disease after allogeneic stem cell transplantation leads to increased regulatory $\mathbf{T}$ cell level

Franziska Modemann ${ }^{I}$, Francis Ayuk ${ }^{I}$, Christine Wolschke $^{I}$, Maximilian Christopeit ${ }^{I}$, Dietlinde Janson ${ }^{1}$, Ute-Marie von Pein ${ }^{1}$, Nicolaus Kröger ${ }^{1}$

${ }^{1}$ University Medical Center Hamburg-Eppendorf, Oncology, Hematology and Bone Marrow Transplantation, Hamburg, Germany

Background: Second and third line therapies for steroid refractory acute Graft versus Host disease (aGvHD) after allogeneic stem cell transplantation (ASCT) are still lacking. Ruxolitinib, a selective Januskinase $1 / 2$ inhibitor could show high efficacy in aGvHD, as well as Extracorporeal Photopheresis (ECP). Here we report a single center experience of combining both therapeutic approaches in severe steroid refractory aGvHD with additional analysis of immune status of these patients to elucidate direct effects of this treatment on immune response.

Methods: From June 2015 to February 2017, 18 patients (77.8\% male, $22.2 \%$ female, median age: 58.5 years, $\mathrm{r}: 21-$ 73) with steroid refractory aGvHD of lower GI-tract after ASCT were treated with Ruxolitinib and Extracorporeal photopheresis as third, fourth or fifth line therapy. Some patients showed additional aGvHD of skin $(n=7)$, liver $(n=6)$ or upper GI-tract $(n=2)$. All patients had an overall grade III (50\%) or IV aGvHD (50\%). Steroid refractoriness was defined as no improvement in 7 days or aggravation after 5 days of steroid treatment.Median start of Ruxolitinib or ECP was day 86.5 after ASCT (r: 35-257). Medianduration of Ruxolitinib therapy was 59.8 days (r: 14-192) with a median start dosage of $20 \mathrm{mg}$ per day $(2 \times 10 \mathrm{mg}$; r: $10-20$ $\mathrm{mg}$ ). All patients started with 2 ECP treatments per week with an individual reduction of treatment frequency. Median number of ECP treatments was 20.5 (r: 2-71) with a median frequency of ECP therapy once a week (r: 0.52.1). Cytomegalovirus (CMV) status of all patients and immune status of ten patients (lymphocyte count with CD4 $+\mathrm{T}$ helper lymphocyte and regulatory $\mathrm{T}$ cell count) were collected previously, after four weeks of starting combined treatment and four weeks after stopping the treatment.

Results: One-year estimated overall survival (OS) of all patients was $50 \%$ with a median estimated OS of 314 days. 3 patients died because of relapse of underlying disease, one of severe therapy refractory aGvHD of lower GI tract and 5 due to infection complications in aGvHD refractory setting. 
Overall response was $55.5 \%$ (complete remission rate: $44.4 \%$, partial remission rate: $11.1 \%) .72 .2 \%(n=13)$ of the patients had cytopenia CTC I-III during the treatment, no grade IV cytopenia was reported. CMV reactivation during Ruxolitinib occured in $66.7 \%$ of cases $(n=12)$. Tapering of steroids could be performed rapidly with a medium reduction time of 1.75 days for reducing to half of the dosage.Remarkably, regulatory $\mathrm{T}$ cells significantly increased during combined Ruxolitinib/ECP treatment compared to regulatory $\mathrm{T}$ cell count before treatment $(\mathrm{p}=0.02)$ and after stopping treatment, regulatory $\mathrm{T}$ cell count decreased again $(p=0.02$, see figure). Significant changes in whole lymphocyte count or in CD4 $+\mathrm{T}$ helper cell count were not observed.

Conclusions: Treatment of severe steroid refractory aGvHD with Ruxolitinib plus ECP could show a high complete remission rate of $44.4 \%$ with an one year OS of $50 \%$. Detecting increased regulatory $\mathrm{T}$ cell count during the treatment underlines its direct effects on immune response and encourages to pursue this promising therapeutic approach.

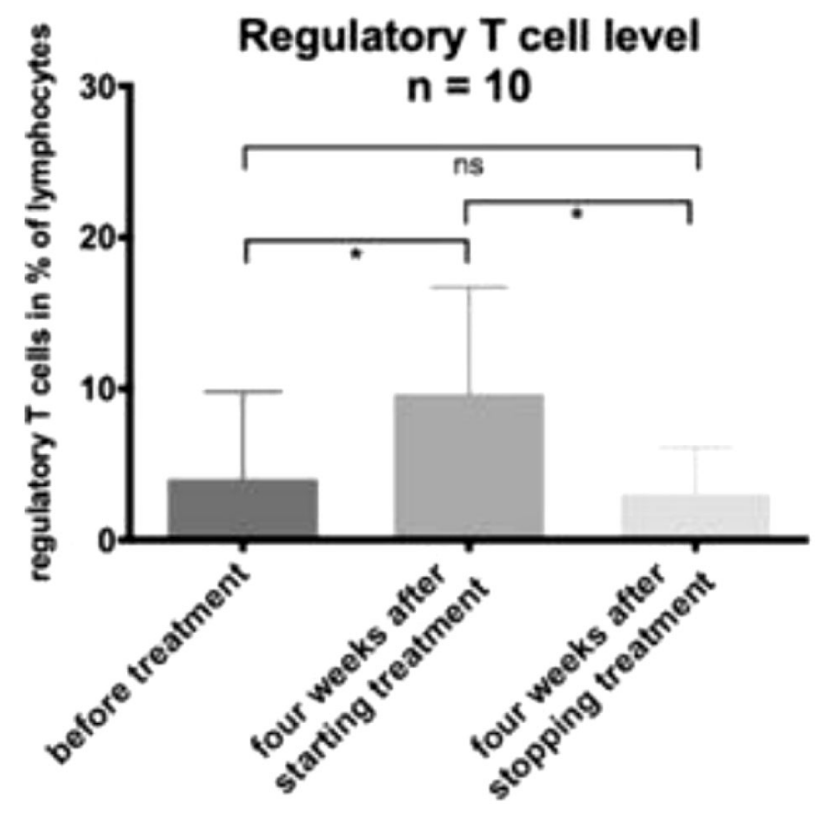

[[P267 Image] 1. Regulatory T cell count]

Disclosure: Nicolaus Kröger received Novartis research grant and honorarium. The other authors have nothing to declare.

\section{P268}

Extracorporeal photopheresis for acute graft-versus host disease and its effect on overall survival through reduction of immunosuppression
Polona Novak ${ }^{1}$, Samo Zver ${ }^{1}$, Matevž Škerget ${ }^{1}$, Saša Anžej Doma $^{1}$, Erik Rupnik ${ }^{1}$, Marko Cukjati ${ }^{2}$ Primož Poženel ${ }^{2}$, Ana Milojković, ${ }^{2}$,Matjaž Sever ${ }^{1}$

${ }^{1}$ UKC Ljubljana, Ljubljana, Slovenia, ${ }^{2}$ Blood Transfusion Centre Ljubljana, Ljubljana, Slovenia

Background: Due to increased immunosuppression infections remain the main cause of death followed by higher risk of relapse in patients treated for acute graft versus host disease (aGvHD) after allogeneic stem cell transplant (SCT). Here we report a single-centre experience with extracorporeal photopheresis (ECP) for acute GvHD that was introduced in order to reduce steroid treatment. Comparison of overall survival (OS) for patients on ECP and patients that received standard first line therapy for aGVHD was performed.

Methods: We retrospectively analysed 62 patients (28\%) with acute GVHD grade II-IV treated from January 2010 to October 2018 out of total 221 allogeneic SCT in that period. All patients received calcineurin inhibitors or sirolimus while receiving steroid treatment for aGvHD. Twenty-five patients $(40 \%)$ received ECP with steroid lowering intent. We defined response as (1) reduction of steroid dose for at least $50 \%$ from baseline while not adding another immunosuppresive agent and (2) not repeating second steroid treatment if the ECP was started after lowering of steroids to prevent aGVHD flare. We checked separately patient responsive and refractory/dependent to steroids. On average patients received ECP procedure once weekly.

Results: Tapering of immunosuppressive therapy as defined was successful in $13(52 \%)$ out of 25 patients in ECP group. In a group of patients without ECP 14 (37\%) patients had steroid refractory or steroid dependent aGVHD compared to 11 (44\%) patients in ECP group. Four (16\%) patients with steroid refractory or dependent aGVHD showed improvement in ECP group compared to only one $(2,7 \%)$ in non ECP group. Twenty $(74 \%)$ patients died due to infectious complication and $7(26 \%)$ due to relapse in non ECP cohort. In ECP cohort 10 (77\%) patients died due to infection and $3(23 \%)$ due to relapse. Median OS was 5 months in non ECP group (r., 1-99) compared to 21 months (r., 2-76) in ECP group and OS of $20 \%$ at 5 years in non ECP compared to $35 \%$ in ECP cohort was observed. Patients with aGvHD treated with ECP and faster steroid tapering had longer OS compared to patients without ECP $(p=0,03)$.

Conclusions: ECP enables successful tapering or withdrawal of steroid therapy in many patients, even in those who are steroid refractory or steroid dependent. Reduction of immunosuppression leads to reduced incidence of infection and relapse which translates into a better overall survival. 
Disclosure: Nothing to declare

\section{P269}

\section{Ruxolitinib: An effective JAK1/2 inhibitor in salvage} therapy of refractory chronic GVHD

\section{Marion Simonet $^{1}$, Ana Berceanu ${ }^{1}$, Etienne Daguindau ${ }^{1,2}$, Yohan Desbrosses $^{1}$, Eric Deconinck ${ }^{1,2}$, Fabrice Larosa ${ }^{1}$}

${ }^{1}$ University Hospital of Besancon, Besancon, France, ${ }^{2}$ Univ. Bourgogne Franche-Comté, INSERM, EFS BFC, UMR 1098, Interactions Hôte-Greffon-Tumeur/Ingénierie Cellulaire et Génique, Besancon, France,

Background: The curative potential of allogenic stem cell transplantation is hampered by Graft-Versus-Host disease (GVHD). Pre-clinical study showed an efficacity of JAK1/2 inhibitor, ruxolitinib, in treatment of steroidrefractory GVHD.

Methods: We reported in this monocentric retrospective study, ruxolitinib response and follow up of 44 cases of chronic GVHD (cGVHD) not improved with standard immunosuppressive therapy. Complete organ response (CR) was defined as the resolution of clinical manifestations of cGVHD in a specific organ. Very good response partial (VGPR) was defined as an improvement of clinical manifestations of cGVHD with more than $50 \%$ decrease of corticosteroid, while a partial response (PR) was associated with less than $50 \%$ decrease of corticosteroid. Treatment failure was defined by the absence of improvement of cGVHD, deterioration of cGVHD in any organ by at least one stage, the development of cGVHD manifestations in a previously unaffected organ, and the use of any additional agents to control the disease.

Results: Median age at transplant was 52 years (range, 19-66). 59\% of patients presented an acute myeloid leukemia. Donor type was sibling $(\mathrm{n}=12)$, unrelated $(n=24)$ or haploidentical $(n=6)$.Two patients benefited a cord blood transplant. Patients received either myeloablative $(64 \%)$ or reduced intensity $(36 \%)$ conditioning regimens. Stem cell source was peripheral blood for $75 \%$ of patients. Patients presented mild $(n=11)$, moderate $(n=21)$ or severe $(n=12)$ cGVHD according to NIH score. Median number of regimens prior to ruxolitinib was 1 (range, 0-6), among those corticosteroids $(\mathrm{n}=36)$. Median follow-up after ruxolitinib was 18 months (range, 4-32). Overall responses rate (ORR) at 1 month was $84 \%$ with $34 \% \mathrm{CR}$, $6 \%$ VGPR and $43 \%$ PR (Figure 1). $13 \%$ of patients failed at 1 month after introduction of ruxolitinib. The rate of CR increased with time : $45 \%$ at 3 months $(n=38), 53 \%$ at 6 months $(\mathrm{n}=30)$ and $65 \%$ at 12 months $(\mathrm{n}=23)$. But VGPR rate was rather stable at 3 months (24\%), at 6 months (33\%) and at 12 months $(22 \%)$ vs $6 \%$ at 1 month. Among the patients under steroids, 21 (58\%) patients discontinued steroids. The 12-months overall survival (OS) and diseasefree survival (DFS) after ruxolitinib was 89\% (80\%-99\%, 95\% CI) and $86 \%(76 \%-96 \%, 95 \%$ CI), respectively. Severe cytopenia (grade 3 and 4) was observed in 12 patients. After introduction of ruxolitinib, 4 patients presented bacterial infections, 3 patients presented an invasive pulmonary aspergillosis and 1 patient developped a pneumocystis. Cytomegalovirus reactivation requiring preemptive treatment was observed in 7 patients. No toxicities required withdrawal of ruxolitinib.

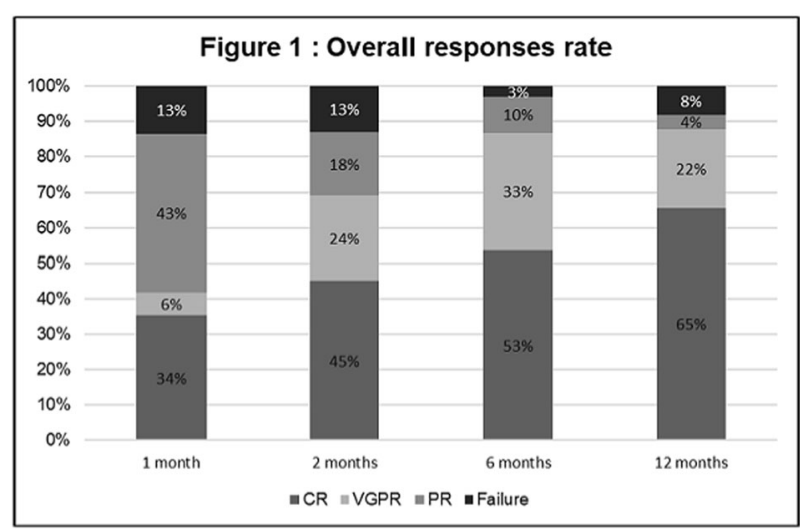

[[P269 Image] 1. Figure 1: Overall Response Rate of chronic GVHD under ruxolitinib therapy]

Conclusions: Ruxolitinib is concidered as efficient and safe to treat chronic GVHD in second line. Prospective study is currently underway.

Disclosure: Nothing to declare

\section{P270}

Mesenchymal stem cell therapy for steroid refractory acute graft versus host disease

\section{Sema Anak ${ }^{1}$, Yöntem Yaman ${ }^{1}$, Kürşat Özdilli ${ }^{1}$, Hulya Bilgen $^{1}$, Ebru Tuğrul Saribeyoğlu' ${ }^{2}$, Volkan Hazar ${ }^{3}$, Fatma Nur Ayman ${ }^{1}$, Murat Elli ${ }^{1}$}

${ }^{1}$ Medipol University, Istanbul, Turkey, ${ }^{2}$ Hospital CarlThiem-Klinikum Cottbus, Pediatric Hematology Oncology, Istanbul, Turkey, ${ }^{3}$ Medical Park Hospitals, Pediatric Hematology Oncology, Istanbul, Turkey,

Background: Acute graft-versus-host disease (aGVHD) is a serious complication of allogeneic hematopoietic stem cell transplantation. Steroid response, as first-line therapy, occurs in more than half of the patients. Mortality rates in patients, especially in grade IV steroid-resistant acute graft versus host disease, exceed $90 \%$. The reported complete 
and partial response to mesenchymal stem cells (MSC), as second-line therapy, varies from $15 \%$ to $82 \%$ in acute GVHD patients. We report our experience using MSCs to treat refractory aGVHD.

Methods: The study was a retrospective single center study. All data were collected from patients' files. Twenty patients were enrolled (age ranging from 7 months to 19 years) between April 2014 and April 2018.

Results: Five of these patients received reduced intensity conditioning and 15 patients received myeloablative regimens before HSCT. One haploidentical, 1 autologous, 1 cord blood, 14 MUD, 3 MSD transplantations were performed. The patients were eligible if they developed grades II-IV aGVHD. All patients were treated with standard first-line treatment with corticosteroids and at least one second-line therapy. The definition of steroid resistant aGVHD considered as either no response to steroid treatment lasting at least 7 days or progression during treatment of at least one grade within the first 72 hours. Prophylactic treatment with calcineurin inhibitors continued at therapeutic dose level. Totally, 77 doses of MSCs were infused. The median dose of MSC was $1.02 \times 10^{6}$ cells per $\mathrm{kg}$ body weight. The median duration between the diagnosis of aGVHD and initiation of MSCs therapy was 16 days (range: 4-241). The received MSC doses ranged from one to seven. None of our patients had severe side-effects during infusions of MSCs. Overall, complete response (OCR) was obtained in 10 patients, partial response in 7 patients and no response (NR) was documented in 3 patients. In our study group, the complete response rates in liver, gastrointestinal, skin aGVHD were $20 \%, 35 \%, 72 \%$ respectively. Four patients $(20 \%)$ died in 90 days after using MSCs from complications of aGVHD. Eleven of 20 patients $(55 \%)$ were still alive with a median follow-up of 558 days (range:171989 days) after first MSCs infusion. One year estimated probability of overall survival for patients achieving OCR and partial remission/no remission in 90th day of MSCs were $87.5 \%$ and $20 \%$, respectively.

Conclusions: In conclusion, MSCs appears to be a safe and effective treatment option for pediatric patients with steroid refractory aGVHD.

Disclosure: Nothing to declare

\section{P271}

Effect of extracorporeal photopheresis on production of serum elafin in chronic graft versus host disease

\section{Arun Alfred ${ }^{1}$, Charlotte Burton ${ }^{1}$, Kathryn Goddard ${ }^{1}$, Nichloas Matthews ${ }^{I}$}

${ }^{1}$ The Rotherham Foundation Trust, Rotherham, United Kingdom
Background: Extracorporeal photopheresis (ECP) is a second line therapy for steroid refractory, dependent or intolerant chronic GVHD (cGVHD). In order to guide ECP there is an unmet need for predictive and diagnostic biomarkers. Elafin is a serine-protease inhibitor primarily produced by epithelial cells, particularly keratinocytes in inflammatory skin diseases and plasma and epidermal elafin have been identified as biomarkers of skin $\operatorname{GVHD}(1,2)$. Since Skin cGVHD is noted for a particularly high response rate to $\mathrm{ECP}$, we conducted a study to investigate whether ECP affects the production of elafin.

Methods: Serum samples were collected from 72 cGVHD patients (39 male /33 female; age range: 25-74) and 17 age-matched healthy controls (10 male / 7 female) before ECP and at 3 month intervals up to 1 year. Patients had GVHD affecting skin (61/72), mucosal membranes (16/ $72)$, liver (14/72), joints (8/72), gut (17/72), eye (8/72), genital (4/72), and respiratory involvement (4/72). Serum elafin was assessed by ELISA (R\&D Systems). Data were analysed using GraphPad Prism 6. Statistical tests performed include 2-tailed Mann-Whitney, Pearson's correlation test, and 2-way ANOVA with repeat measures, as appropriate.

Results: Chronic GVHD patients presenting for ECP had significantly elevated serum levels of elafin $(\mathrm{P}=0.0029$; median of $22 \mathrm{ng} / \mathrm{ml}$, IQR $15-45 \mathrm{ng} / \mathrm{ml}$ ) compared to healthy controls (median of $14 \mathrm{ng} / \mathrm{ml}$, IQR 9.6-18ng/ml). While $85 \%$ of patients had skin involvement, only $40 \%$ had elafin levels above the IQR of healthy controls. Where disease scores were available $(\mathrm{n}=25)$ there were no significant correlations with Modified Rodnans $(r=0.19)$ or NIH BSA scores $(\mathrm{r}=0.37)$.Sub-analysis was performed by grouping cGVHD patients according to quartiles of serum elafin at pre-ECP baseline. Retrospective analysis of patients after 12 months of ECP $(n=66)$ revealed that those with serum elafin levels in the upper quartile (elafin ${ }^{\text {hi }}$ ) pre-ECP (min-max: 46$117 \mathrm{ng} / \mathrm{ml}$ ), showed a significant reduction after 3 months of therapy ( $<<0.05$; mean $+/$ - SD $: 72 \mathrm{ng} / \mathrm{ml}+/-26 \mathrm{ng} / \mathrm{ml}$ vs $53 \mathrm{ng} / \mathrm{ml}+/-23 \mathrm{ng} / \mathrm{ml}$, respectively), which was sustained up to 12 months of ECP $(\mathrm{P}<0.0001$; mean $+/-\mathrm{SD}: 37 \mathrm{ng} /$ $\mathrm{ml}+/-20 \mathrm{ng} / \mathrm{ml})$. In contrast, patients with elafin levels below the upper quartile (elafin ${ }^{\text {lo }}$ ) showed no significant change (mean +/- SD: $21 \mathrm{ng} / \mathrm{ml}+/-11 \mathrm{ng} / \mathrm{ml}$ vs $26 \mathrm{ng} / \mathrm{ml}$ $+/-20 \mathrm{ng} / \mathrm{ml}$, respectively). Of note, pre-ECP patients with elafin below the median received significantly more corticosteroid (CS) than those above, $(\mathrm{P}<0.05$; mean +-SD: $27+/-18 \mathrm{mg} / \mathrm{d}$ vs $16+/-16 \mathrm{mg} / \mathrm{d}$, respectively), which was significantly reduced after 3 months of ECP $(\mathrm{P}<0.001$; to $13+/-10 \mathrm{mg} / \mathrm{d}$ ), while CS dose was not significantly changed in elafin ${ }^{\text {hi }}$ patients until 6 months $(\mathrm{P}<0.05$; mean +/-SD: $16 \mathrm{mg} / \mathrm{d}$ +/- $16 \mathrm{mg} / \mathrm{d}$ vs $6 \mathrm{mg} / \mathrm{d}+/-5.5 \mathrm{mg} / \mathrm{d}$, respectively). 
Conclusions: Consistent with recent data, we found that serum elafin is significantly elevated in a subset of cGVHD patients compared to healthy controls, but did not correlate with skin disease scores. ECP administration was associated with a reduction in serum elafin in the Elafin ${ }^{\text {hi }}$ subset. Further, elafin ${ }^{\text {lo }}$ and elafin ${ }^{\text {hi }}$ patients tolerated different rates of ECP-mediated tapering of CS immunosuppression suggests pre-ECP elafin measurements may have predictive value.

References: 1.Paczesny S. et al 2010. Sci.Transl.Med2: 13ra2 2. Bruggen, $\mathrm{M}-\mathrm{C}$ et al 2015. J.Invest.Dermatol. 135;999

\section{Disclosure:}

Dr N.C Matthews-Research Grant from Mallinckrodt ltd.

Dr A.Alfred-Speaker fees,reseracg grant from Mallinckrodt ltd.

\section{P272}

Ruxolitinib use in graft versus host disease in pediatric hematopoietic stem cell transplant patients. Two centres experience

Yasmina Mozo', María Isabel Benitez ${ }^{2}$, Blanca Rosich ${ }^{1}$, David Bueno ${ }^{1}$, Luisa Sisinni, María Luz Uría ${ }^{1}$, Laura Alonso $^{2}$, Cristina Díaz de Heredia ${ }^{2}$, Antonio PérezMartínez $^{1}$

${ }^{1}$ Hospital Universitario La Paz, Madrid, Spain, ${ }^{2}$ Hospital Universitari Vall d'Hebron, Barcelona, Spain

Background: Allogenic hematopoietic stem cell transplantation (HSCT) is a potential curative treatment for many malignant and no malignant hematologic diseases, primary immunodeficiencies and some metabolic and deposit diseases in children. Graft versus host disease (GVHD) is a major cause of morbidity and a leading cause of non-relapse mortality. Corticosteroids are the standard first-line systemic treatment for both acute and chronic GVHD, whereas no second line option for corticosteroid-refractory patients is standardised. Ruxolitinib is a potent inhibitor of JAK 1/ 2 showing significant responses in refractory GVHD patients in recent reports.

Methods: We present two centres experience with Ruxolitinib for GVHD treatment in pediatric patients. The study was conducted in two spanish pediatric HSCT centres, Hospital Vall d'Hebron (Barcelona) and Hospital Universitario La Paz (Madrid). All patients receiving Ruxolitinib since the drug was available were included for retrospective analysis.

Results: Between March 2017 and December 201819 pediatric patients with acute or chronic GVHD with refractoriness to corticosteroids were treated with
Ruxolitinib, in 22 different episodes (one patient received it in 3 different moments, and one patient received it in 2).

Patient's sex at birth was female in 11 and male in 8 cases. Median age at HSCT was 10,94 years $(0,79-18,11)$. Primary disease was malignant in 11 patients and non malignant in 8 .

Median time of GVHD diagnosis was 47,5 days (6-525). All GVHD episodes were treated with corticosteroids as first line, with maximum doses between $1-5 \mathrm{mg} / \mathrm{kg} /$ day (the main dose used was $2 \mathrm{mg} / \mathrm{kg} /$ day, 15/22 episodes). Patients received a median number of 3,5 (2-7) previous lines of treatment including steroids before starting Ruxolinib; they were extracorporeal photopheresis (19/22 episodes), sirolimus (7/22), mesenchymal cells (5/22), micofenolate (4/22), tacrolimus, etanercept and infliximab (3/22 episodes each), imatinib (2/22), rituximab and low dose methotrexate (1/ 22 each).

Ruxolitinib initiation was indicated for acute gut refractory/steroid dependant GVHD in 9 episodes and chronic multisystemic in 7 episodes. Other indications were chronic lung (3/22 episodes), chronic skin (2/22) and acute skin GVHD (1/22). Median post-HSCT time of Ruxolitinib start was 300 days. Doses ranged between 2,5-10 mg/12h depending on age, weight, and tolerance (hematologic and liver toxicities). Average duration of treatment was 186 days (11-616). Complete response (CR) rate was 18,1\%, global partial response (PR) 72,7\%, and no response (NR) $9 \%$ (progression in one patient and recent treatment start in other patient). Mean time to maximum response was 4 weeks. Treatment stop cause was CR in 2 cases, infection in 4 , liver toxicity in 1 . No severe side effects directly related to Ruxolitinib treatment were described.

Conclusions: Ruxolitinib has been recently introduced as second line strategy for rescuing corticosteroid-refractory GVHD in pediatric patients. While results of randomized trials are lacking, we present our experience (two centres). The main indications for starting treatment were acute gut and chronic multisystemic GVHD. Most patients achieved some grade of response (partial or complete), allowing stopping or tapering corticosteroids. Toxicity profile appears to be acceptable.

Disclosure: Nothing to declare.

\section{P273}

Stability of tacrolimus concentration early after allogeneic hematopoietic stem cell transplantation reduces the risk of acute GVHD

Motohito Okabe ${ }^{I}$, Takanobu Morishita ${ }^{I}$, Tomoe Ichiki ${ }^{1}$, Yuka Kawaguchi', Yoonha Lee ${ }^{I}$, Marie Oobiki, Miyo Goto $^{1}$, Masahide Osaki ${ }^{1}$, Hiroaki Araie ${ }^{1}$, Tatsunori Goto ${ }^{1}$, Yukiyasu Ozawa ${ }^{I}$, Koichi Miyamura ${ }^{I}$ 
${ }^{1}$ Japan Red Cross Nagoya first hospital, Hematology, Nagoya, Japan

Background: Tacrolimus is used as an immunosuppressive drug after allogeneic hematopoietic stem cell transplantation (allo-HSCT). It is well known that early concentration level of tacrolimus is correlated with the risk of acute graft versus host disease (aGVHD), however, whether range of standard derivation (SD) of early tacrolimus concentration after alloHSCT also affect to the risk of aGVHD still remains unknown. Here, we investigate the correlation between the range of SD of early tacrolimus concentration after donor hematopoietic cells engraftment and the development of aGVHD.

Methods: We retrospectively assessed 207 patients who underwent allo-HSCT in our hospital from 2010-2017. All patients received standard GVHD prophylaxis by continuous intravenous (iv) TAC with starting dose of $0.02 \mathrm{mg} /$ $\mathrm{kg}$ /day from 1 day before allo-HSCT (day -1) and iv methotrexate on day $1,3,6$ at dose of $10 \mathrm{mg} / \mathrm{m}^{2}, 7 \mathrm{mg} / \mathrm{m}^{2}, 7$ $\mathrm{mg} / \mathrm{m}^{2}$, respectively. TAC dosage was adjusted to target the serum concentration of $8-12 \mathrm{ng} / \mathrm{ml}$ until at least day 30 and then tapered. To evaluate the SD of weekly tacrolimus concentration, the range of SD of tacrolimus concentration at day1-7 (week-1), day8-14(week-2), day15-21(week-3) and day22-28(week-4) were calculated. The difference of the range of SD between the 2 groups that develop or did not develop aGVHD was compared by using MannWhitney $U$ test. Multivariate analysis was performed by using multiple logistic regression analysis. Patients had given written consent allowing the use of medical records for research, in accordance with the Declaration of Helsinki, and the Institutional Review Board approved the study.

Results: There were 114 males and 93 females and the median age was 45 years (range, 16 to 68 years). The risks of disease were low-standard in 141 and high in 66 pts. The number of donors were in 6 HLA-identical sibling, 13 in HLA-mismatched related donor, 81 in HLA-matched unrelated donor and 107 in HLA-mismatched unrelated donor. Thirty-seven patients developed aGVHD (Grade I-II; 28, GradeIII-IV; 9 patients). As a result, the wide range of SD at week-3 significantly increased the risk of aGVHD (aGVHD- group; $1.178 \pm 0.8159 \mathrm{ng} / \mathrm{ml}$, aGVHD+ group; $1.447 \pm 0.7359 \mathrm{ng} / \mathrm{ml}, \mathrm{p}=0.02$ ). Multivariate analysis demonstrated that narrow range of $\mathrm{SD}$ of tacrolimus concentration at week-3 reduce the risk of aGVHD $(\mathrm{OR}=4.19 ; 95 \% \mathrm{CI}: 1.59-14.80 ; \mathrm{p}=0.005)$. There were no correlation between gender, age, disease status, HLA with the development of aGVHD.

Conclusions: The range of SD at week-3, an engraftment phase of donor hematopoietic cells, was significantly correlated with the development of aGVHD. Fine tuning of early tacrolimus concentration with narrow range of SD reduces the risk of aGVHD, resulting in improvement of the overall survival after allo-HSCT.

Disclosure: Nothing to declair

\section{P274}

\section{Ruxolitinib treatment for steroid-refractory graft- versus-host disease}

Han-Seung Park ${ }^{I}$, Je-Hwan Lee ${ }^{I}$, Jung-Hee Lee ${ }^{I}$, Eun-Ji Choi $^{1}$, Miee Seol ${ }^{1}$, Young-Shin Lee ${ }^{1}$, Young-Ah Kang ${ }^{1}$, Mijin Jeon ${ }^{1}$, Kyoo-Hyung Lee ${ }^{1}$

${ }^{1}$ Asan Medical Center, University of Ulsan College of Medicine, Seoul, Korea, Republic of

Background: Steroid-refractory graft versus-host disease (GVHD) is one of the most lethal complications after allogeneic hematopoietic cell transplantation. Recent studies have shown that ruxolitinib, a Janus kinase 1/2 inhibitor, is effective in patients suffering from GVHD. Here, we report a retrospective result of ruxolitinib treatment for steroid-refractory GVHD.

Methods: All patients had received cyclosporine and a short course of methotrexate as GVHD prophylaxis. Antithymocyte globulin was added for unrelated or mismatched familial donor HCT. Ruxolitinib $5 \mathrm{mg}$ twice daily was added to immunosuppressive treatment in patients with steroid-refractory GVHD.

Results: A total of 27 patients with GVHD (acute, 8, including 3 patients with donor lymphocyte infusion [DLI]related; and chronic, 19) were included in the analysis. All patients had grade 3/4 acute GVHD or severe chronic GVHD at the time of ruxolitinib treatment.

Six $(75.0 \%)$ of 8 patients with acute GVHD responded to ruxolitinib, including 3 with complete response (CR). The median time to response was 13.5 days (range, 8-25). Nineteen patients received ruxolitinib for severe chronic GVHD, with the median of 3 involved organs (range 2-5). Fourteen patients $(73.7 \%)$ showed response to ruxolitinib, including $4 \mathrm{CRs}$. The median time to response was 24 days (range, 12-138). Five responders discontinued ruxolitinib and 9 patients are still on the agent.

After a median follow-up duration of 8.7 months, 5 died ( 2 from relapse of disease, 3 from infection). The 1-year survival probability was $70.1 \%$. Eleven of 20 responders discontinued ruxolitinib. GVHD relapsed in 3 of 11 patients at 14,35 , and 149 days after ruxolitinib discontinuation. Thrombocytopenia $(12 / 27$, Grade $3 / 4 ; 4)$ was the most common adverse event of ruxolitinib. During treatment, 4 with grade 3/4 infectious adverse events occurred; 2 pneumonias, 1 brain abscess, and 1 liver abscess.

Conclusions: Ruxolitinib treatment seems to be effective for the treatment of steroid-refractory GVHD including 
long-standing chronic GVHD. The agent was well tolerated and relatively safe.

Disclosure: Nothing to declare.

\section{P275}

IL6-receptor antibody tocilizumab as salvage therapy in the treatment of severe chronic gvhd after stem cell transplantation: A retrospective analysis

Anna-Sophia Kattner ${ }^{1}$, Ernst Holler ${ }^{I}$, Barbara Holler ${ }^{1}$, Sebastian Klobuch ${ }^{I}$, Daniela Weber ${ }^{1}$, Danilo Martinovic ${ }^{1}$, Matthias Edinger ${ }^{I}$, Wolfgang Herr ${ }^{1}$, Daniel Wolff ${ }^{l}$

${ }^{1}$ University Hospital Regensburg, Hematology \& Oncology, Regensburg, Germany

Background: Severe chronic graft-versus-host disease (cGvHD) remains the most relevant factor affecting survival and long-term quality of life after allogeneic hematopoietic stem cell transplantation (HCT). Besides corticosteroids there is no established therapy for cGvHD and many of the used immunosuppressive agents may lead to significant toxicity incl. infectious complications. Tocilizumab (an IL6-receptor antibody) has shown efficacy in acute GvHD and cGvHD. We retrospectively analyzed the efficacy and safety of patients having received tocilizumab for treatment of advanced cGvHD at our center between the years 2015 and 2018.

Methods: 9 patients with severe steroid refractory cGvHD and a median age of 50 years (range: 21-62 yrs) having received at least two prior lines of therapy for cGvHD (range: 2-8 regimens) were treated with tocilizumab for at least one cycle (q4w, dosage: $8 \mathrm{mg} / \mathrm{kg}$ iv, maximum: $800 \mathrm{mg}$ ) with a median number of 4 cycles (range: 1-22). $\mathrm{NIH}$ consensus criteria grading for cGvHD and the immunosuppressive regimen were noted at the time of the first tocilizumab administration and after 3, 6 and 12 months of therapy. All patients received additional concomitant immunosuppressive agents already given at least 4 weeks without response before start of tocilizumab. No new immunosuppression (IS) was added in parallel to tocilizumab and response assessment was stopped at start of any additional new IS. All patients had received peripheral stem cell allografts. GvHD prophylaxis consisted of a calcineurin inhibitor in combination with methotrexate or mycophenolate and in case of unrelated donors ATG was added. 8/9 patients had quiescent onset of cGvHD, one patient developed de novo cGvHD. The median number of days between HCT and onset of cGvHD was 215 (89-545). The median number of days between HCT and initiation of tocilizumab therapy was 1033 (510-1749) days. At cGvHD onset, 7/9 patients had mild cGvHD and 2/9 patients had moderate cGvHD. The thrombocyte count was $<100 / \mathrm{nl}$ in
5/9 patients. Organs involved at initiation of tocilizumab therapy were skin $(100 \%$, all grade 3$)$, eyes $(78 \%)$, mouth $(67 \%)$, fascia (67\%), lungs $(44 \%)$ and genitals (22\%). 5/9 patients are still receiving tocilizumab at the time of analysis.

Results: As tocilizumab was given fairly recently in most patients, 6- and 12-month follow-up was only reached in 3/ 9 patients (33\%). At three-month follow-up after initiation of tocilizumab therapy, $4 / 9$ patients $(44 \%)$ showed partial remission, 2/9 patients stable disease (22\%), and 2/9 patients progressive disease $(22 \%)$ of cGvHD. Maximal response was partial remission $(56 \%)$, stable disease $(11 \%)$ and progressive disease (22\%). 3 patients required subsequent new immunosuppressive treatment. One patient has not yet reached 3-month follow-up. During tocilizumab therapy none of the patients suffered recurrence of underlying malignancy. Two patients developed significant respiratory infection and one patient developed soft tissue infection, all requiring antibiotic treatment and pausing of tocilizumab administration, hospital admission was not required. The OS and RFS was $100 \%$ with median follow up of 8.5 months (range 2-12 months).

Conclusions: Tocilizumab appears to be a promising treatment option in advanced cGvHD but further evaluation within a phase II trial is required.

Disclosure: Nothing to declare

\section{P276}

Prospective analysis of immune reconstitution in patients after alemtuzumab-mediated $T$ cell depletion as predictive marker for acute GVHD

\section{Saskia Hendriks ${ }^{I}$, Pascal Wölfinger ${ }^{I}$, Diana Kriege ${ }^{I}$, Matthias Theobald $^{1}$, Eva Maria Wagner-Drouet ${ }^{I}$}

${ }^{1}$ University Medical Center Mainz, Mainz, Germany

Background: Allogeneic hematopoietic stem cell transplantation (HSCT) is for many patients suffering from AML the only curative treatment option. One major complication is graft versus host disease (GvHD), caused by donor immune cells attacking healthy tissue.

Regulatory $\mathrm{T}$ cells (Treg) have been getting huge attention during the past years because of their important role in maintaining immune balance. Here we collected peripheral blood samples from 11 patients at different time points after HSCT to investigate immune-reconstitution of Treg as predictive marker for the development of GvHD.

Methods: We collected blood samples from 11 patients in the course of allogeneic HSCT prospectively once a week from $d+7$ up to $d+200$. All patients received conditioning regimen with Fludarabine and Melphalan, combined with 
Alemtuzumab for $\mathrm{T}$ cell depletion. 9 patients developed acute GVHD in the later course.

After isolation of PBMC's we performed FACS multicolor staining of $\mathrm{T}$ cell and NK cells. Treg were identified as $\mathrm{CD}^{+}{ }^{+} \mathrm{CD} 4^{+} \mathrm{CD} 25^{++} \mathrm{Foxp}^{+}$, NK cells were characterized as $\mathrm{CD} 3{ }^{\text {neg }} \mathrm{CD}^{-} 6^{+} \mathrm{CD} 16^{+}$and divided in NK cell subpopulation due to their expression of $\mathrm{CD} 56^{\mathrm{dim}}$ or $\mathrm{CD} 56^{\text {high }}$.

Results: 1. CD52 ${ }^{\text {neg }} T$ cells: all patients developing acute GVHD in the later course showed significant elevated levels of $\mathrm{CD} 4^{+} \mathrm{CD} 52^{\text {neg }} \mathrm{T}$ cells, especially $\mathrm{CD} 52^{\text {neg }}$ Treg at $\mathrm{d}+50$. 2. CD52 ${ }^{\text {neg }}$ Treg / CD8 ${ }^{+} \mathrm{CD} 52^{+} \mathrm{T}$ cells: one patient not developing acute GVHD showed lots of CD52 ${ }^{\text {neg }}$ Treg but missed $\mathrm{CD} 8{ }^{+} \mathrm{CD} 52^{+}$effector $\mathrm{T}$ cells. We recently showed that $\mathrm{CD} 8{ }^{+} \mathrm{CD} 52^{\text {neg }}$ effector $\mathrm{T}$ cells are of impaired effector function. These data suggest that CD52 $2^{\text {neg }}$ Treg are only of relevance combined with functional $\mathrm{CD} 8{ }^{+} \mathrm{CD} 52^{+}$effector $\mathrm{T}$ cells in the development of aGVHD. 3. T cell marker: Patients without aGVHD showed elevated expression of Garp on Treg. Garp was significantly higher expressed on $\mathrm{CD}^{2}{ }^{+}$Treg, indicating a better suppressive capacity of $\mathrm{CD} 52^{+}$Treg. This was detected throughout from $\mathrm{d}+50$ until $d+200$. Tigit and ILT3 showed a heterogeneous expression profile without significant differences between the two groups. 4. NK cells: We detected a higher ratio of $\mathrm{CD}^{++} / 5^{+\mathrm{CD} 56^{\mathrm{dim}}}$ NK-cell population in patients without. We could also show that Tigit is mainly expressed on CD56 ${ }^{\mathrm{dim}}$ NK cells.

Conclusions: We and others showed reconstitution of $\mathrm{CD}^{\text {neg }} \mathrm{T}$ cell subsets after Alemtuzumab mediated $\mathrm{T}$ cell depletion - our data on effector T cells showed an impaired effector function for CD52 ${ }^{\text {neg }} \mathrm{CD} 4$ and $\mathrm{CD} 8 \mathrm{~T}$ cells. Recently we presented Data on impaired suppressive capacity of CD52 ${ }^{\text {neg }}$ Treg and the association with acute GVHD retrospectively (Wölfinger EBMT 2018, ASH 2017). Here we provide prospective Data on patients after the use of Alemtuzumab in the context of HSCT: Our preliminary data suggest that the total amount of CD52 $2^{\text {neg- }}$ Treg and the ratio of $\mathrm{CD} 52^{\text {neg }}$ Treg to $\mathrm{CD} 8^{+} \mathrm{CD} 52^{+}$Treg on $\mathrm{d}+50$ after allogenic HSCT could predict aGvHD. This data may be a basis for immune monitoring of patients at $d+50$ to evaluate their risk for aGVHD and could lead to the use of prophylactic Treg DLI in the context of Alemtuzumab mediated $\mathrm{T}$ cell depletion.

Disclosure: Medac - travel support, Novartis - consultancy fee, Pfizer - consultancy fee, Shire consultancy fee

\section{P277}

\section{Abstract withdrawn}

\section{P278}

Factors affecting nutritional status in chronic GVHD
Dina Ljubas Kelecic ${ }^{1}$, Irena Karas ${ }^{I}$, Lana Desnica ${ }^{I}$, Zinaida Peric ${ }^{I}$, Tamara Vukic ${ }^{I}$, Darija Vranesic Bender ${ }^{I}$, Ana Barisic ${ }^{1}$, Andreja Horvat ${ }^{\prime}$, Antonela Samardzic ${ }^{l}$, Lucija Kraljevic ${ }^{1}$, Ivan Sabol ${ }^{2}$, Nadira Durakovic ${ }^{1}$, Ranka Serventi Seiwerth ${ }^{1}$, Radovan Vrhovac ${ }^{1}$, Drazen Pulanic ${ }^{l}$, Steven Pavletic ${ }^{3}$, Zeljko Krznaric ${ }^{1}$

${ }^{1}$ University Hospital Center Zagreb, Zagreb, Croatia, ${ }^{2}$ Rudjer Boskovic Institute, Zagreb, Croatia, ${ }^{3}$ Center for Cancer Research National Cancer Institute, Bethesda, MD, United States

Background: Multiple factors such as disease activity and severity, therapy and/or dietary habits can cause changes in nutritional status independently or by interacting with each other. Presence of malnutrition or significant weight loss in chronic GVHD (cGVHD) patients was reported in literature up to $43 \%$. The aim of this cross-sectional study was to identify factors that affect nutritional status in cGVHD patients.

Methods: Nutritional status in patients with cGVHD treated at the University Hospital Center Zagreb, Croatia from 2015 to 2018 was assessed. Anthropometric measurements (height, body weight (BW), body mass index (BMI)) and clinical validated tool patient-generated subjective global assessment (PG-SGA) (where patients were categorized as well-nourished (PG-SGA A), moderately malnourished (PG-SGA B) or severely malnourished (PG-SGA C)) were used. All patients were evaluated according to 2005 NIH criteria for cGVHD diagnosis. Descriptive and correlation analysis were preformed.

Results: In total, 44 adult cGVHD patients were included in the study, 23 women (52.3\%), median age 47 (18-65) years, with mild cGVHD in $6(13.64 \%)$, moderate in 24 (54.55\%) and severe in $13(29.55 \%)$ patients. According to the PG-SGA rating 19 (43.18\%) patients had PG-SGA A, 19 (43.18 \%) PG-SGA B and 6 (13.64\%) had PG-SGA C, giving a total malnutrition or risk of malnutrition prevalence of $56.82 \%$. The mean BMI was $23.83 \pm 4.3 \mathrm{~kg} / \mathrm{m}^{2}$ with correlation to PG-SGA rating ( $\mathrm{r}=0.446, \mathrm{p}=0.0024)$. Malnutrition according to the BMI (defined as BMI $<21 \mathrm{~kg} / \mathrm{m}^{2}$ ) was found in 8 patients $(18.18 \%)$. BW changes $(10 \%$ or more in 6 months) were significant in 14 patients (31.82\%). According to the PG-SGA assessment tool, oral symptoms reported by 31 patient $(70.45 \%)$ and decreased appetite reported by 12 patients $(27.27 \%)$ were associated with oral cGVHD NIH score $(\mathrm{r}=0.536, \mathrm{p}=0.0002 ; \mathrm{r}=0.441$, $\mathrm{p}=0.0027$ ) but not with BW or BMI. Gastrointestinal (GI) symptoms assessed with SGA, were generally mild with no correlation to GI cGVHD NIH score. No significant association was found between nutritional status and other NIH cGVHD scores. Corticosteroid therapy present in 12 (27.27\%) correlated with PG-SGA rating ( $\mathrm{r}=0.494$, 
$\mathrm{p}=0.0006)$ but not with BW, BMI or appetite changes. In 23 patients $(52.7 \%)$ with altered PG-SGA rating, BMI, appetite and body weight changes, dietary counseling and oral nutritional supplementation were initiated.

Conclusions: Oral symptoms, decreased appetite and corticosteroid therapy in our cGVHD patients were associated with altered nutritional status according to the PG-SGA, but not with BMI. Therefore, PG-SGA might be a more sensitive tool in assessment of changes of the nutritional status and detection of patients at risk of malnutrition than BMI since it includes different factors like physical examination, presence of GI symptoms and corticosteroid therapy in its scoring system. Nutritional counseling and support are important in cGVHD patients especially in presence of oral symptoms.

Disclosure: Nothing to declare.

\section{P279}

Assessment of inflammatory activity of sclerodermoid chronic graft versus host disease, using high-frequency ultrasonography plus doppler-ultrasound and serum biomarkers methods

\section{María Suárez-Lledó ${ }^{1}$, Priscila Giavedoni ${ }^{1}$, Teresa Solano $^{1}$, Gonzalo Gutierrez-Garcia ${ }^{1}$, Laura Rosiñol ${ }^{1}$, Gerardo Rodriguez-Lobato ${ }^{I}$, Ana Moreno-Castaño ${ }^{I}$, Alexandra Pedraza-Navarrete ${ }^{1}$, Alejandra Patricia Martínez-Roca ${ }^{1}$, Francesc Fernandez-Aviles ${ }^{1}$, Alvaro Urbano-Ispizua ${ }^{1}$, Jose Manuel Mascaro ${ }^{1}$, Montserrat Rovira $^{1}$, Carmen Martinez ${ }^{1}$}

${ }^{1}$ Hospital Clínic de Barcelona, Barcelona, Spain

Background: Sclerotic skin changes are common features in chronic graft versus host disease (cGVHD). One of the most challenging aspects in the diagnosis and management of sclerodermoid cGVHD (scGVHD) is the differentiation between reversible symptoms related to active cGVHD and nonreversible symptoms related to residual permanent damage such as long-standing fibrosis. Although several candidate biomarkers of cGVHD inflammatory activity have been proposed, none of them are currently validated. Therefore, there is a need for the development of more quantifiable and reproducible measurements tools to guide clinical decisions. We report our experience evaluating the usefulness of high-frequency ultrasonography (HFUS) plus Doppler ultrasound (Doppler-US) and serum fibrosis biomarkers to determine the inflammatory activity of scGVHD.

Methods: We report 6 patients with scGVHD. HFUS plus Doppler-US were performed at diagnosis of scGVHD and at different time-points after treatment initiation. Serum hyaluronic acid and pro-colagen-III were measured as fibrosis biomarkers simultaneously with HFUS and Doppler-US. NIH
cGVHD 2014 Consensus Conference diagnosis criteria, scoring system, and response criteria were used to assess global and organ-specific cGVHD, and to measure overall response to therapy. Abnormal ultrasound findings were defined as the presence of $\geq 1$ of the following: hypoechogenic dermis, dermo-epidermal junction effacement, hypoechogenicity of septa and/or hyperechogenicity of lobules in hypodermis, hypoechogenic fascia, or myositis, for HFUS; and, vessels thicker than $1 \mathrm{~mm}$ in dermis and/or hypodermis, systolic pressure $>10 \mathrm{~cm} / \mathrm{sec}$, and index of vascular resistance $>0.75$, for Doppler-US. Inflammatory activity was classified as mild, moderate and severe according to the severity of Doppler-US findings.

Results: HFSU showed abnormal findings in all patients at diagnosis with no changes except in two patients along the treatment follow-up. Inflammatory activity by DopplerUS was observed in 5/6 patients at diagnosis ( 1 mild, 3 moderate, 1 severe). Four patients responded to treatment (2 complete responses, $\mathrm{CR}$, and 2 partial responses, $\mathrm{PR}$ ), one presented clinical improvement less than PR, and one, progressive disease. All patients with clinical response had also a P-ROM improvement or normalization. All patients achieving a response showed normalization $(n=2)$ or improvement $(n=2)$ of Doppler-US findings. The patient with clinical improvement less than PR and the patient with progressive disease showed persistence of inflammatory Doppler-US findings. Most patients had normal or light increase of pro-collagen levels at diagnosis and no significant changes were observed during follow-up. Levels of hyaluronic acid tended to be very high in patients with progressive scGVHD (patients 2 and 5) and tended to decrease or normalize in those who responded to therapy (patients 1, 2, and 3).

Conclusions: In this exploratory study, HFSU was a reliable method for evaluating sclerotic skin changes in scGVHD. Doppler-US showed a good correlation with disease activity and response to treatment. Serum hyaluronic acid levels might be a biomarker of disease activity that deserves further investigation. HFSU plus Doppler-US is a useful, non-invasive, repeatable device in monitoring patients suffering from scGVHD. According to our results, Doppler-US may be a more sensitive parameter than HFSU in assessment inflammatory activity of scGVHD.

\section{Disclosure:}

María Suárez-Lledó received a grant from DKMS-Spain Foundation.

Other authors have Nothing to declare

\section{P280}

Post-transplant cyclophosphamide versus antithymocyte-globulin in HLA-matched unrelated and haploidentical transplantation for hematologic malignancies 
Myung Won Lee ${ }^{I}$, Ik Chan Song ${ }^{I}$, Yoon Seok Choi ${ }^{1}$, Su Jin Lee ${ }^{1}$, Seon Hui Cho ${ }^{l}$, Deog Yeon Jo ${ }^{1}$

${ }^{1}$ Chungnam National University Hospital, Daejeon, Korea, Republic of,

Background: Post-transplant cyclophosphamide(PTCy) and antithymocyte-globulin(ATG) are the most commonly used regimens for the prophylaxis of graft-versus host disease(GVHD). We compared these two regimens in HLAmatched unrelated (MUD) and haploidentical transplantation for hematologic malignancies.

Methods: We retrospectively analyzed the consecutive adult patients with hematologic malignancies who received MUD and haploidentical transplantation at Chungnam National University Hospital between January 2013 and January 2018. Patients who received second transplantation and had refractory disease were excluded.

Results: This study included 45 patients with median age of 54 (range, 18-71) years: 29 (64.4\%) patients received MUD transplant ( 8 and 21 patients in PTCy and ATG group, respectively), and 16 (35.6\%) patients received haploidentical transplant (11 and 5 patients in PTCy and ATG group). Graft source was peripheral blood stem cell in all patients. Median follow-up duration was 15.5 months (range, 0.5-58.0). In MUD transplant, the estimated 20-months survival rate were $85.7 \%$ in PTCy vs. $80.7 \%$ in ATG ( $\mathrm{p}=0.835)$, the 20 -months relapse rate were $37.5 \%$ in PTCy vs. $35.0 \%$ in ATG $(p=0.663)$, the cumulative incidence of grade 2 to 4 acute GVHD were $25.0 \%$ in PTCy vs. $22.2 \%$ in ATG $(\mathrm{p}=0.706)$, and the estimated 20-month extensive chronic GVHD rate were $25.0 \%$ in PTCy vs. $16.7 \%$ in ATG $(p=0.902)$. In haploidentical transplant, the estimated 20-months survival rate were $55.4 \%$ in PTCy vs. $40.0 \%$ in ATG $(\mathrm{p}=0.936)$, the 20 -months relapse rate were $42.9 \%$ in PTCy vs. $33.3 \%$ in ATG ( $\mathrm{p}=0.328)$, the cumulative incidence of grade 3 to 4 acute GVHD rate were $29.9 \%$ in PTCy vs. $33.3 \%$ in ATG ( $\mathrm{p}=0.686)$, and the estimated 12 month extensive chronic GVHD rate were $25.0 \%$ in PTCy vs. $50.0 \%$ in ATG $(\mathrm{p}=0.575)$. Patients receiving PTCy had significantly longer neutrophil engraftment time than those receiving ATG in haploidentical transplant [median(range); 17.0(14.0-21.0) days vs. 14.0(13.016.0) days, $\mathrm{p}=0.005]$.

Conclusions: PTCy might be a good option for the prophylaxis of GVHD in HLA-matched unrelated transplant as well as haplo-identical transplant.

Disclosure: Nothing to declare

\section{P281}

Early fam therapy for post allo-HSCT bronchiolitis obliterans syndrome
Nagore Argoitial, Carmen Tejado ${ }^{I}$, Nerea Uresandi ${ }^{I}$, Lourdes Aguirre ${ }^{I}$, Maria Jose Pizarro ${ }^{I}$, Clara Lombardi, Laida Ondarra ${ }^{I}$, Maria Araiz ${ }^{I}$, Carlos Vallejo ${ }^{I}$

${ }^{1}$ University Hospital Donostia, Stem Cell Transplant Program, Donostia, Spain

Background: bronchiolitis obliterans syndrome (BOS) is a potential major complication after allogeneic hematopoietic stem cell transplantation (HSCT). Attributed to an allo-immune reaction against the small airways, $\mathrm{BO}$ is considered a pulmonary manifestation of chronic GVHD. Reported incidence of BOS ranges from 5 to $12 \%$, and BOS-attributed mortality as high as $30 \%-80 \%$. A few years ago, a new therapeutic approach with Fluticasone, Azithromycin, and Montelukast (FAM) was described (Norman BC, et al. BMT 2011). Our aim was to analyze the outcomes of pts who developed BOS and were precociously treated with the FAM scheme.

Methods: all the 209 allo-HSCT performed in our center from January 2015 and July 2018 were included in the analysis. Baseline diseases were: 69 AML, 49 LPD, 31 MDS, 28 ALL, 16 MPD, 10 MM, and 6 BMF. Day +100 and day +365 overall mortality were $9,1 \%$ and $24,4 \%$, respectively. Rest of characteristics of the series are shown in table. FAM therapy was systematically started when any patient was first diagnosed with BO.

Results: eleven patients $(5,3 \%)$ were diagnosed with BOS. At diagnosis of BOS, the pts exhibited a FEV1 $80 \%$ of predicted (median FEV1: 74\%; range; 54-86\%) and/or a decline $>10 \%$ from pre-HSCT . At day $+100,6$ pts had already the syndrome. Two of them died before the end of the first year: one due to invasive zygomycosis (CNS plus pulmonary) and the other to baseline disease progression. At day $+365,5$ more pts had BOS. Two more pts with BOS died at 15 and 17 months post-HSCT due to baseline disease progression. At the close of the analysis, 7 of the 11 pts were alive. So, with a median follow-up of 29 months (range: 844), mortality and BOS-attributable mortality of the pts with the complication were $36,4 \%$ and $9,1 \%$, respectively.

Conclusions:

1) BOS is an infrequent but very severe complication of allo-HSCT;

2) BOS seems to be less frequent in pts with prophylactic pre-transplant rATG or post-transplant cyclophosphamide, as well as in pts undergoing transplantation with $\mathrm{BM}$ (compared to PBSC).

3) Early diagnosis and therapy are critical to minimize the BOS-attributable mortality.

Disclosure: Nothing to declare

P282

Donor lymphocyte infusion for patients with relapsed hematologic malignancies: The comparison between 
related HLA-matched and HLA-haploidentical hematopoietic stem cell transplantation

Yu-Hsiang Chang ${ }^{1}$ Xiu-Wen Liao ${ }^{I}$, Jia-Hau Liu ${ }^{1}$, Ming Yao $^{1}$, Bor-Sheng Ko ${ }^{1}$, Chien-Ting Lin ${ }^{1}$, Chi-Cheng Li ${ }^{1}$, Cheng-Hong Tsai ${ }^{1}$, Shang-Ju Wu ${ }^{1}$, Hui-Hsuan Huang ${ }^{1}$, Tai-Chung Huangl, Hsin-An Houl, Wen-Chien Chou ${ }^{I}$, Feng-Ming Tien ${ }^{l}$, Yun-Chu Lin ${ }^{1}$, Meng-Yao Lu ${ }^{1}$, KaiHsin Lin ${ }^{1}$, Jih-Luh Tang ${ }^{1}$

${ }^{1}$ National Taiwan University, Taipei, Taiwan, Republic of China,

Background: Donor lymphocyte infusion (DLI) is an established treatment for patients with hematological malignancies relapsed after allogeneic hematopoietic stem cell transplantation (HSCT). However, it is associated with an increased risk of graft-versus-host disease (GVHD) and modest anti-tumor activity. Compared to the infusion of nonmobilized lymphocytes, granulocyte colony-stimulating factor (G-CSF)-primed DLI might induce a stronger anti-tumor effect and reduce the risk of infusion-induced GVHD. Due to the limited experience of G-CSF primed DLI in patients relapsed after haploidentical HSCT, we conducted a retrospective study of all patients at our hospital who received DLI for the relapsed hematological diseases following related HLA-matched or HLA-haploidentical HSCT.

Methods: The Institutional Research Board approved the study. We identified 94 patients with hematological malignancies receiving DLI following related allo-HSCT at National Taiwan University Hospital between 1999 and 2018 Aug. The infusate was obtained from the cryopreserved specimen, which had been collected and stored at multiple aliquots at the same time as the initial haploidentical peripheral stem cell graft. Patients received DLI for either hematological relapse, preemptive or prophylactic treatment. Univariate and multivariate analysis was performed using Cox proportional hazard regression model.

Results: For the 61 patients following related HLAmatched and the 33 patients following HLA-haploidentical HSCT received 119 and 72 doses of DLI, respectively. In comparison, the median CD3+ cell dosage of haplo-DLI is significantly lower $(P=0.0224)$ than that of DLI from sibling donors, with median cell dosage $0.45 \times 10^{7} / \mathrm{kg}$ (range, $0.05-12 \times 10^{7} / \mathrm{kg}$ ) and $1.1 \times 10^{7} / \mathrm{kg}$ (range, 0.04$9.78 \times 10^{7} / \mathrm{kg}$ ), respectively. The median time to DLI from initial sibling HSCT and haplo-HSCT was 152 days (range, 13-3357 days) and 155 days (range, 13-946 days), respectively. Overall, 12 (20\%) of the 61 patients following sibling HSCT developed grade 2-4 acute GVHD after DLI, whereas $12(36 \%)$ of the 33 patients receiving haplo-HSCT developed grade 2-4 acute GVHD after DLI $(\mathrm{p}=0.1460)$. Importantly, for patients receiving DLI with $\mathrm{CD} 3+$ cell dosage less than $1 \times 10^{7} / \mathrm{kg}$, there is no difference in the risk of developing grade 2-4 acute GVHD between patients receiving DLI from sibling or haplo donors (Figure 1A). Interestingly, for patients receiving DLI with $\mathrm{CD} 3+$ cell dosage more than or equal to $1 \times 10^{7} / \mathrm{kg}, 4(50 \%)$ of the 8 patients following haplo-HSCT developed grade 2-4 acute GVHD after DLI, significantly more than 5 (14\%) of the 37 patients following sibling HSCT developed grade 2-4 acute GVHD after DLI (Figure 1B). The cumulative incidence of grade 2-4 acute GVHD at day 100 after haplo-HSCT and sibling HSCT were 50\% (95\% CI: $0.13-0.79)$ and $13.5 \%$ (95\% CI: 0.05-0.27), respectively (Figure $1 \mathrm{~B}, P=0.0146$ ).

A

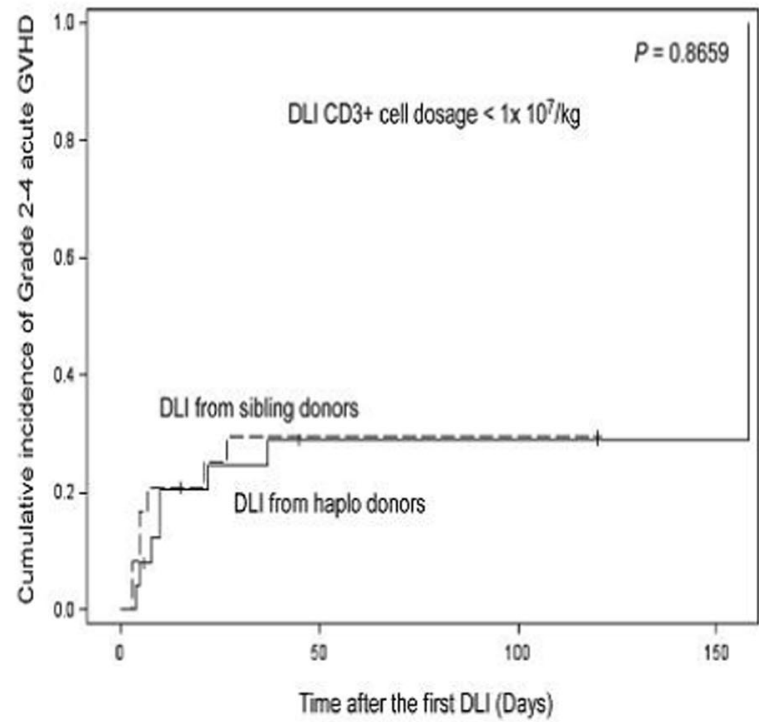

B

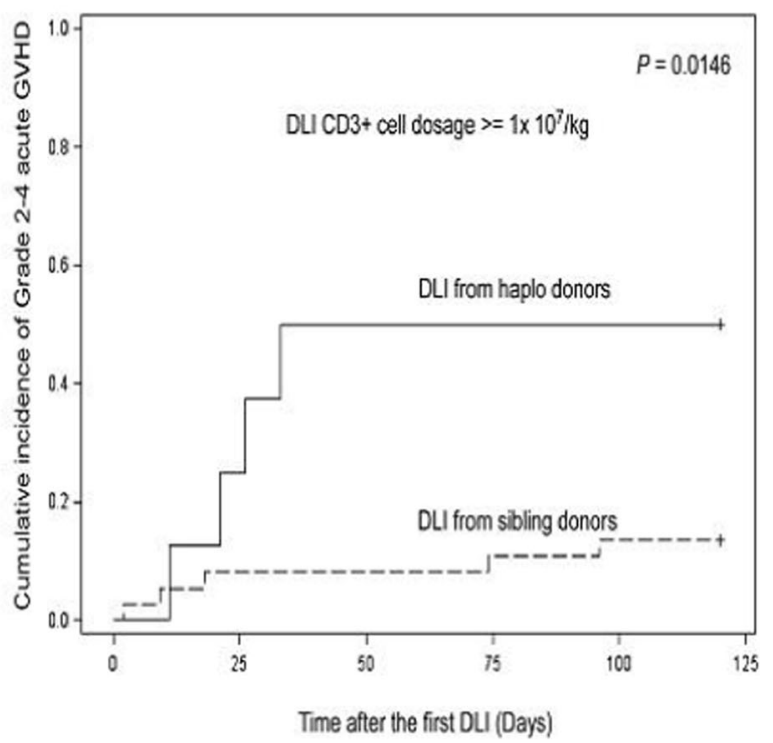

[[P282 Image] 1. Figure 1. Cumulative incidence of grade 2-4 acute GVHD.] 
Conclusions: Our study shows that the administration of G-CSF mobilized DLI is feasible after haploidentical HSCT for relapsed hematological malignancies. However, DLI with $\mathrm{CD} 3+$ cell dosage more than or equal to $1 \times 10^{7} / \mathrm{kg}$ in patients receiving haplo-HSCT is associated with significantly higher risk of developing acute GVHD than DLI from the sibling donors.

Disclosure: The authors declare no competing financial interests.

\section{P283}

Evaluation of a new closed extracorporeal photopheresis system

\section{Jeffrey Winters ${ }^{1}$, Edwin Burgstaler ${ }^{1}$}

${ }^{1}$ Mayo Clinic, Transfusion Medicine, Rochester, MN, United States

Background: The Fresenius Phelix is a UVA irradiation device used to photoactivate MNC collected on the Amicus. The system is closed, utilizing a special MNC kit and modified instrument software. The preliminary results of a phase I safety trial involving three patients (12 treatments) with chronic graft vs. host disease are presented.

Methods: Reasons for transplantation for the patients ages 37, 61 and 62 years were: Acute myelogenous leukemia, myelodysplastic syndrome, and myelodysplastic syndrome with PNH. Stem cell source was peripheral blood with a 10/10 match for all. Each developed chronic skin GVHD. Inclusion criteria included WBC and PLT counts $\geq$ 1000 and $25 \times 10^{9} / \mathrm{L}, \mathrm{GFR} \geq 30 \mathrm{ml} / \mathrm{min} / \mathrm{BSA}$, and AST 10120 unit/L. Exclusion criteria included active GI bleeding, NYHA cardiac disease greater than grade III, and the presence of light-sensitive diseases. Amicus software 4.51 and Phelix software 1.0 were used. Settings included: 80 $\mathrm{ml} / \mathrm{min}$ max draw rate, $2000 \mathrm{ml}$ fixed cycle volume, 1.25 $\mathrm{mg} / \mathrm{kg} / \mathrm{min}$ citrate infusion rate, and 12:1 ACD-A ratio. Venous access was peripheral or subcutaneous port. Target UVA dose was $1.5 \mathrm{~J} / \mathrm{CM}^{2}$ and 8-methoxypsoralen dose was $3.4 \mathrm{ml}$.

Results: The following mean $+\mathrm{SD}$ procedure results were obtained: $2,341 \pm 14 \mathrm{ml}$ whole blood with ACD-A drawn, $191+3 \mathrm{ml}$ ACD-A used, $691+127 \mathrm{ml}$ saline used, $91+$ 5 minutes procedure time, and $\overline{4,881} \pm 419 \mathrm{ml}$ total blood volume. Minor alarms $(\mathrm{N}=4)$ on the Amicus and no alarms on the Phelix were encountered. All 14-day aerobic and anaerobic cultures were negative and mean endotoxin levels were $0.425+0.1752 \mathrm{EU} / \mathrm{ml}$. Mean pre/post CBC and plasma hemoglobin levels were: 12.5/11.8 WBC, 9.9/9.5 neutrophils, 0.06/0.05 basophils, 0.21/0.15 eosinophils, 1.06/1.03 lymphocytes, $1.23 / 1.06$ monocytes, $314 / 293$ platelets $\times 10^{9} / \mathrm{L}, 40 /$ $37 \% \mathrm{HCT}, 13.3 / 12.2 \mathrm{~g} / \mathrm{dl} \mathrm{Hgb}$, and 26.8/23.4 mg/dl plasma hemoglobin. Plasma hemoglobin delta in the product was $0.00+0.001$ grams and the subject was $-0.15+0.70$ grams. Collected product Hct. Mean $1.95 \pm 0.255 \%$. Yields are in the table. Adverse events included one each: Acute respiratory failure, respiratory failure, muscular weakness, musculoskeletal discomfort, and peripheral swelling. Three of four events occurred in one patient two weeks after the study procedure. None of the adverse events were considered related to the procedure or investigational product. The patient who experienced acute respiratory failure was removed from the study because of death due to pneumonia, felt to be unrelated to the procedure.

Conclusions: Results indicate the new closed photopheresis system is capable of collecting sufficient MNC and irradiating the cells producing high lymphocyte apoptosis, with minimal alarms and adverse reactions.

\begin{tabular}{|c|c|c|c|c|c|c|}
\hline Parameters & $\mathrm{N}$ & Mean (SD) & Parameter & $\mathrm{N}$ & Collect & Treated \\
\hline $\begin{array}{l}\text { *Inhibition of } \\
\text { Proliferation (\%) }\end{array}$ & 12 & $95.37(2.41)$ & $\begin{array}{l}\text { *Lymphocyte } \\
\text { Proliferation }(\%)\end{array}$ & 12 & $34.86(12.24)$ & $1.43(0.59)$ \\
\hline $\begin{array}{l}\text { MNC Collection } \\
\text { Efficiency } 1(\%)\end{array}$ & 8 & $55.33(8.22)$ & $\begin{array}{l}\text { *Lymphocyte } \\
\text { Viability }(\%)\end{array}$ & 12 & 72.07 (12.10) & $23.37(9.56)$ \\
\hline $\begin{array}{l}\text { WBC Yield } \\
\left(\mathrm{x} 10^{9}\right)\end{array}$ & 11 & $2.85(1.12)$ & $\begin{array}{l}\text { *Lymphocyte } \\
\text { Apoptosis (\%) }\end{array}$ & 12 & $36.01(13.52)$ & $94.72(4.45)$ \\
\hline $\begin{array}{l}\text { MNC Yield } \\
\left(\times 10^{9}\right)\end{array}$ & 8 & $2.78(0.88)$ & & & & \\
\hline $\begin{array}{l}\text { Lymphocyte } \\
\text { Yield }\left(\mathrm{x} 10^{9}\right)\end{array}$ & 8 & $1.11(0.55)$ & & & & \\
\hline $\begin{array}{l}\text { Monocyte Yield } \\
\left(\mathrm{x} 10^{9}\right)\end{array}$ & 8 & $1.66(0.65)$ & & & & \\
\hline $\begin{array}{l}\text { Granulocyte } \\
\text { Yield (x109) }\end{array}$ & 8 & $0.37(0.21)$ & & & & \\
\hline $\begin{array}{l}\text { Platelet Yield } \\
\left(\times 10^{11}\right)\end{array}$ & 11 & $0.471(0.1504)$ & & & & \\
\hline
\end{tabular}

[[P283 Table] 1. *72 Hour Means (SD)]

Clinical Trial Registry: NA

Disclosure: Research supported by Fresenius Kabi

\section{P284}

Profile of acute graft-versus-host-disease of the gastrointestinal tract following allogeneic haematopoietic cell transplantation from a tertiary care center in India

Arijit Nag ${ }^{1}$, Vivek. S Radhakrishnan', Mita

Roychowdhury ${ }^{2}$, Jeevan Kumar', Saurabh Jayant Bhave ${ }^{1}$, Sayak Chaudhuri ${ }^{3}$, Reena Nair ${ }^{I}$, Mohandas K Mallath', Mammen Chandy

${ }^{1}$ Tata Medical Center, Clinical Hematology and Hematopoietic Cell Transplantation, Kolkata, India, ${ }^{2}$ Tata Medical Center, Clinical Hematology and Hematopoietic Cell Transplantation, Kolkata, India, ${ }^{3}$ Tata Medical Center, Data Manager, Clinical Hematology and Hematopoietic Cell Transplantation, Kolkata, India, ${ }^{4}$ Tata Medical Center, Kolkata, India 
Background: A frequent complication of Allogeneic Hematopoietic Cell Transplantation (Allo-HCT) accounting for significant transplant related morbidity and mortality, is acute Graft Versus Host Disease (GVHD) of the gastrointestinal system. Severe manifestations of acute GVHD of the gut portends a poor prognosis in patients after AlloHCT.

Methods: We conducted a chart review of the patients who underwent Allogeneic HCT at our center between November 2011 and October 2018. The clinical profile of the patients experiencing Acute GVHD of the gastrointestinal system, including treatment received and outcomes were analysed by standard statistical methods to determine baseline- clinical as well as treatment related predictors for the development of Acute Gut GVHD.

Results: 228 patients underwent Allo-HCT for different indications, of whom $63(27.63 \%)$ patients developed acute GVHD. 42 (18.42\%) patients had Acute GVHD of the gastro-intestinal system. The median age was 26.5 years (range: 2-57). Of the 42,30 (71.4\%) patients were male and $12(28.6 \%)$ were females. $13(31 \%)$ patients underwent Allo-HCT for benign haematological diseases (Transfusiondependant Thalassemia: 9, Severe Aplastic Anaemia: 3, Inherited Bone Marrow Failure Syndromes: 1). 29 (69\%) patients had malignant haematological disorders (AML: 13, ALL: 7, CML:5, Myelofibrosis:1, Relapsed Refractory Non-Hodgkin's Lymphoma: 1). Of the patients with GVHD, 24 (57.1\%) underwent Matched Related Donor Allo-HCT, 6 (14.3\%) underwent Matched unrelated donor Allo-HCT while $12(28.6 \%)$ patients had Haploidentical Donor Allo-HCT. 31 (73.8\%) received Myeloablative conditioning regimen, while $11(26.2 \%)$ patients were conditioned with Reduced-Intensity Conditioning regimens. $37(88.1 \%)$ patients received Peripheral Blood stem cell grafts while $5(11.9 \%)$ received Bone marrow grafts. Graft Vs Host Disease Prophylaxis used was Cyclosporine and injectable short-course Methotrexate in 30 (71.4\%) patients, Tacrolimus and Methotrexate in 1 (2.4\%) patient, and PostTransplant Cyclophosphamide along with Tacrolimus and Mycophenolate Mofetil in 11 patients (26.2\%). Grade 1/2 Gut GVHD occurred in 18 patients (Grade1: 7 (16.7\%), Grade 2: $11(26.2 \%)$ ), while Severe (Grade 3/4) Gut GVHD occurred in 24 patients (Grade 3: 15 (35.7\%), Grade 4: 9 $(21.4 \%)) .10(23.8 \%)$ of the 42 patients also had acute GVHD of the skin or Liver. 37 patients $(88.09 \%)$ could be treated and controlled with Methyl-prednislone monotherapy, 5 patients had steroid refractory GVHD of whom 3 patients $(7.14 \%)$ could be salvaged with additional drugs (Infliximab:2; Tacrolimus:1); 2 patients (4.77\%) had refractory acute Gut GVHD and could not be salvaged despite more than three lines of therapy. At the time of reporting, 26 patients $(61.9 \%)$ of the 42 are alive. 11 patients died due to transplant related mortality, while 5 patients developed relapsed disease. On binary logistic regression analysis, no baseline clinical or treatment related predictor (Disease indication, Disease status at transplant, Transplant Type, Graft Source, Type of conditioning) could be identified for developing acute GVHD of the gastrointestinal system.

Conclusions: Acute GVHD of the Gastro-intestinal system is a significant cause for morbidity in Allo-HCT patients at our centre. Further studies are warranted in our cohort, and a prospective analysis of gut microbiome analysis, faecal multi-drug resistance organism surveillance, conditioning related toxicity and antibiotic usage is ongoing.

Clinical Trial Registry: Not applicable

Disclosure: The authors declare no potential conflicts of interest

\section{P285}

\section{Benefits and precautions of ruxolitinib in steroid-} refractory acute GVHD

\section{Dae Sik Hong ${ }^{1}$, Se Hyung Kim ${ }^{2}$, Sung Hee Lim ${ }^{1}$, Chan Kyu Kim ${ }^{1}$, Jong Ho Won ${ }^{4}$, Seong Kyu Park ${ }^{1}$}

${ }^{1}$ Soonchunhyang University Bucheon Hospital, Bucheon, Korea, Republic of, ${ }^{2}$ Soonchunhyang University, Hematology, Bucheon, Korea, Republic of, ${ }^{4}$ Soonchunhyang University Seoul Hospital, Hematology, Seoul, Korea, Republic of

Background: Corticosteroids are the standard first-line treatment option for patients with acute graft-versus host disease (GVHD), but approximately half of patients become refractory to steroids and require second-line treatment. Ruxolitinib has the potential to treat GVHD in steroidrefractory (SR) patients based on retrospective clinical data. The ongoing prospective trials are currently enrolling patients to evaluate the therapeutic potential of ruxolitinib for GVHD.

Methods: We analyzed retrospectively clinical experience with ruxolitinib in patients $(n=15)$ with grade $2 \sim 4$ steroid-refractory acute GVHD patients compared with the control group not receiving ruxolitinib. In addition, immune status was evaluated about 6 weeks $\sim 8$ weeks after the administration of ruxolitinib using flow-cytometry. Ruxolitinib was used as a third option for SR GVHD, combined with previously used immunosuppressive drugs. And steroids were gradually decreased according to the symptoms and discontinued. Patients received ruxolitinib $5 \mathrm{mg}$ twice daily (BID), with increase to $10 \mathrm{mg}$ BID if hematologic parameters are stable and no treatmentrelated toxicities. 
Results: Fifteen patients all were assessable for response. Seven patients achieved a complete response, 5 had a partial response, and 3 had no response at 8 weeks after the first ruxolitinib dose. Overall response rate was $75 \%$. Three were treatment failures. Most adverse effects were manageable, except infectious complications. Infectious complications were occurred in about $73 \%$ patients $(n=11)$, resulting in two deaths. Common cause of infectious events included cytomegalovirus $(n=5)$, herpes-zoster $(n=2)$, Epstein-Barr virus $(n=2)$, fungal infection $(n=2)$, pneumocystis jiroveci $(n=2)$, bacterial infections $(n=1)$, and pneumonia of unknown origin $(n=1)$. T cell counts tended to decreased in the group with ruxolitinib compared with the control group, especially CD4 cell counts.

Conclusions: Ruxolitinib is effective in controlling SR GVHD and can lead to clinical benefits. However, we need to be aware of the infectious complications because ruxolitinib may lead to increased risk of opportunistic infections or reactivation of latent infections. In addition, common infectious complications are presumed to involve $\mathrm{T}$ cell dysfunction.

Clinical Trial Registry: None

Disclosure: Nothing to declare

\section{P286}

Surgical intervention of partial bowel obstruction due to gastrointestinal GVHD in children after hematopoietic stem cell transplantation: A single center experience

Zofia Szmit', Jowita Fraczkiewicz', Matgorzata Salamonowicz ${ }^{1}$, Monika Mielcarek-Siedziuk ${ }^{1}$, Krzysztof Katwak $^{I}$, Mateusz Stachowiak ${ }^{1}$, Elżbieta WawrzyniakDzierżek ${ }^{1}$, Monika Rosa ${ }^{1}$, Marek Ussowicz', Ewa Gorczyńska ${ }^{1}$

${ }^{1}$ Wroclaw Medical University, Wrocław, Poland

Background: Graft versus host disease (GvHD), being one of most common life-threatening complication post HSCT, contributes significantly to morbidity and mortality. When affecting gastrointestinal tract (GI) it is the major cause of death in early period post HSCT. Due to widespread tissue involvement in most patients diagnosed with GI GvHD, surgical treatment is rarely considered.

Methods: Among 972 allo-HSCT performed in Department of Pediatric Hematology, Oncology and Bone Marrow Transplantation in Wroclaw, Poland during years 19962018, 291 (29,9\%) cases were diagnosed with GI GvHD. In this study we present 3 cases $(1 \%)$ which were referred to and benefit from surgical approach.

\section{Results:}

1. Male, 4 years old underwent HSCT from matched unrelated donor (MUD) due to chronic myelogenous leukemia (CML) and subsequent molecular relapse succesfully treated with donor lymphocyte infusion, followed by aGvHD (skin and gut involvement, grade IV). Extensive immunosuppression (steroids, mycofenolate mofetile, ATG, OKT3) resulted in significant resolution of aGvHD symptoms. However aggravating severe abdominal pain and lack of gut movement suggesting bowel obstruction. Due to presence of acute abdomen patient was immediately directed for laparotomy. Resection of constricted bowel segment followed by 2 subsequent laparotomies for secondary obstruction provided complete resolution of abdominal symptoms. After 16 years of follow-up patient is alive and well.

2. Eleven years old male was diagnosed with skin and gut grade IV aGvHD on day +84 post MUD-HSCT performed due to acute myelogenous leukemia (AML). He received pronlonged immunosuppressive treatment including steroids, antibodies, MSC and ECP which led to resolving of skin leasions and diarhoea. Nevertheless patient was suffering from severe paroxysmal abdominal pain and incidentally vomiting. CT enterography showed partial small bowel constriction. After numerous surgical consultations, eventually on day+ 503 patient underwent laparotomy with constricted bowel resection. Histopatological examination of resected tissue revealed moderate GvHD. Immunosuppersion was tapered to low dose of steroids with ECP. For now, 2 years post HSCT patient is alive, rarely experiencing mild abdominal cramps

3 . Fourteen years old female developed severe abdominal pain and high volume diarhoea on day +24 post MUDHSCT performed for severe anaplastic anemia (SAA). Despite extensive immunosuppression (steroids, anti-TNF, anti-IL2 antibodies) patient condition did not improved. Through consistent stomach pain, suspected subileus confirmed by CT enterography, laparotomy was performed (day+158). Resection of inflamated and obstructed bowel was made. Microscopic evaluation confirmed prior GvHD diagnosis therefore immunosuppression including CsA and tapered doses of steroids was continued. Complete resolution of abdominal symptoms was almost immediately achieved post-surgery, however 2 months after recurrent abdominal cramps were observed and are now well controlled by pain killers.

Conclusions: Commonly GI GvHD is diffused inflammatory process. However in some cases it may be localized and may lead to partial bowel constriction. In case of severe and prolonged stomach pain, despite of partial resolving of other GvHD symptoms, ileus should be considered. CT enterography may be useful for diagnosis confirmation. In those patients, surgical intervention may improve quality of life or even be a salvage approach.

Disclosure: Nothing to disclose 


\section{P287}

Is there any impact of the uric acid levels during the preand early post-graft infusion period, on the GVHD occurence and allotransplant outcome?

\section{Panayotis Kaloyannidis ${ }^{1}$, Eshrak Al Shaibani, Rawan Omari $^{1}$, Solaf Kanfar ${ }^{1}$, Deia Awami ${ }^{1}$, Khalid Bakhit ${ }^{1}$, Ashraf Al Suhebah ${ }^{1}$, Reem Khalili, Abdulhamid Liacini, Hani Al Hashmi ${ }^{1}$, Khalid Al Anezi ${ }^{I}$}

${ }^{1}$ King Fahad Specialist Hospital, Dammam, Saudi Arabia

Background: Uric Acid (UA) belongs to the damageassociated molecular patterns (DAMPs), which recently considered as important contributors on the acute Graft vs Host Disease (aGvHD) pathogenesis. Currently, only two published studies evaluated the impact of UA on aGvHD incidence, with totally conflicting results. So apparently, the role of UA as aGvHD mediator is questionable. We herein assessed the association between serum UA levels and the GvHD incidence and transplant outcome.

Methods: We retrospectively evaluated 42 patients, aged of 36.8(16.7-61.6) years, who underwent allogeneic stem cell transplantation (alloSCT) from full-matched sibling donors for acute leukemia $(n=31)$, very severe aplastic anemia/PNH $(n=5)$, lymphoma $(n=3)$, myelodysplastic/ myeloproliferative syndrome $(\mathrm{n}=3)$. Thirty-two patients were in remission at the time of alloSCT (CR1: 23, CR2: 7, beyond CR2: 2). For a better and more accurate assessment of the UA levels on the aGvHD incidence, unlike to the other published studies which evaluated the UA levels only at day 0 , we evaluated the UA levels in different time points during the the peri-transplant period (at the conditioning regimen initiation, and at days $0,+7$ and +14$)$. Because the majority of our patients developed aGvHD within the 15-30 days post-transplant, we did not incorporated in the study the of UA levels beyond the +14 day. We also investigated the effect of the UA on survival and the non-relapse mortality (NRM). The vast majority of patients received allopurinol from the $1^{\text {st }}$ day of conditioning regimen till day -1. The independent T-test, Kaplan-Meir method and logrank test were used in the statistical analysis.

Results: The median UA levels were 4.2, 2.5, 2.7 and 3.2 $\mathrm{mg} / \mathrm{dl}$ at days $-7,0,+7$ and +14 respectively. For the statistical analysis purposes, we grouped our patients as low-UA if they had values $<3 \mathrm{mg} / \mathrm{dl}$ or high-UA if they had $>3 \mathrm{mg} / \mathrm{dl}$. This threshold was chosen based on the UA values from all the collected samples $(n=175)$. Finally $16 /$ $42(38 \%)$ patients developed aGvHD; 14(33\%) were assessed as $\mathrm{gr} \geq \mathrm{II}$, while $7(16 \%)$ as gr III-IV. The incidence of the aGvHD gr $\geq \mathrm{II}$ was similar (ranged from $30-35 \%$ ) in both groups of patients (low-UA and high-UA) and for all the estimated time points (days $-7,0,+7,+14$ ). We noticed a better 2-years overall survival for patients with low-UA (75\% vs. $63 \%$ ) however without any statistical significance. Ten patients succumbed to NRM causes; 6/10 deaths attributed to GvHD complications. The NRM was assessed higher in the high-UA group (38\% vs. $18 \%$ ) but also this difference was not statistically significant.

Conclusions: Though our study bears the limitations of the small number of patients and the retrospective origin, at least to our knowledge is the first which evaluates the impact of UA levels at different time points in the peritransplant period, on the aGvHD incidence. In our study the UA levels did not influence the incidence or the severity of aGvHD. The higher NRM rates for patients with UA $>3.0$ $\mathrm{mg} / \mathrm{dl}$ merits further evaluation. Definitely, the role of UA on the alloSCT outcome will be clarified through well designed prospective trials.

Disclosure: No conflict of interest

\section{P288}

Buccal mucosa autograft and other treatment modalities for male genital chronic graft versus host disease - case series

Lana Desnica ${ }^{1}$, Tvrtko Hudolin ${ }^{1}$, Dinko Hauptman ${ }^{1}$, Ranka Serventi-Seiwerth ${ }^{1}$, Nadira Durakovic ${ }^{1}$, Zinaida Peric $^{1}$, Radovan Vrhovac ${ }^{1}$, Steven Z Pavletic ${ }^{2}$, Drazen Pulanic $^{1}$

${ }^{1}$ University Hospital Centre Zagreb, Hematology, Zagreb, Croatia, ${ }^{2}$ National Cancer Institute, National Institutes of Health, Bethesda, MD, United States

Background: In contrast to women, genital chronic Graft versus Host Disease (cGVHD) in men has not been evaluated or scored until recently. In the new 2014 NIH criteria for diagnosis of cGVHD (Jagasia et al, BBMT), phimosis or urethral scarring or stenosis are listed as diagnostic signs to establish cGVHD diagnosis. Thus, reported incidence of male genital cGVHD likely underestimates magnitude of the problem. Patients commonly under-report symptoms or signs and sexual function issues and clinicians often underdiagnose male genital cGVHD. So far only twelve publications, mostly case reports, and one original paper than included 155 patients (cross-sectional study), reported on male cGVHD. Symptoms and manifestations included inflammatory balanoposthitis, phimosis, lichen sclerosis, Peyronie's disease or urethral stenosis and strictures. In this study case series of male genital cGVHD manifestations and treatment were presented, including treatment of urethral stricture with buccal mucosa autograft.

Methods: From 2013-2017, 317 patients received allogeneic hematopoietic stem cell transplant at University Hospital Centre Zagreb; 81 (41 female, 40 male) were 
diagnosed with cGVHD. Clinical examinations and NIH scoring were done by a multidisciplinary cGVHD team on an IRB approved research observational protocol.

Results: Five male patients (12\%) had genital cGVHD manifestations presented by urethral stricture in $4 / 5$ patients and phimosis requiring surgical treatment in one patient. All five patients had simultaneously cutaneous, oral, and/or ocular cGVHD manifestations. The first patient underwent urethroplasty of bulbomembranous part of urethra with termino-terminal anastomosis and urethroplasty of penile part of urethra with buccal mucosa autograft - BMG (dorsal onlay) that resulted in significant improvement of symptoms and normal miction afterwards. Biopsy of the urethra showed mononuclear infiltration in lamina propria consistent with cGVHD. Biopsy of the buccal mucosa was done prior to surgery and was negative for cGVHD involvement. The second patient underwent urethrotomy due to circular strictures, but symptoms reappeared again and he is now candidate for BMG. In two patients urethral dilatation was done, and the fifth patient presented with phimosis requiring circumcision, resulted in significant improvement of symptoms.

Conclusions: Male genital cGVHD is an underrecognized and under-reported manifestation. Patients after allo-HSCT need to be actively asked about their genital symptoms and sexual function, especially if they are diagnosed with other mucocutaneous or ocular cGVHD. Multidisciplinary approach, early recognition and frequent follow-up is necessary for timely start of treatment. New methods, such as BMG for cGVHD patients with urethral stricture seem promising and should be further investigated.

Disclosure: Nothing to declare.

P289 Abstract withdrawn.

\section{P290}

Heracles: A phase II single-arm prospective study to assess the efficacy of fecal microbiota transfer in the treatment of steroid refractory gastro-intestinal AGVHD post allo-HSCT

\section{Florent Malard $^{\text {I, Ernst Holler }}{ }^{2}$, Andrea Bacigalupo ${ }^{3}$, Maria Bieniaszewska ${ }^{4}$, Emilie Plantamura ${ }^{5}$, Corentin Le Camus $^{5}$, Ronald Carter ${ }^{5}$, Mohamad Mohty ${ }^{1}$}

${ }^{1}$ Hôpital Saint Antoine, Service d'Hématologie et Thérapie Cellulaire, Paris, France, ${ }^{2}$ Universitätsklinikum Regensburg, Studienzentrale der Klinik und Poliklinik für Innere Medizin III, Regensburg, Germany, ${ }^{3}$ Gemelli Hopsital, Roma, Italy, ${ }^{4}$ Uniwersyteckie Centrum Kliniczne w Gdańsku, Klinika Hematologii i Transplantologii, Gdansk, Poland, ${ }^{5}$ MaaT Pharma, Lyon, France
Background: Steroid-refractory acute graft-versus-host disease (SR-aGVHD) is associated with an $80 \%$ mortality rate and reduced quality of life (QoL). So far, there is no approved standard of care for aGVHD second-line treatment. There is an urgent need to identify effective therapy for SR-aGVHD to improve patients' outcomes. Fecal Microbiota Transfer (FMT) might be beneficial to substantially improve the prognosis. Higher gut microbial diversity is strongly associated with increased survival in GvHD patients. Recent studies reported promising results of SR-aGVHD patients treated with FMT. Further evaluation to confirm the efficacy and safety of FMT for aGVHD is warranted. The ongoing phase 2 study (HERACLES) investigates the efficacy of allogeneic FMT in the treatment of patients with SR-aGVHD. HERACLES was launched after the ODYSSEE study showed promising results in the reconstruction of gut microbiota diversity after induction chemotherapy with FMT in Acute Myeloid Leukemia patients. We expect that FMT-based biotherapeutic drugs could be effective treatments to contain SR-aGVHD, and thereby reduce the risk of life-threatening complications after allogeneic HSCT.

Methods: HERACLES is a single-arm, multicenter prospective trial in 5 European countries. Patients aged $\geq 18$ years-old, who underwent allogeneic Hematopoietic Stem Cell Transplantation (allo-HSCT) and developed a first episode of Stage 3 or 4 aGVHD with gut predominance resistant to a first-line steroid therapy are eligible for inclusion. Main exclusion criteria comprise the use of other second-line GVHD therapy, patients with grade IV hyperacute GVHD, late onset aGVHD, and overlap chronic GVHD and aGVHD after donor lymphocyte infusion. Patients receive a first MaaT013 enema within 5 days after SR diagnosis (V1) and 2 additional ones 1 week apart (V2/ V3) from each other. MaaT013 is a highly-diverse, microbiome-rich enema formulation obtained from pooled, rigorously screened faeces from healthy donors, manufactured with a standardized process using the signature MaaT Microbiome Restoration Biotherapeutic (MMRB) platform.

At inclusion (V1), before each dosing (V2,3), and 28 days post inclusion (V4), patients' faeces and blood are collected. Safety monitoring will be performed with corresponding blood analyses. Exploratory measures on faeces include characterization of gut microbiota composition and evolution, impact of MaaT013 on metabolism, and gut inflammation. Immune system phenotyping will be performed by flow cytometry on peripheral blood mononuclear cells, and by ELISA assay on plasma. Patients' QoL will be assessed using a standard, EQ-5D-5L questionnaire.

The primary objective is to assess the efficacy of MaaT013 by evaluating complete response (CR, according to modified Glucksberg criteria) and Very Good Partial Response (VGPR, defined by Martin et al., BBMT, 2009) 
28 days post-inclusion (Primary follow-up). Secondary objectives include FMT safety assessment and evaluation of FMT impact on several endpoints, such as overall, relapsefree or GVHD-free survival and chronic GVHD evaluation, as well as multi-drug resistant bacteria carriage. Patients will be followed-up untill year after inclusion.

Overall, 32 patients are planned to be enrolled and treated, to assess overall response rates and MaaT013's safety profile.

Results: Study was launched in May 2018.

Conclusions: Enrolment is ongoing. ClinicalTrials.gov Id: NCT03359980.

\section{First results expected: Q4 2019}

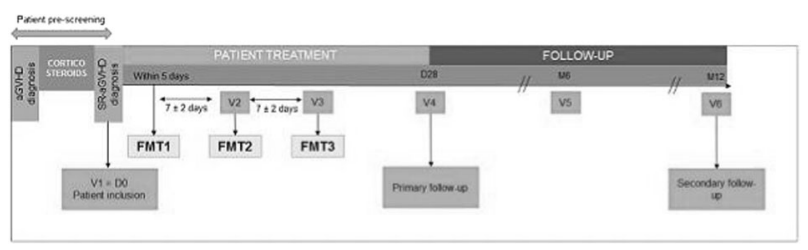

[[P290 Image] 1. HERACLES study flow chart]

Clinical Trial Registry: ClinicalTrials.gov Id: NCT03359980

Disclosure: Emilie Plantamura, Corentin Le Camus and Ronald Carter are MaaT Pharma employees.

Mohamad Mohty is scientific co-founder of MaaT Pharma and received hororaria and consulting fees.

\section{P291}

Steroid-refractory graft versus host disease in a case series of hodgkin lymphoma previously treated with nivolumab

Chiara Cigana ${ }^{I}$, Michela Cerno ${ }^{I}$, Vera Radici ${ }^{I}$, Fabrizia Colasante $^{I}$, Roberta De Marchi ${ }^{1}$, Alessandra Sperotto ${ }^{1}$, Antonella Geromin ${ }^{1}$, Renato Fanin ${ }^{1}$, Francesca Patriarca $^{l}$

\footnotetext{
${ }^{1}$ Azienda Ospedaliera Universitaria - Udine, Clinica Ematologica, Udine, Italy
}

Background: Anti-programmed cell death protein 1 (PD1) monoclonal antibodies can be used as "bridge to" a subsequent allogeneic hematopoietic stem cell transplantation (HSCT) in patients with relapsed/refractory Hodgkin's Lymphoma (HL). This strategy has been reported to be effective, but a frequent onset of steroid-refractory Graft versus Host Disease (GVHD) was also reported. We report 3 clinical cases of patients affected by HL undergoing allogeneic HSCT after having been treated with Nivolumab.
Methods: The 3 patients of 48, 18 and 42 years respectively had advanced HL and had relapsed after a previous autologous (2) or allogeneic (1) HSCT. They underwent a rescue therapy with 18, 12, 16 Nivolumab cycles respectively, depending on the time of partial response achievement and the availability of a donor.

Two patients received a thiotepa-fludarabinecyclophosphamide conditioning, ATG-based prophylaxis and PB cells from unrelated donors. The third patient received BM cells from an haploidentical donor using the "Baltimora" nonmieloablative platform.

Results: At a follow-up of 11, 12, 15 months after HSCT, respectively, all patients achieved and maintained a complete remission by PET-CT scans.

All the 3 patients developed acute GVHD on day +32 , +54 and +107 , respectively.

Patient 1 progressed to grade IV acute GVHD with hepatic and intestinal involvement unresponsive to first line $2 \mathrm{mg} / \mathrm{kg}$ steroid therapy and second line etanercept plus extracorporeal photopheresis (ECP). Third line therapy with ruxolitinib partially controlled the GVHD.

GVHD onset in patients 2 and 3 was preceeded by a prolonged fever without microbiological findings. Patient 2 developed hepatic grade II GVHD with high transaminase levels, initially responsive to steroid therapy, then it progressed to gut requiring second line therapy with etanercept.

Patient 3 progressed to severe chronic GVHD with skin involvement and resulted unresponsive to steroids and ECP and it was partially controlled by ruxolitinib.

Immune reconstitution was delayed in all 3 patients: at 6 months post transplantation CD3 levels were $80 / \mu \mathrm{L}, 17 /$ $\mu \mathrm{L}$ and $127 / \mu \mathrm{L}$ and CD4 levels were $36 / \mu \mathrm{L}, 16 / \mu \mathrm{L}$ and $65 /$ $\mu \mathrm{L}$ respectively.

Only patient 2 , that underwent haploidentical transplant and received post-trasplant cyclophosphamide (PT-Cy), is off of immunosuppressive treatment at 11 months after HSCT, without evidence of GVHD and no history of infections. Out of the 2 patients receiving PBSC from unrelated donors and ATG prophylaxis, patient 1 developed a disseminated fusariosis on day +180 and died of CNS fusarium localization 1 year after HSCT, despite targeted antifungal therapy. Patient 3 had pulmonary aspergillosis, sepsis by multidrug resistant Psuedomonas Aeruginosa and otomastoiditis: at +15 months after HSCT, he is on ruxolitinib treatment with skin clinical partial response.

Conclusions: This case series confirms that Nivolumab as "bridge to transplant" is effective in appropriately selected patients. However, risk of acute GVHD and delayed immune reconstitution may require a careful consideration at the moment of planning the transplant. A possible advantage of PT-Cy GVHD platform and haploidentical donors should be addressed in larger studies. 
Disclosure: Nothing to declare

P292

Treatment with etanercept for corticosteroid refractory acute graft-versus-host disease. A single centre experience

Anna Torrent ${ }^{I}$, Maria Jose Jimenez-Lorenzo ${ }^{1}$, Christelle Ferrá $^{1}$, Mireia Morgades ${ }^{1}$, Susana Vives ${ }^{1}$, Miriam Moreno $^{I}$, Montserrat Batlle ${ }^{I}$, Blanca Xicoy ${ }^{\prime}$, Juan Manuel Sancho ${ }^{I}$, Laura Abril ${ }^{1}$, Gladys Ibarra ${ }^{1}$, Albert Oriol $^{I}$, Marta Peña ${ }^{1}$, Marta Sitges ${ }^{1}$,Josep Maria Ribera ${ }^{I}$

${ }^{1}$ Institut Català d'Oncologia-Hospital Germans Trias i Pujol, Josep Carreras Leukemia Research Institute, Badalona, Spain

Background: Acute graft-versus-host disease (aGVHD) is the most important complication after an allogeneic hematopoietic stem cell transplant (HSCT). No standard secondline treatment has been established for the corticosteroid refractory aGVHD. The anti-TNF $\alpha$ agents are a good option of treatment for these patients, especially when lower GI tract is involved.

Methods: From April 2010 to July 2017 we reviewed the outcome of 19 patients with steroid-refractory (SR) aGVHD treated with etanercept as at least, second line treatment. Etanercept dose was $25 \mathrm{mg}$ twice a week for the first 4 weeks, followed by 4 weekly doses.

Results: Median age was 52 years (range 15-69 years), and 12 patients $(63 \%)$ were male. Fourteen patients (74\%) had a non-advanced disease status at HSTC. Eleven patients $(59 \%)$ received a myeloablative conditioning, and the stem cell source was peripheral blood in 18 patients (95\%). Sixteen patients $(84 \%)$ were $8 / 8$ HLA matched. The characteristics of the 19 patients, their aGVHD stage previous to rescue treatment with etanercept and their outcome are shown in Table 1. Seventeen patients (89\%) had a classic aGVHD while 2 had a late-onset aGVHD. Etanercept was given as a $2^{\text {nd }}, 3^{\text {rd }}$ and $4^{\text {th }}$ line in $3(16 \%), 10$ $(53 \%)$ and $6(31 \%)$ patients respectively. The median doses of etanercept administered were 7 (range 1-12), and just 4 patients (20\%) completed the 12 doses planned treatment, of whom 3 were alive at 38, 24 and 18 months from the onset of rescue treatment. Complications during etanercept treatment were: infection $(n=9$ [47\%]: gram negative bacilli $[n=6])$, grade 2-4 neutropenia $(n=8)$ and grade 3-4 thrombocytopenia $(n=6)$. Etanercept was indicated as a rescue treatment due to: progression after 3 days of aGVHD treatment $(\mathrm{n}=1)$, no response after 7 days of treatment $(\mathrm{n}=11)$, no complete remission after 14 days of treatment $(n=3)$ and relapse due to decrease corticosteroid doses $(n=4)$. At the end of treatment 1 patient achieved a complete response and 3 patients a partial response, all of them are alive. These 4 patients received etanercept as a $2^{\text {nd }}$ $(n=3)$ and 3th line $(n=1)$ treatment, all of them had lower GI aGVHD without any other organ significantly involved. Causes of death were: aGVHD with or without infection in 15 patients (78\%) and leukemia relapse in 1 patient.

Conclusions: Although if etanercept is an option for treatment of SR aGVHD in some patients, their prognostic remains poor and more effective alternative strategies are needed. A prompt initiation of etanercept as a rescue treatment for SR aGVHD is crucial to improve the prognosis.

\begin{tabular}{llll}
\hline Characteristics & & $\mathrm{N}(\%)$ & \\
\hline $\begin{array}{l}\text { Haematologic } \\
\text { disease }\end{array}$ & AML/ALL/MDS & $11(59) / 1(5) / 3(16)$ & \\
Conditioning & TBI-CY/BU-CY/ & $6(67) / 7(37) / 3(16) /$ & \\
regimen & FLU-BU/others & $3(16)$ & \\
GVHD & CsA-MTX CSA- & $11(58) 5(26) 3$ & \\
prophylaxis & MTX-ATG Other & $(16)$ & \\
aGVHD grade & Beginning & Pre- etanercept & $\begin{array}{l}\text { Post- } \\
\text { etanercept }\end{array}$ \\
Global aGVHD & 0/2/9/5/3 & $0 / 0 / 1 / 10 / 8$ & $1 / 1 / 1 / 1 /$ \\
0/1/2/3/4 & & & 15 \\
Skin GVHD 0/ & $3 / 1 / 9 / 4 / 2$ & $7 / 2 / 5 / 4 / 1$ & $10 / 0 / 2 / 3 /$ \\
1/2/3/4 & & & 4 \\
Hepatic GVHD & 15/1/2/1/0 & $13 / 1 / 3 / 1 / 1$ & $10 / 0 / 2 / 3 /$ \\
0/1/2/3/4 & & & 4 \\
Gut GVHD 0/ & $3 / 4 / 6 / 3 / 3$ & $1 / 0 / 2 / 8 / 8$ & $1 / 1 / 2 / 1 /$ \\
1/2/3/4 & & & 14 \\
\hline
\end{tabular}

[[P292 Table] 1. Table 1]

Disclosure: DKMS Foundation, PI14/01971 (Instituto Carlos III) and SGR288 (GRC) Generalitat de Catalunya.

\section{P293}

Conversion from cyclosporine to tacrolimus therapy is feasible or not?

\section{Koray Yalcin ${ }^{1}$, Gulsun Karasu ${ }^{1}$, Safiye Suna Celen ${ }^{1}$, Dayanat Pashayev', Suar Caki Kilic ${ }^{1}$, Volkan Hazar ${ }^{1}$, Akif Yesilipek ${ }^{I}$}

${ }^{1}$ Medical Park Göztepe Hospital, İstanbul, Turkey

Background: Although both cyclosporine (CSA) and tacrolimus are calcineurin inhibitors, CSA is more widely used in pediatric hematopoetic stem cell transplantation (HSCT) as a prophylactic drug for acute graft versus host disease (aGvHD). There are some clinical experience but very few data about the clinical efficacy of conversion to tacrolimus. Here, we present our single center data on this arguable topic. 
Methods: This study involves the data of 71 pediatric HSCT patients in Medical Park Göztepe Hospital between 2014-2018. All 71 patients had prophylactic CSA therapy and for various reasons CSA was converted to tacrolimus therapy. Most of the patients had this conversion due to aGvHD. As steroid is the first line therapy for aGvHD, conversion to tacrolimus is done concurrently at the start of steroid therapy (within 72 hours after the start of steroid). And also, patients who had any other immunosupressive therapy for aGvHD are excluded. Response is defined as resolution of symptoms within 7 days after conversion.

Results: Mean age of the study population is 130 months (5-266 months), male/female ratio is 1,3 (40/31), donor types are MUD 48 patients (67\%), MFD 18 patients (26\%), haplo 5 patients $(7 \%)$ and mean conversion time is 32 days (1-142 days).

The rationales for conversion are aGvHD for 40 patients, unproper CSA plasma levels for 7 patients, allergic reaction for 6 patients, nephrotoxicity for 6 patients, hepatotoxicity for 5 patients, severe headache for 2 patients, high arterial blood pressure for 2 patients and one each for refractory vomiting, autoimmune thyroiditis and visual disturbance.

The subgroup analysis of aGvHD patients reveals that mean conversion time for aGvHD is 41 days (8-142 days) and there are only 11 responders whose aGvHD resolve completely (\%27) after conversion.

All of the patients had proper tacrolimus levels after conversion due to unproper CSA levels and also patients in allegic reaction, severe headache, visiual disturbance and refractory vomiting group responded to conversion completely but only one of the 6 patients in nephrotoxicity group responded and also 3 of the 5 patients in hepatoxicity group responded. The only one patient suffered from autoimmune thyroiditis did not respond to conversion.

Conclusions: In this study, it is obvious that there are response to conversion for some specific adverse effects of CSA and tacrolimus is a good alternative for the patients who have unproper CSA levels. Conversely, the high percentage (\%73) of non-responders shows that it is not feasible to make a conversion to tacrolimus for acute GvHD.

Disclosure: Nothing to declare

\section{P294}

Vascular leak syndrome occured post HSCT and review the art literature

\section{Tian Zheyuan ${ }^{1}$, Xv Kaihong ${ }^{1}$, Ouyang Guifang ${ }^{1}$}

${ }^{1}$ Ningbo First Hospital, Zhejiang, China

Background: Capillary leak syndrome is caused by the dysfunction of the vascular endothelial cells, and is characterized by weight gain,generalized edemas,unresponsive to diuretic treatment,and hypotension.It usually develops in the first 15 days post HSCT.And it is of great difficuty to distinguish from other complications which are occured post the allo-HSCT. To diagnose this complication at the early stage, it is very difficulty.

Methods: A 34-year-old man was admitted to Ningbo first hospital for its abnormal in the peripheral blood .He was diagnosed with AML-M5 by the classical morphology and immunophenotype.Cytogenetic evaluation showed a normal 46, XY(20). The patient achieved CR with induction therapy including Idarubicin, Cytarabine and Etoposide. After consolidation therapy,an allo-HSCT from HLA identical related dornor(33-year-old male, donorrecipient matched by high resolution HLA typing at HLAA, -B, -C, DRB1, and DQB1, 10/10 matches) was performed.The recipient received conditioning with busulfan, $4 \mathrm{mg} / \mathrm{kg} / \mathrm{day}$ injection for 3 days; cyclophosphamide, $50 \mathrm{mg} / \mathrm{kg} /$ day injection for 2 days; cytarabine, $2 \mathrm{~g} / \mathrm{m} 2 /$ day injection for 1 day; semustine, $250 \mathrm{mg} / \mathrm{m} 2 /$ day orally for 1 day; Donor peripheral blood stem cells (PBSC:MNC: $6.43 \times 109 / \mathrm{L}$, CD34+:4.05 $\times 106 / \mathrm{L}$ ) were mobilized, pheresed and administered to the recipient. GVHD prophylaxis consisted of traditional cyclosporine, short-course methotrexate $(15 \mathrm{mg} /$ $\mathrm{m} 2$ at day $+1,10 \mathrm{mg} / \mathrm{m} 2$ at days $+3,+6$, and +11$)$ and Cyclosporin A injection $5 \mathrm{mg} / \mathrm{kg}$ qer day was mot reduced untill the hematopoietic reconstitute sucessfully . On day +19 , complete donor chimerism was acheieved. The CsA was gradually reduced and tapered.On day +150 , the patients was manifested with increasing in the time and volume of the faeces, he was diagnosed with $\mathrm{II}^{\circ} \mathrm{GVHD}$ (gut).The standarded does of immunosuppressive drug including methylprednisolone and Cyclosporin A was administrated. The immunosuppressive drug was gradually reduced when the GVHD was controlled.On day+271, the patient felt distress and the distress was not related to with the exercise,the temperature was normal,and he did not gain weight.There was no edema in the body.Laboratory test including routine blood test,C-reactive protein,procalcitonin,blood gas analysis,CMVDNA,EBVDNA was normal. The CT scan shows that 1the pleural is filled up with water, and could not be enlarged promptly,2There is pericardial effusion in the body.Pulmonary function test shows that 1 reduced function in ventilation and diffusion fuction. The laboratory test of the pleural effusion was normal,the blast cell was not detected in the pleural effusion,the CD34+ cell count was below the dectable level,the next generation sequencing for minimal residual disease shows that there was no gene mutation .Thus, post capillary leak syndrome was considered .Sirolimus was adopted and taken the place of Cyclosporin A,Immunoglobulin was adminstrated to reduce the edema. 
Results: Taking together comprehensively,the effusion in the pleural and cardiac was absorbed well.

Conclusions: Occurance of capillary leak syndrome is rare,there is limited data about capillary leak syndrome. Comprehensively, the mechanism of CLS has not been totally identified.And there is no standard treatment to treat the complication.At present,the CLS of this patient was absorbed well by administrating Sirolimus,closely followup is needed.

Disclosure: Nothing to declare

Graft-versus-host disease - preclinical and animal models

\section{P295}

\section{Short-term KRP203 and posttransplant} cyclophosphamide for graft-versus-host disease prophylaxis

Emi Yokoyama ${ }^{1}$, Daigo Hashimoto ${ }^{1}$, Takahide Ara ${ }^{1}$, Eko Hayase $^{I}$, Takanori Teshima ${ }^{1}$

${ }^{1}$ Hokkaido University Faculty of Medicine, Hematology, Sapporo, Japan

Background: Post-transplant high-dose cyclophosphamide (PTCY) in combination with other immunosuppressants such as calcineurin-inhibitors (CIs) has been increasingly used as GVHD prophylaxis after HLA-haploidentical or matched hematopoietic stem cell transplantation (HSCT). However,CIs could hamper reconstitution of regulatory $\mathrm{T}$ cells (Tregs) and tolerance induction after HSCT, facilitating us to develop novel CI-free/PTCY-based GVHD prophylaxis. In the current study, we developed a novel GVHD prophylaxis in which PTCY was combined with short-term administration of KRP203, a selective agonist of sphingosine-1-phosphate receptor type 1 (S1PR1), using murine models of MHC haploidentical bone marrow transplantation (BMT).

Methods: B6D2F1 (H-2 $\left.{ }^{\mathrm{b} / \mathrm{d}}\right)$ recipients were lethally irradiated and transplanted with bone marrow cells and splenocytes from allogeneic B6 $\left(\mathrm{H}-2^{\mathrm{b}}\right)$ donors. CY at a dose of $50 \mathrm{mg} / \mathrm{kg}$ was intraperitoneally injected into the recipients on day +3 , and KRP at a dose of $1.0 \mathrm{mg} / \mathrm{kg}$ was orally administrated daily from day 0 to day +4 after BMT. Donor $\mathrm{T}$ cells in the target organs and secondary lymphoid organs were evaluated by flow cytometric analysis. Plasma levels of TNF- $\alpha$ were determined using cytometric beads array. To evaluate graft-versus-leukemia (GVL) effects, recipient mice were intravenously injected with luciferase-transduced P815 cells (P815-luc) on day 0 , and in vivo bioluminescence imagingwas conducted weekly after BMT.
Results: Severe GVHD was developed in allogeneic recipients and all mice died by day 50 after BMT.PTCY alone at a dose of $50 \mathrm{mg} / \mathrm{kg}$ significantly ameliorated GVHD and $30 \%$ of PTCY-treated allogeneic recipients survived. Oral administration of KRP203 alone enhanced contraction of donor $\mathrm{T}$ cells in the lymph nodes and also ameliorated GVHD as has been previously shown with multi-S1PR agonist, fingolimod. Next, we tested if shortterm KRP203 on days 0 to +4 added to PTCY enhances anti-GVHD effects of PTCY. We found that survivals of PTCY+KRP203 group were significantly prolonged compared to those of PTCY-alone group (Figure A). Plasma levels of TNF-a, clinical GVHD scores (Figure B), and donor T-cell infiltration into the target organs such as the gut and skin were also significantly reduced in PTCY +KRP203 group compared to PTCY-alone group (Figure C and D). Unlike CIs, addition of KRP203 to PTCY promoted Treg reconstitution after BMT. Finally, bioluminescence imaging demonstrated that proliferation of P815-luc injected on day 0 was significantly delayed in PTCY +KRP203-treated allogeneic recipients compared to control mice transplanted only with T-cell depleted bone marrow cells, suggesting that significant GVL effects persisted in PTCY+KRP203-treated recipients.

Conclusions: A combination of short-term KRP203 and PTCY is a promising novel calcineurin-free GVHD prophylaxis in MHC-haploidentical SCT.
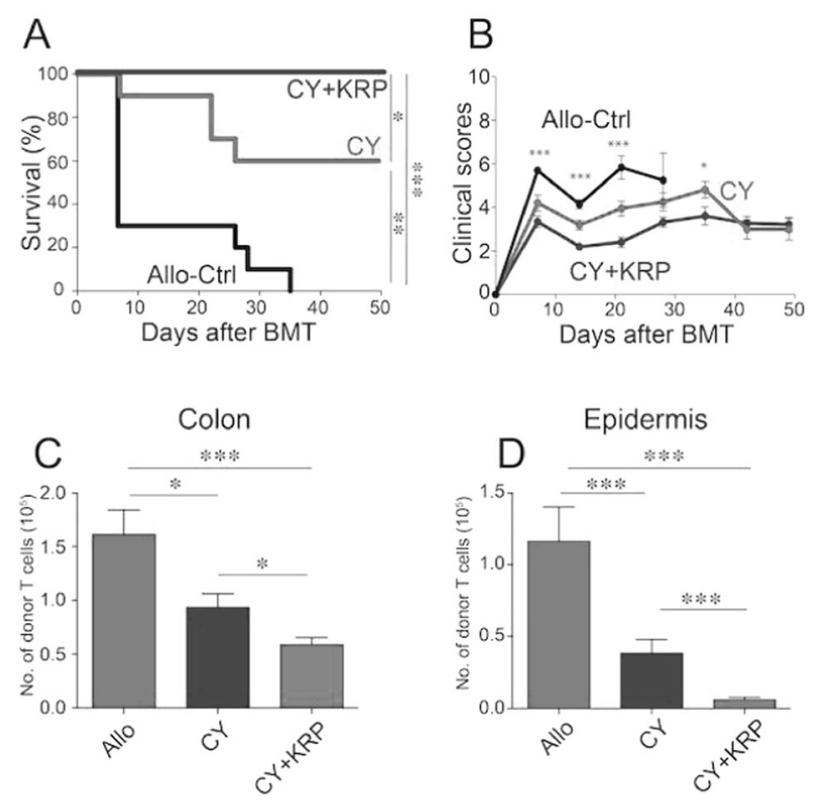

[[P295 Image] 1. (A) OS, (B) Clinical GVHD scores, (C, D) FCM analysis of donor T cells in the gut and skin.]

Disclosure: T.T is a consultant of Novartis Pharma AG and received a research grant from Novartis Pharma AG. 


\section{P296}

donor invariant natural killer $\mathbf{T}$ cells promote immune tolerance through PD-L1 ${ }^{\mathrm{HIGH}}$ dendritic cells and expansion of $\mathrm{CD}^{+} \mathrm{CD}^{+} 5^{+} \mathrm{FOXP3}^{+}$regulatory $\mathrm{T}$ cells

\section{Hannes Schmid ${ }^{1}$, Simona Jahnke ${ }^{I}$, Felix Kettemann ${ }^{1}$, Silke Dürr-Störzer ${ }^{1}$, Hildegard Keppeler ${ }^{1}$, Corina Schneidawind $^{I}$, Dominik Schneidawind ${ }^{I}$}

${ }^{1}$ University Hospital Tübingen, Eberhard Karls University, Tübingen, Germany

Background: Graft-versus-host disease (GVHD) is a major cause of significant morbidity and mortality after allogeneic hematopoietic cell transplantation (HCT). Invariant natural killer $\mathrm{T}$ (iNKT) cells are potent regulators of immune responses and are associated with a reduced GVHD incidence after allogeneic HCT in humans (Malard et al. 2016). We recently showed that donor iNKT cells can be expanded ex vivo and that they are able to prevent activation and proliferation of alloreactive donor $\mathrm{T}$ cells while promoting efficient graft-versus-leukemia effects (Schmid et al. 2018). However, the underlying mechanisms how human iNKT cells induce immune tolerance after allogeneic HCT are not fully understood.

Methods: Monocyte-derived dendritic cells (DCs) were cultured in a mixed lymphocyte reaction with MHCmismatched $\mathrm{T}$ cells and culture-expanded iNKT cells. Tcell activation and proliferation was analyzed by multiparametric flow cytometry and released cytokines were measured via multiplex analysis. Transwell assays and imaging flow cytometry were performed to elucidate cellcell interactions. Bead-controlled flow cytometry-based cytotoxicity assays were used to evaluate DC apoptosis. Apoptotic DCs were then purified by fluorescence-activated cell sorting to investigate their tolerogenic potential to prime regulatory $\mathrm{T}$ cells (Tregs).

Results: The addition of iNKT cells to mixed lymphocyte reactions resulted in a significantly reduced activation and proliferation of MHC-mismatched T cells. Transwell assays and imaging flow cytometry revealed a cell contactdependent mechanism between iNKT cells and DCs leading to apoptosis with increasing DNA fragmentation of DCs over time. Interestingly, various fluorescence-activated single cell sorted iNKT-cell subsets were all able to induce apoptosis of host DCs. Multiplex analysis revealed that DCs triggered iNKT-cell release of cytotoxic factors like perforin, granzyme B and granulysin. Blocking the iNKTcell receptor engagement with a CD1d antibody prevented iNKT-cell degranulation as well as the subsequent induction of host DC apoptosis. Inhibition of cytotoxic factors also abrogated apoptosis of DCs. In turn, sorted apoptotic DCs induced tolerogenic DCs characterized by a high expression of PD-L1 in mixed lymphocyte reactions. Such tolerogenic DCs promoted the expansion of $\mathrm{CD} 4{ }^{+} \mathrm{CD} 25^{+} \mathrm{FoxP} 3^{+}$Tregs and prevented activation and proliferation of MHCmismatched $\mathrm{T}$ cells.

Conclusions: We propose a novel mechanism how culture-expanded human iNKT cells prevent GVHD after allogeneic HCT. Host DC apoptosis through donor iNKT cells induces a tolerogenic immunoenvironment characterized by PD-L1 ${ }^{\text {high }}$ DCs and expanding donor Tregs inhibiting activation and expansion of alloreactive donor $\mathrm{T}$ cells. Our findings pave the avenue for clinical translation of adoptively transferred culture-expanded iNKT cells in humans.

Disclosure: Nothing to declare

\section{P297}

Abstract already published.

\section{P298}

Vasoactive intestinal polypeptide synthesis induced in recipient somatic tissues prevents GVHD in allo-BMT

\section{Edmund Waller ${ }^{\text {, Rohan Dhamsania }}{ }^{1}$, Sruthi} Ravindranathan ${ }^{1}$, Yiwen $\mathrm{Li}^{\mathrm{I}}$

${ }^{1}$ Emory University Winship Cancer Institute, Atlanta, GA, United States

Background: While both donor and host-type hematopoietic cells regulate GvHD in allo-BMT, the role of nonhematopoietic host tissues in the initiation and maintenance of GvHD is less clear. Vasoactive Intestinal peptide (VIP) is a neuropeptide with potent immune-regulatory activity that is induced by inflammation and expressed by neurons, myeloid cells and lymphoid cells. Our objectives were to study the role of VIP synthesis by recipient tissues on donor $\mathrm{T}$ cell activation and GvHD in allo-BMT.

Methods: We used two MHC mis-matched allo-BMT models, B10.BR-->B6 (CD4-driven GVHD) and Balb/c->B6 (CD8-driven GVHD), irradiating VIP-wildtype (WT) and VIP-knock-out (VIP-KO) B6 mice with 11Gy on day -1 and transplanting with $5 \times 10 \mathrm{E} 6 \mathrm{~T}$ cell depleted (TCD) bone marrow (BM) plus $0,1 \times 10 \mathrm{E} 6$, or $3 \times 10 \mathrm{E} 6 \mathrm{~T}$ cells from MHC mis-matched donors. B6-->B6 radiation chimeras lacking VIP in either hematopoietic or non-hematopoietic cells were created by performing B6 WT-->VIP-KO and VIP-KO-->WT syngeneic transplants. Production of VIP in transplant recipients was visualized with immunefluorescent staining of tissues using an anti-VIP antibody and by visualizing the VIP gene promoter activity in transgenic B6 mouse harboring a BAC-containing VIP promoter upstream of GFP. 
Results: VIP-KO mice transplanted with allogeneic TCD $\mathrm{BM}$ alone had increased graft rejection with lower levels of donor chimerism and 33\% day 75 survival compared with $73 \%$ survival of WT recipients. Transplanting TCD BM plus $3 \times 10 \mathrm{E} 6$ donor $\mathrm{T}$ cells from B10.BR or Balb/c donors in VIP KIO mice led to $>95 \%$ donor chimerism and significantly increased GVHD-mortality compared with WT recipients, with $0 \%$ vs $40 \%$ survival in the B10.BR-->B6 model ( $\mathrm{p}<0.01$ ), and $0 \%$ vs $73 \%$ survival in the Balb/c-$>$ B6 model $(\mathrm{P}<0.01)$. Donor-derived T cells in VIP-KO recipients had significantly higher Th1 and Th17 polarization, with higher ROR $\gamma \mathrm{t}$ in both CD4+ $(\mathrm{p}<0.0001)$ and $\mathrm{CD} 8+(\mathrm{p}<0.0001) \mathrm{T}$ cells, and higher frequencies of IFN- $\gamma$ $(\mathrm{p}<0.001)$, TNF- $\alpha(\mathrm{p}<0.05)$, and IL2 $(\mathrm{p}<0.01)$ in CD4+ and $\mathrm{CD} 8+\mathrm{T}$ cells compared to WT recipients. B10.BR-$>\mathrm{B} 6$ second allogeneic transplantation of radiation chimeras caused lethal GVHD mortality in VIP-KO-->VIP-KO and WT-->VIP-KO mice, but not in WT-->WT or VIP-KO-$>$ WT B6 mice, demonstrating the protective effect of VIP was due to synthesis by non-hematopoietic recipient cells. Immunofluorescent imaging of allo-BMT recipients showed marked up-regulation of VIP in lungs post-transplant and high VIP production within neurons innervating the lungs. Finally, we demonstrated that short-term administration of VIP (10mcg/day) from day 0 to day 10 prevented GvHDmortality in VIP-KO recipients transplanted with B10.BR-$>$ B6 MHC donor BM \& T cells.

Conclusions: The absence of VIP in recipient cells led to increased graft rejection in the absence of donor $\mathrm{T}$ cells and increased lethal GVHD when donor $\mathrm{T}$ cells were transplanted, indicating VIP induced post-transplant regulates allo-reactivity of host graft-rejecting lymphocytes and donor GVHD-causing $\mathrm{T}$ cells. The protective effect of parenteral VIP administration suggests VIP-mimetics represent a novel approach to prevent and treat GVHD. These data also suggest a mechanism of action for the mitigation of GvHD by alpha-1 anti-trypsin (AAT) whereby AAT inhibits the proteolytic inactivation of endogenous VIP.

Disclosure: Dr. Waller reports personal fees and other support from Cambium Medical Technologies, grants from Celldex, personal fees from Kalytera, grants and personal fees from Novartis, grants and non-financial support from Pharmacyclics, and equity ownership in Cerus Corporation and Chimerix outside the submitted work. In addition, Dr. Waller has intellectual property related to VIP signaling that has been licensed to Cambium Oncology in which he holds equity.

\section{P299}

Low-density neutrophils expansion is associated with acute graft versus host disease in allogeneic hematopoietic stem cell transplant patients
Olivia Marini ${ }^{1}$, Patrizia Scapini, Marco Antonio Cassatella $^{1}$, Pierpaolo Langanella ${ }^{1}$, Alessia Orsini, Angelo Andreini', Beatrice De Marco ${ }^{1}$, Mauro Krampera $^{I}$, Fabio Benedetti ${ }^{1}$, Cristina Tecchio ${ }^{1}$

${ }^{1}$ University of Verona, Medicine - Section of General Pathology, Verona, Italy,

Background: Low-density neutrophils (LDNs) are distinguished from normal-density neutrophils (NDNs) by their anomalous sedimentation within the mononuclear cell fraction after density gradient centrifugation of peripheral blood (PB). By analysing LDNs and NDNs from G-CSFstimulated donors or lymphoma patients, we have previously demonstrated that, depending on physiopathological conditions, immature $\mathrm{CD} 6 \mathrm{~b}^{+} \mathrm{CD} 10^{-} \mathrm{LDNs}$ can promote $\mathrm{T}$ cell survival and IFN- $\gamma$ production, while mature CD66b ${ }^{+} \mathrm{CD} 10^{+} \mathrm{LDNs}$ can exert immunosuppressive proprieties. Aim of this study was to establish the frequency of CD66b ${ }^{+} \mathrm{CD} 10^{-}$and/or $\mathrm{CD} 66 \mathrm{~b}^{+} \mathrm{CD} 10^{+} \mathrm{LDNs}$ in $\mathrm{PB}$ of allogeneic hematopoietic stem cell transplant (HSCT) patients throughout immune reconstitution, and verify their potential correlation with acute graft versus host disease (aGVHD).

Methods: Patients undergoing HSCT in our Institution between December 2015 and June 2018 were prospectively enrolled in the study upon informed consent and after Institutional Board approval. Criteria of inclusion were age $\geq 18$ years and absence of rheumatologic or viral diseases. PB samples were collected at day $+21,+42,+60,+90$ and +180 after HSCT and any time within day +180 in case of GVHD, before first-line therapy. Eight healthy donors (HDs) were enrolled as control. Mononuclear, polymorphonuclear, and whole blood cells were analysed by flow cytometry after CD45 Vioblue, CD16 APC-Cy7, CD11b PE-Cy7, CD10 PE, CD66b FITC staining. CD66b ${ }^{+}$LDNs were expressed as percentage of $\mathrm{CD} 45^{+} \mathrm{PB}$ mononuclear cells (PBMCs) or $\mathrm{CD} 45^{+}$whole blood cells and were further characterized based on CD10 expression. CD66 ${ }^{+}$NDNs, expressed as percentage of $\mathrm{CD} 45^{+}$whole blood cells, were also analysed for CD10 staining.

Results: 39 patients (M/F 25/14, median age 47) were enrolled in the study. Patients received HSCT from HLAidentical (13) or haploidentical (6) related and from HLAidentical unrelated (20) donors.

After a median time of 33 (15-95) days, 13 patients developed grade II-IV aGVHD. No patients were receiving G-CSF at aGVHD onset. The scheduled assessments were interrupted in aGVHD patients at the beginning of first-line treatment and in 4 patients relapsed of their primary malignancy. No patients developed de novo late-acute or chronic GVHD.

Starting from day +21 the frequency of LDNs within $\mathrm{CD} 45^{+}$PBMCs was higher in all patients as compared to 
HDs. The 25 patients that did not develop aGVHD showed a decreasing frequency of $\mathrm{CD}^{2} 6 \mathrm{~b}^{+} \mathrm{CD} 10^{-} \mathrm{LDNs}$, with a progressive increase of $\mathrm{CD} 6 \mathrm{~b}^{+} \mathrm{CD} 10^{+} \mathrm{LDNs}$, from day +21 to +180 . Interestingly, patients with aGVHD showed a significantly higher frequency of $\mathrm{CD} 6 \mathrm{~b}^{+} \mathrm{CD} 10^{-} \mathrm{LDNs}$ as compared to patients without aGVHD throughout the same time lapse (i.e. from day +21 to +90$)$ (83.15 vs 50.8, $\mathrm{p}=0.027$ ). Consistently, patients with aGVHD had a significantly lower frequency of $\mathrm{CD} 6 \mathrm{~b}^{+} \mathrm{CD} 10^{+} \mathrm{LDNs}$ (12.1 vs 50.05, $\mathrm{p}=0.0014$ ). The frequency of mature $\mathrm{CD}_{66} \mathrm{~b}^{+} \mathrm{CD}_{10}{ }^{+} \mathrm{NDNs}$ was normal in all patients since day +21 .

Conclusions: LDNs are more represented in HSCT patients than in HDs, with a significant expansion of the $\mathrm{CD} 6 \mathrm{~b}^{+} \mathrm{CD} 10^{-}$subpopulation (with a parallel decrease of the $\mathrm{CD} 6 \mathrm{~b}^{+} \mathrm{CD} 10^{+}$subpopulation) in patients with aGVHD as compared to those without aGVHD. According to the previously demonstrated $\mathrm{T}$ cell activating function of $\mathrm{CD} 6 \mathrm{~b}^{+} \mathrm{CD} 10^{-} \mathrm{LDNs}$, it is tempting to speculate that the expansion of this subpopulation may contribute to aGVHD development.

Disclosure: Nothing to declare

\section{P300 \\ LKB1 modulates regulatory $T$ cell homeostasis during acute GVHD}

\author{
Xiuhua Su ${ }^{1}$, Erlie Jiang ${ }^{1}$ \\ ${ }^{1}$ Institute of Hematology, Chinese Academy of Medical \\ Science and Peking Union Medical College inTianjin, \\ Tianjin, China
}

Background: Acute graft-versus-host disease (aGVHD) is a major complication after allogeneic hematopoietic stem cell transplantation (allo-HSCT) which has negative impact on the morbidity and mortality of the patients. Accumulating evidences suggest that abnormalities of Foxp3 + Regulatory $\mathrm{T}$ (Treg) cells contributed to the pathogenesis of GVHD, but the underlying molecular mechanisms still remain largely unknown.

Methods: In this study, we enrolled all the 40 patients treated with allogeneic HSCT at the Institute of Hematology, Chinese Academy of Medical Sciences between 2016 and 2018,as well as 10 age-matched healthy adults as control samples. The ratio of Tregs in PB and BM of healthy controls (HCs) and patients with and without aGVHD was determined by flow cytometry. The transcription profile between Tregs from patients with or without acute GVHD was measured,the pathway enrichment analyses were performed by the Kyoto Encyclopedia of Genes and Genomes (KEGG) pathway database and geneset enrichment analysis (GSEA).The expression of Lkb1 at transcript levels and protein levels was measured by realtime PCR and analyzed by the NanoPro1000TM system. A series of functional assays in vitro were performed to assess the function and stability of Tregs from patients with and without aGVHD.Meanwhile, to assume the affect of Lkb1 on GVHD outcome, we established a murine transplant model,which recipient Balb/c animals were transplanted with the same amount of mixture made by BM, CD4 +CD25-Tcon cells from C57BL/6 and CD4+ Foxp3 YFP+ Tregs from either Foxp3CreLkb1f/f or Foxp3Cre mice.

Results: In this study, we demonstrated that BM had decreased frequencies of Tregs, accompanied with a reversed lower ratio of Tregs frequencies between BM and $P B$ in aGVHD patients. Meanwhile, the number and function of Tregs in bone marrow also affected hematopoietic reconstitution. Futhermore,to elucidate these mechanisms which regulate Tregs homeostasis, we examined the role of Lkb1 on Tregs in patients with aGVHD and in aGVHD murine model. Studies demonstrated that Lkb1deficient Tregs lost Foxp3 expression and weaken suppressor function during aGVHD. Transcriptional profiling and pathway analysis revealed that NF-kB signaling activation and the impairment of a wide spectrum of immunosuppressive genes in aGVHD Tregs. Further mice experiments suggested that CNS2 methylation might lead to the instability of Tregs in aGVHD group. Transplantation with marrow grafts from Foxp3CreLkb1f/fmice exacerbates GVHD lethality.

Conclusions: These studies indicate that Lkb1 is a critical homeostatic regulator for Tregs during aGVHD. Targeting of Lkb1 therefore represents a novel therapeutic strategy that promote immune tolerance to mitigates the severity of aGVHD.

Disclosure: National Program on Key Basic Research Project (973 Program)

\section{P301 \\ Role of aryl hydrocarbon receptor in intestine after allogeneic HSCT in mice}

\author{
Won-Sik Lee ${ }^{I}$, Soung-Min Lee ${ }^{1}$, Sj-Kil Seo ${ }^{1}$ \\ ${ }^{1}$ Inje University, Busan Paik Hospital, Hemato-Oncology, \\ Busan, Korea, Republic of
}

Background: Aryl hydrocarbon receptor (AhR) is a ligandactivated transcription factor that is activated by various small molecules from the diet, microorganisms, host metabolism, and xenobiotic toxic chemicals. The function of AhR has been demonstrated as a crucial regulator in intestinal homeostasis. Here, we investigated the regulatory role of $\mathrm{AhR}$ in intestine of recipients after allogeneic hematopoietic cell transplantation in mice. 
Methods: Wild-type (WT) B6 (H-2 $\left.{ }^{\mathrm{b}}\right), \mathrm{IDO}^{-/-}\left(\mathrm{H}-2^{\mathrm{b}}\right)$ and $\mathrm{AhR}^{-/-}\left(\mathrm{H}-2^{\mathrm{b}}\right)$ mice were lethally irradiated and transplanted with $5 \times 10^{6} \mathrm{TCD}-\mathrm{BM}$ plus $2 \times 10^{6} \mathrm{~T}$ cells from BALB/c donor mice. AhR activation in colon tissue of recipients was determined by the AhR target genes CYP1A1 and CYP1B1 expression using real-time PCR. The recipient mice were monitored every other day for survival and clinical score. Histopathology and pathogenic effector cytokine levels in colon tissue were analyzed for evaluating AhR function.

Results: We observed that CYP1A1 was constitutively expressed in the colon tissue of naïve recipient mice. Although the expression levels were increased by TBI conditioning, the additive up-regulation of its levels with donor T cell alloreactivity was not observed. In contrast, CYP1B1 expression was markedly induced in the colon tissue by donor $\mathrm{T}$ cell alloreactivity. We further observed that the CYP1B1 expression was significantly decreased in the colon of IDO-/- recipients with donor $\mathrm{T}$ cell alloreactivity, but CYP1A1 was not changed. IDO-/- and AhR-/recipient mice showed higher histopathological score for intestinal GVHD and increasing pathogenic cytokine levels in the colon compared with WT mice.

Conclusions: Our results demonstrate that AhR-induced target gene profiles might be differently induced in intestine by ligand dependent manner after HSCT, which affect intestinal GVHD.

Disclosure: Nothing to declare.

\section{P302}

\section{Abstract already published.}

\section{P303}

\section{Abstract withdrawn.}

\section{P304}

In vitro platelet activation evaluation in allogeneic hematopoetic stem cell transplanted patients in response to haemostatic stimulation and cytomegalovirus stimulation

Anne Pouvaret ${ }^{1,2}$, Jérôme Cornillon ${ }^{3}$, Sylvie Pillet ${ }^{1,2}$, Emmanuelle Tavernier ${ }^{3}$, Marie-Ange Eyraud ${ }^{2}$, CharlesAntoine Arthaud ${ }^{2}$, Denis Guyotat ${ }^{3}$, Hind HamzehCognasse $^{2}$

${ }^{1}$ CHU Saint Etienne, Infectious Diseases, Saint-Priest-enJarez, France, ${ }^{2}$ Université Jean Monnet, GIMAP, SaintEtienne, France, ${ }^{3}$ Institut Cancérologie Lucien Neuwirth, Saint-Priest-en-Jarez, France

Background: Platelets have been recently recognized as actors of the immune continuum. Thanks to their secretion of immune mediators and their interactions with innate and adaptative immune cells, they contribute to host defense against invading pathogens like viruses. Platelet immune response to their interaction with Cytomegalovirus (CMV) has never been studied in allogeneic hematopoietic stem cell transplanted (HSCT) patients. Those patients may develop graft-versus-host disease (GvHD), complication of which one of the risk factor is CMV reactivation. The resultant inflammatory platelet response during the high-risk period of GvHD after allogeneic HSCT remains unknown. Our study aimed to characterize spontaneous platelet activation during the $2 \mathrm{~d}$ and $3 \mathrm{~d}$ months after allogeneic HSCT, and in response to haemostatic stimulation and CMV stimulation.

Methods: We compared a group of healthy volunteers to a group of allogeneic HSCT patients followed between the $30^{\text {th }}$ and the $90^{\text {th }}$ days after HSCT. Platelet activation was determined by the platelet surface expression of CD62P and CD63 using flow cytometer after stimulation by an haemostatic agent, Thrombin-receptor activating Peptid (TRAP) and after stimulation by CMV glycoprotein B. The inflammatory response was determined by the detection of immune mediators, RANTES, CD62Ps, PF4, CD40L and CCL3, using the ELISA technique in the stimulated platelet supernatants.

Results: No platelet activation or molecules release were observed after stimulation by CMV glycoprotein B in both groups. RANTES and CD62Ps baseline levels are spontaneously higher in allogeneic HSC patients than in healthy volunteers. Platelets from allogeneic HSCT patients can be activated after haemostatic stimulation and release CD62Ps and RANTES. In this situation, platelets release more CD62PS, RANTES and PF4 than platelets from healthy volunteers.

Conclusions: Although no platelet activation was detected in response to CMV glycoprotein B stimulation, our study revealed a chronic platelet activation condition during the $2 \mathrm{~d}$ and $3 \mathrm{~d}$ months after allogeneic HSCT with an haemostatic inducible hyper-responsiveness. This leads to the release of molecules with immune-modulating properties involved in the pathophysiology of GvHD. As we move further away from the HSCT, that phenomenon seems to gradually weaken.

Clinical Trial Registry:

NCT03009708, FIPALLOC

https://clinicaltrials.gov/ct2/show/NCT03009708

Disclosure: Nothing to declare

\section{P305}

Efficient process and characteristics of umbilical cordderived mesenchymal stromal cells as a feasible source for anti-inflammatory therapy 
Tokiko Nagamura-Inoue ${ }^{1}$, Atsuko Takahashi ${ }^{1}$, Akiko Hori $^{1}$, Yuki Yamamoto ${ }^{1}$, Fumitaka Nagamura ${ }^{2}$, Arinobu Tojo $^{3}$

${ }^{1}$ Institute of Medical Science, University of Tokyo, IMSUT CORD, Tokyo, Japan, ${ }^{2}$ Institute of Medical Science, University of Tokyo, Tokyo, Japan, ${ }^{3}$ Institute of Medical Science, University of Tokyo, Research Hospital, Tokyo, Japan

Background: Recently, umbilical cord (UC) has become attracted source of mesenchymal stromal cells (MSC), because of abundant sources and ease of collection of fetal origin without invasive process for the donor and low immunogenicity with immunosuppressive ability and tissue repair potency. Objectives of this study were to explorer the efficient and safe products and to evaluate the antiinflammatory potency of UC-MSCs for the application of acute graft versus host disease (GVHD).

Methods: Informed consent was obtained from mothers planning to have cesarean sections. UC tissue was cut and once cryopreserved. The safety assessment including infections and baby's health and development were done after 6 months of birth, and performed small-scale quality test of the frozen UC. Then we initiated to isolate master UC-MSCs from frozen-thawed UC by an improved explant method, which was passed for quality test. The master UCMSCs were cryopreserved once and thawed and expanded until P4. Product cells were cryopreserved in original serum-free cryoprotectant DBA-D solution. Mixed lymphocyte reaction (MLR) assay co-cultured with UC-MSCs was carried out using responder mononuclear cells (MNC) stained with CFSE, and proliferation and cytokine secretion were analyzed by flowcytometry.

Results: UC-MSC cultured showed significantly higher proliferation ability compared with those from bone marrow-derived MSCs, and positive for CD105, CD73, CD90, and negative for CD45, HLA-DR. CD80, and CD86 were negative even in the high concentration of IFN- $\gamma$, while BM-MSCs became positive for HLA-DR. PD-L2 was constitutively expressed in UC-MSC, while PD-L1 was induced by the addition of IFN- $\gamma$. In MLR, responder T cell proliferation triggered by allogeneic dendritic cells was inhibited efficiently by 3rd party derived UC-MSCs, in which was induced IDO, PGE2, HGF, and TGF- $\beta$ analyzed by RT-PCR, and inhibited IFN- $\gamma$ and TNF- $\alpha$ in the supernatant by cytokine beads array. UC-MSCs migrated toward the TNF- $\alpha$ treated MNC and increased regulatory $\mathrm{T}$ cells incidence in peripheral mononuclear cells by the coculture.

Conclusions: These results demonstrated that cryopreserved UC are feasible and efficient source of MSCs and frozen-thawed UC-MSCs have high anti-inflammatory potency and we have now started phase I physician's initiated clinical trial for treatment resistant severe acute GVHD.

Clinical Trial Registry: UMIN000032819 https://uploa d.umin.ac.jp/cgi-open-bin/ctr/index.cgi

Disclosure: This study was supported by Grants-in-Aids for Scientific Research from the Japan Agency for Medical Research and Development /Ministry of Health, Labor and Welfare (18bk0104070h0002; 18be0104015h0002).

\section{P306}

Evaluation of the amicus extracorporeal photopheresis (ECP) system in healthy human subjects

Katherine Radwanski, Jennifer Weitgenant ${ }^{1}$, Flora Ayeni $^{1}$, Gregory Coultas ${ }^{1}$, Lan Nguygen ${ }^{1}$, Cheryl Heber ${ }^{1}$, Danielle Badgley ${ }^{1}$, Jeanne Cunningham ${ }^{1}$, Perla Unzueta ${ }^{I}$, Zahra Ali ${ }^{I}$

${ }^{1}$ Fresenius Kabi LLC, Lake Zurich, IL, United States

Background: A new protocol is under development on the AMICUS Separator that enables the device to perform ECP procedures. The AMICUS Separator is used with a photoactivation device, disposable kit and 8-MOP to provide ECP therapy in a closed system. The objective of this study was to evaluate the safety and performance of the investigational AMICUS ECP System in healthy human subjects.

Methods: An IRB-approved written informed consent was obtained from 17 subjects (12 male, 5 female). The AMICUS ECP System processed either 500, 2000 or 4000 $\mathrm{mL}$ whole blood ( $\mathrm{n} \geq 5$ per arm) using double-needle access and ACD-A anticoagulation at a 12:1 WB:AC ratio. After MNC collection was completed, the subject was disconnected from the device. 8-MOP $(3.4 \mathrm{~mL}, 20 \mu \mathrm{g} / \mathrm{mL})$ was injected directly into the collected $\mathrm{MNC}$ product and saline (approximately $200 \mathrm{~mL}$ total), which was photoactivated with 1-2 J/ $/ \mathrm{cm}^{2}$ UVA light. Post photoactivation, the AMICUS Separator reinfused the treated MNCs into a transfer pack. Subject laboratory and safety parameters were evaluated; in vitro evaluations were performed on subject whole blood, collected MNCs, treated MNCs, and reinfused cells. Lymphocyte and monocyte analysis were performed on samples purified using density gradient separation and cultured for up to 3 days post treatment.

Results: In 17 procedures, median (range) WB processed was 2016.0 (509 - 4024) $\mathrm{mL}$ using $172.0(51-333) \mathrm{mL}$ of ACD-A. Procedure time was 93.0 (66 - 119) minutes, including photoactivation. No adverse events were reported. Subjects' vital signs and hematology values were unremarkable and within expected values. The WBC count of the collected MNCs was $13.50(3.3-30.2) \times 10^{3} / \mu \mathrm{L}$, comprised of 77.10 (47.9 - 87.0) \% lymphocytes, $15.50(7.0-36.8) \%$ 
monocytes and $5.70(2.9-25.5) \%$ granulocytes and platelet count was $94.0(70-169) \times 10^{3} / \mu \mathrm{L}$. Using pre-WBC counts, MNC collection efficiency was $66.34(53.4-96.4) \%$. WBC viability and free hemoglobin levels in treated MNCs showed no evidence of cell death or lysis during photoactivation. Treated MNCs were not substantially different than collected MNCs in WBC, RBC, and platelet counts. Additional analysis demonstrated the collected MNCs had a median hematocrit of 2.40 (1.8-2.9). Trends in lymphocyte and monocyte apoptosis/viability during culture were as expected for the Amicus ECP system. Lymphocyte viability in collected MNCs was maintained for up to 72 hours in culture while lymphocyte viability in treated MNCs declined substantively over time. Minimal increases in lymphocyte apoptosis occurred in the collected MNCs at 72 hours in culture while substantive increases were noted in the treated MNCs. Lymphocyte proliferation at 72 hours post stimulation with phytohaemagglutinin-A was significant in collected MNCs 62.30 (39.0 - 79.7) \%, but negligible in treated MNCs 0.85 (0.6 - 6.3) \%. Inhibition of proliferation in the treated versus collected MNCs at 72 hours was $98.58(90.8-99.1) \%$, with all values above $90 \%$.

Conclusions: The safety and in vitro assessments of the AMICUS ECP System in healthy human subjects were as expected, with lymphocyte assay results comparable to values reported for other ECP technologies.

Disclosure: Nothing to Declare, all authors are employees of Fresenius Kabi

\section{P307}

The influence of pathogen reduction with riboflavin/ ultraviolet and gamma-irradiation on proliferation and viability of lymphocytes from whole blood

\section{Yana Bayzyanova ${ }^{1}$, Nikolay Starostin ${ }^{1}$, Irina Kumukova ${ }^{1}$, Elena Osipova ${ }^{I}$, Pavel Trakhtman ${ }^{I}$}

${ }^{1}$ Dmitry Rogachev National Research Center of Pediatric Hematology, Oncology and Immunology, Moscow, Russian Federation

Background: Transfusion of white blood cells (WBC) causes a number of transfusion reactions and complications, for example transfusion-associated graft versus host disease (taGVHT), which still does not have effective treatment and is a fatal complication of transfusions. The only effective method of preventing taGVHT is irradiation of blood components with ionizing radiation (X-ray or gamma radiation). But the use of ionizing radiation sources has a number of technical and material difficulties. The emergence of pathogen reduction technologies (PRT) in blood components targeted by nucleic acids has opened the possibility of using these technologies as an alternative to irradiating of blood components. Several PRT demonstrated effective inactivation of $\mathrm{WBC}$ in platelet concentrates and blood plasma. So, determination of the influence of PRT based on the combined effect of riboflavin (RF) and ultraviolet (UV) on the viability and proliferating potential of lymphocytes in whole blood is important.

Methods: Samples of whole blood were obtained in 35 healthy volunteers. Each sample was divided into three unequal parts: untreated control, gamma irradiated, and treated by RF and UV PRT (Mirasol, Terumo BCT Inc.). Mononuclear cells (MNC) were CFSE stained, viability and proliferating activity were tested at intervals of 24 hours for 3 consecutive days by flow cytometry. Statistical analysis was performed with XLStat 7.0. Levels of significance were calculated by Mann-Whitney test, expressed as P-values $(\mathrm{P}<0,05)$.

Results: The median viability of MNC after application of both methods of treatment was over $85,0 \%$ on day 0 and decreased to day 3 - median percentage of viable MNC were $84,0 \%$ (control group), 69,0\% (after gamma irradiation) and $63,0 \%$ (RF/UV PRT). The median of spontaneous proliferative activity on day 3 of untreated and gamma irradiated MNC did not differ (1,8\% and 2,1\% respectively, $\mathrm{p}<0,05$ ). Phytohemaglutenin (PHA) induced proliferation on day 3 in gamma-irradiated samples was significantly lower in comparison with control group (4,0\% and 45,0\% respectively, $\mathrm{p}<0,01)$. In samples treated with $\mathrm{RF} / \mathrm{UV}$, spontaneous and stimulated proliferating cells was not detected. Median percentage of proliferating MNC was less than $0,2 \%$. The use of this PRT on whole blood, as well as gamma irradiation, significantly reduces the viability of lymphocytes during storage for 3 days.

Conclusions: Inactivation of WBC using RF/UV PRT is a useful and very necessary bonus for a number of reasons. In one procedure two effects are achieved: infectious and immunological safety. The use of PRT on whole blood gives the potential for obtaining pathogen-reduced and immunological safety components of blood, which reduces their material cost and staff loading. The use of RF/UV system does not have such complex security requirements and difficulties in servicing as the use of sources of ionizing radiation. The results demonstrate a promising potential for using this technology as an alternative to irradiation

Disclosure: Nothing to declare

\section{P308}

Influence of patients's serum after allogeneic stem cell transplantation on $T$ cell proliferation and TREG function 
Pascal Woelfinger ${ }^{I}$, Saskia Hendriks ${ }^{I}$, Diana Kriege ${ }^{I}$, Matthias Theobald ${ }^{1}$, Tobias Bopp ${ }^{2}$, Eva Maria WagnerDrouet $^{I}$

${ }^{1}$ Johannnes Gutenberg University Mainz, Haematology, Oncology, Pneumology, UCT, Mainz, Germany, ${ }^{2}$ Johannnes Gutenberg University Mainz, Institute for Immunology, Mainz, Germany

Background: Acute or chronic graft versus host disease (a/ cGVHD) is one of the major complications after allogeneic hematopoietic stem cell transplantation (aHSCT). Application of regulatory $\mathrm{T}$ cells (Treg) as "immunosuppressive DLI" to prevent or treat GVHD is investigated in clinical trials. Here we ask the question, if there could be clinical conditions (e.g. cytokines or drug effects) limiting the efficacy of this approach. To face this problem we tested the influence of patients's serum on T cell proliferation and Treg function.

Methods: Lymphocytes from healthy donors were incubated with T cell medium (90\% AIM V $+10 \%$ serum + IL2/OKT3) containing serum from healthy donors or serum derived from patients after aHSCT with or without GVHD $(n=10)$. Next we evaluated the suppressive function of Treg by performing Treg suppression assays, also comparing serum from patients suffering from GVHD versus serum obtained from healthy donors $(n=8)$. Proliferation of CFSE stained T cells was measured after 5 days. To test the effect of immunosuppressive drugs on Treg we performed Treg suppression assays after incubation of Treg with Corticosteroids or Tacrolimus or the combination of both drugs.

Results: Serum of patients with acute or chronic GVHD had a negative effect on $\mathrm{T}$ cell proliferation. To avoid bias tests were performed with samples from patients without or only with low levels of immunosuppressive drugs. Incubation with serum of patients without GVHD or with serum of healthy individuals showed no differences in $\mathrm{T}$ cell proliferation.

Treg from healthy donors showed a stronger antiproliferative capacity when incubated with serum derived from patients with GVHD. Treg previously incubated with immunosuppressive drugs showed no decreased suppressive capacity.

Conclusions: Components of serum from GVHD patients seem to have an antiproliferative effect on $\mathrm{T}$ lymphocytes itself. This fact might influence the clinical course of GVHD, but should not be a limiting factor for therapeutic application of Treg DLI. Even the systemic treatment with immunosuppressive drugs e.g. corticosteroids or calcineurin-inhibitors should not diminish the Treg application.
In a next step we will analyze serum components responsible for this immunosuppressive effect with multi cytokine assays and proteomic analysis. The aim of our project is to develop new strategies to avoid GVHD and to optimize clinical settings for Treg DLI.

Disclosure: Nothing to declare

Granulocyte and osteoclast disorders

\section{P309}

Denosumab as a first line treatment for post- stem cell transplant hypercalcemia in osteopetrosis

Hemalatha Doss ${ }^{I}$, Heather Weerdenburg ${ }^{I}$, Sam Whiting ${ }^{I}$, Ponni Sivaprakasam ${ }^{I}$, Colin G Steward ${ }^{I}$, Christine P Burren $^{1}$, Adam Gassas ${ }^{I}$

${ }^{1}$ Bristol Royal Hospital for Children, Bristol, United Kingdom

Background: Hypercalcaemia can be very severe following stem cell transplant (SCT) in some osteopetrosis patients. Denosumab is a fully human monoclonal antibody that binds the cytokine RANKL (receptor activator of NFKB ligand), an essential factor initiating bone turnover. RANKL inhibition blocks osteoclast maturation, function and survival, thus reducing bone resorption. We describe the effective management of hypercalcaemia in a patient with RANK mutation osteopetrosis who received a haploidentical SCT.

Methods: Our patient was diagnosed with osteopetrosis at 2 year of age with a defect in the TNFRSF11A gene which codes for RANK and received a maternal haploidentical SCT aged 4 years. The patients calcium levels were monitored regularly post SCT. Denosumab was administered for hypercalcaemia as per laboratory reports or clinical symptoms. The drug was diluted with water for injection to make $6 \mathrm{mg} / \mathrm{ml}$ solution to facilitate subcutaneous administration.

Results: Significant hypercalcaemia emerged on day +18 with a level of $3 \mathrm{mmol} / \mathrm{L}$ and treated with hyper-hydration and diuretics. This was ineffective in reducing the hypercalcaemia; therefore denosumab was initiated on day +20 post-transplant. Initial dosing was determined using the only available paediatric case report at $0.13 \mathrm{mg} / \mathrm{kg}$. A repeated larger dose of $0.19 \mathrm{mg} / \mathrm{kg}$ was given 4 days later due to an inadequate response (calcium decreased from $3.9 \mathrm{mmol} / \mathrm{L}$ to $3.80 \mathrm{mmol} / \mathrm{L}$ ). The calcium decreased to $1.93 \mathrm{mmol} / \mathrm{L}$ after this dose. Four weeks later a third dose was required at $0.26 \mathrm{mg} / \mathrm{kg}$ as the calcium level had increased to $4.1 \mathrm{mmol} / \mathrm{L}$. The dose was further increased to $0.32 \mathrm{mg} / \mathrm{kg}$ for another four doses and then further increased to $0.65 \mathrm{mg} / \mathrm{kg}$ for another 3 doses and repeated every 7 weeks. Normalisation, but not excessive drop in 
calcium was achieved with these larger doses. Over the 9 month follow up post-transplant there were three admissions lasting less than 24 hours for symptoms of hypercalcaemia. These were managed with denosumab administration and hyper-hydration. The remaining doses were given in an outpatient setting.

Conclusions: Denosumab can be safely used as a first line agent in treating post stem cell transplant hypercalcemia in patients with osteopetrosis. A dose of $0.3 \mathrm{mg} / \mathrm{kg}$ is required as an initial starting dose in order to control hypercalcemia. This is a new higher dose than previously suggested by the original report. Denosumab can be effective even after dilution and safely given in children weighing less than $10 \mathrm{~kg}$.

Disclosure: Nothing to Declare

\section{P310}

Granulocytic sarcoma occurring after cord blood (CB) transplantation: A case report

\section{Oscar Borsani ${ }^{1}$, Anna Amelia Colombo ${ }^{1}$, Roberta Sciarra $^{1}$, Michele Ciboddo ${ }^{1}$, Daniela Caldera ${ }^{1}$, Paolo Bernasconi ${ }^{I}$}

${ }^{1}$ University of Pavia. San Matteo Hospital, Pavia, Italy,

Background: Disease recurrence within the first six months post-transplant frequently occurs in patients with an advanced disease at the time of HSCT. However, the occurrence early post-transplant of a disease different from that originally diagnosed is a very rare event. Herein we describe such a patient who underwent a CB transplant for a pro-B acute lymphoblastic leukemia (ALL) and developed a granulocytic sarcoma of recipient's origin 67 days posttransplant.

Methods: The clinical, laboratory and molecular aspects of this Italian male patient who developed such a complication were collected and presented in order to discuss the origin, clinical outcome and management of this very rare post-transplant event.

Results: A 68-years-old man affected by a high-risk chromosomally abnormal, Ph1-, MLL- pro-B (EGIL B-I) ALL relapsed during maintenance treatment, nonresponsive to re-induction chemotherapy, in second complete remission (II CR) after Blinatumumab treatment received a female CB transplant. According to Sorror's and EBMT scores he was considered a high-risk transplant. The patient and the CB unit were sex-mismatched, shared the same blood groups and were both $\mathrm{CMV}+/ \mathrm{EBV}+\mathrm{He}$ received a TBF conditioning regimen that was followed by the infusion of $0.54 \times 10^{5} / \mathrm{kg} \mathrm{CD} 34+\mathrm{CB}$ cells. GVHD prophylaxis consisted of rabbit ATG, Cyclosporine A (CsA) and mycophenolate mophetyl (MMF). Neutrophil engraftment occurred on day +28 , whereas platelets were never $>20.000 / \mu l$. On day +67 a $2 \mathrm{~cm}$ bulged area became apparent on the left parietal region of the skull. An echotomography showed that the lesion adhered to the bone without infiltrating it and lacked blood vessels and suggested that it may be either a site of disease relapse or an area of infection. At the same time a bone marrow (BM) aspiration showed morphological $\mathrm{CR}$ confirmed by immune-phenotypic studies and X-Y FISH a complete chimera. Since the patient was still febrile no biopsy was performed, but on day +94 the axial diameter of the lesion that on a CT scan showed the same appearance revealed by the previous echo-tomography increased to $4 \mathrm{~cm}$. Thus, the lesion was surgically removed and histological examination showed CD33+, CD14+/-, CD163+/-, CD45/LCA+/-, CD21-, CD23-, CD35-, CD207-, and S100- neoplastic cells whose phenotype suggested a granulocytic sarcoma rather than a histiocytic sarcoma. Immuno-chemistry confirmed this suggestion by showing a nuclear NPM1 positivity. FISH studies demonstrated that these neoplastic cells were of recipient's origin. A novel BM aspiration showed CR confirmed by immune-phenotypic studies and FISH revealed a complete chimera. Since the patient was still pancytopenic due to anti-CMV treatment, radiotherapy with $18 \mathrm{~Gy}$ in nine fractions were given and the lesion completely resolved.

Conclusions: A granulocytic sarcoma of recipient's origin occurring three months after a $\mathrm{CB}$ transplant is a very rare and unusual event. In order to explain such a complication we suggest that granulocytic sarcoma cells were dormant but already present at the time of pro-B ALL diagnosis and survived not only the initial ALL treatment but also the CB transplant conditioning regimen. We can't exclude that immune-suppressive treatments given early post-transplant might have promoted the outgrowth of these neoplastic cell population.

Disclosure: Nothing to declare

Haemoglobinopathy and inborn errors of metabolism

\section{P311}

Abstract already published.

\section{P312}

Addition of fludarabine on to anti-thymocyte globulin, busulfan and cyclophosphamide conditioning improves outcomes in low-risk matched-related bone marrow transplantation in children with severe thalassaemia

Stalin Ramprakash ${ }^{1,2}$, Commondoor P Raghuram ${ }^{1,3}$, Deepa Trivedi ${ }^{4}$, Priya Marwah ${ }^{5}$, Rajpreet Soni ${ }^{5}$, Rajat Kumar Agarwal ${ }^{6}$, Rakesh Dhanya ${ }^{6}$, Lawrence Faulkner $^{1,6,7}$ 
${ }^{1}$ People Tree Hospital, Bangalore, India, ${ }^{2}$ Aster CMI Hospital, Paediatric Haematology Oncology, Bangalore, India, ${ }^{3}$ Aster CMI Hospital, Bangalore, India, ${ }^{4}$ Care Institute of Medical Sciences, Ahmedabad, India, ${ }^{5}$ South East Asia Institute for Thalassemia, Jaipur, India, ${ }^{6}$ Sankalp India Foundation, Bangalore, India, ${ }^{7} \mathrm{Cure} 2 \mathrm{Children}$ Foundation, Florence, Italy

Background: Matched-related bone marrow transplantation (BMT) may cure over $80 \%$ of low-risk children with severe thalassemia (ST) defined as a thalassemia syndrome with inability to keep a spontaneous hemoglobin $>7 \mathrm{~g} / \mathrm{dL}$. Very long-term follow up studies have shown how the standard Busulfan-Cyclophosphamide (BuCy) regime may be associated with normalization of Health-Related Quality of Life (HRQoL), no second malignancies in the absence of chronic GVHD, and birth rates comparable to the control population. However, BuCy may be associated with high rejection rates hence increasing immunosuppression by adding anti-thymocyte globulin (ATG) and/or Fludarabine during conditioning may be justified.

Methods: This study retrospectively compares matchedrelated BMT outcomes in two groups of low-risk ST patients conditioned with either only ATG (Protocol 2) or Fludarabine and ATG (Protocol 1) added to the Bucy backbone. We only included fully matched transplants with either sibling or mother as donor and excluded father donors based on our previous experience (Ramprakash et al, Bone Marrow Transplantation 2017) in this analysis. A total of 139 consecutive first BMTs and 3 second BMTs from different matched related donor where performed in PTH, CIMS and SEAIT collaborating BMT centers in the Indian subcontinent. From Dec 2013 to Nov 2016, 45 patients were transplanted after conditioning with ATG-BuCy while between March 2017 and August 2018, 97 patients received Flu-ATG-BuCy. ATG dose was $4 \mathrm{mg} / \mathrm{kg}$ in all patients except patients with splenomegaly $\geq 3 \mathrm{~cm}$ from costal margin and/or sex-mismatched/maternal donor in whom ATG was increased to $7 \mathrm{mg} / \mathrm{kg}$. All patients were younger than 15 years and had no hepatomegaly (liver $\leq 2 \mathrm{~cm}$ from costal margin) at BMT.

Results: Actuarial overall survival (OS) in the ATGBuCy and Flu-ATG-BuCy groups is $89 \%$ and 98\%, thalassemia-free survival (TFS) $75 \%$ and 93\%, GVHDfree and Thalassaemia-free survival (GTFS) at a median follow up of 23.5 and 10.6 months was $72.6 \%$ and $93.3 \%$ months respectively, which is a significantly improved outcome by log-rank statistics ( $\mathrm{p}=0.002$ ) in the Flu-ATG$\mathrm{BuCy}$ group. There was no significant difference between the groups in pre-transplant characteristics and posttransplant complications except for the following: Median cell dose more in $2^{\text {nd }}$ group with total nucleated cell dose of 8.7 vs $6.4 \times 10^{8}$ cell $/ \mathrm{kg}$ with $\mathrm{p}<0.0001 ;$ CSA taper started later in the new protocol (184 day vs. $152 \mathrm{p}=0.0005$ ); Median age at BMT (7.2 vs. 4.6 years, $\mathrm{p}=0.001)$; Number of Pre-BMT transfusions $(\mathrm{p}=0.01)$ and Ferritin at BMT (2.214 vs. $1.599 \mathrm{ng} / \mathrm{mL}, \mathrm{p}=0.002$ ) were higher in the second group; Day 30 and 60 chimerisms were also significantly higher in new protocol $(\mathrm{p}=0.02$ and 0.03 respectively).

There was a trend towards increased incidence of VenoOcculsive Disease (VOD) and Posterior Reversible Encephalopathy Syndrome (PRES) on the second group but this difference did not reach statistical significance.

Conclusions: Adding Fludarabine and targeted dose increase of ATG in the standard BuCy context seems to significantly improve outcomes of thalassaemia transplants without contributing to excessive GVHD or infectious complications. This protocol can be easily administered in low resource setting without major additional costs.

Clinical Trial Registry: Not applicable

Disclosure: Nothing to Declare

\section{P313}

Outcomes of allogeneic hematopoietic stem cell transplantation in patients with paroxysmal nocturnal hemoglobinuria compared to paroxysmal nocturnal hemoglobinuria-aplastic anemia syndrome

Limin Liu ${ }^{1}$, Huifen Zhou ${ }^{1}$, Qingyuan Wang ${ }^{1}$, Huiying Qiu $^{1}$, Xiaowen Tang ${ }^{1}$, Yue Han ${ }^{1}$, Chengcheng $\mathrm{Fu}^{1}$, Zhengming Jin ${ }^{1}$, Suning Chen ${ }^{1}$, Aining Sun ${ }^{1}$, Miao Miaol, Depei $\mathbf{W u}^{1}$

${ }^{1}$ First Affiliated Hospital of Soochow University, Suzhou, China

Background: Paroxysmal nocturnal hemoglobinuria (PNH) is an acquired clonal disorder of the hemopoietic stem cells for which the only curative treatment is allogeneic hematopoietic stem cell transplantation. However, there are still few reports on the outcomes of allogeneic hematopoietic stem cell transplantation (allo-HSCT) in patients with PNH compared to paroxysmal nocturnal hemoglobinuria-aplastic anemia (PNH-AA) syndrome. Our study aimed to compare the outcomes of allo-HSCT for PNH with PNH-AA syndrome.

Methods: The clinical data of $46 \mathrm{PNH}$ patients received allo-HSCT $(\mathrm{PNH}=16$, PNH-AA $=30$ ) in our center from July 2007 to June 2018 were analyzed retrospectively to compare the outcomes of PNH group with PNH-AA group. The clinical data including 28 male patients and 18 female patients, the median age was 29 years (range 6-54). All patients had received various treatments before transplantation such as steroids, androgens, cyclosporine (CsA), antithymocyte globulin, and growth factors. The median 
interval from PNH diagnosis to HSCT was 6 months (range $3-240$ ). The conditioning regimen was modified BU/CYbased regimen in haploidentical donors and unrelated donors, CsA, mycophenolate mofetil (MMF) and shortterm methotrexate (MTX) were administered for graftversus host disease (GVHD) prophylaxis. Patients with matched sibling donors were treated with the FLU/CYbased regimen and CsA were administered for GVHD prophylaxis.

Results: There were no differences of baseline between the 2 groups $(\mathrm{P}>0.05)$ except gender and haploidentical donors. The median values of absolute nucleated cell counts were $10.58(3.83-13.83) \times 10^{8} / \mathrm{kg}$ in the PNH group and $10.81(3.96-33.40) \times 10^{8} / \mathrm{kg}$ in the PNH-AA group $(\mathrm{P}=$ $0.668)$. The median doses of $\mathrm{CD} 34^{+}$cells infused were 5.00 $(3.14-8.42) \times 10^{6} / \mathrm{kg}$ and $3.57(1.97-6.17) \times 10^{6} / \mathrm{kg} \quad(\mathrm{P}=$ $0.002)$, respectively. All patients attained complete engraftment, no patient occurred graft failure. The median time for myeloid engraftment were 11 (range, 7-14) days in the PNH group and 12 (range, 10-26) days in the PNH-AA group (P $=0.003)$. The median time for platelet engraftment were 13 (range, 11-16) days and 18 (range, 12-75) days $(\mathrm{P}=0.002)$, respectively. With a median follow-up of 36 (4-132) months in the PNH group and 26 (4-75) months in the PNH-AA group $(\mathrm{P}=0.428)$. In $\mathrm{PNH}$ and $\mathrm{PNH}-\mathrm{AA}$ groups the incidences of grade I-IV acute graft-versus-host disease (aGVHD) were $12.50 \%$ and $33.30 \%(\mathrm{P}=0.121)$, grade IIIV aGVHD were $6.25 \%$ and $20.00 \%(P=0.209)$; chronic GVHD were $12.50 \%$ and $35.67 \%(\mathrm{P}=0.274)$, moderatesevere chronic GVHD were $0.00 \%$ and $14.39 \%(\mathrm{P}=$ 0.146). In haplo-HSCT and MSD groups the incidences of infection were $37.50 \%(6 / 16)$ and $33.33 \%(10 / 30)(\mathrm{P}=$ 0.777). No patient occurred early death and relapse. 3-year estimated overall survival (OS) of PNH and PNH-AA groups were $100.0 \% \pm 0.0 \%$ and $85.7 \% \pm 6.6 \%(\mathrm{P}=$ 0.141 ), GVHD-free and failure-free survival (GFFS) were $100.0 \% \pm 0.0 \% 、 78.7 \% \pm 7.7 \%(\mathrm{P}=0.067)$.

Conclusions: The preliminary results indicated that alloHSCT is a feasible choice for PNH with favorable outcomes, time for myeloid and platelet engraftment in PNH group were faster than PNH-AA group. There were no differences in OS and GFFS between PNH group and PNHAA group.

Disclosure: no disclosure

\section{P314}

Pattern of calcineurin inhibitor-associated neurotoxicity in sickle cell disease patients receiving a stem cell transplantation

Katharina Kleinschmidt ${ }^{l}$, Juergen Foell ${ }^{I}$, Johgannes Schulte $^{2}$, Ernst Holler ${ }^{1}$, Matthias Edinger ${ }^{1}$, Daniel Wolff ${ }^{I}$, Petra Hofmann ${ }^{1}$, Anja Troeger ${ }^{1}$, Peter Lang ${ }^{3}$, Beatrix
Pfirstinger $^{I}$, Charalampos Aslanidis ${ }^{1}$, Pauline Dibbern ${ }^{I}$, Angelika Eggert ${ }^{2}$, Selim Corbacioglu ${ }^{1}$

${ }^{1}$ University Hospital Regensburg, Regensburg, Germany, ${ }^{2}$ University Hospital Charité, Berlin, Germany, ${ }^{3}$ University Hospital Tuebingen, Tuebingen, Germany

Background: Allogeneic HSCT with a MSD represents currently the only curative option for sickle cell disease (SCD), limited by a donor availability $<20 \%$. Neurotoxicity (NT) contributes significantly to HSCT-associated morbidity and mortality. Calcineurin-inhibitor (CNI) associated NT ranges from $4.2 \%-28.8 \%$ (severe NT 4\%-11\%). The elevated incidence of NT in SCD (around 30\%) might be triggered by the systemic vasculopathy of SCD, with the brain being the primary target. Although both cyclosporine A (CsA) and tacrolimus (FK506) have a proinflammatory effect, it is more pronounced in CsA. Infusion modalities also might impact (10.3\% after bolus injections versus 3.3\% after continuous infusion).

Methods: In a pilot study, we compared T-cell depleted haploidentical HSCT (T-haplo HSCT) with MSD HSCT in patients (pts) with advanced stage SCD, using almost identical conditioning regimens. 32 pts (3-31 years; yrs) with homozygous SCD or $\mathrm{HbS} 0 /+\beta$-Thal were treated between 2012 and 2018. Nine pts received a MSD bone marrow graft, 23 pts received 24 T-haplo-HSCT (1 second T-haplo due to graft rejection). Immunosuppression consisted of either CsA (6 MSD, 16 T-haplo) or FK506 (3 MSD, 8 T-haplo), in combination with mycophenolate mofetil (MMF). FK506 was administered as a 20-hours continuous infusion, CsA as 4-hours bolus injections; both target level adjusted (CsA: 100-120 ng/ml; FK506: 5-8 ng/ $\mathrm{ml}$ ). Duration of immunosuppression was $>6$ months in Thaplo-SCT and $<6$ months in MSD, depending on chimerism.

Results: CNI-related NT was observed in $36.4 \%$, severe NT (PRES, visual disturbance, aphasia) in $21.2 \%$. NT was more prevalent in MSD $(\mathrm{n}=5,55.5 \%)$ than in T-haplo $(\mathrm{n}=7,29.2 \%)$. The incidence of NT was identical under CsA $(8 / 22 ; 36.4 \%)$ and FK506 $(4 / 11 ; 36.4 \%)$, however the majority of severe NT (all PRES) occurred with CsA. Complete recovery of NT was achieved in all pts either spontaneously or after switching to FK506/everolimus or withdrawal of FK506. Moreover, 66.6\% of pts with NT were $>18 \mathrm{yrs}$, and $91.6 \%>12 \mathrm{yrs}$, suggesting an increased risk with age. Only $37.5 \%$ of pts with pre-existing cerebrovascular disease experienced post-HSCT NT. Of note, $57.1 \%$ of pts with severe NT also developed mild acute GvHD. The overall (OS) and disease-free survival (DFS) with a median follow-up of 17 months in T-haploHSCT and 22 months in MSD HSCT was $91 \%$ vs. $100 \%$, respectively. 
Conclusions: Our data confirm an elevated NT risk in SCD pts following allo-HSCT. Importantly, the incidence of NT seems to be related to age ( $91 \%$ of pts with NT were $>10$ yrs), donor source (MSD 55.5\% vs. T-haplo 29.2\%) and type of CNI inhibitor where almost all severe NT (71.4\%, particularly all PRES) was observed under CsA. Continuous infusion of FK506 vs. bolus injections of CsA might have levelled concentration peaks. The NT observed with CsA could be the consequence of predominantly CsArelated vascular toxicity inflicting pre-damaged vessels in SCD. The mechanism of action could be related to other systemic endotheliopathies such as VOD, TAM and aGvHD, which was observed in $57.1 \%$ of pts with severe NT, compared to an overall aGvHD rate of $30 \%$.

Disclosure: Nothing to declare

\section{P315}

Effectiveness of hydroxyurea and supertransfusions as pre-transplant risk-reduction strategy in matched related thalassaemia transplants

Stalin Ramprakash ${ }^{1,2}$, Jayasree Cherukat ${ }^{3}$, Commondoor P Raghuram $^{2,3}$, Deepa Trivedi ${ }^{4}$, Priya Marwah ${ }^{5}$, Rajpreet Soni $^{5}$, Rajat Kumar Agarwal ${ }^{6}$, Rakesh Dhanya ${ }^{6}$, Lawrence Faulkner, 3,7

${ }^{1}$ People Tree Hospital \& Aster CMI Hospital, Paediatrics, Bangalore, India, ${ }^{2}$ Aster CMI Hospital, Bangalore, India, ${ }^{3}$ People Tree Hospitals, Bangalore, India, ${ }^{4}$ Care Institute of Medical Sciences, Ahmedabad, India, ${ }^{5}$ South East Asia Institute for Thalassemia, Jaipur, India, ${ }^{6}$ Sankalp India Foundation, Bangalore, India, ${ }^{7}$ Cure2Children Foundation, Florence, Italy

Background: Matched-related bone marrow transplantation (BMT) may cure over $80 \%$ of low-riskchildren with severe thalassemia (ST) defined as a thalassemia syndrome with inability to keep a spontaneous hemoglobin $>7 \mathrm{~g} / \mathrm{dL}$. It is well known that patient status at the time of transplant is critical in predicting transplant outcome. Liver size $>2$ $\mathrm{cm}$ is an established adverse prognostic factor in terms of transplant-related mortality and, in our own experience, a spleen size $\geq 3 \mathrm{~cm}$ from costal marginis associated with increase rejection rates (Blood 2017 vol. 130 no. Suppl 1 1944). Optimising liver and spleen size prior to transplant is likely to improve transplant outcomes.

Methods: We retrospectively reviewed the effectiveness of our strategy to reduce liver and spleen size pre-transplant using hydroxyurea, super-transfusion and intensive iron chelation. We considered liver size $<2 \mathrm{~cm}$ and spleen size less than $3 \mathrm{~cm}$ below costal margins as good risk features. Liver biopsies were not performed thus Pesaro risk classification could not be assigned. All transplant candidates were started on hydroxyurea for a minimum of 3 months and pre-transfusion haemoglobin was maintained $>7 \mathrm{gm} / \mathrm{dl}$ while on hydroxyurea. If the child had hepatospenomegaly at enrollmentand no improvement in liver and spleen size after an adequate trial of hydroxyurea (minimum of 3 months of treatment achieving maximum dose of $50 \mathrm{mg} / \mathrm{kg}$ day or tolerable haematological toxicity, i.e. neutrophil count between 1000 and $1500 / \mu \mathrm{L}$ and/or platelet count between 100.000 and $150.000 / \mu \mathrm{L}$ ) patients were given a trial of supertransfusion maintaining haemoglobin above $12 \mathrm{~g} / \mathrm{dL}$ ) for a minimum of 3 months prior to declaring the patient as having failed downstaging.

Results: Out of 119 transplants across 3 collaborating centers in India, 85 patients had no hepatosplenomegaly at enrolment and hence were not actively downstaged. Twelve patients were excluded due to inadequate information on their records. All of the remaining 22 patients with enlarged liver and/or spleen were downstaged to low-risk features. All patients received adequate hydroxyurea trial among which seven (32\%) patients required super transfusion in addition to maximal hydroxyurea. Out of the 22 patients 18 $(82 \%)$ were successfully down-staged with the above strategy and proceeded to transplant as low-risk patients. Among the remaining $4(25 \%)$ patients 3 had liver $>2 \mathrm{~cm}$ and one had a spleen $\geq 3 \mathrm{~cm}$ only. There was significant improvement in liver and spleen size from the time of enrollment to transplant ( $\mathrm{p}$ value 0.0004 and 0.0002 respectively by Wilcoxon test for paired samples - two tailed) with median duration of downstaging of 9 months (range 2- 27 months). There was no significant difference in Overall survival (OS) and Disease-Free Survival (DFS) by log rank test between the downstaged group and those who did not have hepatosplenomegaly at enrollment ( $p$ value 0.590 .64 respectively).

Conclusions: In the majority of children with thalassaemia and high transplant risk features liver and spleen size can be reduced pre-transplant using hydroxyurea and supertransfusions thereby decreasing transplant risk.

Disclosure: Nothing to Declare

\section{P316}

Abstract already published.

\section{P317}

Abstract withdrawn.

\section{P318}

Longitudinal analysis of the effect of hematopoietic cell transplantation on ocular disease in children with mucopolysaccharidosis i shows ongoing disease progression 
Brigitte van den Broek ${ }^{1}$, Jens Achterberg ${ }^{1}$, Jaap Jan Boelens $^{1,2,3}$, Michelle van Egmond-Ebbeling ${ }^{I}$, Peter van Hasselt $^{1}$

${ }^{1}$ University Medical Center, Utrecht University, Utrecht, Netherlands, ${ }^{2}$ Memorial Sloan Kettering Cancer Center, Pediatric Bone \& Marrow Transplantation Program, New York City, NY, United States, ${ }^{3}$ Princess Máxima Center for Pediatric Oncology, Utrecht, Netherlands

Background: Corneal clouding is seen in nearly all patients with Mucopolysaccharidosis-1 (MPS-1) causing visual impairment. Hematopoietic cell transplantation (HCT) is able to stabilize disease in many organs including the brain. However, residual disease in peripheral tissues is often described. Therefore, the aim of this study was to determine the long-term effect of HCT on ocular disease in MPS-1 patients.

Methods: Corneal clouding (grade 0-4) and visual acuity (Decimal scale) were prospectively collected from all consecutive MPS-1 patients treated with HCT between 2003 and 2018 at the UMC Utrecht. The primary outcomes of interest, the effect of time on corneal clouding and visual acuity, were analyzed using a linear mixed model. The correlation between corneal clouding and visual acuity was analyzed with Pearson's rho. Other parameters studied were clinical phenotype, age at time of transplantation and hematological enzyme level after transplantation. Other outcomes of interest analyzed included intra-ocular pressure, refraction, and macula and lens abnormalities.

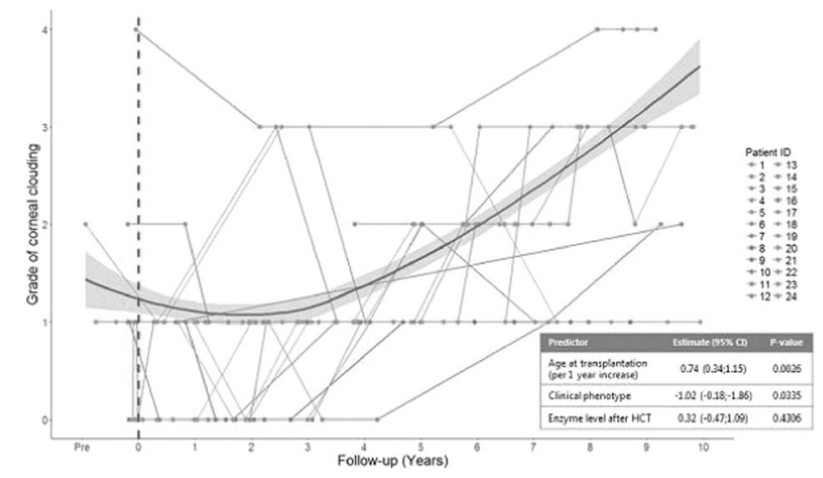

[[P318 Image] 1. Figure 1. Corneal clouding per individual patient $(O D)$ and the effect of time (blue line).]

Results: 24 successfully engrafted MPS-1 patients were included (92\% with $>95 \%$ chimerism and normal enzyme levels after HCT). Corneal clouding stabilized during the first years after HCT, but increased rapidly beyond three years (figure 1). Other predictors for increased corneal clouding were age at time of transplantation $(0.74,95 \% \mathrm{CI}$
$0.34: 1.15 ; \mathrm{p}=0.0026)$ and clinical phenotype $(-1.02,95 \% \mathrm{CI}$ $-0.18:-1.86 ; \quad p=0.0335)$. Visual acuity also worsened significantly over time $(-0.03,95 \%$ CI $-0.06:-0.007$; $\mathrm{p}=0.01)$. Corneal clouding was strongly negatively correlated with visual acuity $(\rho-0.60, p=7.12 \mathrm{e}-11)$.

Conclusions: After initial stabilization, ongoing ocular disease is seen in MPS-1 patients despite successful HCT. This hallmarks the shortcomings of current standard therapies. New therapies that overcome the weak spots of current therapies are necessary to improve the late outcomes of these patients.

Clinical Trial Registry: N.A.

Disclosure: B.T.A.v.d.B. was supported by a research grant from the Sylvia Toth Charity Foundation, the Hague, the Netherlands, while working on this study. The sponsors of this study are public or nonprofit organizations that support science in general. They had no role in gathering, analyzing, or interpreting the data. All authors would like to thank all parents and patients for participating in this study.

All authors state they have no competitive (financial) interests in this study.

\section{P319}

Haploidentical hematopoietic stem cell transplantation compared to matched-sibling donor transplantation for paroxysmal nocturnal hemoglobinuria

Limin Liu ${ }^{I}$, Huifen Zhou ${ }^{I}$, Qingyuan Wang ${ }^{I}$, Huiying Qiu $^{I}$, Xiaowen Tang ${ }^{1}$, Yue Han ${ }^{1}$, Chengcheng $\mathrm{Fu}^{1}$, Zhengming Jin ${ }^{I}$, Suning Chen ${ }^{I}$, Aining Sun ${ }^{I}$, Miao Miaol, Depei $\mathbf{W u}^{1}$

${ }^{1}$ First Affiliated Hospital of Soochow University, Suzhou, China

Background: Paroxysmal nocturnal hemoglobinuria (PNH) is an acquired clonal disorder of the hemopoietic stem cells for which the only curative treatment is allogeneic hematopoietic stem cell transplantation. Haploidentical donor hematopoietic stem cell transplantation (haplo-HSCT) is now increasingly applied as a curative therapy for patients with hematologic diseases. However, there are still few reports on the use of haplo-HSCT for the treatment of PNH. Our study aimed to compare the outcomes of haplo-HSCT with matched-sibling donor transplantation (MSD-HSCT) for PNH.

Methods: The clinical data of $40 \mathrm{PNH}$ patients received HSCT (haplo-HSCT $=25$, MSD-HSCT $=15$ ) in our center from July 2007 to May 2018 were analyzed retrospectively to compare the outcomes of haplo-HSCT group with MSDHSCT group. The clinical data including 23 male patients and 17 female patients, 13 classical PNH and 27 PNH-AA syndrome, the median age was 29 years (range 6-54). All 
patients had received various treatments before transplantation such as steroids, androgens, cyclosporine (CsA), antithymocyte globulin, and growth factors. The median interval from PNH diagnosis to SCT was 6 months (range 3-240). The conditioning regimen was modified BuCybased regimen in haplo-HSCT group, CsA, mycophenolate mofetil (MMF) and short-term methotrexate (MTX) were administered for graft-versus host disease (GVHD) prophylaxis. Patients with MSD-HSCT were treated with the FluCy-based regimen and CsA were administered for GVHD prophylaxis.

Results: There were no differences of gender, age, patients of PNH-AA and median time from diagnosis to transplantation between the 2 groups (På 0.05). The median values of absolute nucleated cell counts were 10.74 (4.80$22.86) \times 10^{8} / \mathrm{kg}$ in the haplo-HSCT group and $12.19(5.14$ $17.25) \times 10^{8} / \mathrm{kg}$ in the MSD-HSCT group $(\mathrm{P}=0.866)$. The median doses of $\mathrm{CD} 34^{+}$cells infused were 3.57 (0.68-7.80) $\times 10^{6} / \mathrm{kg}$ and $4.00(3.02-8.42) \times 10^{6} / \mathrm{kg} \quad(\mathrm{P}=0.151)$, respectively. All patients attained complete engraftment, no patient occurred graft failure. The median time for myeloid engraftment were 12 (range, 9-26) days in the haplo-HSCT group and 11 (range, 7-15) days in the MSDHSCT group $(P=0.065)$. The median time for platelet engraftment were 19 (range, 11-75) days and 13 (range, 11$25)$ days $(P=0.027)$, respectively. With a median followup of 26 (4-65) months in the haplo-HSCT group and 36 (4132) months in the MSD-HSCT group $(\mathrm{P}=0.294)$. In haplo-HSCT and MSD-HSCT groups the incidences of grade I-IV acute graft-versus-host disease (aGVHD) were $32.00 \%$ and $20.00 \%(\mathrm{P}=0.343)$, grade II-IV aGVHD were $16.00 \% 、 13.33 \% \quad(\mathrm{P}=0.759)$. chronic GVHD were $30.69 \%$ and $24.62 \%(\mathrm{P}=0.418)$, moderate-severe chronic GVHD were $12.73 \%$ and $7.14 \%(\mathrm{P}=0.522)$. In haploHSCT and MSD groups the incidences of infection were $32.00 \%(8 / 25)$ and $26.67 \%(4 / 15)(\mathrm{P}=1.000)$. No patient occurred early death and relapse. 3-year estimated overall survival (OS) of haplo-HSCT and MSD-HSCT groups were $86.5 \% \pm 7.3 \%$ and $93.3 \% \pm 6.4 \%(\mathrm{P}=0.520)$, GVHD-free and failure-free survival (GFFS) were $78.3 \% \pm 8.6 \%$ and $92.9 \% \pm 6.9 \%(\mathrm{P}=0.250)$.

Conclusions: The preliminary results indicated that haplo-HSCT is a feasible choice for PNH with favorable outcomes, haplo-HSCT and MSD-HSCT had similar therapeutic efficacy.

Disclosure: no disclosure

\section{P320}

Pres in BMT for thalassemia major in India: Lower incidence and limited impact

Rajat Kumar Agarwal', Priya Marwah ${ }^{2}$, Rajpreet Soni ${ }^{2}$, Stalin Ramprakash ${ }^{3}$, C P Raghuram ${ }^{3}$, Deepa Trivedi ${ }^{4}$,
Amit Sedai ${ }^{1}$, Kumari Ankita ${ }^{1}$, Lalith Parmar ${ }^{1}$, Rakesh Dhanya $^{1}$, Lawrence Faulkner ${ }^{1,5}$

${ }^{1}$ Sankalp India Foundation, Bangalore, India, ${ }^{2}$ South East Asia Institute for Thalassemia, Jaipur, India, ${ }^{3}$ People Tree Hospitals, Bangalore, India, ${ }^{4}$ Care Institute of Medical Sciences, Ahmedabad, India, ${ }^{5}$ Cure2Children Foundation, Florence, Italy

Background: Posterior reversible encephalopathy syndrome (PRES) is a relatively common complication seen after blood or marrow transplantation (BMT) for hemoglobinopathies with a reported frequency of $10-19 \%$. PRES has also been associated with poorer survival rates. Severe hemoglobinopathies are one of the most frequent indications for BMT in the developing world, particularly in India. Given the risk of rejection in multiply transfused patients and the need to minimize GVHD risk, immunosuppression post-BMT for these non-malignant conditions can be particularly intense and prolonged. We sought to measure the incidence and impact of PRES in developing countries.

Methods: We analysed 194 successive transplants for thalassemia using Protocol 1 (ATG-BuCy+CSA/MMF or CSA/MTX) maintaining cyclosporine A (CSA) blood levels $100-150 \mathrm{ng} / \mathrm{mL}$ for 74 patients and Protocol 2 (FLU-ATG$\mathrm{BuCy}+\mathrm{CSA} / \mathrm{MTX})$ maintaining higher CSA levels post, i.e. $150-250 \mathrm{ng} / \mathrm{mL}$ for 120 patients from fully matched donors with G-CSF-primed bone marrow. For 3 patients this was the second transplant from a different matched related donor. PRES was confirmed with brain CT/MRI for all patients.

Results: All recipients who had PRES had sibling donors, 5 males and 2 females. Age median 7.4 (IQR 5.6-8 years). The frequency of PRES was 3.6\%; disease free survival for patients who had PRES was 100\%. PRES resolved completely in all. CSA was switched to MMF in 5 patients who had received MTX and were on CSA only at the time of PRES occurrence, while CSA was stopped but MMF continued in 1 patients taking CSA/MMF combination and CSA was continued for 1 patient.

Three patients with PRES had grade 2 acute GVHD, 1 had grade 1 GVHD and none developed chronic GVHD. CSA levels at the time of PRES were a median of $133 \mathrm{ng} / \mathrm{mL}$ (IQR: 89 to194) with 1 patient having $419 \mathrm{ng} / \mathrm{mL}$. Three patients had PRES while they were thrombocytopenic. Hypertension stage 2 was observed in four patients, stage 1 in one patient, one patient was not hypertensive and in one patient blood pressure values were not available. Two patients were on methylprednisolone 1 and $1.3 \mathrm{mg} / \mathrm{kg} / \mathrm{day}$ and one was on dexamethasone $10 \mathrm{mg} / \mathrm{m} 2 /$ day. One patient was started on CSA again after the PRES episode and within 2 weeks had another one while on CSA (level $30 \mathrm{ng}(\mathrm{mL}$ ), methylprednisolone $1.5 \mathrm{mg} / \mathrm{kg} / \mathrm{day}$ and Ruxolitinib for GVHD. 
Protocol 2 had statistically significant improvement in disease free survival from $67 \%$ to $91 \%(\mathrm{p}<0.001)$ with probability of occurrence of PRES increasing from $1.4 \%$ to $5.0 \%(\mathrm{P}=0.17$, see Figure 1$)$, yet had a benign course in all patients.

Conclusions: Not stopping immunosuppression may have been the key factor which could explain why we have better outcomes with PRES than what is reported. Intensifying immunosuppression pre-BMT did lead to more PRES, albeit not significantly, and yet it was quite manageable. Even with addition of fludarabine our PRES incidence is lower than previously reported.

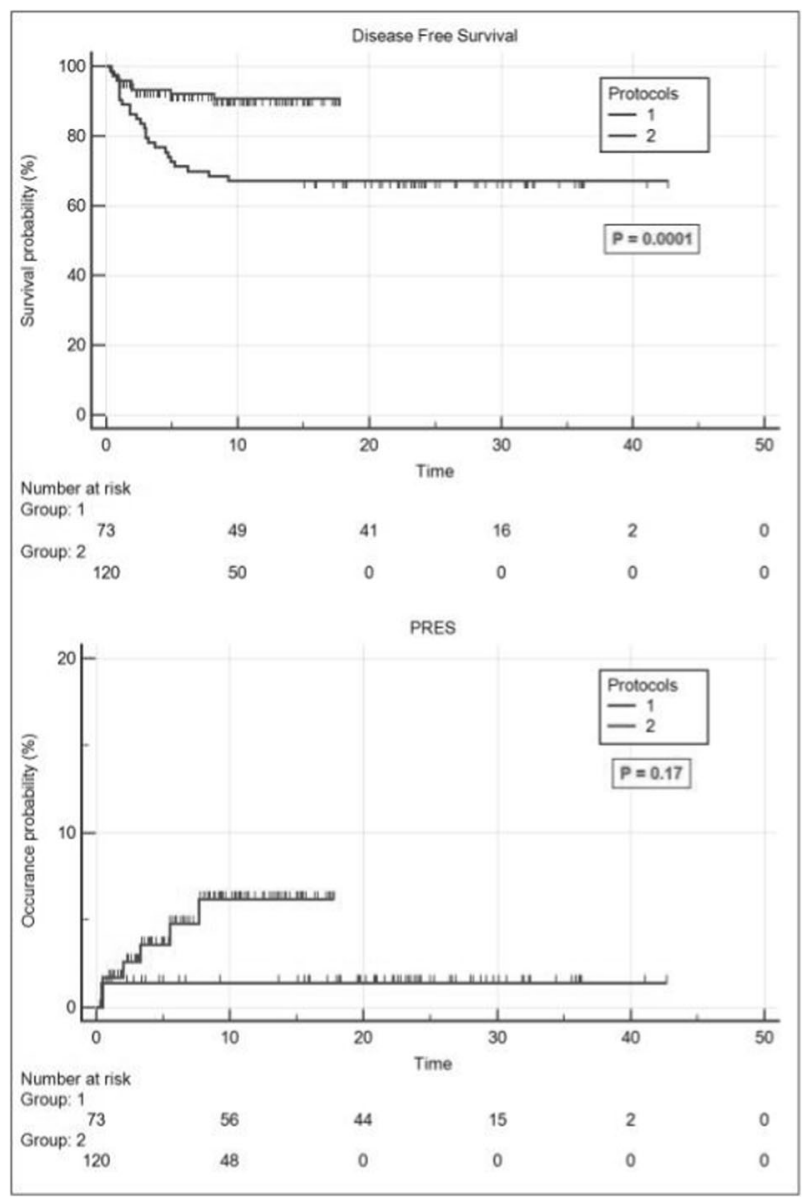

[[P320 Image] 1. Figure 1: Comparing Protocol 1 and 2 for Disease free survival and occurrence of PRES]

Clinical Trial Registry: N/A

Disclosure: None

\section{P321}

Improvement of white matter integrity and structural connectivity in the brain of patients with sickle cell disease after allogeneic hematopoietic stem cell transplantation

Thalita Cristina de Mello Costa ${ }^{1}$, Rodolfo ChiariCorreia ${ }^{1}$, Carlos Ernesto Garrido Salmon ${ }^{1}$, Luiz Guilherme Darrigo Junior ${ }^{1}$, Renato Cunha ${ }^{1}$, Carlos Eduardo Setanni Grecco ${ }^{I}$, Fabiano Pieroni', Joana Teresa Bisinella de Faria ${ }^{1}$, Ana Beatriz Pereira Lima Stracieri $^{1}$, Juliana Bernardes Elias ${ }^{1}$, Daniela Moraes ${ }^{1}$, Maria Carolina Oliveira ${ }^{1}$, Antonio Carlos Santos ${ }^{1}$, Belinda Pinto Simões ${ }^{1}$

${ }^{1}$ University of São Paulo, Ribeirão Preto, Brazil

Background: Sickle-cell diseases (SCD) are a group of genetic hemoglobin disorders marked by brain vasculopathy. Allogeneic hematopoietic stem cell transplantation (HSCT) is a curative option able to stop vascular disease progression. Diffusion-tensor imaging (DTI) is a magnetic resonance imaging (MRI) technique sensitive to the Brownian motion of water molecules and cellular environment. This microscopic quantitative technique is able to detect white matter (WM) alterations before a conventional MRI. The aim of this study was to use DTI to evaluate axonal damage and structural connectivity in the brain of patients with SCD submitted to HLA-identical sibling allogeneic HSCT.

Methods: Sixteen SCD patients with no extensive vasculopathy detected by conventional MRI (11 male, age range: 9 - 33 years) and 17 age-matched healthy controls (10 male, age range: 6 - 31 years) participated in this prospective study. MRI acquisitions were performed in a $3 \mathrm{~T}$ scanner two times for patients (before and 1-5 years after HSCT) and at a single moment for controls. From DTI acquisitions, fractional anisotropy (FA), mean (MD), radial (RD) and axial diffusibility (AD) were calculated in the WM of the whole brain. Structural connectivity was also analyzed, based on graph theory, obtaining efficiency, length path and clustering coefficients of the brain network. An ANOVA test was applied to analyze FA differences among controls and patients, before and after HSCT. A paired two-tailed t-test was used to determine statistical significance of changes in the FA, diffusivity mean values and network parameters before and after HSCT.

Results: Mean FA was lower in patients before HSCT than controls $(\mathrm{p}=0,038)$ and increased after HSCT being not statistically different when compared to controls (controls $=0,3504$; patients before HSCT $=0,3328$; patients after HSCT $=0,3422$; Post hoc Dunnett's test Error 0,03; ANOVA test). When patients were compared before and after HSCT, MD and RD decrease after HSCT (p $=0,038$ and 0,047 , respectively). On the other hand, FA increased $(\mathrm{p}=0,044)$. After HSCT, efficiency was higher 
$(\mathrm{p}=0,023)$ and path length index was lower $(\mathrm{p}=0,027)$ than at study entry (table 1).

Conclusions: This study indicates that, before HSCT, patients with SCD present axonal damage not detectable by conventional MRI, when compared to healthy controls. We also suggest that HSCT is able to promote axonal recovery and reorganization. Partial diffusivity recovery could be associate to a still unidentified mechanism of myelin regeneration. In the future, longer follow up and comparisons with other forms of treatment are required.

\begin{tabular}{lllll}
\hline Parameter & $\begin{array}{l}\text { Patients } \\
\text { Before HSCT }\end{array}$ & $\begin{array}{l}\text { Patients } \\
\text { After HSCT }\end{array}$ & $\begin{array}{l}t \\
\text { Value }\end{array}$ \\
\hline FA & 0,3328 & 0,3422 & $-2,20$ & $0,044^{*}$ \\
MD & 0,8180 & 0,7976 & 2,28 & $0,038^{*}$ \\
RD & 0,6784 & 0,6558 & 2,17 & $0,047^{*}$ \\
AD & 1,1057 & 1,0918 & 1,77 & 0,098 \\
Length Path & 0,8366 & 0,8334 & 2,45 & $0,027^{*}$ \\
Efficiency & 0,2578 & 0,2591 & $-2,52$ & $0,023^{*}$ \\
Clustering & 0,2831 & 0,2831 & 0,01 & 0,99 \\
\hline
\end{tabular}

[[P321 Table] 1. DTI and Structural Connectivity Parameters (Paired-T test)]

Disclosure: Nothing to declare

\section{P322}

Degree of matching determines disease-free survival and alloreactive complications in unrelated blood and marrow transplantation for haemoglobinopathies

\section{Angharad Pryce ${ }^{I}$, Farah O'Boyle ${ }^{I}$, Kirstin Lund ${ }^{I}$, Toni Petterson $^{1}$, Sandrine Bremathas ${ }^{1}$, Sandra Loaiza ${ }^{1}$, Richard Szydlo $^{I}$, Leena Karnik ${ }^{1}$, Josu de la Fuente ${ }^{I}$ \\ ${ }^{1}$ Imperial College London, London, United Kingdom}

Background: BMT is a well-established treatment modality for haemoglobinopathies, limited by the availability of related donors. Unrelated transplantation has historically shown variable outcomes driven by GvHD and toxicity, and usually restricted to 10/10 matches, but the impact of reduced toxicity conditioning regimens is yet to be known.

Methods: From 2011 to 2018 twenty-five consecutive unrelated bone marrow transplants were conditioned with fludarabine $160 \mathrm{mg} / \mathrm{m}^{2}$, treosulfan $42 \mathrm{~g} / \mathrm{m}^{2}$, thiotepa $10 \mathrm{mg} /$ $\mathrm{kg}$ and ATG (Thymoglobulin) $11.25 \mathrm{mg} / \mathrm{kg}$ if the source of stem cells was marrow $(n=21)$ or PTCy if PBSC $(n=4)$. Endogenous haemopoiesis was suppressed pretransplantation for a minimum of 8 weeks. GvHD prophylaxis was provided with ciclosporin/sirolimus and MMF. Thirteen patients were transplanted for $b$ thalassaemia major, one of a thalassaemia major and 11 sickle cell disease. The median age was 8 years $(2$ 19). Ten patients were $10 / 10$ matched (7 thalassemia and 3 sickle) and 15 patients had a 9/10 match (7 thalassaemia and 8 sickle). The median cell dose was $3.88 \times 10^{8} \mathrm{TNC} / \mathrm{kg}$ (range 1.38 - 13.3) and $5.22 \times 10^{6} \mathrm{CD} 34+/ \mathrm{kg}$ (range 1.10 27.41). The median survival was 13.4 months $(0.7-68.3)$. Patients with thalassaemia were Pesaro class I or II (Pesaro class III patients were intensively chelated pretransplantation to return to class I or II). Patients with sickle cell disease were transplanted for stroke or recurrent vaso-oclusive crises and/or acute chest syndrome not responding to hydroxycarbamide.

Results: All patients engrafted and achieved evidence of donor haemopoiesis on day +28 and achieved transfusionindependence and donor haematological values, but subsequently one $9 / 10$ patient with thalassaemia suffered secondary graft failure on day +75 after macrophage activation syndrome. Median neutrophil engraftment was 13 days (range 9 to 19) and 12 days $(9-22)$ for $10 / 10$ and $9 /$ 10 patients respectively. Patient with sickle cell disease had the platelet count maintained $>50 \times 10^{9} / \mathrm{L}$ at all times. The median platelet engraftment $>50 \times 10^{9} / \mathrm{L}$ was 31 days (range 21 to 53) and 40 days (range 15 to 86 ) $10 / 10$ and $9 / 10$ patients respectively.

There were three deaths, all in the 9/10 matched group: two with thalassaemia (day +257 due to idiopathic pneumonia syndrome and day +102 due to MAS) and one with SCD (day +43 due to IPS). There were different trends of complications seen by degree of matching that did not segregate otherwise by disease.

Conclusions: In conclusion, unrelated BMT for haemoglobinopathies with reduced toxicity regimens is feasible. Whilst GvHD caused significant morbidity during the transplant period, other alloreactive/endothelial complications (VOD, macrophage activation syndrome, idiopathic pneumonia syndrome) were only seen in the $9 / 10$ transplants. Disease-free survival, dependent on transplantrelated mortality, and lack of long-term toxicity, including chronic GvHD, are determined by the degree of matching. $10 / 10$ matched transplants have excellent long-term outcomes with no chronic GvHD >18 months and can be considered for patients without a related donor; whereas 9/ 10 transplant have significant toxicity and mortality, warranting a haploidentical approach.

Disclosure: No conflict.

\section{P323}

Long-term safety and efficacy of lentiglobin gene therapy in patients with transfusion-dependent $\beta$-thalassemia following completion of the phase 1/2 northstar study 
Suradej Hongeng ${ }^{1}$, Alexis Thompson ${ }^{2,3}$, Janet Kwiatkowski $^{4,5}$, John Rasko ${ }^{6,7,8}$, Gary Schiller ${ }^{9}$, Elliott Vichinsky $^{10}$, Morris Kletzel ${ }^{2,3}$, P. Joy Ho ${ }^{6,7}$, Usanarat Anurathapan ${ }^{1}$, Manfred Schmidt ${ }^{11}$, Marina Cavazzana $^{12,13,14}$, Philippe Leboulch ${ }^{1,15,16}$, Briana Deary $^{17}$, Ying Chen ${ }^{17}$, Mohammed Asmal ${ }^{17}$, Mark Walters $^{10}$

${ }^{1}$ Mahidol University, Ramathibodi Hospital, Bangkok, Thailand, ${ }^{2}$ Ann and Robert H. Lurie Children's Hospital, Chicago, IL, United States, ${ }^{3}$ Northwestern University, Chicago, IL, United States, ${ }^{4}$ Children's Hospital of Philadelphia, Philadelphia, PA, United States, ${ }^{5}$ Perelman School of Medicine University of Pennsylvania, Philadelphia, PA, United States, ${ }^{6}$ Royal Prince Alfred Hospital, Camperdown, Australia, ${ }^{7}$ University of Sydney, Camperdown, Australia, ${ }^{8}$ Centenary Institute, Gene and Stem Cell Therapy Program, Camperdown, Australia, ${ }^{9}$ David Geffen School of Medicine at UCLA, Los Angeles, CA, United States, ${ }^{10}$ UCSF Benioff Children's Hospital, Oakland, CA, United States, ${ }^{11}$ GeneWerk GmbH, Heidelberg, Germany, ${ }^{12}$ Hospital Necker - Enfants Malades, Paris, France, ${ }^{13}$ IMAGINE Institute, Université Paris Descartes, Sorbonne Paris Cité, Paris, France, ${ }^{14}$ Biotherapy Clinical Investigation Center, Groupe Hospitalier Universitaire Ouest, Paris, France, ${ }^{15}$ Brigham \& Women's Hospital and Harvard Medical School, Boston, MA, United States, ${ }^{16}$ Institute of Emerging Diseases and Innovative Therapies (iMETI), CEA, INSERM, Université Paris-Sud, Paris, France, ${ }^{17}$ bluebird bio, Inc., Cambridge, MA, United States

Background: Patients with transfusion-dependent $\beta$-thalassemia (TDT) may benefit from gene therapy involving $\beta$-globin gene addition to hematopoietic stem cells (HSCs) enabling production of functional hemoglobin ( $\mathrm{Hb}$ ). LentiGlobin gene therapy contains autologous CD34+ HSCs transduced ex vivo with the BB305 lentiviral vector encoding $\beta$-globin with a T87Q substitution under transcriptional control of the encoding $\beta$-globin locus control region. The safety and efficacy of LentiGlobin was evaluated in adults and adolescents with TDT in the 2-year phase 1/2 Northstar study (HGB-204; NCT01745120).

Methods: Patients with TDT $(\geq 100 \mathrm{~mL} / \mathrm{kg} / \mathrm{year}$ of red blood cells [RBCs] or $\geq 8 \mathrm{RBC}$ transfusions/year) received G-CSF and plerixafor for HSC mobilization. To generate drug product (DP), CD34+ HSCs were transduced with the BB305 lentiviral vector. Patients underwent single-agent, myeloablative busulfan conditioning, were infused with the DP, and were followed for safety and efficacy.

Results: Eighteen patients have been treated in the completed Northstar study. As of 14 September 2018, patients had a median follow-up of 38.9 (min - max: 29.3 48.1) months. The median age at consent was 20 (min - max: 12 - 35) years including 15 patients $\geq 18$ years old. Patients received a median cell dose of 8.1 (min - max: 5.2 18.1) CD34+ cells $\times 10^{6} / \mathrm{kg}$ with a median DP vector copy number $(\mathrm{VCN})$ of 0.7 (min - max: 0.3 - 1.5) vector copies/ diploid genome. The median liver iron content (LIC) at baseline was 5.7 ( $\min$ - max: 0.4 - 26.4) $\mathrm{mg} \mathrm{Fe} / \mathrm{g} \mathrm{dw}$. Outcomes by age and baseline iron status will be presented.

The median time to neutrophil and platelet engraftment was 18.5 (min - max: 14 - 30) and 39.5 (min - max: 19 191) days, respectively. Four patients had platelet engraftment $\geq$ Day 60 and four patients had platelet counts of $\leq$ $100 \times 10^{9} / \mathrm{L}$ at Month 12 . None of these patients had $\geq$ grade 3 bleeding events post-LentiGlobin infusion.

Transfusion independence (TI, defined as weighted average $\mathrm{Hb} \geq 9 \mathrm{~g} / \mathrm{dL}$ without $\mathrm{RBC}$ transfusions for $\geq$ 12 months) was achieved in $8 / 10$ patients with non- $\beta^{0} / \beta^{0}$ genotypes and $3 / 8$ patients with $\beta^{0} / \beta^{0}$ genotypes. In patients who achieved TI, total $\mathrm{Hb}$ at last visit was $9.1-14.1 \mathrm{~g} / \mathrm{dL}$. LIC increased from baseline in patients who achieved TI by a median of $55.6 \%$ and $12.5 \%$ at Month 12 and 24 then decreased from baseline by a median of $9.2 \%$ and $44.4 \%$ at Month 36 and 48, respectively.

Non-hematologic grade $\geq 3$ adverse events post-infusion in $\geq 3$ patients included stomatitis, febrile neutropenia, pharyngeal inflammation, and irregular menstruation. There was no transplant-related mortality, vector-mediated replication competent lentivirus, or clonal dominance. Two patients experienced grade 3 serious veno-occlusive liver disease (Table 1). Events resolved following treatment with defibrotide and were attributed to myeloablative conditioning.

Conclusions: In the Northstar study, $80 \%$ of patients with TDT and non $-\beta^{0} / \beta^{0}$ genotypes and $38 \%$ of patients with $\beta^{0} /$ $\beta^{0}$ genotypes achieved transfusion independence. The safety profile of LentiGlobin remains consistent with myeloablative busulfan conditioning. Longer time to platelet engraftment was observed in some patients, but no graft failure was reported.

\section{Clinical Trial Registry: ClinicalTrials.gov NCT01745120}

Disclosure: AT: funding: Amgen, Baxalta/Shire, bluebird bio, Celgene, Novartis, Biomarin, La Jolla Pharmaceutical; consultant: bluebird bio. JK: consultant and research funding: bluebird bio, Agios Pharmaceuticals; research funding: Novartis, Apopharma, Terumo. JR: consultant: Cynata, Spark, Novartis, Rarecyte, and Imago Biosciences; honoraria: Gilead, GSK, Takeda, Cynata, Pfizer, Spark, Novartis, Celgene, bluebird bio; speaker's bureau: Abbvie; investigator for clinical trials sponsored by bluebird bio; Director of Pathology at Genea; board of directors: Cure the Future Foundation and International Society for Cellular Therapy; scientific patron: FSHD Global Research Foundation; Chair: Gene Technology 
Technical Advisory for Australia. GS: research funding: Astellas Pharma and bluebird bio; advisory board: Astellas Pharma. EV: honoraria, research funding, consultant, board member: Global Blood Therapeutics; board member: bluebird bio; research funding: Protagonist. PJH: board member and honoraria: Amgen, Janssen, Novartis; honoraria: Takeda; travel funds: Takeda and Celgene. MS: employee: GeneWerk GmbH and German Cancer Research Center; consultant: bluebird bio. PL: consultant, board member, equity ownership, patents, and royalties: bluebird bio. BD, MA: employees of and hold stock in bluebird bio. YC: consultant: bluebird bio. MW: consultant: bluebird bio, bioverativ, Trucode, Editas, Sangamo Therapeutics; medical director: ViaCord Processing Lab and AllCells, Inc. SH, MC, MK, and UA: nothing to declare.

\section{P324}

Treosulfan based preparative regimen does not improve transplant outcomes in patients with sickle cell disease

Bushra Al-Abri ${ }^{1}$,Murtadha Al-Khabori ${ }^{2}$, Mohammed AlHuneini $^{2}$, David Dennison ${ }^{2}$, Abdulhakim Al-Rawas ${ }^{2}$, Khalil Al-Farsi ${ }^{2}$, Yasser Wali ${ }^{2}$, Salam Al-Kindi ${ }^{2}$

${ }^{1}$ Oman Medical Specialty Board, Muscat, Oman, ${ }^{2}$ Sultan Qaboos University Hospital, Muscat, Oman,

Background: Sickle Cell Disease (SCD) is an inherited hemoglobin disorder associated with high morbidity and mortality. Currently, allogeneic Hematopoietic Stem Cell Transplantation (HSCT) is the only curative therapy for SCD. Transplant outcomes with Thiotepa, Treosulfan and Fludarabine (TTF) preparative regimen are encouraging but this regimen has not been directly compared to other preparative regimens in SCD. We therefore planned to compare the event free probability for death, rejection and high grade acute Graft versus Host Disease (aGvHD) between TTF and Busulfan and Fludarabine (BF) regimens.

Methods: In this retrospectively cohort study, we included all patients with SCD who received allogeneic HSCT at our center or who were transplanted in other centers and referred to ours for follow up before day 100 . Patients were transplanted between July 2007 and December 2017. We used Kaplan-Meier curve to estimate the event free probability for death, rejection and high grade aGvHD (Grades 3-4). Cox regression was used to assess the impact of the preparative regimen on these outcomes.

Results: A total of 61 patients were included with a median age of 20 years (Interquartile Range [IQR]: 14-26) and a median hemoglobin of $10 \mathrm{~g} / \mathrm{dL}$ (IQR: 9-10). Sixtytwo percent were males. The proportion of patients who had splenectomy, stroke and acute chest syndrome was $34 \%$, $23 \%$ and $57 \%$ respectively. All patients received peripherally collected hematopoietic stem cells from a matched sibling donor with a median stem cell dose of $6 \mathrm{x}$ $10^{6} / \mathrm{Kg}$ (IQR: 5.1-8.8). Most patients, 95\%, received cyclosporine or tacrolimus based aGvHD prophylaxis. Most patients received $\mathrm{TTF}(41 \%)$ or $\mathrm{BF}(53 \%)$ preparative regimens. All patients in the BF group received ATG. The median follow-up time was 44 months (Range: 2 -127). Four patients died during the follow-up period with an OS of 93\% (95\% Confidence Interval [CI]: 87\%-100\%) at 5 years. The OS was not different (HR 1.3, p = 0.82) between the TTF $(91 \%)$ and the BF (94\%) regimens. The probability of high grade aGvHD free survival at day 100 was $91 \%$ (95\% CI: 84-99) for all patients. This probability was $85 \%$ in the TTF group and $93 \%$ in the BF group and the difference was not statistically significant (HR 2.2, $\mathrm{p}=0.39$ ). The rejection free survival at 12 months was $93 \%$ (95\% CI: 87-100) for all patients. No patients in the TTF group rejected while the rejection free survival at 12 months for the $\mathrm{BF}$ group was $90 \%$. This was not statistically significant $(\mathrm{p}=0.07)$.

Conclusions: In patients with SCD undergoing allogeneic HSCT from a matched sibling donor, the TTF preparative regimen is not associated with improved OS, rejection free or high grade free aGvHD survival when compared to the BF preparative regimen. Larger studies are needed to confirm these findings.

Disclosure: Nothing to declare.

\section{P325}

Novel strategy for haploidentical hematopoietic stem cell transplant in sickle cell disease

Gaurav Kharya ${ }^{1}$, Pratibha Dhiman ${ }^{1}$, Vikrant Bhar ${ }^{1}$, Anil Khetrapal $^{1}$, Urvashi Kotwal, Vidhi Gupta ${ }^{1}$, Atish Bakane $^{I}$

${ }^{1}$ Artemis Hospital, Gurugram, India,

Background: Sickle cell disease (SCD) remains associated with high risks of morbidity and early death. Even best of supportive care fails to improve quality of life considerably. Hematopoietic stem cell transplant is the only curative option for selected group of patients. Donor availability is always a challenge with $<20 \%$ patients having suitable HLA matched family donor and alternative donor transplant are limited with challenges of graft failure or graft versus host disease. We share our experience of using a novel strategy for haploidentical donor transplant (HHSCT) in SCD.

Methods: 9 consecutive patients suffering from SCD who underwent HHSCT between Jan 2018 till date were enrolled in the study. All 9 underwent autologous backup (target dose $>5 \times 10^{6} / \mathrm{kg}$ ) followed by pre-transplant immune suppression (PTIS) 2 cycles at 3 weekly intervals using 
fludarabine @30mg/m2/day(D1-D5) + cyclophosphamide@1000mg/m2/day(D1) + dexamethasone@20mg/m2/ day(D1-D5) along with hypertransfusion (target $\mathrm{Hb}$ 11$13 \mathrm{gm} / \mathrm{dl})$, hydroxyurea $(20 \mathrm{mg} / \mathrm{kg} / \mathrm{day})$ and azathioprine $(2 \mathrm{mg} / \mathrm{kg} /$ day) from day -60 . The graft was mobilized using GCSF@10mcg/kg/day(D1-D5) + Plerixafor@0.24mg/kg s/ c on D5 6-8 hours before the PBSCH. Conditioning included Thiotepa $10 \mathrm{mg} / \mathrm{kg}$ in two divided doses (D-7), fludarabine $30 \mathrm{mg} / \mathrm{m} 2$ (D-6 to D-2), cyclophosphamide 14.5 $\mathrm{mg} / \mathrm{kg}$ (D-5, D-4), TBI 2Gy with thymic shielding (D-1), rATG (Genzyme Thymoglobulin $1.5 \mathrm{mg} / \mathrm{kg}$ (D-9 to D-7). GVHD prophylaxis included PTCy $50 \mathrm{mg} / \mathrm{kg} /$ day on D3 and 4, sirolimus (target levels 10-15ng/ml) (till 9-12 months post HSCT) and MMF (till D35) starting from D5.

Results: The median age of patient's was 7 years (range 3-22 years). Before transplantation all patients had repeated episodes of one or other complication warranting a transplant, non-responsive to hydroxyurea. Six had maternal donors, 2 paternal and 1 sibling. Median age of the donor was 41 years (range 19-51 years). All were DSA negative with a cutoff MFI of $>2000$ IU. All patients received $10 \times 10^{6} / \mathrm{kg}$ CD34 cells irrespective of harvested dose which ranged from $\left(10.13-34.82 \times 10^{6} / \mathrm{kg}\right)$. Median CD3 dose was $16.59 \times 10^{7} / \mathrm{kg}$ (range $10.44-41.9 \times 10^{7} / \mathrm{kg}$ ). All patients engrafted with median time to neutrophil engraftment 13 days (range 12-15 days) and median time to platelet engraftment 13 days (range 11-16 days). Median duration of hospital stay was 30 days (range 24-35 days). One patient had cytokine release syndrome needing tocilizumab. Five had engraftment syndrome treated with short course of steroids. Two had CMV reactivation needing treatment with ganciclovir/valganciclovir. Acute GVHD grade II was seen in one patient. Till date of analysis none had features compatible with chronic GVHD. Of the 9 patients, 8 are alive without sickle cell disease with Lansky/ Karnofsky scores of 100. At median follow up of 164 days (range 61-271) the probabilities of survival, SCA-free survival, and transplant-related mortality after transplant were $88.9 \%, 88.9 \%$, and $11.1 \%$, respectively. One patient died due to MDR Klebsiella sepsis after being discharged initially while he was receiving IV ganciclovir on day care basis. He had full donor chimerism. None of the patient had primary or secondary graft failure.

Conclusions: Pre-transplant immune suppression and upfront use of plerixafor for graft mobilization decreases the risk of graft failure and graft versus host disease leading to overall better survival in HHSCT for sickle cell disease.

Disclosure: None.

\section{P326}

\section{Combined haematopoietic stem cell transplant and enzyme replacement therapy in wolman disease: Outcomes and challenges}

Jane Kinsella ${ }^{1}$, Denise Bonney ${ }^{1}$, Helen Campbell ${ }^{1}$, Robert Wynn $^{1}$, Simon Jones ${ }^{1}$

${ }^{1}$ Royal Manchester Children's Hospital, Manchester, United Kingdom

Background: Infantile lysosomal acid lipase deficiency more commonly known as Wolman disease - is an autosomal recessive lysosomal storage disease, characterised by storage of cholesterol esters in the liver, spleen and gastrointestinal tract. These children present under the age of 6 months and traditionally had a poor prognosis, with almost all being dead by the age of 12 months. Bone marrow transplant has been used to correct disease manifestations, but limited by high procedure-related mortality with the significant co-morbidities. The survival has changed over the past few years due to pharmacological enzyme replacement therapy but still presents challenges for these patients and their clinicians. In these children haematopoietic stem cell transplant we have offered BMT with enzyme replacement therapy, in certain specific circumstances.

Methods: Four children with Wolman disease being treated with enzyme replacement therapy, limited by alloantibody, or poor venous access, received treosulfan-based, myeloablative conditioning with serotherapy followed by a matched haematopoietic stem cell transplant: two family donors, one sibling donor and one unrelated donor.

Results: Three of the four children survived transplant. They have continued to receive enzyme replacement therapy but at reduced dose and frequency with improved tolerability. They have continues to grow and develop. Growth and gastrointestinal histology is improved for children having received transplant compared to those receiving enzyme replacement alone. Monitoring of peripheral blood chimerism has shown a disease-associated engraftment defect, with mixed chimerism in the 3 surviving patient.

Conclusions: Haemopoietic stem cell transplant is a suitable treatment option in children with Wolman disease in whom receiving enzyme replacement therapy is not possible because of venous access, sensitisation or cost reasons. It improves their tolerability of the enzyme treatment and allows for a reduction in enzyme dose and frequency. However, the results of engraftment are not as good as expected for a transplant with myeloablative conditioning and a matched donor. An engraftment defect has been observed in lysosomal acid lipase deficient animal models. A further understanding of this poor engraftment in children with Wolman disease is required as to determine whether the risks of transplant is beneficial in these patients and for the consideration of future treatment options including gene therapy. 
Disclosure: Nothing to declare

P327

Immune reconstitution of thalassemia major patients after 1 year of hematopoetic stem cell transplantation

Funda Erol Cipe ${ }^{I}$, Ceyhun Bozkurt ${ }^{I}$, Basak Adakli Aksoy $^{I}$, Selime Aydogdu ${ }^{I}$, Gurcan Dikme ${ }^{I}$, Tunc Fisgin ${ }^{I}$

${ }^{1}$ Altinbas University, Bahcelievler Medical Park Hospital, Istanbul, Turkey

Background: Thalassemia major is the most common transfusion dependent hemolytic anemia in the world. The absent or reduced production of the $\beta$-chain of hemoglobin causes severe ineffective erythropoiesis, massive erythroid hyperplasia in the bone marrow and extramedullary hematopoesis occurs. Patients require regular transfusion therapy lifelong. Currently, the only proven curative treatment of thalassemia is allogeneic stem cell transplantation (SCT).

Methods: We evaluated the immune reconstitution results of 23 patients at 1 year after hematopoetic stem cell transplantation at our pediatric bone marrow transplantation center between January 2015 and December 2018. All patients were not receiving any immunosuppressive treatment at least for 3 months and they have normal lymphocyte counts, immunoglobulin levels and transfusion independent. Lymphocyte subtypes and chimerism percentages and the relationship with the donor type were evaluated at 1 year of transplantation.

Results: Ages of transplantation was ranged between 117 years (Median: 5 years). Seven (30\%) of them was male. Matched unrelated donor type was chosen in 7 patients while others (16 patients) were transplanted from family matched donor (Matched sibling: 9 patients, Matched family: 7 patients). All patients received myeloablative conditioning regimen containing busulfan/treosulfan, cyclophosphamide, thiotepa and fludarabine. Follow up time was between 12-47 months (Mean: $24 \pm 10$ months). In 9 patients, whole bone marrow product was used while peripheral stem cell harvest in remaining patients. CD3 levels were found low in only 4 patients, in normal patients mean was $59 \% \pm 14 \%$. CD4 levels were severely low in 18 patients while CD8 in only 1 patient. CD8 levels were increased in total 13 patients in as compensatory. CD4/CD8 ratios were very low in all patients (Range: 0.2-0.7). B cells $(\mathrm{CD} 19+)$ were low in 3 patients while immunoglobulin levels were normal. Chimerism values between 55-99\% (Mean: $93 \pm 11 \%$ ). Donor and product types did not differ in $\mathrm{CD} 3+$ lymphocyte reconstitution at 1 year $(\mathrm{p}=0.15$, $\mathrm{P}=042$ respectively). All patients were alive and well at 1 year after transplantation.
Conclusions: After 1 year of transplantation, although patients are in well condition regarding to infection frequency and transfussion dependency, it was seen that their lymphocyte subtypes reconstitution could not be achieved enough as in normal children. We can conclude that low CD4+ cell levels were an expected finding in almost all patients. So, these patients may have a tendency to suffer serious bacterial and viral infections, and close follow up be required in terms of infections as long as CD4 levels continue to be low. Immunoglobulin replacement therapy did not required even in patients with low B cell levels.

Disclosure: Nothing to declare

\section{P328}

Phase 2 international, multicentre trial to assess haploidentical AB T-cell depleted stem cell transplantation in patients with sickle cell disease with no available sibling donor

Juergen Foell, Daniel Wolff ${ }^{1}$, Anja Troeger ${ }^{I}$, Katharina Kleinschmidt ${ }^{l}$, Beatrix Pfirstinger ${ }^{1}$, Matthias Edinger ${ }^{1}$, Charalampos Aslanidis $^{1}$, Petra Hofmann ${ }^{1}$, Ernst Holler ${ }^{1}$, Norbert Ahrens', Susanne Ellinger ${ }^{1}$, René Schmidt', Wofgang Herr', Selim Corbacioglu ${ }^{1}$

${ }^{1}$ Universitätsklinikum Regensburg, Regensburg, Germany, ${ }^{2}$ University Hospital Muenster, Institut für Biometrie und Klinische Forschung, Muenster, Germany

Background: Sickle cell disease (SCD) is an inherited disorder with an estimate of 300,000 affected newborns per year worldwide. Allogeneic hematopoietic stem cell transplantation (HSCT) with a matched sibling donor (MSD) is currently the curative standard of care for SCD patients (pts). However, MSD availability is $<20 \%$. A T-cell depleted haploidentical HSCT (T-haplo-HSCT) from a relative, mostly a parent, expands the donor availability while exhibiting low GvHD rates and thus could offer cure to the remaining $80 \%$ of SCD patients. In a pilot study, comparing T-haplo-HSCT with MSD HSCT in advanced stage SCD, using almost identical transplant regimens for both. The overall (OS) and disease-free survival (DFS) was $90 \%$ vs. $100 \%$, respectively.

Methods: These results led to the design of a clinical trial to assess TCD-haplo-HSCT prospectively which aims to demonstrate that a HSCT from a haploidentical relative is not inferior to a MSD HSCT with regard to major outcome parameter. This phase 2, prospective, stratified, open-label study is targeting enrollment of 212 patients aged 1-35 years with homozygous $\mathrm{HbS}$ disease or heterozygous $\mathrm{HbSC}$ or $\mathrm{HbS} 0 /+\beta$-Thal suffering from severe or moderate SCD related complications. Inclusion criteria are clinically 
significant SCD related complications such as stroke, silent crisis, pathological angio-MRI, transcranial doppler (TCD) velocity $>200 \mathrm{~cm} / \mathrm{s}, 2$ or more episodes of acute chest syndrome (ACS) in a lifetime, chronic transfusion dependency, transfusion-refractory allo-immunization and others. Pts fulfilling inclusion criteria will be stratified according to donor availability. Pts with a MSD will receive a bone marrow graft, pts requiring an alternative donor will be transplanted with an aß/CD19 depleted graft from a haploidentical family donor. The conditioning regimen for both groups will be identical with the exception that antithymoglobulin (ATG-Neovii ) is given upfront in Thaplo-HSCT versus day -3 to -1 in MSD. Chemotherapy consists of thiotepa, fludarabine and treosulfan. Posttransplant immunosuppression will consist of mofetil mycophenolate and tacrolimus for a duration $>6$ months in T-haplo-HSCT and $<6$ months in MSD, depending on chimerism. (EudraCT number: 2018-002652-33)

Results: Primary efficacy endpoint: Event free survival (EFS). Event is defined as incidence of acute GvHD, grade III - IV, chronic GvHD, rejection (graft failure) or death (for any reason). Key secondary endpoint(s) are OS, DFS, graft failure, hematological and immunological reconstitution, quality of life (QOL) assessment and fertility. The primary null hypothesis is: EFS of SCD patients treated with THaplo-HSCT is non-relevantly inferior to EFS in the MSD arm.

Conclusions: Results will help to determine if an $a / \beta$ depleted T-haplo-HSCT can be considered equivalent to MSD HSCT with regard to DFS, adverse events and safety, in order to offer this form of cure to the majority of patients with SCD.

Disclosure: Nothing to declare

\section{P329}

\section{Hit three birds with one stone: Successful stem cell} transplantation from one family donor to three siblings

\section{Başak Adaklı Aksoy', Selime Aydoğdu', Gürcan Dikme ${ }^{I}$, Azize Mergen ${ }^{1}$, Ceyhun Bozkurt', Tunç Fışgın ${ }^{1}$}

${ }^{1}$ Istinye University, Medical Park Hospitals, Bahçelievler, Istanbul, Turkey

Background: Thalassemia major is a single gene related hereditary hemoglobinopaty required life long regular transfusions with chelation therapies. Stem cell transplantation is the only curative treatment of the disease. Three sibling thalassemia patients 13,4 and 3 years old were succesfully transplanted from their only donor,fully matched seventeen years old sister at our center.

Methods: In August 2016, three thalassemic siblings were admitted to hospital for stem cell transplantation from a full match donor, their 17 years old sister. The patients' general health conditions and specific health issues due to thalassemia were checked extensively. It was decided to perform first transplant to older sister whom the disease and transplant complications are expected more intense due to prolonged transfusion and chelation therapy. The oldest daughter of family, healthy, was planned to accompany her sisters in transplantation unit so parents can take care the others and organize this period for whole family.

Results: The 13 years old sibling was first admitted to bone marrow transplantation unit in July 2017. The conditioning regimen was busulfan, fludarabine, cyclophosphamide and thiotepa with Antithymocyteglobulin(ATG) and defibrotide prophylaxis was given. The healthy donor was admitted to hospital and received G-CSF for 5 continuous days before harvesting. Stem cells were collected peripherally on day 0 and viable CD34 + cells were $2211 / \mathrm{uL}$. Patient received 5,5 x10e6/kg stem cell and the other cell products were divided into 4 parts according to other recipients' weight. No infusion problems were recorded in stem cell transfusion. GVHD prophylaxis was given with cyclosporin and methotrexate. Severe sinusoidal obstruction syndrome was observed and successfully managed with supportive therapy. Neutrophils were engrafted +11 . day, and platelets were on day 64 . Full blood chimerism results were $\% 98$ in day 30, \%99 in day 60 and $\% 98$ in day 180 consecutively. After 3 months from first transplant the 4 years old sister was admitted to hospital on October 2017. Same conditioning with defibrotide prophylaxis and GVHD prophylaxis were given and 6,8 $\mathrm{x} 10 \mathrm{e} 6 / \mathrm{kg}$ peripherally derived and previously frost stem cell was infused without any complications. Mild sinusoidal obstruction syndrome was observed and managed with supportive therapy successfully. Neutrophils were engrafted +11 . day, and platelets were on day 16. Full blood chimerism results were $\% 99$ in day 30,\%99 in day 60 and $\% 99$ in day 180 consecutively.

The third transplant was performed on January 2018 with the same conditioning and prophylaxis regimen. Although defibrotide was used mild SOS was observed and treated with supportive therapy with success. Neutrophils were engrafted +10 . Day, and platelets were on day 27. Full blood chimerism results were $\% 99$ in day 30, \%99 in day 60 and \%99 in day 180 consecutively.

Conclusions: The patients are being followed for over a year after first transplat, neither adverse nor GVHD symptoms were observed. We presented this case for being a unique example for match family donor transplant and the first successful example from one donor to three recipients.

Disclosure: Nothing to declare 


\section{P330}

Hematopoietic stem cell transplantation in congenital dyserythropetic anemia type II: A case report

\section{Vedat Uygun ${ }^{1}$, Roberta Russo ${ }^{2}$, Gülsün Karasu', Hayriye Daloglu$^{1}$, Achille Iolascon ${ }^{2}$, Akif Yeşilipek ${ }^{1}$}

${ }^{1}$ Medical Park Antalya Hospital, Antalya Merkez, Turkey, ${ }^{2}$ University of Naples Federico II and CEINGE- Advanced Biotechnologies, Naples, Italy

Background: There is no guideline for the treatment of congenital dyserythropoietic anemia (CDA) type II patients and they are mostly being followed-up for transfusion based on individually determined target hemoglobin levels and iron chelation according to thalassemia guidelines.However the only known curative option in CDA II patients is hematopoietic stem cell transplantation (HSCT). Only few published case reports of allogeneic HSCT in CDA II patients are available.

Methods: Herein, we report a patient transplanted for CDA II who resulted with success.

Results: Our female patient admitted for anemia at $3^{\text {rd }}$ month of birth and was transfused every 4-5 months from $6^{\text {th }}$ months to 3.5 years of age. Since investigations directed towards hemoglobinopathies or membrane defects like hereditary spherocytosis were unremarkable, she was not transfused for 6 years after the age of 3.5-years because hemoglobin level was constant over $7 \mathrm{~g} / \mathrm{dl}$. Her BM examination showed erythroid hyperplasia and feature of dyserythropoiesis with a few binucleated erythroblasts. It was decided to follow-up the patient with a diagnosis of CDA II. After the age of 10-years, the need for transfusion started again for every 4 to 5 months which led the parents of our patient to request for bone marrow transplantation, however, the diagnosis was not definite, and because of the insufficient data for the transplantations for CDA II patients, it was decided to go on to follow-up. Nevertheless, after 2 years, the frequency of transfusion gradually increased to every 2-3 weeks, and bone marrow transplantation was brought into question again. At that time, genetic examination was started and $S E C 23 B$ gene was analyzed by direct sequencing. HSCT decision from her HLA 10/10 matched brother, carrying $S E C 23 B$ mutation in heterozygous state, was taken. In the preparation regimen, busulfan $(\mathrm{Bu})$ at a myeloablative weight adjusted dose (4 days), $200 \mathrm{mg} / \mathrm{kg}$ cyclophosphamide (Cy) (4 days), and $30 \mathrm{mg} / \mathrm{kg}$ antithymocyte globulin (ATG fresenius) were used. Graftversus-host disease (GVHD) prophylaxis was with cyclosporin A started on day -1 and short-term methotrexate on day $+1,+3$ and +6 . She was transplanted with $\mathrm{BM}$ with a dose of total nucleated cells $=7.1 \times 10^{8} / \mathrm{kg}$ and $\mathrm{CD} 34=3 \times 10^{6} \%$ $\mathrm{kg}$. Neutrophile and platelet engraftment were achieved at
+20 and +38 , respectively. Indeed, grade 4 hemorrhagic cystitis due to $\mathrm{BK}$ virus and a moderate veno-occlusive disease prolonged platelet transfusion days which concealed the exact engraftment day of platelet. The patient was discharged on day 45 with no more need for any transfusion and followed up as a complete chimeric with no type of GVHD since then. Now, she is 20 years old, under regular surveillance at our transplant centre without any symptoms.

Conclusions: HSCT data in CDA II patients is still insufficient, however based on data from TM patients with similar treatment approaches in TD CDA II patients, it is seen that the HSCT is reliable and effective.

Disclosure: Nothing to declare

Hematopoietic stem cells

\section{P331}

Blinatumomab and allo-HSCT - based therapies in children and adults with $\mathrm{MRD}^{+}$and $\mathrm{R} / \mathrm{R}$ B-all, experience from a single center (CIC 725)

Inna Markova', Sergey Bondarenko ${ }^{1}$, Olesya Paina ${ }^{1}$, Polina Kozhokar', Anastasia Frolova', Ildar Barkhatov ${ }^{1}$, Elena Babenko ${ }^{1}$, Alexander Alyanskii', Kirill Ekushov', Tatyana Gindina ${ }^{1}$, Elena Darskay ${ }^{1}$, Bella Aubova ${ }^{1}$, Elena Semenova ${ }^{I}$, Ivan Moiseev ${ }^{I}$, Ludmila Zubarovskaya', Boris Afanasyev ${ }^{1}$

${ }^{1}$ First State Pavlov Medical University of Saint Petersburg, Raisa Gorbacheva Memorial Research Institute for Pediatric Oncology, Hematology and Transplantation, Saint Petersburg, Russian Federation

Background: The prognosis after frontline therapy in BALL patients have improved due to monoclonal antibodies (CD20, CD19, CD22) and approximately $90 \%$ of patients achieve complete remission. In relapsed and refractory (R/ R) B-ALL and also in $\mathrm{MRD}^{+}$outcomes are relatively poor. Disease-free survival (DFS) in this cohort is 10-20\%. In this cohort allo-HSCT is indicated and complete remission before transplantation is crucial for prognosis. Conventional chemotherapy is associated with high failure rate and significant toxicity. Immunotherapy with monoclonal antibodies and CAR-T are more promising approaches. The aim was to evaluate the efficacy (frequency of responses, OS, DFS) and toxicity, especially neurotoxicity and cytokinerelease syndrome, of a bispecific monoclonal antibody blinatumomab in patients both children and adults with persistence of minimal residual disease $\left(\mathrm{MRD}^{+}\right)$or $\mathrm{R} / \mathrm{R} \mathrm{B}-$ ALL as a bridge to allo-HSCT.

Methods: This study included 120 patients with high risk B-ALL blinatumomab treated in 2013-2018, among them 14 pts $(12 \%)$ with $\mathrm{t}(9 ; 22), 10(8 \%)$ with $\mathrm{t}(4 ; 11)$, with MLL $11(9 \%), 84$ pts $(70 \%)$ who were refractory to previous 
chemotherapy, $66(30 \%)$ after allo-HSCT from deferent type of donors. Median age was 21 y.o. (range $5 \mathrm{~m}-71 \mathrm{y} . \mathrm{o})$, 55 children $0-18$ y.o. (46\%) and 65 adults $>18$ y.o. (54\%). R/R ALL had 63 pts $(52 \%), \mathrm{MRD}^{+}-57$ pts (48\%), median days of follow up were 227 (18-720). Blinatumomab was applied as 28-day cycles followed by a 14-day off-period before the start of the following cycle. Majority pts received one cycle $(\mathrm{N}=94,78 \%)$. In $\mathrm{R} / \mathrm{R}$ ALL group dose was of 9 $\mathrm{mcg} / \mathrm{d}$ during the first 7 days and afterwards $28 \mathrm{mcg} / \mathrm{d}$. Patients with weight less than $45 \mathrm{~kg}$ received $5 \mathrm{mcg} / \mathrm{m}^{2} / \mathrm{d}$ and $15 \mathrm{mkg} / \mathrm{m}^{2} / \mathrm{d}$ accordingly. In MRD group dose was 15 $\mathrm{mcg} / \mathrm{m}^{2} / \mathrm{d}$.

Results: The frequency of responses to blinatumomab was higher in $\mathrm{MRD}^{+}$pts in comparison R/R ALL pts $(85 \%$ vs $62 \% \mathrm{p}=0.007)$. In $\mathrm{MRD}^{+}$pts $\mathrm{CR} \mathrm{MRD}^{-}$was achieved in 47 pts $(82.5 \%), 10$ pts $(17.5 \%)$ were MRD+ after blinatumomab. Two-year OS in this group was $61 \%$. Twenty pts (34\%) received allo-HSCT. In RR ALL pts CR MRD $^{-}$was achieved in 30 pts (48\%), 9 pts (14\%) were MRD + after blinatumomab, 24 pts (38\%) had no hematological response . Two-year OS in R/R ALL was $43 \%$. Fifteen pts (24\%) received allo-HSCT. OS in CR $\mathrm{MRD}^{-}$patients who received allo-HSCT was not significantly different in comparison with patients who received blinatumomab as a monotherapy ( $84 \%$ vs $71 \%, \mathrm{p}=0.08)$. No significant differences in DFS were observed at two years in $\mathrm{CR} \mathrm{MRD}^{-}$pts depending status of the disease before therapy- MRD vs R/R (66\% vs 59\%).

Of the reported adverse events, febrile fever was the most common 91pts (76\%), neutropenia 43 (35\%), thrombocytopenia $46(38 \%)$, infection $32(26 \%)$, neurotoxicity 29 $(24 \%)$, cytokine-release syndrome $8(7 \%)$. All complications were reversible.

Conclusions: Blinatumomab is effective option in patients with high risk B-ALL especially in the group with MRD persistence after previous chemotherapy and facilitates effective bridging to HSCT. Blinatumomab therapy is generally well tolerated.

Disclosure: Nothing to declare

\section{P332}

single-cell RNA sequencing reveals enhanced hematopoietic specification from human embryonic stem cells in three-dimensional hydrogel

\section{Wei Cong', Wang Bingsheng ${ }^{1}$, Xu Yulin ${ }^{1}$, Shan Wei ${ }^{1}$, Li Xue $^{1}$, Li Xia ${ }^{1}$, Yu Xiaohong ${ }^{1}$, Huang He ${ }^{I}$ \\ ${ }^{1}$ Bone Marrow Transplantation Center, The First Affiliated Hospital, Zhejiang University, Hangzhou, China}

Background: Hematopoietic stem cells (HSCs) obtained from pluripotent stem cells (PSCs) are emerging as a potential source for HSCs transplantation. We have utilized induced PSCs (iPSCs) to generate transplantable hematopoietic cells within 3D hematopoietic niche comprising nanofibers and stromal cells. Here we address the transcriptional regulation of differentiated cells from human embryonic stem cells (ESCs) using self-assembling peptide hydrogel without stromal cells, and compare with embryoid body (EB) culture system.

Methods: ESC differentiation was induced in EB culture system or three-dimensional (3D) hydrogel culture system. The engraftment potential of differentiated cells was evaluated by flow cytometry. CD $34^{+}$cells from mobilized peripheral mononuclear blood cells (mPBMCs) or differentiated from ESCs at different times (day 7, day 10, day 14) were purified by fluorescent-activated cell sorting. Sorted cells were captured on medium-sized microfluidic chips using the Fluidigm C1 Single Cell Auto Prep System. Sequencing was performed by HiSeq X Ten.

Results: Self-assembling peptide hydrogel formed a 3D scaffold for cell culture, the pore diameter of which ranged from 50 to $200 \mathrm{~nm}$. Compared to EB culture system, ESCs in 3D culture system differentiated more potently. The differentiated cells from 3D system were short-term engrafted in the NOG mice, and myeloid cells, B cells and T cells could all be detected in peripheral blood after transplantation. However, the engraftment was not obtained in differentiated cells from EB culture system. We obtained and analyzed 301 ESCs, $554 \mathrm{CD} 34^{+}$cells from EB culture system, 440 CD34 ${ }^{+}$cells from 3D culture system, and $218 \mathrm{CD} 34^{+}$cells from mPBMCs. The cells were divided into 11 cluters (Figure 1A). In both differentiation systems, the $\mathrm{CD} 34^{+}$cells from day 7 were more heterogeneous than $\mathrm{CD}_{3}{ }^{+}$cells from day 10 and day 14 (Figure 1B). However, CD34 ${ }^{+}$cells from mPBMCs were more homogeneous, probably because the differentiated $\mathrm{CD} 34^{+}$cells contained several cell lineages, including hematopoietic cells, endothelial cells and mesenchymal cells. There is transcriptional overlap between individual $\mathrm{CD} 34^{+}$cells from $\mathrm{EB}$ and $3 \mathrm{D}$ culture systems. However, we found that cluster 3 , which is composed mainly of CD $34^{+}$cells from 3D at day 14 and day 10, expressed similar level of several hematopoietic regulator as HSC, such as TAL1, LMO2, ERG (Figure 1C). The cluster 6, which is almost the $\mathrm{CD} 34^{+}$cells from 3D at day 7, also expressed the highest GATA2 among the clusters from differentiated cells (Figure 1C).

Conclusions: Our study demonstrates that 3D hydrogel culture system facilitates hematopoietic specification of ESCs.

Disclosure: Nothing to declare

\section{P333}

Higher CD34+ cell dose increases overall survival in the setting of dual T-lymphocyte suppression with ATG and PTCY in matched related and unrelated donor alloSCT 
Maria Rhida Bautista ${ }^{1}$, Maria Queralt Salas ${ }^{1}$, Eshetu Atenafu ${ }^{1}$, Shruti Prem ${ }^{1}$, Wilson Lam ${ }^{1}$, Arjun Datt Law ${ }^{1}$, Fotios Michelis ${ }^{1}$, Santhosh Thyagu ${ }^{1}$, Dennis Dong Hwan Kim $^{I}$, Jeffrey Howard Lipton ${ }^{1}$, Auro Viswabandya ${ }^{1}$, Rajat Kumar $^{1}$

${ }^{1}$ Messner Allogeneic Transplant Program, Princess Margaret Cancer Centre, University Health Network, University of Toronto, Toronto, Canada

Background: There is no consensus on the CD34+ donor cell numbers required for optimal outcomes in allogeneic stem cell transplant (alloSCT). There is controversy on the benefits or harm in higher cell dose for alloSCT. This study aims to evaluate the impact of CD34+ cell dose in alloSCT patients receiving reduced intensity conditioning (RIC) combined with anti-thymoglobulin (ATG) and posttransplant cyclophosphamide (PTCy) using related (MRD) and 10/10 and 9/10 matched unrelated donors (MUD).

Methods: This is a single-centre retrospective analysis of 140 adult patients who received alloSCT for hematologic malignancies between October 2015 and May 2018. All received RIC using fludarabine $\left(30 \mathrm{mg} / \mathrm{m}^{2} /\right.$ day: day -5 to $-2)$, busulfan $\left(3.2 \mathrm{~kg} / \mathrm{m}^{2} /\right.$ day: day -3 and -2$)$ and total body irradiation (200 cGy: day -1). All patients also received rabbit-ATG ( $4.5 \mathrm{mg} / \mathrm{kg}$ : day -3 to -1$)$, PTCy $(50 \mathrm{mg} / \mathrm{kg} /$ day: day $+3,+4)$ and cyclosporine (from day +5 ). Unmanipulated peripheral blood stem cells were infused on day 0 .

Analyses were done using 2 thresholds:

(1) an arbitrary CD34+ cell dose of $6 \times 10^{6} / \mathrm{kg}$ (as this was our target dose) and

(2) cell dose according to quartiles $(<5.67,5.67-7.98$, $7.99-11.34$ and $\geq 11.35 \times 10^{6} / \mathrm{kg}$ ).

Results: Median CD34+ cell dose was $7.98 \times 10^{6} / \mathrm{kg}$. Median follow up was 19 months (range 5-35). Median neutrophil engraftment was 16 (range 13-31) days and platelet engraftment was 21 (range 11-83) days.

A cell dose greater than $6 \times 10^{6} / \mathrm{kg}$ was associated with an increased overall survival (OS) at 1 year $(71.3 \%$; $95 \% \mathrm{CI}$, 62.3-80.3 vs 46.7\%; 95\% CI 30.3-63.1; $p=0.018$, Figure 1). The higher dose was also associated with shorter platelet engraftment time $(p=0.016$, Figure 2$)$. There was no significant difference in neutrophil engraftment, nonrelapse mortality (NRM), relapse free survival (RFS), Grade II-IV acute graft versus host disease (aGVHD) and moderate to severe chronic graft versus host disease (cGVHD), (Table 1).

Analyses using quartile cell dose thresholds showed a trend towards decreased OS with a cell dose of < $5.67 \times 10^{\wedge} 6 / \mathrm{kg}$, however this was not statistically significant (Figure 1). Higher CD34+ cell doses were associated with shorter platelet engraftment time ( $p=0.005$, Figure 2$)$. There was no significant difference in neutrophil engraftment, NRM, RFS, aGVHD and cGVHD (Table 2).

Conclusions: CD34+ cell dose greater than $6 \times 10^{6} / \mathrm{kg}$ significantly increases overall survival in the setting of RIC and dual T-lymphocyte suppression with ATG and PTCy in MRD and MUD alloHSCT. Further studies in a larger number of patients and longer follow up are recommended to validate these findings.

Disclosure: Nothing to declare

\section{P334}

Optimal CD34 + cell dose count in the setting of dual Tlymphocyte suppression with ATG and PTCY in haploHSCT

Maria Queralt Salas ${ }^{1}$, Maria Rhida Bautista ${ }^{1}$, Eshetu G Atenafu $^{1}$, Shruti Prem ${ }^{1}$, Arjun Datt Law ${ }^{1}$, Wilson Lam ${ }^{1}$, Dennis (Dong Hwan) Kim ${ }^{1}$, Fotios V Michelis ${ }^{1}$, Santhosh Thyagu $^{1}$, Jeffrey Howard Lipton ${ }^{1}$, Auro Viswabandya ${ }^{1}$, Rajat Kumar ${ }^{1}$

${ }^{1}$ Princess Margaret Cancer Centre, Hans Messner Allogeneic BMT Program, Toronto, Canada

Background: We aimed to evaluate the impact of CD34+ cell dose using reduced intensity conditioning (RIC) regimen combined with anti-thymoglobulin (ATG) and post-transplant cyclophosphamide (PTCy) for haploidentical stem cell transplant (haploHSCT) for different indications.

Methods: Fifty two adult patients were included. Median CD34+ cells requested for infusion were $6 \times 10^{\wedge} 6 / \mathrm{Kg}$. All patients received the same RIC regimen including fludarabine $(30 \mathrm{mg} / \mathrm{m} 2 /$ day day -5 to -2$)$, busulfan $(3.2 \mathrm{~kg} / \mathrm{m} 2 /$ day day -3 and -2 ), and total body irradiation (200 cGy) (day -1) combined with rabbit-ATG (4.5 mg/kg: day -3 to -1$)$, PTCy $(50 \mathrm{mg} / \mathrm{kg} /$ day: day $+3,+4)$, and cyclosporine. Unmanipulated peripheral blood stem cells were infused. Last followup was November 2018. Median follow-up was 13 months (range 5-26).

Median cell dose count infused was $9.83 \mathrm{CD} 34+/ \mathrm{Kg}$. We arbitrarily divided the cohort in two groups with $\mathrm{CD} 34+$ dose of $>8 \times 10^{\wedge} 6 \mathrm{CD} 34 / \mathrm{Kg}$ as cut-off point.

Results: Findings are summarized in Figure1. The infusion of more than $8 \times 10^{\wedge} 6 \mathrm{CD} 34 / \mathrm{Kg}$ dose had a significant worse impact on overall survival (OS) $(\mathrm{P}=0.022)$, relapse-free survival (RFS) $(\mathrm{P}=0.042)$ and cumulative incidence of acute GVHD $(\mathrm{P}=0.000)$. Chronic GVHD could not be compared between the two cohorts due to the different median follow-up.

Conclusions: The infusion of a CD34+ cell dose count higher than $8 \times 10^{\wedge} 6$ cells $/ \mathrm{kg}$ had a significant adverse impact in overall survival and grade II-IV acute GVHD in the 
setting of RIC and dual T-lymphocyte suppression with ATG and PTCy for haploHSCT.

Disclosure: Nothing to declare

\section{P335}

Long-term thymic activity and immune-reconstitution after haplo-identical allografting with post-transplant cyclophosphamide

Giuseppe Lia $^{1,2}$, Sara Butera ${ }^{1,2}$, Andrea Evangelista ${ }^{3}$, Laura Tosti ${ }^{1,2}$, Lorenzo Comba ${ }^{1,2}$, Silvia Cena ${ }^{1,2}$, Lucia Brunello $^{1,2}$, Milena Gilestro ${ }^{1}$, Marina Ruggeri ${ }^{1}$, Daniela Oddolo $^{I}$, Simona A. Caltagirone ${ }^{I}$, Vittorio E. Muccio ${ }^{I}$, Elona Saraci ${ }^{1}$, Moreno Festuccia ${ }^{1,2}$, Giovannino

Ciccone $^{3}$, Enrico Maffini, ${ }^{1,2}$, Mario Boccadoro ${ }^{1,2}$, Luisa Giaccone $^{1,2}$, Paola M. Omede ${ }^{I}$, Benedetto Bruno ${ }^{I}$

${ }^{1}$ A.O.U. Città della Salute e della Scienza di Torino, Torino, Italy, ${ }^{2}$ University of Torino, Torino, Italy, ${ }^{3}$ A.O.U. Città della Salute e della Scienza di Torino, Clinical Epidemiology, Torino, Italy

Background: The use of post-transplant Cyclophosphamide (PTCY) has expanded the application of $\mathrm{T}$ repleted haploidentical stem cell transplantation (haploHSCT). In this setting, to investigate thymus role in longterm clinical outcomes, evaluation of immune reconstitution kinetics was performed.

Methods: Twenty-nine patients (median age 53) were enrolled. Blood samples were collected before conditioning and at 1, 3, 6, 12, 18, 24 months after Haplo-HSCT. Analyses of CD4 and CD8 T-cell subsets by flow-cytometry were correlated by generalized linear models with RealTime PCR (RT-PCR) quantification of signal joint T-cell receptor excision DNA circles (sjTRECs), specific marker of naive T-cells thymopoiesis. A) Naive; b) central; c) memory; and d) revertant CD4 and CD8 T-cells were defined as follows: a) CD45RA+CD62L+; b) CD45RO +CD62L+; c) CD45RO+CD27-; and d) CD45RA+/45RO + , respectively. SjTRECs RT.PCR was performed on genomic DNA (100 ng) extracted from sorted CD4 and CD8 T-cells.

Results: A gradual increase in absolute numbers of all CD4 and CD8 T cell subsets and of sjTRECs copies from the first month up to 2 years post-transplant was observed (Figure 1). However, at 2 years, CD4 and CD8 T-cell levels and sjTRECs levels were lower than those observed in healthy donors. sjTRECs kinetics was associated with the increase in CD4 naive T-cells (overall, $\mathrm{p}<0.002$ ). This correlation suggests that most of CD4 naive T-cells derives from thymic re-education of donor precursor stem cells, whereas CD8 naive T-cells undergo peripheral expansion after thymic production. Furthermore, an increase in CD4 revertant memory T-cells was also significantly correlated with sjTRECs kinetic (p 0,041). Central and effector memory T-cells showed a faster thymic-independent expansion in both CD4 and CD8 Tcells. Interestingly, sjTRECs levels and thymic dependent immune-reconstitution were higher in a cohort of 63 patients undergoing HSCT from HLA identical donors (manuscript in preparation). Clinical outcomes and thymic function were correlated starting at 6 months after HSCT. Lower thymic output was significantly associated by multivariate analysis with low pre-transplant TRECs values ( $\mathrm{p}$ 0,002 and $\mathrm{p}<0,001$ in CD4 and CD8, respectively), moderate-severe chronic graft-versus-host disease (GVHD; $p<0,001$ in CD8), and age ( $\geq 50$ years, $p$ 0,006 in CD8).

Conclusions: The thymus, despite age-dependent involution, substantially contributes to T-cell reconstitution after haplo-HSCT. Chronic GVHD and older age were significantly correlated with reduced thymic function. Overall, lower production of sjTRECs after haploHSCT as compared after HLA identical sibling HSCT may partly be due to a higher degree of "mismatching" of MHC molecules during thymic re-education.

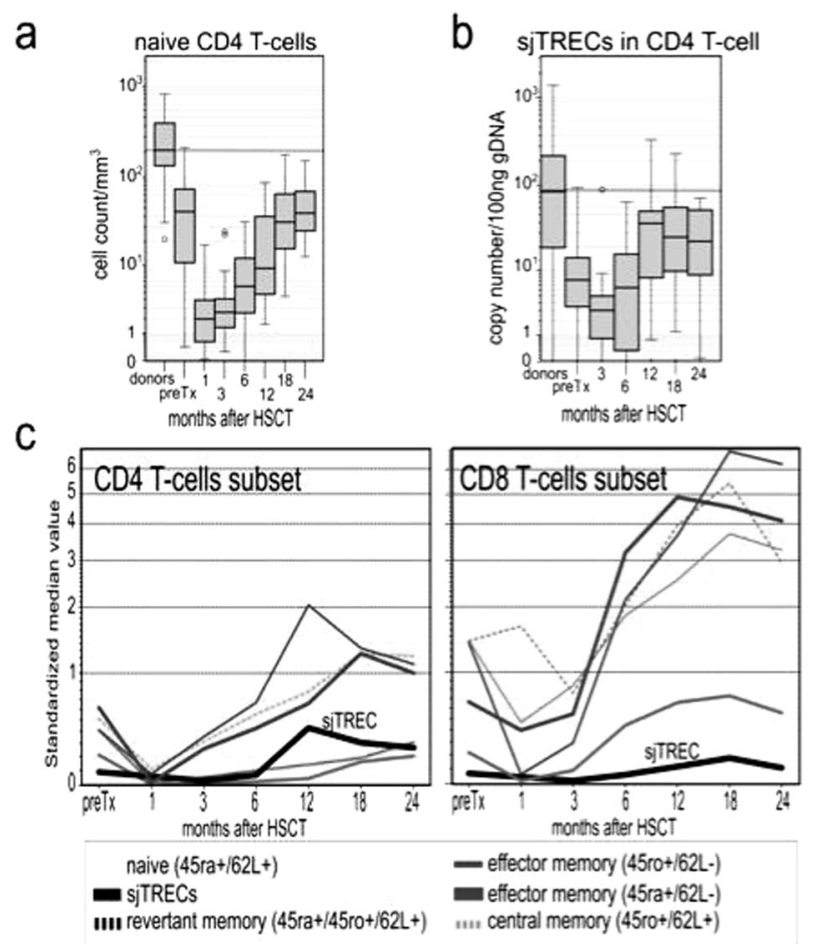

Figure 1: a) CD4 naive T-cell count af different time point after HSCT b) STRECS cooy number varation in CD4 T-cells after HSCT (dashed line = median heathy donor levels). c) Standardized value of CO4 and CD8 T-cell subsets and siTRECS reconstitution at different time - points after HSCT. ( $1=$ donor median value).

[[P335 Image] 1. Figure 1] 


\section{Disclosure:}

Boccadoro Mario Honoraria and research funding from: Sanofi, Celgene, Bristol-Myers Squibb, Novartis, Amgen, Janssen; Honoraria from AbbVie; Research funding from Mundipharma

Benedetto Bruno Honoraria from: Gilead, Pfizer, Hospira; Honoraria and research funding from Celgene; Research funding from Pierre Fabre, Adienne, Hospira italia, MSD Italia

\section{P336}

Risk score can predict long term outcome of allogenic HSCT in AYA with philadelphia negative all treated with an adult based chemotherapy protocol

Mona Hassanein, Afnan Alsabbagh, Hussein Albarqi, Ahmad Alshomar, Mohammed Assiri, Syed Osman Ali Ahmed, Ali Alahmari, Saud Alhayli, Mahmoud Aljurf, Fahad Almohareb, Fahad Alsharif, Hazza Alzahrani, Naeem Chaudhri, Shahrukh Hashmi, Walid Rasheed, Marwan Shaheen, Feras Alfraih, Riad Elfakih, Amr Hanbali

King Faisal Specialist Hospital \& Research Centre, Riyadh, Saudi Arabia

Background: The use of Allogenic Hematopoietic Stem Cell Transplantation (HSCT) in the treatment of Adolescents and Young Adults (AYA) with Philadelphia negative ALL is decreasing with the adoption of pediatric inspired protocols to treat this age group and the incorporation of minimal residual disease assessment in the routine care of ALL patients. Previously, its use was defined mainly by disease risk features at presentation.

Methods: A study on 209 AYA (age 14-39 years), who underwent Allogenic HSCT at our institute for Philadelphia negative ALL, between February 2005 and December 2015. All the studied patients received CALGB based adult chemotherapy protocol for induction, and underwent a Matched Related Donor (MRD) transplant with CY/TBI conditioning and MTX/CSA as GVHD prophylaxis. The patients were eligible for Allogeneic HSCT, if they have a MRD plus one or more of the following risk factors: (1) Age > 30 years, (2) High presenting WBC count ( $>30$ for BALL, >100 for T-ALL), (3) High risk Immuno-phenotyping (Pro-B, Pro-T, Early T, and Mature T), (4) Bulky splenomegaly or bulky lymphadenopathy, (5) High risk cytogenetics $(4 ; 11,1 ; 19$, low hypodiploidy/near triploidy, complex), (6) CNS involvement, (7) Relapsed or refractory disease at D28 of induction. In this study, we investigated the impact of those different risk factors on the long term outcome of Allogeneic HSCT.
Results: The median OS of our studied patients was not reached at 12.5 years, with a median DFS of 8.3 years (Figure 1). In a univariate analysis, relapsed or refractory disease prior to transplant was the only independent risk factor for OS and DFS (p-value $=0.36$, and 0.01 respectively) (Figure 2). In addition, patients who had 3 or more risk factors $(41,19.6 \%)$ prior to transplant had a significantly lower long term outcome compared to patients, who had one $(100,47.9 \%)$ or two risk factors $(68,32.5 \%)$ with a median OS of 23 months, and a median DFS of only 11 months ( $\mathrm{p}$-value $=0.32$, and 0.009 respectively) (Figure 3).

Conclusions: Our results show that the long term outcomes of HSCT in AYA with Philadelphia negative ALL treated on an adult type chemotherapy regimen, were significantly better in patients who showed a good response to initial therapy and a limited poor prognostic factors at presentation, with worsening of DFS as the number of poor prognostic features increase. We can conclude that, using this risk score can be helpful in predicting the outcome of Allogenic HSCT in AYA with Philadelphia negative ALL treated with adult type chemotherapy protocol.

Disclosure: No conflict of interest

\section{P337}

A prospective single center survey on donor-specific anti-HLA antibodies and desensitization strategy in patients undergoing an allogeneic stem cell transplant

Ursula La Rocca', Anna Paola Iori', Maria Paola Perrone $^{2}$, Paola Cinti ${ }^{2}$, Maria Gozzer ${ }^{2}$, Mahnaz Shafii Bafti $^{2}$, Walter Barberi', Luisa Quattrocchi, Claudio Cavallari $^{2}$, Paola Gesuiti ${ }^{2}$, Roberto Lattanzi ${ }^{2}$, Luca Laurenti $^{2}$, Gabriella Girelli ${ }^{2}$, Robin Foà ${ }^{1}$

${ }^{1}$ Policlinico Umberto I, Sapienza University, Hematology, Rome, Italy, ${ }^{2}$ Policlinico Umberto I, Sapienza University, Immunohematology and Transfusion Medicine, Rome, Italy

Background: In the setting of hematopoietic stem cell transplantation (HSCT), considering the risk of poor engraftment or graft failure (GF), the detection of antibodies (Ab) directed against donor specific HLA loci (DSA) represents a contraindication to proceed with the same donor, suggesting the search of other donors. In many cases, there is not sufficient time to search for alternative donors and it is necessary to plan an immunosuppressive strategy to decrease the DSA level, thus reducing the risk of GF. To date, there is no consensus on desensitization standards to manage DSAs in HSCT. The aim of this study was to determine the incidence of anti-HLA $\mathrm{Ab}$ and DSAs in hematologic patients candidate to an allogeneic HSCT, and 
the efficacy of our desensitization protocol. Here, we present an update of the results obtained with our strategy.

Methods: Between August 2014 and September 2018, we prospectively screened for DSA 140 consecutive patients candidates to an allogeneic HSCT. Anti-HLA Ab research was carried out using the Luminex bead assay (Lifecode Screen and LSA I/II-Immucor). The results were expressed as mean fluorescence intensity (MFI); MFI $>1000$ was considered positive. In case of a mismatched related donor, a flow cytometric crossmatch test (FCXM) was performed. If the patient had DSAs and only one available donor, a desensitization strategy was employed, scheduled with Rituximab on day -15 , single-volume plasmapheresis procedures (PP), usually on day -9 and -8 , intravenous immunoglobulins on day -7 , infusion of HLA selected platelets for DSA absorption in case of persistent antibodies directed against class I HLA antigens. The aim of this schedule was to avoid interferences with chemotherapy and anti-T-cell globulins, infused during condition regimen

Results: Since August 2014, 140 patients have been prospectively screened. Thirty-three patients (23.6\%) showed anti-HLA Ab and 9 of them (6.4\%) had DSAs: 6 were treated with the desensitization strategy, applied according to the MFI score and the FCXM result, and all of them obtained an engraftment; in 2 cases, an alternative donor was selected and in 1 case the research for an alternative donor is still underway. DSA detection was performed every 7 days after HSCT for the first month and 60, 180 and 365 days following HSCT. Neither a DSA rebound nor other complications were observed during the follow-up.

Conclusions: Our prospective analysis underlines the high frequency of anti-HLA antibodies detection in hematologic patients, confirming the necessity to routinely evaluate the presence of DSAs before an allogeneic mismatched HSCT. Our desensitization schedules based on the combination of PP, rituximab, IVIG and platelet absorption proved successful in reducing DSAs. We confirm the necessity of a prospective multicenter collaboration to better define the role of DSAs against each HLA locus and the critical MFI cut-off level associated with a higher risk of GF. Transplant and transfusion specialists should joint to define a consensus for a standard desensitization strategy.

Disclosure: Nothing to declare

\section{P338}

CD34 + stem cells selection and CD3 $+T$ cells addback from mud in paediatric recipients: A single center experience

\section{Fulvio Porta ${ }^{1}$, Richard Fabian Schumacher ${ }^{1}$, Elena Soncini $^{1}$, Giulia Carracchia ${ }^{1}$, Vincenzo Pintabona ${ }^{1}$, Elisa}

Bertoni $^{\text {I, Chiara Gorio }}{ }^{1}$, Annalisa Agostini, ${ }^{1}$, Pierpaolo Dal Canton ${ }^{1}$, Rosanna Ceresoli, Marta Comini', Alessandra Beghin ${ }^{2}$, Federica Bolda ${ }^{2}$, Arnalda Lanfranchi ${ }^{2}$

${ }^{1}$ Children's Hospital, ASST Spedali Civili, Brescia, Italy, ${ }^{2}$ Stem Cell Laboratory, Clinical Chemistry Laboratory, ASST Spedali Civili, Brescia, Italy

Background: Only $25 \%$ of patients have an HLA-identical sibling, so in the absence of a suitable related donor, Matched Unrelated Donor (MUD) is a good alternative source for Hematopoietic Stem Cell Transplantation (HSCT) in patients with haematological and non-haematological diseases.

The most frequent technique used for counterbalance partial incompatible HSCT is CD34+ selection that is associated with sustained engraftment and effective reduction of $\mathrm{T}$ cells that minimizes GvHD. On the other hand, this approach could delay immune reconstitution and increase risk of viral and fungal infection. In MUD setting the use of PBSC is the procedure that most centers have recently adopted. This implies the infusion of a relevant higher number of $\mathrm{T}$ cells 5 to 10 times more as compared with Bone Marrow (BM). Since in our centre most part of our patients are primary immunodeficiencies, we applied a procedure to minimize the risk of severe GvHD infusing a controlled number of $\mathrm{CD} 3$ positive cells.

Methods: We report data about 91 paediatric patients who received 92 MUD HSCT (1 patient received 2 HSCT) between 2001 and 2018 in the BMT Unit of the Children's Hospital of Brescia.

Patients received conditioning, according to the European Group for Bone Marrow Transplantation (EBMT) and the European Society for Immunodeficiencies (ESID) Guidelines.

CD34+ selection has been realized by a Milteny column with an ideal addback of $\mathrm{CD} 3$ positive cells of $30 \times 10^{6} / \mathrm{Kg}$.

Stem cell source was BM in 62 cases and PBSC in 30 cases.

Results: Median patients age at transplant was 2 years (range 2.6 months-17 years). The mean number of infused cells were: $12 \times 10^{6} / \mathrm{Kg} \mathrm{CD} 34+$ and $36 \times 10^{6} / \mathrm{Kg} \mathrm{CD} 3+$ in BM product, while $20 \times 10^{6} / \mathrm{Kg} \mathrm{CD} 34+$ and $39 \times 10^{6} / \mathrm{Kg}$ CD3 + in PBSC.

Mean time for engraftment was day 15 post-HSCT. As concerns acute GvHD overall incidence $64.1 \%$ (59/92) of the children presented this complication, but only $11 \%$ (7/ 59) presented GvHD grade III and none GvHD grade IV, while chronic GvHD presented in 7.6\% (6 limited, 1 extensive/92). While acute GvHD incidence and severity weren't significantly different between BM recipients and 
PBSC recipients, the cases of chronic GvHD were prevalently in the latters.

No major infections presented in the post-transplant period and immunological reconstitution both cellular and humoral was completed by 12 months.

Overall survival at 10 years is $75 \%$ (23/92).

Conclusions: The results obtained show how it is possible control severity of GvHD if an addback of a controlled number of CD3 + lymphocytes. Acute GvHD wasn't severe and only few children presented with limited chronic GvHD.

The method allows to graft primary immunodeficiencies patients even with PBSC without infusing too many T cells. In fact, especially in very young children, the number could be excessive and risky. Nevertheless in case of an oncohaematological patient, GvL effect is preserved.

Disclosure: Nothing to declare

\section{P339}

Hematopoietic stem cell transplantation (HSCT) for dyskeratosis congenita (DC): Experience in 28 patients transplanted in a single institution in Brazil

Samantha Nichele ${ }^{1,2}$, Carmem Bonfim ${ }^{1,2,3}$, Lisandro Ribeiro $^{1,2}$, Gisele Loth ${ }^{1,3}$, Larissa Medeiros ${ }^{1}$, Michel Oliveira $^{1}$, Ana Luiza Rodrigues ${ }^{3}$, Cilmara Kuwahara ${ }^{3}$, Adriana Koliski', Rebeca Mousquer', Daniela Marinho ${ }^{1}$, Vaneuza Funke ${ }^{1}$, Rodrigo Calado ${ }^{4}$, Ricardo Pasquini ${ }^{1,2}$

${ }^{1}$ Federal University of Parana, Curitiba, Brazil, ${ }^{2}$ Hospital Nossa Senhora das Graças, Curitiba, Brazil, ${ }^{3}$ Hospital Infantil Pequeno Principe, Curitiba, Brazil, ${ }^{4}$ University of São Paulo, Ribeirao Preto, Brazil

Background: DC is a rare genetic disorder that results from a defective telomere length maintenance and is characterized by mucocutaneous features, bone marrow failure (BMF) and a high predisposition to cancer and pulmonary fibrosis. BMF remains the major cause of mortality and the HSCT is the only definitive treatment to restore hematopoiesis but is limited by a high incidence of treatmentrelated mortality.

Methods: A retrospective analysis of 28 patients (pts) with DC who underwent HSCT at the Bone Marrow Transplantation Unit in the Clinical Hospital of Federal University of Paraná, Brazil, between July-1993 and November-2017.

Results: 15 boys and 13 girls, with a median age of $14 y$ (3 - 30y) received a HSCT from a MDS $(n=7)$, MUD $(n=17)$ or MMRD (haploidentical, $n=4)$. 27pts received bone marrow (BM) and $1 \mathrm{pt}$ received a cord blood unit (CBU). The median of TNC infused was $4,76 \times 10^{8} / \mathrm{Kg}$ (range $2,26-10,12 \times 10^{8} / \mathrm{Kg}$ ) and in the $\mathrm{CBU}$ was $6,5 \times 10^{7}$ /
$\mathrm{Kg}$. Two pts received a myeloablative preparatory regimen with busulfan (Bu) $12 \mathrm{mg} / \mathrm{Kg}+$ cyclophosphamide $(\mathrm{Cy})$ $120 \mathrm{mg} / \mathrm{Kg}$ or fludarabine (Flu) $150 \mathrm{mg} / \mathrm{m}^{2}+$ antithymocyte globulin (ATG). The remaining pts received a $\mathrm{RIC}$ regimen with Cy200mg/Kg $(\mathrm{n}=5)$, Flu150mg/m² + Cy60mg/Kg + ATG5mg/Kg $(n=17)$, and Flu $150 \mathrm{mg} / \mathrm{m}^{2}+\mathrm{Cy} 30+$ TBI200rads ( $n=4$, haplo). Graft versus Host Disease (GVHD) prophylaxis consisted of cyclosporin (CSA) and methotrexate or steroids (CBU) and post-transplant $\mathrm{Cy}+$ $\mathrm{CSA}+$ mycophenolate mofetil in the haploidentical transplants. 26 of 27 evaluable pts engrafted with a median time to neutrophil recovery of 20 days (range:13-36 days). One patient experienced primary graft failure (haplo) while second graft failure occurred in other 3pts. All these 4pts went a second HSCT and 3 survived. Acute GVHD grade II-IV occurred in 6 of 26 pts at risk. Moderate to severe chronic GVHD occurred in 6 pts with 5 cases occurring in pts who had previously presented acute GVHD. Overall survival (OS) was 53,6\% at a median follow-up of 6y. The $5 y$ OS was slightly better in MSD transplants compared to the others $(54,5 \% \times 52,9 \% \mathrm{p}=0,053)$. Causes of early death include adenovirus sepsis $(n=1)$, toxicity to preparatory regimen and sepsis $(n=2)$, primary graft failure $(n=1)$. 13pts remain alive between 1-16y after HSCT with a median FU of $8 \mathrm{y}$. Among them only 1pt has developed organ involvement by the underlying disease: hepatopulmonary syndrome (HPS). 7pts died due to pulmonary fibrosis $(n=1)$, liver fibrosis $(n=1)$, GI bleeding $(n=1)$, HPS $(n=2)$; cGVHD and sepsis $(n=1)$, infection $(n=1)$, and $2 p t s$ were lost to FU.

Conclusions: Early mortality from BMF can be reduced by HSCT, but late outcomes remain a consequence of the underlying disease. Long term FU is essential in order to detect late complications related to the HSCT procedure or the underlying disease.

Disclosure: Nothing to declare

\section{P340}

Single intra-bone cord-blood transplantation with a treosulfan-based regimen, ATG-free and sirolimusbased gvhd prophylaxis: fast hematopoietic engraftment and immune-reconstitution in 20 patients

Elisabetta Xue ${ }^{1}$, Maria Teresa Lupo Stanghellini, Fabio Giglio $^{I}$, Raffaella Greco ${ }^{I}$, Daniela Clerici ${ }^{I}$, Simona

Piemontese ${ }^{1}$, Lorenzo Lazzari ${ }^{1}$, Fabio Serpenti ${ }^{1}$, Andrea Assanelli, Magda Marcatti ${ }^{I}$, Fabio Ciceri ${ }^{I}$, Jacopo Peccatori $^{1}$

${ }^{1}$ San Raffaele Scientific Institute IRCCS, Milan, Italy

Background: Cord Blood Transplants (CBT) require less stringent HLA-matching, compared to peripheral blood 
stem cell or bone marrow. However, CBT has been associated with delayed engraftment and immune reconstitution, especially if in vivo T-cell depletion, such as antithymoglobulin (ATG), is used.

Methods: From 2010 to 2018, 20 patients with high-risk diseases received intra-bone infusion of unwashed single CB unit with an ATG-free GvHD prophylaxis; 7 were in active disease at CBT and 8 had received prior allogeneic stem cell transplantation. Median age was $44 \mathrm{y}$ [range (r) 22-65]. Conditioning regimen was myeloablative, with Treosulfan and Fludarabine in all, intensified with Melphalan in 15 or with 4 Gy TBI in 4 . HLA matches was $4 / 6,5 / 6$, $6 / 6$ in 12,5 and 3 cases, respectively. GvHD prophylaxis included Sirolimus and Mycophenolic acid (MMF).

Results: After thawing, median CD45+ cells was $1.39 \mathrm{x}$ $10^{7} / \mathrm{kg}$ [r 0.69-5.9], median CD34+ cells $0.08 \times 10^{6} / \mathrm{kg}[\mathrm{r}$ 0.04-0.23], and median CD3+ cells $3.05 \times 10^{6} / \mathrm{kg}$ [r 1.29 5.9]. Of the 16 evaluable patients all engrafted with a sustained full donor chimerism at day 100. Median time to neutrophils $(16 / 16$, ANC $>500 / \mu \mathrm{L}$ for 3 consecutive days) and platelet engraftment $\left(14 / 16\right.$, PLT $>20 \times 10^{3} / \mu \mathrm{L}$ for 7 consecutive days) were 27 [r 16 -59] and 52 days [r 25221], respectively. None rejected the transplant.

Five patients died for transplant related causes $(\mathrm{IFI}=3$, $\mathrm{GvHD}=1$, bacterial sepsis $=2$ ), for a day-100 TRM of $25 \%$. Relapse rate was $37.5 \%$ (6/16), median time 290 days [r 89650]; 3 pts received a subsequent SCT, whereas 3 died from relapse.

Seven out of 16 patients suffered from all grades acute GvHD, including peri-engraftment syndrome, median time 19 days [r 11-59], only $2 / 7$ were III/IV grade aGvHD. Viral reactivation were seen in 15 patients $(\mathrm{CMV}=8, \mathrm{HHV} 6=13$, $\mathrm{ADENO}=2$ ), median time 26 days [ $\mathrm{r} 9-39$ ].

Immune-reconstitution of CBT patients (Tables 1a-b) was compared with two cohorts of patients transplanted at our Center from any adult donor with (81) or without (126 patients, including post-transplant cyclophosphamide cohort) ATG in association with Sirolimus and MMF. Profiles of immune-reconstitution at day $90-180$ 365 showed a better CD4+ recovery at any time-point in both CBT and no-ATG versus ATG cohort, with no statistic significant difference in the first 2 cohorts. Moreover, CD4 $+/ \mathrm{CD} 8+$ ratio at any time point was better in the CBT cohort vs the no-ATG cohort. B cell recovery was faster in the CBT cohort; immunoglobulin recovery was superimposable across different platforms.

Focusing on late events ( $>180$ days from CBT), 3/12 pts experienced EBV reactivation, median time 586 days [362655] treated with rituximab, and 1 experienced late HHV6 and CMV reactivation, both solved at last visit. Sirolimus was withdrawn after a median of 188 days [r 64-394]. Only 1 patient developed severe chronic GvHD, solved at last visit.
Overall, after a median follow-up of 330 days [r 9-1311], 11 pts are alive and well.

Conclusions: Our data confirm that intra-bone CBT without in-vivo T-cell depletion is associated with fast hematopoietic engraftment and immune-reconstitution, with very low rate of chronic GvHD and late infective events.

\begin{tabular}{|c|c|c|c|}
\hline & 3 months & 6 months & 12 months \\
\hline $\mathrm{CD} 3+/ \mu \mathrm{L}$ & $222[18-1001]$ & $691[109-1262]$ & $1044[241-1910]$ \\
\hline $\mathrm{CD} 3 \mathrm{CD} 4+/ \mu \mathrm{L}$ & $177[13-366]$ & 321 [95-666] & 664 [194 - 1027] \\
\hline $\mathrm{CD} 3 \mathrm{CD} 8+/ \mu \mathrm{L}$ & $41[5-635]$ & $291[14-622]$ & $334[47-882]$ \\
\hline $\mathrm{CD} 19+/ \mu \mathrm{L}$ & $54[5-187]$ & $295[1-1887]$ & $418[0-2368]$ \\
\hline $\mathrm{NK} / \mu \mathrm{L}$ & $173[36-430]$ & $188[62-730]$ & $195[51-562]$ \\
\hline \multicolumn{4}{|c|}{$\begin{array}{l}\text { a: Median values of } \operatorname{CD} 3+/ \mu \mathrm{L}, \mathrm{CD} 3 \mathrm{CD} 4+/ \mu \mathrm{L}, \mathrm{CD} 3 \mathrm{CD} 8+/ \mu \mathrm{L}, \mathrm{CD} 19+/ \mu \mathrm{L} \text { and NK cells } / \mu \mathrm{LL} \text { at } 3 \\
6 \text { and } 12 \text { months with respective ranges. Normal values range as follows: } \mathrm{CD} 3+605-2460 / \mu \mathrm{L} \\
\operatorname{CD} 3 \mathrm{CD} 4+493-1666 / \mu \mathrm{L}, \mathrm{CD} 8+224-1112 / \mu \mathrm{L}, \mathrm{CD} 19+75-520 / \mu \mathrm{L}, \mathrm{NK} 73-654 / \mu \mathrm{L} \text {. }\end{array}$} \\
\hline & 3 months & 6 months & 12 months \\
\hline lgG g/L & $3.33[0.22-8]$ & $4.8[2.4-12.15]$ & $6.31[2.19-8.4]$ \\
\hline $\operatorname{lgM} \mathrm{g} / \mathrm{L}$ & $0.22[0-0.9]$ & $0.66[0.07-3.17]$ & $0.69[0.14-1.09]$ \\
\hline $\operatorname{lgA} \mathrm{g} / \mathrm{L}$ & $0.15[0.04-0.89]$ & $0.24[0.03-1.09]$ & $0.56[0.13-1.59]$ \\
\hline
\end{tabular}

[[P340 Image] 1. Immune-reconstitution after CordBlood Transplant]

Disclosure: Nothing to declare

\section{P341}

Biomimetic reconstruction of tissue structures using the example of the hematopoietic stem cell niche for the in vitro amplification of human hematopoietic stem cells

Lisa Marx-Blümel ${ }^{1,2}$, Jessica Frey ${ }^{1,2}$, Jürgen

Sonnemann ${ }^{1,2}$, Andreas Schober ${ }^{3}$, Dana Brauer ${ }^{3}$, Gregor Schlingloff ${ }^{3}$, Sukhdeep Singh ${ }^{3}$, Jörg Hampl ${ }^{3}$, Frank Weise $^{3}$, Nora Lindig ${ }^{1,2}$, Christian Marx ${ }^{4}$, Zhao-Qi Wang ${ }^{4}$, Birgit Perner, James F. Beck ${ }^{1}$

${ }^{1}$ Children's Clinic, University Hospital, Jena, Germany, ${ }^{2}$ Research Centre Lobeda, University Hospital, Jena, Germany, ${ }^{3}$ Institute of Micro- and Nanotechnologies MacroNano ${ }^{\circledR}$, Nano-Biosystem Technology, Technical University Ilmenau, Ilmenau, Germany, ${ }^{4}$ Leibniz Institute on Aging - Fritz Lipmann Institute (FLI), Jena, Germany

Background: A promising improvement of hematopoietic stem cell transplantation (HSCT) may lie in the transplantation of high numbers of pluripotent stem cells to minimize the time span between transplantation and immunological reconstitution. Hence, an ex vivo platform is needed that supports HSC proliferation before application and, at the same time, the maintenance of pluripotency by diminishing HSC differentiation into lineage-specific progenitor cells.

Methods: To artificially model the natural HSC niche in vitro, we used 3D bone marrow (BM)-like scaffolds 
made of polydimethylsiloxane (PDMS). These structures are based on a human long bone cross section as a representative of the BM. Human cryoconserved HSCs were cultured in distinct cultivation systems for 14 days under different conditions. Cell counting and FACS analyses at day 14 were conducted. For characterization of the cultivated HSCs, we used antibodies against CD34 alone or in combination with antibodies against CD38, CD90, CD45RA and CD49f.

Results: For optimization of culture conditions for human HSCs, a commercially available medium was supplemented with a panel of cytokines and valproic acid. We found a significant increase in the number of CD34+ HSCs by simultaneously increasing their vitality using the 3D system compared with conventional 2D culturing. A further improvement was achieved by introducing a silicon oxidecovering of the 3D PDMS structures, suggesting that hydrophilic surface properties offer superior attachment for semi-adherent HSCs. For a more precise characterization of the cultivated HSCs, we introduced a panel of FACS markers reflecting the immaturity of the amplified HSC. Surprisingly, with increasing immaturity of the cultivated HSC, non-covered 3D PDMS revealed to be best suited for amplification: Cell number of vital immature HSCs was increased after cultivation on non-covered 3D PDMS compared with silicon oxide-covered 3D PDMS and the 2D system.

Conclusions: By establishing a 3D scaffold according to the human BM, we found a platform mimicking the natural niche of human HSC which is suitable to amplify human HSCs in vitro and support their vitality, pluripotency and ability for self-renewal.

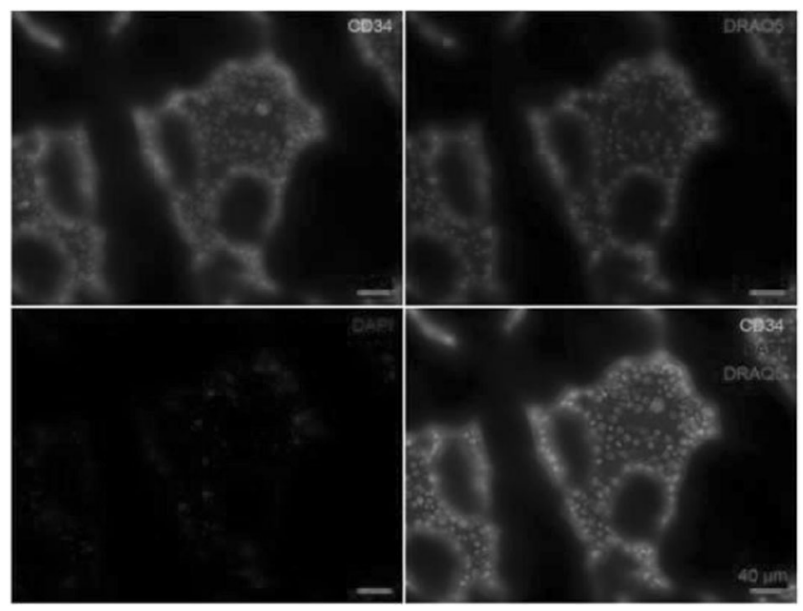

[[P341 Image] 1. Figure 1: Human hematopoietic stem cells cultured on silicon oxide-covered $3 D$ polydimethylsiloxane]
With the introduction of SiOn covering of 3D PDMS structures the maintenance of CD34+ HSCs could be further improved. Despite the better conservation of CD34 + HSCs by using silicon oxide-covered 3D PDMS, we found that immature human HSCs obviously prefer more hydrophobic conditions found on non-covered 3D PDMS.

Disclosure: Nothing to declare.

\section{P342}

\section{Abstract already published.}

\section{P343}

Improvements in neutrophil engraftment following changes in freezing method

Wendy Merrison ${ }^{1}$, Sarah Ward ${ }^{1}$, Shazia Aref ${ }^{1}$, Joanne Berridge $^{I}$, Rik Lewin ${ }^{1}$, Katherine Hodgson ${ }^{2}$, Alexander Martin $^{2}$, Ann Hunter ${ }^{2}$

${ }^{1}$ University Hospitals of Leicester, Stem Cell Laboratory, Leicester, United Kingdom, ${ }^{2}$ University Hospitals of Leicester, Haeematology, Leicester, United Kingdom

Background: In the setting of autologous haematopoietic progenitor cell (HPC) transplants for haematological disorders, peripheral blood stem cells are routinely collected via apheresis and cryopreserved. Leicester Royal Infirmary had been using a controlled rate freezer (CRF) to cryopreserve cellular therapy products up until 2017.

In 2017 a literature review of cryopreservation techniques was undertaken, since the CRF required replacement. This review found consistent evidence that cryopreservation using minus $80^{\circ} \mathrm{C}$ is comparable to $\mathrm{CRF}$, and engraftment times should not be negatively affected by changing to a more simplified method of freezing. There would also be cost saving benefits from switching from a CRF to minus $80^{\circ} \mathrm{C}$ freezers.

Methods: As a result two minus $80^{\circ} \mathrm{C}$ freezers were purchased following the acceptance of a Preparation Process Dosier (PPD) which was prepared for the Human Tissue Authority. Validation was carried out, and from January 2018 stem cell laboratory at the LRI switched from the $\mathrm{CRF}$ method to minus $80^{\circ} \mathrm{C}$ for cryopreservation of cellular therapy products. Briefly, cells are frozen using $10 \%$ DMSO (Wak-Chemie) in 20g/l Human Albumin Solution (Grifols) in Cyrobags (Origen Biomedical, Inc). The cells are transferred on cold packs to the minus $80^{\circ} \mathrm{C}$ freezer. Cells are packaged in between stainless steel heattransfer plates. The plates are placed within a bubble wrap bag, and are placed in a rack within the minus $80^{\circ} \mathrm{C}$ freezer, which allows air to circulate freely around each bag. In one plate a EL-USB-TC thermocouple data logger (Thermosense) is inserted between the bag and stainless steel plate, 
to record the freezing profile. This data is downloaded after each run. After an overnight freeze at minus $80^{\circ} \mathrm{C}$, the cells are subsequently removed from the bubble wrap and plates, and are transferred to minus $150^{\circ} \mathrm{C}$ freezers the following morning.

Results: A total of 40 patients have had autologous stem cells frozen using this method so far this year. In addition to engraftment data for neutrophils, post-freeze trypan blue viabilities were also compared to the previous year. During 2017 a total of 51 patients, who had all their cells cryopreserved, underwent 72 collections. The post freeze median viability was $90 \%$ (75-98\%). A total of median neutrophil engraftment was 12.0 days, with a median CD34 dose infused of $4.0 \times 10^{6} / \mathrm{kg}$. During 2018 so far, 40 patients have undergone 57 collections. Median viability is $95.5 \%$ (82-98\%). Subsequent median neutrophil engraftment is 11 days, with a median CD34 dose infused of $4.1 \times 10^{6} / \mathrm{kg}$.

Conclusions: Ongoing savings of approximately $£ 6400$ per annum have been made by changing our procedure. The benefit of changing to a simplified method of freezing has also resulted in a reduction in staff working overtime. More importantly, this simplified cryopreservation method has resulted in an improvement in neutrophil engraftment times since changing the cryopreservation technique from the previous method using CRF to mechanical freezing using a minus $80^{\circ} \mathrm{C}$ freezer.

Disclosure: Nothing to declare

\section{P344}

Low doses of granulocytes in the apheresis product predict a better outcome of autologous hematopoietic stem cell transplantation in multiple myeloma patients

\section{Marta Peña ${ }^{1}$, Eva Alonso ${ }^{2}$, Christelle Ferrà ${ }^{1}$, Mireia Morgades $^{1}$, Marta Sitges ${ }^{1}$, Mireia Franch ${ }^{1}$, Georgina Gener $^{I}$, Mireia Santos $^{1}$, Eloi Cañamero ${ }^{I}$, Andrea Espasa ${ }^{1}$, Martina Comes ${ }^{I}$, Susana Vives ${ }^{1}$, Miriam Moreno ${ }^{1}$, Anna Torrent $^{I}$, Gladys Ibarra ${ }^{1}$, Laura Abril ${ }^{1}$, Montserrat Batlle $^{1}$, Blanca Xicoy ${ }^{1}$, Juan Manuel Sancho ${ }^{1}$, Albert Oriol $^{1}$, Josep Maria Ribera ${ }^{1}$, Joan Ramon Grifols ${ }^{2}$}

\section{${ }^{1}$ Institut Català d'Oncologia-Hospital Germans Trias i Pujol, Josep Carreras Leukemia Research Institute, Clinical Hematology, Badalona, Spain, ${ }^{2}$ Banc de Sang i Teixits, Hospital Germans Trias i Pujol, Blood Bank, Badalona, Spain}

Background: High-dose chemotherapy with autologous stem-cell transplantation (ASCT) remains the standard consolidation therapy for multiple myeloma (MM). Peripheral blood stem cell collection may be contaminated with large quantities of granulocytes and its consequences on the outcome of ASCT are still unclear. On the other hand, the effect of performing apheresis with high levels of monoclonal component (MC) on outcome is unknown. The objective of this study was to analyze the effect of total nucleated cells (TNC) and granulocytes count (considered as contaminating components of apheresis products) as well as the influence of MC in the apheresis product on outcome of ASCT in MM.

Methods: Eighty-two patients diagnosed with MM were mobilized with filgrastim $10 \mu \mathrm{g} / \mathrm{kg} /$ day (plus plerixafor if insufficient mobilization of CD34+ cells on day 4). Apheresis collection was performed with $\mathrm{CMNc}$ program by Spectra Optia cell separator. CD34+ count was carried out according to ISHAGE protocol (target: $\geq 2 \times 10$ E6 CD34 $+/ \mathrm{Kg})$. Subsequent cryopreservation were performed according to the local protocol.

Results: The medians (range) of collected CD34+, TNC and granulocytes were $4.69 \times 10^{6} / \mathrm{Kg}(1.69-15.54), 9.35 \times 10^{8} /$ $\mathrm{Kg}(2.16-19.66)$ and $13.7 \times 10^{9} / \mathrm{Kg}(1.4-90.6)$, respectively. The medians (range) of infused $\mathrm{CD} 34+, \mathrm{TNC}$ and granulocytes were $2.99 \times 10^{6} / \mathrm{Kg}(0.89-10.93), 6.46 \times 10^{8} / \mathrm{Kg}$ (1.93-18.60) and $8.30 \times 10^{9} / \mathrm{Kg}(1.1-86.0)$, respectively. A successful collection after first line therapy was performed in $94 \%$ of patients. Treatment for MM was continued after carrying out apheresis in $40 \%$ of patients, as per protocol. A significant reduction of MC was observed prior to ASCT, indicating a further improvement in responses after apheresis $(\mathrm{p}=0.005)$. An optimal response (CR or VGPR) at the time of apheresis was achieved in $45 \%$ of patients and a suboptimal response (partial or minimal response) was observed in the remaining 55\%. Undergoing apheresis in optimal response did not result in lower number of TNC or granulocytes in the harvest. The subtype of MC did not influence on the number of TNC and granulocytes in the product of apheresis. No differences in collected TNC and granulocytes were observed when plerixafor was used as mobilizing agent. The type of chemotherapy given prior the apheresis did not have influence on the characteristics of the apheresis product.

A significant improvement in overall survival (OS) probability $(95 \% \mathrm{CI})$ was observed when low TNC (< $\left.6.46 \times 10^{8} / \mathrm{Kg}\right)(80 \%$ [65\%-95\%] versus $55 \%$ [37-73\%], $[\mathrm{p}=0.032])$ and low granulocytes $\left(<8.30 \times 10^{9} / \mathrm{Kg}\right)(87 \%$ [75\%-99\%] versus 49\% [30\%-68\%] [p=0.010]) were infused (Figure 1A). Lower incidence of relapse $(\mathrm{p}=0.044) \quad$ (Figure 1B) and non-relapse mortality $(p=0.040)$ was observed in patients who received low granulocyte count in the graft. No significant correlation was observed between the time of engraftment and the number of TNC or granulocytes infused. Similarly, no increase in the frequency of the engraftment syndrome was observed when higher number of TNC or granulocytes were infused. 
Conclusions: In our series, low doses of granulocytes in the product of apheresis predicted a better outcome of ASCT in MM patients. The amount of MC at the time of apheresis did not have influence in the characteristics of the harvest. Efforts for avoiding contamination in grafts are important for its impact on outcome of ASCT in MM patients.

Disclosure: DKMS Foundation, PI14/01971 (Instituto Carlos III) and SGR288 (GRC), Generalitat de Catalunya.

\section{P345}

Abstract withdrawn.

\section{P346}

First experience of plerixafor and G-CSF addition to conditioning regimen for $\mathrm{TCR} \alpha \beta+/ \mathrm{CD} 19+$ graft depleted stem cell transplantation in patients with chronic granulomatous disease

\section{Svetlana Kozlovskaya ${ }^{1}$, Svetlana Radygina ${ }^{1}$, Irina Shipitsina ${ }^{1}$, Anna Livshits ${ }^{1}$, Alexandra Laberko ${ }^{1}$, Larisa Shelihova ${ }^{1}$, Galina Novichkova ${ }^{I}$, Alexey Maschan ${ }^{1}$, Michael Maschan ${ }^{I}$, Dmitriy Balashov ${ }^{I}$}

${ }^{1}$ Dmitry Rogachev National Research Center of Pediatric Hematology, Oncology, Immunology Ministry of Healthcare of Russian Federation, Moscow, Russian Federation

Background: Our initial experience (from 2012 to 2017 years) with hematopoietic stem cell transplantation (HSCT) from MUD with TCR $\alpha \beta+/ C D 19+$ graft depletion in patients with CGD showed high rate of secondary graft dysfunction, the incident of graft rejection was 29\%. To improve the outcome, we hypothesized that the use of plerixafor and G-CSF as additional agents in conditioning regimen would offers advantages and better outcomes.

This trial was registered at www.clinicaltrials.gov (NCT03547830)

Methods: Between April 2018 and September 2018, 5 patients with CGD underwent allogeneic HSCTs from mached unrelated donors with TCR $\alpha \beta+/$ CD19 + graft. The conditioning regimen included - treosulfan $42 \mathrm{~g} / \mathrm{m} 2$, fludarabine $150 \mathrm{mg} / \mathrm{m} 2$, thiotepa $10 \mathrm{mg} / \mathrm{kg}$, G-CSF 50 $\mathrm{mcg} / \mathrm{kg}$ and plerixafor $720 \mathrm{mcg} / \mathrm{kg}$. All patients received rabbit ATG - Timoglobulin ${ }^{\circledR}$ ("Genzyme Europe B.V.", The Netherlands) $5 \mathrm{mg} / \mathrm{kg}$ for serotherapy. In all cases, TCRab +/CD19+ graft depletion was used with the immunemagnetic method (Miltenyi Biotec, Bergisch Gladbach, Germany) according to the manufacturer's instructions. Stem cell source was G-CSF mobilized peripheral blood stem cells in all cases. Posttransplant GVHD prophylaxis was not performed. Minimal follow up was 90 days after HSCT.
Results: Neutrophil and platelet engraftment occurred at 12-17 days post-HSCT in all patients. 3 patients had full donor chimerism in whole blood and in 2 cases we observed predominantly donor mixed chimerism at last follow up. All patients had full donor chimerism in $\mathrm{CD} 15+$ compartment and mixed chimerism in $\mathrm{CD} 3+$ lineage, but it stable without any sign of graft dysfunction. Acute GVHD is verified in 1 of 5 cases and was limited to skin grade 2 . All patients are alive for periods from 3 to 8 months post-HSCT with good graft function without severe clinical problems.

Conclusions: We presented first experience of G-CSF and plerixafor addition to conditioning regimen before HSCT with TCR $\alpha \beta+/ C D 19+$ graft depletion in CGD patients. We suppose, the preliminary results are encouraging, as the frequency of primary and secondary graft dysfunction in patients from this group is not observed today, there are no significant toxic complications, as well as clinically severe manifestations of GVHD.

Currently, the recruitment of patients is continuing, and estimation of the rates of immune reconstitution and a more detail analyses will be evaluated later.

Clinical Trial Registry: www.clinicaltrials.gov NCT03547830

Disclosure: Nothing to declare

\section{P347}

Blood group incompatibility in haplo-identical stem cell transplantation: Favor or unfavorable effect on the erythrocyte recovery?

Güldane Cengiz Seval ${ }^{1}$, Atilla Uslu ${ }^{1}$, Selami Koçak Toprak $^{1}$, Sinem Civriz Bozdağ ${ }^{I}$, Meltem Kurt Yüksel ${ }^{1}$, Önder Arslan ${ }^{1}$, Günhan Gürman ${ }^{1}$, Pervin Topcuoğlu ${ }^{1}$

${ }^{1}$ Ankara University School of Medicine, Hematology, Ankara, Turkey

Background: Introduction - It is believed that ABOincompatibility is of minor importance in allogeneic stem cell transplantation (allo-SCT) and that the clinical outcome is equivalent to ABO-compatible SCT. Therefore, we performed a single center retrospective study to characterize the impact of ABO-incompatibility on the outcome of haploidentical stem cell transplantation (Haplo-SCT).

Methods: This analysis included 27 consecutive patients who underwent haplo-SCT for various hematological malignancies at our center between October 2010 and May 2018. We used our institutional database to evaluate details and characteristics of patients and transplant outcomes.

Results: Demographic features of the patients and donors have been summarized in Table 1. All of the patients had advanced hematological disease with a high risk of relapse (22 patients with acute leukemia). Out of 24 patients, early 
transplant-related mortality was seen in this cohort of 5 patients. The remaining 19 patients were followed in 1 and 42 months. Donor type ABO group switch was observed in a median of 45 days (30-60 days) after transplant. We were not able to show any statistical difference in terms of blood group switch between minor and major $\mathrm{ABO}$ incompatible transplant. The median red blood cell (RBC) transfusions in the first 30 days for the $\mathrm{ABO}$ compatible and incompatible transplants were median 4 units (range, $0-11$ ) and median 4 units (range, 3-12) $(p=0.6)$. No statistical difference was also encountered for the RBC transfusion need for stem cell source, peripheral blood vs bone marrow. A total of 15 patients were followed up for reticulocyte engraftment. The median time for reticulocyte engraftment was 19 days (range, 15-60) for all patients. Reticulocyte engraftment was tended to be faster in minor $\mathrm{ABO}$-mismatched group

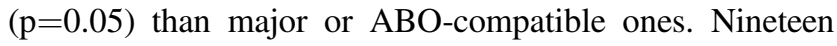
patients achieved independence from $\mathrm{RBC}$ support after a median time of 44 days (range, 11-61 days) in ABOcompatible patients, 25 days (range, 7-52 days) in minor ABO-incompatibilityand 34 days (range, 20-57 days) in major $\mathrm{ABO}$-incompatibilitygroup, respectively $(\mathrm{p}>0.5)$. The engraftman kinetics due to major and minor ABOincompatibilitytransplants were presented in Table-2. Pure red cell aplasia was not developed in our cohort.

Conclusions: The present single center study provides new evidence for the importance of the ABO system for erythrocyte recovery in haploidentic stem cell transplantation. It's important to note that, randomize prospective and larger studies are warranted.

Disclosure: Nothing to declare

\section{P348}

Low dose of anti-T lymphocyte globulin protects against severe forms of graft versus host disease in patients undergoing allogenic stem cell transplantation

Joan Mora Barrios ${ }^{1}$, Miguel Angel Cortés ${ }^{1}$, David Gómez Lamas $^{I}$, Yolanda González ${ }^{I}$, Sara Fernández Luis ${ }^{1}$, Almudena Cabero ${ }^{1}$, Cristina Fernández, ${ }^{1}$, Sara García Ávila ${ }^{1}$, Mercedes Colorado Araujo ${ }^{1}$, Mónica López Duarte $^{1}$, Guillermo Martín Sánchez ${ }^{1}$, Miriam Sánchez Escamilla $^{1}$, Irati Ormazabal ${ }^{2}$, Lucrecia Yáñez San Segundo ${ }^{1}$, José Luis Arroyo ${ }^{3}$, Rocío Lobeira Rubio ${ }^{I}$, Andrés Insunza ${ }^{1}$, Enrique M. Ocio ${ }^{1,4}$, Aranzazu Bermúdez ${ }^{1,4}$

${ }^{1}$ Hospital Universitario Marqués de Valdecilla, Hematology, Santander, Spain, ${ }^{2}$ Hospital Universitario de La Princesa, Hematology, Madrid, Spain, ${ }^{3}$ Banco de Sangre y Tejidos de Cantabria, Santander, Spain, ${ }^{4}$ IDIVAL (Research Institute of Marques de Valdecilla), Santander, Spain
Background: Graft versus host disease (GvHD) is the most important complication after allogeneic stem cell transplantation (alloSCT). Optimal dose of different anti-Tlymphocyte globulin (ATG) formulations in this setting has not been established yet. The aim of this study was to analyze the impact of a low dose of ATG-Fresenius (ATGF) in alloSCT outcomes.

Methods: We analyzed 57 adult patients who received an alloSCT for hematologic malignancies from October 2012 to March 2018. The GvHD prophylaxis included a total dose of $21 \mathrm{mg} / \mathrm{kg}$ (7 mg/kg on day -3, -2 and -1) of ATG-F for patients who received a graft from peripheral blood, an unrelated donor; with a mismatch, and/or were older than 55 years; associated to a calcineurin inhibitor and mycophenolate mofetil/short course-methotrexate. Statistical analysis was performed using SPSS v.22 and R software.

Results: Median age was 57 years (18-70) and $66.7 \%$ of patients were males. Seventy-four percent of patients underwent myeloablative conditioning. The stem cell source was peripheral blood in 51 patients $(89.5 \%), 80 \%$ were from unrelated donors (28\% mismatched). Seventeen (29.8\%) patients had high risk CMV status (D-/R+) (see image 1B). Engraftment was observed in 54 patients $(94.7 \%)$. Primary graft failure occurred in 3 patients (2 myelofibrosis, 1 AML). Twenty (39.2\%) out of 51 evaluable patients developed grade 2-4 acute GvHD. The cumulative incidence of severe aGvHD and moderatesevere chronic GvHD were 8.8\% (95\% CI, 3.2-17.9\%) and $35.2 \%$ (95\% CI $22.7-47.9 \%$ ), only 5 (8.7\%) patients developed severe cGvHD. Twenty-nine patients $(56.8 \%)$ discontinued immunosuppression before the first year of transplant. The median duration of immunosuppression for patients with moderate-severe cGvHD was 488 days (2072046).

At 2 years non-relapse mortality (NRM) was $21.9 \%$ (95\% CI, 12.0-33.7\%). Thirty-nine (68\%) patients developed relevant infectious complications. Two (3\%) patients died within the first 30 days due to gram negative blood stream infection. Eleven (19.2\%) had at least two episodes of cytomegalovirus (CMV) reactivation between day 30 and 100. Three (5\%) patients developed CMV gastrointestinal disease, $2(3 \%)$ had probable invasive fungal infection and 1 (1.7\%), post-transplant lymphoproliferative disorder associated to Epstein Barr Virus.

With a median follow up of 28 months for alive patients (3-67), the GvHD and relapse free survival (GRFS) at one year, overall survival (OS) and progression free survival (PFS) at two years were $47.6 \%$ (95\% CI, 42.8-52.2\%), 59\% (95\% CI, 54.5-63.2\%) and 52\% (95\% CI, 46.9-56.5\%), respectively. The relapse incidence at two years was $26.3 \%$ (95\% CI 14.7-39.4\%). Complete remission at transplant was associated with better long term survival ( $80 \%$ at 2 years, $\mathrm{p}$ $<0.01$ ). HLA disparity did not affect OS (see image $1 \mathrm{~A}$ ). 
Conclusions: The use of low doses of ATG-F is protective against severe forms of acute and chronic GvHD in a cohort with high prevalence of unrelated donors and a high median age. This strategy showed good results in GRFS, OS and PFS in a population at high risk for developing GvHD or relapse.

Disclosure: Nothing to declare

\section{P349}

Performance parameters of a ngs-product for chimerism monitoring - applicable in patients after hematopoietic stem cell transplantation

Linnéa Pettersson ${ }^{1}$, Sofie Vonlanthen ${ }^{2}$, Mehmet Uzunel ${ }^{1}$, Francesco Vezzi', Karin Alwegren ${ }^{2}$, Anders Hedrum ${ }^{1}$, Dan Hauzenberger ${ }^{2}$

${ }^{1}$ Devyser AB, Stockholm, Sweden, ${ }^{2}$ Karolinska University Hospital, Stockholm, Sweden

Background: A complication of hematopoietic stem cell transplantation (HSCT) is relapse of the underlying malignant disease. To detect relapse as early as possible, different methods to determine mixed chimerism (MC) have been developed and are currently used in laboratories worldwide. The aim of this study was to evaluate the performance of a novel NGS-based chimerism method and compare it to previously established molecular techniques for chimerism analysis.

Methods: For this purpose, samples from patients with mixed chimerism (MC) with increasing amounts of recipient DNA were analyzed and compared using realtime PCR of insertions/deletions (indels), fragment analysis of short-tandem repeats (STR) and NGS of indels.

Results: Whereas real-time PCR displayed excellent sensitivity down to $0,01 \% \mathrm{MC}$, but poor precision above $20 \%$, fragment analysis exhibited good precision with limited sensitivity $(>2,5 \%)$. In contrast, NGS chimerism demonstrated good sensitivity, with a limit of detection (LOD) of $0,1 \% \mathrm{MC}$, and precision throughout the whole spectrum of patient/donor mixed chimerism.

The NGS chimerism product (Devyser Chimerism) exhibited at least three (average eight) and at least two (average 5) informative genetic markers (indels), suitable for monitoring mixed chimerism of patients with their corresponding matched unrelated (60) or related (56) donor samples. In order to establish the performance of the separate techniques for determination of mixed chimerism on retrospective patient samples, a cohort of 27 patient monitoring samples (3-7 weeks post-HSCT) with low (< $5 \%$ ), intermediate or high mixed chimerism $(>20 \%)$ were included and analyzed. DNA from all monitoring samples was extracted from sorted cell fractions.
Conclusions: The results show that although all evaluated techniques are suitable for monitoring patient/donor chimerism after allogeneic hematopoietic stem cell transplantation (HSCT), only the NGS chimerism product exhibits high sensitivity (LOD $0,1 \%$ ) and a broad dynamic range (detection range $0,1-100 \%$ ) with good precision and accuracy throughout the whole spectrum of mixed chimerism (\% patient/donor). In addition, the NGS chimerism product employ 24 non-population dependent highly informative genetic markers providing stable resolution power and thus suitable for monitoring mixed chimerism.

Disclosure: Dan Hauzenberger is medical adviser at Devyser AB and shareholder in Devyser Holding

P350

Donor-related factors affecting the results of unrelated transplantations in children with non-malignant diseases

Natalia Sidorova ${ }^{1}$, Alexey Slinin ${ }^{2}$, Kirill Kirgizov ${ }^{1}$, Ekaterina Pristanskova ${ }^{1}$, Veronika Konstantinova ${ }^{1}$, Aleksandra Burya ${ }^{1}$, Marina Persiantzeva ${ }^{2}$, Elena Skorobogatova ${ }^{1}$

${ }^{1}$ RDKB, Moscow, Russian Federation, ${ }^{2}$ Dmitry Rogachev National Medical Research Center of Pediatric Hematology, Oncology and Immunology, Moscow, Russian Federation

Background: The number of children with non-malignant diseases that can be cured by allogeneic stem cells transplantation is growing every year. The choice of an optimal unrelated donor requires the study of factors affecting the outcome of treatment in this group of patients.

Methods: During the period 2003-2017 we performed 99 matched unrelated donors hematopoetic stem cells transplantations in children with non-malignant deseases. Gender distribution: male - 59\% $(n=58)$, female - $41 \%$ $(n=41)$. Age median - 5,8 years old (8 months - 17). Stem cell source: bone marrow - $80 \%(n=81)$, peripheral blood stem cells $-19 \%(n=19) .82$ patients $(83 \%)$ received $10 / 10$ matched unrelated donors hematopoetic stem cells transplantation and 17 patients (17\%) - 9/10 matched unrelated donors hematopoetic stem cells transplantation. Differences in the antigen blood system: single group $41 \% \quad(n=40)$, minor $32 \%(n=31)$, major $20 \%(n=18)$, mixed $11 \%(n=9)$. Age of donor: $18-25$ years old - $25 \%(n=16), 26-35-42 \%$ $(n=27), 36-45-23 \% \quad(n=15), 46$ and more $11 \%(n=7)$. Gender differences in donor/recipient: male/female $25 \%$ $(n=24)$, female/male $18 \%(n=18)$, one sex $57 \%(n=56)$. We also took into account the impact of gender difference and cytomegalovirus serostatus in the donor/recipient pair.

Results: In 10/10 group the estimated probability of overall 2-years survive was $76 \%$ and in the $9 / 10$ group 2- 
years survive was $63 \%$. The increase in donor age of 10 years reduces the 2 -years survive by $9-11 \% \quad(p=0,117)$, however, the 2-years survive from donors over 46 years old was $100 \%$. We have found no difference between 2-years survive in transplants from donors that are compatible/ incompatible with the antigen blood system, cytomegalovirus serostatus, or the gender differences in donor/ recipient. In the study of donor-related factors, we found the negative impact of an human leucincompatibility (9/10) on the incidence of chronic GVHD - 29\% ( $\mathrm{p}=0.019)$. The combination of cytomegalovirus positive serostatus of the donor and the negative status of the recipient increases the risk of primary graft rejection up to $50 \%$, in comparison with others $(\mathrm{p}=0.001)$.

Conclusions: Our study showed the role of genetic matching on the HLA system between the patient and the unrelated donor, and the donors age value. 10/10 transplants have better outcome and lower incidence of severe a. GvHD and ch. GVHD. Younger donor increases 2-years survive, but there is a significant increase in 2-years survive if the donor is over 46 years old.

Disclosure: Nothing to declare

\section{P351}

Allogeneic stem cell transplantation in chronic myelomonocytic leukemia. A single center experience

\section{Alejandro Avendaño Pita ${ }^{I}$, Ana Africa Martín-López ${ }^{I}$, Marta Martin-Izquierdo², Jesús María Hernández- Sánchez ${ }^{2}$, Sara Urrutia-Rodriguez ${ }^{3}$, Beatriz Albarrán ${ }^{4}$, Juan Carlos Castillo ${ }^{5}$, Daniel Rivera ${ }^{1}$, Javier Carrillo Checa $^{I}$, Eduardo Sobejano ${ }^{1}$, Victor Higuero ${ }^{1}$, Daniel Presa Morales ${ }^{I}$, Raul Azibeiro ${ }^{I}$, Marta Fonseca ${ }^{I}$, Beatriz Rey $^{I}$, Estefania Perez ${ }^{I}$, Monica Cabrero ${ }^{1}$, Lucía Lopez- Corral $^{l}$, Fermín Sánchez-Guijo ${ }^{1}$, María Dolores Caballero Barrigon ${ }^{1}$, Félix López Cadenas ${ }^{1}$, Jesús María Hernandez-Rivas ${ }^{1}$, María Díez-Campelo ${ }^{I}$}

${ }^{1}$ Hospital Universitario de Salamanca, Salamanca, Spain, ${ }^{2}$ Instituto Biosanitario de Salamanca (IBSAL), Salamanca, Spain, ${ }^{3}$ Complejo Asistencial Universitario de León, Leon, Spain, ${ }^{4}$ Hospital General Rio Carrión, Palencia, Spain, ${ }^{5}$ Complejo Asistencial de Segovia, Segovia, Spain

Background: Allogeneic stem cell transplantation (ASCT) is the only curative strategy in chronic myelomonocytic leukemia (CMML

Methods: Twenty of the 1000 patients who underwent to an ASCT in our center until June 2018 were CMML. We retrospectively analyzed this subgroup with IBM_SPSS statistics (version 21). We performed next generation sequencing using the Illumina sequence capture enrichment methodology with a laboratory design panel of 117 genes
Results: Clinical and biological characteristics are summarized in table 1. ASCT characteristics are specified in image 1.In terms of hematopoietic recovery, 20 patients (100\%) reach granulocytic graft and 19 (95\%) platelet graft with a median of 19 (12-28) days and 13,5 (10-36) respectively. Regarding chimerism, 18 (90\%) patients reach full donor chimerism in bone marrow and $16(80 \%)$ in peripheral blood.

\begin{tabular}{|c|c|c|c|c|c|}
\hline $\begin{array}{l}\text { Clinical } \\
\text { characteristics }(n=20)\end{array}$ & $\mathrm{N}(\%)$ & $\begin{array}{l}\text { Median } \\
\text { (range) }\end{array}$ & $\begin{array}{l}\text { Biological } \\
\text { characteristics }\end{array}$ & $\mathrm{N}(\%)$ & $\begin{array}{l}\text { Median } \\
\text { (range) }\end{array}$ \\
\hline Age (years) & & \multirow[t]{5}{*}{$\begin{array}{l}56,5 \\
(45-69)\end{array}$} & $\begin{array}{l}\text { Cytogenetic } \\
\text { alterations }\end{array}$ & $10(50 \%)$ & \\
\hline Sex -Male-Female & $\begin{array}{l}14(60 \%) \\
6(40 \%)\end{array}$ & & $\begin{array}{l}\text { Cytogenetic risk } \\
\text { (GESMD) - High } \\
\text { - Intermediate - } \\
\text { Low }\end{array}$ & $\begin{array}{l}3(15 \%) 5 \\
(25 \%) \\
12(60 \%)\end{array}$ & \\
\hline $\begin{array}{l}\text { Phenotype }(\mathrm{N}=19) \\
\text { Myelodysplastic } \\
\text { Myeloproliferative }\end{array}$ & $\begin{array}{l}7(35 \%) \\
12(60 \%)\end{array}$ & & $\begin{array}{l}\text { Molecular } \\
\text { alterations }\end{array}$ & $19(95 \%)$ & \\
\hline $\begin{array}{l}\text { WHO } 2016 \\
\text { classification - } \\
\text { CMML-0 - CMML-1 } \\
\text { - CMML-2 }\end{array}$ & $\begin{array}{l}7(35 \%) \\
3(15 \%) \\
9(45 \%)\end{array}$ & & $\begin{array}{l}\text { Median } \\
\text { mutations }\end{array}$ & & $\begin{array}{l}3,5(0- \\
7)\end{array}$ \\
\hline $\begin{array}{l}\text { Leukemic } \\
\text { transformation }(\mathrm{LT}) \\
\text { pre ASCT }\end{array}$ & $9(45 \%)$ & & $\begin{array}{l}\text { High molecular } \\
\text { risk mutations - } \\
\text { ASXL1 - NRAS - } \\
\text { RUNX1 } \\
\text { - SETBP1 }\end{array}$ & $\begin{array}{l}4(20 \%) 3 \\
(15 \%) 6 \\
(30 \%) \\
3(15 \%)\end{array}$ & \\
\hline \multirow[t]{4}{*}{ Time to LT (months } & & \multirow[t]{4}{*}{$\begin{array}{l}3,77(1- \\
10)\end{array}$} & $\begin{array}{l}\text { CPSS }(n=19)- \\
\text { Low/int-1 - Int-2/ } \\
\text { High }\end{array}$ & $\begin{array}{l}6(30 \%) \\
13(65 \%)\end{array}$ & \\
\hline & & & $\begin{array}{l}\text { CPSS-mol (n=19 } \\
\text { - Low/int-1 - Int- } \\
\text { 2/High }\end{array}$ & $\begin{array}{l}4(20 \%) \\
15(75 \%)\end{array}$ & \\
\hline & & & $\begin{array}{l}\text { Number of } \\
\text { previous } \\
\text { treatments }\end{array}$ & & $2(1-3)$ \\
\hline & & & $\begin{array}{l}\text { Previous } \\
\text { treatments - } \\
\text { Cytoreduction - } \\
\text { HMA - Intensive } \\
\text { Chemotherapy } \\
\text { (IC) - HMA - } \\
\text { HMA + IC - } \\
\text { IC + HMA }\end{array}$ & $\begin{array}{l}12(60 \%) \\
11(55 \%) \\
14(70 \%) \\
5(25 \%) \\
5(25 \%)\end{array}$ & \\
\hline
\end{tabular}

[[P351 Table] 1. Table 1 (Clinical and biological characteristics)]

Nine patients (45\%) relapsed with a median of 6,3 months (1-87) with different strategies at this point: in all cases we modulated immunosuppression, in 2 cases as the unique strategy, in 2 cases with donor lymphocyte infusion (DLI), in 3 cases we employed hypometilating agents (HMA) and in 2 cases with intensive chemotherapy, reaching CR only in two patients, one of them after DLI and the other one after HMA and consolidation with a second ASCT.

Eleven patients $(55 \%)$ died being the relapse the main cause $(64 \%)$. Transplant related mortality (TRM) at +100 were $5 \%$ and global TRM were $15 \%$. In the last follow-up, 9 patients $(45 \%)$ are still alive, $8(89 \%)$ in CR and $1(11 \%)$ in relapse situation. 
With a median follow-up of 58 months (5-138), the event free survival (EFS) were 19 months (5-32) and the overall survival (OS) were 56 months (0-116).

We observed advantage in terms of OS in those patients that reach $\mathrm{CR}$ at +100 post ASCT and in those who develop chronic GVCHD ( $p=0.065$ and $p=0.012$ respectively)

Conclusions: ASCT is still the only curative option despite the high relapse rates. To reach $\mathrm{CR}$ at +100 post ASCT and the development of chronic GVHD seems that they confer advantage in terms of OS. The importance of knowing the molecular profile of the entities that we consider for ASCT.

Disclosure: Nothing to declare

\section{P352}

HPC preparation using spinning membrane filtration for DMSO removal from multiple cryopreservation bags: A first experience

Vanessa Agostini', Mirna Selva ${ }^{1}$, Serena Mangianti, Marina Ratta ${ }^{1}$, Lia Zornetta ${ }^{1}$, Irene Brognara ${ }^{1}$, Francesco Lanza ${ }^{2}$

${ }^{1}$ Processing Laboratory Metropolitan Transplantatio Programme AUSL Romagna, Clinical Pathology, Pievesestina di Cesena, Italy, ${ }^{2}$ Head Section of Hematology and SCT, Ravenna, Italy

Background: Washing of HPC products prior to use is a known method for DMSO and dead cell debris removal. Products collected from cell separators in a 2016 study by Lecchi et al. have median final volumes of $420 \mathrm{ml}$ inclusive of cryopreservative solution with a maximum of $1542 \mathrm{~mL}$ and a minimum of $73 \mathrm{~mL}$ in a sample size of 912 . This study documents a first experience of a cell processing lab seeking to integrate process automation technology to wash and volume reduce products which can account for the initial material source volume variability, product characteristics, and number of bags.

Methods: Here we report the pre-clinical assessment of the lab's initial work with the LOVO Cell Processing System for a 5 product experience over 2 days with 1 machine. This study used products intended for destruction. The workflow used parallel and sequential processing schedule. After water-bath thawing, bags were sampled, weighed to determine volume, and subsequently connected to LOVO or pooled into a transfer pack and then connected to LOVO. The bags were then diluted $1: 1$ at $50 \mathrm{ml} / \mathrm{min}$ with LOVO at +4-8C using 6\% Hydroxyethylstarch 130/0.4 (Voluven, Fresenius Kabi) and processed using a 3 cycle procedure. After processing the bags were weighed for volume, sampled, and stored in a $4-8 \mathrm{C}$ refrigerator in their
LOVO final product bags. Samples were assessed from $\mathrm{T}=0$ to $\mathrm{T}=24$ hours.

Results: CD34+ viability and absolute counts were determined using flow cytometry. Processing duration and solution volume consumed was determined by the LOVO's sensors and confirmed by the operator. Data is presented as a percentage relative to the post-thaw values. Note, the values presented are not total process yields. The results focus on the LOVO processing step.

Conclusions: The operators easily integrated into the software to drive the machine. The machine demonstrated it's flexibility with a wide-range of volumes, cell-inputs, and number of bags. The LOVO produced products which meet our specifications in a quick and reliable manner. Further work on this platform will be performed to validate and qualify this system for production use.

\begin{tabular}{|c|c|c|c|c|c|c|c|c|c|}
\hline $\begin{array}{l}\mathrm{N} . \\
\text { of } \\
\text { bags }\end{array}$ & Method & $\begin{array}{l}\text { Starting } \\
\text { volume } \\
(\mathrm{mL})\end{array}$ & $\begin{array}{l}\text { Final } \\
\text { Volume } \\
(\mathrm{mL})\end{array}$ & $\begin{array}{l}\text { Post } \\
\text { wash } \\
\text { CD34+ } \\
\text { recovery } \\
\text { T0 }(\%)\end{array}$ & $\begin{array}{l}\text { Post } \\
\text { wash } \\
\text { CD34+ } \\
\text { recovery } \\
\text { T24 (\%) }\end{array}$ & $\begin{array}{l}\text { Post } \\
\text { wash } \\
\text { CD34+ } \\
\text { viability } \\
\text { T0 (\%) }\end{array}$ & $\begin{array}{l}\text { Post } \\
\text { wash } \\
\text { CD34+ } \\
\text { viability } \\
\text { T24 (\%) }\end{array}$ & $\begin{array}{l}\text { Source } \\
\text { processing } \\
\text { time } \\
\text { automated } \\
\text { (minutes) }\end{array}$ & $\begin{array}{l}\text { Wash buffer } \\
\text { consumed }(\mathrm{mL})\end{array}$ \\
\hline 1 & NA & 30 & 100 & $96 \%$ & $67 \%$ & $99 \%$ & $91 \%$ & 17 & 460 \\
\hline 3 & Pooled & 363 & 123 & $90 \%$ & $89 \%$ & $89 \%$ & $90 \%$ & 28 & 658 \\
\hline 1 & NA & 81 & 103 & $99 \%$ & $95 \%$ & $97 \%$ & $92 \%$ & 19 & 560 \\
\hline 2 & Parallel & 68 & 108 & $100 \%$ & $100 \%$ & $98 \%$ & $98 \%$ & 22 & 607 \\
\hline 2 & Sequential & 203 & 150 & $100 \%$ & $100 \%$ & $97 \%$ & $98 \%$ & 37 & 958 \\
\hline
\end{tabular}

[[P352 Table] 1. CD34+ recovery and viability postwash]

Disclosure: No conflict

\section{P353}

Oral mucositis prevention in allogeneic hematopoietic stem cell transplantation with the photobyomodulation: A single center case series

Michela Ansuinelli, Luisa Quattrocchi ${ }^{1}$, Walter Barberi', Roberto Pippi', Giulia Galassi', Mattia Brescini', Gianluca Tenore ${ }^{1}$, Federica Rocchetti ${ }^{1}$, Ahmed Mohsen ${ }^{1}$, Alessandro Del Vecchio ${ }^{\text {, Sara Pepe }}{ }^{I}$, Antonella Bruzzese ${ }^{1}$, Ursula La Rocca ${ }^{1}$, Umberto Romeo ${ }^{1}$, Anna Paola Iori ${ }^{1}$

${ }^{1}$ Policlinico Umberto I, Sapienza University, Italy,

Background: Oral mucositis $(\mathrm{OM})$ is an early adverse event of allogeneic hematopoietic stem cell transplantation (HSCT) that occurs approximately in $85 \%$ of cases, negatively affecting quality of life of patients. Photobiomodulation (FBM) has been proposed by the Multinational Association of Supportive Care in Cancer and the International Society of Oral Oncology for the prevention and treatment of chemotherapy-induced $\mathrm{OM}$, as a result of its anti-inflammatory, biomodulator and tissue repair 
properties. The aim of this study was to evaluate prospectively the efficacy of a FBM protocol for the prevention of OM in patients undergoing a HSCT.

Methods: All patients consecutively who underwent a HSCT at our Center from 201X onwards received five weekly FBM sessions with a bidiodic laser (Lumix $2^{\circledR}$, Prodent, Italy), which simultaneously emitted at $650 \mathrm{~nm}$ and $910 \mathrm{~nm}$ with a power of $89 \mathrm{~mW}$ and energy of $4 \mathrm{~J}$ per point. The procedure started the day before the beginning of the conditioning regimen up to the tenth day post-transplant. The laser was applied in a defocused mode on each of the mucosal surfaces (12 areas). At each session, the morphine dose, the OM level (according to the WHO scale) and pain through a Numerical Rating Scale (NRS) were recorded.

Results: 27 consecutive patients (19 male/8 female) submitted to a HSCT were analyzed. The median age was 44 years (range 4-66). Eighteen patients had acute leukemia, 3 myelodysplastic syndromes, 6 lymphoproliferative diseases. The median number of treatment lines before HSCT was 2 (range 1-5). At transplant, 13 patients had advanced disease. The myeloablative conditioning regimen MAC (Thyotepa, Busulphan, Fludarabine) was employed in 17 patients; the same conditioning, with a reduced dose of Busulphan (RIC), was infused in 10 patients. Seven patients $(26 \%)$ had no evidence of OM. The incidence of grade II-IV $\mathrm{OM}$ was $65 \%$ in the group of patients receiving MAC and the median duration 12 days (range 3-28); grade 4 OM was observed, for 1 day, in 1 patient. In the RIC group the incidence of $\mathrm{OM}$ was $50 \%$, the median duration 11 days (range 7-16); no patient had evidence of grade IV OM. In the whole population, the maximum NRS value was 4 . Morphine administration was required in 23 patients, due to the occurrence of non-oral complications.

Conclusions: In our experience, prophylaxis with FBM to prevent or reduce OM was safe and effective, compared to results of previous experiences reported in the literature, which used no prevention against this complication that negatively affects the quality of life of transplanted patients. Further studies on a large series of are necessary to confirm our results.

Disclosure: Nothing to declare

\section{P354}

The impact of genetic risk stratification in AML and MDS patients on overall survival after allogeneic hematopoietic stem cells transplantation: A single-center study

Ewa Karakulska-Prystupiuk ${ }^{1}$, Agnieszka Stefaniak ${ }^{2}$, Joanna Drozd-Sokotowska ${ }^{l}$, Anna Pastwińska ${ }^{l}$, Elżbieta Chmarzyńska ${ }^{2}$, Piotr Boguradzki, Monika Paluszewska ${ }^{I}$, Jarostaw Biliński ${ }^{1}$, Jadwiga Dwilewicz-trojaczek ${ }^{I}$, Wiestaw Wiktor Jędrzejczak ${ }^{1}$, Grzegorz Basak ${ }^{1}$
${ }^{1}$ Medical University of Warsaw, Warsaw, Poland, ${ }^{2}$ Medical University of Warsaw, Cytogenetics Laboratory, Public Pediatric Teaching Hospital, Warsaw, Poland,

Background: Cytogenetic abnormalities are an essential part of prognostic systems in myeloid malignancies before hematopoietic stem cell transplantation (HSCT), however, their role in posttransplantation prognosis is unknown. The aim of this study was to assess the prognostic impact of genetic risk stratification of AML and MDS patients on posttransplantation course, which could be an additional tool in making decisions regarding preemptive therapy.

Methods: A retrospective analysis covering patients treated with allo-HSCT between 2012 and 2018.

Cytogenetic studies included karyotyping (C- and Gbanding) and fluorescence in situ hybridization (FISH). The number of analyzed cells exceeded European Cytogenetics Association guidelines (for each FISH at least 600 interphase nuclei were analyzed). Cytogenetic risk group in AML was assessed based on the ELN 2017 criteria.

Patients with MDS were stratified into three groups; favorable (good and very good prognostic score), intermediate, and adverse (poor and very poor) prognostic score according to IPSS-R 2012.

Results: 99 patients (82-AML, 17- MDS) were enrolled. 71 patients received myeloablative and 28- reduced intensity conditioning. The donors were unrelated for 71 patients and related for 28 .

6 patients $(7 \%)$ belonged to the favorable, $55(67 \%)$ intermediate and 21 (26\%- adverse category in AML group and respectively: $7(41 \%), 3(18 \%)$ and $7(41 \%)-$ in MDS group.

The estimated 1y overall survival (OS) reached $91.7 \%$ (95\%CI: 53.9-98.8) in good, 82.8\% (95\%CI: 69.4-90.7)intermediate and $71.1 \%(95 \% \mathrm{CI}: 50.4-84.4)$ in poor category and 3-y OS: $74.6 \%$ (95\%CI: 40-91), 73.3\% (95\%CI: 58.1-83.7) and 56\% (95\%CI: 35.6-72.2)(p = $0,14597)$, respectively.

Interestingly, the poorest survival was in patients with monosomy of chromosome 7 , which was present in 6 patients of whom 5 succumbed to refractory disease, while all patients who had deletion of long arm of chromosome 7 (del 7q)- are alive at the time of writing of this report after a median follow-up of 34 months (21-73).

Relapse was diagnosed in 31 patients $(31 \%)$, including; $13(42 \%)$ with adverse, 15 (48\%) with intermediate and 3 $(10 \%)$ with favorable cytogenetic risk.

Among 12 patients with a complex karyotype and/or cytogenetic evolution prior HCT: 8 patients (67\%)relapsed, including 6 (50\%)- who died.

Follow-up cytogenetic studies in relapse after transplantation were performed for 24 patients; 4 of them (17\%) had clonal evolutions of the original karyotype with additional 
abnormalities- (50\% died) and 7 (29\%) had new aberrations in cells without primary changes (all died).

In 18 patients $(18 \%)$ (8 unfavorable, 8 intermediate group) cytogenetic relapse was diagnosed by FISH analysis and they were treated with azacitidine (+/- DLI) achieving CR $(\mathrm{N}=9,50 \%)$, stabilization- $(\mathrm{N}-=3,16 \%)$, transient response $(\mathrm{N}=1)$, while 5 deceased).

Conclusions: Cytogenetic studies in patients after transplantation may facilitate assessment of mortality.

Karyotype may undergo cytogenetic evolution after alloHSCT. Patients with monosomy of chromosome 7 seem to have a particularly poor prognosis. Transplanted patients are vulnerable to new cytogenetic alterations.

Disclosure: Nothing to declare

\section{P355}

Abstract already published.

\section{P356}

Efficacy and safety of oral NEPA (netupitant/ palonosetron) in patients with haematological malignancies receiving high dose conditioning regimen and hematopoietic stem cell transplantation (HSCT)

Clara de Risi ${ }^{1}$, Anna Mele ${ }^{1}$, Domenico Pastore ${ }^{2}$, Antonino Greco $^{1}$, Giuseppina Greco ${ }^{1}$, Silvia Sibilla ${ }^{1}$, Rosa De Francesco $^{1}$, Mariarosaria Morciano ${ }^{1}$, Eleonora Prete ${ }^{1}$, Stefania Citiso ${ }^{1}$, Eleonora La Rosa ${ }^{1}$, Alessandro Spina ${ }^{2}$, Francesca Merchionne $^{2}$, Vincenzo Pavone ${ }^{I}$

${ }^{1}$ Card. G. Panico Hospital, Haematology, Tricase, Italy, ${ }^{2}$ Ospedale Perrino, Haematology, Brindisi, Italy

Background: Second generation serotonin receptor antagonists (5-HT3RA) and neurokinin 1 receptor antagonists (NK-1RA) have improved the prevention of acute and delayed Chemotherapy Induced Nausea and Vomiting (CINV) in patients undergoing Multiple-Days (MDs-CT) or High-Dose Chemotherapy (HD-CT).

Methods: The primary end-points were the rate of Complete Response (CR), defined as no emesis and no nausea without rescue medications, for both acute (CR-24) and delayed (CR 25-120) CINV and rate of post-transplant complications until discharge. We prospectively analyzed 61 patients undergoing Autologous (85\%) and Allogeneic (15\%) Stem Cell Transplantation and receiving CINV prophylaxis with NEPA and dexamethasone (schedules shown in fig.1). In our series, 30 patients $(49 \%)$ were female. Patients median age was 55 years (20-74). The most frequent diagnosis were Myeloma (46\%) and Lymphoma (39\%), while $15 \%$ of patients were diagnosed with AML or MDS. Myeloma patients received one day HD-CT with Melphalan (75\% Mel200/25\% Mel140). Lymphoma patients were conditioned with FEAM $(87,5 \%)$ or TT_FLU_EDX (12,5\%) HD-CT. Busulfan-based MDs-CT regimen was offered to AML/MDS patients.

Results: The incidence of CR-24 and CR 25-120 observed was $82 \%$ (50/61) and 47,5\% (29/61), respectively. More than grade 1 nausea and vomiting (according to CTAE-4), was reported in 36\% (22/61) and 5\% (3/61) of patients, respectively. Female sex was associated with an increased risk of acute (HR 19,6; p 0,037 95\% CI 7.9802.191) but not delayed (HR 1,58; p 0,06 95\% CI 200-2.042) CINV. Similar rate of CR24 and CR25-120 was observed in One-day HD-CT ( $82 \%$ and $50 \%$ ) compared to MDs-CT (89\% and $42,5 \%)$ group (pNS). Median lenght of stay was 23 days (15-51). No case of cardiotoxicity and no exitus was observed. The incidence of febrile neutropenia was $61 \%$ (70\% FUO; $22 \%$ sepsis; $18 \%$ pneumonia). Only one patient experienced an aGvHD on day +9 . Neutrophil $(>1000 / \mathrm{mcL})$ and Platelet $(>50000 / \mathrm{mcL})$ recovery occurred in median on day 11 (9-26) and on day 15 (4-45) respectively.

Conclusions: NEPA seems to be safe and effective in preventing acute and delayed CINV in patients receiving both one day HD-CT and MDs-CT as conditioning regimen for HSCT. More studies are needed to define the better 5HT3RA and NK-1RA combination and the better schedule in transplant setting.

Disclosure: "Nothing to declare

\section{P357}

Endothelial impaired function in post allotransplant setting: Evaluation of risk factors in a monocentric experience

Clara De Risi ${ }^{1}$, Anna Mele ${ }^{1}$, Eleonora Prete ${ }^{1}$, Giuseppina Greco $^{1}$, Silvia Sibilla ${ }^{1}$, Rossella De Francesco ${ }^{1}$, Antonino Greco $^{I}$, Stefania Citiso ${ }^{1}$, Eleonora La Rosa ${ }^{1}$, Maria Rosaria Morciano ${ }^{1}$, Vincenzo Pavone ${ }^{I}$

${ }^{1}$ Card. G. Panico Hospital, Haematology, Tricase, Italy

Background: VOD and TA-TMA represent two early endothelial complications occurring after allogeneic stem cell transplantation (SCT) sharing many pre-transplant risk factors. The aim of our study is to evaluate the impact of donor graft composition, engraftment kinetics and infections on the development of these endothelial complications (EC).

Methods: We retrospectively reviewed 55 consecutive SCT recipients at our institu- tion between January 2015 and June 2018. The median age was 48 years (range 17-65). Acute leukemia was diagnosed in 33 patients $(60 \%)$. Complete remission was documented in $63 \%$ of patients at transplant. Donor source was from HLA mismatched 
donor in $53 \%$ and from unrelated donor in $56 \%$ of the patients. HBV positive patients were $13 \%$ of the sample. Conditioning regimen was busulfan based in $63 \%$ of patients. Ursodeoxycholic acid and unfractionated heparin were given to all patients as VOD prophylaxis. Cyclosporine was used as GvHD prophylaxis. lymphocytic subpopulation analysis (CD3+, CD4+, CD8+ and CD16+/CD56 + ) and CD34+ cells count on the donor graft were performed using BD Facs Cantoll. All patients had routine monitoring for EBV and CMV PCR, hemolysis tests, creatinine and electrolyte panels, proteinuria, complete blood count, blood pressure and Schistocytes by direct examination until day +100 . The Fisher's exact test was used to compare categorical variables, while continuous variables were analyzed with Anova test. Diagnosis of VOD and TA-TMA were carried out by using EBMT and Cho criteria respectively.

Results: The incidence of very severe VOD and TMA was $13 \%(7 / 55)$ and $14,5 \%(8 / 55)$ respectively. CMV reactivations with viral load over $30.000 \mathrm{cv} / \mathrm{ml}$ was $27 \%$ and $5 \%$ in patients with and without EC, respectively ( $\mathrm{p}$ 0,041; HR 2,97 p0,0055 95\%CI 1,38-6,4). The median day to neutrophils (Ns) engraftment $(500 / \mathrm{ml}$ and $1000 / \mathrm{ml})$ was 14 vs 17 and 14,5 vs 18 in VOD/TMA group vs control group (p0,03 and 0,027, respectively). More rapid neutrophils engraftment ( $\mathrm{Ns}>500 / \mathrm{ml}$ and $\mathrm{Ns}>1000 / \mathrm{ml}$ within 13 days) was related to a higher risk of EC with a HR of 2,67 (p 0,015; 95\%CI 1,2-5,9) and 2,94 (p 0,006; 95\% CI 1,4-6,3). Patients with EC received a donor graft with a higher median numbers $(x 10 \mathrm{e} 6 / \mathrm{Kg})$ of CD3 + and CD8+ $(\mathrm{p}>0,05)$ and a lower numbers $(\mathrm{x} 10 \mathrm{e} 6 / \mathrm{Kg})$ of NK cells $(\mathrm{p}>0,05)$. Patients who received a CD8 + cells count $>12 \times 10 \mathrm{e} 6 / \mathrm{Kg}$ and NK cells count $<2,5 \times 10 \mathrm{e} 6 / \mathrm{Kg}$ presented a Relative Risk of EC of 2,37 (p 0,036; 95\% CI 1,06-5,3) and 2,35 (p 0,004; 95\% CI 1,02-5,4), respectively. There were no differences with respect to the other analyzed variables between patients who developed VOD/TMA compared with those who did not. (Pic_01)

Conclusions: CMV viremia, early neutrophils engraftment and donor NK and CD8 + cells infused are associated with the risk of VOD and TMA. Very few studies evaluated the link between these variables and the risk of developing such two complications. It could be interesting to investigate these relationships on larger series.

Clinical Trial Registry: Not applicable

Disclosure: Nothing to declare

\section{P358}

When the last hope turns out to be just as good as best: haploSCT following TBF conditioning, PT cyclophosphamide and tacrolimus as GVHD prophylaxis
Albert Esquirol ${ }^{1}$, Rodrigo Martino ${ }^{I}$, Irene Garcia Cadenas $^{1}$, Ana Garrido ${ }^{1}$, Silvana Novelli ${ }^{1}$, Miquel Granell $^{1}$, Silvana Saavedra ${ }^{1}$, Carolina Moreno ${ }^{1}$, Javier Briones $^{1}$, Salut Brunet ${ }^{1}$, Jorge Sierra ${ }^{1}$

${ }^{1}$ Hospital de la Santa Creu i Sant Pau, Barcelona, Spain

Background: Hematopoietic stem cell transplantation is an effective therapy for a variety of severe hematological diseases. In last decades, haploidentical SCT (haploSCT) followed by PTCy as GvHD prophylaxis has been reported as a valid alternative for patients who lack an HLA matched donor. We therefore analysed outcomes with this.

Methods: 38 patients without HLA-matched donor received a haploSCT between 04/13 and 08/18. Thiotepa $5 \mathrm{mg} / \mathrm{kg}$ (2 days), fludarabine $50 \mathrm{mg} / \mathrm{m} 2$ (3 days) and oral busulfan $1 \mathrm{mg} / \mathrm{kg} / 6 \mathrm{~h}$ (3 days, with PKD dose adjustments) was used as conditioning regiment; in patients $>55$ years busulfan administration was limited to two days. GvHD prophylaxis consisted of cyclophosphamide $50 \mathrm{mg} / \mathrm{kg}$ on day +3 and +4 , and tacrolimus as a continuous iv infusion from day +5 . All patients received PBSC as the stem cell source. Outcomes analysed were overall survival (OS), progression free survival (PFS); cumulative incidences (CI) of GvHD, relapse and non-relapsed mortality (NRM).

Results: Median ages was 54 (range 25-71). 53\% male and $47 \%$ female. Diagnoses were: AML 20 (53\%), MDS 7 (18\%), ALL 5 (13\%), HL and NHL 5 (13\%), and 1 MM $(3 \%) .37 \%$ ( $\mathrm{N} \mathrm{14})$ were transplanted in early disease status, while most cases $(63 \%)$ were in advanced status, including, second/third CR $(29 \%, \mathrm{~N} 11)$, one $(3 \%)$ in aplasia without progressive disease and $23 \%$ (N 9) had active/progression disease, $8 \%$ (N 3) had stable disease; four cases were second aloSCT. Thus, $50 \%$ of patients had a high or very high rDRI and 50\% had intermediate. EBMT score was $\leq 4$ was in $58 \%$ of patients ( $\mathrm{N} \mathrm{22}$ ). The donor was as son/ daughter in $50 \%, 29 \%$ a sibling and $21 \%$ a patient's mother.

Median time to neutrophil $(0.5 \times 10 \mathrm{e} 9 / \mathrm{L})$ and platelet $(>20 \times 10 \mathrm{e} 9 / \mathrm{L})$ recoveries were +20 and +27 days, respectively (G-CSF was not used). Only one patient had a primary graft failure attributed to anti-HLA donor specific antibodies. Median follow up in survivor is 22 months (range 2-63).

Overall survival is $81 \pm 6.5 \%$ (at 12 months) and $61 \pm 9 \%$ (at 63 months). PFS is $67 \pm 8 \%$ and $52 \pm 9 \%$ respectively. The cumulative incidence of relapse was $20 \%$ and $35 \%$, respectively, while NRM is $13 \%$ at 63 months.

Day +120 , grade $2-4$ acute GvHD were $13 \%$, while mild/ moderate and severe chronic GvHD were $6 \%$ and $3 \%$ respectively.

EBMT $\leq 4$ and first aloSCT were the only variables to clearly impact 60-months OS in univariate analysis. A combined covariate of ebmt $\leq 4$-no prior aloSCT vs other 
patients showed a 60 month OS $81 \pm 13 \%$ vs $38 \pm 12 \%$ (p 0.001 ), PFS $65 \pm 13 \%$ vs $35 \pm 11 \%$ (p 0.014 ) and NRM $0 \%$ vs $29 \%$ (p 0.008) but without impact on relapse $34 \%$ vs $35 \%$ (p 0.68).

Conclusions: HaploSCT with an age-adapted TBF conditioning regimen, PBSC and PTCy followed by tacrolimus, led to very encouraging results, mainly in patients with a low EBMT score and as a first aloSCT. Although formerly considered as a last aloSCT strategy, we now agree that the time has come to compare this strategy with HLA MUD (and even elderly sibling donors) in ongoing prospective randomized multinational trials.

Disclosure: Nothing to disclosure

\section{P359}

Autologous hematopoietic stem cell transplantation in acute myeloid leukaemia: Long-term outcome

Azizan Sharif', Tan Sen Mui ${ }^{1}$, Ong Tee Chuan ${ }^{1}$, Sharifah Shahnaz Syed Abdul Kadir ${ }^{1}$, Ho Kim Wah ${ }^{1}$, Law Kian Boon $^{2}$

${ }^{1}$ Hospital Ampang, Ampang, Malaysia, ${ }^{2}$ Hospital Ampang, Clinical Research Centre, Ampang, Malaysia

Background: Autologous Hematopoietic Stem Cell Transplantation (AutoSCT) for Acute Myeloid Leukaemia (AML) is increasing becoming a viable option for an increasing number of patients due to limited availability of matched sibling or unrelated donor for Allogeneic Hematopoietic Stem Cell Transplantation (AlloSCT). We examined the relevant long-term outcomes in our local patient cohort.

Methods: We retrospectively reviewed the data for all AutoSCT done for AML in our centre over a 17-years period between $1^{\text {st }}$ January 2001 until $31^{\text {st }}$ Dec 2017 from our electronic record. Patients with Acute Promyelocytic Leukaemia (APML) were excluded from this analysis. Patients were further stratified based on the number of high risk features present; not achieving complete remission (CR) following induction chemotherapy, high presenting total white cell count $\left(\mathrm{WBC}>100 \times 10^{6} / \mathrm{ml}\right.$, adverse cytogenetics (example: complex cytogenetics) and adverse molecular mutations (example: FLT3-ITD \& MLL gene arrangement). Outcome data including mortality (Overall survival (OS) and non-relapse mortality (NRM)) and morbidity (leukaemia free survival (LFS)) were recorded and analysed.

Results: A total of 64 patients were identified. Median age at diagnosis is 34-years old. The cohort comprised of 34 males and 30 females. The overall median OS and median LFS is 3.9 years and 2.2 years respectively. The NRM is $1.6 \%(1 / 64)$. There was no difference in the median OS and median LFS for the patients achieving CR following induction chemotherapy and those not in CR following induction chemotherapy; 4.4 years versus 3.9 years $(\log$-rank, $\mathrm{p}=0.9)$ and 3.4 years versus 2.1 years (Log-rank, $p=0.9)$ respectively. The median OS were statistically significant for patients with zero versus one and two and more high risk features present; 10.2 years versus 3.7 years versus 2.2 years (Log-rank, $\mathrm{p}=0.4$ ) respectively. However, the median LFS were not statistically significant for these three patient cohorts; 3.6 years versus 1.9 years versus 1.4 years $(\log -r a n k, p=0.7)$ respectively.

Conclusions: In our patient cohort, AutoSCT appeared to be a feasible option for patents with AML without matched sibling or unrelated donor available.

Disclosure: None to declare

P360

Hematopoietic recovery and transfusion need after haploidentical transplant in beta-thalassemia patients

\section{Tahir Shamsi ${ }^{\text {, Saqib Ansari }}{ }^{\text {, Tasneem Farzana }}{ }^{1}$}

${ }^{1}$ National Institute of Blood Disease \& Bone Marrow Transplantation, Karachi, Pakistan

Background: At present, the thalassemia free survival after matched-related and unrelated donor transplant is about 80$90 \%$.Despite of bone marrow donor registries and cord blood banks, $20-25 \%$ of the patients still do not find a suitable matched donor. Haploidentical stem cell transplant (HISCT) is an alternative transplant option for these patients. The aim of this study wasto determine the outcome of HISCT in thalassemic patients.

Methods: Between 2014-2018, 16 thalassemic patientsunderwent HISCT. The median age of patients was5 (18)years with male preponderance $(\mathrm{n}=10,62.5 \%)$. Across the gender and $\mathrm{ABO}$ mismatch transplants were done in $43.7 \%$ and $25 \%$ of patients. Stem cell source was bone marrow in $5(31 \%)$ while peripheral blood in $11(69 \%)$ of patients. Mean stem cell dose was $5.6 \pm 2.9 \times 10^{6}$ cells $/ \mathrm{kg}$ and mean volume of product was $188 \pm 60.58 \mathrm{ml}$. Preparative regimen included Anti-thymocyte globulin, Busulfan, Fludarabine and Cyclophosphamide.Graft versus host disease (GvHD) prophylaxis comprised of posttransplant Cyclophosphamide on day $+3 \&+4$ followed by Tacrolimus and Mycophenolate mofetil. Patients were observed for hematopoietic recovery (neutrophil and platelet engraftment) and transplant related mortality including acute and chronic GVHD for skin, gut, liverand lungs, primary and secondary graft failure and infectious complications. 
Results: Nine $(56.25 \%)$ of sixteenpatients were engrafted with full donor chimerism. Twelve $(75 \%)$ patients belonged to Pesaro class I and $4(25 \%)$ toClass II patients. Median time to neutrophil and plateletengraftment were13(11-20) and16(12-36)days respectively. Average number of packed red cell and platelet transfusions were $4.35 \pm 7.54$ and 20.8 \pm 19.18 respectively.Primary graft failure was observed in 3 $(19 \%)$ and secondary graft failure was observed in $4(25 \%)$ patients. Two patients received a second dose of stem cells and they engrafted at 20and 32 days of infusion respectively. 2 of 3 patients with primary graft failure died, one with sepsis (day +23) and the other because of intracranial bleeding (day +21$)$. Acute GvHDof gut and skin (grade II-III) was observed in 2 patients each, within first 100 days post-transplant. None of the patients had grade IV GvHD. Cytomegalovirus reactivation occurred in $50 \%$ of patients, all of them received pre-emptive therapy with intravenous Ganciclovir. None of them developed CMV disease. Invasive fungal infection was not observed in any of the patient. Culture proven bacterialinfection was documented in $62 \%$ of patients requiring intravenous antibiotics during first 100 days post-transplant.Overall survival and relapse free survival were $81.25 \%$ and $56.25 \%$ over a median follow-up of 500 (21-1757) days.

Conclusions: Haploidentical transplant is a suitable modality for thalassemic patients lacking a full matched donor in Pakistan. In view of our results, we suggest that thalassemia patients should be offered HISCTas an option for cure.

Clinical Trial Registry: Not applicable

Disclosure: Nothing to declare

\section{P361}

Gemtuzumab ozogamicin at low doses does not produce significant toxicities during induction therapy and after hematopoietic stem cell transplantation in adults with acute myeloid leukemia

Marta Peña ${ }^{1}$, Christelle Ferrà ${ }^{1}$, Ana Garrido ${ }^{2}$, Rosa Coll ${ }^{3}$, Mireia Morgades ${ }^{1}$, Marta Sitges ${ }^{1}$, Susana Vives ${ }^{1}$, Anna Torrent $^{1}$, Miriam Moreno ${ }^{1}$, Montserrat Batlle ${ }^{1}$, Laura Abril $^{1}$, Gladys Ibarra ${ }^{1}$, Juan Manuel Sancho ${ }^{1}$, Blanca Xicoy $^{1}$, Albert Oriol ${ }^{1}$, David Gallardo ${ }^{3}$, Jorge Sierra ${ }^{2}$, Josep Maria Ribera ${ }^{1}$

${ }^{1}$ Institut Català d'Oncologia-Hospital Germans Trias i Pujol, Josep Carreras Research Institute, Clinical Hematology, Badalona, Spain, ${ }^{2}$ Hospital de Sant Pau, Clinical Hematology, Barcelona, Spain, ${ }^{3}$ Institut Català d'Oncologia-Hospital Josep Trueta, Clinical Hematology, Girona, Spain

Background: Gemtuzumab ozogamicin (GO) is an antiCD33 monoclonal antibody with significant activity in de novo and relapsed/refractory $(\mathrm{R} / \mathrm{R})$ acute myeloid leukemia (AML). A relevant side effect consists of hepatotoxicity and especially sinusoidal obstruction syndrome (SOS). The objective of this study was to analyze tolerability of GO during the induction and reinduction therapy in patients with AML, and its possible impact on subsequent hematopoietic stem cell transplantation (HSCT).

Methods: From 2004 to 2017, 24 patients who had received $\mathrm{GO}$ in three hospitals were collected and their medical records were retrospectively reviewed.

Results: Fourteen patients diagnosed with de novo AML received GO $\left(3 \mathrm{mg} / \mathrm{m}^{2}\right)$ on day +1 in combination with standard chemotherapy (idarubicin and cytarabine, $3 \times 7$ schedule) as induction therapy. Hyperbilirubinemia (bilirubin $>1.5 \mathrm{UNL}$ ) was detected in 4 patients and increase of aspartate aminotransferase (AST) (>2.5 UNL) in 1. Twelve patients achieved complete remission (CR) and one was refractory ( 1 not evaluated). In the $R / R$ setting, 10 patients diagnosed with AML $(n=8)$, biphenotypic acute leukemia $(n=1)$ and acute promyelocytic leukemia $(n=1)$ received $\mathrm{GO}$ as 3 rd or subsequent rescue therapy either as monotherapy $(n=4)$ or in combination with cytotoxic chemotherapy $(n=5)$. Prior HSCT was performed in 5 patients (autologous [ $n=2]$, allogeneic $[n=3])$. Rescue therapy was indicated for refractoriness $(n=2)$, relapse $(n=5)$, partial response $(n=1)$ or absence of donor $(n=2)$. Four patients received 3 doses of GO ( 3 $\mathrm{mg} / \mathrm{m}^{2}$ ) and 3 patients, one dose. Hyperbilirubinemia $(>1.5 \mathrm{UNL})$ was observed in 1 patient and increase in AST (>2.5 UNL) in 2 patients. Seven patients achieved CR, 1 was refractory, 1 obtained partial response and 1 died early during induction).

Thirteen patients received subsequent HSCT (autologous [ $n=4]$, allogeneic $[n=9])$ after GO therapy (10 in the de novo $\mathrm{AML}$ and 3 in the $\mathrm{R} / \mathrm{R}$ group). The reasons for not performing HSCT in the remaining 11 patients were: low cytogenetic risk $(n=1)$, active chronic graft versus host disease (GvHD) in previous HSCT $(n=1)$, early death during treatment $(n=3)$, relapse $(n=1)$, severe complications in rescue treatments $(n=1)$, and unknown $(n=4)$. The conditioning regimen was myeloablative $(n=8)$, non-myeloablative $(n=4)$ and sequential $(n=1)$, and the donors were matched sibling $(n=6)$ or unrelated $(n=3)$. Cyclosporine, methotrexate and thymoglobulin were administered as GvHD prophylaxis in 7 patients and cyclosporine, mycophenolate and thymoglobulin in 3 . Hyperbilirubinemia was observed in 2 patients belonging to the de novo AML group. Death after HSCT occurred in 10 patients due to infection $(n=5)$, relapse $(n=3)$, GvHD $(n=1)$ and traffic accident $(n=1)$. Three patients are currently alive in remission. No SOS was observed in any patient. 
Conclusions: In both de novo and $\mathrm{R} / \mathrm{R}$ AML the administration of low dose GO is feasible and does not have impact on subsequent HSCT outcome. Although some degree of hepatotoxicity was observed, no cases of SOS were observed, either before or after HSCT.

Disclosure: Supported by grants from: Asociación Española Contra el Cáncer, AECC (GC16173697BIGA), Instituto Carlos III (PI14/01971 FI), 2017-SGR288 (GRC), CERCA Program from Generalitat de Catalunya, and "La Caixa" Foundation.

\section{P362}

Outcome of allogeneic hematopoietic stem cell transplantation in patients with benign hematological disorders

\section{Saqib Ansari ${ }^{I}$, Tahir Shamsi ${ }^{1}$, Uzma Zaidi ${ }^{1}$, Saima Siddiqui $^{\text {, }}$ Tasneem Farzana ${ }^{1}$}

\footnotetext{
${ }^{1}$ National Institute of Blood Disease \& Bone Marrow Transplantation, Karachi, Pakistan
}

Background: Allogeneic hematopoietic stem cell transplantation is a potentially curative treatment modality for hematological disorders. We evaluated the outcome of patients suffering from benign hematological disorders, including aplastic anemia, fanconi's anemia and thalassemia after matched related allogeneic transplantation.

Methods: All patients having hematological disorders including aplastic anemia (AA), beta thalassemia (BTM), fanconi's anemia (FA) and severe combined immune deficiency disorder (SCID) with HLA identical related donors who underwent allogeneic transplantation were included. Donors were given G-CSF at a dose of $10 \mu \mathrm{g} /$ $\mathrm{kg} /$ day daily for four days prior to harvest. The conditioning regimens for thalassemia included cyclophosphamide (CY) + busulfan (BU) in 21 (20\%), BU + CY + Thiotepa in 4 (4\%) and BU + CY + Antithymocyte Globulin (ATG) in $81(76 \%)$. Conditioning regimens for aplastic anemia included, Fludarabine (FLU) + CY in 38 (33\%), FLU + ATG in $28(25 \%)$ and CY in $38(33 \%), \mathrm{CY}+\mathrm{ATG}$ in 9 $(8 \%)$ patients. For Fanconi's Anemia FLU + ATG in 12 (52\%), FLU in $2(9 \%), \mathrm{CY}+\mathrm{ATG}$ in $4(17 \%)$ and FLU+ $\mathrm{CY}+\mathrm{ATG}$ in $5(22 \%)$. FLU + ATG in 2(25\%) and Cy given in $4(50 \%)$ and no conditioning regimen was offered to $2(25 \%)$ patients with SCID.

Results: A total of 250 allogeneic transplants were performed for benign hematological disorders including AA $(n=113)$, BTM $(n=106)$, FA $(n=23)$ and SCID $(n=8)$ from 2011 to July 2018. Median age was 7.5 years (range 0.5 48). Across the gender and ABO blood group transplants were $109(43.6 \%)$ and $80(32 \%)$. The median time to neutrophil and platelet recovery was 13 days (range: 9-46) and 18 (range: 10-35). Primary and secondary graft failure was observed in $34(13.6 \%)$ and 29 (11.6\%). Overall survival in aplastic anemia $(63 / 113,56 \%)$, beta thalassemia $(82 / 106,77 \%)$,fanconi's anemia $(15 / 23,65 \%)$ and severe combined immune deficiency disorder $(6 / 8,75 \%)$. Eighty four patients expired (33.6\%) among them 41 patients expired within 100 days post transplant Main cause of deaths included sepsis $(31 \%)$, multi organ failure $(6 \%)$ and Gut GvHD (5\%)

Conclusions: In developing world scenario where non malignant disorders are leading cause of morbidity and mortality. Bone Marrow Transplantation has been successfully implemented with better long term diseases free survival and quality of life.

Clinical Trial Registry: Not applicable

Disclosure: Nothing to disclose

\section{P363}

\section{Burden of chronic diseases at the end of life in} hematopoietic cell transplant survivors

Shaykhah Alotaibi', Riyad Elfakih ${ }^{1}$, Shahrukh Hashmi,

${ }^{1}$ King Faisal Specialist Hospital and Research Center, Riyadh, Saudi Arabia, ${ }^{2}$ Mayo Clinic Rochester, Minnesota, MN, United States

Background: Hematopoietic cell transplantation (HCT) remains the only curative therapy for many diseases, yet transplant survivors carry an unusually high burden of morbidities, primarily because of exposure to intense chemotherapy, radiation and /or GVHD. This study aimed to evaluate the burden of chronic diseases at the end of life after allogeneic-HCT and to identify the disability-adjusted life years (DALY).

Methods: The PubMed, MEDLINE, and OVID databases were queried utilizing specific MeSH terminology (post, allo stem cell, hematopoietic, bone marrow, transplantation).We collected data on the impact of the HCT on the variables affecting survivor's health in all aspects.The rates of late complications were compared to the risks in the general population (United States).

Results: A total of 7 studies fulfilled the selection criteria totaling to 6619 patients (Table 1). Median OS at 5-year mark varied widely between studies from $19 \%$ to $92 \%$. Majority of the patients at 10-year mark were found to have new comorbidities thereby indicating a huge burden of late effects at the end of life, though exact DALYs could not be calculated due to incomplete data.

HCT survivors were found to have higher risk of premature arterial disease (PAD) at $6.8 \%$ compared to the general population, however GVHD or the addition of TBI to the conditioning regimen were not found to be 
significantly associated with PAD. Regarding the risk of new cancers, the cumulative incidence of their development at 5 and 10 years was 1.71 , and 3.61 , respectively.

Increased risks compared with the general population were seen for some solid cancer including cancers of the lip: $\mathrm{P}=0.02$, tonsils: $\mathrm{P}=0.05$, oropharynx: $\mathrm{P}<0.01$, bone: $\mathrm{P}<$ 0.01 , soft tissue: $\mathrm{P}<0.01$, and vulva: $\mathrm{P}=0.01$.

With respect to mental health, depression was prevalent in $(10.8 \%)$ survivors, in whom $(82.5 \%)$ were still on antidepressants at the last follow-up. Cognitive impairment and other psychiatric disorders were found in $(2.4 \%)$ and $(2.7 \%)$ survivors, respectively.

The most common cause of NRM in the first 5 years was GVHD. However, after 10 years, the leading cause of death in those conditioned with MAC regimen was secondary cancer, but in the RIC group, new cancers and GVHD contributed equally.

Conclusions: HCT survivors remain at risk of significant complications which lead to premature death and their burden of comorbidities at the end of life is significantly more than that of general population.

\begin{tabular}{|c|c|c|c|c|c|}
\hline Authors & $\begin{array}{l}\text { sample } \\
\text { size }\end{array}$ & $\begin{array}{l}\text { median } \\
\text { age }\end{array}$ & female $\%$ & OS & TRM \\
\hline AboMourad et al. & 429 & 39 & 45.7 & $92 \%$ at 5 years & $\begin{array}{l}37 \% \\
\text { at } 1 \text { year }\end{array}$ \\
\hline Teixeira et al. & 99 & 35 & 39.4 & $\begin{array}{l}41.3 \% \text { at } \\
2 \text { years }\end{array}$ & $\begin{array}{l}37.4 \text { at } \\
2 \text { years }\end{array}$ \\
\hline Shimoni et al. & 1423 & 55 & 45 & $\begin{array}{l}100 \% \text { at } 2 \\
\text { years } 85 \% \text { at } 5 \\
\text { years (estimate }\end{array}$ & $\begin{array}{l}35 \% \text { MAC, } \\
20 \% \text { RIC at } \\
10 \text { years }\end{array}$ \\
\hline Koh et al. & 42 & 39 & 42 & $19 \%$ at 5 years & $\begin{array}{l}38 \% \text { at } \\
5 \text { years }\end{array}$ \\
\hline Ringdén et al. & 4269 & 53 & 41 & & \\
\hline Le et al. & 92 & 35 & 40 & $\begin{array}{l}41 \% \text { at } \\
10 \text { years }\end{array}$ & \\
\hline Tichelli et al. & 265 & 27 & 45 & $\begin{array}{l}71 \% \text { at } \\
20 \text { years }\end{array}$ & \\
\hline
\end{tabular}

[[P363 Table] 1. Table 1: Final selection of studies with outcomes]

Disclosure: Nothing to declare

\section{P364}

Hyperbaric oxygen therapy for post-transplant BK virus associated hemorrhagic cystitis in children and adolescents

\section{Oded Gilad ${ }^{1,2}$, Asaf Yanir ${ }^{1,2}$, Anat Yahel ${ }^{1}$, Shai Efrati ${ }^{2,3}$, Yair Bechor ${ }^{2,3}$, Jerry Stein ${ }^{1,2}$}

${ }^{1}$ Schneider Children's Medical Center of Israel, Petach Tikva, Israel, ${ }^{2}$ Sackler School of Medicine, Tel Aviv University, Tel Aviv, Israel, ${ }^{3}$ Assaf Harofeh Medical
Center, Sagol Center for Hyperbaric Medicine and Research, Zerifin, Israel

Background: Late onset Hemorrhagic cystitis (HC) is a common complication of hematopoietic stem cell transplantation (HSCT) frequently associated with reactivation of $\mathrm{BK}$ virus (BK-HC).There is no consensus as to the best therapy for $\mathrm{BK}-\mathrm{HC}$, and many different treatments have been reported. Hyper baric oxygen therapy (HBOT) is used as primary or adjuvant therapy in diverse clinical situations involving hypoxic injury to tissues and has been explored as a useful tool in treating $\mathrm{BK}$ - HC.

We report our experience with HBOT in combination with non-invasive supportive care in children and adolescents suffering from BK-HC following allogeneic HSCT.

Methods: The computerized database of Schneider Children's Medical Center of Israel was reviewed for all patients aged 0 to 21 years who underwent HSCT between January 2000 and June 2018 and developed BK-HC. HBOT therapy consisted of 2 hours sessions at 2 atmospheres, with patients breathing oxygen by mask. Parents accompanied patients during the treatment.

Results: Fourteen patients with a variety of underlying diseases received (HBOT) for treatment of BK-HC following HSCT. The initial treatment for children with BK-HC at our center prior to 2008 included continuous bladder irrigation and intravesicular instillation of various medications. Beginning in 2008, we adopted a non-invasive strategy that included the administration of oral anticholinergics (oxybutinin), systemic pain management, hyperhydration and the administration of weekly Cidofovir with Probenecid. HBOT was administered to patients who failed the above regimen. With this protocol, the average time of starting HBOT dropped from 13 (prior to 2008) to 9.8 days.

The median onset of HC was 33 days post HSCT. All patients were receiving immunosuppressive treatment at the onset of BK-HC. All patients suffered from macrohematuria with blood clots (grade III cystitis), 12 (92.3\%) experienced severe dysuria and $11(84.6 \%)$ urgency. BK viruria was present in all patients, and concurrent $\mathrm{BK}$ viremia was detected in $80 \%$ of those who were tested.

Patients reported symptomatic improvement at a mean of 3.6 days following the initiation of HBOT. No patient experienced serious adverse effects due to HBOT, but two patients required insertion of tympanic ventilation tubes. Eleven of our 13 patients $(84.6 \%)$ experienced complete remission of $\mathrm{BK}-\mathrm{HC}$ following $\mathrm{HBOT}$, with an overall response rate of $92.3 \%$ (12/13 patients).Eight of our patients $(61.5 \%)$ eventually succumbed due to either HSCT complications or disease relapse.

Conclusions: Hyperbaric oxygen therapy is a safe, effective, non-invasive and well tolerated treatment 
modality for BK -HC and should be considered for first line therapy for this complication of HSCT.

Clinical Trial Registry: .

Disclosure: Nothing to declare

\section{P365}

Haploidentical hematopoietic stem cell trasplantation with MACS: A feasible treatment for hematological malignancies in a peruvian pediatric hospital

\section{Carlos Vidurrizaga-De Amezaga ${ }^{l}$, Sergio Murillo-} Vizcarra ${ }^{2}$, Karina Rojas-Fernandez ${ }^{2}$, Carolina BenavidesVallve $^{I}$, Karina Pinto-Gossin ${ }^{1}$, Oscar Davila Carlin ${ }^{1}$

${ }^{1}$ V\&C Biotec SAC, Cell Therapy Laboratory, Lima, Peru, ${ }^{2}$ Instituto Nacional de Salud del Niño de San Borja, Lima, Peru

Background: Every year, almost one thousand cases of hematological malignancies in pediatric population are reported in Peru. Allogeneic Hematopoietic Stem Cell Transplantation (allo-HSCT) is an alternative strategy in many of these cases. Only between $20-30 \%$ of the pediatric population that requires a HSCT has a compatible Human Leukocyte Antigen (HLA) donor. The remaining 70\% have to access international donor registries, extending the awaiting time and conditioning the progress of the disease.

Allo-HSCT has the potential to help children with several hematological disorders with non-compatible HLA donor. HLA genotypically identical sibling donors are the best option when pursuing an HSCT. Nevertheless, patients' alternative sources of stem cells could be obtained from an haploidentical donor like one of their parents.

The Haploidentical Transplantation Program with MACS was implemented in Peru in 2016 to reduce the risk of Graft-versus-Host Disease (GvHD), support the immune system reconstitution and to expand pool of donors. It allows patients to access a treatment that is efficient and safe, as shown in the depletion of positive TCR $\alpha / \beta+$ and $\mathrm{B}$ cells procedures for allo-HSCT.

Methods: The mobilized leukapheresis products $(n=19)$ of haploidentical healthy donor was washed to remove platelets and preparations were performed according to Miltenyi's CliniMACS ${ }^{\circledR}$ manual for TCR $\alpha / \beta+$ and CD19+ cell depletion.

Analysis of the initial leukapheresis product and TCR $\alpha / \beta$ + and CD19+ depleted graft (target and non-target product) was performed using flow cytometer. Cells were analyzed for $\mathrm{CD} 3+, \mathrm{CD} 45+, 7-\mathrm{AAD}, \mathrm{CD} 20+, \mathrm{TCR} \alpha / \beta+, \mathrm{TCR} \gamma / \delta$ ,$+ \mathrm{CD} 34+$ and $\mathrm{CD} 133+$ with fluorochrome-labeled antibodies from Miltenyi Biotec using a NovoCyte Cytometer.
Results: The results obtained with the ex vivo T-cell depleted allo-HSCT procedures show an overall survival (OS) over 70\% with an IC95\% at the end of the first year with low incidence of GvHD. MACS of TcR $\alpha / \beta+$ and CD19+ cells are effective $(\log \mathrm{P} 3.9$ and $\log \mathrm{P} 3.3$, respectively) obtaining minimum levels of depleted lymphocytes within clinical established parameters for diverse pathologies. In addition, $\mathrm{TcR} \alpha / \beta+$ and $\mathrm{CD} 19+$ cell depletion does not significantly affect hematopoietic stem cell populations such as CD34+ and CD133+ cells or $\mathrm{TcR} \gamma / \delta+$ cell population. CD34+ and TCR $\gamma / \delta$ cells are highly recovered $(93.15 \%$ and 88.76 , respectively), which contributes with a better engraftment after allo-HSCT.

Conclusions: Peruvian results oscillated within European ranges with an OS over $70 \%$. Our data suggests that the MACS method is an efficient, effective and safe strategy for haploidentical HSCT which has a remarkable cost-benefit ratio and makes it viable in countries of the Latin American region with Peruvian socio-economic characteristics. Evaluation of genoresistance for viral reactivation treatment has been implemented as a strategy to improve OS. Better results are achieved in patients after allo-HSCT with the validation of these tests in Peru.

Preliminary pharmacoeconomic evaluations allow us to establish Magnetic Activated Cell Sorting (MACS) as a promissory strategy compared to other alternatives for haploidentical HSCT.

It is necessary to increase the number of procedures in order to confirm efficacy and safety of MACS in a larger population.

Disclosure: All authors have no conflicts of interest.

\section{P366}

Abstract already published.

\section{P367}

Micro-costing study of hematopoietic stem cell transplantation in two hospital institutions from southern of Brazil

Claudia Caceres Astigarraga ${ }^{1,2}$, Ana Paula Beck da Silva Etges $^{3}$, Ivaine Thais Sautier Sartor ${ }^{4}$, Tatiana Schnorr Silva ${ }^{4}$, Luciane Beatriz Kern ${ }^{4}$, Regina Kuhmmer Notti, Dora Fraga Vargas ${ }^{4}$, Lisandra Rigoni Della Costa ${ }^{1,2}$, Mariana Pinto Pereira ${ }^{1,2}$, Fabiano Barrionuevo ${ }^{4}$, Liane Esteves Daudt ${ }^{1,2}$, Alessandra Paz ${ }^{2}$, Luciane Nascimento Cruz $^{4}$, Maicon Falavigna ${ }^{4}$, Carisi Anne Polanzyck ${ }^{3,4,5}$

${ }^{1}$ Hospiital Moinhos de Vento, Porto Alegre, Brazil, ${ }^{2}$ Hospital de Clinicas de Porto Alegre, Porto Alegre, Brazil, ${ }^{3}$ IATS-National Health Technology Assessment Institute, CNPq, Porto Alegre, Brazil, ${ }^{4}$ Hospital Moinhos de Vento, 
Escritório de Projetos PROADI, Porto Alegre, Brazil, ${ }^{5}$ Hospital de Clinicas de Porto Alegre, Porto Alegre, Brazil

Background: Hematopoietic stem cell transplantation (HSCT) is a potentially curative treatment indicated for patients with onco-hematological, hereditary and immunological diseases. Considering the increase of patients indicated to the HSCT and the lack of knowledge about the costs resulting from this treatment, is important to identify and detail the resources consumed in each phase of HSCT and provide knowledge to the public health brazilian system. We aimed measure the total cost of related HSCT, based on micro-costing study of patients assisted in two hospitals in the South region of Brazil.

Methods: HSCT costs were estimated using the timedriven activity based costing method (TDABC), which measured cost of services / products based on actual consumption of resources. We collected data from medical records of 12 patients submitted to allogeneic HSCT in 2017 from public and private (philanthropic) hospitals. We interviewed professionals involved in the TCTH activities, we performed chrono-analysis and, we consulted financial and administrative systems reports of hospitals. In order to compare costs according to clinical complications observed in patients, we grouped into two ranges of complexity: low/ medium and high. The study was divided into stages: HSCT processes mapping; costs measurement; and analysis of results. Finally, the costs were compared: by activity, by resource and by hospital. This study was financed by PSIDUHS, by an agreement signed between Ministry of Health and Moinhos de Vento Hospital, through adjustment term number: 04 / 2014, and approved by Research Ethics Committees.

Results: From the HSCT processes mapping, the following steps were defined: (I) hospitalization; (II) conditioning; (III) transplantation; (IV) period of aplasia; (V) engraftment; (VI) observation; (VII) pre- and medical discharge. Seven patients were classified in low / medium complexity level, with hospitalization median time of 41 days and an median cost of USD 66,278.29, whereas the other five patients, classified as high complexity, presented median time of 101 days and median cost of USD 300,367.13. The HSCT costs evaluation identified that steps II and IV presented greatest cost in high complexity patients. Lower complexity patients presented, in steps II and IV, median costs of USD 44,274.52 while in higher complexity USD 100,226.26. In addition, median costs of materials and drugs were USD 13,926.02 and USD $181,094.15$ in lower and higher complexity patients.

Conclusions: TDABC method allowed the identification of the moment when patients consume the most resources. Of all the HSCT stages, periods of conditioning and aplasia presented higher costs, representing $76.90 \%$ of the total hospitalization value. In these stages, higher complexity patients presented three times higher the median cost. The resources that had the greatest impact were medicines and medical materials, costing 120 times more than lower complexity patients. Conclusion: This study allowed a detailed identification of the HSCT costs in patients with different complexity ranges in two hospitals from southern Brazil. Therefore, the identification of service demand regarding the clinical complexity, allows the generation of important information for the management of the best care in the health service.

Disclosure: Nothing to declare

\section{P368}

\section{Successful transplantation in Irba deficiency with multiple infections}

Vincenzo Pintabona $^{I}$, Giulia Carracchia ${ }^{1}$, Elena Soncini, ${ }^{1}$, Pierpaolo Dal Canton', Annalisa Agostini', Rosanna Ceresoli $^{1}$, Arnalda Lanfranchi, Fulvio Porta ${ }^{1}$

\section{${ }^{1}$ ASST Spedali Civili Brescia, Brescia, Italy}

Background: Immunodeficiency due to LRBA deficiency is characterized by hypogammaglobulinemia and autoimmunity. Hemolytic anemia, lymphadenopathy, autoimmune hepatitis and, above all, autoimmune enteropathy are the fundamental characteristics of these patients together with the history of recurrent invasive infections. The therapy includes immunosuppressants, endovenous immunoglobulins. Bone marrow transplantation is the final therapy of these patients, especially in the most serious cases and in recent years, also on the light of increasingly targeted conditioning regimes, it is associated with ever better prognosis.

Methods: We present the case of Caterina, an 8-year-old patient with LRBA deficiency, diagnosed at 5 years of age for a history of hypogammaglobulinemia, recurring invasive, bacterial and fungal infections and a picture of autoimmunity represented by AHIA, myelitis (C3-C5), autoimmune hepatitis and enteropathy in treatment with abatacept and sirolimus. It also presents leptin deficiency lipodystrophy. She presented to our observation for ostemielitis in multiple outbreaks (tibia, femur and left knee) secondary to sepsis from MRSA. Broad spectrum antibiotic therapy and curettage surgery were performed during hospitalization.After 5 months of broad-spectrum antibiotic therapy (daptomycin, rifampicin, ceftaroline, cotrimoxazole, levofloxacin, dalbavancin) there was resolution of the infections.Because of the seriousness of the disease it is therefore decided to subject Catherine to Hematopoietic Stem Cell Transplantation. 
Results: Therefore were performed bone marrow transplant from MUD after conditioning at reduced intensity, delayed in 9 days, with treosulfan, fludarabine and thiotepa. Prophylaxis for GVHD was performed with ATG $(5 \mathrm{mg} / \mathrm{kg}$ / day), sirolimus (already practiced for enteropathy) and MMF.

Because of the hepatic picture, we also performed prophylaxis for VOD with defibrotide for 21 days.

Transplantation was performed by peripheral stem cells with $18 \times 10^{\wedge} 6 \mathrm{CD} 34 / \mathrm{kg}$ and $30 \times 10^{\wedge} 6 \mathrm{CD} 3 / \mathrm{kg}$. The patient had always presented good general conditions with engrafment of the PMN to the D +13 and the PLT to the D +11 . The chimerism at $\mathrm{D}+20$ was $100 \%$ donor both on PMN and on PBL. Prophylaxis for GVHD was changed on $\mathrm{D}+7$ by replacing the sirolimus with tacrolimus for the appearance of grade I cutaneous GVHD .

Conclusions: We have successfully performed bone marrow transplantation in patients with LRBA deficiency. The new antibiotic molecules, used to induce infectious remission, the new low-intensity regimens, the prevention of the most fearful complications (VOD) have been the key to success in such complicated case. The high number of CD34 cells infused with a controlled number of CD3 were the key then of rapid engraftment with minimal GVHD readily controlled by the immunosuppressant.

Disclosure: No conflict of interest

\section{P369}

Haploidentical stem cell transplantation may be an option for hematological malignancies in urgent conditions

Osman Ilhan ${ }^{1}$, Guldane Cengiz Seval, Atilla Uslu', Sinem Civriz Bozdag ${ }^{1}$, Selami Kocak Toprak ${ }^{1}$, Meltem Kurt Yuksel, Pervin Topçuoglu ${ }^{1}$, Onder Arslan ${ }^{1}$, Muhit Ozcan $^{I}$, Taner Demirer ${ }^{1}$, Hamdi Akan ${ }^{1}$, Meral Beksac ${ }^{I}$, Gunhan Gurman ${ }^{1}$

${ }^{1}$ Ankara University School of Medicine, Hematology, Ankara, Turkey

Background: In the absence of HLA-matched related donor, allogeneic stem cell transplantation from haploidentical donors are potential alternatives for patients with hematological malignencies with an indication to allogeneic stem cell transplantation.

Herein, we retrospectively assessed the outcome of haploSCT for patients with refractory hematological malignancies.

Methods: This analysis included 27 consecutive patients who underwent haplo-SCT for various hematological malignancies at our center between October 2010 and May 2018. We used our institutional database to evaluate details and characteristics of patients and transplant outcomes.

Results: Demographic features of the patients and donors have been summarized in Table 1. All of the patients had advanced disease with a high risk of relapse. The majority of patients underwent haplo-SCT from their parents. Out of 24 patients, early transplant-related mortality was seen in this cohort of 5 patients. Four patients treated with second haplo-SCT and recovered hematopoiesis after second transplant. The remaining 19 patients were followed in a median of 4 months. Donor type ABO group switch was observed in a median of 45 days (30-60 days) after transplant. The median time for engraftment was 19 days (range, 15-60) for all patients. After the first transplant, 9 patients developed acute GVHD (37.5\%) with 7 patients having grade II-III acute GVHD. Five (18.5\%) had chronic GVHD, none of them with extensive manifestation. The prepative regimen was relatively well tolerated with limited regimen-related toxicity. CMV reactivation occurred in 11 patients (40.7\%) during the follow-up of the study. Eight patients (29.6\%) relapsed after a median of 132 days post transplant (range, 45-588 days). CR was achieved in 17 (63\%) patients after haplo-SCT. Mean estimated 5-year OS and PFS are $66.7 \% \pm 0.9 \%$ and $92.3 \% \pm 0.7 \%$, respectively.

Conclusions: Given the growing data on the similarity of outcomes after HLA-matched and haploidentical SCT, further studies are required to determine whether factors may be more important for donor selection than HLAmatching.

Clinical Trial Registry: -

Disclosure: Nothing to declare

\section{P370}

Outcome of allogeneic stem cell transplantation for hodgkin and non-hodgkin lymphoma: Single center experince from Turkey

Ayșe Uysal', Hale Bülbül', Nur Akad Soyer ${ }^{2}$, Mahmut Tobu $^{2}$, Murat Tombuloglu ${ }^{2}$, Guray Saydam ${ }^{2}$, Filiz Vural ${ }^{2}$

${ }^{1}$ Republic of Turkey, Ministry of Health, Health Sciences University, Trabzon Kanuni Training and Research Hospital, Hematology, Trabzon, Turkey, ${ }^{2}$ Ege University Medical Faculty, Hematology, Izmir, Turkey

Background: Allogeneic SCT (alloSCT) is generally optionally treatment choice for young and fit patients with relapsed/refractory lymphoma who were heavily pre-treated and after the failure of autologous stem cell transplantation (ASCT). Relapse after ASCT is associated with a poor prognosis and alloSCT is a potentially curative therapy for lymphomas which have relapsed after ASCT. 
Methods: In this study, we evaluated 19 patients with HL and NHL who had treated with allo-HSCT between November 2014 and December 2018 in Ege University Adult Hematology Transplantation Unit.

Results: Patients, disease and transplant characteristics were illustrated in table. Histologic subtype of NHL was evaluated as $\mathrm{T}$ cell lymphoma $(\mathrm{n}=8 ; 61,5 \%)$, mantle cell $(\mathrm{n}=2 ; 15,4 \%)$, diffuse large B-cell lymphoma $(\mathrm{n}=2 ; 15,4 \%)$ and B-cell lymphoma, unclassifiable, with features intermediate between DLBCL and classical Hodgkin lymphoma $(n=1 ; 7,7 \%)$. All histologic subtype of HL was determined as nodular sclerosing. The median number of prior treatments before allo-HSCT was 3 (range, 1-4). Twelve $(63,2 \%)$ patients had refractory disease, $3(15,4 \%)$ patients were in complete remission and $4(21,1 \%)$ patients were in partial remission before allo-HSCT. The median time from diagnosis to alloSCT was 24 (range, 8-144) months. Peripheral stem cell was used for stem cell source in all of them. Total body irritation plus fludarabine plus cyclophosphamide and busulfan plus cyclophosphamide were preferred most frequently for conditioning as non-myeloablative and myeloablative, respectively. Neutrophil engraftment was occurred median of 17 (range, 10-21) days. Graft versus host disease (GVHD) prophylaxis was applied all of them and cyclosporine plus methotrexate was preferred most frequently $(n=16 ; 84,2 \%)$. GVHD was occurred in $68,4 \%$ of them (42,1\% acute GVHD, 31,6\% chronic GVHD and $7,7 \%$ both). Veno-occlusive disease (VOD) was occurred in $2(10,5 \%)$ patients. Transplant related death was observed in $5(26,3 \%)$ patients. Overall survival (OS) and disease-free-survival (DFS) were evaluated as median 9 (range, 0-45) and 7 (range, 0-45) months, respectively. Analyze of OS and DFS was illustrated in figure. Six patients are alive without disease.

\begin{tabular}{|c|c|c|c|}
\hline \multicolumn{2}{|l|}{$\begin{array}{l}\text { Patients } \\
\text { Characteristics } \\
\mathrm{n}=19(100 \%)\end{array}$} & \multicolumn{2}{|l|}{$\begin{array}{l}\text { Transplant Characteristics } \\
\mathrm{n}=19(100 \%)\end{array}$} \\
\hline Median age (years) & $44(20-63)$ & $\begin{array}{l}\text { Donor type Related/ } \\
\text { Unrelated }\end{array}$ & $\begin{array}{l}14(73,7 \%) / \\
5(26,3 \%)\end{array}$ \\
\hline $\begin{array}{l}\text { Gender Male/ } \\
\text { Female }\end{array}$ & $\begin{array}{l}12(63,2 \%) / \\
7(36,8 \%)\end{array}$ & $\begin{array}{l}\text { Conditioning regimen } \\
\text { Myeloablative/ } \\
\text { Nonmyeloablative }\end{array}$ & $\begin{array}{l}7(36,8 \%) \\
12(63,2 \%)\end{array}$ \\
\hline Histology HL/ NHL & $\begin{array}{l}6(31,6 \%) / \\
13(68,4 \%)\end{array}$ & Median stem cell dose & $\begin{array}{l}6,39 \times 106 \\
(4,17- \\
10 \times 106)\end{array}$ \\
\hline $\begin{array}{l}\text { Advanced (III/IV) } \\
\text { Stage at presentation }\end{array}$ & $13(68,4 \%)$ & $\begin{array}{l}\text { Neutrophil engrafment Yes/ } \\
\text { No }\end{array}$ & $\begin{array}{l}15(78,9 \%) / \\
4(21,1 \%)\end{array}$ \\
\hline Prior ASCT & $12(63,3 \%)$ & $\begin{array}{l}\text { Acute GVHD/ } \\
\text { Chronic GVHD }\end{array}$ & $\begin{array}{l}8(42,1 \%) / 6 \\
(31,6 \%)\end{array}$ \\
\hline
\end{tabular}

[[P370 Table] 1. Patient, diease and transplant characteristics]

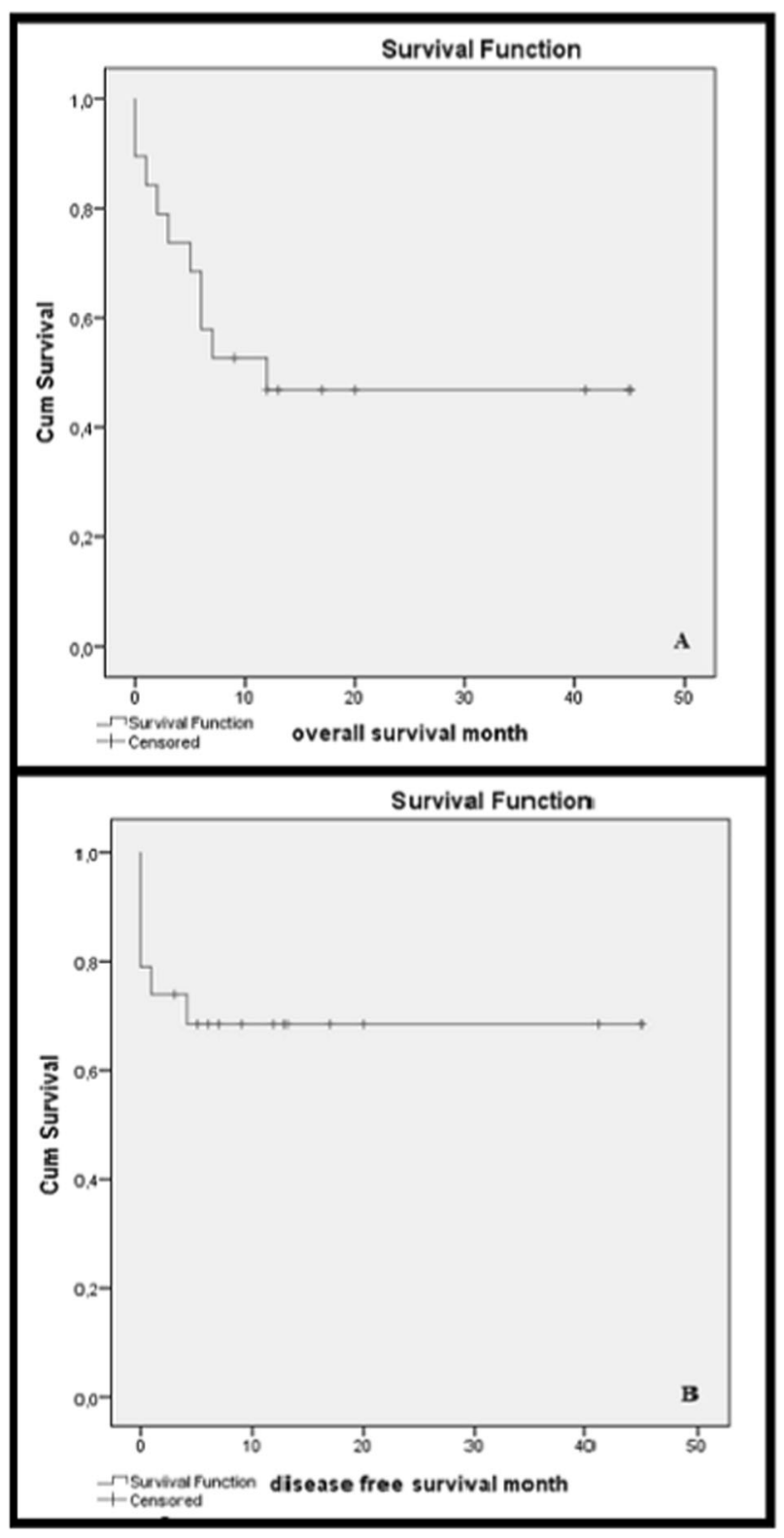

[[P370 Image] 1. Figure:Overall survival (A) and disease-free survival $(B)$ after allogeneic transplantation]

Conclusions: In recent years, the increased options of treatment for lymphoma especially the use of targeted therapies had improved life expectancy. However, R/R lymphoma or after the failure of ASCT have poor prognosis. In this group allo-HSCT may be the only curative approach. The patient group should be selected well because of the risk of transplant-related mortality.

Disclosure: The authors declare no conflicts of interest.

\section{P371}

Successful treatment for relapsed adult Philadelphia- 
positive acute lymphoblastic leukemia after bone marrow transplant with ponatinib

\section{Giulia Palazzo ${ }^{I}$, Giovanni Pisapia ${ }^{I}$, Tiziana Urbano ${ }^{I}$, Addolorata Tamburrano ${ }^{I}$, Patrizio Mazza ${ }^{I}$}

${ }^{1}$ Ospedale S.G. Moscati, Ematologia, Taranto, Italy

Background: The overall survival of patients with Philadelphia chromosome positive acute lymphoblastic leukemia $(\mathrm{Ph}+\mathrm{ALL})$ who have undergone allo-HSCT exceeds 50\%, with risk of relapsed in half of patients thus improvement of postremission therapy is crucial.

Methods: We describe the case of 44 year old a patient with relapsed Philadelphia-positive acute lymphoblastic leukemia post unrelated bone marrow transplant treated with ponatinib +117 days after transplant he experienced molecular, minimale residual desease relapse and initial graft failure.

After a rapid tapering of immunosuopressive therapy was underwent a therapy with ponatinibat dose of $45 \mathrm{mg} / \mathrm{die}$

Results: Afther a month of therapy we observed a rapid decrease in minimal residual disease on molecular assessment with an MMR of P190-BCR-ABL/ABL non detectable confirmed by bone marrow revaluations at days +190 , +222 nd +244 after the salvage therapy.

The patient has not had experienced of graft-versus-host disease, Ponatinib treatment was well tolerated and considered safe with easily manageable side.

Conclusions: Maybe in the era of tyrosine kinase inhibitors (TKIs), philadelphia chromosome positive acute lymphoblastic leukemia ( $\mathrm{Ph}+\mathrm{ALL})$ it could benefit from a combined treatment between transpalnt and TKIs however more studies are needed to confirm these hypotheses.

Disclosure: nothing to declare

Immunodeficiency diseases and macrophage

\section{P372}

Allogeneic hsct in HIV-1 infected patients with hematological disorders: Multicenter experience from GETH (Spanish group of HSCT and cell therapy)

\section{Mi Kwon ${ }^{1,2}$, Rebeca Bailén ${ }^{1,2}$, Pascual Balsalobre ${ }^{1,2}$, Manuel Jurado ${ }^{3}$, Arancha Bermúdez ${ }^{4}$, Jon Badiola ${ }^{3}$, Albert Esquirol ${ }^{5}$, Pilar Miralles ${ }^{1,2}$, Elisa López- \\ Fernández ${ }^{3}$, Lucrecia Yáñez ${ }^{4}$, Mercedes Colorado ${ }^{4}$, Jaime Sanz ${ }^{6}$, José Luis Piñana ${ }^{6}$, Nieves Dorado ${ }^{1,2}$, Laura \\ Solán $^{1,2}$, Carolina Martínez-Laperche ${ }^{1,2}$, Ismael Buño ${ }^{1,2}$, Javier Anguita, ${ }^{1,2,7}$, José Luis Díez-Martín ${ }^{1,2,7}$}

${ }^{1}$ Hospital General Universitario Gregorio Marañón, Madrid, Spain, ${ }^{2}$ Instituto de Investigación Sanitaria Gregorio Marañón, Madrid, Spain, ${ }^{3}$ Hospital Universitario Virgen de las Nieves, Hematology and Hemotherapy, Granada, Spain, ${ }^{4}$ Hospital Marqués de Valdecilla, Santander, Spain, ${ }^{5}$ Hospital de la Santa Creu I Sant Pau, Hematology and Hemotherapy, Barcelona, Spain, ${ }^{6}$ Hospital Universitari i Politècnic La Fe, Hematology and Hemotherapy, Valencia, Spain, ${ }^{7}$ Universidad Complutense de Madrid, Madrid, Spain

Background: Although a number of patients with HIV infection and hematological disease have successfully undergone allogeneic HSCT together with combination anti-retroviral therapy (cART), short and long-term outcomes remain not well known. We report the largest Spanish experience of HIV-infected adult patients with high-risk hematological malignancies with allogeneic HSCT.

Methods: We retrospectively reviewed 22 HIV-positive patients who received allogeneic HSCT between 1999 and 2018 in 5 Spanish centers within GETH (Grupo Español de Trasplante Hematopoyético y Terapia Celular).

Results: Baseline and transplant characteristics of patients are shown in Table 1. Median age was 44 years and $77 \%$ of the patients were men. The most frequent underlying malignancies were non-Hodgkin lymphoma $(9$, $41 \%)$ and AML (7, 31\%). In half of the patients an HLAidentical sibling was the donor; and in the other half, an alternative donor was used. Peripheral blood was used as graft source in $86 \%$ of the transplants. At the time of HSCT, all patients had been receiving suppressive cART for a median of 6 years and only 2 of them showed detectable plasma HIV RNA, one of them because of poor adherence to cART together with the accumulation of multiple resistance mutations; and the other patient had detectable HIV RNA at low levels $(<150$ copies $/ \mathrm{mL})$. All patients received cART throughout the transplant procedure, being temporally stopped in two patients due to significant mucositis. After a median follow-up of 65 months (8112), 5-year overall survival (OS) and event-free survival (EFS) were $46 \%$. NRM was $14 \%$ at 12 months and relapse was $24 \%$ at 24 months. Grade II-IV aGvHD rate was $40 \%$, and moderate/severe cGvHD rate was $41 \%$ at 24 months. A significant proportion of patients (68\%) showed infectious complications with viral infections as the most frequent cause. Two patients had invasive aspergillosis and one patient presented disseminated tuberculosis. Causes of death included infections (50\%), relapse (43\%) and toxicity (7\%). Among the 6 patients who died due to infections, 3 had severe chronic GVHD and were under immunosuppressive therapy. Two patients showed severe toxicity related to drug interaction with anti-retroviral therapy. All survivors except one showed undetectable HIV load at last follow-up after HSCT. 
Conclusions: Allogeneic HSCT is an effective therapy for high-risk hematological malignancies in patients with HIV infection, providing long-term disease free survival together to long-term HIV suppression with cART. However, drug interactions with anti-retroviral agents, occurrence of GVHD, and frequent infectious complications account for a complex procedure in this population. Selected HIV-infected patients with hematological malignancies should be considered for allo-HSCT when indicated, in experienced centers, with a multidisciplinary care.

Disclosure: Nothing to declare

\section{P373}

unrelated umbilical cord blood transplantation for primary immunodeficiency: An improved experience in China

Xiaowen Zhai ${ }^{1}$, Xiaowen Qian ${ }^{1}$, Hongsheng Wang ${ }^{1}$, Wenjin Jiang ${ }^{1}$, Ping Wang ${ }^{1}$, Xiaochuan Wang ${ }^{1}$, Jinqiao Sun ${ }^{1}$, Ying Huang ${ }^{I}$

${ }^{1}$ Children's Hospital of Fudan University, Shanghai, China,

Background: Primary immunodeficiencies (PID) are rare diseases often associated with genetic defects in the immune system, predisposing individuals to recurrent infections and increased risk of allergy, autoimmunity and malignancy. Allogeneic haematopoietic stem cell transplantation (HSCT) has been successfully used as a curative therapy for most severe forms of PID. Because PID is a genetic disease, $<25 \%$ of these children will have a healthy, human leukocyte antigen (HLA) matched sibling donor available, and umbilical cord blood grafts from unrelated donors are a suitable alternative cell source. We report the results of umbilical cord blood transplantation (UCBT) performed in 112 patients with PID between 2014 and 2018 at Children's Hospital of Fudan University in China.

Methods: 112 patients included chronic granulomatous disease (CGD, $n=39$ ), severe combined immunodeficiency (SCID, $\mathrm{n}=23$ ), interleukin-10 receptor-a deficiency (IL10RaD, $\mathrm{n}=27$ ), Wiskott-Aldrich syndrome (WAS, $\mathrm{n}=7$ ), leukocyte adhesion deficiency ( $\mathrm{LAD}, \mathrm{n}=5$ ), severe congenital neutropaenia ( $\mathrm{SCN}, \mathrm{n}=3$ ) and other immunodeficiencies $(n=8)$. All patients were assessed by clinical immunologist to confirm clinical phenotype and genetic diagnosis. Median age of 112 patients was 13 months (range, 2 to 144 months), and median body weight was 8.75 $\mathrm{kg}$ (range, 3.2 to $36 \mathrm{~kg}$ ). All patients received a $\leq 3 / 10$ HLA alleles-mismatched cord blood unit, 16 were HLA fully matched, 28 were $9 / 10$ matched, 49 were $8 / 10$ matched and 19 were 7/10 matched. Median nucleated cells of the cord blood were $14.23 \times 10^{7} / \mathrm{kg}$ (range, 3.55 to $51.5 \times 10^{7} / \mathrm{kg}$ ), and median CD34+ cells were $3.86 \times 10^{5} / \mathrm{kg}$ (range, 0.73 to $30.27 \times 10^{5} / \mathrm{kg}$ ).

Results: Median follow-up time was 13 months (range, 1 to 57 months), the overall survival rate at 1 year for all patients was $74.6 \%$, and was $83.7 \%, 78.3 \%$ and $60 \%$ for CGD, SCID and IL-10RaD, respectively. 27 patients died, Most deaths $(19 / 27,70.4 \%)$ occurred in +100 days after transplantation, the main cause of death was infection (24/ 27, 88.9\%). 87/112 (77.7\%) patients engrafted, median time of neutrophil engraftment was 24 days (range, 11 to $60 \mathrm{~d}$ ), and median time of platelet engraftment was 36 days (range, 9 to 59 d). The cumulative incidence of grade 3-4 acute GVHD was $8.9 \%$, and that of chronic GVHD was $10.7 \%$.

Conclusions: Unrelated UCBT should be considered for PID patients without an HLA -matched sibling donor. Effective control of infection before and after transplantation is important for improving survival.

Disclosure: Nothing to declare

\section{P374}

Single centre outcome of HSCT for dock 8 deficiency

Peter McNaughton ${ }^{1}$, Su Han Lum ${ }^{1}$, Stephen Owens ${ }^{1,2}$, Terry Flood, ${ }^{1}$, Sophie Hambleton ${ }^{1,2}$, Andrew Cant ${ }^{1}$, Mary Slatter $^{1,2}$, Andrew R Gennery ${ }^{1,2}$

${ }^{1}$ The Great North Children's Hospital, Newcastle upon Tyne, United Kingdom, ${ }^{2}$ Institute of Cellular Medicine, Newcastle University, Newcastle upon Tyne, United Kingdom

Background: Dedicator of cytokinesis (Dock 8) deficiency causes a combined immune deficiency characterised by recurrent bacterial infections, susceptibility to viral infection, eczema, food allergies, vasculitis and increased risk of malignancy. Due to the high morbidity and mortality of the disease HSCT has been increasingly offered to patients as a potentially curative therapy ${ }^{1}$.

Methods: We retrospectively reviewed the outcomes of HSCT for patients with Dock 8 deficiency at Great North Children's hospital Newcastle upon Tyne between 2011 and 2018 (5 in published reference).

Results: Ten patients with Dock 8 deficiency were treated with HSCT (Median age 7.5y range 4.5-16.8y). Median duration of follow up was 4.5years (range 1.3-6.5y). There were a range of donor sources (3 MSD, 3 MUD and 4 TCR $\mathrm{ab} / \mathrm{CD} 19+$ depleted haploidentical), conditioning regimens (6 Treo-Flu, 4 Treo-Flu-Thiotepa) and serotherapy (5 alemtuzumab, 3 ATG+Rituximab, 2 none). One patient who received a $\mathrm{CD} 3+\mathrm{TCR}$ alpha beta/CD19+ depleted haploidentical transplant received add back T-cells with caspacide molecular safety switch (Bellicum 
Pharmaceuticals). Skin only aGVHD occurred in 3/10 patients (1x stage $1,2 \mathrm{x}$ stage 3 ). No patients had cGVHD.

Overall survival was $60 \%(6 / 10)$. Survival was comparable regardless of donor source. All deaths occurred within 13 months of transplant. The 4 patients who died had significant burden of disease pre-transplant: 1 patient had chronic liver failure secondary to cryptosporidial sclerosing cholangitis, 1 had a cirrhotic liver secondary to cryptosporidium, cerebral vasculitis, an axillary aneurysm and aortic vasculitis requiring grafting of an ascending aortic aneurysm, 1 was PN dependent for failure to thrive with a history of cryptosporidium infection and 1 had candida in a BAL pre-transplant. Causes of death in these patients were: respiratory failure $(\mathrm{n}=1)$, progressive encephalopathy $(\mathrm{n}=1)$, multi-organ failure with septic shock and encephalopathy $(n=1)$ and multiorgan failure and septic shock after treatment for TMA $(n=1)$. Two of these patients had reactivation of cryptosporidium prior to their death. Pretransplant cryptosporidium was associated with mortality (Graph 1).

One patient who survived had suffered from stroke pretransplant. One suffered from a basilar artery aneurysm 7 years post-transplant at 19 yo. At the time of latest follow up donor chimerism was $100 \%$ in 5/6 survivors and high level mixed in the other(100\% CD3, 89\% CD15 and 92\% CD19).

Conclusions: This single centre study of HSCT for patients is consistent with literature indicating that HSCT is a potentially curative therapy for patients with DOCK 8 deficiency. The increased morbidity associated with Cryptosporidial infection is likely to be a consequence of overall disease burden rather than an infection specific effect. This does however highlight the improved outcomes of transplant prior to development of multiple comorbidities and suggests that HSCT should be considered early. It is unclear whether the late occurrence of vascular complications after transplant were caused by a manifestation of disease which is not corrected by transplant or a result of vascular injury sustained pre-transplant.

Reference: AydinSE et al. Hematopoietic stem cell transplantation as treatment for patients with DOCK8 deficiency. J Allergy Clin Immunol Pract. 2018 Nov 1. pii: S2213-2198(18)30706-2. doi: 10.1016/j. jaip.2018.10.035. [Epub ahead of print]

Disclosure: Nothing to declare

\section{P375}

identification of infants with non-SCID immune deficiency by newborn screening for SCID - the memorial sloan kettering cancer center experience

Susan Prockop ${ }^{1}$, Barbara Spitzer ${ }^{1}$, Michael Walsh ${ }^{1}$, Julie Ruggiero $^{I}$, Jennifer Kennedy ${ }^{I}$, Farid Boulad ${ }^{I}$, Maria
Cancio $^{I}$, Kevin Curran ${ }^{1}$, Nancy Kernan ${ }^{I}$, Anndromachi Scaradavou ${ }^{I}$, Jaap Jan Boelens ${ }^{I}$, Richard O'Reilly ${ }^{I}$

${ }^{1}$ MSKCC, New York, NY, United States

Background: Newborn Screening (NBS) for Severe Combined Immune Deficiency (SCID) has become universal in the United States based on evidence that early identification of infants with SCID can translate into superior survival. New York State established NBS by identification of T-cell Receptor Excision Circles (TREC) in DNA from Guthrie cards. Infants with abnormal NBS are referred for repeat testing. Those confirmed as abnormal are referred for secondary evaluation. The first infant with SCID identified by NBS in New York State was born in December 2010. Between December 2010 and December 2018 we evaluated 35 infants referred to our institution for abnormal SCID NBS. Here we describe the diagnosis and treatment of this cohort.

Methods: Referred infants underwent testing for: immune phenotype ( $\mathrm{ab}, \mathrm{gd}$, naïve and memory $\mathrm{T}$ cell, B cell and NK cell numbers); functional activity of $\mathrm{T}$ and NK cells; maternal engraftment; adenosine deaminase (ADA) and purine nucleoside phosphorylase (PNP) enzyme activity; and genetic testing. Those with a confirmed diagnosis of SCID underwent either allogeneic hematopoietic stem cell transplant (HCT) or (if eligible) gene therapy (GT). Infants identified as having ADA deficiency as the etiology of their SCID received enzyme replacement therapy prior to proceeding to definitive therapy.

Results: Twenty-three (66\%) infants were confirmed to have SCID. Three (13\%) of these infants had a family history of SCID but would not have been identified without NBS. In addition, one infant, born prematurely at 28 weeks, was diagnosed as having PNP deficiency only after developing infections. This infant was identified by NBS but repeat testing at 32 weeks gestation was normal likely due to support of transient $\mathrm{T}$ cell production from exogenous enzyme provided by red cell transfusion.

Twelve infants with confirmed low TRECs had a nonSCID diagnosis: 5 with transient lymphopenia of infancy who normalized TREC, immune phenotype and function, 1 with prenatal exposure to 6-mecaptopurine (6-MP), 3 genetically confirmed with DiGeorge Syndrome, and 3 with prolonged lymphopenia. Of the three with prolonged lymphopenia, two had recurrent infections: one ultimately diagnosed with Ataxia Telangiectasia and one with absent TREC but near normal number of T cells, normal PHA but no specific antigen responses, and absent $\mathrm{B}$ cells who will be undergoing transplant in the near future. The third continues with absent TREC, short telomeres, low numbers of $\mathrm{a} / \mathrm{b} \mathrm{T}$ cells, presence of $\mathrm{g} / \mathrm{d} \mathrm{T}$ cells, vaccine responses and freedom from infection with no identified genetic etiology. 
Conclusions: In summary, $30 \%$ of the patients referred to MSK with confirmed abnormal NBS for SCID have a nonSCID diagnosis. There is no uniform collection of data for these infants and the threshold trigger for repeat testing varies from state to state, so the incidence of significant non-SCID disorders identified will also likely vary from state to state. Although our institution specific experience is biased, as most infants had confirmation of a low number of TREC prior to referral, the significant number of disorders in the non-SCID cohort emphasizes the importance of full evaluations and follow-up for these infants.

Disclosure: None of these relate to the work being presented. Susan Prockop - research funding Mesoblast and Atara Biotherapeutics. Nancy Kernan - research funding Jaz Pharmaceuticals. Richard O'Reilly research funding and royalties Atara Biotherapeutics. Kevin Curran consulting Juno Pharmaceuticals, Novartis. J.J. Boelens Avrobio, Magenta, Chimerix and BlueBird Bio

\section{P376}

Younger age and early thymopoiesis are associated with lower incidence of post-transplant autoimmune cytopenia in children with primary immunodeficiency conditioned with fludarabine and treosulfan

Su Han Lum, Sabeena Selvarajah ${ }^{1}$, Angela DayaMartinez $^{I}$, Peter Mcnaughton ${ }^{I}$, Terry Flood ${ }^{I}$, Stephen Owen $^{1}$, Sophie Hambleton ${ }^{1,2}$, Andrew Cant ${ }^{1}$, Andrew $R$ Gennery $^{1,2}$, Mary Slatter ${ }^{1,2}$

${ }^{1}$ Great North Children's Hospital, Newcastle upon Tyne, United Kingdom, ${ }^{2}$ Institute of Cellular Medicine, Newcastle University, Newcastle upon Tyne, United Kingdom

Background: Post-transplant autoimmune cytopenia (AIC) is challenging and associated with substantial morbidity and mortality. We aimed to study the cumulative incidence (CI) of post-HCT autoimmune cytopenia (AIC) and its predictors in a cohort of children with primary immunodeficiency (PID).

Methods: In this retrospective study, we included 199 children with PID who underwent their first HSCT with fludarabine(F)-Treosulfan(T) \pm Thiotepa(Thio) at Great North Children's Hospital from 2007-2017. Main outcomes of interest were the CI of AIC and its predictors. Fine-andGrey regression models were used to analyse predictors of AIC, considering death as a competing event. Variables included were age at transplant $(<2.5$ years vs $>2.5$ years $)$, gender, diagnosis (SCID vs immune-dysregulatory disorders vs other PIDs), pre-transplant AIC, pre- and posttransplant respiratory virus, donor (MFD vs MUD vs MMFD/MMUD vs haploidentical donor), ABO incompatibility, conditioning (FT vs FTThio), serotherapy (none vs alemtuzumab $0.3-0.6 \mathrm{mg} / \mathrm{kg}$ vs alemtuzumab $0.9-1.0 \mathrm{mg} / \mathrm{kg}$ vs ATG), stem cell source (marrow vs PBSC vs cord vs exvivo $\mathrm{T}$ depleted $\mathrm{PBSC}$ ), infused stem cell doses (TNC, CD34 and CD3), aGvHD (none vs any aGvHD), cGvHD (none vs any cGvHD), viral infections (CMV/adenovirus/ EBV/HHV6 viraemia), chimerism (full vs mixed chimerism (WB $<95 \%$ ) within first year post-HCT). Impact of thymopoiesis using naïve $\mathrm{T}$ cell recovery was studied.

Results: Median age at transplant was 2.4 years (range, 0.11-18.3 years). Primary diagnoses were SCID (22\%), immune-dysregulatory disorders $(28 \%)$ and other PIDs (50\%). Donors were MFD (21\%), MUD (48\%), MMFD/ MMUD (16\%) and haploidentical parents (15\%). Stem cell sources were marrow (30\%), unmanipulated PBSC (38\%), ex-vivo T-depleted PBSC (23\%) and CB (9\%). $16 \%$ received additional thiotepa and $87 \%$ had $\mathrm{CSA} / \mathrm{MMF}$ as GvHD prophylaxis. Median duration of follow-up of survivors was 2.9 years (range 0.2 to 10.2 years). 5-year OS for the entire cohort was 81\%. 6-month and 1-year CI of AIC were 5\% and 13\%. Of 21 developed AIC, $17(80 \%)$ had AIHA, $3(15 \%)$ had AIHA \pm ITP and 1 (5\%) had AIHA \pm ITP \pm AIN. Median onset of AIC was 6.2 months post-HCT (range 0.2-12.8 months). Patients were treated with a median of 3 treatment modalities (range, 1-4). One (5\%) had steroid, 8 (38\%) had steroid+high-dose-IVIg, $8(38 \%)$ had steroid+high-dose-IVIG+rituximab, $1(5 \%)$ had steroid +high-dose-IVIG+sirolimus and $3(14 \%)$ had steroid +high-dose-IVIg + rituximab+sirolimus. The median time to resolution in $18(85 \%)$ who achieved remission after first AIC was 6.5 months (range 1.4-28.6 months). 1 had one relapse and 2 had two relapses. 2 died after development of AIC (1 aspergillus pneumonia; 1 multi-organ failure). Of 19 (91\%) surviving patients after AIC, 4 had on-going AIHA at median of follow-up 2.5 years post-HCT (range 0.9-4.8 years). On univariate completing-risk analysis, age at transplant $>2.5$ years $(p=0.02)$ and pre-transplant AIC $(p=0.02)$ were associated with higher incidence of AIC (Figure 1a-Ic). On Fine-and-grey models, only age at transplant (HR 3.08, 95\%CI 1.12-8.49, $p=0.03$ ) was independently associated with AIC. Of 157 with complete immune reconstitution data, naïve $\mathrm{T}$ cells $>100$ cells $/ \mathrm{mL}$ at 6 months post-HCT was associated lower incidence of AIC (HR 0.36, 95\%CI 0.15-0.91, $p=0.03$ ) (Figure 1d)

Conclusions: Younger age and thymopoiesis were associated lower incidence of AIC in children with PID after HCT

Clinical Trial Registry: None

Disclosure: None

\section{P377}

Abstract withdrawn. 


\section{P378}

Non-myeloablative matched sibling donor peripheral blood stem cell transplant is curative for human hemeoxygenase-1 deficiency

\section{Satya Prakash Yadav ${ }^{1}$, Sagar Nivargi, Anil Sharma ${ }^{1}$, Dhwanee Thakkar ${ }^{1}$, Neha Rastogi ${ }^{1}$}

${ }^{1}$ Medanta The Medicity, Gurgaon, India

Background: Human heme-oxygenase-1 (HO-1) deficiency has been reported to present with tetrad of anemia, nephritis, inflammation and asplenia and is fatal if not treated. Its an auto-inflammatory disorder. Macrophages/ Monocytes express HO-1 and are engaged in recycling of red cells. Human HO-1 deficiency results in intravascular hemolysis and severe damage to the endothelial system, kidneys, and other organs. Transplantation of either healthy wild type macrophages or new macrophages produced by SCT from healthy donor has been proven to be curative for HO-1 deficiency in mice. In 2018, we had reported first successful allogeneic SCT for human HO-1 deficiency. Here we report second successful non-myeloablative MSD HSCT for a child with HO-1 deficiency.

Methods: A 9-yr-old-girl presented with complaints of fever, anemia and severe hypertension. In the past, at the age of 7 years she was admitted for high fever for 1 month and needed blood transfusion for the first time for severe anemia and had high platelets and high ferritin. She was treated as macrophage activation syndrome with prednisolone alone and later cyclosporine was added. She had short stature, abnormal facies but normal development. Hemoglobin $9 \mathrm{~g} / \mathrm{dl}$, urine for haemoglobin was positive, platelets 1055, 000/ul, ferritin $2568 \mathrm{mcg} / \mathrm{L}$ and urine albumin $4+$ and Urine RBC 20-30/hpf. Ultrasound and CT scan abdomen showed asplenia. A diagnosis of HO-1 deficiency was suspected. Mutation analysis showed homozygous missense mutations in exon2 (R44X) on chromosome $22 q 12$, which would result in the absence of the functional HO-1 protein. Both parents were carriers of this mutation. We managed her over next 6-years with prednisolone, hydroxyurea and mycophenolate mofetil (MMF). However she remained steroid dependent. HLA-typing confirmed her healthy unaffected 13-year-old brother to be a fully matched donor. At the age of 16 years she was taken up for MSD SCT after taking informed consent. She weighed just $16 \mathrm{~kg}$. We conditioned her with Alemtuzumab- $0.8 \mathrm{mg} / \mathrm{kg}$, Fludarabine- $160 \mathrm{mg} / \mathrm{m} 2$, Cyclophospamide- $29 \mathrm{mg} / \mathrm{kg}$ and Total body irradiation 2 Gray. We infused 9 million $/ \mathrm{kg}$ peripheral blood stem cells from her brother. Graft-vs.-Host disease (GVHD) prophylaxis consisted of tacrolimus \& MMF.

Results: She tolerated procedure very well. Her entire hospital stay was uneventful and lowest platelet count recorded was 30,000/ul. Her neutrophils engrafted on day +13 and she was discharged on day +19 . His urine albumin was nil by day +7 . She had no GVHD. Her chimerism on day +22 showed $97 \%$ donor cells, on day +60 was $98 \%$ and on day +180 was $98 \%$ donor. Now he is day +210 post-SCT and doing well. She has no evidence of hemolysis, proteinuria, hypertension, fever. She has normal ferritin and platelets. She has gained $3 \mathrm{~cm}$ height and $5 \mathrm{~kg}$ weight in last 7 months. She had no viral reactivation and her immune recovery at 6 months post SCT is good.

Conclusions: Non-myeoablative allogeneic MSD SCT is a curative treatment option for human HO-1 deficiency.

Disclosure: Nil

\section{P379}

Two decades of excellent transplant survival in children with chronic granulomatous disease: A report from a supraregional immunology transplant centre in Europe

Su Han Lum ${ }^{1}$, Terry Flood ${ }^{1}$, Sophie Hambleton ${ }^{1,2}$,
Stephen Owen ${ }^{1}$, Nevenka Cigroski, Andrew Cant,
Andrew R Gennery ${ }^{1,2}$, Mary Slatter ${ }^{1,2}$

${ }^{1}$ Great North Children's Hospital, Newcastle upon Tyne, United Kingdom, ${ }^{2}$ Institute of Cellular Medicine, Newcastle University, Newcastle upon Tyne, United Kingdom

Background: Haematopoietic stem cell transplantation (HSCT) confers life-long curative therapy for chronic granulomatous disease (CGD). The ability of donor-derived neutrophils to replace recipient's defective neutrophils makes HSCT a superior therapy compared to conventional standard of care using antimicrobial therapy.

Methods: We examined the outcome of children with CGD who received a first HSCT at Great North Children's Hospital from 1998 to 2017. Outcomes included overall survival (OS), event-free survival (ES), toxicity endpoints, autoimmune disease, long-term survival and graft function. Cox proportional-hazard models were used to analyse predictors of OS and ES. Variables included for predictor analysis were age at transplant, donor, stem cell source, stem cell doses and conditioning.

Results: $=55$ children were included in this analysis. Median age of transplant was 5.3 years (range, 0.6-18.0 years). $45(82 \%)$ had X-linked and 10 (18\%) autosomal recessive CGD. Twenty (36\%) had matched family donor, $31(56 \%)$ had unrelated donor and $4(8 \%)$ had parental haploidentical donor. Prior to 2007, various conditioning regimens were used, with $21(38 \%)$ patients undergoing conditioning with pharmacokinetic guided intravenous (IV) busulfan $(\mathrm{Bu})$ and IV cyclophosphamide with or without serotherapy. From 2007, the conditioning regimen was switched to Flu-Treosulfan-Alemtuzumab with GvHD 
prophylaxis using ciclosporin (CSA) and mycophenolate mofetil (MMF) for family and unrelated donors $(n=24$, 44\%). Flu-Treosulfan-Thiotepa-ATG-Rituximab was used for CD3 TCR alpha-beta CD19 depleted haploidentical grafts $(n=4,7 \%)$. Ten $(20 \%)$ patients had grade II-IV acute GvHD while 5 had $(9 \%)$ had grade III-IV acute GvHD. None had chronic GvHD.

The 5-year OS for the entire cohort was $89 \%$ (95\% CI, $67-95 \%$ ) (Figure 1). Analysis by age at transplant revealed a 5 -year OS of $100 \%$ for children transplanted at $<=5$ years of age and $81 \%(95 \% \mathrm{CI}, 60-92 \%)$ for the children $>5$ years of age $(p<0.04)$ (Figure 2$)$. The OS was comparable between match family donor $(88 \%, 95 \% \mathrm{CI}, 61-97 \%)$ and unrelated donor transplant $(89 \%, 71-95 \%)$ (Figure 3). All four haploidentical transplants were successful. The 5-year ES for the entire cohort was 77\% (95\% CI 62-87\%). None of the variables was associated with ES. All seven patients with slipping chimerism received a successful second transplant. The five deaths were all due to transplantrelated complications (2 multi-organ failures; 1 pulmonary haemorrhage; 1 graft IV acute GvHD; 1 post-transplant lymphoproliferative disease). The median age at transplant of deceased patients was 10.0 years (range 8.4 to 18 years).

The 1-year and 5-year cumulative incidence of autoimmune diseases were $9 \%$ and $12 \%$ respectively. Three $(5 \%)$ had immune cytopenia while $3(5 \%)$ had autoimmune endocrinopathy ( 2 thyroid dysfunction; 1 type 1 diabetes mellitus). The median age of long-term survivors was 14 years (range, 2 to 36 years) with the median duration of follow-up of 6.5 years (range, 0.32 to 19.5 years). There was no late death in the entire cohort. The median donor myeloid chimerism was $100 \%$ (range 23 to $100 \%$ )

Conclusions: Despite the limitations of a single centre study, our findings confirm that HSCT is a safe and longlasting curative therapy for children with CGD

Disclosure: None

\section{P380}

Non - medical challenges in the diagnosis and transplantation of patients with primary immune deficiency: An experience from a tertiary care center in India

\section{Sagar Bhattad ${ }^{1}$, Stalin Ramprakash ${ }^{1}$, Raghuram CP${ }^{1}$, Chetan Ginigeri ${ }^{1}$, Fulvio Porta ${ }^{1,2}$}

${ }^{1}$ Aster CMI Hospital, Pediatrics, Bangalore, India, ${ }^{2}$ Civil Hospital, Brescia, Italy

Background: Primary Immune Deficiencies (PID) are increasingly being recognized in several parts of India. Despite being diagnosed, many patients fail to receive optimal care due to financial and social constraints.
Methods: Case records of patients diagnosed and treated (including hematopoietic stem cell transplants) for PID diseases during Feb 2018 - Nov 2019 at Aster CMI Hospital, Bangalore, India were analysed. Factors leading to deferred or suboptimal care were assessed in detail.

Results: 65 patients with various PIDs were diagnosed during the study period (details in table). 35 of them warranted a hematopoietic stem cell transplant (HSCT) as definitive curative treatment. A total of 7 children received 9 HSCT. 2 of them died while 5 of them are alive and well. 17 children (13 with Severe Combined Immune Deficiency) died before a HSCT could be carried out. 12 of them were critically ill at presentation, while 5 were stable but deferred further treatment citing financial and social constraints. 11 children needing transplant continue to remain on follow-up and have not been transplanted to date (4 of them have significant financial constraints, 3 families are not convinced about the need for transplant and 4 of them are being prepared for transplant).

Table: (SCID - Severe Combined Immune Deficiency, VODI- Veno-Occlusive Disease with Immunodeficiency, CGD - Chronic granulomatous disease, HLH - Hemophagolymphohistiocytosis, WAS - Wiskott Aldrich Syndrome, XLT - X linked thrombocytopenia, LAD- Leukocyte adhesion deficiency, MSMD - Mendelian Susceptibility to Mycobacterial Disease, XLA - X linked Agammaglobulinemia, CVID - Common Variable Immune Deficiency, APECED - Autoimmune Polyendocrinopathy Candidiasis Ectodermal Dystrophy, CMCC - Chronic MucoCutaneous Candidiasis, AT - Ataxia telangiectasia).

Conclusions: We present our experience from a developing country and discuss non-medical factors leading to suboptimal care in children with PID. Only $20 \%$ children warranting HSCT could be transplanted in our cohort. Among those where HSCT is potentially curative $48 \%$ of children died before HSCT could be offered. Transplants in developing countries pose unique challenges due to the absence of government funding and/or universal insurance coverage. In addition to delay in diagnosis and critical state of patients at admission, financial and social factors significantly contributed to poor outcome.

Disclosure: None

\section{P381}

The outcome of hematopoietic stem cell transplantation (HSCT) in pediatric patients with hemophagocytic lymphohistiocytosis (HLH) in Korea

Jun Eun Park', O Kyu Noh', Jaesung Heo ${ }^{1}$, Hyeon Jin Park $^{2}$, Heri Kim ${ }^{3}$, Jong Jin Seo ${ }^{3}$

${ }^{1}$ Ajou University School of Medicine, Suwon, Korea, Republic of, ${ }^{2}$ Center for Pediatric Oncology, National 
Cancer Center, Pediatrics, Goyang, Korea, Republic of, ${ }^{3}$ University of Ulsan College of Medicine, Pediatrics, Seoul, Korea, Republic of

Background: HSCT is an established treatment modality for familial HLH (FHL), recurrent or refractory HLH despite chemoimmunotherapy, and CNS disease.

Methods: The Korea Histiocytosis Working Party retrospectively collected nation-wide data from the patients diagnosed with HLH and underwent allogeneic HSCT between 2009 and 2017. The clinical characteristics and treatment outcomes of the patients were analyzed.

Results: A total of 44 patients were enrolled. There were 31 patients with FHL (4 FHL2, 26 FHL3, and 1 FHL4), 7 infection associated HLH, and 6 secondary HLH of unknown cause.

All the patients were treated with HLH-2004 protocol, and 30 patients achieved complete response (CR) after treatment for 8 weeks, while 14 did not. The main reasons for receiving transplantation were FHL in 26, reactivation in 17 , and refractory disease in 1 . The conditioning regimens were busulfan-based in 16 patients, fludarabine-based in 4, treosulfan-based in 7, and busulfan/fludarabine-based in 17. Stem cell sources used for HSCT were from peripheral blood in 36 patient, cord blood in 7, and bone marrow in 1 . The donor types of HSCT were unrelated donor in 33 patients and related in 11 (7 matched sibling donor, 4 haploidentical donor, 1 partially matched donor). The causes of death of 7 patients were disease reactivation/ progression in 3, acute GVHD with/without VOD in 3, and graft failure in 1. Five year overall survival rates were $82.4 \%$, respectively. The disease status at the time of HSCT was $\mathrm{CR}$ in 37 patients, and non-CR in 7. The 5-year survival rate of patients who received HSCT in CR was $87 \%$ and $63 \%$ for patients transplanted while in non-CR status $(\mathrm{p}=0.046)$. Patients who received HSCT using peripheral blood stem cells had a better 5-year survival rate of $86 \%$ compared to $75 \%$ of patients who received cord blood stem cells, significantly. The presence of neurologic symptoms, disease status after intial 8 week therapy, conditioning regimen, and CD 34 positive cell count did not have statistically significant impact on survival.

Conclusions: HSCT improved the survival of patients who had familial, or relapsed, or refractory HLH in the Korean nation-wide HLH registry. These results are similar to other reports in the literature. The disease status at the time of HSCT and the stem cell source of the transplant were the important prognostic factors that affected the survivals of the HLH patients who underwent HSCT.

Clinical Trial Registry: No registry number.

Disclosure: To the best of our knowledge, the named authors have no conflict of interest, financial or otherwise

\section{P382}

Hematopoietic cell transplantation with reduced intensity conditioning regimen using fludarabine/ busulfan and fludarabine/melphalan for primary immunodeficiency diseases

Akira Nishimura ${ }^{1}$, Yuki Aoki', Yasuyoshi Ishiwata', Maiko Inoue ${ }^{1}$, Takuya Ichimura ${ }^{2}$,Junichi Ueyama ${ }^{3}$, Kazuaki Matsumoto ${ }^{1}$, Kento Inoue ${ }^{1}$, Haruka Hiroki ${ }^{1}$, Shintaro Ono ${ }^{1}$, Tsubasa Okano ${ }^{1}$, Mari Tanaka ${ }^{1}$, Satoshi Miyamoto $^{1}$, Miho Ashiarai, Reiji Miyawaki, Chika Yamagishi $^{1}$, Mari Tezuka ${ }^{1}$, Teppei Okawa ${ }^{1}$, Akihiro Hoshino $^{1}$, Akifumi Endo ${ }^{1}$, Masato Yasuhara ${ }^{4}$, Takahiro Kamiya ${ }^{1}$, Noriko Mitsuiki ${ }^{1}$, Toshiaki Ono ${ }^{1}$, Masakatsu Yanagimachi $^{1}$, Takeshi Isoda ${ }^{1}$, Daisuke Tomizawa ${ }^{1,4}$, Masayuki Nagasawa ${ }^{1}$, Michiko Kajiwara', Masatoshi Takagi $^{1}$, Shuki Mizutani ${ }^{1}$,Hirokazu Kanegane ${ }^{1}$, Kohsuke Imai $^{1}$, Tomohiro Morio ${ }^{1}$

${ }^{1}$ Tokyo Medical and Dental University, Tokyo, Japan, ${ }^{2}$ Yamaguchi University Hospital, Yamaguchi, Japan, ${ }^{3}$ Tottori University Hospital, Tottori, Japan, ${ }^{4}$ National Center for Child Health and Development, Children's Cancer Center, Tokyo, Japan,

Background: Primary immunodeficiency disease (PID) is congenital disorders of innate or acquired immune system. Hematopoietic cell transplantation (HCT) was a treatment option for PIDs with life-threatening infections or immune dysregulations. Reduced intensity conditioning (RIC) was increasingly used to prevent complications in HCT, but optimized regimens have not been established. We performed HCT for PIDs with RIC using fludarabine and busulfan (FluBU) or melphalan (FluMel) according to the guidelines of European Society for Immunodeficiencies (ESID) / European Society for Blood and Marrow Transplantation (EBMT), and assessed the efficacy and safety of these RIC.

Methods: From April 2004 to December 2017, 42 PID patients underwent RIC-HCT using FluBU or FluMel in TMDU were analyzed retrospectively. The AUC of BU was set to $30 \mathrm{mg}$ *hour/L for severe combined immunodeficiency disease (SCID) and $60 \mathrm{mg} *$ hour/L for non-SCID. Overall survival (OS) was analyzed.

Results: The median age at HCT was $2.0(0.3-21)$ years old ( 35 male and 7 female patients). FluBU was used for 23 patients (7 SCID, 9 combined immunodeficiency disease (CID), 3 ectodermal dysplasia (EDA), and 4 severe congenital neutropenia $(\mathrm{SCN})$ ) and FluMel was used for 19 patients (8 SCID, 6 CID, 4 XIAP deficiency, and 1 EDA). Anti-thymocyte globulin was used in 8 patients of FluBU group and 7 patients of FluMel group. Cord blood in 21 and bone marrow in 21 was used for donor sources. 
Matched donor was used for 7 and 8 patients in FluBU and FluMel groups (30.4\% and $42.1 \%$ ), respectively. Median follow up period was 2.2 years. Two years-OS of all patients, FluBU group patients and FluMel group patients was $79.6 \%, 89.8 \%$ and $64.1 \%$, respectively. Neutrophil engraftment was $92.9 \%, 91.3 \%$ and $94.7 \%$ (all patients, FluBU group and FluMel group). In SCID, all 7 patients in FluBU group achieved engraftment and survived. Seven out of 8 in FluMel group achieved engraftment, but 1 patient had secondary graft failure and 3 patients died. In nonSCID, 14 out of 16 in FluBU group achieved engraftment, but 6 patients had secondary graft failure. All 11 non-SCID patients in FluMel group were engrafted and survived. Two and 7 patients in FluBU group and FluMel group suffered from severe acute graft-versus-host disease (grade III-IV). Ten patients had hemophagocytic lymphohistiocytosis (HLH). Viral reactivation or infection was observed in 20 patients, and resolved in all but one patient.

Conclusions: The RIC-HCT using FluBU or FluMel was advantagous for neutrophil engraftment, and FluBU for SCID and FluMel for CID with immune dysregulation may be an effective opinion. FluBU regimen needs to be improved for secondary graft failure in non-SCID. Prevention of $\mathrm{HLH}$ after transplantation using dexamethasone palmitate will be considered.

Disclosure: Nothing to declare.

\section{P383}

Survival after hematopoietic stem cell transplantation with TCR $\alpha \beta / C D 19$ graft depletion in older children with primary immunodeficiencies

\section{Alexandra Laberko ${ }^{I}$, Elena Gutovskaya ${ }^{I}$, Irina Shipitsina ${ }^{1}$, Svetlana Radigina ${ }^{1}$, Svetlana Kozlovskaya ${ }^{I}$, Elvira Sultanova ${ }^{1}$, Larisa Shelikhova ${ }^{1}$, Michael Maschan $^{I}$, Anna Shcherbina ${ }^{I}$, Alexei Maschan ${ }^{I}$, Dmitry Balashov $^{I}$}

${ }^{1}$ Dmitry Rogachev National Medical Research Center of Pediatric Hematology, Moscow, Russian Federation

Background: TCR $\alpha \beta / C D 19$ depletion is a graft engineering method that proved valuable in increasing the survival rate after hematopoietic stem cell transplantation (HSCT) in patients with primary immunodeficiencies (PID). Decreased survival rate in older patients with PID was previously reported after transplantations with nonmanipulated grafts.

Methods: 148 patients with various PID (excluding classic SCID) who received allogenic HSCT with TCR $\alpha \beta /$ CD19 graft depletion from 2012 to September 2018 in our center were analyzed. The median age at HSCT was 3,5 years (range 0,43-17,63). Patients were divided into 3 age groups: 0-6 years - 95 patients, 6-12 years - 30, 12-18 years
- 23. 112 patients received HSCT from matched unrelated, 31 - haploidentical donors, 5 - siblings. Conditioning regimens with 1-2 alkylating agents and antithymocyte globulin were used in all patients. 113 patient received short courses of various posttransplant immunosuppressants. Median follow up after HSCT is 1,95 years (range 0,046,3 years).

Results: Overall survival (OS) in 148 patients was 0,85 (95\% CI $0,79-0,91)$. We observed similar OS in the younger age groups: $0,89(95 \% \mathrm{CI} 0,83-0,96)$ in $0-6$ years and 0,89 $(95 \%$ CI $0,78-1,0)$ in 6-12 years of age. Seven of 23 patients in older group (12-18 years of age) died, the OS was only 0,65 (95\% CI 0,43-0,86), $\mathrm{p}=0,065$. All patients from older age group who died had combined PID: 1 - Wiscott-Aldrich syndrome, 1 - undefined PID, 1 - IL2RG deficiency, 1 DCLRE1C deficiency, 1 - Nijmegen breakage syndrome (NBS), 1 - Kabuki syndrome, 1 - ICF syndrome. The median time of death after HSCT was 0,64 years (range $0,26-1,58)$.

Six of those had transplant-related mortality (TRM). Five patients had HSCT-associated viral infections: 2 - CMV pneumonias, 3 - ADV infections (1 - fulminant hepatitis, 2 -multiorgan). Interestingly, 2 of them had prolonged history of disseminated viral infections (ADV), with the reduction of viral load in blood and other fluids and tissues upon treatment. One patient with Kabuki syndrome after HSCT developed HHV8 associated Kaposi sarcoma, was successfully treated. Eventually all 3 patients with reduction of viral infection and sarcoma symptoms developed multiorgan failure with some clinical and laboratory evidences of endothelium cell damage syndrome. One patient with NBS died of high grade lymphoma progression.

Conclusions: HSCT with TCR $\alpha \beta / \mathrm{CD} 19$ depletion demonstrates high survival rate in 148 patients with various PID. In our group patients' age older than 12 years predisposes to decreased posttransplant survival. Patients with combined PID are at higher risks of posttransplant mortality. We conclude that at least in some of our patients with prolonged history of viral infections after HSCT the cause of death could be multiorgan failure due to endothelium cell damage syndrome resulting from persistent inflammation and drug toxicity effects.

Disclosure: No conflict of interest to disclose

\section{P384}

Unrelated umbilical cord blood transplantation with two different conditioning regimen for chronic granulomatous disease: Single-institution experience in China

Xiaowen Qian ${ }^{1}$, Xiaowen Zhai ${ }^{1}$, Hongsheng Wang ${ }^{1}$, Wenjin Jiang ${ }^{1}$, Ping Wang ${ }^{1}$, Jinqiao Sun ${ }^{1}$, Xiaochuan Wang $^{1}$ 
${ }^{1}$ Children's Hospital of Fudan University, Shanghai, China,

Background: Chronic granulomatous disease (CGD) is a primary immunodeficiency (PID) caused by a mutation in 1 of the 5 subunits of the nicotinamide dinucleotide phosphate (NADPH) oxidase, which leads to a reduction in the microbicidal activity of phagocytic cell. Starting at an early age, CGD patients suffer from severe recurrent infections, as well as inflammatory events. Allogeneic hematopoietic stem cell transplantation (HSCT) is a curative option for CGD in patients with insufficient benefit from supportive care and prophylactic antibiotics. We reported a series of 39 patients with CGD who underwent Unrelated umbilical cord blood transplantation (UCBT) at our center.

Methods: In this retrospective study, we observed a series of 39 consecutive UCBT performed at our center in children with CGD between 2015 and 2018.Median age at transplantation was 17 months (range, 4 to 144 months), Median body weight was $10 \mathrm{~kg}$ (range, 6 to $34 \mathrm{~kg}$ ). 32/39 patients received a myeloablative conditioning regimen consisting of busulfan, fludarabine, cytarabine, cyclophosphamide and G-CSF, 7/39 patients received another myeloablative conditioning regimen consisting of busulfan, fludarabine, cyclophosphamide and ATG. Prophylaxis for graft-versus-host disease (GVHD) was tacrolimus.

Results: Engraftment occurred in 29/39 (74.4\%) patients. $10 / 39(25.6 \%)$ patients occurred graft failure, all of them received a myeloablative conditioning regimen without ATG. Median time to neutrophil and platelet engraftment were 24 (rang, 14-46) and 36.5 (rang, 15-51) days. 22/39 $(56.4 \%)$ patients developed acute GVHD, with 4/39 (10.3\%) episodes of grade III-IV aGVHD. Chronic GVHD occurred in $3 / 29$ patients $(10.3 \%)$. At a median follow-up of 17 months (rang, 1 to 44 months), the overall survival rate was $83.7 \%$, and event-free survival rate was $66.7 \%$.

Conclusions: Unrelated UCBT should be considered as potential curative methods in children with CGD. CGD patients who used myeloablative conditioning regimen with ATG shows better graft and survival.

Disclosure: Nothing to declare

\section{P385}

Successful use of interferon-gamma for refractory disseminated candida tropicalis infection in a pediatric patient with acute myeloid leukemia and a heterozygous CARD9 mutation

\section{Katharina Kleinschmidt ${ }^{1}$, Juergen Foell, Claudia Huegli $^{I}$, Beatrix Pfirstinger ${ }^{I}$, Anja Troeger ${ }^{I}$, Selim Corbacioglu ${ }^{I}$}

${ }^{1}$ University Hospital Regensburg, Regensburg, Germany
Background: Invasive fungal infections (IFI) remain a major cause of treatment-related morbidity and mortality in AML patients. Although not uncommon, the presentation of unusually severe clinical features might be indicative for an underlying immunodeficiency. Caspase-associated recruitment domain 9 (CARD9) is recognized to have a crucial role in effective antifungal response, leading to Th1 and Th17 differentiation and to the initiation of the inflammatory cytokine cascade. Particularly interferon-gamma (IFNg) increases macrophage activity. Patients with homozygous CARD9 mutations are known to have a significantly increased susceptibility to life-threatening systemic candidiasis. However, some sequence variants may lead to increased IFI-susceptibility even in heterozygosity, e.g. under immunosuppression. IFN- $\gamma$ has been described as an additive treatment option because of its immune stimulating effect on the leukocyte immune response in a situation of immunological "blindness".

Methods: Here, we report the case of an 11-year old male with AML M6 with a severe systemic candida tropicalis infection, unresponsive to triple-antimycotic regimen, leading to multi-organ failure. He was discovered to bear a heterozygous CARD9 mutation, and IFN- $\gamma$ immunotherapy leaded to complete response of all disseminated infections.

Results: The patient developed septic fever immediately after the first chemotherapy cycle. Unexpectedly, candida tropicalis was confirmed in the blood culture within 24 hours. Liposomal amphotericin B (Ambisome ${ }^{\circledR}$ ) was started immediately, however candida rapidly disseminated to lungs, liver, spleen, kidneys and CNS despite extended antimycotic therapy with caspofungin, voriconazole and fluconazole. The patient was splenectomized due to massive infiltration (Figure 1). Genetic testing for mycosis predisposition revealed a heterozygous mutation in the CARD9 gene, inherited from the father (c.809a>T(p.Glu270Val)). IFN- $\gamma$ treatment was started (100 $\mu \mathrm{g}$ subcutaneously, 3 times per week), leading to an almost complete response of disseminated infections. Due to the severe infection, chemotherapy had to be interrupted after one course. However, bone marrow remained in complete remission for almost one year. The patient experienced altogether two relapses requiring an unrelated allogeneic and a haploidentical HSCT. Under combined IFN- $\gamma$ and ambisome/ fluconazole prophylaxis no further mycosis was observed despite extensive and prolonged immunosuppression.

Conclusions: IFI in AML patients are common, however an unusual presentation in presumably immune competent individuals should raise the suspicion for immunodeficiencies. In our case, an unexpected early candida-sepsis was completely unresponsive to an adequate multi-agent treatment. While IFN- $\gamma$ is used in adults as an immune stimulatory cytokine, little data are available for children. To our knowledge, this is the first case of successful IFN- $\gamma$ 
treatment of a pediatric AML patient with disseminated candida sepsis, bearing a CARD9 mutation. Given the elevated mortality risk for IFI, and the apparently safe and well-tolerated application of IFN- $\gamma$, an adjuvant immunotherapy might be considered. Further studies are needed to define the indication and duration of this kind of adjunctive immunotherapy. Moreover, considering the wide heterogeneity of genetic mutations involved in IFI-susceptibility, genome-wide expression profiling might be useful for pediatric cancer patients, as the identification of specific immune pathways might help to identify individual IFIsusceptibility in order to improve the outcome of those high-risk patients.

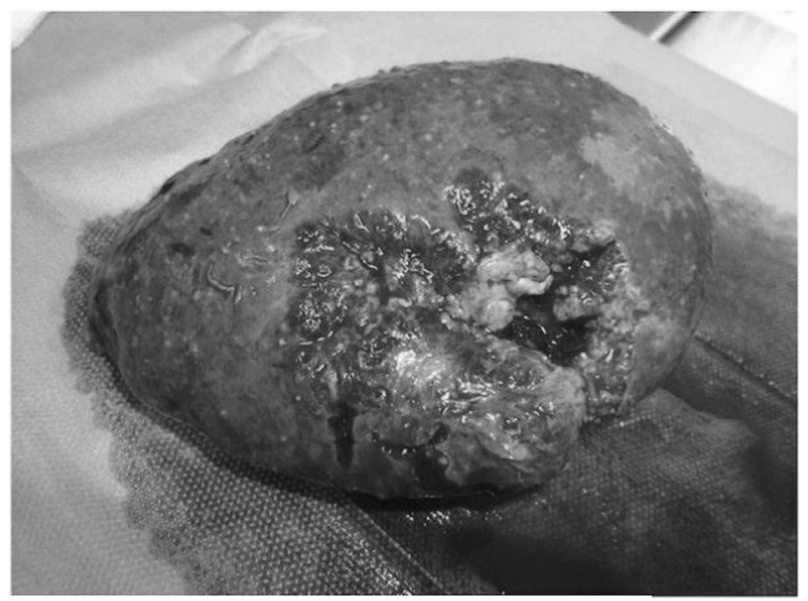

[[P385 Image] 1. Figure 1. Massive candida tropicalis infiltration of the spleen]

Disclosure: Nothing to declare.

\section{P386}

Successful haploidentical stem cell transplant with post transplant cyclophosphamide in chronic granulomatous disease

\section{Satya Prakash Yadav ${ }^{1}$, Anil Sharma ${ }^{1}$, Sagar Nivargi ${ }^{1}$, Dhwanee Thakkar ${ }^{1}$, Neha Rastogi ${ }^{1}$}

${ }^{1}$ Medanta The Medicity, Gurgaon, India.

Background: Chronic granulomatous disease (CGD) is curable by allogeneic hematopoetic stem cell transplant (HSCT). Recent reports of haploidentical donor HSCT with with post transplant cyclophosphamide (PTCy) from family donors in pediatric primary immune deficiencies have shown encouraging results. However, it has not been reported in CGD. Here we describe successful haploidentical HSCT in a child with CGD with myeloablative conditioning and PTCy.
Methods: A 3 year-old, male child diagnosed with CGD showed oxidative activity $0.3 \%$ by dihydrorhodamine (DHR) test. He had no matched related or unrelated donor available so underwent haploidentical HSCT after taking informed consent of the parents in May 2018. Donor was his 5/10 HLA matched healthy elder sister (oxidative activity $55 \%$ by DHR). He has had multiple admissions for recurrent pneumonia prior to HSCT. The conditioning was with Rituximab $100 \mathrm{mg} / \mathrm{m} 2 \mathrm{IV}$ on day -8, Thiotepa $10 \mathrm{mg} /$ $\mathrm{kg} / \mathrm{dose}$ intravenous (IV) for 1 day (Day-7), Busulfan 3.2 $\mathrm{mg} / \mathrm{kg} / \mathrm{dose}$ daily IV for 4 days (Day -6 to -3 ) and Fludarabine $40 \mathrm{mg} / \mathrm{m} 2 /$ dose daily IV for 4 days (Day -6 to -3) and rabbit anti-thymoglubulin (thymoglobulin) $1.5 \mathrm{mg} /$ $\mathrm{kg} /$ dose daily for 3 days (Day-4 to -2 ). Peripheral blood stem cells ( 8 million $/ \mathrm{kg} \mathrm{CD} 34+$ cells) were harvested from his sister and transfused to the patient on Day 0. Graft vs. Host disease (GVHD) prophylaxis was with PTCy $50 \mathrm{mg} /$ $\mathrm{kg}$ on Day+3 \& 4, intravenous cyclosporine from day-3 (targeting levels $150-250 \mathrm{ng} / \mathrm{ml}$ ) and MMF from day +5 .

Results: His neutrophils engrafted on day +15 and platelets on day +17 . Chimerism on day $+30,100$ and 6 months was fully donor. He developed no acute or chronic GVHD. At 6 months his lymphocyte counts showed CD4280/ul, CD8-670/ul, CD19-15/ul and CD16/56-120/ul. His had no viral reactivation. He is disease free and GVHD free on day +190 post HSCT and is on tapering doses of cyclosporine. His DHR test showed oxidative activity of 42 $\%$ on day +100 .

Conclusions: Its feasible to do haploidentical HSCT with PTCy technique in children with CGD.

Disclosure: Nil

\section{P387}

Hematopoietic stem cell transplantation for primary immune deficiencies: Experience from a tertiary medical center - Instituto Nacional de Pediatría

Rosa Maria Nideshda Ramírez Uribe ${ }^{I}$, Haydeé Del Pilar Salazar Rosales ${ }^{1}$, Alberto Olaya Vargas ${ }^{1}$, Gerardo López Hernández ${ }^{1}$, Maria De los Ángeles Del Campo Martínez ${ }^{1}$

${ }^{1}$ Instituto Nacional de Pediatria, Mexico City, Mexico

Background: Primary immune deficiencies (PID) are a functional disorder of inheritance immune system that increase predisposition to infectious disease in number and severity. The incidence is 1: 10,000 birth live; its immunological dysregulation may increase the predisposition of autoimmune diseases and malignancy, the latter being more frequent (4-25\%). At present, the only curative treatment is hematopoietic stem cells transplant (HSCT).

Methods: We describe all patients transplanted with Primary Immune Deficiencies at Instituto Nacional de 
Pediatria. The conditioning regimen depended on the type donor and pathology: myeloablative $(41.07 \%)$, reduced intensity $(37.5 \%)$ and non myeloablative $(21.43 \%)$ without modification statistically significantly in overall survival.

Results: A total of 71 patients were included from 1998 to January/2017. Severe combined immunodeficiency (SCID) is the pathology most frequently transplanted (Figure 1).

Seventy three percent have molecular diagnosis, and $44.83 \%$ have cases of family PID. The most used sources were umbilical cord blood (UCB) with $42.6 \%$ and peripheral blood (39.3\%), however the trend of the source of obtaining it has been modified a few years ago (Figure 2).

The median graft was 14 days for UCB, 16 days for bone marrow (BM) and 12 days for peripheral blood (PB) (Figure 3)

The main complications are infectious (bacterial $48.3 \%$ and viral $39.7 \%$ ) and non-infectious as pre-graft syndrome (23.1\%).

Conclusions: The overall survival was $54.1 \%$ Survival according to pathology was: $100 \%$ Chediak Higashi syndrome, 68\% SCID, 66.7\% Griselli syndrome, 66.7\% hyper IgM syndrome, 60\% WAS, 57\% CGD, 50\% Hemophagocytic lymphohistiocytosis.

Disclosure: Ramírez-Uribe Rosa Maria Nideshda, Salazar-Rosales Haydeé, Olaya-Vargas Alberto, LópezHernández Gerardo, del Campo-Martínez Maria de los Àngeles We wish to confirm that there are no known conflicts of interest associated with this abstact, the only financial support was provided by Mexican Associations that helping children wiht cancer in a few patients.

\section{P388}

Long-term outcome following hematopoietic stem cell transplantation of Wiskott-Aldrich syndrome in a single institute

Mamoru Honda ${ }^{1,2}$, Yukayo Terashita ${ }^{1}$, Minako
Sugiyama $^{1}$,Yuko Cho $^{1}$, Akihiro Iguchi

${ }^{1}$ Hokkaido University Faculty of Medicine, Pediatrics, Sapporo, Japan, ${ }^{2}$ Japan Self Defense Forces Sapporo Hospital, Pediatrics, Sapporo, Japan

Background: Wiskott-Aldrich syndrome (WAS) is an Xlinked disorder of hematopoietic cells, characterized by thrombocytopenia with small platelets, eczema, and immunodeficiency. Hematopoietic stem cell transplantation (HSCT) is the only curative treatment, and it is recommended to be performed as soon as WAS is diagnosed. Myeloablative conditioning before HSCT is recommended because there is a high risk of development of autoimmune disease in patients with mixed chimera after HSCT.
However, there are few reports about late complications such as pubertal development and eruption of teeth in patients with WAS receiving HSCT. Thus, we evaluated late complications in patients with WAS receiving HSCT at Hokkaido University Hospital.

Methods: We reviewed medical records of 8 male patients with WAS who received HSCT between 1995 and 2017.

Results: Mean age at HSCT was 1.4 (range, 0.6-6.8) years, and median follow-up time after HSCT was 13.8 (range 2.9-23.4) years. Conditioning regimen in all patients comprised busulfan at $4 \mathrm{mg} / \mathrm{kg}$ for 4 days and cyclophosphamide at $60 \mathrm{mg} / \mathrm{kg}$ for 2 days or $50 \mathrm{mg} / \mathrm{kg}$ for 4 days. Additionally, anti-thymocyte globulin at $2.5 \mathrm{mg} / \mathrm{kg} /$ day for 1-2 days was administered in 6 patients. Engraftment, normal platelet count, and complete chimera were confirmed in all patients. No patients showed complications such as severe chronic graft-versus-host disease, autoimmune disease, short stature $(\leq-2.0 \mathrm{SD})$ and second malignancy. However, high IgE level was observed in 4 patients. Pubertal development has been confirmed in 6 patients. Lack of complete eruption of permanent teeth has been observed in 3 patients who received HSCT at age of $<$ 2 years.

Conclusions: Although this was a small-cohort study in a single institute, complete chimera has been achieved in all patients who received HSCT with busulfan-based myeloablative conditioning. However, late complications such as male infertility and incomplete eruption of permanent teeth remain major problems.

Disclosure: Nothing to declare

\section{P389}

Umbilical cord blood transplantation in children with leukocyte adhesion deficiency type I: Single center experience in China

Xiaowen Qian ${ }^{1}$, Xiaowen Zhai ${ }^{1}$, Ping Wang ${ }^{1}$, Hongsheng Wang $^{1}$, Wenjin Jiang ${ }^{1}$, Jinqiao Sun ${ }^{1}$, Xiaochuan Wang ${ }^{I}$

${ }^{1}$ Children's Hospital of Fudan University, Shanghai, China,

Background: Leukocyte adhesion deficiency type I (LADI) is a rare primary immunodeficiency disease. As a autosomal recessive inherited disease, LAD-I is caused by mutations in the ITGB2 gene result in defective CD18 molecules on the surface of leukocytes, which causing leukocytes cannot normally adhesion and migrate to inflammatory sites. Clinical features of LAD-I patients mainly include delayed umbilical cord separation, recurrent infection and marked leukocytosis. Allogeneic hematopoietic stem cell transplantation (allo-HSCT) is a curative therapy for LAD-I patients. We report the results of 
umbilical cord blood transplantation (UCBT) performed in 5 patients with LAD-I between 2016 and 2018 at a single center.

Methods: We performed unrelated umbilical cord blood transplantation (UCBT) in 5 consecutive children with LAD-I. Median age of 5 children was 13 months (range, 8 to 131 months), and median body weight was $13 \mathrm{~kg}$ (range, 8 to $24.3 \mathrm{~kg}$ ). All patients received myeloablative conditioning regimen consisting of busulfan, fludarabine and cytarabine. Prophylaxis for graft-versus-host disease (GVHD) was tacrolimus. All patients received a $\leq 2$ HLA alleles-mismatched cord unit, 1 was HLA fully matched, 3 were 9/10 matched, 1 was 8/10 matched. Median nucleated cells of the cord blood were $14.23 \times 10^{7} / \mathrm{kg}$ (range, 4.6 to $20.62 \times 10^{7} / \mathrm{kg}$ ), and median CD34+ cells were $3.87 \times 10^{5} \%$ $\mathrm{kg}$ (range, 1.95 to $5.77 \times 10^{5} / \mathrm{kg}$ ).

Results: All patients engrafted, median time of neutrophil engraftment was 24 days (range, 13 to $28 \mathrm{~d}$ ), and median time of platelet engraftment was 33 days (range, 32 to $56 \mathrm{~d}$ ). Median follow-up time was 13 months (range, 2 to 25 months), all 5 patients were alive with continuous completely donor engraftment, and achieved complete clinical remissions. 4/5 patients developed grade II/III acute graft-versus-host disease (GVHD), and 1/5 patients developed chronic GVHD with skin.

Conclusions: It is the first successful unrelated UBCT for LAD-I children in China. Our data shows UCBT provided excellent outcome for patients with LAD-I.

Disclosure: Nothing to declare

\section{P390}

\section{Excellent outcome using 'NKTM' enriched hematopoietic stem cell transplants for patients with inborn errors of immunity}

\section{Poh Lin Tan', Frances Yeap ${ }^{1}$, Clarice Chan', Krista Francisco $^{1}$, Mariflor Villegas ${ }^{1}$, Allen Yeoh ${ }^{1}$ \\ ${ }^{1}$ National University Hospital, Paediatrics, Singapore, Singapore}

Background: With improved diagnostics and earlier diagnosis, more than $90 \%$ of patients with inborn errors of immunity (IEI) especially those with primary immunodeficiencies transplanted in experienced centres are cured. Infective deaths however remains a major challenge. We hypothesize that NK and T memory cells (NKTm) enriched stem cell grafts provide equivalent survival compared to unmanipulated stem cell grafts regardless of HLA match grade.

Methods: Retrospective review of 23 HSCTs in 18 patients with IEI transplanted between 1996 and June 2018.
Results: Majority of patients in the 2 cohorts had significant infective co-morbidities at the time of HSCT with patients in the later cohort entering HSCT earlier. Patients in the later cohort were sicker at HSCT. Final engraftment occurred in all except 1 patient who received a HLA mis-matched cord blood HSCT. Graft failures occurred in 6 patients ( 2 in earlier and 4 in later cohort); 3 of these patients received unmanipulated HSCTs from HLA mis-matched unrelated donors (1 CB, 2 BM). Second HSCT were with same donors in 3 and different donors in 2 patients. No grade II to IV acute GVHD or extensive GVHD occurred. One patient (CBT) died of infections/ non-engraftment. All 9 patients in the later cohort compared to 7 of 9 patients in earlier cohort are alive, engrafted and cured. Performance status were $100 \%$ in all alive patients. Of the 9 patients in the later cohort, 7 (6 HLA mis-matched related and 1 HLA matched related donors) received 'NKTm' enriched HSCT. In the 6 HLA mis-matched 'NKTm' enriched HSCTs, patients received high CD34+, $\mathrm{CD} 3+\mathrm{CD} 45 \mathrm{RO}+$ and NK cell doses, with median of 16.88 (range, 5.1 - 36.5), 113.9 (range, 3.3 - 232.6) and 89.0 (range, 22 - 654) $\times 10^{6} / \mathrm{kg}$, respectively. No invasive infections occurred in these patients and immune reconstitution in T, B, NK compartments were complete at 1 year after HSCT with CD4 > 200 by 6 months and TCR $\alpha$ b > 1500 by 1 year after HSCT.

\begin{tabular}{lll}
\hline $\begin{array}{l}\text { Transplant } \\
\text { Characteristics }\end{array}$ & 1996 to 2013 & 2014 to 2018 (N =9) Ref:1 \\
\hline $\begin{array}{l}\text { Median age } \\
\text { at HSCT }\end{array}$ & 56 (range, 5 to & 54 (range, 6 to \\
Diagnoses & WAS: 1; SCID (IL2RG): & WAS/ B cell LPD: 1; \\
& 1; CD40LG: 3; CGD: 1; & SCID (Omenn): 1; \\
& LAD1: 1; HLH: 2 & CD40LG: 1; CGD: 1 \\
& & XIAP/ HLH: 1; FHL3: 1; \\
& & Crohn's (IL10R): 1; DKC: \\
& 1; HLH: 1 \\
$\begin{array}{l}\text { Donor } \\
\text { relatedness }\end{array}$ & Unrelated donor: 7 & Related donor: 8; \\
$\begin{array}{l}\text { HLA } \\
\text { matching }\end{array}$ & Matched: 3; & Matched: $2 ;$ \\
$\begin{array}{l}\text { Stem cell } \\
\text { sources }\end{array}$ & UCB: 5; BM: 1; PBSC: 3 & UCB: 0; BM: 2; PBSC: 7 \\
$\begin{array}{l}\text { Graft } \\
\text { engineering }\end{array}$ & Unmanipulated: 9; & Unmanipulated: 2; \\
$\begin{array}{l}\text { Preparative } \\
\text { regimens }\end{array}$ & Majority Busulfan based & Majority \\
$\begin{array}{l}\text { GVHD } \\
\text { prophylaxis }\end{array}$ & CSA \pm MTX in all & Fludarabine based \\
\hline
\end{tabular}

[[P390 Table] 1. Table 1: Transplant Characteristics]

Conclusions: Touzot et. al. ${ }^{2}$ were the first to use CD45RA depleted grafts with CD34+ selected HSCT successfully in HLA mis-matched settings in 5 patients with 
primary combined immunodeficiency. In our preliminary experience comparing patients who received unmanipulated HSCT and those who received CD3 and CD45RA depleted stem cell grafts enriching for NK and T memory cells, we have shown equivalent excellent survival outcome in patients who were sicker at HSCT.

\section{References:}

1. Lee et. al. WAO journal 2012

2. Touzot et. al J of Allergy Clin Immunol 2015

Clinical Trial Registry: NA

Disclosure: Nil

Infectious complications

\section{P391}

\section{Abstract withdrawn}

\section{P392}

The financial burden of antiviral therapy post hematopoietic stem cell transplantation, in view of T-cell immunotherapy

Fani Zervou ${ }^{I}$, Anastasia Papadopoulou ${ }^{1}$, Zoi Bousiou, Andriana Lazaridou ${ }^{I}$, Maria Alvanou ${ }^{1}$, Ioannis Batsis ${ }^{1}$, Varnavas Constantinou ${ }^{1}$, Michalis Iskas ${ }^{1}$, Maria Ganidou $^{1}$, Ioanna Sakellari', Achilles Anagnostopoulos ${ }^{I}$, Evangelia Yannaki ${ }^{1}$

1“George Papanikolaou” Hospital, Thessaloniki, Greece

Background: Viral infections contribute to significant morbidity and mortality after allogeneic hematopoietic cell transplantation (allo-HSCT), increasing both the human and the financial cost. Antiviral agents are often ineffective or/ and associated with toxicity.

Methods: In view of T-cell anti-viral immunotherapy in Greece, we evaluated the actual cost of conventional pharmacotherapy for $\mathrm{CMV}$, EBV and BKV reactivations after allo-HSCT, by calculating the costs of (i) the antiviral agents, (ii) the treatment (excluding transfusions) of antiviral drug primary toxicity (e.g. graft failure, cytopenias, renal or hepatic dysfunction) and secondary toxicity (e.g. leukopenia-associated bacterial infections), iii) the treatment (excluding transfusions, ie for BK cystitis) of infectionrelated complications, iv) the transfusions due to treatmentrelated toxicity (ie cytopenias) or infection-related complications (ie, BK cystitis), v) the inpatient or outpatient daily care. Notwithstanding that blood and its products, as a common natural good, are provided free in our country, the costs related to blood and platelet collection, processing, storage, laboratory testing and infusions were included in our model.

Results: The treatment cost of CMV, EBV and BKV reactivations/infections for the first six months post allo-
HSCT was evaluated in $38 / 51$ patients who reactivated viruses and were transplanted between 10/2015-11/2016 from matched related (17/51), matched unrelated (20/51), mismatched unrelated (8/51), haploidentical (5/51) and mismatched related donors (1/51). We detected $29 \mathrm{CMV}$, $35 \mathrm{EBV}$ and $18 \mathrm{BKV}$ infections/reactivations in 20, 32 and 17 patients respectively, with a mean of $2 \pm 0.4$ infection per patient from all three viruses (1-7/patient). Of note, 22/38 patients experienced reactivations from more than one virus, requiring repeated treatments with antiviral agents and/or rituximab. The cost of antiviral agents for all CMV, EBV and BKV reactivations/infections was $78,656 €, 58,504 €$ and $11,331 €$ respectively $(3,146 €, 2,089 €$ and 1,416 $€ /$ patient, respectively). The treatment cost of toxicity related to antiviral drugs and infection-related complications was $70,358 €(4,309 € /$ patient $)$ excluding transfusions and $676,107 €(28,171 € /$ patient $)$ including transfusions. In particular, the cost of transfusions for BKV hemorrhagic cystitis reached 22,751€/patient. Repeated (1-5) and/or prolonged $(\Delta \mathrm{m} 38 \mathrm{~d}$, range $6-150 \mathrm{~d})$ hospitalizations were needed, up to a total of 745 and 159 days of inpatient hospitalization and short-term outpatient treatment, respectively. Hospitalizations further increased the cost of inpatient and outpatient post-transplant care by $81,640 €$ and $8,000 €$ respectively $(2,634 €$ and $500 € /$ patient, respectively), onthe basis of a, rather underestimating the true cost, fixed, unified hospitalization fee (60€/day and 200 $€ /$ day).

Conclusions: Overall, in a six-month study period, the treatment of CMV, EBV and BKV infections substantially increased the cost of post-transplant care by $956,283 €$ (27,322€/patient). The actual cost is undoubtedly higher as the hospitalization fee for transplant recipients is largely underestimated in Greece. Considering not only the hematopoietic but also the solid organ transplant recipients, the financial burden of antiviral treatment for national economies is enormous. Given that antiviral pharmacotherapy is often associated with suboptimal efficacy, toxicity, development of drug resistance, reactivation recurrences and repeated hospitalizations, it is expected that a one-time treatment with multi-virus-specific $\mathrm{T}$ cells, able to expand in vivo and provide a long-lasting protection without significant toxicity, will serve as a powerful and costeffective treatment over conventional pharmacotherapy.

Disclosure: Nothing to declare

\section{P393}

EBV reactivation associated post-transplant lymphoproliferative disease, clonal gammopathy and outcomes in allogeneic T-cell depleted stem cell transplants for myeloid malignancies 
Varun Mehra', Eleanor Barnwell', Ute Boczek', Stefani Widya $^{2}$, Adrian Choy ${ }^{2}$, Alessandra Bisquera ${ }^{1}$, Kavita Raj $^{1,3}$, Donal Mclornan ${ }^{1,3}$, Shreyans Gandhi', Austin Kulasekararaj ${ }^{1}$, Hugues de Lavallade ${ }^{1}$, Victoria Potter ${ }^{1}$, Ghulam Mufti, ${ }^{1,2}$ Antonio Pagliuca ${ }^{1}$

${ }^{1}$ Kings College Hospital, London, United Kingdom, ${ }^{2} \mathrm{GKT}$ School of Medicine, London, Haematology, London, United Kingdom, ${ }^{3}$ Guy's and St Thomas' Hospital, Haematology, London, United Kingdom

Background: Ebstein Barr Virus is frequently reactivated (EBV-R) in immunocompromised allogeneic haematopoietic stem cell transplant(HSCT) patients following $\mathrm{T}$ cell depletion(TCD). Incidental observations of monoclonal gammopathy(MG or M-protein) post-HSCT have also been reported. We report on incidence \& outcomes of EBV-R, PTLD and association with M-protein in a large cohort of in-vivo TCD allogeneic HSCTs and provide quantitative underpinning to decisions about pre-emptive treatment for post-transplant lymphoproliferative disease (PTLD).

Methods: This is a single-centre retrospective analysis of 293 consecutive patients who underwent TCD allo-HSCTs for myeloid malignancies between January 2012-June 2016. EBV-DNA was monitored frequently on whole blood samples with standardised quantitative real-time PCR. Serum protein electrophoresis was routinely tested with immunoglobulin subclasses identified by immunofixation electrophoresis. Histological confirmation of PTLD was based on standard WHO diagnostic criteria ('proven'), while those without biopsy were classed as 'probable' based on clinical \& radiological criteria as defined by ECIL-6 guidelines.

Results: Majority of patients had AML(n-152/293) and MDS(n-107/293) with a median age of 58 years(range 2276). Median follow up of survivors was 32 months(range 465). Majority of patients(n-220/293;75\%) developed EBV$\mathrm{R}$ with a median time of 79 days[Inter quartile range(IQR) 27-160 days] \&higher cumulative incidence with ATG(n132) versus Alemtuzumab $(n-161)(\mathrm{p}<0.001)$. Figure-1a shows schematic representation of EBV and PTLD events (cumulative incidence of $6.8 \%(95 \%$ CI-4.0\%-10.6\%) at 12 months). Significantly higher peak EBV DNA viral load(EVL) were noted in patients with PTLD(p-<0.001). Development of post-HSCT MG was observed in $29 \%$ (n85/292). ROC curve identified peak blood EVL>150,000 copies/ml significantly correlated with risk of developing PTLD (sensitivity-70.6\%, specificity-79.4\%;AUC-0.82,p< $0.001)$. Based on these estimates, subgroup of patients with no EBV-R(n-72/292), peak EVL $<150,000$ ( < 150k)copies/ $\mathrm{ml}(\mathrm{n}-165 / 292) \quad \& \quad>150,000(>150 \mathrm{k})$ copies/ml(n-55/292) were categorised in 6 groups along patients with/without MG accordingly (Groups 1-6;Figure-1b). Patients with
EBV-R had significantly better OS [5-year OS of 52\% vs $35 \%$ (no EBV-R);Log-rank $\mathrm{p}<0.001$ ], with this survival benefit mainly driven by subgroup of patients with lower EVL $(<150 \mathrm{k})(\mathrm{p}<0.001)$. PTLD patients had trend towards inferior 3-year OS(15\% vs 54\%;p-0.051). Patients with MG had a significantly better OS irrespective of degree of EVL (Group 1-3,p< 0.001).We report a 'sweet spot' of low EVL $\&$ presence of $\mathrm{MG}$ in these patients, with a clear survival advantage compared to those with no EBV-R and/or no Mprotein (Group-2 5-year OS 62\% vs $27 \%$ in Group-6; HR0.15;95\%CI:0.06-0.34;p< 0.001;Figure1b). Overall cumulative incidence of relapse (CIR) was $28 \%$ (95\%CI:23-37) and non-relapse-mortality(NRM) of 24\%(95\%CI:18.6-30) at 5 years. Multivariate analysis(MVA) revealed absence of M-protein,high EVL ( $>150 \mathrm{k}$ copies/ml) or no EBV-R and absence of any GVHD as significant factors for high CIR. Similarly, high EVL or no EBV-R, absence of M-protein and ITU admission were significant predictors of high NRM.

Conclusions: This study adds to our understanding of role of EBV viraemia \& associated MG in TCD-HSCTs while highlighting its significant impact on risk of PTLD, OS, NRM \& CIR. Low EBV burden and development of MG is protective with significantly better survival outcomes and we recommend pre-emptive approach of using Rituximab for EBV-R /PTLD is best employed at higher EBV burden (e.g. $>150 \mathrm{k}$ copies/ml DNA) in high risk patients and be prospectively evaluated in future studies.

Clinical Trial Registry: n/a

Disclosure: Nothing to declare

\section{P394}

Impact of early candidemia on the long-term outcome of allogeneic hematopoietic stem cell transplant in non leukemic patients: An outcome analysis on behalf of IDWP

Simone Cesaro ${ }^{1}$, Gloria Tridello ${ }^{1}$, Nina Simone Knelange $^{2}$, Nicolaas Schaap ${ }^{3}$, Murray Martin ${ }^{4}$, John Snowden $^{5}$, Ram Malladi ${ }^{6}$, Per Ljungman ${ }^{7}$, Eric Deconinck $^{8}$, Tobias Gedde-Dahl', N.H. Russell ${ }^{10}$, Gerard Socié $^{11}$, Patrice Chevallier ${ }^{12}$, Johan Maertens ${ }^{13}$, Tsila Zuckerman $^{14}$, Bruno Lioure ${ }^{15}$, Akin Abayomi ${ }^{16}$, Eefke Petersen $^{17}$, J.J. Cornelissen ${ }^{18}$, William Arcese ${ }^{19}$, Didier Blaise $^{20}$, Noel Milpied ${ }^{21}$, Jean Yves Cahn ${ }^{22}$, Mahmoud Aljurf $^{23}$, Liesbeth de Wreede ${ }^{24}$, Catherineherine Cordonnier $^{25}$, Rafael de la Camara ${ }^{26}$, Dina Averbuch ${ }^{27}$, Malgorzata Mikulska ${ }^{28}$, Jan Styczynski ${ }^{29}$

${ }^{1}$ Azienda Ospedaliera Universitaria Integrata Verona, Verona, Italy, ${ }^{2}$ EBMT Data Office, Leiden, Netherlands, ${ }^{3}$ Nijmegen Medical Centre, Nijmegen, Netherlands, ${ }^{4}$ Leicester Royal Infirmary, Leicester, United Kingdom, 
${ }^{5}$ Sheffield Teaching Hospitals NHS Trust, Sheffield, United Kingdom, ${ }^{6}$ University Hospital Birmingham NHSTrust, Birmingham, United Kingdom, ${ }^{7}$ Karolinska University Hospital, Stockholm, Sweden, ${ }^{8}$ Hopital Jean Minjoz, Besancon, France, ${ }^{9}$ Oslo University Hospital, Rikshospitalet, Oslo, Norway, ${ }^{10}$ Nottingham University Hospital NHS Trust, Nottingham, United Kingdom, ${ }^{11}$ Hopital St. Louis, Paris, France, ${ }^{12} \mathrm{CHU}$ Nantes, Dept. D'Hematologie, Nantes, France, ${ }^{13}$ University Hospital Gasthuisberg, Leuven, Belgium, ${ }^{14}$ Rambam Medical Center, Haifa, Israel, ${ }^{15}$ Techniciens d'Etude Clinique Suivi de Patients Greffes, Strasbourg, France, ${ }^{16}$ Constantiaberg Medi-Clinic, Cape Town, South Africa, ${ }^{17}$ University Medical Centre Regensburg, Utrecht, Netherlands, ${ }^{18}$ Erasmus MC Cancer Institute, Rotterdam, Netherlands, ${ }^{19}$. Tor Vergata“ University of Rome, Rome, Italy, ${ }^{20}$ Programme de Transplantation\&Therapie Cellulaire, Marseille, France, ${ }^{21} \mathrm{CHU}$ Bordeaux, Pessac, France, ${ }^{22} \mathrm{CHU}$ Grenoble Alpes - Université Grenoble Alpes, Grenoble, France, ${ }^{23}$ King Faisal Specialist Hospital \& Research Centre, Riyadh, Saudi Arabia, ${ }^{24}$ LUMC, Dept of Biomedical Data Sciences, Leiden, Netherlands, ${ }^{25}$ Service d'Hématologie Clinique et de Thérapie Cellulaire Pôle CITI CHU, Hospital Henri Mondor, Créteil-Paris, France, ${ }^{26}$ Hospital de La Princesa, Madrid, Spain, ${ }^{27}$ Pediatric Infectious Diseases, Hadassah University Hospital, Jerusalem, Israel, ${ }^{28}$ Ospedale San Martino, Genova, Italy, ${ }^{29}$ University Hospital, Collegium Medicum UMK, Bydgoszcz, Poland

Background: To assess the incidence of, and risk factors for, Candida infection in the first 100 days post-allogeneic hematopoietic stem cell transplantation (HSCT) and the impact on long-term survival.

Methods: Outcome analysis of 50,188 patients, $61 \%$ male, median age 46 years (range $0-80$ ), with diagnosis of hemoglobinopathies in $3176(6.3 \%)$, bone marrow failure in 7626 (15.2\%), lymphoma in 17743 (35.4\%) and myelodysplastic/myeloproliferative disesases in 21643 (43.1\%) patients who underwent HSCT from 2000 to 2015: 420 with candidemia by day +100 , and 49,768 without candidemia.

The incidence of 100-day candidemia was estimated by using the cumulative incidence method. The univariate and multivariate risk factor analysis for 100-day candidemia was performed with the cause-specific Cox regression model.

The occurrence of candidemia was analyzed as a timedependent covariate. The overall survival and non-relapse mortality after day +100 were assessed in a land-mark setting, this analysis was restricted to patients surviving to day +100 post transplant.
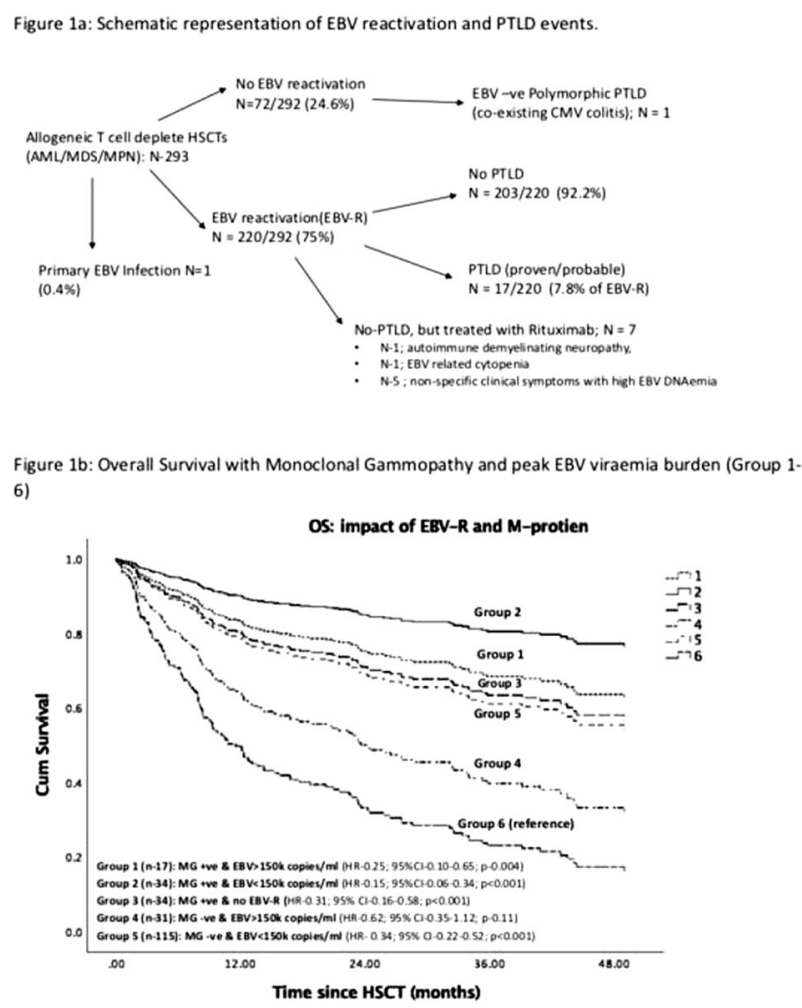

\section{[[P393 Image] 1. Figure la-1b]}

Results: The incidence of candidemia by day +100 was $0.85 \%$ (95\% C.I. $0.77-0.93)(420 / 50,188)$ and occurred at a median of 17 days post-HSCT (range -7-100). Considering the candidemia within 100-day from HSCT as a time dependent covariate, a higher 100-day non-relapsemortality (NRM) (HR 3.47 (2.75-4.38), p < 0.0001), and a lower 100-day overall-survival (OS) (HR 3.21, 95\% CI 2.67-3.85), $\mathrm{p}<0.0001$ ) were obtained from the Cox model for patients with candidemia. Factors significantly associated with candidemia occurrence in the multivariate analysis were: gender female, increased age at HSCT, bone marrow failure, lymphoma or myelodysplastic/myeloproliferative diagnosis, bone marrow or cord blood stem cell source, T-cell depletion, less recent year of HSCT. Among patients alive at day +100 , the 5-year NRM and OS with and without candidemia were $28.2 \%$ vs. $18.8 \%, \mathrm{p}<0.0001$, and $50.5 \%$ vs. $60.7 \%, \mathrm{p}<0.0001$, respectively, after a median follow-up of 4.3 years (95\% CI 4.3-4.4) (Figure 1). In multivariate analysis, the occurrence of a candidemia episode within day +100 was an independent risk factor for higher NRM, HR 1.52 (1.18-1.97), $\mathrm{p}=0.0013$, and lower OS, HR 1.30 (1.08-1.57), $\mathrm{p}=0.0061$.

Conclusions: despite the general improvements in prophylaxis and treatment, the occurrence of early postHSCT candidemia had a negative impact on transplant outcome as showed previously in leukemic patients. 
Disclosure: Nothing to declare.

\section{P395}

Abstract already published.

\section{P396}

Carbapenem-resistant enterobacteriaceae colonization importance in the risk of CRE bacteremia and mortality in stem cell transplant (HSCT) and acute leukemia patients

Marcia Garnica ${ }^{1,2}$, Marco A F Bellizze $e^{1}$, Priscila G A de Jesus $^{1}$, Rafaela R C Gomes ${ }^{1}$, Filipe M Akamine ${ }^{1}$, Alan J Marçal $^{1}$, Luzinete CO Rangel ${ }^{2}$, Andreia Assis ${ }^{2}$, Marcia Rejane Valentim ${ }^{2}$, Angelo Maiolino ${ }^{1}$

${ }^{1}$ Universidade Federal do Rio de Janeiro, Internal Medicine, Rio de Janeiro, Brazil, ${ }^{2}$ Complexo Hospitalar de Niteroi CHN, Niteroi, Brazil

Background: Spread of infections due to Carbapenemresistant Enterobacteriaceae (CRE) is a worldwide phenomenon and has been associated with high mortality and clinical complications. Gut translocation is the most important portal of entry of bacteria during neutropenia, and CRE gut colonization is a possible risk factor for bacteremia during neutropenia. Goals: In the present study, we describe the frequencies of CRE colonization and analyzed its relationship with development of CRE bacteremia and mortality in two different scenarios: stem cell transplant patients (HSCT) and Leukemia patients.

Methods: Prospective cohorts of HSCT (from 2012 to 2017) and Leukemia patients (from 2016 to 2018). HSCT patients were analyzed from conditioning until discharge (pre-engraphment phase) and leukemia patients from first induction chemotherapy until last intensification. If an HSCT was performed in Leukemia patient the patient was censored in leukemia cohort and included in HSCT cohort. All patients had rectal swabs performed weekly during hospitalization for the identification of CRE colonization. Patients with at least one positive swab (CRE colonization group) were compared to patients with no documentation of colonization (controls). The outcomes analyzed were bacteremia due to CRE, and overall mortality.

Results: There were 493 HSCT performed during the study (408 [83\%] autologous and 85 [17\%] allogeneic). Multiple myeloma and Non-Hodgkin Lymphoma were the most frequent baseline diseases $(\mathrm{N}=251 ; 51 \%$, and $\mathrm{N}=88$; $18 \%$ ), respectively. CRE colonization was documented in $10 \%(\mathrm{~N}=50)$, and it was more frequent among Allogeneic HSCT and Leukemia patients ( $p<0.001$ for both). CRE colonized patients had longer hospitalization (25 vs. 20 days, $\mathrm{p}<0,001$ ), higher frequency of CRE bacteremia
( $6 \%$ vs. $0.2 \% ; \mathrm{p}=0.004)$, and mortality ( $16 \%$ vs. $2.4 \%, \mathrm{p}<$ $0,001)$ compared to non-colonized HSCT. Negative and positive predicted values for CRE bacteremia were

$6 \%$ and $99 \%$, respectively. Thirty-one patients were analyzed in Leukemia cohort, accounting to 92 hospitalizations (median 3 hospitalizations per patient, ranging from 1 to 6). The median age was 58 years, and $90 \%$ AML vs. $10 \%$ ALL. CRE colonization was documented in eight (26\%), with a median time from leukemia diagnosis and colonization of 93 days ( 9 - 503 days). CRE bacteremia was documented only in colonized patients $(25 \%$ vs. zero; $\mathrm{p}=0,046$ ). All eight colonized patients were submitted to other cycles of chemotherapy after colonization, in one of them CRE bacteremia relapsed.

Conclusions: A routine surveillance of CRE colonization showed colonization frequencies from $10 \%$ to $25 \%$ in HSCT and Leukemia patients respectively and was effective to stratify CRE bacteremia risk as the predictive negative value was over $95 \%$. Colonization had association with CRE bacteremia and overall mortality. Efforts to minimize risks for colonization and mortality are necessary. The information of surveillance can be a tool to improve adequacy in empirical febrile neutropenia therapy in HSCT and Leukemia patients.

Disclosure: Nothing to declare

\section{P397}

Whether prophylactic antiviral treatment is necessary in HBSAG-NEGATIVE/HBCAB-positive patients receiving allogeneic hematopoietic stem cell transplantation

\section{Yibo Wu ${ }^{1}$, Yamin $\operatorname{Tan}^{1}$, Yanmin Zhao ${ }^{1}$, Jimin Shi, Yuanyuan Zhu', Weiyan Zheng ${ }^{1}$, Jian $\mathbf{Y u}^{1}$, He Huang ${ }^{1}$, Yi Luo ${ }^{I}$}

${ }^{1}$ First Affiliated Hospital of Zhejiang University School of Medicine, Bone Marrow Transplant Center, Hangzhou, China

Background: The incidence of hepatitis B virus infection is high to $6.2 \%$ in Asian population, So there is more and more attention to the risk of hepatitis $B$ virus(HBV) reactivation in the hepatitis B core antibody positive patients during chemotherapy, anti-CD20 monoclonal antibody, HSCT, or other intense immunosuppressive drug therapy (ISDT). Hepatitis B Core Antibody is associated with a significant risk of $\mathrm{HBV}$ reactivation in patients undergoing HSCT. However, There are remain uncertain that the effect of anti-HbsAg antibodies in hepatitis B virus reactivation among the hepatitis B core antibody positive patients undergo HSCT. We aim to investigate the role of the antiHBs and the necessity of anti virus in hepatitis B surface 
antigen(HBsAg) negative, hepatitis B core antibody positive patients during HSCT.

Methods: we enrolled 791 hematological malignant patients received HSCT in our center from 2008 to 2016. we classified $665 \mathrm{HBsAg}$ negative and undetectable HBV DNA patients into 4 groups as anti-HBc(-)anti-HBs(-) $(\mathrm{n}=189)$, anti-HBc(-)anti-HBs(+) $(\mathrm{n}=176)$, anti-HBc $(+)$ anti-HBs(-) $(\mathrm{n}=49)$, and anti-HBc $(+)$ anti-HBs $(+)(\mathrm{n}=251)$.

Results: HBV reactivation was identified in 16 patients (2.4\%) after HSCT. There was a significant difference in HBV reactivation rate in anti-HBc(+)anti-HBs(-) (12.2\%) vs anti-HBc $(+)$ anti-HBs $(+)(2.8 \%)(\mathrm{p}=0.01)$ and anti-HBc $(+)$ anti-HBs(-) (12.2\%) vs anti-HBc(-)anti-HBs(-) (0.0\%) $(\mathrm{p}=0.000)$, anti-HBc(+)anti-HBs(-) $(12.2 \%)$ vs anti-HBc(-) anti-HBs $(+)(1.7 \%)(\mathrm{p}=0.004)$, but not among anti-HBc $(+)$ anti-HBs(+) (2.8\%) and anti-HBc(-)anti-HBs(-) (0\%) and anti-HBc(-)anti-HBs(+) (1.7\%). Whereas there were no difference according to the donor viral profile $(\mathrm{p}=0.774)$. The median time of HBV reactivation in HBsAg negative patients accepted HSCT was 645 (455-1957) days after HSCT. All of the patients with HBV reactivation have been controlled with Nucleos(t)ide Analogues drugs, and 5 of them achieved reverse seroconversion which detect persistent anti-HbsAg antibodies in their bodies.

Conclusions: The anti-HbsAg antibodies negative and anti-HBc positive patients have the highest risk of $\mathrm{HBV}$ reactivation after HSCT in resolved HBV patients. The anti$\mathrm{HbsAg}$ antibodies play a protective role in resolved HBV patients receiving HSCT. We recommend not prophylactic anti hepatitis B virus in $\mathrm{HBsAg}$ negativity and anti-HbsAg antibodies positive patients following hematopoietic stem cell transplantation.

Disclosure: Nothing to declare

\section{P398}

Donor EBV-positive serostatus increases the risk of chronic GVHD in patients with non-malignant hematological disorders: Infectious diseases working party EBMT study

Jan Styczynski ${ }^{1}$, Gloria Tridello ${ }^{2}$, Lidia Gil ${ }^{3}$, Per Ljungman $^{4}$, Malgorzata Mikulska ${ }^{5}$, Steffie van der Werf ${ }^{6}$, Nina Knelange ${ }^{7}$, Diana Averbuch ${ }^{8}$, Gerard Socie ${ }^{9}$, Hendrik Veelken ${ }^{10}$, Jean-Hugues Dalle ${ }^{11}$, Mahmoud Aljurf $^{2}$, Alphan Kupesiz ${ }^{13}$, Yves Bertrand ${ }^{14}$, Abdelghani Tbakhi' $^{15}$, Boris Afanasyev ${ }^{16}$, Marie-Pierre Ledoux ${ }^{17}$, Helene Labussière-Wallet ${ }^{18}$, Xavier Poire ${ }^{19}$, Johan Maertens $^{20}$, Eefke Petersen ${ }^{21}$, Patrice Chevalier ${ }^{22}$, Noel Milpied $^{23}$, John A. Snowden ${ }^{24}$, Ibrahim Yakoub-Agha ${ }^{25}$, Jan Cornelissen ${ }^{26}$, Nicalaas Schaap ${ }^{27}$, Carlo Dufour ${ }^{28}$, Regis Peffault de Latour ${ }^{9}$, Arjan Lankester ${ }^{10}$, Simone Cesaro $^{2}$
${ }^{1}$ Collegium Medicum UMK, Bydgoszcz, Poland, ${ }^{2}$ Azienda Ospedaliera Universitaria Integrata, Verona, Italy, ${ }^{3}$ University of Medical Sciences, Poznan, Poland, ${ }^{4}$ Karolinska University Hospital, Stockholm, Sweden, ${ }^{5}$ Ospedale San Martino, Genova, Italy, ${ }^{6}$ EBMT, Leiden, Netherlands, ${ }^{7}$ EBMT Office, Leiden, Netherlands, ${ }^{8}$ Haddasah Hebrew University Medical Center, Jerusalem, Israel, ${ }^{9}$ Hopital St. Louis, Paris, France, ${ }^{10}$ Leiden University Hospital, Leiden, Netherlands, ${ }^{11}$ Hôpital Robert Debre and University Paris 7 - Paris Diderot, Paris, France, ${ }^{12}$ King Faisal Specialist Hospital \& Research Centre, Rijadh, Saudi Arabia, ${ }^{13}$ Akdeniz University Medical School, Antalya, Turkey, ${ }^{14}$ Institut d’Hematologie et d Oncologie Pediatrique, Lyon, France, ${ }^{15}$ King Hussein Cancer Centre, Amman, Jordan, ${ }^{16}$ First State Pavlov Medical University, St. Petersburg, Russian Federation, ${ }^{17}$ Hopitaux Universitaires de Strasbourg, Strasbourg, France, ${ }^{18}$ Centre Hospitalier Lyon Sud, Lyon, France, ${ }^{19}$ Cliniques Universitaires St. Luc, Brussels, Belgium, ${ }^{20}$ University Hospital Gasthuisberg, Leuven, Belgium, ${ }^{21}$ University Medical Centre, Utrecht, Netherlands, ${ }^{22} \mathrm{CHU}$ Nantes, Nantes, France, ${ }^{23} \mathrm{CHU}$ Bordeaux, Pessac, France, ${ }^{24}$ Sheffield Teaching Hospitals NHS Trust, Sheffield, United Kingdom, ${ }^{25} \mathrm{CHU}$ de Lille, LIRIC, INSERM U 995, Université de Lille, Lille, France, ${ }^{26}$ Erasmus MC Cancer Institute, Rotterdam, Netherlands, ${ }^{27} \mathrm{Nij}-$ megen Medical Centre, Nijmegen, Netherlands, ${ }^{28}$ Instituto Giannina Gaslini, Genova, Italy

Background: In patients with acute leukemia (AL), donor EBV (Epstein-Barr Virus) seropositive status significantly increases the risk of development of acute and chronic graftversus-host disease (GVHD) after allo-HSCT, while it has no influence on other transplant outcomes (overall survival, OS; rejection-free survival, RFS; rejection incidence, RI; non-rejection mortality, NRM) (Styczynski et al, J Clin Oncol, 2016). No data are available on the impact of EBV serostatus on transplant outcomes in patients with nonmalignant hematological disorders.

Objective: We analyzed the influence of the recipient's (R) and donor's (D) EBV serostatus on transplant outcomes (OS; RFS; RI; transplant-related mortality, TRM; acute GVHD, aGVHD; chronic GVHD, cGVHD) in patients with nonmalignant hematological disorders undergoing allo-HSCT.

Methods: 2230 allo-HSCTs performed between 1997 and 2016 for acquired bone marrow failure $(70.6 \%)$ or hemoglobinopathies $(29.4 \%)$, with $\mathrm{BM} \pm \mathrm{CB}(75.8 \%)$ or $\mathrm{PB} \pm \mathrm{CB}+\mathrm{BM}(24.2 \%)$ as a stem cell source were included in this retrospective Registry megafile IDWP EBMT study.

Results: DEMOGRAPHICS: The median age of recipient was 17.7 years (range: $0.1-78$ ), and $50.8 \%$ were children. $79.0 \%$ recipients and $75.4 \%$ donors were $\mathrm{EBV}$-seropositive. $67.8 \%$ had HSCT from a matched family donor, $4.6 \%$ from a mismatched family donor, and $27.6 \%$ from an unrelated 
donor. T-cell depletion was performed in vivo in $82.2 \%$, and ex vivo in $6.6 \%$ patients. Conditioning regimen was myeloablative in $63.7 \%$, RIC in $36.3 \%$. Median follow-up was 4.7 years (95\% C.I. 4.3-5.0). TRANSPLANT OUTCOMES: EBV-seropositive recipients in comparison to EBV-seronegative recipients had lower OS $(85.4 \%$ vs $88.4 \%, \mathrm{p}=0.035)$, and higher NRM $(10.0 \%$ vs $6.4 \%$, $\mathrm{p}=0.018)$. No other significant differences were found for: RI, RFS, and acute or chronic GVHD with respect to EBV pretransplant serostatus donor and/or recipient. MULTIVARIATE ANALYSIS: EBV serostatus as a risk factor did not reach significance, while a trend towards higher risk of development of cGVHD (HR=1.31; 95\%CI 0.97-1.78; $\mathrm{p}=0.081)$ and better survival $(\mathrm{HR}=0.78 ; 95 \% \mathrm{CI}$ 0.59-1.04; $\mathrm{p}=0.087$ ) in allo-HSCT from EBV-seropositive donors. Allo-HSCT in EBV-seropositive recipients had a trend towards lower risk of development of cGVHD ( $\mathrm{HR}=0.75$; 95\%CI 0.56-1.02; $\mathrm{p}=0.066$ ). When 4 subgroups (R-/D-, R-/ $\mathrm{D}+, \mathrm{R}+/ \mathrm{D}-, \mathrm{R}+/ \mathrm{D}+\mathrm{EBV}$ serology) were analyzed, the EBV serostatus had no significant impact on OS, RFS, RI, TRM and development of acute or chronic GVHD.

Conclusions: Allo-HSCT from EBV-seropositive vs EBV-seronegative donors are at $31 \%$ higher risk of chronic GVHD in patients with non-malignant hematological disorders undergoing allo-HSCT, however this difference is non-significant in multivariate analysis.

Disclosure: Nothing to declare.

\section{P399}

Influenza 2017/ 2018 in outpatient HSCT-recipients: Single center surveillance

\section{Markus Ditschkowski, Melanie Fiedler ${ }^{1}$, Rashit Bogdanov ${ }^{I}$, Vesna Klisanin ${ }^{1}$, Michael Koldehoff ${ }^{1}$, Lambros Kordelas ${ }^{1}$, Rudolf Trenschel ${ }^{\text {, Dietrich Beelen }}{ }^{1}$}

${ }^{1}$ University Hospital Essen, West-German Cancer Center, Essen, Germany

Background: Influenza remains a major risk for immunocompromised patients (pts) after allo-SCT. However, data on influenza and the effectiveness of vaccination in the setting of allo-SCT is scarce. Our aims were to evaluate the incidence of influenza-subtypes confirmed by positive swabs in outpatient SCT-recipients during flu epidemic 2017/ 2018 in a German transplant Center and to estimate vaccination rates or determine effectiveness of vaccination in consideration of immune parameters, immunosuppressive therapy and time after SCT, respectively.

Methods: Between January 2018 and April 201895 of $664(14 \%)$ outpatient HSCT-recipients showed clinical signs of flu and received influenza swab-testing with consecutive PCR-analysis for influenza subtypes A, B, A
(H1N1) pdm0e9. In this cohort (51 male, 44 female) the median follow-up post-transplant was 680 days and 63 pts (66\%) received immunosuppressive treatment.

Results: Twenty-eight (30\%) pts (19 male, 9 female) were tested positive (group 1) for subtype A ( $\mathrm{n}=4,14 \%)$, B $(\mathrm{n}=18,64 \%)$ or $\mathrm{A}(\mathrm{H} 1 \mathrm{~N} 1)(\mathrm{n}=6,21 \%)$ while $67(70 \%) \mathrm{pts}$ (32 male, 35 female) were negative (group 2). Vaccination rate in group $1(32 \%)$ was significantly lower compared to group $2(51 \%, \mathrm{p}=0.002)$. The median time after transplantation (790 vs 565 days), T-cell counts (349 vs $296 / \mu \mathrm{l}$ ), Bcell counts (162 vs $135 / \mu \mathrm{l})$, IgG-level $(8,3$ vs $7,6 \mathrm{~g} / \mathrm{l})$, proportion of immunosuppressed pts ( $75 \%$ vs $63 \%)$, male/ female ratio was not significantly different between groups 1 and 2 . Within group 1 influenza subtypes were similarly distributed in vaccinated and not vaccinated pts (A $11 \%$ vs $16 \%$, B $67 \%$ vs $63 \%$, A (H1N1) $22 \%$ vs $21 \%$ ). Pts. with subtype B infection had higher levels of T- (482 vs $274 / \mu \mathrm{l})$ and B-cells (232 vs 108/ $\mu \mathrm{l}$ ) and a longer follow up from SCT (1610 vs 470 days) compared to subtype A / A (H1N1) infection but differences were not significant.

Conclusions: Influenza could be proven in one third of all tested pts. Dominance of B and A (H1N1) pdm0e9 subtype occurrence corresponded to the flu epidemic dissemination in the German population. The most important protective factor for outpatient SCT recipients was influenza vaccination.

Disclosure: Nothing to declare

\section{P400}

Abstract already published.

\section{P401}

CMV infection in the haploidentical transplant (HAPLO) with post-transplant cyclophosphamide (PTCY): One center's experience

Almudena Cabero Martínez ${ }^{1}$, Sara Fernandez Luis ${ }^{1}$, Cristina Fernández Martínez ${ }^{1}$, Sara García Ávila ${ }^{1}$, Andrés Insunza Gaminde ${ }^{1}$, Miriam Sánchez Escamilla ${ }^{2}$, Joan Manuel Mora Barrios', Yolanda González Romero ${ }^{1}$, David Gómez Lamas ${ }^{1}$, Lucrecia Yáñez San Segundo ${ }^{1}$, Mercedes Colorado Araujo ${ }^{I}$, Mónica López Duarte ${ }^{1}$, Javier Núñez Céspedes ${ }^{1}$, Guillermo Martín Sánchez ${ }^{1}$, Amaia Casado Fernández ${ }^{3}$, Miguel Ángel Cortés Vázquez $^{I}$, Carlos Richard Espiga ${ }^{l}$, Enrique Ocio San Miguel $^{1}$, Maria Aranzazu Bermúdez Rodríguez ${ }^{I}$

${ }^{1}$ Hospital Universitario Marqués de Valdecilla, Hematología, Santander, Spain, ${ }^{2}$ Instituto de Formación e Investigación Marqués de Valdecilla (IDIVAL), Hematología, Santander, Spain, ${ }^{3}$ Hospital Universitario de Galdakao, Hematología, Bilbao, Spain 
Background: CMV infection is one of the most frequent complications after HAPLO. Some risk factors are well known but the best strategy (prophylactic or preemptive treatment) to mitigate this complication is not still well defined.

The primary endpoint in our study is to describe incidence and risk factors to develop CMV infection or disease in HAPLO. As secondary objective we analyzed efficacytoxicity of treatment and CMV related mortality.

Methods: We analyzed 60 patients who underwent HAPLO in our center between May 2012 and May 2018. All of them received PTCy (D+3 and D +4$)$, tacrolimus and mycophenolate as graft versus host disease prophylaxis.

A preemptive therapy based on viral load was applied. Treatment was started when $>1000 \mathrm{UI} / \mathrm{mL}$ of CMV were detected in one determination or $>500 \mathrm{UI} / \mathrm{mL}$ in two consecutive determinations. CMV analyses were made in plasma using Cobas PCR technique ${ }^{\circledR}$ and positive viral load cut-off point was $137 \mathrm{UI} / \mathrm{mL}$. The CMV viremia was determined weekly until $\mathrm{D}+100$ and then every two weeks until immune reconstitution.

Results: The CMV infection and disease incidence at D +100 was $61.3 \%$ (38 episodes) and $19.4 \%$ (12 episodes), respectively. CMV disease was digestive $(\mathrm{n}=8)$, pulmonar $(n=2)$, neurologic $(n=1)$ and disseminated $(n=1)$. The median time to first CMV infection was 39.5 days (20-151).

Thirty-six patients had at least one episode of CMV infection: 24 of them (66.7\%) had one episode, 7 (19.4\%) had two episodes and $5(13.9 \%)$ had 3 or more episodes, respectively.

Only pre-transplantation CMV status was significantly associated with CMV infection $(\mathrm{p}<0.001)$. Risk factors are shown in image 1 .

The median viral load in first CMV infection and disease was $8314 \mathrm{UI} / \mathrm{mL}$ (542-51158) and $24842 \mathrm{UI} / \mathrm{ml}$ (271126279), respectively $(\mathrm{p}=0.02)$. The median counts of $\mathrm{CD} 4$ lymphocytes at $\mathrm{D}+100$ in CMV infection and disease were $262 / \mathrm{mm} 3$ and $120 / \mathrm{mm} 3$, respectively $(\mathrm{p}=0.09)$.

Preemptive therapy for the first 2 episodes of CMV infections $(\mathrm{n}=48)$ was valganciclovir $(58,3 \%)$, ganciclovir (35.4\%) or foscarnet $(6.3 \%)$, reaching a complete viral load clearance in $77 \%$, with a median time to response of 19.2 days (6-34) and a median treatment duration of 21 days (2-39). Grade III-IV toxicity (mainly hematologic) was observed in $55.6 \%(\mathrm{n}=28), 30 \%(\mathrm{n}=17)$ and $16.7 \%(\mathrm{n}=3)$, respectively. Three patients had an UL54 mutation, one of them with clinical and microbiological resistance to the 3 mentioned drugs.

Three patients (5\%) had a graft failure secondary to CMV infections. Five patients (8.3\%) died as consequence of CMV infection: 3 before $\mathrm{D}+100$ secondary to CMV disease (2 pulmonar, 1 disseminated) and 2 after $\mathrm{D}+365$ due to graft failure and infectious complications.
With a median follow up of 12.5 months, overall survival at 18 months for patients who had CMV infection was $58.9 \%$ compared to $66.5 \%$ for those who had no infection $(\mathrm{p}=0.08)$.

Conclusions: A high incidence of CMV infection in HAPLO with PTCy was shown in our series and it contributed to mortality in $8.3 \%$ of patients. Only CMV status $(\mathrm{D}-/ \mathrm{R}+$ and $\mathrm{D}+/ \mathrm{R}+$ ) was significantly associated with higher risk of infection. Identification of high risk patients and new prophylactic and treatment strategies may improve these results.

Disclosure: Nothing to declare.

\section{P402}

Weekly screening for multidrug-resistant organisms identifies high number of colonizations in patients undergoing stem cell transplantation

Silvia Monsalvo ${ }^{1}$, Pascual Balsalobre ${ }^{1}$, Nieves Dorado ${ }^{1}$, Laura Solan ${ }^{1}$, Rebeca Bailen ${ }^{1}$, Marina Machado ${ }^{1}$, Maria Dolores Vigil, Javier Anguita ${ }^{1}$, Jose Luis Diez-Martin ${ }^{1}$, Mi Kwon ${ }^{I}$

${ }^{1}$ Hospital Gregorio Marañon, Madrid, Spain

Background: Multidrug-resistant organisms (MDRO) are a challenge in patients undergoing stem cell transplantation (SCT) which often result in an increase in mortality. Current literature defines only screening at the time of work-up. The aims of the study were to assess the rate of MDRO colonization with weekly screening, rate of infection and the associated mortality in patients undergoing SCT.

Methods: Consecutive patients admitted at the SCT unit between January-18 to November-18 were reviewed. Only first admission was analysed. Screening consisted of rectal and perineal swap on admission and weekly until discharge. In case of detection of MDRO, patients were isolated and infection control strategies were applied.

Results: 67 patients were analysed, median age 53 years $(18-70)$. $65 \%$ were male $(n=44)$. Median duration of hospitalization was 30 days(16-133). 293 swabs were performed, with a median of 4 swaps/patient (1-17). Patient characteristics are shown in Table 1. 60 patients (90\%) spiked fever in a median of 9 days after admission (1-26). $13 \%(\mathrm{n}=9)$ had previous documented MDRO colonization. Median neutrophil engraftment was 15 days (95\% CI 14$15)$,

In $24 \%$ patients $(\mathrm{n}=16)$ of patients had a positive screen: in $8(50 \%)$ patients at baseline and in $8(50 \%)$ patients were detected for the first time beyond baseline screen. Cumulative incidence of colonization at 7 days was $10.4 \%$ (95\%CI 3.2-17.6), at 15 days $18 \%$ (95\%CI 8.227.8 ), and at 30 days $19.5 \%$ (9.9-29.1\%) (Figure 1). MDRO 
identified were: 8 with extended-spectrum beta-lactamases producing E. Coli (ESBL-EC), 4 multidrug-resistant Pseudomonas aeruginosa (MR-PS), 3 vancomycin resistant Enterococci (VRE) and 1 patient with carbapenemaseproducing (CP) Citrobacter freundii. 7/16 colonized patients developed MDRO infection (44\%): 4 patients MR-PS, site of infection was 2 urinary tract infection (UTI), 1 urethritis, 1 genital ulcer. Two patients were treated with ceftolozane/ tazobactam, 1 with meropenem+amikacin;2 patients ESBLEC both UTI treated with meropenem; 1 patient CPcitrobacter freundii UTI treated with ceftazidime/avibactam. In $75 \%$ patients $(12 / 16)$ antibiotic treatment at febrile episode was guided by positive screening. No MDRO related ICU admission or mortality was observed.

In $76 \%$ patients $(\mathrm{n}=51)$ screening was negative for MDRO. 2 MDRO-infections were detected in this group: 1 multidrug resistant ESBL-EC bacteremia, treated with meropenem and 1 MR-PS bacteriemia and pneumonia on treated with ceftolozane/tazobam + meropenem+amikacin. Both patients required ICU admission, 1 patient died. Overall cumulative incidence of MDRO-infection at 30 days was $11 \%$ (95\%CI 3.4-16.8)

Conclusions: Weekly screening for MDRO identified a high number of MDRO colonizations, with first detection beyond baseline in $50 \%$ of the cases, allowing to apply early strategies of infection control. $75 \%$ of our cohort could benefit from guided treatment by the time of the febrile episode. Early identification of MDRO colonization might have helped to reduce MDRO related mortality, since none of the colonized patients died due to MDRO associated infection or needed ICU admission. However, these findings should be confirmed with further studies, comparing baseline with weekly MDRO screening strategies.

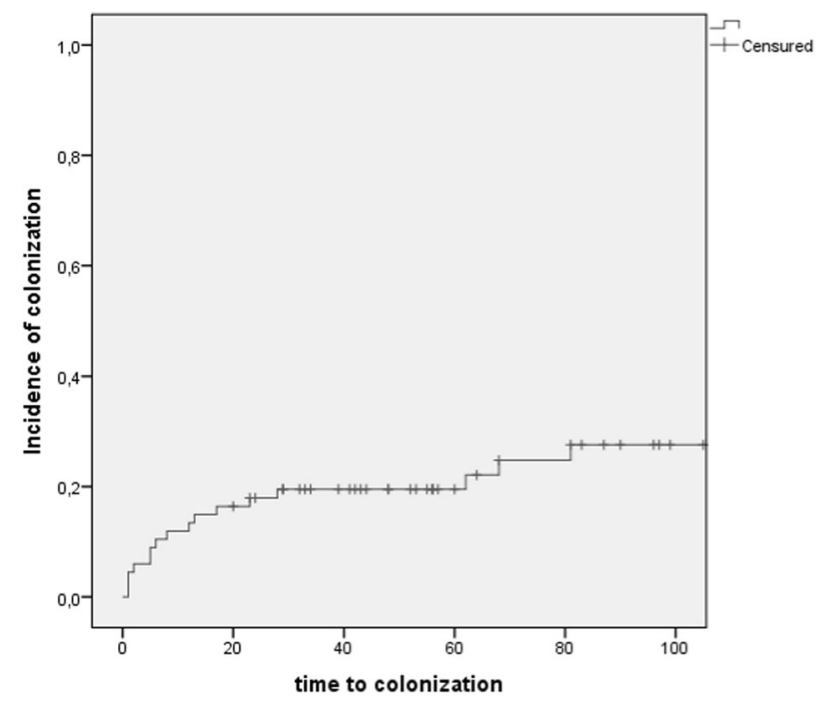

[[P402 Image] 1. Incidence MDRO-colonization]
Underlying Acute leukemia $\mathrm{n}=23$ (34\%); Multiple myeloma $\mathrm{n}=16$ disease (24\%); Lymphoproliferative disorder $n=12(18 \%)$; Myelodysplastic syndrome $n=6(9 \%)$ Others $\mathrm{n}=10(15 \%)$

Transplant Autologous $\mathrm{n}=24(36 \%)$; Allogeneic $\mathrm{n}=43(64 \%)$

Donor in Haploidentical $\mathrm{n}=31(72 \%)$; Matched sibling $\mathrm{n}=5$ alloSCT $\quad(12 \%)$; Matched unrelated $\mathrm{n}=16 \%)$

\section{[[P402 Table] 1. Patient Characteristics]}

Disclosure: Nothing to declare

\section{P403}

Hepatitis e virus is a rare but potentially serious infection in allogeneic hematopoietic stem cell transplant recipients

Lisa Swartling ${ }^{1,2}$, Rickard Nordén ${ }^{3}$, Ebba Samuelsson ${ }^{4}$, Helene Norder ${ }^{4}$, Johan Westin ${ }^{4}$, Ksenia Boriskina ${ }^{1}$, Jonas Mattsson $^{2}$, Davide Valentini ${ }^{1}$, Elda Sparrelid ${ }^{2}$, Per Ljungman $^{1,2}$

${ }^{1}$ Karolinska University Hospital, Stockholm, Sweden, ${ }^{2}$ Karolinska Institutet, Stockholm, Sweden, ${ }^{3}$ Sahlgrenska University Hospital, Gothenburg, Sweden, ${ }^{4}$ University of Gothenburg, Gothenburg, Sweden

Background: Hepatitis E virus (HEV) can cause chronic infection and liver cirrhosis in immunocompromised individuals. There is limited data on HEV infections in patients undergoing allogeneic hematopoietic stem cell transplantation (HSCT). The aim of this study was to investigate the frequency and clinical importance of HEV in a Swedish cohort of HSCT recipients.

Methods: We analyzed serum samples from 262 HSCT patients (241 adults and 21 children), collected 6 months after HSCT. HEV IgG and IgM were detected by ELISA (Dia.Pro ${ }^{\circledR}$ ), HEV RNA by reverse transcriptase PCR, and quantification of HEV RNA was performed by digital PCR. In all patients, who were positive for HEV-RNA and/or serology at 6 months, also samples collected at the time of HSCT from both the patients and their donors were analyzed. In the HEV RNA positive patients, additional samples were analyzed to determine the duration of viremia. Three HEV RNA negative controls were selected for each case of HEV infection, matched for age, diagnosis, conditioning regimen and donor type.

Results: HEV RNA was detected in 8/262 (3.1\%) patients. In three of the patients HEV RNA was positive during a period of 3-8 months, and two of these patients were infected already at the time of HSCT. In five patients HEV-RNA was positive, at a low level, only at 6 months. 
11/262 (4.2\%) patients had detectable HEV IgG and/or IgM, whereof eight patients were HEV RNA negative.

In 4/8 (50\%) patients with HEV infection (HEV RNA positive) alanine aminotransferase (ALT) was $>3$ upper limit of normal (ULN), in $1 / 8(12.5 \%)$ patients $>1.5 \mathrm{ULN}$, and in $3 / 8(37.5 \%)$ patients ALT was normal, at 6 months after HSCT. Bilirubin was elevated $>1.5 \mathrm{ULN}$ in $1 / 8$ $(12.5 \%)$ patients, and $>3 \mathrm{ULN}$ in no patient at 6 months after HSCT. Two patients died with ongoing signs of hepatitis and HEV RNA detected in blood. One of them developed acute liver failure, at the time interpreted as drug toxicity, and died of multi-organ failure. The other patient died of unrelated causes. The remaining six patients had cleared the infection at 7-24 (median 8.5) months after HSCT.

Active GVHD was present at 6 months after HSCT in 5/8 $(62.5 \%)$ patients with $\mathrm{HEV}$ infection, involving the liver in 3 of these patients. Corticosteroid treatment was ongoing in $6 / 8(75 \%)$ patients; the mean dose during the 14 preceding days was $>0.5 \mathrm{mg} / \mathrm{kg}$ in $1 / 8(12.5 \%)$ patient, $0.25-0.5 \mathrm{mg} /$ $\mathrm{kg}$ in $3 / 8(37.5 \%)$ patients, and $<0.25 \mathrm{mg} / \mathrm{kg}$ in $4 / 8(50 \%)$ patients.

HEV infection correlated to elevated ALT > 1.5 ULN, OR 8.3 (CI 1.4-49.9, $\mathrm{p}=0.02$ ) and > 3 ULN, OR 11 (CI 1.581.6, $\mathrm{p}=0.02$ ) at 6 months, but not at 3 months, after HSCT, compared to HEV RNA negative controls.

Conclusions: HEV infection was detected in $3.1 \%$ of patients tested at 6 months after HSCT and was correlated to abnormal ALT. Spontaneous clearance was common but one patient died in acute liver failure, where HEV may have contributed. HEV infection is a differential diagnosis in patients with elevated ALT 6 months after HSCT.

Disclosure: Nothing to declare

\section{P404}

Monitoring of $\mathbf{T}$-cell responses to viral-coded antigens in pediatric patients receiving TCR $\alpha \beta$-depleted haploHSCT followed by BPX-501 cell administration

\section{Stefano Di Cecca ${ }^{1}$, Mattia Algeri ${ }^{1}$, Simone Biagini ${ }^{1}$, Elia Girolami $^{1}$, Pietro Merli ${ }^{1}$, Federica Galaverna ${ }^{1}$, Annalisa Ruggeri $^{\text {, }}$, Valentina Bertaina ${ }^{I}$, Franco Locatelli, ${ }^{1,2}$, Giuseppina Li Pira ${ }^{1}$}

${ }^{1}$ IRCCS Bambino Gesù Children's HospitalRome, Italy, ${ }^{2}$ Sapienza University, Rome, Italy

Background: $\alpha \beta$ T-cell-depleted haplo-HSCT is an effective option for children with hematological disorders in need of an allograft. However, recovery of adaptive immunity is impaired in these patients. Thus, in order to accelerate immune reconstitution, we developed a novel approach based on post-transplant infusion of a titrated number of donor $\mathrm{T}$ cells, transduced with the suicide gene inducible-Caspase-9, iC9 (BPX-501 cells, Sponsor Bellicum Pharmaceuticals ${ }^{\circledR}$; NCT02065869). We previously reported on immune recovery of 108 children transplanted at our institution, showing that BPX-501 cells infused after $\alpha \beta$ T-cell-depleted haplo-HSCT expand in-vivo and persist over time, contributing to fasten adaptive immunity recovery (Merli, ASH2017). Here, we report the results of lymphoproliferation assay to viral-encoded antigens to assess Tcell function in patients transplanted with this approach.

Methods: We evaluated 142 children, 78 male and 64 female. Median age at transplant was 5.7 years (range 0.318). Patients had either malignant (69 children) and nonmalignant (73) disorders. No patient was given any posttransplant graft-versus-host disease prophylaxis. Nine children were enrolled in the phase I portion of the trial consisting of 3 cohorts receiving escalating doses of BPX501 cells. The remaining 133 patients (phase II portion) received the recommended dose of $1 \times 10^{6} \mathrm{BPX}-501$ cells $/ \mathrm{kg}$ identified in phase I. BPX-501 cells were infused at a median of 17 days post-HSCT (range 10-82). Antigendriven activation of peripheral mononuclear cells was evaluated by lymphoproliferation assay with ${ }^{3} \mathrm{H}$-thymidine pulsing at $\mathrm{d}+4$ and harvesting at $\mathrm{d}+5$. Stimuli included PHA or CMV, EBV and AdV whole viral lysate. Results are given as stimulation indexes (SI, cpm stimulated sample/cpm unstimulated control). Thresholds for positive response were arbitrarily set at SI $>3$ for viral-encoded antigens and at SI $>5$ for mitogenic stimulation with PHA. Fractions of responders are indicated in the figure.

Results: Patients were analyzed from $d+30$ to $d+720$ post-HSCT. PHA responders (A) increased to $80 \%$, while CMV (B), EBV (C) and AdV (D) responders were 61\%, $52 \%$ and $61 \%$ at 2 years after haplo-HSCT. Responses to EBV and AdV antigens were slightly delayed but improved over time. Responses to PHA and to CMV (E,F) were analyzed in the CMV-reactivating and CMV-non reactivating groups (CMV-yes/CMV-no). Significant differences in PHA response were observed at $d+60$ and $d+270$. Moreover, increased CMV responses were observed in CMVreactivators at $\mathrm{d}+270, \mathrm{~d}+360$ and $\mathrm{d}+720$, with approximately $100 \%$ of responders at $\mathrm{d}+720$, as opposed to CMVnon reactivators which comprised $50 \%$ responders. Neither primary disease, age nor TBI during the conditioning regimen influenced proliferative capacity of the two subgroups (not shown).

Conclusions: We showed a rapid recovery over time of T-cell function after $\alpha \beta$ T-cell-depleted haplo-HSCT followed by BPX-501 cells administration. When patients were grouped according to CMV reactivation (previously demonstrated as a strong driver of immune reconstitution), a significant difference in the number of responders among the patients experiencing viral reactivation was observed 
using the CMV lysate only but not the immunodominant pp65 protein (not shown), suggesting that other viral antigens account for increased T-cell responses. Results of T-cell function after BPX administration complements the phenotypic data we already reported.

Clinical Trial Registry: NCT02065869

Disclosure: Nothing to declare.

\section{P405}

Letermovir in hematopoietic cell transplantation: Beyond the label

\author{
Andrew Lin ${ }^{1}$, Molly A. Maloy ${ }^{1}$, Valkal Bhatt ${ }^{1}$, Lauren \\ DeRespiris ${ }^{I}$, Meagan Griffin ${ }^{I}$, Carmen Lau ${ }^{I}$, Anthony J. \\ Proli $^{1}$, Juliet N. Barker ${ }^{1}$, Brian C. Shaffer ${ }^{I}$, Sergio A. \\ Giralt, Ann A. Jakubowski,
}

Espernaza B. Papadopoulos ${ }^{1}$, Genovefa A. Papainolaou ${ }^{1}$, Susan K. Seo ${ }^{1}$, Miguel-Angel Perales ${ }^{1}{ }^{1}$ Memorial Sloan Kettering Cancer Center, New York, NY, United States

Background: Cytomegalovirus (CMV) is associated with significant morbidity and mortality in allogeneic hematopoietic cell transplantation (allo-HCT) patients (pts). Cumulative incidence of CMV infection in high-risk patients such as CD34-selected or haploidentical HCT have been reported as high as $61.8-84.5 \%$ and $53-81 \%$, respectively. Letermovir (LTV) was approved in 11/2017 for prophylaxis (ppx) in CMV-seropositive recipients $(\mathrm{R}+)$ of allo-HCT. Since 12/2017, LTV ppx was implemented at our center for both primary and secondary ppx. We report our real-world experience.

Methods: Adult CMV R+ allo-HCT pts who initiated LTV as primary and/or secondary prophylaxis were identified between 1/1/2018 and 6/30/18. Cord blood transplants were excluded. The primary outcome was the incidence of clinically significant CMV infection (CMV viremia requiring preemptive treatment or CMV disease). Pts were followed through 9/2018.

Results: 53 pts initiated LTV. 69.8\% pts were at high risk for CMV reactivation and disease (primarily ex vivo T-cell depleted HCT $[\mathrm{n}=18 ; 34 \%]$ or haploidentical T-replete HCT $[\mathrm{n}=12 ; 22.6 \%])$. The most common indication for HCT was acute myeloid leukemia $(n=16 ; 30.1 \%)$ and the majority of patients received myeloablative conditioning ( $\mathrm{n}$ $=34 ; 64.2 \%) .39$ pts $(73.5 \%)$ received LTV as primary ppx after HCT, with a median day of LTV initiation of D+7 (range D+7-D+40). At LTV initiation, 34 pts had an undetectable CMV DNA, and 4 had CMV $<137 \mathrm{IU} / \mathrm{mL}$. Clinically significant CMV infection requiring preemptive treatment occurred in 2 of 39 pts $(5.1 \%)$. One patient was treated with valganciclovir (VGV) for persistent CMV < $137 \mathrm{IU} / \mathrm{mL}$ and received LTV as secondary ppx. A 2nd patient developed persistently detectable CMV $(<137$ IU/ $\mathrm{mL}$ ) and breakthrough CMV viremia with a mutation in UL56 at site C325YLTV successfully treated with VGV. The median duration of primary LTV ppx was 116 days (54-221), with primary ppx continuing beyond 14 weeks post HCT in 29 pts. The median additional follow-up in patients who discontinued LTV was 40 days (0-154), without clinically significant CMV infection to date. An additional 14pts (15 pts overall; $28.3 \%$ ) received LTV as secondary ppx after CMV pre-emptive therapy. The median duration of secondary LTV ppx was 127 days (18-270), with no reactivation. LTV was not discontinued due to toxicity or intolerance in any patient. CMV outcomes are summarized in figure 1 . All-cause mortality for the $53 \mathrm{pts}$ over the observational period was $11.3 \%$.

Conclusions: Primary LTV ppx significantly reduced CMV reactivation, and high-risk patients may benefit from extended prophylaxis. In patients who received preemptive therapy for CMV, use of secondary $\mathrm{ppx}$ showed no recurrent CMV reactivation. LTV is well tolerated. Additional studies are needed to determine optimal ppx duration and to clarify role of secondary CMV ppx in high-risk alloHCT. The future standard of care will likely include extended primary ppx and secondary ppx and result in decreased morbidity and mortality associated with CMV.

Disclosure: Andrew Lin - Nothing to declare, Molly A. Maloy - Nothing to declare, Valkal Bhatt - Nothing to declare, Lauren DeRespiris - Nothing to declare, Meagan Griffin - Nothing to declare

Carmen Lau - Nothing to declare, Anthony J. Proli Nothing to declare, Juliet Barker - Angiocrine Bioscience (research funding), Nohla Therapeutics (clinical trial regulatory oversight \& consultancy), Gamida Cell Ltd (Research funding \& consultancy), Magenta Therapeutics Inc (Review Panel)

Brian Shaffer - Nothing to declare, Sergio A. Giralt Consultant:AMGEN, Actinuum, Celgene, Johnson \& Johnson, JAZZ pharmaceutical, Miltenyi, Takeda, Novartis, KITE, SPECTRUM Pharma

Ann A. Jakubowski - Nothing to declare, Esperanza B. Papadopoulos - Aveo (Ownership Interest), BG medicine (Ownership Interest), Biogen (Chairman of the Board and Ownership Interest), Exelixis (Chairman of the Board and Ownership Interest), Proteostasis (Ownership Interest), Regulus (Chairman of the Board and Ownership Interest), Evelo (Ownership Interest), Apellis (Ownership Interest), Leap Therapeutics (Ownership Interest), Genovefa A. Papanicolaou - Nothing to declare

Susan K. Seo - Nothing to declare, Miguel-Angel Perales - MolMed (Advisory Board), NexImmune (Advisory Board), Abbvie (Advisory Board), Bellicum (Advisory Board), Incyte (Advisory Board), Nektar Therapeutics (Advisory Board), Novartis (Advisory Board), Merck 
(Consultancy), Medigene, Servier, Incyte (Research funding),Miltenyi (Research Funding)

\section{P406}

Letermovir for prophylaxis of cytomegalovirus (CMV) infection or disease in allogeneic het recipients: Experience of secondary prophylaxis in the French compassionate program

Christine Robin ${ }^{1}$, Sophie Ducastelle-Lepretre ${ }^{2}$, Anne Thiebaut $^{3}$, Regis Peffault de la Tour ${ }^{4}$, Patrice Ceballos ${ }^{5}$, Marion Masure ${ }^{6}$, Thierry Allavoine ${ }^{7}$, Nathalie Benard ${ }^{8}$, Marie Therese Rubio ${ }^{9}$, Ibrahim Yacoub-Agha ${ }^{10}$, Amir Guidoum $^{8}$, Sophie Alain ${ }^{11}$, Catherine Cordonnier ${ }^{12}$

${ }^{1}$ APHP Henri Mondor Hospital and UPEC, Hematology, Creteil, France, ${ }^{2}$ Hospices Civils de Lyon, Hematology, Lyon, France, ${ }^{3}$ Centre Hospitalo-Universitaire de Grenoble, Hematology, Grenoble, France, ${ }^{4}$ APHP Saint-Louis Hospital, Paris, France, ${ }^{5}$ Hopital Saint-Eloi, Hematology, Montpellier, France, ${ }^{6}$ ICTA PM, Fontaine les Dijon, France, ${ }^{7}$ Former employee of MSD France, Lyon, France, ${ }^{8} \mathrm{MSD}$ France, Courbevoie, France, ${ }^{9} \mathrm{CHRU}$ Nancy Brabois, Hematology, Vandoeuvre les Nancy, France, ${ }^{10} \mathrm{CHU}$ de Lille, Hematology, Lille, France, ${ }^{11} \mathrm{CHU}$ Dupuytren, Centre National de Référence CMV, Limoges, France, ${ }^{12}$ APHP Henri Mondor Hospital and UPEC, Hematology, Créteil, France

Background: CMV infection and disease impair survival after allogeneic HCT. Letermovir potently inhibits CMVterminase complex. In a large phase III trial (Marty et al. 2017), letermovir primary prophylaxis (PP) has been shown to reduce clinically significant CMV infection with a favorable safety profile. Letermovir PP will improve the outcome of seropositive patients. However, patients who did not benefit from PP and experienced $\geq 1$ CMV episode (infection or disease) after HCT may be candidate to secondary prophylaxis (SP). Indeed half of them will have $\geq 1$ recurrent episode after pre-emptive treatment (PET). Letermovir is available since November 2017 as part of the French early access program for PP and SP. We report the outcome of patients who benefited from letermovir SP in the context of this program.

Methods: Letermovir is granted, in a restrictive manner, by the French drug agency (ANSM) on a case-by-case basis for prophylaxis of CMV episode, in CMV-seropositive adult allogeneic HCT recipients. SP patients should have a negative baseline CMV PCR, have already experienced $\geq 1$ CMV episode, in the context of a potentially harmful PET according to physicians. Planned letermovir daily dose was $240 \mathrm{mg}$ in case of concomitant cyclosporine and $480 \mathrm{mg}$ otherwise. All patients were routinely screened by blood or plasma CMV PCR.

Results: Between November 2017-July 2018, 57 patients received letermovir in the early access program, 22 for PP, and 35 for SP. Among the 35 SP patients, 6 had previous CMV disease (gut: 5; CNS: 1). Mean age was $55 \pm 13$ years, $\mathrm{M} / \mathrm{F}$ ratio was $22 / 13$. The SP cohort included one cord blood and 10 haplo-identical HCT. Main diagnoses were acute leukemia (46\%) and myelodysplastic syndrome $(26 \%)$. The conditioning regimen was myeloablative in $47 \%$ and included ATG in $61 \%$. Based on available data (6 missing data, $\mathrm{md})$, previous GvHD was present in $22(76 \%)$ patients, and active at letermovir initiation in 17 (59\%). Thirty two (91\%) patients were planned to receive immunosuppressants. Donor's CMV serology was negative in $12 / 15$ (80\%) (20 md). At baseline, CMV PCR was detectable in $1 / 35$ patients. Letermovir was initiated a median of 195 days (IQR: 154-308) after transplant for a mean duration of $112 \pm 47$ days. Only one (3\%) patient developed CMV breakthrough. The median follow-up from letermovir initiation was 103 days. Among the 57 patients exposed to letermovir prophylaxis, two patients permanently discontinued because of letermovir-related adverse events (acute GvHD and nephropathy for one, loss of appetite, pruritus, diarrhoea and weight loss for the other); two deaths occurred with no causal relationship to letermovir. Data were consistent with the known safety profile of letermovir.

Conclusions: Letermovir is or will be soonly available in most European countries for CMV prophylaxis in HCT recipients. Pending its routine use, letermovir used as SP was well tolerated and effective, with only $1 / 35$ patients developing a breakthrough infection. In this high-risk population for CMV recurrence, letermovir may provide a safe bridge between PET and specific immune reconstitution, pending tapering or discontinuation of immunosuppressants. Whether SP may improve survival deserves further studies.

Disclosure: Thierry Allavoine is a former employee of MSD France, Nathalie Benard and Amir Guidoum are employees of MSD France, Marion Masure is an employee of ICTA PM, Sophie Alain and Catherine Cordonnier have participated in advisory boards and have been members of the speaker bureau of MSD. Ibrahim Yacoub-Agha has received honoraria from MSD, Other authors: nothing to declare

\section{P407}

Real-world data on letermovir prophylaxis for cytomegalovirus reactivation after allogeneic hematopoietic cell transplantation: A single center experience 
Patrick Derigs ${ }^{1}$, Maria-Luisa Schubert ${ }^{1}$, Paul Schnitzler ${ }^{1}$, Carsten Müller-Tidow ${ }^{I}$, Peter Dreger ${ }^{I}$, Michael Schmitt ${ }^{I}$

${ }^{1}$ Heidelberg University Hospital, Heidelberg, Germany,

Background: Reactivation of cytomegalovirus (CMV) still contributes substantially to morbidity and mortality after allogeneic hematopoietic cell transplantation (alloHCT). Recently, letermovir became available as the first drug approved in Europe for prophylaxis of CMV reactivation in seropositive patients who have undergone alloHCT. Letermovir is neither myelo- nor nephrotoxic, and significantly reduced the incidence of $\mathrm{CMV}$ reactivation in a pivotal phase III trial (NEJM 2017;377:2433). Therefore we adopted letermovir prophylaxis according to the label as standard policy in our institution: In seropositive recipients letermovir was initiated after engraftment and continued until day +100 or CMV reactivation. The aim of the present study was to investigate if the favorable trial results could be reproduced under real-world conditions.

Methods: The study cohort consisted of the first seropositive 35 patients who received letermovir prophylaxis at our institution (between March and August 2018). These were compared with a control cohort transplanted between August 2017 and March 2018 before the advent of letermovir. Study and control cohorts were matched for CMV donor/recipient sero-status, underlying disease and donor type source of stem cells and application of ATG. CMV viremia was monitored by a quantitative PCR twice a week during the inpatient period and weekly thereafter. Patients reactivating CMV prior to engraftment were not considered as event in both groups.

Results: No major side effects of letermovir intake were observed. With altogether 5 reactivation events, the cumulative rate of $\mathrm{CMV}$ reactivation on day +100 was $14 \%(95 \% \mathrm{CI} 1-45 \%)$ in the letermovir cohort and thus significantly lower than in the control group (20 events, $58 \%$ (95\%CI 42-71\%); HR 0.23 (0.10-0.51); $\mathrm{p}=0.0003$ ). The median time to reactivation was 53 days for the control group and not reached for the letermovir group. The cumulative number of days on valganciclovir before $\mathrm{d}$ +100 was $151 \mathrm{~d}$ for the 35 letermovir patients vs $689 \mathrm{~d}$ for the 35 control patients. There were no hospitalizations for foscavir administration in the letermovir group compared to 5 hospitalizations in the control group. There were 2 deaths before $\mathrm{d}+100$ in the letermovir group (one PD, one NRM) and 3 deaths in the control group (all PD).

Conclusions: This observational study confirms the safety and efficacy of letermovir for the prophylaxis of CMV reactivation in seropositive patients after alloHCT in a real-world setting. Our results are in good concordance with the phase III trial. Although letermovir appeared to reduce the need for therapeutic valganciclovir and foscavir tremendously, larger samples with longer follow-up are needed to assess the impact of letermovir prophylaxis on non-relapse and overall mortality as well as on resource consumption.

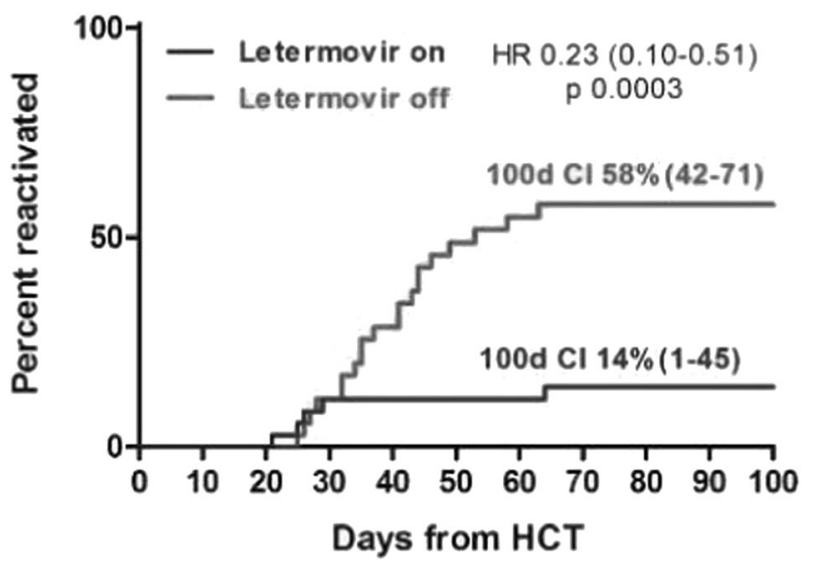

[[P407 Image] 1. Cumulative incidence of CMV reactivation by letermovir prophylaxis ( $n=35$ per cohort)]

Disclosure: Patrick Derigs, Maria-Luisa Schubert and Paul Schnitzler have nothing to declare.

Carsten Müller-Tidow, Peter Dreger and Michael Schmitt are members of a national advisory board of MSD Germany.

\section{P408}

CMV outcomes and health care resource utilization in CMV-seropositive $(\mathbf{R}+)$ hematopoietic stem cell transplant (HCT) recipients managed with pre-emptive therapy (PET)

Jiaqi Fang ${ }^{1}$, Phaedon D. Zavras ${ }^{1}$, Yiqi Su ${ }^{1}$, Amit D. Raval $^{2}$, Yuexin Tang ${ }^{2}$, Miguel-Angel Perales ${ }^{3,4}$, Sergio Giralt $^{3,4}$, Genovefa Papanicolaou ${ }^{1,4}$

${ }^{1}$ Memorial Sloan Kettering Cancer Center, Infectious Disease Service, New York, NY, United States, ${ }^{2}$ Merck \& Co., Inc., Kenilworth, NJ, United States, ${ }^{3}$ Memorial Sloan Kettering Cancer Center, Bone Marrow Transplant Service, New York, NY, United States, ${ }^{4}$ Weill Cornell Medical College, Cornell University, New York, NY, United States

Background: CMV viremia occurs in $40 \%-80 \%$ of CMV R $+\mathrm{HCT}$ recipients. PET use has reduced the risk of CMV end-organ disease (EOD) and associated mortality; However, PET use may lead to substantial antiviral use and healthcare resource utilization. Limited real-world data are available on the outcomes of PET. Therefore, we aimed to examine CMV outcomes (EOD, resistance), CMV-related 
mortality by Day (D)180 and healthcare resource utilization between PET and No-PET groups among CMV R+ recipients undergoing first HCT.

Methods: We conducted a retrospective cohort study of adults, CMV R+ recipients of first peripheral blood or marrow allograft at MSKCC identified from March 2013 through December 2017. Data was extracted from electronic medical records and HCT databases. $\mathrm{CMV}+$ recipients were monitored weekly by quantitative PCR assay starting on D14 through D180 post HCT. Use of antiviral therapy for CMV viremia defined PET. High CMV risk (HR) comprised T-cell depleted (TCD) HCT by CD34+-selection regardless of donor HLA match or conventional HCT from mismatched or haploidentical donors; Low risk (LR) included conventional HCT from matched related donors. CMV EOD was scored by standard criteria. CMV resistance mutations were confirmed by sequencing (Viracor-Eurofins). Length of stay (LOS) for HCT admissions and readmissions were identified through D180. Stratified analyses were performed to examine outcomes by PET use and CMV risk.

\begin{tabular}{|c|c|c|c|c|c|c|c|c|c|}
\hline & $\begin{array}{l}\text { Overall } \\
\text { PET } \\
(\mathrm{n}=208)\end{array}$ & $\begin{array}{l}\text { Overall } \\
\text { No-PET } \\
(\mathrm{n}=160)\end{array}$ & P-value & $\begin{array}{l}\text { Low CMV } \\
\text { Risk PET } \\
(\mathrm{n}=58)\end{array}$ & $\begin{array}{l}\text { Low CMV } \\
\text { Risk No-PET } \\
(\mathrm{n}=118)\end{array}$ & $\begin{array}{l}\text { P- } \\
\text { value }\end{array}$ & $\begin{array}{l}\text { High CMV } \\
\text { Risk PET } \\
(\mathrm{n}=150)\end{array}$ & $\begin{array}{l}\text { High CMV } \\
\text { Risk No- } \\
\text { PET }(\mathrm{n}=42)\end{array}$ & $\begin{array}{l}\mathrm{P} \text { - } \\
\text { value }\end{array}$ \\
\hline $\begin{array}{l}\text { LOS for admission } \\
\text { for HCT in days, } \\
\text { mean (SD) }\end{array}$ & $34.3(18.6)$ & $29.9(15.5)$ & 0.0001 & $36.0(22.1)$ & 30.7 (17.6) & 0.02 & $33.3(16.7)$ & $27.7(6.4)$ & 0.02 \\
\hline $\begin{array}{l}\text { Number of } \\
\text { patients with } \\
\text { readmission(s), } \\
(\%)\end{array}$ & $\begin{array}{l}112 \\
(53.8 \%)\end{array}$ & $53(33.1 \%)$ & 0.00005 & $34(58.6 \%)$ & $39(33.1 \%)$ & 0.001 & $78(52.0 \%)$ & $14(33.3 \%)$ & 0.03 \\
\hline $\begin{array}{l}\text { Number of } \\
\text { readmissions, (\%) }\end{array}$ & $180(100 \%)$ & $78(100 \%)$ & & $53(100 \%)$ & $57(100 \%)$ & & $127(100 \%)$ & $21(100 \%)$ & \\
\hline CMV & $40(22.2 \%)$ & $0(0 \%)$ & N/A & $4(7.5 \%)$ & $0(0 \%)$ & N/A & $36(28.3 \%)$ & $0(0 \%)$ & $\mathrm{N} / \mathrm{A}$ \\
\hline $\begin{array}{l}\text { Non-CMV } \\
\text { Infections }\end{array}$ & $41(22.8 \%)$ & $21(26.9 \%)$ & & $12(22.6 \%)$ & $18(31.6 \%)$ & & $29(22.8 \%)$ & $3(14.3 \%)$ & \\
\hline GrHD & $15(8.3 \%)$ & $6(7.7 \%)$ & & $8(15.1 \%)$ & $4(7.0 \%)$ & & $7(5.5 \%)$ & $2(9.5 \%)$ & \\
\hline Other & $84(46.7 \%)$ & $51(65.4 \%)$ & & $29(54.7 \%)$ & $35(61.4 \%)$ & & $55(43.3 \%)$ & $16(76.2 \%)$ & \\
\hline $\begin{array}{l}\text { Readmission rate } \\
\text { (per 1,000 patient- } \\
\text { days) }\end{array}$ & 5.2 & 3.1 & 0.00006 & 5.5 & 3.0 & 0.002 & 5.1 & 3.2 & 0.04 \\
\hline $\begin{array}{l}\text { Readmission LOS } \\
\text { (per } 1,000 \text { patient- } \\
\text { days) }\end{array}$ & 93.4 & 54.2 & $\mathrm{~N} / \mathrm{A}$ & 95.6 & 53.4 & N/A & 92.6 & 56.3 & $\mathrm{~N} / \mathrm{A}$ \\
\hline
\end{tabular}

[[P408 Table] 1. Hospital length of stay (LOS) and readmissions by CMV risk category and receipt of PET]

Results: Of 368 patients including 76 LR and $192 \mathrm{HR}$ (81.3\% TCD), 273 (74\%) patients developed CMV viremia; $208(56.5 \%)$ patients started PET with an average of 36 days post HCT (including 33\% and 78\% of LR and HR respectively). By D180, 17 (4.7\% of entire cohort or $8 \%$ among PET group) patients developed EOD (gastrointestinal disease in 12, retinitis in 1 , pneumonitis and encephalitis in 2 patients each); Among PET group 7 (3.4\%) patients developed CMV resistance: (val)Ganciclovir 5 patients, Cidofovir 1 patient and Cidofovir+Foscarnet 1 patient. Overall, PET group had longer mean LOS for HCT (34.3 vs 29.9 days, $p=0.0001)$ and higher rates of readmission (5.2 vs 3.1, $\mathrm{p}=0.00006)$. Similar findings were observed in stratified analysis by CMV risk (Table). 40 (22.2\%) of readmissions in PET group were associated with
CMV. By D180, CMV accounted for $17 \%$ of infectionrelated deaths.

Conclusions: Among PET group, nearly 1 in 12 patients developed CMV EOD; PET use was associated with longer inpatient stay and greater rates of readmissions compared to no PET use. Findings highlights the need for improved strategies for CMV management.

Clinical Trial Registry: NA

Disclosure: The study was funded by a grant from Merck \& Co., Inc. Genovefa A. Papanicolaou has received research funding and honoraria from Astellas, Chimerix, Clinigen, Merck, and Siemens and has been an investigator for Chimerix, Merck and Shire. Amit Raval is an employee of Merck and Co, Inc. Yuexin Tang is an employee of Merck and Co, Inc. Miguel Angel Perales has received honoraria from Abbvie, Bellicum, Incyte, Merck, Novartis, Nektar Therapeutics, and Takeda. He has received consulting fees from Servier and Medigene, MolMed and NexImmune. He has received research support for clinical trials from Incyte and Miltenyi Biotec. All other authors have nothing to disclose.

\section{P409}

Impact of CMV prophylaxis on rates of rehospitalization in adult CMV seropositive allogeneic HSCT recipients: Experience from the letermovir phase 3 clinical trial

\section{Shahrul Mt-Isa', Hong Wan', Cyrus Badshah ${ }^{2}$, Valerie Teal $^{2}$, Randi Leavitt ${ }^{2}$, Jonathan Schelfhout ${ }^{2}$ \\ ${ }^{1}$ MSD Ltd, Hoddesdon, United Kingdom, ${ }^{2}$ Merck \& Co., Inc., Kenilworth, NJ, United States}

Background: In a Phase III randomized, double-blind, placebo-controlled study of CMV-seropositive post-HSCT recipients, letermovir prophylaxis significantly reduced the incidence of clinically significant CMV infection through week 24. The objective of this research was to assess the impact of CMV prophylaxis on rates of rehospitalization in adult CMV seropositive allogeneic HSCT recipients from the letermovir phase 3 clinical trial.

Methods: Rehospitalization was recorded as an exploratory endpoint in the clinical trial at end of treatment (Week14), time of primary endpoint (Week24) and through an extended follow-up period (Week48). CMV-related rehospitalization was assessed in the trial. Prespecified analyses describe the observed rates of rehospitalization for the letermovir and placebo groups at the specified times. Fine-Gray cumulative incidence function(CIF) regression models were used to explore the rate of all-cause, and CMV-related rehospitalization accounting for the competing risk of mortality. A multiple linear regression model 
was used to describe the cumulative length of stay (LOS) for all-cause rehospitalizations that occurred through Week48 (excluding time of initial transplant stay).

Results: Observed rates of all-cause rehospitalization were lower for the letermovir group compared to placebo at end of treatment $(36.6 \% \mathrm{vs} .47 .6 \%)$, time of primary endpoint (48.6\%vs.55.3\%), and through extended follow-up (55.7\%vs.60.6\%). The CIF regression model demonstrated rates of all-cause rehospitalization were significantly lower through Week $14(\mathrm{HR}=0.72 ; \mathrm{p}=0.021)$ but did not reach significance at Week24 $(\mathrm{HR}=0.81 ; \mathrm{p}=0.109)$ or Week 48 ( $\mathrm{HR}=0.84 ; \mathrm{p}=0.173)$; and CMV-related rehospitalizations were significantly reduced at Week $14(0.6 \%$ vs.7.1\%; $\mathrm{HR}=0.09 ; \mathrm{p}=0.001)$, Week $24(2.8 \% \mathrm{vs} .7 .6 \% ; \mathrm{HR}=0.36$; $\mathrm{p}=0.015)$, and Week 48

(3.1\% vs. $8.8 \% ; \mathrm{HR}=0.34 ; \mathrm{p}=0.007)$. The adjusted mean cumulative LOS was shorter for letermovir than placebo but did not reach statistical significance( 3.1 fewer days $\mathrm{p}=$ $0.333)$.

Conclusions: Letermovir was shown to significantly reduce the rate of clinically significant CMV infection in a placebo-controlled randomized clinical trial. These analyses suggest that there is also a reduction in the rate and cumulative days of rehospitalization. This trial was not sufficiently powered to detect differences in this exploratory endpoint. Nonetheless, these data provide valuable insights into the economic burden of CMV. Real world data and findings from future clinical trials are needed to better understand the nature of the association between CMV and rehospitalizations.

Clinical Trial Registry: NCT\#: NCT02137772

EudraCT\#: 2013-003831-31

Disclosure: Funding for this research was provided by Merck Sharp \& Dohme Corp., a subsidiary of Merck \& Co., Inc., Kenilworth, NJ, USA.

All authors are employees of MSD

\section{P410}

Impact of fluoroquinolone prophylaxis on bloodstream infection incidence after allogeneic stem cell transplantation

Chiara Maria Dellacasa ${ }^{1}$, Luisa Giaccone ${ }^{1}$, Sara Butera ${ }^{1}$, Elisa Santambrogio ${ }^{I}$, Roberto Passera ${ }^{1}$, Semra Aydin ${ }^{1}$, Lucia Brunello $^{1}$, Danilo Faraci ${ }^{1}$, Chiara Frairia ${ }^{1}$, Giorgia Iovino ${ }^{I}$, Sara Manetta ${ }^{I}$ Benedetto Bruno ${ }^{1}$, Alessandro Busca $^{1}$

${ }^{1}$ AOU Città della Salute e della Scienza di Torino, Torino, Italy,

Background: Fluoroquinolone (FQ) antibacterial prophylaxis in patients undergoing allogeneic hemopoietic stem cell transplantation (HSCT) may reduce bloodstream infections (BSI) during the pre-engraftment phase. However a widespread use of FQ may have an impact on the emergence of multidrug resistant (MDR) bacteria. Based on this observation, antibacterial prophylaxis with $\mathrm{FQ}$ has been withheld in our Stem Cell Transplant Center from June 2016 onwards. Aim of this study was to evaluate whether the absence of FQ prophylaxis may lead to a higher incidence of BSI during the first 30 days after HSCT.

Methods: All consecutive patients with hematologic disorders who received HSCT at our Center between January 2013 and August 2018 were included. Among the 278 evaluable patients, 172 received levofloxacin as antibacterial prophylaxis (group A) while $106 \mathrm{did}$ not receive any FQ prophylaxis (group B). Baseline characteristics were similar in the two groups, except for the number of patients with advanced disease (34\% in group A and $47 \%$ in group B, p 0,042). Median duration of neutropenia was 16 days (range 9-44) in group A and 14 days (range 4-31) in group B. A positive rectal swab for Carbapenem-resistant Enterobacteriaceae (CRE) was detected in 3 patients in group A and 8 patients in group B.

Results: Overall, BSI was detected in 58 patients $(20,9 \%), 29(16,9 \%)$ in group A and $29(27,4 \%)$ in group B $(p=0,048)$. The median onset of BSI was 8 days post transplant (range 0-28), without significant differences between the two groups. In univariate analysis, FQ prophylaxis (OR 0,54; $95 \%$ IC $0,30-0,97)$ and bone marrow stem cell source (OR 2,08; 95\% IC 1,05-4,12) were significant factors associated with the risk of BSI. Gramnegative bacteria accounted for $44,8 \%(n=13)$ of BSI in group $A$ and $65,5 \%(n=19)$ in group $B$, and gram-positive bacteria for $48,3 \%(n=14)$ of BSI in group A versus $27,5 \%$ $(n=8)$ in group $B$, without statistically significant differences $(\mathrm{p}=0,16)$. Polymicrobic BSI were $6,9 \%$ $(\mathrm{n}=2)$ in group A and $6,9 \%(\mathrm{n}=2)$ in group B. MDRgram negative BSI were detected in 4 patients $(14 \%)$ in group A and in 6 patients $(20,7 \%)$ in group B (overall, 2 CRE, 7 ESBL producing enterobacteriaceae and 1 MDRPseudomonas). Death attributable to BSI occurred in 6 of 58 patients (10,3\%); 5 of these patients did not receive FQ prophylaxis, but 2 of them had both a pre transplant KPC colonization and active disease at transplant. Neither antibacterial prophylaxis $(\mathrm{p}=0,98)$ nor BSI $(\mathrm{p}=0,4)$ had a significant impact on overall survival (OS).

Conclusions: The preliminary data of our study show that FQ prophylaxis is associated with a reduced incidence of BSI, in particular Gram-negative infections, with no impact on OS. The limitations of our study may be the different group sizes and the retrospective nauture of the study. Whether antibacterial prophylaxis should be avoided in the pre-engraftment period in still a matter of debate and needs to be evaluated in larger prospective studies. 
Disclosure: Nothing to disclose.

\section{P411}

Invasive fungal infection after peripheral blood nonmanipulated haploidentical SCT with post-transplant cyclophosphamide

\section{Gillen Oarbeascoa $^{1}$, Nieves Dorado ${ }^{1,2}$, Laura Solan ${ }^{1,2}$, Rebeca Bailen ${ }^{1,2}$, Pascual Balsalobre ${ }^{1,2}$, Carolina Martinez-Laperche $^{1,2}$, Ismael Buño ${ }^{1,2}$, Javier Anguita ${ }^{1,2}$, Jose Luis Diez-Martin ${ }^{1,2,3}$, Mi Kwon $^{1,2}$}

${ }^{1}$ Hospital General Universitario Gregorio Marañón, Hematology, Madrid, Spain, ${ }^{2}$ Instituto de Investigación Sanitaria Gregorio Marañón, Madrid, Spain, ${ }^{3}$ Universidad Complutense de Madrid, Madrid, Spain

Background: Incidence and outcome of Invasive Fungal Infection (IFI) are not well characterized in the setting of peripheral blood, non-manipulated haploidentical stem cell transplantation with postransplant cyclophosphamide (HaploSCT). The aim of the study was to analyze incidence and risk factors of IFI in patients who underwent HaploSCT at our institution.

Methods: 132 consecutive patients who underwent peripheral blood HaploSCT with postransplant cyclophosphamide between 2011 and 2017 at our centre were reviewed. IFI was classified according to EORTC definitions. Proven and probable IFI were included.

Results: Patients' characteristics are shown in Table 1. Primary antifungal prophylaxis was performed with micafungin from day -1 until oral intake, followed by posaconazole until day +35 . Patients on steroid treatment for GVHD received prophylaxis with micafungin or posaconazole. $92 \%$ of patients obtained neutrophil engraftment. Twenty-two episodes of IFI were observed in 20 patients: 10 proven and 12 probable, with a cumulative incidence of IFI of $17 \%$ at 500 days. Most commonly isolated organism was Aspergillus spp $(n=5)$, followed by Candida spp ( $\mathrm{n}=4: 1$ C. kruseii and 3 C. parapsilosis), and Fusarium spp $(n=2)$. Isolated cases of Inonotus spp, Mucor spp and Trichosporon Ashii were observed. Pulmonary involvement was the most frequent clinical presentation $(\mathrm{n}=10)$, followed by fungemia $(\mathrm{n}=5: 4$ Candidemia, 1 Trichosporon Ashii) and skin-pulmonary involvement $(n=2)$. Among patients with lung involvement, 10 showed probable IFI: 5 with elevated serum galactomannan and 3 positive galactomannan in bronchoalveolar lavage (BAL). There were 2 patients without galactomannan, one with a positive BAL culture for Penicillum spp and the other with an Aspergillus spp.

Median time to IFI diagnosis was 21 days. Thirteen cases were diagnosed in the pre-engraftment period, 4 after engraftment and 5 cases after day +30 . Among patients with late IFI, median time to development was 220 days. All of them were associated with GVHD ( 3 grade III-IV acute GVHD and 2 moderate/severe chronic GVHD). IFI outcome was favorable in 13 out of the 22 IFI. Treatment was liposomal amphotericin B in 7 cases, voriconazole in 4 and combined treatment (amphotericin B and azole) in 6 . There were 7 IFI related deaths, with a cumulative incidence of IFI related death of $6.4 \%$. Prior transplant (OR 4.5, p< 0.01), particularly alloHSCT was associated to IFI development (OR 8.2, $\mathrm{p}<0.01)$. Patients with previous alloHSCT presented IFI mainly from molds: 3 Aspergillus, 2 Fusarium, Inonotus, Trichosporon and Mucor. There were also 2 candidemia episodes. No other factors were significantly associated to IFI occurrence.

Conclusions: In our experience, cumulative incidence of IFI in the setting of HaploSCT with posttransplant cyclophosphamide was similar than observed in previous studies in alloSCT. Having received a previous SCT, especially alloSCT, was the most significant factor related to IFI development. This high risk population should be closely monitored and could benefit from prophylaxis with azoles.

Disclosure: Nothing to declare.

\section{P412}

RSV infection in HSCT recipients: Experience from one single center

Anna Beatriz C. de Souza ${ }^{1}$, Paula Moreira da Silva ${ }^{1}$, Gustavo Fernandes Silva ${ }^{1}$, Anderson Joao Simioni ${ }^{1}$, Lilian Perilio Zanetti ${ }^{l}$, Juliana R. Do Prado Moreno ${ }^{1}$, Leila Cibele S. de Oliveira ${ }^{I}$, Iago Colturato ${ }^{I}$, Fernada Leite de Souza Franceschi', Ana Cláudia Ferrari dos Santos ${ }^{I}$, Elen Monteiro Adati ${ }^{I}$, Mair Pedro de Souza ${ }^{I}$, Vergilio Antonio Rensi Colturato ${ }^{1}$, Clarisse Martins Machado $^{1,2}$

${ }^{1}$ Amaral Carvalho Foundation, Jau, Brazil, ${ }^{2}$ University of São Paulo, Institute of Tropical Medicine, São Paulo, Brazil

Background: Respiratory syncytial viruses (RSV) circulate yearly all over the world, especially during fall and winter in temperate climates. In HSCT recipients, RSV infections have a wide spectrum of manifestations ranging from selflimited upper respiratory tract infections (URTI) to severe lower respiratory tract infections (LRTI) and death. We reviewed 134 cases of laboratory-confirmed RSV infection in HSCT recipients presenting to the HSCT Program of Amaral Carvalho Foundation from January 2009 to June 2018. In our HSCT center, around 200 procedures are performed yearly. 
Methods: RSV infection was diagnosed in nasal wash (NW) or bronchoalveolar fluid (BAL) by DFA (Millipore, USA) or PCR (Seeplex, Seegene, KOR). URTI and LRTI were defined according to ECIL-4 guidelines. Death from all causes was assessed within 90 days after RSV infection and was attributed to RSV if the patient had persistent or progressive RSV infection with respiratory failure at the time of death. Neutropenia and lymphocytopenia were defined as an absolute neutrophil count (ANC) $<500 / \mathrm{uL}$ and absolute lymphocyte count (ALC) < 200/uL, respectively.

Results: Median number of confirmed RSV infections per year was 12, ranging from 5 to 34 . An outbreak of RSV was detected in 2017, possibly due to a lack of compliance with contact precautions in the unit. Median patients' age was 26 (1-65) years and time to RSV infection was day 80 (-11 to 1837). Twenty-three patients (pts) had received an autologous transplantation (17.2\%) and 111 were allogeneic HSCT recipients $(82,8 \%)$. Median time to engraftment was 15 days, ranging from 10 to 27 days. At RSV diagnosis, 108 pts presented with URTI (80.6\%) and 26 with LRTI (19.4). Surprisingly, around $18 \%$ of the auto HSCT recipients had RSV pneumonia at diagnosis. Variables significantly associated with LRTI at diagnosis were MUD HSCT (No/ Yes, OR 0.42; CI95 0.20-0.89); ANC < 500/uL (OR 2.75; CI95 1.01-7.45); ALC < 200/uL (OR 3.25; CI95 1.12-9.45); and recent or pre-engraftment HSCT (No/Yes, OR 0.38 CI9 0.14-0.98). Among the 108 pts with URTI at diagnosis, 19 progressed to LRTI (17.6\%). Forty-four of the 134 pts died $(32.8 \%)$ and mortality rate was significantly higher in pts with LRTI in comparison with pts with URTI $(53.8 \%$ versus $27.8 \%, \mathrm{p}=0.011)$. Death was attributed to RSV in 11 of the 44 pts who died (25\%).

Conclusions: Autologous HSCT recipients are also at risk of LRTI caused by RSV. Risk of RSV LRTI is higher in MUD HSCT, infection acquired pre-engraftment or early after HSCT, and low neutrophil and lymphocyte counts. Continued education is necessary to sustain compliance to contact precautions in HSCT units.

Disclosure: Nothing to declare

\section{P413}

Risk factors associated with measles immunity after allogeneic hematopoietic cell transplantation

\section{Christine Robin ${ }^{1}$, Alice-Andree Mariaggi ${ }^{2}$, Rabah Redjoul $^{1}$, Mathieu Leclerc ${ }^{\text {, Florence Beckerich }}{ }^{1}$, Ludovic Cabanne ${ }^{I}$, Sebastien Maury ${ }^{I}$, Flore Rozenberg ${ }^{2}$, Catherine Cordonnier ${ }^{I}$}

${ }^{1}$ APHP Henri Mondor Hospital and UPEC, Créteil, France, ${ }^{2}$ APHP Cochin Hospital and University Paris Descartes USPC, Paris, France
Background: Measles is a life-threatening infection after allogeneic HCT. Due to the decreased coverage of vaccination in many countries, the disease reappears, increasing the risk of outbreaks worldwide. Allogeneic HCT recipients have been shown to be seropositive for measles in roughly $40-50 \%$ of the cases 3 years after transplant. However, these data were obtained before the 2000's from HCT populations mainly conditioned with myeloablative (MA) regimens. Our aim was to assess measles immunity before considering vaccination in a cohort of HCT survivors including patients conditioned with reduced intensity (RIC) or non-MA regimens.

Methods: Allogeneic HCT adult recipients who had not been vaccinated for measles since HCT were routinely screened for measles immunity. Measles IgG titers were determined with a chemiluminescence immunoassay (Liaison Measles IgG Kit, Liaison XL analyser, Diasorin, Italy). Patients were considered to be seropositive if the IgG titer was $\geq 16.5 \mathrm{UA} / \mathrm{mL}$. Risk factors for seropositivity were analyzed. Qualitative variables were described as numbers (\%) and compared using the chi-2 test or Fisher exact test as appropriate. Quantitative variables were described as median or mean (range) and compared using the KruskallWallis test. ORs were estimated separately for factor yielding a $P$-value $<0.20$ in the univariate analysis using logistic regression models.

Results: Eighty-six patients, transplanted 1.5 to 38 years (mean: 13,5 years) ago, were included. The mean age was 53 years (range: 21-79), the sex ratio M/F: 0,5. The underlying diseases were acute leukemia: 49 (57\%), myelodysplastic syndrome: 5 (6\%), lymphoproliferative diseases: 17 (19.5\%), myeloproliferative neoplasms: 11 (13\%) and non-malignant diseases : 4 (4.5\%). The HCT was performed from an HLA-identical donor in 52, an unrelated donor in 30 , and a cord-blood in 4.

Conditioning regimen were MA in $48(56 \%)$, RIC in 20 (23\%) and non-MA in 18 (21\%) patients

No patient had experienced measles or had received measles vaccination since transplant.

Fifty-seven of the 86 (66\%) patients were seropositive for measles. Measles seropositivity was not associated with conditioning regimen, patient age at transplant, patient age at time of assessment, donor age at transplant, lymphocyte count or gammaglobulin levels, or type of transplant (HLAid. vs others) measles vaccination before transplant or previous measles before transplant. The only parameters significantly associated to seropositivity were absence of previous GvHD (any type or severity, $\mathrm{p}=0,033$ OR 0,31 $[0,10-0,94])$, and absence of previous extensive chronic GvHD (OR 0,28 [0,09-0,87] p0,027).

Conclusions: Sixty-seven percent of allogeneic HCT are seropositive for measles at a median of 7 years after HCT before vaccination. The only risk factor strongly associated 
with seronegativity is extensive chronic GvHD. In patients without extensive chronic GvHD, the seropositivity is unpredictable. Therefore, a systematic assessment of antimeasles Ab titers is warranted, as recommended in the more recent guidelines, in order to avoid unnecessary vaccination.

Disclosure: Nothing to declare

\section{P414}

Plasma extracellular vesicles in cytomegalovirus reactivation after haploidentical stem cell transplantation

Giuseppe Lia ${ }^{1,2}$, Clara Di Vito ${ }^{3}$, Lucia Brunello ${ }^{1,2}$, Jacopo Mariotti $^{4}$, Elisa Zaghi ${ }^{3}$, Marta Tapparo ${ }^{5}$, Stefania Bruno ${ }^{5}$, Giovannino Ciccone ${ }^{1}$, Mario Boccadoro ${ }^{1,2}$, Andrea Evangelista $^{1}$, Giovanni Camussi ${ }^{5}$, Luca Castagna ${ }^{4}$, Domenico Mavilio $^{3,6}$, Benedetto Bruno ${ }^{1,2}$

${ }^{1}$ A.O.U. Città della Salute e della Scienza di Torino, Torino, Italy, ${ }^{2}$ University of Torino, Torino, Italy, ${ }^{3}$ Humanitas Clinical and Research Center, Rozzano, Milan, Italy, ${ }^{4}$ Humanitas Clinical and Research Center, Rozzano, Milan, Italy, ${ }^{5}$ Molecular Biotechnology Center, University of Torino, Torino, Italy, ${ }^{6}$ University of Milan, Milan, Italy

Background: Cytomegalovirus (CMV) reactivation is a frequent complication after hematopoietic stem cell transplantation (HSCT). Extracellular Vesicles (EVs) have emerged as a promising new category of biological biomarkers in different scenarios, including inflammation, tissue damage, cancer and viral infections. We recently reported on the potential use of serum EVs as biomarkers of aGVHD (Lia G. et al. Leukemia (2018) 32, 765). Here, we investigated the potential correlation of CMV reactivation with plasma EVs in post-transplant Cyclophosphamide (PTCY) haploidentical-HSCT (Haplo-HSCT).

Methods: Plasma samples were collected after mononuclear cell separation at given time-points (pre-transplant, on day $0,3,7,14,21,28,35,45,60,75$ and 90 after HaploHSCT) and EVs were extracted by a protamine-based precipitation method and their concentration and dimension were characterized by Nano-tracking Particle Analysis (Nanosight). After extraction, EVs were analyzed by flowcytometry (Guava EasyCyte Flow Cytometer) with a panel of 14 antibodies (CD44, CD138, CD146, KRT18, CD120a, CD8, CD30, CD106, CD25, CD26, CD31, CD144, CD86, and CD140a).

Results: Thirty-two patients with hematological malignancies underwent haplo-HSCT between 2011 and 2015 . CMV reactivation was observed in 20/32 (62,5\%) and occurred at a median of 50 (range: 10-275) days after transplant. Preliminary analysis (17/32 patients) showed that CD140a fluorescence (platelet-derived growth factor receptor- $\alpha$ or PDGFR- $\alpha$ ), CD30 fluorescence (Ki-1 antigen) and CD144 fluorescence (VE-Cadherin) were associated with an increased risk of CMV reactivation (OR 2.67 $\mathrm{p}=0.045$; OR $3.11 \mathrm{p}=0.011$; OR $2.37 \mathrm{p}=0.08$ ), whereas CD31 (platelet endothelial cell adhesion molecule, PECAM-1) concentration level was associated with a decreased risk of CMV reactivation (OR 0.26, $\mathrm{p}=0.032$ ). All these biomarkers showed a signal change before CMV reactivation (an increase with CD140a, CD30 and CD144, a reduction with $\mathrm{CD} 31$ ). (Figure 1).

Conclusions: We observed a potential association of 4 EVs membrane proteins with CMV reactivation: CD140a, CD30, CD144 and CD31. These proteins are crucial for endothelium and immune cells interaction. CMV can infect different cell types including endothelial cells (Bentz GL. PNAS 105(14) 2008). Moreover, CD140a (PDGFR- $\alpha$ ) has been shown to function as an entry receptor for CMV expressing gH/gL/gO complex (Wu Y. et al. PLoS Pathog 13(4) 2017). We plan to implement our analysis characterizing EVs contents (miRNAs) and will be applied to investigate other viral reactivations (e.g. Epstein Barr Virus and Human Herpes Virus 6).
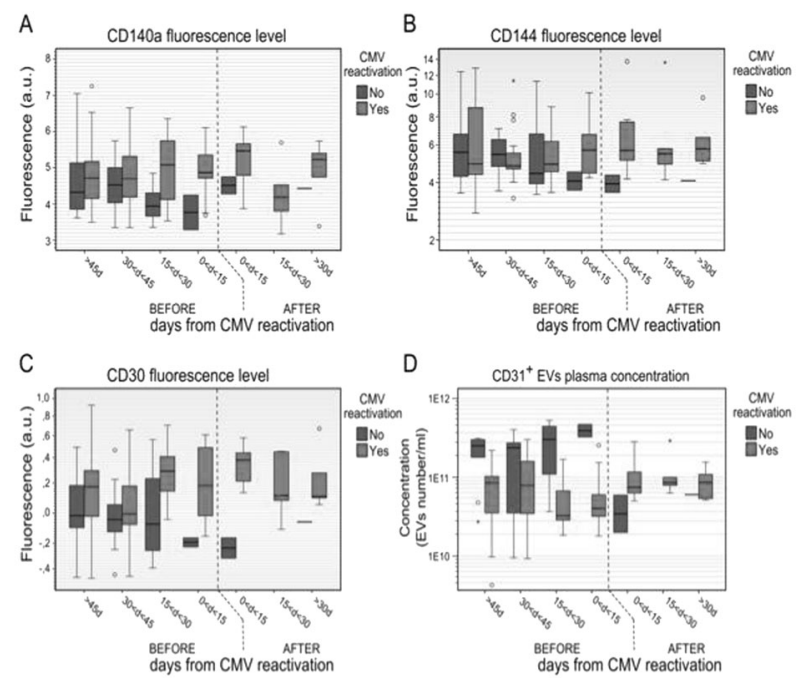

Figure 1: Signal level before and after CMV reactivation. Fluorescence levels variation for A) CD140a, B) CD144 and C) CD30. D) CD31 concentration of positive EVs in plasma. EV measurements in patients with CMV reactivation (red) and in patients without CMV reactivation (blue).

\section{[[P414 Image] 1. Figure 1]}

Disclosure: Boccadoro Mario Honoraria and research funding from: Sanofi, Celgene, Bristol-Myers Squibb, Novartis, Amgen, Janssen; Honoraria from AbbVie, Research funding from Mundipharma

Benedetto Bruno Honoraria from: Gilead, Pfizer, Hospira, Honoraria and research funding from Celgene, Research funding from Pierre Fabre, Adienne, Hospira italia, MSD Italia 


\section{P415}

The clinical value of Q-PCR for cytomegalovirus gastrointestinal infection in hematopoetic allogeneic transplantation

Apostolia Papalexandri ${ }^{1}$, Fotini Kika ${ }^{1}$, Mihalis Iskas ${ }^{1}$, Stella Bouziana ${ }^{1}$, Natassa Konstantinou ${ }^{1}$, Angeliki Giannakopoulou ${ }^{1}$, Georgia Konstantinidou ${ }^{1}$, Evdoxia Koravou $^{1}$, Panagiota Zerva ${ }^{1}$, Zoe Boussiou ${ }^{1}$, Despina Mallouri $^{1}$, Ioannis Batsis ${ }^{1}$, Stylianni Papaemmanouil, Tasoula Touloumenidou ${ }^{1}$, Ioanna Sakellari ${ }^{1}$, Achilles Anagnostopoulos ${ }^{1}$

${ }^{1}$ G. Papanikolaou Hospital, Thessaloniki, Greece

Background: Cytomegalovirus (CMV) infection and disease, serious complications after allogeneic hematopoietic cell transplantation (allo-HCT) target the gastrointestinal (GI) tract commonly. Diagnosis of GI CMV disease relies mostly on endoscopy and GI biopsy; the diagnostic role of CMV viral load in GI tissue has not yet been established.

Methods: To explore the value of CMV DNA extracted from GI tissue for the diagnosis of CMV gastroenteritis, we retrospectively evaluated 71 patients, aged 17-67 (median 44.8 years) who received allo-HCT from sibling(26), matched unrelated(40) or haploidentical donors(5), after receiving myeloablative(56) or reduced intensity conditioning(15). They all underwent endoscopy for gastrointestinal symptoms between 2012-2018. CMV DNA from tissue samples and parallel blood samples were measured by QPCR. Positive CMV DNA on the tissue was considered CMV GI infection.CMV GI disease was proven with the identification of CMV inclusion bodies or positive immunehistochemical staining using anti-CMV antibodies.

Results: Overall, 91 endoscopic tests were performed (55 gastro-,36 colonoscopies) at a median of 73 days (IQR:145) post transplantation. Symptoms included nausea, vomiting, diarrhea, abdominal pain and weight loss. CMV DNA was positive in 41/91 tissue samples: median 536 copies/ml, range: $11-131 \times 10^{\wedge} 6$. Only half patients (22/41) had concurrent CMV viremia (plasma viral load $>100 \mathrm{c} / \mathrm{ml}$ ). CMV GI infection was not correlated to the type of transplant, acute or chronic GvHD. GI CMV disease was documented by biopsy in 13 patients. CMV DNA of the tissue, but not the plasma viral load, was a predictor of biopsy positivity (OR: 1.6, 95\%CI: 1.1-1.8, $\mathrm{p}=0.006$ ). Thirty-six out of 41 CMV DNA positive patients received specific treatment for at least 10 days. Symptoms resolved in $21 / 36$ patients $(60 \%)$ and the GI viral load was not a significant factor to predict cure. GI GvHD was diagnosed in 42/91 patients, among which 45\%(19/42) with CMV DNA positivity. Median OS was 453 days (95\%CI: 297608) for patients with CMV infection, similar to those without (median OS: 890, 95\%CI: 80-1699 days, $\mathrm{p}=\mathrm{ns}$ ). We studied separately endoscopies of the upper $(55 / 91)$ or lower GI tract (36/91). There was no significant relationship between CMV gastritis proven by biopsy and CMV DNA levels in gastric tissue. However, the viral load of the colon was a predictor of CMV enteritis (OR: 1.9, 95\%CI: 1.9-3, $\mathrm{p}=0.007)$. The AUROC of the Q-PCR was 0.849 (95\%CI: 0.659 to 1 ), the sensitivity was $85.7 \%$ and the specificity was $78.6 \%$ with a cutoff value of 370 copies/ml DNA.

Conclusions: Pathognomonic findings in the biopsy remain the gold standard for the diagnosis, especially for the upper GI tract. However, when the lower GI tract is involved, quantification of CMV viral load in the tissue may be a valuable tool to support the diagnosis. Positivity of CMV DNA of the GI tissue, in linearity to the CMV viremia, may guide to preemptive treatment for prevention of CMV disease .

Disclosure: Nothing to declare

\section{P416}

Impact of clostridium difficile infections on survival and gvhd after allogeneic hematopoietic cell transplantation - a multicentre observational study of the polish adult leukemia group (PALG)

Agnieszka Piekarska ${ }^{1}$, Patrycja Mensah-Glanowska ${ }^{2}$, Małgorzata Sobczyk-Kruszelnicka ${ }^{3}$, Joanna DrozdSokołowska ${ }^{4}$, Alicja Sadowska-Klasa', Lidia Gil ${ }^{5}$

${ }^{1}$ Medical University of Gdansk, Gdansk, Poland, ${ }^{2}$ Collegium Medicum, Jagiellonian University, Cracow, Poland, ${ }^{3}$ Maria Sklodowska-Curie Institute - Oncology Center, Gliwice Branch, Gliwice, Poland, ${ }^{4}$ Medical University of Warsaw, Warsaw, Poland, ${ }^{5}$ Poznan University of Medical Sciences, Poznan, Poland

Background: Clostridium difficile infection (CDI) is caused by $\mathrm{CD}$ overgrowth in antibiotic-disturbed intestinal microbiota. Antibiotics targeting unselectively beneficial for T-regulatory cell formation strains of Clostridiales may increase pro-inflammatory processes in the guts promoting or augmenting the development of graft vs. host disease (GVHD). The efficacy of CDI treatment has impact on the persistence of inflammation which might influence the alloreactive reactions.

Methods: We retrospectively and, from 2016, prospectively analyzed the data from 5 transplant centers concerning CDI occurrence, treatment efficacy, and GVHD development. The study included 77 patients with hematological malignancies who underwent allogeneic hematopoietic cell transplantation (alloHCT) between 2012-2018.

Results: Median time to CDI was 14 days post-alloHCT with detection of both toxins $\mathrm{A}$ and $\mathrm{B}$ in $57 \%$ of cases. 
Disturbance of intestinal microbiome was confirmed by a $59 \%$ rate of colonization with multidrug-resistant bacteria (MDRB). The CDI symptoms resolved with the negative toxins after the first line treatment in $76.6 \%$ of patients. The median time to remission and therapy duration was 8 and 10 days, respectively. Fifteen therapeutic failures were observed after treatment with metronidazole (10), vancomycin (2) and a combination therapy (3). Eleven patients responded to second line treatment. Thirty-seven (48\%) patients died due to infections (17), relapses (10) and GVHD/infections (10). We noted recurrent CDI in 6 cases. Eight patients died with active CDI. We observed occurrence or exacerbation of GVHD in 35 (44\%) patients following CDI, including 25 cases with gut involvement (GI-GVHD). Treatment with metronidazole and failure of the first line therapy increased the development or escalation of GI-GVHD $(\mathrm{p}=0.03$ and $\mathrm{p}<0.001$, respectively). The duration of CDI exceeding 10 days also had impact on the GI-GVHD incidence $(\mathrm{p}=0.002)$.

Conclusions: 1. Patients colonized with MDRB are at high risk of CDI. 2. High mortality due to infections and/or GVHD in patients with CDI. 3. Due to lower efficacy and harmful immunomodulatory impact, metronidazole should not be the first line treatment in CDI post-HCT. 4. Emphasis must be put on fast CDI resolution to interrupt a vicious circle of the intestinal inflammatory processes.

Disclosure: Nothing to declare

\section{P417}

\section{Establishing optimal preemptive cytomegalovirus} therapy threshold post allogeneic HCT in a patient population with high prevalence of seropositive status

\section{Moussab Damlaj', Farhan Khalid', Samer Ghazi', Bader Alahmari $^{1}$, Inaam Shehab Eddine ${ }^{1}$, Isam Mahasneh', Ayman Alhejazi, Ahmad Alaskar ${ }^{1}$, Mohsen Alzahrani ${ }^{1}$}

\footnotetext{
${ }^{1}$ King Abdulaziz Medical City, Oncology, Riyadh, Saudi Arabia
}

Background: Preemptive therapy (PET) for cytomegalovirus (CMV) reactivation post allogeneic hematopoietic stem cell transplantation (HCT) was shown to decrease the incidence of CMV disease. However, the optimal PET threshold is unknown and there are significant toxicities associated with anti-CMV therapy. At our institution, we initiate PET at CMV DNA titer above 1000 copies $/ \mathrm{mL}$ (1560 IU/mL). Our aim was to examine the efficacy of this approach including the incidence of spontaneous clearance in a population with high prevalence of CMV seropositive status.
Methods: After due IRB approval, patients that underwent allogeneic HCT were identified and records retrospectively extracted.CMV reactivation was defined as the first detectable viral titer post HCT from plasma samples whereas clearance of viremia as the first date of two negative PCR values obtained at least 1 week apart. CMV monitoring was initiated post HCT performed at least weekly during the first 100 days and every 2-4 weeks thereafter. A high sensitivity assay Abbott RealTime CMV was used with detection threshold of 20 copies/mL (31.2 $\mathrm{IU} / \mathrm{mL}$ ). Analysis was computed using JMP v. 14.0.1

Results: A. Baseline characteristics: A total of 195 patients were identified and included with a median follow up of 18.1 (0.7-95.6) months. Median age was 26 (14-63) years and $58 \%$ were male. Indication for HCT was for a malignant disorder in $77 \%$ of cases. The majority had a matched related donor (87\%) and CMV IgG was positive in both donor and recipient in $98 \%$ of cases. Myeloablative conditioning was given to $117(60 \%)$ and $109(56 \%)$ received TBI. In vivo $\mathrm{T}$-cell depletion was given to 76 (39\%); ATG in 39 (20\%) and alemtuzumab in 37 (19\%). B. CMV Reactivation and PET: A total of 178 (91\%) patients had a positive CMV PCR with median days to reactivation post HCT of 17 (1-1187); 129 (66\%) patients had peak $\mathrm{CMV}$ titer $<1000$ copies/mL (low titer) whereas the remaining $49(25 \%)$ had a peak titer $\geq 1000$ copies $/ \mathrm{mL}$ (high titer). Patients with high titer were more likely to be older $(\mathrm{p}=0.019)$, have malignant disease $(\mathrm{p}=0.019)$, haploidentical or unrelated donor $(\mathrm{p}<0.0001)$ and higher incidence of aGvHD grade II-IV $(\mathrm{P}=0.003)$ as shown in the table. Median peak titers for the low and high groups were 111 vs. 4638, respectively ( $\mathrm{p}<0.0001) .120(93 \%)$ patients with low titers cleared spontaneously with median time to clearance of 40 days (4-188), 1 (1\%) received anti CMV therapy and the remaining died with active viremia (range 49-561 copies $/ \mathrm{mL}$ ) with active disease. One patient in the high titer group developed CMV disease. 2-year OS and CI-NRM was $67.9 \%$ vs. $55.4 \%(\mathrm{p}=0.1)$ and $8 \%$ vs. $19.1 \%(\mathrm{p}=0.034)$ in the low and high titer groups, respectively.

Conclusions: CMV reactivation was high in this cohort however of low titer viremia in over $70 \%$. A PET threshold of 1000 copies/mL (1560 IU/mL) appears desirable as it was associated with spontaneous clearance in almost all patients while minimizing treatment related toxicity. Validation of these observations is warranted.

Clinical Trial Registry: N/A

Disclosure: Nothing to declare

\section{P418}

Rezafungin prophylactic efficacy in a mouse model of pneumocystis pneumonia 
Melanie T. Cushion ${ }^{1,2}$, Taylor Sandison ${ }^{3}$, Alan Ashbaugh $^{1,2}$

${ }^{1}$ University of Cincinnati College of Medicine, Cincinnati, $\mathrm{OH}$, United States, ${ }^{2}$ Cincinnati VAMC, Cincinnati, OH, United States, ${ }^{3}$ Cidara Therapeutics, Inc., San Diego, CA, United States

Background: The risk of Pneumocystis pneumonia often warrants antifungal prophylaxis for recipients of blood and marrow or solid organ transplantation. However, complications such as myelosuppression, nephrotoxicity, and intolerance with the existing standard, trimethoprim/sulfamethoxazole (TMP/SMX), may hinder or interrupt prophylaxis.

Rezafungin (RZF) is a novel echinocandin in development for prevention of invasive fungal disease caused by Candida, Aspergillus, and Pneumocystis species in blood and marrow transplant patients. RZF has a favorable safety and tolerability profile and a low risk of drug-drug interactions. Furthermore, the stability and pharmacokinetics of RZF allow for once-weekly dosing and broad distribution to the lung and other target organs.

RZF was shown to prevent in vitro Pneumocystis biofilm formation and to reduce the viability of mature biofilms. A previous prophylactic study was conducted using a broader range of RZF doses. In the current study, the efficacy of RZF was evaluated to better understand the minimum doses necessary to prevent Pneumocystis growth in a mouse model.

Methods: $\mathrm{C} 3 \mathrm{H} / \mathrm{HeN}$ mice were immunosuppressed (dexamethasone $4 \mathrm{mg} / \mathrm{L}$ in acidified drinking water) and then infected intranasally with $P$. murina $\left(2 \times 10^{6} / 50 \mu \mathrm{L}\right)$. Given the slow growth of $P$. murina, test agents were administered at the same time mice were inoculated to test for prophylactic efficacy. Mice received intraperitoneal injections of either vehicle (control/steroid [C/S]), TMP/ SMX $50 / 250 \mathrm{mg} / \mathrm{kg} / 3 \mathrm{x} /$ week (wk), caspofungin $5 \mathrm{mg} / \mathrm{kg}$ once daily, or RZF $5 \mathrm{mg} / \mathrm{kg}$ or $0.5 \mathrm{mg} / \mathrm{kg}$ once daily, $1 \mathrm{x}$, or 2x/wk. After a 6-week dosing period, mice were sacrificed and lung homogenates were processed for analysis to quantify the nuclei (trophic) and asci (cyst) forms of Pneumocystis.

Prophylaxis efficacy was based on reduction of organism burden compared with C/S. Nuclei and asci counts were log transformed and analyzed by ANOVA; individual groups were compared by the Student-Newman-Keuls $\mathrm{t}$ test. Survival rates were compared using GraphPad Prism v5.

Results: All mice in the RZF groups had significantly reduced nuclei and asci burdens compared with the $\mathrm{C} / \mathrm{S}$ group, and all but the lowest doses of RZF $(0.5 \mathrm{mg} / \mathrm{kg} 1 \mathrm{x}$ or $2 \mathrm{x} / \mathrm{wk}$ ) worked as well as TMP/SMX at reducing nuclei levels. Similarly, all RZF groups except for the $0.5 \mathrm{mg} / \mathrm{kg}$ $1 \mathrm{x} /$ wk group showed reductions in asci levels comparable to that of TMP/SMX. The survival rates were not statistically different between treatment groups.

Conclusions: RZF demonstrated potent in vivo efficacy for prophylaxis against Pneumocystis in an in vivo mouse infection model at dose regimens much lower than the human equivalent Phase 3 regimen. These data support the development of RZF for the prevention of invasive fungal infections including Pneumocystis pneumonia.

\section{Disclosure:}

Melanie T. Cushion: research funding (Cidara Therapeutics)

Taylor Sandison: employee, stockholder (Cidara Therapeutics)

Alan Ashbaugh: Nothing to declare.

\section{P419}

Risk analyses of EBV reactivation after allogeneic hematopoietic stem cell transplantation in Chinese population

Yuhua $\mathrm{Ru}^{1,2}$, Ziling $\mathrm{Zhu}^{1,2}$, Yang $\mathrm{Xu}^{1,2}$, Suning $\mathrm{Chen}^{1,2}$, Xiaowen Tang ${ }^{1,2}$, Yue Han ${ }^{1,2}$, Huiying Qiu ${ }^{1,2}$, Chengcheng $\mathrm{Fu}^{1,2}$, Jia Chen ${ }^{1,2}$, Depei $\mathrm{Wu}^{1,2}$

${ }^{1}$ The First Affiliated Hospital of Soochow University, Suzhou, China, ${ }^{2}$ Jiangsu Institute of Hematology, Suzhou, China

Background: Immunocompromising period following allogeneic hematopoietic stem cell transplantation (alloHSCT) may allow opportunistic pathogens to thrive and result in fatal complications. Epstein-Barr virus (EBV) infects more than $90 \%$ of Chinese population, and its reactivation after HSCT is one of the major concerns due to the increased risk of EBV diseases and post-transplant lymphoproliferative disease. With the development of infection prophylaxis and supportive care after HSCT, demographic data on EBV reactivation post-HSCT needs to be updated.

Methods: We retrospectively analyzed the data of patients who received allo-HSCT between July 2011 and July 2014 in the First Affiliated Hospital of Soochow University. Quantitative PCR (Q-PCR) was used to monitor EBV-DNA load in peripheral blood dynamically. Ganciclovir (pre-HSCT) followed by acyclovir was given as viral prophylaxis. The treatment protocol for EBV reactivation consisted of tapering of immunosuppressive agents, antiviral agents (including ganciclovir and sodium phosphonatel), and rituximab for persistent positive patients. 


\begin{tabular}{llll}
\hline Factors & & Cases & $\begin{array}{l}\text { EBV reactivation } \\
\text { Incidence }\end{array}$ \\
\hline Sex & female & 362 & $20.2 \%$ \\
& male & 528 & $19.3 \%$ \\
Age & younger & 410 & $23.7 \%$ \\
& than 30 & & \\
HLA- & older than 30 & 480 & $19.3 \%$ \\
mismatched & & 261 & $33.0 \%$ \\
ATG use & & & \\
Acute GVHD & grade 1-2 & 656 & $27.9 \%$ \\
& grade 3-4 & 224 & $23.7 \%$ \\
Chronic GVHD & & 478 & $23.8 \%$ \\
\hline
\end{tabular}

\section{[[P419 Table] 1. Characteristics of patients]}

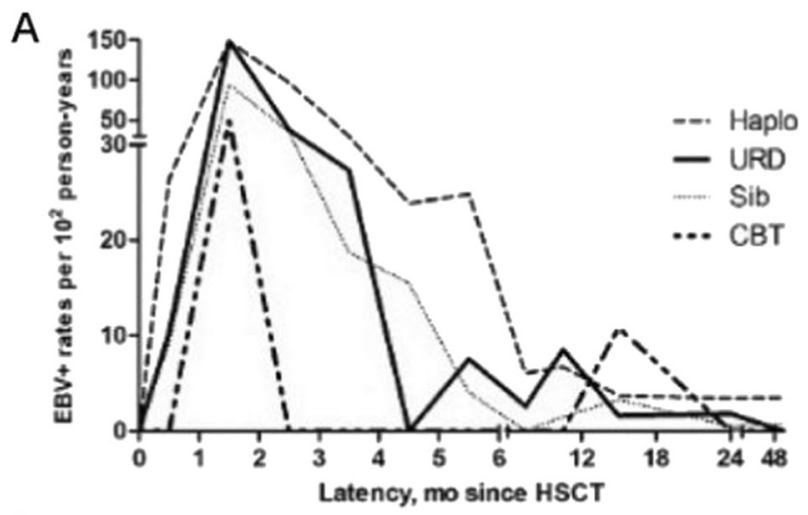

B

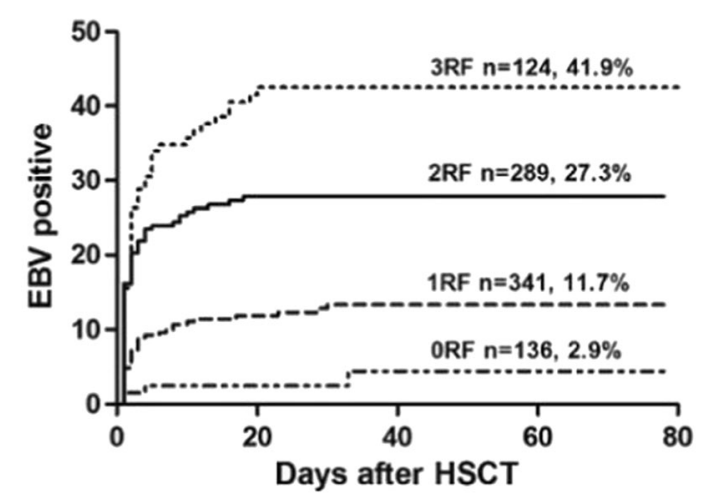

[[P419 Image] 1. Incidence of EBV reactivation]

Results: Totally 890 cases from most of the provinces in China were enrolled (characterized in Table 1), among whom EBV reactivation developed in 175 recipients. Most reactivation events (95.4\%) occurred in the first year postHSCT, with a peak of 113.8 incidence rates per 100 personyears at the second month. Besides, more episodes of lateonset reactivation occurred in patients receiving grafts from haploidientical donors (Figure 1A) . Multivariate analyses revealed that the major impactors of EBV reactivation included ATG as GVHD prophylaxis ( $\mathrm{p}<0.001)$, HLAmismatched donor $(\mathrm{p}=0.001)$ and the appearance of chornic GVHD ( $\mathrm{p}=0.042)$. Cumulative incidence of EBV reactivation was low (2.9\%) among 890 patients with no major risk factors, but increased to $11.7 \%, 27.3 \%$ or $41.9 \%$ with 1,2 , and 3 major risk factors, respectively (Figure 1B). There was no statistical difference of overall survival between people with or without $\mathrm{EBV}$ reactivation $(\mathrm{p}=0.871)$.

Conclusions: We concluded that there are similar EBV reactivation impactors in Chinese population compared to literatures, including ATG use, HLA-mismatched donor and the appearance of chronic GVHD. Additionally, incidences of EBV reactivation increased significantly with the accumulation of risk factors. However, EBV reactivation had no impact on overall survival in current virus management protocol.

Disclosure: Nothing to declare

P420

Abstract withdrawn.

P421

Changes in gut microbiome composition in patients undergoing HSCT colonized by ESBL bacteria and treated with carbapenems

Silvia Corcione ${ }^{I}$, Benedetto Bruno ${ }^{2}$, Lucia Brunello ${ }^{2}$, Alessandro Busca ${ }^{2}$, Gabriele Bianco ${ }^{3}$, Rossana Cavallo ${ }^{3}$, Giovanni di Perri', Francesco Giuseppe de Rosa ${ }^{1}$

${ }^{1}$ University of Turin, Torino, Italy, ${ }^{2}$ University of Torino, Stem Cell Transplant Program, A.O.U. Città della Salute e della Scienza di Torino, Turin, Italy, ${ }^{3}$ Città della Salute e della Scienza, Molinette, Turin, Italy,

Background: Several studies have shown loss of diversity of the gut microbiome in association with significant gut injury following hematopoietic stem cell transplantation (HSCT). Prolonged broad spectrum antibiotic use further promotes loss of microbiome diversity and increases the risk of intestinal colonization by multi-drug-resistant (MDR) bacteria. Aims of this study were to prospectively evaluate the overall changes in gut microbiome composition after HSCT and differences in patients colonized by MDR bacteria and treated with carbapenems.

Methods: We performed a prospective observational study evaluating the gut microbiota of 20 hematological patients undergoing HSCT, from admission (T0) through day +28 (T5). Fecal microbiota was assessed by $16 \mathrm{~S}$ amplicon-based sequencing. Clinical, and microbiological data as well as fecal samples were collected every $7^{\text {th }}$ day from admission. 
Results: One-hundred fecal samples were analyzed. Overall, we found a progressive decrease of bacterial richness from $\mathrm{T} 0$ to $\mathrm{T} 5$, with a significant reduction of Blautia, Ruminococcus and Dorea species, which are strictly associated with the production of short chain fatty acids (SCA) (Fig.1). Moreover, in the 30\% (no.6) of patients who were colonized by ESBL bacteria, we observed a significant reduction of Clostridium spp and Bifidobacterium species. As for antibiotic therapies, carbapenems were used as second line treatment of febrile neutropenia in 50\% (no 9) of cases, usually associated with aminoglycosides. In patients treated with meropenem, a strong decline of Blautia and Ruminococcus species was observed. This finding suggests a correlation between carbapenem regimens and increase of pro-inflammatory bacterial strains in the gut.

Conclusions: Our data support the hypothesis that loss of intestinal commensals that produce short-chain fatty acids may increase dysbiosis. Moreover, for the first time we report significant and progressive alterations in the composition of Blautia, Ruminococcus and Bifidobacterium species in patients treated with meropenem and colonized by ESBL bacteria, respectively. Our findings offer potential modifiable targets to reduce risk of colonization by MDR bacteria and to promote a carbapenem-sparing approach in the HSCT setting.

Clinical Trial Registry: not applicable

Disclosure: This study was supported by internal funding

\section{P422}

Citomegalovirus reactivation in patients with haploidentical stem cell transplantation with posttransplant cyclophosphamide

Javier Ortiz ${ }^{1}$, Nieves Dorado ${ }^{1,2}$, Laura Solan ${ }^{1,2}$, Rebeca Bailen $^{1,2}$, Pilar Catalan ${ }^{1}$, Pascual Balsalobre ${ }^{1,2}$, Carolina Martinez-Laperche $^{1,2}$, Ismael Buno ${ }^{2}$, Javier Anguita ${ }^{1,2}$, Jose Luis Diez-Martin ${ }^{1,2,3}$, Mi Kwon ${ }^{1,2}$

${ }^{1}$ Hospital General Universitario Gregorio Marañon, Hematologia, Madrid, Spain, ${ }^{2}$ Instituto de Investigación Sanitaria Gregorio Marañón, Madrid, Spain, ${ }^{3}$ Universidad Complutense de Madrid, Madrid, Spain

Background: CMV is associated with significant morbidity after allogeneic hematopoietic stem cell transplantation. Strategies to prevent CMV-related complications include universal prophylaxis and preemptive therapy, more widely spread. Antivirals used for CMV reactivation (CMV-R) produces major toxicities and costs. Rate and characterization of CMV-R after haploidentical transplantation with post-transplant cyclophosphamide (Haplo PT-Cy) is scarce. Our goal was to analyze CMV-R rate after Haplo PT-Cy, outcome, complications associated to therapy, and to identify risk factors.

Methods: One hundred Haplo PT-Cy transplants using peripheral blood as stem cell source performed between 2011 and 2016 in our center have been retrospectively reviewed. GVHD prophylaxis consisted of PT-Cy $50 \mathrm{mg} /$ $\mathrm{kg} / \mathrm{day}$ on days +3 and +4 , MMF and CsA from day +5 for all cases.

CMV PCR was performed in a biweekly basis during admission for transplant and treatment, and weekly thereafter. CMV-R was considered with any CMV DNA level by PCR assay above 100 copies $/ \mathrm{ml}$. Prior four consecutive negative weekly PCRs were needed to consider a new reactivation episode. Preemptive strategy was applied in all cases.

Data collected in relation to CMV-R included: CMV serostatus of donor/recipient (D/R), number of CMV reactivations, length of each reactivation, antiviral treatment used, need for admission to receive treatment and adverse events related to CMV reactivation and/or antiviral treatment.

Results: Patients characteristics are summarized in Table 1. Among 100 patients, 78 of them with positive CMV serology, 128 episodes of CMV-R were detected. Seventysix patients (76\%) had at least one CMV-R in a median of 30 days after transplant. None of them had CMV disease or die as a consequence of CMV-R. Median duration was 22 days (15-37). Valganciclovir or ganciclovir was used in 101 episodes (79\%). Foscarnet was used in 43 episodes (34\%). Six of the episodes occurred after initial discharge, and required re-admission for treatment, with a median length of hospitalization of 16 days (6-27). Cytopenias requiring transfusion or G-CSF support occurred in 36 episodes $(36 \%)$ treated with ganciclovir or valganciclovir. Three of them needed further CD34+ cells booster for graft rescue. Mild acute renal failure and genital ulcers were found in $21(49 \%)$ and $5(11,6 \%)$ events treated with foscarnet, respectively. No cases of severe renal failure were observed.

Serological status different than negative/negative $(\mathrm{N} / \mathrm{N})$ (p 0.001) and older age (46 vs 38 years, p 0.02) were significantly associated with CMV-R. No relationship was observed with gender, disease, donor relationship, conditioning, GvHD or cells infused. More than 2 reactivations were more frequent among patients with grade II-IV acute GvHD (aGvHD) and moderate-severe chronic GvHD (cGvHD).

Conclusions: In our experience, rate of CMV-R after unmanipulated Haplo PT-Cy, using PBSC as stem cell source, is considerably high. A significant proportion of patients presented complications associated with CMV-R and its treatment. 
CMV serological status other than $\mathrm{N} / \mathrm{N}$ and older age are associated with high risk of CMV-R. Patients with grade IIIV aGvHD are at higher risk of multiple reactivations. This population could be benefited from primary prophylaxis, in order to decrease treatment's complications, re-admissions and costs.

Disclosure: Nothing to declare.

\section{P423}

Impact of infectious events occurring during the first hundred days after hsct for hematological malignancy: A monocentric retrospective study over a five-year period

Marie-Pierre Ledoux ${ }^{1}$, Célestine Simand ${ }^{1,2}$, Karin Bilger ${ }^{1}$, Annegret Laplace ${ }^{1}$, Bruno Lioure ${ }^{I}$

${ }^{1}$ Hôpitaux Universitaires de Strasbourg, Hematology, Strasbourg, France, ${ }^{2}$ Institut de Génétique et de Biologie Moléculaire de la Cellule, Illkirch-Graffenstaden, France

Background: Patients undergoing hematopoietic stem cells transplantation (HSCT) for hematological malignancy often present with infectious events in the early stages of the procedure, some of which having a documented impact on the outcome of the graft. For instance, cytomegalovirus (CMV) has been shown by some authors to have a protecting effect against relapse, whose features remain to be elucidated.

Methods: We conducted a retrospective monocentric study regarding the outcome in terms of graft versus host (GvHD), relapse and survival of 224 consecutive patients over a period of 5 years, whether they presented or not with an infectious event by day 100 among the following: CMV viremia, Epstein-Barr virus (EBV) viremia, Human herpes virus 6 (HHV6) viremia, BK virus (BKV) viruria, bacterial bloodstream infection (BSI) or invasive fungal infection.

Results: A high proportion of CMV seropositive recipients underwent a viral reactivation of $\mathrm{CMV}$ by day 100 of the HSCT: $81 \%$ if the donor is seronegative and $74 \%$ if the donor is seropositive. We observed that CMV wasn't associated with a lower relapse rate in our cohort, and data weren't sufficient to conclude firmly, but showed a trend towards a worse acute GvHD (hazard ratio HR 2.08, pvalue 0.06). No significant correlation was found for EBV viremia. Occurring in $26 \%$ of our patients and mostly with an early timing, HHV6 strongly correlated with worse acute GvHD (HR 3.25, p-value < 0.001) but its impact on survival was not significant. BKV (27\% of our patients) and BSI ( $46 \%$ of our patients) both correlated with poorer outcome in terms of overall survival (logrank $<0.001$ and 0.014 respectively) although not significantly associated with relapse or acute GvHD. Fungal infections were too rare events to draw any conclusion.

Conclusions: Thus, contrary to many studies, we found no protection against relapse induced by CMV, although the trend for worse acute GvHD was obvious. The mechanisms behind this discordance could include early treatment, but remain to be studied. Whether HHV6 is a cause rather than a consequence of acute GvHD or its treatment is debated, but the correlation is strong and the sequence of events suggests HHV6 might act as a trigger for GvHD. The association between BKV viruria and a higher mortality is in contrast with previous observations, and the lack for association with GvHD and relapse could suggest BKV is a surrogate for poor immune recovery and therefore other causes of non-relapse mortality. In addition to the direct lethal risk of bacteriemia, BSI also are a promoter of late non-relapse mortality through indirect toxicity.

Through the expansion of immune effectors they promote, one could assume that infectious events play a role in GvHD and GvL, and therefore have an interference with relapse. However, the association between each infectious event and outcome remains to be clarified to guide our prophylactic and therapeutic choices by a better understanding of the bright and dark sides of infectious events.

Disclosure: Nothing to declare

\section{P424}

Abstract already published.

P425

A drug-drug interaction (DDI) study between rezafungin and nine probe drugs show no relevant pharmacokinetic change

\section{Voon Ong ${ }^{1}$, Michael Boily ${ }^{2}$, Hong Wong ${ }^{2}$, Taylor Sandison ${ }^{1}$, Shawn Flanagan ${ }^{1}$}

${ }^{1}$ Cidara Therapeutics, Inc., San Diego, CA, United States, ${ }^{2}$ Altasciences, Laval, Canada

Background: Rezafungin (RZF) is a novel echinocandin in Phase 3 development for treatment of candidaemia and invasive candidiasis and for antifungal prophylaxis against invasive fungal diseases caused by Candida, Aspergillus, and Pneumocystis in blood and marrow transplant patients. RZF is differentiated by stable, prolonged pharmacokinetics $(\mathrm{PK})$ that allow for once-weekly dosing and a PK-pharmacodynamic (PD) profile correlating with efficacy. Clinical in vivo evaluations of drug interaction potential were performed proactively to assess the risk of drug-drug interactions (DDIs) with respect to the Phase 3 dose of $400 \mathrm{mg}$ once weekly and known PK exposure in healthy individuals. 
Methods: This open-label study of 26 healthy inpatients assessed DDIs between RZF (as perpetrator) and drugs known to have interactions with CYP enzymes and transporters (probe drugs): repaglinide (CYP2C8), metformin (OCT/MATE), rosuvastatin (BCRP/OATP), pitavastatin (OATP), caffeine (CYP1A2), efavirenz (CYP2B6), midazolam (CYP3A4), and digoxin (P-gp), as well as tacrolimus, a drug likely to be coadministered with RZF. An initial dose of RZF $600 \mathrm{mg}$ was administered on the first dosing day, to approximate a steady state plasma concentration of multiple once-weekly $400-\mathrm{mg}$ doses, followed by 2 once-weekly $400-\mathrm{mg}$ doses on days 10 and 15 . Probe drug cocktails containing $\geq 2$ drugs were administered, once before and once after RZF administration, on a schedule designed to allow for washout between doses and to limit interactions with other probe and test drugs.

Samples were analysed to determine respective drug concentrations in plasma (except for tacrolimus which was in whole blood) to characterize the PK profile of each analyte. Area under curve (AUC) and maximum concentration $\left(\mathrm{C}_{\max }\right)$ were calculated from the plasma/blood concentration-time profiles by noncompartmental analysis. Ln-transformed PK parameters were statistically analysed using an Analysis of Variance model. The ratio of geometric least squared means between each substrate drug when administered with and without RZF and corresponding $90 \%$ confidence intervals (CIs) were calculated for lntransformed $\mathrm{C}_{\max }$ and AUC.

Results: When RZF was given concomitantly with the probe drugs, six of nine substrates (metformin, pitavastatin, caffeine, efavirenz, midazolam, and digoxin) statistically demonstrated the absence of drug-drug interaction, as their 90\% CI were all included within the default $80-125 \%$ noeffect boundary. Three substrates had the upper (repaglinide and rosuvastatin) or lower (tacrolimus) bounds of their CI falling just outside of this range (Figure 1), and these changes are considered unlikely to be clinically significant.

Conclusions: No meaningful PK interactions were observed between RZF and 9 drugs known to have DDIs and/or likely to be coadministered with RZF. These findings provide evidence that no dose adjustment is expected when RZF is co-administered with these commonly used drugs, which stand in contrast with the DDI complications widely associated with azole antifungals.

Disclosure: Voon Ong: employee, stockholder (Cidara Therapeutics), Michael Boily: employee (Altasciences), Hong Wong: employee (Altasciences), Taylor Sandison: employee, stockholder (Cidara Therapeutics), Shawn Flanagan: employee, stockholder (Cidara Therapeutics)

\section{P426}

Abstract withdrawn.
P427

Efficacy of letermovir prophylaxis in cytomegalovirus seropositive allogeneic hematopoietic stem cell transplant recipients receiving in-vivo T-cell depletion

Rosy Priya Kodiyanplakkal, Maxwell Brown ${ }^{1}$, Danielle Guarneri $^{1}$, Rosemary Soave ${ }^{1}$, Alexander Drelick, Adrienne Phillips ${ }^{I}$, Sebastian Mayer ${ }^{I}$, Usama Gergis ${ }^{I}$, Jing-Mei Hsu ${ }^{I}$, Tsiporah Shore ${ }^{I}$, Thomas Walsh ${ }^{I}$, Catherine Small, Koen Van Besien ${ }^{1}$

${ }^{1}$ Weill Cornell Medical College, Cornell University, New York, NY, United States

Background: Cytomegalovirus (CMV) continues to cause morbidity following allogeneic hematopoietic stem cell transplantation (HSCT). Letermovir is a newly approved drug for CMV prophylaxis in CMV-seropositive allogeneic HSCT recipients. However, there is a paucity of data for its efficacy in patients receiving in-vivo T-cell depletion (TCD).

At Weill Cornell Medical Center, we perform in-vivo TCD with alemtuzumab for related and HLA-identical unrelated transplants, and anti-thymocyte globulin for umbilical cord blood transplant supported by third party accessory cells (haplo-cord transplant).Although these drugs reduce the frequency of Graft-Versus-Host-Disease (GVHD), they significantly delay T-cell immune reconstitution post HSCT, and may cause higher rates of CMV reactivation. Our historical rate of CMV reactivation in CMV seropositive recipients receiving high dose valacyclovir prophylaxis is approximately $35 \%$. Therefore, we implemented letermovir for CMV prophylaxis in February 2018.

The primary aim of this study is to determine the incidence of $\mathrm{CMV}$ infection (defined as CMV viremia warranting treatment or development of end-organ disease) in TCD CMV seropositive allogeneic HSCT patients who received letermovir prophylaxis.

Methods: This is a single center, retrospective cohort study to determine the incidence of CMV infection in adult, CMV-seropositive recipients receiving letermovir prophylaxis after in vivo TCD HSCT with ATG or alemtuzumab for GVHD prophylaxis. All included subjects were at least 100 days post-transplant.

Results: 31 allogeneic HSCT transplant recipients met inclusion criteria. Median age was 60 years, IQR $[47,67]$ and $48 \%$ were male. Eight $(26 \%)$ had a matched related donor, six (19\%) had a matched unrelated donor, and 17 (55\%) were haplo-cord transplants. Their underlying malignancy and conditioning regimens are summarized in Table 1.17 (55\%) received ATG and 14 (45\%) received alemtuzumab for GVHD prophylaxis. 
Median follow up time for survivors is 141 days, IQR $[107,187]$. The incidence of CMV infection in the first 100 days post-transplant was $3 \%$ as only one patient reactivated with detectable CMV viremia. This same patient developed CMV pneumonitis with documented UL 56 resistance, and was successfully treated with ganciclovir. The incidence of CMV infection within the first 150 days post-transplant was 5\% (1/19 patients).

Six patients (19\%) developed acute GVHD in the first 100 days, and one (3\%) had relapse of their malignancy. Five patients (16\%) died within 100 days post-transplant, but none of these deaths were CMV related.

\begin{tabular}{llc}
\hline Underlying Malignancy & Acute Myeloid Leukemia & $18(58)$ \\
& Myelodysplastic Syndrome & $6(19)$ \\
& Non Hodgkins Lymphoma & $2(6)$ \\
& Acute Lymphocytic Leukemia & $4(13)$ \\
& Other & $1(3)$ \\
Donor Type & HLA-Identical Sibling & $8(26)$ \\
& HLA-Identical Unrelated & $6(19)$ \\
& Haplo-Cord & $17(55)$ \\
Conditioning Regimen & Reduced Intensity Conditioning & $27(87)$ \\
& Myeloablative Conditioning & $4(13)$ \\
\hline
\end{tabular}

[[P427 Table] 1. Table 1: Characteristics of Cytomegalovirus Seropositive Hematopoietic Stem Cell Transplant Recipients Receiving Letermovir Prophylaxis $(N=31)]$

Conclusions: Letermovir prophylaxis resulted in a low rate of $\mathrm{CMV}$ infection in this uniquely high-risk, in-vivo TCD allogeneic HSCT population.

Disclosure: Dr. Usama Gergisis reports being on the speaker bureau for Merck. Dr. Catherine Small reports research support paid to her institution from GlaxoSmithKline, ViiV, Abbott, Merck, Gilead, Chimerix, Shire, Schering, and Ablynx. Dr. Thomas Walsh reports research support paid to his institution from Amplyx, Astellas, Merck, Scynexis, Allergan, Medicines Company, Lediant, Tetraphase, and T2Biosystems

\section{P428}

Specific features of anti-adenovirus T-cell response in donor peripheral blood: The activity of IFN-gamma production and association with MHC class I and II

Ruslan Nikolaev ${ }^{I}$, Yana Bayzyanova ${ }^{I}$, Victoria Kiseleva ${ }^{I}$, Maria Efimenko ${ }^{1}$, Natalia Khripkova ${ }^{I}$, Julia Panchenko ${ }^{I}$, Rimma Khismatullina $^{l}$, Sergei Blagov ${ }^{\prime}$, Olga Shragina ${ }^{l}$, Elena Osipova ${ }^{I}$, Mikhail Maschan ${ }^{1}$
${ }^{1}$ Dmitry Rogachev National Research Center of Pediatric Hematology, Oncology and Immunology, Moscow, Russian Federation

Background: Infectious complications caused by endogenous adenovirus (AdV) are common and associated with morbidity and mortality rates in patients after hematopoietic stem cell transplantation (HSCT). AdV infections occur in about $3 \%$ to $20 \%$ of HSCT recipients, with significantly higher rates in pediatric patients. A better understanding of adenoviral-specific T-cells (AdvT) response in donors can serve as a basis to develop more effective strategies for antiviral therapy.

Methods: Frequencies of cytomegalovirus (CMV)- and AdV-specific $\mathrm{T}$ cells were determined by enzyme-linked immunospot (ELISPOT) assays with AdV5 Hexon and CMVpp65 respectively in 80 health donors. We used $3 \times 10^{5}$ of mononuclear cells (MNC) per well in ELISPOT assays. All donors were divided into 3 groups according to the number of spots per well (SPW) as follows: high responders (HR) ( $\geq 50$ spots; $\mathrm{n}=32$ ), low responders $(\mathrm{LR})(>10$ and $<$ 50 spots; $n=39)$, nonresponders (NR) $(\leq 10$ spots; $n=9)$. The average spot area of AdV- and CMV-specific lymphocytes was calculated by ImmunoSpot ${ }^{\circledR}$ MultiPlate AutoCount ${ }^{\mathrm{TM}}$. $\mathrm{CD} 45 \mathrm{RA}+$ and $\mathrm{CD} 45 \mathrm{RO}+\mathrm{T}$-cells were generated by immunomagnetic negative selection. HLA typing for class I and II was performed by Sequence Specific Oligonucleotides technology. Statistical analysis was performed using GraphPad Prism v7.00 software. Levels of significance were calculated by Mann-Whitney rank-sum test, expressed as $\mathrm{p}$-values $(\mathrm{p}<0.05)$.

Results: The median frequency per well of AdvTs were 72 in HR group, 24 in LR group, 4 in NR group. The median SPW of CMV-specific T cells in donors MNC were 147 and didn't differ between 3 groups. Antiviral activity may depend not only on the amount of AdvT but also on their ability to produce IFN $\gamma$. The average spot area for AdvT did not differ between HR, LR and NR groups and were $22,4,23,1$ and $19,1 \mathrm{~mm}^{2}$ respectively. The median of the average spot area for anti-CMV T-lymphocytes was equal to $13,5 \mathrm{~mm}^{2}$. Thus, the frequency of AdvT was lower than CMV-specific T-cells, but AdvT have the ability to produce more IFN $\gamma$ per cell $(\mathrm{p}<0.0001)$. In order to evaluate the distribution of the AdvT between naive and memory $\mathrm{T}$ cell compartments, we evaluated response to $\mathrm{AdV}$ in preselected CD45RA + and CD45RO + fractions of T-cells in a group of 17 donors. The median frequency of AdvT in unfractionated MNC was 87; the median frequency of AdvT in CD45RA and CD45RO fractions were 2 and 73, respectively. The amounts of CD45RA and CD45RO Tcells were normalized to their amounts in MNC. We evaluated the impact of HLA-alleles on the anti-AdV response of $\mathrm{T}$-cells in different groups and found 2 
association: HLA-A $* 1$ with HR group $(\mathrm{P}$-value $=0,0043$; $\mathrm{RR}=1,954 ; 95 \% \mathrm{CI}: 1,285$ to 2,604$)$ and HLA-A*31 with LR group ( $\mathrm{P}$-value $=0,0251 ; \mathrm{RR}=1,889 ; 95 \% \mathrm{CI}: 1,135$ to $4,426)$.

Conclusions: In this study the frequency of donors with AdvT is $88,75 \%$ which corresponds to the reported frequency of AdV-seropositive people (95\%) in population of Russia. AdvT are exclusively CD45RO-positive cells. The analysis of AdvT in potential HSCT donors will allow to determine more accurately the amounts and functional activity of specific antiviral T-lymphocytes administered to patient and optimize antiviral therapy.

Disclosure: Nothing to declare

P429 abstract withdrawn.

\section{P430}

Chemotaxis and exhaustion of $\gamma \delta \mathrm{T}$ cells in the allografts are associated with $\mathrm{CMV}$ reactivation after hematopoietic transplantation

Haitao Gao ${ }^{1}$, Lan-Ping $X u^{1}$, Ming Wang ${ }^{1}$, Yazhe Wang ${ }^{1}$, Xiao-Jun Huang ${ }^{I}$, Jiangying Liu ${ }^{I}$

${ }^{1}$ Peking University People's Hospital, Peking University Institute of Hematology, Beijing Key Laboratory of Hematopoietic Stem Cell Transplantation, Beijing, China

Background: Cytomegalovirus (CMV) reactivation and its related diseases remain the most common and serious complications in patients who underwentallogeneic hematopoietic stem cell transplantation (alloHSCT). We previously reported that the incidences of total and refractory CMV reactivation reached approximately $90 \%$ and 50\% after haploidentical HSCT. While majority of studies in the literatures focused on the adaptive $\mathrm{CD}^{+} \alpha \beta$ T cellsand NK cells in anti-CMV immunity, increasing evidences highlighted the important role of $\gamma \delta \mathrm{T}$ cells in this context. A progressive and prolonged expansion of $\mathrm{V} \delta 1^{+} \mathrm{T}$ cells in response to CMV reactivation was observed after alloHSCT. The effect of $\mathrm{V} \delta 1^{+} \mathrm{T}$ cells associated with CMV clearance has been reported in vitro and in vivo. In contrast to the reconstituted $\gamma \delta \mathrm{T}$ cells post transplantation, whether the phenotypes of $\gamma \delta \mathrm{T}$ subsets in allografts correlate to CMV reactivation in HSCT recipients have not been documented.

Methods: The proportions and phenotypes of $\gamma \delta \mathrm{T}$ cells were detected inallografts those were unmanipulated GCSF-mobilized bone marrow (BM) and peripheral blood (PB) harvests from 20 donors for haploHSCT. BM grafts were collected by aspiration on the fourth day of G-CSF treatment (filgrastim, $5 \mu \mathrm{g} / \mathrm{kg} / \mathrm{day}$ ), and $\mathrm{PB}$ grafts were obtained on the fifth day by leukapheresis. Immunophenotyping for $\gamma \delta \mathrm{T}$-cell subpopulations, including the expression of $\mathrm{CD} 3, \mathrm{CD} 4, \mathrm{CD} 8, \mathrm{TCR} \gamma \delta, \mathrm{TCRV} 81$, TCRV82, HLA-DR, NKG2D, CXCR4, CCR5, PD1, ki67, IFN $\gamma$, TNF $\alpha$, and IL-17, was performed using flow cytometry. For detection of the intracellular cytokines, $\mathrm{BM}$ and $\mathrm{PB}$ grafts were pre-stimulated with $1 \mathrm{x}$ Cell Stimulation Cocktail (500x, eBioscience). CMV DNA in the peripheral blood of recipients was routinely monitored by quantitative PCR. The association of $\gamma \delta$ T-cell contents in allografts with CMV reactivation in haploHSCT recipients was analyzed using the Mann-Whitney $U$ test and Spearman test. All calculations were performed using SPSS 22.0 statistical software.

Results: We found that the proportions of total $\gamma \delta \mathrm{T}$ cells, and V $\delta 1$ and V $\delta 2$ subsets in both BM and PB grafts for $\mathrm{CMV}+$ and CMV- recipients were comparable. Neither the expression of HLA-DR nor NKG2D in the allografts were significantly different in correlation to CMV reactivation after HSCT. The productions of intracellular cytokines of $\gamma \delta$ $\mathrm{T}$ subsets did not varied in $\mathrm{BM}$ and $\mathrm{PB}$ grafts for $\mathrm{CMV}+$ and CMV- recipients. Interestingly, the proportions of CXCR4+V81 and CCR5+V81 cells in BM grafts for CMV + recipients were significantly higher than those for CMVrecipients $(\mathrm{P}=0.012$ and 0.045 , respectively). Meanwhile, PMA-stimulated ki67+V81 cells in BM grafts for CMV+ recipients were less than those for $\mathrm{CMV}$ - recipients $(\mathrm{P}=$ 0.039). In parallel, the concentration of PD1+V $\delta 1$ cells in $\mathrm{PB}$ grafts for $\mathrm{CMV}+$ recipients were significantly higher than those for CMV- recipients $(\mathrm{P}=0.023)$.

Conclusions: This study is the first to connect the chemotaxis and exhaustion of $\gamma \delta \mathrm{T}$ cells in grafts to the risk of CMV infection after allogeneic HSCT. Future studies should explore how the expressions of chemokines and exhaustion marker on the effector $\gamma \delta \mathrm{T}$ cells in allografts facilitate CMV replication and/or dissemination in the setting of hematopoietic transplantation.

Disclosure: All authors do not have conflicts of interest.

This study is supported by the National Natural Science Foundation of China (Grants No.81770191 and No.81670167) and the Foundation for Innovative Research Groups of the National Natural Science Foundation of China (Grant No.81621001).

\section{P431}

Invasive candidiasis in children after hematopoietic stem cell transplantation

Inna Markova ${ }^{1}$, Marina Popova ${ }^{1}$, Yulia Rogacheva ${ }^{1}$, Anastasia Froloval, Alisa Volkoval, Olga Pinegina ${ }^{1}$, Tatyana Bogomolova ${ }^{2}$, Asmik Gevorgayn ${ }^{1}$, Olesya Paina ${ }^{1}$, Tatyana Bykova ${ }^{1}$, Maria Vladovskaya ${ }^{I}$, Elena Semenova, Nikolay Klimko ${ }^{2}$, Ludmila Zubarovskaya ${ }^{1}$, Boris Afanasyev $^{I}$ 
${ }^{1}$ First State Pavlov Medical University of Saint Petersburg, Raisa Gorbacheva Memorial Research Institute for Pediatric Oncology, Hematology and Transplantation, Saint Petersburg, Russian Federation, ${ }^{2}$ I.I. Mechnikov North-Western State Medical University, Saint-Petersburg, Russian Federation

Background: Despite the development and introduction into clinical practice of new diagnostic algorithms and modern anti-infective drugs, the risk of severe infectious complications in recipients of hematopoietic stem cells remains high and affects the results of hematopoietic stem cell transplantation (HSCT).

Data on the prevalence and structure of invasive candidiasis in children after HSCT are insufficient. The aim was to study the epidemiology of invasive candidiasis (IC) after hematopoietic stem cell transplantation (HSCT) in large pediatric cohort of patients for the 7 years in Raisa Gorbacheva Memorial Research Institute of Children Oncology, Hematology and Transplantation (CIC725).

Methods: In 2009 - 2016 yy have been performed 754 HSCT in children: 494 allogeneic and 260 autologous. A retrospective study included 22 cases of IC after HSCT. EORTC/MSG 2008 criteria were used for the diagnosis of proven IC as well as to evaluate response to therapy.

Results: Incidence of IC was 2,9\%: allo-HSCT - 3\% $(n=15)$, auto-HSCT - 2,7\% $(n=7)$. The etiology: C. parapsilosis $50 \%$, C. albicans $27 \%$, C. krusei $14 \%$, Candida tropicalis $5 \%$, Candida dubliniensis $4 \%$. The most frequent underlying diseases was acute leukemia $45 \%(n=10)$. The median age was 8 y.o. [ 3 month - 18 years]. The median day of onset of IC after allo-HSCT was 63 [4-243], auto-HSCT - 12 [3-20]. Febrile fever was the main clinical symptom; septic syndrome develops in $32 \%$ cases. Antifungal therapy was with echinocandins - 23\%, lipid ampho B - 27\%, azoles (fluconazole, voriconazole) - 32\%, without therapy (the early mortality) - $18 \%$. Overall survival (OS) at 30 days from diagnosis of invasive candidiasis was 50\%. The central venous catheter (CVC) removal was the only factor significantly improved OS $(70 \%$ vs $33 \%$, $\mathrm{p}=0,035)$.

Conclusions: Incidence of invasive candidiasis in children after hematopoietic stem cell transplantation was $2.9 \%$. The main etiology agent was C. parapsilosis. Invasive candidiasis infections most often affect leukemia patients, developed later after allo-HSCT than auto-HSCT. Overall survival at 30 days from the diagnosis was $50 \%$. Removing of CVC improved overall survival in children with invasive candida infections after HSCT.

Disclosure: Nothing to declare

\section{P432}

Small intestinal bacterial overgrowth evaluation in patients after allogeneic hematopoietic stem cell transplantation

Oleg Goloshchapov ${ }^{1}$, Maksim Kucher ${ }^{1}$, Ruslana Klementeva ${ }^{1}$, Aleksandr Shcherbakov', Aleksandr Shvetcov', Alexei Chukhlovin ${ }^{1}$, Boris Afanasyev ${ }^{1}$

${ }^{1}$ First State Pavlov Medical University of Saint-Petersburg, Russia; Raisa Gorbacheva Memorial Institute of Children Oncology, Hematology and Transplantation, Saint-Petersburg, Russian Federation

Background: Graft versus host disease (GvHD) and virusassociated enteropathy in allogeneic hematopoietic stem cell transplantation (allo-HSCT) may cause severe quantitative and qualitative composition changes of intestinal microbiota, leading to the development of small intestinal bacterial overgrowth (SIBO) on the background of immunodeficiency, which can have a negative impact on treatment effectiveness. The gold standard for diagnosis and the main criterion for SIBO is the detection in the jejunum aspirate $>10^{5} / \mathrm{ml}$ bacteria and/or the appearance of colonlike microbiota in small intestine. It is also acceptable to use an alternative non-invasive technique - hydrogen breath test, which could be especially important in patients with severe mucositis, grade III-IV and thrombocytopenia grade IV. SIBO diagnosis in the setting of the gastrointestinal tract damage and dysfunction in patients treated with allo-HSCT is insufficiently studied.

Methods: The study included 7 patients with acute myeloid leukemia $(n=3)$, acute lymphoblastic leukemia $(\mathrm{n}=1)$, myelodysplastic syndrome $(\mathrm{n}=1)$, non-Hodgkin's lymphoma $(n=1)$, Hurler syndrome $(n=1)$, who underwent allo-HSCT from an unrelated $(n=4)$ and haploidentical donor $(n=3)$, and which were complicated by enteropathy development. In 2 cases, the enteropathy reason was a combination of intestinal GvHD and viral colitis (HHV-6), in 5 cases - viral colitis (HHV-6). All patients had esophagogastroduodenoscopy with species aspiration from descending part of the duodenum and feces collection, with further bacteria PCR identification. Hydrogen breath test was performed also in which patients were treated with oral lactose $2 \mathrm{~g} / \mathrm{kg}$ with subsequent hydrogen assessment after 30 and 60 minutes. The study was performed in the period from 20 to 741 days after allo-HSCT.

Results: According to feces analysis data, colon microbiota composition significantly differed from the reference values. At the same time total bacterial mass of the duodenum was less in comparison with colon microbiota: $2 \mathrm{E}+08(2 \mathrm{E}+7 / 8 \mathrm{E}+9)$ and $9 \mathrm{E}+10(9 \mathrm{E}+7 / 3 \mathrm{E}+12)$, respectively $(\mathrm{p}<0.004)$. Quantitative composition of the duodenal 
microbiota was comparable to that of colon: Lactobacillus spp. $4 \mathrm{E}+5(1 \mathrm{E}+5 / 2 \mathrm{E}+7)>1.5 \mathrm{E}+5(1 \mathrm{E}+5 / 4 \mathrm{E}+11)$, $(\mathrm{p}=0.48) ;$ Bifidobacterium spp. $6 \mathrm{E}+6(1 \mathrm{E}+05 / 3 \mathrm{E}+7)<$ $7.5 \mathrm{E}+6(2 \mathrm{E}+5 / 1 \mathrm{E}+9),(\mathrm{p}=0.423) ;$ Escherichia coli $7 \mathrm{E}+5$ $(1 \mathrm{E}+5 / 2 \mathrm{E}+6)>5 \mathrm{E}+5(4 \mathrm{E}+5 / 9 \mathrm{E}+5),(\mathrm{p}=0.2) ;$ Bacteroides fragilis group $6 \mathrm{E}+6(0 \mathrm{E}+0 / 8 \mathrm{E}+9)>5 \mathrm{E}+6(2 \mathrm{E}+5 / 3 \mathrm{E}$ $+12),(\mathrm{p}=1.0)$; Faecalibacterium prausnitzii $1 \mathrm{E}+6(0 \mathrm{E}+0 /$ $1 \mathrm{E}+7)<1.5 \mathrm{E}+6(1 \mathrm{E}+5 / 3 \mathrm{E}+10),(\mathrm{p}=0.54)$, indicating the presence of SIBO. In this case, the hydrogen breath test was completely uninformative: basal values - $0.035(0.01 / 0.06)$ ppm, hydrogen concentration in 30 minutes - $0.04(0.01 /$ $0.06) \mathrm{ppm}$, in 60 minutes - $0.02(0.01 / 0.07) \mathrm{ppm}$, which is less than in healthy volunteers.

Conclusions: Quantitative composition of the duodenal and colon microbiota is similar in the case of intestinal GvHD and/or virus-associated enteropathy in allo-HSCT patients, which may be of diagnostic value for SIBO confirmation. The hydrogen breath test is an uninformative method for SIBO identification in patients after allo-HSCT.

Disclosure: Nothing to declare

\section{P433}

Brincidofovir (CMX-001) for refractory adenovirus infections after stem cell transplantation: A single-center experience

\section{Johannes Schulte ${ }^{1}$, Patrick Hundsdörfer ${ }^{1}$, Sebastian Voigt ${ }^{1}$}

${ }^{1}$ Charité - Universitätsmedizin Berlin, Berlin, Germany

Background: Adenovirus (AdV) infections or reactivations frequently occur in the pediatric hematopoietic stem cell transplant (SCT) setting and these infections contribute to increased morbidity and mortality. The nucleotide analog Cidofovir might be effective in reducing AdV load, however, nephrotoxicity is a considerable side effect. The new antiviral compound Brincidofovir (BCV, CMX-001), a lipid-conjugate nucleotide analog with broad-spectrum antiviral activity in vitro, has been reported to be effective in cases where Cidofovir treatment was unsuccessful.

Methods: Data of eight pediatric patients undergoing SCT for malignant and nonmalignant indications were analyzed. All patients were weekly monitored for AdV viremia by PCR. In case two consecutive positive AdV PCR results indicating a viral copy number $>1000 / \mathrm{ml}$ were documented, patients received a weekly dose of Cidofovir. If no reduction of $\mathrm{AdV}$ load was seen within two weeks after the commencement of treatment or side effects demanded Cidofovir discontinuation, BCV was obtained through an emergency expanded access programme.

Results: Eight pediatric patients developed AdV viremia with maximum viral loads ranging between 9350 and
4430000 copies/ml. Six patients had C type AdV and two patients had non $\mathrm{C}$ type $\mathrm{AdV}$ infections. Five patients had viral co-infections: two had an additional CMV infection, one had an Epstein-Barr virus (EBV) and Herpes simplex virus co-infection, one patient had an EBV co-infection and one patient had a BK virus co-infection. All eight patients initially received Cidofovir, however, a substantial decrease in AdV load could not be observed in any patient after a two-week administration course. Except in one patient who had extensive intestinal graft-versus host disease (GvHD), $\mathrm{AdV}$ infection was cleared in all patients within three weeks after the beginning of $\mathrm{BCV}$ treatment. In addition, all coinfections were cleared. No nephrotoxicity or other side effects were observed.

Conclusions: $\mathrm{BCV}$ was effective in all but one patient. Oral BCV might not be effective in advanced upper gut GvHD, especially when applied via a gastric tube, yet this was observed in only one patient. Eventually, without nephrotoxic side effects, BCV could be an useful alternative to Cidofovir.

Disclosure: Nothing to declare.

\section{P434}

Increased incidence of viral reactivation in $\mathbf{T}$ cell replete haploidentical stem cell transplantation with posttransplant cyclophosphamide

Ioannis Tsonis ${ }^{1}$, Chara Giatra ${ }^{1}$, Zois Mellios ${ }^{1}$, Frini Karaolidou', Tatiana Tzenou', Eirini Grispou $^{1}$, Maria Bouzani, Zoi Poulopoulou $^{1}$, Eirini Bika ${ }^{1}$, Maria-Eleni Karatza $^{1}$, Vasiliki Delistrati ${ }^{1}$, Vasiliki Varsama ${ }^{1}$, Maria Katsareli $^{1}$, Kimon Fountoulis ${ }^{1}$, Stavros Gigantes ${ }^{1}$, Nikolaos Harhalakis ${ }^{1}$, Dimitrios Karakasis ${ }^{1}$, Ioannis Baltadakis ${ }^{1}$

${ }^{1}$ Evangelismos General Hospital, Athens, Greece

Background: The use of post-transplant high-dose cyclophosphamide (PTCY) has overcome the need for extensive depletion of $\mathrm{T}$ lymphocytes from haploidentical donor grafts, which traditionally resulted in severe and prolonged immunosuppression. However, reconstitution of cellular immunity may be delayed even after $\mathrm{T}$ cell replete haploidentical stem cell transplantation (haplo-SCT) with PTCY. The study of the incidence and severity of viral reactivation is therefore relevant to the outcomes of haplo-SCT with PTCY.

Methods: Our study enrolled 42 patients (women/men, 19/23), who underwent T cell replete haplo-SCT from 12/ 2013 to 10/2018 and achieved hematopoietic engraftment. Median age at transplant was 53.5 years (range, 19-70). The underlying disease was AML $(n=17)$, ALL $(n=9)$, MDS $(n=9)$, myelofibrosis $(n=4), \operatorname{CML}(n=2)$, or CLL $(n=1)$. 
The conditioning regimen was myeloablative $(n=31)$, reduced-intensity $(n=10)$ or non-myeloablative $(n=1)$. Peripheral blood was the graft source in the majority of cases $(n=29)$ and bone marrow in the remaining $(n=13)$. Recipient/donor cytomegalovirus (CMV) serostatus was -/$(n=2),-/+(n=4),+/-\quad(n=8)$, or $+/+\quad(n=28)$. The combination of tacrolimus and mycophenolate mofetil was administered in addition to PTCY for prevention of graftversus-host disease. CMV, Epstein-Barr virus (EBV), and human herpesvirus-6 (HHV-6) reactivation was monitored by real-time quantitative PCR (RQ-PCR) in blood twice weekly post haplo-SCT. BK virus (BKV) reactivation was assessed by RQ-PCR in urine and/or blood specimens in cases with symptoms suggestive of BKV-associated hemorrhagic cystitis (HC).

Results: With a median follow-up time of 25 months (range, 1-56), the cumulative incidences (CIN) of relapse and non-relapse mortality (NRM) were $15.5 \%$ (95\% CI, $6.2-28.6 \%)$ and $33.3 \%(95 \%$ CI, 18.9-48.2\%) at 2 years, respectively. Median disease-free (DFS) and overall survival (OS) were 53.3\% (95\% CI, 39.5-71.9\%) and $58.4 \%(95 \% \mathrm{CI}, 44.6-76.5 \%)$ at 2 years, respectively. The CIN of CMV reactivation/infection (>100 copies/ml) reached $75.7 \%(95 \% \mathrm{CI}, 58.8-86.4 \%)$ at 3 months. CMV infection developed in 30 out of 40 patients who were at risk, whereas recurrent $\mathrm{CMV}$ reactivation was observed in 15 patients with a median number of 2 episodes (range, 2-6) per patient. The median total duration of antiviral therapy for CMV infection was 27 days (range, 14-199). CMV disease (pneumonia) was documented in 2 patients. The CIN of EBV reactivation $(>1,000$ copies/ml) was $50.9 \%(95 \% \mathrm{CI}, 34.2-65.3 \%)$ at 12 months. No case of EBV-related post-transplant lymphoproliferative disorder was observed, however preemptive therapy with rituximab was required in 2 patients with rapidly increasing EBV viral load. HHV-6 reactivation $(>1,000$ copies $/ \mathrm{ml})$ was observed in 5 patients (CIN, $12.3 \%$ at 6 months; 95\% CI, 4.4-24.5\%), with none of them requiring specific therapy. BKV-related $\mathrm{HC}$ occurred at a CIN of $23.9 \%(95 \% \mathrm{CI}, 12.3-37.7 \%)$ at 6 months. Cystoscopy for bladder hemostasis was required in 4/11 and nephrostomy in $1 / 11$ patients with $\mathrm{HC}$.

Conclusions: Despite preservation of non-alloreactive memory T cells, haplo-SCT with PTCY is associated with substantial rates of viral reactivation (especially CMV and $\mathrm{BKV}$ ) resulting in the need for prolonged antiviral therapy and considerable morbidity as well. Therefore, strategies to prevent viral reactivation and disease are still warranted in haploidentical stem cell transplantation.

Disclosure: Nothing to declare.

\section{P435}

Adenoviral infections in adult T-cell depleted allogeneic stem cell transplants for myeloid disorders: Pre-emptive approach and impact on outcomes

Varun Mehra', Giulia Simini', Eleanor Barnwell', Ute Boczek $^{1}$, Stefani Widya ${ }^{2}$,Adrian Choy ${ }^{2}$, Kavita Raj ${ }^{1,3}$, Donal Mclornan', Austin Kulasekararaj', Hugues de Lavallade ${ }^{1}$, Victoria Potter ${ }^{1}$, Judith Marsh ${ }^{1}$, Ghulam Mufti $^{1}$, Antonio Pagliuca ${ }^{1}$

${ }^{1}$ Kings College Hospital, Haematology, London, United Kingdom, ${ }^{2}$ GKT School of Medicine, London, Haematology, London, United Kingdom, ${ }^{3}$ Guy's and St Thomas' Hospital, Haematology, London, United Kingdom

Background: Adenovirus(AdV) infections are a wellrecognised cause of morbidity and mortality in children and adults receiving an allogenic stem cell transplant(HSCT).The reported incidence of $\mathrm{AdV}$ infection is higher(6\%-42\%) in paediatric HSCT than in adults(3-27\%), but we currently lack accurate data of AdV infection burden among adults.Cidofovir has been extensively used as a pre-emptive anti-adenoviral therapy and is current standard of care.We present our single centre experience of $\mathrm{AdV}$ incidence and outcomes with pre-emptive approach in adult patients receiving $\mathrm{T}$-cell depleted(TCD) HSCTs for myeloid disorders.

Methods: This is a single-centre retrospective analysis of 332 consecutive HSCT patients for myeloid disorders including AML, MDS, MPN \& Aplastic Anaemia between January 2012-June 2016 using ATG or Alemtuzumab based TCD.AdV screening was performed in all patients with standardised real time quantitative PCR on weekly basis during standard risk period.

\begin{tabular}{|c|c|c|c|c|}
\hline \multirow{2}{*}{$\begin{array}{l}\text { Patient } \\
\text { Characteristics } \\
(n-332)\end{array}$} & \multirow[t]{2}{*}{ Factors } & \multicolumn{2}{|c|}{ Adenovirus (n-No } & \multirow{2}{*}{ p value } \\
\hline & & 42) & $\begin{array}{l}\text { Adenovirus } \\
(\mathrm{n}-290)\end{array}$ & \\
\hline $\begin{array}{l}\text { Median age in } \\
\text { years (range) }\end{array}$ & & $52(17-70)$ & $57(17-76)$ & $0.20 *$ \\
\hline Patient Gender & r Male/ Female & $27 / 15$ & $192 / 98$ & $0.80 \#$ \\
\hline $\begin{array}{l}\text { T-cell } \\
\text { depletion }\end{array}$ & ATG/ Alemtuzumab & b17/ 25 & $121 / 169$ & $0.88 \#$ \\
\hline Disease & $\begin{array}{l}\text { Aplastic Anaemia/ } \\
\text { AML/ MDS/ MPN }\end{array}$ & $7 / 20 / 11 / 4$ & $\begin{array}{l}32 / 131 / \\
97 / 30\end{array}$ & $0.65 \#$ \\
\hline $\begin{array}{l}\text { Patient } \\
\text { Age at BMT }\end{array}$ & $\leq 60$ years $/>60$ years & $s 29 / 13$ & $180 / 110$ & $0.38 \#$ \\
\hline HLA status & $\begin{array}{l}\text { Matched Unrelated/ } \\
\text { Mismatched } \\
\text { Unrelated/ Matched } \\
\text { Sibling }\end{array}$ & $22 / 6 / 14$ & $193 / 36 / 61$ & $10.16 \#$ \\
\hline Cells Source & $\mathrm{PB} / \mathrm{BM}$ & $42 / 0$ & $284 / 6$ & $0.35 \#$ \\
\hline
\end{tabular}

[[P435 Table] 1. Baseline Characteristics] 


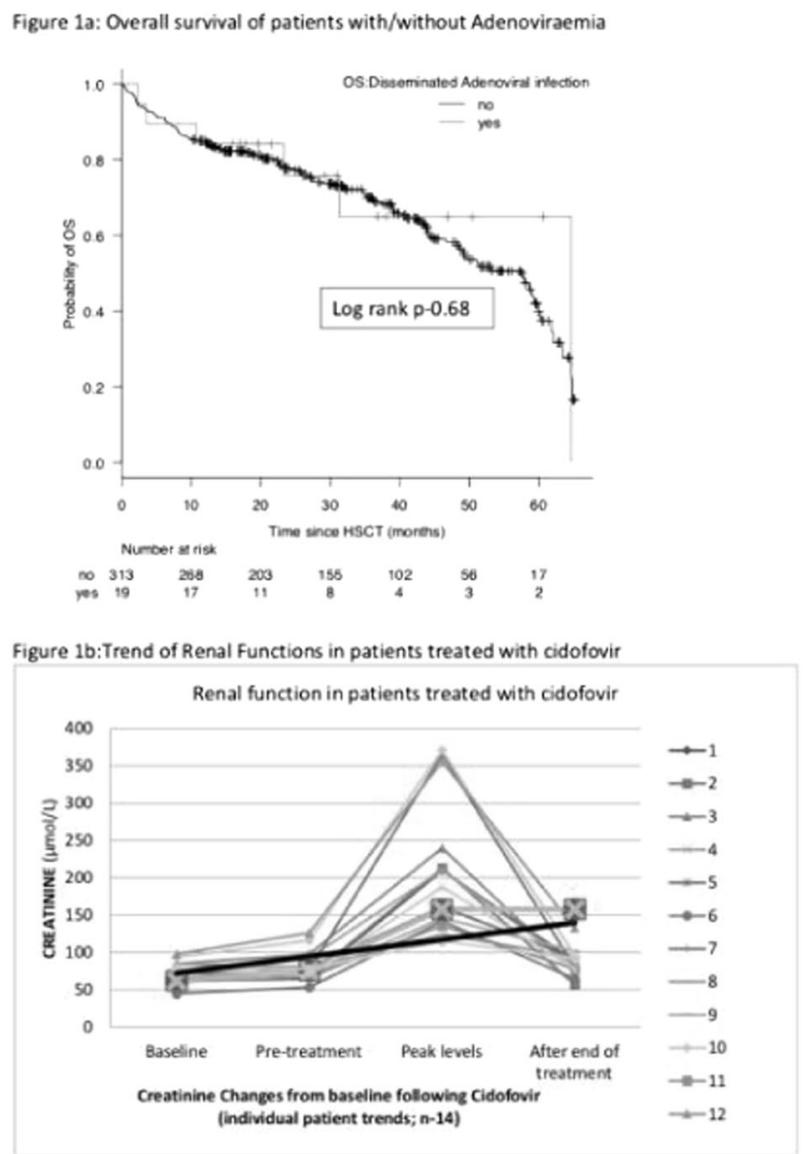

[[P435 Image] 1. Figure 1A-1B]

Results: Baseline characteristics (Table1) of patients were similar across both cohorts with or without AdV infection. Overall $12.6 \%(\mathrm{n}-42 / 332)$ patients were positive for AdV DNA on atleast one of the sanctuary sites(upper respiratory airway,blood,faeces,urine) and 45\%(n-19/42) of these experienced disseminated infection(defined by $\mathrm{AdV}$ in $\geq 2$ sanctuary sites or rising AdV DNA copies in blood), while 2 developed typical adenoviral disease (pulmonary). Among patients with disseminated infection,majority had $\mathrm{AdV}$ in gastro-intestinal(42\%),10.5\% in genitourinary and $21 \%$ as both sanctuary site of infection,in addition to blood viraemia (85\% of all cases).Cumulative incidence of $\mathrm{AdV}$ infection was $10.6 \%(95 \%$ CI:7.6-14.2\%) at 12 months with median time of 108 days(IQR:19-304 days) to detect AdVDNA post HSCT.Overall survival(OS) at 5 years for whole cohort was 41\%(95\%CI:31-50;Median OS-57months) with no statistical difference between patients with disseminated AdV infection vs those with none(log rank; p-0.68;Fig-1a). Overall cumulative incidence of non-relapse mortality (NRM) was 23\%(95\%CI:18-28\%) and relapse(CIR) was $25.5 \%(95 \% \mathrm{CI}: 19-32 \%)$ at 5 years,but no statistical difference noted between patients with disseminated AdV infection \& those with none(NRM:p-0.31;CIR: p-0.50;Gray
test).Pre-emptive therapy with Cidofovir $(3 \mathrm{mg} / \mathrm{kg}$ weekly IV infusion for 2 weeks and fortnightly thereafter until infection free) was required in 33\%(14/42) of symptomatic AdV infection patients and 52\%(10/19) with disseminated infections.One patient required brincidofovir therapy for refractory disease,but one patient died due to severe sepsis, before AdV specific therapy could be given.Remaining patients were monitored and all self-recovered on cessation of immunosuppression.All patients treated with cidofovir developed renal impairment(defined by atleast $>25 \%$ increase in baseline creatinine),however majority(73\%) recovered their renal function near their baseline(Fig-1b).

Conclusions: AdV infection remains a significant cause of morbidity in adult HSCT patients, however pre-emptive management with cidofovir has improved OS and NRM despite use of TCD conditioning.Renal toxicity remains common with cidofovir but with use of intermediate doses, majority do recover their renal functions.

Clinical Trial Registry: $\mathrm{n} / \mathrm{a}$

Disclosure: Nothing to declare

\section{P436}

\section{Abstract withdrawn.}

\section{P437}

Invasive fungal diseases before and after allogeneic hematopoietic stem cell transplantation in patients with hodgkin's lymphoma

Marina Popova ${ }^{1}$, Yuliya Rogacheva ${ }^{1}$, Alisa Volkova ${ }^{1}$, Inna Markoval, Aleksandr Shvetcovi, Ilya Nikolaev', Oleg Goloshchapov', Svetlana Ignatieva ${ }^{2}$, Tatyana Bogomolova $^{2}$, Andrey Kozlov ${ }^{1}$, Kirill Lepik', Yurii Zalyalov ${ }^{1}$, Lilia Stelmakh ${ }^{1}$, Askim Gevorgayn ${ }^{1}$, Anastasia Beynarovich $^{\boldsymbol{I}}$, Evgenia Borzenkova ${ }^{\boldsymbol{I}}$, Elena Kondakova ${ }^{\boldsymbol{I}}$, Elena Darskaya ${ }^{1}$, Maria Vladovskaya ${ }^{1}$, Natalia Mikhailova $^{I}$, Sergey Bondarenko ${ }^{I}$, Ivan Moiseev ${ }^{I}$, Ludmila Zubarovskaya ${ }^{I}$, Nikolay Klimko ${ }^{1,2}$, Boris Afanasyev ${ }^{I}$

${ }^{1}$ First Pavlov State Medical University of Saint-Petersburg, Raisa Gorbacheva Memorial Institute of Children Oncology, Hematology and Transplantation, Saint-Petersburg, Russian Federation, ${ }^{2}$ I.I. Mechnikov North-Western State Medical University, Ministry of Health of Russia, SaintPetersburg, Russian Federation

Background: Publications on invasive fungal disease (IFD) in lymphoma patients are limited especially after alloHSCT. There are no data on outcome of allo-HSCT in lymphoma patients with prior IFD. This study focuses on epidemiology of IFD before and after allo-HSCT in children and adults with Hodgkin's lymphoma (HL). 
Methods: Single center prospective study included 86 patients with classical $\mathrm{r} / \mathrm{r}$ HL who received allo-HSCT from 2002 to 2018. The median age was 27 (13-49) y.o., children (< 18 yo) $-13 \%$. Allo-HSCT from MUD was performed in $45,4 \%(n=39)$, MRD - 24,4\% ( $n=21)$, MMUD - 15,1\% $(\mathrm{n}=13)$, haplo $-15,1 \%(\mathrm{n}=13)$, with RIC $(100 \%)$ and predominantly PTCY-based GvHD prophylaxis (71\%). Primary antifungal prophylaxis was fluconazole in $85 \%$, secondary - voriconazole (100\%). EORTC/MSG 2008 criteria for diagnosis and bronchoscopy before allo-HSCT in pts with CT-scan lung lesions were used. "Active IFD" means IFD diagnosed just before HSCT. Median follow-up time was 12 months [1-71].

Results: Incidence of IFD before allo-HSCT was 12,8\% $(\mathrm{n}=11)$. IFD prior to HSCT were invasive aspergillosis (IA) with lungs involvement. Antifungal therapy before alloHSCT was used in $81,8 \%$ pts with median duration 2 months. Complete response to antifungal therapy was in $45,4 \%$ pts, partial response or stabilization - 36,4\%, and $18,2 \%$ pts had an "active IFD". After allo-HSCT all pts received voriconazole as an antifungal therapy or secondary prophylaxis. Cumulative incidence of relapse or progression of IA after allo-HSCT was 18,2\% with the median 49 day [19-79] after HSCT, which were successfully treated with voriconazole in post HSCT period. Incidence of IFD after allo-HSCT for naïve patients was $17,6 \% \quad(n=13 / 74)$. Etiology of IFD after allo-HSCT was IA - 69\%, invasive candidiasis (IC) - 15\%, mucormycosis - $8 \%$ and $8 \%$ combined IFD caused by Aspergillus fumigatus + Rhizopus stolonifer. The median day of onset of IFD after allo-HSCT was day +114 [1-489] and was associated with post-HSCT relapse of HL $(p=0,04)$. The main site of infection were lungs (88\%), the main clinical symptom - febrile fever $(100 \%)$. Antifungal therapy was used in all patients: voriconazole $-59 \%$, micafungin - $17 \%$, posaconazole $8 \%$, lipid amphotericin B $-8 \%$ and combination lipid amphotericin B with caspofungin - 8\%. Overall survival (OS) at 12 weeks from the diagnosis of IFD after alloHSCT was $80 \%$. The 2-year OS in children and adult with HL after allo-HSCT was 73,3\%. Development of IFD after allo-HSCT do not decrease the 2-year OS rate $(69,2 \%$ vs $74 \%, \mathrm{p}=0,77)$. The impact of prior IFD on 2 -year OS in allo-HSCT recipients was not statistically significant in all group $(63,6 \%$ vs $74,7 \%, p=0,47)$, and separately in children and adults.

Conclusions: Incidence of IFD in children and adults with Hodgkin's lymphoma before allo-HSCT was $12,8 \%$. Incidence of IFD after allo-HSCT in patients with Hodgkin's lymphoma was $17,6 \%$. The major etiology agents as before as after allo-HSCT were Aspergillus spp. IFD was a late complication after allo-HSCT and associated with post-HSCT relapse. Despite the high incidence IFD before or after allo-HSCT didn't influence the outcome in children and adults with Hodgkin lymphoma.

Disclosure: Nothing to declare

\section{P438}

Cytomegalovirus infection among pediatric patients undergoing hematopoietic stem cell transplant in an endemic area - report from a tertiary care center in Saudi Arabia

Murtada Alsultan ${ }^{1}$, Khawar Siddiqui', Mouhab Ayas ${ }^{1}$, Amal Al-Seraihy', Abdullah Al-Jefri', Ali Al-Ahmari', Hawazen Al-Saedi ${ }^{1}$, Awatif Al-Anazi, Ibrahim AlGhemlas $^{\text {I }}$

${ }^{1}$ King Faisal Specialist Hospital \& Research Centre, Pediatric Hematology / Oncology, Riyadh, Saudi Arabia

Background: Morbidity and mortality due to cytomegalovirus (CMV) post-Hematopoietic Stem Cell Transplant (HSCT) have decreased after the introduction of antiviral prophylaxis and pre-emptive treatment strategy for CMV viremia. The incidence of CMV infection post-HSCT is still high, ranging between 29-65\%. Moreover, limited data about pediatric CMV infection post-HSCT and outcomes. Our community has high CMV positive serostatus, which is a known risk for CMV infection or reactivation. We conducted a study to explore the incidence and outcome of CMV infection among post-HSCT children.

Methods: Medical Records of pediatric patients (Age $\leq$ 14 years) undergoing single allogeneic HSCT from January 2014 to December 2016, at King Faisal Specialist Hospital and Research Centre, Riyadh, Saudi Arabia, were reviewed. All patients with active CMV infection or disease before and during transplant were excluded. A total of 307 patients were included in the study; 160 were female. Median age at HSCT was 5 years. Recipient CMV serostatus was positive in 213 patients before HSCT, and 232 donors were CMVpositive. The Recipient-donor (R/D) serology was $63.7 \% \mathrm{R}$ $+/ \mathrm{D}+, 15.3 \% \mathrm{R}+/ \mathrm{D}-, 13.7 \% \mathrm{R}-/ \mathrm{D}+$, and $7.3 \% \mathrm{R}-/ \mathrm{D}-$. Indication for HSCT was Immune Disorders 24.1\%, Hemoglobinopathies 20.8\%, Bone Marrow Failure 20.5\%, Malignant Disorders 20.5\%, Histiocytic 8.1\%, and Metabolic Disorders 5.9\%. Source of stem cells was Bone marrow in 223, Cord blood in 74 and Peripheral Blood Stem Cell in 10 cases. Donor was matched related among 198, Unrelated matched/mismatched in 74, haploidentical 24 , and Related with 1-Antigen mismatch in 11 . Total body irradiation (TBI) based conditioning was used for 47 patients, while ATG was used in 169 patients.

Results: Out of a total of 307 patients, 101 patients developed CMV infection post-HSCT (32.9\%). Incidence in female recipients was high ( $38.1 \%$ versus $27.2 \%$, p-value 
0.042). Both recipient and donor CMV serology positive $(42.5 \%)$ developed CMV infection (p-value < 0.001). However, no CMV infection in both recipient and donor negative group (R-/D-). The incidence of CMV infection post-HSCT was high in patients received TBI based conditioning (22 out of 47, 46.8\%, p-value 0.027 ), and in haploidentical transplant with $66.7 \%$ (p-value 0.008). Source of stem cells, myeloablative versus nonmyeloablative conditioning, ATG use in conditioning and aGVHD, did not exhibit significant association with CMV infection.

In multivariable setting, when adjusted for primary indication for transplant, Donor HLA Type, TBI based conditioning and Recipient and Donor CMV Serology at transplant, haploidentical donor (Odds ratio: 3.491, PValue: 0.041) and Donor-Recipient CMV sero-positivity (Odds ratio: 2.228, P-Value: 0.007) were found to be significant risk factors. CMV infection resolution rate was $83.2 \%$ (84). With a median follow-up time of 32.9 \pm 1.5 months from infusion, five-year overall survival of CMV infected group was lower $(0.658 \pm 0.087)$ as compared to non-CMV infected $(0.706 \pm 0.032, \mathrm{P}-\mathrm{V}$ alue: 0.285$)$.

Conclusions: Incidence of CMV infection post-HSCT in our center is comparable to other centers. Our data suggest that donor-recipient $\mathrm{CMV}$ positive serostatus, haploidentical Donor, and use of TBI based conditioning necessitate close attention and surveillance.

Clinical Trial Registry: N/A

Disclosure: None

\section{P439}

Infections during neutropenia: Epidemiology, outcomes and risk factor analysis in $\mathbf{1 6 6}$ allogeneic hematopoietic stem cell transplantations in one year at one center

\section{Markus Samek ${ }^{1}$, Laura Berneking ${ }^{1,2}$, Claudia Langebrake ${ }^{1}$, Christine Wolschke ${ }^{1}$, Francis Ayuk ${ }^{1}$, Nicolaus Kröger ${ }^{1}$, Maximilian Christopeit ${ }^{1}$}

${ }^{1}$ University Medical Center Hamburg-Eppendorf, Hamburg, Germany, ${ }^{2}$ University Medical Center Hamburg-Eppendorf, Institute of Medical Microbiology, Hamburg, Germany,

Background: During allogeneic hematopoietic stem cell transplantation (allo-SCT), infections significantly contribute to morbidity and mortality. We performed a prospective analysis of allo-SCT in 2015 in order to assess epidemiology, risk factors for and outcome of infections during neutropenia.

Methods: Data were recorded prospectively using a predefined questionnaire.

Results: In 2015, 163 consecutive patients, $37.4 \%$ female, median age 59 (range 18-79) years received 166
allo-SCT. Median duration of leukopenia $<1 \mathrm{Gpt} / \mathrm{L}$ was 14.5 days (range 4-43 days). FUO occurred in 118/166 patients $(71.1 \%)$. Fever preceded CRP increase $(20.5 \%)$, CRP was increased before fever $(36.1 \%)$, or co-occurred $(26.5 \%)$. FUO in neutropenia (117 patients) showed a median duration of 2 (range 1-11) days. Severe sepsis developed in 95, septic shock in 26 patients. Blood cultures (BC) were drawn on a median of 4 (range 1-38) days. In 34 patients at least $1 \mathrm{BC}$ became positive. 23/7/2/2 patients had $1 / 2 / 3 / 4$ positive BC. For $7 / 34$ patients with positive $B C$, the same species grew >1x: 3x Staphylococcus epidermidis, $2 \mathrm{x}$ Candida parapsilosis, $1 \mathrm{x}$ each Cryptococcus neoformans and Pseudomonas aeruginosa. During hospital stay, 94 patients received $\geq 1 \mathrm{tCT}$. Of these, $34 / 35 / 15 / 5 / 1 / 1 / 3$ patients had $1 / 2 / 3 / 4 / 5 / 6 / 7$ scans. In 64/94 patients, $\geq 1$ tCT showed pulmonary infiltrates, with 'possible'/'probable' IFI in $38 / 11$ patients. $89 \mathrm{BAL}$ were performed for 51 patients. 50 patients had $\geq 1$ BAL result positive. The top 5 species discovered were Coagulase-negative staphylococci (31x), Yeast (27x), Enterococcus species (26x), Aspergillus species $(14 \mathrm{x})$ and Streptococcus viridans (10x). Cytomegalovirus was seen $5 x .121 / 27 / 10 / 4 / / 3 / 1$ patients received 1/2/ 3/4/6/9 CVC, consecutively. $1^{\text {st }}$ CVC lasted $25.6 \pm 8.5$ days, $2^{\text {nd }} \mathrm{CVC} 13.3 \pm 6.2$ days. In 86 patients, $\geq 1 \mathrm{CVC}$ was analyzed for microbial growth, $10 \mathrm{CVC}$ showed growth, 5/ 10 CVC Staphylococcus epidermidis, 2 Candida parapsilosis, 1 each Candida glabrata, Enterococcus faecalis and Enterococcus faecium. Excluding mucositis, 84 patients (50.6\%) were found to have a documented infection by diagnostic investigation. All but 13 patients received antibiotic therapy according to the standard operating procedures of the department. 129/75/42/20 patients received $1^{\text {st }} / 2^{\text {nd }} / 3^{\text {rd }} / 4^{\text {th }}$ line empiric antibiotic therapy. Discharge was possible for 145 patients. Reasons for death were infection in 19 patients, other causes in 2 patients. Cumulative incidence of death by infection after one year was $16.6 \%$ (95\%CI 11.3-22.7\%). FUO in neutropenia was influenced by duration of neutropenia $</ \geq 12$ days $(30.8 \%$ vs $82.7 \%$, $\mathrm{p}<.001$ ) only but not by the CD34+ content $/ \mathrm{kg}$ $\mathrm{BW}$ of the patient, CMV IgG status/ matching, type of conditioning, ECOG, age $</ \geq 59$ years, or underlying disease. Documented infection was influenced by duration of neutropenia $</ \geq 12$ days $(\mathrm{p}<.001)$, an ECOG of $0 / 1$ compared to ECOG of $2(78.6 \%$ vs $47.7 \%, \mathrm{p}=.027)$ and the CMV IgG status of the patient (positive $57.7 \%$ vs negative $40.6 \%, \mathrm{p}=.029)$. CD34+ content, conditioning or underlying disease again showed no impact.

Conclusions: Infections still contribute to morbidity and mortality after allo-SCT. The diagnostic results of an elaborate workup around an infection are poor. Further research has to focus on identifying novel diagnostic methods. Careful observance of risk factors for infections 
might help in better choosing patients at risk for severe infections and tailoring antibiotic prophylaxis.

Disclosure: Nothing to declare

\section{P440}

Toxoplasmosis disease after allogeneic stem cell transplantation: A single-centre experience over ten years

Alexandra Pedraza ${ }^{1}$, Maria Suárez-Lledó ${ }^{1}$, Gonzalo Gutiérrez-García ${ }^{1}$, Celia Cardoso ${ }^{2}$, Laura Rosiñol Dachs ${ }^{1}$, Carmen Martínez Muñoz ${ }^{1}$, Gerardo Rodriguez- Lobato ${ }^{1}$, Alexandra Martinez, ${ }^{1}$, Maria Eugenia Valls ${ }^{2}$, Montse Rovira $^{1}$, A Adan ${ }^{2}$, Francesc Fernandez-Avilés ${ }^{1}$

${ }^{1}$ Hospital Clínic, IDIBAP, Josep Carreras Leukemia Research Institute, Barcelona, Spain, ${ }^{2}$ Hospital Clínic, Barcelona, Spain,

Background: Toxoplasmosis is a rare and underestimated complication following allogeneic stem cell transplantation (allo-SCT) with an often fatal course. This is in part due to limited diagnostics relying mainly on imaging and detection of parasite DNA by PCR. We present here eleven cases of Toxoplasma disease following allo-SCT.

Methods: We retrospectively analyzed 534 consecutive adult patients who received an allo-SCT in our Bone Marrow Transplant Unit between July 2008 and July 2018. Eleven $(2 \%)$ of these patients were diagnosed of Toxoplasma disease. The main characteristics of the patients are shown in table 1. All patients, except two cord blood, have received peripheral blood stem cells. Fludarabine-based conditioning regimes were used in all patients. Only the two cord blood patients received Thymoglobulin in the conditioning. Graft-versus-host disease (GVHD) prophylaxis consisted on tacrolimus plus mycophenolate mofetil in $5(46 \%)$ patients and post-transplant cyclophosphamide followed by tacrolimus in $4(36 \%)$. Before the allo-SCT was performed the IgG/IgM Toxoplasma serology of the recipient and donor. We reviewed the absolute lymphocyte count (ALC) and $\mathrm{CD}^{+}$lymphocyte count within four weeks prior to the diagnosis of toxoplasmosis, and if the patients took effective primary prophylaxis for this parasite. Toxoplasma disease was defined as the presence of Toxoplasma infection plus clinical, radiological or pathological evidence. Toxoplasma disease was considered the main cause of death when no other major life-threatening infection or other potential fatal complication occurred immediately before death.

Results: Median (range) age (years) of the eleven patients diagnosed with Toxoplasma disease was 54 (19-69). For pancytopenia, no patient received trimethoprimsufmethoxazole (TMP-SMZ) but pentamidine for
Pneumocystis jirovecii-pneumonia (PcP) prophylaxis in 10 cases and atovaquone in one. Toxoplasma serology pretransplant was positive ( $\operatorname{IgG}+/ \operatorname{IgM}-)$ in ten of the eleven patients. All donors were seronegative (IgG-/IgM-) except two. Toxoplasmosis was diagnosed a median (range) of 85 days (35-330) post allo-STC. The clinical presentations were as cerebral-encephalitis $(n=4)$, chorioretinitis $(n=3)$, pneumonitis $(n=2)$ and disseminated toxoplasmosis $(n=2)$. One case, patient and donor seronegative pre-transplant, was presented as a primary infection in form of chorioretinitis. All three patients with chorioretinitis were diagnosed after day +100 of allo-SCT. At the time of Toxoplasma disease, 8 of $11(73 \%)$ of patients had an ALC $<500$ cells/ $\mu$ l and all of them with immunosuppressive therapy and corticosteroids for acute or chronic GVHD. We had CD4 ${ }^{+}$lymphocyte count only in four patients and in three of them was $<200$ cells $/ \mu L$. Eight of the eleven (73\%) patients died, with a median (range) of 21 days (9$65)$ since diagnosis of toxoplasmosis, and in 6 of them the Toxoplasma disease was the main cause of death.

Conclusions: In our series, the incidence of Toxoplasma disease after allo-SCT is low and is related to high mortality, in accordance with what has been reported by other groups. Positive pre-transplant serology and GVHD and its treatment were factors strongly related with toxoplasmosis. We encourage the use of TMP-SMZ instead of pentamidine for PcP-pneumonia prophylaxis in patients seropositive for Toxoplasma gondii pre-transplant.

Clinical Trial Registry: no

Disclosure: no

\section{P441}

BK virus - associated hemorrhagic cystitis after stem cell transplantation in children

Katerina Kaisari', Anna Paisiou ${ }^{1}$, Christina

Oikonomopoulou', Eleni Ioannidou', Anna

Komitopoulou $^{1}$, Ioulia Peristeri' ${ }^{1}$, George Vessalas ${ }^{1}$, Eugenios Goussetis ${ }^{1}$, Vassiliki Kitra ${ }^{1}$

${ }^{1}$ Agia Sophia Children's Hospital. Elpida Oncology Center, Athens, Greece

Background: BK virus associated hemorrhagic cystitis is a significant complication after bone marrow transplantation. Data about its epidemiology in children are scarce. We retrospectively analyzed the incidence, the severity and the risk factors that contribute to the manifestation of this complication in a pediatric population.

Methods: During a 10-year period (January 2008 - June 2018) we performed in our center 376 allogeneic transplantations, 237 for malignant hematological diseases and 139 for non-malignant. The majority of our patients 
received myeloablative conditioning regimens. Diagnostic criteria of hemorrhagic cystitis were the detection of the virus with PCR in urine samples and/or in blood samples, in combination with hematuria and lower urinary tract symptoms (dysuria, urinary frequency, urgency, suprapubic pain) that couldn't be attributed to any other reason. We defined the hemorrhagic cystitis as severe when one of the following factors was present: formation of clots and continuous bladder irrigation, obstructive uropathy with creatinine elevation or need for urological intervention.

Results: A total of 376 patients with median age 7,2 years $(0,5-20)$ were studied. 37 children $(10 \%, 95 \%$ CI, 7,2-13,3) manifested BK virus associated hemorrhagic cystitis with median age 12,9 years $(5,1-18,5)$. Onset of cystitis occurred at a median time of 27 days (day +-day +52 ) after transplantation. In 17 children cystitis was severe. The median duration of symptoms was 27 days (8-200). The median time of hospitalization for children with severe cystitis was 70 days (29-242) whereas for those who didn't manifest cystitis was 45 (25-184). In 25 of the 37 patients we examined the presence of the virus not only in urine but also in blood samples. In 11 of them the test was positive and almost half of them (6) manifested severe cystitis. The risk factors that were examined were age, administration of antithymocyte globulin, type of disease, graft source, type of donor and the presence of acute Graft versus Host Disease (aGvHD). In multivariable analysis, independent risk factors for the manifestation of hemorrhagic cystitis were age $>6$ years old (HR: 8,46, 95\% CI, 2,86-25, p< 0,001 ), transplantation for malignant disease (HR: 2,26, 95\% CI, 0,97-5,27, $\mathrm{P}=0,05)$ and the presence of aGvHD (HR: $4,04,95 \% \mathrm{CI}, 2,01-8,1, \mathrm{P}<0,001$ ). The overall survival of children with hemorrhagic cystitis was $46,3 \%$ vs $69,3 \%$ of those who didn't manifest this complication, but in multivariable analysis for survival cystitis wasn't a statistically significant risk factor.

Conclusions: According to our results, stem cell transplantation in children $>6$ years old who suffer from a malignant disease and the presence of aGvHD consist independent risk factors for the manifestation of BK virus associated hemorrhagic cystitis. The identification of the risk factors of this serious complication will contribute to better management of transplanted patients. Further research through prospective trials can contribute to the better understanding of the pathophysiology of hemorrhagic cystitis and to the establishment of appropriate diagnostic and therapeutic guidelines.

Disclosure: Nothing to declare

\section{P442}

Impact of natural killer cell reconstitution on outcomes in patients with early cytomegalovirus reactivation after allogeneic hematopoietic stem cell transplantation
Heiwa Kanamori', Taiki Andol, Yasufumi Ishiyama ${ }^{1}$, Takayoshi Tachibana ${ }^{\text {, Masatsugu Tanaka }}$, Hideaki Nakajima $^{2}$

${ }^{1}$ Kanagawa Cancer Center, Yokohama, Japan, ${ }^{2}$ Yokohama City University School of Medicine, Yokohama, Japan

Background: Cytomegalovirus (CMV) reactivation influences survival after allogeneic hematopoietic stem cell transplantation (SCT) and induces natural killer (NK) cell expansion. We evaluated NK cell reconstitution and clinical outcomes following early CMV reactivation after SCT.

Methods: Lymphocyte subsets were measured by flow cytometry on day 100 in patients with hematologic malignancies undergoing SCT between January 2009 and December 2017 at Kanagawa Cancer Center, excluding patients with graft failure or death within 100 days. CMV reactivation was defined as initiation of preemptive CMV therapy following pp65 antigenemia surveillance.

Results: The subjects were 152 males and 94 females with a median age of 51 years (range: 18-69 years). The median follow-up period for survivors was 3.2 years (range: 0.8-9.6 years). There were 128 patients with acute myeloid leukemia, 64 with acute lymphoblastic leukemia, 32 with myelodysplastic syndromes, and 22 with other diseases. At transplantation, 166 patients were standard risk and 80 were high risk. Myeloablative conditioning and reduced-intensity conditioning were employed in 99 and 147 patients, respectively. Bone marrow transplantation, peripheral blood stem cell transplantation, and cord blood transplantation was performed in 98, 36, and 112 patients, respectively. CMV reactivation occurred in 141 patients $(57 \%)$ at a median of 45 days (range: 15-93 days) after SCT. Grade IIIV acute GVHD and chronic GVHD affected 89 patients $(36 \%)$ and 80 patients (33\%), respectively. Among all patients, 5-year overall survival (OS), cumulative nonrelapse mortality (NRM), and cumulative relapse (CIR) rates were $66 \%, 10 \%$, and $33 \%$, respectively. In patients without CMV reactivation (CMVR-) versus patients with CMV reactivation (CMVR+), 5-year OS, NRM, and CIR were $79 \%$ vs. $55 \%$ ( $<<0.001$ ), $3 \%$ vs. $16 \%$ ( $\mathrm{p}=0.005$ ), and $27 \%$ vs. $38 \%(\mathrm{p}=0.142)$, respectively. Among all patients, the median level of CD3-CD56+ cells, CD16 +CD57- cells, and CD16+CD57+ cells on day 100 was

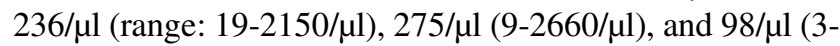
$1767 / \mu 1)$, respectively. NK cell subsets showed no significant differences between CMVR- and CMVR + patients. When patients were divided into low and high groups at the median level of each NK cell subset, CMVR + patients with high CD3-CD56+, CD16+CD57-, or CD16+CD57+ cells showed significantly better 5 -year OS than those with low cells $(72 \%$ vs. $35 \%, \mathrm{p}<0.001 ; 69 \%$ vs. $40 \%, \mathrm{p}<0.001$; $66 \%$ vs. $45 \%, \mathrm{p}=0.001$, respectively). High CD3-CD56+ 
cells were significantly associated with lower NRM (7\% vs. $26 \%, \mathrm{p}=0.002$ ), while high CD16+CD57- cells were significantly associated with lower CIR (26\% vs. $50 \%, \mathrm{p}=$ 0.007). Multivariate analysis confirmed these NK cell subsets as prognostic factors in CMVR+ patients.

Conclusions: NK cell reconstitution may contribute to improved transplantation outcomes in subgroups of CMVR + patients.

Disclosure: Nothing to declare

\section{P443}

Pharmacokinetic-pharmacodynamic analyses of dose selection for rezafungin prophylaxis against invasive fungal infections in blood and marrow transplantation

Janice Brown', Elizabeth A. Lakota', Shawn Flanagan', Taylor Sandison ${ }^{3}$, Voon Ong ${ }^{3}$, Christopher M. Rubino ${ }^{2}$

${ }^{1}$ Stanford University Medical Center, Stanford, CA, United States, ${ }^{2}$ Institute for Clinical Pharmacodynamics, Schenectady, NY, United States, ${ }^{3}$ Cidara Therapeutics, Inc., San Diego, CA, United States

Background: Rezafungin (RZF) is a novel echinocandin in development for prevention of invasive fungal infections caused by Candida, Aspergillus, and Pneumocystis spp. in patients at high risk of infection. RZF has demonstrated in vivo prophylaxis efficacy and low risk of drug-drug interactions. Furthermore, the stability and PK profile of RZF allow for once-weekly dosing.

$\mathrm{RZF}$ is also in development for treatment of candidemia and invasive candidiasis using a dosing regimen of RZF $400 \mathrm{mg}$ followed by $200 \mathrm{mg}$ once-weekly, which achieved $>90 \%$ target attainment against Candida. While lower doses might be useful to prevent Candida and Pneumocystis, invasive aspergillosis is a different challenge.

We evaluated RZF dosing for prophylaxis against Aspergillus fumigatus in blood and marrow transplant (BMT) patients using PK/PD simulations of the treatment dosing regimen.

Methods: A previous population PK model was refined using data from Phase 1 and Phase 2 trials of IV RZF (NONMEM Vers 7.2). Stepwise forward selection $(\alpha=$ $0.01)$ and backward elimination $(\alpha=0.001)$ were used to assess for relationships between interindividual PK variability and covariates, such as age, sex, BSA, albumin, liver and renal function markers, and infection status. The final model was validated by comparing model-based predictions to observed data.

The model and demographic data from 100 BMT recipients at Stanford Medical Center were used for Monte Carlo simulation $(n=2,000)$ of expected RZF concentrationtime profiles in BMT patients receiving IV RZF $400 \mathrm{mg}$ on
Week 1 followed by $200 \mathrm{mg}$ weekly x 11 . Of the 100 patients included in the demographic dataset, 39 were female (mean values at baseline: age, 58 years [19-75 years]; weight, $69.8 \mathrm{~kg}$ [42-123 kg]). The median (range) $\mathrm{BSA}$ in the demographic dataset was $1.84 \mathrm{~m}^{2}$ (1.27-2.61), and albumin was $3.35 \mathrm{~g} / \mathrm{dL}(1.8-4.43 \mathrm{~g} / \mathrm{dL})$. Free-drug concentration-time profiles were evaluated $(97.4 \%$ human protein-binding) relative to the $A$. fumigatus minimal effective concentration required to inhibit $100 \%$ of isolates tested $\left(\right.$ MEC $_{100}$; JMI 2014-2017 SENTRY international surveillance data).

Results: The population PK model was a linear, 4compartment model with zero order IV input. Albumin, sex, infection status, and body surface area were statistically significant predictors of interindividual variability; clinical significance of these factors was not determined. The model provided precise, unbiased fits to the observed data ( $\mathrm{r}^{2}=0.993$ observed vs individual-predicted concentrations).

RZF plasma free-drug concentrations at weeks 1, 2, and 12 were above the $A$. fumigatus $\mathrm{MEC}_{100}(0.03 \mathrm{mg} / \mathrm{L})$ for the entire dosing interval in $98.0 \%, 92.0 \%$, and $90.2 \%$ of simulated patients, respectively, and in $\geq 99.8 \%$ for all 12 weeks based on the $\operatorname{MEC}_{90}(0.015 \mathrm{mg} / \mathrm{L})$.

Conclusions: These data modelled from BMT patients support the RZF dosing regimen of $400 \mathrm{mg}$ IV followed by $200 \mathrm{mg}$ once-weekly for prophylaxis against A. fumigatus. Current antifungal prophylaxis may be limited by toxicity, DDIs, or patient factors such as mucositis. The PK of RZF and its spectrum, safety, tolerability, and lack of DDIs may address current unmet needs in IFI prophylaxis for BMT and other immunocompromised patients.

Disclosure: Janice Brown: Research funding, Cidara Therapeutics, Elizabeth Lakota: Research funding, Cidara Therapeutics, Shawn Flanagan: Employment, Cidara Therapeutics, Taylor Sandison: Employment, Cidara Therapeutics, Voon Ong: Employment, Cidara Therapeutics, Christopher Rubino: Research funding, Cidara Therapeutics

\section{P444}

Is fungal prophylaxis necessary in non myeloablative peripheral blood stem cell allogeneic transplantation in the pre-engrafment period?

Julien Vaidie ${ }^{1}$, Jean-Baptiste Woillard ${ }^{1}$, Stéphane Girault $^{1}$, Marie-Laure Dardé ${ }^{1}$, Arnaud Jaccard ${ }^{1}$, Daniel Ajzenberg $^{I}$, Bernard Bouteille ${ }^{I}$, Pascal Turlure ${ }^{I}$

${ }^{1}$ CHU Limoges, Limoges, France

Background: Non myeloablative peripheral blood stem cell transplantation (PBSC), by limiting toxicity, can be proposed to elderly patients or patients with comorbidities. However, fungal infections remain a key issue that can 
negatively impact outcome, and increase duration and cost of hospitalization. Systematic fungal prophylaxis have demonstrated benefits in outcome in the context of myeloablative conditioning but are not currently in reduced intensity conditioning allograft with PBSC. Fluconazole prophylaxis is currently recommended in this situation (ECIL).

Methods: Primary objective of this retrospective study was to evaluate fungal infection incidence after allograft procedure in patients who received a non myeloablative allograft with PBSC in Limoges University Hospital between june 2009 and june 2018. Patients received Fludarabine $30 \mathrm{mg} / \mathrm{m} 2 /$ day between D-6 and D-2 before allograft and Busulfan $3.2 \mathrm{mg} / \mathrm{kg} / \mathrm{day}$ at D-4 and D-3. GvH prophylaxis consisted in rabbit anti-lymphocyte serum at the dose of $2.5 \mathrm{mg} / \mathrm{kg}$ at D-2 and D-1, and ciclosporin at the beginning dose of $3 \mathrm{mg} / \mathrm{kg}$ per os twice a day. Mycophenolate mofetil was adding for patients with HLA-matched or mismatched unrelated donors.

Patients did not systematically receive antifungal prophylaxis during the neutropenic pre-engraftment period. When patients had fever during more than 72 hours, an empirical fungal treatment (caspofungine) was added to empirical antibiotics. As soon as neutropenic recovered and in the case of apyrexia without microbiologic documentation, antimicrobial treatments were stopped while in the case of microbiologic documentation, treatments were adjusted to germ in term of dosing and time of administration following recommendations.

However, some patients received antifungal azole prophylaxis during the neutropenic pre-engraftment period in case of history of previous invasive aspergillosis (IA), or a nasal colonization by Aspergillus.

In post-engraftment period, posaconasole prophylaxis was administered for patients with systemic corticotherapy for acute graft-versus-host disease.

Results: 91 patients were evaluated (median [min-max] age of 60 [33-71] years). $20 \%$ of patients received an HLAidentical related donor, $69 \%$ an HLA-matched related donor and $11 \%$ an HLA-mismatched unrelated donor. The five years overall survival and survival without relapse or GvHD were $70 \%$ IC95 [59\%-81\%] and 62\% respectively IC 95 [52\%-73\%]. The median time for neutrophil recovery was 15 days. 79 patients did not receive prophylaxis and only 12 patients received systematic fungal azole prophylaxis in the pre-engraftment period. Two patients received an empirical treatment by caspofungine. Only 1 IFI was documented during the neutropenic period : candida krusei in blood culture. In the post engraftment period, 37 patients with acute GVHD treated by corticotherapy received an antifungal prophylaxis by posaconazole and only 1 patient had a probable IA at day 100 despite prophylaxis by posaconasole.
Conclusions: Except for patients with previous history of IFI, our results provide additional arguments against systematic fungal prophylaxis after reduced intensity conditioning with PBSC allogenic transplantation in the pre-engraftment period with a very low incidence of invasive fungal infections. In post-engrafment period, posaconazole prophylaxis is required for patient with GVHD treated by corticotherapy.

Disclosure: No Disclosure

\section{P445}

Determination of letermovir serum concentrations in daily clinical practice using a new high-performance liquid chromatography method

\section{Nora Isberner', Diana Schirmer ${ }^{1}$, Sabrina Kraus ${ }^{1}$, Götz Ulrich Grigoleit ${ }^{l}$, Hartwig H. F. Klinker ${ }^{I}$}

${ }^{1}$ University of Würzburg Medical Center, Würzburg, Germany

Background: Letermovir is a new antiviral drug approved for prophylaxis of cytomegalovirus (CMV) infection and disease in $\mathrm{CMV}$-positive adult allogeneic hematopoietic stem cell transplant recipients. Since Letermovir is a substrate of the hepatic uptake transporter OATP1B1/3, coadministration of OATP inhibitors (e.g. cyclosporine) leads to a clinically relevant increase in drug exposure. Interindividual genetic differences may have an additional effect. By contrast, changes in plasma concentration due to metabolism play a minor role. Knowledge of Letermovir exposure in daily clinical practice is scarce. We established a high-performance liquid chromatography (HPLC) assay for determination of Letermovir concentration and evaluated first Letermovir serum concentrations in a routine clinical setting.

Methods: A simple, rapid and sensitive method using HPLC with a diode-array detector (DAD) was developed and validated for the quantification of Letermovir in human serum using Sorafenib as internal standard. After pretreating serum samples by liquid-liquid extraction with tert-Butyl methyl ether, separation was achieved on a X-Terra RP-18 column (Dimension $150 \times 2.1 \mathrm{~mm}, 5 \mu \mathrm{m}$ ) at $30^{\circ} \mathrm{C}$ using gradient elution with a mobile phase of $20 \mathrm{mM}$ ammonium bicarbonate $\mathrm{pH} 7.8$ (mobile phase solvent-A) and acetonitrile: $20 \mathrm{mM}$ ammonium bicarbonate $\mathrm{pH} 9.2$ (mobile phase solvent-B). Samples were eluted at a flow rate of $0.3 \mathrm{ml} /$ min throughout the 20-minute run. UV wavelength mode was used, detection was at $260 \mathrm{~nm}$.

Results: The calibration curve was linear $(r>0.99)$ in a concentration range of $25-5000 \mathrm{ng} / \mathrm{ml}$ for Letermovir. The HPLC assay established for Letermovir determination showed a high rate of accuracy and precision with an 
intraday variability of -9.21 to $12 \%$ (accuracy) and 0.31 to $3.75 \%$ (precision) and an interday variability of -2.6 to 6.9 $\%$ (accuracy) and 3.74 to $7.41 \%$ (precision), respectively.

16 Letermovir serum concentrations of 9 patients ( 8 male / 1 female, mean age 66.3 years) were determined in daily clinical practice. The mean concentration was $4752 \mathrm{ng} / \mathrm{ml}$ (median $4163 \mathrm{ng} / \mathrm{ml}$, standard deviation $3925 \mathrm{ng} / \mathrm{ml}$, range $89-11452 \mathrm{ng} / \mathrm{ml}$ ).

Conclusions: The newly developed HPLC method is useful for the determination of Letermovir concentrations. Patient samples analyzed in a routine clinical setting demonstrated considerable interindividual variability. All measured concentrations were above the EC50 of Letermovir. Monitoring the concentration of Letermovir could help to prevent over- or underexposure, especially in patients with polypharmacy which is frequent in allogeneic hematopoietic stem cell transplant recipients.

Disclosure: Nora Isberner: nothing to declare; Diana Schirmer: nothing to declare; Sabrina Kraus: travel grant: Jazz Pharmaceuticals; Götz Ulrich Grigoleit: travel grant: medac GmbH; Hartwig H. F. Klinker: Grants: Abbvie, BMS, Gilead, Janssen, MSD; Lecture fees / advisory boards: AbbVie, BMS, Gilead, Hexal, Janssen, MSD

\section{P446}

\section{Impact of cmv replication in overall survival in} allogeneic HSCT recipients

\section{Elena Sola Aparicio ${ }^{1}$, Laura Cardeñoso ${ }^{1}$, Patricia Lopez Pereira $^{1}$, Patricia Cadahía Fernandez ${ }^{2}$, Silvia Granja Torrecillas $^{1}$, Belen Fernandez Caso ${ }^{1}$, Rafael de la Camara $^{1}$}

${ }^{1}$ Hospital Universitario de la Princesa, Madrid, Spain, ${ }^{2}$ Hospital de Pontevedra, Pontevedra, Spain

Background: The use of preemptive strategy (PET) has lowered the incidence of CMV disease in allo-SCT to 5$10 \%$. Nonetheless the use of this strategy implies that more than $50 \%$ of seropositive patients will replicate CMV. Several studies have shown that CMV replication is detrimental for patient survival although the viral load related to this bad outcome variates among studies.

Objective: To analyse thel impact of CMV replication in overall survival (OS) in allo-HCT patients.

Methods: To analyse the impact of CMV replication in OS we perform a unicentric, retrospective study on 117 consecutive first allo-HSCT patients transplanted between Jan-2015 and Oct-2018 with a median follow-up of 398 days (5-1266). All patients were monitored post-HCT with real time PCR Cobas-Taqman ${ }^{\circledR} /$ Cobas $6800^{\circledR}$ (rtPCR) in plasma. The cut-off for inception of PET was $150 \mathrm{IU} / \mathrm{ml}$. CMV mutations (UL54/UL97gene), were studied in plasma samples by Sanger sequencing, median CMV Viral load $3130 \mathrm{IU} / \mathrm{ml}(565-23900)$.

Results: Patients (117): women/men (49\%/51\%), median age was 55 years (range 18-71). 101 identical allogeneic SCTs (86\%), 16 haploidentical SCTs (14\%). Donors were related in 60 cases $(51 \%)$ and 57 (49\%) unrelated. Progenitors source was $98 \%$ peripheral blood and $2 \%$ bone marrow. CMV status was $(\mathrm{D}+/ \mathrm{R}+)$ in $51 \%(\mathrm{n}=60)$, $(\mathrm{D}+/ \mathrm{R}-$ ) in $9 \%(n=11),(D-/ R+)$ in $26 \%(n=31)$ and $(\mathrm{D}-/ \mathrm{R}-)$ in $11 \%$ $(\mathrm{n}=13)$, unknown 2 cases.

Positive PCRs were detected in 63 patients: one episode in 41 (65\%); 2 episodes in $15(24 \%)$ and 3 to 5 episodes in 7 patients $(11 \%)$. Fifty-five patients $(87 \%)$ received preemptive therapy. Fourteen episodes (25\%) were refractory/ probable refractory CMV infections (according to the criteria of Chemaly R. CID 2018). A resistant mutation (UL54 gene) was detected in one patient with refractory infection Patients that developed CMV infection had an inferior non-significant OS at 3 years $(61,3 \%$ VS $76,8 \%$ Log-rank P 0,346). Those patients that received PET for CMV had a significant inferior OS compared with those that replicate CMV but didn't receive preemptive therapy (55,6\% vs $100 \%, \log$ rank $\mathrm{p}=0.04)$. OS of patients that received PET was inferior compared with those without PET (with or without infection) $(55,6 \%$ VS $79,9 \%$, Logrank P 0,05). No difference in survival was found for those patients treated pre-emptively that were refractory vs no refractory (53\% vs $57,1 \%$, Log-rank P 0,51$)$.

Conclusions: Patients that received preemptive therapy had a significant inferior overall survival compared with those that didn't replicate and those that replicate CMV but didn't receive preemptive therapy. This reinforce the relevance of prophylactic strategies for CMV with drugs with good safety profile like letermovir that in a randomised trial proved to decrease the need for preemptive therapy.

Disclosure: Rafael, de la Camara: has received grants from Astellas, Gilead, Janssen, Merck, Novartis and Pfizer

\section{P447}

Clinical evaluation of stenotrophomonas maltophilia infection in allogeneic hematopoietic stem cell transplant recipients - retrospective single-center data analysis

Agnieszka Tomaszewska ${ }^{1,2}$, Barbara NasitowskaAdamska ${ }^{I}$, Magdalena Tormanowska ${ }^{I}$, Anna Kuziak ${ }^{I}$, Ewa Mik ${ }^{l}$, Kazimierz Hataburda ${ }^{I}$

${ }^{1}$ Institute of Hematology and Transfusion Medicine, Warsaw, Poland, ${ }^{2}$ Medical University of Warsaw, Warsaw, Poland

Background: Stenotrophomonas maltophilia (S. maltophilia) is an opportunistic multi-antibiotic resistant Gram- 
negative bacillus that causes severe infections associated with high morbidity and mortality in immunocompromised patients. The aim of our study was to determine incidence, characteristics and outcome of S. maltophilia infection in patients (pts) who underwent allogeneic hematopoietic stem cell transplantations (allo-HSCT) in Institute of Hematology and Transfusion Medicine between October 2010 and November 2018.

Methods: We retrospectively evaluated incidence, clinical features and outcome of S. maltophilia infections in 338 consecutive patients with median age-42 years (range 1971), who underwent allo-HSCT from unrelated donors - 238 (70.4\%), matched sibling donors - $87(25.7 \%)$ and haploidentical donors - $13(3.9 \%)$ in our center. S. maltophilia was detected by culture-based microbiological tests. Invasive infection was defined by isolation $\mathrm{S}$. maltophilia from cultures in the presence of both clinical symptoms and signs of infection - blood stream infection (BSI), pneumonia with or without pulmonary haemorrhage. The only colonization status was defined as S. maltophilia culture-positive samples in the absence of infection symptoms. In vitro susceptibility tests to antibiotics were performed.

Results: 21 pts $(6.2 \%)$ with median age-46 years (range 19-66) with S.maltophilia culture positive samples were identified. 19 (90.5\%) underwent allo-HSCT from unrelated donors, 1-from matched sibling donor and 1-from haploidentical donor. Among them BSI developed in 7 pts $(33.3 \%)$, pneumonia in 4 pts $(19 \%)$-with fulminant and fatal pulmonary hemorrhage in 3 pts $(14.3 \%)$. All patients with pneumonia demonstrated BSI. Positive sputum cultures were detected in 6 pts, in 4 pts hemoptysis was observed. The rest of isolated strains were identified as colonization (throat - in 3 pts, stool - in 9 pts). All 7 patients with invasive S. maltophilia infection before pathogen identification demonstrated persistent fever despite of the use of broadspectrum antibiotics (carbapenems, glycopeptides, aminoglycosides, colistin), prophylactic antifungals and antivirals. All of them received fluoroquinolone (ciprofloxacin) as a standard antibacterial prophylaxis before neutropenic fever occurred. All 3 patients (100\%) with BSI, pneumonia and pulmonary hemorrhage died before engraftment (ANC - $0.0 \mathrm{G} / \mathrm{L}$ ) - 2 of them during 4872 hours from the onset of a positive blood culture for $\mathrm{S}$. maltophilia. The C-reactive protein (CRP) concentration before identification of $\mathrm{S}$. maltophilia invasive infection was $>26 x-69 x$ upper normal limits (UNL). Susceptibility to antibiotics of isolated strains from blood and sputum was respectively: $86 \%$ and $100 \%$ for ceftazidime, $100 \%$ and $100 \%$ for trimethoprim-sulfamethoxazole, $64 \%$ and $50 \%$ for levofloxacin; while $56 \%$ and $50 \%$ strains were resistant to ciprofloxacin. 1-year overal survival (OS) and 2-y OS for this group was $52.4 \%$ and $47.6 \%$ respectively compared with $77.2 \%$ and $68.3 \%$ for group without $\mathrm{S}$. maltophilia infection.

Conclusions: S. maltophilia invasive infections are associated with high morbidity and mortality in alloHSCT recipients especially in the period from conditioning therapy to engraftment. An exposure to broad-spectrum antibiotics in the treatment of neutropenic fever or confirmed bacteremia of other etiology is one of risk factors of breakthrough S. maltophilia infections. Empiric therapy against $\mathrm{S}$. maltophilia in selected patients in risk of such infection before pathogen identification may be lifesaving procedure.

Disclosure: Nothing to declare.

\section{P448}

Role of CMV reactivation following allogeneic stem cell transplantation in preventing relapses in patients with acute myeloid leukemia

Nataraj Kolar Srinivasarao ${ }^{1}$, Shilpa Prabhu ${ }^{1}$, Sharat Damodar $^{1}$, Sunil Bhat ${ }^{1}$, Shobha Badiger ${ }^{1}$, Hamza Dalal, VM Annapandian ${ }^{I}$

${ }^{1}$ Mazumdar Shaw Cancer Center, Bangalore, India

Background: Cytomegalovirus(CMV) reactivation is common in patients undergoing Allogeneic Stem Cell transplantation. It has been shown recently that CMV reactivation is associated with reduced risks of relapse in patients undergoing Allogeneic Stem Cell Transplantation for AML. However the analysis of CIBMTR data did not show any effect of CMV reactivation on relapse. With this background we conducted an analysis of patients suffering from AML who are undergoing Allo-SCT for their long term disease free survival with respect to CMV reactivation.

Methods: After obtaining permission from hospital medical records committee, we retrospectively analysed data from electronic medical records of patients undergoing Allo-SCT for AML at our center between January 2013 to August 2018. Patients who underwent Matched sibling, Matched unrelated and partially matched Allo SCT were included.

All patients underwent CMV monitoring with weekly PCR starting from the time of engraftment till D+100 following Allo SCT. Value of $\geq 1000$ copies $/ \mathrm{mcl}$ was considered as cut off for initiation of treatment in matched sibling donor transplant but in unrelated donor or partially 
matched donor transplants, $\geq 500$ copies/mcl was used as cut off for initiation of pre emptive therapy.

Results: Total of 96 patients were included in study. Median age was $28.01 \pm 17.09$ years (2-69yrs). $60(62.5 \%), 30(31.3 \%)$ and 6(6.3\%) patients underwent Matched Sibling, Haplo (Partially matched) and MUD transplantation respectively. Median follow up was 15 months(1-73months). (Table 1)

Acute GVHD (Grade2-4) was observed in 50(52.1\%) of patients.

CMV reactivation occurred in 70(72.9\%) of patients.

Overall survival at last follow up was $63.5 \%$ (61/ 96patients).

$17(17.7 \%)$ patients relapsed during follow up. Relapse free survival at at last follow up was $61.5 \%$.

$64(90.1 \%)$ of 70 patients who had CMV reactivation didn 't relapse, whereas 10(40\%) of 25 patients who didn't have CMV reactivation relapsed which was statistically strongly significant $\mathrm{P}<0.001$. (Figure 1)

Similar results were seen in recently published paper from Japanese Society For Hematopoietic Cell Transplantation (JSHCT) Transplantation-Related Complication Working Group.

Conclusions: 1. CMV reactivation following Allo SCT had beneficial effect on preventing relapse in patients with AML. 2. Probable immune activation resulting due to CMV reactivation may result in better graft versus leukemia effect preventing subsequent relapses.

Fig 1b: Effect of CMV reactivation on disease remission of AML patients after allogenic stem cell transplantation

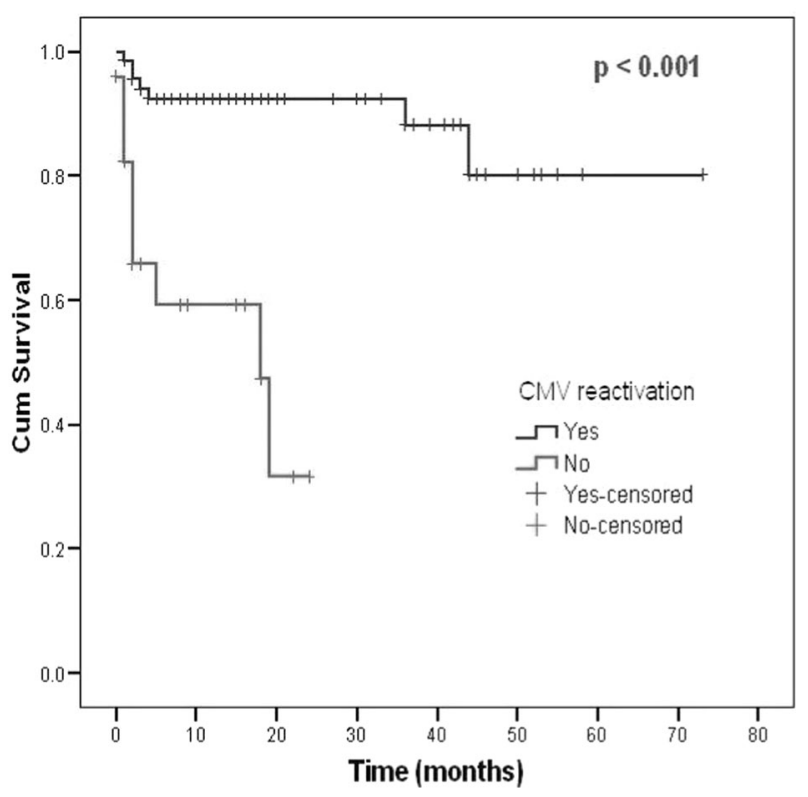

[[P448 Image] 1. Disease free survival with respect to $C M V$ reactivation. Kaplan Meir Curves]

\begin{tabular}{ll}
\hline Parameters & $\begin{array}{l}\text { N }(\%) \text { or Mean } \pm \\
\text { SD n=96 }\end{array}$ \\
\hline Gender (M / F) & $\begin{array}{l}65(66.7 \%) / 31 \\
(32.3 \%)\end{array}$ \\
Age (Years) & $28.01 \pm 17.09$ \\
HLA type Matched Sibling Donors (MSD) & $60(62.5 \%) 30(31.3 \%)$ \\
Haplo-identical Matched Unrelated & $6(6.3 \%)$ \\
Donors (MUD) & \\
ABO match Compatible Major mismatch & $59(61.5 \%) 20(20.8 \%)$ \\
Minor mismatch & $17(17.7 \%)$ \\
Acute GVHD & $50(52.1 \%)$ \\
Chronic GVHD & $9(9.4 \%)$ \\
CMV reactivation & $70(72.9 \%)$ \\
Disease status Under Remission Relapse & $79(82.3 \%) 17(17.7 \%)$ \\
Overall survival at last follow-up & $61(63.5 \%)$ \\
\hline
\end{tabular}

[[P448 Table] 1. Table 1. Baseline Demographics and outcomes]

Clinical Trial Registry: Not Applicable

Disclosure: Nothing to declare

P449

HHV-6 reactivation evaluated by digital polymerase chain reaction and its association with dynamics of CD134-positive $T$ cells after allogeneic stem cell transplantation

Hitomi Nakayama ${ }^{1}$, Rie Yamazaki ${ }^{1}$, Jun Kato ${ }^{1}$, Yuya Koda $^{I}$, Masatoshi Sakurai ${ }^{1}$, Ryohei Abe ${ }^{I}$, Chieko Sumiya $^{1}$, Kohei Shiroshita ${ }^{1}$, Shinichiro Okamoto ${ }^{1}$, Takehiko Mori ${ }^{1}$

${ }^{1}$ Keio University School of Medicine, Tokyo, Japan

Background: Human herpesvirus 6 (HHV-6) causes lifethreating central nervous system disorders such as encephalitis after allogeneic hematopoietic stem cell transplantation (HSCT). Recent studies showed that CD134, a member of the tumor necrosis factor receptor superfamily, has been implicated as a specific receptor of HHV-6B, and that its expression levels in CD4-positive $\mathrm{T}$ cells after HSCT could be related to the reactivation of HHV-6.

Real-time quantitative polymerase chain reaction analysis (qPCR) is the most commonly used method for detecting and evaluating HHV-6 reactivation after HSCT, but more sensitive detection method is required. We recently developed a new monitoring method for HHV-6 reactivation using digital PCR (dPCR) which provides high sensitivity of detecting HHV-6 DNA in clinical samples.

In this prospective study, we evaluated the relationship between HHV-6 reactivation monitored by $\mathrm{dPCR}$ and 
expression of $\mathrm{CD} 134$ on $\mathrm{CD}^{+}{ }^{+} \mathrm{T}$ cells before and after allogeneic HSCT.

Methods: Thirty-four patients who underwent allogeneic HSCT for hematological diseases at Keio University Hospital (Tokyo, Japan) between January 2017 and March 2018 were consecutively enrolled into this study. Peripheral blood samples of the patients were obtained before the conditioning (PRE), the day of transplant (Day 0), and weekly during the first month after transplantation (Days 7, 14, 21, and 28). HHV-6 viral load in plasma was quantitatively measured by dPCR. The primers and a probe of dPCR for HHV-6B were selected from immediate-early 1 (IE-1) protein transactivator region (U90). We evaluated the relationship between HHV-6 reactivation and the serial expression rates of $\mathrm{CD} 134$ in $\mathrm{CD} 4^{+} \mathrm{T}$ cells $(\mathrm{CD} 134 / \mathrm{CD} 4$ ratio) measured by flow cytometry before and after HSCT.

Results: Median age of the patients was 51.5 years. Onethird of patients received cord blood as a stem cell source. HHV-6 reactivation was detected in 23 patients (68\%) with dPCR. A comparison of CD134/CD4 ratio between the patients with and without HHV-6 reactivation after HSCT revealed that CD134/CD4 ratio was significantly higher in patients with HHV-6 reactivation than those without before conditioning (median [range], 3.8\% [0.4-25.6] vs. $1.5 \%$ [0.4-3.7], $P<0.01$ ) and at the day of transplant (Day 0, $8.9 \%$ [0.0-40.0] vs. $0.2 \%$ [0.0-4.4], $P<0.01$ ). In contrast, there was no such significant difference after transplant (Days 7 to 28). In multivariate analysis, higher CD134/CD4 ratio before conditioning (odds ratio $(\mathrm{OR})=10.5,95 \%$ confidence interval $(\mathrm{CI}): 1.3-85.1, P=0.03)$ and stem cell source from human leukocyte antigen mismatched donor (including all cord blood transplantation cases) $(\mathrm{OR}=15.4$, 95\%CI: $2.0-121.0, P=0.04$ ) remained to be significantly associated with the incidence of HHV-6 reactivation.

Conclusions: Higher CD134 expression rate in $\mathrm{CD}^{+}$ $\mathrm{T}$ cells before HSCT was associated with higher risk of HHV-6 reactivation, which could be a promising marker for predicting HHV-6 reactivation after allogeneic HSCT. Careful observation and monitoring may be needed in CD134 highly expressed patients. It is a subject of further research to clarify the role of $\mathrm{CD} 134^{+} \mathrm{CD} 4{ }^{+} \mathrm{T}$ cell in HHV6 reactivation.

Disclosure: Nothing to declare.

\section{P450}

Successful treatment of BK virus hemorrhagic cystitis after allogeneic transplantation with intravescical cidovofir

Maria Liga $^{1}$, Nikos Spyridis ${ }^{1}$, Dimitris Aggelinas ${ }^{1}$, Chara Korovila $^{I}$, Kalliopi Lekka ${ }^{I}$, Evangellia Triantafyllou ${ }^{1}$, Panagiotis Bountouris ${ }^{1}$, Markos Marangos ${ }^{1}$, Alexandros Spyridonidis $^{1}$
${ }^{1}$ University Hospital of Patras, Patras, Greece

Background: BK virus related hemorrhagic cystitis (BKV$\mathrm{HC}$ ) represents e serious complication after allogeneic stem cell transplantation (allo-HCT). There are no clearly defined treatment guidelines for the treatment of BKV-HC. The data on the effectiveness of intravescical instillation of cidovofir) (ICI) to manage severe bleeding are very limited. Aim of this study was to evaluate the efficacy of ICI, to manage grade III-IV BKV-HC.

Methods: Criteria for the administration of ICI (Vistide) were grade III-IV (clinically significant hematuria with clots) BK- related hemorrhagic cystitis after allo-HCT which showed no improvement after symptomatic therapy with hyperhydration and bladder irrigation. Cidofovir was diluted in $100 \mathrm{~mL}$ of normal saline and installed via a Foley catheter which was blocked for 1 hour. Not knowing the level of absorption of the drug we decided to give probenecid prophylaxis in all patients. ICI was repeated weekly according to severity of symptoms. Urine and plasma BKV viral loads were quantified by RQ-PCR

Results: Six patients (median 38 years, 29-51) received ICI after allo-HCT. Patients had haematological malignancies (AML 2, ALL 3, MDS 1), received busilfex-based myeloablative conditioning and a graft (PBSC 5, BM 1) from a 7/8 HLA-matched (4pts), 8/8 (1pt) or haploidentical (1pt) donor. Median time for the onset of BKV-HC after allo-HCT were 53.5 days (range 32-117). All patients were under standard cyclosporine prophylaxis and none of the patients had any signs of acute GvHD at the time of onset of HC. The median PCR-BKV viral load at the onset of BKV$\mathrm{HC}$ in urine and plasma were $5.75 \times 10^{8}$ (range $0.15 \times 10^{8}$ $59 \times 10^{8}$ ) and 141.5 (range $0-255$ ), respectively. The median maximum PCR-BKV viral load in urine and plasma were $37.5 \times 10^{8}$ (range $4.7 \times 10^{8}-450 \times 10^{8}$ ) and 1.100 (range 0400.000), respectively. Five patients had impaired renal function (median eGFR $49 \mathrm{ml} / \mathrm{min}$, range 33-53) at first ICI which was probably multifactorial. The median dose of intravesical cidofovir was $5 \mathrm{mg} / \mathrm{kg}$ (range $2.5-5 \mathrm{mg} / \mathrm{kg}$ ) and a median number of 3.5 instillations (range 2-7) were given. In $5 / 6$ cases symptoms of cystitis improved dramatically and hematuria resolved. Virological response (at least $1 \mathrm{log}$ reduction) was observed in all 5 cases. Two patients experienced relapse of hemorrhagic cystitis and were retreated with ICI which resulted in resolution of the symptoms and the hematuria. No deterioration of renal function of other systemic adverse effects were observed. After a median follow up of 190.5 days after transplantation (range 105-360), 3/6 patients are alive without cystitis symptomatology and 3 died ( 1 due to relapse and 2 due to TRM).

Conclusions: In this retrospective study we propose that local therapy of BKV-HC with ICI is safe and has high 
clinical and virological response rates. The administration of ICI after allo-HCT should be controlled in prospective randomized trials.

Disclosure: Nothing to declare

\section{P451}

\section{Strong impact of pre-HSCT recipient/donor} cytomegalovirus (CMV) serostatus on survival of alloHSCT

Lourdes Aguirre ${ }^{1}$, Clara Lombardi, Laida Ondarra ${ }^{1}$, Mila Montes ${ }^{1}$, Teresa Artola ${ }^{1}$, José Ferreiro ${ }^{1}$, María Araiz $^{1}$, Brígida Esteban ${ }^{1}$, M. José Pizarro ${ }^{1}$, Carlos Vallejo $^{1}$

${ }^{1}$ University Hospital Donostia, San Sebastián, Spain

Background: Since CMV-preemptive therapy approach was implemented, CMV disease frequency is very low. However, CMV reactivation and the need of using nephrotoxic plus/less myelotoxic drugs is very frequent. In addition to the toxicity of the medications to avoid CMV disease, other potential adverse effects of CMV have been mentioned in medical literature. In this study, we wanted to estimate how recipient/donor serologic status influences the outcome of allo-HSCT in our most recent series of patients.

Methods: the population analyzed for this report is the all 224 patients who underwent allo-HSCT during the 4-year period from October 2014 September 2018 in our Unit. Median age at transplant was 52 years (range: 7-69). One hundred and thirty were male $(58 \%)$ and 94 were female (42\%). Baseline diseases were: 83 AML, 51 LPD, 29 ALL, 29 MDS, $14 \mathrm{MPD}, 12 \mathrm{MM}$, and $6 \mathrm{BMF}$. Donor was unrelated in 120 transplants $(54,5 \%)$ and was family in 104 $(45,5 \%)$ (including 34 haplo-identical). Conditioning regimen was RIC in 121 procedures (54\%) and intensive in 103 (46\%). Stem cell source was PB in $210(93,7 \%)$ and BM in 14 cases (6,3\%). Median follow-up was 23 months (range: $3-50)$. Patient's and donor's CMV IgG were positive in 188 $(82,6 \%)$ and $92(58,5 \%)$, respectively. Recipient/donor serology was +/- (risk group 1) in $62(27,7 \%),+/+$ (risk group 2) in $126(56,3 \%),-/+$ (risk group 3$)$ in $6(2,6 \%)$ y -/(risk group 4) in $30(13,4 \%)$.

Results: two pts underwent a second transplant before day +100 due to graft failure. Overall mortalities $(\mathrm{OM})$ at days +100 and +365 of the rest of the series (222 pts) are shown in table. The highest risk group (recipient CMV + I donor CMV -) exhibited more than double $\mathrm{OM}$ at day +100 and more than four times $\mathrm{OM}$ at day +365 , when compared with pts at lowest risk (recipient CMV -). Those striking differences were mainly due to NRM. OM for risk group II (recipient $\mathrm{CMV}+/$ donor CMV +) was intermediate.
Conclusions: in our studied population, mainly adult patients, the combination of CMV-seropositive patient with a CMV-seronegative donor had a very clear adverse impact on HSCT outcome. As a result, we considered that the election of a CMV-positive donor for a CMV-positive patient continues to be strongly advisable, whenever is possible. On the other hand, once Letermovir has proved to be efficient and well-tolerated and has been licensed for prophylaxis of CMV in high risk recipients, this approach appears to be very attractive to try to avoid the adverse impact of recipient CMV-seropositivity, particularly when finally chosen donor is CMV negative.

Disclosure: Nothing to declare

\section{P452}

An active surveillance and an early and individualized management is critical to avoid mortality from respiratory viral infections in allo-HSCT recipients

Maria Jose Pizarro ${ }^{I}$, Maria Lourdes Aguirre ${ }^{I}$, Maider Valencia $^{1}$, Maria Carmen Tejado ${ }^{1}$, Maider Rojo ${ }^{1}$, Maria Del Puy Aparicio ${ }^{1}$, Itziar Otero ${ }^{1}$, Anne Arambarri', Milagros Montes ${ }^{1}$, Juan Carlos Vallejo Llamas ${ }^{1}$

${ }^{1}$ University Hospital Donostia, San Sebastian, Spain

Background: Respiratory viral infections (RVIs) are frequent among the general population. In transplant recipients, RVIs are known to cause an important morbidity and potential mortality. For this reason and several others, as the need of preventing other pts from contagious or avoiding misdiagnosis with other infections processes, a high index of suspicion of VRIs is necessary. During the last few years, we have implemented an active and systematic surveillance policy orientedto early detection and management of RVIs in the HSCT recipients.

Methods: The population analyzed for this report is the 175 patients who underwent allo-HSCT from January 2015 through March 2018 in our Unit. Median age at transplant was 51 years (range: 7-69). One hundred and four were male (59.4\%) and 71 were female $(40,6 \%)$. Baseline diseases were: 64 AML, 38 LPD, 23 ALL, 19 MDS, 15 MPD, 10 MM, and 6 BMF. Donor was unrelated in 92 transplants $(52.6 \%)$ and was family in 83 (47.4\%) (including 29 haplo-identical). Conditioning regimen was reduced in 91 procedures $(52 \%)$ and intensive in $84(48 \%)$.Stem cell source was PB in 163 (93.1\%) and $\mathrm{BM}$ in 12 pts (6.9\%).Median follow-up was 27 months (range: 8-46); at the close of the analysis, majority of the series $(89.1 \%)$ had a follow-up superior to one year from HSCT. A throat swab(TS) was taken from every patient with any, even minor, respiratory symptoms. The respiratorysamples were tested whith a complete PCR panel of human respiratory viruses: rhinovirus (RV), influenza $A$ and $B$ virus 
(IV-A, IV-B), parainfluenza virus (PIVs 1-4), respiratory syncytial virus (RSV), metapneumovirus (MPV), coronavirus $(\mathrm{CoV})$, adenovirus (AdV), and bocavirus (BoV).

Results: Day +100 overall mortality of the series was $8,6 \%$. Day +365 overall mortality was $25,1 \%$ (13,7\% nonrelapse mortality -NRM-, and $11,4 \%$ progression/relapse mortality). Causes of NRM reflected in table 1 . No patients died due to RVIs. From $1^{\text {st } J u l y ~} 2016$ through $30^{\text {th }}$ June 2018 (a 24-month period), $581 \mathrm{TS}$ samples were obtained from 129 pts $(73,7 \%)$. The median number of samples/patient was 3 (range: 1-17).A total of 162 (1-8) RVIs episodes were diagnosed in 87 pts $(49,7 \%)$. The median presentation of the first RVI was at the day +226 (3-924) post-HSCT. The viral distribution was: $47 \mathrm{RV}(26.6 \%), 36 \mathrm{IV}$ (20.3\%), 33 PIV (18.6\%), $28 \mathrm{RSV}$ (15.8\%), $12 \mathrm{MPV}$ (6.8\%), $11 \mathrm{CoV}$ $(6.2 \%), 9 \mathrm{AdV}(5.1 \%)$, and $1 \mathrm{BoV}(0.6 \%)$.There were 13 mixed (two or more viruses) RVI episodes. The temporary distribution of VRI episodes is shown in figure 1.

Conclusions: 1) Symptomatic infections due to respiratory viruses are very frequent among the allo-HSCT recipients. 2) A high level of suspicion, as well as an early and systematic screening and management policy, are critical to avoid potential attributable mortality and the nosocomial spread of RVIs among the transplant recipients. 3) In our series, rhinovirus, parainfluenza and adenovirus might be detected at any moment of the year; the rest of the viruses showed a clear seasonal pattern (November to April).
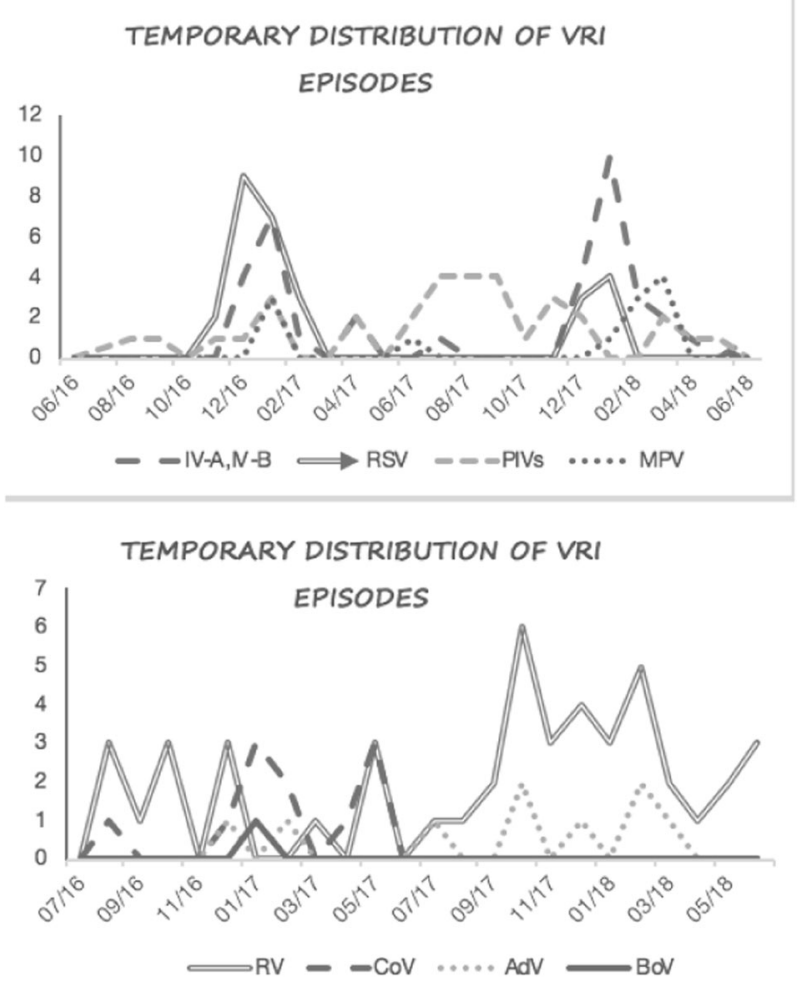

[[P452 Image] 1. figure 1]
Disclosure: Nothing to declare

\section{P453}

Re-exposition of trimethoprim-sulfamethoxazole after allogeneic haematopoietic stem cell transplantation in patients with prior hypersensitivity

\section{Claudia Langebrake ${ }^{1}$, Christine Wolschke ${ }^{1}$, Maximilian Christopeit $^{1}$, Francis Ayuk ${ }^{1}$, Nicolaus M. Kröger ${ }^{1}$}

${ }^{1}$ University Medical Centre Hamburg-Eppendorf, Hamburg, Germany,

Background: Trimethoprim-sulfamethoxazole (TMPSMX) is the most suitable drug for prophylaxis against Pneumocystis pneumonia and infections with Toxoplasma after allogeneic haematopoietic stem cell transplantation (allo-HSCT). Allergic reactions or hypersensitivities, mainly exanthemas, occur in about 3-5\% of the patients, usually resulting in the use of alternative prophylactic drugs (e.g. pentamidine or atovaquone). It has been hypothesised that allergies might be cured with allo-HSCT.

Methods: We conducted a retrospective chart review of patients with TMP-SMX re-exposition after allo-HSCT from December 2017 to September 2018. Follow-up is current as of December 2018.

Results: Six patients (f/m: 4/2, median age: 45 years, range: 25 - 66 years) with a history of TMP-SMX hypersensitivity prior to allo-HSCT were re-exposed to TMP-SMX after engraftment of a matched related (MRD, $n=1$ ) or matched unrelated (MUD, $n=5)$ donor. Median time to re-exposition was 18.5 (range: 11-341) days after allo-HSCT with one oral dose of TMP-SMX.

In four patients, TMP-SMX was tolerated without any signs of hypersensitivity reactions and has been continued for a median of 231 days (range 87-315) until last followup. One patient (MUD, re-exposition at $\mathrm{d}+341$ ) experienced pruritus and erythema some hours after tablet intake. Another patient (MUD, re-exposition at d+18) developed an exanthema one day after re-exposition which was later diagnosed as a cutaneous GvHD.

Conclusions: Re-exposition of TMP-SMX in patients with prior hypersensitivity is feasible after allo-HSCT. After successful re-exposition, patients can be treated with the best-studied drug for prophylaxis of infections with Pneumocystis and Toxoplasma.

Disclosure: Nothing to declare

\section{P454}

Brincidofovir for adenoviremia in paediatric HSCT for primary immune deficiency 
Peter McNaughton ${ }^{1}$, Brigid MacKenzie ${ }^{1}$, Su Han Lum ${ }^{1}$, Terry Flood $^{1}$, Sophie Hambleton ${ }^{1,2}$, Andrew R Gennery ${ }^{1,2}$, Mary Slatter ${ }^{1,2}$, Stephen Owens ${ }^{1,2}$

${ }^{1}$ The Great North Children's Hospital, Newcastle upon Tyne, United Kingdom, ${ }^{2}$ Institute of Cellular Medicine, Newcastle University, Newcastle upon Tyne, United Kingdom

Background: Reactivation of adenovirus is a severe complication of HSCT associated with significant morbidity and mortality, particularly for children with primary immune deficiency (PID). The only drug currently licensed to treat adenovirus infection is cidofovir. Brincidofovir is a lipidlinked derivative of cidofovir which has been shown to be a safe and effective alternative treatment to cidofovir. There is limited data describing the use of brincidofovir in patients undergoing HSCT for primary immune deficiency.

Methods: We reviewed all patients who received brincidofovir after undergoing HSCT for primary immune deficiencies between 2016 and 2018 at the Great North Children's Hospital, Newcastle Upon Tyne, UK.

Results: Of 78 patients transplanted for PID, 11 developed significant adenoviraemia (14\%). All were treated with cidofovir initially but 6 were switched to brincidofovir because of a failure to respond or because of renal toxicity. Of these, 4 resolved their adenoviraemia within 21 days of commencing treatment (figure 1).

Donor sources were TCR alpha/beta/CD19 depleted haplo-identical $(n=4)$, TCR alpha/beta/CD19 depleted MMUD $(n=1)$ and 12/12 MUD $(n=1)$. Patients were conditioned with Treosulphan/Fludarabine/Thiotepa/ATG/ Rituximab ( $n=4)$, Treosulphan/Fludarabine/ATG/Rituximab $(n=1)$ or Treosulphan/Fludarabine/Alemtuzumab/ GCSF/Plerixafor $(n=1)$. Occurrence of aGVHD and treatment of aGVHD are outlined in table 1.

Patient 5 died +111 days post-transplant of multi-organ failure, severe thrombotic microangiopathy and sepsis. Although Patient 2 initially responded to brincidofovir, reactivation occurred after cessation of treatment; severe diarrhoea precluded the reintroduction of brincidofovir and the adenoviraemia persisted with poor immune reconstitution. Treatment with addback $\mathrm{T}$ cells was attempted however the patient died 194 days post-transplant after a cerebral haemorrhage. Patient 3 had long-standing chronic diarrhoea which was thought not severe enough to warrant cessation of brincidofovir treatment.

Conclusions: The complete resolution of adenoviraemia in 4/6 patients who had previously failed to respond to prior therapy with cidofovir suggests that brincidofovir may be an effective treatment option for adenoviral reactivation post-HSCT for PID. However, resolution of adenoviraemia is influenced by many other factors, including the adequacy of immune reconstitution, the degree of induced immune suppression and the presence of comorbidities such as GVHD. Due to the small sample size it was difficult to assess the relative importance of these factors in this cohort. Brincidofovir was well tolerated however its effectiveness may have been limited by poor gastrointestinal function in one patient (Patient 3) and could not be used after a viral reactivation in another for the same reason. Further studies of the use of brincidofovir in this specific cohort are needed to clarify the role and effectiveness of this treatment.

\begin{tabular}{|c|c|c|c|c|c|}
\hline & Age/Sex & Diagnosis & aGVHD & $\begin{array}{l}\text { GVHD } \\
\text { Treatment }\end{array}$ & Outcome \\
\hline $\begin{array}{l}\text { Patient } \\
1\end{array}$ & 1y Male & $\begin{array}{l}\text { MHCII } \\
\text { Deficiency }\end{array}$ & Grade I skin & Topical steroids & Survived \\
\hline $\begin{array}{l}\text { Patient } \\
2\end{array}$ & 6y Male & $\begin{array}{l}\text { MHCII } \\
\text { Deficiency }\end{array}$ & $\begin{array}{l}\text { Grade II } \\
\text { skin, } \\
\text { Grade I gut }\end{array}$ & $\begin{array}{l}\text { Systemic } \\
\text { steroids }+ \\
\text { ciclosporin }\end{array}$ & Died D+194 \\
\hline $\begin{array}{l}\text { Patient } \\
3\end{array}$ & 9m Male & $\begin{array}{l}\text { Common } \gamma \\
\text { chain SCID }\end{array}$ & Grade I skin & $\begin{array}{l}\text { Systemic } \\
\text { steroids }\end{array}$ & Survived \\
\hline $\begin{array}{l}\text { Patient } \\
4\end{array}$ & 8y Male & $\begin{array}{l}\text { IRF8 } \\
\text { deficiency }\end{array}$ & Grade I skin & $\begin{array}{l}\text { Systemic } \\
\text { steroids }+ \\
\text { ciclosporin }\end{array}$ & Survived \\
\hline $\begin{array}{l}\text { Patient } \\
5\end{array}$ & 13y Male & $\begin{array}{l}\text { DOCK8 } \\
\text { deficiency }\end{array}$ & $\begin{array}{l}\text { Grade I- } \\
\text { II gut }\end{array}$ & none & Died D +111 \\
\hline $\begin{array}{l}\text { Patient } \\
6\end{array}$ & 11y Male & STAT1 GOF & none & none & Survived \\
\hline
\end{tabular}

[[P454 Table] 1. Demographics, diagnosis, occurence of $a G V H D$, treatment of $a G V H D$ and outcome for patients treated with brincidofovir]

Disclosure: Stephen Owens is a principle investigator for the Chimerix AdAPT study of brincidofovir and was paid a stipend for acting as an advisor on the earlier Chimerix AdVANCE study of the clinical epidemiology of Adenovirus infection in patients undergoing HSCT

All other authors - Nothing to declare

\section{P455}

Cytomegalovirus reactivation following allogeneic stem cell transplantation (allo-HSCT)

Hanane Bouarab ${ }^{1}$, Malek Benakli, Farih Mehdid', Mounira Baazizi ${ }^{1}$, Nadia Rahmoune ${ }^{1}$, Dina Ait Ouali, Sara Zerkout ${ }^{1}$, Kahina Tarhi ${ }^{1}$, Rachida Belhadj ${ }^{1}$, Sabrina Akhrouf', Imene Abderahim ${ }^{1}$, Chahrazed Benchouk ${ }^{1}$, Farida Harieche ${ }^{I}$, Rose-Marie Hamladji ${ }^{I}$, Redhouane Ahmed Nacer ${ }^{1}$

${ }^{1}$ Pierre and Marie Curie Center, Hematology-Bone Marrow Transplantation, Alger, Algeria

Background: There is a high prevalence of CMV seropositivity in Algerian population. Because of high morbidity and mortality in pts who underwent allo SCT with 
CMV reactivation, effective surveillance and timely treatment using anti-viral therapy s required. The risk of CMV reactivation depends on the type of stem cell source, immunosuppression (IS) and serological status of the donor/ recipient pair.

Methods: over a 24 months period (from 01/01/2016 to 31/12/2017), 254 pts underwent allo-HSCT for malignant or non-malignant hematology diseases of which 246 pts are evaluated for this study. CMV reactivation was observed in 66 pts (26.82\%) (AML: 33 pts, ALL: 8 pts, CML: 4 pts, multiple Myeloma: 2 pt, NHL skin: 1 pt, primary myelofibrosis: 1 pt, Aplastic anemia: 14 pts, Fanconi anemia: 2 pts, $\beta$-thalassemia: $1 \mathrm{pt}$ ), with a median age of 30 years (4-54), sex ratio (M/F) of 1.75. Allo-HSCT done with sibling donors: 51 pts, Haplo-identical donors: 14 pts and Pheno-identical donor: 1 pt. All pts were treated by chemotherapy alone with myéloablative conditioning (MAC) in 41 pts and reduced intensity (RIC) in 25 pts. All pts received peripheral blood stem cells with an average rate of CD34 + cells: $7,8.10^{6} / \mathrm{kg}$ (3.4-12.7). Additional bone marrow graft was used in 7 pts that received a haploidentical graft without PT-cy. GVH prophylaxis associated cyclosporine (cSA) and methotrexate (sibling and phenoidentical); cSA-MTX-MMF or cSA-MTXCyclophosphamid (haplo-identical). Before transplantation, donor/recipient pair is at high risk reactivation in 65 pts (98.4\%). Detection of CMV reactivation done by Antigenaemia pp65 or by quantitative PCR weekly for the first 3 months and during an IS treatment for acute or chronic GVHD. Pre-emptive therapy is initiated by Ganciclovir as soon as positivity of antigenaemia or increased viral load in PCR.

Results: A first reactivation occurred on average day 44 (16-83) in 66 pts $(26.82 \%)$ of which 32 pts under Corticotherapy for acute GVHD (27 pts), Thrombotic Micro-Angiopathy (1 pt) and renal failure (4 pts) or due to a reinforced IS for Haplo-identical transplantation (5 pts). One pt with chronic GVHD presented a late reactivation 18 months after transplant. Twenty pts presented a $2^{\text {nd }}$ reactivation on average day $135(106-180)$ and 8 pts a $3^{\text {rd }}$ reactivation on average day 136 (130-143). Pre-emptive treatment is introduced in the first episode by a viral DNA polymerase inhibitor (Ganciclovir: 22 pts; Valganciclovir: 43 pts, Foscarnet: $1 \mathrm{pt}$ ). The negativity of antigenaemia is observed on average at 5 days of treatment (3-7). Second line treatment was required in 14 pts $(22 \%)$ due to resistance $(12 \mathrm{pts})$, severe cytopenia $(1 \mathrm{pt})$ or renal failure $(1 \mathrm{pt})$. The onset of severe cytopenia imposed a dose reduction (4 pts) or a therapeutic stop (6 pts) before 15 days. Two pts received additional maintenance treatment for negativation delay. Three pts $(4.5 \%)$ died from CMV infections resistant to antiviral treatment (pneumonia: 2, Colitis: 1).
Conclusions: CMV infection is a serious complication after allo-HSCT. In the absence of vaccination, the systematic monitoring for CMV reactivation is strongly recommended for the establishment of a rapid and effective preemptive treatment.

Disclosure: Nothing to declare

\section{P456}

Abstract withdrawn.

\section{P457}

Urinary tract complications after BK virus infection in children undergoing hematopoietic stem cell transplantation

Malgorzata Salamonowicz ${ }^{I}$, Jowita Fraczkiewicz ${ }^{I}$, Ewa Gorczynska ${ }^{1}$, Elzbieta Wawrzyniak-Dzierzek ${ }^{1}$, Anna Krol $^{1}$, Marek Ussowicz ${ }^{1}$, Danuta Wendycz-Domalewska ${ }^{2}$, Blanka Rybkal, Renata Ryczan-Krawczyk ${ }^{1}$, Bernarda Kazanowska ${ }^{1}$, Alicja Chybicka ${ }^{1}$, Krzysztof Kalwak ${ }^{1}$

${ }^{1}$ Medical University Wroclaw, Wroclaw, Poland, ${ }^{2}$ Laboratory for Molecular Diagnostic, Medical University, Wroclaw, Wroclaw, Poland

Background: BK virus infection is one of the most common infectious complications after hematopoietic stem cell transplantation (HSCT), which results in hemorrhagic cystitis (HC) in significant number of patients. The aim of this study was to evaluate the incidence of BKV infection, outcome and risk factors for BKV infection in children undergoing HSCT.

Methods: Between 2016 and 2017 a total number of 128 patients underwent allogenic HSCT. Median age was 10.3 years (range $0,5-25.0$ years). There were 62 boys and 66 girls.

Results: In transplanted group, episodes of BKV reactivation occurred in 54 patients $(42 \%)$. In 26 cases only urine colonization (C) found before HSCT. In this group in 10 patients $(39 \%)$ virus was transmitted from urine to the blood (B) . Dysuria and/or $\mathrm{HC}$ were observed in $4 / 26(15 \%)$ patients. All of them (100\%) had urine and serum involvement. In 28 cases BKV replication was found after HSCT (3 -cases detected in urine, 25 cases-both serum and urine). Dysuric syndromes and/or HC were found in $5 / 28$ of cases (18\%)- all in patients with serum and urine involvement. Urinary tract was always first location of the virus. There was no case of isolated serum reactivation. The incidence of BK infection was higher in patients older than $>5$ yrs $(p<0.05)$, transplanted from family donor (MSD) $(\mathrm{p}<0.05)$. MUD recipients had more often both serum and urine reactivation $(p<0.05)$ than isolated urine involvement. Sex, day of neutrophil recovery, conditioning 
regimen, or use of total body irradiation were not significant risk factors for $\mathrm{BKV}$ infection, or $\mathrm{HC}$. Six patients were treated with Cidofovir (range 1-4 doses) with good response. There was no death due to evident BKV infection.

Conclusions: $\mathrm{BKV}$ reactivation remains one of the most frequent infectious complication in children undergoing allogeneic HSCT. Most of patients experienced mild infection and age $<5$ years was the positive prognostic factor influencing its incidence. BKV monitoring and prompt treatment of $\mathrm{HC}$ resulted in excellent outcome. We observed surprisingly high rate of new BKV replication after HSCT.

Disclosure: Nothing to declare

\section{P458}

Infectiousn complications in patients with multiple myeloma, malignant lymphoma and germ cell tumor receiving autologous blood stem cell transplantation - a retrospective single center analysis

\section{Katharina Engel', Enrico Schalk', Martin Mikusko', Thomas Fischer ${ }^{1}$, Denise Wolleschak ${ }^{1}$}

\footnotetext{
${ }^{1}$ Otto-von-Guericke University, Klinik für Hämatologie und Onkologie, Magdeburg, Germany
}

Background: High-dose chemotherapy (HD-CT) and auto PBSCT have been the standard therapy for multiple myeloma (MM) for more than two decades, despite a wide range of new therapeutic options. Recurrent/refractory malignant lymphomas and recurrent/metastatic germ cell tumors (GCT) also benefit from this intensive therapy. In comparison to allogeneic transplantation, this treatment is known for lower complication rates, e.g. infections. However previous studies have schown that treatment related toxicity may not be underestimated and depending on the conditioning regimen used.

Methods: We retrospectively analyzed 193 patients (225 cases) who underwent HD-CT plus auto PBSCT between 2011 and 2017 in a single-center study. To anlyze the incidence of infections depending on the conditioning regimen, we formed the following categories based on the AGIHO: no infections, neutropenic fever, sepsis and severe sepsis.

Results: The median age in this analysis was 59 years; $74.2 \%$ were male. The most frequent diagnosis was MM $(62.7 \%)$ receiving high dose melphalan (MEL), followed by malignant lymphoma $(26.7 \%)$ receiving BEAM (BCNU, etoposide cytarabine, melphalan) and relapsed/metastatic germ cell tumours (GCT) $(9.8 \%)$ receiving high dose carboplatin/etoposide (CE).

$8 \%$ of all patients developed severe sepsis, 5 patients had to be ventilated and 2 patients died. Sepsis was documented in $25.3 \%$ of all cases (57 cases). The majority of patients (58.2\%, 131 cases) developed neutropenic fever and $8.4 \%$ (19 cases) didn't have any infection complications. The BEAM conditioning regimen showed the highest tendency to result in a septic course (43.3\%), followed by $\mathrm{CE}$ (27.3\%) and MEL (24.1\%). The most commonly documented pathogen in 153 blood cultures was S. epidermidis (41.7\%), followed by E. coli (18.5\%) and S. mitis (9.9\%). Only in one blood culture we detected a multi-resistant pathogen (3MRGN E. coli). P. aeruginosa was detected in 4 blood cultures $(2.6 \%)$, L. monocytogenes in $2(1.3 \%)$ and $\mathrm{S}$. aureus in $6(4 \%)$.

$77.8 \%$ of all patients developed diarrhea, only in $10.9 \%$ of these cases we could detect $\mathrm{C}$. difficile. The conditioning regimen shows no significant effect on the incidence of $\mathrm{C}$. difficile.

The mean neutropenic period was 13.5 days in malignant lymphoma patients, followed by 10.8 in MM patients and 8.7 days in GCT patients. The hospital discharge, calculated from the day of transplantation, was significantly different: for malignant lymphoma the mean was 20.8 days, for MM 19.6 days and for GCT 14.5 days.

Conclusions: Our data correspond to former published results by many groups. The BEAM regimen shows the highest infectious complication rate followed by $\mathrm{CE}$ and MEL. The duration of neutropenia and hospital stay depends on the conditioning regimen. The type of infectious complication doesn't effect the progression free- and overall survival in our analysis.

Disclosure: Nothing to declare.

\section{P459}

Impact of donor and recipient cytomegalovirus serostatus on outcomes of unrelated allogeneic haematopoietic stem cell transplantation

Cheng Kiat Lawrence $\mathrm{Ng}^{1}$, Kim Siang Jeffrey Quek ${ }^{1}$, Yun Xin Chen ${ }^{1}$, Chandramouli Nagarajan', Colin Diong Phipps $^{1,2}$, Dixon Grant ${ }^{1}$, Wei Inng Francesca Lorraine Lim $^{1}$, Jing Jing Lee ${ }^{I}$, Yuh Shan Lee ${ }^{1}$, Hein Than ${ }^{1}$, Su Ming Yvonne Loh ${ }^{1,3}$, Yeow Tee Goh ${ }^{1}$, Ying Khe William Hwang ${ }^{1}$, Yeh Ching Linn', Ban Hock Tan', Thuan Tong Tan $^{1}$, Yew Leng Aloysius Ho ${ }^{1}$

${ }^{1}$ Singapore General Hospital, Singapore, Singapore, ${ }^{2}$ Mount Elizabeth Hospital, Parkway Cancer Center, Singapore, Singapore, ${ }^{3}$ Raffles Medical Center, Raffles Cancer Center, Singapore, Singapore,

Background: Cytomegalovirus (CMV) is an important cause of morbidity and mortality in allogeneic haematopoietic stem cell transplant (HSCT) patients. The aim of our study is to evaluate the outcomes of our CMV seropositive 
recipients who received grafts from seropositive unrelated donors $(D+R+)$ compared with grafts from seronegative unrelated donors (D-R+).

Methods: This is a retrospective single center study on a series of CMV seropositive recipients who underwent HSCT from unrelated donors between Febuary 2012 to July 2018. A total of 96 patients were analyzed. Their clinical course and laboratory results were reviewed for evidence of CMV reactivation and/or CMV disease. We defined CMV infection as detection of CMV reactivation or primary infection by antigenaemia or polymerase chain reaction (PCR) assays, but was not accompanied by signs and/or symptoms suggestive of a systemic disease. CMV disease occurred when CMV was isolated from any site in association with organspecific signs and/or symptoms. Monitoring for CMV infection commenced upon engraftment (approximately Day +14 ). Peripheral blood samples were sent twice a week for CMV antigenaemia or CMV quantitative PCR. The duration of twice weekly monitoring was at least about 100 days. Longer monitoring was performed in patients who experienced CMV infection after HSCT.

Results: All patients received graft-versus-host-disease (GVHD) prophylaxis using Anti-Thymocyte globulin (ATG) at $4.5 \mathrm{mg} / \mathrm{kg}$ in addition to cyclosporin or tacrolimus. Among the entire cohort of 96 patients, 72 (75\%) had CMV infection, including $23(82.1 \%)$ out of 28 patients from the $\mathrm{D}-\mathrm{R}+$ group and $49(72 \%)$ out of 68 patients from the $\mathrm{D}+\mathrm{R}$ + group. 16 patients (57.1\%) from the $\mathrm{D}-\mathrm{R}+$ group and 19 patients $(27.5 \%)$ from the $\mathrm{D}+\mathrm{R}+$ group had $\geqq 2 \mathrm{CMV}$ reactivation above the threshold for preemptive therapy respectively; $\mathrm{p}=0.007 .11$ patients developed CMV disease, $6(21.4 \%)$ from the D-R+ group and $5(7.4 \%)$ from the D $+\mathrm{R}+$ group. CMV resistance to both foscarnet and ganciclovir was detected in 3 patients (10.7\%) from the $\mathrm{D}-\mathrm{R}+$ group but none from the $\mathrm{D}+\mathrm{R}+$ group. 2 patients died due to CMV disease, both were from $\mathrm{D}-\mathrm{R}+$ group. 2 year overall survival (OS) were $60 \%$ versus $55 \%$ for $\mathrm{D}-\mathrm{R}+$ group and $\mathrm{D}+\mathrm{R}+$ group respectively; $\mathrm{p}=0.706$. Median survival was not reached at 2 years. 2 year non-relapse mortality (NRM) were 35\% for D-R+ group and $24 \%$ for D $+\mathrm{R}+$ group respectively; $\mathrm{p}=0.37$.

Conclusions: The incidence of recurrent CMV infection was higher in the $\mathrm{D}-\mathrm{R}+$ group compared to the $\mathrm{D}+\mathrm{R}+$ group. There were no statistically significant differences between the 2 groups in terms of OS and NRM. However, there was a trend towards higher NRM in the D-R+ group compared to $\mathrm{D}+\mathrm{R}+$ group. Our findings suggest that for matched unrelated HSCT, it may still be important to select a seropositive donor for a seropositive recipient.

Disclosure: None
P460

Late-onset varicella-zoster virus infections in patients submitted to allogeneic stem cell transplantation who are in long term complete remission and free of immuno-suppressive treatment

\section{Matteo Parma ${ }^{1}$, Andrea Aroldi, Elisabetta Terruzzi, Marilena Fedele ${ }^{I}$, Elisa Diral ${ }^{I}$, Pietro Pioltelli ${ }^{I}$, Carlo Gambacorti Passerini ${ }^{I}$}

${ }^{1}$ Ospedale San Gerardo, Clinica Ematologica dell Universita Milano-Biocca, Monza, Monza, Italy

Background: It's known that some patients submitted to allogeneic stem cell transplantation (ASCT) could present a greater susceptibility to infection even when they are in long term complete remission or potentially cured. This fact is related to the dynamic of immunological recovery that is variable in every single patients and it is dependent from many factors: the haematological disease, the conditioning regimen, the age of patient and donor, the number of stem cell and lymphocytes infused, the anti-GvHD prophylaxis, the use of anti-Thimoglobulins and others.

In clinical practise we can observe patients who are potentially cured, who tapered and stopped the immunosuppressive treatment months or years ago and who are suddenly graved from opportunistic infections. The largest part of these infections is represented from Varicella-Zoster Virus (VZV) cutaneous eruption.

Methods: In this report we retrospectively analysed a monocentric cohort of 38 patients submitted to ASCT for haematological malignancies from a median time of 30 months. All of them were free of disease. They stopped the immuno-suppressive treatment in a median time of 270 days after ASCT (range: 160-1642) and did not present later chronic GvHD needing treatment neither other moderate or severe chronic post transplant complications nor other diseases. Prophylactic treatment with anti viral agents (Acyclovir or Valacyclovir) has been conducted simultaneously to immuno-suppressive treatment and for a period ranging between 1 to 6 months after its suspension.

In this cohort of patients we considered the incidence of VZV eruption occurred after the suspension of the immunosuppressive treatment, and we analysed the immunological recovery in terms of lymphocytes sub-population after 6 , 12, 18 and 24 months from ASCT.

Results: Of these 38 patients considered, 10 developed at least one VZV manifestation. All the VZV presentation were cutaneous, we did not observe neurological, ophthalmic or visceral presentation. All the VZV manifestation occurred in patients who ended the anti-viral prophylaxis. Median time of presentation was 575 days after ASCT (range: 174-1642) 
The remaining 28 patients did not present VZV manifestation nor other kind of opportunistic infection despite the absence of anti-viral prophylaxis.

The analysis of lymphocyte sub-population after 6-12-18 and 24 months did not show a significant difference in $\mathrm{B}, \mathrm{T}$, T4, T8 and NK lymphocytes in the different post transplant period.

Conclusions: VZV reactivation seems not to be correlated with the number of the different lymphocyte subpopulations in the post transplant period. Actually it is not possible to distinguish patients more suitable of VZV reactivation on the basis of lymphocyte sub-populations analysis, so anti-viral prophylaxis should be prolonged for a medium period after suspension of immuno-suppressive drugs. In absence of anti viral prophylaxis a careful clinical surveillance should be performed in order to treat early eventual VZV manifestations.

Disclosure: No conflict of interest

\section{P461}

Ganciclovir vs valganciclovir to prevent viremia and cytomegalovirus disease in allogeneic stem cell transplant

\section{Karen D. Pérez-Gómez ${ }^{1}$, Brenda Lizeth Acosta- Maldonado $^{I}$, Liliana M Rivera-Fong ${ }^{I}$, Andrea I Milan- Salvatierra', Yayra M Pichardo-Cepín ${ }^{1}$, Perla K Acosta- Maldonado ${ }^{1}$, Luis M Valero-Saldaña ${ }^{I}$}

\section{${ }^{1}$ National Cancer Institute, México City, Mexico}

Background: Infection and disease cytomegalovirus (CMV) are common problems in patients undergoing hematopoietic stem cell transplantation (HSCT). CMV infection has a high overall seroprevalence, therefore, during the first 100days post-HSCT, it is important to prevent reactivation of CMV. The international clinical recommendation is the use of ganciclovir as prophylaxis in HSCT patients; however, the cost of this treatment is not accessible for our population. In this respect it has been used as an alternative Valganciclovir because of its lower cost and oral administration. Our study's aim was to assess the response and safety of valganciclovir in comparison with ganciclovir to prevent viremia and cytomegalovirus disease in patients undergoing allogeneic HSCT

Methods: A retrospective study was performed on patients who receive an HSCT-allo between January 2014 and August 2018. Participants were enrolled in two groups according to prophylaxis treatment:

(a) ganciclovir $5 \mathrm{mg} / \mathrm{k}$ once daily and

(b) valganciclovir 450mg twice daily for 7 days pretransplant, at day +100 ; viremia was measured by PCR.
Demographic and clinical information was collected from medical records and furthermore analyzed in SPSS v21.

Results: Sixty-eight patients were enrolled in the study, $54 \%$ male, the median age was 34 years (19-61) with the following diagnoses: acute lymphoblastic leukemia $44 \%$, acute myeloblastic leukemia $26.5 \%$, granulocytic chronic leukemia $17.6 \%$, myelodysplastic syndrome $4.4 \%$, dendritic cell neoplasia $4.4 \%$, and aplastic anemia $2.9 \%$. Ninety-one percet of the patients received a transplant from an identical HLA donor and $8.8 \%$ received a haploidentical transplant.

Thirty-four patients received Ganciclovir (G1) and thirtyfour Valganciclovir (G2). Median age was 39 vs28 years $(\mathrm{p}=0.022)$, intermediate risk CMV $85 \%$ vs $76(\mathrm{p}=0.048)$, associated bacterial infections was $15 \%$ vs $32 \%(\mathrm{p}=0.15)$, and fungal infections $6 \%$ vs $9 \%$ respectively $(\mathrm{p}=0.5)$. The reactivation by CMV was presented in $21 \%$ vs $15 \%$ respectively $(\mathrm{p}=0.70)$. There were no significant differences in fever, bacterial isolation, dysfunction or graft failure, presence and degree of acute or chronic GVHD and relapse of the disease. The most relevant characteristics and complications are described in table 1. Within the whole group there were 28 deaths, $53 \%$ in the Ganciclovir group and $32 \%$ in Valganciclovir group $(\mathrm{p}=0.14)$, overall survival 1 -year was $66 \%$ vs $80 \%(\mathrm{p}=0.58)$ respectively; in both groups $72 \%$ was associated with relapse and $28 \%$ associated with transplantation.

\begin{tabular}{llll}
\hline Characteristics & $\begin{array}{l}\text { Ganciclovir } \\
\mathrm{n}=34 \mathrm{n}(\%)\end{array}$ & $\begin{array}{l}\text { Valganciclovir } \\
\mathrm{n}=34 \mathrm{n}(\%)\end{array}$ & $\mathrm{p}$ \\
\hline $\begin{array}{l}\text { Viral load>1000 } \\
\text { Viral load (median- }\end{array}$ & $\begin{array}{l}7(21) \\
\text { range) }\end{array}$ & $5(15)$ & 0.70 \\
$\begin{array}{l}\text { Viremia (days) / } \\
\text { median(range) }\end{array}$ & $59 \pm 26$ / 65(28- & $30 \pm 14$ /38(14-40) & 0.067 \\
$\begin{array}{l}\text { Disease (days) } \\
\text { Pneumonitis }\end{array}$ & $70 \pm 26$ & $38 \pm 15$ & 0.32 \\
relate CMV & $3(9)$ & $1(3)$ & $0.021^{*}$ \\
$\begin{array}{l}\text { Myelosuppression } \\
\text { Neutrophil }\end{array}$ & $2(6)$ & $4(12)$ & 0.30 \\
recovery (days) & $12 \pm 5$ & $14 \pm 4.4$ & 0.33 \\
$\begin{array}{l}\text { Toxicity Hepatic } \\
\text { Toxicity Renal }\end{array}$ & $2(6)$ & $4(11)$ & 0.20 \\
\hline
\end{tabular}

[[P461 Table] 1. Table1. Relevant characteristics and complications.]

Conclusions: Ganciclovir and Valganciclovir were effective in preventing the reactivation of CMV, the only statistically significant difference was that the presentation of the disease appeared earlier in the Valganciclovir group. No difference in toxicity between the groups was identified.

Disclosure: None declared 


\section{P462}

Invasive pulmonary aspergillosis after allogeneic hematopoietic stem cell transplantation. Study over a period of 3 years

\section{Mounira Baazizi' ${ }^{I}$,Malek Benakli ${ }^{1}$, Farih Mehdid ${ }^{1}$, Dina Ait Ouali ${ }^{1}$, Hanane Bouarab ${ }^{1}$, Nadia Rahmoune ${ }^{1}$, Rachida Belhadj ${ }^{1}$, Sara Zerkout ${ }^{1}$, Kahina Tarhi ${ }^{1}$, Sabrina Akhrouf ${ }^{I}$, Imene Abderahim ${ }^{I}$, Chahrazed Benchouk ${ }^{I}$, Farida Harieche ${ }^{1}$, Rose-Marie Hamladji ${ }^{1}$, Redhouane Ahmed Nacer ${ }^{1}$}

${ }^{1}$ Pierre and Marie Curie Center, Alger, Algeria

Background: Invasive pulmonary aspergillosis (IPA) is a severe and serious complication that occurs in the immediate post-transplant period due to severe neutropenia or late usually following prolonged corticosteroid therapy during treatment of graft-versus-host disease (GVHD). The objective of this study is to analyze the epidemiological, diagnostic and evolutionary characteristics of this major complication over a period of 3 years.

Methods: From January 2015 to December 2017, 392 patients (pts) received an allogeneic hematopoietic stem cell transplantation (allo HSCT) for malignant and nonmalignant haematological diseases. During the transplant procedure, anti-infectious prophylaxis consisted of pts isolation, digestive decontamination, Fluconazole and Aciclovir. Secondary prophylaxis done for pts with prior history aspergillosis. During the follow-up, a standard chest $\mathrm{X}$-ray is performed systematically at each control or in case of clinical signs a thoracic CT scan is requested from suspicion. The diagnosis of IPA is made according to the criteria of the EORTC-MSG based on the predisposing criteria of the host and clinico-radiological criteria (possible infection). Galactomannan antigen and histopathology criteria are not common practice.

Results: A total of 29 IPA episodes (7\%) were identified in 26 pts (AML: 15, AA: 5, ALL 3, CML 2, MM 1) of median age 36 (8-56), sex ratio: 0.44. All of them had transplantation from a family donor (geno-identical: 22, haplo-identical: 4) with conditioning by chemotherapy alone and a graft of CSP (24 pts) and peripheral stem cells-bone marrow (2 pts). All pts had at least one predisposing risk factor: antecedent of aspergillosis (3 pts), prolonged neutropenia> $10 \mathrm{~d}$ ( 5 pts), acute GVHD (8 pts), chronic GVHD (4 pts), prolonged corticosteroid therapy $\geq 0,3 \mathrm{mg} / \mathrm{kg} /$ day exceeding 21 days (12 pts). The diagnosis of API was possible on average at J172 (20-930) after appearance of clinical signs (in all cases) and evocative radiological in 25 cases (in 4 cases, the standard chest $\mathrm{x}$-ray was normal). At the time of thoracic CT scan, 11 pts (37\%) had characteristic signs: halo sign (6 pts), crescent sign (1 pt) and cavity (4 pts). Other minor radiological signs are found in the other pts. Empirical first-line antifungal therapy was started as monotherapy in 14 pts (Voriconazole: 8, Caspofungin: 6 pts) or in combination in 12 pts. A secondline treatment was required in 10 pts for failure after an average duration of 7 days (1-28). Three pts presented a second episode after an average delay of 5 months (3-6) with a favorable evolution of resumption of thetreatment. Fourteen pts $(54 \%)$ are alive with complete resolution after a median treatment time of 10 months (2-19). Twelve pts (46\%) died rapidly on average 14 days after diagnosis (IPA 11, relapse of his disease: 1 )

Conclusions: IPA occurring after an allograft of alloHSCT is a severe complication with high mortality. It is essential, in each case, to identify the pts with risk factors, perform a thoracic CT-scan, send serum serology for apergillus galactomannan antigenand start specific treatment as soon as possible while waiting to be able to reinforce the diagnosis by direct examination or sputum or brochoalveolar lavage with aspiration.

Disclosure: Nothing to declare

\section{P463}

CMV specific immunoglobulins for CMV pneumonitis post stem cell transplantation: A review of safety and efficacy

\section{Luke Carter-Brzezinski ${ }^{1}$, Fiona Dignan ${ }^{1}$, Muhammad Saif $^{1}$, Elizabeth Davies ${ }^{1}$, Eleni Tholouli ${ }^{1}$}

${ }^{1}$ Manchester Royal Infirmary, Haematology, Manchester, United Kingdom

Background: CMV (Cytomegalovirus) has a prevalence varying between $45-100 \%$. Its pathogenicity is relatively low in the general population, usually resulting in a selflimiting viral illness. In an immunosuppressed host, infection can lead to life threatening illness. Disseminated CMV infection can manifest in a number of organs and is diagnosed using internationally accepted criteria. In the post solid organ and stem cell transplant (SCT) setting, it is postulated that it is viral reactivation, rather than primary reinfection that leads to $\mathrm{CMV}$ viraemia. Prevention of reactivation requires the presence of a competent immune system, mediated by T-cells. This accounts for the increased incidence in intensive and T-cell depleting SCT conditioning regimens.

Despite improved outcomes following the introduction of CMV monitoring by PCR and pre-emptive treatments (current UK guidance), CMV pneumonitis still carries a high mortality. The use of CMV specific immunoglobulins (CMVIG) for the treatment of this complication is generally not recommended post chemotherapy or SCT in 
haematological cancers due to lack of evidence. However, CMVIGs are widely used in the setting of CMV reactivation post solid organ transplants. We report the use of CMVIG in patients with suspected CMV pneumonitis at a single UK centre. The aims of this retrospective study were to establish safety and review efficacy in this highly immunocompromised group of patients.

Methods: Data was collected retrospectively on the use of CMVIG in patients with haematological cancers post SCT or chemotherapy alone between 2007 and 2017 at Manchester Royal Infirmary, UK. All patients included had CMV positive PCR in blood (and or from bronchoscopy), as well as high resolution CT imaging evidence of CMV infection.

The data was sourced from pharmacy database and crossreferenced with a departmental list. For each patient identified, case notes and prescriptions were sourced. Data collected included patient baseline characteristics, timing of treatment, number of doses of CMVIG and outcome.

Results: Eight patients received CMVIG for suspected CMV pneumonitis. Seven patients were post SCT and one patient was severely immunosuppressed with chemotherapy alone. Median age was 40 years (range 16-68). The CMVIG regimen used was $4 \mathrm{ml} / \mathrm{kg}$ of Cytotect on days $0,2,4$ and 6 , followed by $2 \mathrm{ml} / \mathrm{kg}$ every four days until resolution of symptoms. There were no infusion related reactions observed. Patients received a median of 4 doses of CMVIG. Four out of 8 patients responded to the treatment and showed full recovery but only 3 are alive and well to date.

Conclusions: This study shows that the use of CMVIG is safe in the post-SCT setting of acutely unwell patients with multi-organ failure. Despite limitations of retrospective studies, there appears to be benefit for the use of CMVIG in our patient population, with $50 \%$ of patients showing a full recovery from that episode. Allogeneic SCT plays a confounding role in the outcome of patients although the numbers in our study are small.

There is clearly a need for better treatments of CMV pneumonitis. CMVIG is a promising treatment but further studies are needed to identify the optimal dosing regimen and provide evidence of efficacy.

Disclosure: Biotest- Honaria

P464 Abstract withdrawn.

\section{P465}

Antifungal prophylaxis with fluconazole or fluconazole followed by posaconazole in high risk patients in allogeneic setting

Nuno Miranda ${ }^{1}$, Catarina Nunes ${ }^{2}$, Catalina Gomez ${ }^{1}$, Vera Gomes $^{I}$, Sofia Jorge ${ }^{I}$, Gilda Teixeira ${ }^{1}$, Isabelina Ferreira $^{I}$, Fernando Leal-da-Costa ${ }^{I}$, Manuel Abecasis ${ }^{I}$
${ }^{1}$ Instituto Português de Oncologia de Lisboa Francisco Gentil (IPOLFG), Lisboa, Portugal, ${ }^{2}$ Centro Hospitalar de Lisboa Ocidental (CHLO), Lisboa, Portugal

Background: The risk of fungal infection related to allogeneic transplantation is a well-known cause of morbidity and mortality. The main agents implicated are yeast during the neutropenic period and filamentous fungi after this period.

Methods: We decided to evaluate the effectiveness of a prophylactic regimen containing fluconazole since day -3 . After discharge fluconazole was kept until day or 75 or switched to posaconazole in high-risk patients. Patients with GvHD under steroids were kept under prophylaxis. The group of high risk patients was defined by one of the following variables: 1- Non related donors 2- ATG, campath or fludarabine in the conditioning 3- Presence of GvHD with need of steroids above $0.5 \mathrm{mg} / \mathrm{kg}$ We have analyzed the patients submitted to alloBMT during 2016 and 2017. All patients were first admitted to an isolation room with HEPA filters.Patients under secondary prophylaxis were excluded.

Breakthrough fungal infections during the first year and toxicity leading to discontinuation was evaluated.

Results: Sixty six patients were included with 67 transplants. Male/female ratio was $36 / 30$. The age range was 0.6-64 yo with a median of 37. Malignant (53) and nonmalignant (13)diagnosis were included. Donor type was Related (16) Haploidentical (9) and non-related (42). The conditioning regimen includes ATG in 21, campath in 5 and fludarabine in 41 . Fourteen patients were treated after discharge with fluconazole and 52 with posaconazole. Three patients fluconazole were switched to micafungin for hepatic toxicity, two cases to amphotericin due to persistent fever and in one case to caspofungin for a proven fungal infection (candida parapsilosis in blood stream in day +18 ). After discharge and during the first year of follow-up a single case of possible fungal infection was diagnosed, in a patient with GvHD with a lung nodule.

Conclusions: During the neutropenic period after transplantation the main risk of fungal infection is associated with candidiasis. The greatest risk of aspergillosis occurs later and have a significant relation with GvHD. Except for candida parapsilosis the main source of yeasts are the GI tract. The main source of aspergillus are aerosolized particles retained by HEPA filters. In patients without a previous episode of fungal infection the main risk of filamentous fungi occurs only after discharge.

We conclude that fluconazole alone or followed by posaconazole in high risk patients is a feasible and effective regimen for primary prophylaxis, in allogeneic transplantation.

Disclosure: No conflits of interest to declare 


\section{P466}

BK virus-associated hemorrhagic cystitis in pediatric hematopoietic stem cell transplant recipients

\section{Fahra Smith ${ }^{1}$, Julia Palma ${ }^{1}$, Paula Catalan ${ }^{I}$, Cristian Sotomayor ${ }^{1}$, Patricio Garcia ${ }^{1}$}

${ }^{1}$ Luis Calvo Mackenna Children's Hospital, Santiago, Chile

Background: BK virus-associated hemorrhagic cystitis (BKV-HC) has emerged as a serious infection after hematopoietic stem cell transplantation (HSCT). It is characterized by painful hematuria due to hemorrhagic inflammation of the urinary bladder mucosa, this causes significant morbidity, prolonged hospital care with extensive nursing requirements and increases in healthcare costs.

The purpose of this study is to determine the incidence, risk factors, and duration of treatment in our center.

Methods: We performed a retrospective review of HSCT patients at Luis Calvo Mackenna Children's Hospital in Santiago, Chile diagnosed with BKV-HC, from 1st January 2016 to 30th November 2018.

We investigated the incidence, risk factors and duration of treatment of $\mathrm{BKV}-\mathrm{HC}$ in paediatric patients undergoing HSCT over a 35 months period. BKV-HC was defined as $\mathrm{BK}$ virus (BKV) detection in urine by PCR testing in association with clinical symptoms and hematuria grade 2 or higher. Sixty-seven patients were trasplanted during this period.

Results: Eleven patients were diagnosed with BKV-HC at our institution, only one with BK viremia. The cumulative incidence of BKV-HC in our series was $16 \%$. All of them were treated with cidofovir. The median age at diagnosis was 9 years old (range: 3-13 y.o.). The median time from HSCT to hemorrhagic cystitis (HC) was 40 days (range: 25- 240 days), the median length of treatment was 9 weeks (range: 2-36). All patients received myeloablative conditioning regimens and used cyclophosphamide (100\%); ten $(90 \%)$ were unrelated cord blood transplant recipients and nine $(81 \%)$ used antithymocyte globulin. A concomitant viral reactivation (CMV/VH6) was demonstrated in six (56\%) patients. No patient died due to BKV-HC or its complications, but in the follow up three patients died, one in relapse and two of other post transplant's complications.

Conclusions: BKV-HC is the result of a complex interaction between patient characteristics, donor type and conditioning regimen intensity. These patients experienced significant morbidity and prolonged treatment. In our cohort BKV-HC of all patients but one were transplanted with an unrelated umbilical cord blood unit, all of them received myeloablative conditioning regimen with cyclophosphamide and most of them received anti-thymocyte globulin. We also observed frequently co-existence of viral infections from Herpes family as CMV and VH6. The main limitations of this work are its retrospective nature and it's from a single center. More studies are necessary to better understand the epidemiology and risk factor associated with BKV-HC and the morbidities associated with its treatment.

Disclosure: Nothing to declare

\section{P467}

How we manage HHV-6 reactivation in the posttransplant setting

\section{Oscar Borsani, Anna Amelia Colombo ${ }^{1}$, Daniela Caldera ${ }^{\text {, Paolo Bernasconi }}{ }^{1}$}

${ }^{1}$ University of Pavia, San Matteo Hospital, Pavia, Italy

Background: HHV-6 encephalitis is a life-threatening complication in the post-transplant setting and it develops in about $1 \%$ of patients receiving traditional HSCT. Several risk factors were described. A differential diagnosis between HHV-6 encephalitis and other neurological complications is extremely important but often not-easy to achieve because of the highly heterogeneous clinical and radiological features and complexity of interpretation, especially in transplanted patients. Here we described vignettes that represent and highlight distinct problems in the diagnosis and management of transplanted patients with suspected HHV-6 reactivation.

Methods: We collected the clinical, laboratory and radiological (electroencephalogram, brain MRI and brain CT) data of transplanted patients who developed a neurological syndrome suspected for HHV-6 reactivation. HHV-6 was detected on serum and CSF using RT-qPCR.

Results: 1) A 61-years-old patient developed a diffuse erythema and subsequent encephalitic syndrome following HSCT. The brain MRI revealed clear signs of limbic encephalitic and searching for HHV-6 on serum and CSF revealed 150.000 copies $/ \mathrm{mL}$ and 220.000 copies $/ \mathrm{ml}$ respectively. An antiviral therapy was started but no clinical benefit was achieved.

2) A 59-years-old patient developed a typical neurological syndrome without brain MRI findings of encephalitis and with no evidence of skin involvement. The lumbar puncture and CSF analysis showed a total of 49.200 HHV-6 DNA copies/mL. Antiviral therapy with Ganciclovir and Foscarnet was promptly started with clinical improvement and a drastically reduction of HHV-6 DNA on both CSF and serum. A new brain MRI revealed an acute limbic encephalitis.

3) A slight neurological syndrome consisting of confusion and amnesia developed in a 49-years-old-patient. Brain MRI findings were compatible with a Wernicke syndrome, but no improvement of neurologic symptoms were obtained 
with thiamine supplementation. CSF analysis did not revealed HHV-6 DNA, which was detected at low copies number on serum analysis. A second brain MRI was conclusive for limbic encephalitis, so an antiviral therapy with Foscavir was started and radiological but not clinical improvement was noted. The patient died after few days.

4) In the last case we present a 52-years-old patient who developed a clinical picture of encephalopathy (i.e. amnesia, ataxia, drowsiness, weakness, depression) with rapid progression to coma after seventy-eight days from HSCT. A brain MRI showed a slight contrast enhancement in parietal-occipital regions. During the recovery phase from conditioning-induced cytopenia, an increasing in serum HHV-6 DNA was detected. Searching for HHV-6 DNA on donor's follicles showed a chromosomally integrated HHV-6 (ciHHV-6). Cyclosporin A (CsA) was interrupted and neurological improvement was observed in the following hours: a diagnosis of PRES was made.

Conclusions: HHV-6 encephalitis should be suspected in transplanted patients with a clinical syndrome of encephalopathy. PCR detection of HHV-6 DNA in CSF associated with either typical brain MRI abnormalities or a clinical diagnosis of nonspecific encephalopathy must lead to the urgent initiation of systemic antiviral treatment. If an increase of both serum HHV-6 DNA and WBC is detected, a ciHHV-6 should be confirmed. PRES is an important differential diagnosis in transplanted patients which developed an encephalitic syndrome.

Disclosure: Nothing to declare

\section{P468}

\section{Abstract withdrawn.}

\section{P469}

cytomegalovirus reactivation and outcome of antiviral therapy in hematopoietic stem cell transplantation recipients; A single center study from Pakistan

\section{Muhammad Farhan', Tariq Ghafoor ${ }^{\text {, Tariq Mahmood }}$ Satti ${ }^{1}$, Qamar-Un-Nisa Chaudhry ${ }^{1}$, Syed Kamran Mehmood $^{1}$, Raheel Iftikhar Hashmi ${ }^{1}$, Mehreen Ali Khan ${ }^{1}$

\author{
${ }^{1}$ Armed Forces Bone Marrow Transplant Centre, Raw- \\ alpindi, Pakistan
}

Background: Cytomegalovirus (CMV) infection is a major cause of morbidity and mortality after hematopoietic stem cell transplantation (HSCT). It causes end-organ disease, multi-organ dysfunction syndrome, graft failure, increased susceptibility to infections and GVHD. Greatest risk of CMV infection in a seropositive host is the reactivation of latent virus.
Methods: A prospective descriptive study performed at Armed Forces Bone Marrow Transplant Centre, Rawalpindi, Pakistan from Dec 2016 to Sep 2018. Hundred consecutive patients who underwent HSCT were followed with weekly CMV DNA quantitative PCR from engraftment till day 100 for CMV reactivation. Patients in whom CMV PCR showed more than 2000 copies/ml were treated with antiviral therapy. Factors associated with CMV reactivation, outcome of antiviral therapy and effect of CMV on transplant outcome is studied.

Results: Out of 100 cases, 82 were HLA matched siblings, 15 were matched family donors and 3 were haploidentical transplants There were 66 males and 34 females. Mean age was $11.9 \pm 8.9$ years. Fourty-two transplants were done in thalassemia, 35 in aplasia, 09 in leukemias and 14 in other hematological disorders and immune deficiencies. Ninety-eight recipients and all the donors were CMV seropositive before HSCT. CMV reactivation was seen in 81 patients and 42 of them had CMV viral load more than 2000 copies $/ \mathrm{ml}$ and 39 patients had CMV viral load less than 2000 copies/ml. Nineteen patients had no CMV reactivation. Mean time to reactivation since transplant was $36 \pm 19$ days. Valganciclovir was given in 36 patients due to ease of administration and six patients were treated with ganciclovir during their hospital stay. Only one patient had resistant disease. Mean time to clear viremia was $20 \pm 12.8$ days. The patients having viral load less than 2000 copies/ml, subsequently cleared CMV without any treatment. Antiviral agents; ganciclovir and valganciclovir were equally effective for treating CMV infection with $99 \%$ efficacy, however, more adverse effects were seen with ganciclovir. Myelosuppression I-III was seen in $24 \%$ patients treated with valganciclovir and in $46 \%$ treated with valganciclovir. Renal impairment I-II was seen in $14 \%$ of valganciclovir and $20 \%$ of ganciclovir treated patients. Steroid administration was strongly associated with CMV reactivation $(p=0.003)$. No statistically significant association was found with the use of ATG, GVHD, underlying disease, $\mathrm{ABO}$ or gender mismatch. OS was $81.0 \%$ and $93.1 \%$ in with and without CMV Reactivation $(\mathrm{p}=0.1)$ and DFS was $83.3 \%$ and $96.6 \%$ in with and without CMV Reactivation $(\mathrm{p}=0.06)$

Conclusions: CMV reactivation was seen in $81 \%$ of the transplant recipients, this is higher compared to the western world due to high CMV seropositivity is this region. Steroids administration in post-transplant period significantly increase the risk of CMV reactivation. Preemptive therapy with valganciclovir effectively treats CMV reactivation with acceptable side effects. Viral threshold for treatment should be decided considering the regional endemicity. CMV adversely affects the transplant outcome in terms of DFS and OS.

Disclosure: No conflict of interest. 


\section{P470}

Acute nephritis requiring nephrectomy caused by adenovirus (HADV) and human polyomavirus BK (BKPYV) following allogeneic hematopoietic stem-cell transplantation in a patient with $\mathrm{PH}+$ all

\section{Giorgia Saporiti ${ }^{I}$, Sara Uceda ${ }^{I}$, Federica Grifoni, Stefania Piconi $^{2}$, Nicola Fracchiolla ${ }^{1}$, Elena Tagliaferri ${ }^{1}$, Luca Baldini ${ }^{I}$, Giovanna Lunghi ${ }^{I}$, Francesco Onida ${ }^{1}$}

${ }^{1}$ Fondazione IRCCS Ca' Granda Ospedale Maggiore Policlinico di Milano - University of Milan, Milano, Italy, ${ }^{2}$ A.O. Luigi Sacco, Milano, Italy

Background: Adenovirus infection represents an important cause of morbidity and mortality after allogeneic hematopoietic stem cell transplantation (allo-HSCT), with no established therapy. Although different organs may be affected by disseminated HAdV infections, kidney involvement has been rarely reported. Co-infection of HAdV and $\mathrm{BKPyV}$ are common complication in patients undergoing allo-HSCT, but recent studies demonstrate that BKPyV may facilitate the replication of HAdV and lead to elevated viremia with increased virulence and serious clinical consequences.

Here we report a case of an adult patient who required a monolateral nephrectomy due to HAdV pyelonephritis as an early complication of allo-HSCT for Philadelphia-positive acute lymphoblastic leukemia ( $\mathrm{Ph}+\mathrm{ALL})$.

Methods: In September 2014, an ethiopian gentleman was diagnosed with $\mathrm{Ph}+\mathrm{ALL}$ at the age of 24 years. $\mathrm{He}$ was treated with polychemotherapy in association with the tyrosin kinase inhibitor imatinib mesylate achieving a complete remission (CR). One year later, due to disease relapse with CNS involvement, he was started on vincristine and dexamethasone plus imatinib treatment and in April 2017 he was referred to our BMT Center from Ethiopia. Upon confirmation of the p210 $\mathrm{Ph}+\mathrm{B}$-ALL diagnosis, therapy with the Scr/Abl dual inhibitor dasatinib associated to intrathecal chemotherapy was started and a salvage treatment with inotuzumab ozogamicin followed by an allogeneic HSCT from a HLA-identical brother was planned. Having achieved a documented molecular CR disease status, in June 2017 the patient underwent alloHSCT following the fludarabine-melphalan reduced-intensity conditioning regimen. Graft-versus-host prophylaxis included anti-thymocyte globulin, cyclosporine and mycophenolate mofetil

Results: On day +24 post-transplantation the patient developed macro-hematuria due to hemorrhagic cystitis and a CT scan unveiled a left pyelonephritis with marked kidney enlargement. Kidney microbial investigations were all negative.
At the same time, HAdV viremia with very high copy number $(>25000000 \mathrm{cp} / \mathrm{ml})$ was documented and also elevated BKPyV (>25000000 $\mathrm{cp} / \mathrm{ml})$ viruria and viremia $(6400 \mathrm{cp} / \mathrm{ml})$. The genotyping of HAdV evidenced serotype B11 mainly involved in infections of the urinary tract. Treatment with cidofovir was immediately started; nonetheless, due to rapid clinical worsening despite maximal antibiotic therapy, on day +50 a left nephrectomy was performed, which led to a subsequent progressive resolution of the clinical symptoms and negativization of HAdV and $\mathrm{BKPyV}$ viremia and viruria. PCR Real time performed on the kidney tissue unveiled very high concentration of HAdV copy number.

Conclusions: Acute pyelonephritis due to disseminated HAdV infection may represent a possible cause of severe complication following allo-HSCT. Monitoring of HAdV copy number is helpful to evaluate infection severity and response to treatment. Co-infection of HAdV and BKPyV in immunocompromised patients should be always considered likely to worsen clinical course and outcome.

Disclosure: Nothing to declare

\section{P471}

Analysis of febrile neutropenia in allogeneic hematopoietic stem cell transplantation (allo-HSCT)

Sara Zerkout ${ }^{1}$, Malek Benakli, Farih Mehdid ${ }^{1}$, Mounira Baazizi $^{1}$, Nadia Rahmoune ${ }^{I}$, Dina Ait Ouali, Hanane Bouarab $^{1}$, Rachida Belhadj ${ }^{1}$, Kahina Tarhi ${ }^{1}$, Chahrazed Benchouk $^{1}$, Farida Harieche ${ }^{1}$, Rose-Marie Hamladji ${ }^{1}$, Redhouane Ahmed Nacer ${ }^{I}$

${ }^{1}$ Pierre and Marie Curie Center, Alger, Algeria

Background: Infection is a major cause of morbidity and mortality in patients (pts) receiving an allo-HSCT. Its severity is related primarily to the depth and duration of neutropenia. Febrile neutropenia (FN) is defined as a neutrophil count below 500 cells $/ \mathrm{mm}^{3}$ and a fever $\geq 38.3^{\circ} \mathrm{C}$ at a single measurement or $\geq 38^{\circ} \mathrm{C} 2$ times at one hour intervals. The objective of our study is to analyze the epidemiological, clinical, biological characteristics of febrile episodes (FE) occurred in 136 pts who benefited an allo-CSH over a period of 2 years.

Methods: From January 2016 to December 2017, 136 allo-HSCT were performed in 136 pts including 106 sibling-HLA identical, 26 haplo-identical and 04 phenoidentical for essentially acute leukemia (68 pts, 50\%), acquired and congenital aplasia (45 pts, 33\%). The median age is 22 years (3-59) and sex-ratio (M/F): 1.72 . Prophylaxis consisted on isolation sterile room with laminar flow, digestive decontamination, fluconazole and Aciclovir. Nine pts $(6.6 \%)$ were infected at the time of hospitalization 
(cellulitis 03, pneumoniae 02, bacterial angina 01 , veinitis 01, bronchial pneumonia 01, furuncle cutaneous 01) requiring treatment with antibiotics. Conditioning regimen is myeloablative in all pts. Anti-thymocyte globulin is used in 61 pts $(44.8 \%)$. Peripheral blood stem cells (PBSC) are used in 117 pts (86\%) with an average level of CD34+ cells: $7,5.10^{6} / \mathrm{Kg}$ (2.1-19.8), bone marrow (BM) in 5 pts with a mean level of nucleated cells: 3.4 X $10^{8} / \mathrm{Kg}(0.8-3.6)$ and the association of PBSC-BM in 14 pts (haplo-identical). At each FE, are practiced: chest X-ray, procalcitonin test, blood culture, microbiological study of urine and stool (if diarrhea).

Results: All pts showed aplasia with an average duration of 16 days (6-49), neutrophil engraftment was observed at day 18 (9-31). One hundred and twenty-nine pts (94.8\%) presented $205 \mathrm{FE}$ with an average of 1.5 per pt. Eleven pts (8\%) had $3 \mathrm{FE}$ or more. Forty nine $(23,9 \%) \mathrm{FE}$ are clinically documented (digestive: 19, skin: 14, pulmonary: 11, urinary: 03, Oto-Rhino-Laryngology: 02). The blood cultures are made at $195 \mathrm{FE}, 136 \mathrm{FE}$ are microbiologically documented (66 \%): gram-positive bacteremia in $77 \%$ (mainly coagulase negative staphylococci) and gramnegative bacilli in $23 \%$ of cases. Procalcitonin test performed during $157 \mathrm{FE}$ : normal (32 cases), probable infection (90 cases), probable sepsis (15 cases), severe sepsis (19 cases) and septic shock (one case). Empirical double antibiotic therapy is initiated in 120 pts without waiting for the results of the microbiological study. This association was sufficient in 15 pts $(11 \%)$. The transition to a second line was needed in 114 pts $(83.8 \%)$ and third line in 82 pts $(60 \%)$. Antifungal is added in 36 cases $(27 \%)$. Eight pts benefited from G-CSF. The evolution is favorable in $202 \mathrm{FE}(98.5 \%)$, apyrexia obtained after an average of 3.3 days (1-22). Three pts died (2\%) by severe sepsis on a durable aplasia, 2 of which had a cellulitis before the conditioning.

Conclusions: FE increase morbidity and mortality in alloHSCT so prophylactic measures are essential. Empirical antibiotics treatment has to be instituted very quickly in the absence of documentation.

Disclosure: Nothing to declare

\section{P472}

Abstract withdrawn.

\section{P473}

Efficacy of CMV prophylaxis with letermovir early after transplantation

Atsushi Satake ${ }^{1}$, Masaaki Hotta ${ }^{1}$, Ryo Saito ${ }^{1}$, Akiko Konishi $^{1}$, Hideaki Yoshimura ${ }^{1}$, Takahisa Nakanishi, Shinya Fujita ${ }^{1}$, Tomoki Ito ${ }^{1}$, Kazuyoshi Ishii ${ }^{1}$, Shosaku Nomura ${ }^{1}$
${ }^{1}$ Kansai Medical University, Osaka, Japan

Background: Cytomegalovirus (CMV) infection remains a common complication after allogeneic hematopoietic stem cell transplantation (aHSCT), which results in increased morbidity and mortality. Letermovir is a novel anti CMV drug that inhibits the CMV-terminase complex. The purpose of this retrospective study is to elucidate the efficacy and safety of CMV prophylaxis with letermovir early after aHSCT in clinical practice.

Methods: We retrospectively analyzed the incidence of CMV infection, CMV disease, preemptive therapy, adverse events through week 14 after aHSCT, the rates of engraftment and overall survival. All patients underwent aHSCT in our institution for hematopoietic malignancies between May 2018 and Nov 2018. Data collected in this study included patient's characteristics such as age, sex, disease status, donor source and CMV disease risk. CMV infection was evaluated by CMV antigenemia. This study was approved by the Research Ethics Committee of the Faculty of Medicine, Kansai Medical University.

Results: Thirteen patients (male 9, female 4) underwent aHSCT and received CMV prophylaxis with letermovir. The median age was 54 years (range, 18-65 years). Overall, 10 of 13 patients $(76.9 \%)$ were considered to be at high risk for CMV, including 5 patients $(38.5 \%)$ with haploidentical donors, and 1 (7.7 \%)with mismatched, unrelated donors. All patients began letermovir from day 0 after aHSCT, and achieved engraftment (median 17, 13-22 days). No patient developed CMV disease and required preemptive therapy. One patient died of treatment-related mortality, and 2 patients died of acute GVHD. Although one patient discontinued letermovir before day 100 after aHSCT because letermovir was suspected to be a cause of persistent nausea, severe adverse events were not observed.

Conclusions: It is still unknown whether CMV prophylaxis with letermovir improves OS and reduces TRM; however, our data suggests that CMV infection is considerably inhibited by administration of letermovir early after aHSCT.

Clinical Trial Registry: Not applicable.

Disclosure: The authors declare noconflicts of interest for this study.

\section{P474}

Cytomegalovirus reactivation. Risk factors after allogeneic hematopoietic cell transplantatioN

Florencia Otermin ${ }^{1}$, Alberto Rubén Cremona ${ }^{1}$, Santiago José Ramirez Borga ${ }^{1}$, Pedro Pessacq ${ }^{1}$, María Milagros Szelagowski, Teofilo Miguel De Luca ${ }^{1}$, Javier Farina ${ }^{1}$

${ }^{1}$ Hospital Italiano de La Plata, La Plata, Argentina 
Background: Cytomegalovirus (CMV) is cause of increased morbidity and mortality after transplantation of hematopoietic cells. The pathogenesis of CMV disease or infection is complex with multiple interactions with the immune system, mainly in acute and chronic graft-versus-host disease (GVHD). The aim of this study is to analyze the risk factors for the reactivation of $\mathrm{CMV}$ in patients undergoing allogeneic hematopoietic cell transplantation (HCT).

Methods: Prospective descriptive study of the risk factors for the reactivation of $\mathrm{CMV}$ in the described population. Univariate and multivariate analysis of the predisposing factors were performed: donor graft, treatment with corticosteroids, use of antithymoglobin, serologic status, conditioning regimen and the presence of GVHD.

Results: During the period between August 2014 until January 2017, 71 patients were evaluated. $42.25 \%$ (n:30) had reactivation of CMV. Average reactivation was 58 days post transplant. Both (recipient and the donor) had positive CMV IgG in $78.9 \%$. In the univariate analysis, the reactivation of $\mathrm{CMV}$ was associated with haploidentical transplantation ( $\mathrm{p}:<0.01$ ), with the use of corticosteroids ( $\mathrm{p}$ : $<0.01$ ) and GVHD (p: <0.01). In the multivariate analysis, the haploidentical transplant maintained its statistical significance in comparison with the related allogeneic transplant (p: 0.0012, OR:7.07; IC95\%:2.4-20.6) as well as the use of corticosteroids (p: 0.0014, OR:9.25; IC95\%:2.6-32.4). 100\% of patients receiving corticosteroid treatment had grade II / III GVHD. The serologicac status, myeloablative conditioning regimen and the use of ATG showed no statistically significant association.

Conclusions: In patients undergoing allogeneic transplantation, were found as risk factor to reactivation, those who received haploidentic transplantation and treatment with corticosteroids. Another risk factor that showed greater reactivation was the presence of GVHD.

Disclosure: nothing to declare

\section{P475 \\ Rhizopus oryzae infection following allogeneic stem cell transplant}

\section{Jennifer Clay ${ }^{1}$, Richard Barton ${ }^{1}$, Jennifer Ratner', Maria Gilleece $^{1}$, Timothy Collyns ${ }^{1}$}

${ }^{1}$ Leeds Teaching Hospitals NHS Trust, Haematology, Leeds, United Kingdom

Background: We report the successful management of an invasive, posaconazole resistant Rhizopus oryzae infection in a patient with graft failure following allogeneic transplant.

Methods: A 37 y/o male was referred for allogeneic transplant following 3 cycles of induction therapy for AML with complex karyotype and AXSL1 mutation having achieved complete remission following the first cycle of chemotherapy. His first induction cycle was complicated by a perianal myeloid sarcoma which became infected and required surgical drainage and formation of a defunctioning colostomy.

Results: Following 2 allogeneic transplants, the first complicated by secondary graft failure and the second by primary graft failure he presented with two skin lesions, with a third lesion adjacent to his stoma developing shortly after admission. All lesions were erythematous with central necrosis and progressed rapidly in size over 48 hours. Biopsy of the skin and para-stomal lesions revealed fungal mycelia, with culture subsequently identifying Rhizopus oryzae. Initial treatment was with liposomal amphotericin B $5 \mathrm{mg} / \mathrm{kg} /$ day followed by dose escalation to $10 \mathrm{mg} / \mathrm{kg} /$ day due to the development of new skin lesions. The patient had been taking posaconazole (tablet) prophylaxis since his first allogeneic transplant and peripheral blood drug levels checked at the time of admission were therapeutic confirming that this was a breakthrough fungal infection. Consequently posaconazole was stopped and isavuconazole added to the treatment regimen. Surgical assessment was undertaken but surgery was deferred on the basis of high risk due to the extent of the infection and the patient's profound pancytopenia.

The organism was tested for in vitro susceptibilitiy and found to be resistant to posaconazole (MIC $>16 \mathrm{mg} / \mathrm{l}$ ), with borderline resistance to isavuconazole (MIC $16 \mathrm{mg} / \mathrm{L}$ ) and sensitive to Amphotericin B (MIC 0.25mg/L) (PHE Mycology Reference Laboratory, England). Isavuconazole was therefore stopped and the patient was managed with liposomal amphotericin B along with daily granulocyte infusions. He underwent a third allogeneic transplant using a different unrelated donor and stable engraftment was achieved. Post transplant there was initially an increase in the size of the para-stomal lesion, but no new skin lesions developed. Following engraftment he underwent resection of the stomal lesion, with primary closure and re-siting of his stoma. Amphotericin B was replaced by isavuconazole prophylaxis on discharge and he continues to make an excellent recovery.

Conclusions: Whilst Aspergillus species remain the most common cause of invasive fungal infections in allogeneic transplant patients, other species including the Mucorales are seen, and generally associated with poorer outcomes. Whilst there are standardised methodologies for susceptibility testing, fungi specific cut offs based on clinical outcomes are only available for a limited number of species/ antifungal agents. In this case, susceptibility testing demonstrated resistance to posaconazole which was consistent with the clinical presentation of invasive infection despite therapeutic levels of posaconazole. It is also worth 
noting that an estimated $45 \%$ of $R$. oryzae isolates in the UK are resistant to posaconazole. Treatment with high dose Amphotericin B resulted in improvement in small skin lesions with stabilisation of the larger stomal lesion until count recovery allowed surgical resection.

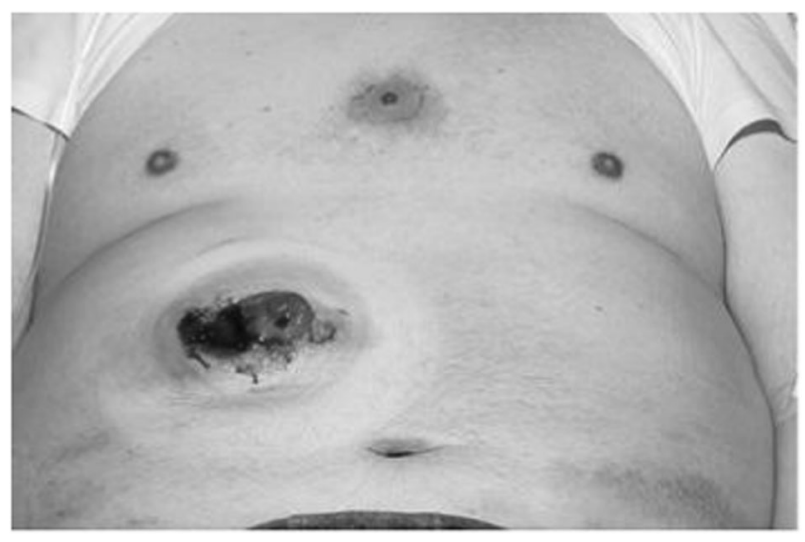

[[P475 Image] 1. Para-stomal and chest wall lesions]

Disclosure: Speakers fees from Gilead Sciences

\section{P476}

Delayed immune reconstitution and subsequent infections can be improved with a viral prophylaxis in a pediatric hospital post haploidentical HSCT with clinimacs device: Peruvian experience

\section{Carlos Vidurrizaga-De Amezaga, Carolina Benavides- Vallve $^{1}$, Sergio Murillo-Vizcarra ${ }^{2}$, Karina Rojas- Fernandez ${ }^{2}$, Karina Pinto-Gossin ${ }^{1}$, Oscar Davila Carlin ${ }^{1}$}

${ }^{1}$ VC Biotec, Cell Therapy Laboratory, Lima, Peru, ${ }^{2}$ Instituto Nacional de Salud del Niño de San Borja, Lima, Peru

Background: Total depletion of innate and adaptive immune cell populations occurs after intensive chemotherapy and hematopoietic stem cell transplantation (HSCT). Both $\mathrm{T}$ and $\mathrm{B}$ lymphocyte pools are restored slower that myelomonocytic populations. HSCT patients are at high risk for bacterial and viral infections at early terms (< 100 days) post-transplant. The reconstitution of the immune system depends on the time required for stem cell recruitment, differentiation, expansion, maturation and release into the bloodstream. Restoration terms for myeloid cells after HSCT are usually defined as the 1st day with neutrophil count of $\geq 0.5 \times 10^{\wedge} 9 / \mathrm{L}$ with mean recovery terms of 12 to 20 days. High occurrence of cytomegalovirus (CMV) in HSCT patients mostly result from reactivation of a latent virus acquired in early childhood. However, delayed immune reconstitution and subsequent infections such as
CMV, adenovirus (ADV) or Herpes 6 (HHV-6) diseases are not unusual and still constitute a major cause of death in Peru.

Methods: Peruvian pediatric patients $(n=22)$ diagnosed with aplastic anemia, MDS, AML or ALL underwent a haploidentical HSCT performed with the CliniMACS device. Patients treated were separated in two groups. The group of patients who received viral prophylaxis (Ganciclovir) was compared to the group that did not receive any prophylaxis treatment. Viral reactivation was confirmed by PCR test twice a week and clinical signs within 15 days after HSCT.

Results: In the group that didn't received prophylactic treatment, engraftment occurred close to day 10 post haploHSCT and none of the patients developed GvHD (graft versus host disease). Nevertheless, incidences of CMV, HHV-6 and BKV infections before day 15 post haploHSCT were still high. An overall survival (OS) over 65\% with an IC 95\% was reached at the end of the first year.

On the other hand, the group of patients that received prophylaxis with Ganciclovir did not developed GvHD and reached the engraftment close to day 10 with a very low viremia incidence after the first month post haplo-HSCT. All viral reactivations were caused by CMV and the OS was over $91 \%$ with an IC $95 \%$ at the end of the first year. Previous prophylaxis to both the donor and the receptor with Ganciclovir $(5 \mathrm{mg} / \mathrm{kg}$ ) every 12 hours before and during the conditioning regimen has allowed a better control of viral reactivation.

Conclusions: The attempts to improve immune function and reduce nonrelapse mortality from infectious complications without increasing GvHD have focused on a partial T cell depleted graft, such as T cell depletion (TCR $\alpha / \beta)$. This graft retains a large numbers of effector cells, such as TcR $\gamma / \delta$ and natural killer cells. However, delayed immune reconstitution and subsequent infections are a big issue.

A novel partial $\mathrm{T}$ cell depletion strategy such as depleted naïve $T$ cells (CD45RA $+T$ cells) could enhance the recovery of immune function after haplo-HSCT because donor pathogen memory $\mathrm{T}$ cells from the donor are retained. It is necessary to increase the studies and the database to set the scheme of previous prophylaxis to the recipient to contain the viral reactivation and to help a rapid immune reconstitution.

Disclosure: No conflict of interest is declared

\section{P477}

Posaconazole vs fluconazole eficacy and safety of antifungal prophylaxis on patients with allogenic stem cell transplantation. Experience of a mexican center

Andrea I Milan-Salvatierra ${ }^{I}$, Brenda Lizeth AcostaMaldonado ${ }^{I}$, Liliana M Rivera-Fong ${ }^{I}$, Yayra M Pichardo- 
Cepín ${ }^{1}$, Karen D. Pérez-Gómez ${ }^{1}$, Mauricio I RodriguezPereira $^{I}$, Perla K Acosta-Maldonado ${ }^{1}$, Luis M ValeroSaldaña ${ }^{1}$

\section{${ }^{1}$ National Cancer Institute, México City, Mexico}

Background: Invasive fungal infection is a main cause of morbimortality after allogenic stem cell transplantation. Fluconazole is the standard prophylaxis treatment, and posaconazole is considered the election drug on patients with graft versus host disease (GVHD). The objective of this study was to evaluate the efficacy and safety at day +100 between posaconazole and fluconazole on patients with allogenic stem cell transplantation (HSCT-allo).

Methods: A retrospective study was performed on patients who receive an HSCT-allo between June 2016 and June 2018. Participants were enrolled in two groups according to prophylaxis treatment: (a) Fluconazole 200mg every 24 hours and (b) Posaconazole 200mg every 8 hours to the day $100+$.

Information was recovered from the medical records.

Results: Thirty-four patients were included, of them with the following diagnoses: Acute leukemia (27), granulocytic chronic leukemia (4), dendritic cell neoplasia (2), aplastic anemia (1). $80 \%$ of the patients received a transplant from an identical HLA donor and $20 \%$ received a haploidentical transplant.

Mean age's patients was 32 years (19-57). Prophylaxis with Posaconazole was performed on $44 \%$ of the patients with identical HLA and $86 \%$ on haploidentical group; the rest of the patients received Fluconazole. The posaconazole group presented: Fever 61\%, mucositis GI-II 94\%, gastrointestinal toxicity GI-II $67 \%(\mathrm{p}=0.006)$, hepatic toxicity $11 \%$, kidney toxicity $17 \%$, oral candidiasis $100 \%$.

During this period none of the patients presented invasive fungal infection in any group. There were 2 deceases, one on each group and none related to a fungal infection. The overall survival was of the $92 \%$ versus $93 \%$ on the posaconazole group and the fluconazole group respectively.

\begin{tabular}{lll}
\hline Toxicity & Posaconazole $\mathrm{n}=18$ Fluconazole $\mathrm{n}=16$ \\
\hline Mucositis & $94 \%$ & $81 \%$ \\
Gastrointestinal & $67 \%$ & $19 \%$ \\
Hepatic & $11 \%$ & $12.5 \%$ \\
Kidney & $17 \%$ & $25 \%$ \\
Hemorrhagic & $22 \%$ & $6.7 \%$ \\
Invasive fungal infection +1000 & 0 \\
\hline
\end{tabular}

[[P477 Table] 1. Table 1. Toxicity according to the prophylaxis]
Conclusions: The prophylaxis with posaconazole and fluconazole is effective on the prevention of invasive fungal infection on the first 100 days. The toxicity was similar on both groups. Posaconazole can be effective on the prevention of the haploidentical type. Is necessary to continue following the patients with infection risk on a long-term period associated with the chronic GVHD.

Disclosure: None declared

Lymphoma

\section{P478}

Is there still a role for allogeneic stem-cell transplant in de novo/transformed DLBCL in the CAR-T cells era? Long-term follow-up of a single centre unit

Laura Prieto-García ${ }^{1}$, Miguel Alcoceba ${ }^{1,2}$, Estefanía Pérez-López ${ }^{1}$, Lucía López-Corral ${ }^{1,2}$, Manuel Delgado ${ }^{1}$, Ana África Martín ${ }^{1}$, Almudena Navarro-Bailón ${ }^{1}$, Lourdes Vázquez $^{1}$, Luis G. Díaz ${ }^{1}$, Pilar Tamayo ${ }^{1}$, Mónica Baile ${ }^{1}$, Miriam López-Parra ${ }^{I}$, Mónica Cabrero ${ }^{I}$, Alejandro Martín $^{1,2}$, M. Dolores Caballero ${ }^{1,2}$

${ }^{1}$ University Hospital of Salamanca (HUS/IBSAL), Salamanca, Spain, ${ }^{2}$ CIBERONC, Madrid, Spain

Background: Allogeneic stem-cell transplant (allo-SCT) is a curative option in patients younger than 65-70 years with diffuse large B-cell lymphoma (DLBCL) - de novo or transformed from previous indolent B-cell lymphoma relapsing or non-candidates to standard salvage therapy including autologous stem cell transplant (auto-SCT). Limitations of allo-SCT include no donor availability or chemorefractory disease at transplant, as well as morbidity and mortality associated with the procedure. Many patients will not reach the transplant. Recently, two Phase II trials with CD19 CAR-T cells in de novo and transformed DLBCL patients have reported a best response of 55\% and 40\% complete response (CR) (Neelapu et al NEJM 2017, Schuster et al, NEJM 2017), have stablish the indication of this new cell therapy in patients relapsing or refractory after two lines of therapy. These results open the question whether allo-SCT should still be offered to these patients.

Methods: We aimed to define the role of allo-SCT in refractory or relapsing after two lines de novo or transformed DLBCL patients, and its comparison with ZUMA-1 CAR-T cells trial (Neelapu et al NEJM 2017). We analyse long-term allo-SCT results in 40 de novo $(\mathrm{n}=23)$ or transformed DLBCL $(n=17)$ out of the 1000 allo-SCT performed in our institution between October 1995 to October 2018.

Results: Patients and transplant characteristics are summarized in Table 1. Complete response (CR) at 100 days was 67,5\% and $83 \%$ of them remain in CR at 12 months. With a 
median follow-up of 46 months, 5-year progression-free survival (PFS) was 54\% and 5-year overall survival (OS) $48 \%$, with a 3-year transplant-related mortality of $18 \%$. Refractoriness at the time of the transplant was associated with a poorer prognosis, with only 2 out of 9 refractory patients being long term survivors (Figure 1).

Similar results were reported for ZUMA-1 trial, with a best response of $55 \% \mathrm{CR}$ retained in $79 \%$ of them at 12 months. With a median follow-up of 15 months, 18-months PFS was $41 \%$ and 18-months OS 53\%. Patients characteristics did not differ in our series and ZUMA-1, except that all the patients in ZUMA-1 were refractory prior to therapy (Table 1).

\begin{tabular}{lll}
\hline & $\begin{array}{l}\text { Allo-SCT, } \mathrm{n} \\
(\%) \mathrm{n}=40\end{array}$ & $\begin{array}{l}\text { ZUMA-1, } \mathrm{n} \\
(\%) \mathrm{n}=101\end{array}$ \\
\hline $\begin{array}{l}\text { Age, years median (range) } \\
\text { Previous lines } \geq 3\end{array}$ & $58(23-76)$ & $58(23-76)$ \\
$\begin{array}{l}\text { Relapse after auto-SCT } \\
<12 \text { months }\end{array}$ & $34(85)$ & $70(69)$ \\
$\begin{array}{l}\text { Best response (day +100 in allo- } \\
\text { SCT): 1) Complete Response 2) }\end{array}$ & $\begin{array}{l}(2,5) ; 6 \\
\begin{array}{l}\text { Partial Response 3) Stable disease } \\
\text { / Progression 4) No evaluable }\end{array}\end{array}$ & $\begin{array}{l}(15) ; 6(15) \\
(28) ; 16\end{array}$ \\
\hline
\end{tabular}

[[P478 Table] 1. Table 1: Patient and transplant characteristics]

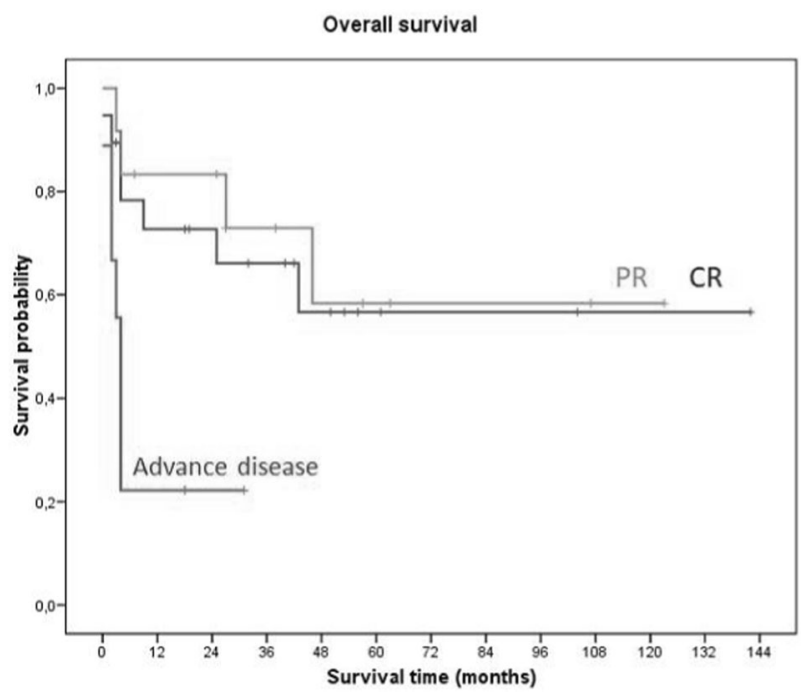

[[P478 Image] 1. Figure 1: Overall survival according to disease status at trasplant]

Conclusions: Although very few patients with de novo or transformed DLBCL are offering an allo-SCT (4\% of all alloSCT), this is a curative option in chemosensitive patients and with more mature data and longer follow-up than with CAR$\mathrm{T}$ therapy; for these reasons, it should still be offer to these poor prognosis patients. Moreover, almost all patients have now available donor, better graft-versus-host disease prophylaxis will decrease TRM and morbidity, and new therapies will make more patients in sensitive disease before allo-SCT. Therefore, allo-SCT and CAR-T cells are strategies to be discussed in every young patient with available donor.

Disclosure: Honoraria as advisor or speaker from Gilead (MDC, AM), Roche (MDC, AM), Celgene (MDC, AM), Kite (MDC), Janssen (MDC, AM, MA), Amgen (MDC), Nordic (MDC), Jazz (MDC), MSD (MDC), Bristol (MDC), Novartis (MDC), Takeda (MDC), Servier (AM), and Morphosys (AM) is reported. MDC has a grant from Gilead. Other authors declare no conflicts of interest.

\section{P479}

A baseline national study of allogeneic transplantation for high grade B-cell lymphoma (HGBL): An outcome baseline prior to the introduction of a CAR-T program

Rachel Brodie ${ }^{1}$, Greg Lee ${ }^{1}$, Valerie Broderick ${ }^{1}$, Elizabeth Higgins $^{1}$, Richard Flavin ${ }^{1}$, Nicole Gardiner ${ }^{1}$, Patrick Hayden $^{1}$, Paul Browne ${ }^{I}$, Eibhlin Conneally ${ }^{1}$, Emma Tuohy ${ }^{I}$, Catherine Flynn ${ }^{1}$, Elisabeth Vandenberghe ${ }^{I}$, Christopher Laurence Bacon ${ }^{1}$

${ }^{1}$ St James's Hospital, Dublin, Ireland,

Background: Patients with either primary refractory or relapsed refractory Diffuse Large B Cell Lymphoma, including tFollicular Lymphoma (tFL) and High-Grade BCell Lymphoma, with $M Y C$ and BCL-2 and/or BCL-2 rearrangements, often referred to as Double Hit Lymphoma (DHL), have a poor outcome with conventional therapy and a published overall survival (OS) of $23.3 \%$ to $58 \%$ (Niitsu et al, Johnson et al). These patients were considered for allogeneic transplantation from 2007 and the outcomes were reviewed prior to the introduction of a CAR-T program for relapsed or refractory Large B-Cell Lymphoma.

Methods: Consecutive patients transplanted for HGBL (excluding Burkitts lymphoma) between 2007-2017 in the EBMT database were included. Data collected included age, sex, pathology subtype (HGBL (including subtypes), tFL, DHL), disease status at SCT, conditioning (MA vs BEAM Cam vs Flu-Mel-Cam/ATG), engraftment, day 100 outcome, TRM, OS and PFS and eligibility for EMEA licensed indication of CAR-T therapy.

Results: Fifty patients (29M, 21F) with a median age of 47 at diagnosis and 50 at SCT were included. The subtypes included HGBL $(\mathrm{n}=29)$, tFL $(\mathrm{n}=11)$ and DHL $(\mathrm{n}=10)$. Indications for SCT were: primary refractory $(n=17)$, relapse $<12$ months after primary treatment $(\mathrm{n}=12)$, previous autologous-SCT $(n=10)$ and DHL $(n=11)$. The median lines of therapy was 4 (range 1 to 6 ). Conditioning 
used was CyTBI $n=21, \mathrm{Bu} / \mathrm{Cy} \mathrm{n}=1$, Etop/TBI $\mathrm{n}=1$, FluBuCy $n=1$, BEAMCam $n=7, F M C / T, n=19$. All patients engrafted with Neutrophil $>1.010^{9} / \mathrm{L}$ at median 15 days and platelets $>2010^{9} / \mathrm{L}$ at median 17 days. The day 100 mortality was $8 \%$ (progressive disease $4 \%$, NRM 4\%) with a 5 year OS of $56 \%$ and mortality due to progressive disease $22 \%$ and NRM 16\%. Disease subtype influenced outcome with an OS for primary refractory HGBL, relapsed HGBL, tFL and DHL respectively of 53\%, 58\%, 57\% and 89\%. 23 patients were eligible for a licensed CAR-T product.

Conclusions: The outcome of these high risk HGBL patients have an acceptable OS of 56\%, with relapsed disease being the commonest cause of mortality. Patients with DHL have a particularly good outcome in this series; recent evidence indicates that some of these patients with a non-immunoglobulin gene associated MYC translocation could be managed more conservatively (ASH Sehn). The outcomes achieved with allogeneic-SCT in this series will provide a baseline for outcome assessment with a CART program.

Disclosure: Nothing to Declare

\section{P480}

Cessation of immune checkpoint inhibitors therapy in R/ R Hodgkin lymphoma: Should we consider HSCT?

\section{Kirill Lepik, Liudmila Fedorova ${ }^{I}$, Natalia Mikhailova ${ }^{I}$, Elena Kondakova ${ }^{1}$, Yuri Zalyalov ${ }^{1}$, Evgeniya Borzenkova ${ }^{I}$, Vadim Baykov, Luibov Tsvetkova ${ }^{I}$, Lilia Stelmakh ${ }^{I}$, Boris Afanasyev ${ }^{I}$}

${ }^{1}$ Pavlov First Saint Petersburg State Medical University/ Raisa Gorbacheva Memorial Institute of Children's Oncology, Hematology and Transplantation, Saint Petersburg, Russian Federation

Background: Immune checkpoint inhibitors (ICI) allow to achieve a durable remission in patients with resistant or refractory $(\mathrm{r} / \mathrm{r})$ classical Hodgkin lymphoma. However, the information about optimal duration of therapy and the prognosis of the patients after ICI cessation is limited (Manson, Blood 2018). Therefore, the optimal role of hematopoietic SCT (HSCT) in this patient group is not defined. Our aim was to determine remission duration in patients who discontinued ICI monotherapy after achieving complete remission (CR).

Methods: This analysis included 20 patients (5 male/15 female) aged 19 to 47 (median 32 years) with $\mathrm{r} / \mathrm{r}$ classical Hodgkin lymphoma who were treated with nivolumab (3 $\mathrm{mg} / \mathrm{kg}$ every 14 days) and achieved CR. Response was assessed by positron-emission tomography/computed tomography (PET/CT) using LYRIC criteria every 3 month.
After nivolumab therapy had been stopped the patients received no other treatment and disease was assessed every 3 months by PET/CT. Median follow-up after therapy discontinuation was 20 (10-21) months.

Results: At the moment of therapy initiation $14(70 \%)$ patients had stage 4 disease, $11(55 \%)$ patients had progressive disease (PD), 4 (20\%) patients had stable disease, $3(15 \%)$ patients had partial remission and $2(10 \%)$ complete remission; $12(60 \%)$ patients had B-symptoms and ECOG score $>1$. The median number of previous therapy lines was 5 (3-10). Before nivolumab initiation high dose chemotherapy with autologous SCT was performed in 9 patients (45\%) and $8(40 \%)$ received brentuximab vedotin. The median number of nivolumab cycles was 25 (18-30). CR was achieved after median of 6 (6-18) cycles. The median duration of therapy after achievement of CR was 7 (1-15) months. At the time of analysis, all patients were alive, $8(40 \%)$ out of 20 patients relapsed after therapy discontinuation. The median progression-free survival (PFS) for the total group was not achieved. Among patients with relapse, the median time before PD was 11 (5-20) months. After relapse all patients were retreated with nivolumab monotherapy or with chemotherapy combination. One patient achieved complete remission; 1 - partial remission; 1 - indeterminate response type 2. Other patients are continuing the therapy and their response has not yet been evaluated.

Conclusions: While complete response was maintained in some patients at median follow up of 20 months after nivolumab therapy cessation, the PFS plateau was not reached. We report that patients with relapse after nivolumab discontinuation sustained sensitivity to nivolumab and achieved a response during retreatment with nivolumab monotherapy or with chemotherapy combination. In patients with unsatisfactory response to nivolumab retreatment, HSCT option should be considered.

Disclosure: Nothing to declare

\section{P481}

High dose chemotherapy with autologous stem cell transplantation in primary central nervous system lymphoma: Data from the japan society for hematopoietic cell transplantation (JSHCT) registry

Eisei Kondo ${ }^{1}$, Takashi Ikeda ${ }^{2}$, Koji Izutsu ${ }^{3}$, Dai Chihara ${ }^{4}$, Risa Shimizu-Koresawa ${ }^{1}$, Nobuharu Fujiii, Tomoyuki Sakai $^{6}$, Tadakazu Kondo ${ }^{7}$, Kohmei Kubo ${ }^{8}$, Yuichi Kato ${ }^{9}$, Takashi Akasaka ${ }^{10}$, Takahiro Fukuda ${ }^{3}$, Tatsuo Ichinohe $^{11}$, Yoshiko Atsuta ${ }^{12,13}$, Junji Suzumiya ${ }^{14}$, Ritsuro Suzuki $^{14}$

${ }^{1}$ Kawasaki Medical School, Kurashiki, Japan, ${ }^{2}$ Shizuoka Cancer Center, Nagaizumicho, Japan, ${ }^{3}$ National Cancer 
Center Hospital, Tokyo, Japan, ${ }^{4}$ National Cancer Institute, Bethesda, MD, United States, ${ }^{5}$ Okayama University Hospital, Okayama, Japan, ${ }^{6}$ Kanazawa Medical University, Uchinada, Japan, ${ }^{7}$ Kyoto University, Kyoto, Japan, ${ }^{8}$ Aomori Prefectural Central Hospital, Aomori, Japan, ${ }^{9}$ Yamagata Unversity School of Medicine, Yamagata, Japan, ${ }^{10}$ Tenri Hospital, Tenri, Japan, ${ }^{11}$ Hiroshima University, Hiroshima, Japan, ${ }^{12}$ Japanese Data Center for Hematopoietic Cell Transplantation, Nagoya, Japan, ${ }^{13}$ Nagoya University Graduate School of Medicine, Nagoya, Japan, ${ }^{14}$ Shimane University Hospital, Izumo, Japan

Background: High-dose chemotherapy (HDT) with autologous stem cell transplantation (ASCT) has been shown to improve prognosis of patients with central nervous system (CNS) lymphoma. Whereas the common regimen of HDT for PCNSL in the Europe and the US is thiotepa-based regimen, e.g. BCNU-Thiotepa, TBC (Thiotepa-Busulfan-Cyclophosphamide), thiotepa-based regimen was only available before discontinuation of thiotepa in 2009 in Japan. We report the results of ASCT for PCNSL from the Japan Society for Hematopoietic Cell Transplantation (JSHCT) Registry.

Methods: Data from the JSHCT registry were retrospectively analyzed. 102 patients with PCNSL who received first HDT/ASCT between 2006 and 2015 were evaluated. Distribution differences of clinical characteristics between groups were analyzed with Fisher's exact or MannWhitney U tests. Overall survival (OS) and progression free survival (PFS) were calculated using Kaplan-Meier method. Two-group analysis of the cumulative incidence of relapse was conducted using the Grey test. Factors were analyzed in univariable analysis, and all factors with $\mathrm{P} \leq .1$ were retained in the multivariable model. All $\mathrm{P}$ values were 2 sided, and values were regarded statistically significant if $\mathrm{P}<.05$.

Results: Median age was 54 months (range 20-74) with 15 patients over 64 years of age; 38 males and 64 females. ECOG-Performance Status (PS) at diagnosis was better (PSO1) in 86 patients and poor (PS2-4) in 16 patients. Serum lactate dehydrogenase (LDH) levels at diagnosis were elevated in 17 patients. Karnofsky PS and Cerebrospinal fluid (CSF) protein concentration at diagnosis were not collected in the registry. 71 patients were in complete remission (CR), 21 patients were in partial response (PR), and 7 patients were stable disease (SD) or progressive disease (PD) at the time of HDT/ASCT. After HDT/ASCT, additional 20 patients achieved CR. With median follow-up period of 44 months, the 5-year OS and PFS were 54.9\% and 38.4\%, respectively. The was no significant difference in OS and PFS between upfront and salvage HDT/ASCT. Since thiotepa, a key agent in HDT/ASCT for PCNSL, has been unavailable after the discontinuation in Japan, the HDT regimens used were not uniform. Thiotepa-containing HDT was received by
16 out of 32 patients before 2010, but by 2 out of 70 patients after 2011. Thiotepa-containing HDT showed improved PFS $(\mathrm{P}=.019)$, lower relapse $(\mathrm{P}=.042)$ and a trend toward a survival benefit. In the multivariate analysis, non-complete remission at HDT/ASCT was an independent predictor for OS $(\mathrm{HR}=2.40,95 \% \mathrm{CI}: 1.25-4.58, \mathrm{P}=.008)$ and thiotepacontaining HDT remained significant for PFS $(\mathrm{HR}=0.42$, 95\% CI:0.19-0.95, $\mathrm{P}=.038$ ).
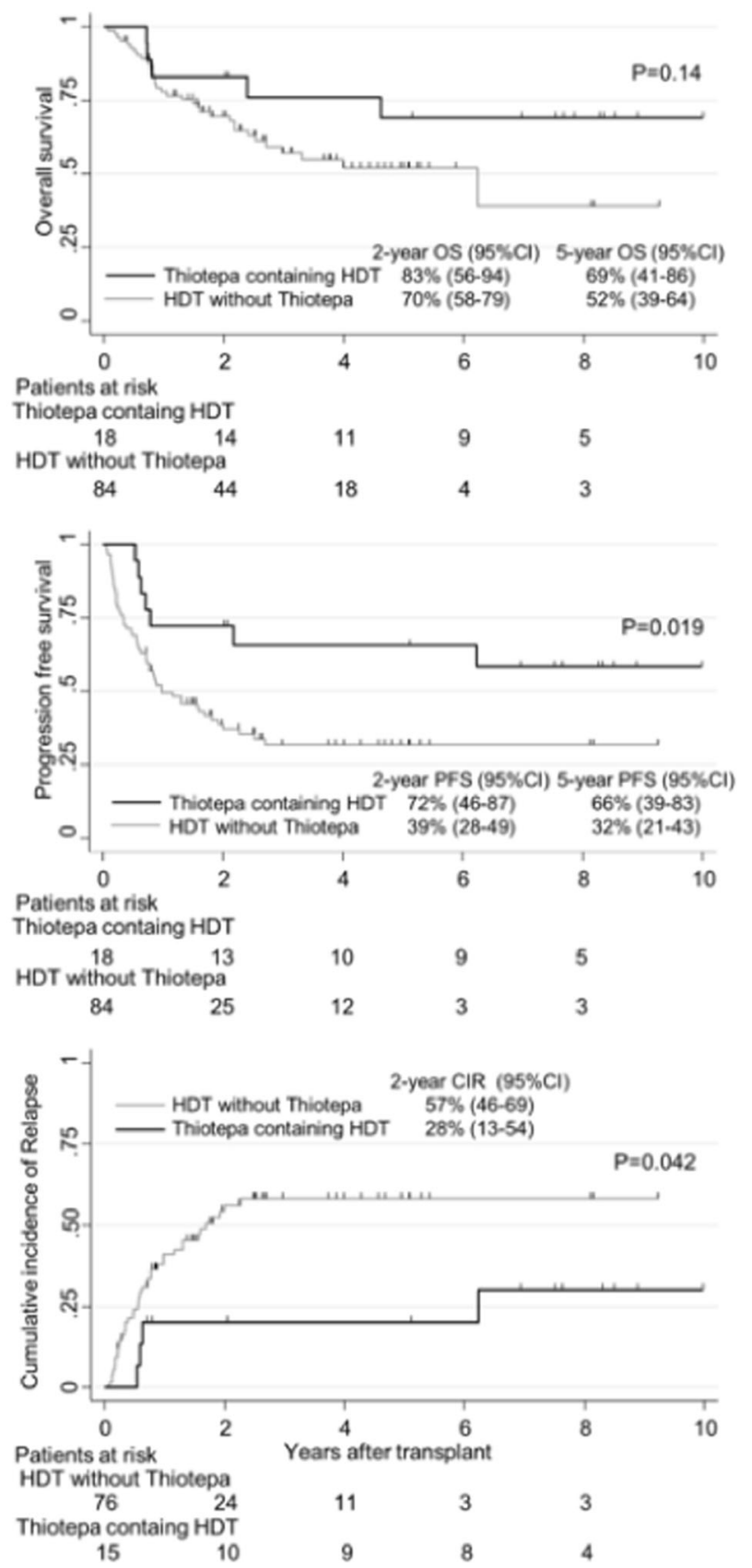

[[P481 Image] 1. OS $(A), P F S(B)$ in all patients $(n=102)$ and cumulative incidence of relapse in $C R$ patients $(C$; $n=91)$ ] 
Conclusions: Our results confirm the activity of thiotepacontaining regimen for HDT/ASCT in PCNSL patients. Currently a pharmaceutical company re-develops thiotepa for new approval of HDT/ASCT in pediatric solid cancer and adult lymphoma in Japan (JapicCTI-163433). Further evaluation with the thiotepa by prospective clinical trials is warranted.

Disclosure: Nothing to declare

\section{P482}

Allogeneic hematopoetic stem cell transplant for patients with advanced T-cell-non-hodgkin lymphomas

Radwan Massoud ${ }^{1,2}$, Christine Wolschke ${ }^{1}$, Gaby Zeck, Tatjana Zabelina ${ }^{I}$, Evgeny Klyuchnikov ${ }^{I}$, Maximilian Christopeit $^{1}$, Francis Ayuk ${ }^{1}$, Nicolaus Kröger ${ }^{1}$

${ }^{1}$ University Medical Center Hamburg-Eppendorf, Hamburg, Germany, ${ }^{2}$ American University of Beirut Medical Center, Bone Marrow Transplant Program, Beirut, Lebanon

Background: T-cell non-Hodgkin lymphomas (T-NHL) are rare diseases and they are associated with worse prognosis when compared to their B-cell counterparts. Allogeneic stem cell transplantation (allo-SCT) may have a curative potential for these patients due to the graft versus lymphoma effect. However, data is limited on the efficacy of allo-SCT for these diseases.

Methods: We identified 53 patients (32\% females; median age: $43 \mathrm{y}$; range,4-67) with T-NHL that underwent allo-SCT at University Hospital Eppendorf between 1992 and 2017. Twenty-one patients (underwent allo-SCT from a matched sibling donor (MSD) and 32 (60\%) from a matched unrelated donor (MUD). Sixteen patients had PTCL (30\%), $n=8(15 \%)$ anaplastic large-cell lymphoma (ALCL), $\mathrm{n}=8(15 \%)$ angioimmunoblastic large cell lymphoma, $\mathrm{n}=8(15 \%)$ adult T-cell leukemia/lymphoma, $\mathrm{n}=5$ (9\%) hepatosplenic gamma/delta T-cell lymphoma, $\mathrm{n}=4$ (8\%) enteropathy associated T-cell lymphoma, $\mathrm{n}=2(4 \%) \mathrm{T}$ cell-prolymphocytic leukemia, and $\mathrm{n}=1(2 \%)$ each extranodal T/NK- cell lymphoma, cutaneous T-cell lymphoma as underlying diagnosis. The median Ann Arbour stage at diagnosis was 4 (range, 1-4). Ten patients (19\%) had bone marrow involvement at diagnosis. All patients were heavily pretreated, 17 (32\%) patients relapsed post autologous stem cell transplant (APSCT) and one patient post allo-SCT. Fifteen patients (28\%) were transplanted in complete remission $(\mathrm{CR})\left(10\right.$ in $1^{\text {st }} \mathrm{CR}, 2$ in $\left.2^{\text {nd }} \mathrm{CR}\right), \mathrm{n}=13(25 \%)$ in partial remission (PR), and $n=21(40 \%)$ with advanced disease. Most of the patients received myeloablative conditioning $(91 \%)$. Thirty-eight $(72 \%)$ patients received Total body irradiation based regimens and 15 (28\%) received chemotherapy based regimens. Twenty patients
(38\%) received Anti-T-lymphocyte globulin (ATLG Neovii), and most patients (87\%) received G-CSF mobilized peripheral stem cells.

Results: Overall, 49 patients $(92 \%)$ had neutrophil engraftment (median days: 13; range,9-36). At day 100, the cumulative incidences of grade II-IV and grade III-IV acute GVHD were $42 \%$ and $15 \%$, respectively. After a median follow up of 12 months (range, 1-171) the cumulative incidences of chronic GVHD was $12 \%$ distributed evenly between limited and extensive. Twenty nine patients (55\%) achieved CR after allo-SCT. Median overall survival (OS) and disease free (PFS) survival were 44 months and 12 months respectively. The 3 year OS and PFS were $50 \%$ and $43 \%$ respectively. Fourteen $(26 \%$; $95 \%$ CI [0.15-0.40]) deaths were due to non relapse mortality (NRM) and 15 patients (28\%; 95\% CI [0.17-0.42]) died due to disease progression. Patients with a male donor had improved OS compared to those with a female donor (3 year OS male 56\%, Female 34\%; $\mathrm{p}=0.038$ ). Patient gender, disease subtype, bone marrow involvement, type of allo$\mathrm{SCT}$, donor, patient CMV status, $\mathrm{ABO}$ incompatibility, disease stage at diagnosis, previous transplant, disease status at transplant, conditioning regimen, ATG and stem cell source had no effect on OS, PFS, NRM, and post transplant complications.

Conclusions: Acknowledging the retrospective nature, our study shows that allo-SCT induces high rates of complete remission, and may have a curative potential even in diseases relapsing post ASCT. However our findings need to be confirmed in larger prospective studies.

Disclosure: No funding, no conflict of interest

\section{P483}

Abstract already published.

\section{P484}

At-home autologous stem cell transplantation in lymphoma patients: Clinical impact of non-G-CSF administration post-transplant

Alexandra Martínez-Roca ${ }^{1}$, Gerardo Rodriguez-Lobato ${ }^{I}$, Gonzalo Gutierrez-Garcia ${ }^{1,2}$, Maria Suárez-Lledó ${ }^{1}$, Montserrat Rovira ${ }^{1,2}$, Carmen Martínez Muñoz ${ }^{1,2}$, Laura Rosiñol $^{1,2}$, Alexandra Pedraza-Navarrete ${ }^{I}$, Ana Belen Moreno-Castaño ${ }^{1}$, Cristina Gallego ${ }^{1}$, Ana Hernando ${ }^{1}$, Susana Segura ${ }^{I}$, Alvaro Urbano-Ispizua ${ }^{1,2}$, Francesc Fernández-Avilés ${ }^{1,2}$

${ }^{1}$ Institute of Haematology and Oncology; Hospital Clínic, IDIBAPS, Barcelona, Spain, ${ }^{2}$ Institut Josep Carreras, Barcelona, Spain 
Background: Severe neutropenia remains the main cause of morbidity and mortality after autologous stem cell transplantation (ASCT). G-CSF administration after ASCT is a common practice, performed to reduce the duration of neutropenia and its complications. In a previous work in patients with multiple myeloma managed at home after ASCT, we did not observe a deleterious clinical impact in those patients that did not receive G-CSF post-transplant (Martinez-Cibrian N. et al, BMT 2016). Despite the fact that lymphoma patients receive a more intensive conditioning regimen that multiple myeloma patients, we hypothesized that the use of G-CSF in lymphoma patients managed at home during the aplasia phase of ASCT does not provide a significant clinical benefit.

Methods: 93 lymphoma patients were managed at-home since day +1 of ASCT. Between February 2010 and July 2016, 68 patients received at-home G-CSF $5 \mu \mathrm{g} / \mathrm{kg}$ per day since day +7 until their ANC reached $1 \times 10^{9} / \mathrm{L}$ (G-CSF group) and, since August 2016, 25 patients did not receive G-CSF (non-G-CSF group). All patients were conditioned with BEAM and received prophylaxis with a quinolone, fluconazole, aerosolized pentamidine and low-dose acyclovir (HVS +). In all cases we added primary prophylaxis with piperacillin-tazobactam $4.5 \mathrm{~g} / 8 \mathrm{~h}$ i.v., using a portable intermittent infusion pump (IIP), from an absolute neutrophil count $\left(\right.$ ANC) $<0.5 \times 10^{9} / \mathrm{L}$ until the first day of fever or until attaining an ANC of $1 \times 10^{9} / \mathrm{L}$. First-line therapy at home of neutropenic fever (NF) was refrigerated meropenem $1 \mathrm{~g} / 8 \mathrm{~h}$ i.v using a portable IIP. Fever was an indication of immediate visit to the hospital, and those patients presenting with focal infection or signs of severe sepsis were admitted. Other indications for readmission were: willingness of the patient or caregiver; uncontrolled nausea, vomiting or diarrhea and mucositis requiring total parenteral nutrition or i.v. morphics.

Results: The main characteristics of the patients are shown in table 1 . There were no differences between groups with respect to gender, diagnosis, stage of disease, comorbidity index (HCT-CI), source of stem cells (peripheral blood) and $\mathrm{CD} 34^{+}$cell dose infused. The median (range) age (years) was 44 (19-71) in G-CSF group and 52 (22-66) in non-G-CSF group $(\mathrm{p}=0.03)$. Duration of neutropenia less than $0.5 \times 10^{9} / \mathrm{L}$ was significantly longer in non-G-CSF group, with a median of 11 days (range 619), compared with 8 (range 6-17) in G-CSF group (p < $0.0001)$. Severe neutropenia, less than $0.1 \times 10^{9} / \mathrm{L}$, was also longer in the non-G-CSF group (8 days (4-11) vs. 7 (5-13); $\mathrm{p}=0.014)$. No differences were observed in the time to platelet engraftment. G-CSF post-transplant avoidance did not influence the incidence of neutropenic fever, the first day and duration of fever, the incidence and severity of oral mucositis, bacterial infections documented and number of readmissions. The median duration of the whole procedure at-home was 1 day shorter in the G-CSF group (14 vs. 15 days; $\mathrm{p}=0.12$ ).

Conclusions: The policy of not administering G-CSF post-ASCT in our home-based program for lymphoma patients, that include intensive bacterial prophylaxis, did not have a deleterious impact on the main results reviewed, which suggests that elimination of its use can be achieved.

Disclosure: Nothing to declare

\section{P485}

Haploidentical non-manipulated stem cell transplant in patients with non-hodgkin lymphoma: Spanish experience

Nieves Dorado ${ }^{1,2}$, Mi Kwon ${ }^{1,2}$, Carlos Solano ${ }^{3}$, Isabel Sánchez Ortega ${ }^{4}$, Lucia López Corral ${ }^{5}$, Christelle Ferra ${ }^{6}$, Inmaculada Heras ${ }^{7}$, Maria Jesús Cascón ${ }^{8}$, Rafael Cabrera $^{9}$, Arancha Bermúdez ${ }^{10}$, Pau Montesinos ${ }^{11}$, Antonia Sampol ${ }^{12}$, Jose Antonio Pérez Simón ${ }^{13}$, Carmen Martín $^{14}$, Santiago Jimbra ${ }^{15}$, Pilar Herrera ${ }^{16}$, Karem Humala $^{17}$, Victor Noriega ${ }^{18}$, Jose Luis Diez-Martín ${ }^{1,2,19}$

${ }^{1}$ Hospital General Universitario Gregorio Marañón, Madrid, Spain, ${ }^{2}$ Instituto de Investigación Sanitaria Gregorio Marañón, Madrid, Spain, ${ }^{3}$ Hospital Clinico Universitario de Valencia, Valencia, Spain, ${ }^{4}$ ICO-Hospital Duran i Reynals, Barcelona, Spain, ${ }^{5}$ Hospital Universitario de Salamanca, Salamanca, Spain, ${ }^{6}$ Hospital Universitario Germans Trias i Pujol, Badalona, Spain, ${ }^{7}$ Hospital General Universitario Morales Meseguer, Murcia, Spain, ${ }^{8}$ Hospital Universitario Carlos Haya, Málaga, Spain, ${ }^{9}$ Hospital Universitario Puerta de Hierro, Madrid, Spain, ${ }^{10}$ Hospital Universitario Marqués de Valdecilla, Santander, Spain, ${ }^{11}$ Hospital Universitario La Fe, Hematology, Valencia, Spain, ${ }^{12}$ Hospital Universitario Son Espases, Palma de Mallorca, Spain, ${ }^{13}$ Hospital Universitario Virgen del Rocio, Hematology, Sevilla, Spain, ${ }^{14}$ Hospital Universitario Reina Sofia, Córdoba, Spain, ${ }^{15}$ Hospital Universitario Doctor Negrín, Las Palmas de Gran Canaria, Spain, ${ }^{16}$ Hospital Universitario Ramón y Cajal, Madrid, Spain, ${ }^{17}$ Hospital Universitario La Paz, Madrid, Spain, ${ }^{18}$ Complejo Hospitalario Universitario A Coruña, A Coruña, Spain, ${ }^{19}$ Universidad Complutense de Madrid, Madrid, Spain

Background: Allogeneic hematopoietic stem cell transplantation (alloSCT) is a potential curative option for patients with relapsed or refractory non-Hodgkin Lymphoma (NHL). Haploidentical stem cell transplantation is a valuable option for patients who lack HLA_identical matched donor in this setting. However, experience with HaploSCT with postransplant cyclophosphamide (Pt-Cy) is scarce among patients with NHL. 
The aim of this study was to analyze the Spanish experience with patients diagnosed of NHL who received HaploSCT with Pt-Cy.

Methods: Sixty patients who received HaploSCT with Pt-CY in 17 Spanish centers from 2012 to 2016 were analyzed. Patients were followed-up until 2017.

GVHD prophylaxis consisted in cyclophosphamide 50 $\mathrm{mg} / \mathrm{kg} / \mathrm{d}$ on days +3 and +4 , and MMF and a calcineurin inhibitor from day +5 .

Results: Patients' characteristics are summarized on Table 1. Median age of patients was 50,75\% male, and diagnosed from T cell lymphoma (37\%). Most of them didn't achieve complete response prior to transplant (55\%), but only $15 \%$ with active disease. Up to $63 \%$ of patients had received previous transplant, from which $5 \%$ was an allogeneic transplantation.

Source of stem cells was mainly peripheral blood (90\%), and reduced intensity conditioning was the preferred $(82 \%)$ regimen. Donors were $43 \%$ siblings (26), 38\% offspring (23), and $18 \%$ parents (11). Median neutrophil and platelet engraftment was 18 (16-21) and 28 (20-42) days, respectively.

Acute GVHD grade II-IV rate was 55\%, with only 6 patients developing grade III-IV (10\%). Chronic GVHD rate was $17 \%$, and only in $3(5 \%)$ was extensive.

Median follow-up was 14 (3-45) months. The 2-year overall survival and event free survival was $43 \%$ and $39 \%$, respectively. The 2-year cumulative incidence of relapse was $14 \%$ and 2-year cumulative incidence of NRM was $22 \%$.

Conclusions: Relapsed/refractory NHL are aggressive entities with a fatal course in a short period of time. HaploSCT with Pt-Cy permit a new treatment option among these patients, with acceptable outcomes. More studies are needed with a larger cohort of patients and longer follow-up to confirm these results.

Disclosure: Nothing to disclose.

\section{P486}

Higher SUV at pre-transplant and day 100 posttransplant pet scan both independently predict inferior survival in patients with diffuse large $B$ cell lymphoma

\section{Malvi Savani ${ }^{1}$, Mehmet Gencturk ${ }^{1}$, Ryan Shanley ${ }^{1}$, Zuzan Cayci $^{1}$, Christopher Wilke ${ }^{1}$, Erica Warlick ${ }^{1}$, Daniel Weisdorf ${ }^{I}$, Claudio Brunstein ${ }^{1}$, Veronika Bachanova ${ }^{1}$ \\ ${ }^{1}$ University of Minnesota Medical Center, Minneapolis, MN, United States,}

Background: Autologous stem cell transplant (auto-HCT) can cure some patients with relapsed diffuse large B-cell lymphoma (DLBCL) but relapse occurs in about $50 \%$ of patients. While our center and others utilize routine surveillance imaging post-transplant, the utility in this setting is unclear. Imaging is costly and exposes patients to radiation. Novel interventions are now available for patients relapsing after auto-HCT making early disease recognition crucial to intervene prior to clinical progression. Hence, we studied impact of post- auto-HCT surveillance (18)F-FDG-PET CT at day 100 on transplant outcomes.

Methods: We analyzed a cohort of 131 consecutive autoHCT recipients with relapsed/refractory DLBCL who then underwent pre-transplant PET/CT and surveillance PET CT at day 100 (Interquartile range (IQR): 97-103 days) postHCT at the University of Minnesota Medical Center. Univariate analysis was performed to analyze PET parameters including Deauville score (D), standardized uptake values (SUV), total lesion glycolysis (TLG) and total metabolic tumor volume (TMTV) as predictors of relapse and survival after auto-HCT. In addition, we assessed outcomes of patients with clinically versus radiographically detected relapsed DLBCL after auto-HCT. Other pre-HCT factors analyzed included age, gender, conditioning regimen, performance status, consolidation radiation therapy, TMTV, SUV, TLG.

Results: Five-year cumulative incidence of relapse after auto-HCT was 50\% (95\%CI 39 to 59) and overall survival (OS) was 51\% (95\% CI 41 to 63). Twelve (9\%) relapsed prior to day 100. D-score for 91 patients with d100 PET/CT were D1 (22\%), D2 (55\%), D3 (0\%), D4 (10\%), D5 (13\%) with median survival in years for D1, D2, D4 and D5 of 6.0, $6.8,4.7$, and 1.2, respectively. Mean SUV varied from 1.53 (D1) to 17.9 (D5). SUV was predictive of relapse and OS. Risk of relapse increased with doubling of SUV; 2-fold higher SUV increased HR by 1.77 (95\%CI 1.34-2.33; $\mathrm{p}=$ 0.01). Mortality increased with doubling of SUV in both pre-HCT (2-fold increase in SUV associated with HR 1.46 [95\% CI 1.1 to 1.8 ]; $\mathrm{p}=0.01$ ) as well as post-HCT PET (HR 1.7 [95\% CI 1.3 to 2.3]; $\mathrm{p}=0$ ) irrespective of the bulk of tumor.

In addition, risk of death was 4 times higher in D5 patients relative to D1 (HR 4.10 [95\% CI $=1.56$ to 10.77]; $p \leq 0.01)$. Patients with D5 $(n=12)$ had higher TMTV (137 $\left.\mathrm{cm}^{3}\right)$ compared to D4 $\left(\mathrm{n}=9\right.$, TMTV $\left.12.5 \mathrm{~cm}^{3}\right)$.

The hazard ratio for death following relapse was 2-fold higher (HR 1.8 [95\% CI 0.9 to 3.4]; $\mathrm{p}=0.08$ ) if relapse was detected clinically versus only radiographically over a median follow-up time period of 3.3 years. Other pretransplant patient and disease characteristics did not significantly influenced the outcomes.

Conclusions: In patients with R/R DLBCL undergoing auto-HCT, surveillance PET/CT at day 100 identified patients with poor survival $\sim 1$ year. Higher SUV in both pre-transplant as well as post-HCT PET was predictive of 
increased mortality. These patients may benefit from novel treatments.

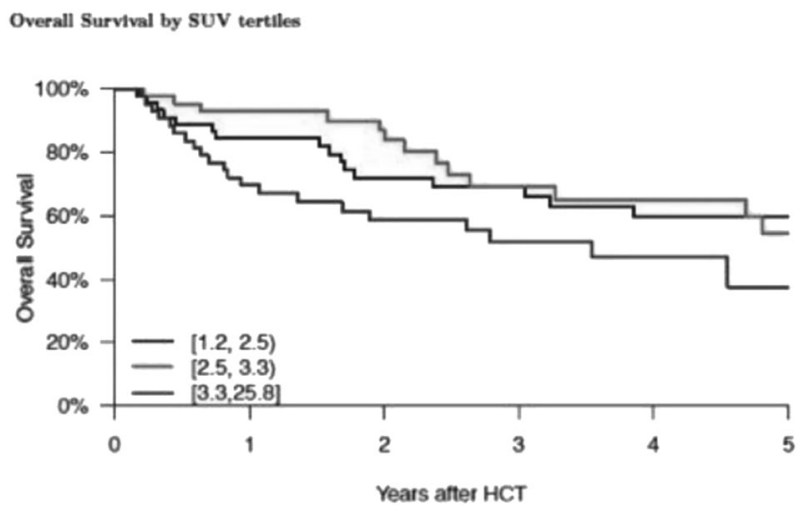

[[P486 Image] 1. Overall survival by SUV tertiles]

Disclosure: Nothing to declare

\section{P487}

The role of immune checkpoint inhibitors in allogeneic stem cell transplantation for patients with relapsed/ refractory classical hodgkin lymphoma

Boris Afanasyev', Anastasiya Beynarovich ${ }^{1}$, Natalia Mikhailova ${ }^{1}$, Kirill Lepik', Elena Kondakova ${ }^{1}$, Evgenia Borzenkova ${ }^{1}$, Andrey Kozlov', Juri Zalyalov ${ }^{1}$, Lilia Stelmakh ${ }^{1}$, Elena Darskaya ${ }^{1}$, Ivan Moiseev ${ }^{1}$

${ }^{1}$ First Pavlov State Medical University of Saint Petersburg, Saint Petersburg, Russian Federation

Background: The programmed-death 1 blockade with nivolumab has demonstrated encouraging efficiency for relapsed and refractory classical Hodgkin lymphoma (r/r cHL). However, remissions are not durable in significant group of patients. Allogeneic hematopoietic stem cells transplantation (allo-HSCT) is a potentially curative option for patients with $\mathrm{r} / \mathrm{r} \mathrm{cHL}$. After introduction of novel reduced-intensity conditioning (RIC) regimens, such as fludarabine-bendamustine (FluBe) (Khouri, Blood 2014), the posttransplant cyclophosphamide-based GVHD prophylaxis (PTCy) (Luznik et al., BBMT 2008) the outcome of allo-HSCT have improved. There are concerns about the risks of nivolumab treatment before and after allo-HSCT, due to the risk of heavy GVHD, thus the place of immune checkpoints inhibitors is not yet defined. This report include analysis of our center experience of nivolumab treatment in patients with $\mathrm{r} / \mathrm{r}$ HL before and after alloHSCT.

Methods: We retrospectively evaluated the results of alloHSCT in 86 patients with $\mathrm{r} / \mathrm{r} \mathrm{cHL}$ who had undergone transplant from 2002 to 2018. The analysis included patients received the FluBe conditioning and PTCy GVHD prophylaxis. In group A patients $(n=20)$ received bridge therapy with Nivolumab and in group B patients $(n=34)$ received bridge therapy with Brentuximab vedotin or chemotherapy-based bridges. Time from the last Nivolumab administration to alloHSCT was at least 2 months.

Results: At the time of analysis, median follow-up was 12 (1-20) months for group A, and 15 (1-64) months for group $B$. There was no difference in two-year OS $(p=0,39)$ with significantly better EFS $(\mathrm{p}=0,025)$ for group A versus group B: $95 \%$ and $95 \%$ vs $85,3 \%$ and $62 \%$ respectively. Relapse incidence was $0 \%$ for group A versus $26,5 \%$ in group $B(p=0,025)$.

Cumulative incidence of non-relapse mortality at 2 years was 5,0\% and $13,8 \%$ in group A and group B, respectively $(\mathrm{p}=0,631)$. There was no difference in grade II-IV (44\% vs $27 \%, \mathrm{p}=0.23)$ and grade III-IV $(22 \%$ vs $13 \%, \mathrm{p}=0.3)$ aGVHD, as well as extensive chronic GVHD (21\% vs $28 \%$, $\mathrm{p}=0,83$ ) in groups $\mathrm{A}$ and $\mathrm{B}$, respectively.

Ten patients with relapse after alloHSCT were treated with different doses $(0,5-3 \mathrm{mg} / \mathrm{kg})$ of nivolumab in CIC725 center. At the median follow up of 16 mo (0,6-28) all patients remain alive.

The objective response to therapy was assessed in 7 patients noted in all patients $(100 \%)$, disregard the dose of the nivolumab: $\mathrm{CR}$ in $29 \%$, and $\mathrm{PR}$ in $71 \%$. The response was lost in four patients, which required nivolumab retreatment. None of the patients developed GVHD after nivolumab administration.

In this analysis, there was also no correlation between dose of nivolumab and incidence and severity of adverse events.

Conclusions: AlloHSCT in combination with immune checkpoints inhibitors is a good option for patients with $r / r$ cHL. Consideration for immune-mediated toxicities and the potential for increased graft-versus-host disease remain important. Early data suggest that nivolumab may be an efficient therapy in patients with $\mathrm{r} / \mathrm{r} \mathrm{cHL}$ relapse after alloHSCT. Further research needed.

Disclosure: The authors declare no conflicts of interest.

\section{P488}

Long term follow-up after stem-cell transplant in transformed DLBCL: A single centre experience

Laura Prieto-García ${ }^{1}$, Sara Alonso-Álvarez', Miguel Alcoceba $^{1,2}$, Manuel Delgado', Norma Gutiérrez ${ }^{1,2}$, Lucía López-Corral ${ }^{1,2}$, Estefanía Pérez-López ${ }^{1}$, Mónica Baile ${ }^{1}$, Mónica Cabrero ${ }^{1}$, Ana África Martín ${ }^{\prime}$, Almudena Navarro-Bailón ${ }^{1}$, Luis G. Díaz ${ }^{1}$, Pilar Tamayo ${ }^{1}$, Lourdes Vázquez, $^{1}$, Alejandro Martín ${ }^{1,2}$, M. Dolores Caballero ${ }^{1,2}$ 
${ }^{1}$ University Hospital of Salamanca (HUS/IBSAL), Salamanca, Spain, ${ }^{2}$ CIBERONC, Madrid, Spain

Background: Transformation to diffuse large B-cell lymphoma (DLBCL) is considered to be one of the most unfavourable events of lymphoma natural history with poorer outcome as compared to de novo DLBCL (AlonsoÁlvarez et al, BJH 2017). In patients suitable for salvage therapy, hematopoietic stem-cell transplantation (SCT) could be an option, although its role is not well stablished. We analyse indication and outcome after transplant in transformed DLBCL at a single Reference Transplant Unit.

Methods: Out of 2565 total of transplants performed at our Unit between 1995 and $2018-1564$ autologous and 1001 allogeneic- 51 were DLBCL transformed from an indolent NHL. Of them, 36 received an autologous SCT (ASCT) and 15 an allogeneic SCT (allo-SCT).

Results: Median age was 60 years old (range 40-69) and 52 (range 35-65) for patients receiving ASCT and allo-SCT, respectively. All ASCT received BEAM as a conditioning regimen and most of the patients in the allo-SCT group received a fludarabine/melphalan combination (73\%). GVHD prophylaxis consisted on tacrolimus/sirolimus combination in $87 \%$ and calcioneurin plus methotrexate in $13 \%$. Regarding transplant disease status, $28(78 \%)$ of the ASCT patients were transplanted in complete response (CR). In the allo-SCT group, 11 (73\%) patients had received three or more treatment lines before transplant and 13 patients $(87 \%)$ had received a previous ASCT, being $11(73 \%)$ in CR, 3 in partial response (PR) and 1 in progressive disease. Transplant related mortality (TRM) was $5.6 \%$ in the ASCT and 27\% In the allo-SCT group. Overall survival (OS) and progression-free-survival (PFS) at 25 months were 94\% (OS), 76\% (PFS) for patients receiving ASCT and 63\% (OS) and 56\% (PFS) for alloSCT. With a median follow up of 57 months for patients receiving an ASCT, 21 (58\%) remain in CR. In the alloSCT group median follow up is 24 months for the whole group and 50 months for alive patients; 9 patients are alive and disease free and 6 have died, 4 due to TRM (28\%). Regarding progression, $12(33 \%)$ have progressed after autologous transplant and 2 after allo-SCT.

Conclusions: Indication for hematopoietic SCT in transformed DLBCL is stablished in few patients. Only $2 \%$ of the patients in our transplant Unit receive a transplant due to transformed lymphoma, corresponding to a $2.3 \%$ of autologous activity and $1.5 \%$ of allogeneic activity. According to our results transplant should be considered a curative option. Most of our patients were transplanted in $\mathrm{CR}$, so new agents trying to reach best response before transplant should be considered.

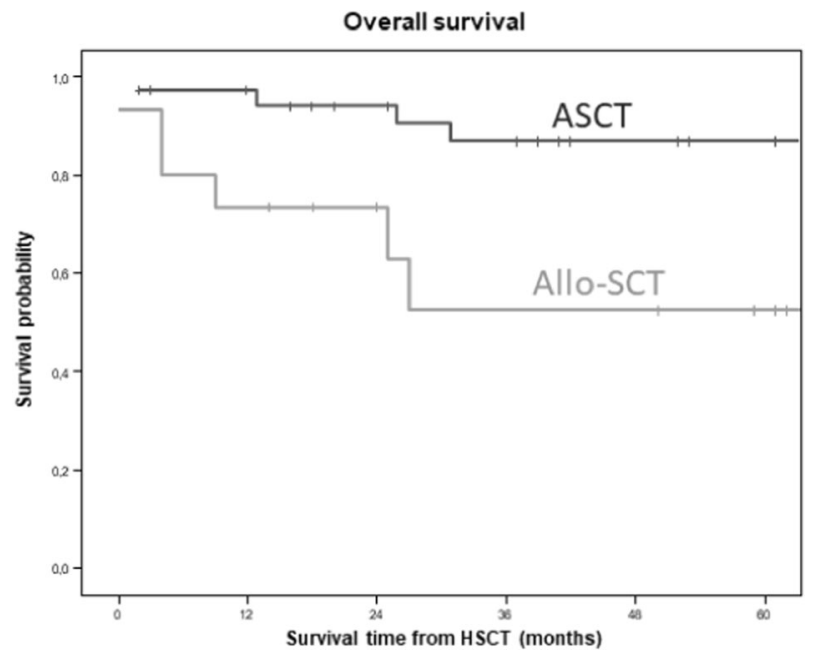

[[P488 Image] 1. Figure 1: Overall survival in tFL by autologous (blue) and allogeneic (green) stem-cell transplant]

Disclosure: Honoraria as advisor or speaker from Gilead (MDC, AM), Roche (MDC, AM), Celgene (MDC, AM), Kite (MDC), Janssen (MDC, AM, MA), Amgen (MDC), Nordic (MDC), Jazz (MDC), MSD (MDC), Bristol (MDC), Novartis (MDC, SAA), Takeda (MDC), Servier (AM), and Morphosys (AM) is reported. MDC has a grant from Gilead. Other authors declare no conflicts of interest.

\section{P489}

Trends in transplant for lymphomas in the Czech Republic

Eva Konirova ${ }^{1}$, Antonin Vitek ${ }^{2}$, Marta Krejci ${ }^{3}$, Edgar Faber $^{4}$, Katerina Steinerova ${ }^{5}$, David Belada ${ }^{6}$, Jan Novak ${ }^{7}$, Juraj Duras $^{8}$, Petr Sedlacek ${ }^{9}$, Veronika Valkova ${ }^{2}$, Andrea Janikova ${ }^{3}$, Ludek Raida ${ }^{4}$, Pavel Jindra ${ }^{5}$, Pavel Zak ${ }^{6}$, Tomas Kozak ${ }^{10}$, Marie Trnkova ${ }^{1}$, Michal Karas ${ }^{5}$, Marek Trneny ${ }^{I}$

${ }^{1}$ General University Hospital in Prague, Prague, Czech Republic, ${ }^{2}$ Institute of Hematology and Blood Transfusiion, Prague, Czech Republic, ${ }^{3}$ Faculty Hospital Brno, Brno, Czech Republic, ${ }^{4}$ Faculty Hospital Olomouc, Olomouc, Czech Republic, ${ }^{5}$ Faculty Hospital Plzen, Plzen, Czech Republic, ${ }^{6}$ Faculty Hospital Hradec Kralove, Hradec Kralove, Czech Republic, ${ }^{7}$ Faculty Hospital Kralovske Vinohrady, Prague, Czech Republic, ${ }^{8}$ Faculty Hospital Ostrava, Ostrava, Czech Republic, ${ }^{9}$ Motol University Hospital, Prague, Czech Republic, ${ }^{10}$ Faculty Hospital Kralovske Vinohrady, Praha, Czech Republic

Background: Hematopoietic stem cell transplantation (HSCT) is an established procedure in lymphoma 
management. However, differences in patient's characteristics as well as frequency of HSCT indication in different lymphoma subtypes have been observed in the last decade. The aim of this study was retrospective analysis of HSCT for lymphomas in Czech Republic.

Methods: Data of adult patients transplanted between years 1993-2016 were retrospectively analyzed using EBMT database.

Results: Between 1993 and 2016, 2816 autologous HSCT (ASCT) were performed in 2651 patients (1511 men, $57 \%$ ) with different lymphoma subtypes. The median age was 49 years (range 18-75). Out of these, 2078 (78\%) were patients with non-Hodgkin lymphoma (NHL), 569 (21\%) with Hodgkin lymphoma (HL). The NHL group comprised of diffuse large B-cell lymphoma (DLBCL, $36 \%$ ), follicular lymphoma (FL, 18\%), mantle cell lymphoma (MCL, 16\%) and T-NHL (9\%). The frequency of ASCT in lymphomas increased from 1993 to 2000 and has been constant since 2000 (120-130 transplants per year). Differences in frequency of ASCT were observed among lymphoma subtypes - decreasing numbers of DLBCL and FL and increasing numbers of T-NHL and MCL, with ASCT as part of the induction therapy. Between 1996 and 2016 a total of 329 allogeneic HSCT (alloSCT) were performed in 319 patients (200 men, 63\%). Median age was 46 years (range 19-66). Out of these $257(81 \%)$ were patients with NHL, 61 (18\%) HL. The most common NHL subtypes were FL (27\%), MCL (22\%), T-NHL (22\%) and DLBCL (16\%). In the last 10 years the number of alloSCT for lymphoma is fluctuating around 20 per year. The median age at ASCT was significantly higher in the years 20102016 vs 1993-2000 [54.5 (18.1-74.8) vs. 40.9 (18.6-72.5), p $<0.0001$, fig 1], while the increase at alloSCT [46.5 (21.3$65.5)$ vs $41.6(20.3-64.5)]$ did not reach statistical significance $(\mathrm{p}=0.07)$. With median follow up for alloSCT, $5 \mathrm{y}$ probability OS for patient transplanted in the later period 2010-2016 was in relapsed DLBCL 25.7\%, in FL 77.4\%, in HL $51.0 \%$ and in MCL $45.7 \%, 5$ y OS for ASCT as part of first line therapy in the same period was in MCL $78.1 \%$ and in T-NHL $48.2 \%$. OS was significantly better in all patients who underwent ASCT in the years 2010-2016 vs 1993$2000(70 . \%$ vs. $57.5 \%, \mathrm{p}<0.0001)$ and there was a trend towards better OS in patients after alloSCT (with $44.7 \%$ vs $23.5 \%, \mathrm{p}=0.1084$ ) (fig 2).

Conclusions: HSCT remains important treatment modality for lymphomas in the era of targeted antibody and molecular therapy and we can transplant older patients due to better supportive treatment.

Acknowledgment: PROGRESS Q28-9 UK from the Czech Ministry of Education Youth and Sports

Disclosure: Nothing to declare

\section{P490}

Could salvage regimens intesification safely overcome the lymphoma disease resistance? A retrospective comparative study between DICEP [Dose intensified cyclophoshamide,/Etoposide/ Cisplatin) versus ESHAP (Etoposide/ Cisplatin/Cyrtarabine/ Methylprednisolone)

Panayotis Kaloyannidis $^{1}$, Eshrak Al Shaibani, Mohamad Darweesh $^{I}$, Nihad Mokhtar ${ }^{1}$, Rawan Omari', Solaf Kanfar $^{I}$, Enas Mutahar ${ }^{I}$, Ayman Ablulhassan ${ }^{I}$, Salman Harbi $^{1}$, Mohamad Kawari, Reem Khalili, Ann Estanislao $^{1}$, Heba Raslan ${ }^{1}$, Asif Moinuddin ${ }^{1}$, Ioannis Apostolidis $^{1}$, Khalid Al Anezi ${ }^{1}$, Hani Al Hashmi ${ }^{1}$

${ }^{1}$ King Fahad Specialist Hospital, Dammam, Saudi Arabia,

Background: Disease chemosensitivity to salvage treatment has been proven to be a major predictive factor for a favorable outcome after autologous stem cell transplantation (ASCT) for patients with refractory lymphomas. Therefore the importance of effective and safe salvageregimens is indisputable.

Methods: We retrospectively compared the outcomes in terms of safety and efficacy, in 67 (HL:36, NHL:31) patients, with a median age of $34.5(16-75)$ years, who received as $1^{\text {st }}$ salvage either DICEP [Cyclophoshamide $\left(1750 \mathrm{mg} / \mathrm{m}^{2}\right)$, Etoposide $\left(350 \mathrm{mg} / \mathrm{m}^{2}\right)$, Cisplatin $(35 \mathrm{mg} /$ $\left.\mathrm{m}^{2}\right)$, days $\left.1-3,(\mathrm{n}=23)\right]$ or the widely used regimen ESHAP $(n=44)$. Rituximab was additionally given to all CD-20 positive lymphoma patients. The statistical analysis based on the independent T-test, Kaplan Meir method and logrank test.

Results: The reason for salvage treatment was primary induction failure (PIF, $\mathrm{n}=34)$, early relapse $(<12$ months post induction-remission therapy $\mathrm{n}=14$ ) and late relapsed disease $(n=19)$. More specifically, $19 / 23$ patients $(83 \%)$ in the DICEP-group, and 29/44 patients (65\%) in the ESHAPgroup were assessed with PIF or early relapsed disease, however this difference was not statistically significant. Both regimens were well tolerated and no major organ toxicities were noticed. Eleven patients $(48 \%)$ from the DICEP-group, while only 4(10\%) from the ESHAP-group developed febrile infections. All patients were successfully managed with the appropriate treatment and only one, from the ESHAP-group, required for short period admission to the intensive care unit.

After 1 cycle of DICEP and 2 cycles of ESHAP the disease response was re-assessed by PET/CT scan. The overall response rate ( $>50 \%$ tumor reduction) was significantly superior for the DICEP-regimen, reaching $92 \%$ (21/23 patients) vs. 64\% (27/44 patients) for ESHAPregimen $(\mathrm{p}=0,003)$. Eleven patients $(48 \%)$ from the DICEP-group and 14(30\%) from the ESHAP-group 
achieved complete metabolic remission according to PET/ $\mathrm{CT}$ criteria $(\mathrm{p}=\mathrm{ns})$. The median hospitalization period was 20(5-25) days for the DICEP-group compared to 10(10-19) days for the ESHAP-group. However, for the ESHAPgroup, an additional median of 20(6-33) hospitalization days were required, since 12 of the non-responders patients received a $2^{\text {nd }}$ salvage before ASCT. The mobilization and stem cell collection was successful for both groups, though significant higher number of CD34+ cells were collected in the DICEP-group $\left(17.2 \times 10^{3} / \mathrm{kg}\right.$ vs. $\left.5.4 \times 10^{3} / \mathrm{kg}, \mathrm{p}=0,001\right)$.

All but two patients (due to refractory disease) underwent ASCT. Noticeably, the median period from $1^{\text {st }}$ salvage treatment to ASCT was significantly shorter for the DICEPgroup (64 vs. 128 days, $\mathrm{p}=0,013$ ), apparently because 12 non-responders patients from ESHAP-group treated with a $2^{\text {nd }}$ salvage. The 3 -years overall and progression free survival were similar for DICEP- and ESHAP-groups ( $95 \%$ vs. $88 \%$ and $70 \%$ vs $80 \%$ respectively). Two heavily pretreated patients from the ESHAP-group developed secondary myelodysplastic syndrome post ASCT

Conclusions: In our series of patients both regimens proved to be safe. Interestingly, despite the fact that more patients in DICEP-group had poor risk disease the DICEPregiment was significantly more effective, resulting thus in an earlier ASCT, less exposure to chemotherapeutic agents, that might led in less long-term toxicity. Nevertheless, prospective trials with large series of patients are needed to define the role of DICEP in the salvage treatment setting.

Disclosure: no conflict of interest

\section{P491}

Clinical significance of autologous hematopoietic stem cell transplantation with ex vivo purging using clinimacs in T-cell lymphomas

Seok-Goo Cho ${ }^{1}$, Young-Woo Jeon ${ }^{1}$, Seugyun Yoon ${ }^{1}, G i$ June Min', Sung-Soo Park', Silvia Park ${ }^{1}$, Jae-Ho Yoon ${ }^{1}$, Sung-Eun Lee ${ }^{I}$, Byung-Sik Cho ${ }^{I}$, Ki-Seong Eom ${ }^{1}$, YooJin Kim ${ }^{1}$, Hee-Je Kim ${ }^{1}$, Seok Lee ${ }^{1}$, Chang-Ki Min ${ }^{1}$, JongWook Lee ${ }^{1}$

${ }^{1}$ Catholic Hematology Hospital, Seoul St. Mary's Hospital, College of Medicine, The Catholic University of Korea, Seoul, Korea, Republic of

Background: Although autologous hematopoietic stem cell transplantation (auto-HSCT) is one of the best curative strategies for patients with chemosensitive T-cell lymphoma, major limitation remains a tumor contaminated graft-related relapse or residual disease after chemotherapy. Several purging methods were introduced in auto-HSCT for these limitations, however there are few studies of ex vivo purging of the autograft in lymphomas, especially T-cell lymphoma. Therefore, we retrospectively analyzed 59 consecutive T-cell lymphoma patients receiving auto-HSCT with/without ex vivo purging.

Methods: Among them, 33 patients underwent autograft manipulation with ex vivo purging by $\mathrm{CD} 34+$ cells selection using a CliniMACS device.

Results: With median follow-up duration of 42 months (range, 6-121 months), 3-year overall survival (OS; 73.8\% vs. $49.0 \%, \mathrm{p}=0.017$ ) and 3-year progression-free survival (PFS; $75.8 \%$ vs. $52.4 \%, \mathrm{p}=0.039$ ) in a purged and unpurged group, respectively. Transplant-related mortality was observed in both groups ( 2 patients of a purged group and 1 patient of an unpurged group). Neutrophil (10 vs. 9 days, $\mathrm{p}=0.240$ ) and platelet ( 30 vs. 24 days, $\mathrm{p}=0.055$ ) recovery were similar in both group and there was no engraftment failure. On subgroup analysis according to upfront and salvage auto-HSCT, while survival outcomes were improved by stem cell purging in the upfront autoHSCT (OS with $\mathrm{p}=0.039$ and PFS with $\mathrm{p}=0.047$ ), there were no different survival outcomes in salvage auto-HSCT. The unmanageable late-infectious complications were few in both groups except for predominantly cytomegalovirus reactivation in a purged group (3 vs. 1 patient).

Conclusions: Although cohort was a small number, ex vivo graft-purging method was feasible and safe in T-cell lymphomas. And this purging strategy observed the more favorable survival outcomes in the upfront auto-HSCT than salvage setting. Therefore, further randomized studies are needed to determine the firm efficacy of $\mathrm{CD} 34+$ purification with the large number of patients in auto-HSCT for T cell-lymphomas.

Disclosure: Nothing to declare

\section{P492}

Nivolumab-based regimens in relapsed or refractory non hodgkin lymphomas: The role of hematopoietic stem cells transplantation

Natalia Mikhailova ${ }^{1}$, Olesya Smykova ${ }^{1}$, Kirill Lepik ${ }^{1}$, Elena Kondakova ${ }^{1}$, Evgeniya Borzenkova ${ }^{1}$, Yuriy Zalyalov $^{1}$, Liliya Stelmakh ${ }^{1}$, Elena Darskaya ${ }^{1}$, Ivan Moiseev ${ }^{1}$, Boris Afanasyev ${ }^{1}$

${ }^{1}$ First I. Pavlov State Medical University of St. Petersburg/ Raisa Gorbacheva Memorial Institute of Children's Oncology, Hematology and Transplantation, Saint Petersburg, Russian Federation

Background: The outcome of patients with relapsed or refractory Non Hodgkin Lymphomas ( $r / r$ NHL) remains dismal and therapeutic options for these patients are limited. Nivolumab-based regimens may be promising treatment for this group of patients. 
Methods: We analyzed data of 18 patients with r/r NHL, among them n11 with Diffuse large B-cell lymphoma (DLBCL), n5 with Primary mediastinal B-cell lymphoma (PMBCL), n1 with Gray zone lymphoma (GZL) and n1 with Gamma-Delta peripheral T-cell lymphoma (PTCL), who received nivolumab-based regimens. The median age was 37 years (range, 18 - 64 years). Most of the patients n14 (78\%) had a primary chemoresistant disease, the rest patients $n 4(22 \%)$ had a relapse. The median of lines of prior therapy was 3 lines (range, 2-6 lines). All sixteen patients with DLBCL and PMBCL received 1 - 3 cycles of nivolumab in combination with bendamustine, gemcitabine and rituximab (BeGeRN). The patient with GZL received 5 cycles of nivolumab in combination with brentuximab vedotin and EPOCH. And the patient with PTCL received 10 cycles of nivolumab monotherapy.

Results: At median follow up 8 months (3-16) objective response (OR) after nivolumab-based regimens was noted in $\mathrm{n} 10(56 \%)$ patients, complete response (CR) and partial response (PR) in $\mathrm{n} 9(50 \%)$ and $\mathrm{n} 1$ (6\%) patients, respectively. CR observed in $\mathrm{n} 4$ patients with DLBCL, n3 with PMBCL, n1 with GZL, n1 with PTCL. And PR observed in 1 patient with DLBCL. Two responding patients with DLBCL underwent auto-HSCT. And four responding patients (n1 DLBCL, n1 PMBCL, n1 GZL, n1 PTCL) received allogeneic hematopoietic stem cells transplantation (allo-HSCT). The median duration of response for all n10 patients with OR was 5 (range: 3-16 +) months. Among n4 patients who achieved OR without HSCT, only $n 1$ remain in CR. Two patients who received auto-HSCT had a relapse. One patient with DLBCL improved the response after allo-HSCT from PR to CR, and all four patients with allo-HSCT remain in CR. The probabilities of 1-year OS and PFS rates were $49 \%$ and $31 \%$, respectively.

Conclusions: Nivolumab-based regimens can lead to an objective response in $56 \%$ patients with r/r NHL. However, the durability of response to therapy is not long. Nivolumab- based regimens can be used as bridge to alloHSCT

Disclosure: There are no conflicts of interest to disclose

\section{P493}

\section{Laboratory and imaging markers for prediction of outcome in aggressive non-hodgkin lymphoma undergoing autologous stem cell transplantation}

\section{Ana Pilar Gonzalez-Rodriguez ${ }^{1}$, Joud Zanabili ${ }^{1}$, Pilar} Palomo $^{1}$, Ana Julia Gonzalez-Huerta ${ }^{I}$, Aida Menendez Parron $^{2}$, Javier Zambrano ${ }^{1}$, Soledad Gonzalez-Muñiz ${ }^{1}$

${ }^{1}$ Hospital Universitario Central de Asturias, Hematology. Stem Cell Trasplantation, Oviedo, Spain, ${ }^{2}$ Universidad de
Oviedo, Hematology. Stem Cell Trasplantation, Oviedo, Spain

Background: Patients with aggressive non-Hodgkin lymphoma (NHL) who relapse after autologous stem cell transplantation have a dismal outcome and could benefit from radiotherapy, allogeneic stem cell transplantation or experimental treatments. Systemic inflammatory parameters at diagnosis have demonstrated to be useful to predict lymphoma evolution.

Methods: We conducted a retrospective review of patients with aggressive NHL who underwent autologous stem cell transplantation (ASTC) to evaluate the relationship between LDH, $\beta 2$-microglobulin, inflammatory parameters (lymphocyte (ALC) and monocyte count (AMC), ferritin or C-reactive protein) and imaging techniques before and on day +100 post-ASTC and progression free survival (PFS), as well as the role of residual disease directed radiotherapy (RT).

Results: One hundred and sixty patients with aggressive NHL received ASCT as consolidation treatment in our center between 2000 and 2017. The most common diagnosis was diffuse large B-cell lymphoma (DLBCL). One hundred and nine patients received upfront ASCT for high risk DLBCL (defined as age-adjusted IPI 2-3) $(\mathrm{n}=28)$ or for having received two or more lines to obtain first complete remission $(n=28)$, for T-cell lymphoma $(n=27)$ and for mantle cell lymphoma $(n=26)$. The rest was performed in relapsed lymphomas. Forty-seven patients (29\%) relapsed and PFS was 110 months.

Pretransplant response was evaluated with CT scan in 66 patients (11 of this with partial remission (CT-PR) and 94 patients were evaluated with FDGPET/CT (27 were pretransplant positive (PET1); of these, 18 patients maintained positivity at day 100 after ASTC (PET2). PFS in patients with CT-PR was 46 months, in PET1 positive ones 99 months and in PET2 positive ones 21 months. Univariate analysis showed PET2 positivity as the most accurate predictor of relapse (HR 3,13, $\mathrm{p}=0,004)$ followed by AMC at day $+100(\mathrm{HR} 1,002, \mathrm{p}=0,13)$, albumin at day +100 (HR 0,93, p=0,03), LDH at day $+100(\mathrm{HR} 1,002, \mathrm{p}<$ 0,001) and pretransplant ALC/AMC ratio (HR 1,068 $\mathrm{p}=0,008)$. Multivariate analysis only demonstrated an association with PET2 positivity (HR 7,78) $\mathrm{p}<001$ and LDH in day +100 (HR 1,008) $\mathrm{p}=0,003$ with PFS.

Five and ten years overall survival were $61 \%$ and $37 \%$ in PET2 negative patients vs 25 and $5 \%$ in PET2 positive ones $(\mathrm{p}<0,01)$.

Eight out of 19 patients with PET2 positivity did not relapse. Salvage radiation therapy was used in 7 patients with positive residual mass and 5 of them did not relapse. Two patients relapsed: one patient had residual mass and 
another had remote affectation from primary site and could be considered as progression before day +100 .

Conclusions: Post ASCT FDGPET/ CT is superior to conventional $\mathrm{CT}$ in predicting outcome in aggressive lymphoma after ASTC.

Pre and post ASCT systemic inflammatory parameters didn't help to improve the relapse risk prediction.

Addition of consolidative RT after ASTC has demonstrated improvement in PFS in patients with PET positivity. It would be neccesary to develop randomized trials to assess the role of RT in residual disease in advanced aggressive NHL with insufficient response to systemic treatment with PET response evaluation.

Disclosure: Nothing to declare

\section{P494}

Long term outcome of patients with lymphoid malignancy who underwent high dose chemotherapy followed autologous hematopoietic cell transplantation at a single institution over 20 years Joanna Romejko-Jarosinska ${ }^{1}$, Ewa Paszkiewicz-Kozik ${ }^{1}$,
Lukasz. Targonski ${ }^{1}$, Lidia Poptawska ${ }^{1}$,Jan Walewski

${ }^{1}$ Maria Sklodowska-Curie Cancer Institute and Oncology Center, Warszawa, Poland

Background: High dose chemotherapy (HDT) and autologous hematopoietic cell transplantation (auto-HCT) is a standard of care for relapsed/refractory lymphoma patients (pts) or it is used as a consolidation for myeloma and high risk lymphoma patients in first line treatment. We retrospectively evaluated long-term outcome including late effects and risk factors in patients with lymphoid malignancy who underwent auto-HCT.

Methods: We collected data from 926 consecutive patients with Hodgkin lymphoma (HL) $(\mathrm{n}=326)$, aggressive B lymphoma (DLBCL) $(n=186)$, myeloma $(n=199)$, indolent lymphoma $(\mathrm{n}=30)$, mantle cell lymphoma $(\mathrm{n}=122)$ and peripheral $\mathrm{T}$ cell lymphoma $(\mathrm{n}=63)$ who underwent auto-HCT at our institution between 1997 and 2017. At transplant median (range) age was 46 (17-71) years, clinical stage III/IV was found in 499 of lymphoma pts, complete remission, partial remission and stable/ progressive disease occurred in 462(50\%), 397 (43\%), 67 (7\%) pts, respectively. BEAM regimen was used in 549 pts (59\%), Mel200 in 200 pts (23\%) and other myeloablative regimens in 177 pts (18\%).

Results: Median time to progression was 29 months (range 1 - 222). Five year and 10-year progression free survival (PFS) were 62\% [95\%C.I.(59\%, 67\%)] and 45\% [95\%C.I. $(41 \%, 49 \%)]$, respectively. With a median of 47 month (range:1- 232) follow-up, 5-year and 10-year overall survival
(OS) from the date of transplant were 67\%[95\%C.I. $(64 \%, 70 \%)]$ and 58\%[95\%C.I.(54\%,62\%)], respectively. Partial remission or stable disease at transplant, clinical stage III or IV, and age more than 60 , were identified as risk factors associated with inferior OS and PFS in univariate and multivariate analysis. Histopathologic diagnosis was not a risk factor for OS and PFS ( $\mathrm{p}=\mathrm{NS})$. The outcome of patients who underwent auto-HCT between 1997-2003 was inferior to the outcome of patients treated in 2004-2010 or 2011-2017. 5year OS was $53 \%, 68 \%, 73 \%(\mathrm{p}<0.001)$ and 5 year PFS was $43 \%, 54 \%, 55 \%(\mathrm{p}<0.01)$, respectively. We recorded 37 (4\%) cases of second primary cancer (18 solid tumors and 19 hematologic cancers). Acute cardiotoxicity occurred in 5 patients from 1 to 15 years after transplant, and required heart transplant in 2 patients. 326 patients $(35 \%)$ died. The main causes of death were progressive disease in 271 pts (89\%), second primary malignancy in 22 pts $(6.7 \%)$ Treatment related mortality was $0.8 \%$ ( 8 pts), and mortality within 100 days was $22(2,4 \%)$.
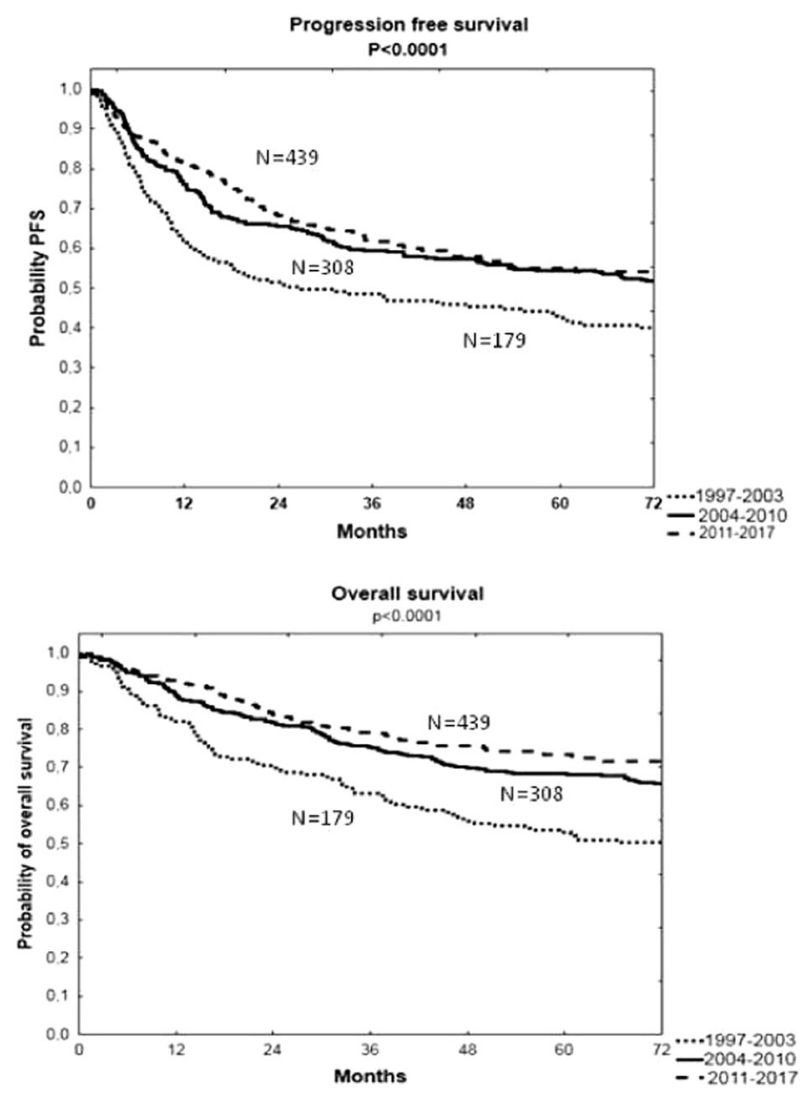

[[P494 Image] 1. PFS and OS in patients underwent HDT and auto-HCT 1997-2003, 2004-2010, 2011-2017]

Conclusions: More than $45 \%$ of patients who underwent HDT and auto-HCT had long term survival without progressive disease. Older age, non-complete remission at 
transplant, advanced stage are associated with poor outcome. Patients recently transplanted had a better outcome than patients transplanted before 2004 .

Disclosure: Nothing to declare

\section{P495}

Outcomes after haploidentical and matched related HSCT in lymphoma do not differ significantly: A single center study

Nadira Durakovic ${ }^{1,2}$, Zinaida Perié ${ }^{1,2}$, Lana Desnica ${ }^{2}$, Ranka Serventi-Seiwerth ${ }^{2}$, Sandra Bašić Kinda ${ }^{2}$, Ivo Radman-Livaja ${ }^{2}$, Alen Ostojić ${ }^{2}$, Ante Vulićc ${ }^{2}$, Dražen Pulanić, $^{1,2}$, Pavle Rončević ${ }^{2}$, Zorana Grubić, ${ }^{2}$, Igor Aurer $^{1,2}$, Radovan Vrhovac ${ }^{1,2}$

${ }^{1}$ University of Zagreb, School of Medicine, Internal Medicine, Zagreb, Croatia, ${ }^{2}$ UHC Zagreb, Zagreb, Croatia

Background: Allogeneic HSCT still offers patients with relapsed/refractory lymphoma the best chance of long-term survival. In most such patients timing of HSCT is crucial, therefore a related donor is preferred. We analyzed acute and chronic GVHD incidence, relapse and overall survival, but also time to immunosuppression (IS) discontinuation and hematopoietic recovery comparing transplantation using haploidentical (haplo) and matched related donors (MRD) in single center in this indication.

Methods: In the time period between 5/2011 and 5/2018 at UHC Zagreb, Croatia, 10 MRD and 13 haplo transplantations in lymphoma were done, 15 for Hodgkin and 8 for NHL. All patients transplanted from haploidentical donors received PTCY. Data were computed using the $\mathrm{R}$ package. The probability of GVHD was calculated using the cumulative incidence method and subgroups were compared using the Gray test.

Results: Median age was 38 (19-62) years; 36 (19-62) in haplo and 41 (25-56) in MRD group. Four patients were in $\mathrm{PR}$ and 9 in CR in haplo group, while in MRD group 5 patients were in CR and 5 in PR. In haplo group 12 patients (92\%) received bone marrow (BM) and only 1 (8\%) peripheral blood stem cells (PBSC). In MRD group all patients received PBSC. All patients in haplo group received NMA ("Baltimore") conditioning with PTCY while in MRD group 9 patients $(90 \%)$ received FluBu2ATG, and only one received FluTBI as conditioning protocol. In haplo group $85 \%$ patients were previously treated with autologus transplantation, $80 \%$ in MRD group. There was no significant difference in time to IS discontinuation, 149 and 155 days in haplo and MRD group, respectively. Patients after haplo recovered slower, recovering ANC after 22.6 days (95\% CI, 17.7-27.4) and $16.5(95 \%$ CI, 13.9-19) $(\mathrm{p}=0.04)$ and recovering platelets after 34.6 days (95\% CI, 15.5-53.7) and 10.8 (95\% CI, 9.212.4) $(\mathrm{p}<0.01)$ in haplo and MRD group.

With a median follow up of 469 days, overall survival was $83 \%$ (95\% CI, 63-100) in haplo and $80 \%$ (95\% CI, 59$100)$ in MRD group. TRM was $8 \%$ in haplo and $20 \%$ in MRD group. Cumulative incidence of aGVHD II-IV was $31 \%$ (95\% CI, 9-56) and 32\% (95\% CI, 3-49) in haplo and MRD group, respectively $(\mathrm{p}=0.84)$. Cumulative incidence of cGVHD requiring treatment was $22 \%$ (95\% CI, 3-54) and MRD 20\% (95\% CI, 0-62) in haplo and MRD group, respectively $(\mathrm{p}=0.46)$. All cases of $\mathrm{cGVHD}$ developed after DLI. Cumulative incidence of relapse was $32 \%$ (95\% CI, 958 ) and 38\% (95\% CI, 7-72) for haplo and MRD group, respectively ( $\mathrm{p}=0.77)$.

Conclusions: We found no significant difference in overall survival, relapse incidence, aGVHD and cGVHD incidence between these two groups. Hematopoietic recovery was slower after haploidentical transplantation, but it did not influence TRM as it was higher after MRD. Even though limited in number, this data contribute to the growing body of evidence that use of haploidentical donors, particularly in lymphoma setting, is as worthy as using matched related donors and should be at least second choice in donor selection, and in older patients (with older donors) probably the first one.

Disclosure: Nothing to declare.

\section{P496}

Adjuvant involved field radiotherapy post autologous stem cell transplantation for refractory/relapsed lymphomas results in favorable outcome with low toxicity: A single center experience

Panayotis Kaloyannidis ${ }^{1}$, Eshrak Al Shaibani, Rawan Omari $^{1}$, Eman Debawy ${ }^{1}$, Deia Awami ${ }^{1}$, Solaf Kanfar ${ }^{1}$, Mohamad Darweesh ${ }^{1}$, Enas Mutahar ${ }^{1}$, Ayman Ablulhassan ${ }^{1}$, Nihad Mokhtar ${ }^{1}$, Khalid Bakhit ${ }^{1}$, Salman Harbi $^{1}$, Jenifer Bacal', Ahmed Al Buali, Afra Dayel, Ioannis Apostolidis ${ }^{I}$, Khalid Al Anezi ${ }^{l}$, Hani Al Hashmi ${ }^{I}$

${ }^{1}$ King Fahad Specialist Hospital, Dammam, Saudi Arabia,

Background: Involved field radiotherapy (IFRT) to previous bulky or localized residual disease, is a widely used treatment approach to minimize the risk of relapse post autologous stem cell transplantation (ASCT). However, the proper time for irradiation treatment remains controversial. Adjuvant IFRT (adj-IFRT) in pre-ASCT period could cause undesirable toxicity which might delays or even cancel the ASCT resulting in increased risk of relapse, or could affect the marrow environmental and marrow niche resulting thus in impaired engraftment. On the other hand, the ajd-IFTR in the early post-ASCT period, upon marrow recovery, offers a 
potential advantage by delivering irradiation after sufficient disease response, without affecting the engraftment. In this retrospective study we evaluated the safety and efficacy of the IFRT as adjuvant treatment in patients who had previously treated with ASCT for relapsed or refractory lymphomas.

Methods: Twenty-three patients (Hodgkin $=14$, NonHodgkin=9), aged of 34(16-76) years, underwent ASCT, for primary refractory $(n=15)$ or relapsed $(n=8)$ disease. Patients who had bulky disease at the time of relapse or those with residual mass post salvage treatment, were considered as candidates for adj-IFRT, early (within 2-3 months) after documentation of autologous stem cells engraftment. All patients proceeded to ASCT with chemosensitive disease after a median of 2 lines of salvage therapy. At the time of ASCT 20 patients (80\%) had residual disease while $4(20 \%)$ evaluated to be in complete remission. The preparative regimens were: single-agent Melphalan ( $\mathrm{n}=9)$, Busulfan-Etoposide-Melphalan $(\mathrm{n}=7)$, BEAM $(\mathrm{n}=5)$ and Bendamustin-Etoposide-CytarabineMelphalan $(\mathrm{n}=3)$. Filgrastim was given till neutrophills recovery, while prophylaxis against bacteria, fungus, viruses and PCP were administered till the completion of adj-IFRT.

Results: All patients engrafted promptly and successfully. No patient experienced any severe toxicity or active infection before adj-IFTR. Though our plan was to proceed with adj-IFRT within 3 months post ASCT, finally it was delivered after a median of 4.5 (2-7) months; the median radiation dose was 30(24-36) Gy. Ten patients received radiotherapy in the mediastinum, 9 in the abdomen/pelvis/ inguinal area 3 in the neck, and 1 in the left leg. The adjIFRT was well tolerated. No patient experienced toxicity grade $>3$ and none required hospitalization. Currently, after a median follow-up of 2(2-5) years, 19/23 patients are alive and well; the 5-years overall and progression free survival rates are $75 \%$ and $55 \%$ respectively. Four patients died; 2 due to relapsed disease and 2 heavily pretreated patients due to secondary myelodyspalstic syndrome

Conclusions: In our study, the adj-IFRT in the early post transplant period demonstrated a safe and well-tolerated profile. Taking into consideration the poor risk status of our patients (residual disease post salvage regimen or bulky disease at the time of relapse), the promising overall and progression free survival rates suggested that adj-IFRT post ASCT is also an effective approach. Well designed trials are needed to clarify the role and the appropriate time of radiotherapy in the ASCT setting

Disclosure: No conflict of interest

\section{P497}

Adverse prognostic impact of pre-transplant neutrophil/ lymphocyte ratio in lymphoproliferative disorders
Asena Dikyar ${ }^{1}$, Zeynep Arzu Yegin ${ }^{1}$, Ferda Can ${ }^{1}$, Görkem Yazıcl $^{1}$, Zübeyde Nur Özkurt ${ }^{1}$, Ramazan Öcal ${ }^{2}$, Münci Yağcl ${ }^{I}$

${ }^{1}$ Gazi University Faculty of Medicine, Ankara, Turkey, ${ }^{2}$ Gülhane Training and Research Hospital, Hematology, Ankara, Turkey

Background: The role of inflammatory cascade in tumor microenvironment has been demonstrated in several studies. As a part of this issue, elevated neutrophil/lymphocyte ratio (NLR) was shown to be associated with an adverse prognosis, particularly in solid tumors. The aim of this study is to determine the impact of pre-transplant NLR on early transplant complications, as well as post-transplant relapse and survival.

Methods: A total of 119 lymphoma patients [median age: 49(18-71) years; male/female: 64/55] who underwent autologous hematopoietic stem cell transplantation (HSCT) were included in this retrospective study.

Results: The initial diagnosis was Hodgkin lymphoma (HL) in 35 (29.4\%), B-cell non-Hodgkin lymphoma (NHL) in $67(56.3 \%)$ and T-cell NHL in $17(14.3 \%)$ patients. Of 116 patients who were evaluated for pretransplant disease status, 52 patients $(44.8 \%)$ were in complete remission, 45 patients $(38.8 \%)$ were in partial remission and 19 (16.4\%) patients had refractory disease. Median pre-transplant NLR was found to be 3.42(0-97). When the study population was divided into two subgroups as "low-" and "high-NLR", based on median NLR value, number of febrile days were found to be relatively higher in the low-NLR group $(p=0.051)$. A positive correlation was demonstrated between NLR and lactate dehydrogenase levels $(\mathrm{r}=0.218 ; \mathrm{p}=0.03)$; and NLR and ferritin levels $(\mathrm{r}=0.277 ; \mathrm{p}=0.003)$. At a median follow-up of 24 (1-264) months, overall survival (OS) was found to be better in the low-NLR group without statistical significance [25(1-264) vs 13(1-91) months; $\mathrm{p}=0.069]$. In univariate analysis, pre-transplant NLR represented a significant impact on OS $(\mathrm{p}=0.021)$. Other prognostic factors were age $(\mathrm{p}=0.041)$, platelet engraftment $(p=0.018)$, post-transplant relapse $(p=0.009)$ and pre-transplant ferritin level $(\mathrm{p}<0.001)$. The permanent impact of ferritin on OS was confirmed in multivariate analysis $(\mathrm{p}=0.008)$.

Conclusions: In this study, an adverse impact of elevated pre-transplant NLR on OS was demonstrated in autologous HSCT recipients with lymphoma. As a predictor of prognosis, NLR may be considered as a safe and cost-effective parameter. Further studies are required in order to use this predictor in routine clinical practice. 


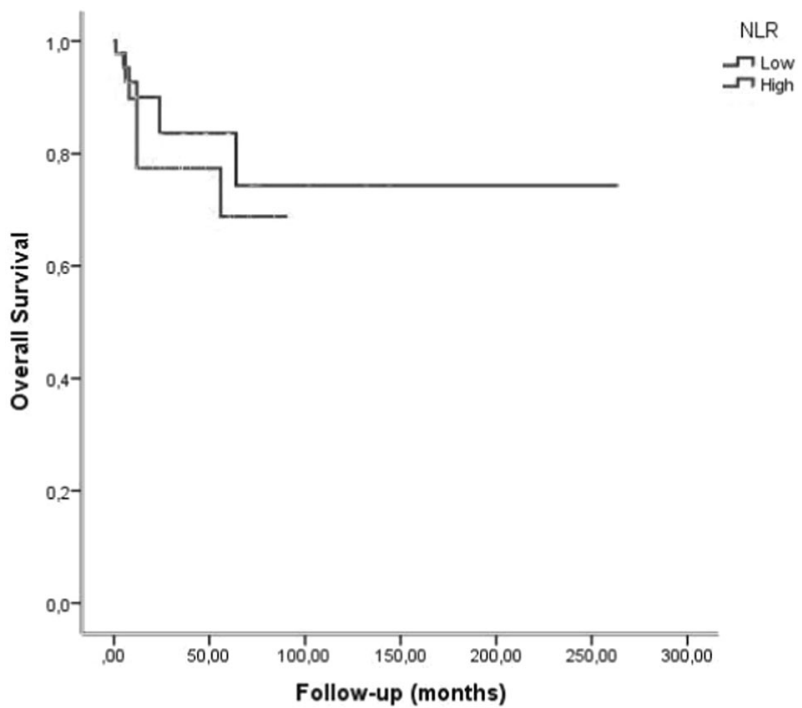

[[P497 Image] 1. Impact of Pre-transplant NLR on Overall Survival $(p=0.021)]$

Disclosure: Nothing to declare

\section{P498}

Safety and efficacy of 4 cycles of brentuximab vedotinas consolidation after autologous peripheral stem cell transplantation in relapsed/refractory Hodgkin's lymphoma

Jeries Kort ${ }^{1}$, Amanda Chidiac ${ }^{1}$, Radwan Massoud ${ }^{1}$, Rola El Sayed ${ }^{I}$, Nohra Ghaoui ${ }^{1}$, Rita Nehme ${ }^{I}$, Ali Bazarbachi ${ }^{I}$, Jean El-Cheikh ${ }^{I}$

${ }^{1}$ American University of Beirut, Beirut, Lebanon

Background: BrentuximabVedotin(BV) is a chimeric anti CD30 IgG1 antibody, conjugated to synthetic antitubulinmomomethylauristatin. $\mathrm{BV}$ is approved for the treatment of classical Hodgkin lymphoma (HL) in relapse either after autologous stem cell transplantation (ASCT) or after two lines of combination chemotherapy in transplant ineligible patients. The AETHERA trial revealed increased PFS when BV is used as maintenance therapy for 16 cycles in high risk patients after ASCT. However, this schedule is associated with a high cost and significant toxicity particularly in term of peripheral neuropathy.

Our primary objective is to assess the efficacy of 4 cycles Brentuximab as consolidation therapy after ASCT for relapsed/refractory (R/R) HL. Secondary objectives include side effects, progression free survival (PFS), and overall Survival (OS).

Methods: This is a retrospective single center analysis approved by the IRB of the American University of Beirut
Medical Center. We included in this study consecutive patients with R/R HL who underwent ASCT between 2014 and 2018, and received BV consolidation post-ASCT.

Results: We identified 18 consecutive adult patients with $\mathrm{R} / \mathrm{R}$ HL treated with $\mathrm{BV} 1.8 \mathrm{mg} / \mathrm{kg}$ IV every 3 weeks as consolidation therapy after ASCT. The indications for BV consolidation was primary refractory disease in 11 patients $(61 \%)$, early relapse in 6 patients (33\%) (after a median time of 10 months; range, 3-11) andextranodalinvolvement in one patient $(6 \%)$.

The median number of lines of therapy pre-ASCT was 3 (range, 2-5). The median time to BV initiation post-ASCT was 76 days (range, $35-188$ ). Patients received a median of 4 cycles (range, 3-4) of BV post-ASCT. After a median follow up of 30 months (range, 8-50), five (28\%) patients relapsed after ASCT. The median time to relapse was 7 months (range, 4-21). Median PFS and OS were not reached. We did not observe any significant toxicities during or after therapy.

Conclusions: 4 cycles of BV consolidation after ASCT seem to be safe and effective in preventing relapse, however our findings need to be confirmed with larger prospective studies.

\begin{tabular}{ll} 
Relapse rate & $5(28 \%)$ \\
Time (months) to progression post ASCT & $7(4-21)$ \\
Relapse while on BV & $2(40 \%)$ \\
Median follow up (months) from diagnosis & $47(20-66)$ \\
Median follow up (months) from transplant & $30(8-50)$ \\
2 years PFS & $70 \%$ \\
2 years OS & $100 \%$ \\
Median PFS & $\mathrm{NR}$ \\
Median OS & $\mathrm{NR}$ \\
\hline
\end{tabular}

[[P498 Table] 1. Outcomes]

Disclosure: Nothing to declare

\section{P499}

Disease status at transplantation determines outcome of allogeneic HSCT for patients with Hodgkin's lymphoma

Andrzej Frankiewicz ${ }^{1}$, Tomasz Czerw ${ }^{1}$, Wlodzimierz Mendrek ${ }^{I}$, Jacek Najda ${ }^{I}$, Malgorzata Ociepa ${ }^{I}$, Maria Sadus-Wojciechowska ${ }^{I}$, Malgorzata SobczykKruszelnicka $^{1}$, Jerzy Holowiecki ${ }^{1}$, Sebastian Giebel ${ }^{I}$

${ }^{1}$ Center of Oncology - Institute, Maria Sklodowska Curie, Gliwice Branch, Gliwice, Poland

Background: The prognosis of patients with relapsed/ refractory Hodgkin's lymphoma who fail salvage 
chemotherapy or who progress after autoHSCT is poor. Despite introduction of novel agents like brentuximab vedotin (BV) or nivolumab, alloHSCT appears the most effective treatment option with curative potential. The goal of this study was to evaluate efficacy of alloHSCT for HL, including patients pre-treated with novel agents.

Methods: Between years 2012-2018, 45 patients (including 23 males) with HL were treated with alloHSCT in MSC Institute of Oncology in Gliwice, Poland. Median age was 32 (19-57) years. Median lines of preceding chemotherapy was $4(2-8) ; 34(76 \%)$ patients had been pre-treated with autoHSCT, $24(53 \%)$ with radiotherapy, $17(38 \%)$ with BV, $7(16 \%)$ - with nivolumab. Disease status at alloHSCT was as follows: CR-19, PR-10, NR-16. Patients were treated with HSCT from either HLA-matched sibling donor (MSD, $\mathrm{n}=21$ ), unrelated donor (URD, $\mathrm{n}=18$ ) or haploidentical donor $(\mathrm{n}=6)$. Conditioning was myeloablative in $21(47 \%)$ cases. Peripheral blood was used as a source of stem cells.

Results: All but one patient engrafted with median time of neutrophil recovery of 14 (9-20) days. The incidence of grade 2-4 and grade 3-4 acute GVHD was $16 \%$ and $11 \%$, respectively, while the incidence of chronic GvHD was $43 \%$. The probabilities of OS and PFS at 2 years were $54 \%$ $(+/-12 \%)$ and $55 \%(+/-8 \%)$, respectively. The incidences of progression and transplant-related mortality were $31 \%$ and $14 \%$, respectively. The $2 \mathrm{y}$ PFS rates were $42 \%$ for MSD, $72 \%$ for URD and $62 \%$ for haploidentical donors. In a univariate analysis PFS was affected by recipient gender (female $-75 \%$, male $-38 \%, \mathrm{p}=0.009$ ) and disease status at alloHSCT (CR - 78\%, PR - 47\%, NR - 38\%, $\mathrm{p}=0.09)$. In a multivariate model the disease status other than $\mathrm{CR}$ was the only factor associated with increased risk of treatment failure (reverse PFS) - $\mathrm{HR}=3.13$, 95\%CI 1.45-6.76, $\mathrm{p}=0.004$. Neither donor type nor conditioning affected long-term outcome.

Conclusions: Results of alloHSCT for patients with relapsed/refractory HL are determined by disease status at transplantation. Efforts should be done to reduce tumour burden before transplantation, optimally to achieve CR.

Disclosure: Nothing to declare

\section{P500 \\ Brentuximab vedotin and anti-PD1 blockade optimize survival in chemo-refractory hodgkin lymphoma patients}

Ioanna Sakellari, Zoi Boussiou ${ }^{1}$, Eleni Gavriilaki ${ }^{1}$, Aris Chatziioannidis $^{I}$, Ioannis Batsis ${ }^{I}$, Despina Mallouri ${ }^{1}$, Varnavas Constantinou ${ }^{1}$, Michail Iskas ${ }^{1}$, Niki Stavroyianni, Antonia Syrigou ${ }^{1}$, Anastasia Marvaki, Maya Pilavaki ${ }^{I}$, Styliani Papaemmanuil, Achilles Anagnostopoulos ${ }^{1}$
${ }^{1}$ George Papanicolaou General Hospital, Thessaloniki, Greece

Background: Brentuximab vedotin (BV), nivolumab and pembrolizumab have been assigned to chemorefractory Hodgkin lymphoma treatment. Impact of these agents on disease-free-survival after autologous stem cell transplantation (ASCT) remains under investigation. Aim of the study is to compare BV- and nivolumab-treated patients with a control group.

Methods: Clinical characteristics and outcomes of chemo refractory Hodgkin lymphoma patients who underwent ASCT during 2011-2017.

Results: A total of 56 patients (33 men; 21 women, median age 37 years old, 19-65) were treated with BV: 24 pre-transplant, 30 post-transplant and 7 pre- and posttransplant. Pre-transplant BV patients had primary refractory disease or early relapse in the majority $(92 \%)$. Post-transplant treatment occurred in the context of relapsed/refractory disease in 27 patients; $12(40 \%)$ had an allogeneic stem cell transplant. Among them, 6 had additional chemotherapy and 2 nivolumab, gaining a complete metabolic response. In the 15 rest of patients, change of treatment due to eventual BV failure occurred. $\mathrm{BV}$ was administered as a maintenance treatment in 10 patients. In six of them BV had already been administered pre-transplant as well. Out of maintenance treatment patients, 2 relapsed and subsequently received nivolumab. Two patients died due to prior chemotherapy complications, whereas 13 are currently on nivolumab treatment. PET-based response was available in 6 patients, 4 having a complete metabolic response (CMR) and 2 a partial metabolic response. Stable disease was achieved by CTbased response in the rest patients. No major toxicities were observed. One patient presented with grade 2 asymptomatic hypothyroidism and one with grade 3 anemia attributed to non-inflammatory upper gastrointestinal blood loss.

In total, 20 patients received anti-PD1 treatment, all post BV failure. With a median follow-up of 34.3 (1.5-202.2) months, 5-year overall survival (OS) was $65.9 \%$ in patients treated only with $\mathrm{BV}$ compared to $78.2 \%$ in patients treated with additional anti-PD1 treatment $(\mathrm{p}=0.356$, Figure). Median OS for patients treated only with BV was 113.5 months, whereas median OS has not been reached for patients that received anti-PD1 treatment.

Conclusions: BV pre or post-transplant and anti-PD1 treatment post-transplant after BV failure have outstanding results in chemo refractory lymphoma patients. Treatment sequence in allogeneic transplantation eligible patients remains to be further studied.

Disclosure: Nothing to declare 


\section{P501}

Abstract withdrawn.

P502

Durable long-term survival in patients with lymphoid neoplasms and low disease risk index post reducedintensity allogeneic transplantation

Ioanna Sakellari, Zoi Bousiou ${ }^{I}$, Andrianna Lazaridou, Eleni Gavriilaki', Ioannis Batsis ${ }^{1}$, Despoina Mallouri, Michail Iskas ${ }^{1}$, Christos Demosthenous ${ }^{1}$, Niki Stavroyianni, Anastasia Marvaki ${ }^{1}$, Magda Pilavaki ${ }^{1}$, Stylianni Papaemmanouil ${ }^{1}$, Achilleas Anagnostopoulos ${ }^{I}$

${ }^{1}$ G. Papanicolaou Hospital, Thessaloniki, Greece

Background: Allogeneic hematopoietic cell transplantation (allo-HCT) with reduced-intensity conditioning (RIC) has been used in heavily pretreated lymphoma patients with the promise of decreased treatment-related mortality. Despite improvements in outcomes of patients with lymphoid neoplasms, several new agents emerge as potential therapies. Therefore, we aimed to describe our long-term experience in patients with Hodgkin (HL), non-Hodgkin lymphomas (NHL) and chronic lymphocytic leukemia (CLL) post allo-HCT.

Methods: In this retrospective study, we enrolled consecutive patients who underwent allo-HCT for lymphoid neoplasms in our institution (2001-2018).

Results: In total, 50 patients (male:female $=35: 25$ ) aged 36 (15-64) years, underwent allo-HCT for HL $(n=24)$, NHL $(n=21)$ and CLL $(n=5)$. The majority of patients were diagnosed at stage IV (48\%); $34 \%$ had bone marrow involvement and $66 \%$ had undergone autologous HCT. Most patients were heavily pretreated (median lines $=4$, range 1-11), 21 of them had received more than 4 treatment lines and at the time of transplantation only 14 had complete response, while 9 had partial response and 27 were refractory. According to Disease-Risk Index (DRI), patients were stratified at low $(\mathrm{n}=11,23.4 \%)$, intermediate $(\mathrm{n}=12$, $25.5 \%)$, high $(n=20,42.6 \%)$ or very high $(n=4,8.5 \%)$ category. Among patients with Hodgkin lymphoma, Brentuximab vedotin was administrated in 7 and 4 of them were effectively bridged to allo-HCT.

All patients received RIC, mainly Fludarabine (150mg/ $\mathrm{m}^{2}$ )-Cyclophosphamide $\left(2 \mathrm{~g} / \mathrm{m}^{2}\right)$ in CLL and NHL and Thiotepa $(10 \mathrm{mg} / \mathrm{kg})$-Fludarabine $\left(120 \mathrm{mg} / \mathrm{m}^{2}\right)$-Cyclophosphamide $(60 \mathrm{mg} / \mathrm{kg})$ in HL from matched sibling $(\mathrm{n}=27)$, matched $(n=15)$ or mismatched unrelated $(n=8)$ donors. Graft-versus-host disease (GVHD) prophylaxis consisted of cyclosporine or tacrolimus and mycophenolate mofetil or short- term methotrexate and additional low-dose antithymocyte globulin $(5 \mathrm{mg} / \mathrm{kg})$ in unrelated donors. Peripheral blood was the main cell source (48/50) and median number of CD34+ cells infused was $6.37 \times 10^{6} / \mathrm{kg}$ (1.33-14.5). Two patients succumbed to advanced underlying disease before engraftment; while the other engrafted successfully. Median time until neutrophil and platelet engraftment was 10 and 12 days respectively. Eighteen patients (36.7\%) developed acute GVHD (grade III-IV, $\mathrm{n}=5$ ), steroid sensitive in 10 $(62.5 \%)$ and 11 relapsed. One-year cumulative incidence (CI) of extensive chronic GVHD was $78.2 \%$, and 13 patients required more than one additional line of immunosuppression (range 1-5). Ten patients presented CMV reactivation successfully treated with antiviral medication and 1 patient died from HSV7 encephalitis. With a median follow of 3 years (1-16 years), 10-year OS was $40.4 \%, 10$-year non-relapse mortality CI $23.4 \%$ and 10 year DFS $32 \%$. There was no difference in survival according to original disease (5-year OS for $\mathrm{NHL}=60.2 \%$, $\mathrm{HL}=46.7 \%, \mathrm{CLL}=31 \% \%, \mathrm{p}=0.67)$. Multivariate analysis revealed high and very high DRI as the single predicting factor of OS (HR 9.69, CI 1.55-60.55, $\mathrm{p}=0.015$ ), when assessing impact of disease, DRI, prior treatment lines, gender and bone marrow infiltration at diagnosis.

Conclusions: Our data suggest that RIC allo-HCT provides encouraging survival rates, potentially offering the chance of cure, with acceptable long-term mortality in selected high-risk patients with lymphoid neoplasms. DRI that is mainly associated with disease stage at transplant independently affects survival. Therefore, continued efforts are necessary for clinical application of novel agents aiming to lower disease stage pre-transplant.

Disclosure: Nothing to declare

\section{P503}

Nivolumab after allogeneic hematopoietic stem cell transplantation in relapsed / refractory hodgkin's lymphoma (RR-HL) - A single center experience

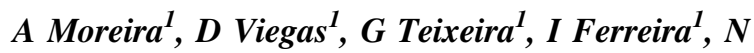
Miranda $^{I}$, F Moita $^{I}$, AS Jorge ${ }^{I}$, MJ Gutierrez ${ }^{I}$, F Leal da Costa $^{1}$, M Abecasis ${ }^{1}$

${ }^{1}$ Instituto Portugues de Oncologia de Lisboa Francisco Gentil, Lisboa, Portugal

Background: Allogeneic Hematopoietic Stem Cell Transplantation (allo-HSCT) is a therapeutic option for patients (pts) with RR-HL. Nivolumab, an anti-PD1 antibody with efficacy in the treatment of RR-HL, has been used in relapsed patients after allo-HSCT. The increased risk of Graft-versus-Host Disease (GvHD) has been discussed in this setting.

Methods: In this series, we intend to present a retrospective analysis of the pts with RR-HL treated with nivolumab after allo-HSCT. 
Results: Six pts were identified, with a median age of 30 years [6-35] at diagnosis: five with HL nodular sclerosis and 1 with lymphocyte depletion. The median number of therapeutic lines prior to allo-HSCT was 5 [4-10]; four pts were previously treated with Brentuximaband two pts had been submitted to high dose chemotherapy with autologous bone marrow support.

At the time of allo-HSCT, 4 pts had progressive disease (DP), 1 was in partial response and 1 in complete response (CR).

Five allo-HSCT were performed with a related donor, 4 of wich were haploidentical (2 parents, 1 sibling and 1 descendant) and 1 with an unrelated donor (10/10). Prophylaxis for GvHD was performed with tacrolimus and mycophenolate mofetil (with post-transplant cyclophosphamide in haploidentical allo-HSCT).

On day +100 evaluations, 5 pts had a CR and 1 patient (pt) had DP. The median time to relapse after allo-HSCT was of 12 months.

At the time of initiation of nivolumab, 5 pts were under steroid therapy for disease control, without other immunosuppressive therapy. The median time between allo-HSCT and the beginning of nivolumab was 16 months. The initial dose was 1 $\mathrm{mg} / \mathrm{kg}$ (associated with corticosteroid therapy), escalated up to $3 \mathrm{mg} / \mathrm{kg}$ biweekly, according to patient's tolerance.

After the start of nivolumab, 3 patients, with previous GvHD manifestations, presented a worsening of the cutaneous GvHD, which required an escalation of immunosuppressive therapy. As toxicity, 1 pt had a grade 4 pneumonitis, $1 \mathrm{pt}$ had a grade 4 encephalitis/hypophysitis, 1 pt had a grade 4 pancreatitis, 2 pts had headache (grade 1 and 3), 2 pts had a grade 2-3 cutaneous reation.

With a median follow-up of 13 months since nivolumab treatment, the overall response rate was 100\%: $1 \mathrm{pt}$ obtained $\mathrm{CR}$ and 5 pts partial remission. Nevertheless, there were 2 deaths after the onset of nivolumab: $1 \mathrm{pt}$ at 4 monts with DP and another one due to acute myocardial infarction at 16 months.

At the time of analysis, 3 pts maintained response under nivolumab treatment (median cycles 26) and 1 pt had therapy suspended because of toxicity.

Conclusions: These results demonstrate the high probability of achieving response with nivolumab treatment in patients with RR-HL relapsing after allo-HSCT, but adverse events of grade 4 were frequent in this small group, and the treatment toxicity was significant.

Disclosure: Nothing to declare

\section{P504}

\section{Clinical features and treatment outcomes of patients with Asian variant of intravascular large B-Cell lymphoma}

Seok Jin Kim ${ }^{1}$, Sang Eun Yoon ${ }^{1}$, Duck Cho ${ }^{1}$, Young Hyeh $\mathrm{Ko}^{1}$, Won Seog Kim ${ }^{1}$

${ }^{1}$ Samsung Medical Center, Sungkyunkwan University School of Medicine, Medicine, Seoul, Korea, Republic of

Background: Intravascular large B-cell lymphoma (IVLBCL) is a rare form of large B-cell lymphoma with pathological findings of intravascular proliferation and/or sinusoidal involvement of lymphoma cells. According to their geographic distribution, IVLBCL could be dichotomized into Asian and Western variants. Compared with the Western variant, where skin involvement was common, the Asian variant was reported to involve more frequently the liver, spleen and bone marrow, and hemophagocytic lymphohistiocytosis is more common in Asian variant. Diagnosis of IVLBL is still difficult because of the lack of overt lymphadenopathy and peripheral blood involvement. Thus, timely diagnosis and immediate treatment remain as a challenge to improve outcomes for patients with the Asian variant. Therefore, we analyzed the clinical features and treatment outcomes of patients with the Asian variant of IVLBCL.

Methods: We analyzed 46 patients who were diagnosed with IVLBCL between 2001 and 2018. All patients were treated with rituximab, cyclophosphamide, doxorubicin, vincristine, and prednisone (R-CHOP).

Results: Forty-six patients were diagnosed with IVLBCL, and their median age at diagnosis was 62 years (range: $34-82$ years). Male patients predominated $(\mathrm{n}=26,57 \%)$, and $\mathrm{B}$ symptoms were present in 31 patients $(78 \%)$. Hepatomegaly and/or splenomegaly were observed in 26 patients $(57 \%)$, whereas lymphadenopathy was less common $(n=22,48 \%)$. Bone marrow and liver were the most commonly involved extranodal organs (54\%, and $48 \%$, respectively) and were the most common sites of biopsy for diagnosis in this study. All patients received R-CHOP as a first-line treatment after diagnosis with a median number of six cycles (range one to eight). At the end of treatment, 31 patients achieved a complete response (CR), whereas eight patients showed progression. Six patients died after the first or second cycle of R-CHOP, and the causes of death were treatment-related adverse events including cytopenia, infectious complications, and pulmonary hemorrhage. Upfront ASCT was done for two patients including one patient with CNS involvement at diagnosis, and these patients were still alive at the time of analysis without evidence of relapse. On the other hand, the outcome of six patients undergoing salvage ASCT after relapse was poor; thus, only one patient was alive. Likewise, patients with disease progression at the end of treatment with R-CHOP showed dismal prognoses even after salvage chemotherapy except for one. At a median follow-up of 47.0 months (95\% confidence interval, CI 25.0-69.0), the median overall survival was 45.0 months (95\% CI 25.8-64.2). 
Conclusions: The treatment outcome of patients with the Asian variant of IVLBCL is still not satisfactory. Although upfront autologous stem cell transplantation might be effective for selected patients at high-risk of relapse, its role is still not clear, either. Thus, further study should be warranted to develop more effective strategies for diagnosis and treatment.

Clinical Trial Registry: Not applicable

Disclosure: Nothing to disclare

\section{P505}

Stem cell transplantation as consolidation in peripheral T-cell lymphoma

Catalina Gomez ${ }^{1}$, Isabelina Ferreira ${ }^{1}$, Filipa Moita ${ }^{1}$, Gilda Teixeira ${ }^{1}$, Nuno Miranda ${ }^{1}$, Sofia Jorge ${ }^{1}$, Maria João Gutierrez ${ }^{1}$, Fernando Leal da Costa ${ }^{1}$, Manuel Abecasis $^{1}$

${ }^{1}$ IPOLFG, Lisboa, Portugal

Background: Peripheral T-cell lymphomas (PTCLs) are about $10 \%$ of non-Hodgkin's lymphomas usually with an aggressive clinical course and unfavorable prognosis.Given their heterogeneity, consensus on the best first-line treatment and the role of autologous/allogeneic (ASCT/alloSCT) stem cell transplantation as consolidation is controversial.

Methods: We evaluated the overall survival (OS), progression-free survival (PFS) and toxicities of a cohort of patients with PTCLs submitted to ASCT/alloSCT intensification at our institution between January 2000 and July 2018.

OS was calculated from the date of diagnosis until death. PFS was measured from transplant until relapse, progressive disease or last follow-up. OS and PFS rates were estimated using the Kaplan-Meier method and compared with the Log-Rank test.

Results: Twenty-six patients were identified, 16 female (61\%), median age was 46 years (range: 22 to 62). Ninetytwo percent of patients presented with advanced-stage disease at diagnosis (Ann Arbor stage III or IV), 38\% with B symptoms. According to the 2016 WHO classification, histologic PTCL subtypes included angioimmunoblastic Tcell lymphoma $(\mathrm{n}=12)$; PTCL not otherwise specified ( $\mathrm{n}$ $=6)$; anaplastic large cell lymphoma, ALK-negative $(\mathrm{n}=5)$; anaplastic large cell lymphoma, ALK-positive $(\mathrm{n}=1)$; nodal peripheral T-cell lymphoma with TFH phenotype (n 2). Extranodal NK/T-cell lymphoma, nasal type and primary cutaneous subtypes were excluded. The ageadjusted IPI (aaIPI) was low/intermediate low in 15 patients $(57 \%)$ and intermediate high/high in 11 patients $(43 \%)$.

Twenty-seven transplants were performed (19 ASCT, 8 alloSCT); 18 were consolidation in 1st response (16 ASCT and 2 alloSCT) with 16 in Complete Remission (CR) and 2 in Partial Remission (PR). Nine transplants were performed as consolidation of $2^{\text {nd }}$ response ( 3 ASCT and 6 alloSCT) with 4 in $\mathrm{CR}$ and 5 PR. In 1 patient alloSCT was performed after ASCT, due to early relapse ( $<12$ months). BEAM regimen was used in ASCT as conditioning and FluMEL in alloSCT. All patients engrafted, the median time to leukocyte recovery $>1,000 / \mu \mathrm{L}$ was 14 days (range, 11 to $25)$. Four of the 8 pts $(50 \%)$, submitted to alloSCT had chronic graft versus host disease which was the most relevant complication of this analysis.

Considering the whole cohort, the median follow-up was 50.5 months (range, 2 to 217). The estimated 5-year OS and PFS were $77 \%$ and $39 \%$, respectively.

Seven patients relapsed ( 6 early) all after ASCT, there were no relapses after alloSCT, however, the results were not statistically significant between the alloSCT and ASCT groups; the 5-year OS rates were $87 \%$ and $62 \%(\mathrm{p}=0,16)$ and the 5year PFS rates were $85 \%$ and $59 \%(\mathrm{p}=0,26)$ respectively.

For the all patients treatment-related mortality (TRM) was 3,7\%; 7 patients died, 6 with progressive disease (ASCT) and 1 for hepatic toxicity (alloSCT) before D+100.

Conclusions: The results of this retrospective study, taking into account the adverse risk profile of the population, suggest that autologous/allogeneic stem cell transplantation as an effective and safe option for the consolidation of patients with PTCLs.

These results need to be validated in prospective studies, including a larger number of patients.

Disclosure: No conflict of interest to declare

\section{P506}

Allogeneic stem cell transplantation in elderly lymphoma patients: Single center experience

\section{Zafer Gulbas ${ }^{I}$, Elif Birtas Atesoglu ${ }^{I}$, Meral Sengezer ${ }^{I}$, Imran Dora ${ }^{1}$, Cigdem Eren ${ }^{1}$, Suat Celik ${ }^{1}$, Demet Cekdemir ${ }^{1}$}

${ }^{1}$ Anadolu Medical Center, Hematology, Kocaeli, Turkey

Background: Autologous stem cell transplantation is used as consolidation therapy in relapsed lymphoma patients. However, outcome of lymphoma patients relapsing after autologous stem cell transplantation is poor and allogeneic stem cell transplantation which can be curative is used in the transplant eligible patients in this setting. Besides, allogeneic stem cell transplantation can be an option before autologous stem cell transplantation in some high risk patients. In this study, we aimed to compare the survival rates of lymphoma patients older than 60 years of age and patients aged 50-60 who had undergone allogeneic transplantation in our center.

Methods: We collected the data of lymphoma patients older than 50 years of age who had undergone allogeneic 
transplantation in our center and analyzed the results by grouping them into 2 , namely the ones between $50-60$ years of age and the ones over 60 years of age.

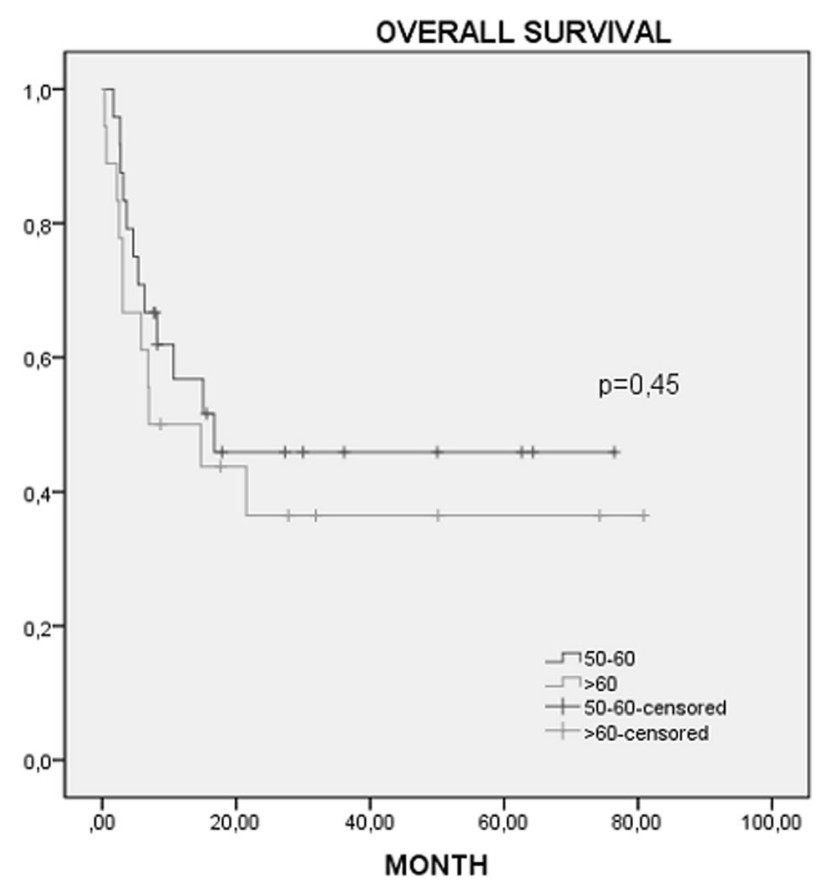

[[P506 Image] 1. Figure 1:Overall Survival]

\begin{tabular}{lllll}
\hline & $50-60 y$ & $>60 y$ & $\mathrm{p}$ \\
\hline $\mathrm{n}$ & 24 & 18 & \\
Lymphoma type Non-Hodgkin Lymphoma & 213 & 162 & 1 \\
Hodgkin Lymphoma & & & \\
Comorbidity index $01-2 \geq 3$ & 8151 & 7101 & 0,89 \\
Neutrophil engraftment time,day, median & $14(11-$ & $14(12-$ & 0,93 \\
(range) & $27)$ & $25)$ & \\
Platelet engraftment time,day, median (range) & $16(11-$ & $13(8-$ & 0,26 \\
& $61)$ & $31)$ & \\
Acute GVHD & $13(\%$ & $3(\%$ & 0,01 \\
& $54,2)$ & $16,7)$ & \\
100 day mortality & $4(\%$ & $6(\%$ & 0,28 \\
& $16,7)$ & $33,3)$ & \\
\hline
\end{tabular}

[[P506 Table] 1. Table1: Characteristics of the patients]

Results: There were 42 patients over the age of 50 who had undergone allogeneic stem cell trasplantation with the diagnosis of lmphoma between 2011 and 2018. 18 of these patients were over 60 years of age. 37 patients had NonHodgkin lymphoma and 5 patients had Hodgkin lymphoma. The characteristics of the patients are summarized in Table 1. Patients' comorbidity indexes were calculated with Augmented HCT-CI which includes patients' pretransplant ferritin, albümin and thrombocyte counts as a variable. No difference could be found between groups regarding neutrophil and platelet engraftment times and comorbidity indexes. However, acute graft versus rate and documented bacterial infection rate during the hospitalization period were higher in the 50-60 years age group $(\mathrm{p}=0,01) .100$ day mortality rate and non-relapse mortality rate were not different between groups. More importantly, Progression free survival(PFS) and overall survival(OS) of patients in the 50-60 years age group and over 60 years of age group were not different $(\mathrm{p}=0,45)$ (Figure 1)

Conclusions: In the present study, although the number of patients is low, we showed that lymphoma patients over 60 years of age have similar outcomes and transplant related toxicity as the patients between 50 to 60 years of age. PFS and OS were very close in this study. We think that this may be due to low relapse rate in the patients and high mortality rate in relapsing patients. In conclusion, allogeneic stem cell transplantation which has a curative potential may be employed in transplant eligible elderly lymphoma patients

Disclosure: Nothing to declare

\section{P507}

Similar survival trends in transformed follicular lymphoma and DLBCL after ASCT. Single center cohort with a long follow-up

\section{Javier Arzuaga Méndez ${ }^{1}$, Maite Moreno Gámiz ${ }^{I}$, Juan Carlos García Ruiz ${ }^{1}$}

${ }^{1}$ Hospital Universitario Cruces, Hematology, Baracaldo, Spain

Background: Follicular Lymphoma (FL) histologic transformation consist on the development of an aggressive lymphoma, usually a diffuse large B cell lymphoma (DLBCL). Histological transformation has been considered to have poor prognosis. In pre-rituximab era median OS ranged between 1 and 2 years, however, in recent series of patients treated with chemotherapy plus rituximab, the outcome of transformed FL has improved, especially in those that receive Autologous stem cell transplantation (ASCT), who reach 5-year OS up to $75 \%$ in some series.

Methods: We have retrospectively studied 19 consecutive patients undergoing ASCT for transformed FL between 2006 and 2015 in a tertiary center in the Basque Country, Spain. Patients were considered to have a transformed FL if they were diagnosed of a DLBCL and they have previous history of FL or histological evidence of a FL in another location.

These patients were compared to a retrospective cohort of 38 DLBCL patients with high IPI or stage that received ASCT in first remission according to our institution strategy. 
PFS and OS were calculated from the time of the ASCT. In the case of transformed FL, both relapses of the aggressive or indolent lymphoma were considered. Survival analysis was performed with Kaplan-Meyer estimator

Results: A total of 19 transformed FL and 38 DLBCL patients were studied, with a median follow up of 41.4 and 51.7 months respectively. Patient characteristics are described in table 1 .

\begin{tabular}{lll}
\hline & Transformed FL & DLBCL \\
\hline $\begin{array}{l}\text { Sex (male, } \\
\text { female) }\end{array}$ & $14(74 \%), 5(26 \%)$ & $25(66 \%), 13(34 \%)$ \\
Age & $52.8(37-71)$ years & $50(19-68)$ years \\
Stage & I-II: $4(21 \%)$ III-IV: & I-II: $10(26 \%)$ III-IV: \\
& $15(79 \%)$ & $28(74 \%)$ \\
IPI & $0-1: 7(37 \%), 2: 2(11 \%), 0-1: 4(10 \%), 2: 9(24 \%), 3:$ \\
& $3: 6(31 \%), 4-5: 4(21 \%)$ & $13(34 \%), 4-5: 12(32 \%)$ \\
$\begin{array}{l}\text { Follow-up, } \\
\text { median } \\
\text { (range) }\end{array}$ & $41.4(35-136)$ months & $51.7(27-122)$ months \\
\hline
\end{tabular}

\section{[[P507 Table] 1. Table 1: Patient characteristics]}

3-year PFS was 68\% in transformed FL and $81 \%$ in DLBCL, and 3-year OS was $81 \%$ and $84 \%$, respectively (picture 1). There were no significant differences in PFS or OS between this two groups $(\mathrm{p}=0.44)$. In both groups all relapses occurred in the first three years after ASCT. Among the patients with transformed FL 6 relapses were observed. Five of them (83\%) were aggressive relapses, while only one patient presented relapse as an indolent lymphoma (FL histological grade $3 \mathrm{~A}$ with an aggressive clinical course).

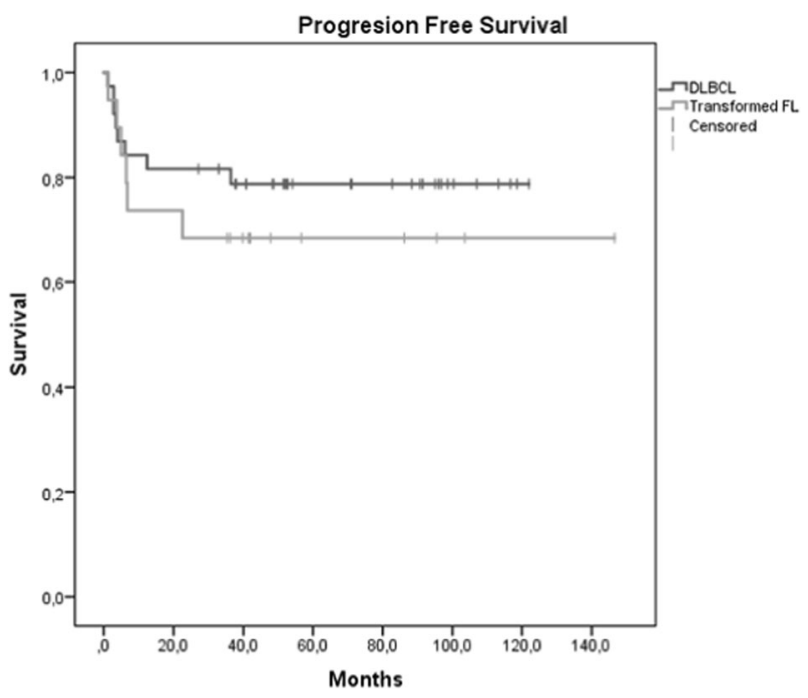

[[P507 Image] 1. Image 1: Transformed FL and DLBCL PFS after ASCT]
Conclusions: In our experience, ASCT in transformed FL offers good results, similar to those in DLBCL. FL presents a natural course akin to that of DLBCL, with relapses occurring early and survival reaching a plateau. This data suggests that some patients with transformed FL can be cured after ASCT.

Disclosure: Nothing to declare.

\section{P508}

Safety and efficacy of intensive preconditioning regimen containing cladribine in autologous peripheral blood stem cell transplantation of refractory and relapsed young highly invasive lymphoma

\section{Xiang-Li Chen ${ }^{1}$}

${ }^{1}$ Henan Provincial People's Hospital, People's Hospital of Zhengzhou University, Zhengzhou, China

Background: Autologous peripheral blood stem cell transplantation (APBSCT) is one of the main treatments for patients with non-Hodgkin's lymphoma (NHL). Effective and safe conditioning regimens can improve the cure rate of NHL. BEAM is the most common pretreatment scheme, but for refractory and relapsed young highly invasive lymphoma, especially for dual-expression DLBCL, pretreatment needs to be strengthened. Studies have shown that the Cladribine (Clad)+Gemcitabine $(\mathrm{Gem})+$ Busulfan $(\mathrm{Bu})$ combination provides synergistic cytotoxicity in lymphoma cell lines.We evaluated the the safety and short-term efficacy of intensive preconditioning regimen containing cladribine $(\mathrm{Clad}+\mathrm{GEM}+\mathrm{BU})$ for refractory and relapsed young highly invasive lymphoma undergoing APBSCT.

Methods: Ten patients with NHL received APBSCT. CA)CTX + Ara-C) therapy followed by G-CSF was used for PBSC mobilization. Sevenr patients received conditioning regimens of BEAM(BEAM group): BCNU 300mg/ $\mathrm{m}^{2} \cdot \mathrm{d}^{-1} \times 1 \mathrm{~d}(-7 \mathrm{~d})$, VP16 $100 \mathrm{mg} / \mathrm{m}^{2} \cdot \mathrm{Q} 12 \mathrm{~h} \times 4 \mathrm{~d}(-6 \mathrm{~d}--3 \mathrm{~d})$, Ara-C $200 \mathrm{mg} / \mathrm{m}^{2} \cdot \mathrm{q} 12 \mathrm{~h} \times 4 \mathrm{~d}(-6 \mathrm{~d}--3 \mathrm{~d})$, Mel $140 \mathrm{mg} / \mathrm{m}^{2} \cdot \mathrm{d}^{-}$ ${ }^{1} \times 1 \mathrm{~d}(-2 \mathrm{~d})$. Three patients received intensive preconditioning regimen containing cladribine (CLGB group): CLad $5 \mathrm{mg} / \mathrm{m}^{2} \cdot \mathrm{d}^{-1} \times 5 \mathrm{~d}(-6 \mathrm{~d}--2 \mathrm{~d})$, GEM $2500 \mathrm{mg} / \mathrm{m}^{2} \cdot \mathrm{d}^{-1} \times 2 \mathrm{~d}(-6 \mathrm{~d}$, -2d), BU0.8mg/Kg Q6h×4d (-6d-3d). Follow-up date expires on December 1, 2018.

Results: The age of 3 patients in CLGB group was 41,12 and 50 years, respectively. Two patients were diagnosed as diffuse large B-cell lymphoma with double expression and one was diffuse large B-cell lymphoma with two recurrences. The patients of BEAM group were all high-risk, relapsed and refractory NHL.All patients were successfully engrafted after infusing APBSC. The average lowest leukocyte in CLGB group and BEAM group were $(0.023$ $\pm 0.023) \times 10^{9} / \mathrm{L}$ VS $(0.237 \pm 0.2033) \times 10^{9} / \mathrm{L}$, respectively 
The average lowest leukocyte in CLGB group was lower than that in BEAM group. The average time to ANC < $0.5 \times 109 / \mathrm{L}$ in CLGB group and BEAM group were $1.33 \mathrm{~d}$ $\pm 1.53 \mathrm{~d}$ VS $3.57 \mathrm{~d} \pm 2.07 \mathrm{~d}$. The average time to $\mathrm{ANC} \geq 0.5 \times 10^{9} / \mathrm{L}$ in CLGB group and BEAM group were $9.0 \mathrm{~d} \pm 1.0 \mathrm{~d}$ VS $10.0 \mathrm{~d} \pm 2.6 \mathrm{~d}$; The average time to PLT $\geq 20 \times 10^{9} / \mathrm{L}$ of CLGB group was not different to that of BEAM group $(8.0 \mathrm{~d} \pm 1.0 \mathrm{~d}$ VS $9.6 \mathrm{~d} \pm 2.2 \mathrm{~d})$ the average time of neutropenia wasn't significantly different in two groups $(8.33 \mathrm{~d} \pm 3.1 \mathrm{~d}$ VS $8.0 \mathrm{~d} \pm 2.1 \mathrm{~d})$. The adverse reactions of gastrointestinal tract and oral mucosa were close in tow groups.VOD, hemorrhagic cystitis, pretreatment-related interstitial pneumonia, Liver and kidney dysfunction were not happened in tow groups. The rate of infectious fever was close in two groups (2/3 VS 4/7). The median followup period in BEAM group was 9 (1 19) months. In the BEAM group, a patient died 20 days after transplantation, because he was diagnosed with recurrent NKT cell lymphoma and intracranial infection caused by severe sinus infection. Another case of BEAM group was diagnosed as double-expressed DLBCL, which relapsed 3 months after transplantation. The remaining patients in EBMT group survived disease-free. The follow-up time of 3 patients in CLGB group were 2 months, 3 months and 9 months respectively. All patients survived without disease.However, the follow-up time is short and needs long-term follow-up.

Conclusions: The treatment of intensive preconditioning regimen containing Cladribine (CLGB) for refractory and relapsed young highly invasive lymphoma undergoing APBSCT is safe. The time of hematopoietic reconstruction is short, and the adverse effects is tolerable for patients with refractory and relapsed young highly invasive lymphoma. The current short-term outcome is good, but the long-term effect need a longer time to follow-up.

Disclosure: This work was supported by National Nature Sciences Found of China (81300417). There is no disclosure of conflict of interest.The all authors name: Xiang-Li Chen, Yu-Zhu Zang, Wen-Hui Zhang, Yin Zhang, Zhong-Wen Liu, Ping-Chong Lei, Jing Yang, Yu-Qing Chen, Kai Sun.

\section{P509 \\ Pembrolizumab in treatment resistant hodgkin disease in child: Clinical case}

Nataliia Kavardakova ${ }^{1}$, Svitlana Donska ${ }^{1}$, Oleg Ryzhak ${ }^{1}$, Julia Basaluk ${ }^{1}$, Sofia Matsiboh ${ }^{1}$, Olena Zinchuk ${ }^{1}$, Elena Kreminska ${ }^{I}$

${ }^{1}$ Association of Pediatric Oncohematologists of Ukraine, Kiev, Ukraine
Background: Small part of children with Hodgkin Disease (HD) demonstrate initial resistance to the standard and even "salvage" chemotherapy and need innovative drugs for the treatment.

Methods: A 15-year girl was diagnosed with classical HD (nodular sclerosis)corresponding to stage II E B (fever $>38^{\circ} \mathrm{C}$ ) in April 2016.After two cycles of OEPA (vincristine, etoposide, prednisone and doxorubicin) and next two cycles COPP (cyclophosphamide, vincristine, prednisone and procarbazine) the patient again had progressive disease. As the patient achieved a partial response (PR) after "Salvage"- therapy with two cycles of IGEV (ifosfamide, gemcitabine, vinorelbine, and prednisone), she received auto-SCT in February 2017 (patient status before auto-SCT was PR). We used CCNU-containing conditioning regimen CEM: Lomustine (CCNU) $300 \mathrm{mg} / \mathrm{m}^{2}$ + Etoposide 1200 $\mathrm{mg} / \mathrm{m}^{2}+$ Melphalan $180 \mathrm{mg} / \mathrm{m}^{2}$. At Day +90 after auto$\mathrm{SCT}$, the patient again demonstrated progression of the disease: PET/CT-examination showed mediastinal tumor mass enlargement with increased left lung nodule simultaneously to short metabolic activity. Patient was under observation. At Day +140 the disease had relapsed and progressed - examination by PET/CT demonstrated multifocal progressive disease with multiple pulmonary lesions and increased metabolism in comparison with the previous PET/CT scan. In July-October 2017, the patient had Salvage chemotherapy with a combination of Brentuximab vedotin (BV) (BV DHAP (dexamethasone, cytarabine, cisplatin) + BV (without chemotherapy due to suspected invasive mycosis) + BV DHAP), however, only partial PET-positive remission was achieved.

Because of many times relapsed and progressed disease Pembrolizumab therapy was started in October 2017: $2 \mathrm{mg} /$ $\mathrm{kg}$ every three weeks four cycles totally. Toxic effects and serious complications during and after therapy by Pembrolizumab were not observed.

In February 2018, after Pembrolizumab \# 4, the patient showed complete metabolic remission of the disease by control PET-CT. In April 2018, the patient received haploSCT with post-transplant HD-Cyclophosphamide. We used conditioning modes with reduced toxicity (Fludarabin $150 \mathrm{mg} / \mathrm{m}^{2}+$ Treosulfan $\left.42 \mathrm{~g} / \mathrm{m}^{2}\right)$, high doses of Cy $(50$ $\mathrm{mg} / \mathrm{kg}$ ) on days +3 and +4 . Tacrolimus and mycophenolate mofetil started on day +5 . MMF was terminated on day 35, Tacrolimus - on day 180. Patient did not have acute and chronic GVHD.

Results: At the moment the patient is alive and still in PET-negative CR with duration more than 9 mo.

Conclusions: Pembrolizumab has demonstrated high activity against resistant HD even after post-auto-SCT progression with good tolerability for the sick child.

Disclosure: Nothing to declare 


\section{P510}

High dose chemotherapy followed by autolougous peripheral blood stem cell transplantation (ASCT) in diffuse large B cell lymphoma (DLBCL)

\section{Redhouane Ahmed Nacer', Nacera Ait Amer', Sabrina Akhrouf $^{l}$, Malek Benakli ${ }^{1}$, Amina Talbi ${ }^{1}$, Farih Mehdid ${ }^{1}$, Rachida Belhadj, Imene Abderahim ${ }^{I}$, Farida Harieche ${ }^{I}$, Rose-Marie Hamladji ${ }^{I}$}

${ }^{1}$ Pierre and Marie Curie Center, Hematology-Bone Marrow Transplantation, Alger, Algeria

Background: High dose therapy(HDT) followed by autologous stem cell transplantation (ASCT) as consolidation treatment allows progression free survival and overall survival improvement in high risk DLBCL.

Methods: Fom may 1998 to December 2016, 150 DLBCL patients (pts) including 32 mediastinal large cell lymphoma (MLCL) pts, underwent HDT with ASCT. Median age is 38,9 years (14 to 67) and sex ratio (M/F) 1.14; Ann Arbor stage III-IV: 104 pts. Before HDT induction chemotherapy (CHOP, C2H2OPA) was instituted and associated with Rituximab in 100 pts $(66,6 \%), 49$ pts $(32,7 \%)$ received more than 2 treatment lines and 16 pts $(10,7 \%)$ received complementary radiotherapy. Transplant disease status before HDT was complete remission (CR) in 93 pts, partial remission in 55 pts (RP) and disease progression in 2 pts. The delay from diagnosis to HDT is 11,7 months (4-103). The HDT protocols used are: Tutshka : 87 pts, Tutshka+VP16 : 42 pts, BAM 12 (Busulfan + Cytarabine+Melphalan) :16 pts et BEAM : 5 pts. All pts received, after thawing, mobilized PBSC obtained by GCSF mobilization $(15 \mu \mathrm{g} / \mathrm{Kg} / \mathrm{d}, 5$ days $)$ alone and froze in liquid nitrogen. The median rate $\mathrm{CD} 34+$ cells infused is 3,6 x $10^{6} / \mathrm{Kg}(0.87-17.36)$. The median follow-up at $12 / 31 / 2017$ is 67 months (1-218).

Results: the median time to graft $\left(\mathrm{PNC}>0.5 \times 10^{9} / 1\right)$ was 13 days (9-18). Ten early deaths were observed including 8 infection (TRM: 6,6\%) and 3 in disease progression at 3 months. After 3 months of HDT 137 pts are assessable including 128 pts in CR $(93,4 \%)$ and 9 pts in PR $(6,6 \%)$. Relapse was observed in 31 pts $(22.6 \%)$ and it was earlier relapse in a period of 24 months in 15 pts (48\%). Deaths were among 39/150 pts (26\%). Persistent $\mathrm{CR}$ was achieved in $112 / 137$ pts $(81,8 \%)$ including $23 / 30$ $(76,6 \%)$ MLCL and 89/107 $(83,1 \%)$ others DLBCL. The overall survival (OS) and event free survival (EFS) at 10 years are respectively $68 \%$ and $64 \%$. The OS and EFS are better in patients who received Rituximab in initial therapy : OS $(79 \%$ vs $54 \%$; p $<0,001)$ et EFS $(79 \%$ vs $46 \% ; \mathrm{p}<0,001)$.
Conclusions: our results are satisfactory and match those of literature. The incorporation of rituximab in the initial treatment before HDT improve survival (OS and EFS).

Disclosure: Nothing to declare

\section{P511}

Exome sequencing of a refractory MCL patient from death back to diagnosis reveals TP53 R175H mutation as the molecular event leading to acquired ibrutinib resistance

\section{Honghu Li ${ }^{I}$, Yuanyuan $\mathrm{Zhu}^{1}$, He Huang ${ }^{I}$}

${ }^{1}$ Zhejiang University; The First Affiliated Hospital, Hangzhou, China

Background: Mantle cell lymphoma (MCL) is a lymphoproliferative disorder accounting for about $5 \%$ of all lymphomas.Its clinical and histological features are heterogeneous. Ibrutinib is an oral inhibitor of Bruton tyrosine kinase (BTK).Although treatment with ibrutinib has has generated a remarkable response in MCL patients, approximately one third of the patients have primary drug resistance while others appear to develop acquired resistance. However, knowledge regarding the molecular events leading to ibrutinib resistance is limited.Herein, we present one patient with refractory MCL, who were insensitive to chemotherapy and then experienced a dramatic improvement with ibrutinib mono-therapy but later developed ibrutinib resistance,ultimately resulting in the deterioration of disease and death.

Methods: We give the patient several examinations including Ultrasound, bone marrow biopsy, lymph node biopsy, Exome Sequencing, Sanger sequencing, and so on. For the treatment of lymphoma, the patient received chemotherapy, including 1 course of $\mathrm{CHOP}$ (cyclophosphamide $1.3 \mathrm{~g}$ day 1 , doxorubicin $40 \mathrm{mg}$ day 1 ,Vinorelbine $40 \mathrm{mg}$ day 1 , and dexamethasone $15 \mathrm{mg}$ from day 1 to 5 ) and 1 course of R-DHAP (rituximab 600mg day0, cytarabine $2 \mathrm{~g}$ q12 day 1, Cisplatin 100mg day 1,dexamethasone $20 \mathrm{mg}$ from day 1 to 5)in succession.Because of the failure to control disease progression, ibrutinib 560mg qd was used until the patient died.

Results: The 64-year-old man initially referred to our hospital for complaints of abdominal pain and distention over 2 months. Ultrasound showed splenomegaly and multiple enlarged retroperitoneal lymph nodes.Excisional biopsy conducted on the right neck lymph node revealed the presence of malignant cells.Immunohistochemically, the neoplastic cells were positive for Bcl2, Bcl6,CD20, CD5, CD79a, CD43, Ki-67(50\%), SOX11, CD21 (FDC) and Cyclin D1 and negative for CD10, CD23 and CD3; Fluorescence In Situ Hybridization(FISH) showed IGH/ 
CCND1,t(11;14) 90\%.Thus a diagnosis of MCL was confirmed. 2 course of therapeutic chemotherapy were applied to the patient but he did not respond well.He suffered recurrent fever, thrombocytopenia, left abdominal pain, splenomegaly and multiple enlarged lymph nodes. Then he received ibrutinib mono-therapy, and experienced a dramatic improvement as his body temperature was controlled, his hemogram became normal and his spleen and lymph node tapered.After about 6 months response of ibrutinib, the disease deteriorated rapidly and he died very soon. Exome sequencing from the patient peripheral blood at this time detected one missense mutation in exon 5 of TP53 at nucleotide 524G $>$ A, resulting in an Argnine to Histidine change at amino acid 175 (p.Arg175His). But Sanger sequencing of the patient bone marrow FFPE sample at the time of original diagnosis did not detect this mutation.

Conclusions: Thus, our study reported a TP53 R175H mutation MCL patient who developed ibrutinib resistance and progressed aggresively, which may open new insight for future effort for alternative therapeutic strategies in ibrutinib-refractory MCL.

Disclosure: Nothing to declare.

Minimal residual disease, tolerance, chimerism and immune reconstitution

\section{P512}

\section{Factors influencing B-cell reconstitution after HSCT}

\section{Nicolaas G. Van Der Maas ${ }^{1}$, Dagmar Berghuis ${ }^{1}$, Hein Putter $^{2}$, Mirjam Van Der Burg ${ }^{1}$, Arjan C. Lankester ${ }^{I}$}

${ }^{1}$ Willem-Alexander Children's Hospital, Leiden University Medical Center, Leiden, Netherlands, ${ }^{2}$ Leiden University Medical Center, Leiden, Netherlands

Background: Immune reconstitution after hematopoietic stem cell transplantation (HSCT) is variable and influenced by different patient, stem cell graft and treatment related factors. Here we used observational data, to investigate longitudinal B cell recovery and the factors influencing this process.

Methods: Between 01-04-2004 and 01-01-2017, 333 children received a single bone marrow (BM), peripheral blood stem cell (PBSC) or cord blood (CB) HSCT for malignant or non-malignant disorders in the Leiden University Medical Center (LUMC). A total of 176 patients were eligible for analysis. Reasons for exclusion were: death within half a year post-HSCT, relapse of disease, use of rituximab and aGVHD.

Peripheral blood samples were obtained for routine analysis at several time points after HSCT. All available blood samples between 0.5 and 2 years were used in the analysis. To assess changes in the CD19+B and CD19 $+\mathrm{CD} 27+$ memory B cell counts over time while accounting for the correlation between the repeated measurements of each patient, we used linear mixed-effects models. Wilcoxon rank test, Kruskal-Wallis test and linear regression were used for univariate analysis.

Results: At one year after HSCT, univariate analysis showed that patients transplanted with a CB graft compared to BM and PBSC had a significantly higher absolute number of $\mathrm{B}$ cells (median $\mathrm{BM}=501$, median $\mathrm{CB}=1402$, median $\mathrm{PBSC}=502$ cells $/ \mu \mathrm{l}, \mathrm{P}=1.4 \mathrm{e}-5)$ and memory $\mathrm{B}$ cells (median $\mathrm{BM}=26$, median $\mathrm{CB}=75$, median $\mathrm{PBSC}=23$ cells/ $\mu \mathrm{l}, \mathrm{P}=9.3 \mathrm{e}-4)$. Recipients with age under 5 years had significantly higher absolute numbers of $\mathrm{B}$ (median $=919$, median $=473$ cells $/ \mu \mathrm{l}, \mathrm{P}=2.9 \mathrm{e}-4$ ) and memory $\mathrm{B}$ cells (median $=54$, median $=22$ cells $/ \mu \mathrm{l}, \mathrm{P}=2.7 \mathrm{e}-5$ ) than above 5 years. Increase in donor age was associated with a decreasing effect on $\mathrm{B}$ cell $\left(\mathrm{R}^{2}=0.35, \mathrm{P}=5.9 \mathrm{e}-14\right)$ and memory $\mathrm{B}$ cell $\left(\mathrm{R}^{2}=0.25, \mathrm{P}=2.1 \mathrm{e}-8\right)$ reconstitution as determined in regression analysis.

Following univariate analysis, we analysed these factors in a mixed effects model to assess the relation with differences in B cell or memory B cell numbers 0.5-2 years after HSCT. In our analysis we found significant decreasing $\mathrm{B}$ cell and memory $\mathrm{B}$ cell numbers with increasing donor age corrected for recipient age and source (both $\mathrm{P}<0.001$ ). Increasing recipient age also showed a significant decrease in $\mathrm{B}$ cell and memory $\mathrm{B}$ cell numbers (both $\mathrm{P}<0.001$ ) but there was no significant influence of donor source (Figure 1).

Conclusions: B cell and memory B cell numbers after HSCT are influenced by donor and recipient age but not by donor source. Older donors and recipients show a decrease in B cells and memory B cells numbers 0.5-2 years after HSCT.

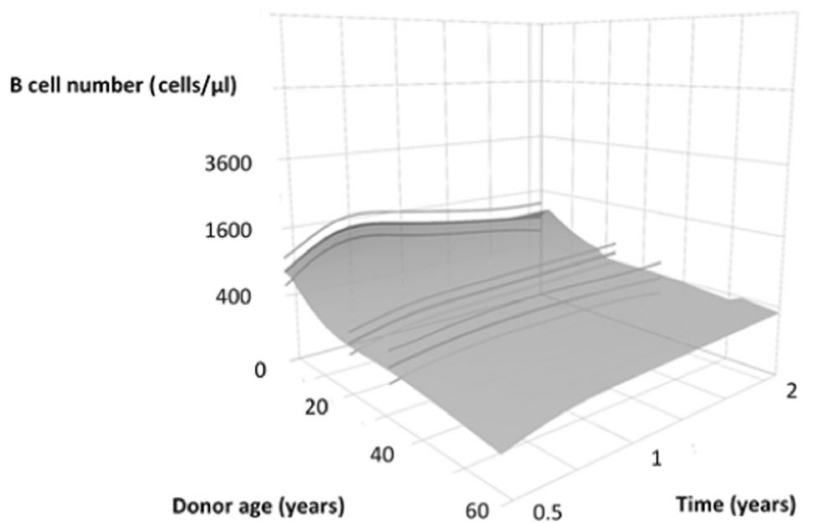

[[P512 Image] 1. Figure 1. B cell development and donor age. Green shows CB, red BM, blue PBSC at mean donor age.] 
Disclosure: Nothing to declare

\section{P513}

Sequence variants and their nature in an unselected cohort of patients with high-risk myeloid disorders

\section{Pia Keske ${ }^{I}$, Anita Badbaran ${ }^{I}$, Nicolaus Kröger ${ }^{I}$, Maximilian Christopeit ${ }^{1}$}

${ }^{1}$ University Medical Center Hamburg-Eppendorf, Hamburg, Germany

Background: Variants revealed by sequencing can be (likely) pathogenic, (likely) benign, or of unclear significance (VUS). The FATHMM-MKL algorithm uses a hidden Markov model to approximate the impact of a sequence variant on the protein $(\mathrm{p} \leq .5$ neutral, $\geq .7$ pathogenic). The distribution of sequence variants and their nature in the general population and in disease cohorts still has to be described in more detail.

Methods: We applied next generation sequencing (PGM, Ion Torrent/ Fischer Lifetechnologies) to an unselected cohort of 414 patients (176 female, 238 male, median age 59 (2-80) years) who had been referred for allogeneic stem cell transplantation due to the presence of a high-risk myeloid disorder. Patients suffered MPN (127), MDS (114), AML (99), CMML (20), MDS/MPN overlap (12), SAA (7), and other, non-malignant hematologic diseases (11).

Results: More than 1,400 sequence variants were called. Amongst 32 genes analyzed in 3 amplicon panels, the TET2 sequence was most frequently altered $(64.7 \%$ of all patients; TET2I1762V (33.8\% of all patients, VUS, FATHMM score .14), TET2L1721W (14\%, .78), TET2P363L (6.8\%, .70), TET2H1778R (5.3\%, VUS)). This was trailed by ASXL1 (26.3\%; ASXL1E1102D (2.7\%) .95), DNMT3A (22.9\%; DNMT3A R882H (3.1\%) .98), CEBPA (18.1\%; CEBPAP198S (16.9\%) VUS), TP53 (15.7\%; TP53P72R (4.1\%) .36), JAK2 (15.5\%; JAK2V617F (15.2\%) .94), IDH1 (14.3\%; IDHIV178I (5.8\%) .99), RUNX1 (11.6\%; RUNX1L56S (1\%) .90), EZH2 (9.2\%; EZH2D185H (5.3\%) 1.0), GATA2 (8.9\%; GATA2A164T (7.2\%) .91), NRAS (7.5\%; NRASG12D (2.4\%) .91), IDH2 (7.5\%; IDH2R140Q (3.6\%) .98), KRAS (6.5\%; KRASR161R (3.1\%) VUS), and KIT (3.9\%; KITM541L (3.9\%) .74).

Patients displayed a median of 3 sequence variants (AML, MPN and CMML patients each 3; MDS, SAA and patients with other, non-malignant hematologic diseases each 4; MDS/MPN overlap patients 5).

Sequence variants found most frequently in AML were CEBPAP198S $(13.2 \%$ of all sequence variants in patients with AML, $\mathrm{p}<10^{-3}$, chi $^{2}$ test), GATA2A164T $\left(6.9 \%, \mathrm{p}<10^{-}\right.$ $\left.{ }^{3}\right)$, KITM541L $\left(2.9 \%, \mathrm{p}<10^{-3}\right)$, TET2H1778R $(2.6 \%$, $\mathrm{p}=.636)$, and $R U N X 1 \mathrm{~L} 56 \mathrm{~S}(0.6 \%, \mathrm{p}=.551) . J A K 2 \mathrm{~V} 617 \mathrm{~F}$
( $11 \%$ of all sequence variants in MPN, $\mathrm{p}<10^{-3}$ ) was primarily found in patients with MPN, and IDHIV178I (2.3\% of all sequence variants in MDS, $\mathrm{p}=.011)$ as well as TET2L1721W $(5.8 \% \mathrm{p}=.080)$ mostly in MDS. TET2P363L $(2.8 \%, \mathrm{p}=.517)$ was seen equally frequent in MDS and in patients with other, non-malignant hematologic diseases. TET2I1762V was seen equally frequent in AML (12.6\% of all sequence variants in AML), MDS (9.6\%) and MPN $(7.9 \%)$. In patients suffering CMML, DNMT3AR882H was particularly frequent $(4.8 \%$ of all sequence variants in CMML, $\mathrm{p}=.064)$. ASXL1E1102D (2.1\% of all sequence variants in other, non-malignant hematologic diseases, $\mathrm{p}=.699), I D H 2 \mathrm{R} 140 \mathrm{Q}(2.1 \%, \mathrm{p}=.677)$ and KRASR $161 \mathrm{R}$ $(4.2 \%, \mathrm{p}=.010)$ were frequent in other, non-malignant hematologic diseases. In SAA, NRASG12D (4\% of all sequence variants in SAA patients; $\mathrm{p}=.005$ ) was frequently found, as were DNMT3AK456fs* (8\%, $\mathrm{p}=.007)$, TET2L1721W (8\%, $\mathrm{p}=.080)$ and TET2I1762V $(8 \%$, $\mathrm{p}=.001)$.

Conclusions: Taken together, these data show that VUS occur with high abundancy in this high-risk cohort of patients, and that they differ in frequency between various myeloid disorders. Further research has to aim at clarifying the in vivo nature of these VUS.

Disclosure: Nothing to declare

\section{P514}

IL-7 and IL-15 levels reflect the degree of T-cell depletion during lymphopenia and drive expansion of effector memory $\mathbf{T}$ cells after allogeneic HSCT

Katrine Kielsen ${ }^{1}$, Lisa V.E. Oostenbrink ${ }^{2}$, Anja M. Jansen-Hoogendijk ${ }^{2}$, Monique M. van Ostaijen-ten Dam ${ }^{2}$, Marianne Ifversen ${ }^{1}$, Carsten Heilmann ${ }^{1}$, Marco W. Schilham $^{2}$, Astrid G.S. van Halteren ${ }^{2}$, Robbert G.M. Bredius $^{2}$, Arjan C. Lankester ${ }^{2}$, Cornelia M. Jol-van der Zijde $^{2}$, Maarten J.D. van Tol', Klaus Müller ${ }^{1}$

${ }^{1}$ Copenhagen University Hospital Rigshospitalet, Copenhagen, Denmark, ${ }^{2}$ Leiden University Medical Center, Leiden, Netherlands

Background: The outcome of allogeneic HSCT is challenged by a delayed and long-lasting imbalanced T-cell reconstitution increasing the risk of acute GvHD, infections and disease progression. Although the role of differentially and functionally distinct T-cell subsets in the development of complications has been addressed, little is known about the factors controlling their recovery.

In this study, we investigated the impact of immuneregulating and homeostatic cytokines on the reconstitution of functionally distinct T-cell subsets and associated clinical outcomes. 
Methods: We included 80 children undergoing allogeneic HSCT for ALL $(n=46)$ or AML $(n=34)$ with a median age of 8.3 years (range: 0.8-17.8). Donors were either MRD $(n=21)$, MUD $(n=45)$ or MMUD $(n=14)$. BM $(n=70)$ or PB $(n=10)$ were used as stem cell source. Conditioning regimens were based on TBI $(n=25)$ or highdose chemotherapy alone $(n=55)$ and included ATG in 58 patients. Thirty age-matched healthy children were included as controls.

Cytokines (IL-7, IL-15, IL-18, SCF, IL-6, IL-2 and TNF$\alpha)$ and active ATG in plasma were longitudinally measured from before conditioning until 3 months after HSCT $(n=80)$ along with an extended phenotyping of T-cell maturation and differentiation by flow cytometry $(n=41)$.

Results: The homeostatic cytokines IL-7 and IL-15 increased from pre-conditioning to peak 1-2 weeks postHSCT and gradually declined thereafter. IL-6 levels were shortly elevated, while IL-18 and SCF remained relatively stable, and IL- 2 and TNF- $\alpha$ levels were below threshold of detection at all time points. The early rise of IL-7 and IL-15 was strongly associated with the degree of T-cell depletion by ATG, while IL-15 also correlated with markers of systemic inflammation. IL-7 and IL-15 levels were significantly higher in children treated with ATG $(P<0.001)$ and correlated with both longer exposure to ATG $(P<0.001)$ and increased levels of active ATG (day +21: IL-7: $r=0.71, \quad P<0.0001$; IL-15: $r=0.61, P<$ 0.0001 ), indicating that high levels of these cytokines reflected more pronounced T-cell depletion during lymphopenia. Higher circulating levels of IL-7 and IL15 were associated with a slow recovery of $\mathrm{CD} 3+, \mathrm{CD} 4+$ and CD8 + T-cell counts at day +30 and +60 post-HSCT $(P<0.05)$, while the remaining cytokines did not correlate with immune reconstitution. Looking into T-cell subpopulations, increased levels of IL-7 and IL-15 during the first month post-transplant were associated with lower numbers of naïve $\mathrm{T}$ cells and correlated with an increased proportion of CD4+ and CD8+ effector memory cells (Figure). No differential effect of cytokines on polarization of CD4+ T cells into Th1, Th2, Th17 cells or Treg cells was found. In ATG-treated patients, IL-7 and IL-15 levels at day +14 were significantly lower in patients developing acute GvHD grade II-IV $(P=0.0007$ and $P=0.0007$, respectively). In the total cohort, increased IL-7 levels were associated with a reactivation of EBV $(P=0.040)$.

Conclusions: These findings suggest that quantification of IL-7 and IL-15 can be indicative for the degree of T-cell depletion during the first weeks after HSCT and predictive of complications. Overall, these results indicate that the lymphopenia-induced elevation of IL-7 and IL-15 is a major driver of the initial expansion of donor T-cells.
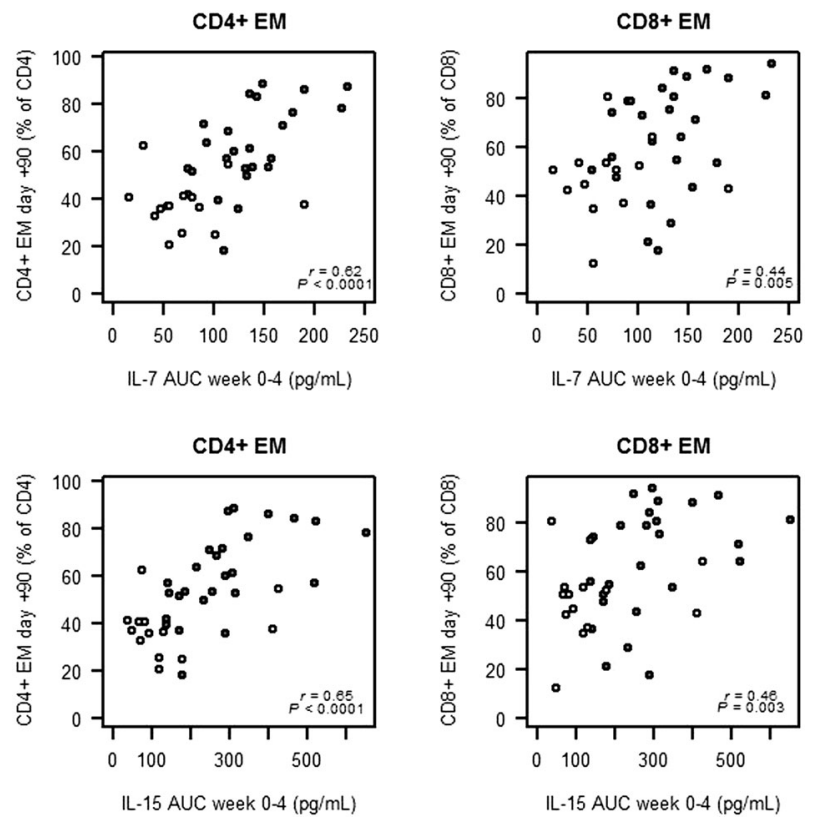

[[P514 Image] 1. Early cytokine levels and effector memory T cells after HSCT with (black) and without (white) $A T G]$

Disclosure: The authors declare no conflict of interest. Financial support was obtained from the Research Council at Copenhagen University Hospital Rigshospitalet, Danish Childhood Cancer Association, Dagmar Marshall Foundation and Netherlands Organisation for Health Research and Development.

\section{P515}

Mathematical kinetic models of immune cell reconstitution and their predictive value for acute graftversus-host disease and relapse after allogeneic hematopoietic stem cell transplantation

\section{Sujun Gao ${ }^{1}$, Long $\mathrm{Su}^{1}$, Xiaoliang $\mathrm{Liu}^{\mathbb{1}}$, Yehui Tan ${ }^{\mathbb{1}}$, Wei Han $^{1}$, Yangzhi Zhao', Yu Liu ${ }^{1}$}

${ }^{1}$ Jilin University, Changchun, China

Background: Mathematical kinetic models were adopted to study immune cell reconstitution after allogeneic hematopoietic stem cell transplantation (allo-HSCT). The associations between acute graft-versus-host disease (aGVHD), relapse and the immune cell reconstitution kinetic models were explored.

Methods: From June 1, 2011 to May 31, 2015, sixty-five patients with hematological malignancies after allo-HSCT were recruited. Peripheral blood was collected on +14 day, +28 day, +42 day and in +2 month, +3 month, +6 month, +9 month, +12 month, +18 month, +24 month. 
Lymphocyte subsets were determined by flow cytometry, including in total $\mathrm{T}$ lymphocytes $\left(\mathrm{CD}^{+}\right)$, helper T cells (CD3 ${ }^{+} \mathrm{CD}^{+}{ }^{+}$, cytotoxic $\mathrm{T}$ cells $\left(\mathrm{CD}^{+} \mathrm{CD}^{+}\right), \mathrm{CD} 4 / \mathrm{CD} 8$ ratio, nature killer (NK) cells $\left(\mathrm{CD}^{-} \mathrm{CD}^{-} 6^{+}\right)$, NKT cells $(\mathrm{CD} 3$ $\left.{ }^{+} \mathrm{CD} 56{ }^{+}\right)$, B lymphocyte $\left(\mathrm{CD} 19^{+}\right)$, naive $\mathrm{T}$ cells $\left(\mathrm{CD}{ }^{+} \mathrm{HLA}-\right.$ $\mathrm{DR}^{+}$), static $\mathrm{T}$ cells $\left(\mathrm{CD}^{+}{ }^{+} \mathrm{HLA}-\mathrm{DR}^{-}\right)$, and regulatory $\mathrm{T}$ cells $\left(\mathrm{CD} 4^{+} \mathrm{CD} 25^{\text {high }} \mathrm{Foxp}^{+}{ }^{+}\right.$. Mathematical kinetic models were calculated for immune cell reconstitution with SPSS.

Results: After allo-HSCT, a logarithmic curve model was observed for $\mathrm{CD}^{+} \mathrm{T}$ cells.

Cubic curve models were observed for $\mathrm{CD}^{+} \mathrm{CD}^{+}$ $\mathrm{T}$ cells, $\mathrm{CD} 4^{+} \mathrm{CD} 25^{\text {high }+} \mathrm{Foxp}^{+} \mathrm{T}$ cell, $\mathrm{CD} 3^{+} \mathrm{HLA}^{+} \mathrm{DR}^{-}$ T cells, $\mathrm{CD}^{+}{ }^{+} \mathrm{CD} 56^{+}$NKT cells, $\mathrm{CD} 19^{+} \mathrm{B}$ cells. CD3 ${ }^{+} \mathrm{CD}^{+}{ }^{+} \mathrm{T}$ cells, $\mathrm{CD} 3{ }^{+} \mathrm{HLA}_{-} \mathrm{DR}^{+} \mathrm{T}$ cells, and $\mathrm{CD} 3{ }^{-} \mathrm{CD} 56^{+}$ NK cells showed $\mathrm{S}$ type curve models. Considering T cells were the major mediators for aGVHD and graft-versusleukemia effect after allo-HSCT. With established immune cell kinetic models, we found that different curve models were observed between patients with and without aGVHD after allo-HSCT. Although the kinetic models were almost the same for leukemia-free and relapsed patients in the first 3 months after allo-HSCT, significantly different kinetic curves could be observed thereafter.

Conclusions: The immune cell reconstitution showed different mathematical curve models after allo-HSCT. Kinetic reconstitution model of certain immune cell was associated with aGVHD and relapse. Hence, mathematical kinetic models of immune cell reconstitution may be potential indictor for predicting aGVHD and relapse after allo-HSCT.

Disclosure: Nothing to declare

\section{P516}

Lineage specific chimerism analysis in pediatric patients following allogeneic hematopoietic cell transplantation (HCT). Influence of age, disease, cytoreduction, graft source and GVHD prophylaxis

Farid Boulad $^{I}$, Eileen Nicoletti ${ }^{I}$, Susan E Prockop ${ }^{I}$, Susan Hsu ${ }^{2}$, Kirsten Fuller ${ }^{1}$, Audrey Mauguen ${ }^{1}$, Nancy A Kernan $^{I}$, Andromachi Scaradavou ${ }^{I}$, Richard J O'Reilly ${ }^{I}$, Jaap Jan Boelens ${ }^{1,3}$

${ }^{1}$ Memorial Sloan Kettering Cancer Center, Pediatric Bone
Marrow Transplant Service, New York, NY, United States,
${ }^{2}$ American Red Cross, Philadelphia, PA, United States,
${ }^{3}$ Princess Máxima Center for Pediatric Oncology, Utrecht,
Netherlands

Background: The outcome of allogeneic HCT is dependent on several variables that include patient age, disease and stage, cytoreduction, graft, graft manipulation, and graft versus host disease (GvHD) prophylaxis. One aspect of
HCT that remains poorly defined and studied is the donor/ host $(\mathrm{D} / \mathrm{H})$ chimerism post HCT. Since 2010, we followed patients with $\mathrm{D} / \mathrm{H}$ lineage specific chimerism post HCT. Analyses were performed by short tandem repeat (STR) polymorphism analysis at the American Red Cross Blood Services (Philadelphia, PA). Studies were performed on blood total leukocytes, myeloid/neutrophil Cells, T-cells, Bcells, and NK-cells.

Methods: In this retrospective study, the charts of 154 consecutive patients who underwent allogeneic HCT between January 2010 to June 2015 on the Pediatric Bone Marrow Transplant Service at MSKCC were retrospectively reviewed. Lineage specific donor chimerism post HCT was studied including $\mathrm{D} / \mathrm{H}$ chimerism trend, and factors with potential impact on chimerism including: age, disease, graft source, and T-cell depletion (TCD). Preliminary analyzes performed on this cohort included Wilcoxon Rank Test and cox proportional hazard analyses.

Results: 137 patients were selected based on the number of analyses. The median age was 11.3 years. Patients had hematologic malignancies $(\mathrm{N}=95)$ or non-malignant hematologic diseases $(\mathrm{N}=27)$, or immune disorders $(\mathrm{N}=15)$. Cytoreduction included TBI- $(\mathrm{N}=42)$, or chemotherapybased regiments $(\mathrm{N}=98)$. Patients were recipients of T-cell depleted marrow or peripheral blood grafts $(\mathrm{N}=101)$, unmodified marrow or peripheral blood grafts $(\mathrm{N}=28)$ or cord blood grafts $(\mathrm{N}=8)$.

Full donor chimerism of myeloid cells, B-cells and NKcells, but not T-cells occurred early post-transplant. There was no difference in the percentage of total donor leukocytes at 3 months vs. 12 months post HSCT $(n=30)$, while the median of donor T-cell chimerism was $51 \%$ at 3 months and $91 \%$ at 12 months post $\mathrm{HSCT}$ ( $\mathrm{p}<$ $0.0001, \mathrm{~N}=42$ ).

Figure 1 shows the impact of different factors including: (A) the use of TBI- or chemotherapy-based regimens, (B) age ( $<$ or $>3$ years), and (C) type of graft (T-cell depleted Vs unmodified Vs Cord Blood). Donor total leukocytes chimerism was significantly lower at 12 months as compared to 3 months for patients $<3$ years of age $(p=0.012)$. For most grafts, full donor chimerism of T-cells occurred early, while for T-cell depleted transplants, it took up to one year to complete. Cord blood grafts were associated with high T-cell donor chimerism throughout the post-transplant period. There was a significant difference in the \% donor T-cells at 3 and 12 months post HCT when comparing $\mathrm{T}$-cell depleted and unmodified grafts $(\mathrm{p}=0.015)$.

Conclusions: This preliminary analysis of lineage specific chimerism post-transplant showed that donor Tcells may take one year to fully recover post-transplant, mostly following T-cell depleted grafts, without intervention. Cord blood grafts were associated with high donor 
chimerism throughout the post-transplant period. Lastly, factors associated with loss of donor chimerism posttransplant were younger age and non-malignant disorders. More in-depth analyses are being performed including the relation of chimerism and HCT outcome.

Disclosure: Eileen Nicoletti - Employee Rocket Pharmaceuticals, Susan Prockop - Investigator Atara Biotherapeutics - Research funding, Susan Prockop - Mesoblast Research funding, Nancy Kernan - Gentium - support; Jazz Pharmaceuticals - Support, Richard O'Reilly - Atara Biotherapeutics - Royalty, Consultancy and Research, Jaap Jan Boelens - Bluebird Bio - Consultancy, Avrobio Consultancy; Jaap Jan Boelens - Chimerix - Consultancy; Magenta - Consultancy

\section{P517}

Loss of mismatched hla as a mechanism of relapse after haploidentical stem cell transplantation

Paula Muñiz ${ }^{1,2}$, Mi Kwon ${ }^{1,2}$, Diego Carbonell ${ }^{1,2}$, María Chicano $^{1,2}$, Julia Suárez-González ${ }^{2,3}$, Cristina Andrés ${ }^{2,3}$, David Serrano $^{1,2}$, Nieves Dorado ${ }^{1,2}$, Laura Solán ${ }^{1,2}$, José Luis Diez-Martín ${ }^{1,2,4}$, Carolina Martínez-Laperche ${ }^{1,2}$, Ismael Buño ${ }^{1,2,3}$

${ }^{1}$ Gregorio Marañón General University Hospital, Madrid, Spain, ${ }^{2}$ Gregorio Marañón Health Research Institute (IiSGM), Madrid, Spain, ${ }^{3}$ Gregorio Marañón General University Hospital, Madrid, Spain, ${ }^{4}$ Complutense University of Madrid, Madrid, Spain

Background: The success of hematopoietic stem cell transplantation (HSCT) lies with the ability of the engrafted immune system to remove residual leukemia cells via a graft-versus-leukemia effect. Despite this, relapse remains the major cause of mortality among patients receiving HSCT. One of the immune evasion mechanisms of leukemic cells to escape from donor $\mathrm{T}$ cell recognition in haplo-HSCT is the genomic loss of the patient specific HLA. It has been described in 20-30\% of acute myeloid leukemia (AML) and myelodysplastic syndrome (MDS) relapses after haplo-HSCT. The aim of this study was to analyze HLA loss in a large cohort of patients who relapsed after T-cell replete haploidentical transplantation with posttransplant cyclophosphamide.

Methods: From December 2007 to September 2018, 180 patients with hematological malignancies who received a haplo-HSCT were recruited. Among them, 31 patients presented a relapse after haplo-HSCT. HLA typing was performed by real-time PCR using HLA-KMR kit (GenDX, Netherlands).Nine patients were excluded from the analysis because the kit employed did not include the recipientspecific HLA. Thus, a total of 22 relapse cases were analyzed. The analysis of chimerism was carried out using short tandem repeat PCR amplification (AmpFISTR SGM Plus, Thermo Fisher, USA) with a sensitivity of $1 \%$.

Results: Genomic loss of the patient HLA occurred in 6/ 22 patients $(27 \%)$ (Table 1). These patients presented different hematological neoplasms. Interestingly, 4 patients presented lymphoid neoplasm (1 acute lymphoblastic leukemia (ALL-T), 1 dentritic cell leukemia (DCL) and 2 hodking's lymphoma (HL)). HLA loss relapses occurred later than classical relapses (370 vs.166 days).

Regarding the treatment received (Table 1), four patients were studied retrospectively. Three of them were treated with donor lymphocyte infusions (DLIs) + chemotherapy or other drugs at the time of the relapse. The other patient did not receive any treatment. In the end, all 4 patients died from disease progression. Prospectively, we detected HLA loss at relapse in other two patients. At the moment of relapse, the first case received brentuximab + Haplo-HSCT from alternative donor and the other case received daratumumab + Haplo-HSCT (pending). Both patients are alive, the first one in complete remission (CR) and the second one in partial remission (PR).

Conclusions: The frequency of HLA loss at relapse is similar in our cohort to what is described in the literature. HLA loss has been identified in patients with lymphoid neoplasms, while this mechanism has not been previously described in such diseases. The analysis of this immune evasion mechanism should be implemented in the routine screening of patients transplanted from haploidentical donors in order to design effective rescue strategies. These treatments should not be based on DLIs or second transplantation with the same donor, instead, alternative donors should be used.

\begin{tabular}{ll}
\hline Diagnosis & AML/ MDS/ DCL/ ALL-T/ HL (2) \\
\hline $\begin{array}{l}\text { Age (Median, range) } \\
\text { Receptor sex (female/male) }\end{array}$ & 48 (27-55) \\
$\begin{array}{l}\text { Time from HSCT to relapse } \\
\text { (days) (Median, range) }\end{array}$ & $370(115-510)$ \\
$\begin{array}{l}\text { Treatment of 4 retrospective } \\
\text { cases. Response }\end{array}$ & $\begin{array}{l}\text { DLIs+Azacitidine; DLIs } \\
\text { +Chemotherapy; DLIs +Brentuximab. }\end{array}$ \\
& $\begin{array}{l}\text { No treatment (1). 4 patients died from } \\
\text { disease progression }\end{array}$ \\
Treatment of 2 prospective & $\begin{array}{l}\text { Brentuximab + alternative haplo- } \\
\text { cases. Response }\end{array}$ \\
& $\begin{array}{l}\text { HSCT. Daratumubab + alternative } \\
\text { haplo-HSCT (pending). CR/PR } \\
\text { respectively. Both patients stay alive }\end{array}$ \\
\hline
\end{tabular}

[[P517 Table] 1. Table 1. Clinical characteristics of patients that relapsed with genomic loss of HLA after haplo-HSCT.]

Disclosure: Nothing to declare 


\section{P518}

Immune reconstitution of CD4 + and NK cells at six months after allogeneic transplantation strongly correlates with survival

\section{Maria Caterina Mico', Chiara Pavoni ${ }^{1}$, Alessandra} Algarotti $^{1}$, Federica Delaini ${ }^{1}$, Anna Grassi ${ }^{1}$, Daniela Taurino $^{1}$, Gianmaria Borleri ${ }^{1}$, Alessandro Rambaldi ${ }^{1,2}$

${ }^{1}$ ASST-PG 23 Bergamo, Bergamo, Italy, ${ }^{2}$ Milano, Milano, Italy

Background: An adequate immune reconstitution (IR) is crucial to reduce transplant toxicity, relapse rate and mortality after allogeneic stem cell transplantation (alloHSCT). The aim of this, single center retrospective study was to investigate the correlation between the recovery of different lymphocyte subpopulations with the main transplant outcomes, including overall survival (OS), disease free survival (DFS) and non-relapse mortality (NRM).

Methods: We analyzed the IR of 177 adult patients (AML $n=62$, ALL $n=41$, MDS $n=24$, NHL $n=9$, HD $\mathrm{n}=2$, CLL $\mathrm{n}=4$, CML $\mathrm{n}=7, \mathrm{MM}, \mathrm{n}=11, \mathrm{MPN} \mathrm{n}=10$ ) who underwent (alloHSCT) between January 2013 and March 2017. Median age at transplant was 52 years (range 17.371.3 ) with male/female ratio of $62 \%$. Donors were HLAidentical siblings $(\mathrm{n}=39,22 \%)$, family haploidentical $(\mathrm{n}=11,6 \%)$, matched unrelated $(109,62 \%)$, mismatched unrelated $(n=11,6 \%)$ and cord blood units $(n=7,4 \%)$. The stem cell source was the bone marrow (BM) in 30 patients $(17 \%)$, the cord blood in $7(4 \%)$ and G-CSF mobilized peripheral blood in 140 (79\%). The conditioning regimen was myeloablative in $99(56 \%)$ transplant, reduced intensity in $75(42 \%)$ and immunosuppressive in 3 (2\%). GVHD prophylaxis was based on calcineurin inhibitors in combination with Methotrexate or Mofetil Mycophenolate. Antilymphocytes Immunoglobulins was used in 145 patients (81\%) (Anti Thymocytes Globulin, ATG Sanofi-Genzyme in 114 or Anti T-lymphocyte globulin, ATLG - Neovii Biotech, in 31). The peripheral blood lymphocyte subsets $(\mathrm{CD} 3+, \mathrm{CD} 3+\mathrm{CD} 4+, \mathrm{CD} 3+\mathrm{CD} 8+, \mathrm{CD} 19+(\mathrm{B}$ cells $)$ and $\mathrm{CD} 16+\mathrm{CD} 56+(\mathrm{NK})$ were analyzed by flow cytometry at 1, 2, 3, 6, 12 and 24 months after HSCT. Post-transplant engraftment was molecularly determined by VNTR analysis.

Results: As detailed in Table 1 the proportion of full donor chimerism analyzed in the peripheral blood $\mathrm{T}$ lymphocytes improved progressively after transplantation and the same pattern was observed when the chimeric status was measured in bone marrow mononuclear cells. To favor the achievement of a full donor chimerism, DLI were performed in 26 patients starting at the median of 96 days after transplant (range:34-447). With a median follow-up observation of 25 months (range 7-61), the one year OS and NRM was $88 \%$ and 5\%, respectively. At 6 months after alloHSCT, the achievement of values higher than 75, 175 and $65 / \mu \mathrm{L}$ for $\mathrm{CD} 4+, \mathrm{CD} 8+$ and $\mathrm{NK}$ cells, respectively was significantly associated to a better OS (Figure 1), DFS $(\mathrm{P}=0.05)$, and to a lower NRM $(\mathrm{P}<0.001$ for $\mathrm{CD} 4+$ and $\mathrm{CD} 8+, \mathrm{P}=0.0034$ for NK). A better lymphoid reconstitution was observed after the use of either a sibling or a haplo donor than a matched unrelated or cord blood donors. The use of ATG was significantly associated with a delayed CD4+ recovery but with a faster NK cells reconstitution.

Conclusions: At six months after alloHSCT, recovery of CD4+ and NK cells predicts survival. Monitoring of immune recovery may help to guide pre and post-transplant treatment strategies.

\begin{tabular}{lllllll}
\hline $\begin{array}{l}\text { Days after } \\
\text { allo HSCT }\end{array}$ & +30 & +60 & +90 & +180 & +360 & +720 \\
\hline CD3+ CD4+ & 64 & 95 & 118 & 165 & 238 & 342 \\
& $(23-$ & $(48-$ & $(68-$ & $(108-$ & $(166-$ & $(254-$ \\
& $146)$ & $147)$ & $180)$ & $266)$ & $350)$ & $458)$ \\
CD56+ CD16+ & 202 & 168 & 180 & 172 & 177 & 237 \\
& $(107-$ & $(93-$ & $(110-$ & $(107-$ & $(106-$ & $(115-$ \\
& $319)$ & $291)$ & $270)$ & $278)$ & $288)$ & $336)$ \\
$\begin{array}{l}\text { Peripheral blood T } \\
\text { cell donor } \\
\text { chimerism }\end{array}$ & $67 \%$ & $75 \%$ & $79 \%$ & $86 \%$ & $96 \%$ & $99 \%$ \\
\hline
\end{tabular}

[[P518 Table] 1. Peripheral blood lymphpid subpopulations (median values $/ \mathrm{mcl}$, with range) and proportion of $B M$ and $P B T$ lymphpcyte chimerism, at different time points]

Disclosure: Nothing to declare

\section{P519}

Immune reconstitution comparison among different transplant settings with post-transplant cyclophosphamide

Nieves Dorado $^{1,2}$, Rebeca Bailén ${ }^{1,2}$, Ana Pérez-Corral ${ }^{1,2}$, Gonzalo Pérez-Balsera ${ }^{1,2}$, Gloria Ruano ${ }^{1}$, Laura Solán ${ }^{1,2}$, Cristina Pascual $^{1,2}$, Pascual Balsalobre ${ }^{1,2}$, Carolina Martínez-Laperche $^{1,2}$, Mi Kwon ${ }^{1,2}$, Jose Luis DiezMartin $^{1,2,3}$, Javier Anguita ${ }^{1,2}$

${ }^{1}$ Hospital General Universitario Gregorio Marañón, Madrid, Spain, ${ }^{2}$ Instituto de Investigación Sanitaria Gregorio Marañón, Madrid, Spain, ${ }^{3}$ Universidad Complutense de Madrid, Madrid, Spain

Background: Immune reconstitution (IR) has a significant impact in hematopoietic stem cell transplantation (HSCT) outcome playing an important role against opportunistic 
infections and disease control. Several groups have demonstrated the independent prognostic value of different lymphocyte subpopulations in HSCT outcomes. Posttransplant cyclophosphamide (PT-Cy) effectively prevents GVHD after HLA-haploidentical hematopoietic stem cell transplantation (Haplo). The use of PT-Cy in HLA matched related (MRD) or unrelated (MUD) donors HSCT, although less explored, has also been introduced.

The aim of this study was to compare the early immune reconstitution after allogeneic HSCT from haploidentical and HLA-matched donors using PT-Cy.

Methods: One hundred and sixty-four HSCT performed in our center were analyzed: 125 Haplo performed between 2011 and 2017 and 39 HSCT from HLA-identical donors (19 consecutive MRD SCT performed with PT-Cy between 2016 and 2017 and 20 MUD SCT with PT-CY between 2014 and 2017). PT-Cy was administered at $50 \mathrm{mg} / \mathrm{kg} / \mathrm{d}$ in days +3 and +4 postransplant, followed by MMF $10 \mathrm{mg} / \mathrm{kg} /$ $\mathrm{d}$ and a calcineurin inhibitor (Ciclosporina A or Tacrolimus) from day +5 ahead.

We retrospectively compared early immune reconstitution at day +30 and day +90 among these three populations. Early IR was assessed through the analysis of lymphocyte subpopulations including total T lymphocytes CD3+, CD4 + and CD8+subpopulations, NK CD3-CD56+ cells, CD56 $+{ }^{\text {bright }}$ immature subpopulation and total B CD19+ lymphocytes.. Lymphocytes subpopulations were determined by multiparametric flow cytometry (FC500 and Navios, Beckman Coulter ${ }^{\circledR}$ ).

Results: All patients, but 1 MUD and 1 Haplo, received PB as stem cell source. 46 patients received prior transplant in Haplo group. Patient's characteristics are shown in table 1 .

Patients who received HSCT from MRD showed the fastest IR, with statistically significant differences compared to Haplo in almost all lymphocyte populations at day +30 $(\mathrm{CD} 3+, \mathrm{CD} 4+, \mathrm{CD} 8+$ and $\mathrm{NK}$ cells), and also in $\mathrm{CD} 3+$, $\mathrm{CD} 8+$ and $\mathrm{B}$ lymphocytes at day +90 . Comparison between Haplo and MUD HSCT showed better IR among Haplo, demonstrated by higher counts in $\mathrm{CD} 3+, \mathrm{CD} 4+$, $\mathrm{CD} 8+$ and NK cell counts at day +30 . No differences were seen at day +90 . (Figure 1). Percentage of immature CD56 $6^{\text {bright }} \mathrm{NK}$ cells was higher in MUD HSCT at +30 , with no differences between Haplo and MRD HSCT.

Conclusions: In our cohort of patients with PT-CY based GVHD prophylaxis regimen, those who received HSCT from MRD showed the earliest immune reconstitution compared to Haplo and MUD at day +30 and +90 . Haplo showed better IR compared to MUD at day +30 . NK maturation at day +30 was a little better among Haplo and MRD HSCT recipients than MUD HSCT patients.

In our experience, using mostly PBSC as graft source, type of donor influenced early IR in PT-Cy based HSCT, with MUD HSCT showing the slowest IR. Further analysis is ongoing to correlate these results to clinical outcome.

\begin{tabular}{llll}
\hline & Haplo (N=125) & MRD (N=19) & MUD (N=20) \\
\hline Age & $46(33-57)$ & $53(42-62)$ & $41(35-52)$ \\
Male & $83(66 \%)$ & $11(58 \%)$ & $9(45 \%)$ \\
AML/ALL diagnosis & $62(50 \%)$ & $9(47 \%)$ & $20(100 \%)$ \\
MAC regimen & $54(43 \%)$ & $9(47 \%)$ & $20(100 \%)$ \\
\hline
\end{tabular}

[[P519 Table] 1. Table 1.- Patients'characteristics]

Disclosure: No disclosure.

P520

Abstract already published.

P521

circulating cell-free DNA (CFDNA) after allogeneic cell transplantation: Characteristics and clinical applications

Miguel Waterhouse ${ }^{1}$, Jesus Duque-Afonso ${ }^{I}$, Dietmar Pfeifer $^{1}$, Justus Duyster ${ }^{1}$, Hartmut Bertz ${ }^{1}$, Jürgen Finke ${ }^{1}$

${ }^{1}$ Freiburg University Medical Center, Freiburg, Germany

Background: Cell-free DNA (cfDNA) isolated from plasma or serum has received increasing interest for diagnostic applications. However, the reported clinical usefulness of cfDNA in patients undergoing allogeneic cell transplantation (HSCT) is scarce.

Methods: The chimeric status both in peripheral blood and in cfDNA obtained from plasma was investigated in 110 patients undergoing HSCT. DNA and RNA were isolated from plasma within four hours of blood draw. Patients were evaluated for chimerism at day $+30,+100$ and +365 post-transplant. A panel of seven microsatellites was amplified by PCR for chimerism detection and PCR products were analysed by capillary electrophoresis. For further cfDNA characterization the micro RNA (miRNA) $200 \mathrm{c}$ was analysed using digital PCR. Mutations frequently used for minimal residual disease assessment such as FLT3ITD, NPM1 and JAK2 were also investigated in cfDNA.

Results: The mean cfDNA concentration in transplanted patients was $469 \mathrm{ng} / \mathrm{ml}$, while in healthy donors used as control group $(\mathrm{n}=20)$ was $119 \mathrm{ng} / \mathrm{ml}$. The mean cfDNA concentration difference between both groups reached statistical significance $(p=0.0197)$. When analysing cfDNA from transplanted patients and in the control group we could not detect DNA fragments larger than $400 \mathrm{bp}$ and the size range of the analysed fragments was between 80 and 200 bp. In 41 out of 110 patients a mixture of donor and 
recipient cfDNA (MC) was detected. With the exception of three patients relapsing after transplant in which MC was detected both in peripheral blood and plasma in the rest of the patients $(n=38)$ MC was detected only in plasma. The mean percentage of recipient cfDNA in the plasma samples was 18\% (range: $1-81 \%$ ). All the patients with acute GvHD (aGvHD) (grade: I-IV) $(\mathrm{n}=15)$ showed MC in plasma at least in one of the time-point tested. No significant difference was found in the mean recipient cfDNA percentage in patients with aGvHD grade I-II when compared with grade III-IV. Meanwhile in the group of patients with chronic GvHD $(n=42)$ MC in plasma was detected in 13 patients. In those patients with clinical improvement of aGvHD $(n=6)$ a decrease in the percentage of recipient cfDNA was observed during treatment. In patients without improvement or even aGvHD worsening $(\mathrm{n}=5)$ stable or increasing recipient cfDNA percentage was detected. Since recipient cfDNA can be detected in patients without transplant-related complications we analysed the miRNA 200c expression in all patients with recipient cfDNA. A significant difference was found in the miRNA 200c expression in patients with aGvHD (mean miRNA 200c: 9.7 miRNA 200c copies $/ 10^{4}$ U6 copies) when compared with patients without GvHD (mean miRNA 200c: 35.4 miRNA copies $/ 10^{4} \mathrm{U} 6$ copies). In those patients with extramedullary AML relapse $(n=3)$ frequent mutations (FLT3-ITD, NPM1) were only detected in the cfDNA fraction.

Conclusions: Longitudinal analysis of cfDNA represents a useful complementary tool in particular for those patients with clinical complications after HSCT.

Disclosure: Nothing to declare

\section{P522}

Comparison of the impact of ATG/PT-CY-based and TCR $\alpha \beta$-depletion as GVHD prophylaxis regimens on the recovery of memory $T$-cell compartment

Natalia Popova ${ }^{1}$, Mikhail Drokov ${ }^{1}$, Yulia Davydova ${ }^{2}$, Nikolay Kapranov ${ }^{2}$, Ekaterina Mikhaltsova ${ }^{I}$, Olga Koroleva $^{1}$, Vera Vasilyeva ${ }^{1}$, Maria Nareyko ${ }^{1}$, Darya Dubnyak $^{1}$, Zoya Konova ${ }^{1}$, Anna Dmitrova ${ }^{1}$, Ekaterina Usikova $^{1}$, Alexey Kazachenok ${ }^{3}$, Yakov Muzalevskii ${ }^{3}$, Denis Kamelskikh ${ }^{4}$,Valentina Dvirnyk, Irina Galtseva ${ }^{2}$, Tatyana Gaponova ${ }^{4}$, Michael Maschan ${ }^{3}$, Larisa Kuzmina ${ }^{I}$, Elena Parovichnikova ${ }^{1}$, Valery Savchenko ${ }^{I}$

${ }^{1}$ National Research Centre for Hematology, Bone Marrow Transplant, Moscow, Russian Federation, ${ }^{2}$ National Research Centre for Hematology, Laboratory of Immunophenotyping, Moscow, Russian Federation, ${ }^{3}$ Dmitry Rogachev National Research Center of Pediatric Hematology, Oncology and Immunology, Institute of Hematology, Immunology and Cell Technologies, Moscow, Russian Federation, ${ }^{4}$ National Research Centre for Hematology,
Blood Cell Processing and Cryopreservation, Moscow, Russian Federation, ${ }^{5}$ National Research Centre for Hematology, Clinical Diagnostics Laboratory, Moscow, Russian Federation

Background: Over recent years haploidentical and HLAmismatched donors have been increasingly adopted as a valid donor source. Modern graft-versus-host disease (GVHD) prophylaxis regimens such as drug-based (antithymocyte globulin (ATG), post-transplant cyclophosphamide (PT-Cy)) or graft-manipulated (TCR $\alpha \beta$-depletion) demonstrate effective prevention of GVHD. Here we report our data about an influence of different GVHD prophylaxis regimens after allo-HSCT with PBSC as a graft source on CD8+ memory T-cells recovery.

Methods: Our study comprised 32 leukemia patients who underwent allo-HSCT with PBSC as a graft source in National Research Center for Hematology, Moscow, Russia. Detailed patients characteristics are presented in Table 1. Peripheral blood samples were collected on day $+30,+60$ and +90 after allo-HSCT. Flow cytometry analysis was performed on BD FACS Canto II (Becton Dickinson, USA) to define T-memory subsets: T-naive and T-stem cell memory (Tnv+Tscm) - CD8+CD45R0-CCR7 $+\mathrm{CD} 28+$; T-central memory (Tcm) - CD8+CD45R0 $+\mathrm{CCR} 7+\mathrm{CD} 28+$; T-transitional memory (Ttm) - CD8 +CD45R0+CCR7-CD28+; T-effector memory (Tem) CD8+CD45R0+CCR7-CD28-; T-terminal effector (Tte) CD8+CD45R0-CCR7-CD28-. Sysmex XE-2100 was used to calculate absolute count of different T- memory cell subsets. Mann-Whitney U test was used for nonparametric data analysis. A p-value less than 0.05 was considered as significant.

Results: Results of Mann-Whitney U test (calculated pvalues) to compare absolute number of T-memory cell subsets in terms of different GVHD prophylaxis regimens are presented in Figure 1. During all follow-up period the number of $\mathrm{Tnv}+\mathrm{scm}$ and $\mathrm{Tcm}$ remains significantly reduced after ATG + PT-Cy or TCR $\alpha \beta$-depletion compared to ATG-based immunosuppressive regimen. On day +30 we observe no difference in the number of $\mathrm{Tnv}+\mathrm{scm}$ and Tcm cells after ATG+PT-Cy or TCR $\alpha \beta$-depletion. Terminally differentiated CD8+ cells (Ttm, Tem, Tte) count is significantly lowered in TCR $\alpha \beta$-depletion patients group in comparison to ATG+PT-Cy. Nevertheless recovery of Tnv+scm and Tcm after PT-Cy is faster than after TCR $\alpha \beta$-depletion.

Conclusions: According to our data the mechanism of PT-Cy is seems to be more selective compared to TCR $\alpha \beta$-depletion due to its transient impact just on $\mathrm{Tnv}+\mathrm{scm}$ and Tcm without affecting on the effector pool. Through this it may lead to delayed reconstitution of adaptive immunity after TCR $\alpha \beta$-depletion compared to using PT- 
Cy. Clinical relevance of the quantitative characteristics of immune recovery in the context of different approaches to GVHD prevention remains to be established.

\begin{tabular}{|c|c|c|c|}
\hline Characteristics & $\begin{array}{l}\text { ATG- } \\
\text { based }(n=10)\end{array}$ & $\begin{array}{l}\text { ATG+PT- } \\
\text { Cy }(n=13)\end{array}$ & $\begin{array}{l}\text { TCR } \\
\alpha \beta \text {-depletion }(n=9)\end{array}$ \\
\hline $\begin{array}{l}\text { Age (median } \\
\text { with range) }\end{array}$ & $42(23-61)$ & $36(20-58)$ & $19(17-57)$ \\
\hline Male / Female & $5 / 5$ & $9 / 4$ & $2 / 7$ \\
\hline ALL / AML & $2 / 8$ & $7 / 6$ & $5 / 4$ \\
\hline CR / Not in CR & $10 /-$ & $11 / 2$ & $8 / 1$ \\
\hline MAC / RIC & $3 / 7$ & $3 / 10$ & $-/ 9$ \\
\hline $\begin{array}{l}\text { Matched related / } \\
\text { Matched unrelated / } \\
\text { Haploidentical / } \\
\text { Mismatched } \\
\text { unrelated }\end{array}$ & $3 / 7 /-/-$ & $-/ 2 / 2 / 9$ & $-/-/ 9 /-$ \\
\hline $\begin{array}{l}\text { Acute GVHD grade } \\
\text { II-IV }\end{array}$ & $5 / 10(50 \%)$ & $\begin{array}{l}4 / 13 \\
(30,8 \%)\end{array}$ & $3 / 9(33,3 \%)$ \\
\hline $\begin{array}{l}\text { Grade II / Grade III / } \\
\text { Grade IV }\end{array}$ & $3(5) / 2(5) /-$ & $\begin{array}{l}1(4) / 2 \\
(4) / 1(4)\end{array}$ & $3(3) /-/-$ \\
\hline
\end{tabular}

[[P522 Table] 1. Table 1. Patients characteristics]

Disclosure: No relevant conflicts of interest to declare.

\section{P523}

\section{Abstract already published.}

\section{P524}

Early peripheral blood immune cells panel impacts on survival after allogeneic haematopoietic stem cell transplantation (HSCT)

Riccardo Boncompagni, ${ }^{1}$ Ilaria Cutini ${ }^{1}$, Stefano Guidi ${ }^{1}$, Antonella Gozzini ${ }^{1}$, Massimo Di Gioia ${ }^{\text {I, Chiara Nozzoli }}{ }^{1}$, Riccardo Saccardi ${ }^{1}$

${ }^{1}$ Careggi University Hospital, Florence, Italy

Background: Immune effector cells, belonging to either innate or acquired immunity, play a key role on preventing disease relapse after allogeneic Haematopoietic Stem Cell Transplantation (HSCT). Most of known immune effector are $\mathrm{CD}^{+}{ }^{+} \mathrm{CD} 8^{+}$T-cells and CD3 CD56 ${ }^{+}$Natural Killer lymphocytes, while $\mathrm{CD}^{+} \mathrm{CD}^{+}$cells act as modulatory and regulatory cells. The early post-HSCT ratio between these cellular subsets may be an indicator of Graft vs-Tumor (GVT) effect.

Methods: We retrospectively revised the immune recovery of 117 allogeneic HSCT performed at our Institution from 2013 to 2017, analysed on peripheral blood by multiparametric flow cytometry lymphocyte subpopulations panel. DIagnosis were Acute Leukemias (69\%), Chronic Myeloproliferative Neoplasms (10\%), Lymphomas (10\%),
Myelodysplastic Syndromes (5\%), Multiple Myeloma (3\%) and Severe Aplastic Anemia (3\%). We established 2 early time-points of evaluation, 30 and 60 days from the graft infusion, to analyse the differences in disease free survival (DFS) and overall survival (OS) between patients according to the $\mathrm{CD}^{+}{ }^{+} \mathrm{CD} 8^{+} \times \mathrm{CD}^{-} \mathrm{CD} 56^{+} / \mathrm{CD}^{+}{ }^{+} \mathrm{CD} 4^{+}$ratio.

Results: Median ratio at +60 days was of 0,5667 . At this time point, patients who showed the ratio higher than the median had both a better DFS (median DFS time not reached vs 12 months; $p=0,018$ ) (Figure 1) and OS (median OS time not reached vs 13 months; $\mathrm{p}=0,014$ ). Likewise, ratio at +30 showed an advantage on DFS ( $\mathrm{p}=$ $0,027)$, and not on OS $(p=0,06)$. Other factors possibly affecting both DFS and OS were analysed in univariate analysis, such as the use of antithymocyte globulin (ATG), conditioning regimen intensity, graft source, HLA-matching and disease status at HSCT, the latter being the only variable with a significantly detrimental impact on both OS and DFS. Disease status was confirmed an independent valriable associated with both DFS and OS as well as +60 ratio both on DFS (Hazard Ratio [HR] - 2,721; $p=0,015$ ) and OS (HR 2,627; $\mathrm{p}=0,022$ ).

Conclusions: Our data show that $\mathrm{CD}^{+} \mathrm{CD}^{+} \times \mathrm{CD} 3^{-}$ $\mathrm{CD}^{+} 6^{+} / \mathrm{CD}^{+} \mathrm{CD}^{+}$ratio assessed at +60 is and independent predictor of transplant outcome, possibly representing a row indicator of anti-leukemic immune surveillance. The integration of this index with other known outcome predictors may help in improving the management of post-transplant phase.

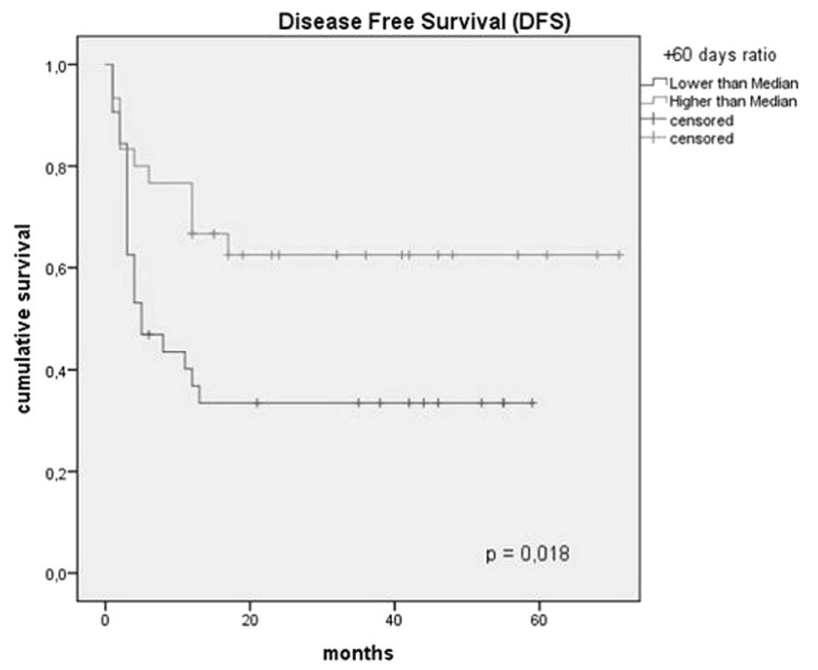

[[P524 Image] 1. Figure 1. Kaplan-Meier survival curves for Disease Free Survival stratified for +60 days ratio.]

Disclosure: Nothing to declare 


\section{P525}

Early recovery of naïve $T$ cells predicts improved survival after allogeneic hematopoietic stem cell transplantation

Valle Gómez García de Soria ${ }^{1}$, Itxaso Portero Sainz, Yaiza Pérez García ${ }^{1}$, Isabel Iturrate Basarán ${ }^{1}$, Lorena Vega Piris ${ }^{1}$, Ana Ramírez Mengíbar ${ }^{1}$, Victor López Huete $^{I}$, Cecilia Muñoz Calleja ${ }^{I}$

${ }^{1}$ La Princesa University Hospital, Madrid, Spain

Background: Allogeneic stem cell transplantation (aloHSCT) is a curative treatment but it is associated with lifethreatening complications. Most deaths are due to relapse, graft versus host disease (GvHD) and infection. The pattern and quality of the immune reconstitution (IR) after transplantation may affect these outcomes. However, there are limited data on the association of the quality of the IR and either the development of GVHD and survival.

Methods: Eighty-five patients who received a non T-cell depleted alo-HSCT in our center from 2011 to 2014 were prospectively studied. Most patients received HLA-identical grafts. Total CD4+ and CD8+ T cells, CCR7+CD4+ and CCR7+CD8+ (which include both naïve and central memory $\mathrm{T}$ cells) and naïve CCR7+CD62L $+\mathrm{T}$ lymphocytes were quantified by flow cytometry. Data were collected at days $+30,+60,+90,+180$ and +360 after alo-HSCT. The association between IR and the GVHD was studied through an ANOVA. For the multivariate analysis, a logistic regression was performed including those confusing clinical variables that were significant in the univariate analysis $(\mathrm{p} \leq 0.10)$. The study of overall survival (OS) versus IR was performed with a cox regression model.

Results: Total CD3 $+\mathrm{T}$ lymphocytes reached normal numbers within the first two months. Median T CD8+ count was 263 cells/ul after one month, which is within the normal range. Conversely, it took nearly one year to get normal counts of CD4+ T cells (542 cells/ul).

The only two clinical parameters conditioning a worse recovery of the $\mathrm{CD} 4+\mathrm{T}$ cells were the previous alosensitization of the donor and the sex, being female donor and male recipient the worst combination for the IR. No parameters influenced the quality of the reconstitution of $\mathrm{CD} 8+\mathrm{T}$ cells. Of note, the age or the HLA status did not influence the quality of the IR.

When the patients were divided into GVHD and no GVHD, we found no differences in the recovery of either the proportion or absolute count of every $\mathrm{T}$ cell subpopulation, including total $\mathrm{T}$ cells as well as naïve/central memory $\mathrm{T}$ cells, both $\mathrm{CD} 4+$ and $\mathrm{CD} 8+$.

Finally, a multivariant analysis confirmed that the absolute counts of $\mathrm{CD} 4+\mathrm{CCR} 7+\mathrm{T}$ cells at day +90 as well as the absolute counts of both $\mathrm{CD} 4+\mathrm{CCR} 7+\mathrm{T}$ cells and naïve $\mathrm{CD} 4+\mathrm{CCR} 7+\mathrm{CD} 62 \mathrm{~L}+$ at day +180 were associated with better OS.

\begin{tabular}{|c|c|c|c|}
\hline & OR & $\mathrm{p}$ value & CI. $95 \%$ \\
\hline $\mathrm{CD} 4+\mathrm{CCR} 7+$ day +90 & 0.96 & 0.001 & $\begin{array}{l}0.95- \\
0.98\end{array}$ \\
\hline $\mathrm{CD} 4+\mathrm{CCR} 7+$ day +180 & 0.86 & 0.014 & $\begin{array}{l}0.77- \\
0.97\end{array}$ \\
\hline $\begin{array}{l}\mathrm{CD} 4+\mathrm{CCR} 7+\mathrm{CD} 62 \mathrm{~L}+(\mathrm{CD} 4+\mathrm{TN}) \\
\text { day }+180\end{array}$ & 0.88 & 0.026 & $\begin{array}{l}0.78- \\
0.98\end{array}$ \\
\hline
\end{tabular}

[[P525 Table] 1. Multivariant analysis of overall survival and immune reconstitution]

Conclusions: In conclusion, neither the development of GVHD nor other relevant parameters seem to play a determinant role in the quality of the IR.

To our knowledge this is the first study which demonstrate a clear association between the recovery of naïve $\mathrm{CD} 4+\mathrm{T}$ cells measured by flow cytometry and the OS.

Disclosure: Nothing to declare

\section{P526}

Abstract already published.

P527

Azacitidine (AZA) for prophylaxis or pre-emptive therapy for myeloid neoplasms after allogeneic stem cell transplantation

Carolina Marini $^{1,2}$, Eolia Brissot ${ }^{1,3}$, Abdulhamid Bazarbachi $^{1}$, Rémy Duléry ${ }^{1,3}$, Simona Sestili ${ }^{1}$, Giorgia

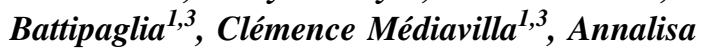
Paviglianiti $^{1}$, Ramdane Belhocine ${ }^{I}$, Françoise Isnard ${ }^{1}$, Simona Lapusan ${ }^{1}$, Rosa Adaeva ${ }^{1}$, Anne Vekhoff ${ }^{l}$, Tounes Ledraa $^{1}$, Ollivier Legrand ${ }^{1,3}$, Myriam Labopin ${ }^{1}$, Agnes Bonnin $^{1}$, Annalisa Ruggeri ${ }^{1}$, Florent Malard ${ }^{1,3}$, Mohamad Mohty ${ }^{1,3}$

${ }^{1}$ Saint Antoine Hospital, AP-HP, Paris, France, ${ }^{2}$ Centro Hospitalar de São João, Porto, Portugal, ${ }^{3}$ Sorbonne University, INSERM UMRs 938, Centre de Recherche Saint-Antoine (CRSA), Paris, France

Background: AZA holds promise in the treatment of relapse after allogeneic transplantation, especially if administered to patients with lower disease burden, on a prophylaxis or pre-emptive basis.

Methods: We present a retrospective study of patients treated with AZA post-transplant in a single centre. Overall, 32 patients were included in this analysis, of 
whom 21 were treated prophylactically and 11 preemptively. Median age was 55 years [range, 15-69] and all patients had a diagnosis of AML or high-risk MDS. Prophylactic treatment consisted of AZA $32 \mathrm{mg} / \mathrm{m}^{2}$ for 5 days in cycles of 28 days. In the pre-emptive setting, 5 patients received $75 \mathrm{mg} / \mathrm{m}^{2}$ for 7 days per cycle and 6 patients $75 \mathrm{mg} / \mathrm{m}^{2}$ for 5 days per cycle. A median of 6 cycles [range, 1-18] was administered in the prophylactic group and of 4 cycles [range, 4-22] in the pre-emptive group. Ten patients also received at least one DLI after the third AZA cycle: 8 patients in the prophylactic group and 2 patients in the pre-emptive one.

Results: During follow-up, 10 patients had significant delays in treatment plan due to transitory mild complications. However, $15 \%$ of patients $(n=5)$ presented infectious complications requiring hospitalisation and $40 \%$ of patients $(\mathrm{n}=10$ in the prophylaxis group and 3 in the pre-emptive group) presented some form of GvHD. In patients who developed GvHD, 3 had to discontinue treatment (all in the prophylaxis group); also 8 patients discontinued treatment due to disease progression. The overall drop-out rate was $37.5 \%(n=12)$. Survival was analysed from initiation of treatment with AZA and median follow-up was 16 months. One-year EFS was $95 \%$ in the prophylaxis group, with only one patient relapsing and no deaths. In the pre-emptive group, the 1-year EFS was $45 \%$ and the median EFS was 6 months; 1-year OS was $70 \%$ and median OS was 24 months.

Conclusions: We conclude that post-transplant AZA treatment is a well-tolerated therapy, but the incidence of side effects remains discordant in the literature. Results in the prophylaxis group are excellent, but patients with positive minimal residual disease treated pre-emptively had a lower outcome with only stabilisation of the disease. Randomised prospective trials are needed to define patients who would benefit the most from this treatment and at what timing, dosage and duration of treatment.

Disclosure: Nothing to declare.

\section{P528}

\section{Abstract already published.}

\section{P529}

Myeloid-derived suppressor cell (MDSC) recovery after allogeneic hematopoietic cell transplantation (ALLOHCT) using post-transplant cyclophosphamide (PTCY) as graft-versus-host disease (GVHD) prophylaxis in children

\author{
Benjamin Oshrine $^{1}$, Patrick Verdugo ${ }^{2}$, Pasquale \\ Innamarato $^{2}$, Shari Pilon-Thomas ${ }^{2}$
}

${ }^{1}$ Johns Hopkins All Children's Hospital, Cancer and Blood Disorders Institute, Saint Petersburg, FL, United States, ${ }^{2}$ Moffitt Cancer Center, Tampa, FL, United States

Background: MDSCs are a heterogeneous group of immature myeloid cells implicated in T-cell regulation; they recover rapidly after alloHCT and may play a role in mediating donor-recipient tolerance, but have not been previously evaluated in the setting of haploidentical transplantation with PTCy.

Methods: Five children (ages 9-17 years) with hematologic malignancies (AML, N=3; ALL, N=2) underwent myeloablative alloHCT using haploidentical family donors and unmanipulated bone marrow grafts, with PTCy-based GVHD prophylaxis. Blood was collected at various time points after transplant. Fresh PBMCs from alloHCT recipients were isolated by Ficoll density gradient centrifugation. M-MDSC (CD14+, HLA-DR-/lo) and PMN-MDSC (CD14-, lineage-, HLA-DR-/lo, CD15+) populations were stained and analyzed by flow cytometry. Additionally, on day 20-22 post-transplant, MDSC were isolated from fresh PBMC by CD15 positive selection and co-cultured with normal donor purified T-cells for MDSC suppressive capacity. MDSC were co-cultured at a 1:1 ratio with Cell Trace Violet loaded T-cells and activated with CD3/CD28 dynabeads for 72-96 hours. T-cells were assessed for proliferation by flow cytometry using Cell Trace Violet dilution and compared to activated T-cells.

Results: All subjects experienced hematopoietic engraftment at a median of 17 days (range 14-20) and demonstrated full donor myeloid chimerism. M-MDSC and PMNMDSC recovery peaked at a median of 22 days posttransplant. The median peak absolute M-MDSC count was $9,828 \mathrm{cell} / \mathrm{mL}$ (range 9,180-41,120/mL) representing a range of $1.5 \%$ to $4.8 \%$ of PBMCs. The PMN-MDSC peak was more robust, with a median absolute peak of 103,730 cells $/ \mathrm{mL}$ (range 11,330-676,800/mL) representing a median of $25.3 \%$ of PBMCs (range 10.3-44.2\%). Of note, the one patient who developed severe, life-threatening GVHD had the lowest absolute and relative PMN-MDSC recovery (11,330 cells $/ \mathrm{mL}$ and $10.3 \%$ of total PBMCs). Recovery of M- and PMN-MDSCs occurred at a similar tempo and magnitude in two recipients of standard GVHD prophylaxis (tacrolimus/methotrexate). However, while MDSCs isolated from PTCy recipients exhibited clear T-cell suppressive capacity, those from the comparison patients did not (see Figure).

Conclusions: MDSCs recover rapidly and robustly after alloHCT using PTCy as GVHD prophylaxis, and may play a role in mitigating GVHD risk by mediating T-cell suppression. This may be a mechanism by which PTCy results in donor-recipient tolerance. 


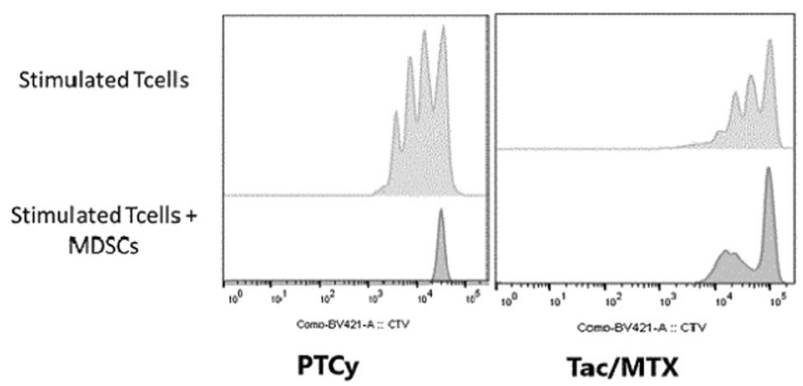

[[P529 Image] 1. MDSC-induced T-cell suppression]

Disclosure: Nothing to declare

\section{P530}

Abstract already published.

P531

Impaired T-cell reconstitution after autologous stem cell transplantation in patients with myeloma and lymphoma in the modern-era

Sonia Bharel ${ }^{\text {, William Johnson }}{ }^{\text {, Thomas Klumpp }}{ }^{1}$, Michael Sun ${ }^{1}$, Adam Binder ${ }^{1}$, Matthew Carabasi ${ }^{1}$, Joanne Filicko-O'Hara ${ }^{I}$,Sameh Gaballa ${ }^{1}$, Margaret Kasner ${ }^{I}$, Dolores Grosso ${ }^{1}$, Ubaldo Martinez-Outschoorn ${ }^{1}$, Neil Palmisiano $^{1}$, Lindsay Wilde ${ }^{1}$, Pierluigi Porcu ${ }^{1}$, Neil Flomenberg $^{1}$, John Wagner ${ }^{1}$, Zachary Lee ${ }^{1}$, Onder Alpdogan

${ }^{1}$ Thomas Jefferson University, Philadelphia, PA, United States

Background: High dose chemotherapy followed by autologous stem cell transplantation (ASCT) offers a cure in the upfront and relapsed setting in both Hodgkin (HL) and nonHodgkin lymphoma (NHL). ASCT also remains standard of care in previously untreated multiple myeloma (MM) patients after induction therapy, if eligible. The availability of new cellular or other immune therapies that can be used after ASCT underscores the potential importance of monitoring immune reconstitution after ASCT.

Methods: Immune reconstitution panels (IRP) were evaluated retrospectively in all lymphoma and MM patients over a 5-year span (2012-2017) whom underwent ASCT at our institution. Patients were included if they had a preASCT measured within 30 days of ASCT and two other IRP at any of the following timepoints (1) day 30-45, (2) day 60-90, and (3) at 1-year post-ASCT. Patients in the lymphoma cohort had their IRP excluded if they had additional treatment within the first year post-ASCT (other than maintenance rituximab). Mononuclear cells from peripheral blood were analyzed by flow cytometry for assessment of lymphocyte phenotype and numbers. Absolute values were compared using the Mann-Whitney U test.

Results: The data on 78 patients were available for analysis (50 MM, 24 NHL, 4 HL). All lymphoma patients were conditioned with BEAM. All MM patients were conditioned with a standard high dose melphalan regimen. The median pre-ASCT absolute CD3 counts in the lymphoma cohort were significantly lower than the MM cohort at 1709 cells $/ \mu \mathrm{L}$ vs 3309 cells $/ \mu \mathrm{L}$, respectively $(P=0.049)$. However, the MM cohort exhibited a greater percent reduction in $\mathrm{CD} 3$ cells on day 30 at $85.3 \%$ vs $63.5 \%$, respectively which continued through day 365 at $79.8 \%$ vs $70.3 \%$, respectively. This led to nonsignificant changes in absolute CD3 count by day 365 at 667 cells $/ \mu \mathrm{L}$ vs 507 cells $/ \mu \mathrm{L}$, respectively $(P=0.39)$ (figure 1$)$. The median absolute CD4 count pre-ASCT for MM and lymphoma cohorts were 2101 cells $/ \mu \mathrm{L}$ and 863 cells $/ \mu \mathrm{L}$, respectively $(P=0.007)$. Similarly, a greater percent reduction in CD4 cells led to comparable absolute counts on day 365 at 310 cells $/ \mu \mathrm{L}$ vs 298 cells $/ \mu \mathrm{L}$, respectively $(P=0.322)$. The failure of post-ASCT CD3 reconstitution to pre-ASCT levels was driven by lack of CD4+ cell recovery, namely $\mathrm{CD} 4+\mathrm{CD} 45 \mathrm{RA}+$ cells with a median of 21 cells/ $\mu \mathrm{L}$ and 20 cells/ $\mu \mathrm{L}$ in the $\mathrm{MM}$ and lymphoma cohorts, respectively at day 365 (figures 2 and 3). This led to markedly diminished CD4:CD8 ratios through day 365 (figure 4).

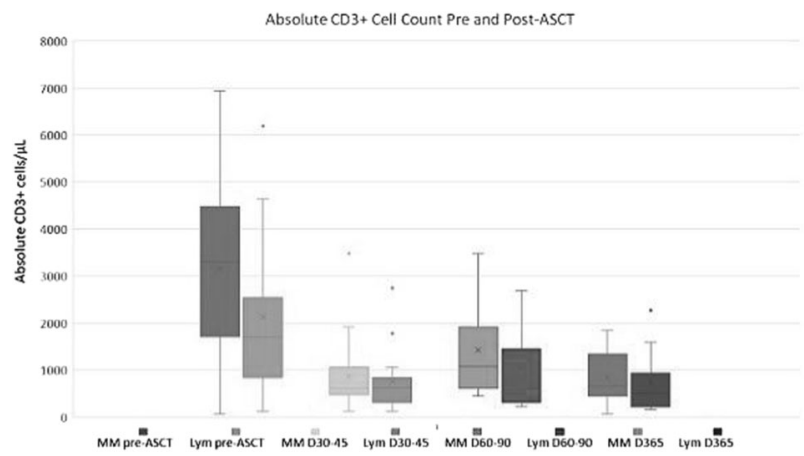

[[P531 Image] 1. Figure 1: Absolute CD3+ Cell Counts Pre and Post-ASCT]

Conclusions: Impaired T-cell reconstitution in both lymphoma and MM continues through 1-year post-ASCT. As shown, a larger percent reduction in median CD3 and CD4 counts through day 365 was appreciated in MM compared to lymphoma leading to the nonsignificant differences in the post-ASCT absolute counts despite significantly higher pre-ASCT counts in the MM cohort. Impaired recovery of CD4 T-cells may increase the risk of opportunistic infections, decrease the response to vaccination and lead to ineffective anti-tumor response. Further prospective and larger retrospective studies like this should 
continue in the modern-era as they may help predict responses to further interventions requiring a robust $\mathrm{T}$-cell repertoire for maximal efficacy such as CAR-T cell and BiTE therapies.

Disclosure: Nothing to declare

\section{P532}

Peri-transplant detection of measurable residual disease by multicolor flow cytometry is highly predictive for relapse in acute myeloid leukemia patients

Evgeny Klyuchnikov ${ }^{I}$, Anita Badbaran ${ }^{I}$, Ulrike FritzscheFriedland $^{I}$, Ute-Marie von Pein ${ }^{I}$, Christine Wolschke ${ }^{I}$, Francis Ayuk ${ }^{l}$, Maximilian Christopeit ${ }^{l}$, Nicolaus Kröger ${ }^{1}$

${ }^{1}$ Hamburg University Hospital, Stem Cell Transplantation, Hamburg, Germany

Background: Presence of measurable residual disease (MRD) prior to allo-SCT has been shown to be predictive for survival in patients in hematological CR of AML. In this study we analyzed the impact of MRD in such patients measured by 8 -color multiparameter flow cytometry (MFC) prior to and on day +100 post-transplant.

Methods: The bone marrow samples immediately prior to allo-SCT and on day +100 post-transplant were retrospectively analyzed. MRD evaluation was carried out with antibodies against: (1) CD34, CD33, CD38, CD15, CD123, CD19, CD45; (2) CD34, CD117, CD56, CD11b, CD14, CD65, CD45; (3) CD34, CD33, CD56, CD2, CD14, CD65, NG2, CD45; (4) CD34, CD33, CD235a, CD61, CD135, CD14, CD45; (5) CD34, CD13, CD117, CD33, CD7, HLADR, CD45; 250.000 events pro tube were recorded.

Results: A number of 55 AML patients (male, $n=35$ ) with median age of 57 years (23-77) in hematological CR prior to allo-SCT were enrolled in the study. We observed lower survival in patients with MRD by MFC pre-transplant (2y OS: $61 \%(41-81 \%)$ vs. $92 \%(82-100 \%), p=0.018)$ due to increased relapse incidence $(35 \%$ (17-53\%) vs. $8 \%$ (0$18 \%), \mathrm{p}=0.009)$. In multivariate analysis, $\mathrm{MRD}^{\mathrm{pos}}$ prior to allo-SCT has strong significant impact on OS (HR 6.5 (1.5 30 ), $\mathrm{p}=0.015$ ). Of 55 patients, a sample both before and on day +100 after transplantation was available in 33 patients. Of those 33 patients, 15 (46\%) were MRD negative prior to transplant and on day +100 (MRD $\left.^{\text {neg/neg }}\right) ; 14$ (42\%) patients were MRD positive prior to transplant and negative at day +100 (MRD $^{\text {pos/neg }}$ ); and $4(12 \%)$ patients were MRD positive at both timepoints (MRD ${ }^{\text {pos/pos }}$ ). DFS and OS for these three groups were as follow: $2 \mathrm{y}$ DFS: $\mathrm{MRD}^{\text {neg/neg: }}$ 93\% (80-100\%), MRD pos/neg. $78 \%$ (56-100\%); MRD ${ }^{\text {pos/pos: }}$ $25 \%(0-75 \%, p=0.006) ; 2 \mathrm{y}$ OS: $\mathrm{MRD}^{\text {neg/neg: }} 100 \%$; MRD ${ }^{\text {pos/neg: }}$ 93\% (79-100\%); MRD ${ }^{\text {pos/pos: }} 25 \%(0-75 \%$, $\mathrm{p}<0.001$ ). Upon multivariate analysis, the MRD status prior to transplant and on day +100 showed strong significant impact on DFS (HR 3.6 (1.3 - 9.9), $\mathrm{p}=0.01$ ) and OS (HR 4.3 (1.3 - 14.5), $\mathrm{p}=0.017)$. We did not observe any significant impact of other factors included in the multivariate analysis (patient's age, patient's sex, and recipient/ donor sex constellation).

Conclusions: MRD positivity prior to allotransplant and at day +100 by MFC is highly predictive for survival after allo-SCT.

Disclosure: Nothing to declaire

\section{P533}

Flow cytometry analysis, T-cell receptor excision circles (TREC) and א-deleting recombination circles (KREC) quantification for immune reconstitution assessment after allogeneic cell transplantation

Miguel Waterhouse ${ }^{1}$, Ingrid Bartsch ${ }^{1}$, Dietmar Pfeifer ${ }^{1}$, Justus Duyster ${ }^{I}$, Hartmut Bertz, Jürgen Finke ${ }^{I}$

${ }^{1}$ Freiburg University Medical Center, Freiburg, Germany

Background: Immune reconstitution is a critical factor for risk assessment of life threatening infections and long-term survival in patients undergoing allogeneic cell transplantation (HSCT).

Methods: Immune cell subsets (CD19, CD4, CD8, CD56, CD25+CD127-) were quantified by flow cytometry. TREC and KREC were quantified simultaneously using droplet digital PCR (dPCR). A total of 31 patients were evaluated. Mean age at transplant was 56 years (range: 1874 years) Samples were obtained before HSCT and at day 30, 100 and 365 after HSCT.

Results: Absolute numbers of CD3 and CD19 cells remained below pre-transplant levels until day 100, increasing further and eventually reaching pre-transplant levels one year after HSCT. Absolute counts of CD4 and CD25+CD127- cells remained below pre-transplant levels beyond one year after HSCT. CD56 cells were characterized by fast reconstitution kinetics, reaching pre-transplant levels already at day 30 . B cells correlated with KREC levels at all time-points tested, whereas $\mathrm{T}$ cells correlated with TREC levels only one year after transplantation. When we compared cell subsets, TREC, KREC levels and the reconstitution kinetics thereof between patients with reduced intensity conditioning $(\mathrm{n}=26)$ or full conditioning $(n=5)$ no significant differences were observed. Patients with pre-transplant TREC levels above the mean (200 TREC copies/ml blood) showed higher TREC levels and a faster T-cell reconstitution after HSCT suggesting that Tcell reconstitution can be predicted by analysing thymic functionality before transplantation. Indeed, in patients with 
a pre-transplant TREC above 200 TREC copies/ml blood, the positive predictive value for an efficient $\mathrm{T}$-cell reconstitution was $0.889(\mathrm{p}=0.012)$. We analysed the recovery kinetics of the cell subsets, TREC and KREC levels in patients with and without transplant-related complications. Patients with either acute graft-versus-host disease or severe infections showed a slower TREC reconstitution when compared with patients without complications.

Conclusions: Our data suggest that the analysis of immune cell subsets together with TREC and KREC quantification can be used to evaluate the immune reconstitution process after HSCT. Pre-transplant TREC levels allow T-cell reconstitution efficiency prediction after HSCT.

Disclosure: Nothing to declare

\section{P534}

Efficacy of donor lymphocyte infusions for persistent mixed chimerism following haematopoetic stem cell transplantation

\section{Henry Braybrook $^{1}$, David Davies ${ }^{1}$, Keith Wilson ${ }^{1,2}$, Emma} Kempshall ${ }^{1}$, Wendy Ingram ${ }^{I}$

\footnotetext{
${ }^{1}$ University Hospital of Wales, Haematology, Cardiff, United Kingdom, ${ }^{2}$ Cardiff University School of Medicine, Cardiff, United Kingdom
}

Background: Falling donor / mixed chimerism after allogeneic haematopoetic stem cell transplant (SCT) is associated with an increased risk of relapse and the potential for graft rejection. Donor lymphocyte infusions (DLI) are often administered in patients with mixed chimerism to achieve full donor chimerism but there is little data on long term outcomes for DLI given for persistent mixed chimerism.

Methods: A retrospective analysis of all patients administered DLI for mixed chimerism between 2008 to January 2017 was performed. All patients were transplanted at the University Hospital of Wales within the South Wales Blood and Marrow Transplant (SWBMT) Programme. Patients were identified by the SWBMT database and additional outcome data gathered by review of patients' medical records.

Results: 58 patients were treated with 111 donor lymphocyte infusions between 2008 and January 2017. Thirty one patients treated for relapse (with or without mixed chimerism) were excluded as was a further patient with a mismatched donor. The rest were 10/10 match. Twenty six patients received a total of 54 donor lymphocyte infusions for mixed chimerism alone. The median age was 63 years (range: $27-71$ ) with $65 \%$ women. Fourteen (54\%) of the patients had sibling donor transplants and twelve
(46\%) from matched unrelated donors. Indications for transplant were: for AML or sAML $(n=16)$, myelofibrosis $(n=4)$, MDS $(n=3)$, Hodgkin lymphoma $(n=1)$, nonHodgkin lymphoma $(\mathrm{n}=1)$ and ALL $(\mathrm{n}=1)$.

Escalating doses of donor CD3 $+\mathrm{T}$ cells were administered commencing at $5 \times 10^{5} / \mathrm{kg}$ to $5 \times 10^{6} / \mathrm{kg}$ then increased at half $\log$ increments according to chimerism results until full donor chimerism was achieved. The median number of doses administered was 2 (range 1-5). The median interval was 94 days (range 48-408). The median dose was $1 \times 10^{6}$ \% kg (range $\left.5 \times 10^{5}-6 \times 10^{7}\right)$.

Seventeen patients $(65 \%)$ achieved full donor chimerism and remained so until most recent follow up (median 25 months, range 12-64). One patient continued to receive DLI after the study period and later reverted to full donor.

Two patients had ongoing mixed chimerism with no evidence of relapse. Two patients relapsed; one of whom later achieved remission. There were six cases of GvHD; acute GvHD (grade II $n=2$, grade III $n=1$ ) and 2 cases of chronic extensive GvHD. One patient had GvHD features consistent with overlap syndrome. A total of five patients died, four due to infection (one in a patient with GvHD) and one due to cardiac toxicity from previous treatment (confirmed on post-mortem).

Conclusions: The results of our single centre study help reinforce the evidence for DLI in establishing full donor chimerism when mixed chimerism is detected in the absence of relapse. Incremental DLI dosing is an effective strategy and associated with a low relapse rate. Caution should still be given to the risk of GvHD following DLI, however the risk appears to be low in this study. Larger prospective studies are ongoing to address the optimal dosing strategy for DLI post-transplant.

Disclosure: Nothing to declare

\section{P535}

Hypomethylating agents for the treatment of relapsed acute myeloid leukemia after allogeneic blood stem cell transplantation: A single center experience

\section{Mariarita Sciume ${ }^{1}$, Giorgia Saporiti, Elena Tagliaferri, ${ }^{1}$, Nicola Fracchiolla ${ }^{1}$, Federica Grifoni ${ }^{1}$, Giorgia Levati ${ }^{1}$, Luca Baldini ${ }^{I}$, Francesco Onida ${ }^{I}$}

${ }^{1}$ Fondazione IRCCS Ca' Granda Ospedale Maggiore Policlinico di Milano - University of Milan, Milano, Italy

Background: Allogeneic blood stem cell transplantation (allo-SCT) is a potentially curative treatment for patients with AML, but relapse still represents the major cause of treatment failure. Hypomethylating agents (HMA) azacitidine (AZA) and decitabine (DAC) have been tested alone or in combination with donor lymphocyte infusions (DLI) in 
the post-transplant period with well-balanced profile of good efficacy and moderate toxicity. We retrospectively evaluated the safety and efficacy of HMA +/- DLI in a reallife cohort of AML patients relapsing after allo-SCT.

Methods: Data from all patients with AML who underwent allo-SCT at our Institution in the last 6 years and subsequently received HMA as a salvage treatment for disease recurrence or preemptively for loss of complete donor chimerism were collected.

Results: Eleven patients with a median age of 64 years (range 41-66) were identified; median time between alloSCT and time to HMA therapy was 10 months (range 4-42). According to ELN genetic risk stratification, 2 patients were classified in the favorable group, 3 in the intermediate-I, 2 in the intermediate-II and 4 in the adverse one. Six patients were treated with AZA, whereas the remaining 5 patients with DAC. The cycles were repeated every 28 days. Ten patients (91\%) started HMA for morphological AML relapse, while one patient received AZA as a sequential treatment after DLI administered for loss of complete donor chimerism. Median number of cycles was 3 (range 1 - 20). Treatment strategy included combination with DLI in 5 patients ( 2 in the DAC cohort, 3 in the AZA cohort), while in one case of FLT3-ITD ${ }^{+}$AML sorafenib was also associated to DAC and DLI. No grade $3 / 4$ toxicities and no acute GvHD occurred.

A clinically significant response was observed in four patients (36\%), all receiving at least 4 cycles of HMA therapy; in particular, a complete remission (CR) was achieved in 3/10 patients treated for morphological relapse, including the one who received the DAC/DLI/sorafenib combination and one (favorable ELN risk) who received AZA alone (not eligible for DLI due to a concomitant lateonset cutaneous grade $2 \mathrm{GvHD}$ ). Of interest, the latter patient also displayed a resolution of the cutaneous GvHD. Full donor chimerism recovery with no GvHD was observed in the patient who received AZA for the progressive donor chimerism loss not responding to DLI alone. With a median follow-up of 7 months (range 4-29), the median OS from HMA treatment in responding patients was 16 months (range 4-29); at the time of data collection responses were maintained in all four patients. Seven patients had died, six from AML progression and one for severe intestinal GvHD occurring after failure of DLI+AZA and a following salvage induction chemotherapy treatment.

Conclusions: Although arising from a limited number of patients, our real-life experience of treatment with HMAs +/- DLI in AML patients relapsing after allo-SCT showed a general very good safety profile and promising antileukemic activity, altogether suggesting a facilitation of the graftversus-leukemia effect (GvL) associated to a possible suppression of the GvH reaction.

Disclosure: Nothing to declare

\section{P536}

Clinical evaluation of immune reconstitution and community-acquired infection after SCT

\section{Akihiro Iguchi ${ }^{1}$, Yukayo Terashita ${ }^{I}$, Minako Sugiyama ${ }^{1}$, Mamoru Honda ${ }^{I}$, Yuko Cho ${ }^{I}$}

${ }^{1}$ Hokkaido University Faculty of Medicine, Pediatrics, Sapporo, Japan

Background: High cure rates in childhood diseases have been achieved by stem cell transplantation (SCT). However, there is little knowledge concerning recovery of the immune system and community-acquired infection after SCT. Here we studied the long-term reconstitution of the immune system and incidences of community-acquired infection after SCT.

Methods: We reviewed medical records for 44 patients (M/F: 31/13, median age: 5 years (range: 1-22years) who were treated in the Department of Pediatrics, Hokkaido University Hospital. We analyzed CD4-positive cell counts, serum immunoglobulin $\mathrm{G}$ (IgG) levels, and incidences of community-acquired infection until 2 years after SCT. Indications for SCT were ALL in 8 patients, AML in 4, AA in 6, NB in 5, RMS in 2, JMML in 1, NHL in 1, CGD in 5, WAS in 3 , XSCID in 2, APDS in 2, CD40LD in 2, and other solid tumor in 3 patients. Stem cell sources were autologous $\mathrm{PB} / \mathrm{BM}$ in 6, allogenic BM in 23 (9 related and 14 unrelated) and allogenic CB in 15 patients. In this study, we excluded patients who relapsed after SCT.

Results: The duration of CD4-positive cell counts $<500 /$ $\mathrm{ml}$ after SCT was $14.7 \pm 11.4$ months in all patients. The durations were $12.6 \pm 7.1$ months in patients with hematologic malignancies, $11.5 \pm 7.3$ months in patients with hematologic disorders such as aplastic anemia and PID, $28.8 \pm 25.9$ months in patients with solid tumor, 16.0 \pm 10.4 months in patients who received autologous SCT, $25.1 \pm 22.2$ months in patients who received related BMT/ PBSCT, 13.8 \pm 6.7 months in patients who received unrelated BMT, and 10.5 \pm 5.8 months in patients who received CBT. The durations of $\mathrm{IgG}<500 \mathrm{mg} / \mathrm{dl}$ after SCT were $14.9 \pm 11.5$ months in all patients, $15.8 \pm 9.7$ months in patients with hematologic malignancies, $11.6 \pm 5.1$ months in patients with hematologic disorders, $11.0 \pm 8.7$ months in patients with solid tumor, $19.0 \pm 18.6$ months in patients who received autologous SCT, $12.0 \pm 8.3$ months in patients who received related BMT/PBSCT, 11.5 \pm 5.7 months in patients who received unrelated BMT, and 15.9 \pm 8.7 months in patients who received CBT. There was a significantly higher incidence of community-acquired infection from 6 months after SCT. There were significant differences in the incidence of community-acquired infections between patients with CD4-positive cell counts of $<500 / \mathrm{ml}$ and 
$>500 / \mathrm{ml} \quad(0.16 \pm 0.17 / \mathrm{months} \quad$ vs $\quad 0.33 \pm 0.31 / \mathrm{months}$, $\mathrm{p}=0.001)$ and between patients with $\operatorname{IgG}$ counts of $<500$ $\mathrm{mg} / \mathrm{dl}$ and $>500 \mathrm{mg} / \mathrm{dl}(0.20 \pm 0.21 / \mathrm{months}$ vs $0.32 \pm 0.35 /$ months, $\mathrm{p}=0.06$ ).

Conclusions: In this study, CD4-positive cell count and IgG value had recovered about 15 months after SCT. In our institute, we have achieved a low incidence of infection by education and medication for patients until recovery of CD4-positive cell count and IgG. However, we found a higher incidence of infection after recovery of CD4-positive cell count and IgG. At 12-15 months after SCT, administration of prophylactic medications such as sulfamethoxazole-trimethoprim were terminated and social comeback such as return to school or work were achieved in most patients. It is possible that the high incidence of community-acquired infection was associated with their comeback. Thus, we should consider additional prevention of infection for patients in this period and further evaluation of immunological markers is needed.

Disclosure: No potential conflicts of interest were disclosed.

\section{P537}

Effect of minimal residual disease before transplantation on the outcome of haplo-identical hematopoietic stem cell transplantation for high-risk acute lymphoblastic leukemia

Yehui Tan', Sujun Gao ${ }^{1}$, Xiaoliang Liu', Long Su', Wei Han $^{1}$, Yu Liu ${ }^{1}$, Yangzhi Zhao ${ }^{1}$

${ }^{1}$ Jilin University, Changchun, China

Background: To analyze the effect of haploid hematopoietic stem cell transplantation (HID-HSCT) on high-risk acute lymphoblastic leukemia (ALL), and to explore the effect of minimal residual disease (MRD) before transplant on the prognosis.

Methods: A retrospective analysis was made on 39 high risk ALL patients accepted HID-HSCT in our hospital from January 2013 to January 2018. The clinical features, stem cell implantation, complications, survival and recurrence were compared between pre-transplant $\mathrm{MRD}^{+}$and $\mathrm{MRD}^{-}$ patients.

Results: All the 39 patients got successfully implanted. The overall survival (OS) was $54.67 \%$, the disease free survival (DFS) was $40.96 \%$, the incidence of acute graft versus host disease (aGVHD) was $53.8 \%$, including $23.1 \%$ II IV degree aGVHD and 2.6\% III IV degree aGVHD. There was no significant difference in stem cell implantation, GVHD, cytomegalovirus and hemorrhagic cystitis between $\mathrm{MRD}^{+}$and $\mathrm{MRD}^{-}$patients. DFS and OS in $\mathrm{MRD}^{+}$ patients were significantly lower than those in $\mathrm{MRD}^{-}$ patients, and the cumulative RR rate increased significantly, there was no significant difference in cumulative TRM.

Conclusions: HID-HSCT was an effective method to treat high risk $\mathrm{ALL}$, but $\mathrm{MRD}^{+}$patients had high recurrence rate and poor prognosis. Strategy Adjustment should be considered to reduce tumor residual and the transplantation strategy should be optimized for these kind of high risk patients, so as to improve survival rate.

Disclosure: Nothing to declare

\section{P538}

\section{IMMUNE RECONSTITUTION IS LINKED WITH LONG-TERM OVERALL SURVIVAL AFTER ALLOGENEIC HEMATOPOIETIC TRANSPLANTATION}

C Lombardi ${ }^{I}, L$ Ondarra ${ }^{I}, J$ Iriondo ${ }^{I}, D$ García $^{I}, L$ Aguirre $^{I}, C$ González $^{I}, N$ Argoitia $^{I}$, M Araiz $^{I}, C$ Vallejo $^{1}$

${ }^{1}$ Hospital Universitario Donostia, San Sebastián, Spain

Background: Lymphocytes are responsible for the cellular and humoral immunity and, consequently, its recovery after allo-HSCT might be linked with the survival after the procedure. The aim of this study was to analyze this hypothesis in our series of patients.

Methods: All the 209 allo-HSCT performed in our center from January 2015 through July 2018 were included in the analysis. Median age was 52 years (range: 7-69). 122 pts were male $(58,4 \%)$ and 87 were female $(41,6 \%)$. Baseline diseases were: 69 AML, 49 LPD, 31 MDS, 28 ALL, 16 MPD, $10 \mathrm{MM}$, and 6 BMF. Donor was unrelated in 113 $(54,1 \%)$, and was family in 96 cases $(45,9 \%)$ (including 31 haplo-identical). Stem cell source was PB in 195 (93,3\%) and $\mathrm{BM}$ in 14 pts $(6,7 \%)$. Conditioning regimen was reduced in 111 procedures $(53,1 \%)$ and intensive in 98 (46,9\%) (including just one non-myeloablative). Overall mortalities at days +100 and +365 (the latter in patients with follow-up superior to 1 year) were $9,1 \%$ and $24,9 \%$, respectively. Median follow-up was 25 months (range: 447). Evolution of absolute lymphocyte counts (ALC) and subpopulations at pre-HSCT and during the first year after allo-HSCT were analyzed.

Results: As shown in table 1, ALC and CD4+ lymphocytes decreased after conditioning therapy, and recovered progressively during the post-HSCT period. At day +365 , majority of patients had $>1000$ ALC $/ \mathrm{mcL}$, clearly improved compared to admission values. CD4+ lymphocytes at day +100 was still very low, but at day +365 around half of the series had $200-500 / \mathrm{mcL}$. We found a strong link between ALC, CD4+ lymphocytes, and CD19 + lymphocytes at days +30 and day +100 with overall survival at day +365 of the series (table 2 ). 
Conclusions: In our series, immunity recovery was a late event for majority of patients undergoing allo-HSCT. In addition, in our experience, the precocity and quality of the ALC, CD4+, and CD19+ cells recovery was clearly linked with long-term survival.

\begin{tabular}{|c|c|c|c|}
\hline & $\begin{array}{l}\text { GLOBAL } \\
\text { MORTALITY } \\
(\mathrm{NRM}+\mathrm{RM}) \\
\end{array}$ & NRM & $\mathrm{RM}$ \\
\hline $\begin{array}{l}\text { ALL PATIENTS } \\
(\mathrm{N}=209)\end{array}$ & $52(24.9 \%)$ & $30(14.4 \%)$ & $22(10.5 \%)$ \\
\hline $\begin{array}{l}\text { LYMPHOCYTES >200 } \\
\text { AT DAY + } 30(N=179)\end{array}$ & $36(20.1 \%)$ & $16(8.9 \%)$ & $20(11.2 \%)$ \\
\hline $\begin{array}{l}\text { LYMPHOCYTES <200 } \\
\text { AT DAY +30 }(\mathrm{N}=17)\end{array}$ & $6(35.3 \%)$ & $5(29.4 \%)$ & $1(5.9 \%)$ \\
\hline $\begin{array}{l}\text { LYMPHOCYTES }>200 \\
\text { AT DAY }+100(N=174)\end{array}$ & $28(16.1 \%)$ & $12(6.9 \%)$ & $16(9.2 \%)$ \\
\hline $\begin{array}{l}\text { LYMPHOCYTES <200 } \\
\text { AT DAY }+100(\mathrm{~N}=10)\end{array}$ & $3(30 \%)$ & $1(10 \%)$ & $2(20 \%)$ \\
\hline $\begin{array}{l}\text { CD4+ LYMPHOCYTES } \\
>200 \text { AT DAY }+100 \\
(\mathrm{~N}=66)\end{array}$ & $6(9.1 \%)$ & $3(4.5 \%)$ & $3(4.5 \%)$ \\
\hline $\begin{array}{l}\text { CD4+ LYMPHOCYTES } \\
<200 \text { AT DAY }+100 \\
(\mathrm{~N}=91)\end{array}$ & $13(14.3 \%)$ & $7(7.7 \%)$ & $6(6.6 \%)$ \\
\hline $\begin{array}{l}\text { CD19 + } \\
\text { LYMPHOCYTES > } 200 \\
\text { AT DAY }+100(N=17)\end{array}$ & $0(0 \%)$ & $0(0 \%)$ & $0(0 \%)$ \\
\hline $\begin{array}{l}\text { CD19 + } \\
\text { LYMPHOCYTES }<200 \\
\text { AT DAY +100 }(\mathrm{N}=113)\end{array}$ & $16(14.2 \%)$ & $10(8.8 \%)$ & $6(5.3 \%)$ \\
\hline
\end{tabular}

[[P538 Table] 1. Table 2. Overall day +365 mortality based on lymphocyte recovery.]

Disclosure: Nothing to declare

\section{P539}

Immune reconstitution including $T$ and $N K$ cells in pediatric hematopoietic stem cell transplantation: A single center experience

\section{Eu Jeen Yang ${ }^{1}$, Kyung Mi Park', Ye Jee Shim², Young Tak Lim ${ }^{1}$}

${ }^{1}$ Pusan National University Children's Hospital, Pediatrics, Yangsan, Korea, Republic of, ${ }^{2}$ Keimyung University Dongsan Medical Center, Pediatrics, Daegu, Korea, Republic of

Background: The reconstitution of $\mathrm{T}$ and Natural Killer (NK) cells after hematopoietic stem cell transplantation (HSCT) strongly influences the outcome of HSCT including viral infection and graft versus-host disease (GVHD). The purpose of this study was to investigate the clinical efficacy of immune reconstitution including $\mathrm{T}$ and NK cells after HSCT in children.

Methods: We reviewed the records of 30 patients who undergoing allogeneic HSCT in Department of Pediatrics, Pusan National University Children's Hospital, from January 2013 to July 2017. The counts of T lymphocyte subsets and NK cells was monitored in peripheral blood by flow cytometric technique during $1,3,6$, and 12 months post-HSCT. Blood samples for cytomegalovirus (CMV) and Epstein-Barr virus (EBV) monitoring were tested by real-time PCR assay.

Results: For total of 30 patients, the mean age was 9.1 years (range, 9 months-19 years), 16 of the patients were boys and 14 was girl. Out of a total 30 patients without pre-HSCT CMV viremia or CMV infection, $10(33.3 \%)$ recipients experienced $\mathrm{CMV}$ infection. The number of $\mathrm{CD} 8^{+} \mathrm{T}$ cells in 3 and 6 months post-HSCT was significantly higher in patients with CMV reactivation compared to patients without (median $1066.11 / \mu \mathrm{L}$ vs. $979.0 / \mu \mathrm{L}, \mathrm{P}=0.004$, and $1047.45 / \mu \mathrm{L}$ vs. $551.44 / \mu \mathrm{L}, \mathrm{P}=0.002)$. In $6(20 \%)$ recipients presented acute GVHD, the number of $\mathrm{CD}^{+}{ }^{+} \mathrm{T}$ cells in 1 and 3 months postHSCT was significantly lower in patients with acute GVHD compared to patients without (median $239.13 / \mu \mathrm{L}$ vs. $365.0 / \mu \mathrm{L}$, $\mathrm{P}=0.045$, and $165.2 / \mu \mathrm{L}$ vs. $344.27 / \mu \mathrm{L}, \mathrm{P}=0.035$ ). The number of NK cells in 1 months post-HSCT was significantly lower in patients with CMV reactivation and acute GVHD compared to patients without $(258.5 / \mu \mathrm{L}$ vs. $501.67 / \mu \mathrm{L}, \mathrm{P}=0.004$, and $162.5 / \mu \mathrm{L}$ vs. $464.88 / \mu \mathrm{L}, \mathrm{P}=0.027$, respectively). In multivariable analysis, acute GVHD was shown to be the decisive factor influencing total $\mathrm{T}$ cells $(P=0.028)$ and $\mathrm{CMV}$ reactivation was independently associated with $\mathrm{CD}^{+}$ T cells $(P=0.026)$. The $\mathrm{CD} 4^{+} \mathrm{T}$ cells counts were associated with prior HSCT history and acute GVHD $(P=0.042$ and $P=0.038)$, and the $\mathrm{CD}^{+} \mathrm{T}$ cells counts were also significantly associated with donor type $(P=0.016)$.

Conclusions: Overall, our study documents that immune reconstitution of $\mathrm{CD}^{+}, \mathrm{CD}^{+}{ }^{+} \mathrm{T}$ cells and NK cells is strongly associated with $\mathrm{CMV}$ reactivation and acute GVHD. Additionally, we show that acute GVHD is influenced by lack of sufficient numbers of NK cells as well as $\mathrm{CD}^{+} \mathrm{T}$ cells early after $\mathrm{SCT}$. $\mathrm{CD} 8^{+} \mathrm{T}$ cells, on the other hand, significantly increase after CMV-reactivation and most likely play an important role in reactivation.

Disclosure: Nothing to declare

\section{P540}

T-cell subpopulation, specific for patients after allogeneic hematopoietic stem cell transplantation

Zoya Konova ${ }^{I}$, Nadia Bykova ${ }^{I}$, Murad Vagida ${ }^{I}$, Larisa Kuzmina ${ }^{I}$, Mikhail Drokov ${ }^{I}$, Grigory Efimov ${ }^{I}$, Elena Parovichnikova $^{I}$, Valery Savchenko ${ }^{I}$ 
${ }^{1}$ National Research Center for Hematology, Moscow, Russian Federation

Background: Curative effect of allogeneic hematopoietic stem cell transplantation (allo-HSCT) depends on the alloreactive T-cell immune response toward residual malignant cells - graft-versus-leukemia reaction. However, alloreactive population has not been phenotypically defined. Recent studies suggest that alloreactive $\mathrm{T}$ cells express both costimulatory and inhibitory receptors simultaneously. Exhaustion caused by the inhibitory signaling dampens Tcell functionality, which could lead to the disease relapse.

Here we aimed to investigate the expression of costimulatory and inhibitory receptors on antigen-experienced $\mathrm{T}$ cells after transplantation, to isolate subpopulation specific for allo-HSCT patients and analyze their T-cell receptor (TCR) repertoire.

Methods: Expression of coinhibitory and costimulatory molecules on PBMCs patients at various time points after allo-HSCT was analyzed for expression of: CD3, CD8, CD4, CD45RA, CCR7, CD95, CD27, CD28, KLRG1, TIGIT, PD1, CD137 and OX40 by flow cytometry and compared to healthy donors. CD3+CD8+CD95-CD27 $+\mathrm{CD} 28+\mathrm{PD} 1+\mathrm{TIGIT}+$ fraction and CD3+ CD8+ control fractions were separated on FACS Aria II cell sorter. Double barcoded cDNA libraries of TCR beta-chains for both fractions were prepared and analyzed by sequencing on Illumina platform. Sequencing results were processed by MiGEC, MiXCR and VDJTools software. Enriched clones were identified by Fisher's exact test $\left(\mathrm{p}>10^{-10}\right)$.

Results: We did not find any significant differences between patients after allo-HSCT and healthy donors in single marker's expression, but, when considering coexpression of co-stimulatory and inhibitory molecules on $\mathrm{T}$ cells we found that $\mathrm{CD} 3+\mathrm{CD} 8+\mathrm{CD} 95-\mathrm{CD} 27+\mathrm{CD} 28$ + PD1+TIGIT + subpopulation was significantly increased in allo-HSCT patients. Moreover it increased with the time since the transplantation (Fig. 1). This population was isolated by cell sorting and alongside with total CD8+ fraction subjected to TCR beta-chain repertoire sequencing. The population contained clones significantly enriched compared with $\mathrm{CD} 8+$ fraction representing potentially alloreactive cells. This hypothesis is further supported by the notion that the level of expression of CD27 and CD28 co-stimulatory molecules is lower in the group of patients who subsequently relapsed, compared with the patients with complete remission, while the expression of inhibitory receptors was high in both groups.

Conclusions: According to our data patients after alloHSCT have a phenotypically distinct T-cell population characterized by simultaneous expression of costimulatory and inhibitory markers. This population contains specifically enriched clones, which may be specific for alloantigens. Further functional assays are needed to confirm the alloreactive potential of this subpopulation. Besides low expression of costimulatory molecules combined with high expression of inhibitory receptors on antigen-experienced T-cells of patients after allo-HSCT might be associated with a disease relapse.

Funding was provided by Russian Science Foundation grant 17-15-01512

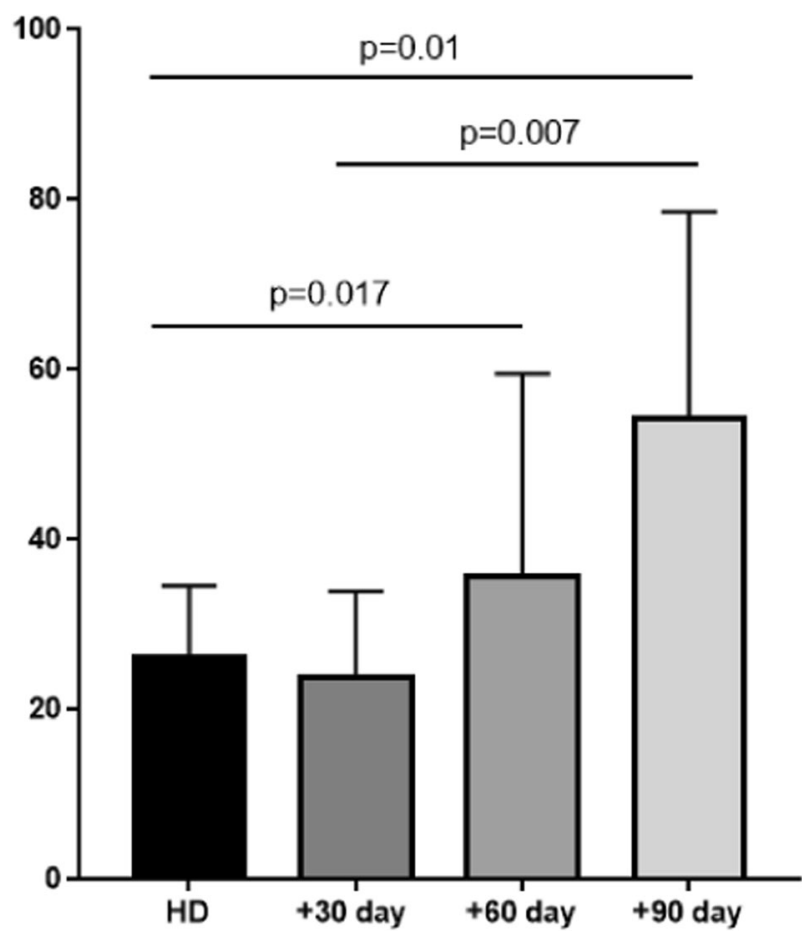

[[P540 Image] 1. T-cell population expressing both costimulatory and inhibitory markers after allo-HSCT.]

Disclosure: No relevant conflicts of interest to declare.

\section{P541}

Efficacy and safety of CD34+ selected stem cell boost (SCB) for poor graft function after haematopoietic stem cell transplantation (HSCT) in children: A centre experience

\section{Giulia Prunotto ${ }^{1}$, Sara Napolitano ${ }^{I}$, Giorgio Antonio Maria Ottaviano ${ }^{2}$, Giuseppe Gaipa ${ }^{3}$, Attilio Maria Rovelli $^{1}$, Adriana Cristina Balduzzi ${ }^{1}$}

${ }^{1}$ Clinica Pediatrica, Universita' degli Studi di Milano Bicocca, Osp. San Gerardo/Fondazione MBBM, Monza, IT, Pediatric Hematology, Monza, Italy, ${ }^{2}$ Università degli Studi di Milano Bicocca, Pediatric Hematology, 
Fondazione MBBM, Monza, Italy, ${ }^{3}$ Ospedale San Gerardo, Laboratorio Stefano Verri, Monza, Italy

Background: Poor graft function (PGF) is a severe complication after HSCT, with a high risk of morbidity and mortality, mainly due to infections. Donor CD34+ SCB seems to offer high chances of haematological recovery, not jeopardized by GvHD. However, pediatric reports remain scarce.

Methods: 19 out of 215 patients undergoing transplantation in our pediatric unit from 2012 to 2018 have been retrospectively evaluated for at least 2 line persistent cytopenia $(\mathrm{Hb}<9.5 \mathrm{~g} / \mathrm{dl}, \mathrm{PLT}<30000 / \mathrm{mmc}, \mathrm{N}<1000 /$ $\mathrm{mmc}$ ) and/or transfusion-dependency beyond 3 months after HSCT in the presence of full donor chimerism. Bone marrow cellularity was evaluated through biopsy as further indicator of PGF.

\begin{tabular}{ll}
\hline Variables & $\mathrm{n}(\%)$ \\
\hline $\begin{array}{l}\text { Gender: Male; Female } \\
\text { Diagnosis: LLA; LMA; }\end{array}$ & $12(79) ; 4(21)$ \\
$\begin{array}{l}\text { Thalassemia; SSA } \\
\text { Median age at HSCT }\end{array}$ & $13,8(6-19)$ \\
(years) & \\
$\begin{array}{l}\text { Donor: MRD/MSD; MUD } \\
\text { Cell source: BM }\end{array}$ & $19(10) ; 13(69) ; 4(21)$ \\
$\begin{array}{l}\text { HSCT CD34+ dose } \\
\text { Cytopenia: } 1 \text { line; } 2 \text { lines; }\end{array}$ & $3.09 \times 10^{\wedge} 6(1.65-5.6)$ \\
S lines; & $(25) ; 5(62.5) ; 1(12.5)$ \\
Median time from & 178 days $(78-206)$ \\
HSCT to SCB & $5.75 \times 10^{\wedge} 6(2.9-14.5)$ \\
SCB CD34+ dose &
\end{tabular}

[[P541 Table] 1. Characteristics of the patients treated with CD34+ boost]

Results: 19 patients, shown in the Table below, would have been eligible for a CD34+ SCB; 74\% had experienced some GVHD and $13(68 \%)$ had at least 1 viral infection/ reactivation. 11 out of 19 received SCB, while 8/19 didn't, either due to donor's refusal $(1 / 8)$ to donate or medical decision (7/8). Bone marrow cellularity was $<5 \%$ in $50 \%$ of the patients who underwent SCB for which the histology was available (8 cases), and $25 \%$ in those who have not been treated (2/8). At 30 days after SCB 10/11 (91\%) patients had hematological response, which was complete in $46 \%$ and partial in $45 \%$ of the patients. Only 1 patient had no response. The infusion was always well tolerated with no adverse events, and no worsening of GvHD. Haematological recovery occurred spontaneously at 30 days after bone marrow biopsy in a significantly lower proportion of patients $(2 / 8,25 \%, p<0.05)$ within the non-SCB group. In two cases platelets engraftment was significantly delayed, up to one year after bone marrow biopsy and in one case thrombocytopenia persists and the patient is still receiving thrombopoietin agonists and red blood cells transfusions at 9 months after bone marrow biopsy.

Conclusions: A stem cell boost matched the goal to yield count recovery in our cohort. Viral infections and GvHD may be possible risk factors for PGF.Bilinear or trilinear cytopenia with transfusion dependency and BOM cellularity $<5 \%$ and full donor chimerism are good indications for $\mathrm{SCB}$, that can provide a significantly earlier hematological reconstitution, without risks of GvHD. Due to the proved early efficacy and safety of CD34+ stem cell infusion, we suggest that this procedure should be taken in consideration in children with severe bone marrow hypoplasia and persistent cytopenia after HSCT.

Disclosure: Nothing to declare

P542

Discrepancies in $\mathrm{ABO}$ and rhesus antigens genotyping data obtained by different laboratory methods in oncohematological patients after hematopoietic stem cell transplantation

Larisa Golovkina ${ }^{1}$, Alla Stremouchova ${ }^{1}$, Tatjana Pushkina $^{1}$, Vadim Surin ${ }^{1}$, Olesya Pshenichnikova ${ }^{1}$, Andrey Sudarikov ${ }^{1}$, Nataliya Risinskaya ${ }^{1}$, Elena Parovichnikova $^{1}$, Larisa Kuzmina ${ }^{1}$, Valeriy Savchenko ${ }^{1}$

${ }^{1}$ National Research Center for Hematology, Moscow, Russian Federation

Background: As allogeneic hematopoietic stem cell transplantation (HSCT) is sometimes performed despite erythrocyte's antigens incompatibility and mismatch, it is essential to carefully track patients' genotypes after it.

Methods: For the study we used erythrocytes $(\mathrm{N}=189)$ and DNA $(\mathrm{N}=20)$ from patients undergoing $\mathrm{ABO}$ - or Rhesus-mismatch HSCT and their donors. We used posttransplant no transfused patients on the periods according transplant protocol by hemagglutination methods in plate and tube using monoclonal antibodies to $\mathrm{ABO}$ and Rhesus antigens (Hematolog, Russia). We extracted DNA with DNA kit (BAG, Germany) and conducted PCR-SSP with kits ABO-Type, RH-Type (BAG, Germany). Chimerism was assessed by the STR-PCR analysis with COrDIS Plus multiplex kit for amplification of 19 polymorphic STRmarkers and amelogenin loci. The fragment analysis was performed on a 3130 Genetic Analyzer. Informative loci were chosen by comparison of pretransplant patient's and donor's DNA. The percentage of donor chimerism was calculated using standard formula. Precise RHCE and ABO genotypes were determined by direct Sanger sequencing. 
Results: We revealed 3 patients with unexpected erythrocyte $\mathrm{ABO}$, Rhesus phenotypes and genotypes after HSCT on +30 ( 2 patients) and +160 days (all patients). Chimerism analyses on STR showed in A.E.Kh. and G.L.V. patients $99 \%$ of donor's DNA and less than $1 \%$ of recipient's one. B.N.A. patient was relapsed and chimerism analysis revealed $95 \%$ of recipient's DNA and $5 \%$ of donor's one.

Using serological methods and PCR-SSP we revealed genotypes $\mathrm{ABO}^{*} \mathrm{~A} 1 \mathrm{~B} 1$; RHD+; RHCE ${ }^{*} \mathrm{CcEe}$ in patient $\mathrm{A}$. E.Kh. before HSCT, ABO ${ }^{*}$ 2O1; RHD+; RHCE ${ }^{*}$ Ccee in her donor, and $\mathrm{ABO}^{*} \mathrm{~A} 1 \mathrm{~A} 2$; RHD+; RHCE ${ }^{*}$ Ccee on $+30 \mathrm{~d}$ after HSCT. Genotype A1A2 was no recipient's neither donor's origin. Direct sequencing did not prove this genotype, but revealed donor's one.On $+160 d$ serological methods and PCR-SSP also revealed donor's genotype in this patient.

Patient B.N.A. had genotypes ABO*O01/O01; RHD+; RHCE ${ }^{*} \mathrm{C}^{\mathrm{w}} \mathrm{cEe}$ before HSCT, her donor - ABO ${ }^{*} \mathrm{~B} 1 \mathrm{O} 1$; RHD + ; RHCE ${ }^{*}$ Ccee. On $+160 d$ this patient relapsed, but Rhesus genotype has been detected as RHD+; $\mathrm{RHCE}^{*} \mathrm{C}^{\mathrm{W}-}$ cee (lack $\mathrm{E}$ gene). Direct sequencing revealed gene RHCE*Ee. ABO genotype was recipient's origin - O1O1.

In patient G.L.V. using serological and PCR-SSP methods we determined genotypes ABO*O01/O01; RHD +; $\mathrm{RHCE}^{*} \mathrm{CCee}$ genotype before HSCT, and $\mathrm{ABO}^{*} \mathrm{~A} 1 /$ O01; RHD+; RHCE ${ }^{*}$ CcEe genotype in her HSC donor. On +30d patient had unexpected genotype ABO*O01/O01 (a lack of $\mathrm{A}$ antigen); RHCE ${ }^{*}$ Ccee (a lack of $\mathrm{E}$ antigen). In order to explain unexpected patient's genotypes after HSCT we sequenced her RHCE and ABO genes and found donor's genotype $\mathrm{CcEe}$; $\mathrm{A} 1 \mathrm{O} 1$ that was in agreement with results of STR analysis. To resolve discrepancies between serological, PCR-SSP and sequencing analysis data we sequenced patient's RHCE cDNA and observed only Ce allele. At present time the molecular basis of selective inactivation one of the two RHCE alleles is not clear. On $+160 \mathrm{~d}$ patient had donor's genotype.

Conclusions: What kind of mechanisms led to discrepancies between results obtained by different laboratory methods are still not clear. An interesting case of expression of only one RHCE allele in patient G.L.V. allows us to suggest involvement of some epigenetic mechanisms like DNA methylation or histone modification in this process.

Clinical Trial Registry: National Research Center for Hematology, Moscow, Russian Federation www.blood.ru

Disclosure: No conflict of interest

\section{P543}

Blinatumomab as a bridging therapy between reinduction and allogeneic stem cell transplantation in relapsed acute B-lymphoblastic leukemia patients with minimal residual disease. Single center experience
Piotr Boguradzki ${ }^{1}$, Michat Górka ${ }^{1}$, Monika Paluszewska ${ }^{1}$, Jarostaw Biliński ${ }^{1}$, Ewa Karakulska-Prystupiuk ${ }^{1}$, Wiestaw Wiktor Jędrzejczak ${ }^{1}$, Grzegorz. Wtadystaw Basak ${ }^{1}$

${ }^{1}$ Medical University of Warsaw, Hematology, Oncology and Internal Diseases, Warsaw, Poland

Background: In relapsed patients with acute B - lymphoblastic leukemia (ALL-B) who achieved complete remission (CR) after re-induction therapy, minimal residual disease (MRD; $\geq 10^{-3}$ ALL-B cells/ul) is often detected. According to available data, such condition varies from $30 \%$ to even $50 \%$ of cases, as assessed by PCR or flow cytometry (FC), while the presence of MRD is the most important risk factor for ALL recurrence. In this abstract, we describe our experience with bridging therapy using blinatumomab infusion after re-induction regimens and before the planned allogeneic stem cell transplantation (alloSCT).

Methods: The procedure was performed in three young men suffering from relapsed Ph (-) ALL-B at the age of 19, 22 and 34 years. In the first case (19yo), relapse with previous MRD accounting for $2.7 \%$ occurred 15 months after CR1 MRD ${ }^{\text {neg }}$. In the next patient (22yo) the second relapse with central nervous system (CNS) involvement occurred 5 months after allo-SCT performed in CR2 (26 months after $\mathrm{CR} 1, \mathrm{MRD}^{\text {neg }}$ ), while in the third patient (34yo), recurrence with CNS and testis involvement occurred 12 years after CR1 $\left(M^{\text {neg }}\right)$. All patients underwent chemotherapy (FLAM, HyperCVAD and DNR/VCR/PegAsp/Dexa regimens respectively) followed by one cycle of blinatumomab (at a dose of $9 \mathrm{mcg} / \mathrm{d}$ on days $1-7$, followed by $28 \mathrm{mcg} / \mathrm{d}$ on days $8-28$ in a continuous infusion) and allo-SCT (using Eto/Cy/TBI/ATG/ conditioning regimen for Ist and IIIrd patient and BuCy2 for IInd patient; using matched unrelated donor (MUD, Ist and IIIrd patient) or matched related donor (IInd patient)). MRD status was assessed after each cycle of blinatumomab by FC.

Results: All patients achieved CR $\mathrm{MRD}^{\text {pos }}$ after reinduction therapy followed by clearance of MRD after blinatumomab course (Tab. 1). The second patient, due to positive MRD 6 months after allo-SCT received 4 donor lymphocyte infusions additionally. During the administration of blinatumomab, no adverse events (AEs) were observed in grade 3 or 4 . One patient developed cytokine release syndrome in grade 1.

The progression free survival, time to positive MRD and follow up are presented in Tab 1 .

Conclusions: The use of blinatumomab as a bridging therapy between re-induction regimens and allo-SCT in patients with ALL-B and MRD ${ }^{\text {pos }}$ appears to be safe and leads to the clearance of MRD which may be crucial in OS 
and PFS prolongation after following allo-SCT. Future studies on larger groups of patients are necessary to confirm this thesis.

\begin{tabular}{llll}
\hline Patient & I & II & III \\
\hline $\begin{array}{l}\text { Relapse after } \\
\text { Time to relapse }\end{array}$ & CR1 & CR2 & CR1 \\
$\begin{array}{l}\text { Re-induction } \\
\text { FLAM }\end{array}$ & $\begin{array}{l}5 \text { months } \\
\text { HyperRCVAD }\end{array}$ & $\begin{array}{l}\text { DNR/VCR/ } \\
\text { PegAsp/Dexa }\end{array}$ \\
$\begin{array}{l}\text { MRD after re- } \\
\text { induction }\end{array}$ & $0.015 \%$ & $1.9 \%$ & $0.1 \%$ \\
$\begin{array}{l}\text { MRD after } \\
\text { Blimatumomab } \\
\text { allo-SCT }\end{array}$ & $0.000 \%$ & $0.000 \%$ & $0.000 \%$ \\
$\begin{array}{l}\text { Time to } \\
\text { positive MRD }\end{array}$ & YES & YES & YES \\
$\begin{array}{l}\text { PFS } \\
\text { Follow up/Alive }\end{array}$ & $\begin{array}{l}\text { NR months } \\
\text { YES }\end{array}$ & 6 months & NR \\
& 16 months & NR \\
\end{tabular}

[[P543 Table] 1. Treatment course and results]

\section{Disclosure: None}

\section{P544}

Immune reconstitution following haploidentical hematopoietic stem cell transplantation: Experience of a single center in Colombia

Claudia Lucia Sossa Melo ${ }^{1,2}$, Angela Maria Peña ${ }^{1,2}$, Sara Inés Jiménez, ${ }^{1,2}$, Claudia Marcela Chalela ${ }^{1}$, Maria LunaGonzalez, ${ }^{1}$, Manuel Rosales ${ }^{2}$, Manuel Ardila-Baéz, ${ }^{1}$, Sergio Serrano $^{1}$, Javier Delgadillo ${ }^{1}$, Luis Antonio Salazar ${ }^{1,2}$

${ }^{1}$ Universidad Autónoma de Bucaramanga, Bucaramangaga, Colombia, ${ }^{2}$ Clinica FOSCAL, Bucaramanga, Colombia

Background: Haploidentical hematopoietic stem cell transplantation (HSCT) is considered an alternative treatment for hematologic malignancies in patients who do not have an HLA-identical sibling donor [1]. Since infections and disease relapse resulting from delayed immune reconstitution (IR) are the most common causes of mortality among patients undergoing haploidentical-HSCT [2], timely IR is essential in the recovery and survival of these patients. The aim of this study is to describe the evolution of IR after haploidentical-HSCT and to estimate survival rates in patients with delayed vs. adequate reconstitution in a single center in Colombia, South America.

Methods: A retrospective cohort study was conducted on 26 consecutive adult haploidentical-HSCT recipients at a tertiary referral center. CD4+cells, CD8+cells, CD3+cells, and immunoglobulins levels were monitored before HSCT, at first month, and then every three months for the first two years post-transplantation. Descriptive statistics were used to analyze patient's clinical characteristics. The KaplanMeier method was used to assess overall survival (OS) and relapse-free survival (RFS) rates.

Results: Twenty-six patients were included $(50 \%$ were male), with a median age of 25.5 years (range 16-59). The most common indication for haploidentical HSCT was acute lymphoblastic leukemia $(n=21,80.8 \%)$, followed by non-Hodgkin lymphoma $(n=3,11.5 \%)$ and myelodysplastic syndrome $(n=2,7.7 \%)$. All patients received GvHD prophylaxis therapy with cyclophosphamide, tacrolimus, and mycophenolate mofetil. Fifteen patients $(57.7 \%)$ presented cytomegalovirus reactivation (25/26 at risk), 5 patients $(19.2 \%)$ Epstein-Barr virus reactivation, and 4 patients $(15.4 \%)$ developed adenovirus infection. Median time to neutrophil engraftment (neutrophils $>0.5 \times 10^{9} / \mathrm{L}$ ) was 15 days (range12-29) for the 23 patients recipients of peripheral blood progenitor cells (PBPCs) and 21 days (range20-27) for the three remaining bone marrow recipients. Platelet engraftment, defined as $>20,000$ platelets/ $\mathrm{mm}^{3}$ untransfused, occurred at a median time of 17 days (range10-38) post-transplantation. Evaluating cellular IR by T-cell subsets, CD4+ cells normal levels were reached by $18.2 \%$ of the patients at nine months post-HSCT, and normal levels of CD8+ cells were reached by $59.1 \%$ and $95.2 \%$ of the patients at 3- and 12-months post-transplantation respectively. Even though only $22.7 \%$ of the patients reached normal CD3 + cells value at 3 months post-HSCT, $76.2 \%$ had normal values after one year of transplantation. Assessing B cell IR, $100 \%$ of transplant recipients reached normal IgG level at three-months post-HSCT. Assessing survival at 4 years post-SCT by cellular IR, OS was $100 \%$ for patients with adequate IR vs. 53.6\% (95\%CI25.3-75.4) for patients with delayed IR, and RFS was 75.0\% (95\% CI12.8- 96.1) for adequate IR vs. 59.4\% (95\%CI32.3-78.7) for delayed IR.

\begin{tabular}{|c|c|c|c|}
\hline Risk Factors & $\begin{array}{l}\text { Adequate IR } 4 \\
(15.4 \%)\end{array}$ & $\begin{array}{l}\text { Delayed IR } 11 \\
(84.6 \%)\end{array}$ & $\begin{array}{l}\mathrm{p}- \\
\text { value }\end{array}$ \\
\hline GvHD & $1(8.3)$ & $11(91.67)$ & 0.54 \\
\hline $\begin{array}{l}\text { Conditioning Regimen } \\
\text { TT-BU-FLU }\end{array}$ & 0 & $1(100)$ & 0.192 \\
\hline $\begin{array}{l}\text { TT-BU-FLU non- } \\
\text { myeloablative }\end{array}$ & 0 & $1(100)$ & \\
\hline Melphalan-TT-FLU & $3(13.6)$ & $19(86.4)$ & \\
\hline BU-FLU & 0 & $1(100)$ & \\
\hline
\end{tabular}

[[P544 Table] 1. Risk factors for delayed IR by cellular immune response]

Conclusions: Myeloid and platelet engraftment was prompt in the present cohort. T-cell IR was delayed in the 
majority of patients. Changes in pre- and post-HSCT therapies need to be studied as strategies to improve time to immune reconstitution.

Disclosure: Nothing to declare

\section{Multiple myeloma}

\section{P545}

Daratumumab as salvage therapy in relapsed/ Refractory multiple myeloma patients after allogenic stem cell transplantation

\section{Sonja Essmann ${ }^{1}$, Ute-Marie von Pein ${ }^{1}$, Evgeny Klyuchnikov ${ }^{I}$, Dietlinde Janson ${ }^{1}$, Stefan Bonmann ${ }^{I}$, Francis Ayuketang Ayuk ${ }^{1}$, Christine Wolschke ${ }^{I}$, Nicolaus Kröger ${ }^{1}$}

${ }^{1}$ University Hamburg-Eppendorf, Hamburg, Germany

Background: Daratumumab is a human monoclonal antibody directed against the glycoprotein CD38 that is overexpressed on the surface of plasma cells in multiple myeloma patients. It is approved as second line therapy either as single agent therapy or in combination with lenalidomide or bortezomib for the treatment of patients with relapsed/refractory multiple myeloma. Despite the curative potential of an allo-SCT, the high relapse rate remains a clinical problem. Data addressing the choice of an optimal salvage therapy regime for these heavily pre-treated patients is missing.

Methods: From April 2016 till November 2018 a total of 22 patients (male, $n=10$ ) with the median age of 64 years (40-72) received daratumumab as a salvage therapy for relapse of multiple myeloma after allo-SCT at the University of Hamburg. Prior to allo-SCT all but one patient had received an autograft, 9 patients even $\geq 2$ autografts and 4 patients also a 1. allograft. The median number of salvage lines post-transplant and prior to first daratumumab infusion was $2(0-4)$. These salvage regimens included cyclophosphamide, etoposide, bortezomib, lenalidomide, pomalidomide and carfilzomib. Daratumumab was started at a median of 19 months (0-43) after relapse/ progress and initiated as single agent therapy in all patients. Concomitantly, 14 patients received either an immunomodulatory drug (lenalidomid, $\mathrm{n}=10$; pomalidomid, $\mathrm{n}=1$ ) or a proteasome inhibitor (bortezomib, $\mathrm{n}=3$ ) during a later course of daratumumab infusions. Combination therapy was initiated when a slow rise of paraprotein and/or free light chains or no response to monotherapy was observed (median at the $11^{\text {th }}$ Infusion).

Results: The median number of infusions was 14 (3-38). Twenty adverse reactions were observed in 13 of 22 (59\%) patients: dyspnea $(\mathrm{n}=5)$, bronchospasm $(\mathrm{n}=2)$ shivering $(n=3)$, cough $(n=2)$, musculoskeletal pain $(n=4)$, acute coronary syndrome $(n=1)$, skin rush $(n=1)$, facial edema $(\mathrm{n}=1)$, pressure on eyes $(\mathrm{n}=1)$. All adverse reactions appeared during the first infusion and were mostly mild or moderate (CTC 1-2, $\mathrm{n}=19$ ). Tolerance of the following infusions improved and in none of the cases therapy had to be stopped due to adverse events. Three patients developed late onset infections (pneumonia, $\mathrm{n}=2$; urinary tract infection, $\mathrm{n}=1$ ) followed by temporarily therapy interruption. With a median follow-up of 14 months after the first administration 20 of 22 patients remain alive (1-year OS $90.9 \%$ ). One patient died due to progress of myeloma and another died due to severe infection/sepsis. 15 of 22 patients responded (68\%; $\mathrm{PR}, \mathrm{n}=5 ; \mathrm{vgPR}, \mathrm{n}=8 ; \mathrm{CR}, \mathrm{n}=2)$ to the therapy with daratumumab. The responses (decrease of paraprotein and/or free light chains $\geq 50 \%$ ) occurred at a median of 7 days (4-372) after the first administration and lasted for 5.0 months $(0.5-26.6)$.

Conclusions: Daratumumab shows an encouraging efficacy and acceptable toxicity profile in patients with relapsed/refractory myeloma after allo-SCT. Further studies are needed to investigate the role of the combination therapy with immunomodulatory drugs or proteasome inhibitors in this setting.

Disclosure: Nothing to declare

\section{P546}

Clonal plasma cell detection by high sensitive flow cytometry in aphaeresis product is poor prognostic and not increased by use of plerixafor alone

Guldane Cengiz Seval ${ }^{1}$, Atilla Uslu ${ }^{1}$, Klara Dalva ${ }^{1}$, Sule Mine Bakanay ${ }^{1}$, Mustafa Merter ${ }^{1}$, Ugur Sahin ${ }^{1}$, Osman Ilhan $^{I}$, Meral Beksac ${ }^{I}$

${ }^{1}$ Ankara University, Ankara, Turkey

Background: In an earlier from our center we have demonstrated that residual clonal plasma cells (cPC) decrease both overall survival (OS) and disease free survival (DFS) (ASH 2018).Plerixafor is a selective antagonist of CXC4 chemokine receptor (CXCR4) and able to mobilize human peripheral blood stem cell (PBSCs) by acting synergistically with G-CSF.The purpose of this study was to evaluate the safety and efficacy of plerixafor in myeloma patients who were proven poor mobilizers and specifically to assess the flow cytometric measurement of residual clonal plasma cells in the apheresis products.

Methods: Patients with a diagnosis of MM who underwent auto HSCT at our center between January 2008- November 2018 were retrospectively analyzed.Out of 164 patients, 16 patients received plerixafor as mobilization regimen due to poor mobilization with G-CSF.PBSC grafts were tested for 
the presence of clonal PCs (cPC) and the number of normal PCs (NPC) by multi-parameter flow cytometry (FCM).The acquisition of the cells was performed using the Navios Flow cytometer (3 laser-10color, BeckmanCoulter).Upon the daily checks of the instrument, $3 \times 10^{6}$ cells for each sample were acquired and the collected data was analyzed using the Kaluza software (BeckmanCoulter,USA).

Results: Patient demographics are shown in Table 1.The majority of patients were male and median age was 62 years in the plerixafor group.The median interval from time of diagnosis to mobilization and follow-up from mobilization were 3.3 months and 14.3 months in plerixafor group, respectively. cPC contamination in the PBSC grafts was detectable in 32 and 7 patients with counts ranging between $0-8.7 \times 10^{-5}$ and $0-0.1 \times 10^{-5}$ in G-CSF alone and G-CSF+Plerixafor groups, respectively $(\mathrm{p}=0.116)$. There were no significant differences in the proportion of the patients with graft contamination between subtypes of MM in both groups. One hundred (GCSF/plerixafor;93/7) patients had pre-ASCT PETCT imaging done with 71 (GCSF/plerixafor;64/7) have active lesion at the time of mobilization. Statistically significant association could not be demonstrated between the disease < $\mathrm{CR}$ status at mobilization and the number of APC in the apheresis product in both groups $(\mathrm{p}>0.05)$. Twelve of 16 patients from plerixafor treatment arm proceeded to transplantation within median 9.5 months.The best overall response to induction treatment is shown in Table 1.Thirtyfour patients from the G-CSF alone arm and 2 patients from the G-CSF+plerixafor arm died during the follow-up $(\mathrm{p}=0.52)$.Disease progression was seen in 48 patients from G-CSF alone group and 6 patients from G-CSF+plerixafor group of the study $(\mathrm{p}=0.56)$.Estimated mean OS was better among patients w/o aPC contamination in plerixafor group, respectively (38.9 $\pm 5.3 \mathrm{mos}$ vs $16.8 \mathrm{mos} ; \mathrm{p}=0.52)$.

Conclusions: Our results on 164 and few plerixafor used patients show that clonal plasma cells are detectable by multiparametric flow more frequently when patients are poor mobilizers and require plerixafor.The clonal PC contamination can be attributed to the myeloma biology as manifested by higher number of lines induction regimens and PET positivity among the plerixafor-required patients. The overall and disease survival was impaired by residual clonal PCs in the graft but not by plerixafor per se. Neither was the content of clonal PCs differed from others. Thus the CXCR4 shared by HSC and myeloma cells do not cause a myeloma mobilization.

Clinical Trial Registry: -

Disclosure: nothing to declare

\section{P547}

Prognostic factors for overall survival after allogeneic hematopoietic cell transplantation in multiple myeloma patients
Joud Zanabili ${ }^{1}$, Pilar Palomo ${ }^{I}$, Soledad González ${ }^{I}$, Ana Julia Goszález ${ }^{1}$, Christelle Castañón ${ }^{1}$, Lucía Rita Morais ${ }^{1}$, Walter Javier Zambrano ${ }^{I}$, Laura Escalada ${ }^{1}$, Carolina Tamayo ${ }^{1}$, Ana Pilar González ${ }^{\mathbb{I}}$

${ }^{1}$ Hospital Universitario Central de Asturias, Hematology, Oviedo, Spain

Background: Allogeneic hematopoietic cell transplantation (allo-HCT) has the potential to cure subgroups of patients with multiple myeloma (MM) but its role is controversial due to high transplant-related mortality. While autologous HCT is well established as consolidation after induction therapy using novel agents, allo-HCT is still considered experimental due to excessive early toxicity.

Methods: We retrospectively studied $45 \mathrm{MM}$ patients (pts); $25(55,6 \%)$ were males with a median age of 52 years (range: 32-60), who underwent allo-HSCT in our center between 2007 and 2018.

Median time between diagnosis and allo-HCT was 40 months (range: 20-143).

Results: The International Staging System (ISS) was ISS I $(33,3 \%)$, ISS II $(28,9 \%)$, and ISS III $(37,8 \%)$. Twentyeight $(62,2 \%)$ pts received cells from unrelated donor and 17 pts from identical siblings. Stem cell source were: peripheral blood $(n=25)$ and bone marrow $(n=20)$. Grouprisk distribution using HCT-CI and PAM index can be seen in figure 1. Response was evaluated at day +100 : CR $(53,3 \%)$, VGPR $(11,1 \%)$, PR $(22,2 \%)$ and SD $(2,2 \%)$; response was not evaluated in 5 pts. Regarding hospitalization, $11(24,4 \%)$ pts and $22(48,9 \%)$ pts needed readmission in the first 100 days and 365 days post-alloHCT, respectively, and median days of hospitalization was 27 days (range: 4-92). Reasons for first re-admission were: infection $(68,2 \%)$, GvHD $(27,3 \%)$ and renal insufficiency $(4,5 \%)$. Twenty-seven (60\%) died and death causes were: infection $(35,6 \%)$, progression $(15,6 \%)$, secondary tumor $(4,4 \%)$ and the remainder, GvHD and intracranial hemorrhage. In addition, 26 pts $(57,8 \%)$ suffered cytomegalovirus (CMV) reactivation.

Median overall survival (OS) was 44 months (range: 1176). Univariate analysis showed that re-admission in first 100 days (HR 4,19; $\mathrm{p}=0,001$ ), re-admission in first 365 days (HR 3,6; $\mathrm{p}=0,001$ ), CMV reactivation (HR 2,38; $\mathrm{p}=0,038$ ), ISS (ISS-I vs ISS-II and III; HR 0,$347 ; p=0,033$ ), days of hospitalization in the first 100 days and response at +100 day (CR plus VGPR vs the remainder; HR 0,143; $\mathrm{p}=0,04)$ were predictor factors for OS. Other factors like karyotype, response before allo-HCT, HLA-mismatch or baseline CMV serostatus, among others, do not impact on OS.

Median OS in pts with low HCT-CI were 56 months (range: 0-156) vs 6 months in intermediate-high HCT-CI 
pts $(p=0,016)$. On the other hand, median OS in pts with high/very high risk group of PAM score was 17 months vs 93 months (range: 37-148) in low/intermediate risk $(\mathrm{p}=0,016)$.

All factors with significant influence on pts survival were included multivariate analysis (Cox regression model) but only re-admission in the first 365 days demonstrated impact on OS (HR 4,082; $\mathrm{p}=0,005$ ).

Conclusions: We analyzed risk factors for survival in MM pts who received allo-HCT. Our study identified disease-related risk factors like ISS and transplantationrelated factors such as HCT-CI and PAM, hospital readmission, days of hospitalization and CMV reactivation that were associated with worse long-term survival. In our series, the most frequent death and re-admission cause was infection, so focusing the efforts in reduction of infection could have a beneficial impact on improvement of survival in MM undergoing allo-HCT.

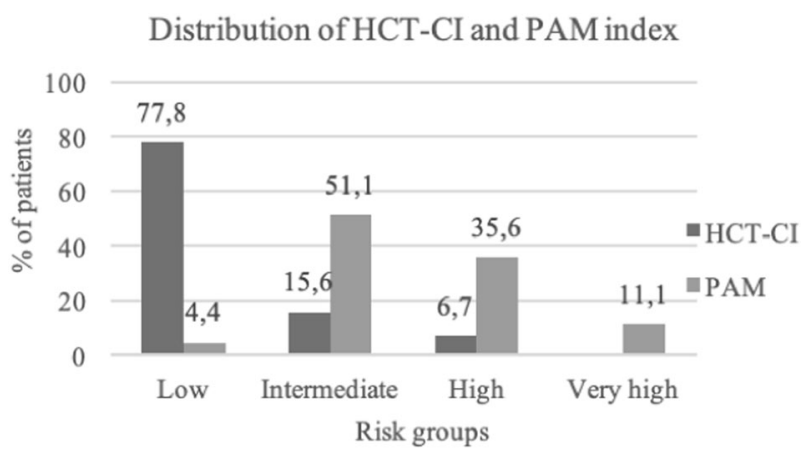

[[P547 Image] 1. Figure 1]

Disclosure: There is no disclosure.

\section{P548}

Novel protocol for autologous hsct in multiple myeloma: Ambulatory chemomobilization and transplantation of fresh hematopoietic stem cells with backup storage

\section{Emilian Snarski ${ }^{1}$, Martyna Maciejewska ${ }^{1}$, Michat Górka ${ }^{1}$, Elżbieta Urbanowska ${ }^{I}$, Magdalena Feliksbrot Bratosiewicz ${ }^{1}$, Karol Lis ${ }^{1}$, Anna Waszczuk Gajda ${ }^{1}$, Kamila Skwierawska ${ }^{1}$, Hubert Heleniak ${ }^{I}$, Mateusz Ziarkiewicz ${ }^{I}$, Grzegorz Niewińnski, Król Matgorzata ${ }^{1}$, Maria Król ${ }^{1}$, Wiestaw Wiktor Jędrzejczak ${ }^{1}$}

${ }^{1}$ Medical University of Warsaw, Warsaw, Poland

Background: Autologous hematopoietic stem cell transplantation (AHSCT) after melphalan conditioning is still a part of standard treatment of multiple myeloma patients. Traditional transplantation of frozen stem cells poses additional risk for the patients connected with DMSO and central venous catheter. The transplantation of fresh cells is an option - however, most mobilization protocols are either low-efficient (G-CSF), expensive (G-CSF + plerixafor) or toxic (standard dose chemomobilization) to directly proceed to transplantation in this fragile group of patients. We describe here the novel combination of ambulatory mobilization with very low doses of Ara-C and G-CSF connected with direct AHSCT with fresh cells.

Methods: The prospectively collected database of patients after AHSCT was searched for patients who underwent AHSCT after chemomobilization with Ara-C and transplantation with fresh cells (FC) and compared with control group of consecutive patients transplanted with standard protocol (SP) (transplantation with frozen cells) between July 2016 and October 2018. Protocol of ambulatory mobilization was: $400 \mathrm{mg} / \mathrm{m}^{2}$ of AraC on days +1 and +2 and G-CSF at the dose $5 \mu \mathrm{g} / \mathrm{kg} / \mathrm{day}$ from day +5 and escalated to $10 \mu \mathrm{g} / \mathrm{kg} /$ day split into two doses +10 to +13 , apheresis started on day +14 (or later) and finished when at least $7.5 \times 10 \mathrm{e} 6 \mathrm{CD} 34+$ positive cells were collected. The collected cells were split in three equal parts:1) for use as fresh transplant 2) frozen for possible $2^{\text {nd }}$ transplant 3) frozen as backup.

Results: There were 48 transplantations with fresh cells and 49 transplantations with frozen cells compared. Both groups had same mobilization protocol - ambulatory low dose ARA-C. The median age and number of transplanted cells was similar in both groups ( 56 vs $57, p=0.99 ; 5.2$ vs $5.3 \mathrm{CD} 34+/ \mathrm{KG}, \mathrm{p}=0.97)$. Median dose of Melphalan was 160 in FC patients and 177 in SP patients $(\mathrm{p}=0.04)$. The WBC engraftment time, PLT 20 engraftment time, hospitalization time, all transfusions, neutropenic fevers, positive blood cultures all favoured transplantation with fresh cells (FC vs SP; 10 days vs 15 days, $\mathrm{p}<0.0001$; 12 days vs 18 days, $\mathrm{p}<0.0001 ; 19$ days vs 34 days, $\mathrm{p}<0.0001 ; 2.9$ vs $9.9, \mathrm{p}<0.0001 ; 26 \%$ vs $68 \%, \mathrm{p}<0.0001$; $4 \%$ vs $46 \%, \mathrm{p}<0.0001)$.

Conclusions: We present novel approach that allows direct AHSCT after chemo mobilization in all patients who are treated with melphalan. We show that it is not only feasible to do AHSCT directly after chemomobilization but also the results may favour this approach when compared with current standard.

Disclosure: Nothing to declare

\section{P549}

Autologous HSCT improves the quality of pre-HSCT response in patients with multiple myeloma

L Ondarra ${ }^{I}$, C Lombardi ${ }^{l}, J$ Iriondo ${ }^{1}$, M Sirvent $^{I}, T$ Hernandez $^{1}$, A Alkorta ${ }^{I}$, A Uranga ${ }^{I}$, JR Furundarena ${ }^{I}, M$ Araiz $^{I}, C$ Vallejo ${ }^{I}$ 
${ }^{1}$ University Hospital Donostia, San Sebastian, Spain

Background: High dose chemotherapy followed by autologous hematopoietic cell transplantation (HSCT) is considered, since the nineties, the standard of care for patients aged less than 70-75 years old with newly diagnosed multiple myeloma (MM). However, the optimal induction treatment prior to HSCT to reduce the tumor burden has changed during the last few years. Improved regimens have shown to be able to increase the quality of the pre-HSCT response, which might subsequently impact on the postHSCT response, which has been proved to be associated with a longer PFS. We recently changed the induction therapy for pts candidates to HSCT. In this analysis, we aimed to check if newer regimens impacted on pretransplant responses, and how auto-HSCT changed the pre-HSCT status.

Methods: All the 117 auto-HSCT for 99 MM patients performed in our center from January 2014 through August 2018 were included in the analysis. Median age was 60 years (range: $38-76)$ ) 54 pts were male and 45 were female. Durie-Salmon stage was distributed as follows: I (9.4\%), II $(37.6 \%)$ and III $(53 \%) ; 13 \%$ had creatinine $>2 \mathrm{mg} / \mathrm{dL}$. ISS was: 1 (41\%), 2 (28.2\%), and 3 (30.8\%). Type of monoclonal component was: $\operatorname{IgG}(51.3 \%)$, light chains (23.9\%), IgA (22.2\%), and non-secretory (2.6\%). $51.3 \%$ had Bence Jones proteinuria. Conditioning regimen was Melphalan $200 \mathrm{mg} / \mathrm{m}^{2}$ in 106 (90.6\%), Melphalan 100-140 $\mathrm{mg} / \mathrm{m}^{2}$ in $9(7.7 \%)$, and other in $2(1.7 \%)$.

Results: Pre-transplant therapy was: VCD in 60 (mostly in 2014-6), VTD/VRD/KRD in 34 (mostly in 2017-8), and others in 23 cases. Status of the disease at transplant was: CR/VGPR in 75, PR in 35, and SD in 7. Distribution of pretransplant response based on the type of induction is shown in table 1. Peri-transplant mortality was $0 \%$. Day +100 mortality was $1.7 \%$ (2 pts), due to progressive disease. As shown in table 2 , all patients (14/14) who obtained CR preHSCT, maintained the response at day +100 post-HSCT. Among the patients in VGPR at HSCT, 20 (32.8\%) became into CR, and 37 (60.7\%) maintained the response. The CR rate at post-HSCT increased $264 \%$ compared to pre-HSCT (37 versus 14 pts). Altogether, after HSCT 44 pts (37.6\%) improved and $65(55.6 \%)$ maintained the pre-HSCT response. During the last period of time, 37 pts started on post-HSCT maintenance/consolidation, mainly with lenalidomide.

\section{Conclusions:}

1) With the new chemotherapeutic schemes, $76.5 \%$ of patients underwent HSCT in CR or VGPR;

2) Majority of pts $(93.2 \%)$ consolidated or improved the pre-HSCT response;

3) CR increased substantially (2.64 times) after transplant;
4) Optimized induction regimens, along with auto-HSCT followed by the recently licensed use of maintenance therapy with lenalidomide, might result in a better PFS of patients with MM.

\begin{tabular}{|c|c|c|c|}
\hline & \multicolumn{3}{|l|}{ Pre-HSCT } \\
\hline & CR/VGPR & PR & SD \\
\hline Global $(\mathrm{N}=117)$ & $75(64.1 \%)$ & $35(29.9 \%)$ & $7(6.0 \%)$ \\
\hline CyBorD (VCD) $(\mathrm{N}=60)$ & $36(60.0 \%)$ & $21(35.0 \%)$ & $3(5.0 \%)$ \\
\hline VTD/VRD/KRD $(\mathrm{N}=34)$ & $26(76.5 \%)$ & $6(17.6 \%)$ & $2(5.9 \%)$ \\
\hline Others $(\mathrm{N}=23)$ & $13(56.5 \%)$ & $8(34.8 \%)$ & $2(8.7 \%)$ \\
\hline
\end{tabular}

[[P549 Table] 1. TABLE 1: Pre-transplant disease status (global and based on induction therapy).]

Disclosure: Nothing to declare

P550 Abstract withdrawn.

\section{P551}

Autologous stem cell transplantation with high dose melphalan conditioning regimen is safe for elderly multiple myeloma patients

Anna Czyz ${ }^{I}$, Agnieszka Szeremet ${ }^{1}$, Aleksandra BoguckaFedorczuk ${ }^{1}$, Maciej Majcherek ${ }^{1}$, Justyna Rybka ${ }^{I}$, Iwona Prajs $^{1}$, Tomasz Wrobel ${ }^{1}$

${ }^{1}$ Wroclaw Medical University, Wroclaw, Poland

Background: Autologous stem cell transplantation (ASCT) is commonly used in treatment of patients over 65 years with multiple myeloma (MM), however the safety and efficacy of this procedure is debatable.

Methods: We conducted a retrospective review of MM patients who underwent ASCT from 2013 to 2018 at our institution. The purpose of this retrospective study was to compare the 100-day mortality, time to engraftment, and incidence of grade 1-4 toxicities in elderly MM patients with younger patients taking into account comorbidity information. Other secondary end points measured were overall survival (OS) and progression-free survival (PFS).

Results: A total of 95 patients were analysed and categorized by age as young patients (37 to $64 \mathrm{y} ; \mathrm{n}=70$ ) or elderly ( 65 to $71 \mathrm{y} ; \mathrm{n}=25$ ). The compared groups did not differ in terms of gender, ECOG, HCT-specific comorbidity index (HCT-CI), and disease status at ASCT. Melphalan in a dose of $200 \mathrm{mg} / \mathrm{m}^{2}$ was used as preparative regimen in $65 \%$ of younger patients, and in $43 \%$ of the elderly ( $\mathrm{p}=$ 0.18). The remaining patients received $140 \mathrm{mg} / \mathrm{m} 2$ of melphalan or lower dose (range, $100-140 \mathrm{mg} / \mathrm{m}^{2}$ ) due to HCT-CI $>2$ or age, on the physician discretion. In the whole 
study group there were no transplant related deaths within the first 100 days of ASCT. Stratifying by age, there was no statistically significant difference concerning febrile neutropenia (FN) incidence, which was observed in $14 \%$ of younger patients, and $17 \%$ of elderly. In contrast, FN was observed more frequently in patients with HCT-CI $>2$ ( $38 \%$ vs $12 \%, \mathrm{p}=0.05$ ). Grade $1-2$ infections were more frequent in older patients ( $35 \%$ vs $5 \%, \mathrm{p}=0.005)$, but no difference was found in grade 3-4 infections incidence rate such as pneumonia, uroinfections and neutropenic enterocolitis ( $25 \%$ vs $35 \%, \mathrm{p}=0.52$ ), nor grade $1-2$ and 3-4 noninfectious toxicities ( $50 \%$ vs $38 \%, \mathrm{p}=0.31$, and $4 \%$ vs $21 \%$, $\mathrm{p}=0.06$, respectively). The median time to granulocyte engraftment was 11 days (range, 9-19 days) in elderly and was comparable with younger patients. The time to platelet recovery was also similar. After the median follow-up of 12 months for survivors, OS at 18 months was $94 \%$ in both groups. PFS at 18 moths was $65 \%$ for younger patients, and $55 \%$ for elderly $(\mathrm{p}=0.86)$. However, the association between PFS and the dose of melphalan used in conditioning was observed. PFS probability at 18 months for patients conditioned with the dose of $200 \mathrm{mg} / \mathrm{m}^{2}, 140 \mathrm{mg} / \mathrm{m}^{2}$ and $100 \mathrm{mg} / \mathrm{m}^{2}$ was $71 \%, 58 \%$ and $21 \%$, respectively $(\mathrm{p}=0.012)$.

Conclusions: Our data show that ASCT in transplant eligible MM patients $\geq 65$ years of age is safe and provides similar outcomes as seen in younger patients.

Disclosure: Nothing to declare

\section{P552}

Is mobilization with chemotherapy effect response in the multiple myeloma?

Guldane Cengiz Seval, Atilla Uslu ${ }^{I}$, Sinem Civriz Bozdag $^{1}$, Meltem Kurt Yuksel', Onder Arslan ${ }^{1}$, Muhit Ozcan $^{1}$, Taner Demirer ${ }^{1}$, Gunhan Gurman ${ }^{1}$, Osman Illhan $^{1}$, Hamdi Akan ${ }^{1}$, Meral Beksac ${ }^{1}$, Pervin Topcuoglu ${ }^{1}$

${ }^{1}$ Ankara University School of Medicine, Hematology, Ankara, Turkey

Background: High dose melphalan therapy with autologous stem cell support is a standart approach in symptomatic multiple myeloma patients. Response rates increased with the novel anti myeloma agents and the use of chemotherapy for stem cell mobilization should be questioned. The purpose of this study is to determine the effect of cyclophosphamide used during stem cell collection on disease response and transplantation results.

Methods: We retrospectively collect data from 270 myeloma patients who underwent autologous stem cell transplantation (ASCT) in Ankara University Medicine Faculty, blood and bone marrow transplantation unit between January 2012 and November 2018. 51 patients who received cyclophosphamide protocol for stem cell mobilization were included in the study. Disease response were determined according to International Myeloma Study Group (IMWG) criteria before and after the cyclophosphamide. Transplant responses and their effects on survival were also indicated.

Results: After the diagnosis of MM, 51 patients (male/ female: 32/19; median age: 59 years (between $40-74$ years)) with median follow-up of 59.1 months (between 7,6 185,8 months) underwent ASCT at a mean of 21,7 $\pm 6,2$ months.. Forty-one patients were evaluated before and after cyclophosphamide (table 1). In $69 \%$ of the patients, the disease response was not altered by cyclophosphamide therapy, and $22 \%$ of the patients improved their response status. Post-transplant response rates of patients who underwent stem cell mobilization with cyclophosphamide are also shown in table-1. The mean survival of the patients was $52,7 \pm 4,8$ months. When patients were grouped according to changes in response status before and after cyclophosphamide; there was no statistical difference between mean calculated survival (improved response, disease progression and stable disease; 59,3 $\pm 9,0$ months, $22,7 \pm 14,7$ months and $46,4 \pm 5,0$ months respectively, $\mathrm{p}=0.425$ )(Figure-1). The rates of 1-year and 3-year overall survival (OS) of the patients with no response to cyclophosphamide treatment were as follows; $74,2 \% \pm 7,9 \%$ and $70,5 \% \pm 8,3 \%$ respectively. Thirteen patients who were followed up median 27 months after transplantation died at an average of $3,4 \pm 2,5$ months; 6 of these deaths were caused by the infection after transplantation.

Conclusions: In our study, it was observed that the use of cyclophosphamide for CD34+ stem cell mobilization did not change the disease response rates by $69 \%$. The posttransplant survival rates of MM patients who had progressive disease after cyclophosphamide use were lower. However, these results warranted confirmed by randomized controlled trials.

Clinical Trial Registry: -

Disclosure: Nothing to declare

\section{P553}

Results of a single center experience: An attempt to augment conditioning regimen in first autologous stem cell transplantation

Guldane Cengiz Seval ${ }^{1}$, Derya Koyun ${ }^{1}$, Ekin Kircali, Selami Kocak Toprak ${ }^{1}$, Muhit Ozcan ${ }^{1}$, Meral Beksac ${ }^{1}$

${ }^{1}$ Ankara University School of Medicine, Ankara, Turkey

Background: The role of high-dose chemotherapy followed by autologous stem cell transplant (ASCT) is in the 
treatment of multiple myeloma (MM) continues to evolve in the era of novel agents. The addition of bortezomib to highdose melphalan (Bor-HDM) has been reported by several groups, and it has been outcome and toxicity profile is comparable to high dose melphalan (HDM) alone. The aim of this retrospective study was to evaluate the outcome of the BOR-HDM conditioning regimen on overall response for patients with MM undergoing first single ASCT at our institution.

Methods: This retrospective single center study reviewed 136 consecutive myeloma patients who had received the first ASCT either with Bor-HDM $(n=15, M / F=11 / 4)$ or single agent $\operatorname{HDM}(n=121, M / F=76 / 45)$ conditioning regimen. In the single agent HDM conditioning regimen, melphalan was administered intravenously at a total dose of $200 \mathrm{mg} / \mathrm{m} 2$ on day -3 and -2 and stem cells were infused on day 0 . In the Bor-HDM group, melphalan $200 \mathrm{mg} / \mathrm{m}^{2}$ was administered on day -2 . Bortezomib was administered intravenously at a dose of $1 \mathrm{mg} / \mathrm{m}^{2}$ on day's $-6,-3,+1$, and +4 as described in a phase 2 study by Intergroupe Francophone du Myé lome (IFM).

Results: All consecutive patients who underwent single ASCT from January 2010 to March 2018 using Bor-HDM as conditioning or HDM were evaluated. Conditioning regimen was HDM in 121 patients and Bor-HDM in 15 patients. Median age was significantly lower in Bor-HDM conditioned ASCT compared to HDM group (62 years vs 52 years, $\mathrm{p}=000$ ). There was no significant difference for MM subtype, ISS stage at diagnosis, prior treatment line among HDM vs Bor-HDM cohorts ( $p>0.5$ ). After a median of 4 cycles of induction chemotherapy, patients in the BorHD exhibited $\geq$ VGPR of $53.8 \%(n=7)$ compared to $56.7 \%$ $(\mathrm{n}=51)$ in the HDM group $(\mathrm{p}=\mathrm{p}>0.5)$. Pre-ASCT immune response (IF (-)) was reported in $22.3 \%$ of patients treated with HDM, higher than that seen in the Bor-HDM group $(6.7 \%)(\mathrm{p}=0.3)$. Nine $(69.2 \%)$ patients achieved post-ASCT immune response (IF (-)) $\geq$ VGPR compared to 92 (78.6\%) in the HDM group. At the time of this analysis, ten patients in the Bor-HDM group and 87 in the HDM group had already died, respectively ( $\mathrm{p}>0.5)$. A total of $5(45.5 \%)$ patients in the Bor-HDM group and 50 (44.2\%) patients in HDM group had already progressed ( $>0.5)$. Estimated mean OS and PFS was shorter for group treated with BorHDM; $48.2 \pm 6.9$ mos and $40.2 \pm 8.5$ mos vs. $76.7 \pm 3.9$ mos and $67.3 \pm 3.8 \mathrm{mos}$, respectively $(\mathrm{p}>0.5$ ) (Figure-1). We could not demonstrate the impact of pre or post transplant $\geq$ VGPR immune response on survival and disease free survival. There was no engraftment failure observed on either treatment group and no worsening peripheral neuropathy was developed in the bortezomib arm.

Conclusions: This single center experience on a small patient pool was able to repeat the prospective randomized study results of IFM. Further studies are warranted to explore this regimen, especially when induction treatment with novel agents are employed, with special emphasis on the high-risk myeloma patients where response rates are good but sustainability remains an issue.

Disclosure: Nothing to disclosure

\section{P554}

The efficacy and safety of bortezomib plus busulfan/ melphalan as conditioning regimen in multiple myeloma undergoing autologous stem cell transplantation: PHASE 1/2 study

Jong Hyuk Lee ${ }^{1}$, Sung-Soo Park ${ }^{2}$, Kihyun Kim ${ }^{3}$, Jae Hoon Lee ${ }^{4}$, Sung Soo Yoon ${ }^{5}$, Yeung Chul Mun ${ }^{6}$, Je-Jung $\mathrm{Lee}^{7}$, Jin Seok Kim ${ }^{8}$, Chang-Ki Min ${ }^{2}$

${ }^{1}$ Seoul St. Mary's Hospital, Internal Medicine, Seoul, Korea, Republic of, ${ }^{2}$ Seoul St. Mary's Hematology Hospital, College of Medicine, The Catholic University of Korea, Seoul, Korea, Republic of, ${ }^{3}$ Samsung Medical Center, Sungkyunkwan University School of Medicine, Seoul, Korea, Republic of, ${ }^{4}$ Gachon University Gil Hospital, Incheon, Korea, Republic of, ${ }^{5}$ Seoul National University Hospital, Seoul, Korea, Republic of, ${ }^{6}$ Ewha Womans University School of Medicine, Seoul, Korea, Republic of, ${ }^{7}$ Chonnam National University Hwasun Hospital, Hwasun, Korea, Republic of, ${ }^{8}$ Yonsei University College of Medicine, Seoul, Korea, Republic of

Background: Bortezomib have a powerful antimyeloma activity and was regarded as backbone of therapy in the past decade but its safety and efficacy as a part of busulfan/ melphalan conditioning regimen of autologous stem cell transplantation is yet to be shown.

Methods: A phase $1 / 2$ trial to explore the safety and activity of a bortezomib on days $-6,-3$, and +1 added to a conditioning regimen with busulfan and melphalan (BuMel, $3.2 \mathrm{mg} / \mathrm{kg} /$ day and busulfan during day -5 and $-3,140 \mathrm{mg} /$ $\mathrm{m}^{2} /$ day of melphalan on the day -2 ), in multiple myeloma (MM) patients who received autologous stem cell transplantation following bortezomib-based induction chemotherapy. In Phase 1 , escalating doses $(0.7,1.0$, and 1.3 $\mathrm{mg} / \mathrm{m}^{2}$ ) of bortezomib with BuMel were administered in each group with three patients. With determined maximum tolerated dose of bortzomib at a $1.3 \mathrm{mg} / \mathrm{m}^{2} /$ day, cohort with 41 patients were analyzed for Phase 2.

Results: In Phase 1, no dose limiting toxicity was observed at a $1.3 \mathrm{mg} / \mathrm{m}^{2} /$ day of bortezomib. In Phase 2 , overall responses at 3 months was shown as $75 \%$ of very good partial response (VGPR) or better and $55 \%$ of complete response (CR), whereas post-transplant overall best response included $83 \%$ of VGPR or better, and $68 \%$ of $\mathrm{CR}$, respectively. With median follow-up duration of 
31.4 months, median progression-free survival (PFS) was 26.8 months. The probabilities of 2 years-PFS and overall survival (OS) were $56.5 \%$ and not estimable, respectively. Especially, high-risk cytogenetics were associated adverse survival outcome compared to standard-risk cytogenetics, respectively (PFS, 12.2 vs. 35.7 months, $\mathrm{p}=0.039$; OS, 26.7 vs. 73.3 months, $\mathrm{p}=0.086$ ). With median 11 days and 10 days for neutrophil and platelet engraftments, any graft failure or delayed engraft was not observed. The common grade 3 or severe non-hematological adverse events included neutropenic fever (73.2\%) and stomatitis (14.6\%). Except three cases with transplant-related mortality due to sepsis, other adverse events were manageable.

Conclusions: These results demonstrate that bortezomib is safe and can be a part of conditioning regimen in combination with BuMel, for patients with transplanteligible multiple myeloma.

Clinical Trial Registry: https://clinicaltrials.gov/ct2/ show/NCT01255527

Disclosure: Nothing to declare

\section{P555}

Allogeneic stem-cell transplantation for relapsed/ refractory multiple myeloma: A single institution retrospective analysis of 25 cases

Nobuhiro Tsukada ${ }^{1}$, Takao Yogo ${ }^{I}$, Junichiro Nashimoto ${ }^{I}$, Kota Sato ${ }^{1}$, Mizuki Ogura ${ }^{1}$, Yu Abe ${ }^{1}$, Kiyoshi Okazuka ${ }^{1}$, Tadao Ishida ${ }^{\text {, Kenshi Suzuki }}{ }^{1}$

${ }^{1}$ Japanese Red Cross Medical Center, Tokyo, Japan

Background: Allogeneic stem-cell transplantation (allo$\mathrm{SCT}$ ) is one of treatment option for patients with multiple myeloma (MM) refractory to novel agents. The reports on allo-SCT for MM are limited and it is an important issue to argue appropriate conditioning regimens and stem-cell sources, and patient population who will benefit from allo-SCT.

Methods: We retrospectively analyzed 25 consecutive patients who received allo-SCT for relapsed and refractory multiple myeloma (RRMM) between Oct 2009 and July 2018 at Japanese Red Cross Medical Center. Characteristics of patients, progression-free survival (PFS), and overall survival (OS) were analyzed.

Results: Median age at allo-SCT was 47 (range 31-61). Twelve patients were male and 13 were female. Myeloma type were $\operatorname{IgG}: 14, \operatorname{IgA}: 3$, IgD: 2, and Bence-Jones: 6 . Stem-cell sources were peripheral blood from HLAmatched related donor (rPBSCT): 6 , bone mallow from HLA-matched unrelated donor (MUD): 4, bone marrow from HLA-mismatched donor (MMUD): 7, and cord blood (CB): 8. Twenty-three of 25 patients received Flu/Mel-base, one patient received $\mathrm{Bu} / \mathrm{Mel}$-based, and one patient received etoposide/cyclophosphamide-based conditioning regimens. Twenty-two patients who transplanted after 2012 received $8 \mathrm{~Gy}$ of total body irradiation (TBI). Responses before allo-SCT were CR: 8, VGPR: 6, PR: 6, SD: 5 . Five-year PFS was $22 \%$ (95\%CI: 6-45) and 5-year OS was 42\% (95\%CI: 16-66). Ten patients died during observation period and causes of death were primary disease: 8 and treatment-related mortality: 2. Patients with VGPR or better before allo-SCT showed significantly better PFS $(p=0.04)$ and OS $(p=0.011)$ as compared with others. Female recipients showed significantly better PFS $(p=0.0034)$ and $\mathrm{OS}(\mathrm{p}=0.035)$ as compared with male recipients. Recipients of MMUD showed significantly better PFS $(p=0.0034)$. Among 15 patients surviving, 10 patients received treatments including maintenance therapy.

Conclusions: The reason for better PFS and OS in female recipients is unknown. It is interesting that recipients of MMUD showed better PFS, suggesting graft-versusmyeloma effects. Allo-SCT can be an effective treatment option if patients and stem-cell sources are appropriately selected.

Disclosure: authors declare that there are no conflicts of interest.

\section{P556}

Second autologous hematopoietic stem cell tranpslant versus chemoimmunotherapy in relapsed multiple myeloma after first transplantation: Single center data

Ferda Can ${ }^{I}$, Ramazan Ocal ${ }^{1}$, Lale Kaynar ${ }^{1}$, Zeynep Arzu Yegin $^{I}$, Münci Yagcl, Rauf Haznedar ${ }^{1}$, Zübeyde Nur Ozkurt

${ }^{1}$ Gazi University Faculty of Medicine, Ankara, Turkey

Background: Combination therapy, mostly triple, followed by autologous hematopoietic stem cell transplantation (auto-HCT) is widely accepted as the first-line standard therapy for multiple myeloma (MM). Despite the availability of agents such as new immunomodulatory drugs (IMIDs), proteasome inhibitors (PIs), histone-deacetylase inhibitors and antibodies, it is still possible to achieve longer and deeper responses, however, multiple myeloma is still not cured and relapse is inevitable. The availability of these novel agents has increased questions for determining optimal treatment of patients with relapse after the first autoHCT.

Methods: We retrospectively analyzed 60 patients who relapsed according to International Myeloma Working Group (IMWG) criteria after 1st auto-HCT. First group [salvage chemotherapy $(\mathrm{CT})](\mathrm{n}=27)$ was treated with only chemoimmunotherapy because of early relapse or refractory 
first auto-SCT (within 6 months), ineligible to second transplantation because of co-morbidity, unwillingness to transplant. Second group $(n=33)$ (salvage transplantion) was treated with second auto-HCT as a salvage therapy. Consolidation and long term maintenance treatments were used in both groups.

Results: There was no difference in sex and age between salvage CT and auto-SCT groups [Female/male: 11 vs 13/ 16 vs 20; Age 55(33-71) vs 59(44-70)]. The best response after salvage auto-SCT was complete remission (CR) in $62,5 \%$, partial remission (PR) in $15,6 \%$ patients, while CR in $44 \%$, PR in $14,8 \%$ patients treated with salvage CT. Progression free survival (PFS) were significantly better in second transplant group (PFS; $71 \%$ on the first year; 46,9 $\%$ on the second year after transplant vs $59 \%$ on the first year; $17 \%$ on the second year after the salvage therapy in chemotherapy patients)[p: 0,001]. Overall survival (OS) in salvage auto-SCT group was longer than salvage CT $(42,3$ $\% 23.7 \%$ ), although it did not reach a statistical significance ( $\mathrm{p}>0.05$ ). Time to achieving the best response after salvage auto-SCT and salvage CT was 1 (0-9) month versus 6,5(215) months $[\mathrm{p}: 0,02]$. Grade 3 or 4 nonhematological toxicities were similar (auto-SCT 19\%, salvage CT 13\%) in both groups.

Conclusions: Salvage auto-HCT may provides longer progression free survival with similar toxicity profile according to chemoimmunotherapy especially in patients with sensitive to first auto-SCT. It is suggested that earlier and better responses, long-term progression free survival can be achieved with salvage auto-SCT. We believe that there will be statistical significance in OS such as PFS by increasing the number of patients. The authors believe that large scale randomized clinical trials are needed for optimal treatment of relapsing multiple myeloma after first autoSCT.

Disclosure: Nothing to declare

\section{P557}

Correlation of the immune response genes polymorphic status with the autograft cellularity in patients with multiple myeloma

\section{Elena Nazarova ${ }^{1}$, Konstantin Vetoshkin ${ }^{1}$, Natalja Minaeva $^{1}$, Jemilija Suhorukova ${ }^{1}$, Natalija Zorina ${ }^{1}$, Sergey Utemov $^{I}$, Natalija Isaeva ${ }^{I}$, Igor Paramonov ${ }^{I}$}

${ }^{1}$ Federal State Budget Institution of Science, Kirov Scientific Research Institute of Hematology and Blood Transfusion of the Federal Medical-Biological Agency, Kirov, Russian Federation

Background: One of the conditions for successful transplantation of autologous hematopoietic stem cells (auto-
HSCT) in patients with multiple myeloma (MM) is the timely recovery of hematopoiesis, which is associated with the quantitative and qualitative characteristics of the graft. One of the key indicators is the content of CD34+ cells in the autograft, which depends on many factors. Some of them are due to previous treatment, others are directly related to the patient: age, stage of the disease, features of the hematopoietic stem cells (HSC) microenvironment. The aim of the study was to assess the influence of the immune response genes on the autograft cellularity in patients with MM.

Methods: A retrospective analysis of the genotyping results was performed. Evaluation of 21 loci in 15 genes immune response and harvesting of autologous HSC in 78 patients with MM has been made. Hematopoietic stem cell mobilization regimen included cyclophosphamide $4 \mathrm{~g} / \mathrm{m}^{2}$ with granulocyte colony-stimulating factor. Genotyping of the immune response genes polymorphic regions was carried out by the polymerase chain reaction with allelespecific primers. The number of CD34+ cells was counted on a 6-color FACS Canto II flow cytometer.

Results: According to the results of the autologous transplant harvesting, two groups of patients were identified. First included 65 patients with an autograft cellularity of more than $2 \times 10^{6} / \mathrm{kg}$ body weight. The second group consisted of 13 patients examined with the number of CD34 + cells in the autograft $\leq 2 \times 10^{6} / \mathrm{kg}$ of the patient's body weight. Comparing the identified haplotypes of the immune response genes with the cellularity of the transplantation material, it was found that the presence of the mutant allele in the homo- and heterozygous haplotypes of the ILI $\beta$ gene (T-511C) increased the chances of harvesting cellular material with a higher content of CD34+ cells in 4 times $(\chi 2=5.04, p=0.02)$, and the carriage of the wild type allele in the homo- and heterozygous state of the TLR2 (Arg753Gln) gene is more than in 15 times $(\chi 2=5.06$, $p=0.02$ ). Currently, it has been shown that single nucleotide or amino acid substitutions in genes can lead to changes in the expression pattern of their final products: increased secretion of interleukin $1 \beta$ (IL-1 $\beta$ ) or changes in the spatial configuration and functionality of the receptors (TLR2). Thus, in the presence of mutations in the $I L 1 \beta$ gene, the enhanced synthesis of IL-1 $\beta$ influences on fibroblasts, immunocompetent, endothelial, epithelial and other cells, by activating hemopoiesis. In turn, the mutational status of the Arg753Gln locus located within the TIR domain of the TLR2 receptor in the cytosol, determines the spatial configuration of the TLR2 acting as a co-stimulatory receptor of $\mathrm{CD} 4+$ cells, which ensure the engraftment of the graft.

Conclusions: Identified haplotypical features of the $I L 1 \beta$ and TLR2 genes in patients with MM may act as predictors of the response effectiveness to mobilization of HSCs in 
their carriers, which may contribute to the mobilization regimen optimization and will contribute to harvesting the optimal cellularity of an autologous graft.

Clinical Trial Registry: None.

Disclosure: Authors declare no conflict of interest.

\section{P558}

Differentiating diffuse from focal pattern on computed tomography: Added values of a radiomics approach

\section{Alberto Stefano Tagliafico ${ }^{1}$, Michele Cea ${ }^{I}$, Federica Rossi $^{1}$, Francesca Valdora ${ }^{I}$, Bianca Bignotti ${ }^{I}$, Giulia Succio $^{1}$, Stefano Gualco ${ }^{1}$, Alessio Conte ${ }^{1}$, Carmela di Grazia $^{I}$, Sara Aquino ${ }^{1}$, Emanuele Angelucci ${ }^{1}$, Alida Dominietto $^{I}$}

${ }^{1}$ IRCCS Ospedale Policlinico San Martino, Genova, Italy

Background: Focal pattern in multiple myeloma (MM) seems to be related to poorer survival and differentiation from diffuse to focal pattern on computed tomography (CT) has inter-reader variability. Therefore the purpose of this study is to assess if a Radiomic approach could help radiologists in differentiating diffuse from focal patterns.

Methods: We retrospectively reviewed imaging data of 70 patients with MM between January 2013 and September 2018 of whom 61(27 men and 34 women; mean age 54.2 \pm 3.7 ) with CT, PET-CT or MRI available before bone marrow transplant. Two general radiologist evaluated in consensus only CT images to define a focal (at least one lytic lesion $>5 \mathrm{~mm}$ in diameter) or a diffuse (lesions $<5 \mathrm{~mm}$, not osteoporosis) pattern. Radiomic analysis on CT thinslice images was then applied with regions of interest (ROIs) done by one Researcher not expert in Medical Imaging or MM blindly to the condition of the patients. The reference standard to differentiate diffuse from focal pattern was done by radiological evaluation of two expert musculosketal radiologists blinded to the clinical data reviewing CT, MRI and PET-CT images. N=104 Radiomics features were extracted and evaluated with an open source software. Mann-Whitney $U$ test for unpaired data with 1000 bootstraps samples was used to compare Radiomics features of diffuse and focal patterns and then feature reduction was done to avoid over-fitting. Receiver Operator Characteristic (ROC) analysis with area under the curve was done to compare Radiologists and Radiomics evaluation against reference standard. Reading time to perform Radiomic analysis was also estimated.

Results: The pathological group included: 22 diffuse and 39 focal patterns. After feature reduction, 9 features were different $(\mathrm{p}<0.05)$ in the diffuse and focal patterns $(n=2 / 9$ features were Shape-based: MajorAxisLength and Sphericity; $n=7 / 9$ were Gray Level Run Length Matrix (Glrlm)).
Mean time to perform Radiomics analysis was at $3( \pm$ 45 minutes) hours per patients. AUC of the 2 General Radiologists versus Reference Standard was 0.68 (95\% CI: $0.53-0.83$ ) $\mathrm{p}=0.20$. AUC of the best 4 features (MajorAxisLength, Median, SizeZoneNonUniformity, ZoneEntropy) were: 0.73 (95\% CI: 0.58-0.88); 0.71 (95\% CI: 0.54-0.88); 0.79 (95\% CI: $0.66-0.92) ; 0.68 \quad(95 \%$ CI: $0.53-0.83)$ respectively.

Conclusions: in MM differentiation between focal and diffuse pattern on CT is difficult, but a Radiomic approach could improve standard radiological evaluation with possible implication for prognostic prediction on the base of CT images. IRB approval: (439REG2016)

Disclosure: Nothing to declare

\section{P559}

Post-transplant cyclophosphamide after conditioning with thiotepa, busulfan and allograft in patients with multiple myeloma relapsing after autograft

Evgeny Klyuchnikov ${ }^{1}$, Dietlinde Janson ${ }^{1}$, Ute-Marie von Pein $^{I}$, Maximilian Christopeit ${ }^{I}$, Francis Ayuk ${ }^{1}$, Christine Wolschke ${ }^{I}$, Nicolaus Kröger ${ }^{1}$

${ }^{1}$ Hamburg University Hospital, Stem Cell Transplantation, Hamburg, Germany

Background: Allo-SCT is a potentially curative therapy for patients with multiple myeloma as it provides a graftversus-myeloma effect and a myeloma-free graft. Due to increased NRM and unclear OS benefit the recent guidelines suggested allo-SCT to be used in context of clinical trials focusing on the high-risk patients and those who relapsed early after autograft. Reduced-intensity conditioning regimens may improve rate of NRM; however, optimal conditioning regimen is still to be determined. Here we studied conditioning regimen with 3 alkylating agents consisting of thiotepa (TT), busulfan (Bu) and GvHD prophylaxis with post-transplant cyclophosphamide (post-Cy) in high risk myeloma patients relapsing after autograft.

Methods: A total of 40 patients $(\mathrm{m}, \mathrm{n}=23)$ with median age of 55 years (range 40-67) underwent an allo-SCT (MUD, $\mathrm{n}=19$; MRD, $\mathrm{n}=12$; MMUD, $\mathrm{n}=6$, haploidentical, $\mathrm{n}=3$ ) during a period from 2014 to 2018 in University of Hamburg. The majority of patients had advanced disease (stage IIIAB, $73 \%$ ) and high-risk cytogenetics (56\%). The median response durartion after autograft was 1.8 years (range 0.5-8.3). The conditioning included TT (cum. dosage $10 \mathrm{mg} / \mathrm{kg}$ ), Bu (median cum. dosage $9.6 \mathrm{mg} / \mathrm{kg}$ i.v. or 6.4 $\mathrm{mg} / \mathrm{kg}$ in elderly patients) and post-Cy (cum. dosage $100 \mathrm{mg} / \mathrm{kg}$, day +3 and +4 ). Eight patients (all of them received allografts from MMUD or haploidentical donors) received additionally fludarabine (FLU, cum. dosage of 
$150 \mathrm{mg} / \mathrm{kg}$ ). A number of eleven patients did not receive any additional immunosuppression except of post-Cy.

Results: After a median follow up of 11.4 months (range 3.3 - 34.5) 28 patients were alive. The 2-year probabilities of PFS and OS were $42 \%(21-63 \%)$ and $65 \%(47-83 \%)$.The cumulative incidences (CIs) of relapse and NRM at 2 years were $28 \%(11-45 \%)$ and $22 \%$ (8-36\%), respectively. Lower serum albumin level at transplantation $(\leq 36 \mathrm{~g} / \mathrm{dL})$ was associated with increased relapses (HR 3.8 (1.2-12.7), $\mathrm{p}=0.028)$ and NRM (HR $7.9(2-30), \mathrm{p}=0.0026)$ and resulted in poorer PFS (HR $7.9(2.4-25.8), \mathrm{p}=0.001)$ and OS (HR 8.4 (1.02-68.6), $\mathrm{p}=0.048)$. MMUD and haploidentical donors were associated with poorer NRM (HR 6.2 (1.9-20.3), $\mathrm{p}=0.0027$ ), and resulted in decreased PFS (HR 8.5 (2.6-29.6), $\mathrm{p}=0.001)$. The high-risk cytogenetic at diagnosis showed no impact on survival. The CIs of acute (grade II-IV) at day +100 and chronic GvHD at 2 years were $26 \%(10-42 \%)$ and $48 \%$ (26-70\%), respectively. Absence of immunosuppressive medication beside postCy was associated with poorer OS (HR 11.7 (2.8-47.6), $\mathrm{p}=0.01$ ).

Conclusions: The conditioning with BU, TT and post-Cy leads to a favorable PFS and OS due to low incidences of relapse and NRM for patients with multiple myeloma relapsing after autografting.

Disclosure: Nothing to declare

\section{P560}

\section{Management of biochemical relapse during} lenalidomide maintenance therapy post asct in multiple myeloma: A descriptive observational study of a single institution

Rola El Sayed ${ }^{1}$, Yolla Haibe ${ }^{1}$, Nour Moukalled ${ }^{1}$, Radwan Massoud ${ }^{1}$, Jeries Kort', Charbel Matar ${ }^{1}$, Ammar Zahreddine ${ }^{1}$, Ali Bazarbachi', Jean El-Cheikh ${ }^{1}$

\section{${ }^{1}$ American University of Beirut, Beirut, Lebanon}

Background: Patients with multiple myeloma (MM) who underwent autologous stem cell transplantation (ASCT), are known to benefit from maintenance therapy post-transplant. With signs of early relapse, physicians can either optimize maintenance therapy or use a new therapeutic regimen.

The purpose of this study is to analyze the clinical outcomes beyond biochemical relapse of patients with MM on maintenance therapy. Time to next treatment (TNT), progression free survival (PFS) and overall survival (OS) are assessed. Different approaches for treatment of relapse such as increase in lenalidomide ( $\mathrm{R})$ dose, addition of dexamethasone (D) or Bortezomib (V) or shift to different lines of treatment are possible.
Methods: Between January 2011 and May 2017, we included 51 patients with MM who underwent ASCT and received Bortezomib/Lenalidomide/Dexamethasone (VRD) consolidation and maintenance therapy, mainly lenalidomide(R) $10 \mathrm{mg} /$ day for 21 days every 28 days.

Results: The median age at transplant was 55 years (4675). Forty-six (90\%) of patients received R maintenance, 3 patients received VRD maintenance for higher risk features. Median duration of R maintenance was 22 months (3-62). R dose was changed for toxicity (grade I-II) in $17(33 \%)$ patients. Twenty-nine (57\%) patients relapsed: $13(25 \%)$ patients were shifted to different treatment protocols (treatment change). 6 patients $(11 \%)$ were kept on the same R maintenance (observation group) and $10(20 \%)$ patients had increased lenalidomide dose with dexamethasone (R/ D group). 6 patients (37\%) of the last 2 groups required change of treatment later. The median follow up was 38 months (1-85). Median TNT was 32 months (7-62). At 2 years, the estimated PFS and OS were 39\% and $97.5 \%$ respectively. The median OS and PFS2 (from change of therapy) were 54 and 29 months for patients in the observation group, versus 52 and 27 months in the R/D group, and 45 and 33 months with treatment change, respectively. No statistically significant difference was noted.

Conclusions: Our small monocentric study is limited by its retrospective design and small sample size.

However, it suggests that increasing lenalidomide dose as well as adding dexamethasone in selected patients can postpone change to different lines of treatment without affecting survival.

Disclosure: Nothing to declare

\section{P561}

Can the drugs used before autologous hematopoietic stem cell transplantation have impact on CMV reactivation that results in decreased os in myeloma patients after ASCT?

Meltem Kurt Yüksel ${ }^{1}$, Güldane Cengiz Seval ${ }^{1}$, Atilla Uslu ${ }^{1}$, Ekin Kırcılı ${ }^{I}$, Derya Koyun ${ }^{1}$, Cemalettin Öztürk ${ }^{1}$, Zehra Narlı Özdemir', Güle Çınar ${ }^{2}$, Sinem Civriz Bozdağ ${ }^{I}$, Selami Koçak Toprak ${ }^{1}$, Pervin Topçuoğlu ${ }^{1}$, Önder Arslan $^{1}$, Muhit Özcan ${ }^{1}$, Günhan Gürman ${ }^{1}$, Taner Demirer $^{1}$, Osman İlhan', Alpay Azap ${ }^{1}$, Hamdi Akan ${ }^{1}$, Meral Beksaç ${ }^{I}$

${ }^{1}$ Ankara University School of Medicine, Hematology, Ankara, Turkey, ${ }^{2}$ Ankara University Faculty of Medicine, Ankara, Turkey

Background: The overall incidence of active CMV infection in patients with multiple myeloma (MM) receiving 
more intensive treatment regimens, such as proteasome inhibitors (PI) and/or immunomodulatory (IMID) agents. We performed a retrospective, single center study to evaluate the incidence, risk factors, and outcomes of CMV infection in patients with MM who underwent ASCT with a high-dose melphalan-based regimen.

Methods: This study involved a retrospective review of all patients with who underwent ASCT between January 2015 and November 2018 at our stem cell transplantation center. A total of 144 consecutive adult patients with a diagnosis of MM (median age at diagnosis: 58, range: $35-$ 77) underwent ASCT following induction treatment with novel agents (PIs and/or IMIDs). All patients received antiviral prophylaxis with acyclovir $600 \mathrm{mg} / \mathrm{day}(\mathrm{n}=104)$ or valaganciclovir $1000 \mathrm{mg} /$ day $(\mathrm{n}=36)$.

Results: Baseline patient characteristics, according to induction treatment, are summarized in Table-1. One hundred-five of the 144 patients $(97.2 \%)$ were CMV IgGpositive before ASCT. Overall, $14.6 \%(n=21)$ of CMVseropositive patients developed at least one episode of CMV viremia (CMV DNA >100 copies/ml) after a median 24 months (range; 3-48 mos) follow-up. Persistent CMV viremia (detectable CMV DNA load in more than 2 sequential plasma specimens) occurred in $4.9 \%$ (7 of 144) of the seropositive ASCT recipients and all of them were preventive treated with ganciclovir $(n=5)$ or valganciclovir $(n=2)$. The time from stem cell infusion to the development of CMV viremia ranged from 3 days to 48 days. None of the patients with untreated viremia developed identifiable CMV sequelae. No case of primary infection in seronegative patients at transplant was observed. Adding to that none of the patients developed CMV disease post ASCT. If we analyzed the subgroups of patients according to induction therapy (PI-based, IMIDs, PI+IMID), the incidence of post-ASCT CMV reactivation was higher but not statistically significant, in patients who received only PI vs PI+IMID (13 (61.9\%) vs 8 (38.1\%); $\mathrm{p}=1.00$ ). In univariate analysis, we could not demonstrate the importance of induction therapy with novel agents the occurrence of a post-ASCT CMV reactivation requiring antiviral treatment. However, statistically significant association found between the disease < VGPR status at ASCT and CMV reactivation $(61.1 \%$ vs. $38.9 \% ; \mathrm{p}=0.035)$. After a median follow-up 14.3 months (range; 1-45.9 months), there was no significant impact on PFS, however there was significant decrease in estimated mean OS who had CMV reactivation when compared to those without CMV reactivation ( $34.1 \pm 4.5$ vs. $41.9 \pm 1.3 ; \mathrm{p}=0.002)$ (Figure-1).

Conclusions: CMV establishes lifelong latency within host cells and in the setting of impaired cellular immunity; CMV may reactivate from latency, disseminate, and directly cause life-threatening disease. Our data suggests that MM patients treated with PI-based induction regimens and immunological response < VGPR at time of ASCT seem to have higher risk of developing symptomatic CMV reactivation. However, further studies on a large number of patients are warranted to clarify these findings.

Clinical Trial Registry: -

Disclosure: Nothing to declare

\section{P562}

Effect of age in autologous stem cell transplantation in patients with multiple myeloma: Experience of a single center

Walter Javier Zambrano Márquez, Laura Escalada Gonzalez, Paola Carolina Tamayo Arroyo ${ }^{1}$, Jose Carlos Iglesias ${ }^{1}$, Paula Lopez ${ }^{1}$, Christel Castañon ${ }^{1}$, Lucia Morais Bras $^{I}$, Antonio Sole Magdalena ${ }^{I}$, Laura Francisca Avila Idrovo ${ }^{1}$, Tamara Arias Fernandez ${ }^{2}$, Daniel Martinez Carballeira $^{1}$, Joud Zanabili Al Sibai ${ }^{1}$, Soledad Gonzalez. Muñiz ${ }^{1}$, Ana Julia Gonzalez Huerta ${ }^{1}$, Ana Pilar Gonzalez $^{I}$, Pilar Palomo Moraleda ${ }^{I}$

${ }^{1}$ Hospital Universitario Central de Asturias, Hematology, Oviedo, Spain, ${ }^{2}$ Centro Comunitario de Sangre y Tejidos de Asturias, Oviedo, Spain

Background: High-dose therapy followed by autologous stem cell transplantation (ASCT) has been shown to prolong survival in patients with multiple myeloma (MM) in randomized trials. However, these trials only include patients aged $<65$ years. Data regarding safety and outcomes in this patient population is lacking.

Methods: The aim of this study was to compare safety profile and outcomes in MM patients younger and older than 65 years-old who underwent ASCT in our Unit from July 2014 to October 2018. Patient's demographics, clinical characteristics, transplant related variables and probability of admission to the Intensive Care Unit (ICU) were analyzed. Patients aged $<65$ and $\geq 65$ years-old would be called M1 and M2, respectively, from now on. Sorror index was used to estimate risk of mortality in the two cohorts.

Results: A hundred and eleven patients with MM underwent ASCT in the study period. Median age was 60.9 years-old (range $35-73$ ) and $53.2 \%$ were male. Thirtythree $(28,8 \%)$ patients were $\geq 65$ years. The probability of having a high risk comorbidity index was similar in both groups (M1 11,7 vs M2 12,1\%). The median cells obtained in the apheresis procedure was $5.35 \times 10^{6}(1,70-15.92)$ in M1 compared to $3.77 \times 10^{6}(1.96-10-48)$ in M2. There were no differences in median admission lenght between the 2 cohorts (M1:19 days vs M2: 20 days). Median days for neutrophil recovery above 1000 was 12 days in both groups with a wider range in M1 (11-23) compared to M2 (11-15). No differences were found in platelet recovery above 
100.000 (M1 20 days vs M2 21 days). Median packed red blood cells and platelets transfusions were $1(0-6)$ and $3(0$ 16), respectively, in M1. In M2 cohort, they were 2 (0-5) and $4(0-14)$, respectively. The incidence of grade 3-4 mucositis in M1 and M2 was $50.6 \%$ and $44.1 \%$, respectively. There were no statistically significant differences in terms of using morphine for pain control between the two cohorts (M1, 63,4\% vsM2, 58,8\%). None patient requiered total parenteral nutrition (TPN) in group M2 and only one in group M1. The incidence of ICU admission was 1.5 times higher in patients aged $\geq 65$ than in patients $<65$ years-old $6,1 \%$ vs $3,9 \%$ ), but differences were not statistically significant $(\mathrm{p}=0.52)$. There were no deaths during the transplant procedure in any of the 2 cohorts

Conclusions: 1) In our series, high-dose therapy followed by autologous hematopoietic cell transplantation in MM patients aged $\geq 65$ was feasible. 2) Transplant procedure in older patients was as safe as in patients $<65$ years-old. 3 ) No differences were found in terms of graft, transfusion support, transplant related complications and length of admission. 4) Age should not be a limiting factor in considering the modality of ASCT in this patient population

Disclosure: Nothing to declare

\section{P563}

The correlation between the kinetics of peripheral blood counts and the response to treatment after high-dose melphalan with stem cell support in multiple myeloma patients

\section{Sigal Grisariu ${ }^{1}$, Sasha Halfin ${ }^{1}$, Moshe Gatt ${ }^{1}$, Vlademir Vainstein $^{1}$, Batia Avni ${ }^{1}$ \\ ${ }^{1}$ Hadassah Hebrew University Medical Center, Jerusalem, Israel,}

Background: The long-term survival of MM patients has dramatically increased in the last 20 years, particularly for younger patients. This is attributable in part to the introduction and development of high dose chemotherapy with melphalan with stem cell support (HDM-ASCT). Currently, frontline ASCT is still considered the standard of care for all eligible patients. Many prognostic factors pre and post transplantation have been identified, e.g.: age, comorbidities, cytogenitcs, response to treatment and disease status prior to and post transplantation. To our knowledge there is no data correlating between kinetics of counts response to melphalan and prognosis. Our aim was to assess the prognostic significance of the neutrophil and platelets decaying counts after high dose melphalan.

Methods - we retrospectively analyzed our cohort of 159 multiple myeloma patients who underwent HDM-ASCT at the Hadassah medical center bone marrow transplant department, between the years 2007-2015. The kinetics of neutrophil and platelet decay during the first two weeks after melphalan administration was fitted using linear and exponential mathematical models.

Methods: we retrospectively analyzed our cohort of 159 multiple myeloma patients who underwent HDM-ASCT at the Hadassah medical center bone marrow transplant department, between the years 2007-2015. The kinetics of neutrophil and platelet decay during the first two weeks after melphalan administration was fitted using linear and exponential mathematical models.

Results: Factors associated with prolonged OS in univariate analysis were: ISS stage $1(\mathrm{p}=0.024), \leq 2$ lines of treatment prior to $\operatorname{ASCT}(\mathrm{p}<0.001)$, favorable cytogenetics $(\mathrm{p}=0.004)$, response to treatment (PR or better, $\mathrm{p}=0.046)$ and rapid linear neutrophil decay $(\mathrm{p}=0.046)$. In multivariate analysis, only $\leq 2$ lines of treatment before HDM-ASCT and rapid linear neutrophils count decay remained statistically significant for OS prolongation. No predictive threshold value of the neutrophil decay incline was found. Improved PFS was associated with $\leq 2$ lines of treatment prior to ASCT, and the response status after HDM-ASCT ( $\mathrm{p}=0.003, \mathrm{p}=0.019)$. Additionally, toxicity evaluation showed prolonged neutropenia to be associated with inferior $\mathrm{OS}(\mathrm{HR}=1.139, \mathrm{p}=0.03)$ and rapid exponential decay of neutrophil counts to correlate with higher incidence of mucositis $(\mathrm{p}=0.013)$. Fast platelet decay was associated with delayed platelet engraftment $(\mathrm{p}<0.01)$

Conclusions: We have shown that rapid linear decay in neutrophil counts predicts better OS without a significant benefit in PFS in MM patients undergoing HDM-ASCT. This discrepancy might reflect the problematic estimation in a retrospective analysis of PFS. Rapid decrease in neutrophils and platelet counts was associated with more toxicity: higher mucositis rate and delayed engraftment, respectively. Therefore a rapid decay of blood counts after HDM-ASCT appears to be an in-vivo phamacodynamic marker of higher efficacy and toxicity of melphalan.

Disclosure: Nothing to declare

\section{P564}

Do we need to freeze hematopoietic cells for autotransplants in patients with myeloma conditioned with melphalan?

\section{Daniel Garcia Belmonte', Beatriz Aguado Bueno ${ }^{1}$, Miguel Herrero Coderch ${ }^{1}$, Rafael de la Camara ${ }^{1}$}

${ }^{1}$ Hospital Universitario Sanitas la Zarzuela, Hematology, Madrid, Spain 
Background: Multiple myeloma (MM) is the most frequent indication of auto-HSCT, representing $51 \%$ of all autoHSCT in 2016 (Passweg JR. BMT 2018; 53:1139-48). Nearly all are performed with peripheral blood progenitor cells (PBPC), and Melphalan $200 \mathrm{mg} / \mathrm{m} 2$ is considered the gold standard conditioning regimen. The standard procedure consists in obtaining progenitors, cryopreserved with Dimethyl sulfoxide (DMSO) and stored and subsequently thawed and re-infused in the patient on day 0 . The procedure of cryopreservation is expensive and has some inherent toxicities (DMSO) and loss of cells during the procedure. Several groups have used non-cryopreserved progenitors showing some benefits compared with cryopreserved transplants, mainly a faster engraftment and a shorter length of hospitalization. Objective: To compare noncryopreserved vs cryopreserved auto-HSCT in MM

Methods: We perform an unicentric, retrospective study on 23 consecutive first auto-HSCT MM patients transplanted with PBPC between Nov-2011 and Oct-2018, and conditioned with high dose melphalan $(200 \mathrm{mg} / \mathrm{m} 2)$. The median follow-up was 1620 days (range: 44-2413). 9 patients received non-cryopreserved and 14 cryopreserved auto-HSCT. Patients characteristics, without differences between non-cryopreserved vs cryopreserved: women/men (15/8); median age was 65 years (range 49-72); in the majority auto-HSCT was done as consolidation after first line therapy (78\%); year of transplant $\leq 2014(61 \%), \geq 2015$ (39\%). The number of infused CD34 cells were not different: median $3.5 \times 10 / \mathrm{kg}$ (range 1.7-11.5) in noncryopreserved patients and $3.75 \times 10 / \mathrm{kg}$ (range 2.1-17) in cryopreserved patients.

Results: We didn't observe significant differences in the day of engraftment between non-cryopreserved vs cryopreserved although always was a little bit faster in the noncryopreserved group with a tendency to faster platelet engraftment (>50000/mm3): >20000 platelets/mm3 (median day: 12 vs 12.5, P 0.2), >50000 platelets/mm3 (median: 13 vs 16 days, $\mathrm{P}$ 0.09); >500 neutrophils/mm3 (median: 13 vs 13.5 days, $\mathrm{P}$ 0.3). The media of days of hospitalization was shorter in non- cryopreserved patients (18 vs 21 days) although not statistically significant (P 0.14). Transplantrelated mortality at day +100 was $0 \%$ in both groups. Overall survival at 5 years was not different: $83.3 \%$ in in non-cryopreserved vs $61.5 \%$ in cryopreserved patients (Kaplan-Meier, Log-rank $\mathrm{P}$ 0.27). The accumulative incidence of relapse at the median follow up (1600 days) was similar: $33.3 \%$ in non-cryopreserved vs $35.9 \%$ in cryopreserved patients.

Conclusions: In our short experience, auto-HSCT with non-cryopreserved PBPC in myeloma patients conditioned with high dose melphalan obtain similar results to those performed with classical cryopreserved PBPC and might has a faster platelet engraftment and shorter length of hospitalization. If no advantages are associated with cryopreservation, the simplicity of using fresh product is appealing.

Disclosure: Nothing to declare

\section{P565}

Abstract withdrawn.

\section{P566}

Long-term results of autolougous transplantation with intensification MEL 200 and BU-MEL in multiple myeloma

Rose-Marie Hamladji ${ }^{1}$, Redhouane Ahmed Nacer ${ }^{1}$, Malek Benakli $^{1}$, Farida Harieche ${ }^{1}$, Amina Talbi ${ }^{1}$, Farih Mehdid ${ }^{1}$, Rachida Belhadj', Mounira Baazizi', Nadia Rahmoune $^{I}$

${ }^{1}$ Pierre and Marie Curie Center, Hematology-Bone Marrow Transplantation, Alger, Algeria

Background: Autolougous transplantation with intensification Melphalan 200 (MEL200) became the most used protocol. Tests involving the association BusulfanMelphalan (BU-MEL) were again recently undertaken concerning the forms of bad cytogenetics prognosis (EHA 2017). It seemed interesting to us to report the long-term results of 2 groups of patients (pts) treated either by MEL200 or BU-MEL.

Methods: This is a retrospective study that analyses the long-term results of 2 groups of pts recruited between 2000 and 2006, all of whom received an induction treatment by VAD followed by an intensification with autograft: -Group 1: Melphalan $200 \mathrm{mg} / \mathrm{m}^{2}, 38 \mathrm{pts}$ including 20 men and 18 women; Sex-ratio 1.1; median age 56 years (39-64); Postinduction Status: complete remission (CR): 12, Partial remission (PR): 14, Refractory: 12. -Group 2: Melphalan $140 \mathrm{mg} / \mathrm{m} 2$ and Busulfan $12 \mathrm{mg} / \mathrm{kg}$ (per os); 49 pts of which 25 men and 24 women; Sex-ratio 1.01; median age 50 years (24-56); Post-induction Status: CR: 16, PR: 17, Refractory: 16. Peripheral blood stem cells were collected by apheresis after mobilization by G-CSF alone. The median rate of CD34 is $4.510^{6} / \mathrm{kg}(0.63-16.6)$. At 30 December 2017, the median follow-up is 50 months (4-194).

Results: A early death is observed in one pt (Group 1) and 5 pts(Group 2). The median delay of aplasia is 14 days (10-22) and 12 days (9-17) respectively. In group 1, among the evaluable pts, $30 / 37(81 \%)$ are in CR, 5 pts in PR and 2 refractory. In Group 2, CR: 35/43 (81\%), PR: 6 and 2 refractory. A relapse is observed in 32 pts/37 (86,5\%) in Group 1 and 35 pts/43 (81\%) in Group 2 with a frequency of $54 \%$ and $41 \%$ respectively in the first 24 months. At 36 months: 13 pts/38 (34\%) in Group 1 and 19 pts/49 (38\%) 
in Group 2 are dead. At 60 months: 20 pts (52\%) and 29 pts (59\%). At 120 months: 31 pts $(81,5 \%)$ and 37 pts $(75,5 \%)$. The overall survival (OS) of the Group 1 and Group 2 pts were $66 \%$ and $62 \%$ at 36 months; $47.5 \%$ and $38.5 \%$ at 60 months; $18 \%$ and $24,5 \%$ at 120 months respectively (without significant difference). The event free survival (EFS) of group 1 and 2 pts were $37 \%$ and $36,5 \%$ at 36 months, $25 \%$ and $13 \%$ at 60 months and $8 \%$ and $15 \%$ at 120 months respectively (without significant difference).

Conclusions: These 2 protocols with equivalent toxicity allow obtaining of long-term equivalent results on the response rate early transplant, on the rate of relapse and on the overall survival. These results are identical to those of Fermand (1999).

Disclosure: Nothing to declare

\section{P567}

Viability of hematopoetic stem cells following storage at $-80^{\circ} \mathrm{C}$ with $5 \%$ dimethylsulfoxide and hematologic recovery in transplanted myeloma patients

Ivan Tonev ${ }^{1}$, Stanislav Simeonov ${ }^{I}$, Marina Ilieva ${ }^{1}$, Ivan Mitkoff ${ }^{I}$, Georgi Arnaudov ${ }^{\prime}$, Penka Ganeva ${ }^{I}$, Yavor Petrov $^{I}$, Branimir Spassov ${ }^{I}$, Milcho Mincheff ${ }^{I}$

${ }^{1}$ National Specialized Hospital for Active Treatment of Haematological Diseases, Sofia, Bulgaria,

Background: Dimethylsulfoxide (DMSO) is a major intracellular cryoprotectant, used for cryopreservation of stem cells. It is toxic to both cells and patients at temperatures above $0^{\circ} \mathrm{C}$. Reduction of this effect is achieved by either washing of cells after thawing or by reduction of DMSO during freezing and storage. The latter requires addition of extracellular cryoprotectants to the freezing media. We assessed the effect of low DMSO concentration and different hematocrits of the frozen cells on cell viability and hematologic recovery in patients, transplanted for multiple myeloma.

Methods: Cells were non-programmed frozen and stored at $-80^{\circ} \mathrm{C}$ in a cryoprotectant solution achieving final concentrations of 5\% DMSO, 3.6\% of hydroxyethyl starch (HES, Weight average molecular weight $450000 \mathrm{Da}$ ) and $3 \%$ of human serum albumin. The cell concentration in the frozen product for the first 77 patients (84 transplantations) varied between $100 \times 10^{6}$ and $250 \times 10^{6}$ cells $/ \mathrm{ml}$. In an attempt to reduce the amount of DMSO infused, for the rest of the patients $(n=172 ; 192$ transplantations $)$ we further decreased the volume of the freezing suspension by removal of the entire plasma.

The average age of the transplanted patients was 55 (35 71). The cells were bedside thawed at $37^{\circ} \mathrm{C}$ water bath. The average cell dose was $2,95 \times 10^{6} / \mathrm{kg}\left(1,3-9,2 \times 10^{6} / \mathrm{kg}\right)$.
Results: Viability of the stem cells following thawing assessed by trypan blue exclusion was 95,34\% (70-99). The hematocrit of the frozen cells had no effect on cell viability (94,80\%(low) vs 95,52\%(high)). The major complaints, if any, during stem cell infusion were coughing and an increase in nausea and vomiting induced by the prior conditioning.

The average time for hematological recovery was 11,66 days (between 9 and 19) for the neutrophils, and 12,17 (between 9 and 20) days for the platelets. There was no significant difference in viability and hematologic recovery (11,86 and 12,60 vs 11,59 and 12,02) between patients receiving cells frozen with low or high hematocrit.

Conclusions: Dimethylsulfoxide, despite its cryoprotective properties, is toxic for stem cells at temperatures above zero $\mathrm{C}$ and induces many side effects (cardiac, neurologic, respiratory, etc.) in the patients. To reduce those side effects we use lower DMSO concentration, high hematocrit resulting in lower volume of the frozen cell suspension, thus reducing the final quantity of DMSO to be infused to the patients. This does not affect the cell viability or the hematologic recovery of patients after transplantation. Our easily performed method for unprogrammed freezing of stem cells with final DMSO concentration $5 \%$ at $-80{ }^{\circ} \mathrm{C}$ is safe, well tolerated, and provides cryopreservation, which allows high viability and stable cell engraftment, while reducing the undesired side effects of DMSO.

Disclosure: Nothing to disclose

\section{P568}

Cell dose and hematologic recovery after autologous stem cell transplantation for multiple myeloma

\section{Ivan Tonev ${ }^{1}$, Stanislav Simeonov ${ }^{1}$, Marina Ilieva ${ }^{1}$, Ivan Mitkoff ${ }^{I}$, Georgi Arnaudov ${ }^{\prime}$, Penka Ganeva ${ }^{I}$, Yavor Petrov $^{I}$, Branimir Spassov ${ }^{1}$, Milcho Mincheff ${ }^{I}$}

${ }^{1}$ National Specialized Hospital for Active Treatment of Haematological Diseases, Sofia, Bulgaria,

Background: The relation between the dose of the infused hematopoietic stem cells and the time of hematologic recovery after autologous transplantation has been investigated in different types of patients. We assessed the strength of this relation in patients with multiple myeloma, transplanted in our center.

Methods: We analyzed the relation between different factors and the hematologic recovery in 277 transplantations performed on 249 patients (138 male and 111 female) with multiple myeloma, who underwent autologous transplants between 2005 and 2018. Twenty eight of them had second (tandem or not tandem) transplantation. 
The conditioning regimen consisted of Melphalan for most of the patients. The average age at the time of transplantation was 55 years $(35-71)$. Patients were transplanted with an average cell dose of $2,95 \times 10^{6} / \mathrm{kg}(1,30$ $-9,20 \times 10^{6} / \mathrm{kg}$ ) for the first transplantation and $2,36 \times 10^{6} / \mathrm{kg}$ $\left(1,64-3,19 \times 10^{6} / \mathrm{kg}\right)$ for the second one (every patient received the same cell dose as for the first) with average cell viability 95,34\% (70 - 99\%), with little difference between first and second transplantation.

Results: The average time for hematological recovery was 11,60 (between 9 and 19) days for the neutrophils, and 12.11 (between 9 and 20) days for the platelets.

We found no correlation between the cell dose and the hematological recovery. There was no difference in the hematopoietic recovery between the first and the second transplantation in the patients, who underwent tandem or two transplantations.

Conclusions: Recovery time is considered by some to be a function of the effective stem cell number. We did not find such correlation, probably because in the analyzed group all the patients, except four of them, received a dose greater than $2 \times 10^{6} / \mathrm{kg}$ cell, which is accepted as a safe dose for autologous stem cell transplantation.

Disclosure: Nothing to disclose

\section{P569}

Plerixafor-mobilized patients have a high risk of noninfectious fever during engraftment after autologous peripheral blood stem cell transplantation

Takao Yogo ${ }^{1}$, Nobuhiro Tsukada ${ }^{1}$, Junichiro Nashimoto ${ }^{1}$, Yui Uto ${ }^{I}$, Kota Sato ${ }^{1}$, Kanji Miyazaki', Mizuki Ogura ${ }^{1}$, Yumiko Yoshiki, Yu Abe ${ }^{I}$, Kiyoshi Okazuka , Tadao Ishida', Kenshi Suzuki ${ }^{1}$

${ }^{1}$ Japanese Red Cross Medical Center, Shibuya, Japan

Background: Plerixafor enables rapid and efficient mobilization of hematopoietic stem cells. However, its impact on adverse clinical events after autologous peripheral blood stem cell transplantation (PBSCT) is not fully understood. Fever is one of the major complications in the preengraftment phase of PBSCT. In this research, we focused on non-infectious fever around the time of bone marrow recovery and investigated whether plerixafor as mobilization therapy plays a role in engraftment fever.

Methods: We reviewed 80 autologous PBSCTs for treatment of multiple myeloma at the Japanese Red Cross Medical Center between 2012-2018. Non-infectious fever was defined as temperature $\geq 37^{\circ} \mathrm{C}$ with onset between two days prior to and two days after engraftment without clinical or microbiological documentation of infection.
Results: Patients were mobilized by cyclophosphamide and filgrastim in $57.5 \%(\mathrm{n}=46)$ and filgrastim and plerixafor in $42.5 \%(\mathrm{n}=34)$. The median number of transfused CD34+ cells were $2.61 \times 10^{6} / \mathrm{kg}$ and $3.45 \times 10^{6} \%$ $\mathrm{kg}$, respectively $(\mathrm{P}=0.002)$. Patients transfused with plerixafor-mobilized grafts had a higher risk of noninfectious fever $(85.3 \%$ vs $63.0 \%, \mathrm{P}<0.05)$. CD34+ cell number or cyclophosphamide pretreatment had no relationship to non-infectious fever. The recovery of lymphocytes was more rapid in plerixafor-mobilized patients $(\mathrm{P}=0.001)$. However, the number of lymphocytes was not associated with non-infectious fever.

Conclusions: Combination of filgrastim and plerixafor as mobilization therapy resulted in an increased risk of noninfectious fever during engraftment comparing to mobilization with cyclophosphamide and filgrastim. While the mechanism remains unclear and requires further studies, plerixafor-mobilized grafts may result in an unintended increase in engraftment fever. Clinicians should be aware of this possibility if patients are transplanted with those grafts.

Disclosure: KS received honorarium outside the submitted work from Janssen, Novartis, Celgene, Ono Pharmaceuticals, Takeda, Fujimoto Pharmaceuticals and SRL. TI received honorarium outside the submitted work from Janssen, Celgene, Ono Pharmaceuticals and Takeda.

\section{P570 Abstract withdrawn.}

\section{P571 Abstract withdrawn.}

\section{Myelodysplastic syndromes}

P572

Abstract already published.

P573

Conditioning regimen with decitabine of allogeneic stem cell transplantation for myelodysplastic syndrome and myeloproliterative neoplasms

Yigeng Cao ${ }^{1}$, Erlie Jiang ${ }^{1}$, Sudong Zhang ${ }^{1}$, Qiaoling Ma ${ }^{1}$, Aiming Pang ${ }^{1}$, Weihua Zhai', Sizhou Feng ${ }^{1}$, Mingzhe $\operatorname{Han}^{1}$

${ }^{1}$ Hematopoietic Stem Cell Transplantation Center, Tianjin, China

Background: Allogeneic hematopoietic stem cell transplantation (allo-HSCT) is a potentially curative treatment for patients with myelodysplastic syndromes (MDS) and myelodysplastic/myeloproliferative neoplasms (MDS/ MPN), but post-HSCT relapse remains the major cause of treatment failure. 
Methods: We assessed the efficacy of a new conditioning regimen consisted of Decitabine (Dec), Busulfan $(\mathrm{Bu})$, Cyclophosphamide (Cy), Fludarabine (Flud) and cytarabine (Ara-c) for allo-HSCT in patients with MDS and MDS/ MPN. Fifty patients were enrolled, including 46 with MDS and 4 with CMML. Patients received Dec $20 \mathrm{mg} / \mathrm{m}^{2} /$ day on days -9 to -5 , combining $\mathrm{Bu} / \mathrm{Cy} / \mathrm{Flu} /$ Ara-c modified preparative regimen.

Results: At a median follow-up of 522 (15-1313) days, the overall survival (OS) was $86 \%$, disease-free survival (DFS) was $78 \%$, and relapse incidence was $11 \%$. The incidence of severe acute (grade III/IV) graft-versus-host disease (GVHD) was 22\%, and that of (predominantly mild) chronic GVHD was $40 \%$. OS at 2 years was $74 \%$ for MDS patients with high risk, $86 \%$ for MDS patients with very high risk, respectively. The survival was delightful in patients with poor-risk mutations, such as TP53 and ASXL1, (86\%) and with three or more gene mutations (77\%). Among the total 12 patients with poor-risk mutations in our research, only one patient $(8 \%)$ with TP53 relapsed and one (8\%) with ASXL1and TET2 died. Result of continuous observation after transplantation, the percentage of NK cells in the peripheral blood of all patients who had received Dec/Flu/Bu/CY/Ara-c conditioning increased at day 28, which may essentially contribute to disease control post-transplantation.

Conclusions: In summary, the addition of a 5-day schedule of decitabine to a Flu/Bu/CY/Ara-c conditioning regimen has proven feasible, with a low level of toxicity and promising early disease control especially in patients with high risk MDS.

Disclosure: There are no conflicts of interest.

\section{P574}

The SFGM-TC MDS score at day 180 is associated with post-transplant outcomes in patients with myelodysplastic syndrome who underwent CD34+ selected allogeneic stem cell transplant

Ana Alarcon ${ }^{1,2}$, Patrick D. Hilden', Molly Maloy ${ }^{2}$, Josel D. Ruiz ${ }^{2}$, Miriam Sanchez-Escamilla ${ }^{2,3}$, Lucrecia Yañez $^{2,4}$, Nerea Castillo ${ }^{2}$, Roni Tamari ${ }^{2}$, Brian Shaffer ${ }^{2}$, Hugo Castro-Malaspina ${ }^{2}$, Sergio Giralt ${ }^{2}$, Miguel A. Perales $^{2,5}$

${ }^{1}$ Hospital Universitario Puerta de Hierro Majadahonda, Madrid, Spain, ${ }^{2}$ Memorial Sloan Kettering Cancer Center, Adult Bone Marrow Transplantation Service, New York, NY, United States, ${ }^{3}$ IDIVAL (Research Institute of Marques de Valdecilla), Santander, Spain, ${ }^{4}$ Hospital Marqués de Valdecilla, Santander, Spain, ${ }^{5}$ Weill Cornell Medical College, Cornell University, New York, NY, United States
Background: Several scoring systems have been developed to estimate outcomes in MDS patients who undergo AlloHCT. However, none of them have been specifically validated in $\mathrm{T}$ cell depleted grafts. The aim of this study is to investigate the prognostic ability of a recently published scoring system (SFGM-TC) in a cohort of patients with MDS who underwent TCD transplants.

Methods: 109 patients underwent a first TCD AlloHCT for MDS from 2007 to 2018. The SFGM-TC score (Caulier et al. Curr Res Transl Med. 2018) is performed at day 100 and ranges from $0-8$, discriminating low (0), intermediate (1-3), and high risk (4-8). Additional analyses were performed at day 180 and day 365. A landmark analysis was done at each time point for the day 100, 180, and 365 analyses, respectively.

\begin{tabular}{|c|c|c|}
\hline $\begin{array}{l}\text { Recipient age, years, } \\
\text { mean }+/- \text { SD }\end{array}$ & & $61+/-10.9$ \\
\hline \multirow[t]{2}{*}{ FAB/WHO Category, n (\%) } & $\begin{array}{l}\text { RA/RARS/RCMD/ } \\
\text { Unclassified }\end{array}$ & $47(43.1)$ \\
\hline & RAEB-1, RAEB-2 & 34 (31.2), 28 (25.7) \\
\hline $\begin{array}{l}\text { IPSS cytogenetic risk } \\
\text { score, } \mathrm{n}(\%)\end{array}$ & $\begin{array}{l}\text { Favorable, Intermediate, } \\
\text { High risk }\end{array}$ & $\begin{array}{l}60(58.3), 23(22.3), \\
20(19.4)\end{array}$ \\
\hline $\begin{array}{l}\text { Bone marrow blast } \\
\text { count. } \mathrm{n}(\%)\end{array}$ & $\geq 5 \%$ & $15(14.6)$ \\
\hline $\begin{array}{l}\text { Grade of acute GVHD until } \\
\text { day } 100 \mathrm{n}(\%)\end{array}$ & 0/I, II, III/IV & $\begin{array}{l}93(85.3), 13(12.6), \\
3(2.9)\end{array}$ \\
\hline $\begin{array}{l}\text { Grade of acute GVHD until } \\
\text { day } 180 \mathrm{n}(\%)\end{array}$ & 0/I, II, III/IV & $\begin{array}{l}81(76.4), 17(16), \\
8(7.5)\end{array}$ \\
\hline $\begin{array}{l}\text { Lack of recovery } \\
\text { platelet, } \mathrm{n}(\%)\end{array}$ & & $1(0.92)$ \\
\hline Relapse before day $100, \mathrm{n}(\%)$ & & $1(0.92)$ \\
\hline Relapse before day $180, \mathrm{n}(\%)$ & & $3(2.75)$ \\
\hline
\end{tabular}

\section{[[P574 Table] 1. Patient's characteristics.]}

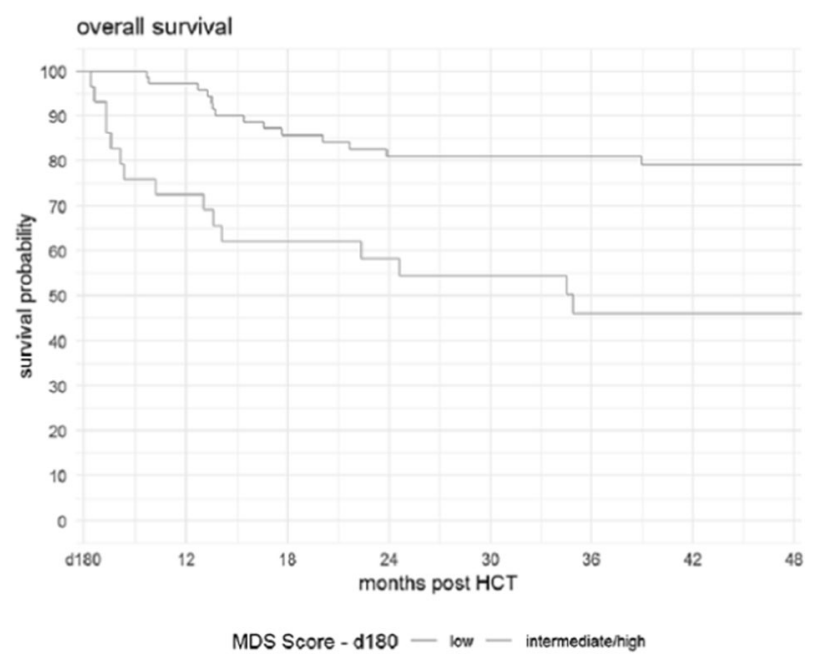

[[P574 Image] 1. The 4.8-year OS rates with landmark day 180] 
Results: Median age was 61 years (range 20 to 72 years), and 64 patients $(59 \%)$ were male. 89 patients $(82 \%)$ had a matched related or unrelated donor. All patients received myeloablative conditioning and ex vivo $\mathrm{T}$ cell depleted PBSC grafts (Table 1). With a median follow-up of 57.5 months $(3.5,114.7)$ among survivors, 1 and 3-year OS was $85.1 \%$ (95\% CI, 76.8-90.6) and 66.9\% (95\% CI, 56.7-75.1), respectively. 70 patients $(64.2 \%)$ were alive at last follow up. At post-transplant day 180, 72 patients had a SFGM-TC low risk and 29 patients an intermediate/high score. In contrast to the analysis at day 100, the SFGM-TC score at day 180 was associated with outcomes. Using the day 181000 score, OS was significantly worse in the intermediate/high risk group (p-value 0.002, HR 3.25 [95\% CI 1.60, 6.60]) (Figure 1) as compared to the low group. There was no significant difference in the SFGM-TC score using a day 100 (p-value 0.117 , HR of Intermediate/high group: $1.89[0.89,4.01]$ ) or day 365 landmark (p-value 0.098, HR of Intermediate/high group: 2.01 [0.88, 4.59]).

Conclusions: In patients with MDS undergoing TCDHCT, the SFGM-TC score at day 180 is significantly associated with survival. The lower incidence of acute GVHD in recipients of CD34-selected transplants and the use of myeloablative condition regimens, with lower relapse, may explain the difference with the original finding that the SFGM-TC was predictive at day 100 in unmodified grafts.

Disclosure: S Giralt: Consultancy: Amgen, Actinuum, Celgene, Johnson\&Johnson, JAZZ pharmaceutical, Milteny, Takeda, Novartis, Kite and Spectrum Pharma. MA Perales: Consultancy: Merck; Ad Board: Incyte, Novartis, Abbvie and Nektar Therapeutics; Member and Scientific advisory board: MolMed and NexImmune; Personal fees and Clinical trial support; Merk: Personal fees; Takeda, Abbvie: and Novartis. L. Yanez reports personal fees from Janssen, Roche, Gilead, Merck and Pfizer. H. CastroMalaspina reports clinical trial research from Alexion. A. Alarcon, P Hilden, M Maloy, JD Ruiz, M.SanchezEscamilla, Nerea Castillo, B. Shaffer and R. Tamari declare no conflict of interests

\section{P575}

Azacitidine for treating relapse after allogeneic hematopoietic stem cell transplantation -multicenter analysis from the Polish adult leukemia group

Joanna Drozd-Sokołowska ${ }^{1}$, Ewa KarakulskaPrystupiuk ${ }^{1}$, Agnieszka Piekarska ${ }^{2}$, Magdalena Dutka ${ }^{2}$, Grzegorz Basak, Anna Waszczuk-Gajda ${ }^{1}$, Krzysztof Madry', Anna Kopińska ${ }^{3}$, Aleksandra Gołos ${ }^{4}$, Joanna Góra-Tybor ${ }^{4}$, Pawet Szwedyk ${ }^{5}$, Eukasz Botkun ${ }^{6}$, Anna Czyż $^{7}$, Sebastian Giebel ${ }^{8}$, Jadwiga Dwilewicz-Trojaczek ${ }^{1}$
${ }^{1}$ Medical University of Warsaw, Warsaw, Poland, ${ }^{2}$ Medical University of Gdansk, Gdansk, Poland, ${ }^{3}$ Medical University of Silesia, Katowice, Poland, ${ }^{4}$ Institute of Hematology and Transfusion Medicine, Warsaw, Poland, ${ }^{5}$ Ludwik Rydygier Hospital, Cracow, Poland, ${ }^{6}$ Medical University of Bialystok, Bialystok, Poland, ${ }^{7}$ Wroclaw Medical University, Wrocław, Poland, ${ }^{8}$ Maria Sklodowska-Curie Institute-Cancer Center, Gliwice, Poland

Background: Allogeneic hematopoietic stem cell transplantation (allo-HSCT)constitutes the standard of carefor eligible patients with myelodysplastic syndromes (MDS) and acute myeloid leukemia (AML). Relapse of the primary disease post allo-HSCT belongs to the major causes of treatment failure, with limited treatment options for this group of patients. Here we report a multicenter analysis by the Polish Adult Leukemia Group analyzing the effectiveness of treatment with azacitidine in this setting.

Methods: Retrospective analysis of patientswho experienced either hematological relapse or progressed to AML after allo-HSCT and were treated with azacitidine for this indication at 7 hematological centers in Poland. The primary end-point was overall survival (OS), the secondary - response rate.

Results: 36 patients, 22 males (61.1\%), median age 52 (range, 15-66), were enrolled. The primary indication for allo-HSCT was AML (28 patients; 77.8\%) and MDS (8; $22.2 \%)$. Eleven patients $(30.6 \%)$ had received azacitidine prior to allo-HSCT. Conditioning intensity was myeloablative in 21 patients $(58.3 \%)$. Median time from alloHSCT to relapse was 5.1 (range, 1.6-93.5) months, while from relapse to commencement of azacitidine - 0.4 (range, 0-14.9) months. EBMT AZA relapse prognostic score [1] was 0 in 7 patients (19.4\%), 1 in $6(16.7 \%)$ and 2 or 3 in 23 patients $(63.9 \%)$. Ten patients $(27.8 \%)$ received other treatment for relapse prior to azacitidine.

Azacitidine was administered most frequently at a dose of $75 \mathrm{mg} / \mathrm{m} 2$ days 1 through 7 (25 patients; 69.4\%) and 100 $\mathrm{mg} / \mathrm{m} 2$ days 1 through 5 (6 patients; 16.7\%). Concomitant donor lymphocyte infusions were administered to 20 patients (55.6\%). Median OS for the whole group was 6.9 months (95\%CI: 4.9-11); with 2-year OS of $17.2 \%$ (95\%CI: 5.1-35.2). For patients stratified according to EBMT AZA relapse prognostic score 2-year OS was: 9.6\% (95\%CI: $0.8-32.3$ ), 33.3\% (95\%CI: 4.6-67.6) and $24.5 \%$ (95\% CI: $1.2-64.2)$ for 2 or 3,1 and 0 points respectively. Survival was statistically shorter for patients with 2 or 3 points in comparison to patients with 0 points $(p=0.027)$. Neither prior-to-allo-HSCT azacitidine use nor other treatment of relapse prior to azacitidine did impact on OS. Eight patients $(22.2 \%)$ obtained response (7 complete remissions; 19.4\%), 5 (13.9\%) stabilization of the disease. Median OS for patients obtaining response was not reached 
during the observation time, 1-year OS was $100 \%$ and 2year OS - 66.7\% (95\%CI: 5.4-94.5). None of these patients received a subsequent allo-HSCT.

The most frequent grade 3, 4 toxicities were thromobocytopenia and neutropenia. Infections developed in 12 patients $(33.3 \%)$, neutropenic fever in $8(19.4 \%)$. Five patients $(13.9 \%)$ either developed or experienced exacerbation of acute graft versus host disease (GVHD), none chronic GVHD.

Conclusions: Azacitidine use is associated with only modest activity in patients who relapse after allo-HSCT. However, in patients who respond to treatment it may allow for a durable disease control.

Disclosure: The authors declare no competing conflicts of interest

\section{Reference:}

[1] Craddock C, Labopin M, Robin M,et al. Clinical activity of azacitidine in patients who relapse after allogeneic stem cell transplantation for acute myeloid leukemia. Haematologica. 2016;101(7):879-83.

\section{P576}

Prognostic somatic mutations in patients with myelodysplastic syndrome treated with hematopoietic stem-cell transplantation

Zixian Liu ${ }^{1}$, Shanbo Cao ${ }^{2}$, Feng Lou $^{2}$, Ying Sun ${ }^{2}$, Chengcheng Wang ${ }^{2}$, Mingzhe Han', Erlie Jiang ${ }^{1}$

${ }^{1}$ Institute of Hematology and Blood Disease Hospital, Tianjin, China, ${ }^{2}$ Beijing Acornmed Biotechnology Co., Ltd., Beijing, China

Background: Somatic mutations in MDS patients are closely related with clinical phenotypes and prognosis in MDS patients. But whether mutations are prognostic for outcomes after allogeneic hematopoietic stem-cell transplantation (allo-HSCT) remains to be elaborated.

Methods: Targeted mutational analysis were performed on samples obtained before transplantation from 134 patients underwent HSCT. We analyzed the relationship of mutations and clinical outcomes.

Results: All 134 patients carried more than one somatic mutations, most frequently in KMT2D(67.16\%), ARID1B (61.94\%), CCDC168(52.24\%), PCLO(47.01\%), ASXL1/2 (46.27\%), SRCAP(44.78\%), U2AF1(42.54\%), DNAH2 (41.79\%), USH2A(41.04\%) and TET2(34.33\%). TP53 mutations were associated with higher IPSS-R risk, complex karyotype and monosomal karyotype. DNAH2 were more frequent in pediatric patients. In univariable analyses, TP53 mutations were related with decreased disease-free survival $(\mathrm{P}=0.047)$; DNAH2 mutations were related with increased disease-free survival (DFS)
( $\mathrm{P}=0.038)$. In multivariable analysis including IPSS-R stratification, GVHD, HCT-CI and candidate genes, DNAH2 mutations were independently associated with better DFS $(\mathrm{P}=0.027)$.

Conclusions: DNAH2 mutations is independently associated with better outcomes in MDS patients treated with allo-HSCT while TP53 may predict unfavorable outcomes. Accounting for these somatic mutations may help better selection of candidates for allo-HSCT among MDS patients.

Disclosure: Nothing to declare

\section{P577}

Pre-transplant treatment strategy in patients with MDS and secondary AML influences response and outcome to salvage therapy for relapse after transplant

\section{Thomas Schroeder ${ }^{I}$, Christina Rautenberg ${ }^{I}$, Kathrin Nachtkamp ${ }^{1}$, Mustafa Kondakci ${ }^{1}$, Rainer Haas ${ }^{1}$, Ulrich Germing $^{I}$, Guido Kobbe ${ }^{I}$}

${ }^{1}$ University of Duesseldorf, Duesseldorf, Germany

Background: There is a controversy among experts if and how patients with MDS and sAML should receive cytoreductive therapy before transplant. While aiming to reduce disease burden in order to lower the risk of relapse after transplant cytoreductive therapy is associated with several drawbacks. Besides a considerable risk for toxicity and mortality preventing patients to proceed to transplant cytoreductive therapy may also favour the selection of resistant clones which may be difficult to treat at relapse.

Methods: To address this hypothesis we retrospectively analysed the response and survival following salvage therapy in 73 patients with MDS and sAML who had relapsed in median 5.6 months (1 to 110 months) after alloSCT according to their pre-transplant strategy (upfront transplantation $\mathrm{n}=3244 \%$; induction chemotherapy [CTX] $\mathrm{n}=2636 \%$; hypomethylating agents [HMA] $\mathrm{n}=1520 \%$ ).

Results: The majority of these 73 patients received salvage therapy with HMA ( $n=58,79 \%$; Aza $n=57$, DAC $\mathrm{n}=1$ ) mostly in combination with DLI, while the remaining received other salvage treatments (intensive chemotherapy $\mathrm{n}=1$, DLI alone $\mathrm{n}=1,2^{\text {nd }}$ transplant $\mathrm{n}=3$, BSC $\mathrm{n}=5$, miscellaneous $\mathrm{n}=2$, missing information $\mathrm{n}=3$ ). When focussing on those patients treated with HMA and DLI it became apparent that a significantly higher proportion of patients in the upfront group (58\%) achieved CR after salvage therapy in comparison to pre-treated patients (10\% $\mathrm{CR}, \mathrm{p}=0.0005$; CTX group 5\% CR; HMA group $18 \% \mathrm{CR}$ ). Accordingly, overall survival (OS) calculated from the time of relapse was significantly longer in patients in the upfront group than in the group of pre-treated patients (2-year OS $59 \%$ vs. $19 \%, \mathrm{p}=0.0001)$. 
Conclusions: Overall, these findings imply that pretransplant therapy may favour the iatrogenic selection of resistant clones, which poorly respond to salvage therapy with HMA and DLI in case of relapse after allo-SCT. Furthermore, the results support the concept that an upfront transplant strategy is a promising alternative for patients with MDS and sAML that can be augmented by salvage therapy with HMA and DLI.

Disclosure: TS and GK received travel support, lecture fees and research funding from Celgene $\mathrm{GmbH}$

\section{P578}

Abstract already published.

\section{P579}

impact of haploidentical HSCT in MDS: Results from Latin American registry

Fernando Barroso Duarte ${ }^{I}$, Talyta Ellen de Jesus dos Santos $^{2}$, Anna Thawanny Gadelha Moura ${ }^{2}$, Yhasmine Delles Oliveira Garcia', Vaneuza Araújo Moreira Funke, Virgílio Antônio Rensi Colturato ${ }^{4}$, Nelson Hamerschlak ${ }^{5}$, Luiz Fernando Lopes ${ }^{6}$, Maria Cristina Martins de Almeida Macedo ${ }^{7}$, Afonso Celso Vigorito ${ }^{8}$, Rodolfo Daniel de Almeida Soares ${ }^{9}$, Alessandra Paz ${ }^{10}$, Mariana Stevenazzi $^{11}$, Lilián Diaz ${ }^{11}$, Abrahao Elias Hallack Neto ${ }^{12}$, Gustavo Bettarello $^{13}$, Breno Moreno de Gusmão ${ }^{14}$, Marco Aurélio Salvino ${ }^{15}$, Rodolfo Froes Calixto ${ }^{16}$, Maria Cláudia Rodrigues Moreira ${ }^{17}$, Gustavo Machado Teixeira $^{18}$, Cinthya Corrêa da Silva ${ }^{5}$, Anderson Joao Simioni $^{4}$, Phillip Scheinberg ${ }^{19}$, Neysimélia Costa Vilela ${ }^{20}$, Romélia Pinheiro Gonçalves Lemes ${ }^{2}$

${ }^{1}$ Hospital Universitário Walter Cantídio, Fortaleza, Brazil, ${ }^{2}$ Universidade Federal do Ceará, Farmácia, Fortaleza, Brazil, ${ }^{3}$ Universidade Federal do Paraná, Bone Marrow Transplant, Paraná, Brazil, ${ }^{4}$ Fundação Amaral Carvalho, Jaú, Brazil, ${ }^{5}$ Hospital Israelita Albert Einstein, São Paulo, Brazil, ${ }^{6}$ Hospital de Câncer Infanto-Juvenil de Barretos, São Paulo, Brazil, ${ }^{7}$ Instituto Brasileiro de Controle do Câncer, São Paulo, Brazil, ${ }^{8}$ Universidade Estadual de Campinas, São Paulo, Brazil, ${ }^{9}$ Natal Hospital Center, Natal, Brazil, ${ }^{10}$ Hospital das Clinicas de Porto Alegre, Porto Alegre, Brazil, ${ }^{11}$ Hospital de Clínicas do Uruguai, Montevideo, Uruguay, ${ }^{12}$ Hospital Universitário de Juiz de Fora, Juiz de Fora, Brazil, ${ }^{13}$ Pietro Albuquerque ICDFBMT São Paulo, Brazil, ${ }^{14}$ Hospital São José,, São Paulo, Brazil, ${ }^{15}$ Universidade Federal Bahia, Salvador, Brazil, ${ }^{16}$ Real Hospital Português de Beneficência em Pernambuco, Recife, Brazil, ${ }^{17}$ CEMO-Instituto Nacional do Câncer, Rio de Janeiro, Brazil, ${ }^{18}$ Hospital das Clínicas da Universidade Federal de Minas Gerais, Belo Horizonte, Brazil, ${ }^{19}$ Hospital São Jose, Hospital Beneficencia Portuguesa de São Paulo, São Paulo,
Brazil, ${ }^{20}$ Hospital do Câncer Infanto-Juvenil de Barretos, Barretos, Brazil

Background: Hematopoietic stem cell transplantation (HSCT) is the only curative procedure for the treatment of myelodysplastic syndrome (MDS), but among several limiting factors for its accomplishment, such as the patient's performance status, is a very relevant issue, i.e., the availability of a compatible HLA donor and, when available, very often the donor's age and comorbidities also constitute factors that hinder this medical conduct. Considering this scenario, the possibility of a haploidentical transplantation (HT) has emerged as an option. In Latin America, HT has been included as a treatment option since 2014. Since then, these patients have been included in the Latin American Registry of Transplantation in Myelodysplastic Syndrome, making it possible to analyze the viability and results of these transplants.

Methods: From October 2012 to October 2018, seventeen (17) patients were transplanted with a haploidentical donor and included in the Latin American Registry. None of these patients had an identical HLA (8/8 match) related or unrelated donor. Data were obtained from the Latin American Registry of HSCT in MDS. The statistical analyses were performed using the software SPSS, version 23.1 and Graphpad Prism version 5.0, with significance being set at $\mathrm{p}<0.05$.

Results: Table 1 shows the patients and their characteristics. All donors were haploidentical. There was a predominance of reduced intensity conditioning, which was performed in 13 patients $(76.5 \%)$, whereas the others received the myeloablative conditioning. Cell source was peripheral blood in $10(58.8 \%)$ and bone marrow in 7 $(41.2 \%)$ of the patients. Graft-versus-host disease (GVHD) prophylaxis in post-HSCT was carried out with cyclophosphamide $50 \mathrm{mg} / \mathrm{kg}$ on $\mathrm{D}+3$ and $\mathrm{D}+5$, cyclosporin from D0 and mycophenolate from D+1. Complete hematologic recovery was achieved in $16(94.1 \%)$ patients. The incidence of grade II-IV acute GVHD was $11.7 \%$, whereas chronic GVHD was $5.8 \%$. One death occurred due to graft failure and none of the patients showed autologous recovery. Three other patients died. One on $\mathrm{D}+210$ due to a fungal infection, the second on $\mathrm{D}+90$ due to sinusoidal obstruction syndrome and a third on $\mathrm{D}+61$ due to pneumonia caused by Pseudomonas. Regarding overall complications, there was a predominance of mucositis (47\%), overall infections (35.2\%) and reactivated CMV in $23.5 \%$ of cases. Of the total number of living patients, 8 (47\%) achieved complete remission and $5(29.4 \%)$ showed disease relapse. The mean follow-up was 39 months (ranging from 5 to 72 months). The lowest probability of disease-free survival at 3 years was 79\% (95\% CI: 71.48 88.51). 
Conclusions: In our country, this procedure has shown to be feasible and we hope to improve it, with better infection control and by acquiring more experience related to the management of these patients.

Chart 1. Survival of patients submitted to haploidentical transplantation.

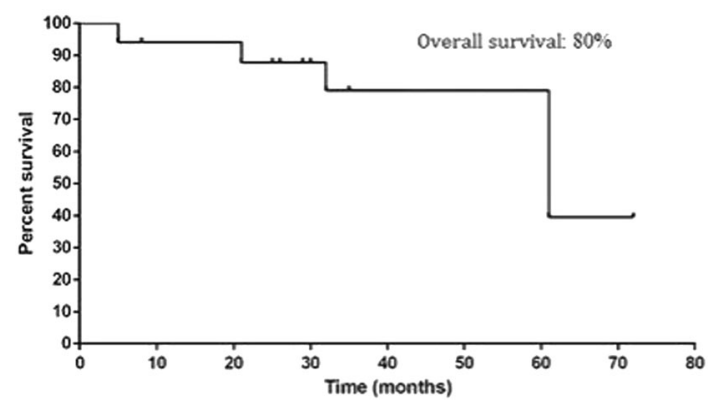

[[P579 Image] 1. Chart 1. Survival of patients submitted to haploidentical transplantation]

\begin{tabular}{ll}
$\begin{array}{l}\text { Number of patients } \\
\text { Age (years) }\end{array}$ & $\begin{array}{l}17 \\
51(2-79)\end{array}$ \\
$\begin{array}{l}\text { Revised IPSS score } \\
\text { (Very High }=3) ;(\text { High }=9) ; \\
\text { (Intermediate }=1) ;(\text { Low }=4)\end{array}$ \\
$\begin{array}{l}\text { Previous demethylating } \\
\text { agents therapy }\end{array}$ & None \\
$\begin{array}{l}\text { Previous chemotherapy } \\
\text { (AML like) }\end{array}$ & No.4\%) \\
No previous treatment & 4 \\
\hline
\end{tabular}

[[P579 Table] 1. Table 1. Characteristics of patients.]

Disclosure: None to be declared.

\section{P580}

MDS relapse presenting as tumour with B-cell precursor markers and meningeosis with myeloid phaenotype 5 years after allogeneic stem cell transplantation for high risk MDS

\section{Josephine Schröder ${ }^{1}$, Anna Laube ${ }^{1}$, Judith Niederland, Christoph Buhl, Stephan Fuhrmann ${ }^{I}$, Bertram Gla $\beta^{I}$, Herrad Baurmann ${ }^{I}$}

\section{${ }^{1}$ HELIOS Clinic Berlin-Buch GmbH, Berlin, Germany}

Background: Extramedullar relapse of MDS is a rare complication after allogeneic stem cell transplantation. We present the case of a 69-year-old woman who was admitted into hospital because of insecure walking. Paresis of both legs, hypaesthesia of the inner thighs, increased effort at urinating, reduced sphincter tonus, central paresis of the right arm and discreet paresis of the right facial nerve were documented at neurological exam. MRI showed a large tumour of the dorsal thorax that immured the adjacent ribs and spine, affected the processus transversus of T9-10 and invaded the spinal canal.

The patient had undergone RIC allogeneic stem cell transplantation five years ago for MDS-EB 2 with complex aberrant karyotype. Following an uneventful course and no signs of GvHD, she had been off immunosuppression since 4,5 years. At the time of the admission the patient had slightly lowered WBC (2,9 Gpt/l) and PLT (111 Gpt/l) and clearly increased LDH (793 U/l).

Methods: Histology of a CT-based biopsy of the paravertebral tumour showed an infiltration of the muscles by blastous cells that were CD45-, CD34-, PAX5-positive, TdT and CD79a were questionably positive. Provisonal diagnosis therefore was lymphoblastic lymphoma, POX tested negative. The bone marrow was hypocellular with increased numbers of mature lymphocytes, but no definite signs of malignancy.

Cerebrospinal fluid revealed 1574 cells $/ \mu$ l with $90 \%$ blasts. Immunotype was CD34, CD117, CD13, CD33, HLA-DR positive, POX and lymphatic markers were negative. Because of this we finally suspected meningeosis leucaemica. We completed the diagnostic workup with genetical and chimaerism tests and compared the result to the patients' MDS before allogeneic stem cell transplantation.

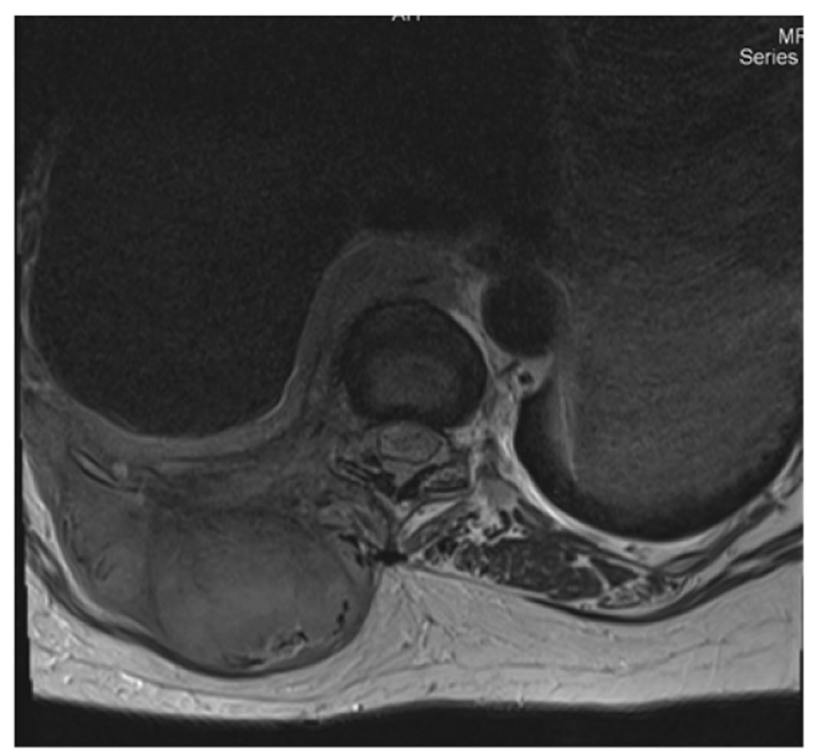

[[P580 Image] 1. MRI scan of the large thoracic tumour]

Results: Cerebrospinal fluid (CSF) cells consisted of $92 \%$ recipient cells, whereas peripheral blood cells were $100 \%$ donor. High risk MDS at transplant displayed a complex 
caryotype including trisomy 8 and tetrasomy 8 , now $10 \%$ of the cells in CSF showed trisomy 8 and $70 \%$ tetrasomy 8 . Chimerism and FISH of the solid tumour could not be performed, coexpression of myeloid markers within the tumour is pending.

Conclusions: In conclusion the patient has meningeosis as a result of exclusively extramedullary relapse of myeloid blasts originating from the initial high risk MDS with blast excess and complex aberrant caryotype. The evolution of a trisomy 8 clone to tetrasomy 8 clone in relapse is linked to extramedullar manifestations. Whether the solid tumour represents myeloid sarcoma with coexpression of lymphoid markers, extramedullary relapse of MDS with lymphoid differentiation or, less likely, a separate lymphobastic lymphoma, is not yet clear.

Disclosure: Nothing to declare

New drug- and cell-based immune therapies

\section{P581}

Abstract already published.

\section{P582}

Longitudinal tracking of WT1-specific $\mathrm{T}$ cells allows to generate a library of WT1-specific $\mathrm{T}$ cell receptors (TCR), for TCR gene editing of acute leukemia

Eliana Ruggiero', Zulma Magnani ${ }^{1}$, Erica Carnevale ${ }^{1}$, Lorena Stasi ${ }^{1}$, Beatrice Claudia Cianciotti ${ }^{1}$, Michela Tassara $^{1}$, Birgit Schultes ${ }^{2}$, Adel Nada ${ }^{2}$,Mark McKee ${ }^{2}$, Andrew Schiermeier ${ }^{2}$, Fabio Ciceri ${ }^{1}$, Chiara Bonini ${ }^{1,3}$

${ }^{1}$ San Raffaele Scientific Institute, Milan, Italy, ${ }^{2}$ Intellia Therapeutics, Inc., Cambridge, MA, United States, ${ }^{3}$ Vita Salute San Raffaele University, Milan, Italy

Background: Adoptive $\mathrm{T}$ cell therapy with genetically engineered $\mathrm{T}$ cells is a potent innovative immunotherapeutic approach for cancer treatment. Unfortunately, the use of $\mathrm{T}$ cells redirected against tumor antigens, is severely limited by 1) the difficulty in identifying appropriate cell surface antigens, that could be targeted by CAR T cells and 2) the paucity of tumor-specific $\mathrm{T}$ cell receptors (TCRs) against shared, oncogenic antigens.

Methods: Focusing on Wilms' Tumor 1 (WT1), a tumorassociated antigen overexpressed by acute myeloid leukemia and several solid tumors, we designed and implemented an innovative protocol for the rapid isolation of WT1-specific $\mathrm{T}$ cells and for the generation and characterization of a library of WT1-specific TCRs displaying different human leukocyte antigen (HLA) restrictions, to be exploited by TCR gene transfer and TCR gene editing. To this aim, we repetitively stimulated $\mathrm{T}$ cells with autologous antigen-presenting cells, including immortalized $\mathrm{B}$ cells, pulsed with overlapping peptides spanning the entire WT1 protein. T cell recognition was assessed by flow cytometry in terms of CD107a expression and IFN $\gamma$ production. Recognized peptides were mapped by a deconvoluting grid and $\mathrm{T}$ cell clonotypes were longitudinally tracked by TCR $\alpha \beta$ sequencing.

Results: We successfully expanded tumor-specific $\mathrm{T}$ cells from 14 consecutive healthy donors, in an average of 4 rounds of in vitro stimulations. The ability of WT1specific $\mathrm{T}$ cells to recognize naturally processed epitopes and their on-target specificity was demonstrated upon coculture with antigen-expressing targets including primary leukemic blasts. Tracking of the TCR $\alpha \beta$ repertoire during culture led to the identification of 20 clonotypes that recognize several tumor-associated peptides and are restricted by more than 5 HLA alleles, including HLA$A * 02: 01$. TCRs were then expressed via genome editing. Briefly, simultaneous editing of endogenous TCR $\alpha$ and $\beta$ chain genes was achieved using CRISPR/Cas9 technology (efficiency $>90 \%$ ), followed by transduction of $\mathrm{T}$ cells with lentiviral vectors encoding WT1-specific TCRs (efficiency $>95 \%$ of $\mathrm{CD}^{+} \mathrm{T}$ cells). Phenotypic characterization of edited $\mathrm{T}$ lymphocytes showed a major enrichment of cells harboring $\mathrm{T}$ stem cell memory properties. Functional validation of the edited $\mathrm{T}$ cells is currently ongoing. Preliminary results of a 6 hours coculture experiment show that TCR edited T cells kill fresh $\mathrm{WT}^{+}{ }^{+}$leukemic blasts, harvested from HLA-matched patients, with an efficiency up to $70 \%$ at an effector to target ratio of 5 to 1 , while no killing of controls is observed.

Conclusions: We set up a protocol enabling consistent and efficient hunting for tumor-specific TCRs with no need for labor intensive $\mathrm{T}$ cell cloning. TCR genes can be easily and rapidly used to redirect $\mathrm{T}$ cell specificity against cancer cells by TCR gene editing.

Disclosure: Chiara Bonini: Research funding from Intellia Therapeutics

\section{P583}

CAR $T$ cell therapy targeting relapsed or refractory CD19+ lymphoid disease with third-generation vector RV-SFG.CD19.CD28.4-1BBZETA

Maria-Luisa Schubert ${ }^{I}$, Anita Schmitt ${ }^{1}$, Leopold Sellner $^{1,2}$, Brigitte Neuber ${ }^{1}$, Angela Hückelhoven-Krauss ${ }^{1}$, Kunz Alexander ${ }^{I}$, Lei Wang ${ }^{I}$, Gern Ulrike ${ }^{I}$, Birgit Michels $^{1}$, Susanne Hofmann ${ }^{1}$, Carsten Mueller-Tidow ${ }^{1,2}$, Dreger Peter ${ }^{1,2}$, Michael Schmitt ${ }^{1,2}$

${ }^{1}$ University Hospital Heidelberg, Heidelberg, Germany, ${ }^{2}$ German Cancer Consortium (DKTK), Heidelberg, Germany 
Background: $\mathrm{T}$ cells genetically engineered to express chimeric antigen receptors (CARTs) directed against CD19 have demonstrated significant efficacy in patients with Iymphoid malignancies including relapsed or refractory ( $r / r)$ B-lineage acute lymphoblastic leukemia (ALL) or $\mathrm{r} / \mathrm{r}$ B-cell non-Hodgkin's lymphoma (NHL). Access to CART treatment for patients in Europe has been limited so far given that the vast majority of CART trials have been performed in the United States and the P. R. of China. Here we present the preliminary results of the first investigator-initiated trial (IIT) CART trial in Germany. HD-CAR-1 (EudraCT No. 2016-00 4808-60; NCT03676504) is a phase I/II trial with in-house CART manufacturing which was initiated in September 2018 at the University Hospital Heidelberg.

Methods: Adult as well as pediatric patients with $\mathrm{r} / \mathrm{r}$ ALL and patients with chronic lymphocytic leukemia (CLL) or NHL including diffuse large B-cell lymphoma (DLBCL), follicular lymphoma (FL) or mantle cell lymphoma (MCL) are treated with autologous T lymphocytes transduced with a third-generation CAR retroviral vector (RV-SFG.CD19.CD28.4-1BBzeta) targeting CD19. The main purpose of HD-CAR-1 is to evaluate safety and feasibility of escalating third-generation CAR $\mathrm{T}$ cell doses $\left(1-20 \times 10^{6}\right.$ transduced cells $\left./ \mathrm{m}^{2}\right)$ after $1 \mathrm{ym}$ phodepletion with fludarabine and cyclophosphamide. Patients are monitored for cytokine release syndrome (CRS), CAR-T-cell related encephalopathy syndrome (CRES) and/or other toxicities. In vivo function, survival and anti-tumor efficacy of CARTs are assessed.

Results: To date, three patients (CLL, DLBCL and MCL, respectively) have been enrolled and subjected to leukapheresis. High numbers of transduced CARTs were harvested on day 10 of culture $\left(82-123 \times 10^{6}\right.$ CARTs). Transduction efficiency ranged between 33 and $42 \%$. CART products were sterile and free from mycoplasms and endotoxins. No production failure occurred and all patients received the CART product. No signs of CRS or CRES > grade 2 have been observed. Assessments of clinical responses are pending and will be presented at the conference along with updated technical results.

Conclusions: For HD-CAR-1, GMP-conform leukapheresis as well as CART manufacturing was effective. Administration, patient monitoring and follow-up were performed in-house providing independency from transport or production sites outside the University Hospital Heidelberg, altogether suggesting that academic CART IITs are feasible in Germany.

Clinical Trial Registry: NCT03676504; EudraCT No. 2016-00 4808-60

Disclosure: Nothing to declare

\section{P584}

Anti-CD19 CAR T-cell therapy post antibody treatment in adult patients with relapsed/refractory precursor Bacute lymphoblastic leukemia

\section{Ivetta Danylesko ${ }^{1}$, Michal Besser ${ }^{2}$, Elad Jacoby ${ }^{3}$, Orit Itzhaki ${ }^{2}$, Noga Shem-Tov ${ }^{1}$, Ronit Yerushalmi ${ }^{1}$, Amos Toren $^{3}$, Avichai Shimoni', Arnon Nagler ${ }^{1}$, Abraham Avigdor $^{1}$}

${ }^{1}$ Chaim Sheba Medical Center Tel Hashomer, Ramat Gan, Israel, ${ }^{2}$ Ella Lemelbaum Institute for Immuno Oncology, Chaim Sheba Medical Center Tel Hashomer, Ramat Gan, Israel, ${ }^{3}$ The Edmond and Lily Safra Children's Hospital, Sheba Medical Center Tel Hashomer, Ramat Gan, Israel

Background: The prognosis of adult patients (pts) with relapsed/refractory (R/R) precursor B- acute lymphoblastic leukemia (ALL) is dismal, including with allogeneic hematopoietic stem cell transplantation (allo-HSCT). Blinatumomab, a bispecific CD19-directed CD3 T-cell engager and inotuzumab ozogamycin (IO), a CD22-directed antibody-drug conjugate revolutionized the field, improving their outcomes. Anti-CD19 chimeric antigen receptor T (CART) cell therapy has led to further progress and improved outcome (Jacoby E; Am J Hematol, 2018). Nowadays, patients with R/R B-ALL can be offered both therapies, but there are limited data on the safety and efficacy of CART - cell therapy post antibody treatment. We detailed our single center experience in this regard.

Methods: This report is a part of a single center, phase $1 \mathrm{~b} / 2$ study on therapy of B-cell malignancies with locally produced CART-cells (NCT02772198). The approach uses autologous T cells with CAR construct that is composed of an anti-CD19 single-chain Fv, CD28 co-stimulatory and CD3-zeta intracellular domains. CD19 expression on the blasts was documented prior treatment in all pts by flow cytometry. All pts received $1 \times 10^{6} / \mathrm{kg}$ CART-cells after lymphodepletion with fludarabin and cyclophosphamide.

Results: Six pts (2 Males and 4 Females) with R/R BALL were enrolled, including one with Ph-positive B-ALL. The median age was 42 years (25-59).

Median number of prior therapy was 4 (3-5). Five pts had prior allo-HSCT. Four pts were given antibodies as the last therapy prior to CART cells. Two pts received blinatumomab resulting in PR in one of them. Two additional pts received IO (1 after failing blinatumomab) achieving MRDpositive $\mathrm{CR}$.

Cytokine release syndrome occurred in all pts and was severe in only one patient who required tocilizumab treatment. This patient was also the only patient who experienced neurotoxicity (grade 3 ), and was treated with dexamethasone. This patient eventually died 18 days post 
infusion of CART cells due to severe pseudomembranous colitis, toxic megacolon and sepsis. All pts had prolonged neutropenia for a median of 14 days (12-18) after the infusion of CART cells.

At day 28 after infusion of CART- cells the CR for the entire cohort was 67\%: three pts with MRD-negative and one with MRD-positive response. Among the four pts who received antibodies prior the CART- cells, one patient had MRD-positive and two pts had MRD-negative response. The patient with $\mathrm{Ph}$ positive B-ALL had progressive disease during the treatment. Two pts were referred to second alloHSCT from other donors. One patient with MRD-negative response relapsed after the second transplant and was treated by salvage therapy. The second patient with MRDnegative response demonstrated prolonged remission (20 months) even without second transplantation. With a median follow-up of 11 months (3-20) the median progression-free and overall survival for the entire cohort were 7.5 and 9 months, respectively.

Conclusions: Autologous anti-CD19 CAR T-cell therapy after debulking treatment with antibodies, including blinatumomab and/or IO, is feasible and results in high response rates in pts with R/R B-ALL. Patients may respond to antiCD19 CAR T- cell therapy even after failure to their last salvage therapy with blinatumomab, which demonstrates similar mechanism of action.

Clinical Trial Registry: NCT02772198

Disclosure: Nothing to declare

\section{P585}

Clonal evolution of acute myeloid leukaemia from $T$ $(12 ; 21)$ acute lymphoblastic leukaemia under immunological selection imposed by CD19 CAR-T therapy

Srividhya Senthil, Denise Bonney ${ }^{I}$, Helen Campbell, Persis Amrolia $^{2}$, Gary Wright ${ }^{2}$, Nicholas Telford ${ }^{2}$, Robert Wynn ${ }^{1}$

${ }^{1}$ Manchester Royal Infirmary, Manchester, United Kingdom, ${ }^{2}$ Great Ormond Street Hospital, NHS Foundation Trust, London, United Kingdom

Background: Genetically engineered $\mathrm{T}$ cells expressing a chimeric antigen receptor (CAR-T) targeting specific antigens present on acute lymphoblastic leukemia (ALL) blasts have generated promising results in children and adults with relapsed and refractory disease. The below report provides an insight of lineage switch occuring as a result of intense immunological selection after CAR-T cell therapy, even with a tumor clone that has no potential for this switch.

Methods: An eight year old Caucasian male with precursor $\mathrm{B}(\mathrm{pB})$ cell lymphocytic leukemia was treated with CD 19 directed CAR-T cell therapy in third remission, and after relapse after previous bone marrow transplantation (BMT).

He was diagnosed with $t(12 ; 21) \mathrm{pB}$ cell ALL at 2 years of age and treated with BFM protocol. He relapsed 8 months after completion of maintenance therapy, and had a 9/10 MMUD bone marrow transplant after etoposide, TBI and Alemtuzumab conditioning therapy. He had cutaneous acute and chronic GVHD but 8 months post-transplant, he relapsed again with $\mathrm{pB}$ cell ALL, with the same cytogenetic and immunophenotypic disease characteristics..

He was treated with lymphodepleting chemotherapy with fludarabine, cyclophosphamide and alemtuzumab followed by infusion of CD19 directed CAR T cells. He developed cytokine release syndrome of grade 1 severity manifested as persistent fever, associated with CAR T cell expansion in the blood. After CAR-T infusion,there was no detectable B cell ALL clone in the marrow by PCR and the cytogenetics were negative for $\mathrm{t}(12: 21)$ translocation.

8 months after the CAR T cell therapy, he was found to have a MRD positive disease which was monitored closely.

Results: We document clonal evolution from CD19 negative, MRD positive disease to AML, with the same Ig rearrangement (the same clonal disease) but with complete myeloid phenotype MPO, CD33, CD13 positive disease. There was cytogenetic evolution of the underlying clone but the original $\mathrm{t}(12 ; 21)$ was retained within the evolved karyotype. Sadly, our patient developed fludarabineneurotoxcity during an attempt to induce AML remission, and further curative-intent chemotherapy was not possible.

Conclusions: There are two case reports of MLL rearranged B-ALL acquiring a clonally related myeloid phenotype associated with CD19- negative escape after CD19 directed CAR T cell therapy,so far. But, this is the first Post CAR-T cell therapy transformation of ALL to AML with ETV6-RUNX1 mutation, which is not recognised to have such lineage-switch potential. Unlike MLL, ETV6-RUNX1 translocation in the pathogenesis of acute myeloid leukemia is not been reported in the literature. The theory behind such transformation is an intense immunological selection of the tumor, driving it to myeloid differentiation with additional clonal cytogenetic events.

Disclosure: Nothing to declare

\section{P586}

IVAC-all-1: interim analysis of a phase I/II clinical study on personalized peptide vaccination based on patient-individual tumor-specific variants in relapsed pediatric acute lymphoblastic leukemia

\author{
Armin Rabsteyn ${ }^{1,2}$, Christopher Mohr ${ }^{3}$, Olaf Witt ${ }^{2,4}$, \\ Roland Meisel $^{2,5}$, Cristiane Chen-Santel ${ }^{6}$, Tobias \\ Feuchtinger $^{2,7}$, Christopher Schroeder ${ }^{8}$, Jakob Matthes ${ }^{8}$,
}


Nicolas Casadei ${ }^{8}$, Patrick Hundsdoerfer ${ }^{9}$, Johannes Schulte $^{2,9}$, Till Milde ${ }^{4}$, Stefan Stevanovic ${ }^{2,10}$, Olaf Riess ${ }^{8}$, Oliver Kohlbacher ${ }^{3}$, Hans-Georg Rammensee ${ }^{2,10}$, Stefan Pfister $^{2,4}$, Rupert Handgretinger ${ }^{11}$, Peter Lang ${ }^{1,2}$

${ }^{1}$ University Children's Hospital Tübingen, Tübingen, Germany, ${ }^{2}$ German Cancer Consortium (DKTK) and German Cancer Research Center (DKFZ), Partner Sites Tübingen, Heidelberg, Düsseldorf, Berlin and München, Heidelberg, Germany, ${ }^{3}$ Institute for Applied Bioinformatics, University of Tübingen, Tübingen, Germany, ${ }^{4}$ Heidelberg University Hospital, Heidelberg, Germany, ${ }^{5}$ Clinic for Pediatric Oncology, Hematology and Clinical Immunology, Heinrich-Heine-University Düsseldorf, Düsseldorf, Germany, ${ }^{6}$ rité University Medicine Berlin, Pediatric Oncology and Hematology, Berlin, Germany, ${ }^{7}$ Dr. von Hauner'sches Children's Hospital, LMU Munich, München, Germany, ${ }^{8}$ Institute of Medical Genetics and Applied Genomics, University of Tübingen, Tübingen, Germany, ${ }^{9}$ Charité University Medicine Berlin, Pediatric Oncology and Hematology, Berlin, Germany, ${ }^{10}$ Institute for Cell Biology, University of Tübingen, Tübingen, Germany, ${ }^{11}$ University Children's Hospital Tübingen, Tübingen, Germany

Background: Acute lymphoblastic leukemia (ALL) is the most common pediatric malignancy. Standard chemotherapy is a successful treatment in $80 \%$ of patients, only about $20 \%$ develop a relapse, however these patients have a dismal prognosis. Prevention of relapse after firstline chemotherapy or stem cell transplantation (SCT) is therefore an urgent clinical need. We established a platform for the design of patient-individual peptide vaccination cocktails by combination of whole exome sequencing of tumor and normal tissue with in silico epitope prediction algorithms for individual patient HLA types. We started clinical translation of this approach by starting a phase I/II clinical trial in 2016 (NCT03559413). Besides feasibility and toxicity assessments, we aim to assess the capability of the peptide vaccination to induce neoantigen-specific $\mathrm{T}$ cell responses in high-risk ALL patients to target residual tumor cells and prevent leukemic relapses.

Methods: Key inclusion criteria are: pediatric patients with ALL who suffered from second relapse after standard therapy or first relapse after SCT. Hematological remission has to be reached prior to vaccination. Nonsynonymous mutations are identified by whole exome and transcriptome sequencing of patient leukemic blasts and healthy reference tissue. HLA binding peptides harboring the altered amino acids are subsequently predicted in silico by algorithms SYFPEITHI, NetMHC and NetMHCpan for the patients' individual HLA type. Vaccine cocktails consisting of 3-5 individual peptides are produced and formulated under GMP conditions. The vaccination schedule is 16 vaccinations over 33 weeks using GM-CSF and Imiquimod as adjuvants. Response to the vaccination is monitored by detection of $\mathrm{T}$ cells recognizing the vaccinated peptides occurring over time in peripheral blood of patients by prestimulation and intracellular cytokine staining.

Results: Until now, 23 patients were recruited, for 17 of those, whole exome sequencing was performed to identify ALL-specific SNVs using a comparative bioinformatics pipeline. We found an average of 118.8 mutations per patient on DNA level. Based on these data, an average of 178 HLA binders derived from neoantigens could be predicted per patient. An average expression of $34.4 \%$ of mutations was assessed by RNA sequencing. In all cases validated mutations could be identified and cocktail design was feasible. Until now, 4 patients received vaccinations. The vaccine was generally well tolerated and no or only mild side effects were observed. Immune monitoring was performed for 2 patients until now. In the first patient, we observed a transient $\mathrm{CD} 4+$ response against one vaccinated MHC Class II ligand and a sustained CD4+ response against the included wildtype control peptide derived from the antigen Survivin. In the second patient, immune monitoring was performed for the first 8 vaccination timepoints, a $\mathrm{T}$ cell response was not measurable at this timepoint of vaccination.

Conclusions: Whole exome sequencing of pediatric ALL patients is feasible and yields small amounts of expressed, tumor-specific mutations. These few mutations are sufficient to predict HLA-binding peptides that can be used to formulate individualized peptide vaccine cocktails. We currently conduct a clinical phase I/II trial in a small cohort of high-risk ALL patients to assess safety, toxicity and immunogenicity.

Clinical Trial Registry: NCT03559413

www.clinicaltrials.gov

Disclosure: Nothing to declare

\section{P587}

Abstract already published.

\section{P588}

Optimization of quantitative real-time polymerase chain reaction (QPCR)-based assessment of vector copy number as safety release criterion for clinical grade CAR T cell therapy

Alexander Kunz ${ }^{1}$, Maria-Luisa Schubert ${ }^{I}$, Ulrike Gern ${ }^{1}$, Leopold Sellner $^{1,2}$, Anita Schmitt ${ }^{l}$, Brigitte Neuber ${ }^{1}$, Lei Wang $^{I}$, Angela Hückelhoven-Krauss ${ }^{I}$, Birgit Michels ${ }^{I}$, Susanne Hofmann ${ }^{1}$, Carsten Müller-Tidow ${ }^{1,2}$, Peter Dreger $^{1,2}$, Michael Schmitt ${ }^{1,2}$ 
${ }^{1}$ University Hospital Heidelberg, Heidelberg, Germany, ${ }^{2}$ German Cancer Consortium (DKTK), Heidelberg, Germany

Background: Chimeric antigen receptor $\mathrm{T}$ cells (CART) are considered as gene therapy medicinal products (GTMP) and genetically modified organisms (GMO). Hence, CARTs manufacturing for clinical application is strictly regulated. Appropriate methods assessing CAR transgene copy number $(\mathrm{CN})$ in a CART product and definition of the frequency of CARTs in treated CART patient are mandatory. Although quantitative real-time PCR-based (qPCR) analysis has been used for this purpose, no standardized procedure to minimize systematic errors and enable comparability has been established yet. Here, we report on a single copy genebased (SCG) duplex (DP) PCR (SCG-DP-PCR) for the determination of the vector copy number $(\mathrm{VCN})$ in CART products as well as patient samples following CART administration. SCG-DP-PCR was validated and compared to the broadly used absolute copy number qPCR (ACN) approach within the framework of a clinical trial treating patients with good manufacturing practice (GMP)-grade CARTs (HD-CAR-1).

Methods: For conventional ACN, primers and probe targeting the CAR vector RV-SFG.CD19.CD28.4-1BBzeta were designed. Standard curves were established via serial dilutions of the SFG.CAR plasmid. Amplification of the standard curve as well as target genomic DNA for VCNdetermination was performed as singleplex (SP) PCR (SPCAR) (method A).

On the same qPCR plate, duplex (DP) qPCR reactions were carried out. Additionally to the components comprised within method A, the experimental setup contained haploid human genomes as well as primers and probe targeting ribonuclease (RNAse) P as human SCG. The amplifications for the SFG.CAR plasmid (DP-CAR) and the RNAseP gene (DP-RNAseP) were performed simultaneously (SCG-DPPCR; method B). SCG-DP-PCR was performed for standard curves and target samples. Target-sample DNA was extracted from CARTs prepared from leukapheresis products of three healthy donors (HD).

Results: For method validation, efficiency and linearity (correlation coefficient) of the qPCR reactions of method A (SP-CAR) and method B (DP-CAR, DP-RNAseP) were assessed by linear regression of the PCR signal to the reference standard curve. Overall, standard curves were only considered valid if a correlation coefficient $\left(\mathrm{R}^{2}\right)$ of above 0.98 and efficiencies of $100 \% \pm 10 \%$ were achieved. VCNs applying method A and B to the same target sample were compared. SP-CAR PCR reaction displayed efficiency of $103.5 \pm 7.1 \% ; 104.2 \% \pm 2.1 \%$ and $99.3 \pm 1.6 \%$ efficiencies were achieved for DP-CAR and DP-RNAse PCR reaction, respectively (Table 1). Applying SCG-DP-
PCR using formula for relative $\mathrm{CN}$ assessment $\left(2^{-\Delta \mathrm{Ct} \text { (DP-CAR }}\right.$ - DP-RNAseP) ) on HD samples resulted in an average of $0.8 \pm$ 0.2 increased $\mathrm{CN}$ when compared to method $\mathrm{A}$

(Table 2).

Conclusions: In terms of efficiency and linearity by linear regression qPCR reactions were comparable. Validation of SCG-DP-PCR was achieved and represents an exact and less error-prone method to fulfil regulatory safety release criteria of CART products. Besides of accurately assessing VCN of transduced cells, SCG-DP-PCR is also a highly robust method to follow-up CARTs in treated patients. Applying this approach, no standard curve is needed, hence significantly economizing required material as well as time.

Disclosure: Nothing to declare

\section{P589}

Modulation of AML-blasts with clinically approved response modifiers to leukemia-derived dendritic cells $\left(\mathrm{DC}_{\mathrm{LEU}}\right)$ ex vivo: DC-, T-cell- and cytokine profiles are predictive for antileukemic T-cell reactivity

Zuzana Fischer', Diana Deen ${ }^{1}$, Anika Hirn ${ }^{1}$, Caroline Plett $^{l}$, Christoph Kugler ${ }^{1}$, Anja Liepert ${ }^{1}$, Markus Freudenreich $^{1}$, Doris Kraemer ${ }^{2}$, Christoph Schmid ${ }^{3}$, Helga Maria Schmetzer ${ }^{I}$

${ }^{1}$ University Hospital Munich-Grosshadern, Munich, Germany, ${ }^{2}$ Municipal Hospital Oldenburg, Oldenburg, Germany, ${ }^{3}$ Municipal Hospital Augsburg, Augsburg, Germany

Background: T-cells' antileukemic responses in AML-pts need to be improved. $\mathrm{DC}_{\text {leu }}$ effectively activate T-cells against leukemic blasts, resulting in blast-lysis ex-vivo. Factors influencing these activities are not known.

Methods: We generated $\mathrm{DC} / \mathrm{DC}_{\mathrm{leu}}$ from AML-blastcontaining mononuclear cells $(\mathrm{n}=74)$ using standard methods (MCM-Mimic/Ca-Ionophore/Picibanil/IFN- $\alpha$, "MNC-methods") and from blast-containing heparinized whole blood $(n=35)$ using modulatory kits (various combinations of 2-3 clinically approved response-modifiers, "WB-kits", patent 102014014993) and correlated statistically (T-/U-test, Pearsons correlation, " means significant) proportions of DC-/T-cell-subtypes/cytokine-profiles with T-cells' antileukemic cytotoxicity (CTX), achieved after mixed lymphocyte culture (MLC) with/without MNCmethod- ("T* $\mathrm{DC}_{\mathrm{MNC}}$ "/" $\mathrm{T} * \mathrm{Bla}_{\mathrm{MNC}}$ ") or WB-kit-treated cultures ("T* $\mathrm{DC} \mathrm{WB}_{\mathrm{WB}} / \mathrm{T} * \mathrm{Bla}_{\mathrm{WB}}$ "). CTX was given as proportions of cases with achieved or "improved" blast-lysis compared to control and as frequencies of viable blasts $\left(b^{2} a_{\text {via }}\right)$ after effector-cell-influence. Pooled data and data obtained with single methods in different cohorts are given.

Results: 1. Generation of $\mathrm{DC} / \mathrm{DC}_{\text {leu }}$ : with a) MNCmethods: $\varnothing 18 \pm 15 \% \mathrm{DC}$ and $\varnothing 11 \pm 10 \% \mathrm{DC}_{\text {leu }}$ and b) WB- 
kits: $\varnothing 13 \pm 8 \% \mathrm{DC}$ and $\varnothing 11 \pm 13 \% \mathrm{DC}_{\mathrm{leu}}$ without induction of blasts' proliferation.

T-cell-proliferation increased (vs uncultured T-cells) after MLC with a) MNC-methods: $\varnothing 34 \pm 38 \%$ vs $\emptyset 7 \pm 9 \%$ and b) WB-kits: $\varnothing 63 \pm 25 \%$ vs $\emptyset 23 \pm 20 \%$. 2. Antileukemic reactivity (T-effector-cell-cytotoxicity after MLC): Pooling all data: a) MNC-methods ("T*DC${ }_{\text {MNC" }}$ vs " $\mathrm{T} * \mathrm{Bla}_{\mathrm{MNC}}$ "): we found a1) blast-cytotoxicity in $\emptyset 70 \%$ (vs $50 \%$ ) of cases with $\varnothing 59 \%\left(v^{2} 117 \%\right)$ bla $_{\text {via }}$, a2) blast-cytotoxicity was improved (vs control) in $86 \%$ with $\varnothing$ decrease of bla $a_{\text {via }}$ of $78 \%$; b) WB-kits ("T*DC $\mathrm{WB}_{\mathrm{WB}}$ " vs " $\mathrm{T} * \mathrm{~B} \mathrm{a}_{\mathrm{WB}}$ "): we found $\mathrm{b} 1$ ) blastcytotoxicity in $\varnothing 80 \%$ (vs $71 \%$ ) of cases with $\emptyset 68 \%$ (vs $56 \%$ ) bla $_{\text {via }}$, b2) blast-cytotoxicity was improved (vs control) in $67 \%$ of cases with $\emptyset$ decrease of $\mathrm{bla}_{\mathrm{via}}$ of $43 \%$. In general, these results could be confirmed with single methods: best MNC-methods were Picibanil and MCM-Mimic, best WBkits were kits containing GM-CSF+Picibanil or Prostaglandins. 3. Correlations: Pooling all data: cases with "improved" lysis (vs "not improved" lysis) were characterized by a) MNC-methods: increased proportions of mature DC/cells (Ø46 $29 \%$ vs $\emptyset 35 \pm 20 \%), D_{\text {leu }} /$ cells $(\varnothing 10 \pm 10 \%$ vs $\varnothing 5 \pm 4 \%)$ and proliferating T-cells $(\varnothing 35 \pm 40 \%$ vs $\varnothing 19$ $\pm 14 \%)$, b) WB-methods: DC/cells $\left(\mathrm{r}=0.781^{\#}\right), \mathrm{DC}_{\text {leu }} /$ cells $\left(\mathrm{r}=0.815^{\#}\right), \mathrm{DC}_{\text {leu }} / \mathrm{bla}(\varnothing 40 \pm 23 \%$ vs $\varnothing 29 \pm 16 \%), \mathrm{DC}_{\text {leu }} / \mathrm{DC}$

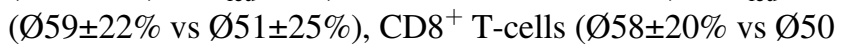
$\pm 24 \%), \mathrm{IFN}-\gamma\left(\mathrm{r}=0.868^{\#)}, \mathrm{MCP}-1 \quad(593 \pm 242\right.$ vs $547 \pm 223$ $\mathrm{pg} / \mathrm{ml})$.

Conclusions: Blasts are regularly converted to $\mathrm{DC}_{\text {leu }}$ in the presence of MNC-methods and WB-kits (simulating the in vivo microenvironment). T-cells' coculture with $\mathrm{DC} /$ $\mathrm{DC}_{\text {leu }}$ after MLC induces and improves antileukemic T-cell activation compared to controls. Blast-cytotoxicity correlates with proportions of $\mathrm{DC} / \mathrm{DC}_{\mathrm{leu}}$ - and T-cell subtypes and released cytokines. These data support a role of antigen presentation by leukemic cells $\left(\mathrm{DC}_{\text {leu }}\right)$ for the stimulation of an immune response in AML in vitro and possibly in vivo.

Disclosure: Nothing to declare

\section{P590}

Evaluation 1 year after the launch of the motion comic immuno-T, explaining patients and their caregivers how immunotherapy strategies work

\section{Chiel Charakhani Nia ${ }^{1}$, Barbara Casteels ${ }^{1}$, Jonas Segaert $^{I}$, Bruno Bollaert ${ }^{2}$, Tessa Kerre ${ }^{I}$}

${ }^{1}$ Ghent University Hospital, Hematology, Gent, Belgium, ${ }^{i 2}$ WahWah vzw, Gent, Belgium

Background: One year ago, the first version of Immuno-T, a motion comic explaining to patients and caregivers how immunotherapy strategies work, was released. People were informed on and inspired to use the application during (inter)national meetings and events for the general public. Meanwhile, the motion comic was further refined and adapted into a second version, based on the evaluations we've collected on the first version. Adaptations included a multi-language tool (currently 6 languages), increased user friendliness, and a new supporting musical score. Also, a website was launched from which the second version could be downloaded on tablet or smartphone (both android and apple) and a new online evaluation form could be filled in. In 10 months, 40 people have evaluated the motion comic online, and these results are presented here, as well as our future plans within the Immuno-T program.

Methods: Through an online questionnaire, 41 participants from Belgium $(n=38)$ and the Netherlands $(n=3)$ have evaluated the Dutch version, and 4 Belgian participants evaluated the French version of the motion comic.

Results: The total group $(n=45)$ consisted of patients $(n=15)$ and their families $(n=2)$, general public $(n=10)$, students $(n=7)$, health care professionals $(n=4)$, researchers $(n=5)$ and kindergarten teachers $(n=2)$. Participants' age ranged from 19 to 80 years, with an even distribution amongst the different generations. The majority of the evaluators $(97,8 \%, \mathrm{n}=44)$ thought the motion comic is a good way to explain immunotherapy to patients. 23 individuals $(51,1 \%)$ felt mainly interested after watching immuno-T, and a total of 32 participants $(71,1 \%)$ felt hopeful or motivated.

Focussing on the patient group $(n=15)$, all of the responders think the Immuno-T motion comic is a good tool to use in a patient-doctor consultation. 11 patients $(73,3 \%)$ felt hopeful and/or motivated after watching the whole motion comic, while 3 of them (20\%) felt combative and $5(33,3 \%)$ felt gripped and intrigued.

As for the new musical score, 35 participants $(77,7 \%)$ think the music is suitable for the app, while 6 evaluators $(13,3 \%)$ think the new music is not or not at all fitted to support the motion comic.

Conclusions: The detailed evaluations allow us to further improve Immuno- $\mathrm{T}$, and aid us in the development of other motion comics we plan to release under the Cancer- $\mathrm{T}$ in motion umbrella.

With the current version of Immuno-T, a single-center pilot study is being set up, to test the efficacy and usability of Immuno-T, based on qualitative research during the experience of the tool, and using validated questionnaires. With this study we want to evaluate the impact of Immuno$\mathrm{T}$ on patient empowerment, and the decision making process. The study protocol will be presented at EBMT.

Disclosure: The development of Immuno-T was partly financially supported by Celyad, Calgene, Novartis, Roche, Amgen, BMS, but these companies did not by any means influence the contents and development of the motion comic. 


\section{P591}

A therapeutic strategy to trespass the blood brain barrier for adoptive NK cell therapy in glioblastoma multiforme induced rat: A preclinical study

\section{Farzaneh Sharifzad ${ }^{1,2}$, Amir Ali Hamidieh ${ }^{3}$, Marzieh Ebrahimi $^{2}$}

${ }^{1}$ Kashan University of Medical Sciences, Kashan, Iran, Islamic Republic of, ${ }^{2}$ Royan Institute for Stem Cell Biology and Technology, ACECR, Cell Sciences Research Center, Tehran, Iran, Islamic Republic of, ${ }^{3}$ Tehran University of Medical Sciences, Children's Medical Center, Tehran, Iran, Islamic Republic of

Background: Glioblastoma Multiforme (GBM) is among the most common and aggressive primary brain tumors with very poor prognosis. According to the Central Brain Tumor Registry of the United States, central nervous system (CNS) tumors in pediatric patients (ages between 0-14 years old) are the second most common malignancies after blood-born malignancies, and the first amongst solid tumors, and known the most common cause of cancer-related deaths. Although hematopoietic stem cell transplantation has been exploited to treat many kinds of malignancies, currently its success rate in GBM is limited. Therefore, the GBM treatment paradigm needs shifting towards more effective treatments such as immune cell therapy. Natural killer (NK) cells have been recognized as potential anti-cancer effector cells, as they can recognize and target tumor cells. Since a small percentage of blood cells are differentiated as NK cells, the number of this group of cells is hardly enough to fight tumors, and so their multiplication and activation would be a potential effective cancer treatment.

Methods: This preclinical study was focused on setting up an optimal protocol for expansion and activation of naïve NK cells and assessing their efficacy towards induced GBM in rat models. Ex-vivo expanded and interlukin-2 (IL-2)and heat shock protein-70 (HSP-70)-treated NK cells have been exploited. After in vitro study and confirming the efficacy of treated cells through cytotoxicity assays, we induced GBM in 6 male Wistar rats (weighted 275-300 gr) using C6 tumor cells injection in rat brain through stereotactic surgery. The tumor formation was proven by MRI imaging. Following tumor establishment, we analyzed the effect of single injection of IL-2- and HSP-70-treated NK cells compared with single injection of non-treated NK cells in two groups of rats.

Results: Systemic intravenous delivery of IL-2- and HSP70-treated NK cells through tail's vein resulted in tumor shrinkage in different time intervals and complete remission in the first group of GBM-induced rat models, whereas in the other group of GBM rats receiving untreated NK cells, the tumor progressed. Therapeutic efficacy of the treated NK cells was ascertained compared with non-treated NK cells considering tumor shrinkage observed in MRI and survival rates between the two model groups.

Conclusions: The amelioration of tumor which has been confirmed by MRI, proved the migration of activated NK cells through blood brain barrier and homing to CNS, and finally targeting GBM tumor cells. Our data suggest that NK-cells treated with IL2/HSP70 may be a promising immune cell-based therapeutic approach towards treating the fatal GBM.

Disclosure: Nothing to declare

P592 Abstract withdrawn.

\section{P593}

Long term sorafenib response for extramedullary FLT3 + AML relapse after allogeneic stem cell transplantation

Elena Sola ${ }^{I}$, Beatriz Aguado ${ }^{I}$, Patricia Lopez-Pereira ${ }^{I}$, Javier Cornago $^{1}$, Ángela Figuera ${ }^{1}$, Adrián Alegre ${ }^{1}$

${ }^{1}$ Hospital Universitario de la Princesa, Hematology, Madrid, Spain

Background: Relapse is the most important cause of failure after allogeneic hematopoietic stem cell transplantation (HSCT) for FLT3-ITD-positive acute myeloid leukemia (AML). Treatment with FLT3 tyrosine kinase inhibitors (TKI) constitutes a promising clinical approach to induce remission without conventional chemotherapy.

Methods: A 50 year-old woman diagnosed with AML secondary to myelodisplastic syndrome (MDS) with NPM1 mutation and internal tandem duplications of the FLT3 gene (FLT3-ITD) in October 2013. After achieving complete remission (CR) with conventional chemotherapy, she received a HLA sibling allogenic-HSCT in February 2014, with BuCy. Four months later, AML relapsed only at medullary level (FLT3 ratio: 0,67 \%), treated with chemotherapy and donor lymphocytes infusions (DLI). She achieved $2{ }^{\text {nd }} \mathrm{CR}$ and developed limited chronic graft-versushost disease (cGVHD). Nine months later (April 2015), she suffered the first extramedullary relapse only, breast and skin. Disappearance of the lesions at all levels was achieved with chemo and radiotherapy. She always had full hematologic donor chimerism.

In December 2015, she referred atypical precordial pain irradiated to the back. Cardiac MRI was performed and several masses were visualized in the pericardial sac, up to 5 $\mathrm{cm}$ in diameter. $\mathrm{BM}$ remained in $\mathrm{CR}$ with full donor chimerism.

Pericardial fluid showed massive infiltration by leukemicFLT3 positive cells (ratio: $0,7 \%$ ). She was not considered 
candidate for further systemic chemotherapy nor radiotherapy, so treatment with FLT3 inhibitor (Sorafenib) was started.

Results: Based on current evidence, Sorafenib was started (in December 2015) at dose of $200 \mathrm{mgr} / 12 \mathrm{~h}$. After first month of treatment, pericardial lesions decreased from 5 to $1.7 \mathrm{~cm}$ in diameter. Subsequent CT showed progressive decrease of lesions until resolution. In last CTs pericardial thickening is almost non-existent, without new lesions.

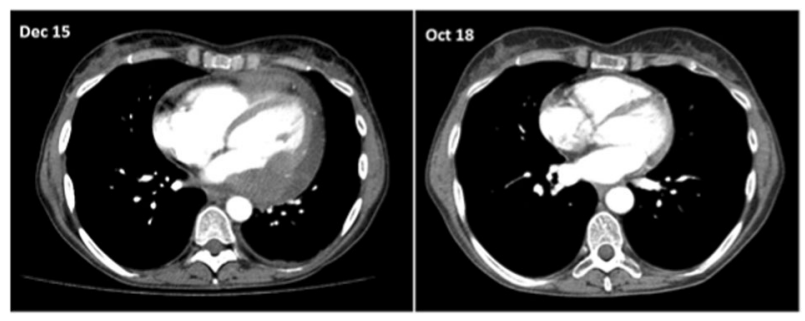

\section{[[P593 Image] 1. Image 1.]}

Since June 2017, Sorafenib dose has been tapered to 200 $\mathrm{mg} / \mathrm{day}$, due to mild skin and GI toxicity. After 3 years of treatment, she maintains CR at medullary and extramedulary levels, with no evidence of a disease that had escaped the mechanisms of action of chemo, HSCT and DLI.

Conclusions: In our patient, treatment with Sorafenib has provided long-term control of this refractory extramedullary disease, even at adjusted doses. Further studies are needed to confirm the efficacy of FLT3 inhibitors in the control of relapses after allo-HSCT, extramedullary disease and its potential role as maintenance agent.

Disclosure: Nothing to declare

\section{P594}

Therapy of posttransplant poor graft function with eltrombobag

\section{Khalid Halahleh ${ }^{1}$, Waseem Allan ${ }^{1}$, Rozan Alfar ${ }^{1}$ \\ ${ }^{1}$ King Hussein Cancer Center, Amman, Jordan}

Background: Posttransplant poor graft function (PGF) is an important complication of allotransplants.Current therapies include molecularly-cloned haematopoietic growth factors, top-up with donor blood, bone marrow or mesenchymal cells or a $2^{\text {nd }}$ transplant. These therapies are only partially effective. Eltrombobag, a thrombopoetin receptor agonist, is effective in aplastic anemia and idiopathic thrombocytopenic purpura (ITP) and may improve posttransplant graft function (Popat et al, ASH 2015).

Methods: We evaluated eltrombobag in 7 consecutive subjects with posttransplant poor graft function.Median age was 47 y (range, 4-60 y). 4 were male. Diagnoses included acute myeloid leukaemia $(\mathrm{N}=1)$, myelodysplastic syndrome $(\mathrm{N}=1)$, aplastic anaemia $(\mathrm{N}=2)$, Hodgkin lymphoma $(\mathrm{N}=2)$ and acute lymphoblastic leukaemia $(\mathrm{N}=1)$. Donors included HLA-identical siblings $(\mathrm{N}=4)$ and HLA-haplotype-matched relatives $(\mathrm{N}=3)$. Pretransplant conditioning was conventional $(\mathrm{N}=3)$ or reduced intensity $(\mathrm{N}=4)$. Grafts were blood $(\mathrm{N}=6)$ or bone marrow $(\mathrm{N}=1)$. Median time to diagnosis of poor graft function was $38.2 \mathrm{~d}$ (range, 17.7-139 d) posttransplant. Median platelets count, $18 \times 10 \mathrm{E}+9 / \mathrm{L}$. 3 subjects had platelets $<20 \times 10 \mathrm{E}+9 / \mathrm{L}$. Median posttransplant follow-up is $8 \mathrm{mo}$ (range, 6-41 mo).

Results: Median interval between diagnosis of poor graft function and starting eltrombobag was $38.2 \mathrm{~d}$ (range, 17.7139d). Median maximum dose was $525 \mathrm{mg} / \mathrm{w}($ range, 350$700 \mathrm{mg} / \mathrm{w}) .5$ subjects responded (71.4\%). Median interval to platelets $>50 \times 10 \mathrm{E}+9 / \mathrm{L}$ was $21 \mathrm{~d}$ (range, 7-30d).

Conclusions: Eltrombobag can reverse posttransplant poor graft function.

Disclosure: Nothing to disclose

\section{P595}

Successful haploidentic bone marrow transplantation with inotuzumab ozogamicin in patient with relapsed and resistant $B$ cell acute lymphoblastic leukemia

\section{Ali Ünal ${ }^{1}$, Serhat Çelik, Zeynep Tuğba Güven ${ }^{1}$, Bülent Eser $^{1}$, Leylagül Kaynar ${ }^{1}$, Mustafa Çetin ${ }^{1}$}

${ }^{1}$ Erciyes Medical School, Hematology, Kayseri, Turkey

Background: Although chemotherapeutic(CT) agents that used in the treatment of acute lymphoblastic leukemia (ALL) increase survival, the results are still weak. Longterm survival with CT's in relapse ALL cases is difficult and the prognosis is very weak. Inotuzumab ozogamicin is an anti-CD 22 monoclonal antibody and it has the potential to reduce the overall toxicity of intensive regimens for ALL, as well as to possibly increase the number of patients who may achieve a state of minimal residual disease.

Methods: 26-year-old male patient was diagnosed with B-cell ALL in December 2017.After the HOELZER KT protocol was started, maintenance treatment was continued. In the fifth month of treatment,FLAG CT protocol was started cause of recurrence was seen on $5 \%$ blast detection in peripheral blood smear. In August 2018, inotuzumab ozogamicin treatment was started and six cures were completed because the patient was not in remission. In September 2018, He had gone Haploidentical bone marrow transplantation from his sibling donor(8/10)with Defibrotid prophylaxis for Veno-Occlusive Disease(VOD)s. He engrafted succesfully and chimerizm was $99.85 \%$ in 30th 
days of transplantation. He is 60th day of transplantation and in a remission.

Results: Bone marrow transplantation cannot be performed since the complete response cannot be achieved in patients with relapse and resistant B-ALL.In these patients, new therapies targeting malignant lymphoblasts are needed. Inotuzumab ozogamicinis a monoclonal antibody drug conjugate that targets CD22 antigen on malignant lymphoblasts.In many studies, it has been shown that Inotuzumab ozogamycin is effective and reliable anti-tumor activity in adults with recurrent and resistant CD22 positive ALL. However, monoclonal antibody drug conjugates have been shown to be associated with VOD's.For this purpose, we used Defibrotid to protect our patient from VOD.

Conclusions: Treatment with combination CT regimens in B-ALL is suboptimal and long-term survival is achieved in only $30-40 \%$ of patients. Targeted molecular therapy and new regimens are needed in relapse and resistant patients.At this point, Inotuzumab ozogamycin is an anti-CD-22 monoclonal antibody, as in our case, it provides remission in recurrent and resistant B-ALL patients and allows patients to complete their treatment with an allogeneic transplant from a fully compatible donor.

Disclosure: Nothing to declare

\section{Non-hematopoietic stem cells}

\section{P596}

Anti-proliferative effects of mesenchymal stem cells (MSCS) derived from multiple sources on ovarian cancer cell lines: An in-vitro experimental study

Charbel Khalil $^{1,2}$, Mayssam Moussa ${ }^{2}$, Rawad Salameh ${ }^{3}$, Jessica Habbouch ${ }^{3}$, Kamal Zahran ${ }^{3}$, Albert Azar ${ }^{3}, \mathrm{Nada}^{2}$ Alaadine $^{2}$, Ahmad Ibrahim ${ }^{3,4}$

${ }^{1}$ Lebanese University - Middle East Institute of Health, Beirut, Lebanon, ${ }^{2}$ Saint Joseph University, Beirut, Lebanon, ${ }^{3}$ Lebanese University - Middle East Institute of Health, Beirut, Lebanon, ${ }^{4}$ Makassed Hospital, Beirut, Lebanon

Background: Mesenchymal stem cells (MSCs) are an attractive consideration for therapeutic cures of many difficult diseases on the cellular-level. Due to the trophic effects of the cytokines and chemokines that they produce, MSCs have shown multiple beneficial properties in the field of oncology. In this study, we will be investigating the effect of MSCs derived from human bone marrow (BM), adipose tissue (AT), and umbilical cord derived MSCs (UCMSCs) on ovarian cancer. To differentiate the MSCs, we performed a comparative analysis between the various sources for proliferative capacity, surface antigen expression, differentiation ability, tumor marker and paracrine activity, and their influence on ovarian cancer cell proliferation.

Methods: Measurements of ovarian tumor marker proteins were computed by ELISA. Proliferative effects, immunomodulatory effects, and apoptosis of the MSCs were measured by the culture and counting of colony formations. Flow cytometry (FCM) was used to measure the variation of the immunophenotyping and cytokine secretions in co-culture, as well as gene expression.

Results: Cells noticeably proliferated without any modifications to their immunophenotype during the third subculture. The colony-forming unit fibroblast (CFU-F) test showed a proliferation of the MSCs along with healthy cells and cancer cell lines with no changes in their phenotype. The supernatant of MSCs showed an increase in cellular death of the OVCAR3 in ovarian cancer cell lines. A reduction in the level of CA-125 $(75-90 \% ; \mathrm{p}=0.769)$ with OVCAR3 in co-culture, and a decline of LDH (10-20\%; $\mathrm{p}=0.03)$ and beta-hCG $(10-20 \% ; \mathrm{p}=0.04)$ were observed in co-culture in CAOV3, SKOV3 and IGROV3 cell lines. A decrease in $\mathrm{CD} 24$ of the cancer cell lines in co-culture with the MSC supernatant showed a reduction of the cancer tumorigenicity and aggressiveness, while the rate of the CD24 and CD44 asserted their stem state. MSC supernatant decreased cell proliferation and MMP-2, MMP-9, and CA125 mRNA expression, while increasing TIMP 1, 2, and 3 . This suggests that MSCs have a role in cell death and inhibition of ovarian cancer cell proliferation. An increase of anti-inflammatory IL-4 and IL-10 cytokines, and a decrease in growth factor GM-CSF along with their proinflammatory INF-a, TNF-a, IL-9, and IL-17a cytokines were also noted.

Conclusions: The gene and cytokine activity indicate a potential therapeutic anti-inflammatory and antiproliferative role of MSCs on ovarian cancer despite their sources. The reduction of CA-125, LDH, and beta-hCG in co-culture, along with the decrease in CD24 and amplified cellular apoptosis demonstrate the beneficial effects of stem cells in ovarian cancer cell lines.

Disclosure: No disclosure

\section{Paediatric issues}

\section{P597}

Abstract already published.

\section{P598}

Comparison of toxicity after hematopoietic stem cell transplant in sickle cell disease between children aged less than 13 years and teenagers aged 13-18 years old

Manon Delafoy ${ }^{1}$,Jean-Hugues Dalle ${ }^{1}$, Bénédicte Neven ${ }^{2}$, Sylvie Chevret ${ }^{3}$, Audrey Grain ${ }^{1}$ 
${ }^{1}$ Hôpital Robert Debré, Paris, France, ${ }^{2}$ Hôpital Necker Enfant Malade, Paris, France, ${ }^{3}$ Hôpital Saint Louis, Paris, France

Background: Hematopoietic stem cell transplant (HSCT) is the only cure in sickle cell disease (SCD) so far. Because of the risk of toxicity, its indication in France is restricted to severe patients with match sibling donor. This study compares the incidence of severe acute toxicity after HSCT, between children aged less than 13 years and teenagers aged 13 to 18 years old, treated for SCD.

Methods: All patients suffering from SCD, aged less than 18 years at transplant, who received HSCT in CHU Robert Debré and Necker, between 01/01/2005 and 12/31/2017, were included. Severe acute toxicity, defined by onset of severe acute GVHd, organ toxicity or infection, was compared between the two groups of age.

Results: 73 patients (59 children and 14 teenagers) were included. All patients received a myeloablative conditioning regimen. Bone marrow from a sibling donor was the main stem cell source $(n=65 ; 89 \%)$. Neither death nor rejection was observed with a median follow-up of 29.6 months (range, 3-152). The incidence of grade III-IV acute GVHd was $12.3 \%$ and was similar between the two groups; no risk factor was identified in univariate analysis. Teenagers had more frequently acute skin toxicity $(21.4 \%$ vs $0 \%$, $\mathrm{p}=0.006$ ). In univariate analysis, patients presenting severe organ toxicity were significantly older than others (9.3 vs 7.5 years old, $\mathrm{p}=0.027$ ). Teenagers were more frequently treated for bacterial $(64.3 \%$ vs $32.2 \%, \mathrm{p}=0.035)$ or $\mathrm{BK}$ virus $(42.9 \%$ vs $6.8 \%, \mathrm{p}=0.002)$ infections. In univariate analysis, patients who developed infection were also significantly older at time of transplant (respectively 9.7 vs 7.5 years old, $\mathrm{p}=0.016$ ). No severe sinusoidal obstruction syndrome was observed. Regarding long-term toxicity, 2 patients presented an extensive chronic GVHd, they were both aged less than 13 years old. No cut-off of age could have been defined.

Conclusions: This study confirms the excellent results of HSCT in SCD, with a 5-year event-free survival and overall survival of $100 \%$. An older age at transplant seems to be associated with more frequent severe acute toxicity. These results are consistent with previous studies and suggest that HSCT should be performed as soon as possible, without any defined "best age". Prospective studies are needed, in order to define the place of each therapeutic in SCD, with the aim of reducing treatment-related toxicity and developing alternative strategies for patients without match sibling donor.

Disclosure: nothing to declare

\section{P599}

New insights into risk factors for transplant-associated thrombotic microangiopathy (TA-TMA) in paediatric HSCT

Reem Elfeky', Giovanna Lucchini', Su Han Lum ${ }^{3}$, Giorgio Ottaviano ${ }^{4}$, Natalia Builes, ${ }^{5}$, Alexandra Battersby ${ }^{3}$, Zohreh Nademi ${ }^{3}$, Austen Worth ${ }^{2}$, Robert Chiesa $^{2}$, Persis Amrolial, ${ }^{1,2}$ Kanchan Rao ${ }^{2}$, Andrew J Cant $^{3}$, Stephen Owens ${ }^{3,6}$, Terry Flood ${ }^{3}$, Sophie Hambleton $^{3,6}$, Sinead Greener ${ }^{3}$, Andrew Gennery, Helen Young ${ }^{3}$, Bilyana Doncheva ${ }^{2}, P$ Walsh ${ }^{6}$, David Kavanagh $^{6}$, Waseem Qasim ${ }^{1,2}$, Mary Slatter ${ }^{3,6}$, Paul Veys $^{1,2}$

${ }^{1}$ UCL Great Ormond Street Institute of Child Health, London, United Kingdom, ${ }^{2}$ Great Ormond Street Hospital, London, United Kingdom, ${ }^{3}$ The Great North Children`s Hospital, Newcastle upon Tyne, United Kingdom, ${ }^{4}$ San Gerardo Hospital/Fondazione MBBM, University of MilanBicocca, Monza, Italy, ${ }^{5}$ Pablo Tobón Uribe Hospital, Medellin, Colombia, ${ }^{6}$ Newcastle University, Newcastle upon Tyne, United Kingdom

Background: TA-TMA is a severe complication that can reduce survival after HSCT. Risk factors have been variably reported in adults although data on children remains scarce. We aimed to identify a risk profile for development of TATMA in children undergoing HSCT.

Methods: We retrospectively reviewed clinical charts of 398 children who underwent 406 HSCTs between 2013-16: at Great Ormond Street Hospital (GOSH) and the Great North Children's Hospital (GNCH). TA-TMA was defined according to revised criteria (Jodele et al. 2013). Risk factors were categorized into patient derived [age, gender, active co-morbidity at D0 of HSCT (uncontrolled viral/ bacterial or fungal infection, pulmonary, cardiovascular instability, steroid therapy $>0.3 \mathrm{mg} / \mathrm{kg}$ beyond D0, BCGiosis or autoimmune disease), transplant related factors (conditioning intensity, stem cell source, HLA-matching, use of Ciclosporin A (CSA) or Tacrolimus (Tac), CD34+ dose, ex-vivo T cell depletion, use of Defibrotide) and postHSCT factors (aGvHD, post-HSCT viral reactivation, venoocclusive disease (VOD) and occurrence of posterior reversible leukoencephalopathy (PRES).

Results: At a median of 7 months post-HSCT, TA-TMA occurred among 21/406 transplants $(5.1 \%)$. There was no reported centre variation $(5.7 \%$ vs $4.6 \%$ in GOSH vs $\mathrm{GNCH} ; \mathrm{p}=0.6)$. Gender, underlying disease - primary immune deficiency (PID) $(\mathrm{n}=273)$ vs haematological disease ( $n=133)$, use of myeloablative $(n=153)$ vs reducedor minimal intensity conditioning $(n=229)$, use of serotherapy or mega doses of CD34 $\geq 10 \times 10 * 6 / \mathrm{Kg}$ did not influence the development of TA-TMA. Donor type: MSD/ $\operatorname{MFD}(n=100)$ vs MUD $(n=129)$ vs MMUD/Haplo-HSCT 
$(\mathrm{n}=170)$ was associated with TA-TMA in $1 \%$ vs $5.8 \%$ vs $7.7 \%$ respectively $(p=0.067)$. The presence of comorbidities at D0 $(n=86)$ was significantly associated with an increased risk of TA-TMA $10.4 \%$ vs $3.7 \%$ in absence of co-morbidity $(n=320) ; p=0.032$. Use of CSA/Tac-based GvHD prophylaxis was associated with less TA-TMA with an incidence of $4 \%$ vs $13 \%$ if these agents were not included $(\mathrm{p}=0.01)$. In univariate analysis TA-TMA was significantly higher among patients with aGvHD grade IIIV $(12 / 138 ; 8.6 \%)$ vs Grade 0-I $(6 / 227 ; 2.6 \%)(\mathrm{p}=0.01)$. PRES was recorded among 10 cases and $50 \%$ of them developed TA-TMA. Two out of the 21 patients with TATMA had pathological gene mutations in their complement pathway. On multivariate analysis the presence of active comorbidity was a risk factor for TA-TMA (OR:6.6; 95\% CI:1.1-37.6; $\mathrm{p}=0.032$ ) while the use of CSA/Tac-based GvHD prophylaxis did not increase the risk for TA-TMA (OR:0.04; CI:0.004-0.39; $\mathrm{p}=0.005$ ). In the presence of comorbidities the use of Defibrotide as prophylaxis or therapy for VOD $(n=86)$ was associated with a drop in the incidence of TA-TMA from $12 \%$ (9/79) in absence of Defibrotide to $0 \%(0 / 7)$. 2- year overall survival was significantly lower among TA-TMA cases (58\%) in comparison to $81.5 \%$ in absence of TA-TMA $(p=0.001)$ (figure 1).

Conclusions: Active co-morbidity is a significant risk factor for TA-TMA. Use of Defibrotide prophylaxis in patients with co-morbidities at the time of HSCT might offer protection against TA-TMA. Surprisingly the use of CSA/Tac based GvHD prophylaxis is not a risk factor for TA-TMA probably through limiting the development of high grades aGvHD. The association between PRES and TA-TMA suggests a common pathway of endothelial damage

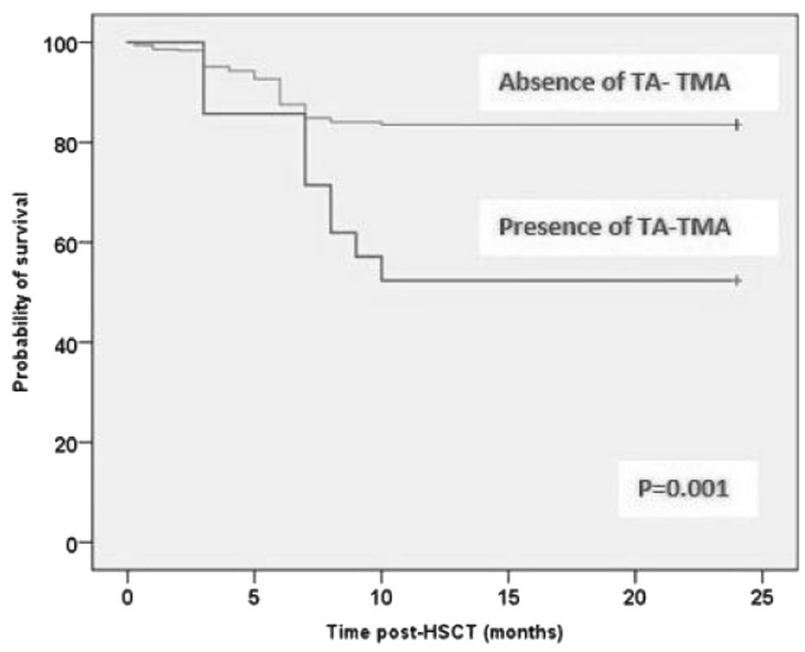

[[P599 Image] 1. Figure 1: Overall survival]
Clinical Trial Registry: Not applicable

Disclosure: Nothing to declare

\section{P600}

TCR $\alpha \beta$-depleted haploidentical hematopoietic cell transplantation for pediatric patients with acute leukemia in remission

\section{Ho Joon $\mathrm{Im}^{1}$, Hyery Kim ${ }^{1}$, Kyung-Nam Koh ${ }^{1}$, Jong Jin Seo ${ }^{I}$, Eun Seok Choi ${ }^{1}$}

${ }^{1}$ University of Ulsan College of Medicine, Asan Medical Center, Pediatrics, Seoul, Korea, Republic of,

Background: We evaluated the outcome of haploidentical HCT (HHCT) using ex vivo $\alpha \beta \mathrm{T}$ cell-depleted (TCD) grafts after reduced-intensity conditioning (RIC) containing low-dose TBI (LD-TBI) in pediatric patients with acute leukemia (AL) in complete remission (CR).

Methods: Between May 2012 and October 2018, 36 patients with acute leukemia (17 ALL and 19 AML) in CR received haploidentical hematopoietic cell transplantation (HHCT) using TCR $\alpha \beta$-depleted graft at Asan Medical Center Children's Hospital. Eighteen patients received HHCT between 2012 and 2015 (earlier time period) and the remaining 18 between 2016 and 2018 (recent study period). The conditioning regimens, the dose of $\alpha \beta+\mathrm{T}$ cells and GVHD prophylaxis are summarized in Table.

Results: All 36 patients achieved a sustained neutrophil engraftment at a median of 10 days (range, 9-13). Of 36 patients, 11 patients ( 8 ALL \& 4 AML) relapsed at a median of 6 months (range, 3-16) after transplant. Of the 11 patients, 10 patients died of disease. One patient died of disseminated tuberculosis at 11 months after transplant, leading to the TRM of $4 \%$ at 1 year. As of December 2018, 25 of the 36 patients survive free of disease at a median follow-up of 21 months (range, 1-77). At a median followup of 35 months (range, 1-80), EFS and OS at 2 years for all patients were $59 \%$ and $67 \%$, respectively. Outcome of HHCT in the recent study cohort was significantly better than that in the earlier study period (EFS of $85 \%$ vs EFS of $44 \%, \mathrm{P}=0.05)$. Among the 17 patients with ALL, the EFS of 8 patients, who received HHCT in early time period after conditioning with TBI of $600 \mathrm{cGy}$, was significantly worse than that of 9 patients, who received in recent study period after a higher dose of TBI at 800 cGy $(13 \%$ vs $83 \%, \mathrm{P}<$ 0.05). The EFSs of AML were similar between the two study groups $(70 \%$ for earlier cohort vs $86 \%$ for recent study, $\mathrm{P}>0.05)$.

Conclusions: In pediatric patients with acute leukemia in $\mathrm{CR}$, our current haploidentical HCT using ex vivo $\alpha \beta$ TCD graft after RIC containing LD-TBI without GVHD prophylaxis is feasible approach with a low TRM. The 
outcome of patients with AML is favorable. In addition, more intensified conditioning regimen with higher dose of TBI markedly improved the outcome of ALL.

\begin{tabular}{|c|c|c|c|c|c|}
\hline Study period & Disease & $\begin{array}{l}\text { Number } \\
\text { of } \\
\text { patients }\end{array}$ & $\begin{array}{l}\text { Conditioning } \\
\text { regimens }\end{array}$ & $\begin{array}{l}\text { Dose } \\
\text { of } \alpha \beta \\
+ \\
\text { T cells }\end{array}$ & $\begin{array}{l}\text { GVHD } \\
\text { prophylaxis }\end{array}$ \\
\hline $\begin{array}{l}2012-2015 \\
\text { (earlier) }\end{array}$ & $\begin{array}{l}\text { ALL/ } \\
\text { AML }\end{array}$ & $8 / 10$ & $\begin{array}{l}\text { TBI600/ } \\
\text { Flu180/ } \\
\text { Cy100/ATG3 }\end{array}$ & $\begin{array}{l}1-5 \times \\
10^{\wedge} 4 / \\
\mathrm{kg}\end{array}$ & $\begin{array}{l}\text { Tacrolimus/ } \\
\text { MMF }\end{array}$ \\
\hline $\begin{array}{l}2016-2018 \\
\text { (recent) }\end{array}$ & $\begin{array}{l}\text { ALL/ } \\
\text { AML }\end{array}$ & $9 / 9$ & $\begin{array}{l}\text { TBI800/ } \\
\text { Flu180/ } \\
\text { Cy100/ATG3 } \\
\text { for ALL } \\
\text { TBI600/ } \\
\text { Flu180/ } \\
\text { Cy100/ATG3 } \\
\text { for AML }\end{array}$ & $\begin{array}{l}\leq 5 \times \\
10^{\wedge} 4 / \\
\mathrm{kg}\end{array}$ & None \\
\hline
\end{tabular}

[[P600 Table] 1. Transplant characteristic of patients]

Clinical Trial Registry: www.clinicaltrials.gov (NCT02014506)

Disclosure: Nothing to declare.

\section{P601}

Gonadal function after busulfan comparing to treosulfan in pediatric patients received allogeneic stem cell transplant. on behalf of paediatric disease $\&$ transplant complications working parties

Maura Faraci', Tamara Diesch', Myriam Labopin ${ }^{3}$, Arnaud Dalissier ${ }^{4}$, Arian Lankester ${ }^{5}$, Andrew Gennery ${ }^{6}$, Mikael Sundin $^{7}$, Duygu Uckan-Cetinkaya ${ }^{8}$, Marc Biering $^{9}$, Brigitte Strahm ${ }^{10}$, Martina Garwer ${ }^{11}$, Ansgar Schulz $^{12}$, Gerard Michel ${ }^{13}$, Giovanna Giorgiani ${ }^{14}$, Bernd Gruhn $^{15}$, Franco Locatelli ${ }^{16}$, Stefano Giardino ${ }^{17}$, Anne Uyttebroeck $^{18}$, Fanny Rialland ${ }^{19}$, Maija Itäla-Remes ${ }^{20}$, Peter Dreger ${ }^{21}$, Peter H Shaw ${ }^{22}$, Victoria Bordon ${ }^{23}$, Paul G. Schlegel $^{24}$, Karin Mellgren ${ }^{25}$, Jose M. Moraleda ${ }^{26}$, Katharine Patrick ${ }^{27}$, Pascale Schneider ${ }^{28}$, Charlotte Jubert $^{29}$, Anita Lawitschka ${ }^{30}$, Nina Salooja ${ }^{31}$, Grzegorz W. Basak $^{32}$, Selim Corbaciuoglu ${ }^{33}$, Rafael Duarte ${ }^{34}$, Peter Bader $^{35}$

${ }^{1}$ Istituto G. Gaslini, Genova, Italy, ${ }^{2}$ University Children's Hospital Basel, Basel, Switzerland, ${ }^{3}$ Hôpital Saint-Antoine, Paris, France, ${ }^{4}$ EBMT Paris Study Office/CEREST-TC, Saint Antoine Hospital, Paris, France, ${ }^{5}$ Willem-Alexander Children's Hospital, Leiden University Medical Center, Leiden, Netherlands, ${ }^{6}$ Children`s Hospital Newcastle upon Tyne, Paediatric Team Children`s Newcastle upon Tyne, United Kingdom, ${ }^{7}$ Astrid Lindgren Children's Hospital, Karolinska University Hospital, Haematology/Immunology
Section, Stockholm, Sweden, ${ }^{8}$ Hacettepe University Faculty of Medicine, Ankara, Turkey, ${ }^{9}$ University Hospital for Children, Utrecht, Netherlands, ${ }^{10}$ University Medical Centre, Paediatric Haematology/Oncology, Freiburg, Germany, ${ }^{11}$ University Hamburg-Eppendorf, Paediatric haematology Clinic and Policlinic of Oncology, Hamburg-Eppendorf, Germany, ${ }^{12}$ University Medical Center Ulm, Ulm, Germany, ${ }^{13}$ Hopital d'Enfants de la Timone Marseille, Marseille, France, ${ }^{14}$ Fondazione IRCCS Policlinico San Matteo, Pediatric Hematology-Oncology, Pavia, Italy, ${ }^{15}$ Jena University Hospital, Jena, Germany, ${ }^{16}$ IRCSS Ospedale Pediatrico Bambino Gesù, University La Sapienza, Rome, Italy, ${ }^{17}$ Institute G Gaslini, Haematology-Oncology, Genova, Italy, ${ }^{18}$ University Hospitals Leuven, Leuven, Belgium, ${ }^{19}$ Pediatric BMT Unit, Nantes, France, ${ }^{20}$ Turku University Hospital, Children's Hospital, Turku, Finland, ${ }^{21}$ University of Heidelberg, Heidelberg, Germany, ${ }^{22}$ The Children's Hospital at Westmead, Sydney, Australia, ${ }^{23}$ Ghent University Hospital, Ghent, Belgium, ${ }^{24}$ University Children's Hospital Würzburg, Würzburg, Germany, ${ }^{25}$ The Queen Silvia's Hospital for Children and Adolescents, University of Göteborg,, Pediatric Hematology and Oncology, Göteborg, Sweden, ${ }^{26}$ Hospital Clínico Universitario Virgen de la Arrixaca, Murcia, Spain, ${ }^{27}$ Sheffield Children's Hospital, Sheffield, United Kingdom, ${ }^{28}$ University Hospital, Rouen, Rouen, France, ${ }^{29}$ Bordeaux University Hospital, Bordeaux, France, ${ }^{30}$ Medical University of Vienna, Vienna, Austria, ${ }^{31}$ Imperial College London, London, United Kingdom, ${ }^{32}$ Medical University of Warsaw, Warsaw, Poland, ${ }^{33}$ University of Regensburg, Regensburg, Germany, ${ }^{34} \mathrm{Hos}-$ pital Universitario Puerta de Hierro Majadahonda, Madrid, Spain, ${ }^{35}$ Goethe-Universität, Universitätsklinikum Frankfurt, Frankfurt, Germany

Background: Gonadal impairment is a severe late effect of myeloablative conditioning regimes with significant impact on quality of life of cancer survivors. The aim of this study was to analyze gonadal function after Busulfan $(\mathrm{Bu})$ or Treosulfan (Treo) containing regimens with regard to pubertal stage.

Methods: This was a retrospective, multicenter study involving patients treated in pediatric EBMT centers between 1992-2012. Patients receiving myeloablative doses of $\mathrm{Bu}$ or Treo as part of HSCT conditioning were eligible for inclusion. Analysis was conducted in two groups according to pubertal status at time of HSCT.

Results: 137 patients (pts) were treated in 25 pediatric EBMT with $\mathrm{Bu}$ or Treo before allogeneic HSCT. The median age at transplant was 11.04 years (range 5-18); 89/ 137 (65\%) were males (m), 48/137 (35\%) were females (f). $89 / 137(65 \%)$ pts were pre-pubertal at HSCT $(\mathrm{f}=27 ; \mathrm{m}=62)$ and $48 / 137(35 \%)$ were post pubertal $(\mathrm{f}=21 ; \mathrm{m}=27) .118 /$ $137(86 \%)$ patients received $\mathrm{Bu}(\mathrm{f}=38 ; \mathrm{m}=80), 77 / 118$ 
$(65 \%)$ were pre-pubertal. 19/137 $(14 \%)(\mathrm{f}=10 ; \mathrm{m}=9)$ received Treo,12/19 (64\%) were pre-pubertal (Figure 1). Females who received Treo in pre-pubertal stage $(n=6 / 6)$ reached more often spontaneous puberty (100\% vs $38 \%$; $\mathrm{p}=0.016)$ compared to pre-pubertal Bu group $(\mathrm{n}=8 / 21)$ and occurrence of menarche was higher in Treo group $(\mathrm{p}<.01)$ Hormonal replacement therapy was given in $13 / 27(48 \%)$ females transplanted in pre-pubertal stage and in $15 / 21$ $(71 \%)$ of those transplanted in post-pubertal stage. $77 / 89$ $(86 \%)$ males were pubertal at last follow-up and 8 of them (10\%) performed sperm analysis (5 oligo-azoospermic, 3 unknown). Three pregnancies were reported in the population group, all received $\mathrm{Bu}$. Regarding the evaluation of hormonal levels in pubertal patients at time of hormonal dosage (median $19.54 \mathrm{yrs}$ ) (66 Bu and 15 Treo), males treated with Treo had significant lower LH levels ( $\mathrm{p}=$ 0.045) compared to $\mathrm{Bu}$ group. Females treated with Treo had significant lower levels of LH and FSH ( $p=0.02$ and $\mathrm{p}=0.0035$ respectively).

Conclusions: Gonadal damage related to Treo was significantly lower compared to $\mathrm{Bu}$. We observed that: females transplanted during pre-pubertal period had spontaneous puberty more frequently after Treo compared to $\mathrm{Bu}$ and that hypogonadism hypergonadotropic was more frequent after $\mathrm{Bu}$ than Treo. These results must be further confirmed on a larger population.

\begin{tabular}{|c|c|c|c|}
\hline Variables & $\begin{array}{l}\text { Entire } \\
\text { Population } \\
\mathrm{n}=137(\%)\end{array}$ & $\begin{array}{l}\text { Busulfan } \\
\mathrm{n}=118(86 \%)\end{array}$ & $\begin{array}{l}\text { Treosulfan } \\
\mathrm{n}=(14 \%)\end{array}$ \\
\hline No Puberty at SCT & $\begin{array}{l}89(64.9) \mathrm{f}=27 \\
(30.3) \\
\mathrm{m}=62\end{array}$ & $\begin{array}{l}77(65.25) \mathrm{f}=21 \\
(27.2) \mathrm{m}=56 \\
(72.7)\end{array}$ & $\begin{array}{l}12(63.1) \mathrm{f}=6 \\
(50) \mathrm{m}=6(50)\end{array}$ \\
\hline Yes Puberty at SCT & $\begin{array}{l}48(35.0) \mathrm{f}=21 \\
(43.7 \%) \mathrm{m}=27 \\
(56.2 \%)\end{array}$ & $\begin{array}{l}41(34.7 \%) \\
\mathrm{f}=17(41.4 \%) \\
\mathrm{m}=24(58.5 \%)\end{array}$ & $\begin{array}{l}7(36.8 \%) \\
\mathrm{f}=4(54.1) \\
\mathrm{m}=3(42.8 \%)\end{array}$ \\
\hline $\begin{array}{l}\text { Age at } \operatorname{HSCT}(\mathrm{y}), \\
\text { median (range) }\end{array}$ & $\begin{array}{l}11.04(5.02- \\
18.55)\end{array}$ & $10.7(5-18.6)$ & $\begin{array}{l}12.2(6.1- \\
18.4)\end{array}$ \\
\hline $\begin{array}{l}\text { Chronic Myeloid } \\
\text { leukemia }\end{array}$ & 41(29.9) & $38(32.2)$ & $3(15.7)$ \\
\hline $\begin{array}{l}\text { Myelodisplastic } \\
\text { syndrome }\end{array}$ & $28(20.4)$ & $23(19.4)$ & $5(26.3)$ \\
\hline $\begin{array}{l}\text { Congenital bone } \\
\text { marrow failure }\end{array}$ & $2(1.4)$ & $2(1.6)$ & 0 \\
\hline $\begin{array}{l}\text { Primary immune } \\
\text { deficiency }\end{array}$ & $59(43.0)$ & $48(40.6)$ & $11(57.8)$ \\
\hline $\begin{array}{l}\text { Hemophagocytic } \\
\text { Lymphohistiocitosis }\end{array}$ & $7(5.11)$ & $7(5.9)$ & 0 \\
\hline
\end{tabular}

\section{[[P601 Table] 1. Characteristic of patients]}

Disclosure: Nothing to declare

\section{P602}

Management of pediatric patients with anti-HLA antibodies undergoing $\mathbf{T}$-cell depleted haploidentical hematopoietic stem cell transplantation

Pietro Merli ${ }^{I}$, Giovanna Leone ${ }^{1}$, Federica Galaverna ${ }^{1}$, Paola Giustiniani ${ }^{1}$, Giovanna Del Principe ${ }^{1}$, Antonella Meschini $^{1}$, Stefania Lazzaro ${ }^{1}$, Mattia Algeri ${ }^{1}$, Annalisa Ruggeri', Enrica Pittaluga ${ }^{1}$, Daria Pagliara', Silvia Bordasco $^{1}$, Carmela Parlavecchio ${ }^{1}$, Elia Girolami, Giuseppina Li Pira', Mauro Montanari', Franco Locatelli, $^{1,2}$

${ }^{1}$ Bambino Gesù Children's Hospital IRCCS, Hematology and Oncology, Cellular and Gene Therapy, Rome, Italy, ${ }^{2}$ Sapienza University, Rome, Italy

Background: Anti-HLA antibodies (aHAb) have been recently recognized as an important risk factor for graft failure (GF), especially in HLA-haploidentical stem cell transplantation (haplo-HSCT). Although, recently, EBMT consensus guidelines have been published [Ciurea, Bone Marrow Transplant 2018], experience in pediatric T-cell depleted (TCD, another well-known risk factor for GF) haplo-HSCT is lacking. In the present study, we report our experience on the use of a desensitization approach based on EBMT guidelines.

Methods: Between June 2017 and August 2018, all patients affected by non-malignant diseases and scheduled for a transplant from an HLA-haploidentical donor after negative depletion of $\alpha \beta \mathrm{T}$ and $\mathrm{B}$ cells as previously described [Li Pira, Biol Blood Marrow Transplant. 2016], were tested for $\mathrm{aHAb}$ with Luminex $^{\circledR}$ solid-phase immunoassay (One Lambda, Thermo Fisher Scientific) 1 month before the HSCT. All patients with a MFI higher than 5000, which is considered a cutoff predicting for GF, were treated with a desensitization protocol based on the use of antiCD20 rituximab $\left(375 \mathrm{mg} / \mathrm{m}^{2}\right.$ before and immediately after the end of plasma-exchange cycle) and plasma-exchange $(\mathrm{PE}) \pm$ infusion of irradiated buffy coat (BC) (if after PE aHAb MFI was still > 5000 MFI). This latter was obtained by the non-target fraction of the $\alpha \beta$ T-cell/B-cell-depletion procedure and consisted of $5 * 10^{7}$ irradiated nucleated cells $/ \mathrm{kg}$ of the recipient; this was infused 2-4 hours before the infusion of the TCD graft. PE was performed with Miltenyi Life 18 TM Apheresis Unit (Miltenyi, BergishGladback).

Results: In the study period, 37 patients received $\alpha \beta \mathrm{T}$ and B-cell depleted haplo-HSCT. Eighteen (48\%) resulted positive for aHAb (MFI> 1000); $14(77 \%)$ of them had an MFI > 5000 for either anti-class I or II aHAb. These patients (see Table I for details) received the sensitization therapy. A median of $3 \mathrm{PE}$ procedure (on consecutive days) per 
patients were performed (range 3-10); median total plasma volume exchanged per procedure was 1.5 (range 1-1.5). Median pre-desensitization MFI of both anti-class I and II aHAb was 9392 (range 1420-22418) and 2637 (range 163613280), respectively. After desensitization therapy, these values were 2810 (range 0-13883) and 1589 (range 0-3680), respectively. Median reduction of anti-class I and II aHAb was 5280 (range 880-10791) and 2414 (range 0-9600) $(\mathrm{p}=0.05)$ (Figure 1). Nine children received the BC before the infusion of the graft. Cumulative incidence of GF was $22.2 \%$ (95\% CI 2.7-37.8) in the whole population, while it was $29.2 \%$ (95\% CI $0.3-49.8)$ in the 14 treated patients. No serious advent events associated with the desensitization therapy was recorded. Most common adverse events were rituximab infusion reaction and (mild) hypotension during PE.

Conclusions: Desensitization therapy for aHAb based on EBMT guidelines is safe in pediatric patients undergoing TCD haplo-HSCT. PE and rituximab seem more effective in reducing the title of anti-class $\mathrm{I}$ aHAb, thus supporting the use of BC infusion before HSCT for those patients with anti-class II aHAb. More data, as well as correlation with other variables (e.g., presence of complement fixing $\mathrm{aHAb}$ ), are needed to better stratify patients at high risk for GF.

Disclosure: Nothing to declare

\section{P603}

Decreased cost of viral specific $\mathbf{T}$ cells (VSTS) compared to anti-viral therapy in pediatric hematopoietic stem cell transplant recipients

\section{Ashley Teusink-Cross ${ }^{1}$, Lauren Hudson ${ }^{1}$, Thomas Leemhuis $^{2}$, Daria Heyenbruch ${ }^{2}$, Carolyn Lutzko ${ }^{1}$, Jeremy Rubinstein ${ }^{1}$, Stella Davies ${ }^{\text {, }}$ Michael Grimley ${ }^{1}$, Adam Nelson ${ }^{1}$}

${ }^{1}$ Cincinnati Children's Hospital Medical Center, Cincinnati, $\mathrm{OH}$, United States, ${ }^{2}$ Hoxworth Blood Center, Cincinnati, $\mathrm{OH}$, United States,

Background: Viral infections significantly contribute to both morbidity and mortality in patients undergoing hematopoietic stem cell transplantation. Traditional antiviral therapy is associated with lack of efficacy, potential toxicity, prolonged hospitalization and increased patient costs. Viral specific T cells can be manufactured from donor blood to treat viral infections post-transplant, and are associated with increased clinical efficacy and low toxicity. We postulated that direct costs of VST therapy are lower compared to traditional anti-viral medications

Methods: VSTs were manufactured according to local protocols and FDA requirements. Total drug cost (as per institutional charges per drug) was calculated for patients who required treatment for viral infections post-HSCT. Manufacturing costs of VSTs are fixed per FDA requirements. Patients who were treated with investigational antiviral medications (including brincidofovir) were excluded from analysis. Patients treated with VSTs +/anti-viral medications over a 2 year period were compared to patients treated only using traditional anti-viral medications, including cidofovir, ganciclovir, valganciclovir, foscarnet and rituximab.

Results: Demographics are shown in Table 1. There were no major differences between the two groups treated. The number of anti-viral medications used in the VST group was lower compared to the anti-viral treatment group. Median cost of VST treatment was significantly lower compared to those threated with traditional anti-viral therapy $(\$ 12,757$ vs $\$ 16,392$, p-value $=0.03)$.

Conclusions: Treatment with VSTs post-HSCT for viral infections was lower in cost compared to anti-viral medical therapy. It is likely that overall costs are further reduced with VSTs due to reduced inpatient hospital time, less monitoring of labs associated with anti-viral medication side-effects and reduced ancillary costs including nursing and pharmacy. More studies are needed to examine these indirect costs further.

\begin{tabular}{lll}
\hline Demographic & VST treatment & $\begin{array}{l}\text { Anti-viral } \\
\text { treatment }\end{array}$ \\
\hline Median Age (years) & $\begin{array}{l}7 \text { (range 1- } \\
26 \text { years) }\end{array}$ & $\begin{array}{l}5.5 \text { (range 2- } \\
19 \text { years) }\end{array}$ \\
Sex $\bullet$ Male $\bullet$ Female & $\bullet 13 \bullet 10$ & $\bullet 10 \bullet 12$ \\
Diagnosis $\bullet$ Marrow Failure $\bullet$ & $\bullet 15 \bullet 7 \cdot 1$ & $\bullet 10 \bullet 8 \bullet 4$ \\
Immunodeficiency $\bullet$ Malignancy & & \\
Median number of anti-viral medications & 1 (range 0-3) & 2 (range 1-5) \\
Median cost of anti-viral therapy & $\$ 296($ range $\$ 0-$ & $\$ 16,392($ range \\
& $\$ 42436)$ & $\$ 146-94055)$ \\
Cost of VSTs & $\$ 12,461$ & NA \\
Total anti-viral cost & $\$ 12,757$ & $\$ 16,392$ \\
p-value & 0.03 & \\
\hline
\end{tabular}

[[P603 Table] 1. Demographics and Results comapring traditional anti-viral therapy to VSTs]

Disclosure: Nothing to declare

\section{P604}

Hematopoietic stem cell transplantation in pediatric DOCK8 deficiency related hiper ige syndrome

Gülcihan Ozek ${ }^{1}$, Serap Aksoylar' ${ }^{1}$, Salih Gözmen², Güzide $\mathrm{Aksu}^{3}$, Neslihan Edeer Karaca ${ }^{3}$, Ferah Genel ${ }^{4}$, Nesrin Gülez $^{4}$, Necil Kütükçüler ${ }^{3}$, Savaş Kansoy ${ }^{1}$

${ }^{1}$ Ege University, Izmir, Turkey, ${ }^{2}$ Dr. Behcet Uz Children's Research Hospital, Izmir, Turkey, ${ }^{3}$ Ege University Pediatric 
Immunology, Izmir, Turkey, ${ }^{4}$ Dr. Behcet Uz Children's Research Hospital Pediatric Immunology, Izmir, Turkey

Background: DOCK8 deficiency is an autosomal recessive primary immunodeficiency (PID) disease caused by loss-offunction mutations in the DOCK8 gene (1). Patients with DOCK8 deficiency present with multiple abnormalities of the immune system, including defective $T$ cell function and impaired production of antigen-specific antibodies. These lead to persistent viral infections of the skin, mucocutaneous candidiasis, recurrent sinopulmonary infections, atopic dermatitis, and other allergic disease, malignancies, and sometimes autoimmunity (2). Hematopoietic stem cell transplantation (HSCT) is currently the only curative treatment option available (3).

Methods: We retrospectively evaluated our patients who underwent allogeneic hematopoietic stem cell transplantation due to DOCK8 deficiency in Ege University Pediatric Stem Cell Transplantation Unit between 2013 and 2018.

Results: We identified 16 patients transplanted at a median age of 7.8 years (range: 2-15.5 years). Of 16 patients; 4 (\%25) received HSCT from matched sibling, 11 (\%68) from unrelated donors and 1 patient from haploidentical donor. We used busulfan-based myeloablative conditioning regimen to 12 patients (\%75), reduced toxicity myeloablative regimen with treosulfan to 2 patients $(\% 12.5)$ and nonmyeloablative regimen to 2 patients (\%12.5). Eight of the recipients received bone marrow, 6 of the patients received peripheral blood stem cells, 2 of the recipients received cord blood as stem cell source.

Fifteen of 16 patients (\%93) had achieved engraftment and median follow-up of patients was 29 months (1-64). Grade III-IV acute graft versus host disease (GVHD) occurred in $13 \%$ of patients and chronic graft versus host disease was seen $18 \%$ of patients. One patient received cord blood from unrelated donor did not engraft and died from septic shock. Four patients died from transplant related toxicity. Our patient's survival was $\% 68 ; 11 / 16$ patients alive.

Conclusions: HSCT is the only curative treatment option for DOCK8 deficiency. In particular, patients with high comorbidity scores have a high risk of toxicity and toxic death. Therefore, reduced toxicity conditioning regimens should be used for these patients.

\section{References:}

1) Zhang, Q. et al. 2009. Combined immunodeficiency associated with DOCK8 mutations. N. Engl. J. Med. 361: 2046- 2055.

2) Aydin SE, Kilic SS, Aytekin C, et al. DOCK8 deficiency: clinical and immunological phenotype and treatment options-a review of 136 patients. J Clin Immunol. 2015; 35:189-198. [PubMed: 25627830
3) Bittner TC, Pannicke U, Renner ED, et al. Successful long-term correction of autosomal recessive hyper-IgE syndrome due to DOCK8 deficiency by hematopoietic stem cell transplantation. Klin Padiatr. 2010; 222:351-355. [PubMed: 21058221]

Disclosure: Nothing to declare

\section{P605}

Eltrombopag use for post hematopoetic stem cell transplantation thrombocytopenia in pediatric patients

\section{Murat Elli ${ }^{I}$, Yöntem Yaman ${ }^{I}$, Kürşat Özdilli ${ }^{I}$, Hulya Bilgen ${ }^{I}$, Aslı Çakır ${ }^{1}$, Şifa Şahin ${ }^{1}$, Sema Anak ${ }^{1}$}

${ }^{1}$ Medipol University, Istanbul, Turkey

Background: Eltrombopag, a low-molecular-weight synthetic nonpeptide thrombopoietin receptor agonist (TPO-R), is a second- generation TPO. It is an oral thrombopoietin mimetic licensed in chronic immune thrombocytopenic purpura that induces platelet maturation and release by binding to c-MPL receptors on megakaryocytes. In a recent study; for patients with refractory SAA, eltrombopag induced a response (at 12 weeks) in at least one hematologic lineage in $44 \%$ patients and $36 \%$ no longer required platelet transfusions. And also $24 \%$ patients became RBC transfusion independent and 36\% had a neutrophil response. Trilineage responses were seen in $24 \%$ of patients; although surprising, this might indicate stimulation of c-MPL receptors on remaining stem cells. Delayed recovery from thrombocytopenia is common after stem cell transplantation. In a study including 12 adult patients, Eltrombopag was used to enhance platelet recovery for post-HSCT thrombocytopenia. It is well tolerated and efficacious offering transfusion independence.

Methods: In our retrospective study, Eltrombopag $(50 \mathrm{mg} /$ day) was started in 19 pediatric patients (age ranging from 4 to 16 years with a median age 12.3 years) for posthematopoietic stem cell transplantation (HSCT) thrombocytopenia. All patients fulfilled the following 3 criteria: (1) undergone hematopoietic stem cell transplantation (HSCT), (2) had improved total leucocyte counts after leucocyte engraftment, (3) had prolonged thrombocytopenia $(<30.000)$ needing platelet transfusion.

Results: Four of the patients have received RIC while 15 patients MA conditioning regimens before HSCT. Two haploidentic, 2 autologous, 7 MUD, 8 MSD transplantations were performed. ET $(50 \mathrm{mg} /$ day) was started in 19 patients who had thrombocytopenia despite neutrophil engraftment on the +30th day of HSCT A reduction in platelet transfusions and a platelet count of more than 30,000 were the primary endpoints. Before ET treatment, bone marrow biopsy was checked in $15 / 19$ patients, $10 / 15$ 
patients had decreased number of megacocyocytes. None of the patients had active bleeding at the start of Eltrombopag but they were all at high risk of bleeding. According to the platelet monitoring, 12 patients had a dose increase starting from the second week. The number of patients in need of platelet transfusions was 7 at the end of the first month; and only 2 at the end of the 2nd month. All patients had a thrombocyte count of more than 30.000 in the third month. In 11 patients, ET was discontinued after 2-11 months. No dose limiting toxicities have been observed.

Conclusions: As a conclusion, ET was found highly effective for postHSCT thrombocytopenia, with no drug related adverse effects. There was a gradual increase in platelet count, and none of the patients had any complication due to thrombocytopenia.

Disclosure: nothing to declare

\section{P606}

ten years outcome of unmanipulated Haplo-HSCT in high risk pediatric ALL AND AML. CIC 725

Olesia Paina ${ }^{1}$, Polina Kozhokar ${ }^{1}$, Anastasia Frolova', Kirill Ekushov ${ }^{1}$, Zhemal Rakhmanova ${ }^{1}$, Maria Galas ${ }^{1}$, Aigul Khabirova ${ }^{1}$, Elena Babenko ${ }^{1}$, Aleksandr Alianskii ${ }^{1}$, Ildar Barkhatov ${ }^{1}$, Elena Semenova ${ }^{1}$, Ludmila Zubarovskaya $^{I}$, Boris Afanasyev ${ }^{I}$

${ }^{1}$ Pavlov First Saint Petersburg State Medical University, R. Gorbacheva Memorial Institute of Children Oncology Hematology and Transplantation, St.Petersburg, Russian Federation

Background: The introduction of Haplo-HSCT expands the possibilities of treating children with unfavorable variants of acute leukemia, having advantages associated with the availability of the donor as soon as possible. Nevertheless, there are still a few unsolved questions that can affect individual outcome of Haplo-HSCT: the source of HSC, optimal conditioning regimens and prophylactic of GVHD and high risk of others complications.

The aim: To evaluate the effectiveness of Haplo-HSCT in children and adolescents with acute leukemia (AL), high risk. Primary end points: long-term overall survival (OS), GVHD-free/relapse-free survival (GRFS). Secondary end points: engraftment rate, incidence of relapse rate, GVHD incidence, risk of infection, transplant relate mortality (TRM).

Methods: From 12.2006 till 12.2016 Haplo-HSCT performed in 106pts with acute leukemia (AL), high-risk, median age 7 y.o. (range 0 - 18), ALL - 63 (59,4\%), AML $43(40,6 \%)$. Complete remission (CR) has 43pts (40,6\%): CR1 21pts(49\%), CR2-13pts(30\%), CR3-9pts(21\%). Resistance disease or resistance relapse (R/R) AL-63pts $(59,4 \%)$.
Conditioning regimens: MAC - «GIAC» 39pts(36,8\%), MAC based on $\mathrm{Bu}(12 \mathrm{mg} / \mathrm{b}$.w. $)+$ Flu $\left(150 \mathrm{mg} / \mathrm{m}^{2}\right)-2 \mathrm{pts}(2 \%)$, MAC reduced toxisity based on Treosulfan $\left(42 \mathrm{~g} / \mathrm{m}^{2}\right)+$ Flu6 pts $(5,7 \%)$. RIC based on $\mathrm{Mel}\left(140 \mathrm{mg} / \mathrm{m}^{2}\right)-40 \mathrm{pts}(37,7 \%)$, $\mathrm{Bu}(8 \mathrm{mg} / \mathrm{b}$.w. $)-18 \mathrm{pts}(17 \%)$. Prophylaxis of aGVHD: with ATG - ATGAM 60mg/b.w.-39pts(36,8\%), posttransplant cyclophosphomide (PTCy) 50mg/b.w. on D+3;D+4-67pts $(63,2 \%)$. Conventional immunosuppressive therapy: Tacro +Sirolimus 47pts(44,3\%) or CsA 59pts(55,7\%). Source of transplant: G-CSF stimulated unmanipulated Haplo-BM +PBSC after positive selected of CD34+-27pts (25,5\%); G-CSF stimulated unmanipulated Haplo-BM - 79(74,5\%). Median dose of CD34+in G-CSF stimulated unmanipulated

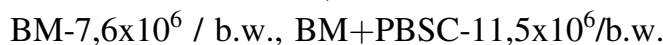

Results: Engraftment rate in all group - 80 pts(75,7\%), median time $\mathrm{D}+24$ (range $\mathrm{D}+14-\mathrm{D}+34)$. Ten years $\mathrm{OS}$ in all group-33,3\%, OS in $1^{\text {st }}-2^{\text {nd }} \mathrm{CR}-64,7 \%$ vs $18,1 \% \mathrm{R} / \mathrm{R}$ $(\mathrm{p}=0,000)$. There are no statistical significant in OS depending on intensity of conditioning regimen MAC vs RIC $36,2 \%$ and $30,5 \%$ and type of disease ALL vs AML $36,5 \%$ and $27,9 \%$ respectively. Incidence of aGVHD in PTCy group $\mathrm{II}^{0}-7 \mathrm{pts}(10,4 \%), \mathrm{III}^{0}-\mathrm{IV}^{0}-6 \mathrm{pts}(9 \%)$, aGVHD in ATG group II ${ }^{0}-14$ pts $(35,9 \%)$, III ${ }^{0}-\mathrm{IV}^{0}-9 \mathrm{pts}(23,1 \%)$; moderate cGVHD: PTCy group-5pts(7,5\%), ATG group-8pts $(20,5 \%)$. Relapse rate in all group-51pts $(48,1 \%)$ with median time D+94 (range D+17-D+1101). Main cause of death-relapse 39pts(48,8\%). TRM in all group-21,6\%: infections-14pts(13,2\%), aGVHD-7pts(8,75\%), toxicity2 pts $(1,9 \%)$. Ten years GRFS in $1^{\text {st }}-2^{\text {nd }}$ CR-35,3\% vs $11,1 \%$ in relapse or resistant disease $(\mathrm{p}=0,003)$.

Conclusions: Unmanipulated Haplo-HSCT in $1^{\text {st }}-2^{\text {nd }} \mathrm{CR}$ in children and adolescents with high risk $\mathrm{AL}$ allows achieving the long-term survival in $64,7 \%$. The use of GCSF stimulated unmanipulated Haplo-BM is associated with a satisfactory rate of engraftment. The main cause of death in our study was relapse after allo-HSCT. The frequency of acute and chronic GVHD was acceptable, 10years GRFS rate of $35,3 \%$ in $1^{\text {st }}-2^{\text {nd }} \mathrm{CR}$ represents good quality of life following unmanipulated Haplo-HSCT and therefore may be recommended as option for use in children and adolescents with high-risk AL.

Disclosure: Nothing to declare

\section{P607}

Isavuconazole use in paediatric hematopoietic stem cell transplantation: The experience of infectious disease working group of Italian association of pediatric hematology oncology (AIEOP)

Nunzia Decembrino ${ }^{1}$, Katia Perruccio ${ }^{2}$, Marco Zecca ${ }^{1}$, Mariadelfina Molinaro ${ }^{I}$, Antonella Colombini ${ }^{3}$, Marta Verna $^{3}$, Elena Soncini ${ }^{4}$, Bianca Maria Goffredo ${ }^{5}$, Maurizio Caniglia $^{2}$, Simone Cesaro ${ }^{6}$ 
${ }^{1}$ Fondazione IRCCS Policlinico San Matteo, Pavia, Italy, ${ }^{2}$ Ospedale Santa Maria della Misericordia, Perugia, Italy, ${ }^{3}$ Fondazione Monza e Brianza per il Bambino e la Mamma Ospedale San Gerardo, Monza, Italy, ${ }^{4}$ ASST Spedali Civili Brescia, Brescia, Italy, ${ }^{5}$ Ospedale Pediatrico Bambino Gesù, Roma, Italy, ${ }^{6}$ Ospedale Donna Bambino Verona, Azienda Ospedaliera Universitaria Integrata, Verona, Italy

Background: Isavuconazole (ISA) is a new triazole approved for IFI treatment in the adult population. Advantages are activity against both moulds and yeasts spp, excellent bioavailability after oral administration without relevant food or gastric $\mathrm{pH}$ effect, a water-soluble prodrug developed to facilitate intravenous administration without nephrotoxic excipients such as $\beta$-cyclodextrin, potentially poor drug-drug interactions. ISA does not currently appear to require TDM. ISA safety and efficacy have not been yet established in children and, in particular, no data are available in the pediatric HSCT setting.

Methods: Italian Association Pediatric Hematology Oncology (AIEOP) multicentric analysis of a cohort of allogeneic HSCT pediatric patients who received ISA as IFI treatment or prophylaxis. Due to the lack of recommended dosing in pediatric patients and a clear target ISA plasma trough-level range, the therapeutic monitoring (TDM) of ISA concentrations was applied by a validated liquid chromatography-tandem mass spectrometry (HLPC-MS/ MS) assay technique. ISA trough plasma concentrations (C0) and 3 hours after drug intake (C3h) were measured.

Results: A total of 16 allo-HSCT recipients were included, (M/F 12/4); median age: 13,1 years, range 5-19, median body-weight $48,7 \mathrm{Kg}$ (range 17-80). ISA was used as IFI treatment in 14 cases and as prophylaxis in 2 patients. Donors were haploidentical in 9 patients, matched-sibling in 4, allogenic-unrelated in 4 cases. According to EORTC criteria, IFI was proven in 3 patients (1 Penicilum, 1 Mucor, 1 Aspergillus fumigatus), probable in 5 and possible in 7 patients. Lungs were the main localization (12 cases), associated with CNS involvement in 4 cases and paranasal sinuses in 3; 1 patient had possible hepatic candidiasis. All patients, but one, received ISA as rescue treatment for previous therapeutic failure (6 AmBisome, 3 Voriconazole, 1 combination therapy, 1 Posaconazole). Seven patients received only IV ISA, 3 received only oral ISA whereas 6 patients received both IV and oral ISA. Patients under 20 $\mathrm{Kg}$ body weight received half ISA dose (100 mg TID loading dose on days 1 and 2, $100 \mathrm{mg} /$ die manteinance). The others received adult schedule; only 5 patients received loading dose. ISA was administered for a median of 83 days (range: 24-240). In 3 patients ISA was administered in combination with Caspofungin. TDM was applied to 5 patients including 1 patient with severe VOD and 1 with renal failure secondary to TA-TMA. The median ISA concentrations were 3.94 (1.57-5.85) $\mathrm{mg} / \mathrm{L}$ and 4.7 (1.298.37) $\mathrm{mg} / \mathrm{L}$ for $\mathrm{C} 0$ and $\mathrm{C} 3 \mathrm{~h}$, respectively. IFI complete remission was achieved in 5 cases, partial remission in 3 ; treatment failure was experienced by 2 patients. In 3 cases fungal lesions remained stable. CTAE grade II-III toxicity was observed during treatment in 3 patients, with increased transaminase and/or creatinine levels which resolved after temporary ISA withdrawal. No drug-drug interactions were observed in 3 patients receiving CSA as GvHD prophylaxis and no modification of CSA daily dose was needed.

Conclusions: Isavuconazole use may be considered in the pediatric population, even in the HSCT setting, for its safety, efficacy, tolerability, no drug-drug interaction. Of course these data deserve further evaluation.

Disclosure: Nothing to declare

\section{P608}

New treosulfan-based conditioning regimens including epigenetic agents in patients with very high-risk neuroblastoma

Igor Dolgopolov ${ }^{1}$, Marina Rubanskaya ${ }^{1}$, Vasily Boyarshinov, ${ }^{1}$, Natalia Subbotina ${ }^{1}$, Vida Dajlidite ${ }^{1}$, Maria Tukhvatulina ${ }^{1}$, Georgy Mentkevich ${ }^{1}$

${ }^{1}$ Pediatric Hematology and Oncology, Moscow, Russian Federation

Background: The pts aged 18 mos. or older with disseminated NB involving bone and bone marrow constitute a group of pts with very poor prognosis. Although the majority of them are responsive to intensive conventional chemotherapy, most eventually relapse with EFS at 5 years of $<10 \%$. At the beginning of the year 2018 we came up with a protocol for this very unfavorable group including epigenetic therapy (5-azacitidine) in the phases of consolidation.

Methods: Seven pts with a median age of $4(2,6-8)$ years completed the protocol and received HDCT with autologous SCT as a consolidation. HDCT included two different epigenetic agent containing regimes according to tumor response to the induction therapy assessed by ${ }^{123} \mathrm{I}$ MIBG and MRI (CT-scan). Three pts revealing large active residual tumor assessed by ${ }^{123} \mathrm{I}-\mathrm{MIBG}$ scan or multiple active bone metastases received a conditioning regimen (regimen A) including ${ }^{131}$ I-MIBG therapy at a dose 4.0-4.4 MBe/kg on d-14, Treosulfan $10000 \mathrm{mg} / \mathrm{m}^{2} / \mathrm{d}$, on d- $6,-5$ and -4 (total $30000 \mathrm{mg} / \mathrm{m}^{2}$ ), Melphalan $70 \mathrm{mg} / \mathrm{m}^{2} / \mathrm{d}$, on d-3,-2 (total $140 \mathrm{mg} / \mathrm{m}^{2}$ ), 5 -azacitidine $75 \mathrm{mg} / \mathrm{m}^{2} / \mathrm{d}$ on d-9 to $\mathrm{d}-5$ (total $375 \mathrm{mg} / \mathrm{m}^{2}$ ). Four pts with CR or VGPR received a «split» conditioning regimen (regimen B) including Treosulfan $10000 \mathrm{mg} / \mathrm{m}^{2} / \mathrm{d}$, on $\mathrm{d} 11$ and -10 , and on d-4 and d-3 (total $40000 \mathrm{mg} / \mathrm{m}^{2}$ ), Melphalan $70 \mathrm{mg} / \mathrm{m}^{2} / \mathrm{d}$, on $\mathrm{d}-3$ and -2 
(total $140 \mathrm{mg} / \mathrm{m}^{2}$ ), and 5 -azacitidine $75 \mathrm{mg} / \mathrm{m}^{2} / \mathrm{d}$, on $\mathrm{d}-14$ to $\mathrm{d}-12$ and on $\mathrm{d}-9$ to $-\mathrm{d}-5$ (total $600 \mathrm{mg} / \mathrm{m}^{2}$ ). A median number of 5.3 (3.7-7) CD34+/kg was infused on $\mathrm{d} 0$.

Results: The median recovery times to $\mathrm{WBC}>1.0 \times 10^{9} / 1$ and to an unsupported $\mathrm{Plt}>20 \times 10^{11} / 1$ were 10 (7-12) and 13 (9-17) days, respectively. All pts experienced grade 4 hematological as well as infectious toxicity assessed by NCI-CTC score. There were 7 episodes of severe organ toxicity of grade 3 occurring in 4 pts. In 4 cases we observed a severe mucositis, in 2 cases GI toxicity and 1 episode of the erythema multiforme occurred. One pt revealed a lifethreatening episode of hypotension of grade 4. No transplant-related death occurred. The median number of transfused RBC and Plt doses was 3 (1-4) and 6 (3-8), respectively. All pts are alive and well without signs of disease progression in complete hematological recovery with a limited follow-up of 5.3 (4- 7) mos. from day 0 of HDCT.

Conclusions: Although it is rather early to evaluate the efficacy of the epigenetic agent's inclusion in the induction and/or consolidation phases of a very high-risk NB treatment, we can assume that, first, the HDCT combining MIBG ${ }^{131}$ and/or high dose of treosulfan with epigenetic agent such a 5-azacitidine was feasible and had an acceptable toxicity. Second, the "split" modality of the treosulfan use in conditioning regimen would permit to increase the total dose of the alkylating agent with no inacceptable toxicity.

Disclosure: Nothing to declare

\section{P609}

Pre-and-post magnetic resonance imaging of hips and knees for detecting osteonecrosis in children undergoing hematopoietic cell transplantation: In whom is it necessary?

\section{Ali Y Suliman ${ }^{1}$, Sue C Kaste ${ }^{2}$, Ying Li ${ }^{1}$, Dinesh Keerthi, Guolian Kang ${ }^{1}$, Brandon M Triplett', Ashok Srinivasan ${ }^{1}$}

${ }^{1}$ St. Jude Children's Research Hospital, Memphis, TN, United States, ${ }^{2}$ St. Jude Children's Research Hospital, Memphis, TN, United States

Background: Osteonecrosis (ON) is a debilitating complication in survivors of allogeneic hematopoietic cell transplantation (HCT). Limited data is available on its course post-transplantation in children. The purpose of our study was to identify recipients of HCT in whom pre-and post-magnetic resonance imaging (MRI) is indicated.

Methods: The retrospective cohort consisted of 436 patients who underwent first allogeneic HCT from 19982014, and prospectively underwent a total of 1092 pre-and post-transplant MRI studies of the hips and knees done annually for 3 years regardless of symptoms. Surviving patients were followed for a median time of 8.33 (range 3.93-14.10) years. Cases of ON were compared to controls matched for age, sex, transplant type, and follow up in a 1:4 ratio for the following variables: ethnicity, underlying disease, ON pre-HCT, conditioning regimen, graft source, bone mineral density Z-scores, body mass index, presence or absence of graft-versus-host disease, steroid use and dosage, and survival status.

Results: Thirty (6.9\%) patients had MRI findings confirming ON post-HCT. All patients with ON except one were more than 10 years of age. Twenty $(67 \%)$ patients were male. ON pre-HCT $(\mathrm{P}<0.0001)$ was the only factor associated with presence of ON post-HCT. Epiphyseal ON was seen in $9(30 \%)$ patients pre-HCT, and $26(87 \%)$ postHCT. Eighteen $(60 \%)$ patients had involvement of more than $30 \%$ of articular surface, and were more likely to undergo surgery $(\mathrm{P}=0.009)$.

Conclusions: The incidence of $\mathrm{ON}$ in this large pediatric cohort was 7\%. The only risk factor for ON post-HCT was pre-existing ON. MRI evaluation for ON pre-HCT is indicated in all patients. MRI evaluation for ON post-HCT is only indicated for patients with ON pre-HCT and symptomatic patients. This will entail cost savings of USD 500,000 per 100 surviving allogeneic HCT patients per year. Patients with more than $30 \%$ involvement of the articular surface need close follow up.

Clinical Trial Registry: None

Disclosure: None

\section{P610}

Impact of rabbit anti thymocyte globulin exposure on immune reconstitution and outcome after stem cell transplantation in children

\section{Anne Kruchen ${ }^{1}$, Valentina Vogelsang ${ }^{1,2}$, Katharina Wustrau', Ingo Müller $^{1,2}$}

${ }^{1}$ University Medical Center Hamburg-Eppendorf, Clinic for Pediatric Hematology and Oncology Hamburg, Germany, ${ }^{2}$ Research Institute Children's Cancer Center Hamburg, Hamburg, Germany

Background: Rabbit anti thymocyte globulin (rATG) has been frequently used for many years as GvHD prophylaxis in pediatric stem cell transplantation. Precise dosing regimens are crucial but remain challenging due to several pharmacological characteristics in children.

Methods: rATG levels were measured in pediatric patients undergoing allogeneic stem cell transplantation after obtaining approval by the local IRB and informed consent by legal guardians. 32 pediatric patients who received either Thymoglobuline ${ }^{\mathrm{TM}}(\mathrm{n}=22)$ or Grafalon ${ }^{\mathrm{TM}}$ (ATG-F) $(n=10)$ as part of their conditioning regimen 
between April 2016 and August 2017 at the University Medical Center Hamburg-Eppendorf, Germany, were included. Total and active rATG plasma levels were analyzed by ELISA and flow cytometry, respectively. Primary endpoint of the study was exposure to rATG. Secondary endpoints included transplant-related mortality, incidence of acute and chronic GvHD, immune reconstitution, chimerism, rejection and viral infections. Patients were monitored at least 100 days post transplantation. Statistical analyses were performed using IBM SPSS Statistics 24 software, or GraphPad Prism 5 software.

Results: Median total Grafalon ${ }^{\mathrm{TM}}$ and Thymoglobuline ${ }^{\mathrm{TM}}$ peak plasma levels were $419.0 \mu \mathrm{g} / \mathrm{ml}$ and $60.4 \mu \mathrm{g} / \mathrm{ml}$, respectively; median active Grafalon ${ }^{\mathrm{TM}}$ and Thymoglobuline ${ }^{\mathrm{TM}}$ peak plasma levels (aPPL) were $77.9 \mu \mathrm{g} / \mathrm{ml}$ and $8.11 \mu \mathrm{g} / \mathrm{ml}$, respectively. Active Thymoglobuline ${ }^{\mathrm{TM}}$ plasma levels showed highly variable pharmacokinetics compared to Grafalon ${ }^{\mathrm{TM}}$. Neither Grafalon ${ }^{\mathrm{TM}}$ nor Thymoglobuline ${ }^{\mathrm{TM}}$ exposure correlated with lymphocyte count prior to transplantation, cell count in the graft (WBC, MNC, $\mathrm{T}$ cells), age, body weight or body surface area (BSA). This is indicative for a saturation effect in both groups. To correlate high or low rATG exposure with clinical outcome parameters, we built two groups within each patient cohort by median aPPL. The incidence of GvHD was not dependent on high or low rATG exposure. Until day 100 post HSCT, 49 viral infections or reactivations (EBV, CMV, ADV, HHV6, BKV) occurred in the 32 patients. Interestingly, ADV infections affected only children with high rATG exposure. The median time to leukocyte engraftment was not significantly longer in the high rATG groups compared to the low rATG groups (17 to 16 days for Grafalon $^{\mathrm{TM}}$, and 14 to 16 days for Thymoglobuline ${ }^{\mathrm{TM}}$ ). There was a decreased and/or delayed recovery of $\mathrm{CD}^{+}$, $\mathrm{CD} 4^{+}$and $\mathrm{CD} 8^{+} \mathrm{T}$ cell reconstitution, but not of $\mathrm{B}$ cells and NK cells in the high Thymoglobuline ${ }^{\mathrm{TM}}$ exposure group compared to the low Thymoglobuline ${ }^{\mathrm{TM}}$ exposure group. Overall survival was not statistically significant with $80 \%$ in the Grafalon ${ }^{\mathrm{TM}}$ and $95.5 \%$ in the Thymoglobuline ${ }^{\mathrm{TM}}$ group without influence of rATG exposure.

Conclusions: High and low exposure to Grafalon ${ }^{\mathrm{TM}}$ or Thymoglobuline ${ }^{\mathrm{TM}}$ did not result in significant differences in outcome parameters as incidence of survival, aGvHD, cGvHD, rejection, or mixed chimerism in this limited cohort. Delayed and decreased immune reconstitution in the high rATG exposure groups did not translate into different clinical outcome parameters. ADV infections only occurred in the high rATG exposure group. Grafalon ${ }^{\mathrm{TM}}$ and Thymoglobuline ${ }^{\mathrm{TM}}$ showed distinct pharmacological and immunological differences in children and larger cohorts are needed to detect clinically significant differences and adjust dosing regimens individually.

Disclosure: Nothing to declare

\section{P611}

Melphalan-based reduced intensity conditioning results in improved outcomes in children undergoing allogeneic hematopoietic cell transplantation for myeloid malignancies compared to myeloablative busulfan-based conditioning

\section{Benjamin Oshrine ${ }^{1}$, Lauren Adams ${ }^{2}$, David Shyr ${ }^{3}$, Gregory Hale ${ }^{1}$, Aleksandra Petrovic ${ }^{4}$}

${ }^{1}$ Johns Hopkins All Children's Hospital, Saint Petersburg, FL, United States, ${ }^{2}$ Johns Hopkins All Children's Hospital, Cancer and Blood Disorders Institute, Saint Petersburg, FL, United States, ${ }^{3}$ University of Utah, Salt Lake City, UT, United States, ${ }^{4}$ Seattle Children's Research Institute, Seattle, WA, United States

Background: The optimal conditioning regimen for allogeneic hematopoietic cell transplantation (alloHCT) in children with myeloid malignancies remains undefined, particularly when reduced-intensity conditioning (RIC) regimens are utilized.

Methods: We performed a retrospective review of children undergoing alloHCT for acute myeloid leukemia (AML) and myelodysplasia-related AML (MDR-AML) over a 10 year period (2008-2018) at our institution, comparing the outcomes of those who received either a busulfan (Bu)- or melphalan/thiotepa (Mel/Thio)-based conditioning regimen.

Results: A total of 49 patients were analyzed. Twentyone received fludarabine/melphalan/thiotepa and 28 received myeloablative busulfan-based conditioning, either in combination with cyclophosphamide $(\mathrm{N}=19)$ or fludarabine $(\mathrm{N}=9)$. ATG was used in 27 patients depending on donor. Recipients of Mel/Thio were selected for RIC regimens due to pre-transplant comorbidities (cardiac dysfunction, $\mathrm{N}=9,3$ requiring peri-transplant milrinone), transplant during chemotherapy-induced aplasia $(\mathrm{N}=5)$, underlying diagnosis of treatment-related AML (t-AML) and significant pre-alloHCT chemotherapy exposure $(\mathrm{N}=5)$. Proportions of patients with de novo AML (Mel/ Thio, 57\%; Bu, 78\%) and MDR-AML (19\% and 21\%) were similar between groups; however, recipients of Mel/Thio were more likely to have t-AML ( $24 \%$ vs $0 \%)$. Cytogenetic and molecular risk factors were similar between groups. The majority of patients were transplanted in CR1 (Mel/Thio, $71 \%$ vs Bu, $68 \%$ ) or CR2 (24\% vs $38 \%$ ). More recipients of Bu conditioning ( $71 \%$ vs $43 \%$ ) were MRD-negative at the time of alloHCT; both groups had comparable proportions of patients with $\geq \mathrm{M} 2$ marrow $(\sim 10 \%)$. Donor types and stem cell sources were similar between groups, except unrelated umbilical cord blood which was more common in $\mathrm{Bu}$ recipients (21\% vs $10 \%)$. There were no graft failures in 
$\mathrm{Mel} / \mathrm{Thio}$ recipients, compared to $14 \%(\mathrm{~N}=4)$ in those receiving Bu-based regimens. Engraftment kinetics and immune reconstitution were similar. Overall acute and chronic GVHD incidence was higher in recipients of Mel/ Thio compared to $\mathrm{Bu}(39 \%$ vs $21 \%$, and $29 \%$ vs $18 \%$, respectively), but rates of grade III-IV acute or extensive chronic GVHD were comparable. VOD requiring treatment was diagnosed in $6(21 \%)$ recipients of Bu conditioning and no Mel/Thio recipients. Median duration of follow-up was 30 months (range 2-70) in the Mel/Thio group, and 18 months (range 1-121) in the $\mathrm{Bu}$ group. Transplantrelated mortality (TRM) was similar in both groups (1 patient), occurring before day 100 . Relapse incidence was comparable (Mel/Thio, 29\% vs Bu, 32\%); however, relapse occurred at a later time in Mel/Thio recipients (median $\mathrm{d}$ +396 vs $\mathrm{d}+137$ ). Overall survival at 1 and 3 years was superior in Mel/Thio recipients (95\% vs $74 \%$, and $75 \%$ vs $50 \%$, respectively).

Conclusions: In our single institution experience, use of a melphalan/thiotepa-based RIC regimen was associated with similar outcomes compared to full-intensity Bu-based conditioning, despite higher risk patient and disease characteristics. The majority of recipients of Mel/Thio conditioning had significant pre-transplant comorbidities, which did not translate into higher TRM. While Mel/Thio recipients had less optimal leukemia control at the time of transplant and high-risk leukemia features (e.g. t-AML), relapse was similar between groups, occurring later in Mel/ Thio recipients, which may have contributed to better overall survival.

Disclosure: Nothing to disclose

\section{P612}

Pubertal outcomes of children transplanted with allogeneic stem cells after myeloablative TBI or busulfan: influence of age, sex, role of chronic GVHD in puberty onset

Sara Weinhard ${ }^{1}$, Arnaud Wiedemann ${ }^{1}$, Camille Cordero ${ }^{2}$, Marie Detrait ${ }^{I}$, Marie-Thérèse Rubio ${ }^{\prime}$, Maud D'AvenyPiney $^{I}$, Audrey Contet ${ }^{I}$, Julie Valduga ${ }^{1}$, Claudine Schmitt $^{1}$, Pascal Chastagner ${ }^{1}$, Bruno Leheup ${ }^{1}$, Carole Legagneur ${ }^{I}$, Cyril Schweitzer ${ }^{1}$, Jean-Hugues Dalle ${ }^{3}$, Béatrice Lebon-Labich ${ }^{1}$, Cécile Pochon ${ }^{I}$

${ }^{1}$ Nantes University Hospital, Nantes, France, ${ }^{2}$ Institut Curie, Paris, France, ${ }^{3}$ Robert Debrè Hospital, Paris, France

Background: Myeloablative conditioning before allogeneic hematopoietic stem cell transplantation (HSCT) during childhood exposes to serious long-term complications, especially gonadal dysfunction. Pubertal issues are less described than other post-HSCT sequelae in childhood.
Methods: Pubertal development and biological gonadal parameters were assessed in a retrospective monocentric cohort of pre-pubertal patients who underwent HSCT after myeloablative conditioning with Total Body Irradiation (TBI) or Busulfan between 1981 and 2017.

Results: Seventy-four patients (28 girls and 46 boys) were included. No spontaneous pubertal development was found in $50 \%$ of girls and $10 \%$ of boys $(\mathrm{p}<0.001)$ and delayed puberty or no spontaneous pubertal development was found in $57 \%$ of girls and $24 \%$ of boys $(\mathrm{p}=0.009)$. Hormone replacement therapy was used in $82 \%$ of girls and $24 \%$ of boys $(\mathrm{p}<0.001)$. In univariate analysis, TBI conditioning $(\mathrm{p}=0.05)$, female sex $(\mathrm{p}<0.001)$, acute GVHD $(\mathrm{p}=0.05)$, extensive chronic GVHD $(\mathrm{p}=0.021)$, steroid treatment $>6$ months $(\mathrm{p}=0.016)$, and malignant diseases $(p=0.016)$ were associated with no spontaneous pubertal development, whereas TBI conditioning $(\mathrm{p}=0.003)$ and extensive chronic GVHD $(\mathrm{p}=0.005)$ were associated with delayed puberty. In multivariate analysis, factors independently associated with no spontaneous puberty onset were female sex $(\mathrm{p}=0.001)$ and age $>10$ years $(\mathrm{p}=0.033)$. Factors independently associated with delayed puberty were extensive chronic GVHD $(\mathrm{p}=0.041)$ and age $>10$ years $(\mathrm{p}=0.031)$. TBI was not an independent risk factor for pubertal complications.

Conclusions: This study confirms the toxicity of myeloablative conditioning on pubertal development and the role of older age and female sex in increased pubertal issues, and suggests a possible role of GVHD in delayed puberty.

Disclosure: Nothing to declare

\section{P613}

Abstract already published.

\section{P614}

Neutrophil elastase activity may serve as a marker for neutrophil extracellular traps formation following stem cell transplantation

\section{Ronit Elhasid, Sivan Berger-Achituv ${ }^{1}$, Hila Rosenfeld- Keidar', Szilvia Baron ${ }^{1}$ \\ ${ }^{1}$ Tel Aviv Sourasky Medical Center - Tel Aviv University, Tel Aviv, Israel}

Background: Post-transplant Infections rise dramatically in patients with quantitative or qualitative neutrophil defects and constitute a major source of morbidity and mortality following hematopoietic stem cell transplantation (HSCT). Neutrophils protect the host from microorganisms via multiple processes including phagocytosis and formation of neutrophil extracellular traps (NETs). Although reactive 
oxygen species (ROS) production seems to be essential for NETs formation, the key enzymes of the process are neutrophil elastase (NE) and myeloperoxidase (MPO).

Methods: NE and MPO activity as well as NETs formation were investigated following HSCT in 11 patients at week 1 to 6 and 30 after neutrophil engraftment. Neutrophils were isolated using EasySep Direct Human Neutrophil isolation kit (StemCell Technologies Inc.) by immunomagnetic negative selection. Enzymatic activity of NE and MPO were measured using colorimetric assays. NETs formation of phorbol 12-myristate 13-acetate (PMA)activated neutrophils was investigated by confocal fluorescence microscopy. All results were compared to those of healthy volunteers. Statistical significance was calculated using one way-ANOVA with Bonferroni post hoc test.

Results: 11 patients (median age of 6.8 years [range 2-22 years]) were investigated, 6 following allogeneic HSCT (2 acute lymphoblastic leukemia, 1 acute myeloblastic leukemia, 2 epidermolysis bullosa, 1 rhabdomyosarcoma) and 5 following autologous HSCT (4 Ewing sarcoma, 1 desmoplastic small round cell tumor). All patients experienced fever and neutropenia. At engraftment, average NE activity was significantly decreased compared to the average value of 50 healthy individuals. NE activity improved week by week in patients, reached the lower reference range at 4 weeks following transplantation (Fig. 1A) and continued to increase. The enzymatic activity of MPO was comparable to the average value of 50 healthy individuals (Fig. 1B) and showed no significant difference between the distinct time points. At neutrophil engraftment, NETs formation was absent and comparable to those of non-activated neutrophils (Fig. 1C). Although NETs formation increased week by week, it did not reach the average of 7 normal controls during the monitored time period. Also linear correlation between NE activity and NETs formation $\left(\mathrm{R}^{2}=0.978\right)$ was demonstrated.

Conclusions: Impaired NE activity following HSCT corresponds to decreased NETs formation and could serve as a marker for NETosis. Strategies to accelerate the recovery of NE function post transplantation might improve NETs formation and thereby induce better infection control. A) The average of $\mathrm{NE}$ activity $(\mathrm{N}=11)$ during 30 weeks following HSCT. Reference range was measured and calculated from measurements of 50 healthy volunteers using. B) The average of MPO activity $(\mathrm{N}=11)$ during 30 weeks following HSCT. Reference ranges were measured and calculated from measurements of 50 healthy volunteers using the quartile method. C) The average of NETosis activity after $100 \mathrm{nM}$ PMA activation for $3 \mathrm{~h}$ $(\mathrm{N}=11)$. Dashed line represents average NETosis of 7 healthy volunteers in the same condition.

Clinical Trial Registry: NA

Disclosure: Nothing to declare

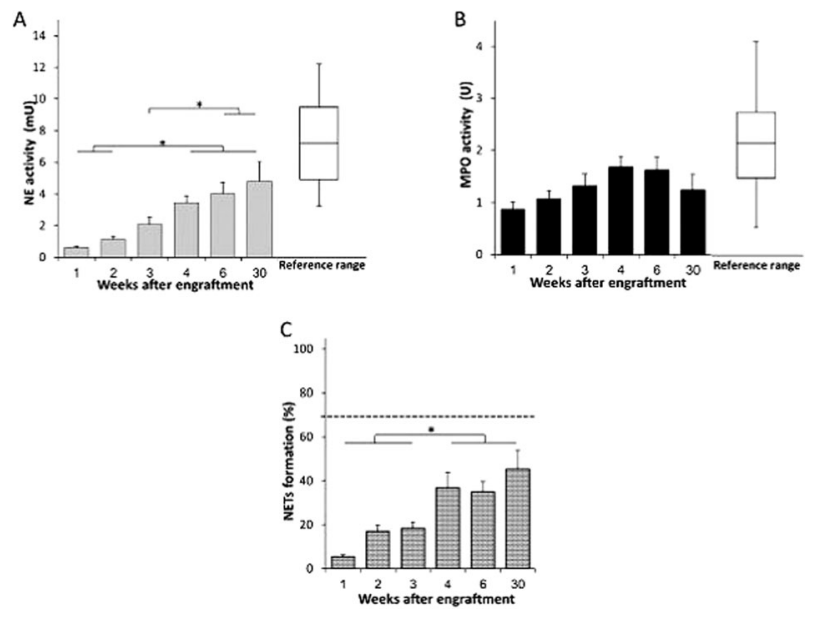

[[P614 Image] 1. Figure 1. NETs formation, meutrophil elastase and myeloperoxidase activity of pediatric patients following HSCT.]

\section{P615}

Abstract already published.

P616

Immune cytopenias after allogeneic hematopoietic stem cell transplantation in children

Victoria Bordon ${ }^{1}$, José Coene ${ }^{2}$, Sophie Van Lancker ${ }^{1}$, Catharina Dhooge ${ }^{I}$

${ }^{1}$ Ghent University Hospital, Ghent, Belgium, ${ }^{2}$ Belgian Red Cross-Flanders, Ghent, Belgium

Background: To have a better understanding of incidence, treatment, outcome and risk factors of immune cytopenia in children after allogeneic HSCT.

Methods: Between January 2010 and September 2018, 105 pediatric allogeneic HSCT have been performed in 99 patients at the Ghent University Hospital (Ghent, Belgium).

Autoimmune hemolytic anemia was defined by a positive direct agglutinin test (DAT).

DAT was performed at moment of engraftment and in case of hemolysis or unexplained anemia.

Platelets antibodies were evaluated in case of no otherwise explained thrombocytopenia.

Results: The cumulative incidence of post allo SCT autoimmune cytopenia is $9.5 \%$ (10/105). In 9 cases there were positive antibodies against red blood cells, and one patient against had antibodies against platelets. Of these 10 cases, only $4(3.8 \%)$ were clinically relevant and needed treatment. The median observation period post SCT for the whole cohort was 36 months $(3-105)$. 
The clinically significant immune cytopenia started at a median time of day +158 and day +113 in the group without symptoms.

The patient who presented the autoimmune thrombopenia developed antibodies against anti-gpIIb/IIIa, this was resolved after 130 days, the treatment consisted intravenous immunoglobulins (Ivig).

Two of the 3 patients with autoimmune hemolytic anemia had IgG mediated antibodies, and 1 had complementmediated DAT. These 3 patients were treated with Ivig, steroids, Rapamune and rituximab. One patient has still DAT positive after 36 months, but clinical stable. The other two are also DAT positive and have some hemolysis, but the follow up is much shorter ( 2 months).

Treosulfan- contained conditioning regimens were more frequently used in patients with significant immune cytopenia.

Conclusions: Immune cytopenia is an infrequent complication after allogeneic HSCT. However, its treatment can be challenging, and the hemolysis can persist for years. The association of Rapamune and rituximab was adequate to treat this problem in our patients. Treosulfan-containing regimes should be monitored for this complication.

Clinical Trial Registry: N/A

Disclosure: No conflict of interest

\section{P617}

Brentuximab vedotin prior to hematopoetic stem cell transplantation in children and adolescents with hodgkin's lymphoma

Andrey Kozlov', Tatiana Iukhta ${ }^{1}$, Asmik Gevorgian ${ }^{1}$, Ilya Kazantzev ${ }^{I}$, Polina Tolkunova ${ }^{I}$, Liubov Tsvetkova ${ }^{I}$, Elena Morozova $^{1}$, Kirill Lepik ${ }^{1}$, Natalia Mikhailova ${ }^{1}$, Ludmila Zubarovskaya ${ }^{1}$, Boris Afanasyev ${ }^{1}$

${ }^{1}$ R.M. Gorbacheva Memorial Institute for Pediatric Oncology, Hematology and Transplantation, I.P.Pavlov Saint Petersburg First State Medical University, Saint Petersburg, Russian Federation

Background: Approaches to the management of refractory and relapsed classical Hodgkin's lymphoma (r-r cHL) are changing and become more effective. The role of anti-CD30 targeted immunochemotherapy with Brentuximab vedotin (BV) has been extensively investigated in adults with r-r cHL and is only to be elucidated in children.

Methods: The study included 14 children and adolescents with r-r cHL that were sucessfully treated with BV-based therapy prior to hematopoetic stem cell transplantation (HSCT). Median age of patients was 16 years (9-19), main histological variant - nodular sclerosis $(93 \%, \mathrm{n}=13)$, advanced stage at diagnosis - $86 \%(n=12)$. Most were heavily pre-treated (median number of previous therapies 4 ) and progression after autologous HSCT was documented in $4(29 \%)$. Refractory disease was diagnosed in $8(57 \%)$ and relapsed in $6(43 \%)$. Among relapsed patients $4(67 \%)$ were with multiple episodes, $1(16.5 \%)$ - early and 1 (16.5\%) -late relapse. Treatment regimens consisted of BV in monotherapy $1.8 \mathrm{mg} / \mathrm{kg}$ triweekly $(\mathrm{n}=7)$ or combination of BV $1.8 \mathrm{mg} / \mathrm{kg}$ on Day 1 with Bendamustine $90-120 \mathrm{mg} /$ $\mathrm{m} 2$ on Days 1 and 2 of 3 -week cycles $(n=5)$ or combination of BV $1.8 \mathrm{mg} / \mathrm{kg}$ on Day 1 with DHAP $(\mathrm{n}=2)$. Median number of BV infusions was 3.5 (2-7). All selected patients achieved complete $(n=5,36 \%)$ or partial remission $(n=9$, $64 \%$ ) prior to HSCT. Consolidation with autologous HSCT was performed in $9(64 \%)$ and with allogeneic HSCT - in 5 $(36 \%)$. Primary end points were overall (OS) and progression free survival (PFS). Response to BV was not assessed in the study as only responders to the BV-based treatment were included.

Results: With median follow-up of 497 days (105-1368) OS and PFS for all patients are $68 \%$ and $66 \%$, respectively. PFS after autologous HSCT and allogeneic HSCT are 70\% and $60 \%$, respectively $(\mathrm{p}=0.7)$ At present moment 10 (71\%) patients are alive and are in remission. Three patients died $(21 \%)$ : disease progression $(n=1)$, postransplant idiopathic pneumonia syndrome $(n=1)$ and posttransplant pneumonia $(n=1)$. BV was generally well tolerated with only mild polyneuropathy in 3 patients $(21 \%)$ as the main reversable documented adverse event.

Conclusions: In prognostically unfavourable heavily pretreated children and adolescents with r-r cHL achievement of response to BV-based therapy prior to HSCT is assosiated with promising rates of OS and PFS.

Disclosure: Nothing to declare

\section{P618}

Allogeneic transplant and disease lessons from MNGIE: The royal manchester children's hospital experience

\section{David Deambrosis', Denise Bonney ${ }^{I}$, Helen Campbell',} Simon Jones ${ }^{1}$, Jane Kinsella ${ }^{1}$, Robert Wynn ${ }^{1}$

${ }^{1}$ Manchester University NHS Foundation Trust, Manchester, United Kingdom

Background: Mitochondrial Neurogastrointestinal Encephalomyopathy (MNGIE) is a rare, autosomal recessive disorder of mitochondrial function with deficiency of Thymidine Phosphorylase enzyme leading to accumulation of harmful substrates, progressive mitochondrial impairment and a multisystem disorder including gastrointestinal failure, peripheral neuropathy and leukoencephalopathy. Untreated, MNGIE leads to death before the $4^{\text {th }}$ decade of life. Haematopoietic stem cell transplantation (HSCT) 
provides a treatment by restoring thymidine phosphorylase function and improving disease manifestations. Here we report the outcomes of 4 affected siblings who underwent transplantation using an unaffected sibling donor to highlight important experiences in the transplant of such a rare condition.

Methods: Four siblings of consanguineous Pakistani descent aged 16, 15, 13 and 5 years underwent myeloablative HSCT using fully HLA-matched (10/10) peripheral blood stem cells harvested in a single apheresis from an unaffected 9 year old sibling. The oldest sibling, a 16 year old male, first presented in 2016 having emigrated from Pakistan with a history of growth failure and abnormal movements. Biochemical, nerve conduction and imaging studies confirmed a diagnosis of MNGIE. Testing on three other siblings identified similar biochemical abnormalities, though the 2 youngest children had minimal clinical manifestations of the disease. Based on the progressive nature of the disease and the availability of a fully matched donor, a decision was made to pursue transplant for all affected siblings.

Results: Due to the severity of their disease, the 2 oldest siblings were transplanted first using a myeloablative conditioning regime of Fludarabine, Thiotepa and Treosulfan with Alemtuzumab. Neutrophil engraftment occurred on day +11 for both, with $100 \%$ donor chimerism achieved. There were no significant transplant related complications. The post-transplant course of the 15 year old sibling was complicated by a major stroke-like event characterised by dramatic imaging changes and requiring ventilation, though no cause was identified and the patient's neurologic deficits have since resolved. Gastrointestinal symptoms have persisted and both remain TPN dependent, though symptomatically have shown gradual improvement. Following the neurologic complications in their older sibling, the 2 younger siblings were conditioned with AUC-Targeted Busulfan and fludarabine plus Alemtuzumab. Neutrophil engraftment occurred on day +11 , with full donor chimerism achieved. Progression to enteral feeding has been much more rapid, with nutrition now fully enteral for both. There were no significant transplant related complications.

Conclusions: Stem Cell transplantation represents the only curative option for MNGIE. Due to its rarity and relative infancy as a condition, little is known of the expected course following transplant or the best approach to transplantation itself. Despite previous challenges with graft failure in MNGIE recipients, we were able to gain rapid and sustained donor engraftment using 2 different myeloablative conditioning regimes with minimal transplant-related morbidity and no mortality. In keeping with previous reports, resolution of established gastrointestinal symptoms has been slow, though the 2 siblings transplanted earlier in their disease course have shown more rapid improvement supporting the role of early recognition and access to transplant. It is essential moving forward that specialised transplantation centres collaborate so as to guide clinicians in the management of such a challenging condition.

Disclosure: There are no conflicts of interest to disclose.

\section{P619}

G6PC3 congenital neutropenia - biology of inflammatory colitis associated with GCSF use, and disease response to allogeneic transplant, a report of 4 cases

Jane Kinsella ${ }^{I}$, Denise Bonney ${ }^{1}$, Helen Campbell ${ }^{I}$, Robert Wynn $^{1}$, Peter Arkwright ${ }^{I}$, Stephen Hughes ${ }^{1}$, Anu Goenka ${ }^{1}$

${ }^{1}$ Royal Manchester Children's Hospital, Manchester, United Kingdom

Background: An autosomal recessive disease, Glucose-6phosphatase catalytic subunit 3 (G6PC3) deficiency is a relatively recently identified cause of chronic severe neutropenia. There can be a spectrum to the disease and patients may also present with non-haematological features including prominent chest veins, cardiac, endocrine or urogenital abnormalities. We describe in our patient cohort a response to GCSF but an inflammatory, incapacitating, biopsyproven colitis associated with that G-CSF response. We have transplanted 3 children with such colitis, and describe a similar colitis with intestinal failure in a fourth.

Methods: We investigate the biology of the neutropenic colitis, and demonstrate necrosis of the stimulated neutrophils. In vitro studies demonstrated that unstimulated neutrophils from patients with G6PC3D exhibited significantly increased production of IL8, reactive oxygen species (ROS) and neutrophil extracellular traps (NETs) alongside significantly higher expression of CD11b, CD66b and CD14. In contrast, neutrophils from patients with G6PC3D produced significantly less ROS, MMP-9, neutrophil elastase and NETs upon stimulation. Neutrophils from patients with G6PC3D also exhibited significantly accelerated apoptosis and secondary necrosis which was exaggerated upon stimulation with live Escherichia coli bacteria but could only be partially rescued with supplemental exogenous glucose.

Results: 3 patients have undergone HSCT for G6PC3 neutropenic enterocolitis (1 unrelated donor and 2 MSD) after Fludarabine treosulfan and thiotepa conditioning therapy. Alemtuzumab was given as as serotherapy. All 3 patients are alive and well, immune suppression has been discotniuned and there is no GVHD with normal organ function, and resolution of colitis. We describe a $4^{\text {th }}$ patient 
with no good donor who has continuing intestinal failure with G-CSF use.

Conclusions: We describe the aetiology of intestinal inflammation and failure with an extensive study of neutrophil biology in this metabolic neutropenia. We describe a novel indication for HSCT in this "G-CSFresponsive neutropenia".

Disclosure: Nothing to declare

\section{P620}

Does body mass index (BMI) pose a risk to outcome for pediatric non-infantile patients undergoing hematopoietic cell transplantation (HSCT)?

Mona Al-Saleh ${ }^{1}$, Khawar Siddiqui, Amal Al-Seraihy, Abdullah Al-Jefri', Ali Al-Ahmari', Hawazen Al-Saedi, Awatif Al-Anazi, Mouhab Ayas ${ }^{1}$, Ibrahim Al-Ghemlas ${ }^{1}$

${ }^{1}$ King Faisal Specialist Hospital \& Research Centre, Pediatric Hematology / Oncology, Riyadh, Saudi Arabia

Background: Obesity among children is a growing health problem. malnutrition or being over-weight can be of prognostic impact among children who need HSCT.

Scientific literature shows a lot of controversy in terms of effect of BMI at the time of infusion on the outcome of HSCT.

Methods: We reviewed data of patients who underwent HSCT at King Faisal Specialist Hospital \& Research Centre between 2010-2016 to correlate BMI with the outcome and complications of HSCT. Transplant naïve recipients with age at infusion between 2-14 years who received HSCT from matched related donor or autologous HSCT, were included in the dataset for analysis. A total of 423 patients' profiles were reviewed of whom $51.1 \%$ were boys. Median age at transplant was 7.5 years. Primary indication of disease was malignancy in $41.6 \%$ followed by hemoglobinopathies $26.7 \%$, bone marrow failures $17.3 \%$ and immune disorders $12.5 \%$. Solid tumors accounted for $29.5 \%$ among malignant disorders. Myeloablative conditioning was used in $97.2 \%$ transplants with $11.8 \%$ regimens containing total body irradiation. Majority of the patients $81.3 \%$ underwent allogeneic transplantation using BM as the source in $80.4 \%$ and PBSC in the remaining $19.6 \%$ cases. Donor was HLAIdentical Sibling in $89 \%$, parents in $10.2 \%$ and others in the remaining $0.9 \%$ patients. Median TNC Dose was $2.55 \mathrm{X}$ $10^{\wedge} 9$ and $\mathrm{CD} 34$ was $5.63 \times 10^{\wedge} 6$ per $\mathrm{Kg}$ of the body weight at the time of infusion. Age and gender specific BMI percentiles were obtained and classified according to the definition of centers for disease control and prevention (CDC); $24.8 \%$ (105) recipients were categorized as UnderWeight, $61.2 \%$ (259) Normal, 5.9\% (25) Over-Weight and remaining $8 \%$ (34) as Obese.
Results: Based on chimeric studies at Day-100, our engraftment rate was $98.3 \%$ (400) out of 407 evaluable cases. Median time to neutrophil recovery was 14-days from infusion and 26-days for platelets. No statistically significant difference was found for engraftment rate on D100 as it was $100 \%$ (25) among Over-Weight, $98.8 \%$ (247) in Normal, 97\% (97) in Under-Weight and 96.9\% (31) in the Obese (P-Value: 0.467). Median time to neutrophil recovery from the infusion date was 15-days in Over-weight patients and 14 in the remaining three groups (P-Value: 0.841). Acute Graft vs. Host Disease (aGvHD) of any grade at Day-100 was recorded in $19.9 \%$ (84). Any-grade aGvHD was more common in Over-Weight $24 \%$ (6), followed by Obese with $23.5 \%$ (8), 21\% (22) in Under-weight and $18.6 \%$ (48) in Normal BMI-category (P-Value: 0.770). Chronic GvHD was more frequent in Over-Weight (14.3\%, $3)$, compared to Under-Weight $(10.5 \%, 9)$ or Normal $(7.6 \%$, 17) as categorized based on BMI values (P-Value: 0.196). With a median follow-up time of $50.4 \pm 3.1$ months from infusion and 89 events of mortality, overall survival by BMI-Categories was $0.797 \pm 0.027$ for Normal, $0.735 \pm 0.094$ for Over-Weight, $0.727 \pm 0.047$ for Under-Weight and 0.753 \pm 0.076 for Obese groups (P-Value:0.739).

Conclusions: No significant association of BMICategorization with respect to transplant outcome was observed among patients at our institution.

Clinical Trial Registry: NA

Disclosure: None

\section{P621 Abstract withdrawn.}

\section{P622}

Single high dose oral vitamin D3 (Stoss therapy) in pediatric patients undergoing HSCT: A proposed solution to vitamin $\mathrm{D}$ deficiency during transplant

Jessica Bodea ${ }^{1}$, Kristen Beebe $e^{1}$, Kyrie Hospodar ${ }^{1}$, Jennifer Stahlecker ${ }^{1}$, Dana Salzberg ${ }^{1}$, Holly Miller ${ }^{1}$, Roberta Adams ${ }^{1}$, Alexander Ngwube ${ }^{I}$, Lucia Mirea ${ }^{2}$

${ }^{1}$ Phoenix Children's Hospital, Center for Cancer and Blood Disorders, Phoenix, AZ, United States, ${ }^{2}$ Phoenix Children's Hospital, Clinical Research Administration, Phoenix, AZ, United States

Background: Patients undergoing hematopoietic stem cell transplant [HSCT] are at increased risk for having suboptimal vitamin D [VD] levels and associated worse outcomes. While up to $70 \%$ of HSCT pediatric patients are VD insufficient, standard repletion and aggressive strategies have failed to attain repletion. Single high-dose oral VD (Stoss therapy) has been used in select populations of chronically ill children where levels are difficult to attain 
with no evidence of toxicity. As benefits of Stoss therapy in HSCT remain unknown, and safety has yet to have been studied extensively in the pediatric population, we hypothesize that Stoss therapy is an effective and safe method to reach and attain sufficient levels of VD in pediatric patients undergoing HSCT.

Methods: This is an ongoing prospective, randomized clinical control trial at Phoenix Children's Hospital that commenced December $1^{\text {st }}$, 2017. Following consent, subjects are randomized to the intervention (Stoss) or control arm prior to HSCT. Stoss therapy consists of a single oral dose of VD (ranging 100,000IU - 600,000IU), given based on baseline 25-hydroxyvitamin D [25(OH)D] level and age, followed by standard weekly supplementation. Subjects enrolled on the control arm receive standard of care based on Endocrine Society Guidelines of weekly VD supplementation. Data collection includes demographics, 25(OH)D levels at baseline, day +30 , and day +100 , VD toxicity (hyperphosphatemia, hyperkalemia and renal calculi), as well comorbidities were collected. At each time point and for each trial arm, the mean $25(\mathrm{OH}) \mathrm{D}$ level and changes from baseline were computed with corresponding $95 \%$ confidence intervals (CIs) to indicate variability.

Results: Presently, 12 subjects have completed baseline assessment, with Day +30 and Day +100 follow-up completed for 11 and 9 of these, respectively. At baseline, the mean $(95 \% \mathrm{CI}) 25(\mathrm{OH}) \mathrm{D}$ was $19.8 \mathrm{ng} / \mathrm{dl}(10.9,28.7)$ among Stoss patients and $18.9 \mathrm{ng} / \mathrm{dl}(10.3,27.4)$ among controls. The mean difference in $25(\mathrm{OH}) \mathrm{D}$ levels between Day +30 and baseline was $25.2 \mathrm{ng} / \mathrm{dl}(12.2,38.3)$ for Stoss, but lower $17.1 \mathrm{ng} / \mathrm{dl}(-1.2,35.5)$ in controls. Between Day +100 and baseline, the mean difference in $25(\mathrm{OH}) \mathrm{D}$ levels increased further among Stoss patients to $33.9 \mathrm{ng} / \mathrm{dl}(-4.5$, 72.4), while the change among controls was $17.4 \mathrm{ng} / \mathrm{dl}$ (1.7, 33.1 ), remaining close to that observed at Day +30 .

Conclusions: Preliminary results are consistent with continued higher increases in $25(\mathrm{OH}) \mathrm{D}$ levels for Stoss therapy at both Day +30 and Day +100 compared to controls. Formal statistical tests will compare changes in 25 $(\mathrm{OH}) \mathrm{D}$ levels between Stoss and controls after complete data collection for 50 subjects, expected by February, 2019.

Clinical Trial Registry: Clinical Trial Registry number NCT03176849

https://clinicaltrials.gov/ct2/show/NCT03176849

Disclosure: None

\section{P623}

Impact of bone marrow transplantation on healthrelated quality of life in children with severe thalassemia in a lower-middle income setting

Harshita Agarwal, Priya Marwah ${ }^{1}$, Rajpreet Soni, Seema Bhattacharyya ${ }^{1}$, Lawrence Faulkner ${ }^{2}$
${ }^{1}$ South East Asia Institute for Thalassemia, Jaipur, India, ${ }^{2}$ Cure2Children Foundation, Firenze, Italy

Background: Patients with Severe Thalassemia (ST), i.e. a thalassemia syndrome characterised by the inability to maintain a spontaneous haemoglobin level above $7 \mathrm{~g} / \mathrm{dL}$, often require regular blood transfusions at an early age which eventually may lead to iron overload and organ damage. Chronic supportive care and related financial burden, often have an adverse impact on patients and their families. Moreover, in India ST is still associated with significant morbidity and mortality.

Methods: The aim of this study was to assess healthrelated quality of life (HRQoL) in ST patients after BMT. Three groups of 30 patients each aged 6 to 12 years were analyzed: Group A consisted of healthy controls, i.e. siblings of children with severe thalassemia; Group B included children with ST on supportive care; and Group C children at least two years after successful BMT for ST. Data was collected using the Pediatric Quality of Life Inventory by direct and non-direct communication. HRQoL scores were compared by two-tailed Mann Whitney t-test.

Results: Total HRQoL scores of transplanted patients were significantly improved compared to those on supportive care and also compared to healthy siblings $(\mathrm{P}<0.0001$ and 0.0002 respectively), the same was true for physical ( $\mathrm{P}$ $<0.0001$ and 0.0003 respectively) and emotional functioning $(\mathrm{P}<0.0001$ and 0.0073 respectively). Social and school functioning of transplanted children were not different from healthy siblings ( $\mathrm{P} 0.5893$ and 0.7603 respectively) while were very significantly improved compared to children with ST on supportive care $(\mathrm{P}<0.0001$ in both cases).

Conclusions: BMT in a lower-middle income setting may be even more impactful compared to high-income regions. Our analysis clearly indicates normalization of HRQoL in all major areas of children transplanted for ST. A possible resilience effect was noted for physical and emotional scores which were improved compared to healthy sibling controls. We could not however quantify the effect of longer-term issues like fertility impairment after BMT which may eventually adversely impact HRQoL, particularly in the Indian culture.

Disclosure: None

\section{P624}

Allogeneic hematopoietic stem cell transplantation in ataxia telangiectasia patients without malignancy

Shahrzad Bakhtiar ${ }^{1}$, Jan Soerensen ${ }^{1}$, Andrea Jarisch ${ }^{1}$, Eva Rettinger ${ }^{1}$, Ralf Schubert ${ }^{1}$, Fabian H Hauck ${ }^{2}$, Stefan Zielen $^{1}$, Michael H Albert ${ }^{2}$, Peter Bader ${ }^{1}$ 
${ }^{1}$ Childrens Hospital Goethe University, Frankfurt, Germany, ${ }^{2}$ Dr. v. Hauner University Children's Hospital, Ludwig-Maximilians-Universität, Munich, Germany

Background: Ataxia telangiectasia (A-T) is a primary immunodeficiency with mutations in ATM-gene. Besides a slowly progressive neurodegenerative course, A-T leads to increased susceptibility to malignancies which affects $25 \%$ of patient (median:12.5 years) with a high mortality mainly due tocomplications of conventional radio-chemotherapy. The incidence of cancer correlates with the extent of immunodeficiency. Patients often develop severe progressive granulomatous skin disease with evidence of vaccine-strain rubella-virus in the lesions. Prolonged survival, neurologic improvement and malignancy prevention was observed in Atm-deficient mice after treatment by syngeneic HSCT. Nevertheless, pre-emptive HSCT is not routinely performed in A-T patients due to concerns about neurodegeneration and toxicity.

Methods: We present three A-T patients with severe immunodeficiency phenotype, undergoing successful HSCT as an individual treatment strategy intending to restore immunodeficiency for long-term malignancyprevention (patient-1) and to treat progressive skin/joint granulomas (Patients-2 and -3).

Results: Patient-1 underwent a reduced intensity conditioning (RIC) regimen at 4 years of age including fludarabine $\left(150 \mathrm{mg} / \mathrm{m}^{2}\right)$, cyclophosphamide $(80 \mathrm{mg} / \mathrm{kg})$, and ATG-Fresenius $(20 \mathrm{mg} / \mathrm{kg} / \mathrm{d})$ which was tolerated well. Hematopoietic engraftment occurred by day +15 . There was an expansion of naïve and memory $\mathrm{CD} 4^{+} \mathrm{T}$-cells and $\mathrm{CD} 19^{+}$cells. While initially a mixed donor chimerism in patient's PBMCs (10-20\% donor) was observed, patient's Tcells $\left(\mathrm{CD}^{+}\right)$reached over $90 \%$ of donor origin over time. At last follow-up (6 years) he is well, without signs of GVHD and organ toxicity, off immunosuppression with normal levels of ATM- protein; his granulomas resolved.

Patient-2 is a 6 year-old male who was transplanted from his HLA-identical sibling, conditioned with fludarabine $\left(150 \mathrm{mg} / \mathrm{m}^{2}\right)$, cyclophosphamide $(120 \mathrm{mg} / \mathrm{kg})$, and ATGFresenius $(20 \mathrm{mg} / \mathrm{kg})$. Hematopoietic engraftment was observed by day +10 . T-cell reconstitution started by day +40 with $>200 \mu \mathrm{l}$ CD3 + T-cells. His mixed chimerism rapidly turned to donor origin $(95 \%$ donor $\mathrm{CD} 3+)$ over time. There was no acute toxicity, however, he developed lumbosacral pain episodes with evidence of urine BK-virus with spontaneous remission. An intermittent metapneumovirus associated pulmonary hypertension was observed with pericardial effusion. Treatment included sildenafil and oxygen. At last follow-up (9 months) patient is well without immunosuppression.

Patient- 3 suffered from recurrent chest infections, failure to thrive and progressive and debilitating rubella positive progressive granulomas of the skin. She received alloHSCT from an HLA-identical family donor at 6 years of age. Conditioning included busulfan $(2.2 \mathrm{mg} / \mathrm{kg})$, fludarabine $\left(150 \mathrm{mg} / \mathrm{m}^{2}\right)$, cyclophosphamide $(40 \mathrm{mg} / \mathrm{kg})$, and alemtuzumab $\left(1 \times 5 \mathrm{mg} / \mathrm{m}^{2}, 3 \times 10 \mathrm{mg} / \mathrm{m}^{2}\right)$. HSCT was complicated by intermittent acute renal failure, CMV reactivation and TMA. Hematopoietic recovery was observed by day +22 . $\mathrm{T}$ - cell chimerism increased rapidly over time $(>90 \%$ donor). At last follow-up (7 months) patient is well, off immunosuppression and IVIG. Her skin granuloma resolved with scarring residues.

Conclusions: Pre-emptive alloHSCT is feasible in A-T when reduced intensity conditioning is used and can correct the immunodeficiency. It might be a treatment option for some A-T patients at high risk of hematological malignancy and severe granulomatous skin disease. To what extent the restored immune system and the increase of ATM-protein in these patients could prevent the development of other malignancies needs to be evaluated further.

Disclosure: Nothing to disclose

\section{P625 Abstract withdrawn.}

\section{P626}

\section{Hematopoietic stem cell transplantation in diamond} blackfan anemia: Brazilian experience

Luiz Darrigo Junior', Gisele Loth', Vergilio Colturato ${ }^{3}$, Adriana Seber ${ }^{4}$, Victor Zecchin ${ }^{5}$, Juliana Folloni', Lisandro Ribeiro $^{7}$, Simone Franco ${ }^{8}$, Ricardo Pasquine ${ }^{2}$, Carmen Bonfim ${ }^{2}$

${ }^{1}$ University Sao Paolo (USP) Ribeirão Preto, Ribeirão Preto, Brazil, ${ }^{2}$ University Federal Paraná (UFPR), Curitiba, Brazil, ${ }^{3}$ Hospital Amaral de Carvalho, Jau, Brazil, ${ }^{4}$ Hospital Samaritano, São Paulo, Brazil, ${ }^{5}$ GRAACC, São Paulo, Brazil, ${ }^{6}$ USP São Paulo, São Paulo, Brazil, ${ }^{7}$ Hospital Nossa Senhora das Graças (HNSG), Curitiba, Brazil, ${ }^{8}$ Hospital da Criança de Brasilia, Brasilia, Brazil

Background: Diamond-Blackfan anemia (DBA) is a rare inherited red cell aplasia caused by an intrinsic defect of erythropoietic progenitors. The main therapeutic approach is based on repeated red blood cell transfusions and/or corticosteroid therapy. Hematopoietic stem cell transplantation (HSCT), a potentially curative treatment for DBA, is indicated for patients that do not respond to first-line therapy.

Methods: The aim of our retrospective study is to report the outcomes of 30 Brazilian DBA patients transplanted between 1990 and 2017 in 9 BMT centers. The median age of the patients was 5 ys (range 1-15) and 60\% were male. Seventeen patients $(57 \%)$ were transplanted with matched related donors (MRD) and thirteen (43\%) from matched and 
mismatched unrelated donors (MUD/MMUD). In the MRD group all patients received bone marrow as HSC source, while in the MUD/MMUD, eight patients received bone marrow and five received cord blood. All patients with incompatibilities (mismatched) were UCB (5/5). Nineteen recipients were conditioned with busulfan plus cyclophosphamide, while the remaining 11 received fludarabine and busulfan, which has been the preferred regimen in Brazil in the recent years. After transplant, most $(n=24)$ of the MRD and MUD recipients received cyclosporine and short course methotrexate as graft versus host disease (GvHD) prophylaxis.

Results: Twenty-two out of the 30 patients were alive and disease-free at a median follow-up of 24 months (range 1 to 213 months). The 3-year overall survival (OS) was 71\% (CI $51-91 \%$ ) (Fig 1). Similar results have been demonstrated in studies from Europe and from the United States. When analyzed according to donor type, OS was 73\% (CI 55$100 \%$ ) and 66\% (CI 43-100\%) in MRD and MUD/MMUD respectively (fig 2). Three out of the 5 patients who were transplanted with UCB died. These results are in agreement with those of previously published data showing worse results in unrelated UCB transplants. Twenty-nine out of the 30 patients engrafted successfully. In 25 of the evaluated patients, the median time to neutrophil engraftment was 20 days (range 10-27). One patient experienced an early death from hemorrhagic shock on day 12 , before neutrophil recovery, and another two patients experienced primary graft failure. Post-transplant chimerism was available for 22 patients. Sixteen had complete chimera ( $>90 \%$ chimerism), while 6 patients presented with mixed chimerism. Acute GvHD was observed in 9 patients (32\%), 6 of which classified as grade IV. Five patients developed chronic GvHD, considered severe in three of them. Eight patients died at a mean of 155 days (range 12-728 days) after HSCT and the main causes of death were infections and hemorrhagic disorders.

Conclusions: HSCT is a potentially curative treatment option for DBA. In the present study, we report the outcomes of 30 patients with DBA transplanted in Brazil with a OS of $71 \%$, with better results in MRD compared to MUD, as expected. Despite the small numbers, we observed lower survival after MUD/MMUD UCB transplantation. Since DBA is a rare disease, international collaborative studies are essential to better understand the benefits of the HSCT in the treatment of these patients.

Disclosure: Nothing to declare

\section{P627}

Treatment of the obliterant bronchiolitis in pediatric allogeneic recipients: Two periods compared
Maura Faraci ${ }^{I}$, Stefano Giardino ${ }^{1}$, Francesca Bagnasco ${ }^{I}$, Donata Girosi ${ }^{1}$, Roberta Olcese ${ }^{I}$, Erica Ricci ${ }^{1}$, Edoardo Lanino $^{1}$

${ }^{1}$ Institute G Gaslini, Genova, Italy

Background: Onset of Obliterans Bronchiolitis (BO) as pulmonar manifestation of chronic graft-versus-host disease changes dramatically the prognosis of children underwent an allogeneic SCT.The aim of this study is to evaluate changing in overall survival (OS) in children affected by $\mathrm{BO}$ after the introduction in our Centre of a specific protocol for BO including imatinib (IMA), montelukast, azitromicin and inhalator budesonide; high-dose intravenous bolus methylprednisolone (bolus MPD, $10 \mathrm{mg} / \mathrm{Kg}$ ) monthly was reserved to severe and not responsive patients.

Methods: Onset of Obliterans Bronchiolitis (BO) as pulmonar manifestation of chronic graft-versus-host disease changes dramatically the prognosis of children underwent an allogeneic SCT.The aim of this study is to evaluate changing in overall survival (OS) in children affected by BO after the introduction in our Centre of a specific protocol for BO including imatinib (IMA), montelukast, azitromicin and inhalator budesonide; high-dose intravenous bolus methylprednisolone (bolus MPD, $10 \mathrm{mg} / \mathrm{Kg}$ ) monthly was reserved to severe and not responsive patients.

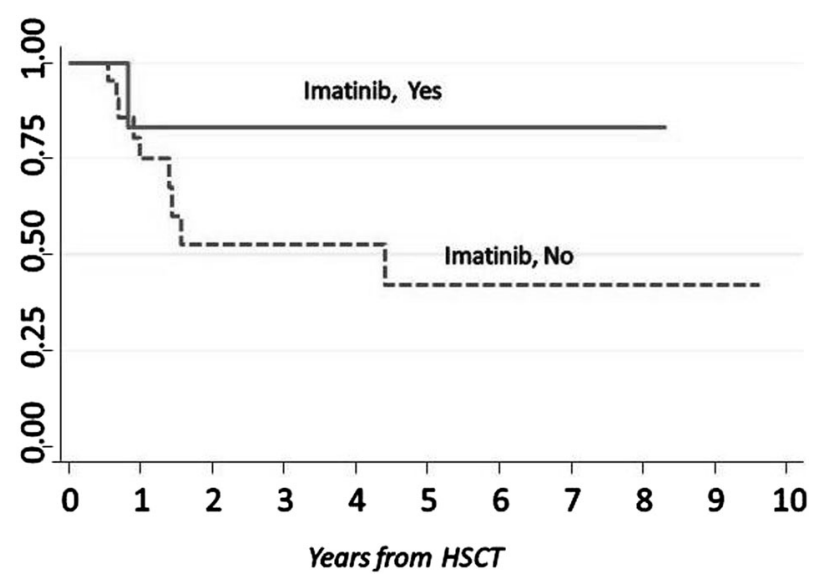

[[P627 Image] 1. Overall survival in the 2 groups]

\begin{tabular}{llll}
\hline Patients & $\begin{array}{l}\text { Median age } \\
\text { HSCT yrs } \\
\text { (ranges) }\end{array}$ & Donor Type & $\begin{array}{l}\text { Conditioning } \\
\text { regimen (CR) }\end{array}$ \\
\hline $\begin{array}{l}\text { Before } \\
2008=11\end{array}$ & $7.8(4.5-16)$ & $\begin{array}{l}\text { Alternative Donor=9 Related } \\
\text { Donor=2 }\end{array}$ & $\begin{array}{l}\text { Myeloablative }=9 \\
\text { Reduced } \\
\text { Intesity }=2\end{array}$ \\
$\begin{array}{l}\text { After } \\
2008=14\end{array}$ & $8.1(1.3-15.3)$ & $\begin{array}{l}\text { Alternative Donor=8 Related } \\
\text { Donor }=4 \text { Haploidentical } \\
\text { Donor=2 }\end{array}$ & $\begin{array}{l}\text { Myeloablative }=9 \\
\text { Reduced } \\
\text { Intensity=5 }\end{array}$ \\
\hline
\end{tabular}

[[P627 Table] 1. Characteristics of children with BO] 
Results: In group 1, the therapies administered for BO included prolonged treatment with steroids in all patients, anti-TNF in 1, azatioprine in 4 ; while in the group 2, all patients received IMA, montelukast and azitromicin, and 4 received i.v. MPD. The median duration of Imatinib therapy was 4 years (0.3-7.1 years). After a median follow-up of 4.4 years (range 0.5-12.1 yrs), 9/11 patients of group 1 (82\%) died with $\mathrm{BO}$ in progress for transplant-related causes. While in the group 2, 2/14 (14\%) died in presence of worsening BO. The estimated OS at 1 year after HSCT was $75 \%$ (95\% CI; 50-89) in group 1 and 83\% (95\% CI, 27-97) in group $2(\mathrm{p}=0.103)$ (Figure 1), while the OS after 4 year decreased at $42 \%(95 \% \mathrm{CI} ; 16-66)$ in the group 1 while remained stable in the group 2.

Conclusions: this experience shows a relevant improving in prognosis of children with $\mathrm{BO}$ with the use of this protocol including IMA, since the significant improving of survival obtained, confirming as reported in adult populations.

Disclosure: Nothing to declare

\section{P628}

\section{Abstract already published.}

\section{P629}

\section{Abstract already published.}

\section{P630}

A case series of patients with posterior reversible encephalopathy syndrome (PRES) following pediatric allogeneic bone marrow transplantation

\section{Ilgen Sasmaz, ${ }^{1,2}$, Bulent Antmen', Barbaros Karagun', Hakan Erkman', Murat Serbest', Duygu Türksoy'}

${ }^{1}$ Cukurova University, Pediatric Hematology, Adana, Turkey, ${ }^{2}$ Acibadem Adana Hospital, Adana, Turkey

Background: Posterior reversible encephalopathy syndrome (PRES) is a neuro-radiological syndrome characterized by headache, seizures, visual disturbance, and altered mental function associated with reversible white matter edema affecting the posterior parietal and occipital lobes of brain. PRES has been reported to occur in association with numerous co-morbidities, including hypertensive encephalopathy, pre-eclampsia, eclampsia, infections, electrolyte imbalance, immunosuppressive drugs, allogeneic bone marrow transplantation (BMT), solid organ transplantation, autoimmune diseases, and high-dose cancer chemotherapy. We report 20 patients who developed PRES during the treatments with Cyclosporine A (CsA), tacrolimus and metilprednisolone, utilized for graft versus host disease
(GVHD) prophylaxis and treatment after pediatric allogeneic BMT.

Methods: Between 2013 and 2018, 249 patients received an allogeneic BMT at Acibadem Adana Hospital Pediatric Bone Marrow Transplant Unit. Twenty cases of PRES were observed in this period. Medical records and magnetic resonance images (MRIs) were evaluated retrospectively.

Results: We presented 20 patients with PRES, age ranging from 2 months to 19 years with a average of 9.5 years. There were ten patients with thalassemia major, two patient with acute lymphoblastic leukemia, three patients with sickle cell disease and one patient with myelodysplastic syndrome, one patient with immune deficiency, two patients with acute miyeloid leukemia, one patient with aplastic anemia. Ten patients were males, ten were female. All patients were treated with CsA or tacrolimus and metilprednisolone for the prophylasix of GVHD. PRES occurred at a median of 90 days (range 5-625). Clinical findings at onset of leukoencephalopathy were hypertension, headache, seizures, visual disturbance, and altered mental function. Eighteen patients alive with normal neurological status. MRI showed abnormalities in all patients including patchy bilateral cortical and subcortical lesions, especially in parieto-occipital lobes.

Conclusions: BMT is associated with several neurological complications that may be underlying diseases, BMT procedure, and severe immunosupression. PRES is an uncommon but serious complication after BMT. We report 20 cases of PRES who received allogeneic BMT for thalassemia major to emphasize the importance of early recognition and institution of appropriate management of PRES during BMT.

Disclosure: Nothing to declare

\section{P631}

Continuous complete molecular remission using three different monoclonal antibodies followed by allogeneic bone marrow transplantation in an infant with chemotherapy-refractory acute lymphoblastic leukemia

\section{Bernd Gruhn ${ }^{1}$, Susan Wittig ${ }^{1}$, Thomas Ernst ${ }^{1}$, Jana Ernst $^{l}$}

${ }^{1}$ University of Jena, Jena, Germany,

Background: A 10-week-old infant was diagnosed with very immature acute lymphoblastic leukemia (ALL) with myeloid markers in a foreign university hospital. At the end of induction therapy according to the current LAL/SHOP protocol $10 \%$ leukemic cells were detectable in the bone marrow. Treatment was changed to fludarabine, cytarabine and granulocyte colony-stimulating factor (FLAG) in combination with liposomal doxorubicin. After this re- 
induction still 5\% leukemic cells were detected in bone marrow, so the bispecific T-cell engager antibody blinatumomab was given. Due to an increasing portion of leukemic cells during the continuous infusion, antibody therapy was stopped and a cycle of clofarabine, cyclophosphamide and etoposide was administered. Unfortunately, still $31 \%$ leukemic cells were detectable afterwards. Because of chemotherapy-refractory leukemia a palliative oral treatment with mercaptopurine was started. However, the parents did not accept the palliative situation and searched for alternative therapeutic options in other university hospitals in Europe. After plenty of refusals the infant was admitted to our hospital five months after diagnosis.

Methods: For molecular characterization genomic DNA was isolated from leukemic cells. A MLL-MLLT3/AF9 rearrangement as a consequence of the translocation $\mathrm{t}(9 ; 11)$ (p22;q23) was detected and used as a marker for minimal residual disease. For further molecular characterization targeted deep next-generation sequencing was performed for a panel of 54 leukemia-associated genes. Interestingly, no mutation was found. To allow precise immunophenotyping of the leukemic cells treatment with mercaptopurine was stopped.

Results: As in the first immunophenotyping the CD33 antigen was found, we administered the anti-CD33 monoclonal antibody gemtuzumab ozogamicin twice within two weeks. Because of the detection of CD38+ leukemic cells after infusion of gemtuzumab ozogamicin, the anti-CD38 antibody daratumumab was given alternating twice within two weeks. Unfortunately afterwards, leukemic cells reappeared being negative for $\mathrm{CD} 33$ und $\mathrm{CD} 38$, but positive for CD22. Therefore, we administered the third antibody, the anti-CD22 monoclonal antibody inotuzumab ozogamicin, whereupon our patient developed a tumor lysis syndrome and a severe bone marrow aplasia. Shortly after, allogeneic bone marrow transplantation from an unrelated donor using a special conditioning regimen consisting of thymoglobulin, busulfan, fludarabine and clofarabine was conducted. Clofarabine was added because an additional antileukemic effect especially in infant ALL with MLL rearrangement was described. After transplantation the patient suffered from a severe hepatic sinusoidal obstruction syndrome with massive ascites, renal and pulmonary dysfunction, but finally the patient recovered completely. The first bone marrow examination 30 days after transplantation revealed a donor chimerism of $100 \%$ and a complete molecular remission using the MLL-MLLT3/AF9 rearrangement as marker for minimal residual disease. In all follow-up bone marrow samples we observed a complete donor chimerism and a complete molecular remission. Currently, eight months after transplantation the patient is in a very good physical condition with normal development according to the age.
Conclusions: A combination of different monoclonal antibodies, in this case three, according to the immunophenotype of the leukemic cells can effectively eliminate leukemic cells in a chemotherapy-refractory leukemia and serve as bridging to transplant to induce a complete molecular remission afterwards even in infants.

Clinical Trial Registry: Not applicable.

Disclosure: Nothing to declare.

\section{P632}

Immunocytokines and FC-optimized antibodies targeting B7-H3 (CD276) in neuroblastoma

Florian Heubach $^{1}$, Patrick Schlegel ${ }^{1}$, Latifa Zekri', Timo Manz $^{2}$, Sabine Schleicher ${ }^{1}$, Armin Rabsteyn ${ }^{1}$, Gundram Jung $^{2}$, Hans-Jörg Bühring ${ }^{3}$, Stephen D. Gillies ${ }^{4}$, Rupert Handgretinger ${ }^{1}$, Peter Lang ${ }^{1}$

${ }^{1}$ University Children's Hospital, Pediatric Hematology and Oncology, Tuebingen, Germany, ${ }^{2}$ Eberhard Karls University Tuebingen, Interfaculty Institute for Cell Biology, Tuebingen, Germany, ${ }^{3}$ University Hospital Tuebingen, Tuebingen, Germany, ${ }^{4}$ Provenance Biopharmaceuticals, Carlisle, MA, United States

Background: B7-H3 (CD276) is thought to act as an immune checkpoint and regulates $\mathrm{T}$ and NK cell responses. It is highly overexpressed on many solid tumors while on healthy tissue protein expression is limited. This makes B7$\mathrm{H} 3$ an interesting target for cancer immunotherapy. In highrisk neuroblastoma patients, targeting disialoganglioside GD2 with the recently approved monoclonal antibody (mAb) CH14.18 after autologous or allogeneic SCT, significantly improves survival. However, GD2 expression is heterogeneous and $\mathrm{CH} 14.18$ causes severe adverse effects. Thus, we evaluated B7-H3 as an alternative or additional target antigen. We investigated different anti-B7-H3 mAb constructs and mAb-cytokine fusions (immunocytokines) for their ability to elicit antibody-dependent cellular cytotoxicity (ADCC).

Methods: Derived from a parent anti-B7-H3 clone five $\mathrm{mAb}$ constructs were engineered: (1) chimeric fused with human IL-2 (cHEK5-IL2), (2) chimeric and Fc-optimized (SDIE) w/o fusion (cHEK5opt), (3) cHEK5opt with human IL-2 fusion (cHEK5opt-IL2), (4) cHEK5opt with human IL-15 fusion (cHEK5opt-IL15) and (5) cHEK5-IL2 produced in YB2/0 (rat myeloma) to create an optimized lowfucose version (cHEX5LF-IL2). The abilities of all six anti$\mathrm{B} 7-\mathrm{H} 3 \mathrm{mAb}$ constructs and the GD2-specific mAb CH14.18 to mediate ADCC were compared in vitro in cytotoxicity assays (RTCA xCELLigence and calcein release assays). Target cells: NB cell lines with high B7H3 but variable GD2 expression (LAN-1, Kelly, SH- 
SY5Y). Effector cells: Human expanded NK cells (eNKs), expanded $\gamma / \delta \mathrm{T}$ cells and patient PBMCs after receiving allogeneic SCT.

Results: Except the parent clone, all anti-B7-H3 mAb constructs mediated ADCC. Target cell lysis of LAN-1 (high expression of both GD2 and B7-H3) mediated by the optimized anti-B7-H3 immunocytokines was comparable or even better than that mediated by $\mathrm{CH} 14.18$ (effectors: eNKs; calculated after 36 hrs.; in ascending order): Targets + effectors w/o mAb (24 \%), parent pHEK5 (29\%), cHEK5opt (44 \%), cHEK5-IL2 (76 \%), cHEK5opt-IL15 (85\%), CH14.18 (90\%) and cHEK5opt-IL2 (97\%). In comparison with all other $\mathrm{mAb}$ constructs the recently produced low-fucose immunocytokine cHEX5LF-IL2 mediated best target cell lysis against SH-SY5Y (effectors: eNKs; calculated after 48 hrs.; in ascending order): Targets + effectors w/o mAb (31 \%), CH14.18 (42 \%), parent pHEK5 (46 \%), cHEK5-IL2 (80 \%), cHEK5opt (85\%), cHEK5opt-IL2 (93\%), cHEK5opt-IL15 (96 \%) and lowfucose cHEX5LF-IL2 (100\%). Using expanded $\gamma / \delta$ T cells of healthy donors, cHEX5LF-IL2 was confirmed to be the most effective mAb construct. Interestingly, cHEK5-IL2 showed comparable target cell lysis - however, lysis was only transient while cHEX5LF-IL2 mediated permanent target cell lysis. Using patient PBMCs after receiving allogeneic SCT, cHEX5LF-IL2 and CH14.18 mediated comparable lysis. Calculated specific lysis of LAN-1 (after 96 hrs.; in ascending order): Targets + effectors w/o mAb (22\%), parent pHEK5 (26\%), cHEK5opt-IL2 (40\%), cHEK5opt (49 \%), cHEK5-IL2 (57 \%), cHEK5opt-IL15 (70 \%), low-fucose cHEX5LF-IL2 (78 \%) and CH14.18 (81\%).

Conclusions: $\mathrm{B} 7-\mathrm{H} 3$ is a suitable target antigen in case GD2 expression is low or absent. Immunocytokines and Fcoptimized mAbs targeting B7-H3 might increase the efficacy of immunotherapy in GD2-negative tumors and in combinatory approaches. Until now, the low-fucose immunocytokine cHEX5LF-IL2 seems to be the most promising anti-B7-H3 construct.

Disclosure: Nothing to declare.

\section{P633}

A phase 1/2 dose-finding, safety, and efficacy study of ibrutinib in paediatric patients with chronic graft versus host disease

\section{Franco Locatelli, Peter Shaw ${ }^{2}$, Ying Luan ${ }^{3}$, Juthamas Sukbuntherng $^{3}$, Justin Wahlstrom ${ }^{3}$, Lori Styles ${ }^{3}$, Paul Carpenter $^{4}$}

${ }^{1}$ Ospedale Pediatrico Bambino Gesù, Sapienza University of Rome, Roma, Italy, ${ }^{2}$ The Children's Hospital Westmead, Sydney, Australia, ${ }^{3}$ Pharmacyclics LLC, an AbbVie company, Sunnyvale, CA, United States, ${ }^{4}$ Fred Hutchinson Cancer Center, Seattle, WA, United States

Background: Paediatric chronic graft versus host disease (cGVHD) is a debilitating condition associated with substantial morbidity and mortality. To date, there are no approved therapies for paediatric patients with cGVHD, and current treatments often lack sufficient efficacy or lead to severe/life-threatening toxicities that limit their effectiveness. Ibrutinib, a first-in-class, once-daily inhibitor of Bruton's tyrosine kinase (BTK), is approved in the US for the treatment of adult patients with cGVHD after failure of $\geq 1$ lines of systemic therapy. This phase $1 / 2$ study will evaluate the use of ibrutinib in paediatric patients with moderate or severe cGVHD.

Methods: This open-label, multicenter, international phase 1/2 study (PCYC-1146) includes patients with moderate or severe cGVHD as defined by the $2014 \mathrm{NIH}$ Consensus Development Project Criteria. It is divided into two parts: Part A will determine the recommended paediatric equivalent dose (RPED) of ibrutinib in patients aged $\geq 1$ to $<12$ years, and Part B aims to evaluate the safety and efficacy of ibrutinib in patients age $\geq 1$ to $<22$ years.

For Part A, patients with cGVHD aged $\geq 1$ to $<12$ years who have failed $\geq 1$ lines of systemic therapy will receive once daily oral ibrutinib at a starting dose of $120 \mathrm{mg} / \mathrm{m}^{2}$ to be escalated up to $240 \mathrm{mg} / \mathrm{m}^{2}$ after 14 days, if no grade $\geq 3$ toxicities occur, until the RPED is determined. For Part B, patients aged $\geq 12$ to $<22$ years with cGVHD who have failed $\geq 1$ lines of systemic therapy or have newly diagnosed cGVHD will receive once daily ibrutinib (420 mg) until one of the following criteria is met: treatment is no longer required; new systemic treatment for $\mathrm{cGVHD}$ is initiated; progression of cGVHD; recurrence of underlying disease; or unacceptable toxicity. Patients with newly diagnosed cGVHD will receive ibrutinib in addition to daily corticosteroids $(0.5-1 \mathrm{mg} / \mathrm{kg}$ prednisone). Patients $<12$ years of age may be enrolled in Part B and treated at the RPED after it is determined in Part A. Key exclusion criteria include uncontrolled active systemic infection or active infection requiring systemic treatment; progressive underlying malignant disease or any post-transplant lymphoproliferative disease; or active hepatitis $\mathrm{C} /$ hepatitis $\mathrm{B}$ virus. Patients must have adequate renal, hepatic, and hematologic function to be enrolled.

The primary endpoint of Part A is the RPED of ibrutinib, as based on pharmacokinetic (PK) data; secondary endpoints include safety and pharmacodynamics (BTK occupancy). The primary endpoints of Part B are PK and safety of ibrutinib in paediatric patients with cGVHD. Secondary endpoints for Part B include response rate at 24 weeks as defined by the 2014 NIH Consensus Development Project 
Criteria; duration of response; overall survival; and late effects on growth, development, and immune reconstitution.

Results: This global study is currently enrolling.

Conclusions: This phase $1 / 2$ study will explore the use of ibrutinib in paediatric patients with cGVHD to potentially meet the high unmet need for proven effective therapies for this population.

\section{Disclosure:}

Franco Locatelli: consultancy/advisory role with Jazz Pharmaceuticals, Amgen, and Pfizer; research funding from Bellicum Pharmaceuticals; Peter Shaw: consultancy/advisory role with Novartis;

Ying Luan: employment with Pharmacyclics LLC, an AbbVie Company; stock ownership in AbbVie;

Juthamas Sukbuntherng: employment with Pharmacyclics LLC, an AbbVie Company, and spouse's employment with Global Blood Therapeutics; stock ownership in AbbVie, Global Blood Therapeutics, Portola; Justin Wahlstrom: employment with Pharmacyclics LLC, an AbbVie Company; stock ownership in AbbVie; Lori Styles: employment with Pharmacyclics LLC, an AbbVie Company; stock ownership in AbbVie; Paul Carpenter: consultancy/advisory role with Incyte and Mallinckrodt; research funding from Mallinckrodt and Pharmacyclics LLC, an AbbVie Company.

Funding: Pharmacyclics LLC, an AbbVie company.

\section{P634}

Cyclophosphamide (80MG/KG), fludarabine, and antithymocyte globulin as preparative regimen for hematopoietic stem cell transplantation in children with severe aplastic anemia

\section{Mohammed Essa ${ }^{I}$, Enas Basher ${ }^{I}$, Rodaina Abujoub ${ }^{I}$, Talal Al Harbi ${ }^{1}$, Khalid Al Jamaan ${ }^{\text {, Ali Al Omari }}{ }^{1}$, Reem Al Sudairy ${ }^{1}$, Abdulrahman Al Sultan ${ }^{1,2}$}

\footnotetext{
${ }^{1}$ King Abdullah Specialist Children's Hospital, King Saud Bin Abdulaziz University For Health Sciences, King Abdullah International Medical Research Center, Riyadh, Saudi Arabia, ${ }^{2}$ King Saud University, Pediatrics, Riyadh, Saudi Arabia
}

Background: Hematopoietic stem cell transplantation (HSCT) is the standard treatment for children with severe aplastic anemia (SAA) who have HLA-identical related donor. There is no standard conditioning regimen for children with SAA secondary to non-fanconi anemia (FA) constitutional bone marrow failure syndromes such as telomeropathies. We report the outcome of a consistent reduced intensity conditioning regimen in patients with idiopathic SAA or inherited bone marrow failure syndromes other than FA who underwent HLA matched related HSCT
Methods: children with SAA underwent HSCT using the following conditioning regimen: fludarabine $175 \mathrm{mg} / \mathrm{m} 2$, cyclophosphamide $80 \mathrm{mg} / \mathrm{m} 2$, and ATG (thymoglobulin) $(10 \mathrm{mg} / \mathrm{kg})$. GVHD prophylaxis included cyclosporin and mycophenolate mofetil. Donors were all matched related and bone marrow was the stem cell source. All patients had normal chromosomal fragility test

Results: a total of 18 children with SAA underwent HSCT, 12 females and 6 males. Average age was 8.1 (range 0.8-13.8 years). All nine patients who were tested for telomere length had short telomeres. Pathogenic or likely pathogenic mutations were reported in 5 patients (2 ERCC6L2, 1 ANKRD26, 1 TINF2, 1 LZTFL1). All donors had normal physical examination, normal $\mathrm{CBC}$, and negative genetic testing if patient mutation is known. All 18 patients engrafted successfully, median time to neutrophil engraftment was 16 (range, 11-29 days) and platelet engraftment 19 (range, 13-45 days). Median infused nucleated cell dose was 3.3 (range,0.9-7.3 X10 $/ \mathrm{kg}$ ) and CD34 cell dose was 6.7 (range, 1.1-13.1 X10\% $/ \mathrm{kg}$ ). None of our patients had acute GVHD and one patient had mild classic chronic GVHD of the skin that was controlled with topical therapy for a short period. Three patients had secondary graft failure in the first-year post transplant. First patient had pancytopenia with loss of donor chimerism and underwent successful second transplant using fludarabine, ATG, and melphalan. The second patient had a nonfunctioning graft despite full donor chimerism suggesting that the related donor might be affected and had silent phenotype. The third patient had

homozygous ERCC6L2 mutation and developed progressive cytopenia with myelodysplastic features few months post-transplant and subsequently underwent myeloablative matched unrelated transplant using busulfan, fludarabine, thiotepa and ATG. However, the patient progressed to have acute myeloid leukemia six months post HSCT. Fifteen patients (83\%) have normal CBC and stable donor chimerism. Median follow-up duration of 1140 days (range 330 -2595 days). One patient with LZTFL1 mutation developed chronic renal impairment five years post HSCT.

Conclusions: HSCT using lower dose cyclophosphamide $(80 \mathrm{mg} / \mathrm{kg})$ as part of fludarabine based regimen was safe and effective in SAA patients with shorter telomeres and described genetic abnormalities. Optimal conditioning regimen in ERCC6L2-associated bone marrow failure needs to be defined. Larger study is needed to confirm our results.

Clinical Trial Registry: Not applicable

Disclosure: Nothing to declare

\section{P635}

Late effects in patients with hemophagocytic lymphohistiocytosis treated with hematopoietic stem cell transplantation: A review of the literature 
Karin Beutel', AnnaCarin Horne ${ }^{2}$, Kanchan Rao ${ }^{3}$, Despina Moshous ${ }^{4,5}$, Itziar Astigarraga ${ }^{6}$, Mahmoud Elzembely $^{7}$, K. Scott Baker ${ }^{8}$

${ }^{1}$ München Klinik Schwabing und Klinikum München Rechts der Isar, TU München, Pediatric Hematology and Oncology, München, Germany, ${ }^{2}$ Karolinska Institute, Stockholm, Sweden, ${ }^{3}$ Great Ormond Street Hospital, NHS Foundation Trust, Blood and Marrow Transplantation, London, United Kingdom, ${ }^{4}$ Hôpital Necker-Enfants Malades, Assistance Publique-Hôpitaux de Paris, Pediatric Immunology, Hematology and Rheumatology, Paris, France, ${ }^{5}$ Institut Imagine and Université Paris Descartes -Sorbonne Paris Cité, INSERM U 1163, Paris, France, ${ }^{6}$ Hospital Universitaria Cruces. IIS Biocruces Bizkaia. Universidad del Pais Vasco UPV/EHU, Pediatrics, Bilbao, Spain, ${ }^{7}$ South Egypt Cancer Institute, Assiut University, Pediatric Oncology, Assiut, Egypt, ${ }^{8}$ Fred Hutchinson Cancer Research Center, Seattle, WA, United States

Background: Hemophagocytic lymphohistiocytosis (HLH) is an inherited or acquired disorder of immunedysregulation. Early diagnosis and immunosuppressive treatment can prevent significant organ-failure. The inherited forms, and some acquired cases can only be cured by hematopoietic stem cell transplantation (HSCT). With modern transplant practices, a significant number of patients survive. The purpose of this literature review was to collect data on the frequency and type of late effects in HLH patients surviving after HSCT and to examine the association with pre-existing HLH conditions (eg. involvement of the central nervous system (CNS) before transplant) and with the pre-transplant conditioning regimens.

Methods: The MEDLINE, EMBASE, Web of Science and PubMed databases were searched, by two librarians at the Karolinska Institutet, between May and September 2016 according to the Preferred Reporting Items for Systematic Review and Meta-analysis (PRISMA) statement. The search terms included "HLH", "FHL", "MAS", multiple terms for "HSCT" and late-effect conditions. Inclusion criteria were publications in English that included children between January 1995 and May 2016. Authors of this review screened all the abstracts of studies against the inclusion criteria.

Results: Only nine papers published between 2006 and 2016, with information on late effects in HLH patients who had undergone HSCT, were identified. Three reports include only small numbers of HLH patients. The remaining 6 papers contain data on 261 long-term survivors with a median follow-up of 5.4 years. Five papers address neurological sequelae with a reported incidence from $7-57 \%$. The highest incidence was found after a thorough neurological assessment of $21 \mathrm{HLH}$ patients compared to matched sibling controls. However, the association with CNS disease before transplant and age at transplant was not clear. Patients with EBVassociated HLH seem to have fewer long-term neurological problems. Non-neurological late effects are described in 4 papers only, with endocrinological problems, namely short stature, being the most frequent. One paper specifically analyzed poor growth, thyroid dysfunction and vitamin D deficiency in a cohort of patients with non-malignant disorders including HLH who had undergone HSCT after a reduced intensity conditioning regimen and found significant abnormalities in all groups.

Conclusions: Data on late effects in HLH patients is scarce and is mostly based on the retrospective evaluation of small national cohorts. The available information indicates that a significant number of patients suffers from problems which affect their daily life, but lack of information does not allow to analyze the association between pre-transplant conditions and long-term sequelae. Therefore, a retrospective comprehensive analysis of patients registered in the EBMT and CIBMTR registries is currently performed. It will be crucial to better define the frequency and type of late effects in a large cohort. This knowledge will aid counselling prior to HSCT, provide guidance for long-term monitoring of these patients, and potentially identify specific risk factors for late effects in this rare patient population.

Disclosure: Nothing to declare.

\section{P636}

Allogeneic stem cell transplantation in patients with mucopolysaccharidosis type II (Morbus hunter)

\section{Bernd Hartz', Nicole Muschol, Matthias Bleeke ${ }^{1}$, Johanna Schrum ${ }^{1}$, Ingo Müller ${ }^{1}$}

${ }^{1}$ Hamburg University Hospital, Hamburg, Germany, ${ }^{2} \mathrm{Ham}-$ burg University Hospital, International Center for Lysosomal Disorders, University Children's Hospital HamburgEppendorf, Hamburg, Germany

Background: Mucopolysaccharidosis type II (Morbus MPS II, Hunter syndrome) is a rare X-linked lysosomal storage disease caused by a deficiency of the lysosomal enzyme iduronate-2-sulfatase. Multiple organs are effected by this severe disease and a neuropathic type of Hunter syndrome can be distinguished from a non-neuropathic course.

Enzyme Replacement Therapy (ERT) is the treatment of choice in non-neuropathic Hunter syndrome, but as the recombinant enzyme does not cross the blood brain 
barrier and neuropathic Hunter syndrome is left untreated. Hematopoietic stem cell transplantation (HSCT) is the standard of care in patients with severe mucopolysaccharidosis (MPS) type I (MPSIH, Hurler syndrome) as early transplantation halts cognitive decline in these patients and significantly improves survival. Only few case studies have been published on the potential benefit of HSCT in MPS II and mostly used busulfan-based conditioning regimens. In one comparative non-randomised multicenter study, HSCT might to be superior compared to ERT. Here, we present our experience in HSCT in three children with Hunter syndrome using a treosulfan-based conditioning regimen.

Methods: A retrospective chart review was carried out in patients, who underwent HSCT for Hunter syndrome. The conditioning chemotherapy regimen included fludarabine, treosulfan, thiotepa and ATG. All patients received bone marrow of either related and or matched unrelated donors. GvHD prophylaxis was performed with CsA and methotrexat.

Results: Three patients with Hunter syndrome were transplanted in our department in 2010. The age was six months, two years and four years, respectively. Bone marrow donors were related in one patient and matched unrelated in two patients. The conditioning therapy was generally well tolerated. Major complications were fever of unknown origin with need for antibiotic therapy and a mucositis. One patient developed a CMV reactivation. All patients engrafted successfully and recovered well from the HSCT. There was no case of acute or chronic GvHD.

In 2018 all three patients are alive. Donor chimerism is complete in one patient; two patients have a mixed donor chimerism. After application of donor lymphocyte infusions in one patient, donor chimerism is stable at a low level of $16 \%$. The donor chimerism of the other patient still slowly declines to currently $50 \%$.

After stem cell transplantation, two patients did not show further progression of the disease and even achieved psycho-motor improvements. Interestingly, one of these patients is the one with the low donor chimerism of $16 \%$. One patient suffers from a further progression of the underlying disease with psycho-motoric agitation, aggressive behavior and loss of speech, that occurred within the first year following HSCT, but neurocognition stabilized thereafter.

Conclusions: We found a beneficial effect of HSCT on the neuropsychological outcome or at least stabilization of neurocognitive function in our patients with a follow-up of eight years. Despite low toxicity of the conditioning regimen, increased donor chimerism may further improve the neurological outcome.

Disclosure: Nothing to declare.
P637

Tandem sct in pediatric solid tumors, other than brain tumors, has no advantage in terms of EFS over single procedure-single center experience

Cristina Georgiana Jercan ${ }^{1,2}$, Ana Maria Bica ${ }^{2}$, Andra Daniela Beldiman $^{2}$, Andreea Nicoleta Serbanica ${ }^{1,2}$, Letitia Elena Radu ${ }^{1,2}$, Alexandra Ionete ${ }^{3}$, Carmen Calugaroiu ${ }^{3}$, Constantin Arion $^{1,2}$, Anca Colita ${ }^{1,2}$

${ }^{1}$ Carol Davila University of Medicine and Pharmacy, Pediatric, Bucharest, Romania, ${ }^{2}$ Fundeni Clinical Institute, Pediatric, Bucharest, Romania, ${ }^{3}$ Fundeni Clinical Institute, Bucharest, Romania

Background: Solid tumors, excepting brain tumors, represent $30 \%$ of all pediatric cancers. Treatment plans include agressive chemotherapy, surgery \pm radiotherapy; for high-risk patients hematopoietic stem cell transplantation (SCT) and immunotherapy. Event free survival (EFS) rates are very low, especially in high risk patients. Studies have shown evidence of dose-dependent evolution, so double or triple SCT have been proposed.

Methods: We retrospectively analyzed results of tandem SCT vs single SCT in pediatric patients with solid tumours treated in Fundeni Clinical Institute, Bucharest, Romania between January 2002 and May 2018. Patients diagnosis were: neuroblastoma (NBL), germ-cell tumour (GCT), Ewing sarcoma (ES), nefroblastoma. Patients were divided into 2 groups according to the number of procedures: $1 \mathrm{st}$ group-single SCT procedure, 2nd group-multiple procedures. Regimens used for stem cell mobilisation were: Topo-Cy for NBL and Epi-Tax for GCT, followed by G$\mathrm{CSF} \pm$ Plerixafor. Conditioning regimens: Bu-Mel and Thiotepa-Cy for pts with NBL, Thio-Tax and ICE for pts with GCT. Patients received antibiotic, antiviral and antifungal prophylaxis, parenteral nutrition and supportive treatment. Patients received consolidation treatment, followed up monthly in the first year, then yearly. Patients were evaluated for residual disease by imaging tests. Parents signed informed consent forms.

Results: We performed 67 SCT procedures to 52 patients: 65.3\% NBL, $17.3 \%$ ES, $11.6 \%$ GCT and $5.8 \%$ nefroblastoma. For this study only patients with NBL and GCT were considered. In 1st group were $79 \%$ of pts, $21 \%$ in 2nd group. Patients were diagnosed, staged and treated according to international protocols. Sex ratio was 18F/34M. Age distribution was $1-4$ y $38 \%$ (20 pts), $4-10$ y $35 \%$ (18 pts), $>10$ y $27 \%$ (14 pts). Peripheral stem cell (PBSC) mobilisation was more difficult in patients with multiple courses of chemotherapy \pm radiotherapy. We found no difference in the period of engraftment following a 2nd or 3rd procedure. Hospitalization and supportive measures increased 
in 2 nd and 3rd procedures (26 to 29 days). Patients with multiple courses of chemotherapy and multiple hospitalizations had increased infectious risk and during the 2nd or 3rd procedures developed various infectious complications.Incidence of severe oral mucositis after the first HSCT was $17 \%$, after tandem HSCT was $69 \%$. NBL patients : 1st group-6/23 patients alive and EFS, 1/23 receives anti G2 treatment; 2nd group-1/7 patients-alive, $2 / 7$ patients-not reached timepoint for $\mathrm{mIBG}$ scan; 4/7 patients-mIBG negative at first, relapsed after $6 \mathrm{mo} ; 1 / 7$ patient deceased due to pulmonary toxicity. GCT patients: 1st group-1/5 patients alive and EF, 1/5 high values in AFP levels and receives metronomic therapy, 3/5 patients deceased due to progressive disease, but only had 2 SCT. Only $1 / 5$ patient had one procedure and died due to progressive disease.

Conclusions: In our study, tandem HSCT in children with solid tumours lead to an increase in survival rates, at least in the first 6 months after SCT. Most patients $(90 \%)$ had progressive or relapsed/refractory disease when referred to our department. Multiple procedures require a higher number of CD34 cells, very hard to achieve in patients with multiple courses of chemo \pm radiotherapy. New approaches have to be considered in these diseases, especially in high risk group.

Disclosure: Nothing to declare

\section{P638}

Antibiotic prophylaxis effectiveness in pediatric allogeneic hematopoietic stem cell transplantation experience from a tertiary care children hospital in over a decade

Erica Ricci $^{1}$, Alessio Mesini ${ }^{2,3}$, Cristina Moroni ${ }^{3}$, Roberto Bandettini $^{4}$, Elio Castagnola ${ }^{3}$, Edoardo Lanino ${ }^{5}$, Stefano Giardino $^{5}$, Maura Faraci ${ }^{5}$

${ }^{1}$ University of Genova, DINOGMI, Genoa, Italy, ${ }^{2}$ University of Genova, DiSSal, Genova, Italy, ${ }^{3}$ Istituto Giannina Gaslini, Genova, Italy, ${ }^{4}$ Istituto Giannina Gaslini, Genova, Italy, ${ }^{5}$ Istituto Giannina Gaslini, Genova, Italy

Background: Antimicrobial prophylaxis for prolonged neutropenia occurred during the pre-engraftment period is a common practice in allogeneic HSCT recipients. Data on its effectiveness are few and generally from cases series and not from randomized clinical trials, especially in children.

Methods: All clinical records of allo-HSCT performed from January $1^{\text {st }} 2007$ to November $30^{\text {th }} 2018$ at HSCTUnit of Istituto G.Gaslini, Genoa-Italy, were retrospectively reviewed. Collected data were underlying diseases, type of donor, antibiotic prophylaxis administration and type, development of fever and pathogen isolated from blood culture, if any, during pre-engraftment neutropenia.
Antibiotic prophylaxis, usually starting together with the conditioning regimen, was categorized in "standard" (with amoxicillin/clavulanate or ampicillin/sulbactam) or "tailored" (when based on previous bacterial isolations or colonizations).

Results: 246 allo-HSCTs were performed in 217 pediatric patients (59\% males) with a median age at HSCT of 8 years (IQR: 4-13; range: 0-22). HSCTs were performed from alternative donor (AD) in $55 \%$ patients, from relative donor (RD) in $25 \%$, and from haploidentical donor in $20 \%$. Table 1 shows the pre-engraftment febrile neutropenia episodes according to type of antibiotic prophylaxis. 224 (91\%) HSCTs received standard prophylaxis, while $20(8 \%)$ the tailored one; only in $2(1 \%)$ did not receive any prophylaxis. Fever occurred in 194 (87\%) of episodes in patients receiving standard prophylaxis, in 19 (95\%) of those treated with tailored prophylaxis and $1(50 \%)$ in the group without prophylaxis; only $13 \%$ of patients who received prophylaxis did not develop fever.In $38 \%$ of patients, the febrile episodes were diagnosed as bloodstream infections: Staphylococcus aureus in 2\%; CoNS in 19\%; Enterococcus spp in 15\%; Enterobacteriaceae in 25\%; Pseudomonas aeruginosa in 11\%; other non-fermenting gram negatives in $2 \%$ and fungi in $10 \%$.

Conclusions: The occurrence of fever in patients who received antibiotic prophylaxis suggested that it could not be effective in prevention of fever related to neutropenia after allo-HSCT. The personalization of prophylaxis could be a possible path to follow these patients.

Disclosure: Nothing to declare

\section{P639}

Study of fear caused by medical manipulations in children 3 to 6 years

\section{Olesya Morozova ${ }^{1}$, Olga Ushakova ${ }^{1}$, Alisa Volkova ${ }^{1}$}

${ }^{1}$ R.M. Gorbacheva Memorial Institute for Pediatric Hematology, Oncology and Transplantation, Rehabilitation, Saint Petersburg, Russian Federation

Background: Cancer itself is a psycho-traumatic situation and may impair child's normal development. During a play children utilize traumatic material associated with pain, discomfort and fear. The involved observation and clinical diagnostic interview methods were used to study this situation.

Methods: A total of 15 patients with leukemia or neuroblastoma were included in the study. Patients' mothers signed an informed consent for participation in the study. Six of study participants were boys and 9 girls, all aged 3 to 6 years. The control group consisted of 18 healthy preschool children ( 2 groups of 9 children aged 5 to 6 years), 8 boys and 10 girls. 
Results: In most of games the role of a doctor was played by a child. Only one child declined to impersonate both a patient and a doctor. Younger children mostly agreed to have for a "patient" a toy (proposed by psychologist or one of child's own), child's mother or a medical psychologist. The game lasted for 15 - 20 minutes. Most patients preferred using real medical consumables and instruments (syringes, adhesive tape, winged infusion sets or, more rarely, pills). Most often a syringe or an adhesive tape was chosen. As known from their mothers, among medical manipulations most unpleasant for children are injections and changing implanted catheter dressing. Also, most healthy preschool children preferred using real medical instruments over toy ones. Group 1 more often used a syringe, a winged infusion set, adhesive tape, gauze or pills. Group 2 most often chose syringe or gauze. Among medical instruments both groups more often chose a phonendoscope or thermometer.One patient refused to cause pain to a "toy patient". Other children sympathized with a "toy patient", stroke injection or dressing location sites or used soothing terms ("wait a little", "it's going to be all right"), wished prompt recovery and hugged their "patients". One child was angry over his "patient" wishing him to "get hurt too". First preschool group children were mostly scolding a toy "patient" for "being guilty of getting sick". Second group children were mostly compassionate, encouraged a "toy patient" telling that "all the procedures are needed to get healthy".

From children's schoolmasters we know that all first group children received vaccination about a week before a test. Children from second group had no injections. Overall attitude towards toy "patients" was more mild in the second group.

Conclusions: 1 . During a play children mostly use the medical devices which cause them most discomfort and/or pain. 2. Manipulating the items children illustrate their own impression of medical procedures, which are most unpleasant. 3. Children may express their negative emotions directed towards medical manipulations via their play actions, these negative reactions may be suppressed in different ways by parents or medical staff. 4 . The intensity of child's own traumatic experience and an attitude of nearby adults may influence the child's attitude towards other patients. 5. The mother's wish for a child to tolerate all medical procedures with ease exceeds real capabilities of a small child.

Disclosure: Nothing to declare.

\section{P640}

\author{
Allogeneic stem cell transplantation in patients with \\ mucopolysaccharidosis type IIIA (Sanfilippo): A case \\ series
}

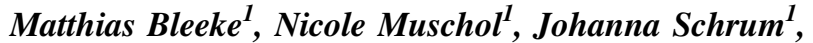
Bernd Hartz, Ingo Müller ${ }^{I}$

\author{
${ }^{1}$ University Children's Hospital Hamburg-Eppendorf,
}

Background: Mucopolysaccharidoses (MPS) are lysosomal storage diseases caused by inborn errors in enzymes required for the degradation of glucosaminoglycanes. Hematopoietic stem cell transplantation (HSCT) is an established therapeutic option for patients with MPS type I, but data on safety and efficacy of SCT in other types of MPS are sparse. We report a small series of three patients with MPS type IIIa (Sanfilippo type A) who underwent HSCT as a salvage therapy since other therapeutic options were unavailable for these patients.

Methods: Allogeneic SCT was performed at the ages of 2, 5 and 4 years, respectively. All three patients received intrathecal enzyme replacement therapy within a clinical trial setting prior to HSCT. The conditioning regimen consisted of treosulfan, fludarabine, thiotepa and thymoglobuline. GvHD-prophylaxis was carried out with CSA and MTX in two patients and CSA and MMF in one patient. Stem cell source was bone marrow in two patients and peripheral blood stem cells in one patient.

Results: The conditioning regimen was well tolerated and all three patients successfully engrafted. Two of three patients had an uncomplicated course without occurrence of acute or chronic graft-versus-host-disease (GVHD). At last follow-up 12 and 15 months after HSCT, both patients are in good condition and show constant progress of psychomotor development. The third patient experienced severe steroid refractory acute GVHD of intestines (stage 4) and skin (stage 3), which resolved under intensive immunosuppression with cyclosporine, mycophenolate and ruxolitinib. Around day 110 after HSCT, this patient showed clinical and biochemical signs of transplant-associated microangiopathy (TMA) with cerebral seizures and acute renal failure. The cerebral MRI showed progressive cerebral atrophy and leukoencephalopathy, also consistent with a progress of the MPS IIIa. At last follow-up 15 months after HSCT, this patient had recovered from TMA and was in a stable clinical condition.

Conclusions: In consideration of the small case number and the short follow-up period in our cohort, allogeneic HSCT might be considered as a salvage therapy for patients with MPS IIIa if other therapeutic options are unavailable for children with this otherwise unfavourable prognosis. However, the early psychoneurological course after transplant seems promising compared to the literature and HSCT could become a treatment option for this rare disease.

Disclosure: Nothing to declare 


\section{P641}

Characterization of a bimodal NF-кB FEEDBACK mechanism in rhabdomyosarcoma (RMS) cells induced by the treatment with SMAC mimetics (SM)

\section{Vinzenz Särchen ${ }^{1}$, Lisa Marie Reindl $l^{2,3}$, Meike Vogler ${ }^{1}$, Thomas Klingebiel ${ }^{2}$, Simone Fulda ${ }^{1,4,5}$, Evelyn Ullrich ${ }^{2,3,5}$}

${ }^{1}$ Institute for Experimental Cancer Research in Pediatrics, Goethe University, Frankfurt am Main, Germany, ${ }^{2}$ University Hospital Frankfurt, Goethe University, Frankfurt am Main, Germany, ${ }^{3}$ Experimental Immunology, Goethe University, Frankfurt am Main, Germany, ${ }^{4}$ German Cancer Consortium (DKTK), Heidelberg, Germany, ${ }^{5}$ German Cancer Research Center (DKFZ), Heidelberg, Germany

Background: Upregulation of inhibitor of apoptosis proteins (IAPs) is one underlying mechanism causing cell death resistance of rhabdomyosarcoma (RMS) cells. Previous studies could characterize an influence of Smac mimetics (SM) not only on the tumor cell side but also on NK cells, resulting in an increased susceptibility of tumor cells towards NK cell-mediated killing and an increased cytotoxicity of the NK cells (Fischer et al. 2017).

Methods: For the identification of underlying molecular mechanisms leading to the increased sensibility of RMS cells, the activation status of different NF-kB signaling pathways were analyzed using western blot analysis and quantitative real time PCR (qPCR). Further, flow cytometry was used to analyze the surface expression of death receptors on either SM treated or untreated RMS cells. The overall effect on cell death induction was measured by PI/Hoechst staining using a fluorescent microscope.

Results: Treatment with SM led to the suspected degradation of IAPs. Followed by the activation of both the canonical NF-kB signaling pathway, indicated by the phosphorylation of $\mathrm{I} \kappa \mathrm{B} \alpha$ and $\mathrm{p} 65$, and the non-canonical NF- $\mathrm{KB}$ signaling pathway, as indicated by the accumulation of NIK and the degradation of p100 to p52. Determination of selected target gene transcription revealed an upregulation of the inhibitor IKB $\alpha$, NIK, p100, IL-8 and at later time points the death receptors TRAIL-R1 and TRAIL-R2. Analysis of gene transcription also led to the finding of neither up- nor downregulation of cIAP1 and p65. To evaluate the involvement of TRAIL-R1 and TRAIL-R2 in the SM induced sensitization towards NK cell-mediated killing, surface expression of both death receptors was analyzed. Treatment with SM led not only to an induced transcription of TRAIL-R1 and TRAIL-R2, but also to an increased surface presentation of TRAIL-R2. Subsequent ligation of TRAIL-R2 by either wt-TRAIL or a specific agonistic antibody (ETR-2) resulted in a significant increase in cell death induction.
Conclusions: The aforementioned analysis of gene transcription hints towards a bimodal feedback mechanism regulating both, the canonical and non-canonical NF- $\mathrm{NB}$ signaling pathway. On the one side, the canonical pathway is negatively regulated by the induced transcription of the inhibitor I $\mathrm{K} \mathrm{B} \alpha$. On the other side, the induced transcription of NIK, p100 and RelB points towards a positive feedback loop of the non-canonical pathway. One mechanism of the increased RMS cell sensitivity might be the induced transcription and surface presentation of the death receptor TRAIL-R2. The involvement of TRAIL receptors is further validated by the cytotoxicity data, illustrating a SM mediated sensitization towards a TRAIL induced cell death induction. This mode of cell death fits to the previous research, were TRAIL transcription could be induced in NK cells by SM treatment.

The graphical abstract shows the transcriptional upregulation of target genes leading to a putative bimodal NF-kB regulation and increased surface presentation of TRAIL-R2 by treatment with Smac mimetics.

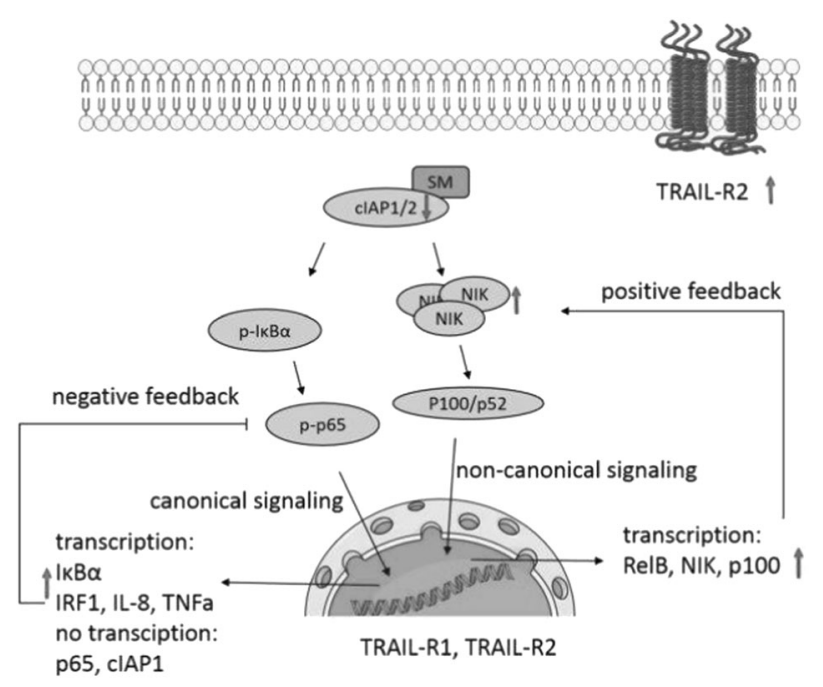

[[P641 Image] 1. SM induced bimodal NF- $k B$ signaling in RMS cells]

Disclosure: Nothing to declare

\section{P642}

Transplantation of unrelated cord blood in pediatric patients with malignant and non malignant diseases

Anna Komitopoulou ${ }^{1}$, Anna Paisiou ${ }^{1}$, Christina Oikonomopoulou $^{I}$, Katerina Kaisari ${ }^{1}$, Eleni Ioannidou, Eftychia Petrakou ${ }^{1}$, Eirini Sfougkataki, Maria

Theodosaki, Maria Ktistaki ${ }^{1}$, Giorgos Vessalas ${ }^{1}$, Ioulia Peristeri $^{1}$, Evgenios Goussetis ${ }^{1}$, Vassiliki Kitra ${ }^{1}$ 
${ }^{1}$ St Sophia Children's Hospital, Athens, Greece

Background: Umbilical cord blood (UCB) is an alternative graft for patients not having a compatible donor. UCB units are rapidly available with less chronic complications for the patients, while immune reconstitution and engraftment is delayed.

Aim: To investigate the outcome of UCB transplantation in pediatric patients with malignant and non malignant diseases

Methods: Data from 30 patients underwent first allogeneic bone marrow transplantation with UCB from 2/1999 until 6/2013 were retrospectively analyzed. Eighteen had malignant disease (MD), of whom 15 in complete hematologic response, and 12 non malignant disease (NMD) (SCID 5, chronic granulomatous disease 1, severe aplastic anemia 2, s.Kostmann 1, osteopetrosis 1, WiskottAldrich1, amegakaryocytic thrombocytopenia 1). The majority of the patients were male, for MD and NMD, as well (M:10/F:8, M:8/F:4, respectively), of median age 6.5 years (range 0.8-11.8 years) and 0.8 years (range 0.4-6.5 years), respectively

Results: All patients but one, received 1 UCB unit. HLA compatibility in antigen/allele level was at least 5/6 and only in 3 patients with MD was 4/6. Conditioning regimens were myeloablative and TBI 12 Gy was given in 4/30. GVHD prophylaxis consisted of cyclosporine and ATG was given in all patients pre-transplantation. Median value of nucleated cells for MD was $3.75 \times 10^{7} / \mathrm{kg}$ (range $2-6.3 \chi 10^{7} /$ $\mathrm{kg}$ ) and for NMD was $11.05 \chi 10^{7} / \mathrm{kg}$ (range $2-27.2 \chi 10^{7} / \mathrm{kg}$ ). Neutrophil and platelet engraftment was achieved in $13 / 18$ and $12 / 18$ patients with MD respectively, in a median time

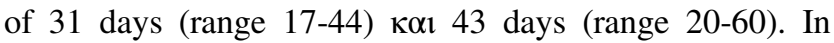
patients with NMD, neutrophil and platelet engraftment was achieved in 7/12 and 6/12 with median day of engraftment 21 days (range 18-28) $\kappa \alpha \iota 29$ days (range 22-70) respectively. Acute GVHD grade II-IV presented in 9/18 patients with MD and 4/12 with NMD, although none had cGVHD. The incidence of viral infections was 22 cases in 11 patients with MD and 9 cases in 6 patients with NMD. Disease relapse occured in $9 / 18$ patients with MD. After a median time of 10 years follow up, overall survival (OS) and event free survival (EFS) for children with MD were $22 \%$ and $18.5 \%$ respectively, while for NMD, OS and EFS were $33 \%$. Treatment related mortality at $d+100$ was $8 \%$ for MD and 50\% for NMD. Among 18 patients with MD, 4 are still alive, while the rest died from relapse (n: 8), viral infections (n: 4), septicemia (n: 1) and $\operatorname{aGVHD}(n: 1)$. Among 12 patients with NMD, 4 are alive, while the rest died from viral infections (n: 2), septicemia (n:4) and multiple organ failure $(n=2)$. The median time of hospitalization for patients with MD was 76 days (range 32-168), whilst for NMD was 63 days (range 28-114).
Conclusions: Transplantation of unrelated UCB in our unit was combined with high TRM in children with NMD and higher probability of relapse for MD.

Disclosure: Nothing to declare

\section{P643}

Serum levels of 5-S cysteinyldopa is associated with stem cell transplantation related complications

\section{Yukayo Terashita ${ }^{1}$, Mamoru Honda ${ }^{1}$, Minako Sugiyama ${ }^{1}$, Yuko Cho ${ }^{1}$, Akihiro Iguchi ${ }^{1}$}

${ }^{1}$ Hokkaido University Hospital, Pediatrics, Sapporo, Japan

Background: Diffuse hyperpigmentation is common in patients who received chemotherapy or stem cell transplantation (SCT). However, there are few reports of the relationship between skin reaction such as pigmentation and the other complications. Pigmentation of the skin is thought to be the result of melanin stagnating in the dermic layer due to increased synthesis of melanin and destruction of the basement membrane due to inflammation induced chemoradiotherapy. Melanin pigments are classified into two types: brown to black eumelanin and yellow to reddishbrown pheomelanin. 5-S Cysteinyldopa (5SCD) is precursors of pheomelanin, and its serum level has been used specific biochemical marker for malignant melanoma. Here, we examined serially 5SCD during the course of SCT to determine association with SCT related complications, because visual evaluation of skin color is difficult, and there have been no reports about 5SCD as SCT related biomarker.

Methods: We prospectively analyzed 41(27 males, 14 females) patients who received SCT between May 2011 and March 2015 in Hokkaido University Hospital. The median age at transplantation of the patients was 7.9 years (range, 0-22). Indication for SCT were acute myelogenous leukemia in 10 patients, acute lymphoblastic leukemia in 9 patients, and other disease in 22 patients; juvenile myelomonocytic leukemia(2), malignant solid tumor(11), immunodeficiency(6), anaplastic anemia(2), and Diamond Blackfan anemia(1). 34 patients received allogeneic SCT and 7 received autologous SCT. Myeloablative conditioning (MAC) was used for 30 patients and reduced intensity conditioning (RIC) was used for 11 patients. Sera were obtained from patients before conditioning therapy, on day $0,+5,+10,+15,+25$ and +40 . All blood samples were centrifuged at $3,000 \mathrm{rpm}$ for $15 \mathrm{~min}$, and stored at $-80^{\circ} \mathrm{C}$ until used. We also examined SCT related complications such as graft-Versus-host disease (GVHD), viral infection, and pre-engraft syndrome (PES). Statistical analyses were completed using the Mann-Whitney $U$ test for unpaired 
samples, and Kruskal-Wallis test for three samples. Each test was performed with a $5 \%$ level of significance.

Results: The average value of $5 \mathrm{SCD}$ reached two peaks, day0 $(21.6 \mathrm{nmol} / \mathrm{L})$ and day $5(21.7 \mathrm{nmol} / \mathrm{L})$, regardless of stem cell source and intensity of conditioning. In all patients, we found that the level of $5 \mathrm{SCD}$ on day0 was associated with viral reactivation $(\mathrm{p}=0.049), 5 \mathrm{SCD}$ on day5 was associated with PES $(\mathrm{p}=0.034)$, and $5 \mathrm{SCD}$ on day40 was associated with malignant disease $(\mathrm{p}=0.04)$. Similarly, in patient who received allogeneic SCT $(\mathrm{N}=34)$, the level of 5SCD on day0 was associated with viral reactivation $(\mathrm{p}=0.048), 5 \mathrm{SCD}$ on day5 was was associated with PES $(\mathrm{p}=0.034), 5 \mathrm{SCD}$ on day 40 was associated with malignant disease $(p=0.04)$. In addition, the level of $5 \mathrm{SCD}$ on day5 was associated with GVHD of skin $(p=0.027)$, the peak level of 5SCD was associated with acute GVHD $(p=0.04)$.

Conclusions: We found that $5 \mathrm{SCD}$ can be a biomarker for SCT-related complications such as aute GVHD. It is presumed that the production of pheomelanin could be induced by inflammatory procedure in SCT.

Disclosure: Nothing to declare

\section{P644}

HSCT in children with bone marrow failure: Outcomes from a single Singapore centre

Prasad Iyer', Michaela Seng ${ }^{1}$, Vijayakumari $K^{1}$, Ah Moy Tan $^{1}$, Mei Yoke Chan ${ }^{1}$, Rajat Bhattacharyya ${ }^{1}$

${ }^{1}$ KK Women's and Children's Hospital, Paediatric Haematology-Oncology, Singapore, Singapore

Background: Children presenting with pancytopenia often present a challenge to the paediatric haematologist. The underlying diagnosis can be hard to establish as many of the inherited bone marrow failure syndromes (IBMFS) can present with protean manifestations. The large majority of patients with bone marrow failure are often diagnosed with idiopathic severe aplastic anaemia (SAA) despite extensive testing. We report our experience of HSCT in patients treated with primary and acquired bone marrow failure.

Methods: We reviewed case notes of all the children who underwent HSCT for bone marrow failure in our centre.

Results: A total of fifteen patients underwent eighteen stem cell transplants in our centre between 2003 and 2016. Three patients were diagnosed with Fanconi Anaemia, one with Hoyeraal-Hreidarsson syndrome, one with paroxysmal nocturnal haemoglobinuria and the remaining ten children had idiopathic SAA. Eight children had matched sibling donor transplant, 1 had a matched related donor, 1 had a matched unrelated donor, 3 had umbilical cord blood transplants and the remainder 5 were haploidentical transplants. Four of the haploidentical transplants were T- cell depleted and one was T-cell replete. One child with Fanconi anaemia had primary graft rejection with cord blood transplant and was successfully rescued with a haploidentical transplant. One child with SAA had primary graft rejection twice (T-cell depleted graft) and then was rescued with an alternate haploidentical donor with a T-cell replete graft. Of the two patients who died, one had a fatal fungal infection ten months after transplant, and the other died due to a severe influenza pneumonitis three and a half years after BMT.

Conclusions: Haematopoietic stem cell transplant outcomes from our centre are comparable to leading centres in the world. The understanding of underlying conditions that present with bone marrow failure has improved our approach and the way we treat bone marrow failure syndromes.

Clinical Trial Registry: Not applicable.

Disclosure: Nothing to declare.

\section{P645}

Antifungal prophylaxis with posaconazole versus fluconazole in children with neutropenia following hematopoietic stem cell transplanation

\section{Bunchoo Pongtanakul ${ }^{1}$, Chonthida Wangkittikal ${ }^{1}$, Nattee Narkbunnam ${ }^{I}$, Kleebsabai Sanpakit ${ }^{1}$}

${ }^{1}$ Mahidol University, PediatricsBangkok, Thailand

Background: Invasive fungal infection (IFI) are common and contribute to mortality in pediatric patients undergoing hematopoietic stem cell transplantation (HSCT).The efficacy of posaconazole oral suspension (POS) compared to fluconazole (FLU) as primary antifungal prophylaxis in this setting was uncertain.

Methods: A retrospective study was performed in children treated with HSCT who received POS or FLU during early neutropenic period until engraftment from January 2000 to December 2017 at Siriraj hospital in Thailand. The efficacy, safety and tolerability of POS were compared to FLU.

Results: There were 66 HSCT recipients (allo-HSCT $62.1 \%$, auto-HSCT $37.9 \%$ ) with mean age of $7.6 \pm 4.3$ years. Most of the patients were thalassemia (34.4\%) followed by hematologic malignancy $(32.2 \%)$ and solid tumor $(16.7 \%)$. Seventeen and 49 cases received POS and FLU, respectively. All of patients in POS group were alloHSCT whereas $48.9 \%$ in FLU group were allo-HSCT. In POS group, 2 cases were diagnosed with suspected IFI and 2 cases were probable IFI with total 4 cases $(23.5 \%)$. In FLU group, 10 cases were diagnosed with suspected IFI and 2 cases were probable IFI with total 12 cases $(24.5 \%)$ which compared 2 groups were not statistically significant 
( $p=0.937$ ). No possible and proven IFI in both groups. In FLU group patients received empirical antifungal treatment more than POS group but no statistical significance (20.4\% VS.11.76\%, $p=0.498)$. Both groups had similar rate of elevated liver function test $(p=0.567)$. No early discontinuation of antifungal prophylaxis for intolerance was found in both groups. Only $26.7 \%$ of patients achieved POS target trough level of $0.7 \mathrm{mg} / \mathrm{L}$ after 7 days of treatment with started dose $4 \mathrm{mg} / \mathrm{kg}$ three times a day.

Conclusions: POS and FLU are comparably effective, safety and tolerability in IFI prophylaxis in neutropenic children treated with HSCT. Defining dose recommendation of POS in this setting requires larger studies.

Disclosure: Nothing to declare

\section{P646}

Hematopoetic stem cell transplantation in children with severe congenital neutropenia

Akif Yeşilipek ${ }^{1}$, Vedat Uygun ${ }^{1}$, Volkan Hazar ${ }^{2}$, Hayriye Daloglu ${ }^{1}$, Seda Irmak Öztürkmen ${ }^{1}$, Suna Celen ${ }^{2}$, Suar Çakı Kılıç2, Gülsün Karasu ${ }^{2}$

${ }^{1}$ MedicalPark Antalya Hospital, Antalya, Turkey, ${ }^{2}$ MedicalPark Göztepe Hospital, İstanbul, Turkey

Background: Severe congenital neutropenia $(\mathrm{SCN})$ is typically characterized by ANC of $<500 / \mu l$, maturation arrest of bone marrow myeloid precursors at the promyelocyte- myelocyte stage, and susceptibility to lethal pyogenic bacterial and fungal infections. SCN is a rare group of disorders resulting from intrinsic defects in myeloid cell proliferation and maturation caused by mutations in several genes; ELANE, HAX1, GFI1, WAS, and G6PC3 are among the most common ones. Almost $10 \%$ of patients are refractory to G-CSF, and the only definitive curative approach for such patients is allogeneic HSCT. The current absolute indications for HSCT is failure to respond to GCSF treatment, or the development of MDS/leukemia in patients with SCN. Here, we present the result of 10 children with SCN who received allogeneic HSCT .

Methods: We retrospectively assessed 10 allogeneic HSCT in children with severe congenital neutropenia. All patients received busulphan (BU) based myeloablative conditioning regimen. Busulphan was used according to weight adjusted dose. In addition, all patients received fludarabine $150 \mathrm{mg} / \mathrm{m} 2$ in five days or cyclophosphamide $200 \mathrm{mg} / \mathrm{kg}$ in 4 days and ATG $30 \mathrm{mg} / \mathrm{kg}$ in 3 days. Cyclosporin-A and MTX were used for graft versus host disease (GVHD) prophylaxis. Donor chimerism was evaluated in either bone marrow or peripheral blood on days $+30,+100$ and +180 .
Results: The median transplantation age of the patients was 49 months (range 11-167 months). Six of them are male. Two of the donors were matched siblings and 8 were unrelated two of which were 1 ag 1 ag mismatched. Stem cell source was bone marrow in 6 patients, peripheral blood in 2 and cord blood in 2 patients. All patients engrafted. The median time of neutrophil and platelet engratment to was 15 (13-34) days and 16(9-90) days, respectively. Graft rejection was experienced in 2 patients, one of them had received unrelated cord blood. All patients are alive, eight of which are with full donor chimerism (between 95-100\%) without any complication (no infection, no GVHD) with a median 40 months (range 24-83 months) follow up. Probability of disease free and overall survival were found $80 \%$ and $100 \%$, respectively.

Conclusions: We concluded that HSCT is a useful treatment for SCN patients, especially those who are unresponsive to GCSF treatment and at high risk for leukemic transformation. However, a larger number of SCN patients and longer follow-up are necessary to identify appropriate conditioning regimens and long-term prognosis.

Disclosure: Nothing to declare

\section{P647}

Eltrombopag use in secondary failure of platelet recovery in pediatric hematopoietic stem cell transplant patients

Blanca Rosich del Cacho ${ }^{1}$, Yasmina Mozo del Castillo ${ }^{1}$, David Bueno $^{I}$, Luisa Sisini ${ }^{1}$, Antonio Perez Martinez ${ }^{1}$

${ }^{1}$ Hospital Universitario La Paz, Madrid, Spain

Background: Prolonged thrombocytopenia (PT) or secondary failure of platelet recovery (SFPR) are a lifethreatening complications that occurs in $20-40 \%$ and 12 $20 \%$ respectively of the patients following allogeneic hematopoietic stem cell transplantation (allo-HSCT). Management strategies, including the use of growth factors, CD34+-selected stem cell boost, mesenchymal stem cell (MSC) transfusion, and second allo-HSCT, are not effective or possible for all patients.

Eltrombopag, is an oral non-peptide thrombopoietin receptor agonist, that leads to signal transduction and results in promoting the proliferation and differentiation of megakaryocytes. Some recently studies show that also can promote haematopoiesis along all three lineages.

Methods: We described our experience in 4 paediatric patients with poor graft function or secondary failure of platelet recovery after allogeneic stem cell transplantation treated with Eltrombopag.

Results: Patients characteristics are detailed in Table 1. All the patients received and allo-HST. The median dose of 
CD34+ cells infusion was $6.43 \times 10 \mathrm{e} 6 / \mathrm{kg}$ (3.95-8.29). Neutrophils engraftment occurred in +15 day (10-21d) and platelets in +26 day (16-42d).

All the patients had an hypoplastic bone marrow with complete chimerism. The median duration from transplantation to SPCF diagnosis was 10 months $(1.5-24 \mathrm{~m})$. One of the patients received a stem cell boost prior to Eltrombopag, without response.

The time onset from sPGF/sFPR diagnosis to initiating Eltrombopag was 16 days (8-32d). Eltrombopag was started at a dose of $1 \mathrm{mg} / \mathrm{kg} / \mathrm{d}$, requiring an increase dose in all cases. The median dose was $50 \mathrm{mg} / \mathrm{d}(25-100 \mathrm{mg})$.

The overall response rate was $50 \%$ (2/4). Two patients achieved complete response (CR), as defined by platelet $\geq$ $50 \times 109 / \mathrm{L}$. Both patients already got neutrophil $\geq 1.0 \times$ 109/L without G-CSF. The time from Eltrombopag initiation to achieving CR was 21 (10-49d) days.

The treatment was given for a median of 81 days (8-203). It was discontinued after 96 and 203 days respectively in the two responder patients. Both patients maintain stable blood counts after discontinuing the treatment.

The non-responders patients had to stop the treatment because of other reasons not related to Eltrombopag. Patient 4 had to be rescued with a CD34+ cells boost with a good response.

Two patients that were in treatment with voriconazole for a fungal infection developed hyperbilirubinemia. There were no grade 3-4 toxicities related to Eltrombopag.

Conclusions: In our experience, according to recently published studies, Eltrombopag is a safe and efficacy drug in the treatment of secondary failure of platelet recovery post-HSCT. It may be used successfully in children. Sometimes higher doses may be considered if no response is achieved.

Further prospective trials are needed to increase the level of evidence and to identify predictors of response.

Disclosure: Nothing to declare

\section{P648 \\ Very slow clearance of busulfan in a child with infant leukemia}

\section{Claudia Langebrake $^{1,2}$, Matthias Bleeke ${ }^{3}$, Ingo Müller ${ }^{3}$}

${ }^{1}$ University Medical Centre Hamburg-Eppendorf, Hamburg, Germany, ${ }^{2}$ University Medical Center Hamburg-Eppendorf, Hospital Pharmacy, Hamburg, Germany, ${ }^{3}$ University Medical Centre Hamburg-Eppendorf, Hamburg, Germany

Background: Busulfan is a drug with a high interindividual variability between dose and exposition. Therefore, it is recommended to perform therapeutic drug monitoring
(TDM) in the context of myeloablative conditioning, especially in children.

Methods: We report on a 7-month old boy $(7.2 \mathrm{~kg}, 66$ $\mathrm{cm}$ ) of caucasian decent born to non-consanguine parents with MLL-rearranged proB-lymphoblastic leukemia. Diagnosis was established one month after birth from peripheral blood and CSF tap showed CNS involvement. Primary chemotherapy was commenced according to the Interfant06 protocol. However, MRD remained positive two months under treatment, leading to an indication for allogeneic stem cell transplantation. In the Interfant-06 protocol, we opted for a conditioning regimen comprised of fludarabine (1.2 $\mathrm{mg} / \mathrm{kg}$ for 5 days), busulfan and thiotepa $(2 \times 5 \mathrm{mg} / \mathrm{kg}$ ). In our institution, busulfan is applied once daily with a target AUC of $85-95 \mathrm{~h} * \mathrm{mg} / \mathrm{l}$ in this very high risk situation. According to body weight, busulfan was given with $5.1 \mathrm{mg} /$ $\mathrm{kg}$ as a three-hour infusion on the first day. Busulfan concentrations in plasma were measured with gas chromatography-mass spectrometry (GC-MS) and AUC was calculated using Bayesian curve fitting.

Results: Exact busulfan quantification was not possible after the first dose due to technical reasons. As the levels were estimated to be very high, we decided to reduce the second dose of busulfan by $25 \%$. This resulted in a very high AUC of $44 \mathrm{~h}^{*} \mathrm{mg} / \mathrm{l}$ for the second dose, so that busulfan was discontinued after two days, because it was calculated that the patient already received busulfan with a cumulative AUC of $90 \mathrm{~h} * \mathrm{mg} / \mathrm{ml}$. Trough levels after the first and second dose were 547 and $572 \mu \mathrm{g} / \mathrm{l}$, respectively. The patient showed a very slow clearance of $2.1 \mathrm{l} / \mathrm{h} / \mathrm{sqm}$, while the volume of distribution was in the usual range $(0.86 \mathrm{l} / \mathrm{kg})$. Bilirubin and liver transaminases were in the normal range at the time of conditioning, while albumin and Quick were decreased

On day +18 after transplantation the patient developed clinical und biochemical signs of venoocclusive disease (VOD). VOD symptoms completely resolved under therapy with defibrotide. Leukocyte engraftment was established on day +14 . Unfortunately, the patients suffered from an early relapse of the leukemia from day +62 . Attempts to induce a second remission with blinatumomab failed. The patient is currently under palliative chemotherapy.

Conclusions: Busulfan TDM is very important especially in infants receiving myeloablative doses of busulfan to prevent under- or over-exposure. There is evidence that high busulfan trough levels contribute to the development of VOD, but anti-leukemic activity of busulfan and CNS permeability make it a valuable drug for very high risk patients in childhood leukemia. Larger patient cohorts are needed to assess the exposure dependent risks of toxicity versus relapse in infants and toddlers.

Disclosure: Nothing to declare 


\section{P649}

Peculiarities of psychosexual development of adolescents after hematopoietic stem cell transplantation

Svetlana Oleshko ${ }^{1}$, Alisa Volkova ${ }^{1}$, Asmik Gevorgian ${ }^{2}$, Olesya Paina ${ }^{3}$, Anna Osipova ${ }^{4}$, Tatyana Bykova ${ }^{3}$, Kirill Ekushov $^{4}$, Ludmilla Zubarovskaya ${ }^{3}$, Inna Markova ${ }^{3}$, Boris Afanasyev ${ }^{3}$

${ }^{1}$ First I. Pavlov State Medical University of St. Petersburg/ Raisa Gorbacheva Memorial Institute of Children's Oncology, Hematology and Transplantation, Rehabilitation Medicine, Saint Petersburg, Russian Federation, ${ }^{2}$ First I. Pavlov State Medical University of St. Petersburg/Raisa Gorbacheva Memorial Institute of Children's Oncology, Hematology and Transplantation, Bone Marrow Transplantation for Pediatric Solid Tumor, Saint Petersburg, Russian Federation, ${ }^{3}$ First I. Pavlov State Medical University of St. Petersburg/Raisa Gorbacheva Memorial Institute of Children's Oncology, Hematology and Transplantation, Saint Petersburg, Russian Federation, ${ }^{4}$ First I. Pavlov State Medical University of St. Petersburg/Raisa Gorbacheva Memorial Institute of Children's Oncology, Hematology and Transplantation, Pediatric HSCT Outpatients, Saint Petersburg, Russian Federation

Background: The transplantation of hematopoietic stem cells (HSCT) is one of the leading methods of treatment in patients with blood system diseases, primary immunodeficiency syndromes and genetic diseases. At the same time, the quality of life in patients in the long-term after HSCT significantly differs from the quality of life of healthy people of the same age.

Deformations in psychosexual development including problems in the gender identity formation cause social isolation of adolescents, which makes their sexual selfrealization impossible and significantly reduces the quality of their life. The purpose of our study was an assessment of the level of gender identity formation of adolescents and psychosexual development correlation to the normal adolescents of the same age.

Methods: In a prospective single-center study in 2018, on the base of the Department of Rehabilitation Medicine Raisa Gorbacheva Memorial Institute of Children's Oncology, Hematology and Transplantation, we conducted a study of 17 families. The respondents were: 1) parents / guardians of patients accompanying them in the process of examination; 2) adolescents who underwent HSCT treatment and undergo planned examinations at the clinic in the posttransplant period (after $\mathrm{D}+100),(\mathrm{n}=17$, of which 8 girls and 9 boys, age $12-17$ years, from the date of HSCT 1 - 5 years).
The following methods were used to assess gender identity: Specially developed questionnaires for teenagers and parents; questionnaire by Sandra L. Bem (Sandra L. Bem, 1974); projective techniques "The Human Picture", "The non-existent animal"; Max Lüscher's color choices test.

Results: The traditional type of gender identity, which characterizes high masculinity among male respondents and high female gender indicators in $100 \%$ of cases, was not revealed. Both among girls and among boys, the androgynous type prevails with a tendency toward femininity.

On average, adolescents see themselves as a bit more courageous than their mothers, with rare exceptions, regardless of gender. This confirms the thesis that we received in a previous study that parents tend to see and encourage complacency of adolescents of both sexes, passivity instead of leadership, dedication and independence. All $100 \%$ of adolescents who participated in the test demonstrate a shift in the theme of aggression, $77 \%$ have some signs of preventing sexual self-determination, abandoning their body, gender, and age. $88 \%$ of patients do not communicate with their peers. In $47 \%$ of them, negative emotions prevail over positive ones. One third of the test participants demonstrate strong support for rest and minimizing their efforts.

Conclusions: The characteristics of family upbringing of adolescents who have undergone HSCT often contribute significantly to limiting their social experience and lead to specific deformities of individuality, including in the sphere of gender identity. We consider advisable to introduce thematic group counseling of parents within the framework of the "Patient's School" in psychological treatment support in the clinic. Early diagnosis of the personal aspects of the psychosexual development of adolescents after HSCT allows for timely identification of individual problems in this area and identification of general trends in the long term after HSCT.

Disclosure: All authors - nothing to disclose.

\section{P650}

Abnormalities in the morphology of the umbilical cord blood obtained at delivery from children who received a diagnosis of cerebral palsy

\section{Maciej Boruczkowski ${ }^{1}$, Izabela Zdolińska-Malinowska ${ }^{2}$, Maciej Rojek $^{2}$, Dariusz Boruczkowski ${ }^{2}$}

${ }^{1}$ Poznan University of Medical Sciences, Chair of Pathomorphology and Clinical Immunology, Poznań, Poland,

${ }^{2}$ The Polish Stem Cell Bank, Warsaw, Poland

Background: The aim of this experiment was to detect abnormalities in the morphology of the umbilical cord 
blood (UCB) obtained at delivery from three children who received a diagnosis of cerebral palsy.

Methods: Immunophenotyping of the UCB leukocyte fraction was performed using multicolor flow cytometry. The procedure was performed according to the protocol by Shatorje and colleagues (1). Briefly, the UCB samples were labeled with specific antibodies and incubated in the dark for 30 minutes. Afterwards, the samples were treated with $5 \mathrm{ml}$ of BD FACS Lysing Solution for 10 minutes to preserve the leukocyte fraction only. The cells were washed using PBS (Roche) and then centrifuged twice (1500 RPM, $4^{\circ} \mathrm{C}, 4$ minutes). The results were analyzed using the FACSDiva software (Becton Dickinson).

Results: We found an increased white blood cell (WBC) count, lymphocyte count, and CD4:CD8 ratio in all UCB samples. One patient had a low NK cell count and percentage, and another had a low B-cell count and percentage. One sample displayed high $\mathrm{T}(\mathrm{CD} 3+)$ and $\mathrm{Th}$ cell $(\mathrm{CD} 4+)$ counts, but with percentages within the limits of the reference values.

Conclusions: We detected elevated WBC and lymphocyte counts in all UCB samples, despite a lack of intrauterine infection symptoms. Many authors have described the pathogenesis of hypoxic-ischemic encephalopathy. Briefly, after an acute hypoxia-ischemia insult, activated resting microglia show macrophage-like activity. This leads to a break-down of the blood-brain barrier, infiltration by peripheral leukocytes, and brain exposure, which further exacerbates inflammation. The role of systemic inflammation is being evaluated in the animal model. It is known that systemic inflammation plays a role in traumatic brain injury (TBI) and is an independent risk factor for poor outcome in isolated TBI patients. On the other hand, M2-phenotype microglia inhibit inflammation and protect neurons from secondary damage and death. However, anti-inflammatory mechanisms in neonates are immature and expose them to extremely intensive inflammation. Therefore, anti-inflammatory agents, including stem cells, may be beneficial in these patients.

Disclosure: Three of four authors are employees of the Polish Stem Cell Bank, Warsaw, Poland

\section{Reference:}

(1) Schatorje' E. J. H., Gemen E. F. A., Driessen_ G. J. A., Leuvenink J., van Hout R. W. N. M., de Vries E. Paediatric Reference Values for the Peripheral $\mathrm{T}$ cell Compartment. Scandinavian Journal of Immunology, 2012, 75, 436-444

\section{P651}

Donor natural killer cell infusion following haploidentical stem cell transplantation in children with recurrent neuroblastoma
Young Bae Choi ${ }^{1}$, Na Hee Lee ${ }^{2}$, Eun Sang $\mathrm{Yi}^{3}$, Ji Won Lee $^{4}$, Eun-Suk Kang ${ }^{5}$, Keon Hee Yoo $^{4}$, Jung Hyun Her ${ }^{6}$, Okjae Kim ${ }^{6}$, Miyoung Jung ${ }^{6}$, Yu-Kyeong Hwang ${ }^{6}, \mathrm{Ki}$ Woong Sung ${ }^{4}$, Hong Hoe Koo ${ }^{4}$

${ }^{1}$ Chungbuk National University Hospital, Pediatrics, Cheongju, Korea, Republic of, ${ }^{2}$ Cha Bundang Medical Center, Cha University, Seongnam, Korea, Republic of, ${ }^{3}$ Korea University Guro Hospital, Korea University College of Medicine, Seoul, Korea, Republic of, ${ }^{4}$ Samsung Medical Center, Sungkyunkwan University School of Medicine, Pediatrics, Seoul, Korea, Republic of, ${ }^{5}$ Samsung Medical Center, Sungkyunkwan University School of Medicine, Seoul, Korea, Republic of, ${ }^{6}$ MOGAM Biotechnology Institute, Yongin, Korea, Republic of

Background: Under the hypothesis that early natural killer cell infusion (NKI) following haploidentical stem cell transplantation (haplo-SCT) will reduce relapse in the early post-transplant period, we conducted a pilot study to evaluate the safety and feasibility of NKI following haplo-SCT in children with recurrent neuroblastoma who failed previous tandem high-dose chemotherapy and autologous SCT.

Methods: We used the high-dose ${ }^{131}$ I-metaiodo benzylguanidine and cyclophosphamide/fludarabine/antithymocyte globulin regimen for conditioning and infused $3 \times 10^{7} / \mathrm{kg}$ of ex-vivo expanded NK cells derived from a haploidentical parent donor on days 2,9 , and 16 posttransplant.

Results: Seven children received a total of 19 NKIs, and NKI-related acute toxicities were fever $(n=4)$ followed by chills $(\mathrm{n}=3)$ and hypertension $(\mathrm{n}=3)$; all toxicities were tolerable. Grade $\geq$ II acute GVHD and chronic GVHD developed in two and five patients, respectively. Higher amount of NK cell population were detected in peripheral blood until 60 days post-transplant compared with reference cohort. Cytomegalovirus and BK virus reactivation occurred in all patients and Epstein-Barr virus in six patients. Six patients died of relapse/progression $(n=5)$ or treatment-related mortality $(\mathrm{n}=1)$, and one patient remained alive.

Conclusions: NKI following haplo-SCT was relatively safe and feasible in patients with recurrent neuroblastoma. Further studies to enhance the graft-versus-tumor effect without increasing GVHD are needed.

Disclosure: Nothing to declare

Regenerative medicine

P652

Repeated administration of G-CSF using stem cell mobilization protocol could induce improvement of 
cognitive functions of children with cerebral palsy: Phase II randomized placebo-controlled study

Young-Ho Lee ${ }^{1}$, Jin Hwa Moon ${ }^{1}$, Hani Koh ${ }^{1}$, Hyun-Joo Jun $^{1}$, Ae-Ri Sung ${ }^{1}$, Mi Jung Kim ${ }^{2}$, Young-Jun Lee ${ }^{1}$, Hyewon Park', Jee Sun Lee ${ }^{3}$, Kyu Bum Lee ${ }^{1}$, Jong-Min Lee $^{1}$, SeJin Hwang ${ }^{1}$, Eunwoo Nam ${ }^{1}$

${ }^{1}$ Hanyang University Medical Center, Seoul, Korea, Republic of, ${ }^{2}$ University of Ulsan, Ulsan, Korea, Republic of, ${ }^{3}$ Seoul Rehabilitation Hospital, Seoul, Korea, Republic of,

Background: We performed phase II randomized placebocontrolled clinical study to reveal the safety and feasibility of repeated granulocyte colony-stimulating factor (G-CSF) administration for improvement of cognitive functions of children with cerebral palsy.

Methods: Forty-four children with non-severe type of cerebral palsy were enrolled, and their age were 2-10 years old. G-CSF $(5 \mu \mathrm{g} / \mathrm{kg})$ was administered for 5 days subcutaneously every 3 months during 18 months. We compared their cognitive functions with the magnetic resonance imaging (MRI) findings and the following tools between before and 18 months after treatment; zoo location and picture memory as working memory index (WMI) in Wechsler Preschool and Primary Scale of Intelligence (WPSSI), receptive and expressive vocabulary test (REVT), and visual motor integration (VMI)/visual perception (VP) test. Mobilized stem cell count and cytokine levels were measured before $(\mathrm{D}+0)$ and after $(\mathrm{D}+5)$ G-CSF administration for 5 days every 3 months.

Results: No significant findings in demography was noticed between G-CSF (G-) and placebo (P-) groups. No serious adverse events were observed during the whole study period. The non-severe adverse events such as urticaria $(n=1)$, itching sense $(n=3)$, bone pain $(n=1)$, headache $(n=1)$, fever $(n=2)$, and stomatitis $(n=1)$ were tolerable. The parents felt the clinical improvements of cognition in 10 cases of G-group and 4 cases of P-group $(p=0.0367)$, of language in 8 cases of G-group and 3 cases of P-group ( $\mathrm{p}=0.0632$ ). In zoo location test, we can not find out the significant score (expressed as age equivalent) differences between G- and P- groups. However, in picture memory test, there were significant improvement of age equivalent of 10 months $(45.60 \pm 19.66 \rightarrow 55.60 \pm 23.27)$ during 18 months of study period in G-group compared to 3 months in P-group ( $p=0.0242)$. In REVT, there were significant improvement of 18 months of age equivalent in expressive tests of G-group $(57.87 \pm 33.66 \rightarrow 76.00 \pm 43.38$, $\mathrm{p}=0.0198)$ compared to 8 months in P-group. No significant findings were noted in receptive test. VMI test showed the increasing tendency of 14 months of age equivalent in G-group $(47.75 \pm 13.22 \rightarrow 61.13 \pm 17.78$, $\mathrm{p}=0.0746)$ compared to P-group. The increment of CD34 + cell counts in peripheral blood were significant in Ggroup compared to P-group. The changed levels of interleukin (IL)-6, IL-10, vascular endothelial growth factor (VEGF) as well as G-CSF were noted in G-group. We also observed the correlation of cognitive function tests and white matter connectoms of several networks using functionally-defined white matter atlases.

Conclusions: The repeated administration of G-CSF using stem cell mobilization protocol is safe and feasible to improve the language and cognitive functions in children with cerebral palsy. Further studies for cellular and paracrine effect of G-CSF and/or mobilized peripheral blood stem cells would be needed.

Clinical Trial Registry: ClinicalTrials.gov NCT02866331

Disclosure: Nothing to declare

Solid tumours

\section{P653}

High-dose chemotherapy with autologous or allogeneic hematopoietic stem cell transplantation for high-risk neuroblastoma patients, a single transplant center experience

Ilya Kazantsev', Asmik Gevorgian', Tatiana Iukhta ${ }^{1}$, Polina Tolkunova ${ }^{1}$, Andrew Kozlov ${ }^{I}$, Tatiana Andreeva ${ }^{1}$, Elena Morozova ${ }^{1}$, Andrey Litvinov ${ }^{1}$, Polina Kuga ${ }^{I}$, Svetlana Safonova ${ }^{1}$, Yuri Punanov ${ }^{1}$, Liudmila Zubarovskaya ${ }^{I}$, Boris Afanasyev ${ }^{I}$

${ }^{1}$ R.M. Gorbacheva Institute of Pediatric Oncology Hematology and Transplantation, I.P. Pavlov First St. Petersburg State Medical University, Saint Petersburg, Russian Federation,

Background: While high-dose chemotherapy (HDCT) with autologous hematopoietic stem cell transplantation (autoHSCT) is an integral part of multimodal therapy for highrisk neuroblastoma (HR NB), there are still subgroups, in which the results are extremely poor. For these patients allografting (allo-HSCT) may offer some hope.

Methods: We summarize the experience of 83 consecutive HR NB patients receiving therapy in our pediatric transplant department in 2008-2017. The median age was 4 years ( 8 months to 22 years). A total of 78 auto-HSCTs and 20 allo-HSCTs were performed. All auto-HSCT recipients were characterized by one or several high-risk features: age of more than 18 months at disease (onset $\mathrm{n}=56$ ), primary disseminated disease $(n=59)$, unfavorable biologic variant $(\mathrm{n}=29)$, poor $1^{\text {st }}$-line therapy response $(\mathrm{n}=16)$ or systemic relapse $(n=9)$. Most patients $(n=75)$ received Bu-Mel 
HDCT (in younger patients oral busulfan was replaced by Busilvex), in 3 primary resistant cases a 5D/5D regimen was used. A total of 20 patients with $1 \mathrm{st}(\mathrm{n}=11)$ or $2 \mathrm{nd}(\mathrm{n}=2)$ chemosensitive relapse, resistant relapse $(n=4)$ or poor mobilizers with locally advanced resistant tumor $(n=3)$ received allo-HSCT from haploidentical donor with fludarabine-based RIC. In 12 cases the transplant was modified via immunomagnetic positive or negative selection, 8 patients received post-transplant cyclophosphamide (post-CY)-based GVHD prophylaxis. GVHD prophylaxis also consisted of calceneurin inhibitors and sirolimus. Thirteen of 20 allo-HSCT recipients received posttransplant immunoadoptive $(n=6)$ or targeted $(n=7)$ therapy.

Results: The 5-year OS and EFS in auto-HSCT recipients was $48 \%$ and $36 \%$, accordingly. All but one patient engrafted with a median time of 17 (11-51) days. Bu-Mel regimen was characterized by acceptable toxicity with most common toxicities being oral mucositis and infectious complications. The VOD/SOS incidence was only $3 \%$. Four patients dies due to infection $(n=2)$, CNS hemorrhage $(\mathrm{n}=1)$, and secondary leukemia $(\mathrm{n}=1)$. According to multivariate analysis the most important prognostic factors were response to $1^{\text {st }}$ line therapy and post auto-HSCT MIBG scan results. The prognosis in initially resistant patients with good response to $2^{\text {nd }}$ or $3^{\text {rd }}$-line therapy was still very poor (all patients relapsed with the median EFS of 12 months). All patients receiving a second auto-HSCT after relapse died due to disease progression. With a median follow up of 6 (1-80) months 9 allo-HSCT recipients are alive, 6 of them with no signs of disease progression. All long-term responders received post-transplant therapy. One patient died due to transplant complications, other deaths were caused by disease progression. There was no obvious difference between outcomes in post-CY based and transplant modification-based transplantations. aGVHD more often developed in modified transplant recipients (8 patients vs 2 in post-CY group, 4 of these cases Gr III-IV), 3 patients in post-CY group had Grade III-IV hemorrhagic cystitis. The median time to engraftment was longer for PTCM group compared to transplant modification group (D +23 vs. $\mathrm{D}+17$, accordingly).

Conclusions: While single HSCT with auto-HSCT is a golden standard in HR NB patients, the relapse rate is still high and the prognosis in relapsed/refractory patients is dismal. The allografting has some limited effectiveness in these cases and post-transplant therapy has a potential for further improvement.

Disclosure: Nothing to declare

\section{P654}

Abstract already published.
P655

Veno-occlusive liver disease (VOD) is frequent but well treatable with early defibrotide administration in children with neuroblastoma receiving high-dose busulfan and melphalan

Peter Svec, Anna Panikova ${ }^{1}$, Petra Keslova ${ }^{2}$, Ivana Bodova $^{1}$, Renata Formankova ${ }^{2}$, Petr Riha ${ }^{2}$, Julia Horakova', Ales Luks², Josef Malis ${ }^{2}$, Pavel Bician ${ }^{3}$, Vladimira Jerabkova ${ }^{2}$, Natalia Galoova ${ }^{4}$, Alexandra Kolenova $^{1}$, Petr Sedlacek ${ }^{2}$

${ }^{1}$ National Institute of Children's Diseases, Bratislava, Slovakia, ${ }^{2}$ Charles University Hospital Motol, Prague, Czech Republic, ${ }^{3}$ Children's University Hospital Banska Bystrica, Banska Bystrica, Slovakia, ${ }^{4}$ University Children Hospital UPJS, Kosice, Slovakia

Background: Using high-dose intravenous busulfan and melphalan (BuMel) prior to autologous stem cell transplantation (SCT) in children with high-risk neuroblastoma, seems to decrease toxicity of the myeloablative regimen, except for VOD. In this multicenter retrospective study we aimed to assess the outcome of BuMel-associated VOD with early defibrotide treatment intervention.

Methods: We retrospectively analyzed 64 children with high-risk neuroblastoma who underwent autologous SCT with i.v. BuMel regimen in Slovakia and Prague, Czech Republic in the period 1/2008 - 10/2018. Busulfan was administered in $\mathrm{q} 6$ hour schedule, with therapeutic drug level monitoring in $84 \%$ of patients. All VOD patients except one were treated with defibrotide starting at a standard dose of $25 \mathrm{mg} / \mathrm{kg} /$ day, given in 4 doses per day. 1 patient was treated with supportive therapy only. Ursodeoxycholic acid was used as prophylaxis in all patients. VOD was established using the modified Seattle clinical criteria (Corbacioglu, Lancet 2012).

Results: The incidence of VOD was 23\% (15/64) in patients treated with intravenous busulfan and melphalan. There was no significant difference in busulfan total dose $/ \mathrm{kg}$ between patients with $(19.2 \mathrm{mg} / \mathrm{kg}(\mathrm{SD}=1,8))$ and without $(18.0 \mathrm{mg} / \mathrm{kg}(\mathrm{SD}=2,4))$ VOD manifestation. VOD developed at a median of 17 days after SCT (range 11 - 22 days). Anicteric forms of VOD were documented, although $73 \%$ patients with VOD (11/15) presented with increased bilirubin. $73 \%$ patients with VOD (11/15) developed ascites but only 4 patients $(27 \%)$ required ascites drainage. No VOD patient received renal replacement therapy and only one needed mechanical ventilation. Importantly, we successfully treated VOD in all patients. Relapse or progression of neuroblastoma was the cause of death in 4 VOD patients $(27 \%)$ who died. 
Conclusions: Despite targeting busulfan levels to decrease toxicity of the regimen, VOD is common (we observed VOD incidence exactly in the range of the SIOPEN HR NBL-1 multicenter study (Ladenstein, 2017)). Early recognition and early treatment with defibrotide seems to be effective in VOD associated with BuMel regimen - none of our 15 patients died due to VOD.

Disclosure: Nothing to declare

\section{P656}

Results of high-dose chemotherapy (HDCT) with autologous hematopoietic stem cell transplantation (auto-HSCT) in the treatment of ewing sarcoma family tumors (ESFT)

Tatiana Iukhta ${ }^{I}$, Ilya Kazantsev ${ }^{I}$, Asmik Gevorgian ${ }^{I}$, Andrey Kozlov ${ }^{I}$, Polina Tolkunova ${ }^{I}$, Tatiana Andreeva ${ }^{I}$, Elena Morozova ${ }^{\text {, }}$, Yury Punanov ${ }^{1}$, Ludmila Zubarovskaya $^{I}$, Boris Afanasyev ${ }^{I}$

${ }^{1}$ R.M. Gorbacheva Memorial Institute for Pediatric Oncology, Hematology and Transplantation, I.P.Pavlov Saint Petersburg First State Medical University, St. Petersburg, Russian Federation

Background: While current dose-intense treatment protocols allow achieving 70-80\% survival in localized ESFT patients, the long-term survival in high-risk cases is still unsatisfactory. Although there is a considerable body of data on high-dose consolidation the real effectiveness and optimal indications for this option are still not completely clarified. Therefore, a large prospective cohort analysis may still yield useful data.

Methods: The whole cohort includes 73 consecutive highrisk ESFT patients with median age of 12 (range 1-23) years receiving HDCT with auto-HSCT in 2007 to 2018 after obtaining $1^{\text {st }}(n=25)$ or $2^{\text {nd }}(n=3) C R, P R(n=35)$, or stable disease $(n=10)$. The high-risk features included lung $(n=52)$, bone $(n=25)$ or bone marrow $(n=11)$ involvement, inadequate local control in primary axial tumors $(n=52)$, large lesions volume or poor treatment response $(\mathrm{n}=52)$, and chemosensitive relapse $(n=9)$. Most patients had several risk factors. Disseminated disease patients were also evaluated according to prognostic score by R.Ladenstein et al. Highdose busulfan-melphalan followed by autologous stem-cell transplantation (HDT/SCT) was used.

Results: The median observation time was 60 (range 3130) months. The 5-year overall (OS) and event-free (EFS) survival were $40 \%$ and $37 \%$, accordingly. Most important outcome predictors were inadequate local control in chemoresistant cases, a primary tumor volume more than $200 \mathrm{~mL}$, more than one bone metastatic site, bone marrow involvement and additional lung metastases. According to prognostic risk score in disseminated disease ESFT patients identified three groups with 5-year OS rates of $49 \%$ for score $\leq 3$ (19 patients), $36 \%$ for score 3 to 5 (27 patients), and $8 \%$ for score $\geq 5$ (12 patients), $(\mathrm{P}<0.01)$.

Conclusions: While Bu-Mel HDCT with auto-HSCT may still be a feasible option with acceptable toxicity for chemosensitive patients with inadequate local control and some primarily disseminated cases it is ineffective in primary resistant or very high-risk metastatic patients.

Disclosure: Nothing to declare

\section{P657}

Efficacy of tandem high-dose chemotherapy with autologous hematopoietic stem cell transplantation in the treatment of infant embryonal brain tumors

Asmik Gevorgian, Polina Tolkunova ${ }^{1}$, Tatiana Iukhta, Elena Morozova', Ilya Kazantsev ${ }^{I}$, Andrew Kozlov ${ }^{I}$, Yury Punanov $^{1}$, Ludmila Zubarovskaya', Olga Zheludkova ${ }^{2}$, Boris Afanasyev ${ }^{I}$

${ }^{1}$ R.M. Gorbacheva Institute of Pediatric Oncology Hematology and Transplantation, I.P.Pavlov First St. Petersburg State Medical University, St. Petersburg, Russian Federation, ${ }^{2}$ Scientific Center of Roentgenoradiology, Moscow, Russian Federation

Background: Embryonal brain tumors are the most common malignancies in infants less than 48 months of age. Histologically characterized as undifferentiated small round cell tumors, all are similarly aggressive, have a tendency to disseminate throughout central nervous system and very poor prognosis. We tried to assess the effectiveness of tandem highdose chemotherapy (HDCT) with autologous hematopoietic stem-cell transplantation (auto-HSCT) in this patient group.

Methods: From 2010 to 2018, 52 infants under 48 months with different primary embryonal brain tumors such as medulloblastoma $(\mathrm{N}=28)$, different PNET NOS $(\mathrm{N}=10)$, pineoblastoma $(\mathrm{N}=3)$, atypical teratoid rhabdoid tumor $(\mathrm{N}=3)$, ETMR ( $=8)$ after surgical resection and induction chemotherapy were planned to receive tandem HDCT with auto-HSCT. Nine patients were conducted only single transplantation because of the development of lifethreatening complications after the first HDCT $(\mathrm{N}=4)$ or the emergence of early disease progression $(\mathrm{N}=5)$. At the moment of HDCT 31 patients were in complete remission (CR), 20 patients were in partial remission (PR) and 1 patient had stable disease (SD). The conditioning regimen for tandem auto-HSCT were: the first HDCT was carboplatin and etoposide, the second was thiotepa and cyclophosphamide, both with intraventricular methotrexate.

Results: The median follow-up is 24 months (range, 6-85). The median time to engraftment after the first auto-HSCT was 
day +15 (range, $8-83$ ), after the second auto-HSCT was day +17 (range, 9-86). Two-year overall survival (OS) was $76 \%$ and disease free survival (DFS) was 68\%. DFS was significantly better among patients with $\mathrm{MB}(95 \%)$ and PNET (75\%) in compared to children with ETMR (55\%), PB $(33 \%)$ and ATRT $(0 \%),(\mathrm{p}=0,01)$. DFS in patients who received tandem auto-HSCT was $75 \%$ in compare to infants who received only one auto-HSCT $(44 \%),(\mathrm{p}=0,000)$. Complications grade 4 (according to COMMON TOXICITY CRITERIA 2014) were observed in $14 \%$ of cases.

Conclusions: Employment of tandem HDCT with autoHSCT in primary infant embryonal brain tumors may be a feasible option for patients after induction treatment. Both conditioning regimens had acceptable toxicity. All patients who had tandem HDCT with auto-HSCT had better OS (75\%) in compare with single HDCT (44\%). Patients with MB and PNET had better prognosis with OS $95 \%$ and $75 \%$, respectively, in compare with other embryonal tumors.

Disclosure: Nothing to declare

\section{P658}

Outcome of high dose chemotherapy and autologous stem cell transplantation in extra ocular retinoblastoma; single center experience

\section{Hanafy Hafez $^{1,2}$, Hossam Elzomer ${ }^{1,2}$, Abdullah Elhussiny ${ }^{2}$, Adel Ali Eldin ${ }^{2}$, Hala Taha ${ }^{2}$, Mohamed Khaled $^{2}$, Alaa Elhaddad ${ }^{1,2}$}

${ }^{1}$ National Cancer Institute,Cairo University, Cairo, Egypt, ${ }^{2}$ Children Cancer Hospital Egypt, CCHE 57357, Cairo, Egypt

Background: Metastatic extra ocular retinoblastoma is carrying a poor prognosis. Therapeutic intensification with high-dose, marrow-ablative chemotherapy and autologous HSCT has been explored, but its role is not yet clear. This study aimed to evaluate the survival outcome of patients with extraocular retinoblastoma post autologous stem cell transplant, treated at single center

Methods: This is a retrospective study included all patients with metastatic extraocular retinoblastoma (stages 4a and 4b) that underwent autologous HSCT at the Children Cancer Hospital Egypt (CCHE) 57357 from November 2010 to July 2017, the treatment protocol was adopted from COG protocol (ARET0321) as all patients received 4 cycles induction chemotherapy followed by consolidation myloablative conditioning, CEM (VP16 $416.6 \mathrm{mg} / \mathrm{m} 2$ x3, Melphalan: $40 \mathrm{mg} / \mathrm{m} 2 \times 4$, Carboplatin: $500 \mathrm{mg} / \mathrm{m} 2 \times 3$ ) and stem cell rescue. Patients data including initial disease characteristics, transplant data, and survival outcomes were collected and analyzed
Results: A total of 11 cases were included with median age of 1.7 years, and male to female ratio 2.66. Nine patients $(81 \%)$ were initially presented by extra ocular disease, while 2 patients were presented by intra ocular disease and progressed to metastatic disease. According to COG staging of extra ocular disease, 4 patients had stage $4 \mathrm{a}$, and 7 were stage $4 \mathrm{~b}$ ( 5 of them had trilateral disease). After induction therapy, 7(63\%) showed complete response and $4(36 \%)$ had $\geq$ partial response. With average CD34 count of $4 \times 10^{6} / \mathrm{kg}$, the median time to ANC and platelet engraftment were 10 days and 19 days respectively, and there was no transplant related mortality. Post-transplant radiotherapy was given only to 2 patients. With median duration of follow up of 32 months, the overall and event free survival rates of whole patients were $88.9 \%$ and $87.5 \%$ respectively

Conclusions: High dose chemotherapy and stem cell transplantation is a promising potential curative option for patients with metastatic extra ocular retinoblastoma

Disclosure: All authors declare; No conflict of interest Stem cell donor

\section{P659}

Does donor kir-genotype impact outcome after unrelated hematopoietic stell cell transplantation for myelodysplastic syndromes or secondary acute myeloid leukemia?

Johannes Schetelig ${ }^{1,2}$, Henning Baldauf ${ }^{3}$, Linda Koster ${ }^{4}$, Michelle Kuxhausen ${ }^{5}$, Falk Heidenreich ${ }^{3,6}$, Liesbeth C. de Wreede $^{2,7}$, Stephen Spellman ${ }^{8}$, Massalski Carolin', Vinzenz Lange, Michel van Gelder ${ }^{10}$, Benedetto Bruno ${ }^{11}$, Francesco Onida ${ }^{12}$, Victoria Potter ${ }^{13}$, Per Ljungman ${ }^{14}$, Nicolaas Schaap ${ }^{15}$, Patrick Hayden ${ }^{16}$, Nicolaus Kröger ${ }^{17}$, Stephanie Lee ${ }^{18}$, Kathy Hsu ${ }^{19}$, Ibrahim Yakoub-Agha ${ }^{20}$, Marie Robin ${ }^{21}$

${ }^{1}$ University Hospital Carl Gustav Carus, Technical University Dresden, Dresden, Germany, ${ }^{2}$ DKMS, Tübingen, Germany, ${ }^{3}$ DKMS, Dresden, Germany, ${ }^{4}$ EBMT Data Office Leiden, Leiden, Netherlands, ${ }^{5}$ CIBMTR (Center for International Blood and Marrow Transplant Research), Minneapolis, WI, United States, ${ }^{6}$ University Hospital Carl Gustav Carus, TU Dresden, Dresden, Germany, ${ }^{7}$ Leiden University Medical Center, Biomedical Data Sciences, Leiden, Netherlands, ${ }^{8}$ CIBMTR (Center for International Blood and Marrow Transplant Research), Minneapolis, MN, United States, ${ }^{9}$ DKMS Life Science Lab, Dresden, Germany, ${ }^{10}$ Maastricht University Medica Center, Maastricht, Netherlands, ${ }^{11}$ A.O.U. Città della Salute e della Scienza di Torino, Torino, Italy, ${ }^{12}$ University of Milano, Milano, Italy, ${ }^{13}$ Kings College Hospital NHS Trust, London, United Kingdom, ${ }^{14}$ Karolinksa University Hospital, Stockholm, Sweden, 
${ }^{15}$ Radboud University - Nijmegen Medical Centre, Nijmegen, Netherlands, ${ }^{16}$ St. James`s Hospital, Dublin, Ireland, ${ }^{17}$ UKE University Medical Center, Hamburg, Germany, ${ }^{18}$ Fred Hutchinson Cancer Center, Seattle, WA, United States, ${ }^{19}$ Memorial Sloan Kettering Cancer Center, New York, NY, United States, ${ }^{20}$ Lille University Hospital, Lille, France, ${ }^{21}$ Hopital St. Antoine, Paris, France

Background: A series of findings suggest that optimizing natural killer (NK) cell reactivity could further improve outcome after allogeneic hematopoietic cell transplantation (alloHCT). This could be achieved by killer cell immunoglobulin-like receptor (KIR) genotype informed donor selection. An enhanced Receptor-Ligand model which used KIR2DS1 and KIR3DL1 donor genotype information to augment NK cell activation and minimize inhibition demonstrated improved survival in one large AML study (Boudreau et al, JCO 2017). Likewise, a second model built on the classification of centromeric and telomeric KIR haplotype motifs, also predicted mortality after alloHCT for AML (Cooley et al, Blood 2010). This joint EBMT and CIBMTR study aimed at validating the two approaches in an independent cohort of patients with MDS or secondary AML.

Methods: Donor samples were retrieved from the Collaborative Biobank (Dresden, Germany) and mapped to patient outcome data extracted from the EBMT and CIBMTR. Genotyping of all KIR genes by sequencing exons $3,4,5,7,8$, and 9 was performed by high resolution amplicon-based next generation sequencing. The impact of the classifiers on time-to-event outcomes was tested in cause-specific Cox regression models adjusted for patient age, a modified disease risk index, performance status, donor age, HLA-match, sex match, CMV match, conditioning intensity, type of T-cell depletion and graft type.

Results: Clinical data from 1704 patients and corresponding donor genotype information were analyzed. The median age at alloHCT was 59.4 years (range, 18.1 to 79.6 years). The indication for alloHCT was MDS for $72 \%$ and sAML for $28 \%$ of patients. Disease risk was low/intermediate and high/very high in $41 \%$ and $59 \%$, respectively. Donors were $10 / 10$ matched for $79 \%$ of patients. Myeloablative, reducedintensity and non-myeloablative conditioning regimens were used in $31 \%, 57 \%$, and $12 \%$ of patients, respectively. Peripheral blood stem cells were the predominant graft source (93\% of patients). ATG was administered in 56\% and alemtuzumab in $9 \%$ of patients. During follow-up after alloHCT 776 patients died. In univariable and multivariable analyses of the whole cohort, overall survival and the cumulative incidence of relapse of patients with KIRadvantageous versus disadvantageous donors were not statistically significantly different. We could not replicate the pattern of outcomes predicted by the KIR3DL1/
KIR2DS1-Receptor-Ligand model and the $\mathrm{B}$ content haplotype model (A/A vs $\mathrm{B} / \mathrm{x}$ ).

Conclusions: Relapse incidence and overall survival after unrelated donor alloHCT could not be predicted using the KIR3DL1/KIR2DS1-Receptor-Ligand model and centromeric/telomeric KIR-motif model in this large cohort of patients with MDS or secondary AML. This points at the possibility of interactions between NK-cell mediated alloreactivity and disease type or procedural variations of alloHCT. Available information on KIR-genes, which have been sequenced but not yet analysed, will be investigated in exploratory analyses.

Disclosure: The authors have nothing to disclose

\section{P660}

Donor specific anti-hla antibodies (DSA) in haploidentical stem cell transplantation with post transplant cyclophosphamide: Risk of graft failure, poor graft function and impact on outcomes

Stefania Bramanti ${ }^{1}$, Valeria Calafiore ${ }^{1}$, Elena Longhi ${ }^{2}$, Jacopo Mariotti ${ }^{1}$, Loretta Crespiatico ${ }^{2}$, Barbara Sarina ${ }^{1}$, Angela Nocco $^{2}$, Chiara De Philippis ${ }^{1}$, Armando Santoro', Luca Castagna ${ }^{1}$

${ }^{1}$ Istituto Clinico Humanitas, Rozzano, Italy, ${ }^{2}$ Fondazione IRCCS Ca' Granda - Ospedale Maggiore Policlinico di Milano, Milano, Italy

Background: The presence of donor-specific anti-HLA antibodies (DSA) is associated with a 10-fold increased risk of Graft Failure (GF) in haploidentical stem cell transplantation (Haplo-SCT). The Consensus Guidelines from the European Society for Blood and Marrow Transplantation (EBMT) set a Mean Fluorescence Intensity (MFI) > 1000 as a cut-off for DSA positivity. In the absence of an alternative donor, it is recommended that patients undergo desensitization therapy, especially with high DSA levels (>5,000 MFI). The aim of this study is to analyze the impact of DSA on risk of GF and poor graft function (PGF), and on major outcomes in a consecutive cohort of patients who were systematically screened for DSA before Haplo-SCT.

Methods: 141 consecutive patients were candidates for unmanipulated Haplo-SCT with post-transplant Cyclophosphamide (PT-Cy) at our center from January 2012 to January 2018 and 135 were analyzed for the presence of HLA antibodies.

Results: 134 patients underwent haplo-SCT. HLA antibodies were detected in 40 patients, 19 of them were DSA, while 21 were non-DSA (NDSA). 10 patients out of 19 with DSA were transplanted using the same donor; 2 underwent a desensitization program before transplant. 


\begin{tabular}{lcll}
\hline & HLA-Ab negative (94 pts) & \multicolumn{2}{l}{ HLA-Ab positive (40 pts) } \\
\cline { 3 - 4 } & & Non DSA (30 pts) & DSA (10 pts) \\
\hline MDS/Acute leukemia & $31(33 \%)$ & $15(50 \%)$ & $8(80 \%)$ \\
Lymphoproliferative disorders & $62(66 \%)$ & $15(50 \%)$ & $2(20 \%)$ \\
Myeloproliferative disorders & $1(1 \%)$ & 0 & 0 \\
Low-Intermediate DRI & $74(79 \%)$ & $22(73 \%)$ & $4(40 \%)$ \\
High-Very High DRI & $20(21 \%)$ & $8(27 \%)$ & $6(60 \%)$ \\
RIC Conditioning Regimen & $46(49 \%)$ & $14(47 \%)$ & $6(60 \%)$ \\
NMA Conditioning Regimen & $40(43 \%)$ & $10(33 \%)$ & $1(10 \%)$ \\
MAC Conditioning Regimen & $8(8 \%)$ & $6(20 \%)$ & $3(30 \%)$ \\
\hline
\end{tabular}

\section{[[P660 Table] 1. Characteristics of patients]}

Only two primary GF (1.4\%) occurred, both without DSA. 20 patients developed a primary PGF (15\%). 3-years OS, 3years PFS and 1-year NRM were analyzed according to the presence of DSA in comparison with negative population. No statistically difference was found. No impact of the presence of DSA on the risk of developing GF and PGF was revealed. Major outcomes of transplant was analyzed separately in patients with PGF and good graft function (GGF). 3-years OS, 3-years PFS and 1year-NRM in GGF and primary PGF populations were $62 \%$ vs $20 \%$ ( $\mathrm{p}<0.0001$ ); $53 \%$ vs $20 \%$ (p< $0.001), 12 \%$ vs $40 \%$ ( $\mathrm{p}=0.009)$, respectively.
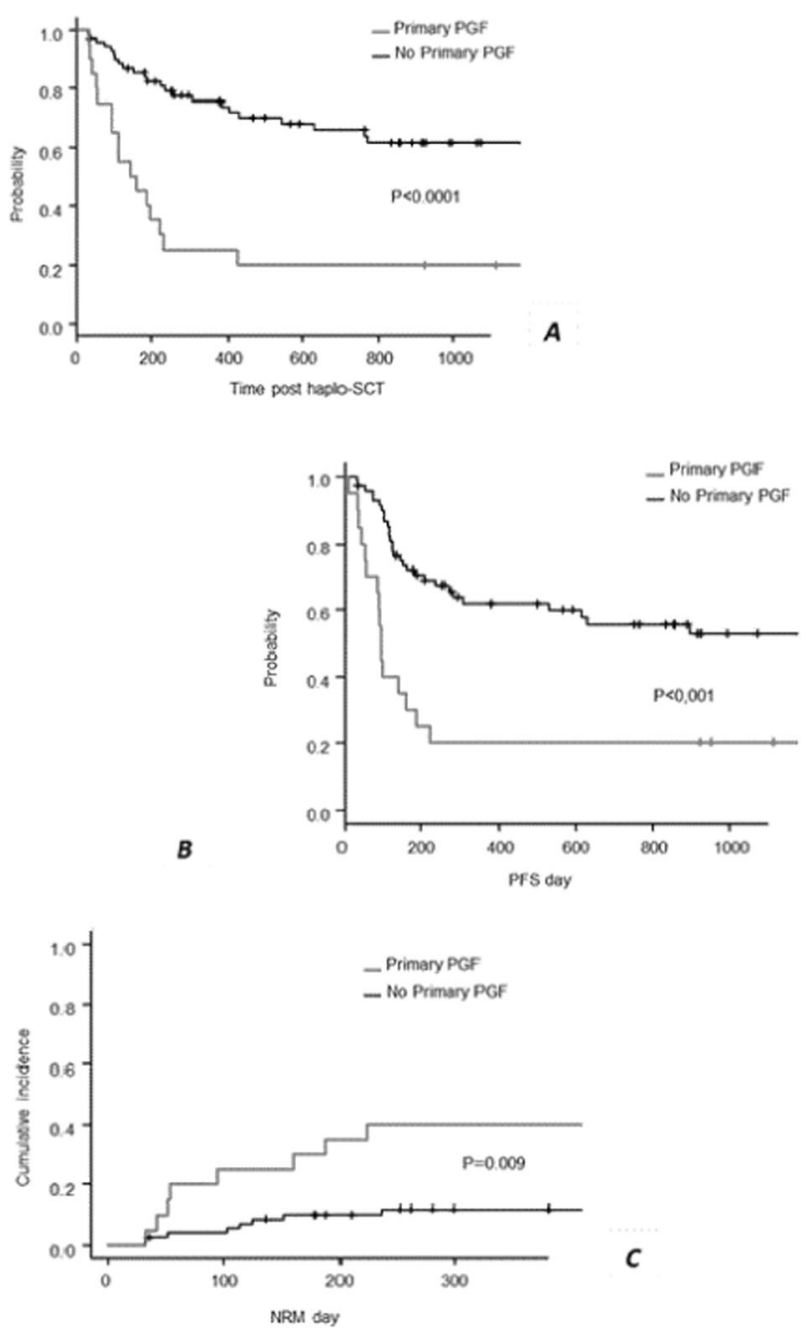

[[P660 Image] 1. OS (A), PFS (B), NRM (C), according to primary $P G F]$

Conclusions: The presence of low level of DSA in the absence of desensitization doesn't correlate with the risk of developing GF and PGF. Patients who experienced a PGF had worse outcomes in comparison with patients with GGF.

Disclosure: Nothing to declare.

\section{P661}

The impact of HLA-DPB1 mismatch in T-cell replete unrelated donor allogeneic stem cell transplantation

Bjorn Christer Linder Gronvold, Maryan Ali ${ }^{1}$, Mats Remberger $^{I}$, Jonas Mattsson ${ }^{I}$, Torstein Egeland ${ }^{I}$, Katrin Lundin', Anders Eivind Myhre ${ }^{I}$, Ingerid Weum Abrahamsen $^{\text {, Dag Heldal }}{ }^{1}$, Ingunn Dybedal ${ }^{l}$, Geir E Tjønnfjord $^{l}$, Yngvar Fløisand ${ }^{I}$, Tobias Gedde-Dahl ${ }^{I}$

${ }^{1}$ Oslo University Hospital, Rikshospitalet, Oslo, Norway

Background: High resolution matching of donor-recipient HLA improves outcome in allogeneic stem cell transplants. Matching for HLA-A, -B, -C, -DRB1 and -DQ is mandatory in our transplant centre, to identify $10 / 10$ or $9 / 10$ matched unrelated donors. High resolution matching for DPB1 has been added over the last 10-15 years. However, the role of DPB1 matching is not yet clearly defined.

Methods: In this study, we retrospectively analyzed the impact of HLA-DPB1 matching on the outcome of T-cell replete allogeneic hematopoietic stem cell transplants with CyA/Mtx- and without ATG as GVHD prophylaxis in patients with hematological malignancies at Oslo University Hospital between 2005 and 2017. 301 patients with an unrelated donor fully matched (10/10) at HLA-A, -B, -C, -DRB1 and -DQB1 loci were included. Further, 87 patientrecipient pairs were also fully matched on DPB1 (12/12); 118 had permissive and 96 had non-permissive mimatches of one or two DPB1 alleles. The Three Groups were comparable with respect to diagnosis, gender, age, cytomegalovirus serostatus and conditioning regimen.

Results: Cumulative incidence of relapse at 5 years were significantly higher in the DPB1 matched pairs compared with the permissive and non-permissive mimatched ones, at $40 \%$ vs $22 \%$ and $13 \%$ ( $<<0.001)$ respectively. Relpase free survival and overall survival were superior in the nonpermissive and permissive DBP1 mismatched groups vs the fully matched, at $60 \%$ and $49 \%$ vs $29 \%(\mathrm{p}=0.01)$ and $59 \%$ and $53 \%$ vs $40 \%(\mathrm{p}=0.09)$ respectively.

No difference in frequency of acute GVHD grade II-IV between the three groups were found; DP match $43 \%$, permissive mismatch $40 \%$ and non-permissive mismatched $48 \%(\mathrm{p}=0.49)$. Neither was there a difference seen in 
GVHD grade III-IV; $12 \%$ vs $17 \%$ vs $11 \%$, respectively. Finally, there were similar outcomes between the three groups regarding chronic GVHD and TRM.

In corrected multivariate analysis, only DP matching had significant influence on mortality and survival.

Conclusions: Our results show a favorable relapse free and overall survival following a MUD allotransplant with a DPB1 permissive or non-permissive mismatched donor compared to a fully DPB1 matched. This is likely due to an increased GvL-effect in DPB1 mismatched groups without the counterbalance of increased acute GVHD and TRM.

Disclosure: Nothing to declare

\section{P662}

A haploidentical may be a better choice than a female genoidentical donor to transplant a patient with high risk acute myelogenous leukemia in first remission

Norbert Gorin ${ }^{1}$, Myriam Labopin ${ }^{1}$, Didier Blaise ${ }^{2}$, Goda Choi $^{3}$, Gerard Socie ${ }^{4}$, Jean Henri Bourhis ${ }^{5}$, Fabio Ciceri, Emmanuelle Polge $^{7}$, Arnon Nagler ${ }^{8}$, Mohamad Mohty ${ }^{I}$

${ }^{1}$ Paris Sorbonne University, Hopital Saint Antoine APHP, Hematology and Cell Therapy and EBMT Paris Office, Paris, France, ${ }^{2}$ Institut Paoli Calmettes, Stem Cell Transplantation, Marseille, France, ${ }^{3}$ University Medical Center Groningen (UMCG) Dept. of Hematology, Groningen, Netherlands, ${ }^{4}$ Hopital St. Louis Paris, France, ${ }^{5}$ Gustave Roussy, Institut de Cancérologie, BMT Service, Villejuif, France, ${ }^{6}$ Ospedale San Raffaele, Stem Cell Transplantation, Milano, Italy, ${ }^{7}$ Paris Sorbonne University, Hopital Saint Antoine APHP, EBMT Paris Study Office, Paris, France, ${ }^{8}$ Chaim Sheba Medical Center Tel_Hashomer, Stem Cell Transplantation, Tel_Hashomer, Israel

Background: A recent study from EBMT comparing matched sibling (MSD) versus haploidentical donors transplantations, showed better outcome with MSD in adult patients with intermediate risk AML in first remission (CR1). However, a female donor to a male recipient transplant combination is a poor prognostic factor and this study did not address the question whether in this situation, a haploidentical donor transplant might do better. The present study compared the outcomes of allografted male patients according to whether they received stem cells from a female MSD or a haploidentical donor, in the intermediate and high risk cytogenetics groups (MRC classification).

Methods: The study included 1066 male patients with cytogenetics transplanted between January 2007 and June 2017 and reported to EBMT. 834 received stem cells from a MSD female donor and 232 from a haploidentical donor (133 male and 99 female). The follow up was 25 months (12-62). We studied separately intermediate and high risk patients. Multivariate analysis was adjusted on factors differing significantly between the 2 groups.

Results: 1-Intermediate risk group: 638 male patients received a female MSD and 160 a haploidentical transplant. The distribution of group characteristics was even except that in the haploidentical transplant group, donors were younger (39 y versus $51 ; \mathrm{p}<0.0001$ ), marrow was more frequently used $(45 \%$ versus $17 \%, \mathrm{p}<0.0001)$ and the interval from diagnosis to transplant was longer (5.4 versus 4.5 months, $\mathrm{p}<0.0001)$. By univariate analysis at two years post transplant, cumulative incidence (CI) of NRM post haplo was higher $(26 \%$ versus $15 \%, \mathrm{p}=0.002)$ and $\mathrm{CI}$ of extensive chronic GVH lower ( $14 \%$ versus $27 \%$; $\mathrm{p}=0.002$ ). LFS post MSD and post haplo were $64 \%$ and $51 \%$ $(\mathrm{p}=0.03)$, OS $69 \%$ and $60 \%$ (NS), GRFS $(43 \%$ and $43 \%$ ). By multivariate analyses the only significant poor risk factors were the haplo-identical transplant for NRM (HR: 1.7 (1.1-2-61)) and the patient age for OS (HR: 1.15 (1.02-1.28; $p=0.02)$. Haploidentical transplantation resulted in less chronic GVHD (HR: $0.43(0.29-0.64)$; $\mathrm{p}<10^{-4}$ ), but a lower LFS (HR: 1.7 (1.1-2.61); $\mathrm{p}=0.04)$. 2-High risk group: 196 male patients received a female MSD and 72 a haploidentical transplant. In the haploidentical group, donors were younger ( 38 y versus $54 ; \mathrm{p}<0.0001$ ), marrow was more frequently used $(42 \%$ versus $11 \%, \mathrm{p}<0.0001)$ and the interval from diagnosis to transplant was longer (5.1 versus 4.3 months, $\mathrm{p}=0.003$ ). By multivariate analysis, haploidentical transplants were associated with a lower relapse incidence (HR: $0,40(0.21-0.75 ; \mathrm{p}=0.004)$, a better LFS (HR: 0,46 (0.28-0.77; $p=0.003)$, OS (HR: 0,43 (0.25$0.75 ; \mathrm{p}=0.003$ ), and GRFS (HR: $0,53(0.34-0.84 ; \mathrm{p}=$ 0.006)(see figure). The only other significant prognostic factor was patient age.

Conclusions: This study shows that in a male patient with intermediate risk AML, a genoidentical sister donor remains associated with a better LFS. In contrast, in a male patient with high risk AML in CR1, a haploidentical donor may be a better choice than an HLA genoidentical sister.

Disclosure: Nothing to declare

\section{P663}

Abstract already published.

\section{P664}

Hematopoietic transplant for older acute leukemia patients: Improved survival with offspring donor in comparison with older-aged matched siblings

\section{Yu Wang ${ }^{1}$, Sheng-Ye Lu ${ }^{1}$, Qi-Fa Liu ${ }^{2}$, De-Pei Wu ${ }^{3}$, Xiao- Jun Huang ${ }^{I}$}

${ }^{1}$ Peking University People's Hospital, Beijing, China, ${ }^{2}$ Nanfang Hospital, Southern Medical University, Guangzhou, 
China, ${ }^{3}$ The First Affiliated Hospital of Soochow University, Hematology, Suzhou, China

Background: Despite the incidence of leukemia increases with age, currently the geriatric population is poorly represented in the standards of care concerning that older adults undergoing hematopoietic cell transplant (HCT) may experience higher transplant-related mortality (TRM). Previous studies have demonstrated that donor age is vital for older patients by affecting TRM and survival. Accordingly a relevant question is whether outcomes can be improved with a younger HLA-haploidentical offspring donor rather than an older HLA-matched sibling (MSD). In our previous multi-center report under ATG $+\mathrm{G}-\mathrm{CSF}$ based protocol for haplo HCT, offspring donor is correlated with lower TRM and higher leukemia free survival (LFS) as compared with older MSD in subgroup analysis for recipients >50years although it did not reach statistical significance. On the contrary, in a recent report from EBMT and CIBMTR under PTCY modality for haplo HCT, among patients aged 55 to 76 years, despite lower chronic graft-versus-host-disease (GVHD), graft failure, TRM, and overall mortality were higher after transplant from offspring compared with an MSD although there were differences in transplant platforms between the 2 groups.

Methods: We extended our multi-center dataset and a matched pair analysis was performed. Outcomes of 142 acute leukemia patients ( $>=50$ years) transplanted in CR1/ CR2 who received HCT from offspring $(n=57)$ or MSD $(n=85)$ between Jan, 2013 and June, 2017 present in the multi-center database were analyzed. Because the patient population was small, a 1:1 ratio matched pair analysis was implemented with the following matching factors: underlying disease (acute myeloid leukemia, acute lymphoblastic leukemia), disease status (CR1/CR2), age and sex of patients, year of transplant, blood group incompatibilities, and sex of donor.

Results: We were able to match 41 offspring with 41 MSD patients. The two matched groups were comparable in baseline characteristics except for donor age due to the family relationship. All patients achieved myeloid recovery with a median time of $14 \mathrm{~d}$ and $12 \mathrm{~d}$ for MSD cohort and offspring group $(\mathrm{p}=0.002)$. The $100 \mathrm{~d}$ platelet recovery rate was $95 \%$ in both groups. The cumulative incidence of grade II-IV acute GVHD in MSD cohort was significantly lower than in offspring group $(12 \%$ vs $37 \%, \mathrm{P}=0.009)$ while the incidence of chronic GVHD in MSD cohort was significantly higher than in offspring group $(51 \%$ vs $27 \%$, $\mathrm{P}=0.013)$. The 3 -year TRM ( $10 \%$ vs $31 \%, \mathrm{P}=0.028)$ were significantly lower in offspring-HCT compared with in MSD-HCT and relapse incidence was comparable (8\% vs $14 \%, \mathrm{P}=0.56)$. As a result, the 3 -year overall survival $(57 \%$ vs $82 \%, \mathrm{P}=0.033)$ and $\operatorname{LFS}(55 \%$ vs $82 \%, \mathrm{P}=0.024)$
(Figure 1) were significantly higher in offspring-HCT compared with in MSD-HCT. In a multivariate analysis, MSD-HCT remained a significant factor for decreased overall survival (HR 2.791(1.141-6.824), $\mathrm{P}=0.024)$ by increased TRM (HR 3.478(1.114-10.856), $\mathrm{P}=0.032)$ in comparison with offspring-HCT.

Conclusions: These data favor a young offspring over an older MSD in patients >50 years. The current analyses confirm non-HLA donor characteristics, rather than HLA disparity, predominantly influence survival in older acute leukemia patients. Validation of these findings requires a prospective trial wherein the transplant platforms can be closely matched.

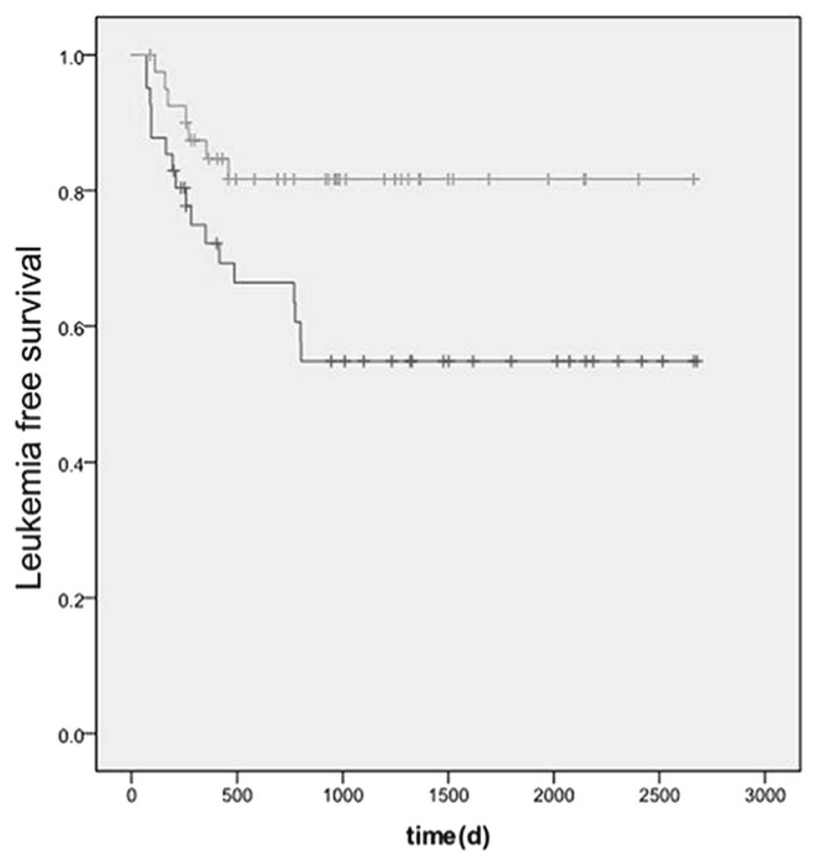

[[P664 Image] 1. Figure1. LFS in offspring-HCT compared with in MSD-HCT (55\% vs 82\%, P=0.024)]

Disclosure: Nothing to declare.

\section{P665}

Impact of sibling donor-recipient sex combinations on rejection after hla-matched bone marrow transplantation for severe thalassemia

Enam Bankas', Rajat Agarwal ${ }^{2}$, Rakesh Dhanya ${ }^{2}$, Lalith Parmar $^{2}$, Amit Sedai ${ }^{2}$, Ankita Kumari', Sadaf Khalid, Stalin Ramprakash ${ }^{3}$, Priya Marwah ${ }^{4}$, Rajpreet Soni ${ }^{4}$, Naila Yaqub ${ }^{5}$, Fatima Itrat ${ }^{5}$, Sarah Gilani ${ }^{5}$, Tatheer Zahra $^{5}$, Lallindra Gooneratne ${ }^{6}$, Ruwangi Dissanayake ${ }^{6}$, Senani Williams ${ }^{7}$, Wasantha Rathnayake ${ }^{7}$, Santanu Sen ${ }^{8}$, Sameer Tulpule ${ }^{8}$, Lawrence Faulkner ${ }^{1,3}$ 
${ }^{1}$ Cure2Children Foundation, Florence, Italy, ${ }^{2}$ Sankalp India Foundation, Bangalore, India, ${ }^{3}$ People Tree Hospitals, Bangalore, India, ${ }^{4}$ South East Asia Institute for Thalassemia, Jaipur, India, ${ }^{5}$ Pakistan Institute of Medical Sciences, Islamabad, Pakistan, ${ }^{6}$ Central Asiri Hospital, Colombo, Sri Lanka, ${ }^{7}$ Nawaloka Hospital, Colombo, Sri Lanka, ${ }^{8}$ Kokilaben Dhirubhani Ambani Hospital, Mumbai, India

Background: Severe thalassemia (ST), i.e. a thalassemia syndrome with inability to keep spontaneous hemoglobin > $7 \mathrm{~g} / \mathrm{dL}$, is a common indication for Bone Marrow Transplantation (BMT) in children in the Middle East and South East Asia. Sex mismatch has been associated with increased risk of solid organ rejection but is not generally considered an important transplant-associated risk factor in the context of fully matched sibling BMT for ST.

Methods: A total of 154 consecutive sibling bone marrow transplants carried out between January 2009 and April 2017 after conditioning with busulfan $(14 \mathrm{mg} / \mathrm{kg}$ oral, not adjusted to serum levels) and cyclophosphamide (200 $\mathrm{mg} / \mathrm{kg}$ ) (2 patients) in addition to either thiotepa $(10 \mathrm{mg} / \mathrm{kg}$ ) (49 patients), or anti-thymocyte globulin (Genzyme $4 \mathrm{mg} /$ $\mathrm{kg}$ or Fresenius $16 \mathrm{mg} / \mathrm{kg}$ on days -12 to -10$)$ (92 patients) and Fludarabine $150 \mathrm{mg} / \mathrm{m}^{2}$ (11 patients) were analysed. All cases received cyclosporine and methotrexate or mycophenolate mofetil as GVHD/rejection prophylaxis. In the thiotepa group methylprednisolone at $0.5 \mathrm{mg} / \mathrm{kg} /$ day was also used during the first 30 days after BMT (Lucarelli protocol 6i). Bone marrow was the source of hematopoietic stem cells in all cases, in the ATG group it was G-CSFprimed $(5 \mu \mathrm{g} / \mathrm{kg} / \mathrm{dose}$ twice daily for 3 to 5 days prior to harvest). All patients were considered low risk based on liver size $<2 \mathrm{~cm}$ from costal margin and age less than 15 years (median 4.2 years, range 0.9 to 14.5), all sibling pairs where HLA-compatible.

\section{Results:}

Sibling Donor-Recipient sex combinations

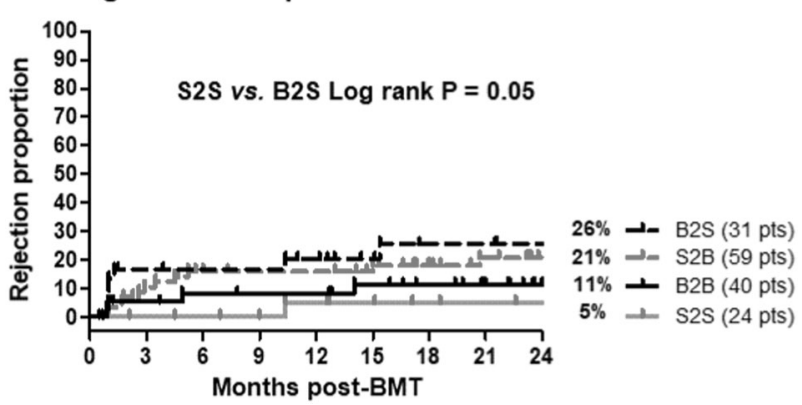

[[P665 Image] 1. Sibling donor-recipient sex combinations.]
The lowest rejection rate (5\%) was observed in the Sister to Sister (S2S) group of 24 cases, followed by Brother to Brother (B2B) group of 40 cases with $11 \%$. In the Sister to Brother (S2B) group of 59 cases, rejection rate was $21 \%$, and $26 \%$ in the Brother to Sister (B2S) group of 31 cases. On univariate analysis the only significant difference at the P 0.05 level by log rank test was B2S vs. S2S groups (rejection proportions of $26 \%$ and $5 \%$ respectively). Interestingly, all 3 patients with rejection and persistent pancytopenia were female recipients of male grafts.

Conclusions: Even though several preparative regimens were employed over an 8-year period, our data suggests that sex mismatch among compatible siblings should be considered as a relevant variable related to BMT decisionmaking. We also recommend to consider autologous back up hematopoietic stem cell collection and storage in sibling sex mismatched transplants, particularly in brother to sister BMTs. Same-sex fully matched related BMT for severe thalassemia might be the best scenario in which reducedintensity preparation strategies aiming at maximizing fertility preservation might be explored.

Disclosure: Nothing to declare.

\section{P666}

Outcomes of $\mathbf{T}$-cell replete hematopoietic cell transplantation from mismatched related or unrelated donors using high dose post-transplant cyclophosphamide based GVHD prophylaxis

\section{Jan Vydra ${ }^{I}$, Markéta St’astná Marková ${ }^{I}$, Veronika Válková ${ }^{1}$, Ludmila Nováková ${ }^{1}$, Barbora Čemusová ${ }^{1}$, Michal Kolár $\check{r}^{1}$, Mariana Hričinová ${ }^{1}$, Robert Pytlik ${ }^{1}$, Petr Cetkovskýl, Antonín Vitek ${ }^{1}$ \\ ${ }^{1}$ Institute of Hematology and Blood Transfusion, Prague, Czech Republic}

Background: High dose post-transplant cyclophosphamide (ptCy) based GvHD prophylaxis overcomes immunological barriers in HLA mismatched donor transplantation. ptCy has been adopted in many centers as de facto standard for HCT from haploidentical donors (haplo HCT). It's use in mismatched unrelated donor transplant (MMUD HCT) is less well established.

Methods: We analyzed retrospectively outcomes of contemporary cohorts of patients who underwent haplo HCT or MMUD HCT using ptCy + cyclosporine (CSA) and mycophenolate mofetil (MMF) at our center. We compared these outcomes with outcomes of cohorts of patients who underwent HCT from matched unrelated donors (MUD) using ATG based GvHD prophylaxis or matched sibling donor (MSD) with CSA and MMF. Patients and donors were considered matched if they 
had 10/10 HLA match or if they had a single DQB1 mismatch (8/8 match). Patients with acute leukemia in CR1, acute lymphoblastic leukemia in CR1 and myelodysplastic syndrome were included in the analysis.

Results: 281 patients were identified in our transplant database and divided into groups according to donor type and use of ptCy- or ATG-based GvHD prophylaxis: MSD/MUD-ATG ( $n=189)$, MMUD-ATG $(n=39)$, haploptCy $(n=25)$, MMUD-ptCy $(n=32)$. Patients in MMUDATG group were younger compared to other groups: median age was 36 years in MMUD-ATG, 43 years in MMUD-ptCy, 53 years in MSD/MUD standard and 53 years in haplo-ptCy groups $(\mathrm{P}=0.002)$. Other baseline characteristics (diagnosis, HCT-CI, regimen intensity, donor age, ABO mismatch, CMV status, graft type) were not significantly different among the groups. Conditioning regimens were $\mathrm{Cy} / \mathrm{Bu}(\mathrm{n}=56), \mathrm{Bu} / \mathrm{Flu}$ $(n=47)$, Flu/Mel $(n=83)$, TBI/Vep16( $n=85)$ or TBI/Flu $(\mathrm{n}=11)$. PtCy was given with Bu/Flu, Flu/Mel or TBI/Flu regimens. Diagnosis was AML in 52\%, ALL in 36\% and MDS in $12 \%$ of patients. $90 \%$ of patients received PBSC grafts, $10 \%$ received unmanipulated bone marrow grafts.

OS at 2 years was $70 \%$ in MSD/MUD-ATG, $76 \%$ in haplo-ptCy, $80 \%$ in MMUD-ptCy and 43\% in MMUDATG groups $(\mathrm{p}=0.0027)$. In a multivariate Cox model, only increasing age (HR 1.42 [95\% CI 1.09-1.85] for every 10years) and MMUD-ATG group (HR 3.51 [95\% CI 2.03 6.06]) were associated with inferior survival. Median time to granulocyte engraftment was 17 days in haplo-ptCy and MSD/MUD-ATG groups, 18 days in MMUD-ptCy and 23 days in MMUD-ATG groups $(\mathrm{p}=0.08)$. Non-relapse mortality was $17 \%, 17 \%, 4.5 \%$ and $42 \%$ in MSD/MUDATG, haplo-ptCy, MMUD-ptCy and MMUD-ATG groups, respectively. Cumulative incidence of acute GvHD grade 3 or 4 was $6 \%, 9.5 \%, 3 \%$ and $12 \%$ after in MSD/MUD-ATG, haplo-ptCy, MMUD-ptCy and MMUD-ATG ( $\mathrm{p}=0.007$ ). Cumulative incidence of chronic GvHD was $26 \%$ in the MSD/MUD-ATG group, $25 \%$ in haplo-ptCy, $45 \%$ in MMUD-ptCy and $20 \%$ in the MMUD-ATG groups $(\mathrm{p}=0.09)$.

Conclusions: Outcomes of HCT from haploidentical related donors and mismatched unrelated donors using posttransplant high dose cyclophosphamide are similar to outcomes of HCT from matched donors and superior to outcomes of a historical cohort of patients who received HCT from mismatched unrelated donors using ATG based GvHD prophylaxis. Larger prospective randomized trials are needed to establish relative importance of pretransplant predictors on HCT outcomes in the era of ptCy based GvHD prophylaxis.

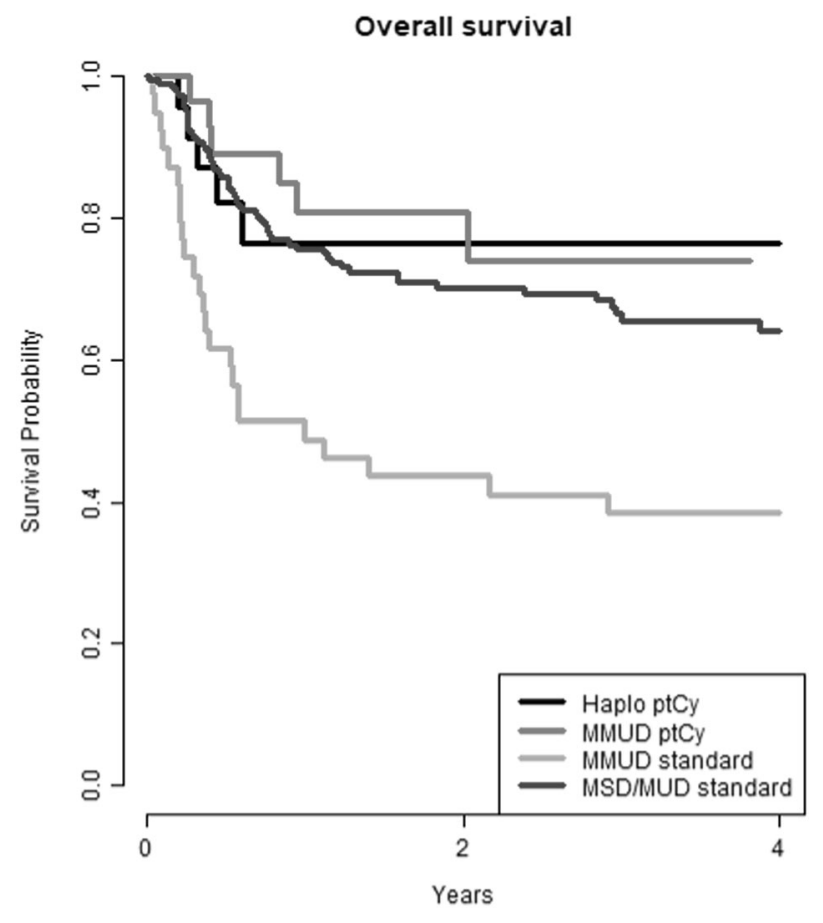

[[P666 Image] 1. Overall survival]

Disclosure: Nothing to declare

\section{P667}

Prevalence of HLA-alloimmunization in patients referred for unrelated stem cell donor search

Daniel Fuerst ${ }^{1,2}$, Uwe Platzbecker ${ }^{3}$, Verena Wais ${ }^{4}$, Donald Bunjes $^{4}$, Eva Wagner-Drouet ${ }^{5}$, Martin Gramatzki ${ }^{6}$, Gerald Wulf ${ }^{7}$, Chrysanthi Tsamadou ${ }^{1,2}$, Hubert Schrezenmeier $^{1,2}$, Joannis Mytilineos ${ }^{1,2,8}$

${ }^{1}$ Institue of Clinical Transfusion Medicine and Immunogenetics Ulm, Transplantation Immunology, Ulm, Germany, ${ }^{2}$ University of Ulm, Institue for Transfusion Medicine, Ulm, Germany, ${ }^{3}$ University of Leipzig, Leipzig, Germany, ${ }^{4}$ University of Ulm, Ulm, Germany, ${ }^{5}$ Johannes GutenbergUniversity Medical Center, Mainz, Germany, ${ }^{6}$ University of Kiel, Kiel, Germany, ${ }^{7}$ Georg-August-University Goettingen, Goettingen, Germany, ${ }^{8}$ DRST-German Registry for Stem Cell Transplantation, Ulm, Germany

Background: HLA-alloantibodies are a major risk factor for engraftment failure in allogeneic hematopoietic stem cell transplantation (HSCT). Particularly, complement fixing, donor specific antibodies were shown to be associated with early engraftment failure. Prospective antibody-screening, although not currently required for donor search, could permit early identification of high risk patients for positive crossmatch. Aim of this study is to set the basis for future 
applicability of antibody-screening-based definition of acceptable mismatches in donor selection, by creating a large prospective antibody-screening database of patients due to receive an HLA-mismatched allogeneic HSCT.

Methods: Patients $(n=2106)$ diagnosed with MDS/MPS, NHL, MM, CLL, CML, anaemia (aplastic anemia, hemoglobinopathies, $\mathrm{PNH}$ ) and $\mathrm{HL}$ were prospectively screened for HLA-antibodies whenever initial donor search indicated that no completely matched donor would be available. Screening was performed with an ELISA class I +II screening assay. All positive screening cases were tested for antigen-specific antibody identification with Luminex SAB, and acceptable mismatches were defined. The results were subsequently considered in donor search and selection. We now report the frequencies of alloimmunization observed in these patients.

Results: The highest rate of alloimmunization was observed in patients from the anaemia disease group (overall 40.5\%) followed by those from the MDS/MPN group (overall 24.8\%). The lowest immunization rates were observed in CLL (overall 6.9\%) and HL (7.0\%) patients. Alloimmunization rates for HLA-class I antigens $(\mathrm{p}<0.001)$ were significantly higher compared to HLA-class II antigens. Overall HLA-class I immunization rates ranged from $2.3 \%$ to $33.3 \%$. HLA-class II immunization rates ranged from $4.7 \%$ to $19.8 \%$ (table 1 ).

Conclusions: Our findings suggest that patients with high transfusion burden like anaemia and MDS/MPN patients have the highest risk of HLA-alloimmunization with $40.5 \%$ and $24.8 \%$ anti-HLA prevalence rates, respectively. Analysis of follow-up data, will enable us to confirm whether prospective definition and consideration of acceptable mismatches in donor selection may lead to similar engraftment failure rates between immunized and non-immunized patients undergoing HLAmismatched HSCT.

\begin{tabular}{lcccc}
\hline Diagnosis & $\mathrm{n} \quad \begin{array}{l}\text { Class-I- } \\
\text { AB pos }\end{array}$ & $\begin{array}{l}\text { Class-II- } \\
\text { AB pos }\end{array}$ & $\begin{array}{l}\text { Any HLA- } \\
\text { AB pos }\end{array}$ \\
\hline MDS/ & 783 & $17.8 \%$ & $13.5 \%$ & $24.8 \%$ \\
MPN & & & & \\
NHL & 590 & $5.6 \%$ & $4.7 \%$ & $8.6 \%$ \\
MM & 316 & $8.9 \%$ & $5.4 \%$ & $11.4 \%$ \\
CLL & 159 & $3.8 \%$ & $5.0 \%$ & $6.9 \%$ \\
CML & 104 & $12.5 \%$ & $6.7 \%$ & $15.4 \%$ \\
Anemia & 111 & $33.3 \%$ & $19.8 \%$ & $40.5 \%$ \\
HL & 43 & $2.3 \%$ & $4.7 \%$ & $7.0 \%$ \\
\hline
\end{tabular}

[[P667 Table] 1. Table: Prevalence of HLA-antibodies in patients referred for unrelated donor search according to disease entity]
Disclosure: The authors declare that no conflicts of interests are present

P668

Comparison of the outcomes with different haploidentical transplantation strategies for hematologic malignancies: Results from Taiwan blood and marrow transplantation registry (TBMTR)

Cheng-Hong Tsai, ${ }^{1,2}$, Chi-Cheng Li $^{3}$, Feng-Min Tien ${ }^{1,2}$, Chien-Ting Lin ${ }^{1,2}$, Szu-Chun Hsu ${ }^{2}$, Bor-Sheng Ko ${ }^{2}$, JihLuh Tang ${ }^{1,2}$, Ming Yao ${ }^{2}$

${ }^{1}$ National Taiwan University, Tai-Cheng Stem Cell Therapy Center, Taipei, Taiwan, Republic of China, ${ }^{2}$ National Taiwan University Hospital, Taipei, Taiwan, Republic of China, ${ }^{3}$ Tzu-Chi Hospital, Hualian, Taiwan, Republic of China

Background: There are two most noteworthy strategies of haploidentical stem cell transplantations (haplo-HSCT), the Baltimore post-transplantation cyclophosphamide (PTCy) with or without anti-thymoglobulin (ATG), and the Beijing G-CSF primed bone marrow (BM) plus peripheral blood stem cells (PBSC) (GIAC). However, the comparison of these two modalities is scarce. In this study, we aim to compare these two approaches for hematological malignancies based on the Taiwan Blood and Marrow Transplantation Registry (TBMTR).

Methods: From July 2012 to June 2018, 161 patients underwent haplo-HSCT, either by GIAC ( $\mathrm{n}=99$, group 1$)$, PTCy with ATG ( $\mathrm{n}=40$, group 2), or PTCy without ATG $(n=22$, group 3), were enrolled. All the grafts in the PTCy group were PBSC, while in the GIAC group were from BMSC and PBSC.

Results: The basic characteristics of these three groups were listed in Table 1. The patients in group 1 were younger than the ones in group 2 and group 3 (35.5 vs. 51.1 vs. 50.1, $\mathrm{P}<0.001)$. Regarding the conditioning regimens, more patients in group 1 received reduced-intensity conditioning compared with group 2 and group 3 (59.6\% vs. $42.5 \%$ vs. $45.5 \%, \mathrm{P}=0.015)$. There was a trend of more patients in group 3 having pre-HSCT advanced stage disease [45.5\% (group 1) vs. $42.5 \%$ (group 2) vs. 63.6\% (group 3), respectively, $\mathrm{P}=0.557]$. With the comparable $\mathrm{CD} 34$ infusion amounts, the neutrophil engraftment time were statistically distinct among these three groups $[\mathrm{D}+12$ (group 1) vs. D+15 (group 2) vs. D+17 (group 3), respectively, $\mathrm{P}<0.001$ ], as well as the platelet engraftment time $(\mathrm{D}+20$ vs. $\mathrm{D}+32$ vs. $\mathrm{D}+33$, respectively, $\mathrm{P}<0.001)$. The patients in group 1 had the most favorable 2-year overall survival (OS) and 1-year non-relapse mortality (NRM) compared with group 2 and group 3 [OS: 52.0\% 
(group1) vs. $34.5 \%$ (group 2) vs. $15.3 \%$ (group 3), respectively, $\mathrm{P}<0.001$; NRM: $16.9 \%, 31.9 \%$, and $35.9 \%$, respectively, $\mathrm{P}=0.048]$. Lethal infection was the major cause of death in all the groups. The cumulative incidence of relapse was the highest in group $3[37.2 \%$ (group 1) vs. $36.5 \%$ (group 2) vs. $65.3 \%$ (group 3), respectively, $\mathrm{P}=0.011]$, which might be partially resulted from preHSCT disease status. As to the graft-versus-host disease (GvHD), the patients in group 1 had more grade II-IV but similar grade III-IV acute GvHD compared with others (grade II-IV: $60.0 \%$ vs. $37.5 \%$ vs. $21.6 \%$, respectively, $\mathrm{P}<$ 0.001 ; grade III-IV: $16.3 \%$ vs. $13.5 \%$ vs. $11.5 \%$, respectively, $\mathrm{P}=0.816)$. The patients in group 1 had similar chronic GvHD rates (including all grade and extensive forms) as group 3 but higher than group 2 [all grade: $65.2 \%$ (group 1) vs. 29.3\% (group 2) vs. 63.3\% (group 3), respectively, $\mathrm{P}=0.056$; extensive form: $45.0 \%$ vs. $8.5 \%$ vs. $44.9 \%$, respectively, $\mathrm{P}=0.042$ ].

Conclusions: Haplo-HSCT with different strategies is a feasible treatment modality for hematologic malignancies in Taiwan. Regarding the retrospective nature and limited patient numbers of this study, further prospective trial is warrant to confirm our findings.

Disclosure: The authors declared no conflict of interest.

\section{P669}

Changing donor or manipulate the same donor - single institute analysis of 477 patients who relapsed among 1493 allogeneic stem cell transplantation

Xiu-Wen Liao', Jia-Hau Liu ${ }^{1,2}$, Ming $\mathrm{Yao}^{2}$, Chi-Cheng $\mathrm{Li}^{3}$, Bor-Sheng Ko ${ }^{2}$, Chien-Ting Lin ${ }^{1,2}$, Cheng-Hong Tsai $^{1,2}$, Shang-Ju Wu ${ }^{2}$, Shang-Yi Huang ${ }^{2}$, Sheng-Chieh Chou $^{2}$, Hui-Hsuan Huang ${ }^{2}$, Tai-Chung Huang ${ }^{2}$, Hsin-An Hou $^{2}$, Wen-Chien Chou ${ }^{2}$, Feng-Ming Tien ${ }^{1,2}$, Yun-Chu Lin $^{2}$, Meng-Yao Lu ${ }^{2}$, Shiann-Tarng Jou ${ }^{2}$, Yung-Li Yang ${ }^{2}$, Hsiu-Hao Chang ${ }^{2}$, Jih-Luh Tang ${ }^{1,2}$

${ }^{1}$ National Taiwan University, Tai-Cheng Stem Cell Therapy Center, Taipei, Taiwan, Republic of China, ${ }^{2}$ National Taiwan University Hospital, Taipei, Taiwan, Republic of China, ${ }^{3}$ Hualien Tzu Chi Medical Center, Taiwan, Hualien, Taiwan, Republic of China

Background: Post-transplant relapse remains the major cause of death of treatment failure. Therapeutic options for relapse after first allogeneic stem cell transplant (1st HSCT) include chemotherapy followed by donor lymphocyte infusion or second allo-HSCT (2nd HSCT) from the original donor or change to another donor. However, there is unclear outcome for different treatment approach. In this retrospective cohort study, we aim to compare the clinical outcome after different treatment strategy for relapse after first Allo-HSCT.
Methods: Between 1992 Jan and 2018 Oct, 1493 consecutive patients receiving $1^{\text {st }}$ HSCT registered to the BMT database in National Taiwan University Hospital were analyzed. Among them, 580 cases had relapsed after first Allo-HSCT. One hundred and three patients who received no treatment after relapse or with incomplete data were excluded. Their transplant data was collected following the EBMT Registry data collection forms and manuals. Overall survival rate and progression free survival rate were performed by the Kaplan-Meier method. Univariate and multivariate analysis were performed using Cox proportional hazard regression model.

Results: Of the 477 patients who experienced relapse after $1^{\text {st }}$ HSCT, total 244 patients $(51 \%)$ received chemotherapy followed by DLI or $2^{\text {nd }}$ HSCT from the same donor (no change group), 36 patient (8\%) received chemotherapy followed by $2^{\text {nd }}$ HSCT from different donors (change group), and 197 (41\%) had conventional chemotherapy alone. The patients in "change group" were younger (median age 29 vs 37, p =.02), and had more patients achieving complete remission $(\mathrm{CR})$ prior to $2^{\text {nd }}$ HSCT ( $44 \%$ vs $10 \%, \mathrm{p}=<.01$ ) than patients in "no change group". After the $2^{\text {nd }}$ HSCT, the CR was $68 \%$ for "no change group" and $91 \%$ for "change group". The progression-free survival at 3-year and 5-year were $10.5 \%$ and $5.9 \%$ (Fig 1A, p = .0438\%), respectively, for "no change group" and $9.4 \%$ and $9.4 \%$, respectively, for "change group". While the overall survival (OS) at 3-year and 5-year were $15.8 \%$ and $9 \%$ (Fig 1B, $\mathrm{p}=.3459 \%$ ), respectively, for "no change group" and $13.5 \%$ and $9 \%$, respectively, for "change group". Those who achieved CR prior to $2^{\text {nd }}$ HSCT had a trend of better OS than those without CR $(17.5 \%$ vs $8.7 \%$ at 3 -year; $8.7 \%$ vs $9.1 \%$ at 5 year, $\mathrm{p}=.0365)($ Fig 1C). There were 1 cases survived for more than 10 years in "change donor group" and 6 cases survived more than 10 years in "no change group". Only one had developed relapse after $2^{\text {nd }}$ HSCT but achieved subsequent remission again.

Conclusions: Our study shows that change donor had similar poor outcome comparing to those using the same donor after the $1^{\text {st }}$ HSCT. Patients who achieved CR before 2nd HSCT had a trend of better OS than those without remission and the long-term survivors were only those who achieved CR prior to $2^{\text {nd }}$ HSCT. Novel therapy for CR induction would be warrant for this poor prognostic population.

Disclosure: Nothing to declare

\section{P670}

Functional relevance of fetal microchimerism in NK cell cytotoxicity against leukemic blasts in children: A role for HLA-C1 and KIR2DL2/S2? 


\section{Lena-Marie Martin ${ }^{1}$, Anne Kruchen ${ }^{2}$, Ingo Müller ${ }^{1,2}$}

${ }^{1}$ Research Institute Children's Cancer Center Hamburg, Hamburg, Germany, ${ }^{2}$ University Medical Center HamburgEppendorf, Hamburg, Germany

Background: Mothers displaying a persistent fetal microchimerism (FM) proved to be the most suitable donor in $\mathrm{T}$ cell-depleted haploidentical stem cell transplantation (hHSCT) in children. We presumed that fetal cells leave an imprint in the mothers' immune system which positively affects recognition and elimination of malignant cells in the child by maternal effector cells. Distinct killer cell immunoglobulin-like receptors (KIR)/HLA constellations are not only associated with reduced relapse rates after HSCT in children, but also supposedly influence the establishment of an FM.

Methods: After approval by the local IRB and obtaining informed consent, we initiated a protocol to elucidate the factors that influence the establishment, persistence and effect of FM. We established a digital droplet PCR (ddPCR) protocol to determine the fetal microchimerism. For differentiation between maternal and fetal cells, biallelic short insertion/deletion polymorphisms were used. KIR and HLA-C genotyping was performed by SSP-PCR. Parental NK cell alloreactivity against the respective leukemic blasts and KIR phenotyping were analyzed by flow cytometry.

Results: We analyzed 45 parents, whose children were treated for hematological diseases at the University Medical Center Hamburg-Eppendorf. A fetal microchimerism was detected in $25 \%$ of the mothers. The amount of fetal cells varies between individuals $\left(8 \times 10^{-6}-9 \times 10^{-4}\right)$. We observed a positive correlation between a persisting FM and HLA-C1 homo- and heterozygous mothers along with a maternal Cen $\mathrm{A} / \mathrm{B}$ and $\mathrm{Cen} \mathrm{B} / \mathrm{B}$ genotype. Additionally, FM positive mothers showed a higher surface expression of the HLA-C1 respective receptors KIR2DL2/S2. The percentage of alloreactive maternal NK cells against fetal cells was higher compared to paternal NK cells; while alloreactivity of FM positive maternal NK cells was similar to NK cells from FM negative mothers.

Conclusions: Persistence of FM was more frequent in mothers carrying at least one HLA-C1 allele and a centromeric $\mathrm{B} / \mathrm{x}$ motif. Phenotypically, FM positive mothers had higher expression of KIR2DL2/S2 indicating a role of these receptors on the persistence of an FM. In vitro, maternal NK cells showed a higher alloreactivity compared to paternal NK cells. There was no difference in alloreactivity whether the mothers were FM positive or negative, suggesting other mechanisms are responsible for the superior outcome in transplantation from FM positive mothers.

Disclosure: Nothing to declare

\section{P671}

Comparison of allogeneic stem cell transplantation results from related or unrelated donors in $\beta$ thalassemia major

Selime Aydoğdu', Azize Mergen ${ }^{1}$, Başak Aksoy ${ }^{2}$, Mila N. Çokluk ${ }^{3}$, Gürcan Dikme ${ }^{1}$, Funda Çipe ${ }^{1}$, Tunç Fuşgın ${ }^{4}$, Ceyhun Bozkurt ${ }^{2}$

${ }^{1}$ Medical Park Hospitals, Bahçelievler, Istanbul, Turkey, ${ }^{2}$ Istinye University, Medical Park Hospitals, Bahçelievler, Istanbul, Turkey, ${ }^{3}$ Altinbas University, Medical Park Hospitals, Bahçelievler, Istanbul, Turkey, ${ }^{4}$ Altinbas University, Medical Park Hospitals, Bahçelievler, Istanbul, Turkey

Background: Although there have been significant improvements with conventional therapies in beta thalassemia major, hematopoietic stem cell transplantation is only curative therapy. Related donors are preferred to diminish transplant risks. In lack of identical related donor, identical unrelated donors are second best choice. In this study, thalassemia major patients transplanted from unrelated donors (MUD) were compared with thalassemic patients transplanted from relative donor (MRD) retrospectively.

Methods: 45 patients who were transplanted between June 2016 and December 2017 in Bahçelievler Medical Park Hospital Pediatric Bone Marrow Transplantation Unit were evaluated retrospectively. All patients were classified according to pesaro risk classification. Thirty four of 45 received busulfan, fludarabine, cyclophosphamide, thioteopa for conditioning, 11patients received myeloablative preparation regimen with treosulfan, fludarabine, thiotepa, cyclophosphamide. All patients were given ATG, cyclosporine and methoteraxate for GVHD prophylaxis. The patients were compared in terms of acute complications in first 100 days, engraftment, chimerism, acute and chronic GVHD after transplantation. Results were evaluated with IBM SPSS Statistics 22 (IBM SPSS) program.

Results: A total of 45 patients, $26(57.8 \%)$ male and 19 (42.2\%) female, aged between 1 and 18 years (median 5 years) were evaluated. Patients were evaluated in two groups as "MUD" $(\mathrm{n}=15)$ and "MRD" $(\mathrm{n}=30)$ groups. There was no difference between groups about given stem cells (MUD 6,16 $\pm 1,07 \times 10^{6} / \mathrm{kg}$ and MRD 6,25 $\pm 1,24 \times 10^{6} \%$ $\mathrm{kg}$ ). Neither significant difference between different Pesaro risk groups in terms of developing acute and chronic GVHD and nor decreased chimerism were detected. Neutrophil engraftment time (16,40 days) in MRD group was significantly longer than MUD group (13,71 days) ( $\mathrm{p}=$ 0.006) but no difference between platelet engraftments were observed. GVHD ratio was $33.3 \%$ in MUD donor group 
and $13.3 \%$ in MRD group and no statistically significant difference was found( $\mathrm{p}>0.005)$. The incidence of engraftment loss in MUD group was $13.3 \%$ and $36.7 \%$ in the MRD group, and there was no statistically significant difference $(\mathrm{p}>0.05)$. The rate of decreased chimerism was found to be significantly higher in the MRD group (50\%) than in the MUD group (6.7\%) (p:0.010; $<<0.05)$. The survival rate was $92.9 \%$ in the MUD group and $96.7 \%$ in the MRD group. The disease-free survival rate was $90.9 \%$ in the MUD group and $50 \%$ in the MRD group. The disease-free survival of MUD group was significantly higher than MRD group (p:0.010).

Conclusions: In our study, transplant related complications and success of transplantation with both MUD and MRDs were found to be similar. It is promising for MUD transplantations to found lower decreased chimerism and similar OS and DFSs. Based on these results, it was concluded that HSCT from non-family donors, especially for patients incompatible with chelation therapy and had organ damage, transplantation from unrelated identical donors can be a good choice. Although the results of our study seem promising, larger patient groups and prospective clinical trials are required.

Disclosure: Nothing to declare

\section{P672}

Haploidentical versus unrelated donor peripheral blood stem cell transplantation for acute myeloid leukemia: Single center experience

\section{Deniz Goren Sahin', Emine Tülay Özçelik ${ }^{2}$, Nurcan $\ddot{O}_{\text {zçelik }}^{2}$, Burcu Kurt ${ }^{2}$, Fehmi Hindilerden ${ }^{3}$, Reyhan Küçükkaya', Mutlu Arat ${ }^{2}$}

${ }^{1}$ Istanbul Bilim University, Istanbul, Turkey, ${ }^{2}$ Florence Nightingale Sisli Hospital, Istanbul, Turkey, ${ }^{3}$ Bakırköy Sadi Konuk EAH, Istanbul, Turkey

Background: Allogeneic stem cell transplantation (alloSCT) remains the most effective curative intent therapy for patients with unfavorable risk acute leukemia. Various donor options are available for the patient who lacks an HLA-matched sibling donor, such as unrelated donors (URD) and HLA-mismatched family (haploidentical) donors. In order to discover the exact role of transplantation type, there are many retrospective analysis, which compared these donor sources, have been reported. Recent studies showed some promising results of haploidentical donor transplantation (HIDT) using post-transplant cyclophosphamide in comparison with unrelated donor. The goal of this study was to compare the outcome of allo-SCT from haploidentical versus matched unrelated (MUD 10/10) or mismatched unrelated donor at a single HLA-locus
(MMUD 9/10) for patients with acute leukemia in remission.

Methods: Ninety-six adult (18-65 years) patients with acute leukemia in first or second remission who underwent allogeneic transplantation with a minimum 100 days follow-up at Florence Nightingale Hospital Hematopoietic Stem Cell Transplantation Center between 2011 and 2017 were included in this study. Patient characteristics and medical records of all patients were reviewed retrospectively. Thirty-eight patients who received haploidentical donor transplantation were compared with 23 patients receiving a MUD $10 / 10$ and 35 receiving a MMUD 9/10. Patients who completed minimum 100 days post-transplantation follow-up were identified as eligible for survival analysis.

Results: The characteristics of the patients and transplant donors in this study are summarized in Table 1. Median age of patients was 39.4 \pm 14 years. Proportion of male patients was $39.1 \%, 74.2 \%$ and $57.8 \%$ for MUD 10/ 10, MMUD 9/10 and HIDT groups, respectively, which is significantly different $(p=0.02)$. The other baseline factors were similar, including patient age, donor age, recipient cytomegalovirus (CMV) status, donor CMV status, graft versus host disease incidence, median neutrophil and platelet engraftment times and disease status at post-transplant $100^{\text {th }}$ day. No significant difference was identified in survival analysis among the MUD 10/10, MMUD 9/10 and HIDT groups, even if they were classified according to primary disease (AML vs ALL) and pre-transplant disease status (CR1 vs CR2). Also, donor CMV status (CMV IgG positivity or negativity) was not an important factor on survival analysis when compared between these three groups $(\mathrm{p}=0.406)$.

Conclusions: In our study population, clinical outcomes of HIDT patients were inferior to MUD 10/10 and MMUD 9/10 groups. When choosing an alternative donor for patients without an available HLA-matched sibling, urgency of transplantation and host/donor features should be considered. We believe that HIDT might be a feasible alternative choice in this subset of patients.

Disclosure: Nothing to declare

\section{P673}

G-CSF primed bone marrow in HLA-haploidentical transplantation using post-transplantation cyclophosphamide (PTCY) could promote tolerance and further reduce risk of GVHD

Nadira Durakovic ${ }^{1,2}$, Zinaida Peric $^{1,2}$, Lana Desnica ${ }^{2}$, Ranka Serventi-Seiwerth ${ }^{2}$, Mirta Mikulić, ${ }^{2}$ Brian Melamed $^{3}$, Alen Ostojic ${ }^{2}$, Dražen Pulanić ${ }^{1,2}$, Pavle Rončević ${ }^{2}$, Zorana Grubić ${ }^{2}$, Radovan Vrhovac ${ }^{1,2}$ 
${ }^{1}$ University of Zagreb, Zagreb, Croatia, ${ }^{2} \mathrm{UHC}$ Zagreb, Zagreb, Croatia, ${ }^{3}$ General Hospital Bjelovar, Bjelovar, Croatia

Background: Use of G-CSF stimulation of bone marrow (BM) donors is beneficial in many aspects; it can enhance TNC yield, but also have an immunomodulatory effect on donor $\mathrm{T}$ cell function, particularly invariant natural killer $\mathrm{T}$ (iNKT) cells expansion as well as APCs. We analyzed outcomes of 34 consecutive patients receiving bone marrow from HLA-haploidentical donors that were stimulated with G-CSF prior to harvest.

Methods: In the time period between 05/2012 and 05/ 2018, 34 patients received bone marrow from donors stimulated with $10 \mathrm{ug} / \mathrm{kg}$ BW of G-CSF on days $-2,-1$ and day of BM collection. Four patients (12\%) received myeloablative (BuCy) conditioning, one $(3 \%)$ received TEC RIC conditioning while $29(85 \%)$ received NMA ("Baltimore") conditioning. All patients received posttransplantation cyclophosphamide (PTCY) on days +3 and +4 , tacrolimus and MMF were started on day +5 . For 2 patients donors were fathers, 11 mothers, 9 siblings and 12 children.

Results: Median age was 43 years (20-63), there were 14 female and 20 male patients. Twelve patients had AML, 10 Hodgkin lymphoma, 5 ALL, 3 MDS, 3 NHL and 1 CML. Median number of infused TNC in graft was $4.7 \times 10^{8} / \mathrm{kg}$ BW (1.8-8.2) and CD34+ cells $1.9 \times 10^{6} / \mathrm{kg} \mathrm{BW}(1-4.5)$.

After median follow up of 397 days (range 26-2139), overall survival was $57 \%$, with median survival of 71 months. Engraftment was established in $29(85 \%)$ patients, $2(6 \%)$ had primary rejection and 3 patients $(9 \%)$ died in sepsis prior to engraftment. Of 29 patients that engrafted, further $3(9 \%)$ patients had secondary rejection, two of them were transplanted again from a haploidentical donor, both using PBSC as a source of graft. Median time to neutrophil recovery (ANC $>500$ ) was 23 days (12-36), while median time to platelet recovery (PLT $>20 \times 10^{9} / \mathrm{L}$ ) was 30 days (12-72) in evaluable patients. Cumulative incidence of aGVHD II-IV was $27.7 \%$ (95\% CI, 13-44); of note is that of 9 patients that developed aGVHD only one had grade III, while remaining 8 patients had grade II. Cumulative incidence of cGVHD requiring treatment was $6.9 \%(95 \%$ CI, 1-20). Cumulative incidence of relapse was $36.1 \%$ (95\% CI, 20-52) and TRM was $25.2 \%$ (95\% CI, 11-41).

Conclusions: The use of G-CSF mobilized BM graft in the HLA-haploidentical setting with PTCY has proven to be useful to us, not only in terms of TNC yield which was more than satisfactory and contributed to adequate hematological recovery, but also in the excellent control of both acute and chronic GVHD, with most patients developing aGVHD of grade II and only one grade III (actually developed only after DLI given for decreasing chimerism).
Comparative studies are of course warranted to prove benefit, but this data contributes to the growing body of evidence that indeed donor stem cell stimulation with GCSF has potentially powerful immunomodulatory effect.

Disclosure: Nothing to disclose

\section{P674}

other-relative donors as a reliable bank for allogeneic hsct in countries with culturally accepted cousin-cousin marriages: A two-year report from a pediatric center in Iran

Amir Ali Hamidieh ${ }^{1}$, Alieh Safari Sharari ${ }^{1}$, Seyed Mostafa Monzavi $^{l}$, Poorya Salajegheh ${ }^{1,2}$, Hajar Nasiri ${ }^{l}$, Nazanin Khodayari $^{l}$, Leila Hejazipour ${ }^{I}$, Hamideh Keykani, Zeynab Nikfetrat ${ }^{I}$, Fahimeh Jaafari ${ }^{1}$, Maged Kheder ${ }^{1}$, Maryam Behfar ${ }^{I}$

${ }^{1}$ Tehran University of Medical Sciences, Children's Medical Center, Tehran, Iran, Islamic Republic of, ${ }^{2}$ Kerman University of Medical Sciences, Kerman, Iran, Islamic Republic of

Background: Although the optimal donors for patients undergoing allogeneic hematopoietic stem cell transplantation (allo-HSCT) are fully-matched siblings, the cousincousin (consanguineous) marriages in some countries have extended the chance to find a matched donor for the HSCTrecipient. In this study, an outcome analysis of transplanted patients receiving stem cells from their relatives other than siblings (other-relatives or non-sibling donors) is provided.

Methods: In this retrospective cross-sectional study, a two-year report of patients who received allo-HSCT from their other-relative donors during September 2016 to September 2018 at the Department of Stem Cell transplantation of Children's Medical Center in Tehran, Iran is presented. The patients were followed up until $1^{\text {st }}$ December 2018.

Results: During this time period, 178 patients underwent HSCT (both autologous and allogeneic) at this center, of which 159 cases received allo-HSCT. Out of allo-HSCT recipients, the donors of stem cells for 51 cases (32.1\%) were their other-relatives. The median (range) age at HSCT was $6(1-11)$ years and the majority of patients were boys (31/51, 60.8\%). According to disease class, the patients were most commonly involved with non-malignant hematologic diseases (18/51 patients, 35.3\%) (Figure). The source of HSCs for most patients (48 cases, 94.1\%) was peripheral blood and for only 3 patients the source was bone marrow. The donors for 50 patients were fully matched and only one patient received the HSCs from a one-locus mismatched donor. HSCT was successful in 50 patients with most of them achieving full chimerism (44 patients, 
$86.2 \%)$ followed by those developing mixed chimerism (6 patients, $11.8 \%)$ and only one patient (2\%) experienced graft failure. Post-HSCT complications included CMV infection in 34 patients $(66.7 \%)$, other infections in 7 (13.7\%), hemorrhagic cystitis in $3(5.9 \%)$ and PRES in 2 (3.9\%). Acute GvHD occurred in 22 patients $(43.1 \%)$ and chronic GvHD in $3(5.9 \%)$. Death occurred in 7 cases and 5 of them were transplant-related, while 1 was due to disease relapse and 1 due to graft failure. The median of overall survival was 469 (40-792) days.

Conclusions: The likelihood of receiving HSCs from an HLA-matched other-relative donor in one-thirds of children undergoing allo-HSCT, with comparable outcomes to sibling and unrelated donors (as evidenced in this study compared with other studies), introduces family bank as a reliable source for pediatric allo-HSCT in countries with culturally accepted cousin-cousin marriages. Hence, for transplant physicians, parental consanguinity would be an indication of an extended search for a potential matched donor among the patient's family.

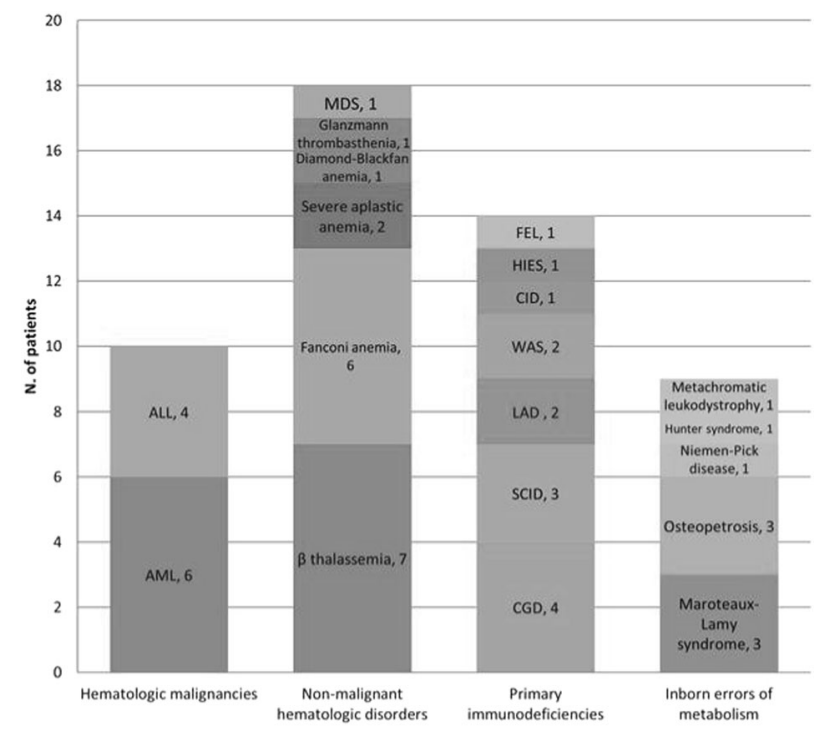

[[P674 Image] 1. Distribution of patients according to disease and disease class]

Disclosure: Nothing to declare.

\section{P675}

Abstract already published.

\section{P676}

Update on the HLA frequency distribution of the portuguese bone marrow donor registry

Eduardo Espada ${ }^{1}$, Dário Ligeiro ${ }^{2}$, Hélder Trindade ${ }^{2}$, João Forjaz de Lacerda
${ }^{1}$ Centro Hospitalar Universitário de Lisboa Norte, EPE Hospital de Santa Maria, Lisboa, Portugal, ${ }^{2}$ Centro de Sangue e Transplantação de Lisboa, IPST, IP, Lisboa, Portugal

Background: We previously presented a low-resolution HLA analysis of CEDACE, the voluntary Portuguese Bone Marrow Donor Registry (EBMT 2011, Poster P1127) and, more recently, its epidemiological characterization (EBMT 2018, Poster B348). Currently, CEDACE is one of the largest bone marrow donor registries in the world, including nearly $4 \%$ of the country's population, twice the number of donors present in 2011. The current work is an update on the most common HLA haplotypes found in CEDACE.

Methods: HLA data was obtained in August 2017; haplotype frequencies were estimated using Arlequin 3.5.2 and distributions were compared among districts and NUTS 2 regions.

Results: Of the 396545 donors in the CEDACE registry, 99.51\% were typed in at least 3 loci (HLA-A/-B/-DRB1), $43.91 \%$ in 4 (including HLA-Cw), and $1.28 \%$ in 5 (including HLA-DQB1); only $0.77 \%$ included high resolution typing in all five loci. In the 3 loci low-resolution analysis, of the 4913 haplotypes with a frequency higher than $0.0001 \%$, the 5, 25 and 150 most common haplotypes accounted for, respectively, $8.9 \%, 23.5 \%$ and $50 \%$ of the haplotypes found in the entire registry. The five most common haplotypes at the low-resolution, HLA-A/-B/DRB1 and HLA-A/-B/-Cw/-DRB1 levels were 01-08-03 (2.978\%), 29-44-07 (1.995\%), 02-44-04 (1.474\%), 33-14$01(1.226 \%)$, and $03-07-15(1.180 \%)$; and $01-08-07-03$ (2.881\%), 29-44-16-07 (1.889\%), 33-14-08-01 (1.194\%), 03-07-07-15 (1.156\%), and 02-44-05-04 (1.004\%), respectively. Since very few donors were typed in 5 loci and at high resolution, haplotype frequency estimation was not as accurate. To account for this, we integrated the relative estimated haplotype frequencies at 5 loci at low and high resolutions with the previously calculated haplotype frequencies at 3 and 4 loci, low-resolution. With this method we were able to estimate the real distribution of 5 loci, high-resolution haplotypes within the 150 most frequent 3 loci, low-resolution haplotypes; therefore, the five most common high-resolution HLA-A/-B/-Cw/DRB1/-DQB1 haplotypes were 01:01-08:01-07:01-03:0102:01 (2.932\%), 29:02-44:03-16:01-07:01-02:02 (1.884\%), 33:01-14:02-08:02-01:02-05:01 (1.167\%), 03:01-07:0207:02-15:01-06:02 (1.083\%), and 23:01-44:03-04:0107:01-02:02 (0.839\%). At the 3 loci, low-resolution level, out of 394621 donors, 167505 individual genotypes were identified, leading to an HLA matching probability at this level of $57.6 \%$; at the 4 loci, low-resolution level, there were 119196 individual genotypes out of 174128 donors, with an HLA matching probability of $31.6 \%$. The haplotype 
frequency distribution varied throughout the country, allowing for analyses of molecular variance and generation of relatively geographically accurate graphical representations of genetic distances between regions and districts.

Conclusions: With the most recent HLA analysis of the Portuguese Bone Marrow Donor Registry we were able to extrapolate high-resolution haplotype frequencies from the most common low-resolution HLA-A/-B/-DRB1 haplotypes (corresponding to $50 \%$ of the estimated haplotypes at that level), which will lead to an optimization of its use, hopefully limiting the time between donor search and allogeneic hematopoietic stem cell transplant.

Disclosure: Nothing to declare.

\section{P677}

Abstract already published.

\section{P678}

Abstract already published.

\section{P679}

Unmanipulated haploidentical donor transplantation compared to identical sibling donor had better antileukemia effect for refractory/relapsed acute myeloid leukemia in not remission status

Yue Lu' ${ }^{1}$, Yan-Li Zhao ${ }^{1}$, Jian-Ping Zhang ${ }^{1}$, Min Xiong ${ }^{1}$, Xing-Yu Cao ${ }^{l}$, De-Yan Liu ${ }^{1}$, Rui -Juan Sun ${ }^{I}$, Zhi-Jie Wei $^{1}$, Jia-Rui Zhou ${ }^{1}$, Dao-Pei Lu ${ }^{1}$

${ }^{1}$ Hebei Yanda Lu Daopei Hospital, Langfang, China

Background: Some study has been confirmed haploidentical-hematopoieticstem-cell transplantation(HIDHSCT) have a stronger anti-leukemia effect compared to identical sibling donor HSCT(ISD-HSCT) in high-risk features .but in refractory/relapsed(R/R) AML patients who not in remission status, it is unclear whether it also augments the GVL effect.

Methods: Wth this background,we conducted a retrospective analysis of 124 patients who underwent HIDHSCT for R/R AML in NR status between April 2012 to december 2016. compared to 24 patients who underwent ISD-HSCT in the same time. 68 (45.0\%) patients had PIF, $83(54.9 \%)$ patients were relapsed refractory to at least one cyles of salvage combination chemotherapy. the median age 27(range,2-69)years,male to female was 93:58. the median disease course was 9(range, 3 to 27 ) months. according to cytogenetic risk stratification, $44(29.1 \%)$ in poor risk,86 $(56.9 \%)$ in intermediate risk, $21(13.9 \%)$ in favourable risk. 36(23.8\%)patients with FLT3-ITD. Myeloablative conditioning regimens were administered with all patients, Busulfan (Bu)plus Cyclophosphamide (Cy)in 18 (11.9\%) patients, 133(88.0\%) patients added chemotherapy before $\mathrm{Bu} / \mathrm{Cy}$, Antithymocyte globulin was used in haploidentical HSCT. unmanipulated bone marrow and peripheral blood stem cells for all patients. Cyclosporine, short-term Methotrexate were employed for GVHD prophylaxis. Mycophenolate mofetil included in HID-HSCT. performed multivariate analysis for all patients of pretransplantation variables and developed a predictive scoring system for survival according to adverse factors.

Results: The total survivor median period of follow up was 46 (20-73) months. HID -cohort had higher 5-year actuarial of $\mathrm{OS}(48.6 \pm 4.6 \%$ vs $25.9 \pm 8.4 \%, \mathrm{P}=0.017)$ and RFS $(41.6 \pm 7.5 \%$ vs $25.9 \pm 8.4 \%, \mathrm{P}=0.019)$ compared to ISD-cohort.these were no difference in 5-year cumulative incidences of NRM $(18.0 \pm 3.8 \%$ and $34.9 \pm 12.6 \%, \mathrm{P}=0.212)$ between them,but the 5-year CIRs were lower in HID group $(55.4 \pm 8.9 \%$ vs $67.3 \pm 9.9 \%, \mathrm{P}=0.021)$ compared to ISD group. compared degrees of HID -HLA disparity to ISD, HID-HLA5/10 subgroup had higher OS $(48.7 \pm 4.6 \%$ vs 25.9 $\pm 8.4 \% \%, \mathrm{P}=0.028)$ and $\mathrm{DFS}(37.6 \pm 5.1 \%$ vs $25.9 \pm 8.4 \% \%$, $\mathrm{P}=0.035)$ to ISD-cohort,also HLA $6 / 10$ subgroup had higher OS $(53.6 \pm 5.1 \%$ vs $25.9 \pm 8.4 \% \%, \mathrm{P}=0.041)$ and $\mathrm{DFS}$ $(53.6 \pm 5.1 \%$ vs $25.9 \pm 8.4 \% \%, \mathrm{P}=0.049)$ to ISD-cohort,but similar OS(42.8 $\pm 6.5 \%$ vs $25.9 \pm 8.4 \%, \mathrm{P}=0.253)$ and $\mathrm{DFS}$ ( $42.8 \pm 6.5 \%$ vs $25.9 \pm 8.4 \% \%, \mathrm{P}=0.259)$ between HID -HLA $\geq 7 / 10$ subgroup and ISD. among different HID HLA 5/10,6/ $10, \geq 7 / 10$ subgroup, these were no difference $\mathrm{OS}(\mathrm{P}=0.635)$ and $\operatorname{DFS}(\mathrm{P}=0.615)$,relapse $(\mathrm{P}=0.438)$.Multivariate analysis showed ISD-HSCT,standard conditioning regimen and less than $50 \%$ proportional reduction in blast percentage preHSCT are three adverse factors associated with OS and RFS. the patients who had a predictive score of 0 had 66.6 $\pm 4.5 \% \%$ OS at 5 years, whereas OS was $44.1 \pm 3.6 \%$ for a score 1 . but only $4.4 \pm 0.2 \%$ OS rate for a score $\geq 2$.

Conclusions: Haploidentical donor compared to identical sibling donor had better anti-leukemia effect in allo-HSCT for $\mathrm{R} / \mathrm{R}$ AML in NR status; pretransplantation variables delineate subgroups who can maximum benefit from transplantation.

Disclosure: None

\section{P680}

Survival and engraftment after haploidentical transplant for severe aplastic anemia (SAA): Experience from a single center in Brazil

Alessandra Johann ${ }^{1}$, Lenisa Raboni ${ }^{1}$, Michel Oliveira, Marco Bitencourt ${ }^{I}$, Caroline Sola ${ }^{I}$, Daniela Setubal ${ }^{I}$, Samir Nabhan ${ }^{I}$, Ricardo Pasquini, Vaneuza Araujo Funke $^{I}$

${ }^{1}$ Federal University of Parana, Hematology, Curitiba, Brazil 
Background: Patients diagnosed with SAA with no sibling donors and who are refractory to immunosupression are candidates to Hematopoietic Stem Cell Transplant using alternative donors. Haploidentical donor transplants has been reported using Cyclophosphamide (Cy) post stem cell infusion as immunephrophylaxis. The present study has the objective of evaluating overall survival and engraftment rates after Haploidentical stem cell transplant for SAA in a reference center.

Methods: 15 SAA adult patients ( $\geq 15$ yo) received HSCT from haploidentical donors from de January/2010 to August/2018. Median age was 19 y (15-28); Donor was the father in six, mother in five and a brother in four cases. Stem cell source was marrow in 13 cases $(87 \%)$. Conditioning: 10 patients $(67 \%)$ received Cy $29 \mathrm{mg} / \mathrm{kg}$, fludarabine $30 \mathrm{mg} / \mathrm{m}^{2}$ e TBI 200cGy. The remaining received the same drugs but radiotherapy dose varied from 300-400cGy, All them received immunephrophylaxis with post transplant $\mathrm{CY} 100 \mathrm{mg} / \mathrm{Kg}$, cyclosporine and $\mathrm{MMF}$. Median of infused cells (TCN) was $4,94 \times 10^{8} / \mathrm{kg}(2,07-$ 12,66).

Results: eight patients engrafted (53\%). Among seven graft failures four received a second haploidentical transplant and one received an unrelated donor transplant as salvage regimen. Two patients were successfully rescued after the second haplo and the others died from infectious complications. Three years overall survival was 55\%. Death causes included: five infections and two lung hemorrhage. Median survival was 93 days (17-1065). No patient had acute graft-versus-host-disease (GVHD) and one patient had mild c-GVHD.

Conclusions: Haploidentical transplant was feasible as therapy for SAA refractory to immunessupression with an overall survival of $55 \%$ in this cohort. Graft failure however is still a problem to be addressed in this setting.

Disclosure: no disclosure

\section{Stem cell mobilization, collection and engineering}

\section{P681}

\section{Abstract already published.}

\section{P682}

Key performance and quality indicators for a successful bone marrow collection

\section{Marco Sampaio ${ }^{1,2}$, Ana Salselas ${ }^{2}$, Fátima Amado ${ }^{2}$, Filipa Bordalo $^{2}$, Sérgio Lopes ${ }^{2}$, Catarina Pinho ${ }^{2}$, Susana Roncon $^{2}$}

${ }^{1}$ Centro Hospitalar e Universitário do Porto, Serviço de Hematologia Clínica, Porto, Portugal, ${ }^{2}$ Instituto Português de Oncologia do Porto Francisco Gentil (IPO), EPE, Serviço de Terapia Celular, Porto, Portugal

Background: Bone marrow (BM) is an important source of hematopoietic stem cells, despite the increasing use of peripheral blood stem cells. BM transplantation requires implementation, development, and coordination of unique quality management systems with evaluation audits, intrahospital and international accreditation and certification processes. Quality of health care is a major focus for providers, patients, and accreditors; so, in this study, we aim to compare the quality of BM harvested at IPO-Collection Centre (ICC) with the quality of $\mathrm{BM}$ received from External Collection Centres (ECC) during these 6 last years.

Methods: This retrospective evaluation included the number of total nucleated cells (TNC) requested by the Transplant Centre, the TNC collected, and the results of BM microbiological analysis performed; donor age, weight and infectious disease markers (IDM); patient demographics and diagnosis. BM collection technique in use at ICC was validated according to JACIE Standards.

We consider successful a collection (SC) with TNC between 75 and $125 \%$ of the requested value, unsuccessful (UC) if lower than $75 \%$ and outstanding (OC) if over $125 \%$.

Results: A total of $106 \mathrm{BM}$ was collected, 99 for allogenic (75 unrelated) and seven for autologous transplant; 21 unrelated BM were received from ECC (nine from Germany, seven from USA and five from Portugal). Patient main diagnosis were severe aplastic anaemia $(n=28)$, acute myeloblastic leukaemia $(n=20)$, and acute lymphoblastic leukaemia $(n=15)$. Donors IDM were all negative and nonreactive.

Mean age ( \pm standard deviation, SD) was $33( \pm 13.2)$ and 31 ( \pm 9.3$)$ years for ICC and ECC donors, respectively. At ICC, we were asked to collect an average ( \pm SD) of $221.7 * 10^{8}( \pm 136.0)$ TNC while ECC were asked for $184.7 * 10^{8}( \pm 105.7)$. We collected $169.7 * 10^{8}( \pm 82.3)$ and received $204.5^{*} 10^{8}( \pm 109.2)$ TNC. Correlation between requested and collected TNC was 0.69 at ICC and 0.56 for ECC.

We had $41.2 \%$ SC and $26.8 \%$ OC meaning an accomplishment of $68.0 \%$. We failed to collect required TNC in $32.0 \%$. Although $85 \%$ of received BM fulfil TNC requirements, $\mathrm{BM}$ processing lowered this value to $55 \%$ due to erythrocyte removal (seven patients with major $\mathrm{ABO}$ incompatibility) and plasma reduction (two patients with ABO minor incompatibility). These steps reduce final TNC available for transplant. Weight difference between donor and patient had no significant impact on final TNC collection performance.

Sixteen BM from ICC (seven Staphylococcus spp., five Propionibacterium acnesand fourCorynebacterium spp.) 
and one from ECC (Staphylococcus spp.)presented positive microbiological results.

Conclusions: BM collection is a challenging strategy because it is a one-time procedure and manually operatordependent technique; simultaneously it is more difficult to control the final cellular content of the BM, which is a risk for donor volume depletion. BM collection is feasible even with donor and recipient weight difference. Poorer performance may be found when higher TNC are requested.

We respond efficaciously when the request is between 200 and $400 * 10^{8} \mathrm{TNC}$ but we fail to accomplish higher TNC values. We must emphasise that ICC TNC demanded was generally lower than ECC. Deciding the appropriate $\mathrm{TNC}$ for each patient remains a dare and an art.

Disclosure: Nothing to declare.

\section{P683}

Impact of adding plerixafor to mobilization protocol in the immune reconstitution of $\mathrm{V} \delta \mathrm{T}$ cells after autologous hematopoietic stem cell transplantation

Adriana Roque ${ }^{1,2,3}$, Isabel Silva ${ }^{4}$, Diana Mota ${ }^{1,2}$, Maria Carolina Afonso $^{1}$, Ana Luísa Pinto ${ }^{1}$, Emília Cortesão ${ }^{1,2,3}$, Ana Isabel Espadana ${ }^{1}$, Ana Bela Sarmento-Ribeiro ${ }^{1,2,3}$, Letícia Ribeiro $^{1,3}$, Artur Paiva ${ }^{3,4}$, Catarina Geraldes ${ }^{1,2,3}$

${ }^{1}$ Centro Hospitalar e Universitário de Coimbra, Coimbra, Portugal, ${ }^{2}$ University of Coimbra, Coimbra, Portugal, ${ }^{3} \mathrm{Cen}$ tre of Investigation on Environment Genetics and Oncobiology (CIMAGO), Coimbra, Portugal, ${ }^{4}$ Centro Hospitalar e Universitário de Coimbra, Coimbra, Portugal

Background: Plerixafor is a CXCR4 antagonist used for CD34+cell mobilization previous to autologous hematopoietic stem cell transplantation (aHSCT) in patients (pts) that failed standard mobilization. Recent evidence suggested that plerixafor affects mobilized cells composition. Besides that, there are few studies investigating the impact of mobilization protocol in immune reconstitution after aHSCT, which can lead to concerns about its impact in recovery. $\gamma \delta \mathrm{T}$ cells are effector/cytotoxic cells involved in immune surveillance, with evidence supporting an anticancer activity for $\mathrm{V} \delta 1+$.

Our aim was to evaluate the impact of plerixafor on engraftment and $\gamma \delta \mathrm{T}$ populations reconstitution.

Methods: We prospectively analyzed 54 pts submitted to aHSCT - 44 with multiple myeloma (MM) and 10 with nonHodgkin's lymphoma (NHL). The mobilization protocol was cyclophosphamide $(1.5 \mathrm{mg} / \mathrm{m} 2)+$ pegfilgrastim $(0.1 \mathrm{mg} /$ $\mathrm{kg}$ ) for $\mathrm{MM}$ and chemotherapy+G-CSF (granulocytecolony stimulating factor) $(5-10 \mu \mathrm{g} / \mathrm{kg} /$ day $)$ for NHL. MM pts who failed mobilization $\left(\geq 4 \times 10^{\wedge} 6 \mathrm{CD} 34+/ \mathrm{kg}\right.$ for $\mathrm{MM}$ [sufficient for two aHSCT] and $\geq 2 \times 10^{\wedge} 6 / \mathrm{kg}$ for NHL) were re-mobilized with plerixafor $(240 \mu \mathrm{g} / \mathrm{kg} / \mathrm{day})+\mathrm{G}-\mathrm{CSF}(10 \mu \mathrm{g} /$ $\mathrm{kg} / \mathrm{day}$ ) (PLEX). Conditioning protocol was melphalan $200 \mathrm{mg} / \mathrm{m} 2$ for MM and BEAM for NHL. The quantification and characterization of $\gamma \delta \mathrm{T}$ cells in peripheral blood samples were performed by flow cytometry based on the expression of $\mathrm{CD} 3 / \mathrm{CD} 45 / \mathrm{V} 81 / \mathrm{V} 82 / \mathrm{V} \gamma 9 / \mathrm{CD} 27$ at 30,60 and 100 days after aHSCT. Percentage (\%) of $\gamma \delta \mathrm{T}$ cells represented the proportion of these cells among all $\mathrm{T}$ cells.

Results: Median age at aHSCT was 61 (40-70) years, $63 \%$ male. Median time from diagnosis to mobilization was 8 (4-52) months, after a median number of therapeutic lines of 1 (1-3); 7pts (13.0\%) received radiotherapy. Seventeen pts $(31.5 \%)$ were re-mobilized with plerixafor $\pm \mathrm{G}-\mathrm{CSF}$ $(25 \% \mathrm{MM}$ vs $60 \% \mathrm{NHL} ; \mathrm{p}=0.056)$. Median number of apheresis sessions was 2 (1-4), being higher in PLEX (1 vs $2 ; \mathrm{p}=0.009)$. No patients failed mobilization after plerixafor rescue. The mean number of $\mathrm{CD} 34+$ cells achieved (and infused) was lower in PLEX $(4.52 \pm 0.50$ vs 2.81 $\pm 0.25 \times 10^{\wedge} 6 / \mathrm{kg} ; \mathrm{p}=0.038$ ), and pts mobilized with plerixafor received a higher dimethylsulfoxide (DMSO) volume $(22.59 \pm 11.17$ vs $69.76 \pm 32.50 \mathrm{~mL} ; \mathrm{p}<0.001)$. There was no difference in febrile neutropenia incidence $(\mathrm{p}=0.766)$, timeto-engraftment $\quad(\mathrm{p}=0.093), \quad$ time-to-neutrophils $>500 / \mu \mathrm{L}$ $(\mathrm{p}=0.174)$ or erythrocyte transfusions $(\mathrm{p}=0.076)$. However, there was more time-to-platelets $>20,000 / \mu \mathrm{L}$ ( 4 vs 8 days; $\mathrm{p}=0.004$ ) and platelet transfusions ( 2 vs 4.5 units; $\mathrm{p}<0.001$ ) in PLEX. PLEX presented higher $\% \mathrm{~V} \delta 1+(0.38 \pm 0.60$ vs $1.15 \pm 1.49 \% ; \mathrm{p}=0.027)$ and lower $\% \mathrm{~V} \gamma 9+\mathrm{CD} 27+(0.15$ \pm 0.07 vs $0.07 \pm 0.06 \% ; \mathrm{p}=0.005)$ at $\mathrm{D} 100$. There was no significant difference in other subpopulations between the two groups, despite a trend to higher $\% \mathrm{~V} \gamma \delta$ in PLEX at day 30 and $100(p=0.064$ and $p=0.059$, respectively). After a median follow-up of 16.6 months, progression free survival (PFS) was lower in PLEX (24.0 vs 19.7 months; $\mathrm{p}=0.003$ ). Response status previous to aHSCT was similar between the two groups. $\gamma \delta \mathrm{T}$ subpopulations did not affected PFS.

Conclusions: Our results showed that pts mobilized with plerixafor need more collection volume (less CD34+cells, higher DMSO). Plerixafor negatively affected platelet recovery, with similar hematologic and immune recovery for the remaining variables. Its use was associated with higher $\% \mathrm{~V} \delta 1+$, suggesting that it induces an antineoplastic phenotype. More studies with larger samples and follow-up period are needed to evaluate plerixafor impact in $\gamma \delta \mathrm{T}$ reconstitution as well as their prognostic value.

Disclosure: Nothing to declare.

\section{P684}

Optimal washout period between bortezomib induction and stem cell mobilization in patients with multiple myeloma - a single center historical prospective analysis 
Efrat Luttwak $^{1,2}$, Yael Chava Cohen ${ }^{1,2}$, Odelia Amit ${ }^{1,2}$, Irit Avivi $^{1,2}$, Svetlana Trestman ${ }^{1,2}$, Esti Rom ${ }^{1,2}$, Rinat Eshel ${ }^{1,2}$, $\operatorname{Ram} \operatorname{Ron}^{1,2}$

${ }^{1}$ Tel Aviv Medical Center, Tel Aviv, Israel, ${ }^{2}$ Sackler Faculty of Medicine, Tel Aviv University, Tel Aviv, Israel

Background: Multiple Myeloma has remained an incurable disease even in the era of novel therapies. Front line treatment typically comprises of induction chemotherapy with 46 cycles of a bortezomib-based regimen, stem cell mobilization, and harvesting of peripheral blood stem cells (PBSC) by apharesis, followed by high dose melphalan with HCT. While brotezomib-based induction regimens have demonstrated no adverse impact on hematopoietic cell harvest number and quality, no study analyzed the impact of timing of the last brotezomib dose prior to collection. In this study we aimed to determine the effect of the timing of the last dose of brotezomib before hematopoietic cell collection and the collection yield.

Methods: This was a single center historical prospective study, including all sequential newly diagnosed patients with myeloma between 2012 and 2017 that were given a bortezomib-based induction therapy ( $\leq 6$ cycles) followed by PBSC collection. We excluded patients who either received $1^{\text {st }}$ line VTD-PACE or lenalidomide-containing regimens. Peripheral blood CD34+ cells were measured on the day of collection. Patients with CD34+ levels of $>10$ cells/ microliter started collection on the same day, while those with lower levels were given plerixafor. We performed regression analyses to analyze the impact of a variety of precollection factors, including days from last bortezomib therapy on the collection yield.

Results: We identified 75 patients who fulfilled the inclusion criteria, Table. Median time from last dose of brotezomib to first leukapheresis was 11 (range, 2-27) days. A statistically significant correlation was found between the days from last dose of brotezomib and both the first collection day-CD34+ cells $/ \mathrm{kg}(\mathrm{r}=0.466, \mathrm{P}<0.001)$, and the total collected $\mathrm{CD} 34+$ cells $/ \mathrm{kg}(\mathrm{r}=0.341, \mathrm{P}=0.03)$, Figure.

The optimal cutoff point as indicated by the ROC curve was 8.5 days according to collection success with sensitivity of $79 \%$ and specificity of $74 \%$, Youden's index 0.52 . In multivariate analysis included other factors affecting collection yield (age, gender, status of disease at collection, and prior radiation) - timing of last dose of brotizomib remained significantly associated with the total collected $\mathrm{CD} 34+$ cells $/ \mathrm{kg}(\mathrm{p}=0.01)$. Increasing age, female gender, and prior radiation were associated with lower collection yield ( $\mathrm{p}=0.006,0.012,0.043$, respectively). Based on this, we developed a model to predict the total collected CD34pos cells $=11.76+0.13$ (timing in days of last dose of brotezomib) - 0.1 (age) - 1.39 (if female) -0.01 ( $\geq \mathrm{PR})-1.35$ (if prior radation).

Conclusions: Timing of last dose of brotezomib is an important factor for predicting a successful collection. A washout period of 9 days is associated with a better collection yield. These results should be further validated in a prospective study.

\begin{tabular}{|c|c|}
\hline Age (median, range) & $63(37-78)$ \\
\hline Gender - male $(\%)$ & $44(59)$ \\
\hline Prior radiation treatment $(\%)$ & $18(24)$ \\
\hline Disease status at collection (\%) & $\geq \mathrm{PR}-51(71.8)$ \\
\hline \multicolumn{2}{|c|}{$\begin{array}{l}\text { Mobilized >2 } 10 \text { million CD34+ cells/ } 75 \text { (100) } \\
\operatorname{Kg}(\%)\end{array}$} \\
\hline \multicolumn{2}{|c|}{$\begin{array}{l}\text { Mobilized }>210 \text { million CD } 34+\text { cells/Kg58(77.3) } \\
\text { on the first day of collection(\%) }\end{array}$} \\
\hline \multicolumn{2}{|c|}{$\begin{array}{l}\text { Total CD34 + cells/kg collected [median6.3(2-16.3) } \\
\text { (range) } 10 \text { million] }\end{array}$} \\
\hline Number of days of leukopharesis (\%) & $\begin{array}{l}1 \text { day- } 28 \text { (37.3), } 2 \text { days }-41 \\
(54.7), 3 \text { days - 6(8) }\end{array}$ \\
\hline
\end{tabular}

[[P684 Table] 1. The baseline characteristics of the patients, their disease and the collection outcome]

Disclosure: Nothing to declare

\section{P685}

Mobilization with plerixafor in "poor mobilizer" related and unrelated donors of HPC-a in case of failed mobilization with G-CSF

\section{AURORA Vassanelli ${ }^{1}$, Daniele Laszlo², Francesca Bonifazi $^{3}$, Patrizia Accorsi ${ }^{4}$, Simonetta Pupella ${ }^{5}$, Letizia Lombardini ${ }^{6}$, Nicoletta Sacchi ${ }^{7}$}

${ }^{1}$ Azienda Ospedaliera Universitaria Integrata, Verona, Italy, ${ }^{2}$ IEO European Oncologic Institute, Milano, Italy, ${ }^{3}$ Policlinico Sant'Orsola - Malpighi, Ematologia - Centro Trapianti, Bologna, Italy, ${ }^{4}$ Ospedale Santo Spirito, Centro Trasfusionale, Pescara, Italy, ${ }^{5} \mathrm{CNS}$ - Centro Nazionale Sangue, Roma, Italy, ${ }^{6} \mathrm{CNT}$ - Centro Nazionale Trapianti, Roma, Italy, ${ }^{7}$ Ospedale Galliera, IBMDR, Genova, Italy

Background: In the allogeneic HPC transplantation, both from related and unrelated donors, the most commonly used source is peripheral blood after mobilization with G-CSF. It is however known that about $2 \%$ of donors are "poor mobilizers"; the rescue strategies are: a third apheretic collection; bone marrow donation.

Methods: In 2016-2017 in Italy a procedure to be adopted in case of failed mobilization of peripheral blood stem cells has been defined and shared between IBMDR, CNT (Transplant National Center) and CNS (Blood 
National Center) and the Scientific Societies SIMTI, SIdEM and GITMO, using Plerixafor, a selective reversible antagonist of the CXCR4 receptor with its binder (the stromal derived factor SDF-1), in combination with standard G-CSF dose. Moreover since 2017, in accordance with this protocol, the Competent Authority (AIFA) has extended the registration of Plerixafor (Law no.648/96), also for the mobilization in "poor mobilizer" healthy donors. Finally, in 2018 the protocol was extended to "poor mobilizer" family donors, making management equivalent in related and unrelated donors. At the time of the donor's informed consent for the donation of HPC, the hypothesis of the lack of mobilization or inadequate collection was illustrated and the possible actions proposed as "back-up donation" were anticipated. Failed mobilization of CSE has been defined as the presence of one of the two criteria: a number of $\mathrm{CD} 34+$ circulating on peripheral blood lower than $20 / \mu \mathrm{L}$ on the 5 th day of stimulation (5D), or the collection of CD34+ $<1.0 \times 106 / \mathrm{kg}$ weight of the recipient at first apheresis. In these cases, a single dose of Plerixafor is administered subcutaneously by health professionals under medical supervision, $0.24 \mathrm{mg} / \mathrm{kg}$ of body weight, 6 11 hours before the start of apheresis.

In case of use Plerixafor due to failed mobilization of HPC-A or collection of an inappropriate number of CD34+, the notification is made by the Collection Center to IBMDR (for both family and non-family donors), and to the recipient Transplant Center : both donor and recipient express their consent; finally once the collection is completed, the Collection Center informs IBMDR, which in turn notifies CNT/CNS/SIMTI/SIDEM/GITMO.

Any adverse reactions/events are notified in real time, based on the SOP specifications and current regulations.

Results: Since the introduction of the national protocol, 7 donors (4 unrelated donors and 3 related donors) were treated, presenting at least one of the two inclusion criteria $(\mathrm{CD} 34<20 / \mu \mathrm{L}$ at $5 \mathrm{D}$ or CD34 $<1 \times 10 \mathrm{e} 6 / \mathrm{Kg}$ after first collection)After use of Plerixafor in all donors, the required dose of CD34 was obtained to ensure successful transplantation, with a sufficient increase in the CD34+ cells. No side effects or adverse reactions related to the administration of Plerixafor occurred.

Conclusions: In cases of failed mobilization in the related and unrelated donor, the use of Plerixafor according to the methods described in the shared protocol between IBMDR, CNS, CNT, SIMTI, SIdEM, GITMO, proved to be safe and effective. This protocol emphasizes the great value of the sharing of procedures between the register, institutions and scientific societies, ensuring the supervision of the process and the protection of the donor and recipient.

Disclosure: Nothing to declare
P686

Optimization of on-demand plerixafor strategy through CD34+ kinetics and $\mathrm{CD34}+/ \mathrm{WBC}$ in cyclophosphamide-based mobilization for multiple myeloma patients

\section{Massimo Di Gioia ${ }^{I}$, Francesca Materozzi ${ }^{I}$, Riccardo Boncompagni $^{1}$, Chiara Innocenti ${ }^{1}$, Chiara Nozzoli ${ }^{1}$, Ilaria Cutini ${ }^{1}$, Stefano Guidi ${ }^{1}$, Antonella Gozzini ${ }^{1}$, Serena Urbani $^{1}$, Riccardo Saccardi ${ }^{I}$}

${ }^{1}$ AOU Careggi, Cell Therapy and Transfusion Medicine, Firenze, Italy

Background: Autologous stem cells transplantation (ASCT) is an effective treatment option for young patients with multiple myeloma (MM). A minority of patients may still experience untoward toxicity due to delayed engraftment. Thus, the current policy in many centers is aimed to increase the target dose of collected $\mathrm{CD} 34^{+}$cells up to an "optimal" level of $4 \times 10^{6} / \mathrm{Kg}$ per procedure. Therefore, an ideal mobilization, aimed to collect 4 to $8 \mathrm{CD} 34^{+}$cells $/ \mathrm{kg}$ in one apheresis, should achieve a number of circulating CD34 + cells $>40 / \mathrm{mcL}$ (very good mobilizers). Plerixafor may help to maximize the $\mathrm{CD} 34^{+}$collection but its use is limited by high cost. We carried out a retrospective analysis aimed to predict the quality of mobilization and develop an algorithm to optimize both timing of collection and use of Plerixafor.

Methods: We retrospectively collected data from 91 mobilization procedure performed in our center between 2006 and 2016 for MM. All received the same mobilization protocol with cyclophosphamide (range 3-4 gr/sqm) and G$\mathrm{CSF} 10 \mathrm{mcg} / \mathrm{kg}$ from +5 . $\mathrm{CD} 34^{+}$cell count was started when White Blood Cells (WBC) count exceeded $1 \times 10^{9} / \mathrm{L}$. Patients were excluded from this analysis if 1) showed a CD34+ count $>40 / \mathrm{mcL}$ (target achieved at first day count) and/or 2) $\mathrm{CD} 34^{+}$count on second day was missing and/or 3) Plerixafor was administered on first day according to previous policies. Sixty-eight patients were evaluable for the study. Univariate and multivariate logistic regression analysis to study $\mathrm{cCD} 34^{+}$kinetics and assess predictors impact on mobilization was carried out. Ratio CD34 $+/$ WBC in first day count, gender, disease category and time from mobilization chemotherapy were also included

Results: Among the 68 patients included in the analysis, the threshold of $40 \mathrm{CD} 34+/ \mathrm{mcL}$ cells on the second day was reached by $35(51,47 \%)$ of patients (GroupA) whilst the remaining $33(24,7 \%)$ failed the target (GroupB). Median (range) WBC $\times 10^{9} / \mathrm{L}$ and $\mathrm{CD} 34^{+} / \mathrm{mcL}$ counts in group A and B were 2,01 (1-5,01) and 21,33 (7,56 - 39,91), $1,08(1-6,46)$ and 3,31 (0,06-31,14) respectively, with a statistically significant differences among group (Mann- 
Whitney $\mathrm{p}=0,01$ and $\mathrm{p}=0,02$ respectively). Only CD34 ${ }^{+} / \mathrm{WBC}$ ratio and $\mathrm{CD} 34^{+} / \mathrm{mcL}$ on first day count had an impact on kinetics and optimal mobilization. Logistic regression model highlight $\mathrm{CD} 34+/ \mathrm{mcL}(\mathrm{OR}=1,270 ; 95 \%$ CI: 1,060 - 1,522) on first count as an independent predictor of second day optimal mobilizer, with AUC of $0.9 \%(0,85-$ $0,99)$ in ROC analysis. Two CD $34^{+}$thresholds were then calculated: $<7,56 / \mathrm{mcl}$ (PPV 0,81; NPV 1,0) that identified poor mobilizer, and $\geq 24,06 / \mathrm{mcl}$ (PPV 1,0; NPV0,59) that exclude probability to fail on second day. For those with a $\mathrm{CD} 34^{+}$count between 7,56-24,06 the $\mathrm{CD} 34+/ \mathrm{WBC}$ ratio $(\mathrm{OR}=1,768,95 \%$ CI: $1,307-2,391)$ was a predictor of optimal mobilization (AUC 0,94; 0,885-0,996); cut-off value was 5,63 (sensibility 94,28; specificity 84,84 )

Conclusions: Assessment of circulating WBC, CD34+ and their ratio at WBC recovery in a chemo-based mobilization is a valid tool to manage the collection strategy and the on-demand use of Plerixafor. We have developed an algorithm aimed to the use of Plerixafor to both rescue poor mobilizers and boost $\mathrm{CD} 34^{+}$count in intermediate mobilizers. The algorithm is currently under validation

Disclosure: Nothing to declare

\section{P687}

The difference in mobilization using etoposide compared with cyclophosphamide

Ka-Won Kang ${ }^{1}$, Byung-Hyun Lee ${ }^{1}$, Eun Sang $\mathrm{Yu}^{1}$, Min Ji Jeon $^{1}$, Dae Sik Kim ${ }^{1}$, Hojoon Choi ${ }^{1}$, Se Ryeon Lee ${ }^{1}$, Hwa Jung Sung ${ }^{1}$, Chul Won Choi ${ }^{\text {, }}$ Yong Park ${ }^{1}$, Seok Jin Kim $^{2}$, Byung Soo Kim ${ }^{1}$

${ }^{1}$ Korea University College of Medicine, Seoul, Korea, Republic of, ${ }^{2}$ Sungkyunkwan University School of Medicine, Seoul, Kosovo, Republic of

Background: Successful autologous stem cell transplantation (ASCT) requires the infusion of a sufficient number of hematopoietic stem cells (HSCs). Peripheral blood (PB) is the most commonly used source of HSCs, therefore, it is important to optimize methods used to mobilize the HSCs. The most clinically used chemotherapeutic agents for effective mobilization are cyclophosphamide and etoposide. Recent published studies suggest that etoposide has a better mobilization effect than cyclophosphamide even at lower doses, but it is not clear why this difference occurs. In this study, we tried to determine whether there is a difference in the mechanism of mobilization between cyclophosphamide and etoposide.

Methods: First, in order to confirm the clinical data for efficacy and toxicity of mobilization, we retrospectively analyzed the data of patients who were diagnosed with lymphoma and performed mobilization using cyclophosphamide or etoposide from January 2011 to December 2018. Second, mesenchymal stem cells (MSC) were primarily cultured from the healthy controls, then treated cyclophosphamide or etoposide at a concentration of $10 \%$ inhibition of cell growth, and cytokine analysis was performed to identify cytokines known to be associated with mobilization. Third, mobilization mouse model using cyclophosphamide or etoposide was generated, total blood was collected at the time of HSCs collection, and cytokine and network analysis (using Ingenuity Pathway Analysis) was performed.

Results: The mobilization yields for cyclophosphamide or etoposide were analyzed. Etoposide miblized a significantly higher median number of $\mathrm{CD} 34+$ cells than cyclophosphamide. The rate of successful or adequate mobilization was also significanctly higher for etoposide in univariate and multivariate analysis (Table 1). In the analysis of toxicity during mobilization, the incidence of neutropenic fever was higher in the cyclophosphamide group $(\mathrm{P}=0.007)$. During mobilization, cyclophosphamide maintained lower WBC counts than etoposide and showed a large increase in WBC counts at the start of collection (Figure 1). The cumulative dose of cyclophosphamide or etoposide in patients who underwent autologous stem cell transplantation did not affect leukocyte (ANC $>1000 / \mathrm{microL}$ or platelet (PLT $>100 \mathrm{k} / \mathrm{microL}$ ) engraftment.

In MSC treated with etoposide at a concentration of $10 \%$ inhibition of cell growth, IL-8, which is a cytokine that promotes hematopoietic stem cell mobilization, were shown a statistically significant increase (Figure 2). In the mouse model of mobilization (Figure 3), the levels of KC, one of the IL-8 homologues in mice, had significantly increased in the etoposide-treated group compared with the levels in the cyclophosphamide-treated group. The levels of other IL-8 homologues, MIP-2 and LIX, also showed increases in the etoposide-treated group compared with those in the cyclophosphamide-treated group; these differences, however, were not statistically significant (Figure 4). Network analysis based on in vivo cytokine results identified that etoposide could promote mobilization in association with matrix metalloproteinase as compared to cyclophosphamide (Figure 5).

Conclusions: Etoposide has a higher mobilization efficacy when compared to cyclophosphamide, which could due to the different mechanisms of mobilization through the elevation of IL8 and the activation of matrix metalloproteinase associated therewith.

Disclosure: This research was supported by a grant of the Korea Health Industry Development Institute (KHIDI), funded by the Ministry of Health \& Welfare, Republic of Korea (grant number : HI17C2072) 


\section{P688}

Efficiency of peripheral blood stem cell collections and factors affecting it. Experience of NHS blood and transplant registry

Marina Karakantza ${ }^{1}$, Olivia Pirret ${ }^{2}$, Khaled El-Ghariani ${ }^{3}$, Therese Callaghan ${ }^{4}$, Helen New ${ }^{5}$, Davina Potok ${ }^{1}$, Rachel Pawson $^{6}$, Jayne Peters ${ }^{7}$, James Griffins ${ }^{2}$

${ }^{1}$ NHS Blood and Transplant, Leeds, United Kingdom, ${ }^{2}$ NHS Blood and Transplant, Bristol, United Kingdom, ${ }^{3}$ NHS Blood and Transplant, Sheffield, United Kingdom, ${ }^{4}$ NHS Blood and Transplantlood and Transplant, Liverpool, United Kingdom, ${ }^{5}$ NHS Blood and Transplant, London, United Kingdom, ${ }^{6}$ NHS Blood and Transplant, Oxford, United Kingdom, ${ }^{7}$ NHS Blood and Transplant, Manchester, United Kingdom

Background: Success of peripheral blood stem cell (PBSC) collections depends on patient biological parameters and stable apheresis device performance. Peripheral blood $\mathrm{CD} 34+$ cell enumeration is the most reliable predictive factor of apheresis yield however, there are some unexpectedly poor CD34+ cell harvests despite successful mobilization. The aim of the study was assess total collections CD34+ yields and factors influencing main apheresis procedure outcomes including collection efficiency (CE).

Methods: Of 2233 consecutive donors covering the period 1-1-2016 to 30-9-2018 were analyzed for the following parameters: pre CD34 count, CD34 yield per procedure, total CD34 dose collected per patient, CD34 collection targets requested by clinical teams. The efficiency of PBSC procedures was determined by calculating the $\mathrm{CE}$ and the correlation coefficient between pre CD34 count and yield per procedure. $\mathrm{CE}$ was correlated to preprocedure WBC, platelet count, pre CD34 count and blood volume processed. All PBSC collections were performed by Optia Spectra across 7 units in UK.

Results: Of the 2233 donors, 1611 were autologous and 622 allogeneic. The autologous donors underwent in total 2543 procedures. The median CD34 target dose for these donors was $4 \times 10^{6} / \mathrm{kg} .799(50 \%)$ achieved the target dose with 1 procedure and $566(35 \%)$ with 2 procedures. The median pre CD34 count was 30/ $\mu$. The median CD34 yield per procedure was $2.54 \times 10^{6} / \mathrm{kg}$ and the median total CD34 dose collected per donor was $5.38 \times 10^{6} / \mathrm{kg} .92(5.7 \%)$ of autologous donors collected a total CD34 dose $<2 \times 10^{6} / \mathrm{kg}$, of those $27(1.7 \%)$ had a pre CD34 count $<10 / \mu l$ and 65 $(4 \%)>10 / \mu l$.

The allogeneic donors underwent in total 878 procedures. The median CD34 target dose for these donors was $5 \times 10^{6}$ \% kg. 381 allogeneic donors (61\%) achieved the target dose with 1 procedure and $221(36 \%)$ with 2 procedures. The median pre CD34 count was 51/ $\mu$ l. The median CD34 yield per procedure was $4.07 \times 10^{6} / \mathrm{kg}$ and the median of total CD34 dose collected per donor was $6.70 \times 10^{6} / \mathrm{kg} .17(2.7) \%$ of allogeneic donors collected a total CD34 dose $<2 \times 10^{6} \%$ $\mathrm{kg}$, of those $3(0.5 \%)$ had a pre CD34 count $<10 / \mu \mathrm{l}$ and 14 $(2.2 \%)>10 / \mu l$. The median CE for autologous donors was 55\% (range 20-166) and for allogeneic donors was $47 \%$ (range12-116). The CE was negatively correlated to WBC $(r=-0.29$ and -0.37$)$ and platelet count $(r=-0.14$ and -0.06$)$ for auto and allogeneic donors respectively, but did not correlate to the pre CD34 and blood volume processed. The correlation coefficient between pre CD34 count and CD34 yield per procedure was $r^{2}=0.67$ for the autologous and $\mathrm{r}^{2}=0.34$ for the allogeneic collections.

Conclusions: The majority of autologous and allogeneic donors achieved the target CD34 dose with one procedure. $94.3 \%$ of autologous and $97.3 \%$ allogeneic donors collected a transplantable CD34 dose of $>2 \times 10^{6} / \mathrm{kg}$. $4 \%$ of autologous and $2.7 \%$ of allogeneic donors did not collect a transplantable dose despite a preCD34 count of $>10 / \mu \mathrm{l}$ indicating suboptimal procedure performance. The $\mathrm{CE}$ was variable and was negatively correlated to the preprocedure WBC and platelet count. The CE and correlation coefficient are lower in allogeneic donors compared to autologous donors.

Disclosure: Nothing to declare

\section{P689}

The outcome of autologous blood stem cell collection and its actual use in real world: the 21st century experience

Kyoungmin Lee ${ }^{I}$, Jung Yong Hong ${ }^{1}$, Dok Hyun Yoon ${ }^{I}$, Jae-Lyun Lee ${ }^{I}$, Shin Kim ${ }^{1}$, Kyoung Min Lee ${ }^{I}$, Jung Sun Park $^{1}$, Cheolwon Suh

${ }^{1}$ Asan Medical Center, University of Ulsan College of Medicine, Seoul, Korea, Republic of

Background: High-dose chemotherapy followed by autologous blood stem cell transplantation (ASCT) is a standard therapy for wide range of hematologic and solid malignancies. Although various methods have been introduced to improve the peripheral blood stem cell (PBSC) mobilization, autologous stem cell collection (ASCC) is not successful in every patient. Furthermore, even if the ASCC is complete, not all of them lead to ASCT. We evaluated the result of ASCC and actual use of PBSC grafts in current practical setting.

Methods: We retrospectively reviewed the all consecutive ASCC procedures performed at the Department of Oncology in Asan Medical Center, Seoul, Korea, between January 2000 and October 2018. The targeted number of 
$\mathrm{CD} 34^{+}$cells was $\geq 5.0 \times 10^{6} / \mathrm{kg}$ of body weight in general, and $\geq 10.0 \times 10^{6} / \mathrm{kg}$ for plasma cell neoplasms (PCN). PBSC harvest was discontinued after at least two days from the initiation of leukapheresis when it seemed that they were not likely to yield $\geq 0.2 \times 10^{6} / \mathrm{CD} 34^{+}$cells/kg per day.

Results: Total 1156 ASCC procedures were carried out in 1072 patients over 18 years, once in 985 patients, twice in 83 patients, three times in 3 patients, and four times in 1 patient. Non-Hodgkin lymphoma (NHL) comprised 56.7\% of all cases $(\mathrm{n}=655)$, followed by PCN $(\mathrm{n}=400,34.6 \%)$, HL $(\mathrm{n}=56,4.8 \%)$, and germ cell tumor $(\mathrm{GCT})(\mathrm{n}=45$, $3.9 \%)$. Overall, the median of $12.4 \times 10^{6} \mathrm{CD} 34^{+}$cells $/ \mathrm{kg}$ (range 0.33-91.6) in PCN and $7.3 \times 10^{6} \mathrm{CD} 34^{+}$cells $/ \mathrm{kg}$ (range 0-172.7) in non-PCN were harvested. There were 98 cases of failed collection with median of $0.7 \times 10^{6} \mathrm{CD} 34^{+}$ cells $/ \mathrm{kg}$ (range 0-5.83), resulting in ASCC success rate of 91.5\% $(\mathrm{n}=1058 / 1156)$. When multiple logistic regression analysis was performed, disease type (NHL vs PCN: OR 3.04, 95\% CI 1.10-8.444, $\mathrm{p}=0.033$; GCT vs PCN: OR $12.01,95 \%$ CI $3.78-38.20, \mathrm{p}<0.001$ ), total number of chemotherapy cycles before ASCC (OR 1.06, 95\% CI 1.004-1.13, $\mathrm{p}=0.035$ ), failure to achieve at least partial response before ASCC (OR 2.97, 95\% CI 1.01-8.73, p = 0.048), total number of days receiving G-CSF for mobilization (OR 1.10, 95\% CI 1.002-1.23, $\mathrm{p}=0.045$ ), and salvage use of plerixafor (OR 3.78, 95\% CI 1.92-7.47, $\mathrm{p}<0.001$ ) were found to be independent factors associated with failure of ASCC. At the end of the study period, $90.9 \%$ of successful collections $(n=962 / 1058)$ were used for ASCT, $4.5 \% \quad(n=48 / 1056)$ were in storage awaiting transplantation, and remaining $4.5 \%(n=48 / 1056)$ were discarded because of patients' death $(n=42)$ before the planned ASCT or patients' refusal to transplantation $(n=6)$. Of note, $69.0 \%(n=29 / 42)$ of those deaths were due to progressive disease.

Conclusions: In this large size of real world cohort, ASCC failure rate reached $8.5 \%$ and $4.5 \%$ of successful collection could not proceed to ASCT. The research has also shown that achieving long-lasting response with lower number of chemotherapy cycles is important to ensuring the success of ASCC as well as leading to ASCT as planned.

Disclosure: Nothing to declare

\section{P690 \\ CD34 + mobilization and collection from four patients with fanconi anemia}

Chloé Couzin ${ }^{1}$, Elisa Magrin ${ }^{1}$, Thierry Leblanc ${ }^{2}$, Alessandra Magnani ${ }^{1}$, Sandra Manceau ${ }^{I}$, Jean-Sébastien Diana $^{I}$, Michaela Semeraro ${ }^{3}$, Jennifer Nisoy ${ }^{I}$, Masselis Jennifer ${ }^{I}$, Maxime Bouabdelli, Leila Raimbaud, Matthieu Bendavid $^{4}$, Jean Soulier ${ }^{5}$, Stephane Blanche ${ }^{4}$, Marina Cavazzana ${ }^{1}$, François Lefrère ${ }^{I}$
${ }^{1}$ Hôpital Necker-Enfants Malades, Assistance PubliqueHôpitaux de Paris, Biotherapy, Paris, France, ${ }^{2}$ Hôpital Robert Debré, Paris, France, ${ }^{3}$ Hôpital Necker-Enfants Malades, Assistance Publique-Hôpitaux de Paris, Clinical Investigation Center, Paris, France, ${ }^{4}$ Hôpital NeckerEnfants Malades, Assistance Publique-Hôpitaux de Paris, Pediatric Immunology-Hematology, Paris, France, ${ }^{5}$ Hôpital Saint Louis, Hematology Laboratory, Paris, France

Background: Fanconi Anemia (FA) is a rare inherited genetic bone marrow (BM) failure syndrome. While abnormal BM cells production occurs very early in life, the usual age of diagnosis is 5-10 years old. Gene therapy (GT) might be an alternative to hematopoietic stem cells (HSC) transplantation, but harvest a large number of autologous HSC remains a challenge. We started a mobilization assay, FancoMob, to evaluate the safety and the efficacy of FA patients' mobilization with granulocyte-colony stimulating factor (G-CSF) and plerixafor. This study is part of the FA's European GT project "EuroFancolen".

Methods: Four patients with FANCA mutations following the inclusion criteria were selected before pancytopenia. To note, FA4 was diagnosed before clinical manifestation through family screening. They received subcutaneous injection of G-CSF (12 $\mu \mathrm{g} / \mathrm{kg}$ twice a day) from D-1 and plerixafor $(0.24 \mathrm{mg} / \mathrm{kg} /$ day $)$ from $\mathrm{D}-4$. The collection protocol targeted $3 \times 10 \mathrm{e} 6 / \mathrm{Kg}$ of $\mathrm{CD} 34+$ cells, based on a predicted future weight in 5 years. $\mathrm{CD} 34+$ cells and white blood cells (WBC) blood count were monitored tightly along the mobilization. Patients with more than 10 CD34 $+/ \mu \mathrm{l}$ or between 5 and $10 \mathrm{CD} 34 / \mu \mathrm{l}$ with a clustered aspect detected by flow cytometry after plerixafor injection underwent apheresis. CD34+ cells were immunoselected from the collection with CliniMACS purification system (Miltenyi) and cryopreserved for further GT manipulation.

Results: The mobilization target was not achieved for the first two included patients (FA1 12 years old and FA2 8 years old). The minimum value of $\mathrm{CD} 34+/ \mu$ l required wasn't obtained for FA1 and the flow cytometry CD34+ aspect was not clustered for FA2. CD34+ cells were mobilized quickly but transitionally after plerixafor injection for the last two patients, FA3 and FA4, 5 and 2 years old respectively. Both patients underwent apheresis procedures. No CD34+ cell rebound was observed after the apheresis was stopped.Collection target was not achieved after four days of collection for FA3. It was obtained the first day for FA4 (Figure 1). Back-up for HSC transplantation could not be cryopreserved because of the limited number of CD34+ cells collected in patients FA3 and FA4. No short-term adverse events were observed. Following CD34+ immunoselection, CD34+ cell purity and recovery were poor but in the normal range described in the literature 
for Fanconi patients (8-20\%)(Table I). One month after the collection hemograms were unchanged.

Conclusions: Our clinical study offer new data showing that mobilization of FA patients with G-CSF and plerixafor is safe and more efficient for younger patients, especially before clinical manifestations of BM failure. Further efforts are required to establish an effective technic to purify the CD34+ cells after harvesting.

\begin{tabular}{llllllll}
\hline & $\begin{array}{l}\text { Pre-CD34 } \\
\text { selection } \\
\text { TNC (10e8) }\end{array}$ & $\begin{array}{l}\text { Pre-CD34 } \\
\text { selection } \\
\text { CD34 } \\
\text { content }(\%)\end{array}$ & $\begin{array}{l}\text { Pre-CD34 } \\
\text { selection } \\
\text { CD34 } \\
+/ \mathrm{kg}(10 \mathrm{e} 6)\end{array}$ & $\begin{array}{l}\text { Post-CD34 } \\
\text { selection } \\
\text { TNC (10e8) }\end{array}$ & $\begin{array}{l}\text { Post-CD34 } \\
\text { selection } \\
\text { CD34 } \\
\text { content }(\%)\end{array}$ & $\begin{array}{l}\text { Post-CD34 } \\
\text { selection } \\
\text { CD34+ } \\
\text { recovery }(\%)\end{array}$ & $\begin{array}{l}\text { Post-CD34 } \\
\text { selection } \\
\text { CD34 } \\
+/ \mathrm{kg}(10 \mathrm{e} 6)\end{array}$ \\
\hline $\begin{array}{l}\text { FA3-1st } \\
\text { apheresis }\end{array}$ & 167 & 0.07 & 0.73 & 0.16 & 8.4 & 11 & 0.08 \\
$\begin{array}{l}\text { FA3-2nd } \\
\text { apheresis }\end{array}$ & 203 & 0.04 & 0.54 & 0.31 & 9.3 & 35 & 0.19 \\
$\begin{array}{l}\text { FA3-3rd } \\
\text { apheresis }\end{array}$ & 163 & 0.02 & 0.21 & 0.09 & 12.6 & 38 & 0.08 \\
$\begin{array}{l}\text { FA3-4th } \\
\text { apheresis }\end{array}$ & 120 & 0.03 & 0.27 & 0.32 & 7.4 & 59 & 0.16 \\
$\begin{array}{l}\text { FA4 } \\
\text { HD }\end{array}$ & 450 & 0.26 & 11.7 & 0.51 & 20 & 8.6 & 1.01 \\
(n=4) mean & $112(99)$ & $2.68(1.93-$ & NA & $1.94(0.92-$ & $97(93-99)$ & $85(77-100)$ & NA \\
\hline
\end{tabular}

[[P690 Table] 1. Table I. CD34+ cell purification from mobilized peripheral blood stem cells collection for FA3's apheresis and FA4 compared to CD34+ cell purification]

Clinical Trial Registry: $\mathrm{N}^{\circ}$ EudraCT : 2014-005264-14 Disclosure: Nothing to declare

\section{P691}

Basal CD34 + cell and platelets count are a strong predictor for mobilized peripheral blood stem cells on the 4th day of G-CSF treatment in donors

Massimo Martinol, Annalisa Pitino ${ }^{2}$, Mercedes Gori², Tiziana Moscato $^{3}$, Virginia Naso ${ }^{3}$, Anna Ferreri ${ }^{3}$, Fabio $^{2}$ Provenzano $^{3}$, Santina Barillà ${ }^{3}$, Barbara Loteta ${ }^{3}$, Cristina Sanguedolce $^{3}$, Antonia Dattola ${ }^{3}$, Giulia Pucci, ${ }^{4}$, Massimo Gentile $^{5}$, Anna Grazia Recchia ${ }^{6}$, Giovanni Tripepi ${ }^{7}$

${ }^{1}$ Stem Cell Transplant Program, Clinical Section, CTMO, Reggio Calabria, Italy, ${ }^{2}$ IFC-CNR, Roma, Italy, ${ }^{3}$ Grande Ospedale Metropolitano BMM, CTMO, Reggio Calabria, Italy, ${ }^{4}$ Stem Cell Transplantation Program, Processing Facility Section, CTMO, Reggio Calabria, Italy, ${ }^{5}$ Ospedale Annunziata, Cosenza, Italy, ${ }^{6}$ Unità di Ricerca Biotecnologica (URB), Aprigliano, Cosenza, Italy, ${ }^{7}$ IFC-CNR, Reggio Calabria, Italy

Background: Mobilized peripheral blood stem cells (PBSCs) have largely replaced bone marrow as the graft source for allogeneic stem cell transplantation. PBSCs mobilization with G-CSF is highly effective even on the 4th day in order to collect enough number of stem cells. A longitudinal, prospective, observational, single-center, cohort study on healthy donors (HDs) was designed to identify predictors of $\mathrm{CD} 34+$ cells on the 4th day.

Methods: As potential predictors of mobilization, age, sex, body weight, height, blood volume as well as white blood cell count, peripheral blood (PB) mononuclear cells, platelet count, hematocrit, and hemoglobin levels were considered. Two different evaluations of $\mathrm{CD} 34+$ cell counts were determined for each donor: baseline (before granulocyte colony-stimulating factor [G-CSF] administration) and in PB after G-CSF administration on day 4. A total of 122 consecutive HDs with a median age of 47.5 years were enrolled.

Results: The median value of CD34+ on day 4 was 43 cells/ $\mu \mathrm{L}$ (IQ 23-68). Basal WBC, Plt and basal CD34+, are significantly higher for group with $\mathrm{CD} 34+$ on the 4th day over the median than below. A multivariate quartile regression analysis, adjusted by gender, age, basal CD34 + and basal Plt, shows a, progressively steeper, relationship between baseline CD34+, basal Plt and CD34+ on the 4th day. The basal CD34+ cut-off for prevision of CD34+ on the 4 th day was $<=2$ cells $/ \mu \mathrm{L}$ and $>=3$ cells $/ \mu \mathrm{L}$ whereas basal platelets count was $<=229 \times 109 / \mathrm{L}$ and $>=230$ x 109/L.

Conclusions: G-CSF can be highly effective in HDs on the 4th day in order to collect enough number of stem cells and we have developed a model for predicting the probability to perform PBSC collection after a short course of G-CSF.

Disclosure: nothing to declare

\section{P692}

Pre-apheresis peripheral blood CD34 + cell counts highly correlates to actual stem cells collected

\section{Gaertner Johannes ${ }^{1}$, Schaefer-Eckart Kerstin ${ }^{1}$, Wendelin} Knut $^{I}$, Dressler Sabine ${ }^{I}$, Kerache Francoise ${ }^{I}$

${ }^{1}$ Klinikum Nuernberg, Medizinische Klinik 5, Nuernberg, Germany

Background: Prediction of stem cell yield on the basis of pre-apheresis CD34+ cell count and the processed blood volume is essential for the planning and executing of the apheresis process.

Methods: Data analyzed included donor weight, complete blood count and CD34+ count on day of collection, total processed blood volume, $\mathrm{CD} 34^{+}$cell dose collected in the apheresis product and the number of aphereses performed. Using the method described by Pierelli et al, predicted CD34 ${ }^{+}$yields were calculated: Predicted CD34 + yield $\times 106 / \mathrm{kg}=($ Benchmark CE $\times$ Volume of blood to be processed $\mathrm{x}$ peripheral CD34+ count per $\mu \mathrm{l}) /$ (Patient's weight in $\mathrm{kg} \mathrm{x}$ metric conversion factor). 
Results: In 2017 we established the method described by Pierelli to predict the CD34+ cell yield. 323 allogenic aphereses were performed in 2017 with this approach. Mean processed volume was 13.71 Liters. The mean CD34+ peripheral count before apheresis was 68/ul, the mean collected $\mathrm{CD} 34+$ count per $\mathrm{kg}$ bodyweight recipient was 6.35. Pearson's correlation coefficient ( $\mathrm{r}$ ) between predicted yield using pre-apheresis CD34+count and actually collected CD34+ cells per kg bodyweight recipient was 0.967 . The mean difference between predicted and collected cell dose was $+4.45 \%$.

With knowledge of the predicted stem cell count, we were able to adjust apheresis procedure. In case of marginal predicted yield compared to the requested cell dose, we increased the blood volume to be processed. This proceeding led to a significant reduction of second day donations in 2017 by $38 \%$ compared to 2016 . In only 3 cases we saw more than $-40 \%$ lower CD34+ doses collected than initially predicted. All 3 donors showed mild iron deficiency with RBC microcytosis, a factor known to affect apheresis procedure.

Conclusions: Pierellis method of calculating the stem cell yield shows a good correlation between pre-apheresis CD34 + count and actual collected stem cells, making planning and adjusting of the apheresis procedure more feasible and reliable. This proceeding led to significant reduction of second day donations. Attention should be paid to iron deficiency anemia, leading to lower than estimated CD34+ dose.

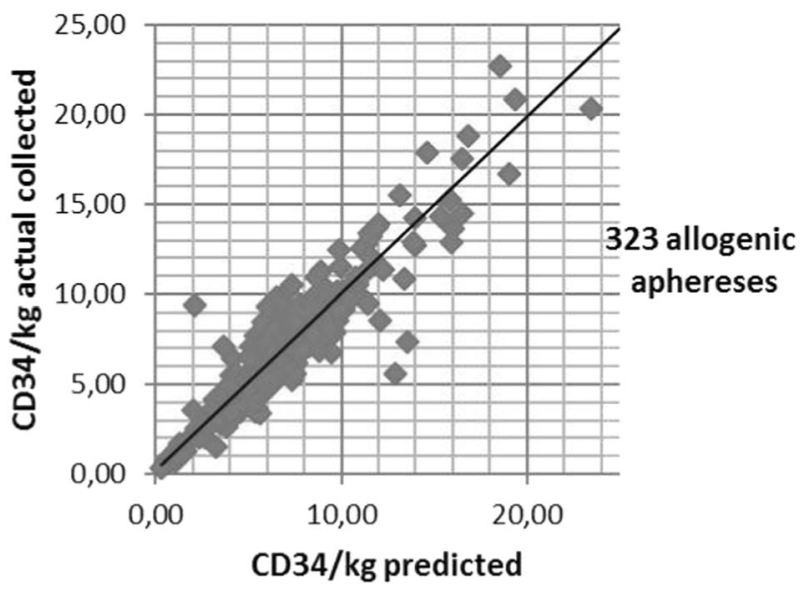

[[P692 Image] 1. Correlation]

Disclosure: none

\section{P693}

Usage and disposal rate of cryostored autologous hematopoietic stem cell grafts from patients with AML,
ALL, MPN and burkitt lymphoma: A 12-year retrospective study

Katharina Kriegsmann ${ }^{1}$, Maurizio Wack', Petra Pavel', Anita Schmitt', Mark Kriegsmann ${ }^{3}$, Felix Krummradt, Thomas Bruckner ${ }^{4}$, Svea Löwhagen ${ }^{5}$, Harald Klüter ${ }^{5}$, Stefan Klein ${ }^{6}$, Carsten Müller-Tidow ${ }^{1}$, Patrick Wuchter ${ }^{5}$

${ }^{1}$ Heidelberg University, Heidelberg, Germany, ${ }^{2}$ IKTZ Heidelberg GmbH, Stem Cell Laboratory, Heidelberg, Germany, ${ }^{3}$ Heidelberg University, Institute of Pathology, Heidelberg, Germany, ${ }^{4}$ Heidelberg University, Institute of Medical Biometry und Informatics, Heidelberg, Germany, ${ }^{5}$ Institute of Transfusion Medicine and Immunology, Medical Faculty Mannheim, Heidelberg University, German Red Cross Blood Service Baden-Württemberg Hessen, Mannheim, Germany, ${ }^{6}$ University Medical Center Mannheim (UMM), Medical Faculty Mannheim, Heidelberg University, Mannheim, Germany

Background: For more than a decade many transplant centers routinely collect and cryopreserve two or more peripheral blood stem cell (PBSC) grafts for a tandem and/ or salvage autologous blood stem cell transplantation (ABSCT) in patients with hemato-oncological diseases. However, subsequent high-dose chemotherapy (HD-CHT) and ABSCT is in many cases not performed for a variety of reasons, specifically in patients with AML, ALL, MPN and Burkitt lymphoma. Data about the actual utilization rate of the cryostored stem cell products are lacking.

Methods: We retrospectively analyzed the collection, storage and disposal practices of PBSC products from a large cohort of patients who were treated at the University Hospital Heidelberg or at the University Medical Center Mannheim during a 12-year period. Disease entities included acute myeloid leukemia (AML, $n=74$ ), acute lymphoblastic leukemia (ALL, $n=22), \quad$ MPN $(n=18$; primary myelofibrosis $[\mathrm{PMF}], \mathrm{n}=9$; chronic myeloid leukemia $[\mathrm{CML}], \mathrm{n}=7$; secondary fibrosis/essential thrombocythemia [ET], $\mathrm{n}=1$; not specified, $\mathrm{n}=1$ ) and Burkitt lymphoma $(n=18)$. Patients between 2001 and 2012 were included and followed until 2016.

Results: An adequate stem cell graft was defined as $\geq 2.0$ $\mathrm{x} 10 \mathrm{Exp} 6 \mathrm{CD} 34+$ cells $/ \mathrm{kg}$ body weight. $97 \%$ of the patients were able to collect at least one stem cell graft and the median number of grafts per patient was 1 (range 0-5). We could demonstrate that only $31 \%$ of all patients who had collected sufficient PBSCs for transplant subsequently underwent an ABSCT. Among the disease entities the actual use of the stored PBSC grafts varied considerably from $0 \%$ to $56 \%$ (Figure 1).

Conclusions: We could identify striking discrepancies between the collection/storage and actual utilization of 
cryopreserved PBSCs. This was associated with considerable efforts for the patients and caused additional treatment costs. On the one hand, having the therapeutic option of an autologous transplantation in the future may represent a clinically relevant advantage. However, a huge number of stem cell products are kept in storage for many years without ever been used for transplantation. Our study provides cause for a careful reevaluation of the current clinical practice, which may help to focus more precisely on patients who actually benefit from a cryostored autologous stem cell graft.

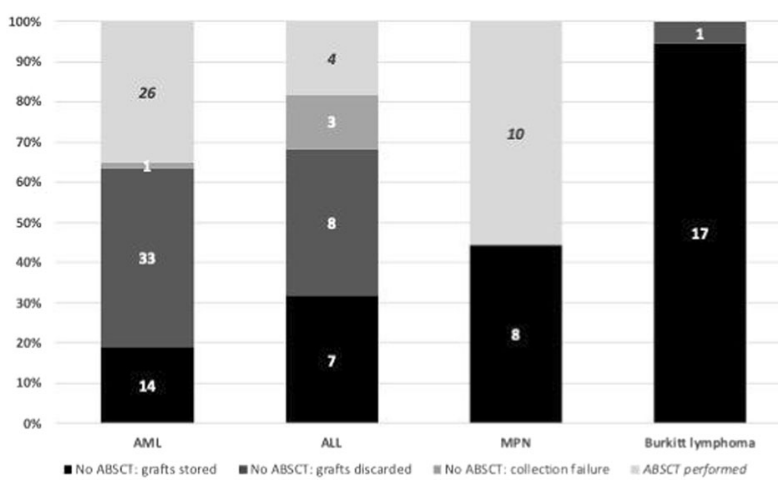

[[P693 Image] 1. Fig. 1: Absolute numbers and relative distribution of stem cell grafts]

Disclosure: The authors confirm that there are no potential conflicts of interest to disclose, except the following: Katharina Kriegsmann: research funding from BMS, Celgene, and Sanofi. Patrick Wuchter: Membership in Advisory Boards for Sanofi-Aventis.

\section{P694}

Reduction of dimethylsulfoxide (DMSO) concentration from $10 \%$ to $5 \%$ in criopreservation of stem cells. Influence on the kinetics of engraftment and tolerance to infusion

Patricia Lopez-Pereira ${ }^{1}$, Beatriz Aguado ${ }^{1}$, Elena Sola ${ }^{1}$, Carmen Cámara ${ }^{I}$, Isabel Vicuña ${ }^{1}$, Lorena Vega ${ }^{I}$, Adrian Alegre $^{1}$

${ }^{1}$ Hospital Universitario de la Princesa, Madrid, Spain

Background: DMSO is the cryoprotectant most used in the cryopreservation of stem cells. It is associated with adverse effects during the infusion of the product, its toxicity being proportional to the volume infused. The most common concentration used has been $10 \%$, although recent publications report that reducing it to $5 \%$ leads to lower rate of side effects without impact on the product or the graft.
Methods: We retrospectively analyzed 207 patients recipients of autologous peripheral blood stem cell transplantation (HCT) in our hospital from January 2009 to September 2017. They are divided into two groups according to the concentration of DMSO used in the freezing $(10 \%$ until September 2014 or $5 \%$ since October 2014).

The baseline characteristics of the patients, the infused product and the graft are shown in Table 1. The CD34 count was performed by flow cytometry. All freezings were performed with a biological freezer for programmed controlled rate cryopreservation and stored in ultrafreezers at $-80^{\circ} \mathrm{C}$.

Results: Both population groups are homogeneous. The t-test was used for statistical analysis. Regarding the CD34 + variable, no statistically significant differences were observed ( $\mathrm{p}=0.636$ ). Neither for the variables leukocyte recovery and platelet recovery $(\mathrm{p}=0.178, \mathrm{p}=0.991$ respectively). The difference in the variable Viability is 5.61 units (CI95\%: [0.61-10.62]) and is statistically significant (p $=0.028$ ) in favor of DMSO $5 \%$.

Regarding adverse effects, $100 \%(\mathrm{n}=4)$ of the serious adverse reactions occurred in the 10\% DMSO group (hypotension and seizures). The mild and moderate ones were similar in both groups, most were mild nausea, vomiting and flushing. Overall, no statistically significant differences were observed due to the low rate of adverse effects found.

\begin{tabular}{lll}
\hline $\begin{array}{l}\text { PATIENTS AND } \\
\text { PRODUCT }\end{array}$ & $\begin{array}{l}\text { DMSO 10\% } \\
(\mathrm{n}=111)\end{array}$ & DMSO 5\% (n=96) \\
\hline GENDER & $\begin{array}{l}56.8 \% \text { male/ } \\
43.2 \% \text { female }\end{array}$ & $\begin{array}{l}47,9 \% \text { male/ } \\
52.1 \% \text { female }\end{array}$ \\
AGE AT HCT & 57 years $(21-70)$ & 58 years $(23-70)$ \\
UNDERLYING DISEASE & $44 / 39.6 \% ; 10 / 9 \% ; 26 / 27.1 \% ; 22 /$ \\
(n/\%): NHL; MM; & $45 / 40.5 \% ; 12 /$ & $22.9 \% ; 38 / 39.6 \% ;$ \\
HL; Others & $10.8 \%$ & $10 / 10.4 \%$ \\
CODITIONING & $44 / 39.6 \% ; 10 / 9 \% ; 26 / 27.1 \% ; 22 /$ \\
REGIMEN (n/\%): BEAM; & $45 / 40.5 \% ; 12 /$ & $22.9 \% ; 38 / 39.6 \% ;$ \\
$\begin{array}{l}\text { Melf } 140 ; \text { Melf } 200 ; \text { Others } \\
\text { VOLUME INFUSED (ml) }\end{array}$ & $49.8 \%(70-2880)$ & $10 / 10.4 \%$ \\
CD34+ x 106 /kg & $3,2(2-15,6)$ & $2,8(2-10)$ \\
VIABILITY(\%) & $64 \%(19.2-99.1)$ & $71.3 \%(19.9-98.2)$ \\
$\begin{array}{l}\text { LEUKOCYTE } \\
\text { RECOVERY (median day } \\
\text { after-HCT) }\end{array}$ & $14(7-67)$ & $17(11-42)$ \\
$\begin{array}{l}\text { PLATELET RECOVERY } \\
\text { (median day after-HCT) }\end{array}$ & $13(8-62)$ & $14(7-43)$ \\
\hline
\end{tabular}

[[P694 Table] 1. Table 1. Characteristics of patients, product and graft.]

Conclusions: In our experience, reduction in concentration of DMSO from 10 to $5 \%$ entails a lower rate of serious 
adverse effects during the infusion in the autotransplant, without worsening the characteristics of the product and with no significant impact on the graft.

Disclosure: Nothing to declare

\section{P695}

The use of biosimilar of filgastrim seems to be less efficacious than original for stem cell mobilization in donors and patients with myeloma or lymphoma

Rocío Parody ${ }^{I}$, Isabel Sanchez-Ortega ${ }^{I}$, Christelle Ferrà ${ }^{l}$, Ramón Guardia ${ }^{2}$, Carme Talarn ${ }^{3}$, Maite Encuentra ${ }^{1}$, Eduard Fort ${ }^{l}$, David Lopez ${ }^{I}$, Morgades $^{I}$, E Alonso ${ }^{I}$, Sandra Ortega ${ }^{1}$, Josep Sarrà ${ }^{3}$ David Gallardo², Josep Maria Ribera ${ }^{I}$, Anna Sureda ${ }^{I}$

${ }^{1}$ ICO - Hospital Duran i Reynals, Barcelona, Spain, ${ }^{2}$ Hospital Josep Trueta, Clinical Hematology, Girona, Spain, ${ }^{3}$ Hospital Joan XXIII, Clinical Hematology, Tarragona, Spain

Background: Biosimilars (BIO) of granulocyte colony stimulating factors (GCSF) were approved several years ago on the basis of some studies that indicated similar efficacy to the already patented GCSF (Neupogen ${ }^{\circledR}$, NEU) both in terms of shortening the neutropenic period after chemotherapy as well as peripheral blood stem cell mobilization in patients with lymphoma and multiple myeloma (MM) treated with autologous stem cell transplantation (auto-HCT). However, all these studies are retrospective and there are still concerns about the real efficacy of BIO and even more about the real benefit on final costs.

Methods: We have retrospectively compared the characteristics of the mobilization procedure in both patients with $\mathrm{MM}$ and lymphomas, and healthy donors that received either NEU or BIO (with no chemotherapy) in 4 University Hospitals in Catalunya from December / 2013 to November 2017. BIO replaced NEU in June 2016 in all 4 institutions. Primary objectives were the mobilization rate (defined as the percentage of patients that achieved $\geq 10 \times 10^{3} / \mathrm{ml} \mathrm{CD} 34$ + cells in peripheral blood on day 4) and the use of plerixafor (PLEX) in each group as pre-emptive strategy. A multivariable analysis of risk factors influencing the use of PLEX and mobilization failure (defined as collection of $<2$ x $10^{6} / \mathrm{kg} \mathrm{CD} 34+$ cells) was also performed.

Results: We treated 216 patients (102 lymphomas and $114 \mathrm{MM}$ ) and 55 healthy donors. Both groups of patients (138 NEU and $78 \mathrm{BIO}$ ) and donors (33 NEU and $22 \mathrm{BIO}$ ) were comparable regarding pre-mobilization general characteristics. There was a trend for a lower median CD34+ peak on day 4 for BIO patients $(17 v s 20$, p value $=0.1)$. A total of 53 patients received PLEX, although 7 of them
(13.2\%) out of strict theoretical indication, CD34+ cells > $10 \times 10^{3} / \mathrm{ml}$ (range 10.5-13.5) and were removed for further analysis $(n=46,31$ in the NEU group and 15 in the BIO group). Median number of CD34+ cells on day 4 was significantly lower in the group BIO who needed PLEX (2.4 vs 4.8 for NEU+PLEX, $\mathrm{p}=002$ ), as well as CD34+ cells finally harvested $\left(2.5 v s 3.3 \times 10^{6} / \mathrm{kg}, \mathrm{p}=0.03\right)$. Mobilization failure rate was higher in BIO group ( $0 v s 20 \%, \mathrm{p}=0.01)$. Regarding no PLEX patients, median number of CD34 + cells on day 4 was also significantly lower for BIO patients (33.4 vs 23.7, $\mathrm{p}=0.03$ ). Risk factors for PLEX use were age, basal disease (lymphoma) and number of prior mobilization therapies. The use of BIO was the only risk factor for mobilization failure patients receiving PLEX [HR 10.3 (95\%CI 1.3-77.8), $\mathrm{p}=0.02]$. With respect to healthy donor mobilization, none of them needed PLEX but 2 cases from the BIO group (9\%) needed more than one apheresis procedures (2 and 3, respectively).

Conclusions: We found a lower efficacy of BIO in the setting of stem cell mobilization of patients with only GCSF both in terms of a lower CD34+ cells peak on day 4 and a lower number of CD34+ cells in final apheresis product. BIO GCSF also seems to be less effective in healthy donors.

Disclosure: No conflict of interest to be declared

\section{P696}

Comparison of $\mathrm{CD34}^{+}$cell mobilization, blood graft composition and recovery after autologous stem cell transplantation (Auto-SCT) in myeloma (MM) compared to non-Hodgkin lymphoma (NHL) patients

Antti Turunen ${ }^{1,2}$, Anu Partanen ${ }^{1}$, Jaakko Valtola ${ }^{1}$, Antti Ropponen $^{3}$, Timo Siitonen ${ }^{4}$, Outi Kuittinen ${ }^{5,6,7}$, Hanne Kuitunen $^{6}$, Mervi Putkonen ${ }^{8}$, Marja Sankelo ${ }^{9}$, Leena Keskinen $^{10}$, Eeva-Riitta Savolainen ${ }^{11}$, Marja Pyörälä ${ }^{1}$, Taru Kuittinen $^{1}$, Raija Silvennoinen ${ }^{12}$, Karri Penttilä ${ }^{13,14}$, Anu Sikiö ${ }^{15}$, Kaija Vasala ${ }^{16}$, Lasse Ågren ${ }^{17}$, Pentti Mäntymaa $^{18}$, Pelkonen Jukka ${ }^{3,18}$, Ville Varmavuo ${ }^{19}$, Esa Jantunen $^{1,2,17}$

${ }^{1}$ Kuopio University Hospital, Kuopio, Finland, ${ }^{2}$ University of Eastern Finland, Clinical Medicine/Internal Medicine, Kuopio, Finland, ${ }^{3}$ University of Eastern Finland, Kuopio, Finland, ${ }^{4}$ Oulu University Hospital, Oulu, Finland, ${ }^{5}$ Kuopio University Hospital, Kuopio, Finland, ${ }^{6}$ Oulu University Hospital, Oulu, Finland, ${ }^{7}$ University of Eastern Finland, Oncology, Kuopio, Finland, ${ }^{8}$ Turku University Hospital, Turku, Finland, ${ }^{9}$ Tampere University Hospital, Tampere, Finland, ${ }^{10}$ Tampere University Hospital, Tampere, Finland, ${ }^{11}$ Oulu University Hospital, Nordlab, Medical Research Center Oulu, Oulu, Finland, ${ }^{12} \mathrm{Helsinki}$ University Hospital, Comprehensive Cancer Center, Hematology, Helsinki, Finland, ${ }^{13}$ Finnish Medicines Agency, Kuopio, Finland, 
${ }^{14}$ Savonlinna Central Hospital, Savonlinna, Finland, ${ }^{15} \mathrm{Cen}-$ tral Hospital of Central Finland, Jyväskylä, Finland, ${ }^{16} \mathrm{Cen}-$ tral Hospital of Central Finland, Jyväskylä, Finland, ${ }^{17}$ North Carelia Hospital District, Joensuu, Finland, ${ }^{18}$ Laboratory Centre of Eastern Finland, Kuopio, Finland, ${ }^{19}$ Kymenlaakso Central Hospital, Kotka, Finland

Background: Auto-SCT is a common treatment in patients with MM or NHL. The aim of the prospective multicenter GOA (Graft and Outcome in Autologous transplantation) study was to investigate the impact of mobilization method used on the cellular composition of collected blood grafts and eventually hematological and immune recovery as well as long-term outcome post-transplant. Altogether 282 patients with MM or NHL transplanted between 5/2012 and $12 / 2016$ at four university hospitals were included. The long-term goal of the study is to evaluate characteristics of optimal blood grafts in regard to post-transplant recovery and outcome.

Methods: Altogether 147 patients with MM undergoing first auto-SCT were compared with 135 patients with NHL. All NHL patients were mobilized with chemotherapy + GCSF, whereas $39 \%$ of MM patients were mobilized with GCSF only $(\mathrm{p}<0.001)$. Mobilization data, graft cellular composition including $\mathrm{CD} 34^{+}$cell subsets and lymphocyte subsets of the blood grafts, post-transplant hematological recovery and outcome were evaluated. The median followup time was 46 months in MM patients and 35 months in NHL patients.

Results: MM patients mobilized $\mathrm{CD}^{+} 4^{+}$cells better (median peak blood CD34 65 vs. $40 \times 10^{6} / \mathrm{L}, \mathrm{p}<0.001$ ). The median number of aphereses was 2 in both groups $(\mathrm{p}=$ 0.09). Altogether $26 \%$ of the NHL patients received plerixafor compared to $12 \%$ in MM patients $(\mathrm{p}=0.002)$. The median number of $\mathrm{CD} 34^{+}$cells collected was higher in MM patients (6.4 vs. $\left.3.9 \times 10^{6} / \mathrm{kg}, \mathrm{p}<0.001\right)$.

The median amount of $\mathrm{CD} 34^{+}$cells (with 7-aminoactinomycin) in the infused graft was $2.35 \times 10^{6} / \mathrm{kg}$ in MM group and $2.5 \times 10^{6} / \mathrm{kg}$ in NHL group $(\mathrm{p}=0.02)$. The grafts contained more NK cells (median 10.1 vs. $6.1 \times 10^{6} / \mathrm{kg}, \mathrm{p}=$ 0.01 ) and $\mathrm{CD} 19^{+}$cells (median 1.69 vs. $0.00 \times 10^{6} / \mathrm{kg}, \mathrm{p}<$ 0.001 ) in MM patients. Neutropenic fever tended to be more common in NHL patients ( $88 \%$ vs. $79 \%, \mathrm{p}=0.06$ ) but MM patients had significantly more bloodstream infections (31 \% vs. $15 \%, \mathrm{p}=0.003$ ). The median duration of hospitalization was longer in the NHL patients $(22 \mathrm{~d}$ vs. 18 $\mathrm{d}, \mathrm{p}<0.001)$ and the NHL patients had significantly more often ICU admissions ( $4 \%$ vs. $0 \%, \mathrm{p}=0.02$ ).

Post-transplant neutrophil engraftment was faster in the NHL group (median $9 \mathrm{~d}$ vs. $12 \mathrm{~d}, \mathrm{p}<0.001$ ). The median time to platelet engraftment was 11 days in both groups. Platelet count was higher in the MM group from day 15 until 1 year after auto-SCT. There were significantly more early deaths ( $<100 \mathrm{~d}$ from the graft infusion) ( $4 \%$ vs. $0 \%$, $\mathrm{p}=0.02)$ and non-relapse deaths $(6 \%$ vs. $0 \%, \mathrm{p}=0.003)$ in the NHL group.

Conclusions: MM and NHL patients differ in terms of $\mathrm{CD} 4^{+}$cell mobilization, graft cellular composition and post-transplant recovery as well as risk of non-relapse death. Thus, the optimal graft may be different in NHL and MM patients.

Disclosure: The study was supported by VTR fund from North Savo Hospital District and study grant from Sanofi.

\section{P697}

Abstract already published.

\section{P698}

Single-center experience in use of plerixafor for autologous stem cell mobilization: Change in practice over 10 years

Sanja Mazić1, Mirta Mikulić ${ }^{1}$, Ines Bojanić ${ }^{1}$, Iva Lucija Burnać $^{1}$, Dubravka Sertić ${ }^{1}$, Sandra Bašić Kinda ${ }^{1}$, Ranka Serventi Seiwerth ${ }^{1}$, Dražen Pulanić ${ }^{1}$, Pavle Rončević ${ }^{1}$, Marijo Vodanović ${ }^{1}$, Dino Dujmović ${ }^{1}$, Igor Aurer ${ }^{1}$, Branka Golubić Ćepulić ${ }^{1}$

\section{${ }^{1}$ UHC Zagreb, Zagreb, Croatia}

Background: Plerixafor has been proven to mobilize human periferal blood stem cells (PBSCs) alone or acting synergistically with granulocyte-colony stimulating factor (G-CSF). It has mainly been used for rescue mobilization after failed regimen of chemotherapy plus G-CSF, but lately preemptive use in poor mobilizers has been established as cost-effective. We aim to show ten years of experience and change in practice with plerixafor use in our center.

Methods: We retrospectively evaluated the outcome of mobilization procedures and leukapheresis collections in our center in the period from January 2009 to October 2018. Practice from the first 5 years, when plerixafor was mainly used as rescue agent after failed attempt, was compared to period from 2014 till present when preemptive use in poor mobilizers (defined as CD34+ cell counts $<10 \times 10^{6} / \mathrm{L}$ blood) was established.

Results: In the period from 2009 to 2013, total of 434 patients underwent collection of autologous PBSCs, and 55 patients required repeated mobilization cycles $(12,7 \%)$. GCSF alone was used in 39 patients and 16 patients (29\%) recieved combination G-CSF with plerixafor. This cohort consisted of 10 males and 6 females with Non-Hodgkin (NHL) and Hodgkin lymphoma (MH); 13 and 3 respectively. We noted 37 unsuccessful mobilizations $(67,3 \%$ in repeated mobilization, $8,5 \%$ in total) of which one patient was from plerixafor group $(6,3 \%)$. 
Starting with 2014 until October 2018, total of 414 patients attempted collection of autologous PBSCs, and 38 poor mobilizers recieved plerixafor during first mobilization cycle. in total, 9 patients required repeated mobilization cycles $(2,2 \%)$ of which 6 were from the plerixafor group. In total 42 patients recieved pleriksafor; 23 females and 19 males, median age 58 (8-71) with following diagnoses: 28 NHL, $5 \mathrm{MH}, 5$ multiple myeloma, 1 neuroblastoma, 1 nephroblastoma, 1 sarcoma Ewing and 1 seminoma. Of 6 repeated mobilizations with plerixafor, 2 patients $(33,3 \%)$ still failed to collect adequate transplant. In this period we had altogether 3 unsuccessful mobilizations $(33,3 \%$ in repeated cycles, $0,72 \%$ in total). This group of patients consisted of 1 male and 2 female patients, median age 42 (33-58), diagnosis of NHL and failure to collect after 4 leukapheresis procedures each. Median number of leukapheresis needed for adequate collection was 2 with preemtive plerixafor use, and 4 in repeated mobilizations.

Conclusions: Our expirience shows that preemtive use of plerixafor in poor mobilizers is efficient and has enhached success of the PBSC collections. Due to drug high cost each institution needs to develop its own algorythm in management of poor mobilizers. The factors contributing to plerixafor mobilization failure still need to be elucidated.

Disclosure: Nothing to declare.

\section{P699}

Platelets recovered from mobilized leukapheresis units obtained from HLA-haploidentical donors fulfill the criteria of a conventional hemocomponent and can be used for transfusion

David Malaspina ${ }^{1}$, Giuseppina Li Pira ${ }^{1}$, Simone Biagini, Elia Girolami', Gianpiero Conflitti ${ }^{1}$, Manuel Broglia ${ }^{I}$, Giuseppe Palumbo', Daria Pagliara', Stefania Gaspari², Franco Locatelli, ${ }^{2,3}$, Pietro Merli ${ }^{2}$

${ }^{1}$ IRCCS Bambino Gesù Children's Hospital, Rome, Italy, ${ }^{2}$ IRCCS Bambino Gesù Children's Hospital, Rome, Italy, ${ }^{3}$ Sapienza University, Rome, Italy

Background: Transplantation of HLA-haploidentical hematopoietic stem cells (haplo-HSCT) is an established procedure for the treatment of several different hematological diseases. One possible strategy to reduce the risk of Graft-versus-Host Disease is represented by the selective depletion of ab T-lymphocytes (coupled with the depletion of CD19+ B-cells in order to reduce the risk of PTLD) using the CliniMACS device (Miltenyi, Bergish-Gladback). Before depletion, leukapheresis units are washed by lowspeed centrifugation, resulting in a platelet (PLT) rich supernatant (PRS) as a by-product generally discarded. We studied the possibility of recovering PLT from PRS of the haplo donor for transfusion to the recipient during the aplasia period occurring after HSCT.

Methods: HSC donors were mobilized with G-CSF (plus Plerixafor in 6 out of 24 donors) as previously described [Locatelli et al, Blood 2017]. Leukapheresis units, obtained with a Spectra Optia device, were washed twice (producing 2 PRS bags) at low speed to remove PLT before starting the ab T-cell/B-cell depletion. The two PRS were leukodepleted by filtration, centrifuged, resuspended and pooled in a total volume ranging from 95 to $360 \mathrm{ml}$ InterSol (IS-PLT) for overnight incubation at $22^{\circ} \mathrm{C}$ with agitation $(60$ cycles $/ \mathrm{min})$. The IS-PLT samples were analyzed for the criteria established by the Italian transfusion law. IS-PLT bags were examined for the following parameters: volume $>40 \mathrm{ml}$, PLT after leukodepletion $>2 \times 10^{11}$, residual leukocytes $<1 \times 10^{6}, \mathrm{pH}>6.4$ at $22^{\circ} \mathrm{C}$ at the end of the 5-day storage period. The sterility was tested using BD Bactec culture vials. The evaluation of the residual leukocytes was performed with the BD LeucoCount Kit. The absolute PLT counts were determined using hemocytometer Sysmex XN2000.

Results: PRS bags from 24 donors were processed to produce IS-PLT units. Median resuspension volume in InterSol was $220 \mathrm{ml}$ (range 95-360). The absolute mean value of PLT counts measured at the end of the storage period was $2.1 \times 10^{11}$ (range 1.0-6.1 $\times 10^{11}$ ). This value was found below the threshold fixed by Italian regulation in 8 cases $(33.3 \%)$. Mean value of residual leukocytes was $1.3 \times 10^{5}$ (range $0-9 \times 10^{5}$ ); the $\mathrm{pH}$ value was always $>6.4$. Sterility was observed in all cases. According to the work of Slichter et al. [New Eng J Med 2010], the number of PLT in the IS-PLT units is sufficient for a median of 1 (range 1-3) transfusion per patient.

Conclusions: We demonstrated that PLTs recovered from leukapheresis bags can be accepted as a conventional hemocomponent according to the parameters fixed by Italian transfusion law and thus can be administered to the haplo-HSCT recipients early after transplantation. This strategy carries several advantages. Indeed, apart from the obvious advantages in terms of reduced costs, IS-PLT can be used to desensitize the recipient by absorption of antiHLA class I antibodies, if present in the recipient. Moreover, this strategy can avoid the risk of sensitizing the transplanted patients with HLA alleles that differ from the donor's ones. Finally, the IS-PLT unit is readily available. A clinical study aimed at testing the use of ISPLT units in transplant recipients will be performed to confirm the clinical efficacy of the approach.

Disclosure: Nothing to declare

\section{P700}

Ultrasound guidance as a powerful tool in increasing 
peripheral vascular access in patients undergoing peripheral blood stem cell collection

\section{Roman Małachowski ${ }^{1}$, Kamil Zieliński ${ }^{1}$, Anna Szmigielska Kapton ${ }^{1,2}$, Mateusz Nowicki ${ }^{1}$, Olga Grzybowska-Izydorczyk ${ }^{1,2}$, Agnieszka Pluta ${ }^{1,2}$, Magdalena Czemerska $^{2}$, Piotr Stelmach ${ }^{1,2}$, Tadeusz Robak ${ }^{1,2}$, Agnieszka Wierzbowska ${ }^{1,2}$}

${ }^{1}$ Copernicus Memorial Hospital in Lodz Comprehensive Center and Traumatology, Hematology, Łódź, Poland, ${ }^{2}$ Medical University of Łódź, Hematology, Łódź, Poland

Background: Central venous catheter (CVC) related complications may lead to high morbidity and mortality. Unlike CVC, peripheral cannulation offers a quick and inexpensive method for safe and non-traumatic vascular access (VA) thus its utilization is strongly recommended whenever possible. The ultrasound (US) guidance for acquiring peripheral VA is a useful tool for reduction or elimination of the need of using CVC for stem cells collection. We have made an attempt to introduce US method in our apheresis unit having no previous experience with US devices. The aim of the study was to measure the decrease of CVC insertions after introducing US and evaluate the quality of VA by comparing average flow rate and confirming that the desired blood volume could be processed.

Methods: The theoretical education involved a free elearning course in peripheral ultrasound-guided VA (PUGVA, USabcd, Aarhus, Denmark). Subsequently, the personnel have implemented knowledge in practical training on gelatine and silicone phantoms and healthy volunteers. The practical activities also included a fiveday course in an apheresis centre with US-guided cannulation experience.

The details concerning VA were recorded, including VA site, cannula size, average inlet flow rate, number of inlet pressure alarms reported by the apheresis device. The procedure details where traditional approach was applied i.e. palpable cannulation and CVCs have been collected. Similarly, the necessary data for procedures where veins were assessed with ultrasound prior to apheresis were recorded.

Results: Before introducing ultrasonography, 58 stem cell collections have been performed in 46 patients. Of all these procedures, 18 were accomplished with CVC $(31 \%)$ and 40 with peripheral VA (69\%). Median cubital vein was the vessel of choice. Out of the 40 peripheral VA procedures, $14(35 \%)$ were problematic, with 10 or more inlet pressure alarms during every procedure.

After the training stage, 34 collection procedures were performed in 25 patients. After introducing US we have observed a significant reduction of the number of CVC insertion required for successful apheresis from $31 \%$ to $9 \%$ $(\mathrm{p}=0.01$; Chi-square test with Fisher's exact). Thirty one procedures were completed with peripheral VA (91\%). Ultrasound device enabled cannulation not only the superficial veins but also for the deeper veins. Cannulation sites included upper arm cephalic vein (39\%), median cubital vein (35\%), upper arm basilic vein $(13 \%)$, median antebrachial vein (13\%). Out of the 34 collections, 13 were considered problematic $(42 \%)$. No difference in an average flow rate was observed between procedures performed peripherally with and without ultrasound usage $(\mathrm{p}=0.34$; $\mathrm{U}$ Mann-Whitney test).

Conclusions: Despite no previous experience in US guidance, we have successfully managed to introduce the new method in our apheresis unit. Within 5 months, we have reduced CVC usage threefold and as the personnel is gaining more experience, we suppose that the CVC usage may be reduced to episodic cases. Despite slightly higher number of pressure alarms, all procedures with ultrasound guidance were completed as planned. Ultrasound guidance is the most important tool for significant increase in peripheral VA usage and may become the only option for patients with difficult VA.

Disclosure: Nothing to declare.

\section{P701}

\section{Abstract already published.}

\section{P702}

Donor blood management in healthy bone marrow donors: A retrospective single institution analysis

Soo-Zin Kim-Wanner ${ }^{1}$, Beate Luxembourg ${ }^{2}$, Nadine Sorg $^{1}$, Carolin Poppe ${ }^{1}$, Milica Bunos ${ }^{1}$, Eva Wingenfeld ${ }^{1}$, Richard Schäfer $^{I}$, Erhard Seifried ${ }^{1}$, Halvard Bönig ${ }^{I}$

${ }^{1}$ German Red Cross and Johann-Wolfgang-Goethe Medical University, Institute of Transfusione Medicine and Immune Hematology, Frankfurt, Germany, ${ }^{2}$ Justus Liebig University Medical Center, Gießen, Germany

Background: Over the last two decades mobilized peripheral blood stem cells (PBSC) have been established as the main source of stem cells because of improved engraftment and no necessity for hospitalization for the donors. Nevertheless, due to the introduction of promising new transplant regimens, especially in the haploidentical transplantation setting bone marrow (BM) donations are regaining importance. Although for both donation methods severe side effects are rarely described, BM collection is associated with considerable blood loss and hence symptoms of acute blood loss are commonly observed. Therefore 
autologous blood are collected routinely in some institutions before donation. Since the collected bone marrow amount depends on the target dose, the WBC yield in the product influences the required bone marrow volume. Therefore we sought to investigate the relationship between collection volume, $\mathrm{RBC}$ volume removal, drop in $\mathrm{Hb}$ and indications for blood transfusion. Furthermore, we assessed WBC and CD34+yields in relationship to various donor parameters and to product volume, in order to find prediction tools for collection volumes.

Methods: 489 allogeneic bone marrow harvests from adult donors were performed at our institution and retrospectively analyzed. Complete blood counts, serum iron and ferritin were assessed at work-up and 4 weeks after donation. The bone marrow product quality including WBC, hematocrit (Hct) and CD34+ cells were assessed by automatic hemocytometry and single-platform flow cytometry with ISHAGE gating.

Results: Besides local pain most of the side effects were related to blood loss. None of the donors received blood transfusions. The mean reduction of hemoglobin levels was $2.5 \mathrm{~g} / \mathrm{dl}$ with a minimum hemoglobin level of $8.9 \mathrm{~g} / \mathrm{dl}$ and a persistent anemia according to WHO criteria after 4 weeks in $7.9 \%$ and pathologically low ferritin levels in $29 \%$. No donor presented symptoms with indication for blood transfusion. The median WBC concentration of the BM product was 26.5/nl (5-95\% percentile:16.77-38.75/ nl) the CD34+cell concentration $186.5 / \mu 1$ (5-95\% percentile: $73.92-346.9 / \mu 1)$. In the linear regression analysis leukocyte counts of the donor before donation correlated significantly with WBC concentration in the product. Thus in order to collect with $90 \%$ certainty the 300 Mio WBC which are a typical per-kg dose for an allogeneic recipient, $16.24 \mathrm{ml}$ of bone marrow must be collected. Collection volume did not systematically affect WBC or CD34+ cell concentration.

Conclusions: Achieving high WBC yields in the bone marrow product allowed for collection of relatively modest $\mathrm{BM}$ volumes, thus protecting donors from excessive blood loss. Acute adverse events were acceptable. Optimization of perioperative management in healthy bone marrow donors may be achieved by good collection technique and reevaluation of WBC yields of each institution to calculate required bone marrow amount. The collection of autologous blood is not indicated. Furthermore stringent pre- and postoperative hemoglobin management is predicted to limit adverse effects.

Disclosure: Nothing to declare.

\section{P703}

Donor-recipient weight ratio predicts successful stem cell mobilization on day four of GCSF mobilization
Ekaterina Selbuz ${ }^{1}$, Maximilian Christopeit ${ }^{2}$, Martina Güllstorf ${ }^{2}$, Susanne Schlichting ${ }^{I}$, Claudia Schubert ${ }^{I}$, Sven Peine $^{1}$, Nicolaus Kröger ${ }^{2}$, Francis Ayuk ${ }^{2}$

${ }^{1}$ University Medical Center Hamburg-Eppendorf, Hamburg, Germany, ${ }^{2}$ University Medical Center Hamburg-Eppendorf, Hamburg, Germany

Background: Peripheral blood stem cells are generally the preferred graft source for allogeneic stem cell transplantation for malignant disease. In most centers first apheresis is performed on day 5 after 8 to 9 doses of granulocyte colony stimulating factor (GCSF) up to $5 \mathrm{ug} / \mathrm{kg}$ twice daily. The dose of GCSF and the number of apheresis procedures required contribute to symptom, travel and time burden donors are put through during the process. We hypothesized that taking donor-recipient weight differences into consideration may help reduce this burden

Methods: A total of 261 healthy donors who donated peripheral blood stem cells on day 4 of GCSF mobilization in the period between January 2015 and August 2018 at the University Medical Center Hamburg-Eppendorf were included in this quality control evaluation. The donors were divided into two cohorts. The impact of donorrecipient weight ratio on stem cell harvest was tested in the training cohort (2015-2016) and validated in the second cohort (2017-2018). For the training cohort, donors were grouped according to donor-recipient weight ratio $<1.0$ vs. 1.0-1.2 vs. $>1.2$. For the purpose of this analysis a stem cells dose of $5 \times 10^{6} \mathrm{CD} 34+/ \mathrm{kg}$ recipient weight was set for successful apheresis.

Results: In the training cohort including 142 donors, 52 (37\%), $36(25 \%)$ and $54(38 \%)$ had a donor-recipient weight ratio of $<1.0,1.0-1.2$ and $>1.2$ respectively. The target stem cells count of $5 \times 10^{6} \mathrm{CD} 34+/ \mathrm{kg}$ recipient weight was achieved in 36 of 52 (69\%), 26 of $36(72 \%)$ and 45 of $54(83 \%)$ donors with donor:recipient weight ratio < $1.0,1.0-1.2$ and $>1.2$ respectively. The cut-off for the validation cohort was therefore set at a weight ratio of 1.2.

In der validation cohort including 119 donors, 75 (48\%) had a weight ratio $>1.2$ while $62(52 \%)$ had a weight ratio $\leq$ 1.2. Overall in this cohort target cell count of $5 \times 10^{6} \mathrm{CD} 34$ $+/ \mathrm{kg}$ recipient weight was reached in $92(77 \%)$ cases. This target was reached in 43 of $62(69 \%)$ of donors with weight ratio $\leq 1.2$ and in 49 of $57(86 \%)$ donors with weight ratio > $1.2, \mathrm{p}=0.03$.

Conclusions: A donor-recipient weight ratio of $>1.2$ is seen in about $40 \%$ of peripheral blood stem cell donations for allogeneic stem cell transplantation. In these cases apheresis on day 4 after 7 doses of GCSF is reasonable. Donors with lower weight ratios should preferentially donate on day 5 after 8 to 9 doses of GCSF.

Disclosure: All authors declare no conflicts of interest 


\section{P704}

Donor risk assessment for a second collection: The impact of time between donations and donor age

Ana Catarina Pinho ${ }^{1}$, Filipa Bordalo ${ }^{1}$, Sérgio Machado Lopes $^{1}$, Sara Ferreira ${ }^{1}$, Fátima Amado ${ }^{1}$, João Mota ${ }^{2}$, Susana Roncon ${ }^{1}$

${ }^{1}$ Instituto Português de Oncologia do Porto FG, EPE, Porto, Portugal, ${ }^{2}$ Centro de Sangue e da Transplantação do Porto, Porto, Portugal

Background: The effect of a second mobilization and collection of peripheral blood stem cells (PBSC) on the cell yield is low, as we previously demonstrated. However, donor safety has been poorly addressed with no changes in the clinical practices.

Methods: Second donations of unrelated and related donors performed between 2001 and 2017 were evaluated $(\mathrm{n}=24)$, including PBSC+PBSC $(\mathrm{n}=18)$, bone marrow $(\mathrm{BM})+\mathrm{PBSC}(\mathrm{n}=5)$ and PBSC+BM $(\mathrm{n}=1)$. Analytical parameters including leukocyte, lymphocyte, hemoglobin and LDH quantification, obtained on the pre-harvest evaluation of first and second donation, were retrospectively analyzed and compared for all donors. The Portuguese Bone Marrow Donors Registry (CEDACE) recommends a time between donations no lesser than 6 months. It also states that in very urgent situations like graft failure, donor should be clinical and analytical cleared and its safety ensured.

In order to evaluate the impact of time between donations, donor population was divided in 3 groups: < 2 months, 2-6 months, $>6$ months; to determine the influence of donor age, donors were divided in 2 groups: < 40 and $\geq 40$ years.

Results: Among the total of 24 donors, 13 were volunteer donors of CEDACE and 11 were familiar. Fifteen second donations were performed because of recipient graft failure and 9 due to disease progression or relapse. At the time of second collection, median donor age was 37 years (range 23-57). The median delay between both collections was 262 days (37-1519). Time between donations did not seem to substantially impact the analytical donor evaluation: leukocytes, lymphocytes, hemoglobin and LDH results are kept within the reference values. However, donors with less than 2 months between donations showed a slight decrease on leukocyte counts $(35 \%)$ and hemoglobin values $(12 \%)$, from the first to the second pre-harvest evaluation. Donor age showed no significant influence on the analytical evaluation. Nevertheless, when considering only the PBSC+PBSC donations, donors with $\geq 40$ years showed a small decrease on lymphocyte counts (19\%).
Conclusions: This study demonstrated that the analytical parameters, chosen based on literature, had no significant changes between first and second donation. However, particular attention should be paid when time between donations is lesser than 2 months or donor age is $\geq 40$ years.

As we concluded that no significant changes were observed in the group of 2-6 months, it is our opinion that the minimum of 6-12 months established by the registries can be shortened to 2 months ensuring donor safety. An accurate donor risk assessment with a larger population should be accomplished in order to strengthen this recommendation.

Disclosure: Nothing to declare.

\section{P705}

Plerixafor modifies the graft composition favorably in $T$ cell replete haploidentical transplant: A pilot study from India

Gaurav Kharya ${ }^{1}$, Atish Bakane ${ }^{1}$, Pratibha Dhiman ${ }^{1}$, Anil Khetrapal $^{\text {, }}$, Vikrant Bhar ${ }^{l}$

${ }^{1}$ Artemis Hospital, Gurugram, India

Background: $\mathrm{T}$ cell replete haploidentical stem cell transplant (HHSCT) is complicated mainly by increased risk of graft failure (GF) and graft versus host disease (GVHD). Conventionally GCSF has been used to mobilize hematopoietic stem cells (HSC). In TCR HHSCT GCSF mobilized graft with megadose of CD34+ cells expose the patient to higher doses of alloreactive $\mathrm{T}$ cells increasing the risk of GVHD. Plerixafor based mobilization gives an advantage of giving high CD34 cell dose limiting exposure to high alloreactive $\mathrm{T}$ cell dose. We share our experience of GCSF + Plerixafor based mobilization for TCR HHSCT.

Methods: 9 consecutive patients suffering from SCD who underwent HHSCT between Jan 2018 till date along with the respective donors were enrolled in the study (Group 1). All 9 underwent pre-transplant immune suppression (PTIS) 2 cycles at 3 weekly intervals using fludarabine+cyclophosphamide+dexamethasone. The graft was mobilized using GCSF@10mcg/kg/day(D1D5)+plerixafor@0.24mg/kg(D5) 6-8 hours before the PBSCH. GVHD prophylaxis included PTCy $50 \mathrm{mg} / \mathrm{kg} /$ day on D3 and 4, sirolimus and MMF starting from D5. Group 2 included 5 historical controls where graft was mobilized using GCSF@10mcg/kg/day(D1-D5). Various parameters pertaining to mobilization, harvest, engraftment, GF and GVHD were assessed between the two groups. 
Results: In Group 1 median age of donor was 41 years (range 19 to 51 years). In Group 2 median age of donor was 31years (range21 to 48 years). Table 1

\begin{tabular}{llll}
\hline Variable analyzed & $\begin{array}{l}\text { Group 1 } \\
(\mathrm{n}=9) \text { mean }\end{array}$ & $\begin{array}{l}\text { Group } \\
(\mathrm{n}=5) \text { mean }\end{array}$ & P value \\
\hline $\begin{array}{l}\text { Preharvest CD34 count } \\
\text { Volume of blood }\end{array}$ & $\begin{array}{l}182.8 \times 106 / \mathrm{kg} \\
\text { processed }\end{array}$ & $\begin{array}{l}96.4 \times 1 \mathrm{ml} \\
10948 \mathrm{ml}\end{array}$ & 0.04 \\
$\begin{array}{l}\text { Duration of procedure } \\
\text { Volume of ACD used }\end{array}$ & $\begin{array}{l}153.44 \mathrm{mins} \\
630.8 \mathrm{ml}\end{array}$ & $234.6 \mathrm{mins}$ & 0.07 \\
CD34 dose collected & $21.08 \times 106 / \mathrm{kg}$ & $11.2 \times 106 / \mathrm{kg}$ & 0.02 \\
CD34 dose infused & $10 \times 106 / \mathrm{kg}$ & $10 \times 106 / \mathrm{kg}$ & $\mathrm{NA}$ \\
CD3 dose infused & $18.59 \times 107 / \mathrm{kg}$ & $35.58 \times 107 / \mathrm{kg}$ & 0.02 \\
$\begin{array}{l}\text { Day on neutrophil } \\
\text { engraftment }\end{array}$ & 13.5 & 13 & 0.52 \\
$\begin{array}{l}\text { Day of platelet } \\
\text { engraftment }\end{array}$ & 12.7 & 13 & 0.66 \\
\hline
\end{tabular}

\section{[[P705 Table] 1. Table 1]}

elaborates other parameters analyzed between the two groups. One patient in Group 1 developed grade II acute GVHD whereas 3 patients in Group 2 developed acute GVHD grade II-IV. At the last follow up no (0/9) patient in Group 1 has any symptoms of chronic GVHD whereas (2/5) patients in Group 2 have features of chronic GVHD (one extensive, one limited).

Conclusions: Our observation suggests that upfront use of plerixafor in combination with GCSF modifies the graft favorably decreasing the risk of graft failure and graft versus host disease both acute and chronic. It also helps the donor by decreasing the total volume processed, amount of ACD exposure and the duration of harvest.

\section{Disclosure: None.}

\section{P706}

Impact of vitamin D levels on peripheral stem cell mobilization in autologous hematopoietic stem cell transplant recipients

\section{Ferda Can', Zeynep Arzu Yegin', Zubeyde Nur Ozkurt', Orhun Akdogan ${ }^{1}$, Lale Aydin Kaynar ${ }^{1}$}

${ }^{1}$ Gazi University Faculty of Medicine, Ankara, Turkey

Background: .A favourable impact of vitamin D levels on hematopoietic stem cells was demonstrated in animal experiments. Human stem cells were also found to be sensitive to vitamin D in ex-vivo studies. The aim of this study is to evaluate the impact of pre-mobilization vitamin $\mathrm{D}$ levels on peripheral stem cell mobilization in patients undergoing autologous hematopoietic stem cell transplantation (AHSCT).

Methods: A total 103 AHSCT candidates [median age: 57(18-75) years; male/female: 54/49] were included in this study. Twenty-seven patients $(26.2 \%)$ were diagnosed as non Hodgkin's lymphoma, 8 patients (7.8\%) Hodgkin's lymphoma, 52 patients $(50.5 \%)$ multiple myeloma, 12 patients $(11.6 \%)$ acute myeloid leukemia, 3 patients $(2.9 \%)$ plasmocytoma and 1 patient (1\%) testis cancer. Premobilization serum 25-hydroxy vitamin D (25-OH D) levels were measured with immunoassay method.

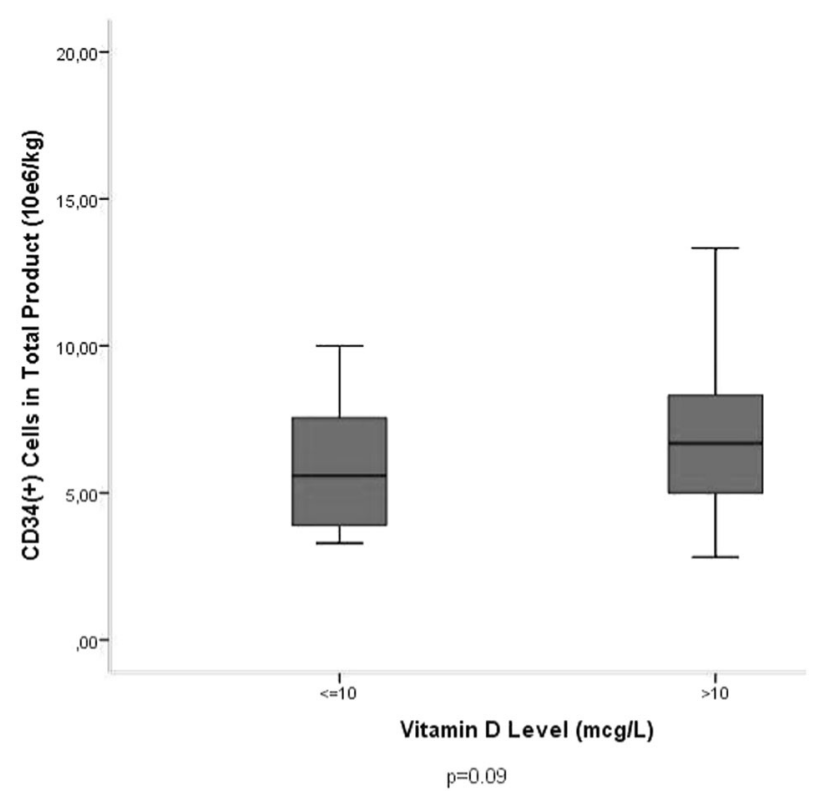

[[P706 Image] 1. Total product CD34+ cell count / Vitamin D levels]

Results: Median vitamin D level was $12(5-76) \mu \mathrm{g} / \mathrm{L}$ in the study population. Peripheral $\mathrm{CD}^{+} 4^{+}$cell count was found to be $27(9-893) \times 10^{6} / \mathrm{kg}$ on median $5(2-18)$ day of granulocyte colony stimulating factor (G-CSF). Median $\mathrm{CD} 4^{+}$cell count was $4.2(0.6-15) \times 10^{6} / \mathrm{kg}$ in the product of the first apheresis, while it was found to be 6.1(2.8$17.2) \times 10^{6} / \mathrm{kg}$ in the total product at the end of median 1 (1-3) apheresis. A total of 16 patients were exposed to radiotherapy before mobilization. Mobilization regimen was G-CSF in 54 patients $(52.4 \%)$ and chemotherapy $+\mathrm{G}-$ CSF in 49 patients $(47.6 \%)$ which mainly contains high dose cyclophosphamide. Plerixafor was used in $36(35 \%)$ patients due to mobilization failure. The total product $\mathrm{CD}_{3} 4^{+}$cell count was found to be significantly higher in myeloma patients compared to lymphoma patients [6.7 (2.9-17.2) vs 5(3-10.1); $\mathrm{p}=0.001]$. Peripheral $\mathrm{CD}^{+} 4^{+}$cell count [34(9-983) vs 24,5(9-130); $\mathrm{p}=0,025]$, first product $\mathrm{CD}^{+}{ }^{+}$cell count $[5.5(0.6-15)$ vs $3.6(1.2-10) ; \mathrm{p}=0,002]$ 
and total product $\mathrm{CD} 34^{+}$cell count $[6.7(02.8-15)$ vs 5.9 (2.9-17.2); $p=0.047]$ were significantly higher in patients receiving chemotherapy $+\mathrm{G}-\mathrm{CSF}$ than G-CSF only. The study group was divided into two groups based on peripheral CD34 (cut-off level: $20 \times 10^{6} / \mathrm{kg}$ ) as well as product CD34 levels (cut-off level: $5 \times 10^{6} / \mathrm{kg}$ ). Vitamin D levels were found to be similar among these groups (p>0.05). Total product $\mathrm{CD} 34^{+}$cell count was found to be relatively lower in patients with vitamin $\mathrm{D}$ levels below $10 \mu \mathrm{g} / \mathrm{L}$ [5.6(3.3-10) vs 6.7(2.8-17.2); $\mathrm{p}=0,09]$. (Figure 1)

Conclusions: Based on its effect on stem cells in in vitro studies, it may be considered that vitamin D may have a favourable impact on stem cell mobilization. Statistically insignificant but relatively lower total product $\mathrm{CD} 34^{+}$cell count in patients who had lower vitamin D levels, which may indicate a role for vitamin $\mathrm{D}$ in stem cell mobilization, needs to be confirmed with larger studies. Considering the high prevalence of vitamin $\mathrm{D}$ deficiency in the general population, the possible role of vitamin $\mathrm{D}$ in hematopoietic stem cell mobilization deserves further consideration.

Disclosure: Nothing to declare

\section{P707}

Hematopoiesis recovery after autologous stem cell transplantation with cryoprotectant DMSO washing from mononuclear concentrate before infusion in patients with hodgkin lymphoma

Natalia Zorina ${ }^{1}$, Natalia Minaeva ${ }^{\mathbb{1}}$, Marina Khorobrykh ${ }^{1}$, Philip Sherstnev ${ }^{I}$, Sergey Utemov ${ }^{1}$, Konstantin Vetoshkin $^{1}$, Natalia Isaeva ${ }^{1}$, Anastasia Endakova ${ }^{1}$

${ }^{1}$ Federal State Budget Institution of Science 'Kirov Scientific Research Institute of Hematology and Blood Transfusion of the Federal Medical-Biological Agency', Kirov, Russian Federation

Background: One of the factors, affecting efficiency of autologous hematopoietic stem cell transplantation (autoHSCT) in Hodgkin lymphoma (HL) patients is early recovery of graft, depending on CD $34+$ cell count and conditions of cell product cryopreservation and storage. It is well known, that dimethylsulfoxide (DMSO), used for cryopreservation, can be cardiotoxic and cause diverse gastrointestinal, pulmonary, kidney, liver side effects and acute hemolysis. Lethal for animals dose 30$40 \mathrm{mg} / \mathrm{kg}$ leads to life threatening arrhythmias and respiratory arrest. In order to improve DMSO toxicity different ways of alternative cryoconservation modes are studied - lower DMSO concentration (5\% VS 10\%), temperature $-80^{\circ} \mathrm{C}$ instead of ultra-low and washing of cell product. Aim of the study is to evaluate the influence of DMSO washing on hematopoietic recovery after autoHSCT.

Methods: Retrospective analysis of hematopoietic recovery of 37 relapse/refractory HL patients after autoHSCT was performed. Mobilization regimen included second line chemotherapy for HL (DHAP, BeGEV, IGEV, ICE) with consecutive G-CSF administration. CD 34+ cells were assessed, using 6-colour flow cytometer FACS Canto II while cell collection, thawing and washing. Cells with $10 \%$ DMSO were stored at $-196^{\circ}$ and washed in 14 cases of transplantation with human albumin-dextran (Reopolyglukin) and centrifugation. Statistical data processing was performed by the $\chi^{2}$ method - Pearson criterion; $\mathrm{p}$ - the level of significance of differences.

Results: Patient groups had no difference in age, disease stage, gender, time from treatment start to autoHSCT and CD34+ cell count $(p>0,05)$. Time to WBC recovery $>1 \times 10^{9} /$ л was 9-29 (median 13,6) days vs 10-34 (median $13,7)$ days, time to platelet $>30 \times 10^{9} /$ л recovery was $11-34$ (median 16,9) days vs 11-58 (median 17,9) days in groups without and with cell washing respectively $(\mathrm{p}=0,507)$. No difference in blood component consumption was observed $(\mathrm{p}=0,546)$. In 18 out of $23(78 \%)$ patients during cell reinfusion without washing nausea, vomiting, arterial hypertension was observed, no reactions were detected after cell washing $(\mathrm{p}=0,03)$.

Conclusions: Washing autologous mononuclear cells from cryopreservant DMSO does not lead to low hematopoietic recovery rate after autoHSCT and can avoid toxicity, thus making autoHSCT more safe.

Disclosure: authors declare no conflict of interests.

\section{P708}

Quality assesment of hematopoietic stem cells autografts after cryostorage, harvested using plerixafor

Irina Kobzeva ${ }^{1}$, Tatiana Astrelina, Anait Davtyan ${ }^{1}$, Elena Sokolova $^{1}$, Zarui Simavanyan ${ }^{1}$, Victoria Nikitina ${ }^{1}$, Yulia Suchkova $^{1}$, Anastasiya Machova ${ }^{I}$, Elena Lomonosova ${ }^{I}$

${ }^{1}$ State Research Center Burnasyan Federal Medical Biophysical Center FMBA of Russia, FMBA of Russia, Moscow, Russian Federation

Background: The introduction of high-dose chemotherapy followed by transplantation of autologous hemopoietic stem cells (HSCs) into the treatment program for multiple myeloma (MM) has significantly increased the frequency of achieving complete remissions and overall survival in patients. To obtain a sufficient amount of HSCs, hematopoiesis is stimulated with granulocyte-macrophage factors (GM-CSF) both in mono mode and after the administration of cytostatics followed by cytapheresis sessions (alone or 
after the cytostatics followed by cytapheresis sessions) . Cryopreservation protocols are used to preserve cells in a viable state, followed by long-term storage of transplants in liquid nitrogen. However, in some patients it is not possible to obtain the necessary amount of HSCs. The inclusion of plerixafor in standard mobilization schemes allows you to prepare the sufficient quantity of HSCs in most patients with MM.

Methods: The study included 18 samples of autografts from 18 patients with MM from 2015 to 2017 (Median $2.1 \pm 0.3$ ). HSCs mobilization was performed on the background of unstable blood formation after high doses of cyclophosphamide $6 \mathrm{~g} / \mathrm{m}^{2}$ with the subsequent administration of G-CSF at a dose of 12-24 $\mu \mathrm{g} / \mathrm{kg}$ (9 samples from 9 patients) and with the addition of plerixaphor at a dose of $0.24 \mu \mathrm{g} / \mathrm{kg}$ (9 samples from 9 patients). Immunophenotype viability of HSCs in autotransplants after cryopreservation were determined by flow cytometry using the ISHAGE protocol on a flow cytometer (FACS CantoII, Becton Dickinson) by expressing surface markers of antibodies against CD34, CD45, CD90, CD38 and staining with 7aminoactinomycin (7-AAD). The colony-forming activity of HSCs (CFU-CFU-mix, CFU-GM, CFU-G, CFU-M) was evaluated in methylcellulose (MethoCult H4435, Stemcell Technologies, Canada) for $1 \times 10^{5}$ transplanted cells for 14 days.

Results: The viability of HSCs in autografts (CD45 + I CD34 + / 7ADD-) after cryopreservation in both groups was $98 \pm 0.7 \%$. In the group of samples using plerixaphor, a higher content of primitive hemopoiesis precursors (primitive cells $)(\mathrm{CD} 34+\mathrm{CD} 90+\mathrm{CD} 38-)$ was detected compared with the control group $(29.2 \pm 2.7 \%$ and $13.2 \pm$ $12 \%$, respectively). The CFU count (CFU-CFU-mix, CFUGM, CFU-G, CFU-M) in the plerixafor group was $100 \pm$ 2.5 per $1 \times 105$ explanted cells, in the control group - $70 \pm$ 1.8 (Figure 1A-D).

Conclusions: The use of plerixafor against the background of standard protocols for the mobilization of HSCs allows to obtain high-quality graft with a higher content of primitive cells and proliferative activity.

Disclosure: no conflict of interest. Nothing to declare.

\section{P709}

Comparison of effectiveness of plerixafor plus G-CSF in poor and very poor-movilizers: Efficacy of the combination of plerixafor and G-CSF in poor-movilizer

Christell Catañón-Fernández ${ }^{1}$, Jose M García-Gala ${ }^{1}$, Eva Martinez-Revuelta ${ }^{I}$, Ana Julia Gonzalez-Huerta ${ }^{1}$, Joud Zanabili Al-Sibai ${ }^{\prime}$, Angeles Fernández-Rodriguez ${ }^{\prime}$, Lucia Morais Bras ${ }^{1}$, Walter Javier Zambrano Márquez, Laura
Escalada González ${ }^{1}$, Paola Carolina Tamayo Arrollo ${ }^{1}$, Enrique Colado-Varela ${ }^{I}$, Soledad González-Muñiz ${ }^{I}$

${ }^{1}$ Hospital Universitario Central de Asturias, Hematologia Hemoterapia, Oviedo, Spain

Background: Poor mobilizers (PM) defined as those with a peripheral blood $\mathrm{CD} 34^{+}$count $\leq 10$ cells $/ \mu \mathrm{l}$ on day +4 is a significant risk factor for mobilization failure. Within these, patients with $<5$ cells $/ \mu$ l are considered as very poor mobilizers (VPM). Use of plerixafor in VPM patient is controversial. The aim of our study is to compare mobilizing and engraftment between PM and VPM who received Plerixafor plus GCSF (P+G-CSF).

Methods: In our center, mobilization with G-CSF at dose of $10 \mu \mathrm{g} / \mathrm{Kg} /$ day was used in all pts. Apheresis were scheduled on day +5 . Plerixafor $(0.24 \mathrm{mg} / \mathrm{kg})$ was added if the number of $\mathrm{CD} 34^{+}$cells on day +4 was $<10 / \mathrm{uL}$ for $2 \times 10^{6} \mathrm{CD} 34^{+} / \mathrm{kg}$ requested (or $<20 / \mathrm{uL}$ for $4 \times 10^{6} \mathrm{CD} 34^{+} / \mathrm{kg}$ ), or if the number of $\mathrm{CD} 34^{+}$cells collected in the first apheresis was $<50 \%$ of $\mathrm{CD} 34^{+}$cells requested.

Between January 2016 and September 2018, 37 out of 157 pts $(23,6 \%)$ received Plerixafor for mobilization. We retrospectively studied 30 pts who mobilized with $\mathrm{P}+\mathrm{G}$ CSFdue to the number of $\mathrm{CD} 34^{+}$cells on day +4 was $<10 /$ uL

Results: Twelve out of 30 pts were PM, 7 were females, median age 55,5 years (range:32-70). Patients' baseline diseases were: 10 non-Hodgkin lymphoma (NHL) $(83,3 \%), 1$ multiple myeloma (MM) and 1 Hodgkin lymphoma. Median CD $34^{+}$cell count on day +4 was $8 /$ uL (range:6-10). There was no mobilization failure. Eighteen out of 30 pts (60\%) were VPM, 9 were females, median age 62,5 years (range:34-69). Patients' baseline diseases were: $10 \mathrm{NHL}(55,5 \%), 7 \mathrm{MM}$ and 1 solid tumor. Median $\mathrm{CD} 34^{+}$cell count on day +4 was $2,5 / \mathrm{uL}$ (range:2-5). Two out of 18 pts $(16,6 \%)$ were considered mobilization failure, in 2 of them did not realized apheresis due to $\mathrm{CD} 34^{+}$cell count on day +5 was $2 / \mathrm{uL}$. No difference was seen between both groups regarding gender, age, patients baseline disease or median $\mathrm{CD} 34^{+}$cells count on day +4 .

VPM needed more apheresis sessions, $5 / 16$ pts required 2 sessions against $1 / 12$ pts in PM ( $\mathrm{p}=$ not significant (ns). We obtained enough cells to carry ASCT in $90 \%$ pts, although mean number of $\mathrm{CD} 34^{+}$cells obtained in VPM was lower than in $\mathrm{PM}\left(4,89 \times 10^{6} / \mathrm{kg}\right.$ vs $6,38 \times 10^{6} / \mathrm{kg}$, respectively) $(\mathrm{p}=\mathrm{ns})$.

Twenty-six pts underwent ASCT and mean number of $\mathrm{CD} 34^{+}$cells infused were $4,27 \times 10^{6} / \mathrm{kg}$ in VPM vs 
$5,18 \times 10^{6} / \mathrm{kg}$ in $\mathrm{PM}(\mathrm{p}=\mathrm{ns})$. Regarding engraftment, the mean time to achieve $\geq 500 / \mu \mathrm{L}$ neutrophils and $\geq 1.000 / \mu \mathrm{L}$ were later in VPM than in PM $(p=0,04)$. The remainder results about engraftment can be seen in table 1.

\begin{tabular}{llll}
\hline Median days to reach: & VPM & PM & p VALUE \\
\hline Neutrophils $\geq 500 / \mu \mathrm{L}$ & 12.77 & 11.09 & 0.04 \\
Neutrophils $\geq 1.000 / \mu \mathrm{L}$ & 13.54 & 11.36 & 0.04 \\
Platelets $\geq 20.000 / \mu \mathrm{L}$ & 24 & 12 & $\mathrm{NS}$ \\
Platelets $\geq 50.000 / \mu \mathrm{L}$ & 39.5 & 18.18 & $\mathrm{NS}$ \\
Median days of hospital stay: & 19.43 & 18.64 & $\mathrm{NS}$ \\
\hline
\end{tabular}

\section{[[P709 Table] 1. Engraftment]}

Conclusions: In our series, 23,6\% pts needed Plerixafor. The use of preemptive and rescue strategy of Plerixafor demonstrate effectiveness in more than $80 \%$ of VPM. Despite the amount of infused $\mathrm{CD} 34^{+}$cells were similar between VPM and PM patients, the median time to neutrophils engraftment was delayed in VPM. In conclusion, VPM could beneficiated of received Plerixafor on day +4 .

Disclosure: García-Gala JM. Honoraria from Sanofi

\section{P710}

Experience using G-CSF and plerixafor for stem cell mobilization in haploidentical transplantation with exvivo $\mathrm{T}$-depletion for pediatric patients

Mercedes Gasior Kabat ${ }^{1}$, Maria Isabel Rivas Pollmar ${ }^{1}$, Antonio Pérez Martínez ${ }^{I}$, Eduardo García Pérez $z^{I}$, Abel Dos Santos ${ }^{1}$, Ana Belen Romero ${ }^{1}$, Antonio Marcos ${ }^{1}$, Irene Panizo Echauri, Raquel De Paz ${ }^{1}$

${ }^{1}$ Hospital Universitario La Paz, Hematology, Madrid, Spain

Background: Healthy donors ocassionally show a poor response to mobilization agents. Plerixafor $+\mathrm{G}-\mathrm{CSF}$ can be a salvage strategy in poor mobilizers. Some series describe the use of plerixafor to collect greater doses of CD34+ cells in hematopoietic stem cell transplantation (HSCT) with Tcell depletion. Plerixafor use in the mobilization protocol could help collecting higher CD34+ dose in indirect T-cell depletion (CD34+ selection) for ex-vivo manipulated haploidentical transplantation, with less number of apheresis and a rapid engraftment.

Methods: Data of fourteen healthy peripheral-blood donors was retrospectively collected. They received 4 days $10 \mathrm{mcg} / \mathrm{Kg} /$ day G-CSF and $0,24 \mathrm{mg} / \mathrm{Kg} /$ day Plerixafor on $4^{\circ}$ day as mobilization treatment. Fourteen pediatric patients (median age 13 years, range 8-15) diagnosed with malignant and no malignant hematological diseases received haploidentical HSCT with $\mathrm{CD} 34+$ selection and CD45RA+ depletion between February 2015 and July 2017 .

Results: One leukoapheresis procedure was performed in all cases. Median processed volume was 11 liters (range 614). Median of CD34+ cells obtained was $13,73 \times 10^{6} / \mathrm{Kg}$ (range 7,8-26,47). After positive selection, $>4 \times 10^{6} / \mathrm{Kg}$ $\mathrm{CD} 34+$ cells were infused in all cases (Figure 1). Neutrophil engraftment was achieved after a median of 15 days (range 10-17). Few donors presented only Plerixafor mild secondary effects.

Conclusions: Our experience showed that a mobilization protocol using G-CSF and standard dose of Plerixafor (compasive use) is a safe strategy that allows collecting great $\mathrm{CD} 34+$ dose in one apheresis procedure. This could be useful for haploidentical transplantation with ex-vivo $\mathrm{T}$ depletion, especially if there's a weight disproportion between donor and patient.

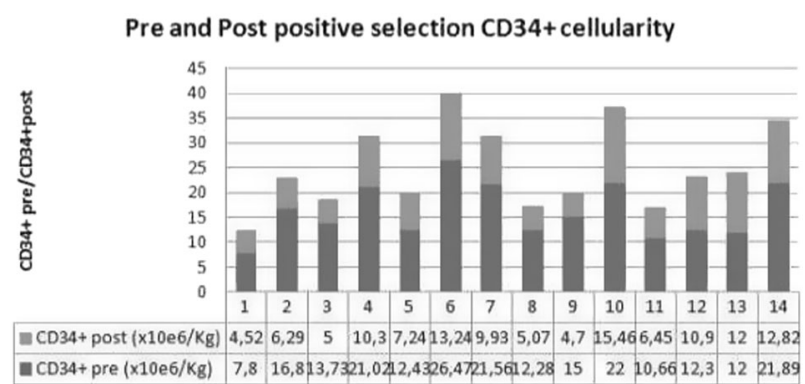

[[P710 Image] 1. Pre and Post positive selection CD34+ cellularity]

Disclosure: Nothing to declare.

\section{P711}

Large volume of cryopreserved hematopoietic progenitor cells infused through peripheral IV Cannula in hematological patients with cardiovascular risk

Ariadna Domenech-Bachiller ${ }^{1}$, Marta Salas-Ortiz, Núria Borràs- Maixenchs ${ }^{1}$, Dolores Herrera-Luque ${ }^{1}$, Montserrat Carreño-Mendez ${ }^{l}$, Montserrat Valverde- Bosch ${ }^{l}$, Gonzalo Gutierrez-García ${ }^{1}$, Montserrat Rovira-Tarrats ${ }^{1}$, Pedro Marin-Fernandez ${ }^{1}$

${ }^{1}$ Hospital Clinic, IDIBAPS, Barcelona, Spain

Background: Administration of large volume of cryopreserved hematopoietic progenitor cells (cHPC) can produce life threatening complications such as cardiac arrest or arrhythmia. Traditionally, central venous catheters (CVC) 
have been the only route used for cHPC administration. The appearance of other catheters types made us to reconsider the exclusive use of the CVC for the infusion of cHPC. We analyzed the use of a peripheral IV cannula (PIVC) as an alternative to CVC for the infusion of cHPC in patients with cardiovascular diseases.

Methods: Medical records of 65 patients who received an ASCT for hematological malignant diseases at the Hospital Clínic of Barcelona from January 2017 to February 2018 were reviewed. Of those, eight were infused through a PIVC due to cardiac impairment related to previous treatments, ischemic cardiomyopathy or amyloid deposition.HPC were obtained from peripheral blood by apheresis after mobilization with G-CSF using ACD-A as an anticoagulant. Cryopreservation was performed with autologous plasma and DMSO $10 \%$ by mechanical means and stored in liquid nitrogen. Analytical controls were performed including hematocrit, total nucleated cells, total polymorphonuclear neutrophils and platelets using the ADVIA 120 analyzer. The CD34 + I CD45 + population was analyzed by flow cytometry following the ISHAGE single-platform protocol. Viability of total nucleated cellularity was carried out by vital staining with Acridine Orange and the specific viability of the CD34 + population through the technique of 7 Aminoactinomycin D. Thawing was performed bag to bag by immersion in a water bath at $37^{\circ} \mathrm{C}$ and transferred to the bedside of the patient for gravity infusion using an infusion set without filter through PIVC of 22 Gauche. Vital sings monitoring performed before, during and the end of the every infusion bag including: blood pressure, heart rate, oxygen saturation, body temperature and central venous pressure. Other aspects assessed during the infusion were pain, cold sensation and signs of extravasation in the area of PIVC insertion. After the infusion, the recovery time of the granulocyte series and platelet were evaluated.

Results: Median volume and bags administered was 480 (360-600) $\mathrm{mL}$ and 4(3-5). The median of total nucleated cells, total nucleated cells / $\mathrm{mL}$ and total CD34+ cells $/ \mathrm{Kg}$ was $462.6 \times 10^{8}$ (329.5-657.8), $107.3 \times 10^{8}$ (64.66-156.7) and 3.24 (2.46-4.6) respectively. Vital signs were within the normal range and allowed to perform the infusion in an average of 20-30 minutes/bag. No patient required stopping the infusion due to pain in the area of peripheral catheter insertion and no extravasations were detected. All patients referred some cold sensation in the insertion vein and its path. Median hematopoietic recovery was 14 (11-19) days for neutrophils and $11(0-19)$ days for platelets, similar to the recovery experienced from patients who received cHPC through CVC.
Conclusions: Based on our data, we conclude that the administration of cHPC, through PIVC and by gravity is safe for the product and for the patient, being the preferred choice for patients suffering from some type of cardiovascular disease.

Disclosure: Nothing to declare

\section{P712}

Depletion of $\mathrm{CD}^{2} 5 \mathrm{RA}^{+}$naive $t$ cells for haploidentic stem cell transplantation

Bremm Melanie $^{1}$, Theresa Krastel, Olga Zimmermann ${ }^{1}$, Katzki Verena ${ }^{1}$, Lisa-Marie Pfeffermann ${ }^{1}$, Jan Sörensen ${ }^{1}$, Halvard Bonig $^{2}$, Claudia Cappel ${ }^{l}$, Thomas Klingebiel, Peter Bader ${ }^{1}$, Sabine Huenecke ${ }^{I}$

${ }^{1}$ University Hospital Frankfurt / Clinic for Children an Adolescent, Frankfurt am Main, Germany, ${ }^{2}$ Institute for Transfusion Medicine and Immunhematology, Frankfurt am Main, Germany

Background: By selective depletion of potentially alloreactive CD45RA ${ }^{+}$cells, $T$ memory cells might be retained in the graft and could mediate pathogen specific immunity. However, CD45RA expression is not restricted to naïve $\mathrm{T}$ cells, but also available on $\mathrm{B}$ cells, NK cells and CD $34^{+}$ stem cells to some extent.

Methods: Within this project we aim to analyze CD45RA expression on stem- and NK cells by flow cytometric analysis to estimate the eventual loss of these cells during CD45RA-depletion. Furthermore, CliniMACS depletion following a one-step approach of direct CD45RAdepletion and a two-step approach with primary CD34selection followed by CD45RA-depletion of the negative fraction was investigated.

Results: CD45RA expression on $\mathrm{CD} 34^{+}$stem cells was in median $16.9 \%$. With a Median of $99.4 \%$ CD45RA expression was measurable on nearly all $\mathrm{B}$ cells, which obviates depletion via CD19. A comparably high CD45RA expression of in median $96.0 \%$ was detected on NK cells. Unfortunately, the amount of NK cells in the CD45RA-depleted product was $0.24 \%$. CliniMACS depletion following one-step approach resulted in a stem cell recovery of $52.0 \%$. Memory $\mathrm{T}$ cell recovery was $24.2 \%$ following one-step and $42.0 \%$ applying two-step approach. Depletion quality measured by log-depletion

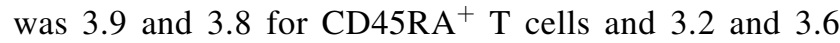
for $\mathrm{CD} 19^{+} \mathrm{B}$ cells for one- and two-step approaches, respectively.

Conclusions: With regard to stem cell recovery, a previous CD34-selection before CD45RA-depletion is recommendable. 
Disclosure: Nothing to declare.

\section{Stem cell source}

\section{P713}

Abstract already published.

\section{P714}

Abstract already published.

\section{P715}

Abstract already published.

\section{P716}

Retrospective review to evaluate the effects of routine filter use on neutrophil and platelet engraftment duration in autologous stem cell transplant patients

Muneer Abidi ${ }^{1,2}$, Jessica Parker, Sami Brake ${ }^{3,2}$, Stephanie Williams ${ }^{1,2}$, Jubran Afzal Khan Rind ${ }^{4}$, Laura Paulsen $^{3}$, Annum Faisal

${ }^{1}$ Michigan State University College of Human Medicine, Internal Medicine, Grand Rapids, MI, United States, ${ }^{2}$ Spectrum Health Cancer Center, Hematology and Oncology, Grand Rapids, MI, United States, ${ }^{3}$ Spectrum Health Office of Research, Grand Rapids, MI, United States, ${ }^{4}$ Mercy Health Saint Mary's Hospital, Geriatric Medicine, Grand Rapids, MI, United States

Background: Current clinical practice of routine use of filters for infusion of autologous hematopoietic cell transplantation (AHCT) at bone marrow transplant centers across North America and Europe is not known. The use of "Y" administration tubing without a filter could possibly increase the risk of infusion of macro-aggregates and cellular debris, which may result in increased side effects.

Methods: We carried out a retrospective chart review of patients (pts) at Spectrum Health who underwent AHCT. Group A (Gp A) pts received AHCT using a "Y" administration tubing with 170-micron filter from 3/2013 $-3 / 2017$. These patients were compared to a control group (Gp B) that received AHCT without filter administration tubing from 3/2014-4/2016. This change in clinical practice occurred due to a change in policy at our transplant center as a result of inorganic particles noticed during cryopreservation. We compared the neutrophil and platelet engraftment duration between these groups. We also studied the length of hospital stay and the effect of filter use on any immediate side effects after infusion. Due to the retrospective nature of the study it was not feasible to evaluate the difference in duration of infusion between these groups
Results: The two groups were similar in their age, gender, primary disease distribution and median number of CD34 stem cells infused (Table). There was no difference in median neutrophil (11 vs 11 days) or platelet engraftment (19 vs 19 days) duration for the filter group and the nonfilter group respectively. The median length of hospital stay was also comparable (17 days). There was no statistically significant difference in the immediate side effects (fever, cough, dyspnea, fluid overload, flushing, nausea, vomiting, hypertension, hypotension and anaphylaxis) or confirmed post-transplant infections (viral, bacterial, fungal) experienced by these two groups.

Conclusions: Our results show that the routine use of filter does not prolong hospital stay, and neutrophil/ platelet engraftment duration, thereby, suggesting that viable stem cells are not affected. On the other hand, filter use failed to demonstrate any appreciable decline in the immediate side effects experienced after AHCT.

\begin{tabular}{|c|c|c|c|}
\hline & $\begin{array}{l}\text { Gp A Filter } \\
(\mathrm{N}=168)\end{array}$ & $\begin{array}{l}\text { Gp B No } \\
\text { Filter }(\mathrm{N}=46)\end{array}$ & $\mathrm{p}$ value \\
\hline Age- Median yr (Range) & $63(25-81)$ & $64(20-77)$ & 0.77 \\
\hline Gender- no. (\%) (male) & $106(63.1 \%)$ & $27(58.7 \%)$ & 0.59 \\
\hline $\begin{array}{l}\text { Primary Disease no. (\%) } \\
\text { Multiple myeloma, Non- } \\
\text { Hodgkin's Lymphoma, } \\
\text { Hodgkin's Lymphoma, } \\
\text { Amyloidosis }\end{array}$ & $\begin{array}{l}95(56.5 \%), 55 \\
(32.7 \%), 13(7.7 \\
\%), 5(3.0 \%)\end{array}$ & $\begin{array}{l}28(60.9 \%), 10 \\
(21.7 \%), 5(10.9 \\
\%), 3(6.5 \%)\end{array}$ & 0.31 \\
\hline $\begin{array}{l}\text { Median CD34 cells } \times 10^{\wedge} 6 \\
\text { cells/kg body weight (range) }\end{array}$ & $3.5(1.8-18.0)$ & $3.5(2.2-9.5)$ & 0.47 \\
\hline $\begin{array}{l}\text { Median Number of HCT } \\
\text { bags Infused (range) }\end{array}$ & $2(1-9)$ & $2(1-8)$ & 0.35 \\
\hline $\begin{array}{l}\text { Median Platelet engraftment } \\
\text { duration in days (range) }\end{array}$ & $19(10-43)$ & $19(12-41)$ & 0.88 \\
\hline $\begin{array}{l}\text { Median Neutrophil } \\
\text { engraftment in days (range) }\end{array}$ & $11(9-19)$ & $11(9-13)$ & 0.51 \\
\hline $\begin{array}{l}\text { Immediate side effects -no. } \\
(\%)\end{array}$ & $42(25.0 \%)$ & $8(17.4 \%)$ & 0.28 \\
\hline Infections (post-transplant) & $45(26.8 \%)$ & $11(23.9 \%)$ & 0.69 \\
\hline
\end{tabular}

[[P716 Table] 1. Patient Characteristics]

Disclosure: All authors have "Nothing to declare"

\section{P717}

A new protocol for isolating mesenchymal stem cells from human placental tissue processed 24 hours after delivery

Silvia Perez-Lopez ${ }^{1}$, Jose García-Gala ${ }^{\mathbb{1}}$, Catalina Fernandez-Plaza ${ }^{I}$, Angeles Fernandez-Rodriguez, ${ }^{I}$, Maria Alvarez-Viejo ${ }^{I}$, Eva Martinez-Revuelta ${ }^{I}$, Marcos PerezBasterrechea $^{1}$, Irene Reguera-Gomez ${ }^{2}$

${ }^{1}$ Hospital Universitario Central de Asturias, Oviedo, Spain, ${ }^{2}$ Universidad de Oviedo, Oviedo, Spain 
Background: Mesenchymal stem cells (MSCs) are selfrenewing multipotent progenitor cells with wide differentiation potential. Their ease of isolation and expansion in vitro as well as their unique regenerative therapeutic properties suggest the use of MSC as an approach for treating several disorders. Extra-embryonic tissues as placenta have been proposed as potential sources of MSCs due to the absence of ethical problems neither risks for the patients. Furthermore, only protocols using fresh placental tissue have been described so far. A protocol for isolating MSCs from delayed-manipulated tissue was designed and tested in order to optimize the use of placental MSCs (MSCs-P) in an advanced therapies context.

Methods: Full term placentas $(n=10)$ were obtained from healthy mothers in Hospital Universitario Central de Asturias (Spain). Informed consent was obtained from each mother prior to delivery. After dissection of $12 \mathrm{gr}$ decidual tissue it was washing with saline (B. Braun, Germany) and cut into small pieces. These biopsies were conserved 24 hours in DMEM media with $1 \%$ antibiotic solution 100X (Gibco, USAUSA) until processing. The day after, tissue was mechanically minced and then enzimatically digested with a combination of $80 \mathrm{UI} / \mathrm{ml}$ DNAse I (Sigma Aldrich, USA) and $0.25 \%$ tripsin-EDTA solution (w/v) (Biochrom, Germany) at $37^{\circ} \mathrm{C}$ for 1 hour. Then, the mixture was filtered with $40 \mu \mathrm{m}$ Cell Strainer (BD Bioscience, USA) and centrifuge at 300xg for 5 minutes. Finally, cells were resuspended in $5 \mathrm{ml}$ of DMEM media suplemented with $10 \%$ FBS and antibiotic, seeded in 24-cm flask and incubated in Forma SteriCult $\mathrm{CO}_{2}$ incubator (Thermo Fisher Scientific, USA) at $37^{\circ} \mathrm{C}$, $5 \% \mathrm{CO}_{2}$. Culture-expanded MSCs cells were phenotipically characterized by flow cytometry (FACS Aria IIu, BD) with antibodies against CD29, CD44, CD73, CD90, CD105, CD166, CD34, CD45, HLA-DR CD14 and CD19 using Mesenchymal Cell Kit (Immunostep, Spain). Afterwards, these cells were differentiated to adipogenic, osteogenic and chondrogenic lineages using StemMACS AdipoDiff Media, StemMACS OsteoDiff Media and NH ChondroDiff Medium (Miltenyi Biotec, Germany) respectively. After three weeks of differentiation cells were fixed in $4 \%$ paraformaldehide (Merck, USA) and analyzed. Adipogenic, osteogenic and chondrogenic differentiation was visualized after staining with Oil Red O, Alkaline Phosphatase and Hematoxilin-Eosin (Sigma-Aldrich, USA).

Results: MSCs-P isolated cells were characterized according to the ISCT criteria for mesenchymal stem cells. They were positive for CD29, CD44, CD73, CD90 and CD105 and negative for CD14, CD34, CD45 and HLA-DR, indicative of a typical MSC phenotype (Figure 1). All the markers showed a high percentage of expression between 84.6 and $99.9 \%$, meaning that MSC population obtained with the designed method was very homogenous. Similarly, staining for the three studied lineages was positive (Figure 1).

Conclusions: The described protocol allows us to obtain MSCs from decidual placental tissue stored and processed 24 hours after the biopsy extraction using a unique enzymatic digestion. This circumstance permits to take advantage of placentas that are discarded after delivery giving us the option to obtain mesenchymal cells that could be used in clinical trials.

Disclosure: Nothing to declare

\section{P718}

Outcomes of umbilical cord transplant in high risk relapsed or refractory acute myeloid leukaemia

\section{Samar Elbahy ${ }^{1,2}$, Denise Bonney ${ }^{1}$, Helen Campbell, David Deambrosis ${ }^{1}$, Robert Wynn ${ }^{1}$}

${ }^{1}$ Royal Manchester Children's Hospital, Manchester, United Kingdom, ${ }^{2}$ Faculty of Medicine, Benha University, Benha, Egypt

Background: High-risk relapsed/refractory acute myeloid leukaemia (AML) is a fatal disease. Allogeneic haematopoietic stem cell transplantation represents the only chance of cure. As the transplant relies on the graft-versusleukaemia $(\mathrm{GvL})$ effect, and if different donors exert different GvL effects, then choosing the right donor assumes great importance. In Manchester, a large BMT centre in the north of England, our practice in such AML has been to choose unrelated cord blood (UCBT), without serotherapy in the conditioning therapy, as our preferred donor cell source.

Methods: We report the results of 16 unrelated UCBT in 15 patients (five boys and ten girls) with high-risk AML, defined as relapsed or refractory disease.

Thirteen patients (81\%) received this as a $1^{\text {st }}$ transplant, two Patients $(13 \%)$ received this as a $2^{\text {nd }}$ transplant for relapsed AML post matched unrelated donor transplant, and one $(6 \%)$ received UCBT twice, once in CR1 and once in CR2. Nine patients $(56 \%)$ had mismatched UCBT, and the rest were fully matched at class-I (HLA-A, -B, and-C) and class-II (HLA-DRB1).

Conditioning was given as Treosulfan, Fludarabine and Thiotepa in half of the patients $(\mathrm{n}=8)$, other Treosulfanbased regimens were used in two patients (12\%), and Busulfan-based regimens were used in six patients (38\%). No serotherapy was given.

Results: The median age at transplant was 5 years (Range, 5months - 15years). Neutrophil and platelet engraftment were achieved in 15 and 12 patients at a median of 16 and 35 days, respectively. 13 patients (81\%) 
had engraftment syndrome. All engrafted patients achieved $100 \%$ donor chimerism, except one patient who had mixed lymphoid chimerism initially, that was corrected spontaneously to $100 \%$ at three months after transplant.

Acute GVHD Grade I-II developed in six patients (38\%), and Grade III-IV developed in three patients (19\%). All cases resolved, except two patients where acute GVHD evolved into Chronic GVHD (One with Grade I skin GVHD which fully resolved, and one with Grade III GVHD gut colitis who was parenteral nutrition dependent till death). Two more patients developed Chronic Grade I skin GVHD and resolved (Chronic GVHD developed in 25\% in total).

Three patients (17\%) developed veno-occlusive disease (VOD), that completely resolved with defibrotide treatment and necessitated ascitic drainage in one of them. Viral reactivations occurred in five patients $(30 \%)$ and were successfully treated.

At a median follow-up of 20 months (Range, seven months - four years), eight patients (50\%) died at a median of 79 (Range, 24 to 230 days), with a transplantrelated mortality of $25 \%$ and relapse-related mortality of $25 \%$. Five patients (31\%) relapsed post-UCBT; four died and one had a successful second UCBT (event-free survival was 44\%). Immune reconstitution in alive patients was achieved at a median of eight months.

Conclusions: Very high-risk patients treated with UCBT with good overall survival and event-free survival, similar to AML treatment rate with low-risk disease.

Disclosure: Nothing to declare

\section{P719}

In haploidentical transplants is the incidence of acute and chronic GVHD strictly related to the stem cell source?

\section{Paolo Bernasconi ${ }^{1}$, Anna Amelia Colombo ${ }^{1}$, Daniela Caldera $^{I}$, Roberta Sciarra ${ }^{I}$, Catherine Klersy ${ }^{I}$, Gianluca Viarengo ${ }^{I}$, Claudia Del Fante ${ }^{1}$, Cesare Perotti ${ }^{1}$, Oscar Borsani $^{l}$}

${ }^{1}$ University of Pavia, San Matteo Hospital, Molecular Medicine, Pavia, Italy

Background: Allo-HSCT from related haplo-identical donors (Haplo-HSCT) with post-transplant high-dose cyclophosphamide is increasingly employed in patients who lack a matched related or unrelated donor. The current standard is to use bone marrow grafts (BM) as peripheral blood stem cell grafts (PBSC) have been associated with an increased risk of acute and chronic GvHD. Thus, the aim of our study was to compare the main transplant outcomes and especially the incidence of acute and chronic GVHD in recipients of BM and PBSC grafts.

Methods: Thirty-five unselected patients with hematologic malignancy who underwent an haploidentical transplant at our Unit between 2011 and 2018 and received BM $(\mathrm{n}=17)$ or PBSC $(\mathrm{n}=18)$ grafts after the same TBF conditioning regimen were analysed in order to assess differences in transplant outcomes.Our GVHD prophylaxis consisted in cyclosporine A (CsA) from day -1 to +180 , a methotrexate "short course" and mycophenolate mofetil (MMF) from day +1 to +28 .

Results: No statistically-significant differences were observed between patients who received BM grafts and those who received PBSC grafts. At transplant fourteen patients were in first complete remission (CR), twelve in advanced $\mathrm{CR}$ and 9 had active disease. According to Sorror's risk, nine patients were low-risk, nine intermediate-risk and seventeen high-risk. Twenty-eight $\mathrm{CMV}+$ patients received the graft from twenty-three $\mathrm{CMV}+$ and five CMV- donors, seven CMV- patients received the graft from five $\mathrm{CMV}+$ and two CMVdonors. Mean age at transplant was 51 years (range 2372), mean donor's age 38 years (range18-39) and mean follow-up 16.9 months (range 1.9-56.7). Median CD 34+ cell dose was $5.2 \times 10^{6} / \mathrm{kg}$ (range1.4-10.4), $3.6 \times 10^{6} / \mathrm{kg}$ (range 1.4-7.7) in BM recipients and $14.0 \times 10^{6} / \mathrm{kg}$ (range 4.2-10.4) in PBSC recipients. Median time to neutrophil recovery ( $>500 / \mu \mathrm{l})$ was 22 days (range 16-39) posttransplant, 23 days (range 18-27) for BM recipients and 20 (range 16-39) for PBSC recipients. Platelet recovery $(>20.000 / \mu 1)$ occurred in all patients except one at a median of 17 days (range 10-151) post-transplant, at a median of 20 days (11-151) post-transplant for BM recipients and at a median of 14 days (range (10-26) for PBSC recipients. Seven patients never reached platelets $>50.000 / \mu 1$. Three patients developed a poor graft function. Acute and chronic GVHD incidence was $28.5 \%$ and $22.8 \%$ and the risks of acute (hazard ratio [HR], 1.04; $P=.955)$ and chronic (HR, 0.85; $P=.816$ ) graft-versus-host disease were similar in the two patient groups. In addition, there were no differences in relapse risks post-transplant (HR, 0.90; $P=.898$ ); relapse-free survival was better with PBSC grafts but this difference did not reach any statistical significance. Finally, no significant differences were noted in overall mortality by graft type (HR, 0.62; $P=.441$ ).

Conclusions: Despite in haplo-HSCT the incidence of acute and chronic GVHD is reported to be higher with PBSC than with BM our small patient series does not confirm this assumption that should be clarified by additional studies. Instead, our data suggest that PBSC 
grafts might be associated with a faster platelet recovery and a better relapse-free survival.

Disclosure: Nothing to declare

\section{P720}

Cord blood stem cell transplantation: Experience in a pediatric bone marrow/stem cell transplant unit in Mexico 'HOSPITAL CIVIL JUAN I. MENCHACA GDL, JALISCO'

Regina Navarro Martín del Campo ${ }^{I}$, Ilse Mayela Delgado Ruiz $^{I}$, Ana Luisa Orozco Alvarado ${ }^{1}$, Maria Magdalena Ortiz Sandoval, Oscar Gonzalez-Ramella ${ }^{1}$, Fernando Sánchez Zubieta ${ }^{1,2}$

${ }^{1}$ Hospital Civil de Guadalajara 'Juan I Menchaca', Guadalajara, Mexico, ${ }^{2}$ Universidad de Guadalajara, Instituto de Investigacion de Cancer Infantil, Guadalajara, Mexico

Background: Since 1988, cord blood (CBU) has become an alternative source of stem cells for transplantation, with approximately 35,000 procedures currently performed. With a 2-year GS of $50 \%$.

Objective: Analyze the outcomes from all the patients transplanted with CBU in our hospital unit.

Methods: Retrospective, longitudinal study. All patients transplanted with CBU in our hospital between 2007 and 2017 were included. We analyzed 40 patients with ages from 7 months to 14 years.

Results: Two of them received doubled cord transplantation the ratio male: female was 1.3: 1. The transplanted pathologies were: Bone marrow failure $35 \%$, immunodeficiency $25 \%$, AML 20\%, ALL $17.5 \%$, Osteopetrosis $2.5 \%$. RIC regimens were used in patients with bone marrow failure and immunodeficiency and myeloablative conditioning regimens were used in patients with malignant hematology diseases. Antithymocyte rabbit globulin (ATG) based serotherapy was used. One case received CBU from a related donor (sister), the rest received unrelated CBU obtained from Centro Nacional de la Trasfusión Sanguínea. The infusion of CD34 + was in a range of 0.11 to of $2.7 \times 10^{6} / \mathrm{kg}$ with and average of $0.43 \mathrm{X}$ $10^{6} / \mathrm{kg}$. Compatibility was $4 / 6$ in $70 \%, 5 / 6$ in $20 \%$ and $6 / 6$ in $10 \%$. Post-thawing cellularity was not measured. The HLA-C was not analyzed. Forty two point five percent of the patients had a successful engraftment; the average time of engraftment was 30 days. Primary graft failure was detected in $57.5 \%$ and secondary graft failure in $10 \%$, for a total success of $32.5 \%$. GVHD was detected in $20 \%$ of patients, of which $90 \%$ was grade I-II and $10 \%$ grade III-IV. The overall mortality was $52.5 \%$. Causes of death were: infection 45\% Relapse 30\%, Hemorrhage 20\% and GVHD 5\%.
Conclusions: The CBSCT continues to be an essential alternative in our patients who required transplantation knowing that this stem cell source allows the procedure to be done with less histocompatibility requirements and it is available immediately, which facilitates the process considering the great diversity that exists within our population. However, in our experience, the CBSCT has shown a higher mortality risk, which can be improved by analyzing the HLA C, choosing in this way the units with better compatibility, and improving cellular dosage since this is key in success.

Disclosure: nothing to declare

\section{P721}

The risk of infection of the umbilical cord is not related to the microbiological status of the umbilical cord blood

\section{Izabela Zdolińnska-Malinowska ${ }^{I}$, Maciej Rojek ${ }^{1}$, Dariusz} Boruczkowski

\section{${ }^{1}$ The Polish Stem Cell Bank, Warsaw, Poland}

Background: A common problem in umbilical cord (UC) banking is the contamination of cords and cord blood (UCB) with maternal fecal bacteria. Recently, some of our clients have raised concerns regarding the rationale and ethics of UC banking in cases of positive umbilical blood culture. To address them, we carried out a statistical analysis assessing the relationship between UCB contamination and UC disqualification in samples sent to Polski Bank Komórek Macierzystych (PBKM, the Polish Stem Cell Bank) in Warsaw, Poland.

Methods: The statistical analysis was carried out on data obtained from samples taken in Poland between 01-Jan2017 and 31-Dec-2017. The samples were collected in hospitals by external midwifes and sent to the PBKM in accordance with the requirements of the American Association of Blood Banks. After arrival in the laboratory, the blood samples were cultured and the UCs were assessed immediately for visual signs of infection, such as odor, altered color, or visible bloom. The status of both kinds of samples was introduced into the PBKM general database. For the purpose of this analysis, the UCs were considered as microbiologically pure if stored, destroyed after storage, or handed over to the PBKM. Samples marked as infected or disqualified for unknown reasons (other than termination of the contract with the customer, viral infection of the mother, and lack of cell growth) were considered as infected. At the time of the statistical analysis, the samples of unknown UCB microbiological culture status were removed from the generated report. The data was summarized as percentages and the odds ratio was calculated. Statistical significance was considered at $\mathrm{p}^{<} 0.05$. 
Results: The odds ratio was 1.78 with a $95 \%$ confidence interval of $0.94-3.37(\mathrm{p}=0.07)$.

Conclusions: The risk of infection of the UC is not related to the microbiological status of the UCB. A possible explanation for this is the presence of antibiotics in the medium used for UC, but not UCB, transport. This means that cryopreservation of UCs from which contaminated cord blood has been obtained is justified.

\begin{tabular}{|c|c|c|}
\hline & $\begin{array}{l}\text { Umbilical cord } \\
\text { infected }\end{array}$ & $\begin{array}{l}\text { Umbilical cord not } \\
\text { infected }\end{array}$ \\
\hline $\begin{array}{l}\text { Umbilical cord blood culture } \\
\text { positive }\end{array}$ & $1.6 \%$ & $98.4 \%$ \\
\hline $\begin{array}{l}\text { Umbilical cord blood culture } \\
\text { negative }\end{array}$ & $0.9 \%$ & $99.10 \%$ \\
\hline
\end{tabular}

[[P721 Table] 1. Number of umbilical cords with symptoms of infection in subgroups separated on the basis of microbiological status of umbilical cord blood.]

Disclosure: All authors are employees of the Polish Stem Cell Bank, Warsaw, Poland.

\section{P722}

Comparison of turkish stem cell coordination center (TURKOK) with Istanbul university bone marrow bank (TRIS); A single center experience in match unrelated donors

Azize Mergen', Selime Aydoğdu², Başak Aksoy ${ }^{3}$, Yunus Emre Savct ${ }^{2}$, Gürcan Dikme ${ }^{2}$, Funda Çipe ${ }^{2}$, Ceyhun Bozkurt ${ }^{3}$, Tunç Fışgın ${ }^{1}$

${ }^{1}$ Altinbas University, Medical Park Hospitals, Bahçelievler, Istanbul, Turkey, ${ }^{2}$ Medical Park Hospitals, Bahçelievler, Istanbul, Turkey, ${ }^{3}$ Istinye University, Medical Park Hospitals,Bahçelievler, Istanbul, Turkey,

Background: Match family donors are the preferable options in allogenic stem cell transplant. However, in the absence of donor relatives match unrelated donors have been an option. In this study, the donor screening, transplant preparation phases of Turkish Stem Cell Coordination Center (TURKOK) and the İstanbul University Bone Marrow Bank (TRIS), were compared.

Methods: The unrelated donor scanning data between March 2015 and November 2018 in Pediatric stem cell transplantation Unit of Altınbas University Bahcelievler Medical Park Hospital were evaluated. 93 unrelated transplants were performed in total. $65 \%$ of these transplants $(n=61)$ were included from the donors of TURKOK registration system and $34,4 \%$ of these transplants $(n=32)$ were included by means of TRIS from donors outside of Turkey. 8 patients (5 TRIS, 3 TURKOK) were excluded from the study in consequence of screening update and postpone of transplantation. The statistics were carried out on a total of 85 patients, $67,1 \%$ of whom were in TURKOK $(\mathrm{n}=57)$ and $32,9 \%$ were transplanted via TRIS $(n=28)$. The day of application to stem cell transplantation unit, reply dates and the transplantation dates were examined for the transplant patients.

Results: In the current study, the average response time of TURKOK was found as $0,91 \pm 2,96$ day (median: 0 ), the average transplant time after receiving a reply was found as $98,24 \pm 63,89$ (median: 85 ) day, the average number of days from date of application to date of transplantation of patients was found as $101,12 \pm 63,17$ (median: 89). The average response time of TRIS was $18,78 \pm 18,36$ (median: 15) day, the average transplant time after receiving a reply from TRIS was 136,82 $\pm 63,84$ (Median: 119) day, average number of days from date of application of TRIS to date of transplantation of patients 155,61 $\pm 72,23$ (Median: 140) day.

The average response time of TURKOK, the average transplant time after receiving a reply from TURKOK and the average number of days from date of application of TURKOK to date of transplantation of patients was shorter than TRIS. The difference between them was found statistically significant $(\mathrm{p}<0.05)$.

Conclusions: In this study, it was determined that the transplantation processes with TURKOK were progressing more rapidly. The rapid progress of the process was attributed to the fact that all donor HLA tissue groups in the TURKOK database were studied in high resolution. In international scans carried out through TRIS, it was thought that the examination of the donor castings coming from bone marrow banks and the time differences between the countries prolong the process. It was thought that HLA tests of the registered donors in the TRIS database and some international bone marrow banks were studied in low resolution but not studied the all HLA loci, the centers wanted high-resolution HLA, and therefore the involvement of social security institutions and payment procedures were among the factors extending this process.

Disclosure: Nothing to declare

\section{P723}

Outcomes of haploidentical transplantation: A singlecenter experience from Turkey

Yusuf Ulusoy $^{I}$, Hale Bülbüil ${ }^{1}$, Eren Arslan Davulcu ${ }^{I}$, Fatma Keklik Karadağ ${ }^{1}$, Ayşenur Arslan ${ }^{1}$, Nur Soyer ${ }^{1}$, Güray Saydam ${ }^{1}$, Mahmut Töb $\ddot{u}^{1}$, Murat Tombuloğlu ${ }^{I}$, Fahri Şahin ${ }^{\text {, Filiz Vural }}{ }^{1}$

${ }^{1}$ Ege University Hospital, İzmir, Turkey 
Background: Allogeneic hematopoietic stem cell transplantation (AHSCT) is being performed for a group of hematologic diseases with a curative intent. Outcomes after AHSCT are influenced by the type of donor used. Haploidentical transplantation is an emerging option when a fullmatched donor is unavailable.

Methods: We retrospectively analyzed our transplants performed between January 2015 and November 2018, investigating outcomes and complications among haploidentical stem cell recipients.

Results: One hundred and nineteen patients underwent AHSCT, 9 of them (7.5\%) were recipient of a haploidentical stem cell and included in this study. One patient diagnosed with acute lymphoblastic leukemia (ALL) were performed a haploidentical AHSCT for two times due to relapse. Among those 9 transplants, 4 of them were diagnosed with acute myeloid leukemia, 2 with ALL, 1 with chronic lymphocytic leukemia, 1 with myelodysplastic syndrome and 1 with Hodgkin lymphoma. The mean age of group was $35.7 \pm 15.2$ years. Three patients ( 2 AML, 1 ALL) were in remission at the time of transplantation. Patients were given a conditioning regimen based mostly on busulfan, fludarabin and total body irradiation with a myeloablative intent. Patients were also given a various combinations of post-transplant cyclophosphamide, calcineurin inhibitors, mycophenolate mofetil and antithymocyte globulin for graft versus host disease (GVHD) prophylaxis; post-transplant cyclophosphamide administered on $6(67 \%)$ of those transplantations. Peripheral blood was the source of stem cells in all patients. Patients were infused with mean $6.15 \pm 0.67 \times 10^{6} / \mathrm{kg}$ of $\mathrm{CD} 34+$ cells. Hematological recovery was achieved with neutrophil engraftment at a mean of $19.2 \pm 2.1$ days and platelet engraftment at a mean of $17.3 \pm 2.7$ days. After a median 8month (0.2-40.6 months) follow up, the cumulative rates of grade 3-4 GVHD, relapse and non-relapse mortality were $11 \%, 28 \%(\mathrm{n}=7)$ and $71 \%$, respectively. One patient died due to relapse, at the end of the follow up two were still alive with remission. Only one patient has died due to chronic GVHD affecting serosa and resulting with a fatal tracheoesophageal fistula. The mean overall survival was $6.9 \pm 2.6$ months in our study.

Conclusions: Haploidentical transplant is a feasible option in hematologic malignancies with novel GVHD prophylaxis approaches, especially post-transplantation cyclophosphamide. However, these results need to be supported with further investigations with a larger patient group.

Disclosure: Nothing to declare
P724

Survival outcomes of unrelated donor source: A small retrospective study

Fatma Keklik Karada $\breve{g}^{I}$, Hale Bülbüll, Nur Akad Soyer ${ }^{1}$, Eren Arslan Davulcu ${ }^{I}$, Ayşenur Arslan ${ }^{1}$, Yusuf Ulusoy ${ }^{1}$, Mahmut Töbü̈ ${ }^{1}$ Murat Tombuloğlu ${ }^{I}$, Fahri Şahin ${ }^{1}$, Güray Saydam ${ }^{\text {, Filiz Vural }}{ }^{1}$

${ }^{1}$ Ege University Hospital, Hematology, Izmir, Turkey

Background: Hematopoietic stem cell transplantation (HSCT) remains the potentially curative treatment modality for patients with hematologic malignancy. However, fully HLA matched related donors are available only $30 \%$ of them. Although having matched or unmatched unrelated donor takes a long time, it is one of important alternative donor source due to its good outcomes compare to matched sibling donors.

Methods: We conducted a retrospective analysis of the patients who underwent HSCT from unrelated donors at Ege University hospital between 2015 and 2018.

Results: A total of 25 patients between 19 and 58 years (median age: 33 years). Transplant was done for following disease: acute leukemia $(n=9,36 \%)$, aplastic anemia $(n=5$, $20 \%)$, lymphoma $(n=4,16 \%)$, myelofibrosis $(n=3,12 \%)$, myelodisplastic syndrome $(\mathrm{n}=2,8 \%)$, chronic myeloid leukemia $(n=2,8 \%)$. Ten donors were from Turkey and fifteen donors were from different countries of Europe and America. Two of 25 donors were $9 / 10$ and the other 23 was 10/10 HLA matched. The conditioning regimen was mostly non myeloablative $(n=17,68 \%)$ while eight patients were treated with myeloablative regimen. Other than two patients who took tacrolimus and mycophenolate mofetile all of them got cyclophosphamide and methotrexate for graft versus host disease (GVHD) prophylaxis. The median time of neutrophil and platelets engrafman were 18 days (range 11-31) and 13,5 days (range 9-36) respectively. Acute GVHD was seen nearly half of the patients $(47,8 \%)$.

Overall survival was $40 \%$ for all patients and 15 of 25 patiens $(60 \%)$ died within first month to 18 months (median 3 months). The mortality rate was more higher for the recipients who had donor source from countries other than Turkey $(30 \%$ vs $80 \% \quad \mathrm{p}=0,018)$. Transplant related mortality was the most common reason of mortality $(\mathrm{n}=7 /$ $15,46,7 \%)$ and other reasons were GVHD (33,3\%), infections and cirrhosis respectively.

Conclusions: We found the mortality rate more higher in the patients whose donors were from out of our country. However, we need to further multicentric and prospective investigations to confirm our hypothesis, it would be related with impact of ethnicity.

Disclosure: Nothing to disclose 


\section{Late-breaking abstracts}

\section{P725}

targeted twice daily busulfan-based ric-conditioning for allogeneic hematopoietic stem cell transplantation in pediatric patients with chronic granulomatous disease: A 10-year experience with the Zurich protocol

Matthias Felber ${ }^{1}$, Mathias Hauri-Hohl ${ }^{1}$, Ulrike Zeilhofer ${ }^{1}$, Federica Achini, Jana Pachlopnik-Schmid ${ }^{2}$, Janine Reichenbach ${ }^{2}$, Seraina Prader ${ }^{2}$, Tayfun Güngör ${ }^{1}$

${ }^{1}$ University Children's Hospital Zurich, Stem Cell Transplantation, Zurich, Switzerland, ${ }^{2}$ University Children's Hospital Zurich, Immunology, Zurich, Switzerland

Background: Chronic granulomatous disease (CGD) needs sufficient myeloablation to avoid graft failure. For this purpose the EBMT Inborn errors working Party currently recommends Treosulfan or Busulfan-based conditioning regimens for CGD-SCT. We analyzed the last 10 years of targeted Busulfan-based RIC-conditioning including engraftment, GvHD rates, chimerism and late term effects in our pediatric SCT center in Zurich.

Methods: Between 2008 and 2018, n=34 consecutive pediatric CGD patients (median age 6 years, range 1-16 years, $n=4$ female, $n=9$ autosomal recessive inheritance) have been transplanted. All patients received therapeutic drug monitoring of twice daily administered iv Busulfan (3 or 4 hour infusions; d-5 to $-\mathrm{d} 2$ ) to achieve a targeted cumulative AUC of $45-70 \mathrm{mg} / \mathrm{L} * \mathrm{~h}$. Fludarabine $(180 \mathrm{mg} /$ sqm; d-8 to -d3) and serotherapy (Thymoglobuline $7.5 \mathrm{mg} /$ $\mathrm{kg}$ total, d-5 to d-3) or Alemtuzumab (0.5-0.6 mg/kg total; d-8- to d-6) were used for immunoablation. Donors were matched unrelated (10/10 HLA; $n=19)$, mismatched unrelated $(9 / 10 \mathrm{HLA} ; \mathrm{n}=7)$, mismatched unrelated (HLA $8 / 10 ; n=1$ ), matched sibling (HLA 6/6; $n=5$ ) and haploidentical parental (HLA 5/10; $\mathrm{n}=2$ ). For 2 patients with haploidentical donor post-transplant cyclophosphamide (d-3 and d-4 with $50 \mathrm{mg} / \mathrm{kg}$ iv each) and upfront ATG-Grafalon (40 mg/kg -12 to-d-9) was used. Stem cell sources were BM $(n=29)$ and PBSC $(n=5)$. GVHDprophylaxis included iv CSA (d-3; continuous infusion) and iv. MMF (d-0, in 2-3 doses).

Results: Follow-up was 6 to 118 months. Good overall engraftment was noted, with $n=1$ secondary graft failure followed by successful retransplantation. In one patient a stem cell boost/DLI was necessary due to decreasing myeloid donor chimerism during EBV reactivation, resulting in rapid myeloid donor reconstitution after intervention. Low rates of GvHD were documented with $n=2$ aGvHD grade IV and $n=2$ mild/limited cGvHD (NIH criteria). With exception of one patient, myeloid donor chimerism at last follow-up was over $82 \%$, mostly over $95 \%$. Overall survival was $31 / 34(91 \%)$. Deaths were due to GI-GvHD $(n=1)$, autoimmune hemolytic anemia/sepsis $(n=1)$ and thrombotic microangiopathy $(n=1)$.

Conclusions: Precision dosing of iv Busulfan in combination with Fludarabine and serotherapy results in excellent outcome of HSCT for pediatric CGD-patients with good engraftment, low overall cGvHD rates and stable, mostly excellent donor chimerism. Graft failure rate was as low as 3\%. Low dose Alemtuzumab prevented GVHD in the majority of patients. This analysis demonstrates that targeted Busulfan-based conditioning is a valid option for pediatric CGD-patients. Serum alemtuzumab or ATG monitoring could further improve GF and GVHD rates in the future.

Disclosure: The authors declare no conflict of interest.

\section{P726}

Abstract already published.

\section{P727}

Young HLA-matched unrelated donors are comparable to matched sibling donors in elderly patients receiving reduced-intensity conditioning: an analysis on behalf of the EBMT scientific council

Roni Shouval', Joshua A Fein ${ }^{1}$, Myriam Labopin ${ }^{2}$, Rafael F. Duarte ${ }^{3}$, Selim Corbacioglu ${ }^{4}$, Peter Bader ${ }^{5}$, Christian Chabannon $^{6}$, Jurgen Kuball', Grzegorz Basak ${ }^{8}$, Régis Peffault de Latour', Arjan Lankeste ${ }^{10}$, Silvia Montoto ${ }^{11}$, John A Snowden ${ }^{12}$, Jan Styczynski ${ }^{13}$, Ibrahim YakoubAgha $^{14}$, Nicolaus Kröger ${ }^{15}$, Mohamad Mohty ${ }^{2}$, Arnon Nagler ${ }^{1}$

${ }^{1}$ Chaim Sheba Medical Center, Tel Aviv University, Hematology and Bone Marrow Transplantation, Ramat Gan, Israel, ${ }^{2}$ Hopital Saint-Antoine, Université Pierre \& Marie Curie, Paris, France, ${ }^{3}$ Hospital Universitario Puerta de Hierro, Mardrid, Spain, ${ }^{4}$ University Hospital Regensburg, Regensburg, Germany, ${ }^{5}$ University Hospital Frankfurt, Frankfurt, Germany, ${ }^{6}$ Institut Paoli-Calmettes, Marseille, France, ${ }^{7}$ UMC Utrecht, Utrecht, Netherlands, ${ }^{8}$ Medical University of Warsaw, Warsaw, Poland, ${ }^{9}$ SaintLouis Hospital, Paris, France, ${ }^{10}$ Leiden University, Leiden, Netherlands, ${ }^{11}$ Barts Health NHS Trust, London, United Kingdom, ${ }^{12}$ Sheffield Teaching Hospitals NHS Foundation Trust, Sheffield, United Kingdom, ${ }^{13}$ Collegium Medicum, Nicolaus Copernicus University Torun, Bydgoszcz, Poland, ${ }^{14} \mathrm{CHU}$ de Lille, Lille, France, ${ }^{15}$ University Hospital Eppendorf, Hamburg, Germany

Background: *RS, JAF, MM, and AN had an equal contribution. 
Older patients are increasingly being transplanted, thanks to improvement in allogeneic hematopoietic stem cell transplantation (allo-HSCT) techniques. Increasing donor age is associated with greater risk for mortality and graftversus-host disease (GVHD). Since sibling donors are of similar age to recipients, we hypothesized that, in older patients, a young matched unrelated donor (MUD) would be comparable to an HLA-matched sibling donor (MSD).

Methods: We retrospectively compared outcomes of alloHSCT from MSD $(n=1797)$ and 10/10 HLA MUD $(n=2212)$ in patients aged $\geq 60$ years with hematological malignancies transplanted between 2006-2015. All patients received reduced-intensity conditioning and graft source was peripheral blood. The primary outcome was overall survival. MSDs served as the reference category and were compared to MUDs split into three age groups $(\leq 25$ $[n=524], 25-40 \quad[n=1072],>40 \quad[n=616]$ years) using univariable analyses and multivariable Cox regression models adjusted for patient, disease, and transplantation features.

Results: The median age of HSCT recipients was 64 years and was similar across groups. Median donor age for MSD was 60 years and 22, 33, and 45 for the MUD age groups $\leq 25,25-40$, and $>40$ years. Acute leukemia was the leading transplant indication (46\%) followed by myelodysplastic syndrome, myeloproliferative neoplasms and indolent non-Hodgkin lymphoma. Disease risk distribution was similar across donor groups (low [48\%], intermediate [42\%], and high [10\%] in the complete population; $\mathrm{p}=0.771$ ). Time from diagnosis to HSCT was longer with MUD compared to MSD and increased with an older age of MUD. In a univariate analysis, overall survival was $49 \%$ (MSD), 50\% (MUD $\leq 25$ ), 45\% (MUD 25-40), and $43 \%$ $(\mathrm{MUD} \geq 40, \mathrm{p}=0.002)$. Corresponding non-relapse mortality (NRM) cumulative incidence was $22 \%, 25 \% ., 31 \%$, and $32.2 \%$ ( $\mathrm{p}<0.001)$ (Figure). GVHD-relapse-free (GRFS) was $29 \%, 30 \%, 26 \%$, and $24 \%(\mathrm{p}=0.012)$. In a multivariable Cox model, young MUD $(\leq 25)$ had a similar risk for mortality compared to MSD (HR 1.00, $\mathrm{p}=0.97$ ), while a monotonic increase in risk was observed with an older donor age (MUD 25-40y: HR 1.17, $\mathrm{p}=0.014$; MUD $\geq 40 \mathrm{y}$ : HR 1.26, $\mathrm{p}=0.001$ ) (Table). Findings were confirmed in a propensity score analysis, matched for key covariates. NRM and Grade 2-4 acute GVHD were consistently higher with MUD, with the greatest risk associated with older MUDs. The hazard for GRFS was higher with MUD aged 25 or higher compared to MSD; risk was not higher with younger MUD.

Conclusions: In older patients receiving reduced intensity conditioning, MSD remain the optimal choice. However, when not available, young MUD provide comparable results.

Disclosure: Nothing to declare

\section{P728}

Pulmonary invasive fungal disease after community acquire respiratory virus infection in allogeneic hematopoietic stem cell transplantation recipients: Results from a prospective observational study

Jose Pinana ${ }^{I}$, Dolores Gomez ${ }^{I}$, Juan Montoro ${ }^{I}$, Ignacio Lorenzo", Ariadna Perez ${ }^{2}$, Estela Gimenez ${ }^{2}$, Eva Gonzalez $^{1}$, Carlos Carretero ${ }^{1}$, Manuel Guerreiro ${ }^{1}$, Miguel Salavert ${ }^{1}$, Juan Carlos Hernandez-Boluda ${ }^{4}$, Jaime Sanz ${ }^{1}$, Carlos Solano ${ }^{2}$ David Navarro ${ }^{2}$

${ }^{1}$ Hospital Universitari i Politècnic La Fe, Valencia, Spain, ${ }^{2}$ Hospital Clinic de Valencia, Valencia, Spain

Background: There is growing evidence that community acquired respiratory virus (CARV) increase the risk of pulmonary invasive fungal disease (IFD) in recipients of allogeneic hematopoietic stem cell transplantation (alloHSCT). To date, there is a lack of knowledge regarding the rate of IFD, risk factors (RFs) as well as the most critical period for the development of a later IFD after CARV infections in allo-HSCT recipients.

Methods: In this prospective observational study, we retrospectively analyzed the effect of CARV on the development of a later IFD in a consecutive cohort of 287 allo-HSCT adult recipients who developed 597 CARV infectious episodes from December 2013 to December 2018. Respiratory virus in upper and/or lower respiratory tract specimens were tested using multiplex PCR panel assays.

Results: Overall, 29 out of 287 allo-HSCT recipients (10\%) developed IFD within 2 months after a CARV episode at median of 21 days (range 0-158 days) from the day of CARV detection. All the IFDs involved the lungs and in 28 cases $(97 \%)$ the diagnostic was IA accomplishing criteria of probable $(n=26)$ or proven $(n=2)$. Of note, 26 out of 29 IFD (91\%) occurred within the first year after transplantation. The overall rate of IFD after CARV episodes was $5 \%$ whereas this rate was higher in recipients developing CARV during the first year of transplant (7\%). IFD was diagnosed in 25 out of 203 with CARV lower respiratory tract disease (LRTD) episodes (12\%) compared to 4 out of $394 \mathrm{CARV}$ upper respiratory tract disease (URTD) $(1 \%)(\mathrm{p}=0.0001)$. Twenty-three out of $133 \mathrm{CARV}$ episodes involving the LRTD during the first year after transplant (17\%) developed IFD. We did not found differences in IFD rates according to the type of CARV identified. Multivariate analysis identified 4 RFs for IFD: the use of ATG as a part of conditioning [odds ratio (OR) 2.7, 95\% confidence interval (C.I.) 1.2-3.4, $\mathrm{p}=0.01]$, CARV LRTD (OR 11.8, 95\% C.I. 3.8-36, $\mathrm{p}=0.0001$ ), CARV infection during the first year of transplant (OR 5.9, 
95\% C.I. $1.7-20.6, p=0.001)$ and voriconazol prophylaxis during CARV (OR 4.2, 95\% C.I. 1.1-115.6, $\mathrm{p}=0.03$ ).

Conclusions: We provide evidence that IFD after CARV infection. Allo-HSCT recipients developing a CARV LRTD during the first year after transplant may benefit from an adequate antifungal prophylaxis and a close monitoring for the development of a later IFD.

Disclosure: Jose Luis Piñana has received both, advisory for preclinical/clinical research and financial support to assist to the Spanish society of hematology annual meeting 2018 from MSD.

\section{P729}

Favorable outcome and engraftment following reducedintensity conditioned allo-HSCT in children with primary haemophagocytic lymphohistiocytosis (HLH) and high-risk langerhans cell histiocytosis ( $\mathrm{LCH})$

\section{Laura M. Moser, Emilia Salzmann-Manrique, Andrea Jarisch, Jan Sörensen, Shahrzad Bakhtiar, Peter Bader}

University Hospital Frankfurt, Frankfurt am Main, Germany

Background: Primary haemophagocytic lymphohistiocytosis (HLH) and high-risk Langerhans cell histiocytosis (LCH) represent two major entities of childhood histiocytoses, which are - although only of rare occurrence - severe in their clinical manifestations. Patients present with multisystemic uncontrolled inflammation and multi-organ involvement requiring diverse courses of immunosuppressive and chemotherapy regimens. Allogeneic haematopoietic stem cell transplantation (alloHSCT) is the only available curative option; however, the cumulative treatment toxicity and the underlying inflammatory disease often result in high organ toxicity and inflammatory complications of transplantation, such as Graft versus Host Disease (GVHD) and/or graft failure. Especially patients with unrelated donors often deal with high transplant-related mortality (TRM) in the setting of conventional intensity conditioning.

Herein, we present the clinical course of the disease and transplant outcome of 14 children diagnosed with primary HLH $(n=12)$ and high-risk LCH $(n=3)$ who underwent alloHSCT at our centre from 02/2003 to 08/2018.

Methods: The HLH cohort consisted of 7 cases of familial HLH (fHLH), 4 cases of Griscelli syndrome, one XIAP-deficient patient and one HLH-patient with inconclusive genetic testing. All HLH patients had developed clinical symptoms prior to transplantation and had been treated according to HLH-protocols 94, 2004 or 2015. Median age at transplantation was 10 months (4 to 128 months). Stem cells were derived from HLA-matched siblings (MSD, $n=4$ ), matched unrelated donors (MUD, $n$ $=9)$ or haploidentical donors $(\mathrm{n}=1)$.

The majority of patients (9/14) received a RIC regimen containing fludarabine, melphalan and thiotepa $(\mathrm{n}=8)$ and fludarabine plus cyclophosphamide $(\mathrm{n}=1)$. Myeloablative treatment $(5 / 14)$ included a treosulfan-based regimen $(n=3)$ and busulfan-containing treatment $(\mathrm{n}=2)$. The entire cohort received serotherapy using either muromonab $(n=3)$, ATGFresenius $^{\circledR}(n=9)$ or alemtuzumab $(n=2)$.

Results: The overall survival of the entire cohort was $78.6 \%(11 / 14)$ on a median follow-up of 9.9 years (Figure $1 \mathrm{~A}+\mathrm{B})$.

All LCH patients, being treated with fludarabine, melphalan and thiotepa, survived transplantation and showed complete remission (3/3). Within the HLH cohort the overall survival was $72.7 \%(8 / 11)$. Fatalities $(n=3)$ included two patients from the myeloablative group and one RIC-treated patient. The cause of death were progressive disease activity during the conditioning phase, leading to multi-organ failure on day +15 despite immunosuppressive treatment $(\mathrm{n}=1)$ and complicated cerebral seizures followed by lung haemorrhage, possibly due to aspiration pneumonia with evidence of Enterococcus faecium, resulting in septic multi-organ failure on day $+4(n=1)$. A third HLH patient developed a sudden cerebral edema and ensuing respiratory insufficiency on day +4 . Whether this was caused by acute neurotoxic damage by fludarabine or a consequence of relapsed HLH could not be conclusively specified. None of our patients suffered from transplant failure or nonengraftment. There was neither severe acute GVHD (IIIIV) nor chronic GVHD observed in this cohort.

Conclusions: Primary HLH and high-risk LCH are lifethreatening medical conditions needing rapid alloHSCT. RIC regimens are well-tolerated and sufficient for proper engraftment and disease clearance.
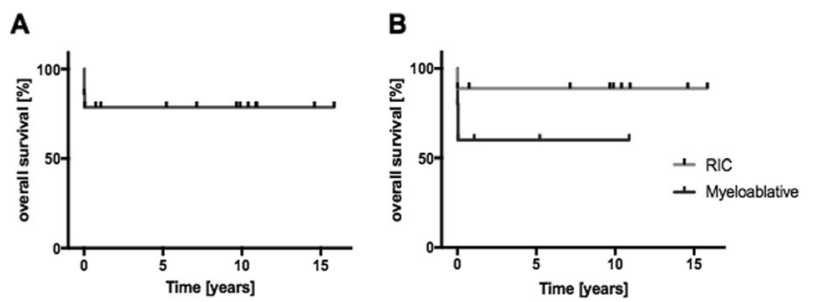

[[P729 Image] 1. OS of all patients (A) and patients treated with RIC $(n=9)$ or myeloablative conditioning $(n=5)(B)]$

Disclosure: The authors have no conflicts of interest to disclose.

P730

Abstract withdrawn. 


\section{P731}

Natural killer cell alloreactive haploidentical stem cell transplantation for multiple myeloma patients

\section{Catharina Elssen ${ }^{1}$, Lotte Wieten ${ }^{1}$, Peter Von dem Borne ${ }^{2}$, Ellen Meijer ${ }^{3}$, Gerard Bos ${ }^{1}$}

${ }^{1}$ Maastricht University Medical Center, Maastricht, Netherlands, ${ }^{2}$ Leiden University Medical Center, Leiden, Netherlands, ${ }^{3}$ Amsterdam University Medical Center, Location VUMC, Cancer Center, Amsterdam, Netherlands

Background: In the past years many new drugs for Multiple myeloma (MM) have been developed and are responsible for a increase in survival. Notwithstanding such progress, MM remains incurable. Results from allogeneic stem cell transplantation (SCT), including haploidentical transplantation, in MM has shown clinical results. However, these responses are only observed in a minority of patients. We hypothesize that this observation might be due to differences in natural killer (NK) cell alloreacitvity, since we have shown in in vivo and in vitro models that mismatched alloreactive NK cells hold the capacity to kill MM cells. The aim of this prospective phase 2 study is to evaluate if KIR-ligand mismatched haploindentical bone marrow transplantation (BMT) with post-transplant cyclophosphamide will improve progression free survival (PFS) in poor risk MM patients.

Methods: Poor risk MM patients, aged $<66$ years were enrolled if they were responsive to their last line of therapy. Poor risk was defined as, high-risk cytogenetics, or relapse within a year after autologous SCT, or treated with three or more previous lines of therapy. A prerequisite of enrolment was the possibility of an NK cell mismatch and availability of a mismatched family donor. Patients were excluded if donor-specific HLA-antibodies were present. Patients received a haploidentical BMT with a non-myeloablative conditioning regimen and post-transplant cyclophosphamide. Primary endpoint is PFS at 1,5 years. Secondary endpoints are engraftment, bone marrow reconstitution, NK cell reconstitution and repertoire, graft versus host disease (GVHD), infections and non-relapse mortality (NRM) at 1,5 years.

Results: In total 12 poor risk patients were included in the study of which 10 could be evaluated for the primary end point. Graft failure and disease progression before transplant rendered the remaining two patients not evaluable. At this interim analysis 7 patients have already reached the 1,5 years of follow up, 5 relapsed within 1,5 years and 2 died due to treatment related infections, without showing progression of disease (20\% NRM). Average time of progression is 105 days (60-270 days). Two of the remaining patients at follow up, still show responsive disease (days 210 en 120). The average time to neutrophil reconstitution is 19 days (14-28 days). All evaluated patients (6/10) show NK cell reconstitution with a mature phenotype in the bone marrow and peripheral blood by day 60. Three patients developed acute GVHD (25\%) of which $2 / 12$ grade I-II aGVHD and 1/12 patient showed a grade IV aGVHD. Treatment related mortality was 3/12 (25\%), which was in all cases due to infectious disease.

Conclusions: Our interim analysis of mismatched haploidentical BMT in MM showed that the treatment is feasible and forms a possible platform for immunotherapeutic strategies. The majority of patients showed an early disease progression. We predefined that with a PFS of $25 \%$ at 1,5 years we would qualify this treatment option successful. With only two patients still in remission this goal will not be achieved and we hypothesize that the late NK cell reconstitution (day 60) is responsible for the lack of response.

Clinical Trial Registry: NCT02519114

Disclosure: Gerard M.J. Bos. CEO CiMaas

\section{P732}

Clinical application of MSC-FFM in pediatric patients after Allo-SCT - A case series

Sümeyye Elgaz, Andrea Jarisch ${ }^{1}$, Sörensen Jan ${ }^{1}$, Eva Rettinger $^{1}$, Andre Manfred Willasch ${ }^{1}$, Selim Kuci ${ }^{1}$, Zyrafete Kuci ${ }^{1}$, Halvard Bönig ${ }^{2}$, Martin Hutter ${ }^{1}$, Peter Bader $^{1}$

${ }^{1}$ University Children's Hospital Frankfurt, Frankfurt, Germany, ${ }^{2}$ Goethe University Medical Center, German Red Cross Blood Center Frankfurt and Institute of Transfusion Medicine and Immunohematology, Frankfurt, Germany

Background: MSCs are known to have immune modulatory capacity and may be effective in the treatment of patients with acute GVHD. However clinical studies yielded inconclusive results which was in part due to the great heterogeneity of the MSC used. The off-the-shelf MSC preparation "MSC-FFM", generated by a proprietary pooling process, selection by plastic-adherence, expansion for an aggregate four weeks followed by cryopreservation until use, is available in Germany through a national marketing authorization. "MSC-FFM" is indicated in steroidrefractory aGvHD, dosed at $1-2 \times 10^{6} / \mathrm{kg}$ BW i.v. in four doses one week apart.

Methods: We report seven consecutive pediatric patients (median age $7.49 \mathrm{y}$ ), who received "MSC-FFM" from unrelated HLA disparate donors between December 2017 and November 2018 in our institution. We gave MSC infusions to 6 patients with steroid-refractory grades III-IV aGVHD and one patient who had therapy-refractory 
thrombocytopenia following allo-SCT concomitant to stem cell boosts. Steroid refractoriness was defined as: progression after three days or no response after 5 days of steroid treatment. The median time from SCT to the onset of aGvHD was $22.5 \mathrm{~d}$ (range 9-122 d), and $11 \mathrm{~d}$ (range 3-40 d) from the onset of aGvHD to the first MSC infusion, respectively.

The majority of our patients $(n=5)$ suffered from a malignant disease and received a graft from a matched unrelated donor $(n=6)$, while one patient had a haploidentical donor. GvHD prophylaxis was performed in all patients except the patient with the haplo-identical graft. All patients with aGvHD were treated with steroids and the patient with thrombocytopenia required regularly transfusions and Romiplostin therapy. The median MSC dose was $1.7 \times 10^{6}$ cells $/ \mathrm{kg}$ BW (minimum $1.1 \times 10^{6}$; maximum 3 $\mathrm{x} 10^{6}$ ). Three patients received 2 MSC doses, two patients 4, one patient 5 and another 7 doses.

Results: At the time point of aGvHD manifestation and MSC application, two patients had CMV reactivation, one patient adenovirus infection and one patient EBVreactivation. By day $28,5 / 6$ aGvHD patients responded to MSC administration: 3 with complete response and 2 with partial response. At the last follow-up (median: 4.42 months, range 0,82- 12.06 months), 4 of 6 patients were alive without acute or chronic GVHD. One patient died soon after MSC treatment with no obvious response in the course of a systemic hyperinflammation syndrome. The other patient although complete responder to MSC-FFM developed fatal adenovirus sepsis. This based on the profound Tcell depletion induced by concomitant application of steroids. The overall survival probability at six month was $66.7 \%$. No acute side effects occurred after MSC infusions. The previously mentioned patient suffering from thrombocytopenia did not need any further transfusions after receiving 2 doses MSCs combined with stem cell boosts while continuing Romiplostin application.

Conclusions: Our data confirm excellent tolerability and high efficacy of the licensed off-the-shelf MSC preparation "MSC-FFM" in pediatric steroid-refractory aGvHD. In our center, current treatment algorithms have escalated "MSCFFM" to the second line, i.e. immediately after steroid refractoriness has been established. Besides immunoregulatory properties, this product might facilitate hematopoietic stem cell engraftment.

Disclosure: Novartis (consultancy: included expert testimony, speaker bureau, Honoraria), Medac (Research Funding, Patents and Royalties), Riemser (Research Funding), Neovii (Research Funding), Amgen (Honoraria)

\section{P733}

Expression of CCR4 modulates migrational properties of in vitro expanded murine regulatory $t$ cells
Laura M. Moser ${ }^{1,2}$, Ulrike Tischler ${ }^{1}$, Christin Riegel ${ }^{1}$, Julia Minderjahn ${ }^{1}$, Rüdiger Eder ${ }^{I}$, Jaqueline Dirmeier ${ }^{I}$, Isabel Zimmermann ${ }^{I}$, Evelyn Röseler ${ }^{I}$, Petra Hoffmann $^{1,3}$, Matthias Edinger ${ }^{1,3}$

${ }^{1}$ University Hospital Regensburg, Regensburg, Germany, ${ }^{2}$ University Hospital Frankfurt, Frankfurt am Main, Germany, ${ }^{3}$ Regensburg Center for Interventional Immunology (RCI), University Regensburg, Regensburg, Germany

Background: Regulatory T cells (Treg) are known for their immunosuppressive function and have proven successful as graft-versus-host disease (GVHD) prophylaxis after allogeneic bone marrow transplantation in a number of preclinical as well as first clinical studies without compromising graft-versus leukemia (GVL) effects. In murine models of acute GVHD lymph node homing capacity via CD62L (L-selectin) proved to be essential for disease prevention. Yet, Treg recruitment from lymph nodes to peripheral sites of ongoing GVHD also seems necessary to achieve maximum protective as well as therapeutic effects. The chemokine receptor CCR4 directs activated T cells to sites of inflammation, thus high CCR4 expression should facilitate Treg homing to affected GVHD target organs. With this project we lay the foundation for future in vivo studies of Treg therapy for GVHD by upregulation of CCR4 expression.

Methods: We performed systematic ex vivo analysis of CCR4 expression on murine naive and memory conventional (Tconv) and regulatory $\mathrm{T}$ cells isolated from spleen, blood, bone marrow, lymph nodes, liver and lung. Cells were stained for characteristic surface and intracellular markers and characterized by multiparametric flowcytometric analysis. CCR4 expression kinetics following stimulation were analysed in Tconv and Treg isolated from murine splenocytes by FACS and polyclonally activated by anti-CD3/CD28-coated beads in the presence of exogenous IL-2. Expression was monitored by daily flow cytometric analysis. CCR 4 overexpression was induced by transduction of expanded Treg with CCR4 mRNA via electroporation. Expression kinetics were monitored by FACS, receptor function was tested in transwell migration assays using CCR4 ligands CCL-17 and CCL-22.

Results: Systematic analyses showed higher CCR4 expression on memory Treg than on their naive counterpart in all examined organs with bone marrow samples displaying the greatest disparity. Memory Treg showed higher CCR4 expression than memory Tconv in all analysed organs, except for lymph nodes where both memory populations revealed equal expression levels. Stimulation of in vitro expanded Treg and Tconv lead to a strong increase in CCR4 expression with maximum levels on $\mathrm{d} 3$ and $\mathrm{d} 2$ respectively, whereas restimulation (d7) 
resulted in no further relevant CCR4 expression on Treg. We performed systematic optimization of stimulation and mRNA-electroporation conditions to reliably achieve highlevel short-term CCR4 expression. Transduction of Treg on d11 of in vitro expansion resulted in a strong CCR4 expression, with maximum levels $2 \mathrm{~h}$ after electroporation and strong CCR4 expression being detectable for at least $8 \mathrm{~h}$. Transwell migration assays showed enhanced migrational properties of mRNA-electroporated Treg towards CCR4 ligands. Analyses performed $2 \mathrm{~h}$ and $16 \mathrm{~h}$ after electroporation showed persistent migration even though measured CCR4 surface expression had already declined significantly.

Conclusions: We showed that high CCR4 expression can be detected on memory Treg in all analysed organs. Since in vitro stimulation of murine Treg did not reliably induce CCR4 expression, we established a protocol for CCR4 mRNA-electroporation. Electroporated cells showed stable short-term CCR4 expression and enhanced migrational properties towards CCR4 ligands in vitro. Future studies will show whether the induction of short-term CCR4 expression will facilitate in vivo homing of adoptively transferred Treg to sites of ongoing GVHD and thus mediate long-term inflammation suppression.

Disclosure: The authors have no conflict to disclose.

\section{P734}

Survival and immune reconstitution of syngeneic, haploidentical and allogeneic hematopoietic stem cell transplantation in ATM-deficient mice

\section{Ruth Pia Duecker', Patrick C. Baer ${ }^{2}$, Stefan Zielen ${ }^{1}$, Ralf Schubert ${ }^{1}$}

${ }^{1}$ Children's Hospital, Goethe-University, Frankfurt, Germany, ${ }^{2}$ Goethe University, Frankfurt, Germany

Background: Hematopoietic stem cell transplantation (HSCT) as it is carried out successfully at other genetic instability syndromes seems to be an encouraging opportunity for a curative therapy to restore immunity and prevent the development of hematologic malignancies in Ataxiatelangiectasia (A-T). However, experience in the conditioning regimen is limited and no transplantation strategy for A-T patients exists, especially in an allogeneic setting. Conditioning regimen and donor selection are critical factors in the clinical setting of HSCT and incur substantial risks, especially in A-T. The aim of this study was (1) to evaluate whether different approaches of HSCT including allogeneic hematopoietic HSCT are feasible in regard to graft versus host response (GvHD) and sufficient concerning immune reconstitution (2) and to de-escalate the toxic effects of the conditioning regimen by reducing the dose of cyclophosphamide (CP).

Methods: $\mathrm{T}$ cells from syngeneic, allogeneic and haploidentical donor mice were used to determine GvHD induced $\mathrm{T}$ cell proliferation in a mixed lymphocyte reaction (MLR). Atm-deficient mice were treated with $\mathrm{CP}$ or reduced $\mathrm{CP}$ in combination with Fludarabine (FLU) and transplanted with $5 \times 10^{6}$ CD90.2 depleted bone marrow donor cells from 129/SvEv GFP-transfected wildtype mice (syngeneic) or from mice of the F1 generation of $129 / \mathrm{SvEv}$ wildtype mice and C57BL/6 mice (haploidentical), or from C57BL/6 mice (allogeneic). Tracking of GFP-positive donor derived cells was performed using flow cytometry and Atm PCR. Oxidative stress and damage were detected by a RT Profiler PCR Array and 8-Hydroxy-2'deoxyguanosine.

Results: MLR resulted in an increased proliferation of allogeneic donor $\mathrm{T}$ cells compared to syngeneic and haploidentical donor cells. Response was lower on dendritic cells isolated from Atm-deficient mice compared to wildtype controls. In vivo results showed the restoration of $\mathrm{T}$ cells in Atm-deficient mice accompanied by a prolonged life span and through reduction of thymic tumors. However, allogeneic stem cell transplantation was accompanied with a higher mortality rate, compared to the haploidentical and syngeneic setting. Decreased antioxidative capacity and a higher DNA-damage were seen in CP treated Atmdeficient mice.

Conclusions: Haploidentical HSCT seems to be a feasible strategy for A-T. Our data provided further evidence for the high sensitivity against ROS-inducing agents in A-T and this fact needs to be taken into consideration in the choice of the host-conditioning strategy.

Disclosure: Nothing to declare

This research received funding from Action for A-T Charity (14GOU01)

\section{P735}

\section{Abstract already published.}

P736

Allogeneic cellular immunotherapy induces selective clearance of refractory childhood cancers: A single center experience

Michael Merker ${ }^{1}$, Michael Torsten Meister ${ }^{1,2}$, Annekathrin Heinze ${ }^{1}$, Andrea Jarisch, Jan Soerensen, Sabine Huenecke ${ }^{I}$, Melanie Bremm ${ }^{I}$, Claudia Cappel ${ }^{I}$, Thomas Klingebiel ${ }^{1}$, Peter Bader ${ }^{I}$, Eva Rettinger ${ }^{I}$ 
${ }^{1}$ University Hospital Frankfurt, Frankfurt, Germany, ${ }^{2}$ Princess Máxima Center for Pediatric Oncology, Utrecht, Netherlands

Background: Prognosis of pediatric patients and young adults suffering from refractory or high-risk soft tissue sarcomas remains poor with limited improvement over the last decades despite multimodal treatment strategies. Replacing the immune system by an allogeneic hematopoietic stem cell transplantation (HSCT) in refractory solid malignancies has been proposed as a potentially curative therapy due to its presumable graft versus tumor effect. Based on this concept we additionally performed consecutive donor-derived lymphocyte infusions in allogeneic HSCT-patients with refractory or relapsed solid malignancy to further increase anti-tumor efficacy post-transplant.

Methods: Pediatric patients with relapsed and/or refractory cancers or with delayed responses to the respective induction therapies were offered donorderived cellular therapies after immune system replacement by an allogeneic HSCT. Cellular immunotherapies comprised of donor lymphocyte infusions (DLI), natural killer (NK) cell or cytokine-induced killer (CIK) cell infusions generated from the original stem cell donors. Allogeneic NK cells were generated from unstimulated leukapheresis by a two-step purification procedure using immunomagnetic CD3 $\mathrm{T}$ cell depletion, followed by NK cell enrichment (CD56+) with or without in vitro IL-2 stimulation and expansion for 9-14 days. For CIK cell generation peripheral blood mononuclear cells were isolated and activated by in vitro cytokine stimulation (INF- $\gamma$, anti-CD3, IL-2 and IL-15) an expanded over 1012 days. Expanded CIK cells represented a heterogeneous population of polyclonal $\mathrm{T}$ cells with in part shared phenotypic and functional properties of NK cells.

Results: Between October $1^{\text {st }} 2003$ and January $1^{\text {st }} 2014$ a total of 18 patients (eight patients with rhabdomyosarcoma, one patient with synovial sarcoma, two patients with Ewing sarcoma, five patients with neuroblastoma, one patient with hepatoblastoma, and one patient with nasopharynx carcinoma) were enrolled. Seven of 18 (39\%) patients in this study had achieved complete remission (CR) before HSCT while another 7 of 18 (39\%) patients had obtained at least very good partial or partial response (VGPR or PR). DLI was applied in 5 patients, NK cell treatment was offered to another 6 patients, while CIK cell therapy was given to 7 patients.

1.5-year probabilities of overall survival (OS) and progression-free survival (PFS) were $16.2 \%$ and $15.9 \%$ for all patients with a median follow up of 8.5 months (range, 1.5-115.1 months). Patients in CR at the time of immune cell therapy (IT) showed estimated 1.5-year OS and PFS of $49.6 \%$ and $42.7 \%$, respectively. The majority of patients relapsed and ultimately succumbed to their diseases with two of $18(11 \%)$ patients still being alive 9.6 and 9.3 years after IT. Cumulative incidence of relapse was $79.8 \%$ at 1.5 years. T cell engraftment and immune reconstitution (IR) was improved by IT, and correlated with treatment response. However, two of 18 heavily pretreated patients (11\%) died due to cumulative treatment-related mortality (TRM). Furthermore, acute graft-versus-host-disease (aGVHD) occurred in 9 of 18 patients (50\%) with aGVHD grade I-II observed in $6(33 \%)$ and aGVHD grade III seen in three $(17 \%)$ patients.

Conclusions: Altogether, the results of this study indicate that allogeneic donor-derived cellular therapy at its current state offers curative benefit in selected refractory childhood cancers but warrants further improvement.

Disclosure: Michael Merker, Michael Torsten Meister, Annekathrin Heinze, Andrea Jarisch, Jan Sörensen, Sabine Huenecke, Melanie Bremm, Claudia Cappel, Thomas Klingebiel and Eva Rettinger have nothing to declare. Peter Bader declares the following potential conflicts of interest: Novartis (consultancy: included expert testimony, speaker bureau, Honoraria), Medac (Research Funding, Patents and Royalties), Riemser (Research Funding), Neovii (Research Funding), Amgen (Honoraria).

\section{P737}

Immune reconstitution post allogeneic stem cell transplantation in pediatric patients with nonmalignant diseases

Emilia Salzmann-Manrique ${ }^{1}$, Sabine Huenecke ${ }^{1}$, Melanie Bremm $^{I}$, Lisa-Marie Pfeffermann ${ }^{1}$, Martin Hutter ${ }^{I}$, Jan Soerensen $^{1}$, Eva Rettinger ${ }^{1}$, Shahrzad Bakhtiar ${ }^{\text {I, Andre }}$ Willasch $^{I}$, Thomas Klingebiel ${ }^{1}$, Peter Bader ${ }^{I}$, Andrea Jarisch ${ }^{1}$

${ }^{1}$ University Hospital Frankfurt, Frankfurt, Germany

Background: Allogeneic stem cell transplantation (alloSCT) is the only curative treatment option for a variety of nonmalignant diseases. The success of allo-SCT is strongly associated with rapid and sustained immune reconstitution (IR). We analyzed the IR in patients who received an alloSCT for nonmalignant diseases.

IR was assessed on days $+30,+60,+90,+180$ and +365 after allo-SCT analyzing leukocytes, lymphocytes, monocytes, $\mathrm{CD}^{+} \mathrm{T}$ cells, $\mathrm{CD} 3^{+} \mathrm{CD} 4^{+} \mathrm{T}$ helper cells, $\mathrm{CD}^{+}{ }^{+} \mathrm{CD} 8^{+}$cytotoxic $\mathrm{T}$ cells, $\mathrm{CD} 3^{-} \mathrm{CD} 56^{+}$natural killer (NK) cells and $\mathrm{CD} 19^{+} \mathrm{B}$ cells.

Methods: We analyzed IR-data of 116 consecutive patients receiving allo-SCTs between September 2001 and 
November 2018. Indications of allo-SCT were hereditary anemias (thalassemia, sickle cell disease, Diamond Blackfan Anemia; HA, $\mathrm{n}=35,30 \%$ ), inherited bone marrow failure syndrome (Fanconi anemia, severe aplastic anemia, others; BMFS, $\mathrm{n}=34,29 \%$ ), hemophagocytic lymphohistiocytosis (HLH, $\mathrm{n}=12,10 \%)$, immunodeficiency (ID, $\mathrm{n}=28,24 \%)$ and metabolic disorders $(\mathrm{n}=7,6 \%)$. The median age at allo-SCT was 7 years (range, $0.2-26$ ) and at diagnosis 0.8 years (range, $-0.5-17.8$ ).

Patients received $1^{\text {st }}$ allo-SCT from MSD/MFD ( $\mathrm{n}=53$, $46 \%)$, MUD $(n=42,36 \%)$, haploidentical mismatch family donors $(n=15,13 \%)$ and MMUD $(n=6,5 \%)$. Conditioning regimens were Busulphan-based $(\mathrm{n}=14, \quad 12 \%)$, Treosulphan-based $(n=45,39 \%)$, Flu-Mel-Thio $(n=35$, $30 \%)$ or others $(n=22,19 \%)$. Graft sources were BM $(n=84,72 \%)$ and PBSC $(n=32,28 \%)$.

In order to consider the age-dependency of IR we normalized each absolute cell count with its corresponding age-specific expected mean values. (Huenecke et al.; Eur J Haematol; 2008)

Results: The IR pattern was similar between the HA and BMFS groups. The $\mathrm{CD} 4^{+} \mathrm{T}$ cells were recovering slightly faster in HA patients compared to the recovery of BMFS patients.

Monocytes and NK cells proliferate very fast. At day +60 half of the patients already reached their respective monocytes reference value except for ID patients, who reached $80 \%$ of the reference value at the end of the first year.

$\mathrm{CD}^{+}{ }^{+} \mathrm{CD}^{+}$cytotoxic $\mathrm{T}$ cells recovered significantly faster in patients with hematologic diseases compared to patients with HLH $(\mathrm{P}<0.001)$ and ID $(\mathrm{P}=0.047)$. Half of the patients reached the reference value of cytotoxic $\mathrm{T}$ cells in the hematologic diseases group at day +365 . By far inferior was the IR for the HLH patients. In this group only $50 \%$ of the patients reached the $45^{\text {th }}$ percentile of the healthy age-matched reference. In the ID group $50 \%$ of all patients reached the $87^{\text {th }}$ percentile of the age-matched reference group at day +365 .

$\mathrm{B}$ cells are profoundly decreased at day +30 in all groups. However, the longitudinal expansion of $\mathrm{B}$ cells was significantly lower both in the ID group and HLH group compared to the hematologic diseases group. At day +365 fifty percent of patients with ID, HLH and hematologic diseases reached the $37^{\text {th }}$ percentile, $52^{\text {th }}$ percentile and the $100^{\text {th }}$ percentile, respectively $(\mathrm{P}<0.001 ; \mathrm{P}=0.027)$.

Conclusions: Allo-SCT is the only curative option for patients with nonmalignant diseases. IR is dynamic and revealed a complex diversity pattern with regard to the original disease. To investigate factors influencing IR after allo-SCT is crucial to improve outcome of these patients.

Disclosure: Nothing to declare.
P738

Allogeneic hematopoietic stem cell transplantation in patients with myelodysplastic syndrome of relatively high-risk groups: curative effect analysis and optimal timing selection

\section{Qianqian Wang ${ }^{I}$, Mingzhe Han ${ }^{1}$}

${ }^{1}$ Institute of Hematology \& Blood Disease Hospital, CAMS \& PUMC, Tianjin, China

Background: Allogeneic hematopoietic stem cell transplantation (allo-HSCT) is the only potential curative therapy for myelodysplastic syndromes (MDS). To choose the right time to transplant, reduce the risk of transplantation and achieve the maximum benefit is very important.

Methods: We retrospectively analyzed 135 patients undergoing allo-HSCT for primary MDS or secondary acute myeloid leukemia (sAML) evolving from MDS of relatively high-risk groups according to the revised International Prognostic Scoring System (IPSS-R) from January 2010 to May 2018 in our hospital. Pediatric patients and patients in very low or low IPSS-R risk stratification at transplant were excluded already.

Results: Among the 135 patients, 133 patients were transplanted successfully. The 3-year overall survival (OS) rate and disease-free survival (DFS) rate was 76.8\% $\pm 4.2 \%$ and $76.7 \% \pm 4.3 \%$ respectively. The 3 -year cumulative relapse rate (RR) and the non-relapse mortality (NRM) rate was $13.9 \% \pm 0.1 \%$ and $18.4 \% \pm 0.1 \%$ respectively. The incidence of grade II-IV acute graft versus host disease (aGVHD) was $25.2 \% \pm 0.1 \%$. For the patients who survived more than 100 days after allo-HCT, the 2 years cumulative incidence of chronic graft versus host disease (cGVHD) was $33.4 \% \pm 0.2 \%$. Univariate analysis showed that the hematopoietic cell transplantation comorbidity index(HCT-CI) and grade III-IV aGVHD are the high risk factors for $\mathrm{OS}(81.2 \pm 4.5 \%$ vs $62.8 \pm 10 \%$, $\mathrm{P}=0.027$ and $81.9 \pm 4.4 \%$ vs $51.1 \pm 11.8 \%, \mathrm{P}<0.001)$. Multivariate analysis demonstrated that grade III-IV aGVHD and HCT-CI are independent risk factors for $\mathrm{OS}(\mathrm{HR}=6.206, \mathrm{P}<0.001,95 \% \mathrm{CI}: 2.529 \sim 15.288$ and $\mathrm{HR}=2.651, \mathrm{P}=0.025,95 \% \mathrm{CI}: 1.127 \sim 6.232$ ). Chemotherapy before transplantation did not improve OS or DFS for patients with bone marrow blast cells more than $10 \%$ at the time of diagnosis.

Conclusions: Allo-HSCT is an effective treatment for MDS patients of relatively high-risk groups. The physical condition of the patients and occurrence of aGVHD are independent risk factors. For intermediate and high risk IPSS-R MDS patients, transplantation before the disease progressed into very high risk can achieve better prognosis, high-risk group can still benefit 
from allo-HSCT. It's not necessary to do chemotherapy before transplantation for patients with bone marrow blast cells more than $10 \%$ at the time of diagnosis.
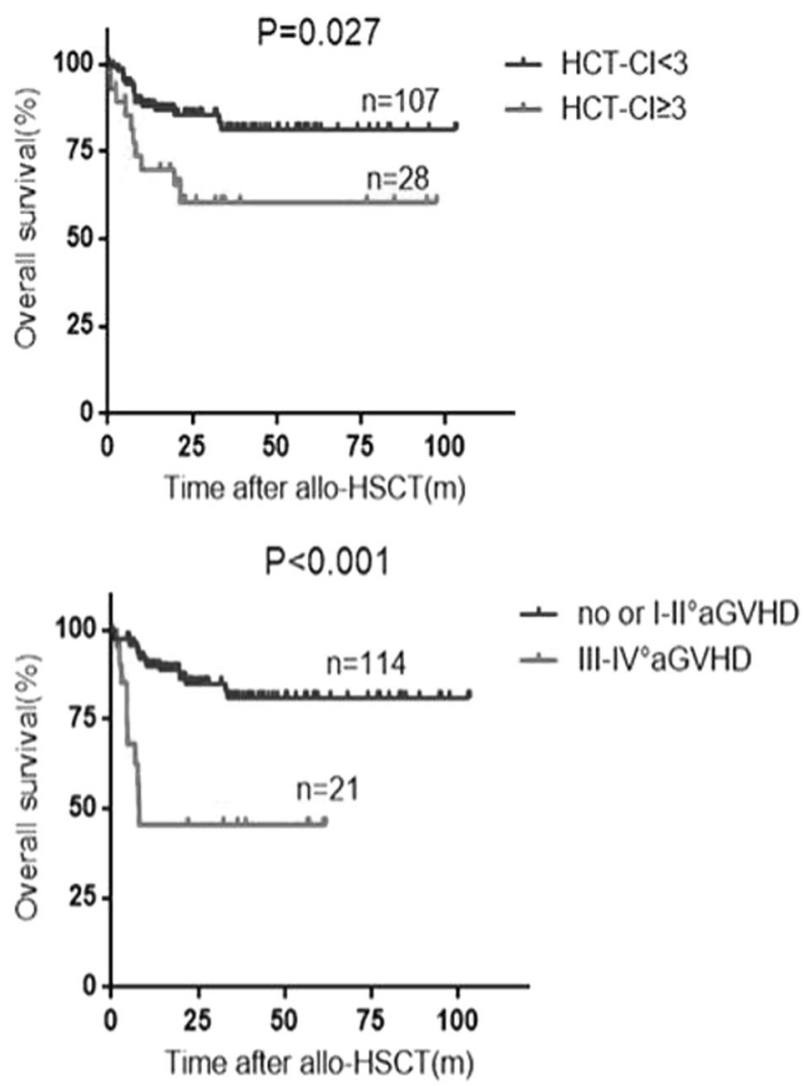

[[P738 Image] 1. Figure 1 The HCI-CT of patients before transplantation and occurance of grade III-IV aGVHD on overall surviv]

\begin{tabular}{lllll}
\hline & & Cases & OS & P value \\
\hline Age & $<50 \mathrm{y}$ & 112 & $78.1 \pm 4.4 \%$ & 0.63 \\
& $\geq 50 \mathrm{y}$ & 23 & $67.6 \pm 13 \%$ & \\
IRSS-R & intermediate & 56 & $83.3 \pm 5.1 \%$ & 0.451 \\
& high & 43 & $82.2 \pm 6.1 \%$ & \\
& very high & 36 & $64.7 \pm 9.3 \%$ & \\
Time from Diagnosis to & $<6 \mathrm{~m}$ & 87 & $81.8 \pm 4.8 \%$ & 0.075 \\
Transplantation & & & & \\
& $\geq 6 \mathrm{~m}$ & 48 & $67.7 \pm 7.8$ & \\
cGVHD & yes & 43 & $84.8 \pm 6.4 \%$ & 0.069 \\
& no & 91 & $74.3 \pm 5.3 \%$ & \\
\hline
\end{tabular}

[[P738 Table] 1. Table 1 Univariate analysis of factors influencing survival]

Disclosure: Nothing to declare

\section{P739}

Treosulfan-based allogeneic haematopoietic stem cell transplantation in children, adolescents and young adults with transfusion-dependent thalassemia

Friedhelm Schuster ${ }^{1}$, Olga Kyrillopoulou ${ }^{1}$, Sujal Ghosh ${ }^{1}$, Florian Babor ${ }^{1}$, Meinolf Siepermann ${ }^{2}$, Hiba Fouz ${ }^{1}$, Stefan Balzer ${ }^{1}$, Arndt Borkhardt ${ }^{1}$, Roland Meisel ${ }^{1}$

${ }^{1}$ Klinik für Kinder-Onkologie, -Hämatologie und -Klinische Immunologie, University of Duesseldorf, Düsseldorf, Germany, ${ }^{2}$ Krankenhaus Neuwerk, Moenchengladbach, Germany

Background: 1. Allogeneic haematopoietic stem cell transplantation (SCT) offers the chance of cure for patients with transfusion-dependent thalassemia (TDT). Based on the non-neoplastic nature of this condition SCT approaches urgently require to prove both efficacious and safe.

Methods: 2. We report on 13 children, adolescents and young adults (median age: 6 years; range 2-28 years) with TDT receiving SCT from an HLA-matched donor (MUD $n=8$, MSD $n=3$, MFD $n=2$ ) in our center from 2011-2017. All patients received the same treosulfan-based conditioning regimen (treosulfan $3 \times 14 \mathrm{~g} / \mathrm{m} 2$, fludarabine $4 \times 40 \mathrm{mg} / \mathrm{m} 2$, thiotepa $1 \times 8 \mathrm{mg} / \mathrm{kg}$ ). GvHD prophylaxis was based on ATG-Fresenius ${ }^{\mathrm{TM}}(3 \times 10 \mathrm{mg} / \mathrm{kg}$, if MUD or MFD as donor), CSA (with taper from day +270 ) as well as MTX (day $1,3,6,11$ ) in $9 / 13$ and MMF in 4/13 patients with MTX toxicity, respectively. Stem cell source was bone marrow in 8 , peripheral blood stem cells in 4 and cord blood in 1 patient. Prior to transplantation 6 children received cytoreductive treatment with azathioprine, hydroxycarbamide and intensified erythrocyte transfusion. Iron elimination therapy was carried out in 12/13 children with deferasirox. Among the 11 patients with available Ferriscan $^{\mathrm{TM}}$ analysis 4 patients showed substantial liver iron overload (liver iron $>8 \mathrm{mg} / \mathrm{g}$ ) despite intensive chelation prior to SCT.

Results: 3. All patients achieved leukocyte engraftment at median day +23 (range 15-39), however two patients required a CD34-selected PBSC boost on day +36 and day +50 based on delayed platelet and/or erythrocyte engraftment. Nine patients exhibited full donor chimerism in the $\mathrm{BM}$ at day +30 , the other 4 showed mixed chimerism with $<5 \%$ autologous cells. On day +360 peripheral blood chimerism was complete in $12 / 13$ patients with the remaining patient exhibiting stable split chimerism with $100 \%$ donor-derived erythrocytes and $60-80 \%$ autologous myeloid cells. Acute GvHD was observed in three patients (grade 2: $\mathrm{n}=1$, grade 3: $\mathrm{n}=2$ ). However, all patients responded to immunosuppressive therapy with steroids \pm ECP and re-initiation of CNI $(n=1)$. One patient suffered 
from rebound GvHD after CNI tapering which was promptly responsive to treatment steroids, FK506 and ECP. Aa one year after SCT $12 / 13$ patients were without GvHD and off all immunosuppression while one single patient was still on taper of immunosuppressant after rebound acute GvHD. No chronic GvHD occurred. SCTrelated toxicity was common with mucositis in all patients (WHO grade 4: $n=5$ ), elevated liver enzymes ( $\geq$ grade 3 : $\mathrm{n}=9$ ) and impaired renal function (GFR 40-60ml/min: $\mathrm{n}=8$ ). Five patients developed neurologic symptoms (seizures $n=2$, PRES $n=1$, pseudotumor cerebri $n=2$ ) which all resolved without sequelae. Overall survival and transfusion-free survival was $100 \%$ with a median observation time of 5 (2-8) years.

Conclusions: 4 . Treosulfan-based conditioning followed by SCT from HLA-matched related or unrelated donors represents a highly efficacious treatment approach for children, adolescents and young adults with TDT and exhibits an acceptable but not negligible safety profile. An individual risk-benefit assessment incorporating hazards such as secondary graft failure, GvHD and long-term toxicity including infertility and $2^{\text {nd }}$ malignancy has to be executed in the informed consent process for every patient and his/her guardians.

Disclosure: "Nothing to declare"

\section{P740}

Abstract already published.

\section{P741}

Early iron chelation with deferasirox might prevent relapse after busulfan plus fludarabine and ATG as a myeloablative conditioning for HLA-identical sibling allogeneic HCT in AML

Nabil Yafour ${ }^{1}$, Faiza Serradj ${ }^{1}$, Soufi Osmani', Mohamed Brahimi $^{1}$, Hafida Ouldjeriouat ${ }^{1}$, Nassima Hakiki ${ }^{1}$, Badra Enta Soltane ${ }^{1}$, Kamelia Amani ${ }^{1}$, Asma Chadli ${ }^{1}$, Leila Charef $^{1}$, Rachid Amar Bouhass ${ }^{1}$, Abdessamad Arabi', Mohamed Amine Bekadja ${ }^{1}$

${ }^{1}$ EHU 1er Novembre 1954 Oran, Hematology and Cellular Therapy, Oran, Algeria

Background: Some published data suggest a positive effect of iron chelators on the risk of post-transplant relapse, with an improvement in overall survival after allogeneic hematopoietic stem cell transplantation (HSCT) (Callens $J$ et al. Exp Med. 2010. Michallet et al. abstract. Blood 2013). Rational based on the hypothesis that iron chelators would have an anti-proliferative effect in acute leukemia and potential leukemia differentiation of blast cells.
Objective: the aim of this study was to determine the role of early iron chelation on relapse incidence after allogeneic HSCT in adult patients with acute myeloid leukemia (AML).

Methods: This is a prospective, single-center study in AML patients in CR, who received allogeneic HSCT from February 2013 to January 2018. Conditioning regimen consisted to intravenous Bu $130 \mathrm{mg} / \mathrm{m}^{2}$ and, Flu $40 \mathrm{mg} / \mathrm{m}^{2}$ D-6 to D-3. GVHD prophylaxis included ATG $2.5 \mathrm{mg} / \mathrm{kg}$ on D-2 and D-1, ciclosporin and methotrexate. All patients received peripheral blood stem cell transplant from an identical HLA-related donor. Iron chelation consisted on deferasirox (Exjade $\left.{ }^{\circ}\right) 10-20 \mathrm{mg} / \mathrm{kg} / \mathrm{day}$, started at day 100, if serum ferritin level $\geq 800-1000 \mathrm{ug} / \mathrm{l}$ and stopped when the level decreased below $600 \mathrm{ug} / \mathrm{l}$ or normalized. Ferritin Serum evaluated every three months. Patients divided into two groups: Group 1 (G1); who did not receive any iron chelators treatment due to ferritin level $<800 \mathrm{ug} / \mathrm{l}$, side effects or compliance problems. Group 2 (G2) consisted of patients who received deferasirox treatment after day 100 . None of patients received prophylactic $D L I$ after transplantation. Patients relapse or dead before day100 were excluded.

Results: 40 patients were included. Median age at transplant was 37 years (18-65), including 26 men and 14 women. Median CD34+/ kg cells and CD3 + lymphocytes infused were $4.51 \times 10^{6}(2.24-7)$ and $2.15 \times 10^{8}(0.004-$ 6.24) respectively. Median time for absolute nuclear cells $>$ $0.5 \times 10^{9} / \mathrm{L}$ was 12 days (5-25), and unsupported platelet counts of at least $20 \times 10^{9} / \mathrm{L}$ was 14 days (10-43). CMV reactivation occurred in $21 \%$ of patients. Acute GVHD grade I-IV and grade III-IV were $44 \%$ and $5 \%$, respectively. Chronic GVHD rate was $44 \%$, including only $8 \%$ of patients with severe grade. At day 100 after transplant, 35/ 40 patients were evaluable ( $\mathrm{G} 1=20 \mathrm{pts}),(\mathrm{G} 2=15 \mathrm{pts})$. Patients were ABO compatible (66\%), had major incompatibility (29\%), and 6\% had minor incompatibility. Median serum ferritin level at day 100, were $1200 \mathrm{ug} / \mathrm{l}$ (833-4449) and $1200 \mathrm{ug} / \mathrm{l}$ (123-1853) in G1 and G2 respectively. In G2, treatment duration with deferasirox was 233days (91-422). Iron chelating treatment, reduced serum ferritin $564 \mathrm{ug} / \mathrm{l}$ (56-1982). With a median follow-up of 34 months, (12-68), disease relapse incidence was higher in patients who did not received iron chelation treatment (G1: 39.8\%) versus those who received oral deferasirox (G2:15.6\%) (Figure 1), but the difference was not statistically significant $(p=0.19)$. Overall survival was $55 \%$ and $67.8 \%(P=0.27)$ in $\mathrm{G} 1$ and G2 respectively.

Conclusions: These results deserve more investigation, and needs to be confirmed by a large randomized trial.

Disclosure: No conflict of interest 


\section{P742}

Sequential chemotherapy followed by allogeneic hematopoietic stem cell transplantation in patients with refractory acute myeloid leukemia

\section{Salvador Tabares ${ }^{1}$, Estefanía García ${ }^{1}$, Rafael Rojas ${ }^{1}$, Josefina Serrano ${ }^{1}$, Jose Ramon Molina ${ }^{1}$, Antonia Villa ${ }^{1}$, Joaquin Sanchez ${ }^{1}$, Carmen Martin ${ }^{1}$, I Concepcion Arroyo ${ }^{1}$}

${ }^{1}$ Hospital Reina Sofia, Cordoba, Spain

Background: Patients with relapsed or refractory acute myeloid leukaemia (AML) have a poor prognosis. Allogeneic hematopoietic stem cell transplantation is the only curative option. However, allogeneic transplantation with active leukemia failed to improve significantly the longterm outcome. To improve the outcome of allo-HSCT in such high-risk and refractory patients, sequential schedule of cytoreduction therapy followed by nonmyeloablative conditioning has been developed.

Methods: To evaluate the outcome of sequential intensified conditioning regimen followed by allogeneic hematopoietic stem cell transplantation (allo-HSCT) for refractory acute myeloid leukemia (AML).

Results: A total of 20 patients with primary or secondary refractory AML transplanted between June 2011 to July 2018 were included. Refractoriness was defined as primary induction failure, relapse within 6 months from induction/ consolidation chemotherapy or second relapse. Median age is 39 years ( 1 to 61). The salvage chemotherapy administered was FLAG-IDA. Two patients did not receive intensive chemotherapy because of no recovery after induction chemotherapy. Seven days after the end of FLAG-IDA, a reduced intensity conditioning consisting of fludarabine, $60 \mathrm{mg} / \mathrm{m} 2$, thiotepa, $5 \mathrm{mg} / \mathrm{kg}$ and busulfan 6,4 $\mathrm{mg} / \mathrm{kg}$ i.v. $(\mathrm{n}=12)$ for haploidentical donors or fludarabine plus busulfan $(n=8)$ for HLA identical sibling or unrelated donors was administered. Graft-versus-host disease (GVHD) prophylaxis consisted of Tacrolimus and Mycophenolate mofetil. The Mycophenolate was withdrawn at day +35 post-transplantation and Tacrolimus at day +100 . Donor lymphocytes (DLI) were infused in patients without aGVHD at Day +120 post-transplantation. Seventeen patients achieved complete donor chimerism, 3 patients progressed early and 1 patient died before engraftment. One of the patients which recovery was with persistent leukemia reached donor chimerism after immunosupression discontinuation. Ten patients are alive in complete remission. Median follow-up of survivors is 28 months (range: 6-43). Five patients died of leukemic progression, 3 as result of GVHD and 1 suffered intracranial hemorrhage. Five patients received prophylactic DLI. The incidence of acute moderate-severe GVHD and moderate-severe chronic GVHD were $70 \%(n=14)$ and $50 \%(n=7)$, respectively. The non-relapse mortality was $21 \%(n=4)$, mainly due to acute GVHD $(n=3)$.

The 2-year cumulative incidence of relapse posttransplantation was $36.8 \%$. The probability of relapse was $57 \% \pm 13 \%$. The 2 -year OS and DFS were $46 \% \pm 12 \%$ and $38 \% \pm 12 \%$

Conclusions: The strategy of sequential chemotherapy followed by alloHSCT \pm prophylactic DLI has an acceptable toxicity profile and improves both the relapse rate and the survival for refractory AML patients.

Disclosure: Nothing to declare

\section{P743}

Verification of mentype digitalquant assay on the quantstudio 3D platform

Patricia Dalheimer $^{1}$, Emilia Salzmann-Manrique ${ }^{1}$, Peter Bader $^{1}$, Hermann Kreyenberg ${ }^{1}$

${ }^{1}$ University Hospital Frankfurt / Clinic for Children an Adolescent, Frankfurt, Germany

Background: The concept of immunological intervention to prevent relapse after hematopoietic stem cell transplantation is associated with the assessment of the chimerism status. Distinguishing patient and donor hematopoiesis is usually performed by STR-PCR, a powerful method developed for forensic purposes. However, this method shows restrictions with respect to detection limit, preciseness, and the possibility of automated read out. Digital PCR could circumvent some of these limitations.

Methods: Recently, validated for the Bio-Rad droplet digital platforms, the Biotype Mentype DigitalQuant Assay was released. The assay uses INDEL polymorphisms on chromosomal DNA to distinguish patient and donor hematopoiesis on a FRET hydrolysis assay basis ("TaqMan assays"). Thus the assay in principle is applicable on the chamber based 3 D digital PCR system (Thermo Fisher). Due to different reaction chemistry and physical properties of thermal transfer between the digital PCR platforms protocols are reasonably not fully compatible which would lead to lower fluorescence intensities and poor signal resolution on the solid chip based Thermo Fisher 3D platform. An adjusted PCR protocol was established and optimized using representative markers, followed by determination of tolerable and optimal amount of input DNA. Specificity, sensitivity and reproducibility testing with artificial mixed samples preceded the extensive verification by comparative measurement of clinical samples $(\mathrm{N}=59)$ and ring-trial samples $(\mathrm{N}=20)$. 
Results: We show an excellent concordance between chimerism assessment on Bio-Rad and $3 \mathrm{D}$ platforms over the complete range of mixture ratios $\left(R^{2}>0,9\right)$ and proof the lower detection limit $(0,1 \%)$ compared to STR-PCR.

Conclusions: Our results promote the transfer of the established Mentype assay to a more diverse instrument portfolio. That will allow to implement the analysis of patient and donor hematopoiesis by digital PCR methods in our lab.

Disclosure: Nothing to declare

\section{P744}

Incorporating fertility counseling in pediatric oncology and stem cell transplantation: Set up of structures and results of the counseling process

\section{Anke Barnbrock ${ }^{1}$, Emilia Salzmann ${ }^{1}$, Konrad Bochennek $^{1}$, Nicole Sänger ${ }^{2}$, Henning Fiegel ${ }^{1}$, Falk Ochsendorf $^{1}$, Sandra Lange ${ }^{1}$, Thomas Klingebiel ${ }^{1}$, Peter Bader $^{I}$, Andrea Jarisch ${ }^{I}$}

${ }^{1}$ University Hospital, Goethe University, Frankfurt, Germany, ${ }^{2}$ University Hospital Bonn, Bonn, Germany,

Background: With the immense progress in therapeutic regimens in pediatric oncology and stem cell transplantation the survivor rates increased up to $80 \%$. At the same time the field of reproductive medicine has achieved substantial advances to offer potential options for fertility preservation. Therefore it is of great importance to implement fertility counseling and fertility preserving (FP) procedures for patients facing gonadotoxic therapy. In the report on the expert meeting of the Paediatric Diseases Working Party (PDWP) of the EBMT in 2017 counseling related to FP opportunities should be offered to each patient receiving stem cell transplantation (SCT), as part of the pre-SCT workup by a dedicated and trained team. Yet there many medical, ethical, structural and financial issues to consider and overcome. We describe the setting up process to enable fertility counseling for all children with newly diagnosed cancer or those facing stem cell transplantation for malignant and nonmalignant diseases in our department of pediatric oncology and immunology/stem cell transplantation.

Methods: At our tertiary care center we assembled a multidisciplinary team involved in fertility preservation (pediatric hematology/oncology, pediatric immunology/ stem cell transplantation, reproductive medicine, andrology, psychology and pediatric surgery). We developed an internal grading system for recommendations regarding fertility preservation based on the current recommendations for fertility preservation of leading societies in this field like EBMT, GPOH and DGGG. It is important to find a consensus within the team for the counseling to ensure reliable counseling. The third step is to implement structures needed for fertility counseling and performance of invasive procedures including legal aspects (AMG). A detailed description of this process is given.

Results: After setting up structures for the counseling process we counseled 140 oncology and stem cell transplant patients (0-18 years) between January 2017 and May 2018 . We analysed data of the patients including age subgroups and disease entities and the results of the counseling process. For those patients undergoing stem cell transplantation the risk of gonadotoxicity is very high, therefore even the very young children underwent fertility preserving procedures in alignment with our recommendations if they suffered from a nonmalignant disease. Currently we discourage tissue preserving in malignant systemic disease due to possible contamination with malignant cells. Postpubertal female patients were more likely to undergo invasive procedures such as ovarian tissue cryopreservation in the case of oncological diseases, while sperm cryopreservation was recommended in all postpubertal male patients. Overall a high percentage of the patients and their family followed our recommendations.

Conclusions: Fertility preservation should be considered as a very important part of the treatment plan for newly diagnosed children and young adults with cancer and those facing stem cell transplantation. Unfortunately there is still a great need for setting up structures in institutions taking care of these patients. In addition fertility preservation sadly lacks funding by health insurance in some countries. With the presentation of our experience and data we want to facilitate incorporation of fertility counseling in other pediatric care centers to provide counseling for pediatric patients in need for fertility preservation.

Disclosure: no conflict of interest

\section{P745}

Comparison of tacrolimus combined with methylprednisolone or methotrexate as graft-versushost disease prophylaxis after umbilical cord blood transplantation

Tomonari Shigemura ${ }^{1}$, Kazuo Sakashita ${ }^{2}$, Eri Okura ${ }^{1}$, Daisuke Morital, Kazutoshi Komori ${ }^{2}$, Takashi Kurata ${ }^{2}$, Koichi Hirabayashi, Shoji Saito ${ }^{I}$, Miyuki Tanaka ${ }^{I}$, Ryu Yanagisawa ${ }^{3}$, Yozo Nakazawa

${ }^{1}$ Shinshu University School of Medicine, Matsumoto, Japan, ${ }^{2}$ Nagano Children's Hospital, Azumino, Japan, ${ }^{3}$ Shinshu University Hospital, Matsumoto, Japan

Background: Unrelated umbilical cord blood (UCB) has increasingly being used as an alternative donor stem cell 
source to allogenic BM or PBSC. UCB units are immediately available for transplantation as they are frozen and banked with defined HLA typing and it has an advantage for patients who need urgent transplantation. In addition, a higher degree of HLA mismatch appears to be acceptable with a comparatively lower risk of acute and chronic GVHD. Meanwhile, a higher incidence of engraftment failure, delayed neutrophil and platelet recovery, and posttransplant immune disorders including pre-engraftment immune reactions (PIR) are major problems in unrelated UCBT.

Methods: In our institute, GVHD prophylaxis in UCBT was changed after March 2013. Between January 2007 and March 2013, thirty-two patients received tacrolimus plus methylprednisolone (Tac/mPSL) and between April 2013 and January 2018, thirty-one patients received Tac plus methotrexate (Tac/MTX) for GVHD prophylaxis. To investigate better GVHD prophylaxis after UCBT, we compared transplant outcomes after UCBT using GVHD prophylaxis with Tac/mPSL $(n=32)$ and Tac/MTX $(n=31)$ in single-pediatric transplantation center.

Results: The cumulative incidence of neutrophil engraftment at day 30 in Tac/mPSL group was $70.1 \%$ and $90.3 \%$ in Tac/MTX group $(p=0.09)$. Median time of neutrophil engraftment was 2 days earlier in Tac/MTX group (17 days) than Tac/mPSL group (19 days). According to PIR, and acute GVHD, Tac/MTX group showed superior outcomes; the incidence of PIR $(p=0.020)$ and the cumulative incidences of acute GVHD at day 100 (38.7 vs $68.8 \%, p$ $=0.045$ for grade II-IV, 9.7 vs $34.4 \%, p=0.021$ for grade III-IV) was significantly lower in Tac/MTX group than in Tac/mPSL. However, the incidences of relapse $(p=0.921)$ and cytomegalovirus viremia $(p=0.908)$, and estimated overall survival $(p=0.87)$ and event-free survival $(p=0.88)$ were comparable between two groups.

Conclusions: Our results indicated that GVHD prophylaxis with Tac/MTX had favorable effects; reduced incidence of RIP and acute GVHD after UCBT without any negative influences.

Disclosure: Nothing to declare

\section{P746}

Transfer of donor regulatory T-cellS after ATG reconditioning cures severe refractory GVHD and leads to long term persistence of regulatory $\mathrm{T}$-cellS in the recipient

Britta Lamottke ${ }^{1}$, Fatih Noyan ${ }^{2}$, Lubomir Arseniev ${ }^{3}$, Lilia Goudeva $^{4}$, Christoph Priesner, Marena Rebekka Niewisch $^{1}$, Karl-Walter Sykora ${ }^{1}$, Matthias Edinger ${ }^{5}$, Britta Eiz-Vesper ${ }^{4}$, Martin Sauer ${ }^{1}$, Britta Maecker-Kolhoff ${ }^{I}$
${ }^{1}$ Hannover Medical School, Pediatric Hematology and Oncology, Hannover, Germany, ${ }^{2}$ Hannover Medical School, Gastroenterology, Hepatology and Endocrinology, Hannover, Germany, ${ }^{3}$ Hannover Medical School, Institute of Cellular Therapeutics, Hannover, Germany, ${ }^{4}$ Hannover Medical School, Institute for Transfusion Medicine, Hannover, Germany, ${ }^{5}$ University Hospital Regensburg, Regensburg, Germany

Background: Despite the development of new immunosuppressive drugs graft-versus-host disease (GvHD) remains a major complication of allogeneic hematopoietic stem cell transplantation (SCT). First line therapy generally consists of steroids, second line treatment employs other immunosuppressive drugs, extracorporal photopheresis (ECP) or mesenchymal stem cells (MSC). Newer approaches to treat GvHD include the use of antibodies or small molecules targeting the signaling cascades that are activated during GvHD. Regulatory T-cells $\left(\mathrm{T}_{\text {reg }}\right)$ have been shown to play a role not only in autoimmune diseases and solid organ transplantation but also in GvHD. Several mouse models showed a decrease of GvHD incidence after $\mathrm{T}_{\text {reg }}$ administration. The few clinical trials regarding the application of $\mathrm{T}_{\text {reg }}$ for the treatment of GvHD are encouraging, however the data is limited.

Methods: Patient: A 14-year-old boy underwent allogeneic SCT for chronic myeloid leukemia refractory to imatinib, dasatinib and nilotinib treatment from his 10/10 HLA identical brother. Freshly derived unmanipulated bone marrow was transplanted after conditioning with of fludarabine $\left(40 \mathrm{mg} / \mathrm{m}^{2} / \mathrm{d}\right.$, day -7 to -4$)$, thiotepa $(2 \times 5 \mathrm{mg} /$ $\mathrm{kg}$, day -3) and melphalan $\left(160 \mathrm{mg} / \mathrm{m}^{2}\right.$, day -2). Cyclosporin A (CsA) and mycophenolatmofetil (MMF) were used for GvHD prophylaxis. Leukocyte regeneration $(>1000 / \mu \mathrm{l})$ was seen on day +15 , granulocyte regeneration $(>500 / \mu \mathrm{l})$ on day +16 and thrombocyte regeneration $(>50.000 / \mu \mathrm{l})$ on day +21 . On day +30 after SCT he developed acute intestinal GvHD that exacerbated to grade $\mathrm{IV}^{\circ}$ (bloody diarrhea, ileus) and did neither respond to steroids, nor to different immunosuppressive drugs such as cyclosporin, tacrolimus, sirolimus, mycophenolatemofetil and ruxolitinib. Extracorporal photopheresis and the administration of immunmodulatory antibodies (adalimumab and tocilizumab) did not succeed either.

Results: By administration of low-dose interleukin-2 (IL2) in vivo induction of $T_{\text {reg }}$ was expected but did not succeed. Finally antithymocyte globuline (ATG, $20 \mathrm{mg} / \mathrm{kg} /$ d) was administered on day +150 to +152 to eliminate the GvHD-triggering cells. Hence, the GvHD declined to grade III. Finally, a decision was made to manufacture $T_{\text {reg }}$ from his stem cell donor. From an unstimulated leukapheresis $T_{\text {reg }}$ were selected by magnetic depletion of $\mathrm{CD} 8^{+} \mathrm{T}$-cells and $\mathrm{CD} 19^{+} \mathrm{B}$-cells followed by positive selection of $\mathrm{CD} 25^{+}$ 
cells on a CliniMACS ${ }^{\circledR}$ Plus device (Miltenyi Biotec). The cell product contained $83 \%$ FoxP3 $^{+}$T-cells. The patient received $3,4 \times 10^{5} / \mathrm{kg} \mathrm{T}_{\text {reg }}$ on day +177 . Subsequently intestinal GvHD decreased and finally resolved. Three months after the first $\mathrm{T}_{\text {reg }}$ transfer the patient got a second $\mathrm{T}_{\text {reg }}$ transfer $\left(3,4 \times 10^{5} / \mathrm{kg}\right)$ on day +281 due to decreasing $\mathrm{T}_{\text {reg }}$ levels. Thereafter $\mathrm{T}_{\text {reg }}$ persisted and there was no recurrence of GvHD. The patient is well with low dose sirolimus and prednisone as the only immunosuppressants and is particularly recovering intestinal function.

Conclusions: This case illustrates an unusually severe acute GvHD after matched sibling SCT. Transfer of donorderived $\mathrm{T}_{\text {reg }}$ was able to cure severe and refractory GvHD after T-cell ablation by ATG. Transferred $\mathrm{T}_{\text {reg }}$ persisted in the recipient for a long period and did not lead to any adverse Events.

Disclosure: no disclosures to declaim

\section{P747}

\section{Allogeneic HSCT for patients with transfusion dependent anemia from matched and mismatched} donors

\section{Julia Fekadu ${ }^{1}$, Andrea Jarisch ${ }^{1}$, Jan Sörensen ${ }^{1}$, Emilia Salzmann ${ }^{1}$, Eva Rettinger ${ }^{1}$, André Willasch ${ }^{1}$, Shahrzad Bakhtiar $^{1}$, Thomas Klingebiel ${ }^{I}$, Peter Bader ${ }^{1}$}

\section{${ }^{1}$ Goethe University Medical Center, Frankfurt am Main,} Germany

Background: Treatment of patients with transfusion dependent anemia like thalassemia major (TM), sickle cell disease (SCD) and Diamond-Blackfan anemia (DBA) has improved over the last decades. For the vast majority of patients, allogeneic hematopoietic stem cell transplantation (HSCT) is the only available curative therapy. For a long time, HSCT has only been performed from HLA-identical sibling donors (MSD) or matched family donors (MFD). However, approximately only $25-30 \%$ of affected patients do have a matched sibling donor, therefore HSCT from 10/ 10 (MUD) and even 9/10 (MMUD) matched unrelated donors has gained importance in recent years.

Methods: 36 patients (age range: 2-27 years) with SCD $(\mathrm{n}=7)$, DBA $(\mathrm{n}=6)$ or TM $(\mathrm{n}=23)$, receiving HSCT from a MSD, MFD, MUD or MMUD between 2010 and 2019 were included in our analysis. 23 patients received transplants from MSD/MFD, 13 patients from MUD/ MMUD. 7 patients were identical for HLA-A, B, CW, DRB1 and DQB1, 6 patients shared only $9 / 10$ genes. We analyzed extended haplotypes including DRB3, DRB4, DRB5 and DPB1 for all patients with thalassemia. 7 pairs showed non-permissive DPB1 mismatch and 1 pair mismatch for DPB1 and DRB4.
Results: Median time for granulocyte recovery was 20 days in patients transplanted from MSD/MFD and 22 days in patients transplanted from MUD/MMUD. Platelet recovery was reached after 22 days after HSCT from MSD/MFD and 24 days after HSCT from MUD/ MMUD.

28/36 (80\%) patients showed complete donor chimerism in all controls. 5/36 (13\%) patients showed low level mixed chimerism up to $20 \%$ during follow up. 1 patient died shortly after HSCT, 1 patient showed slowly increasing mixed chimerism and finally developed autologous recovery and one patient rejected the graft.

Cumulative incidence of grade II-IV acute graft-versushost disease (aGVHD) of MUD/MMUD was 15,2\%, whereas only 2 cases of aGVHD grade I occurred in patients transplanted from MSD/MFD.

As 1 patient rejected the graft from a HLA-identical parent, 1 patient transplanted from a HLA-identical grandparent developed autologous recovery after 1 year and 1 patient transplanted from a MUD lost the graft due to hemophagocytosis, the probability of event-free survival was $89,5 \%$ after HSCT from MSD/MFD and 83,9 \% from MUD/MMUD

Altogether $34 / 36$ patients $(94,4 \%)$ are alive and transfusion-independent with complete donor chimerism two years after HSCT; resulting in an overall survival probability of $93,1 \%$. In contrast, overall survival probability was $100 \%$ in the group of patients transplanted from MSD/MFD and 80,1\% in patients transplanted from MUD/ MMUD after 2 years.

There were 2 patients with thalassemia $(6,9 \%)$ who died from transplantation-related causes. The first patient died 13 days after HSCT from a MMUD due to candida sepsis with pulseless electrical activity resulting from cardiac iron overload. The second patient died 5 months after HSCT from a MUD due to graft failure.

Conclusions: HSCT from MUD and MMUD is a feasible therapy option for patients with transfusion dependent anemia. Nevertheless, it should be noted that iron overload can cause severe complications; therefore, measurement of liver and heart iron concentration through MRI prior to HSCT as well as phlebotomy after transplantation are advisable.

Disclosure: Novartis (consultancy: included expert testimony, speaker bureau, Honoraria), Medac (Research Funding, Patents and Royalties), Riemser (Research Funding), Neovii (Research Funding), Amgen (Honoraria)

\section{P748}

Abstract already published.

\section{P749}

Second autologous stem cell transplantation in relapses 
hodgkin's lymphoma after the first ASCT: Preliminary results of one center

\section{Natalya Milanovich ${ }^{1}$}

${ }^{1}$ State Institution "Minsk Scientific and Practical Center of Surgery, Transplantology and Hematology", Minsk, Belarus

Background: The therapeutic options for patients with Hodgkin's disease who relapse after first high-dose chemotherapy with autologous stem cell $\left(1^{\text {st }}\right.$ ASCT $)$ support are limited. Allogeneic stem cell transplantation in this setting is associated with a high level of transplant-dependent mortality rates in excess of 50-65\%. New agent, such as brentuximab vedotin, have been approved for the treatment of these patients, however, their efficacy to provide longterm control or cure is still unknown. A second autologous stem cell ( $2^{\text {nd }}$ ASCT) has historically been considered as an option only in a small group of patients so the published experience is scarce. We report our institution's experience with second autologous transplants in this patient population.

Methods: We evaluated the outcome of 16 adult patients (7 (44\%) female and $9(56 \%)$ male), who received an $2^{\text {nd }}$ ASCT between 10/2013 and 08/2018. Planned tandem ASCT were excluded. The median age at $2^{\text {nd }}$ ASCT was 32 years (range 21-54), 15 (94\%) patients had a Karnofsky performance score $280 \%$. $12(75 \%)$ patients were in complete remission $(\mathrm{CR})$ and $4(25 \%)$ patients were in partial remission (PR) at day 100 after $1^{\text {st }}$ ASCT. Seven (44\%) relapses within 12 months after $1^{\text {st }}$ ASCT. Patients received a median of $1(0-3)$ treatment lines between $1^{\text {st }}$ ASCT and $2^{\text {nd }}$ ASCT. Only $4(25 \%)$ patients received brentuximab vedotin and none of the patients in our series received checkpoint inhibitors as salvage after $1^{\text {st }}$ ASCT. The median interval from $1^{\text {st }}$ ASCT to relapse/progression was12,9 months (range 2,9-133,3). The median interval from relapse/progression to $2^{\text {nd }}$ ASCT was 9,2 months (range 1,4-46,6). All patients received BEAM as the conditioning regimen for $1^{\text {st }}$ ASCT, and BeEAM as the conditioning regimen for $2^{\text {nd }}$ ASCT.

Results: The median time to neutrophil recovery $\left(>0.5 \times 10^{9} / \mathrm{L}\right)$ after $2^{\text {nd }}$ ASCT were 10 days (range 8-17). Best response at day 100 following $2^{\text {nd }}$ ASCT included CR in $12(75 \%)$ patients and PR in $3(19 \%) ; 1(6 \%)$ had stable disease. $3(19 \%)$ patients received brentuximab vedotin and none of the patients received checkpoint inhibitors after $2^{\text {nd }}$ ASCT. $14(87,5 \%)$ patients are currently alive, with a median follow-up 49,8 months (range 4,2-154,2).

2 patient died after $2^{\text {nd }}$ ASCT. Causes of death were HL progression. The 5-year overall survival was $86 \%$.
Conclusions: The second ASCT in patients with a longterm response after the first ASCT may be the optimal therapeutic option, the effectiveness of which can be enhanced by using new drugs, such as brentuximab vedotin, at all stages of treatment.

Disclosure: Nothing to declare

\section{P750}

Effectiveness of chemo-G-CSF protocols for mobilization of peripheral stem cells in patients with non-hodgkin lymphomas and hodgkin disease-single center experience

Ilina Micheva ${ }^{1}$, Stela Dimitrova ${ }^{1}$, Vladimir Gerov ${ }^{1}$, Trifon Chervenkov $^{2}$, Liana Gercheva ${ }^{I}$, Igor Reznik ${ }^{1}$

${ }^{1}$ University Hospital 'Sveta Marina', Clinic of Hematology, Varna, Bulgaria, ${ }^{2}$ University Hospital 'Sveta Marina', Varna, Bulgaria

Background: High-dose chemotherapy and autologous stem cell transplantation (ASCT) play an important role in achieving long-term remission in patients with nonHodgkin lymphoma (NHL) and Hodgkin disease (HD). Granulocyte colony stimulating factor (G-CSF) combined with high-dose chemotherapy is a frequently used mobilization approach; however, the optimal mobilization strategy has not been determined.The objective of the study was to analyze the mobilizing potential of different regimens used for the collection of peripheral stem cells in patients with relapsed or refractory (R/R) NHL and HD.

Methods: We retrospectively analyzed 40 patients with R/R NHL and HD undergoing stem cell collection after chemo-mobilization in the transplant unit at the University Hospital, Varna. Patients were mobilized after DHAP $(n=18)$, ICE $(n=6)$, cyclophosphamide (CY) $(n=7)$, IGEV $(n=3)$, CHOEP $(n=5)$, and 1patient received VTD-PACE. G-CSFwas given at a dose of $10 \mu \mathrm{g} / \mathrm{kg}$ depending on the protocol. The aim was to collect at least $2 \times 10^{6} \mathrm{CD} 34(+)$ cells/kg body weight.

Results: Forty patients, 29 with NHL (18 DLBCL, 6 PTCL, 4 MCL, 2 FL, 1 PBL) and 11 with HD, median age of 44,5 years, 27 male and 13 female, were mobilized between 2016 and 2018. All patients were stage III and IV at diagnosis. 29 of the patients were mobilized after one line of treatment, six after two lines of treatment and five after 3 and more lines of treatment. At the time of mobilization 29 $(72,5 \%)$ were in complete response, $5(12,5 \%)$ were with partial response and $6(15 \%)$ with progressive disease. Overall $36(90 \%)$ patients achievedat least $2 \times 10^{6} \mathrm{CD} 34(+)$ cells $/ \mathrm{kg}$ after 1-3 apheresis with mean harvest of $8,44 \times 10^{6} \%$ $\mathrm{kg}$ (range 2,0-41,5). Fore patients (3 CY and 1DHAP) failed mobilization.Three patients were successfully mobilized 
after first ASCT. The mean harvest for patients receiving DHAP was $10,02 \times 10^{6} \mathrm{CD} 34(+)$ cells $/ \mathrm{kg}, 3,04 \times 10^{6} \mathrm{CD} 34$ $(+)$ cells $/ \mathrm{kg}$ for $\mathrm{CY}, 8.56 \times 10^{6} \mathrm{CD} 34(+)$ cells $/ \mathrm{kg}$ for IGEV, $6,76 \times 10^{6} \mathrm{CD} 34(+)$ cells $/ \mathrm{kg}$ for ICE, $10,52 \times 10^{6} \mathrm{CD} 34(+)$ cells $/ \mathrm{kg}$ for CHOEP. The patient mobilized with VTDPACE achieved $2,2 \times 10^{6} \mathrm{CD} 34(+)$ cells $/ \mathrm{kg}$ after 2 apheresis. 19 of the patients achieved the target number of $>2 \times 10$ ${ }^{6} / \mathrm{kg} \mathrm{CD} 34+$ cells after 1 apheresis, 15 after two, and 2 after three apheresis. The median time to apheresis was 13 days (8-18) without significant difference between the regimens. The mean WBC count at the time of apheresis was $42,8 \times 10$ ${ }^{9} / \mathrm{L}$ after DHAP, $34,5 \times 10^{9} / \mathrm{L}$ after $\mathrm{CY}, 21,2 \times 10^{9} / \mathrm{L}$ after IGEV, $23,1 \times 10^{9} / \mathrm{L}$ after ICE, $45,8 \times 10^{9} / \mathrm{L}$ after CHOEP. There was correlation between WBC and CD34 harvested cells $(p=0.005)$. Grate $3-4$ thrombocytopenia was found in 8 patient (5 DHAP, 1 ICE, 1IGEV, 1 VTD-PACE). Grate 3 4 anemia was registered in 3 patients (2 DHAP and 1 VTDPACE). No correlation was found between the CD $34+$ harvest and the age, number of previous lines chemotherapy, the response before mobilization, the type of the lymphoma and the clinical stage.

Conclusions: Our results demonstrate that the chemo-GCSF protocols have comparable effectiveness with acceptable toxicity and are superior to CY-G-CSF for mobilizing stem cells in lymphoma patients.

\section{Disclosure: no}

\section{P751}

\section{First experience with letermovir in pediatric Haemato-/} Oncologic patients

\section{Daniela Sperl, Klaus Kubesch ${ }^{2}$, Josef Donnerer ${ }^{3}$, Wolfgang Schwinger ${ }^{1}$, Klara Zach ${ }^{1}$, Herwig Lackner ${ }^{1}$, Martin Benesch ${ }^{I}$, Volker Strenger ${ }^{I}$}

${ }^{1}$ Medical University of Graz, Graz, Austria, ${ }^{2}$ pharm-analyt Labor GmbH, Baden, Austria, ${ }^{3}$ Medical University of Graz, Otto Loewi Research Center, Graz, Austria

Background: Cytomegalovirus (CMV) may cause severe complications in recipients of allogeneic haematologic stem cell transplantation (alloHSCT). Letermovir (LTV, 480/ 240mg daily without/with co-administration of Cyclosporine) was recently licenced only for CMV prophylaxis in adult alloHSCT-recipients. Paediatric data as well as data on CMV therapy are missing so far.

Methods: We administered letermovir $240 \mathrm{mg}$ orally once daily (with no co-administration of Cyclosporine A) to 2 paediatric patients after alloHSCT. EDTA-plasma were occasionally obtained at different time points and frozen for determination of letermovir levels using liquid chromatography/mass spectrometry (LC-MS/MS).
Results: For details on patients, treatment and CMV load see table 1. In short periods of letermovir administration, CMV blood levels became negative in both patients. Considering the lacking safety data in paediatric patients, we stopped letermovir treatment in both patients, when liver parameters increased. In patient 1 hepatopathy turned out to represent histologically proven Graft versus Host Disease (GvHD). In patient 2 liver parameters further increased despite withdrawal for another 4 weeks, however, hepatopathy was only mild and self-limiting. Both patients additionally received other possibly hepatotoxic substances (mycophenolate mofetil and trimethoprim/ sulfamethoxazole).

Letermovir plasma levels were $11.900 \mathrm{ng} / \mathrm{mL}$ (2h), $11.600 \mathrm{ng} / \mathrm{mL}$ (12h), $1.640-2.190 \mathrm{ng} / \mathrm{mL}$ (median 1.990ng/ $\mathrm{mL}, \mathrm{n}=3,24 \mathrm{~h}$ ) and $1.050 \mathrm{ng} / \mathrm{mL}$ (36h after administration).

\begin{tabular}{|c|c|c|c|}
\hline & & patient 1 & patient 2 \\
\hline & $\begin{array}{l}\text { age/sex/ } \\
\text { body weight }\end{array}$ & $6 \mathrm{a} / \mathrm{m} / 21 \mathrm{~kg}$ & $11.5 \mathrm{a} / \mathrm{m} / 25 \mathrm{~kg}$ \\
\hline & $\begin{array}{l}\text { underlying } \\
\text { disease }\end{array}$ & $\begin{array}{l}\text { sickle cell } \\
\text { disease }\end{array}$ & relapsed ALL \\
\hline $\begin{array}{l}\text { alloHSCT } \\
\text { (MSD) }\end{array}$ & $\begin{array}{l}\text { CMV serostatus } \\
\text { donor/receipient }\end{array}$ & $+/+$ & $+/+$ \\
\hline \multirow[t]{5}{*}{$\begin{array}{l}\text { Letermovir } \\
\text { (LTV) }\end{array}$} & $\begin{array}{l}\text { reason to switch } \\
\text { GCV to LTV }\end{array}$ & $\begin{array}{l}\text { sickle cell } \\
\text { nephropathy }\end{array}$ & $\begin{array}{l}\text { refractory CMV } \\
\text { viraemia }\end{array}$ \\
\hline & $\begin{array}{l}\text { CMV load } \\
\text { before/at } \\
\text { LTV start }\end{array}$ & $<10^{\wedge} 2 \mathrm{cp} / \mathrm{ml}$ & $>10^{\wedge} 4 \mathrm{cp} / \mathrm{ml}$ \\
\hline & $\begin{array}{l}\text { CMV load at } \\
\text { LTV stop }\end{array}$ & negative & negative \\
\hline & $\begin{array}{l}\text { LTV levels } \\
\text { (hours after } \\
\text { administration) }\end{array}$ & $\begin{array}{l}2.190 \mathrm{ng} / \mathrm{mL} \\
(24 \mathrm{hr}) ; 1.050 \\
\mathrm{ng} / \mathrm{mL}(36 \mathrm{hr})\end{array}$ & $\begin{array}{l}11.900 \mathrm{ng} / \mathrm{mL}(2 \mathrm{hr}) ; \\
11.600(12 \mathrm{hr}) ; 1.940 \\
\text { and } 1.640 \mathrm{ng} / \\
\mathrm{mL}(24 \mathrm{hr})\end{array}$ \\
\hline & $\begin{array}{l}\text { duration LTV } \\
\text { treatment }\end{array}$ & 10 days & 24 days \\
\hline $\begin{array}{l}\text { liver } \\
\text { parameters }\end{array}$ & $\begin{array}{l}\text { AST/ALT/GGT } \\
\text { (U/L)/bilirubin } \\
(\mathrm{mg} / \mathrm{dL}) \text { at } \\
\text { LTV stop }\end{array}$ & $\begin{array}{l}252 / 275 / 63 / \\
0.22\end{array}$ & $74 / 36 / 204 /$ normal \\
\hline
\end{tabular}

[[P751 Table] 1. Table 1. Patient characteristics and details on treatment and liver parameters.]

Conclusions: During short letermovir treatment, we observed fast resolution of CMV viraemia as well as rising liver parameters in both patients. While elevated liver parameters represented GvHD in 1 patient, a causal relationship with letermovir might be considered in the other patient.

Letermovir peak levels after administration of $240 \mathrm{mg}$ were within ranges reported in adults after administration of $480 \mathrm{mg}$ while trough levels were higher indicating differences in pharmacokinetics in terms of delayed clearance. 
Letermovir is promising, even as a therapeutic agent. More paediatric data are urgently needed.

Disclosure: Nothing to declare

\section{P752}

Development of paroxysmal nocturnal hemoglobinuria in a patient after mudallohsct due to JAK2V617Fpositive myelofibrosis-a case of successful treatment with the second transplantation from another donor

Agnieszka Tomaszewskal, Barbara NasitowskaAdamska $^{2}$, Iwona Solarska ${ }^{2}$, Kazimierz Hataburda ${ }^{2}$

${ }^{1}$ Medical University of Warsaw, Hematology, Oncology and Internal Medicine, Warsaw, Poland, ${ }^{2}$ Institute of Hematology and Transfusion Medicine, Warsaw, Poland

Background: Paroxysmal nocturnal hemoglobinuria (PNH) is a very rare disease associated with $P I G-A$ gene mutations in hematopoietic stem cells. There are only single case reports on evolving myeloproliferative diseases to $\mathrm{PNH}$ in the literature. There is no data concerning development of PNH de novo after allogeneic hematopoietic stem cell transplantation. In our report we describe a patient with recurrence of JAK2V617-positive myelofibrosis 5 years after matched unrelated donor allogeneic hematopoietic stem cell transplantation (MUDalloHSCT) with simultaneous development of clinically significant PNH.

Methods: A 51 year-old-man with a history of MUDalloHSCT in May 2011 due to JAK2V617-positive myelofibrosis secondary to essential thrombocythemia was admitted to our department 5 years later with mild anemia $(\mathrm{Hb}-11.0 \mathrm{~g} / \mathrm{dL})$ and elevated lactate dehydrogenase (1400 $\mathrm{U} / \mathrm{L})$. During last 5 years he remained in complete remission of myelofibrosis with JAK2V617 mutation negativisation and $100 \%$ donor chimerism. Suspecting disease recurrence we performed trephine biopsy confirming myelofibrosis (MF2/MF3) with heterozygous JAK2V617 mutation and in flow cytometry analysis of bone marrow we identified cell membrane defect in myeloid line (loss of CD66c). We decided to perform detailed diagnostic tests on PNH - multiparametric flow cytometry of peripheral blood revealed $89 \%$ granulocytes and $15 \%$ red blood cells with loss of GPI-anchored proteins - PNH clone. These results corresponded with donor chimerism - it was only $20 \%$ of donor DNA in bone marrow and $33 \%$ in blood tests. Molecular analysis didn't revealed any mutations in genes: CALR, ASXL1 and MPL. Finally the diagnosis of myelofibrosis recurrence after MUDalloHSCT with presence of PNH clone was established. The therapy with eculizumab was unreachable. So the second alloHSCT from another matched unrelated donor after fludarabinemelphalan-thymoglobuline-TBI $200 \mathrm{cGy}$ conditioning was performed on 21.10.2016. We didn't observe any complications of this procedure, engraftment was slightly delayed: ANC $>0.5 \mathrm{G} / \mathrm{L}$ on the 29 day and PLT $>50 \mathrm{G} / \mathrm{L}$ on the 50 day.

Results: At present, more than 2 years after the second MUDalloPBSCT, the patient remains in a very good condition with $100 \%$ of the second donor chimerism and without any features of PNH (clone is undetectable) and myelofibrosis.

Conclusions: Presented case is the first in the literature well documented myelofibrosis recurrence after MUDalloHSCT with concurrently development of clinically significant paroxysmal nocturnal hemoglobinuria. The second MUDalloHSCT from another donor was safe and successful treatment strategy in this situation.

Disclosure: Nothing to declare.

\section{P753}

\section{Abstract already published.}

\section{P754}

Cutaneous refractory T-cell lymphoma treated with allogeneic hematopoietic stem cell transplantation

Marcia Silva $^{I}$, Ercole Orlando $^{I}$, Maria Claudia Moreira ${ }^{1}$, Simone Lermontov ${ }^{1}$, Simone Maradei ${ }^{1}$, Yung Gonzaga ${ }^{1}$, Leonardo Arcuri' ${ }^{1}$, Renato Araujo ${ }^{I}$, Decio Lerner ${ }^{I}$

${ }^{1}$ Instituto Nacional de Cancer, CEMO, Rio de Janeiro, Brazil

Background: Folliculotropic mycosis fungoides (fMF) is an aggressive clinical course variant of cutaneous T-cell lymphoma (CTCL) - classic mycosis fungoides (MF) ${ }^{1}$, with distinct clinical and pathological characteristics, and it is less responsive to skin-directed therapies. For diseases in advanced stages, chemotherapy, autologous hematopoietic stem cell transplantation (HSCT) or immunomodulator drugs may provide remissions with limited duration and the treatment remains substantially palliative ${ }^{2,3}$. These dismal results have induced to explore the therapeutical approach with allogeneic hematopoietic stem cell transplantation (HSCT) in such patients. Early studies have shown encouraging results also in patients with advanced disease, suggesting a major therapeutical role played by the graft versus lymphoma (GVL) effect ${ }^{4,5,6}$.

Methods: This is a case report of the use of allogeneic HSCT as a potential cure for cutaneous refractory T-cell lymphoma type folliculotropic mycosis fungoides .

Results: Case presentation : A 31-year-old male patient with refractory subtype B fMF T-cell lymphoma ${ }^{7}$, diagnosed in 2007, clinically characterized by exfoliative erythroderma, widespread plaques on the trunk and limbs, solitary tumor on the right shoulder, pruritus and bilateral 
inguinal lymphadenomegaly. After failure to respond to seven conventional treatment lines: methotrexate, COP (cyclophosphamide, vincristin and prednisone); gemcitabine; PUVA; interferon; acitretin and extracorporeal photopheresis), allogeneic HSCT from an identical HLA male donor was indicated. The non-myeloablative conditioning consisted of fludarabine (200mg / m2), cyclophosphamide (50mg / kg) and total body irradiation(TBI) (400cGy). Prophylaxis of graft versus host disease (GVHD) was performed with cyclosporine $(3 \mathrm{mg} / \mathrm{kg}$ ) and mycophenolate mofetil $(30 \mathrm{mg} / \mathrm{kg})$. After conditioning, there was improvement of pruritus and involution of the skin. Bone marrow infusion occurred on 2/9/2018 (D0). On $d+83$ he presented recurrence of skin lesions of fMF. Donor lymphocyte infusion (DLI) was performed $\left(1 \times 10^{7} \mathrm{CD} 3\right.$ + cells / kg / recipient). He presented oral lichen and diarrhea respectively as manifestations of GVHD on $d+$ 104 and D + 112. As infectious intercurrence, hemorrhagic cystitis occurred by BK virus 3 months after the first DLI and he received conservative treatment and remained without systemic immunosuppression. Nine months after HSCT, a second DLI ( $5 \times 10^{7} \mathrm{CD} 3$ + cells / Kg / receptor $)$ was performed and at this time the patient is without clinical manifestations of fMF or GVHD.

Conclusions: the clinical response of the presented case confirms what has been reported in the literature. CTCLs appear to be particularly susceptible to GVL effect, which makes HSCT a potential cure for advanced CTCLs in eligible patients. The timing to perform HSCT in the clinical course of the disease remains a matter to be settled

Clinical Trial Registry: not applicable

Disclosure: no conflict of interest

\section{P755}

Abstract already published. 